\title{
61st Annual Conference of Indian Society of Hematology \& Blood Transfusion (ISHBT) November 2020
}

\section{Contents}

Oral Abstract Papers

1. Acute Leukemia (Clinical) ...

2. Acute Leukemia (Laboratory)

3. Anemia Including Hemolytic Anemia

Bleeding Disorders

5. Bone Marrow Failure and MDS (Clinical)

Bone Marrow Failure and MDS (Laboratory)

Infections and Support Care

Lymphoma and Myeloma (Clinical)

Lymphoma and Myeloma (Laboratory) .....

Miscellaneous (Laboratory Haematology)...

11. Myeloproliferative Neoplasm

12. Stem Cell Transplantation

13. Thrombosis

14. Transfusion Medicine.

Poster Abstract Papers

1. Acute Leukemia (Clinical)

2. Acute Leukemia (Laboratory)

ORAL Submission.

Lymphoma and Myeloma (Laboratory) (ORAL-667)

\section{Oral Abstract Papers}

\section{Acute Leukemia (Clinical)}

\section{Prognostic Value of Measurable Residual Disease After} Venetoclax and Decitabine in Acute Myeloid Leukemia

Abhishek Maiti ${ }^{* 1}$, Courtney DiNardo ${ }^{1}$, Sa Wang ${ }^{2}$, Jeffrey Jorgensen ${ }^{2}$, Tapan Kadia ${ }^{1}$, Naval Daver ${ }^{1}$, Nicholas Short ${ }^{1}$, Musa Yilmaz ${ }^{1}$, Naveen Pemmaraju $^{1}$, Gautam Borthakur ${ }^{1}$, Prithviraj Bose ${ }^{1}$, Ghayas C. Issa ${ }^{1}$, Alessandra Ferrajoli ${ }^{2}$, Elias J Jabbour ${ }^{2}$, Nitin Jain ${ }^{2}$, Guillermo GarciaManero $^{2}$, Maro Ohanian ${ }^{2}$, Koichi Takahashi ${ }^{2}$, Guillermo MontalbanBravo $^{2}$, Lucia Masarova ${ }^{2}$, Jan Burger ${ }^{2}$, Philip A. Thompson ${ }^{2}$, Srdan Verstovsek $^{2}$, Koji Sasaki ${ }^{2}$, Michael Andreeff ${ }^{2}$, Caitlin R. Rausch ${ }^{2}$, Kathryn S. Montalbano ${ }^{2}$, Sherry Pierce ${ }^{2}$, Hagop Kantarjian ${ }^{1}$, Marina Konopleva ${ }^{1}$, Farhad Ravandi ${ }^{1}$

\author{
${ }^{1}$ Department of Leukemia, ${ }^{2}$ The University of Texas MD \\ Anderson Cancer Center, Houston, United States
}

Aims \& Objectives: Measurable residual disease (MRD) has prognostic utility in acute myeloid leukemia (AML). However, the value of MRD after lower-intensity regimens is less established. We sought to evaluate the prognostic value of MRD after achievement of response with 10-day decitabine and venetoclax (DEC10-VEN).

Patients/Materials \& Methods: We conducted a post hoc analysis of a phase 2 trial to DEC-10-VEN (NCT03404193). MRD was assessed on bone marrow (BM) specimens using 8-color multiparametric flow cytometry (FCM) validated to a sensitivity level of $0.01-0.1 \%$. Newly diagnosed patients with AML were treated with decitabine $20 \mathrm{mg} / \mathrm{m} 2$ for 10-days for induction followed by decitabine for 5-days after CR/ CRi. Venetoclax dose was $400 \mathrm{mg}$ daily or equivalent. Response and relapse were defined using the ELN 2017 criteria. Overall survival (OS) was determined from start of treatment until death or censored at last follow-up (LFU).

Relapse-free survival (RFS) was determined from date of CR/CRi till morphologic relapse, death, or censored at LFU or stem-cell transplantation.

Results: 97 newly diagnosed patients with a response were included in this analysis. Median age was 72 years (interquartile range [IQR] $68-78$ ), 52\% patients had de novo AML, and $64 \%$ patients had adverse-risk AML. $61 \%$ patients had achieved a CR, median time to response was $1.4 \mathrm{mo}$ (IQR 1.1-2.6) and median time to negative MRD was 2.0 months (IQR 0.9-3.1). Patients achieving CR MRDnegative had longer OS compared to those with an inferior response (median OS 25.1 vs. 11.6 months, hazard ratio $[\mathrm{HR}]=0.33$, 95\% CI $0.19-0.58, p<.0005)$. Patients becoming MRD-negative within 1-month had improved OS compared to MRD- positive patients (median OS 25.1 vs. 3.4 months, HR $=0.15$, 95\%CI $0.03-0.64$, $p<.0001)$. Patients becoming MRD-negative by 2 - months had improved OS compared to those remaining MRD-positive (median OS 25.1 vs 7.1 months, HR $=0.24,95 \%$ CI $0.12-0.47, p<.001$ ). Attainment of negative MRD at 2 months after starting therapy conferred similar improvement in RFS (median not reached vs 5.2 mo, $\mathrm{HR}=0.30,95 \%$ CI $0.12-0.78, p<.01$ ). Multivariable analysis showed that OS was independently associated with MRD- status.

Discussion \& Conclusion: MRD-negative status assessed by FCM is associated with improved OS in older/'unfit' patients with AML receiving first-line therapy with DEC10-VEN. 


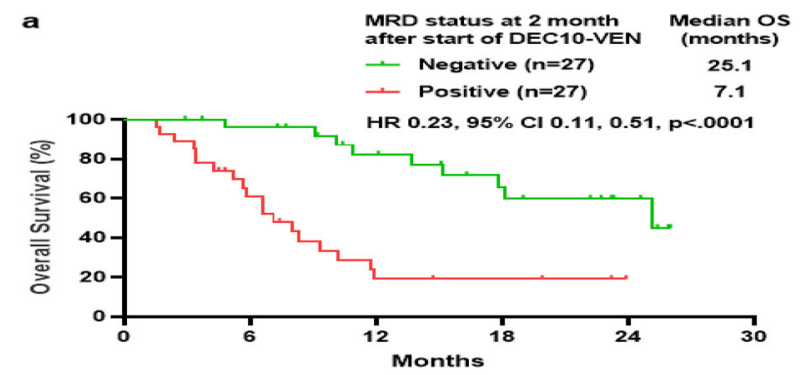

b

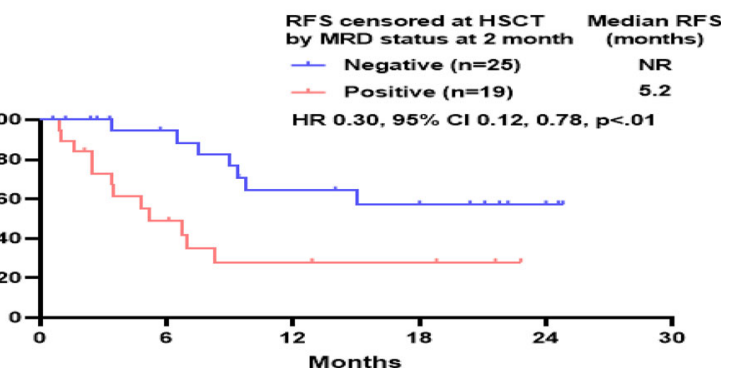

Disclosure of Interest: A. Maiti: None Declared, C. DiNardo: None Declared, S. Wang: None Declared, J. Jorgensen: None Declared, T. Kadia: None Declared, N. Daver: None Declared, N. Short Research Grant from: Division of Cancer Medicine, The University of Texas MD Anderson Cancer Center, M. Yilmaz: None Declared, N. Pemmaraju: None Declared, G. Borthakur Research Grant from: University of Texas MD Anderson Cancer Center, Department of Leukemia, P. Bose: None Declared, G. C. Issa: None Declared, A. Ferrajoli: None Declared, E. J. Jabbour: None Declared, N. Jain: None Declared, G. Garcia-Manero: None Declared, M. Ohanian: None Declared, K. Takahashi: None Declared, G. Montalban-Bravo: None Declared, L. Masarova: None Declared, J. Burger: None Declared, P. A. Thompson: None Declared, S. Verstovsek: None Declared, K. Sasaki: None Declared, M. Andreeff: None Declared, C. R. Rausch: None Declared, K. S. Montalbano: None Declared, S. Pierce: None Declared, H. Kantarjian Research Grant from: The University of Texas MD Anderson Cancer Center, M. Konopleva Research Grant from: National Institutes of Health, National Cancer Institute, F. Ravandi: None Declared.

\section{Ten-Day Decitabine with Venetoclax Versus Intensive Chemotherapy in Relapsed or Refractory Acute Myeloid Leukemia: A Propensity Score Matched Analysis}

Abhishek Maiti ${ }^{* 1}$, Courtney DiNardo ${ }^{1}$, Tapan Kadia ${ }^{1}$, Elias Jabbour $^{1}$, Caitlin Rausch ${ }^{2}$, Naval Daver ${ }^{1}$, Gautam Borthakur ${ }^{1}$, Naveen Pemmaraju ${ }^{1}$, Nicholas Short ${ }^{1}$, Musa Yilmaz ${ }^{1}$, Yesid Alvarado $^{1}$, Kathryn S. Montalbano ${ }^{1}$, Allison Wade ${ }^{2}$, Rita E. Maduike $^{2}$, Julio A. Guerrero ${ }^{2}$, Kenneth Vaughan ${ }^{2}$, Carol A. Bivins ${ }^{3}$, Sherry Pierce ${ }^{2}$, Farhad Ravandi ${ }^{1}$, Hagop Kantarjian ${ }^{1}$, Marina Konopleva ${ }^{1}$

${ }^{1}$ Department of Leukemia, ${ }^{2}$ The University of Texas MD Anderson Cancer Center, ${ }^{3}$ The University of Texas MD Anderson Cancer Center leukemia@mdanderson.org, Houston, United States

Aims \& Objectives: Relapsed/refractory (R/R) acute myeloid leukemia (AML) has poor outcomes and no standard therapy in the absence of actionable mutations. Lower-intensity venetoclax- containing regimens are now standard for older/unfit patients with newly diagnosed AML. However, it is unknown how such regimens compare to intensive chemotherapy (IC) for R/R AML. We compared outcomes of R/R AML treated with venetoclax and decitabine versus IC.

Patients/Materials \& Methods: Venetoclax cohort received decitabine $20 \mathrm{mg} / \mathrm{m} 2$ daily for 10-days for induction and 5-days for consolidation, with venetoclax $400 \mathrm{mg}$ daily or equivalent on a phase II trial (DEC10-VEN, NCT03404193). IC cohort received idarubicin $10 \mathrm{mg} / \mathrm{m} 2 /$ day for 3 -days and cytarabine $1-2 \mathrm{~g} / \mathrm{m} 2 /$ day for 5 -days with either cladribine (CLIA), clofarabine (CIA), or fludarabine (FIA) on phase Ib/II trials (Kadia. ASH 2015 Abstr\# 2541, Short. Leuk Lymphoma 2018). Propensity scores derived from baseline characteristics of age, ECOG PS, ELN 2017 risk group, and number of prior therapies, were used to match DEC10-VEN and IC patients to minimize bias.

Results: 54/55 patients treated with DEC10-VEN were matched to 54/197 patients treated with IC. Median age of the DEC10-VEN cohort was 62 years and baseline characteristics were well balanced between the two groups (Table 1). In IC cohort, CLIA, CIA and FIA were administered in 26, 20 and 8 patients, respectively. Median follow-up for the DEC10-VEN and IC cohorts were 12.5 and 22.5 months, respectively. Overall response rate was significantly higher with DEC10-VEN versus IC $(59 \%$ vs $31 \%$, odds ratio [OR] $3.17,95 \%$ CI $1.44-6.98, p=.23$, Table 1 ) and rate of refractory disease was significantly lower with DEC10-VEN versus IC (28\% vs $54 \%$, OR $0.33,95 \%$ CI $0.15-0.74, p<.01)$. 30-day mortality with DEC10-VEN and IC were 7\% and 9\%, respectively. Median overall survival with DEC10-VEN was comparable to IC (7.1 vs 5.5 month, hazard ratio [HR] $0.72,95 \% \mathrm{CI} 0.46-1.14, p=.16$ ). The median OS after stem-cell transplantation was comparable in DEC10-VEN $(\mathrm{n}=10)$ and IC cohort $(\mathrm{n}=8 ; 19.3$ vs 13.5 month, HR $1.21,95 \% \mathrm{CI}$ $0.35-4.22, p=.1)$.

Discussion \& Conclusion: DEC10-VEN represents an appropriate salvage therapy comparable to IC regimens without venetoclax in $\mathrm{R} / \mathrm{R}$ AML and can serve as a bridge to SCT, and for adding novel therapies in the R/R setting.

Supporting Document:

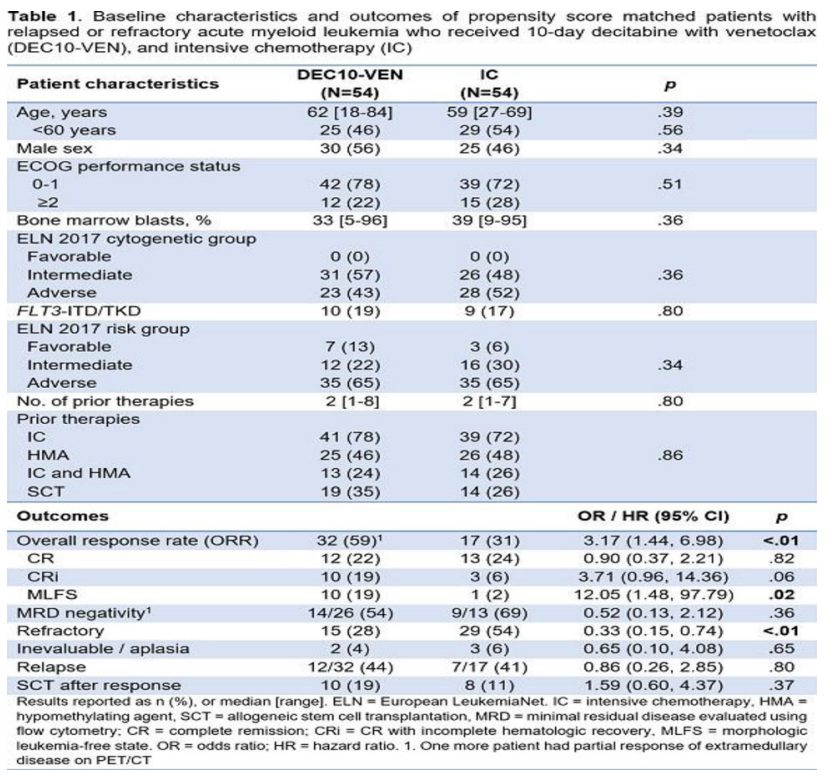

Disclosure of Interest: A. Maiti: None Declared, C. DiNardo: None Declared, T. Kadia: None Declared, E. Jabbour: None Declared, C. Rausch: None Declared, N. Daver: None Declared, G. Borthakur: None Declared, N. Pemmaraju: None Declared, N. Short: None 
Declared, M. Yilmaz: None Declared, Y. Alvarado: None Declared, K. Montalbano: None Declared, A. Wade: None Declared, R. E. Maduike: None Declared, J. A. Guerrero: None Declared, K. Vaughan: None Declared, C. A. Bivins: None Declared, S. Pierce: None Declared, F. Ravandi: None Declared, H. Kantarjian: None Declared, M. Konopleva But No Conflict with: National Institutes of Health, National Cancer Institute, Research Grant from: National Institutes of Health, National Cancer Institute.

\section{Acute Leukemia (Laboratory)}

\section{A Cost-Effective Strategy to Detect BCR-ABL1 Like Acute Lymphoblastic Leukemia (ALL) Using RQ-PCR}

Dikshat G. Gupta ${ }^{* 1}$, Neelam Varma ${ }^{1}$, Shano Naseem ${ }^{1}$, Man Updesh S. Sachdeva ${ }^{1}$, Pankaj Malhotra ${ }^{2}$, Sreejesh Sreedharanunni ${ }^{1}$, Parveen Bose $^{1}$, Minakshi Gupta ${ }^{1}$, Jogeshwar Binota ${ }^{1}$, Palak Rana ${ }^{1}$, Preeti Sonam ${ }^{1}$, Subhash Varma ${ }^{2}$

${ }^{1}$ Hematology, ${ }^{2}$ Internal Medicine, Post Graduate Institute of Medical Education \& Research (PGIMER), Chandigarh, India

Aims \& Objectives: A new provisional entity of "B-lymphoblastic leukemia/lymphoma, BCR-ABL1-like" was introduced in 2016 revised WHO classification. BCR-ABL1-like cases are negative for $\mathrm{Ph}$ chromosome, fusion BCR-ABL1 RNA transcripts and resultant proteins. Their gene expression profile is similar to BCR-ABL1positive ALL. Different approaches are used for detection of BCRABL1-like cases including Gene expression profiling (GEP) or Nextgeneration sequencing (NGS) and TLDA (TaqMan low- density array). TLDA is not commercially available in India. NGS is very labour-intensive and requires sophisticated bioinformatics for analysis. In order to detect BCR-ABL1-like cases, authors describe a cost effective strategy using RQ-PCR to study the expression of 8 selected genes.

Patients/Materials \& Methods: Flowcytometric immunophenotyping and multiplex RT-PCR were performed on 350 B-ALL cases, as part of diagnostic work-up. Further, 12 BCR-ABL1-positive cases were subjected to transcriptome profiling using Affymetrix microarray; then a targeted panel of 50 differentially expressed genes was constructed using nCounter Platform, according to our microarray findings and previously published data. Further using penalised regression, 15 genes were selected. A total of 96 B-ALL cases (16 BCR-ABL1-positive cases and 80 BCR-ABL1-negative cases) were subjected to GEP using nCounter Platform. Finally, 8 genes were selected whose performance was individually analysed with maximum sensitivity and specificity, and quantified the relative gene expression in 216 B-ALL cases (108 BCR-ABL1-positive and 108 BCR-ABL1-negative cases) and were then validated by RQ-PCR.

Results: BCR-ABL1 chimeric fusion transcripts were positive in $30.85 \%(108 / 350)$ cases. Out of 78 ALL patients, 33 were BCRABL1-like ALL, using 15 genes in nCounter Nanostring technology. Out of 108 examined BCR-ABL1-negative cases, 36 were clustered together with 108 BCR-ABL1-positive cases using hierarchical clustering and principal component analysis approach and were categorised as BCR-ABL1-like ALL cases using RQ-PCR. Finally, we detected BCR-ABL1-like ALL cases using 2 different platforms, for the first time in India.

Discussion \& Conclusion: We have devised a simple RQ-PCR method using 8 selected genes for the detection of BCR-ABL1-like ALL cases. This RQ-PCR based approach is very simple, efficient, cost-effective and without any multistep approach. It is very important to detect BCR-ABL1-like ALL cases, so as to provide them the benefit of desirable TKI therapy.
Epigenetic Analysis in a Cohort of Relapsed Pediatric B-Acute Lymphoblastic Leukemia Cases

Prateek Bhatia ${ }^{* 1}$, Minu Singh ${ }^{1}$, Aditya Singh ${ }^{1}$, Pankaj Sharma ${ }^{1}$, Amita Trehan ${ }^{1}$, Neelam Varma ${ }^{2}$

${ }^{1}$ PHO Unit, Pediatrics, ${ }^{2}$ Hematology, PGIMER, Chandigarh, India

Aims \& Objectives: Epigenetic changes play a major role in mediating chemo resistance and relapse in pediatric ALL and hence in current pilot study, we tried to identify DNA methylation, miRNA expression and copy number variations (CNVs) in a cohort of relapse pediatric B-ALL cases.

Patients/Materials \& Methods: DNA methylation, miRNA expression and $\mathrm{CNV}$ analysis was performed in a total of 14,16 and 18 cases as diagnosis-relapse samples. Briefly, DNA methylation was performed using Infinium HumanMethylation850 chip and data analysed using RnBeads. miRNA were sequenced on illumina NextSeq500 platform for $20 \mathrm{M} 75 \mathrm{bp}$ SE reads and analysed by DESeq. CNVs were assessed by MLPA assay using the ALL P-335 probemix kit and analysed by coffalyzer.net.

Results: On methylation analysis, oncogenes MYCN, MYB, and ERBB1 were found hypomethylated (overexpressed) while tumour suppressor gene MDM4 \& BCL11B hypermethylated at relapse. miRNA analysis revealed significant differential expression of miR$128-2-5 p$ and miR-378C (p-4.4e-15 and p-6.4E-12) in relapse samples (Fig $1 \mathrm{a}-\mathrm{b}$ ). CNV analysis revealed frequency of good and intermediate/poor risk CNV profile at diagnosis is nearly equal (40\% vs. $60 \%$ ). However, CDKN2A/2B and IKZF1 gene CNVs if present in initial diagnostic clone usually persist in relapse clone.

Discussion \& Conclusion: Our pilot study is the first to highlight two pro-oncogenic miRNAs (miR-128-2-5p and miR-378C) as novel candidate biomarkers of relapsed B-ALL. However, a significant methylation specific signature could not be identified in relapsed B-ALL and our data results need validation in a larger series of cohort along with correlation of miRNA markers with clinical and disease outcome parameters.

\section{Blast Size Specific Flowcytometric Ploidy Assessment and Its Correlation with Cytogenetic Ploidy}

Karthik Bommannan*1, Jhansi Rani Arumugam ${ }^{1}$, Teena Koshy ${ }^{1}$, Venkatraman Radhakrishnan ${ }^{2}$, Tenali Gnana Sagar ${ }^{2}$, Shirley Sundersingh ${ }^{1}$

${ }^{1}$ Oncopathology, ${ }^{2}$ Medical Oncology, Cancer Institute (W.I.A.), Adyar, Chennai, India

Aims \& Objectives: Numerical chromosomal abnormalities (aneuploidies), present in $\sim 30-50 \%$ of pediatric precursor B-lineage acute lymphoblastic leukemia (B-ALL) patients are commonly identified through a laborious conventional cytogenetic (CG) technique.

Flow cytometry (FCM) can identify both physical and fluorescent properties of cells together, and by using fluorescent nucleic-acid binding dyes, FCM can identify variations in the total nucleic acid content of cells. FxCycle Violet dye (FxCV) is a selective DNA binding dye that permits simultaneous multiparametric immunophenotyping and cell-cycle/ploidy assessment in a single assay. In this study, we tried to analyze the correlation between the DNA content and size of B-lymphoblasts measured by flow cytometric scatter properties. We also tried to develop a novel FCM gating strategy using blast size as a guide to refine the results of FCM ploidy analysis. Patients/Materials \& Methods: Blast size-specific FCM-ploidy was prospectively analyzed using FxCV-dye in 109 pediatric B-ALL 
patients and the results were compared with their concurrent CGploidy status. Size of the blast cells was compared against the size of the lymphoid cells (internal control) present within the sample. BSI was calculated as the ratio between forward scatter geometric mean (FSC-GM) of blast-peak and the FSC-GM of lymphoid cells. DI was calculated as the ratio between GM of FxCV expression in G0/G1 peak of blasts and lymphoid cells.

Results: FCM-ploidy categorization was feasible in $98 \%$ of samples tested and the results were $82 \%$ concordant with CG-ploidy status. We observed significant correlation between DNA content \& blast size $(r=0.823, p<0.001)$, and could demonstrate size differences between diploid vs low-hyperdiploid $(p=0.025)$, diploid vs highhyperdiploid $(p<0.001)$ and low vs high-hyperdiploid blasts $(p=0.007)$. Based on our observations, we speculate that FCMploidy is very sensitive to identify changes in DNA content resulting from large chromosomal aneuploidies. However, it may not be sensitive enough to pick up isolated small-sized chromosomal aneuploidies, especially for chromosome 21 .

Discussion \& Conclusion: FCM-ploidy assessment using FxCV dye is a reliable assay and the results closely concur with CG based ploidy stratification and risk assessment. Using blast size specific DNA content analysis, the results of FCM-ploidy analysis can be further fine-tuned.

\section{Cytogenetic Profile of Indian Patients with pH-Like Acute Lymphoblastic Leukemia}

Purvi Mohanty ${ }^{* 1}$, Dhanlaxmi Shetty ${ }^{1}$, Hemani Jain ${ }^{1}$, Kruti Chaubal ${ }^{1}$, Papagudi Subramanian ${ }^{2}$, Prashant Tembhare ${ }^{2}$, Nikhil Patkar ${ }^{2}$, Nirmalya Moulik ${ }^{3}$, Chetan Dhamne ${ }^{3}$, Hasmukh Jain ${ }^{3}$, Bhausaheb $\mathrm{Bagal}^{3}$, Gaurav Narula ${ }^{3}$, Manju Sengar ${ }^{3}$, Navin Khattry ${ }^{4}$, Shripad Banavali $^{3}$

${ }^{1}$ Dept. of Cancer Cytogenetics, ${ }^{2}$ Dept of Haematopathology, ACTREC, Tata Memorial Center, Navi Mumbai, ${ }^{3}$ Dept. of Medical Oncology, Tata Memorial Hospital, Mumbai, 4Dept. of Medical Oncology, ACTREC, Tata Memorial Center, Navi Mumbai, India

Aims \& Objectives: To determine the prevalence and impact on outcome of cytogenetic alterations in genes involved in kinase and cytokine receptor signaling (ABL1, ABL2, CSF1R, PDGFR-B and CRLF2) in newly diagnosed adult and paediatric B Cell PrecursorALL patients.

Patients/Materials \& Methods: The study cohort included 400 newly diagnosed BCP-ALL (B-NEG) negative for standard BCPALL markers (BCR/ABL1, TCF3/PBX1, ETV6/RUNX1, MLL rearrangements, high hyperdiploidy and hypodiploidy) referred to the Department of Cancer Cytogenetics, ACTREC from June 2018- July 2020 for cytogenetic testing. Fluorescence in situ hybridization (FISH) using breakapart probe was performed to detect ABL1, ABL2, CSF1R, PDGFR-B and CRLF2 gene rearrangements. Post- induction minimal residual disease (MRD) was assessed and Targeted RNA sequencing was carried out to detect fusion partners and unidentified gene fusions.

Results: The median age at diagnosis of our study cohort was $11 \mathrm{yrs}$. Age group wise distribution of 400 B-NEG patients was 190 $(47.5 \%)<10$ yrs, 58 (14.5\%) 10-15 yrs, 110 (27.5\%) 16-39 yrs (adolescents and young adults) and $42(10.5 \%)>39$ yrs of age. Of 400 patients, $6.3 \%$ patients $(25 / 400)$ harbored rearrangements in genes involved kinase and cytokine receptor signaling. 16 patients harbored kinase alterations i.e. ABL1 $(n=2)$, ABL2 $(n=1), \operatorname{CSF} 1 R$ $(n=4)$ and PDGFR-B $(n=9)$ rearrangements and CRLF2 gene rearrangement involved in cytokine signaling was detected 9 cases. $\mathrm{Ph}$ like anomalies were frequently detected in paediatric patients $(\mathrm{n}=18)$ as compared to adult patients $(\mathrm{n}=7)$. Fusion partners identified were ETV6/ABL1, FOXP1/ABL1, EBF1/PDGFRB $(n=3)$, LDB1/CSF1R ( $n=1)$, CRLF2-IGH $(n=3)$, CRLF2-P2RY8 $(n=6)$. Post-induction MRD was positive in cases with EBF1/PDGFRB, CRLF2-IGH, FOXP1/ABL1, LDB1/CSF1R fusion while CRLF2P2RY8 patients showed no evidence of minimal residual disease.

Discussion \& Conclusion: Philadelphia chromosome-like acute lymphoblastic leukemia ( $\mathrm{Ph}$-like ALL) is a newly identified high-risk B-lineage ALL subtype defined on the basis of a gene expression profile that is similar to Ph-positive ALL but lacking BCR-ABL1 fusion. The incidence (15-30\%) and spectrum of genetic alterations of Ph-like ALL differs by geographic location and age. Our study was comparable to Asian cohort studies while contrasting studies from Western population showing an overall lower incidence of genetic alterations reinstating the need for Ph-like status evaluation through whole genomic approach.

\section{Organochlorine Pesticide Residues in Bone Marrow and Sera of Children with Hematological Disorders}

Rachit Khandelwal ${ }^{* 1}$, Pooja Dewan ${ }^{2}$, Sunil Gomber ${ }^{3}$, Mrinalini Kotru $^{4}$, Basu Dev Banerjee ${ }^{5}$, Harendra Shah ${ }^{5}$

${ }^{1}$ Pediatrics, UCMS and GTB hospital dilshad garden, new delhi 110095, Delhi, ${ }^{2}$ Pediatrics, UCMS and GTB hospital dilshad garden, new delhi 110095, Delhi, Delhi, India, ${ }^{3}$ Pediatrics, ucms and gtbh dilshad garden new delhi, delhi, ${ }^{4}$ Pathology,

${ }^{5}$ Biochemistry, UCMS and GTB hospital dilshad garden, new delhi 110095, Delhi, Delhi, India, India

Aims \& Objectives: Primary: To compare the Organochlorine Pesticide (OCP) residues in the sera of children with malignant and nonmalignant hematological disorders (HD) with those in healthy controls. Secondary: (1) To compare the OCP residues in the bone marrow of children with malignant and non-malignant HD. (2) To correlate the OCP residues in serum with that in bone marrow.

Patients/Materials \& Methods: Children $<12$ year with (1) nonmalignant HD ( $\mathrm{n}=30)$; (2) malignant HD ( $\mathrm{n}=30)$; and (3) healthy controls $(n=30)$. Exclusion criteria: Inherited HD (i.e. thalassemia, hemophilia), nutritional HD (like iron deficiency anemia), gross congenital malformations, known chronic diseases (like chronic renal or hepatic disease).Two $\mathrm{ml}$ of peripheral venous blood sample of 90 participants in 3 groups and 48 samples of bone marrow aspirate (30 malignant HD and 18 benign HD) was drawn and stored in EDTA vial at -20 . OCPs were estimated in the stored samples by gas-liquid chromatography.

Results: Serum total OCP concentration was highest in non-malignant HD group (17.29 \pm 3.5$) \mathrm{ng} / \mathrm{dl}$ followed by malignant HD group $(14.47 \pm 3.48) \mathrm{ng} / \mathrm{dl}$ and healthy control $(14.28 \pm 2.42) \mathrm{ng} / \mathrm{dl}$; it was significant between healthy and diseased HD group $(p<0.01)$. Compared to malignant HD group, sera of non-malignant HD group had significantly higher levels (ng/dl) of $\beta$ hexa- chlorohexane $(\beta$ $\mathrm{HCH})[0.53( \pm 0.48)$ Vs $1.15( \pm 0.66) ; p<0.01]$, total $\mathrm{HCH}$ $[2.29( \pm 1.14)$ Vs $3.51( \pm 1.37), p<0.01]$, methoxychlor $[0.80$ $( \pm 0.59)$ Vs $1.46( \pm 1.21) ; p=0.01]$ and total OCPs $[14.47( \pm 3.48)$ Vs $17.29( \pm 3.5) ; p=<0.01]$. Serum heptachlor levels $(\mathrm{ng} / \mathrm{ml})$ were significantly higher in the malignant HD group compared to nonmalignant HD group $[1.34( \pm 1.21) \mathrm{Vs} 1.13( \pm 0.82) \mathrm{ng} / \mathrm{dl}, p=0.02$; OR 20.32 (95\% CI 2.41-171.26); $p=0.01$ ]. No significant correlation was seen between OCP concentration in bone marrow and serum. Discussion \& Conclusion: OCP exposure seems to predispose children to an altered milieu in bone marrow prompting an association between OCP exposure and hematological disease. The link seems to be greater for non-malignant HD as the levels of OCP in sera and bone marrow aspirates was higher in the non-malignant HD group 
compared to malignant HD group. studies have assessed the relationship between parental pesticide or residential exposure and childhood leukemias. Most of these studies are questionnaire based and limited by factors like quality control of self-reports, varied geographic information studies and retrospective in design. Very few studies have estimated the level of pesticides in the blood or bone marrow aspiates. This study was the first of its kind where bone marrow samples of children have been subjected to analysis for pesticide residues levels along with serum samples. Another strength is assessment of exposure to pesticides by measuring the levels in sera or bone marrow aspirate.

\section{Anemia Including Hemolytic Anemia}

\section{Association of HLA Haplotypes and Clinical Heterogeneity in HBE/Beta-Thalassemia}

Motiur Rahaman ${ }^{1}$, Manisha Jain ${ }^{2}$, Gayatri Mukherjee ${ }^{1}$, Praphulla C. Shukla ${ }^{1}$, Prantar Chakrabarti ${ }^{3}$, Tuphan K. Dolai ${ }^{2}$, Nishant Chakravorty $^{* 1}$

${ }^{1}$ School of Medical Science and Technology, Indian Institute of Technology Kharagpur, Kharagpur, ${ }^{2}$ Department of Hematology, Nil Ratan Sircar Medical College and Hospital, ${ }^{3}$ Department of Hematology, Vivekananda Institute of Medical Sciences, Kolkata, India

Aims \& Objectives: HbE/beta-thalassemia is a specific type of betahemoglobinopathy that is remarkable due to its clinical heterogeneity. While in some patients it may present as a mild or asymptomatic condition requiring occasional or no blood transfusion; in others it may present as a life-threatening condition requiring regular and frequent blood transfusions. Although several genetic and environmental factors have been suggested to influence the severity of the disease, there are no specific markers to predict its clinical heterogeneity, thus making it difficult to manage and prognosticate the disease. This study aimed at correlating this clinical heterogeneity with HLA haplotypes.

Patients/Materials \& Methods: The study was approved by Institutional Ethics Committees of IIT Kharagpur and NRS Medical College. Blood samples were collected from patients with $\mathrm{HbE} / \mathrm{beta}-$ thalassemia (2-60 years) and their demographic parameters and medical records were evaluated. The patients were stratified into three different severity groups using Mahidol scoring. Their biochemical data like serum albumin and Alkaline phosphatase (ALP) levels were evaluated. Subsequently, high-resolution NGS- based (Illumina platform) HLA typing of 5 loci (A, B, C, DRB1 and DQB1) was performed and correlated with the disease severity groups.

Results: We have collected blood samples from $\mathrm{HbE} / \mathrm{beta}$-thalassemia patients (age: $19.53 \pm 11.45$ ) years. The study cohort consists of $35 \%$, mild, $44 \%$ moderate and $21 \%$ severe patients. Statistical analysis demonstrated a significant association of serum ALP level with severity of the disease $(p$ value $<0.05)$. High-resolution NGS-based HLA typing revealed the following: HLA-C ${ }^{*} 07$ was significantly associated with moderate patient groups when compared with other two groups [odds ratio $(\mathrm{OR})=0.32$, 95\% confidence interval $(95 \% \mathrm{CI})=0.17-0.60, \quad p<0.0002]$. Similarly, HLADQB1*05 was found to be significantly associated with the mild patient group compared to patients with moderate and severe categories [odds ratio $(\mathrm{OR})=4.31,95 \% \mathrm{CI}=2.23-8.50, p<0.0001$ ]

Discussion \& Conclusion: HLA haplotypes are significantly associated with disease severity and could be used as clinical predictors to understand disease heterogeneity, segmenting cases based on haplotypes/mutations and make clinical and therapeutic decision accordingly.
Endocrine and Metabolic Changes of Thalassemia Patients in Manipur

Khoinaijam Peepa ${ }^{* 1}$, Irom Anil ${ }^{1}$, Ksh A. singh ${ }^{1}$, Tungnung Ginzaniang $^{1}$

\section{${ }^{1}$ Medicine, Jnims, IMPHAL, India}

Aims \& Objectives: (1) To assess the clinical profile of thalassemia patients in Manipur. (2) To assess the endocrine and metabolic changes in thalassemia syndromes. To compare the endocrinal and metabolic changes in iron overloaded vs non-iron overloaded thalassemia syndromes.

Patients/Materials \& Methods: Study design: Observational study. Study population: Patient-attending medicine OPD, JNIMS and patient admitted in Medicine ward of JNIMS,IMPHAL. Study period: The study was carried out for a period of 3 years effective from May 2018 to April 2021.

Inclusion criteria:

(1) Thalassemia major or Thalassemia intermedia. (2) Willing to participate in the study. Exclusion criteria:

(1) Thalassemia minor. (2) Patients who are not willing to participate in study.

Results: The study comprises of 76 patients, 48 males and 28 females. 48 mild-E $\beta$ thalassemia, 20 moderate $\mathrm{E} \beta$ thalassemia, 6 severeE $\beta$ thalassemia and $2 \beta$ thalassemia major.

Mean haemoglobin level were $7.6 \pm 1.7$,with ferritin values ranged from. The mean ferritin level was calculated to be $851.5 \pm 840.1$

Regular blood transfusion was seen only $10.5 \%$ of the patients. $63.1 \%$ of the patients have received less than 50 transfusion and $3.9 \%$ of the patients have received more than 200 transfusion correlating with the severity of the disease.There was significant reduction in the number of blood transfusion which was due to the fact that blood trasnsfusion guidelines were strictly followed as per the thalassemia federation guidelines and proper counselling were done. At present majority of the patients were transfused occasionally and based on the symptoms. 45 patients had spleenomegaly at the time of study. Out of this 9 patients had already undergone splenectomy. 24 (31.5\%) of the patients had Iron overload and majority of the patients belonged to severe and $\beta$ thalassemia major. 2 patients were found to have diabetes mellitus and 3 were found to have pre-diabetes. Short stature(10.6\%), hypothyroidism(4\%) and hypogonadism(4\%) were the three most common complication. 56 of the patients was on hydroxyurea. 45 had responded with increased in hemoglobin $>1 \mathrm{gm} /$ dl after 6 weeks. Gastrointestinal side effect -nausea \& vomiting being the most common sideeffect $(53.5 \%)$ and mucocutaneous changes $11(19.6 \%)$ iand increased creatinine $8(14.2 \%)$. Clinically facial deformities like thalassemic facies, short stature were more in the patients having regular transfusion .

Discussion \& Conclusion: Thalassemia patients have a high prevalence of endocrinological abnormalities. Wide spread screening programme in the localities of Manipur is very essential to prevent from the impact of the syndrome. If we are able to counsel and give awareness to all the thalassemia minors/carries, we will be able to prevent the marriage among the thalassemia minors. 
Susoka: A Self-Screening Mobile Application For Beta Thalassemia Traits

Reena Das ${ }^{1}$, Saikat Datta ${ }^{2}$, Anilava Kaviraj ${ }^{3}$, Soumendra N. Sanyal ${ }^{4}$, Peter Nielsen ${ }^{4}$, Izabela Nielsen ${ }^{4}$, Prashant Sharma ${ }^{1}$, Tanmay Sanyal ${ }^{5}$, Kartick Dey ${ }^{6}$, Subrata Saha ${ }^{* 4}$

${ }^{1}$ Department of Hematology, Postgraduate Institute of Medical Education and Research, Chandigarh, ${ }^{2}$ Department of Clinical Hematology, Anandaloke Hospital, Siliguri, ${ }^{3}$ Department of Zoology, University of Kalyani, Kalyani, India, ${ }^{4}$ Department of Materials and Production, Aalborg University, Aalborg, Denmark, ${ }^{5}$ Department of Zoology, Krishnagar Government College, Krishnagar, ${ }^{6}$ Department of Mathematics, University of Engineering \& Management, Kolkata, India

Aims \& Objectives: The most effective way to combat inherited hemoglobin disorders including beta-thalassemia traits (BTT) is to prevent the birth of children through carrier screening. High-performance liquid chromatography (HPLC) test is often recommended for this purpose, which involves higher cost. Therefore, a cost-effective screening method is essential to identify possible BTT at the initial stage of a screening program and reduce the suspected cases with utmost accuracy to reduce the burden of healthcare resources.

Patients/Materials \& Methods: We proposed a scoring mechanism to classify such disorders with five hematological parameters namely $\mathrm{Hb}, \mathrm{MCV}, \mathrm{MCH}, \mathrm{RBC}, \mathrm{RDW}$ to formulate a scoring mechanism by employing decision trees, Naïve Bayes classifier, and artificial neural network frameworks. We further developed an android application named "SUSOKA", presently available in the Google Play Store for initial screening of BTT. One can easily access SUSOKA to determine whether there is a need for the HPLC test to confirm BTT after entering the values of the five hematological parameters from the respective complete blood count report.

Results: 1076 clinical data were collected from the Department of Hematology at the Postgraduate Institute of Medical Education and Research (PGIMER), Chandigarh, India. The data sat was used to construct the score and determine a threshold. Further 956 clinical data from the same institute were used for validation purpose. The results revealed the specificity and sensitivity of the screening score to be $84.76 \%$ and $100 \%$, respectively for BTT for validation set.

Discussion \& Conclusion: For a sample with no blood transfusions in the recent past or without pregnancy if the application confirms that a person is not a carrier of BTT then there is no need to perform HPLC for further evaluation. The application is presently under trail in different medical units and the responses from the users are satisfactory.

\section{Continuous Intravenous Infusion VS Intermittent Intramuscular Injection of Tramadol For Sickle Cell Vasoocclusive Crisis: An Open Label Randomized Trial}

Baladev P. Kar ${ }^{* 1}$, Pradeep K. Mohanty ${ }^{1}$

${ }^{1}$ General Medicine, VIMSAR, Sambalpur, India

Aims \& Objectives: Sickle cell disease is a global health problem and in India it is prevalent in Gujarat, Maharashtra, Madhya Pradesh, Chhattisgarh, Odisha and Jharkhand. The protean manifestation as vaso occlusive crisis occurs repeatedly and cumbersome to manage and responsive for increased morbidity and mortality. Inadequately managed pain have negative consequences in physiological functions such as autonomic hyperactivity and reduced motility leading to muscle wasting, joint stiffening and decalcification with deleterious psychological changes. As per WHO Analgesic ladder for mild to moderate pain long acting opioid or continuous infusion short acting opioid is required with/without non opioid adjuvant agents. Tramadol is a synthetic codeine analogue which is weak mu opioid receptor agonist. Usual practice is to administer Tramadol intermittently as intramuscular injection which results in breakthrough pain. Ideal way is to infusion, which is not in usual practice.

Patients/Materials \& Methods: The study conducted after Ethical approval and CTRI registration on indoor patients of VIMSAR, Burla with diagnosed case of sickle cell disease having VOC defined as pain involving skeleton, chest, abdomen or all three lasting four hours or more. Study groups were randomly divided either getting Tramadol infusion or intermittent intramuscular injection and monitored four hourly for assessment of pain on visual analogue scale and for breakthrough pain in which adjuvant analgesic Inj Ketorolac/Diclofenac used.

Results: We found in the study that the mean duration of hospital stay, better pain relief, lesser amount of breakthrough pain and lesser cumulative dose of Tramadol in continuous intravenous infusion group compared to the intermittent intramuscular group.

Discussion \& Conclusion: In sickle cell disease vaso occlusive crisis patient appropriate pain assessment and adequate pain management is considered to be standard of care. Given its profound impact in patient physiology and quality of life, the management of pain must be an important in therapeutic intervention. Although Morphine is the most ideal opioid recommended for painful crisis, use is limited due to regulatory prohibition along with Codeine, Oxycodone and Hydromorphone which are not available. Tramadol is as effective as Morphine in mild to moderate pain and ideal way is to use intravenous infusion.

\section{Serum Cystatin C \& Beta-2 Microglobulin as Early Renal Markers in Beta Thalassemia Children}

Jeevitha $\mathbf{G}^{* 1}$, Sunita Sharma ${ }^{1}$, Jagdish Chandra ${ }^{2}$, Smita Tripathi ${ }^{3}$

${ }^{1}$ Department of Pathology, ${ }^{2}$ Department of Paediatrics, ${ }^{3}$ Department of Biochemistry, Lady Hardinge Medical College, New Delhi, India

Aims \& Objectives: To estimate the levels of serum Cystatin $\mathrm{C}$ and serum and urinary $\beta 2$ Microglobulin in children with $\beta$ thalassemia as early markers of renal dysfunction.

Patients/Materials \& Methods: A hospital based descriptive crosssectional study was conducted on $60 \beta$ Thalassemia children having normal S.Creatinine levels [Group A1-30 $\beta$ Thalassemia major (TM) and Group A2-30 $\beta$ Thalassemia intermedia (TI)], aged 2-18 years along with 30 healthy controls (Group B). S.Cystatin C and serum and urinary $\beta 2$ Microglobulin were measured by ELISA (Cystatin C-EIABEST ELISA kit and Demeditec beta-2-microglobulin ELISA DE7610 kit) on the pre-transfusion serum and urine samples in cases. Estimated GFR (eGFR) was calculated using revised Schwartz formula and spot U.Pr/Cr ratio were done.

Results: The mean S.Cystatin $\mathrm{C}$ was significantly higher in cases $(4.05 \pm 1.20 \mu \mathrm{g} / \mathrm{ml})$ than the control group $(1.76 \pm 0.34 \mu \mathrm{g} / \mathrm{ml})$ $(p<0.001)$ with higher levels in thalassemia major than thalassemia intermedia. All thalassemia major cases and $86.6 \%$ of thalassemia intermedia cases had S.Cystatin C levels higher than the upper limit of the normal $(2.44 \mu \mathrm{g} / \mathrm{ml})$. The mean S. $\beta 2$ Microglobulin was significantly higher in cases $(3.95 \pm 1.02 \mu \mathrm{g} / \mathrm{ml})$ than the control group $(2.53 \pm 0.38 \mu \mathrm{g} / \mathrm{ml})(p<0.001)$, with higher levels in thalassemia intermedia than thalassemia major. S. $\beta 2$ Microglobulin $>3.0 \mu \mathrm{g} / \mathrm{ml}$ was observed in $78.33 \%$ of thalassemia major cases and $80 \%$ of thalassemia intermedia cases. The cases had significantly high urinary B2 Microglobulin $(0.40 \pm 0.66 \mu \mathrm{g} / \mathrm{ml}) \quad$ than controls $(0.12 \pm 0.08 \mu \mathrm{g} / \mathrm{ml}) \quad(p=0.001)$ with no significant difference between the two subgroups. S.Cystatin $\mathrm{C}$ had significant positive 
correlation with number of transfusions $(p<0.001)$, S.Creatinine $(p<0.001)$ and S. $\beta 2$ Microglobulin $(p<0.001)$. S.Cystatin C and S. $\beta 2$ microglobulin had significant negative correlation with eGFR ( $p=0.008$ and $p=0.037$ respectively) however no correlation was observed with S.Ferritin.

Discussion \& Conclusion: In the present study, S.Cystatin C and S. $\beta 2$ Microglobulin were elevated even in patients with normal S.Urea and S.Creatinine. Hence, these novel markers can serve as sensitive early predictors of renal dysfunction and to modify treatment protocol for better prognosis in $\beta$ thalassemia children.

\section{Unravelling the Genetic and Phenotypic Heterogeneity of SPTA1 Variants in HE/Hpp Patients Using NGS}

\author{
Tejashree A. More ${ }^{* 1}$, Prabhakar Kedar ${ }^{1}$ \\ ${ }^{1}$ Haematogenetics, ICMR-National Institute of \\ Immunohaematology, Mumbai, India
}

Aims \& Objectives: Hereditary Elliptocytosis (HE) and Hereditary Pyropoikilocytosis (HPP) are heterogeneous red blood cell (RBC) membrane disorders that result from mutations in the genes encoding $\alpha$-spectrin (SPTA1), $\beta$-spectrin (SPTB), or protein 4.1R (EPB41). The resulting defects alter the horizontal cytoskeletal associations and affect RBC membrane stability and deformability causing shortened RBC survival. Here we present the genetic and phenotypic heterogeneity of HE and HPP patients with SPTA1 gene variants.

Patients/Materials \& Methods: Eight patients of unrelated families, with clinically suspected HE or HPP based on their clinical presentation varying from moderate to severe hemolysis and RBC morphological smear showing elliptocytes, few spherocytes, poikilocytes and fragmented cells, with some (6 out of 8 ) requiring regular blood transfusions are included in this study. All the patients showed normal EMA readings. We identified the causative genetic variants by Next Generation Sequencing in 8 patients with clinically suspected HE or HPP and studied genotype-phenotype-correlation.

Results: Targeted next-generation sequencing identified 6 missense, 1 nonsense and 2 splice site SPTA 1 variants, out of which 5 are novel (Table 1). Three homozygous and one compound heterozygous SPTA1 variants causing HPP and 4 heterozygous variants causing HE are identified. The variants have been validated by Sanger sequencing. One patient had compound heterozygous SPTA1 variants resulting in HPP. Of the 8 patients, one has HPP in combination with a heterozygous variant in ANK1 gene causing HS resulting in severe clinical manifestations with phenotypic variability. Almost all of these genetic variants are deleterious, as revealed by the result of the In silico analysis. Most patients had SPTA1 variants within or near the spectrin self- association site. Structural analysis of the SPTA1 variants was performed using the HOPE software. Moreover, genotypic differences justified the phenotypic differences associated with HE/HPP patients.

Discussion \& Conclusion: HE is caused by monoallelic (heterozygous) mutations while HPP, the more severe form, is typically caused by biallelic (homozygous or compound heterozygous) mutations leading to variable phenotypic differences. These results extend the genetic heterogeneity of SPTA1 variants causing HE/HPP. Moreover, causative molecular diagnosis by NGS allows genotype-phenotype correlations in these heterogeneous disorders and may aid in prognosis considerations.
Metabolic Derangements Accompanying Vascular Dysfunction in Children with Beta Thalassemia Major

Sudeep Kumar $^{* 1}$, Kapil Bhalla ${ }^{2}$

${ }^{1}$ Pediatrics, Pt. B.D Sharma, ${ }^{2}$ Pediatrics, Pt. B.D. Sharma PGIMS, Rohtak, India

Aims \& Objectives: An observational study with 60 Thalassemic children to identify vascular dysfunction in preclinical stages.

Patients/Materials \& Methods: 60 children with $\beta$ Thalassemia major were compared with control population. All children underwent USG doppler and CIMT (Carotid Intima Media Thickness) was determined by a single radiologist who was unaware of clinical history of the subjects, it was followed with detailed history, anthropometry, complete hemogram, serum ferritin, lipid profile and Atherogenic index of plasma (AIP calculated as log of total Triglycerides/HDL) and Cardiac risk ratio (CRR: total cholesterol/ HDL) were derived.

Results: The demographic profile was comparable in both groups. AIP in thalassemic children (0.695) was significantly higher than their gender and age matched controls (0.364). Similarly, CIMT was significantly higher in thalassemic children compared to controls $(0.481 \pm 0.051$ vs $0.471 \pm 0.019 \mathrm{~mm})$ on right and $(0.400 \pm 0.051$ vs $0.393 \pm 0.019 \mathrm{~mm}$ ) on left side; $p$ value $<0.001)$. AIP was strongly correlated to BMI, waist hip ratio and Systolic blood pressure. CIMT was found to have strong correlation with age, chronicity of the disease, serum ferritin levels and Diastolic blood pressures. Children with higher AIP were found to have higher CIMT values ( $p$ value $<0.01$ ). AIP was found to be $81.7 \%$ sensitive with a positive predictive value of $87.5 \%$ and CIMT was $90 \%$ sensitive with a negative predictive value of $88.9 \%$ with a cut-off value of $0.415 \mathrm{~mm}$. Discussion \& Conclusion: Thalassemia is a state of iron overload and endothelial dysfunction which leads to arterial remodeling.

The increased oxidative stress causes lipid peroxidation culminating in atherosclerotic changes early in life. The present study found both AIP and CIMT useful in identifying children at risk for early vascular dysfunction prior to any manifestations and can be use to tailor chelation therapy and primary preventive measures.

\section{Does The Heme Oxygenase-1 (Hmox-1) Gene Play Role in Fetal Hemoglobin Induction?}

Priya Hariharan ${ }^{* 1}$, Vrushali Chavan ${ }^{1}$, Anita Nadkarni ${ }^{1}$

${ }^{1}$ National Institute Of Immunohaematology, Mumbai, India

Aims \& Objectives: Inducible heme oxygenase-1 (HO-1) acts as a potential cytoprotective and anti-inflammatory enzyme which is activated at times of inflammation, hypoxia and ischemic condition. It is observed that sickle cell anemia patients with elevated fetal hemoglobin $(\mathrm{HbF})$ levels have a relatively mild clinical course. There is sparse literature on the association of higher $\mathrm{HbF}$ levels leading to reduction in the oxidative stress in SCA patients. Hence, the significance between the HMOX1 gene polymorphisms and the HbF levels have been studied.

Patients/Materials \& Methods: Preliminary screening by performing complete blood count and by HPLC was carried out. Genotyping of 3 genetic variants in the HMOX1 gene was performed in 90 SCA patients and 50 healthy controls by PCR-RFLP, GeneScan fragment analysis, and DNA sequencing.

Results: In order to understand the effect of elevated $\mathrm{HbF}$ levels on the hematological indices, the patients were classified into 2 groups considering the median $\mathrm{HbF}$ levels of $18.1 \%$. The SCA patients with higher $\mathrm{HbF}$ levels, showed improved hematological indices with inverse effect on $\mathrm{HbS}$ levels. It was observed that the TT genotype of 
rs2071746 $(\mathrm{A} \rightarrow \mathrm{T})$ polymorphism (P:0.012) and the long form $(>25$ GT repeats) of rs3074372 (GT)n repeats was found to be linked with increased $\mathrm{HbF}$ levels. We could not find any association of rs2071749 $(\mathrm{A} \rightarrow \mathrm{G})$ polymorphism with the $\mathrm{HbF}$ levels. Further, generalised multifactor dimensionality reduction [GMDR] analysis to understand the interaction of the covariates (SNPs) in elevating the HbF levels showed higher presence of mutant allele $\mathrm{T}$ of $\mathrm{rs} 2071746 \mathrm{~A} \rightarrow \mathrm{T}$ polymorphism in SCA patients in raised $\mathrm{HbF}$ group. Multivariate analysis using different genetic models indicated that the $\mathrm{T}$ allele of rs2071746 $\mathrm{A} \rightarrow \mathrm{T}$ variant was significant for the co-dominant and dominant genetic model with the TT genotype resulting in significant induction of $\mathrm{HbF}$ levels.

Discussion \& Conclusion: As the SCA patients show significant oxidative stress due to hemolysis, the study of polymorphisms in the HMOX1 gene may act as potential independent marker for elevated $\mathrm{HbF}$ levels. In future the cumulative study of the genetic modifiers of $\mathrm{HbF}$ may help in forecasting the disease severity.

\section{Pulmonary Functions in Children With Beta- Thalassemia Major}

Neha Panwar ${ }^{* 1}$, Sunil Gomber ${ }^{1}$, Pooja Dewan ${ }^{1}$, Raj Kumar ${ }^{2}$

${ }^{1}$ Department of Peadiatrics, GTB Hospital, Dilshad Garden, ${ }^{2}$ Department of Pulmonology, VPCI Hospital, New Delhi, India

Aims \& Objectives: $\beta$-thalassemia major is an inherited disorder requiring frequent blood transfusions for maintenance of hemoglobin levels. Due to repeated transfusions, iron overload is noticed in these patients causing iron deposition in various organs like heart, liver, lung and other vital organs. There are no standard guidelines for monitoring of lung parenchymal iron deposition in these patients. Hence, the present study was undertaken to monitor pulmonary function (PFT) of multi transfused thalassemic children and to assess its correlation with iron load (assessed by serum ferritin (SF) and $\mathrm{T} 2{ }^{*} \mathrm{MRI}$ of heart and liver). The study also checked reversibility of lung dysfunction (if any) with intravenous deferoxamine chelation therapy ( 4 weeks).

Patients/Materials \& Methods: This prospective cohort study (November 2017 to April 2019) was carried out in haemato-oncology unit of tertiary care center in Delhi, India on 77 patients. Analysis of PFT and iron load assessment was done. PFT included spirometry, total lung capacity (TLC) by helium dilution test and diffusion capacity by carbon monoxide (DLCO). Indian standards were used for spirometry reference predictive values and western standards were used for TLC and DLCO as Indian standards were not available for same. Intravenous deferoxamine administrated in patients with moderate to severe lung dysfunction for 4 weeks and then, PFT and SF were repeated.

Results: $68.83 \%$ (50/77) patients had lung dysfunction (figure 1), most common defect noticed was diffusional impairment (48/ $50 ; 96 \%)$, second was reduced TLC $(11 / 50 ; 22 \%)$ and none had obstructive pattern. $9 / 11$ patients with restrictive defect had moderate to severely deranged DLCO. PFT and T2* MRI values were inversely correlated with serum ferritin. No correlation was noticed between PFT and T2*MRI values. 17 patients with moderate to severe lung dysfunction were given intravenous deferoxamine (4 weeks) at $40 \mathrm{mg} / \mathrm{kg} / \mathrm{dose}$ and then PFT's were repeated. Significant improvement was noticed in spirometry indices, DLCO and SF with intensive intravenous chelation therapy with deferoxamine for 4 weeks.

Discussion \& Conclusion: All multi-transfused thalassemic patients should undergo Pulmonary Function Test at least once in a year so as to detect lung dysfunction as early as possible and thus decreasing morbidity and mortality in future. No such recommendations are made in major guidelines worldwide.
Supporting Document:

\section{Figure 1: Lung function profile $(n=77)$}

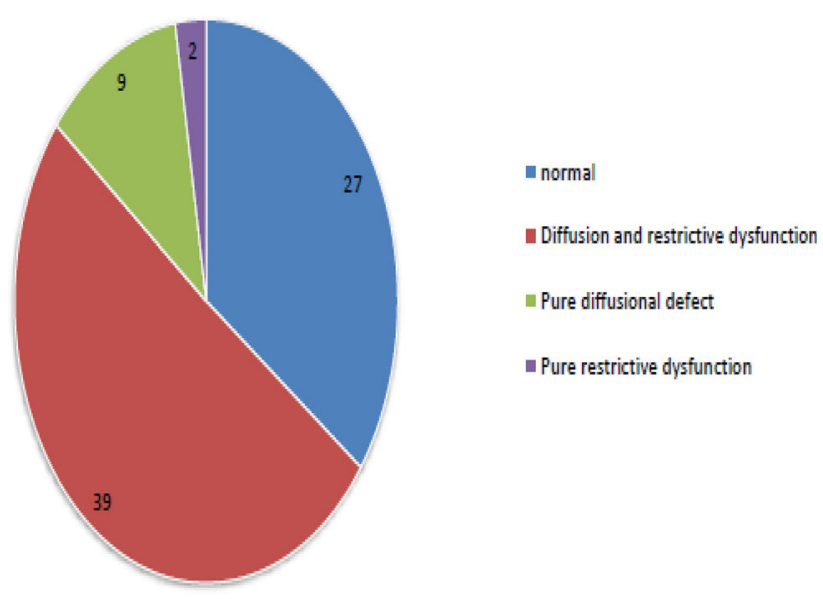

Bleeding Disorders

\section{Can Rotem in Combination with ISTH-BAT Score be} Used as a Screening Test for Factor XIII Deficiency?

Therese V. David*1, Tulasi Geevar ${ }^{1}$, Rutvi G. Dave ${ }^{1}$, Gayathri K. Chellaiya $^{1}$, Ramya Vijayan ${ }^{1}$, Surendar Singh ${ }^{1}$, Sukesh C. Nair ${ }^{1}$

${ }^{1}$ Department of Transfusion Medicine and Immunohematology, Christian Medical College, Vellore, India

Aims \& Objectives: -To calculate sensitivity and specificity of ROTEM parameters for the diagnosis of Factor XIII deficiency. $\bullet$ To calculate the sensitivity and specificity of ISTH-BAT score for the diagnosis of Factor XIII deficiency.

- To calculate the sensitivity and specificity of combined ROTEM parameters and ISTH-BAT score for the diagnosis of Factor XIII deficiency.

- To study the association of bleeding symptoms specifically umbilical stump bleeding for the diagnosis of Factor XIII deficiency. Patients/Materials \& Methods: •In this retrospective study, we analysed patients who were found to have Factor XIII deficiency among patients sent for a detailed coagulation workup from October 2016 -October 2020 in the Hemostasis laboratory of Christian Medical College, Vellore.

- We also included 80 consecutive patients who were found to have no intrinsic hemostatic defect after a detailed coagulation workup from November 2019- October 2020.

- We looked at the bleeding symptoms, ISTH-BAT score and ROTEM parameters in the patient and control group. Factor XIII antigen and activity levels were done to confirm the diagnosis.

- Sensitivity and specificity were calculated.

Results: • 34 cases of Factor XIII deficiency were identified during the study period.

- All cases and controls had normal PT, aPTT, TT and fibrinogen values.

- All patients with Factor XIII deficiency had a significant ISTHBAT score when compared to $26 / 80$ in the control group (sensitivity $=100 \%$, specificity $=67.5 \%$ ). 
- 27/34 patients had positive history for umbilical stump bleeding when compared to $2 / 80$ in the control group (sensitivity $=79.4 \%$, specificity $=97.5 \%$ )

- 26/34 patients had increased maximum lysis (ML $\geq 15 \%$ ) in ROTEM when compared to $7 / 80$ in the control group (sensitivity $=76.5 \%$, specificity $=92.5 \%$ ).

- 33/34 patients had either history of umbilical stump bleed or increased maximum lysis ( $\mathrm{ML} \geq 15 \%$ ) in ROTEM when compared to $9 / 80$ in the control group (sensitivity $=97.1 \%$, specificity $=88.8 \%$ ).

Discussion \& Conclusion: - Factor XIII deficiency cannot be detected using routine coagulation screening tests.

- ROTEM/TEG is possibly the only rapid test to assess hyperfibrinolysis.

- A combination of BAT score, especially history of umbilical stump bleeding and lysis in ROTEM can be used as a screening test for Factor XIII deficiency.

Supporting Document:

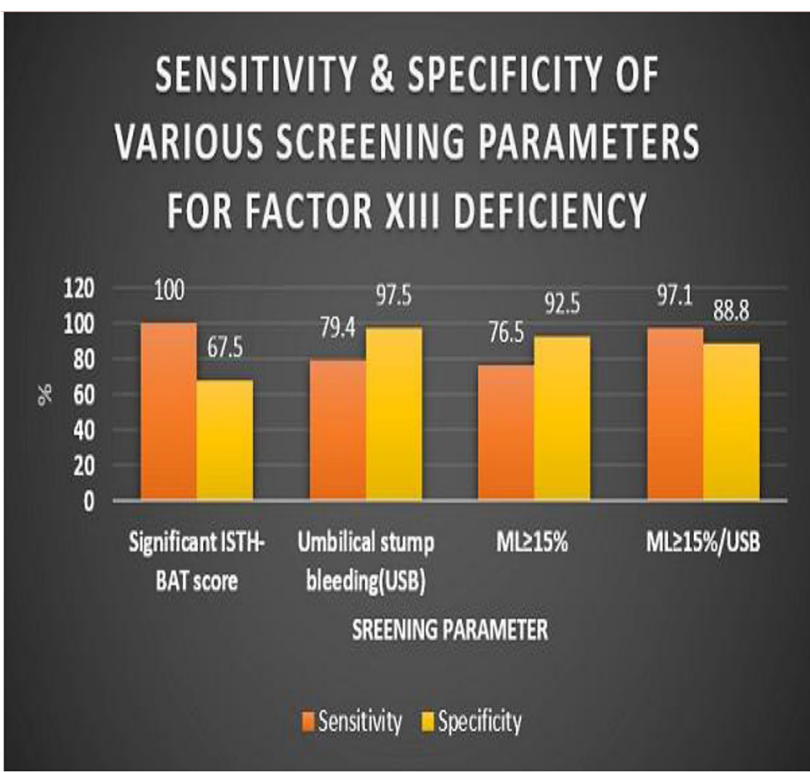

\section{Utility of Rotem and APTT-CWA Analysis to Assess Severity of Hemophilia A}

Deepika Gupta ${ }^{* 1}$, Vandana Arya ${ }^{1}$, Jasmita dass ${ }^{2}$, Nitin gupta ${ }^{3}$, Ajay sharma $^{3}$, Anupam sachdeva ${ }^{4}$, Jyoti kotwal ${ }^{1}$

${ }^{1}$ Hematology, Sir Ganga Ram Hospital, ${ }^{2}$ Hematology, AIIMS, ${ }^{3}$ Clinical hematology, ${ }^{4}$ Pediatric hemato oncology, Sir Ganga Ram Hospital, New Delhi, India

Aims \& Objectives: Nearly $10-15 \%$ of hemophilia A patients show discordance between their factor levels and clinical phenotype. This study has been conducted to study the diagnostic utility of Rotational thromboelastometry (ROTEM) and partial thromboplastin time clot waveform (APTT-CWA) to assess severity in Hemophilia A.

Patients/Materials \& Methods: A total of 66 Hemophilia A cases were recruited. The clinical phenotype was assigned using the criteria previously described. The patients were divided into severe and non severe HA phenotype. Patients were further divided in four sub groups.
Group I: Mild and moderate HA with non severe phenotype $(\mathrm{N}=25)$ Group II: mild and moderate HA with severe phenotype $(\mathrm{N}=6)$ Group III: Severe HA with severe phenotype with inhibitors $(\mathrm{N}=14)$.

Group IV: severe HA with severe phenotype without inhibitors $(\mathrm{N}=21)$

HA was diagnosed by APTT and one stage FVIII assay. ROTEM was performed on INTEM mode. APTT CWA parameters were obtained on ACL TOP 750 and 700 coagulometer using APTT reagent SynthASil. APTT inhibitor screening and Bethesda assay were done for the patients who had incomplete correction on mixing studies.

Results: The first and second derivatives of CWA were lower significantly in severe HA patients. CT and CFT on ROTEM were significantly higher in severe HA. MCF and MA30 was lower in severe vs. non-severe HA. Alpha angle and MAXV were much reduced in severe HA whereas MAXVT was much prolonged in severe HA. However, no statistically significant differences were found between group II and group IV. This implies that patients in group II (mild/moderate HA with severe phenotype) behaved similar to the patients with severe HA without inhibitors and severe phenotype using both APTT-CWA and ROTEM.

Severe HA patients with inhibitors could not be differentiated from severe HA without inhibitors using APTT-CWA. However, we found that these groups can be differentiated using ROTEM parameters including alpha angle, ma30, mcf and maxv which were lowest in inhibitor group. We calculated the cut off values with best sensitivity and specificity for parameters on ROTEM and APTT- CWA. The values for ROTEM are provided in the box.

Discussion \& Conclusion: We found APTT-CWA and ROTEM can distinguish severe from non severe phenotype. Since the tests like Bethesda assay are technically challenging, we found ROTEM can be used to distinguish severe HA with inhibitors from severe HA without inhibitors.

Supporting Document:

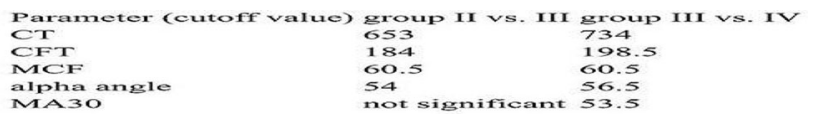

\section{Haemostatic Action of a Topical Foam Based Patch (Velseal-T) in Person with Hemophilia with External Bleeding}

\author{
Anupam Dutta $^{* 1}$, Taniya S. Dutta ${ }^{2}$, Anup K. Das ${ }^{1}$ \\ ${ }^{1}$ Medicine, ${ }^{2}$ Pediatrics, Assam Medical College and Hospital, \\ Dibrugarh, India
}

Aims \& Objectives: Haemophilia is an X-linked congenital bleeding disorder due to deficiency of coagulation factor VIII (in haemophilia A) or factor IX (in haemophilia B) caused by mutations of the respective clotting factor genes. Treatment involves the administration of an appropriate dose of factor concentrate, as soon as possible, in the event of any bleeding episode. In low-resource settings, such as Northeast India, where factor concentrates are not widely available, people with haemophilia $(\mathrm{PwH})$ may bleed profusely even from trivial external injuries, warranting transfusion of blood or blood products. We previously reported on the use of a low cost, foambased haemostatic patch to treat an external bleed in a single patient. In this study, we investigated its use to treat a range of external injuries in PwH presenting at Assam Medical College and Hospital.

Patients/Materials \& Methods: Over 24 months, eligible PwH with external injuries attending our haemophilia clinic were treated with a 
topical haemostatic patch (VELSEAL-T) at the target bleeding site. The time to cessation of bleeding was recorded and the wound sites evaluated after haemostasis to monitor efficacy and safety.

Results: Out of 72 individuals with bleeding disorders who volunteered to participate, 59 cases of external bleeding in $48 \mathrm{PwH}$ were eligible for inclusion in the study. Nine $(15.3 \%)$ had aberration wounds, $24(40.7 \%)$ cut wounds, $21(35.6 \%)$ tooth and/or gum bleeding and five $(8.4 \%)$ bleeding from puncture wounds. The average time required for achievement of haemostasis was $9.9( \pm 4.7)$ minutes. Aberration wounds required the least amount of time for haemostasis at $7.3( \pm 4.4)$ minutes. Cut wounds required a mean time of 8.5 ( \pm 2.9 ) minutes; puncture wounds required $9.0( \pm 3.1) \mathrm{min}$ utes; gum bleeding required the longest time to achieve haemostasis with a mean of $12.7( \pm 5.6)$ minutes.

Discussion \& Conclusion: The use of this topical haemostatic patch has been shown to be beneficial in the treatment of external injuries in $\mathrm{PwH}$, and provides a good treatment option in resource-constrained areas. A larger controlled study would be helpful to further investigate its efficacy and safety.

\section{Assessment of Joints in Patients with Hemophilia}

Bhabani S. Dhal ${ }^{* 1}$, Anupam Dutta ${ }^{2}$, Dhrubajyoti Borpatragohain ${ }^{3}$, Adity Sharma ${ }^{4}$

${ }^{1}$ Department of Medicine, ${ }^{2}$ Assistant Professor, Department Of Medicine, ${ }^{3}$ Associate Professor, Department of Radiodiagnosis, ${ }^{4}$ Professor, Department of Pathology, Assam Medical College and Hospital, Dibrugarh, India

Aims \& Objectives: We studied the clinical profile of all the patients with hemophilia presented to Assam medical college and assessed their joints by integrating clinical, radiological, functional methods.

Patients/Materials \& Methods: The study was carried out on 67 patients with hemophilia during one-year duration. Every patient was subjected through proper history taking, examination and adequate investigations were done. Assessment of joints were done by HJHS2.1, FISH, HEAD-US, PETTERSSON scoring method and necessary correlations among the parameters were done by statistical methods. Patients were treated with adequate factor replacement.

Results: Out of the 67 patients 2 were females, 57 patients had hemophilia A, 9 patients had hemophilia B, 1 patient had acquired hemophilia. Total $38(57.72 \%)$ patients had severe factor deficiency. $24(35.82 \%)$ patients did not have any prior family history. Mean age of our study population was $21.69 \pm 8.24$ years. Joint bleed was the most common manifestation found in $71.70 \%$ patients, with multiple joint involvement in $41(61.19 \%)$ patients. 40 (59.70\%) patients had target joint as knee joint followed by elbow joint in 11(16.41\%) cases and ankle joint in $6(8.95 \%)$ cases. $51(76.12 \%)$ patients were on prophylactic factor replacement therapy. We came across 4 patients with inhibitors during this period. We found mean ABR, AJBR, ATJBR values had a negative correlation with factor activity level ( $p$ value $<0.001$ ). We noticed that FISH score increases with factor activity level where as HJHS, HEAD-US, PETTERSSON SCORE decreases with factor activity level. Significant Positive correlation was established between PETTERSSON score and age, ABR of the patient. We found degree of association between HJHS score was at maximum $(60.62 \%)$ with HEAD-US score, whereas between PETTERSSON and HJHS score it was $57.74 \%$. Statistically significant negative correlation was established between FISH and HEAD-US score.

Discussion \& Conclusion: A combined multimodal approach using HJHS, FISH, HEAD-US, PETTERSSON scoring system can be a cheap, more reliable, quick, accurate, with minimal inter and intra observer reliability option for the consistent assessment of joints and optimizing the management of the destructive changes of the joints in patients with hemophilia, as far as the developing country like India is concerned, where resources are limited.

\section{A Novel In-House Clot Based Assay for Measuring Endogenous Thrombin Potential (ETP) \\ Puja Soni $^{* 1}$, Rucha Patil ${ }^{1}$, Shrimati Shetty ${ }^{1}$ \\ ${ }^{1}$ Haemostasis and Thrombosis, ICMR-NIIH, Mumbai, India}

Aims \& Objectives: Thrombin generation assay (TGA) evaluates total thrombin generation by incorporating both anti and pro- coagulant factors. It is used to assess thrombotic and haemorrhagic risks in patients. It is the most widely used global haemostasis assay (GHA) for monitoring bypassing agent therapy and nonfactor products like Emicizumab, Fitusiran, etc. But owing to the cost, requirement of a dedicated machine, and experts, there is a need for a simple assay that can be correlative to St-Genesia TGA. Aim: To develop and validate an in-house assay for measurement of thrombin.

Patients/Materials \& Methods: Plasma samples with different FVIII levels (<1 IU/ml, $20 \mathrm{IU} / \mathrm{ml}, 30 \mathrm{IU} / \mathrm{ml}, 35 \mathrm{IU} / \mathrm{ml}, 40 \mathrm{IU} / \mathrm{ml}, 100 \mathrm{IU} /$ $\mathrm{ml}$ ) were prepared by using different dilutions of factor concentrate in FVIII depleted plasma to mimic samples with different ETP values. The TGA was done using bleedscreen reagent in a fully automated TGA (St-Genesia, Diagnostica Stago, France). The correlation coefficient (r) was calculated by Bland-Altman analysis and Spearman's method using St-Genesia TGA as the gold standard. Different combinations of pro and anti-coagulants like different APTT reagents, tissue factor: (HemosIL RecombiPlasTin, 6000 pM, IL, USA), phospholipid (Phospholipid vesicles consisted of $20 \mathrm{~mol} \%$ phosphatidylserine, $20 \mathrm{~mol} \%$ phosphatidylethanolamine and $60 \mathrm{~mol} \%$ phosphatidylcholine), heparin (Heparin Inj. $25000 \mathrm{IU} / 5 \mathrm{ml}$, Company, country), thrombomodulin (TM; Thrombomodulin human, Sigma Aldrich, US) and Protac (3U, Enzyme research laboratory, US) in varying dilutions was used as triggering reagent. Results: Correlation of the in-house TGA assay using Protac was found to be significantly correlative to varying FVIII levels $(p<0.05)$ [Figure 1]. Validation of the clotting time(CT) obtained in the in-house TGA assay in 60 different haemostatic samples showed high significant correlation with peak height(ht) $(\mathrm{r}=-0.85, p<0.0001)$ and ETP $(\mathrm{r}=-0.799, p<0.0001)$ obtained by St-Genesia TGA [Figure 2A \& 2B]. The Bland-Altman analysis was done to compare the results of the in-house clot based TGA with increasing peak ht and ETP of StGenesia assay showed a bias of -14.5 and 590.5 which associates with the decreasing CT of clot based TGA assay showing a strong correlation [Figure 2C \& 2D].

Discussion \& Conclusion: An in-house clot based TGA assay has been successfully developed and validated with gold standard StGenesia TGA $(p<0.0001)$. Though preliminary, the in-house clot based TGA assay is, simple, inexpensive (1.35 US-dollars/test) and highly correlative to St-Genesia TGA (44 US-dollars/test). It could definitely be utilized routinely for thrombin generation assessment in various clinical settings. 
Supporting Document:

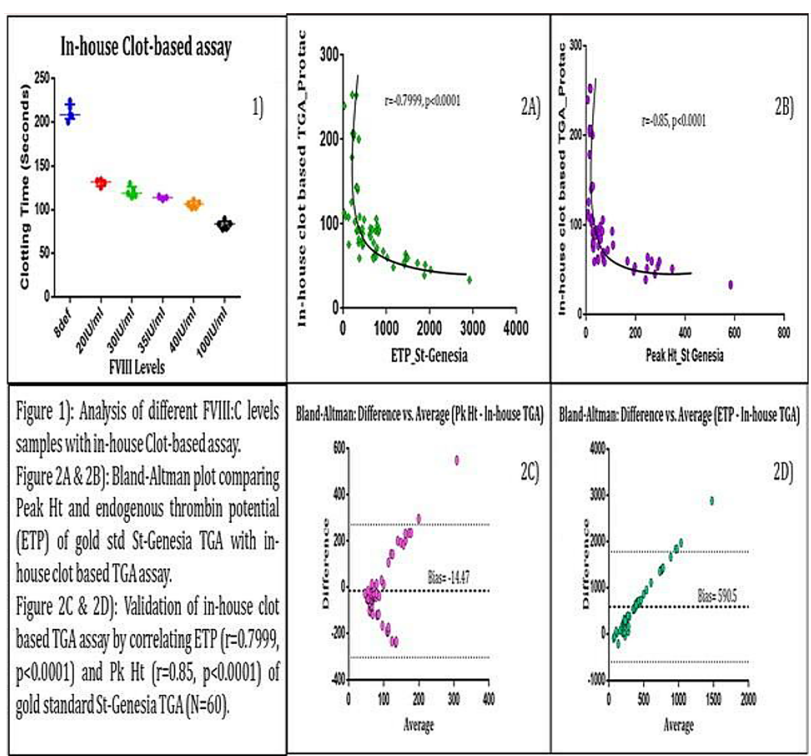

\section{Bone Marrow Failure and MDS (Clinical)}

\section{Addition of Eltrombopag to Immunosuppressive Therapy (ATG, Cyclosporine) in Acquired Aplastic Anemia: A Tertiary Care Center Experience}

Stitha P. Gudala ${ }^{* 1}$, Rishi Dhawan ${ }^{1}$, Mukul Aggarwal ${ }^{1}$, Pradeep Kumar $^{1}$, Tulika Seth ${ }^{1}$, Manoranjan Mahapatra ${ }^{1}$

${ }^{1}$ Clinical Hematology, AIIMS, New Delhi, India

Aims \& Objectives: To assess the responses of Acquired Aplastic Anaemia patients who have received Eltrombopag in addition to Immunosuppressive therapy (ATG + Cyclosporine A) at our tertiary care center.

Patients/Materials \& Methods: We retrospectively analysed the responses of Acquired Aplastic Anaemia patients who have received Eltrombopag, Antithymocyte globulin, and Cyclosporine A from Jan 2019 to March 2020.

Results: Thirty-eight patients were included in our study. The median age is 27.6 years (7-68). Male to Female ratio 1.2:1. 6 patients were less than 13 years of age and, 31 patients were between $>13$ years to $<60$ years and 1 patient was $>60$ years. PNH clone was positive in 6 patients $(15 \%)$. Majority of our patients were Severe Aplastic Anemia (76.3\%), and Non Severe Aplastic Anemia (10.5\%), Very Severe Aplastic Anaemia(13.1\%).All of our patients received Cyclosporine and Danazol before ATG. The median duration from diagnosis to treatment is 12.2 months(1-60 months). Except for one who received Rabbit ATG, everyone received Equine ATG. Eltrombopag was given at a dose of $75 \mathrm{mg}$ in $13.15 \%$ (5) and $150 \mathrm{mg}$ in $86.85 \%$ (33). Complete Response, Partial Response, No response were seen in $15.8 \%, 57.8 \%, 26.5 \%$ respectively. 3 patients expired within three months of treatment, 3 more patients expired by 6 months(1, COVID-19).

Discussion \& Conclusion: Overall Response rates of $75 \%$ in an Indian cohort of acquired aplastic anemia patients treated with ATG and Cyclosporine plus Eltrombopag is comparable to that reported in studies from US and Europe. Median duration of disease prior to ATG based IST was 1 year in our cohort of AA patients.Addition of eltrombopag to ATG and Cyclosporine was able to overcome the delayed initiation of IST in AA patients.Treatment was well tolerated in our patients.

\section{Clinico-Pathological Profile of Patients with Myelodysplastic Syndromes with Bone Marrow Fibrosis}

Sahitya Dangudubiyyam ${ }^{* 1}$, Narasimhapriyan Kannan ${ }^{2,3}$, Mukul Aggarwal $^{2}$, Jasmita Dass², Rishi Dhawan ${ }^{2}$, Ganeshkumar Viswanathan $^{2}$, Pradeep Kumar ${ }^{2}$, Tulika Seth ${ }^{2}$, Manoranjan Mohapatra $^{2}$, Seema Tyagi ${ }^{2}$

${ }^{1}$ Clinical Hematology, ${ }^{2}$ Hematology, ${ }^{3}$ Hematology, AIIMS, DELHI, India

Aims \& Objectives: Marrow fibrosis is found in $10-20 \%$ of patients with Myelodysplastic syndrome. Role of fibrosis in MDS remains unsolved. We conducted this study to compare clinicopathological features in MDS patients with and without marrow fibrosis.

Patients/Materials \& Methods: We retrospectively studied MDS patients between January 2017 till December 2019 at the Department of Hematology, AIIMS, New Delhi. Clinical profile, cytogenetics, molecular profile, bone marrow features, treatment and response, were compared for marrow fibrosis.

Results: 217 patients diagnosed with MDS were enrolled in our study. Median age at diagnosis in our cohort was 51 years(3.5-87). Six patients were less than 18 years of age. Male: Female ratio was 1.7:1. Thirty-six patients had bone marrow fibrosis $(16.6 \%)$. Out of $36,15(42 \%)$ patients had grade $1,13(36 \%)$ had grade 2 and $8(22 \%)$ had grade 3 fibrosis. Median haemoglobin, ANC and platelet counts were $6.9 \mathrm{gm} / \mathrm{dl}$ (IQR 2.9-10.2), 3170/l (IQR 500-14000/l) and 48,500/l (IQR 0.13-6.2 lakhs/1). Median transfusion requirement was 7 packed red blood cells prior to diagnosis. Out of 36 patients, $24(66 \%)$ had dyserythropoiesis, $20(55 \%)$ had dysmyelopoiesis and $24(66 \%)$ had dysmegakaryopoiesis. Cytogenetics were available in 21 patients, of which $2(10 \%)$ had 5Q, 1(5\%) had complex, 3(14\%) had MDS related chromosomal abnormalities other than 5Q and 15(71\%) had normal karyotype. According to WHO classification, 1(3\%) had MDS with isolated 5Q, 8(22\%) had MDS- EB1, 10(28\%) had MDSEB2, 11(30\%) had MDS-MLD, 2(6\%) had MDS -SLD, 3(8\%) had MDS-RS and 1(3\%) had MDS-U. R-IPSS was available in 21 patients, of which $1(5 \%)$ were very low risk, 4(19\%) were low risk, $5(24 \%)$ were intermediate risk, $6(29 \%)$ were high risk and $5(23 \%)$ were very high risk. Treatment details were available for 20 patients. Thirteen $(65 \%)$ patients received hypomethylating agents (azacytidine), $1(5 \%)$ patients received Lenalidomide, 1(5\%) patients underwent hematopoietic stem cell transplant and 5(25\%) received other therapies (danazol, erythropoietin, B12). Response assessment was available in 11 patients of which $2(18 \%)$ attained complete response, $1(9 \%)$ had complete haematological response, $2(18 \%)$ had stable disease, $4(36 \%)$ had progressive disease (according to IWG criteria). Two patients expired and the rest were lost to follow up. There was no significant difference in the above parameters between fibrosis and non-fibrosis group.

Discussion \& Conclusion: Prevalence of marrow fibrosis in MDS patients in our study is comparable to other published studies. Bone marrow fibrosis changes observed in MDS patients did not correlate with IPSS-R in our cohort. 
Bone Marrow Failure and MDS (Laboratory)

\section{Bone Marrow Mesenchymal Stromal Cells Derived Extracellular Vesicles of Severe Aplastic Anemia Patients Exhibit Altered Hematopoiesis Supporting Properties}

Jyotika Srivastava $^{* 1}$, Chandra P. Chaturvedi ${ }^{2}$, Shobhita Katiyar ${ }^{2}$, Khaliqur Rahman ${ }^{1}$, Soniya Nityanand ${ }^{1}$

${ }^{1}$ Department of Hematology, ${ }^{2}$ Stem Cell Research Centre, Sanjay Gandhi Post Graduate Institute of Medical Sciences, Lucknow, India

Aims \& Objectives: Currently there is an increasing interest in studying the bone marrow (BM) hematopoietic niche in aplastic anemia (AA) patients since it maintains the survival, proliferation and differentiation of hematopoietic stem cell progenitor cells (HSPCs). Mesenchymal stromal cells (MSCs) are a key cell of the hematopoietic niche and play a crucial role in hematopoietic homeostasis by interacting with HSPCs. We have recently demonstrated that the BM-MSC of AA patients have altered expression of hematopoiesis regulatory molecules. The BM-MSCs are thought to mediate their functional effects via the release of paracrine factors in biologically active extracellular vesicles (EVs). Therefore, the aim of the present study was to evaluate the hematopoiesis supporting properties of BM-MSC derived EVs from severe AA (SAA) patients and compare it with that of controls.

Patients/Materials \& Methods: Study Subjects: Patients \&Controls: $5 \mathrm{ml}$ of BM aspirate was collected from acquired idiopathic severe aplastic anemia (SAA) patients $(n=6)$ and controls $(n=5)$ after informed consent. Human Umbilical Cord blood (UCB): $25 \mathrm{ml}$ of UCB was collected after informed consent.

Materials \& Methods: EVs were isolated from 3rd passage BMMSC of SAA patients and controls using Exosome Isolation Reagent and characterized by flow cytometric demonstration of exosomal marker CD63 and their particle size and concentration were determined by Nanoparticle Tracking Analysis. To study the defect in the hematopoiesis supporting properties of MSC derived EVs from SAA, UCB derived CD34 + HSC were co-cultured with BM-MSC derived EVs from SAA and controls and the HSC colony forming units (CFU), cell proliferation and apoptosis were studied.

Results: EVs derived from BM-MSC of SAA patients and controls exhibited comparable expression of CD63 exosomal marker and particle size and concentration. HSC co-cultured with EVs from BMMSC of SAA patients in comparison to EVs from BM-MSC of controls, exhibited a significantly reduced number of BFU-E $(p=0.002)$, CFU-GM $(p=0.006)$, reduced cell proliferation $(p=0.031)$ and increased cell apoptosis $(p=0.004)$.

Discussion \& Conclusion: Our results demonstrate that EVs from BM-MSC of SAA patients have impaired hematopoiesis supporting functions, thus highlighting that there is an abnormality of BM-MSC in AA. Their contribution in the pathogenesis of AA needs further evaluation.

\section{Infections and Support Care}

\section{Risk Factors and Outcome Analysis of Covid 19 Infection in Hematological Diseases}

Shiva Kumar Komaravelli ${ }^{* 1}$, Nataraj $\mathrm{KS}^{1}$, Ranganatha $\mathrm{R}^{2}$, Shilpa Prabhu $^{1}$, Bharath Ram ${ }^{1}$, Aditi Shah ${ }^{1}$, Monisha $\mathrm{H}^{1}$, Karthick RG ${ }^{1}$, Sharat Damodar ${ }^{1}$

${ }^{1}$ Hematology, ${ }^{2}$ Pulmonology, Narayana Health City Bangalore, Bangalore, India
Aims \& Objectives: To analyze the factors affecting the severity and outcomes of COVID19 in patients with hematological disorders.

Patients/Materials \& Methods: We reviewed clinical characteristics $\&$ outcomes of COVID-19 with hematological diseases at NH, Bangalore from June 2020 to September 2020. Outcome parameters (Severity \& mortality) were analyzed based on risk factors.

Results: Total 41 patients (21-Malignant, 19-Benign) were included in the study. Baseline characteristics are mentioned in the table. Expired patients mean D-dimer \& Ferritin at admission were higher ( $p=0.001,0.001)$ as compared to recovered group, there was no significant difference in mean ALC, ANC\& CRP. Mortality rate was higher in patients with D-dimer $>500(p=0.001)$, Ferritin $>500$ $(p=0.06)$ at admission. Other factors DM, HTN, ABO group, ANC, ALC \& CRP at admission did not correlate with morality. Overall mortality rate was $20 \%$ (Malignant $-33.3 \%$, Benign $-5.3 \%$ ), in patients with severe disease with malignancy mortality was higher as compared to benign disease $(p=0.02)$. In subgroup analysis of malignant population, Only D-dimer $>2000(p=0.04)$ was correlated with moratlity. Mortality rates were similar in ALL vs AML $(p=1)$, Active vs non active disease $(p=1)$, Leukemia Vs Lymphoma $(p=1) \&$ chemotherapy with in 1 month vs more than 1 month $(p=0.5)$. In overall study population mean D- dimer $(p=0.01)$, mean Ferritin $(p=0.04)$ were significantly higher in patients with severe disease. Patients with D-dimer $>2000(p=0.01)$, ferritin $>1500(p=0.07)$ were associated with severe disease. In sub group analysis of malignant group only D-dimer $>2000(p=0.04)$ was associated with severe disease. Other factors DM, HTN, ABO group, ANC, ALC, CRP, ferritin at admission, disease status \& type of disease, did not correlate with severity of disease.

Discussion \& Conclusion: Only D- dimer and Ferritin has prognostic value in disease severity and mortality among COVID 19 patients wit hematological disorders. But these cutoff values are higher in malignant diseases (D-dimer $>2000$, Ferritin $>1500$ ) as compared to benign diseases(D-dimer $>500$, Ferritin $>500$ ). Other factors such as DM, HTN,ABO group, elevated CRP, neutropenia, lymphopenia, disease status, type of disease \& duration of last chemotherapy are not associated with severity of disease or mortality. Supporting Document:

\begin{tabular}{|c|c|c|}
\hline & Malignant disease $(n-21)$ & Benign diseases $(n-19)$ \\
\hline Age (Median) & $19-70(45)$ & $26-72(55)$ \\
\hline Sex $M / F$ & $14 / 7$ & $13 / 6$ \\
\hline Diagnosis & $\begin{array}{l}\text { AML- 7 } \\
\text { ALL-3 } \\
\text { MPAL-1 } \\
\text { MDS-1 } \\
\text { CLL-1 } \\
\text { Hodgkins Iymphoma -3 } \\
\text { Nonhodgkins lymphoma - } 2 \\
\text { Multiple myeloma - } 3\end{array}$ & $\begin{array}{l}\text { Megaloblastic anemia - } 2 \\
\text { IDA-2 } \\
\text { Anemia of CKD - } 4 \\
\text { ITP - } 1 \\
\text { THALASSEMIA-1 } \\
\text { APLASTIC ANEMIA -2 } \\
\text { Other anemia }-3 \\
\text { Polycythemia - } 4\end{array}$ \\
\hline $\begin{array}{l}\text { Disease status - Active } \\
\text { Remission }\end{array}$ & \begin{tabular}{|l|}
$18(85.7 \% 6)$ \\
$3(14.3 \%)$
\end{tabular} & \\
\hline $\begin{array}{l}\text { Other comorbidities } \\
\text { DM } \\
\text { HTN } \\
\text { CAD/ CKD }\end{array}$ & \begin{tabular}{|l}
$6(28.5 \%)$ \\
$2(9.5 \%)$ \\
$0 / 1(4.7 \%)$
\end{tabular} & $\begin{array}{l}6(31.6 \%) \\
6(31.6 \%) \\
3(15.8 \%) / 4(21 \%)\end{array}$ \\
\hline $\begin{array}{l}\text { Neutropenia (ANC<1500) } \\
\text { tymphocytopenia (ALC< } 1000 \text { ) }\end{array}$ & $\begin{array}{l}7(33.3 \%) \\
10(47.6 \%)\end{array}$ & $\begin{array}{l}1(5.3 \%) \\
6(31.6 \%)\end{array}$ \\
\hline $\begin{array}{l}\text { CRP > } \\
\text { D-Dimer }>500 \\
\text { Ferritin }>500\end{array}$ & $\begin{array}{l}11(52.4 \%) \\
10(47.6 \%) \\
16(76.2 \%) \\
\end{array}$ & $\begin{array}{l}12(61.2 \%) \\
4(21 \%) \\
5(26.3 \%) \\
\end{array}$ \\
\hline $\begin{array}{l}\text { COVID } 19 \text { severity - } \\
\text { Asymptomatic } \\
\text { Mild } \\
\text { Moderate } \\
\text { Severe }\end{array}$ & $\begin{array}{l}4(19 \%) \\
5(23.8 \%) \\
3(14.3 \%) \\
9(42.8 \%)\end{array}$ & $\begin{array}{l}0 \\
8(41 \%) \\
6(31.5 \%) \\
5(26.4 \%)\end{array}$ \\
\hline $\begin{array}{c}\text { Response - Recovered } \\
\text { Expired }\end{array}$ & $\begin{array}{l}14(66.7 \%) \\
7(33.3 \%)\end{array}$ & $\begin{array}{l}18(94.7 \%) \\
1(5.3 \%)\end{array}$ \\
\hline
\end{tabular}

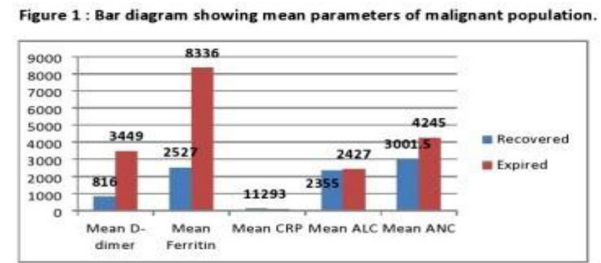




\section{T-Regulatory Cells in Paediatric Tuberculosis}

Ranjith Kumar $^{* 1}$, Richa Gupta ${ }^{2}$, Pooja Dewan ${ }^{3}$, Bineeta Kashyap ${ }^{4}$, Mrinalini Kotru ${ }^{2}$

${ }^{1}$ Junior resident, ${ }^{2}$ Pathology, ${ }^{3}$ Paediatrics, ${ }^{4}$ Microbiology, University college of medical sciences, Delhi, India

Aims \& Objectives: AIM: To evaluate T-regulatory cells(Tregs) in paediatric tuberculosis(TB) patients using flow-cytometry. OBJECTIVES: Primary objective: To estimate the number of Tregs in paediatric TB. Secondary objectives: (1) To compare the number of Tregs in paediatric TB patients with age matched healthy controls. (2) To compare Tregs between severe TB and non severe TB.

Patients/Materials \& Methods: Study enrolled 40 paediatric patients(less than 12 years) newly diagnosed as TB based on clinical, radiological and/or microbiological criteria along with 20 age matched healthy controls(without any history of TB contact in family). TB patients were also classified as severe TB and non severe TB. They were subjected to extensive haematological work-up and flowcytometry based work up for Tregs. Data was analyzed and statistically evaluated using SPSS-PC-19 version.

Results: Tregs were quantified by using two definitions as CD4CD25 high and CD4FOXP3 cells on CD4-T lymphocyte gating. CD4CD25 high Tregs (\%) ranged from 0.55 to 12.8 with a Mean \pm SD of $3.61 \pm 2.98$ in cases and 0.3-6.5 with a Mean \pm SD of $1.29 \pm 1.4$ in controls. CD4FoxP3 Tregs ranged from 0.02 to 13.44 with a Mean \pm SD of $3.56 \pm 2.76$ in cases and 0.33-2.59 with a Mean \pm $\mathrm{SD}$ of $1.57 \pm 0.58$ in controls. There was a significant difference between cases and control group $(p<0.05)$. No statistically significant difference was observed in Tregs between severe TB and non severe TB.

Discussion \& Conclusion: During TB, T cell responses helps in containing Mycobacterium tuberculosis infection invivo but it also causes collateral damage to host tissues. In order to control this damage, immune system sets various mechanisms to counteract the action of $\mathrm{T}$ cells, the most important of which are $\mathrm{T}$ regulatory cells. Therefore, our data of Tregs between TB patients and healthy controls in paediatric population suggests that during active TB and before anti-TB therapy, Tregs were increased in the peripheral blood. Supporting Document:

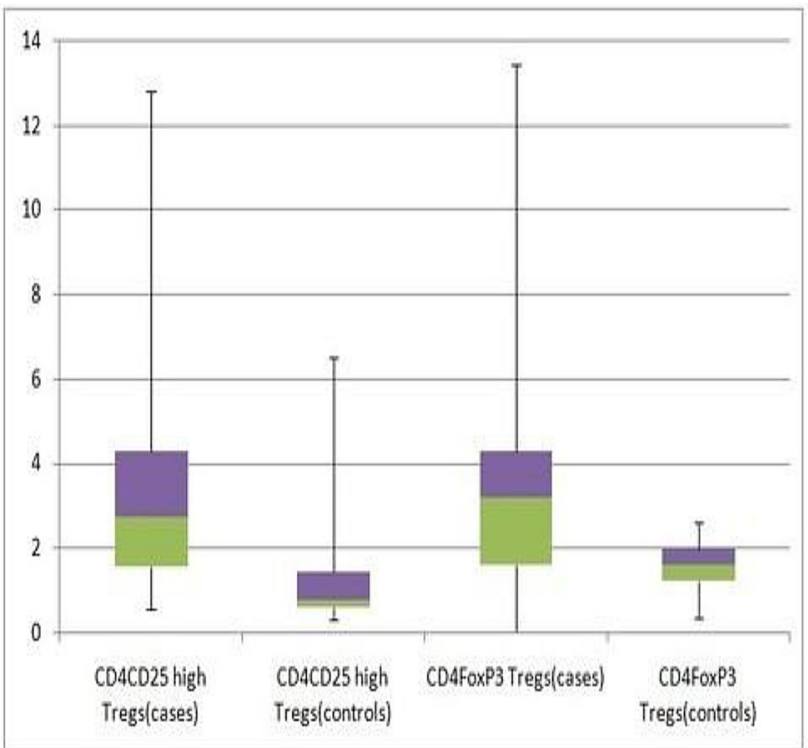

Lymphoma and Myeloma (Clinical)

\section{Clinical Spectrum and Challenges in Management of Patients with Primary CNS Lymphoma}

Charanpreet Singh $^{* 1}$, Arihant Jain ${ }^{1}$, Aastha Takkar ${ }^{2}$, Rajender Kumar $^{3}$, Deepesh Lad ${ }^{1}$, Alka Khadwal ${ }^{1}$, Pankaj Malhotra ${ }^{1}$, Vishali Gupta $^{4}$, Vivek Lal ${ }^{2}$, Ashim Das ${ }^{5}$, Bishan Radotra ${ }^{5}$, Gaurav Prakash ${ }^{1}$

${ }^{1}$ Internal Medicine, ${ }^{2}$ Neurology, ${ }^{3}$ Nuclear Medicine, ${ }^{4}$ Ophthalmology, ${ }^{5}$ Histopathology, PGIMER, Chandigarh, India

Aims \& Objectives: Primary CNS Lymphoma (PCNSL) involves the brain, eyes and rarely the spinal cord. We aimed to study the clinical profile and outcomes of patients with PCNSL at our centre.

Patients/Materials \& Methods: We retrospectively analysed the case records of patients diagnosed with a PCNSL over the course of 10 years from 2010 to 2020 .

Results: A total of 59 patients with PCNSL were identified in the patient records. The median age of the patients was 50 years (Range 22-85 years) with a M:F ratio of 1.4:1. The most common site of involvement was the corpus callosum, thalamus and deep structures of the brain $(50.9 \%)$ and $40 \%$ patients showed multiple lesions on MRI. Concomitant slit lamp examination was done in 25 patients and 8 had additional evidence of an intra-ocular lymphoma. IELSG risk stratification was available for 43 patients and most patients fell into the intermediate risk category $(67.4 \%)$. Only one patient was positive for HIV. Fifty-five patients received therapy at our center for newly diagnosed PCNSL. Thirty-six patients received Modified De-Angelis protocol \pm Rituximab with curative intent. Fourteen of these patients were unable to complete the protocol with the most common cause being development of methotrexate toxicity. Nineteen patients were given non HDMTx based therapy, either due to advanced age or financial limitations and this cohort received temozolamide based combination chemotherapy. Twenty-nine patients $(52.7 \%)$ were able to achieve a complete response at end of therapy. Overall response rates were higher in the HDMTx group $(69.4 \%$ vs $31.6 \%$, $p$-0.007). Fifteen patients experience a relapse during follow up and 22 patients died during follow up. The most common cause of mortality was relapse/progressive disease. The Median EFS and OS of the cohort was 29 months and 40 months respectively.

Discussion \& Conclusion: Apart from presenting the real-world outcome data of consecutively treated PCNSL patients from a tertiary care center in Northern India, we highlight the challenges in managing PCNSL with limited resources in an academic center. Based on our data, the inability of $1 / 3$ rd of the patients to complete De-angelis protocol points towards a need to develop a newer less toxic combination regimen which can be delivered to the patients with borderline fitness levels.

Impact of Depth of Response and MRD Status PostTransplant on Long Term Survival in Multiple Myeloma

Ritu Gupta ${ }^{* 1}$, Nupur Das ${ }^{1}$, Meetu Dahiya ${ }^{1}$, Lata Rani ${ }^{1}$, Anubha Gupta $^{2}$, Akanksha Farswan ${ }^{2}$, Omdutt Sharma ${ }^{1}$, Atul Sharma ${ }^{3}$, Lalit $\mathrm{Kumar}^{3}$

${ }^{1}$ Lab oncology, BRAIRCH, AIIMS, ${ }^{2}$ Department of ECE, Indraprastha Institute of technology, ${ }^{3}$ Medical Oncology, BRAIRCH, AIIMS, NEW DELHI, India

Aims \& Objectives: The number of multiple myeloma (MM) patients achieving complete response (CR) after autologous stem cell transplant (ASCT) has increased substantially with significant impact on long term survival. Herein, we have evaluated MM patients prior to 
and at Day 100 post-ASCT for the effect of disease response on patient survival.

Patients/Materials \& Methods: In this analysis, we evaluated MM patients who underwent ASCT at our centre from 2002 to 2019. The conventional response assessment of the patients was done as per the IMWG criteria for response evaluation. Additionally, multi parametric flowcytometry based minimal residual disease (MRD) assessment was carried out on bone marrow aspirates collected at Day 100 post ASCT. A total of at least 5 million events were acquired and the data was analyzed using Kaluza v2.0 software.

Results: A total of 340 patients of MM who received ASCT at our centre were analysed for the purpose of this study. The risk of progression as well as mortality was higher in patients with $\leq \mathrm{PR}$ as compared to patients who were having response $\geq$ VGPR. $212(62 \%)$ patients improved their response at day 100 post ASCT. The outcome was inferior in patients who did not show any upgradation in their response after ASCT than those showing improvement in response after ASCT in terms of both PFS (166 Vs 312 weeks; HR = 2.2; $\mathrm{CI}=1.58-3.08 ; p<0.005)$ and $\mathrm{OS}(359$ Vs 867 weeks; $\mathrm{HR}=2.98$; $\mathrm{CI}=1.8-4.93 ; p<0.005)$. MRD status at day 100 post ASCT was evaluated in $184 / 340$ patients; $35 \%(n=64)$ were MRD positive and $65 \%(\mathrm{n}=120)$ were MRD negative. MRD positive patients had inferior $\mathrm{PFS}(179$ Vs 324 weeks; $\quad \mathrm{HR}=2.73 ; \quad \mathrm{CI}=1.58-4.72$; $p<0.005$ ) and OS (Not Reached in both groups; $\mathrm{HR}=3.37$; $\mathrm{CI}=$ $1.44-7.88 ; p=0.01$ ) than MRD negative group.

Discussion \& Conclusion: There is a distinct benefit in progression free as well as overall survival commensurate with increasing depth of response before as well as after ASCT in multiple myeloma.Nonetheless our study also demonstrates that post-transplant MRD negativity is an important prognostic factor in MM for long term survival in transplant recipients and signifies the utility of MRD as surrogate endpoint in MM.

Disclosure of Interest: None Declared.

\section{Lymphoma and Myeloma (Laboratory)}

\section{Clinical Implications of Post-Therapy Immunomodulation in Multiple Myeloma Assessed by Flowcytometry}

Nupur Das ${ }^{* 1}$, Ritu Gupta ${ }^{1}$, Meetu DAHIYA ${ }^{1}$, Lata RANI ${ }^{1}$, Atul Sharma $^{2}$, Lalit Kumar ${ }^{2}$, Anubha Gupta ${ }^{3}$, OmDutt Sharma ${ }^{1}$

${ }^{1}$ Lab Oncology, ${ }^{2}$ Medical Oncology, BRAIRCH, AIIMS, ${ }^{3}$ Department of ECE, Indraprastha Institute of Information Technology, NEW DELHI, India

Aims \& Objectives: In this study, bone marrow plasma cells(BMPC) were evaluated for immunophenotypic aberrancies at baseline and during the course of therapy in patients with multiple myeloma (MM).Furthermore, diagnostic immunophenotype (IPT) was compared with post-treatment IPT features to assess the occurrence of immunomodulation after exposure to anti-myeloma therapy.

Patients/Materials \& Methods: Patients diagnosed and/or treated as MM at our centre over a period of four years $(2016-2019)$ and for whom minimal residual disease (MRD) was positive after treatment were retrospectively evaluated in this study. Bone marrow aspirates collected in EDTA were processed for flow cytometric immunophenotyping as per the Euroflow protocol. Two-tube 8-color antibody combination or a single tube 10 -color combination was used to assess the BMPC. A total of at least 5 million events were acquired and the data was analysed using Kaluza v2.0 software.PCwere gated based on expression pattern of CD38, CD138 and CD45. An aberrant plasma cell cluster was defined by presence of $>30$ plasma cells with 3 or more aberrant phenotypes.
Results: A total of 302 samples from 151 patients were evaluated at baseline and follow-up time points. At baseline, the most frequently aberrant IPT markers in decreasing order of frequency were CD19 (88.74\%), CD27 (83.44\%), CD45 (79.47\%), CD56 (70.86\%), CD38 (64.90\%), CD81 (53.64\%), CD117 (10.59\%) and CD138 (9.93\%). At the MRD time-point, the most frequent aberrant IPT markers in decreasing order of frequency were CD19 (84.76\%), CD45 (81.45\%), CD56 (80.79\%), CD27 (76.82\%), CD38 (63.57\%), CD81 (36.42\%), CD138 (13.90\%) and CD117 (12.58\%). Comparison of baseline IPT with post induction MRD positive samples showed loss of CD81 (17.21\%), CD38 (13.90\%), CD45(11.25\%), CD27 (7.28\%), CD19 (5.96\%) and CD56 (4.63\%). Post-therapy gain of expression was most marked in CD38 (15.23\%) followed by CD27 (13.90\%), CD56 (13.24\%), CD81 (10.59\%) and CD45 (9.27\%) cases.

Discussion \& Conclusion: Flowcytometry based abnormal plasma cell identification greatly contributes to the diagnosis of MM, however MRD assessment is challenged by the process of immunomodulation after chemotherapy and thus, simultaneous analysis of multiple markers is required for reliable MRD assessment.

\section{Prognostic Significance of T-Cells and Macrophages in the Tumor Microenvironment of Nodal DLBCL}

Soundarya Ravi*1, Sree Rekha ${ }^{1}$, Debdatta Basu ${ }^{1}$, Smita Kayal ${ }^{2}$ ${ }^{1}$ Pathology, ${ }^{2}$ Medical Oncology, JIPMER, Puducherry, India

Aims \& Objectives: To identify the association of FOXP3 positive T-regulatory cells, cytotoxic T-cells and macrophages with the immunophenotypic subtypes, clinicopathological characteristics, treatment response and survival in nodal diffuse large B-cell lymphoma (DLBCL) patients.

Patients/Materials \& Methods: The clinicopathological and treatment data of 83 DLBCL patients diagnosed and treated at our institute from January 2015 to December 2018 were collected and these patients were followed up till June 2020. Immunophenotypic subtyping of cases was done according to Hans algorithm. CD8, FOXP3 and CD68 immunostains were performed to highlight the cytotoxic T-cells, T-regulatory cells and macrophages respectively on the lymph node biopsies. The distribution of these cells was analyzed in the biopsy. The association of these cells with the immunophenotypic subtypes, clinicopathological characteristics and treatment outcome was studied using independent student t-test and Chi square test. Survival analysis was done using multivariate Cox regression analysis.

Results: There was no significant association between the cells of the tumor microenvironment and the immunophenotypes. DLBCL cases with higher percentage of CD3 positive T-cells and CD8 positive cytotoxic T-cells had significant association with attainment of complete response to treatment. In addition, CD8 positive T-cells more than $6.5 \%$ proved to be an independent predictor of treatment outcome $(p=0.003)$. Multivariate Cox regression survival analysis revealed that cases with 'good' R-IPI prognostic score and 'high CD68 positive macrophages in tumor microenvironment' had significantly longer overall survival.

Discussion \& Conclusion: This study is the first Indian study done to assess the prognostic significance of tumor microenvironment in DLBCL. This study suggests that cytotoxic T-cells and macrophages in the tumor microenvironment of nodal DLBCL have an antitumor effect. Increased cytotoxic T-cells were significantly associated with complete response to treatment. Higher number of macrophages correlated significantly with better overall survival. 


\section{Prognostic Significance of Cytogenetic Aberrations in Bone Marrow of B-Cell Non Hodgkins Lymphoma Patients}

Hemani Jain ${ }^{* 1}$, Dhanlaxmi Shetty ${ }^{1}$, Purvi Mohanty ${ }^{1}$, Hasmukh Jain ${ }^{2}$, Bhausaheb Bagal ${ }^{2}$, Avinash Bonda ${ }^{2}$, Sachin Punatar ${ }^{3}$, Anant Gokarn ${ }^{3}$, Lingraj Nayak ${ }^{2}$, Manju Sengar ${ }^{2}$, Navin Khattry ${ }^{3}$, Papaguddi Subramanian $^{4}$

${ }^{1}$ Cancer Cytogenetics Department, ACTREC, Tata Memorial Center, Navi Mumbai, ${ }^{2}$ Medical Oncology Department, Tata Memorial Hospital, Mumbai, ${ }^{3}$ Medical Oncology Department, ${ }^{4}$ Hematopathology Department, ACTREC, Tata Memorial Centre, Navi Mumbai, India

Aims \& Objectives: Present study was carried out to identify and investigate the incidence of genetic changes which aid in classification and prognosis of B-NHL patients with bone marrow involvement.

Patients/Materials \& Methods: This study included 71 bone marrow specimens of B-cell NHL cases ( $>18$ years of age) referred to Cancer Cytogenetics Dept between January 2019-September 2020. Molecular cytogenetic (FISH) study was carried out using Metasystems LSI IGH, Abott Molecular LSI c-MYC, BCL6 break apart rearrangement probes \& LSI IGH-CCND1, IGH-BCL2 dual colour dual fusion translocation probes. A total of 200 interphase cells were analyzed. The cases were diagnosed according to the 2016 World Health Organization (WHO) classification criteria. Treatment response was assessed at the end of the treatment.

Results: A total of $29 / 71(41 \%)$ cases showed cytogenetic rearrangement. Of these 29 cases, BCL2, c-MYC and CCND1 rearrangement was observed in 7\% (5/71), 3\% (2/71) and 18\% (13/ 71) cases respectively. BCL6 rearrangement was observed in $2(3 \%)$ cases with large B-cell lymphoma. Two cases (3\%) showed concurrent rearrangement of BCL2 and/or CCND1 with BCL6 (without MYC involvement) \& one with BCL2, BCL6 and c-MYC rearrangement, thus confirming as "triple hit lymphoma". IGH translocations were observed in $3(10 \%)$ cases. One case $(1 / 71)$ diagnosed as low grade B-cell NHL revealed IGH-BCL2: $\mathrm{t}(14 ; 18)$, thus classifying the patient as follicular lymphoma. Median age of the cytogenetically rearranged cases was 49 years (range-18-80 years) with male predominance. All the patients had high LDH. These patients received rituximab based chemoimmunotherapy Discussion \& Conclusion: Significant morphological, clinical and biological heterogeneity of B-NHL confirms the existence of several subtypes of the disease with different clinical course. Recurrent chromosomal aberrations are found in individual subgroups which enables risk stratification of B-NHL patients. Although these patients are treated with high-dose chemotherapy, they have very poor prognosis and the disease progresses rapidly. Data from the retrospective and prospective studies will be needed to know the prevalence and characterize individual lymphomas in India. Molecular cytogenetics (FISH) should be made an integral part of routine assessment as it provides valuable information and classifies the disease which helps in better understanding of the pathogenesis of B- NHL and design risk adapted therapies.

\section{Immunophenotypic Comparison of Abnormal and Normal Plasma Cells: A Tertiary Care Center Experience}

Asish Rath ${ }^{* 1}$, Tribikram Panda ${ }^{1}$, Rishi Dhawan ${ }^{1}$, Mukul Agarwal ${ }^{1}$, Pradeep Kumar ${ }^{1}$, Ganesh K. V. ${ }^{1}$, Jasmita Dass ${ }^{1}$, Tulika Seth ${ }^{1}$, Manoranjan Mahapatra ${ }^{1}$, Seema Tyagi ${ }^{1}$

${ }^{1}$ Haematology, AIIMS, New Delhi, New Delhi, India

Aims \& Objectives: To study immunophenotype of abnormal and normal plasma cells.

Patients/Materials \& Methods: Bone marrow aspirates from 34 multiple myeloma cases, 2 MGUS cases and 2 MGRS cases diagnosed in the department of hematology, AIIMS, New Delhi were evaluated for APCs. 7 cases ( 3 cases of metastatic carcinomas and 4 cases of monoclonal gammopathy with significant NPCs) were evaluated for immunophenotype of NPCs. Flow cytometric evaluation was done by a bulk lysis protocol and 6-color antibody panel on BD FACS Canto II (BD Biosciences, San Jose, CA). Analysis was done on BD FACSDiva 8.0.

Results: Mean age of MM patients and MGUS/MGRS patients was 57.8 (mean \pm 2 SD, $42.8-72.8$ years). Male to female ratio in MM and MGUS/MGRS cases were 2.1 and 1:1 respectively. The median number of plasma cells as determined by morphology (Bone marrow plasma cell-BMPC) and flowcytometry (Flowcytometry plasma cellsFPC) were $44.5 \%$, (range, 4\%-96\%) and $15.5 \%$ (range, $0.1 \%-75.9 \%$ ) respectively. There was moderate positive correlation between BMPC and FPC $(\mathrm{r}=0.52, p<0.0013)$. Percentage of normal plasma cell in MM cases ranged from $0.1 \%$ to $3.4 \%$ of total PCs, where as in MGUS/MGRS cases it varied from $11 \%$ to $50 \%$ of total PCs. Results (attached as supporting document).

Discussion \& Conclusion: Our study concluded that both CD81 and CD19 are very sensitive and specific markers to identify APCs. In combination with CD45 and CD56 precise quantification of APCs can be done. A significant higher proportion of NPCs differentiates monoclonal gammopathies from MM cases.

Supporting Document:

\begin{tabular}{|c|c|c|c|c|c|}
\hline Markers & Population & $\begin{array}{l}\text { Strong } \\
\text { positive }\end{array}$ & Dim/partial & Negative & $\begin{array}{l}\text { Sensitivity(\%) } \\
\text { Specificity }(\%)\end{array}$ \\
\hline \multirow[t]{2}{*}{ CD19 } & APCs $(n=38)$ & 0 & 4 & 34 & \multirow[t]{2}{*}{$100 / 85.7$} \\
\hline & NPCs $(n=7)$ & 6 & 0 & 1 & \\
\hline \multirow[t]{2}{*}{ CD45 } & $\operatorname{APCs}(n=38)$ & 4 & 9 & 25 & \multirow[t]{2}{*}{$89.4 / 57.1$} \\
\hline & NPCs $(n=7)$ & 4 & 3 & 0 & \\
\hline \multirow[t]{2}{*}{ CD56 } & $\operatorname{APCs}(n=38)$ & 22 & 7 & 9 & \multirow[t]{2}{*}{ 76.3/57.1 } \\
\hline & $\operatorname{NPCs}(n=7)$ & 0 & 3 & 4 & \\
\hline \multirow[t]{2}{*}{ CD117 } & $\operatorname{APCs}(n=36)$ & 7 & 7 & 22 & \multirow[t]{2}{*}{$38.8 / 100$} \\
\hline & $\operatorname{NPCs}(n=7)$ & 0 & 0 & 7 & \\
\hline \multirow[t]{2}{*}{ CD81 } & $\operatorname{APCs}(n=37)$ & 1 & 03 & 33 & \multirow[t]{2}{*}{$97.3 / 85.7$} \\
\hline & $\operatorname{NPCs}(n=7)$ & 6 & 1 & 0 & \\
\hline \multirow[t]{2}{*}{$\mathrm{CD} 27$} & $\operatorname{APCs}(n=37)$ & 6 & 6 & 25 & \multirow[t]{2}{*}{$83.7 / 57.1$} \\
\hline & $\operatorname{NPCs}(n=7)$ & 4 & 2 & 1 & \\
\hline
\end{tabular}




\section{Miscellaneous (Laboratory Haematology)}

\section{Role of Newer Hematological Parameters in Diagnosis of Sepsis: The Jigsaw Puzzle with Missing Pieces}

\author{
Anish Sharma*1, Kanwaljeet Singh ${ }^{2}$, Ankur Ahuja ${ }^{3}$, Venkatesan \\ Somasundarum $^{3}$, Tathagat Chatterjee ${ }^{1}$ \\ ${ }^{1}$ Department of Lab Sciences and Molecular Medicine, Army \\ Hospital(Referral \&Research), ${ }^{2}$ Dept of Lab Sciences \& \\ Molecular Medicine, ${ }^{3}$ Department of Lab Sciences and Molecular \\ Medicine, AHRR, Delhi Cantt, New Delhi, India
}

Aims \& Objectives: We are presenting this study to highlight the significance of newer parameters like Neut-X, Neut-Y, Neut-Z in sepsis and to do comparative analysis of these biomarkers with CRP in cases of Sepsis.

Patients/Materials \& Methods: In this cross sectional observation study, 79 cases of blood culture proven sepsis and 161cases of healthy cases were study subjects. Analysis of toxic granulation in neutrophils was done in the form of Neut-X (sideward scatter of light) and Neut Y (Sideward fluorescence) in hematology analyser ADVIA 2120I and normal reference range was calculated from the control samples. Neut-Z is the sum of Neut-X and Neut-Y. CRP was analysed using commercially available kit using automated analyser.

Results: Neut $-\mathrm{Z}$ had a better sensitivity $(88.6 \%)$ and specificity (91.9\%) compared to Neut-X and Neut-Y. CRP gave a better sensitivity $(91.9 \%)$ and specificity(97.5\%) compared to Neut -X,Neut -Y and Neut-Z. A combination of both showed increased sensitivity and specificity as compared to individual tests.

Discussion \& Conclusion: Although sepsis is a major cause of morbidity and mortality in ICU setting, its pathogenesis is poorly understood. Hence there is an urgent need of identifying a reliable biomarker for sepsis diagnostics. We want to conclude that combination of the above mentioned markers will help in the early diagnosis of sepsis and hence timely management to reduce the mortality and morbidity.

\section{Comparison of CD71 and Glycophorin A to E-Cadherin by Immunohistochemistry in Bone Marrow Biopsy for Assessing the Erythroid Lineage \\ Anandhi Anbalagan*1, Debdatta Basu ${ }^{1}$, Rakhee Kar ${ }^{1}$ \\ ${ }^{1}$ Pathology, JIPMER, Puducherry, Puducherry, India}

Aims \& Objectives: Introduction:Erythropoiesis gets affected in various haematological conditions. Erythroid precursors need to be delineated from other immature cells whenever morphological difficulty arises. Various immunohistochemical stains are available for this purpose. E-Cadherin, a cell to cell adhesion molecule is marker proven to be highly specific to erythroid lineage in bone marrow biopsies. CD71, a transferrin receptor and Glycophorin A, a transmembrane sialoglycoprotein are known to express in erythroid lineage.

Aim and objective: To compare the immunohistochemical staining pattern of CD71 and Glycophorin A with E-Cadherin in normal and abnormal bone marrow biopsies.

Patients/Materials \& Methods: We studied 159 consecutive bone marrow biopsies with normal or suppressed erythropoiesis, erythroid hyperplasia and dyserythropoiesis. Immunohistochemistry was done with E-Cadherin, CD71 and Glycophorin A. The distribution and pattern of positivity in spectrum of erythroid cells were studied. We have taken E-Cadherin as gold standard and calculated the sensitivity and specificity for CD71 and Glycophorin A.
Results: Overall sensitivity and specificity of CD71 was $97 \%$ and $92 \%$ with a positive predictive value and negative predictive value of 99\% and $75 \%$ respectively. Glycophorin A showed a sensitivity and specificity of $84 \%$ and $100 \%$ respectively and positive predictive value of $100 \%$ and a low negative predictive value of $37 \%$ respectively. CD 71 highlighted early precursors (Sensitivity- 93\% and specificity-92\%) better than Glycophorin A (Sensitivity-8\% and specificity- $1 \%$ ), the latter stained late normoblasts mainly (Sensitivity-89\% and specificity- $87 \%$ ) and also the RBCs.

Discussion \& Conclusion: Our results demonstrate that CD71 has higher sensitivity and specificity in highlighting both early and late erythroid precursors than Glycophorin A and could be used as a good substitute for E-cadherin. Glycophorin A did not prove to be a useful marker in highlighting early erythroid precursors in a bone marrow trephine biopsies and was often associated with obscuration of precursor cells because of staining the red blood cells.

\section{D-Dimer a Potential Biomarker for Severity of Covid 19 Infection: Observational Study from a Tertiary Care Centre in India}

\author{
Vidisha Mahajan ${ }^{* 1}$, Shanaz Khodaiji ${ }^{1}$, Binu Jacob ${ }^{2}$, Ajit Yadav ${ }^{1}$ \\ ${ }^{1}$ Hematology, P.D.Hinduja Hospital, ${ }^{2}$ Hematology, P.D Hinduja \\ Hospital, Mumbai, India
}

Aims \& Objectives: The beginning of 2020 shook the world with the onset of a pandemic caused by SARS-coronavirus disease 2019 (COVID-19). As the disease outcome data from various parts of the world unfolded, disturbances in the pathways of coagulation emerged as a major cause of morbidity and mortality and reports of utility of d-dimer as a prognostic marker emerged. We present an observational retrospective analysis of value of d-dimer in 496 Covid-19 patients in a tertiary care hospital and its correlation with disease severity.

Patients/Materials \& Methods: Records of 496 patients who presented to the hospital between May 2020 to September 2020 with a confirmed diagnosis of COVID-19 were retrospectively analysed. COVID-19 positivity was confirmed based on RT-PCR in all patients and d-dimer was estimated by immunometric assay with a reference range of $0-0.35 \mathrm{mg} / \mathrm{L}$ (Instrument -Nephle plus one). The patients were divided into mild/moderate, severe and the non-survivor outcome groups according to the MoHFW, India guidelines. The Kruskal-Wallis one-way ANOVA was used to evaluate the statistical significance of the D-dimer values at admission and outcome in the various groups. ROC curves were derived to estimate cut off value of d-dimer to differentiate between outcome groups.

Results: 496 patients were studied retrospectively from May 2020 to September 2020.The patients age ranged from 19 to 91 years of which 157 female and 340 males. The D-dimer values of the 3 groups on admission and outcome was compared and was significantly higher in patients belonging to the severe $(2.64+3.39)$ as compared to the mild/moderate $(1.05+1.68)$ group of patients ( $p$ value $<0.00001$ ). The $p$ value for the $\mathrm{D}$-dimer levels at admission and outcome for the non-survivor group were significant by the Wilcoxon signed rank test. ROC curves showed a high accuracy of the D-dimer to discriminate between the survivor and the non-survivor group at outcome (cut off $>2.16 \mathrm{D}-\mathrm{DU} \mathrm{mg} / \mathrm{l}, \mathrm{Se}=82.05$, $\mathrm{Sp}=80.42$, AUC $=0.88)$. The ROC curve with a cut off of $>$ $1.31(\mathrm{Se}=58, \mathrm{Sp}=81, \mathrm{AUC}=0.72)$ at discharge between the mild/moderate and severe group was obtained.

Discussion \& Conclusion: Elevated D-dimer appears to be good predictor of severity of the Covid 19 infection and in addition can be used for monitoring of these patients. 


\section{Immunophenotypic Evaluation of Reactive Lymphoid Cells by Flow Cytometry}

Priyanka Goel $^{* 1}$, Seema Acharya ${ }^{1}$, Sana Ahuja ${ }^{1}$

${ }^{1}$ Pathology, Shri guru ram rai institute of medical and health sciences, Dehradun, India

Aims \& Objectives: (1) To study the various lymphoid populations in reactive lymphoid cells by flowcytometry. (2) To assess percentages of $\mathrm{B} \& \mathrm{~T}$ cell immunophenotypic outliers in reactive lymphoid cell populations Lymphadenopathy is a common presentation in clinical practice. Fine needle aspiration(FNA) is the safest, accurate and minimally invasive diagnostic modality. Its role in diagnosis of leukemia/lymphomas remains controversial. The accuracy rate of Fine needle aspiration cytology(FNAC) has been found to be $79-90 \%$ for high grade NHL and classical HL but is significantly limited in diagnosis of low grade NHL. Heterogeneous populations of lymphocytes often present in lymphoid tissue involved with a malignant lymphoma and a sampling difference in the specimens submitted for histologic examination and flow cytometry cause interpretational complexities. When coupled with immunophenotyping by flowcytometry, the diagnostic accuracy of fine needle aspiration is improved. Assessment of lymphoid cells involve detecting of immunophenotypic $\mathrm{B}$ and $\mathrm{T}$ cell outliers which may also be present in reactive hyperplasia. Patients/Materials \& Methods: A prospective hospital based study was conducted on 30 patients who presented with lymphadenopathy after approval from Institutional Ethics Committee. Patients consent was taken.

Inclusion criteria: (1) Benign lymph node aspirate samples. (2) Benign lymph node scrapings from fresh excision biopsies which were confirmed to be reactive on histopathological examination(Nonlymphoma). (3) Lymphoid cell rich aspirates of metastatic carcinomas.

Exclusion criteria: (1) Samples suggestive of hematolymphoid malignancy on cytomorphology. Lymphoid aspirate was taken in RPMI and evaluation was done by flow cytometry for the overall antigen distribution with focus on outliers.

Results: Various cell populations were assessed in reactive lymph nodes. The lymphoid population in aspirate comprised of $42.18 \%$ of all cells with predominance of T cells $(62.75 \%)$ over B cell $(16.28 \%)$ population. B cell outliers(76.67\%) (23 cases) and $\mathrm{T}$ cell outliers $(60 \%)(18$ cases) were noted which subsequently did not progress to lymphoma.

Discussion \& Conclusion: Outlier flow immunophenotypes are identified in benign reactive lymph nodes. Hence, caution should be excercised not to overdiagnose lymphoma by flowcytometric analysis.

\section{Myeloproliferative Neoplasm}

\section{Diagnostic and Prognostic Utility of Flowcytometric Enumeration of H-Micl Expressing Circulating Leukemic Stem Cells in BCR-ABL1-Negative Myeloproliferative Neoplasms}

Gurleen Oberoi ${ }^{* 1}$, Rishi Dhawan ${ }^{1}$, Jasmita Dass ${ }^{1}$, Suman Lata ${ }^{1}$, Seema Tyagi ${ }^{1}$, Manoranjan Mahapatra ${ }^{1}$

\section{${ }^{1}$ Hematology, AIIMS, New Delhi, India}

Aims \& Objectives: (1) To evaluate the utility of flowcytometric enumeration of circulating CD34 + CD38- h-MICL + cells in the diagnosis of BCR-ABL1 negative Myeloproliferative Neoplasms. (2) To correlate the level of aberrant hMICL expression with DIPSS score. (3) To define a discriminatory cut-off of aberrant hMICL expression at the stem cell level for accurate identification of PMF patients including early prefibrotic myelofibrosis.

Patients/Materials \& Methods: A total of 54 MPN patients, including 15 Polycythemia Vera (PV), 13 Essential thrombocythemia (ET), 13 Overt Myelofibrosis (MF), 8 early prefibrotic stages of Primary MF, and 5 Post-PV/ET MF, who presented in the clinics of hematology department of AIIMS, New Delhi were included in the study. Bone marrow examination and mutational analysis were carried out. Peripheral blood flowcytometric evaluation was done. An analysis template based on 20 control samples were prepared.The subsets analysed were CD34 + (hematopoietic stem cell enriched population), $\mathrm{CD} 34+\mathrm{CD} 38+$ (committed progenitors), $\mathrm{CD} 34+$ CD38- (stem cells), and the aberrant CD34+CD38hMICL + population. The aberrant hMICL expression on CD34 + CD38- subsets were correlated with DIPSS score.

Results: Our study included 54 MPN patients (42 males, 12 females) with a median age of 49.5 years (range: $16-72$ years). A significant difference in distribution of proportion of CD34 + CD38-hMICL + (\%)among the MPN sub-groups was found. The median of CD34 + CD38-hMICL + cells subset in Overt MF, early stage/prefibrotic-MF and Post-PV/ET MF group was 2.8\%, $4.15 \%$ and $3 \%$. respectively, which was significantly more than PV and ET (median $0 \%, p<0.001)$.Discriminatory cut off of Proportion of CD34+ CD38-hMICL + subset $\geq 0.9$ predicted MF with highest sensitivity and specificity of $88 \%$ and $89 \%$ respectively(Table 1 ). This cut off is also found to be applicable to differentiate early/prefibrotic MF from ET. We found a statistically significant positive correlation $($ rho $=$ $0.41, p=0.036$ ) between proportion of CD34 + CD38-hMICL + cells and DIPSS score. For every 1 unit increase in DIPSS Score, CD34 + CD38-hMICL + cells increased by $1.72 \%$.

Discussion \& Conclusion: Subcategorization of MPNs by morphology is challenging due to overlapping features and interobserver reproducibility. Driver mutations do not differentiate subtypes. Distinguishing ET from prefibrotic MF is especially challenging. In this study we propose CD34 + CD38- hMICL + subset is reliable for subcategorization of BCR ABL1-negative MPNs. We defined a cut-off to discriminate MF from non-MF MPNs, especially distinguishing prefibrotic phase of MF from ET. In addition, the prognostic value of CD34 + CD38- hMICL subset vis-a-vis DIPSS score was determined. Thus enumeration of circulating CD34 + CD38h-MICL + cells holds a potential as a diagnostic test, tool for prognostication and a potential target for therapy.

Supporting Document:

\begin{tabular}{|c|c|c|c|c|c|}
\hline Variable & Sensitivity & Specificity & PPV & NPV & $\begin{array}{l}\text { Diagnostic } \\
\text { Accuracy }\end{array}$ \\
\hline $\begin{array}{l}\text { CD34+Cells } \quad(\%) \\
\text { (Cutoff: } 1.1 \text { by ROC) }\end{array}$ & $69.2 \%(48-86)$ & $82.1 \%(63-94)$ & $78.3 \%(56-93)$ & $74.2 \%(55-88)$ & $75.9 \%(62-87)$ \\
\hline $\begin{array}{l}\text { Proportion of } \\
\text { CD34+CD38- Cells } \\
\text { of CD34+Cells ( } \%) \\
\text { (Cutoff: } 15.3 \text { by } \\
\text { ROC) }\end{array}$ & $76.9 \%(56-91)$ & $60.7 \%(41.78)$ & $64.5 \%(45-81)$ & $73.9 \%(52.90)$ & $68.5 \%(54-80)$ \\
\hline $\begin{array}{l}\text { Proportion of } \\
\text { CD34+CD38+ Cells } \\
\text { of CD34+Cells (\%) } \\
\text { (Cutoff: } 84.7 \text { by } \\
\text { ROC) }\end{array}$ & $76.9 \%(56-91)$ & $60.7 \%(41-78)$ & $64.5 \%(45-81)$ & $73.9 \%(52.90)$ & $68.5 \%(54-80)$ \\
\hline $\begin{array}{l}\text { Proportion } \\
\text { CD34+CD38. } \\
\text { hMICL+ } \\
\text { CD34+CD38. Cells } \\
\text { (\%) (Cuttoft: } 0.9 \text { by } \\
\text { ROC) }\end{array}$ & $88.5 \%(70-98)$ & $89.3 \%(72.98)$ & $88.5 \%(70.98)$ & $89.3 \%(72.98)$ & $88.9 \%(77-96)$ \\
\hline
\end{tabular}




\section{CD26 Expression on Circulating CD34+/CD38- Progenitor Population is a Reliable Tool for the Rapid Flow Cytometric Diagnosis of Chronic Myeloid \\ Leukemia}

Khaliqur Rahman ${ }^{1}$, Priyanka Gupta ${ }^{* 1}$, Ruchi Gupta ${ }^{1}$, Dinesh Chandra $^{1}$, Manish Singh ${ }^{1}$, Anshul Gupta ${ }^{1}$, Sanjeev Yadav ${ }^{1}$, Rajesh Kahsyap $^{1}$, Soniya Nityanand ${ }^{1}$

\section{${ }^{1}$ Hematology, SGPGI, Lucknow, India}

Aims \& Objectives: The current focus in chronic myeloid leukemia (CML) research is identification and characterization of leukemic stem cells (LSCs). These cells reside within the CD45(dim)/CD34 +/ CD38-/Lin- fraction and can be differentiated from normal hematopoietic stem cells (HSCs) by expression of some newly identified markers like CD25, CD26 (dipeptidylpeptidase IV), ILR1P and CD93. This study was aimed to evaluate the expression of CD26 on leukemic stem cells of CML using flow cytometry and its utility as a rapid diagnostic tool for CML.

Patients/Materials \& Methods: Peripheral blood samples from suspected cases of CML and other hematolymphoid neoplasm were evaluated for the expression of CD26 on stem cells using a four color antibodies cocktail: CD45/CD34/CD38/CD26. A Lyse stain wash protocol was followed with an aim to achieve 2 million events per tube. Positive expression was considered based on the fluorescence minus one (FMO) control and internal positive controls (CD26 pos T Lymphocytes). Diagnosis of CML was confirmed by presence of BCR-ABL1 fusion by fluorescent in situ hybridisation (FISH) and/or BCR-ABL1 fusion transcript analysis by PCR.

Results: A total of 45 cases were evaluated which included 33 cases of CML and 12 cases of other neoplasm (Ph positive Acute Lymphoblastic Leukaemia $(n=4)$, Acute Myeloid Leukemia $(n=3)$, Primary Myelofibrosis $(n=4)$, Essential thrombocythemia $(n=1)$. The median (range) proportion of CD34+/CD38- component of total leukocytes was $0.3 \%$ (0.05 to 1.6$)$ for the CML and 6.3\% (0.3$31.87 \%$ ) for non CML cases, respectively. The expression of CD26 on CD34 +/CD38- population was detectable in all cases of CML. None of the other haematological neoplasm, including the $\mathrm{Ph}$ positive ALLs demonstrated the expression of CD26. CD26 was expressed in $69.3 \%(10-96.1 \%)$ of CD34 +/CD38- population of CML. Interestingly, One case, which was negative for BCR-ABL1 fusion transcript (p210 and p190) by RT-PCR but positive by FISH (? Atypical transcript) also expressed CD26 (28\%) on the CD34 +/CD38population.

Discussion \& Conclusion: We confirm the specificity of CD26 as a marker for CML LSCs. Flow cytometric analysis of CD26 expression on peripheral blood CD34+/CD38- population can be used as a rapid, reliable and powerful tool for the diagnosis of CML.

\section{Patterns of Ruxolitinib Failure in Patients of Philadelphia Negative Myeloproliferative Neoplasms: Real World Experience from Two Tertiary Care Centers in India}

\author{
Va Arun ${ }^{* 1}$, Arihant Jain ${ }^{1}$, Harshit Khurana ${ }^{2}$, Pankaj Malhotra ${ }^{1}$
}

${ }^{1}$ Hematology, PGIMER, Chandigarh, ${ }^{2}$ Hematology, Command Hospital Pune, Pune, India

Aims \& Objectives: To study patterns of ruxolitinib failure in patients of philadelphia negative myeloproliferative neoplasms. Patients/Materials \& Methods: We retrospectively analyzed the clinical characteristics and outcomes of all $\mathrm{Ph}$ negative MPN patients treated with Ruxolitinib from two tertiary care centers in India till date. Previously published criteria were used to define best spleen response and symptom response(MPN-SAF TSS).

Results: Among the 27 patients who received Ruxolitinib, 18 patients had primary myelofibrosis, 6 patients had post Polycythemia Primary myelofibrosis (Post PV-PMF) while 3 patients received Ruxolitinib for Hydroxyurea intolerant/resistant Polycythemia Vera. Among the 21 patients with Myelofibrosis who had baseline data available, 5 patients had INT-1 IPSS, 10 patients belonged to Intermediate 2 IPSS category, while 6 after patients had high risk IPSS. Among the patients who had Int-2/High risk PMF, the median time from diagnosis to initiation of Ruxolitinib was 5 months. At a median follow up of 49 months after ruxolitinib initiation, 55\% patients had best spleen response. Overall $70 \%$ patients had achieved a symptom response. Among the 27 patients who were started on Ruxolitinib, 6 patients (22\%) had Ruxolitinib failure at the time of data analysis. Reasons of Ruxolitinib failure included intolerance in 7 patients and disease progression in 4 patients. Among the 7 patients who were intolerant to Ruxolitinib, 2 had TB reactivation while 6 patients had grade 3 thrombocytopenia. Among the 8 patients who were taking ruxolitinib through out of pocket expenditure, 2 patients had discontinued the drug due to financial burden despite patient assistance program. The median overall survival of the study population was not reached at median follow up of 49 months. Nonetheless in the entire cohort, 5 patients had died while taking ruxolitinib.

Discussion \& Conclusion: While ruxolitinib treatment leads to improvement in symptom responses in patients with MPN, however $\mathrm{TB}$ reactivation, cytopenias and financial constraints are major reasons for ruxolitinib failure in India.

\section{Stem Cell Transplantation}

\section{Diversity of KIR Repertoire in Western Indian Population: Implication in Donor Selection for HSCT}

Selma Dsilva ${ }^{* 1}$, Meenakshi Singh ${ }^{1}$, Sandeep Dhanda ${ }^{2}$

${ }^{1}$ ACTREC, Navi Mumbai, India, ${ }^{2}$ Saint Jude Children's Research Hospital, Tennessee, United States

Aims \& Objectives: Killer Immunoglobulin like receptor genes are among the most variable genes in the human genome. The frequencies of the individual genes vary among different populations. In a transplant set up, the interaction of donor KIR genes with the patient HLA class I ligand drives NK alloreactivity resulting in graft versus leukemia effect. The aim of this study was to investigate the KIR gene diversity within the Western Indian population in order to assess the ready availability of an appropriate donor for hematopoietic stem cell transplantation.

Patients/Materials \& Methods: HLA typing and KIR genotyping of 161 healthy unrelated adult hematopoietic stem cell donors was performed using the PCR-SSP method. The KIR ligands were calculated using the freely available KIR ligand calculator software. Gene and genotype frequencies were calculated using scripts provided in GitHub and Python packages.

Results: The KIR framework genes were present in all individuals. The frequencies of the KIR major inhibitory genes was $87.6 \%$ for KIR3DL1 and $94.4 \%$ for KIR2DL1 whereas, that of the major activating genes was $52.2 \%$ for KIR2DS1 and $65.8 \%$ for KIR2DS2. A total of 56 different KIR genotypes were observed in the population. The most common genotype observed was the KIR inhibitory genotype ID 1 AA. The HLA ligand $\mathrm{C} 1$ and $\mathrm{Bw} 4$ was present in majority of the individuals.

Discussion \& Conclusion: Our study provides a comprehensive analysis on the KIR and HLA ligand profiles in the Western Indian population. The results show that majority of the Western Indian 
population has an inhibitory KIR haplotype, the plausible cause being avoiding autoimmune destruction. In a transplant setting, NK alloreactivity can ensue good GvL either by loss of inhibition (inhibitory KIR receptor ligand mismatch) or enhanced activation (activating KIR receptor ligand match). The current study shows that finding a donor missing the inhibitory KIR receptor is a rare event owing to its high frequency, hence, selecting a donor based on the KIR activating receptors can ensure good GvL These findings highlight that since it is not possible to control the KIR ligands present on the patient, it is important to include donor KIR genotyping to ensure good transplant outcomes.

\section{Role of Pre-transplant High Resolution Computed Tomography of Chest and Serum Galactomannan Index in Predicting Post-Transplant Invasive Fungal Infections in Allogeneic Hematopoietic Cell Transplant Recipients}

Rintu Sharma ${ }^{* 1}$, Alka Khadwal ${ }^{1}$, Gaurav Prakash ${ }^{1}$, Pankaj Malhotra ${ }^{1}$, Arihant Jain ${ }^{1}$, Aditya Jandial ${ }^{1}$, Valliappan Muthu ${ }^{2}$, Ujjwal Gorsi $^{3}$, Arunaloke Chakrabarti4, Deepesh Lad ${ }^{1}$

${ }^{1}$ Internal Medicine, ${ }^{2}$ Pulmonary Medicine, ${ }^{3}$ Radiology, ${ }^{4}$ Microbiology, PGIMER, Chandigarh, India

Aims \& Objectives: Diagnosis and management of invasive fungal infection (IFI) in allogeneic hematopoietic cell transplant (allo- HCT) recipients is challenging. The role of pre-HCT radiographic plus biomarker screening in predicting the post-HCT IFI is debatable.

Patients/Materials \& Methods: This was a single-center, prospective study from 2014 to 2020 . All consecutive, consenting patients of $>12$ years of age who underwent allo-HCT were included. All patients had pre-HCT high resolution computed tomography (HRCT) chest imaging and serum galactomannan index (GMI). None of them had an active IFI at the time of HCT. All patients received antifungal prophylaxis with oral voriconazole starting from Day +1 of HCT.

Results: A total of 82 patients of age $>12$ years underwent allo-HCT during this period. The median age was 27 years (12-59 years). Underlying diagnoses included AML/MDS 41.4\% ( $\mathrm{n}=34)$, CML $13.4 \%(\mathrm{n}=11)$, AA, ALL $20.7 \%(\mathrm{n}=17$ each) and MPAL, CLL and CMML $1.2 \%$ (1 patient each). Fifty-four $(65.9 \%)$ patients had fully matched related donor, and $28(34.1 \%)$ patients had an alternative donor. Twelve (14.6\%) patients had required antifungal therapy preHCT for probable or proven IFI. Pre-HCT HRCT chest was normal in $51(62.2 \%)$ of patients. The most common abnormality was nodules \pm ground-glass opacities (GGO) in $17(20.7 \%)$ of patients, fibroatelectatic changes in $7(8.5 \%)$, pleuropericardial effusion in 3 $(3.7 \%)$, and cavitary lesion with GGO in $2(2.4 \%)$ patients. Bronchopneumonic pattern with GGOs and patchy lobar consolidation was seen in $1(1.2 \%)$ patient each. GMI was abnormal $(>0.5)$ in 22 $(26.8 \%)$ patients. A total of $33(40.2 \%)$ patients received therapeutic antifungal therapy in the post-HCT period, with $20(24.4 \%)$ receiving it for probable or proven IFI. On univariate analysis, the presence of nodular/cavitary lesions \pm GGO in pre-HCT HRCT chest had a higher incidence of post-HCT probable or proven IFI $(p=0.039)$. Also, patients with pre-HCT antifungal therapy $(p=0.003)$ and an abnormal GMI $>0.5(p=0.02)$ had higher incidence of post-HCT probable or proven IFI. Incidence of IFI in the post-HCT period also significantly impacted day 100 mortality, with 12 deaths (60\%) ( $p=0.001)$. Age, gender, basic diagnosis, type of HCT, or conditioning intensity did not predict post-HCT IFI.

Discussion \& Conclusion: Pre-HCT HRCT chest and GMI predicted probable/proven IFI post-HCT in our study.

\section{Thrombosis}

Influence of Lipoproteins on Thrombotic Risk

Iti Garg ${ }^{* 1}$, Vinay Kumar ${ }^{1}$, Swati Srivastava ${ }^{1}$, Chhavi Rai ${ }^{1}$, Babita Kumari $^{1}$, Uday Yanamandra ${ }^{2}$, Jasjit Singh ${ }^{3}$, Lilly Ganju ${ }^{1}$, Rajeev Varshney ${ }^{1}$

${ }^{1}$ Ministry of Defence, DRDO, DIPAS, ${ }^{2}$ Army Hospital Research \& Referral, Delhi, ${ }^{3}$ Western Command Hospital, Chandimandir, Chandigarh, India

Aims \& Objectives: Venous thromboembolism (VTE) is a multifactorial and potentially fatal disorder that includes deep vein thrombosis (DVT) and pulmonary embolism (PE). DVT is clotting of blood in a deep vein of an extremity (usually calf or thigh) or the pelvis and it is the primary cause of pulmonary embolism. Clinical studies depicted potential link between lipid associated proteins and expression/function of coagulation/fibrinolytic factors among VTE patients but observations are inconsistent and assorted. To get more insight, present study was designed to comprehend association of lipid associated proteins and DVT in human subjects.

Patients/Materials \& Methods: Study was conducted as per institutional ethical guidelines which was duly approved by Indian council of Medical Research (ICMR). Written consent was obtained from each volunteer before recruiting them for the study. Patients with history of pre-existing systemic disease, any prior surgery, vasculitis and malignancy were excluded. Peripheral blood was collected from DVT patients $(n=35)$ along with sex and aged matched healthy subjects $(n=40)$. Basic demographic and physiological information was also collected in both the groups. Quantification of D-Dimer, Lipoprotein (a), Apolipoprotein A1, Platelet Activating Factor-AH (PAF-AH), Paraoxonase1 (PON1), Sphingosine-1-phosphate (S1P), Oxidised HDL (oxHDL), oxidized LDL (oxLDL) was performed by ELISA in both the groups.

Results: There was significant deficiency of natural anticoagulants like Protein-C (55\%), Protein-S (30\%) and ATIII (35\%) among DVT patients having low HDL values. Quantification by ELISA showed that there is significant elevation in plasma levels of D-dimer, Lipoprotein (a), oxHDL and oxLDL in DVT patients in comparison to healthy subjects. Whereas there is significant decline in plasma level of Apolipoprotein A1, PAF-AH, PON1, S1P in DVT patients compared to healthy subjects.

Discussion \& Conclusion: The present study is prospective in nature and provides an evidence towards influence of lipid associated proteins against risk of venous thrombosis. These findings necessitates further clinical studies in larger cohort for further validation and will be helpful in designing distinctive strategies to elevate HDL by life style changes, therapeutics etc. which will certainly reduce the risk of thrombotic complication.

\section{Identification of Epigenetic Methylation Signatures in Patients of Venous Thrombosis (VT)}

Swati Srivastava ${ }^{* 1}$, Iti Garg ${ }^{1}$, Babita Kumari ${ }^{1}$, Uday Yanamandra ${ }^{2}$, Lilly Ganju ${ }^{1}$, Rajeev Varshney ${ }^{1}$

${ }^{1}$ DIPAS, DRDO, ${ }^{2}$ Army Hospital Research \& Referral, R \& R, New Delhi, India

Aims \& Objectives: Venous thrombosis (VT) posses major health problem and has high incidence in several populations worldwide. It is a complex multi-factorial disease that occurs as a result of blood clot formation due to an imbalance of procoagulants and anticoagulant. VT encompasses two clinically interrelated conditions; deep vein thrombosis (DVT) and pulmonary embolism (PE), the later one being 
potentially fatal. The pathogenetic mechanism of VT is not completely elucidated, however there are clear evidences that it occurs by complex interaction of genetic and environmental factors. Epigenetic modifications like DNA methylation, negatively regulate transcriptional networks thereby modulating gene expression under environmental influence and translating it phenotypically. Scanty data is available on methylation patterns during VT event. Aim of the present investigation was to gain insights into pathophysiological mechanism of VT by studying global methylation signatures in VT patients compared to controls.

Patients/Materials \& Methods: Present study involved Human subjects and was conducted in accordance with the ethical guidelines of Indian council of Medical Research after approval from institutional human ethical committee. Patients with history of pre-existing systemic disease, any prior surgery, vasculitis and malignancy were excluded from our study. Gold standard method for bisulfite sequencing was used for quantitative DNA methylation measurements. Briefly, DNA was isolated from peripheral blood of VT patients and controls. After quantitation, library preparation followed by bisulfite conversion and paired end sequencing using Illumina HiSeq platform. After alignment of sequenced reads, methylation state of all cytosine positions were read to identify hyper- methylated and hypo-methylated genes.

Results: Mapping efficiency of paired end reads was in between 78 and $80 \%$, with more that $80 \%$ methylation in $\mathrm{CpG}$ context. Approximately, 80-90\% of methylation changes occurred in intergenic and intronic regions of genes, whereas, smaller proportion was differentially methylated in exonic, 3'region and promoter region of gene. Preliminary analysis revealed that hypo-methylated genes in VT included those involved in cell/platelet adhesion, immune response, membrane receptors and regulatory proteins; whereas those hypermethylated included various transcriptional activators and co-activators and growth factors.

Discussion \& Conclusion: Present global methylation case control study is the first systematic approach to investigate the role of DNA methylation in VT pathophysiology.

Keywords: Methylation, Venous thrombosis, Epigenetic modulation. Disclosure of Interest: S. Srivastava: None Declared, I. Garg: None Declared, B. Kumari Research Grant from: DIPAS, DRDO, U. Yanamandra Research Grant from: DIPAS, DRDO, L. Ganju: None Declared, R. Varshney: None Declared.

\section{Meta-Analysis of RNA Transcripts in Thrombosis and Acute Respiratory Distress Syndrome: An Insight to SARS-CoV-2 Pathological Mechanisms}

\section{Aastha Mishra $^{* 1}$, Shankar Chanchal ${ }^{2}$, Mohammad Zahid ASHRAF ${ }^{1}$ \\ ${ }^{1}$ Biotechnology, JAMIA MILIA ISLAMIA, ${ }^{2}$ Biotechnology, Jamia Millia Islamia, DELHI, India}

Aims \& Objectives: Activated coagulation pathways, endothelial damage, and microvascular thrombotic injuries are observed in the critical COVID-19 patients. Coagulation parameters abnormalities increase the propensities of venous thromboembolism (VTE), a secondary complication to several form of cancers and viral infections such as COVID-19 caused by the novel strain of coronavirus, SARS$\mathrm{CoV}-2$. It has been observed that cytokine burst leading to sepsis drives Acute respiratory distress syndrome (ARDS) in the severe COVID-19 patients that could be worsened further with the accumulation of coagulation factors in the lungs. As a consequence, ARDS associated with thrombosis may have serious implications for treatment and management of critical COVID-19 patients. This study, therefore, makes an attempt to understand the shared transcriptomics signatures among the datasets derived from ARDS and VTE through network-based meta-analysis.

Patients/Materials \& Methods: An integrated gene expression metaanalysis of the two independently publicly available datasets for ARDS and VTE were conducted. A total of two studies were selected for the meta-analysis that met our inclusion criterias. A web interface for integrative meta-analysis, Integrative Meta-Analysis of Expression Data tool was used for the meta-analysis along with the Cytoscape tool for the hub gene analysis.

Results: Our meta-analysis identified a total of 1878 significant DEGs among the datasets, which when subjected to pathway enrichment analysis suggested Inflammation-Coagulation-Hypoxemia convolutions in COVID-19 pathogenesis. The top hub genes of our study gave insights into the various genes known to be participating in the hostvirus-interactions. The top hub genes were tumor protein53 (TP53), Lysine Acetyltransferase 2B (KAT2B), DExH-Box Helicase 9 (DHX9), REL-associated protein (RELA), RING-box protein 1 (RBX1) and Proteasome 20S Subunit Beta 2 (PSMB2).

Discussion \& Conclusion: Perhaps, the host-virus interactions participation of these hub genes in a datasets of ARDS and thrombosis is suggestive of the various strategies deployed by the virus to improve its replication and spreading. These pathological conditions might strengthen a favourable environment for virus and further aids in aggravating its viral load and deterioration of patients.

\section{Transfusion Medicine}

\section{Red Cell Alloimmunization in Multitransfused Patients at a Tertiary Care Teaching Hospital}

Sandhya K. Sinha ${ }^{* 1}$, Sandip Kumar ${ }^{1}$

${ }^{1}$ Pathology, Institute of Medical Science, BHU, Varanasi, India

Aims \& Objectives: Red blood cell alloimmunization is an immune competent host evoked response against the unknown RBCs antigens not present in the body. It occurs mostly after repeated blood transfusion or in pregnancy. Aim of this study to determine the seroprevelance and specificity of RBC antibodies in multitransfused patients, in whom the risk of alloimmunization is high.

Patients/Materials \& Methods: This study was conducted in Department of Pathology and Blood bank of Sir Sunder Lal Hospital, Institute of Medical Science, Banaras Hindu University, Varanasi. Blood was drawn from the patient of thalassemia major,sickle cell disease, haemat-oncology cases,chronic renal failure with haemodialysis, Pregnancy with complications and bad obstetric history,transfusion dependent oncology cases, anemia,bleeding disorder, $\mathrm{Rh}$ incompatible cases, and rest all the cases those requiring multiple blood and its component transfusion. The serum was used for antibody screening and identification test. Three cell antibody screening was performed using antihuman globulin gel cards(ID-Card LISS/ Coombs) and three cell panel (ID-Diacell I,II,III BioRad). Those with positive antibody screening were analyzed further for antibody identification test using 11 cell panel(Set ID-Dia Panel BioRad).

Results: Overall prevalance of RBC alloantibodies was 4\%. Of the 15 specific type of alloantibody identified, most (53.3\%) belonged to $\mathrm{Rh}$ blood group system, followed by Kell blood group(20\%), bombay blood group (13.3\%), and Duffy blood group (6.6\%).

Discussion \& Conclusion: Red cell alloimmunization in multiple transfused patients should not be ignored. To prevent the risk of 
hemolytic reactions and delay the reaction, regular antibody screening and its identification should be done in set interval of time after transfusion. Especially in patient who required long term blood transfusion and in those at higher risk of alloimmunization. RBC transfusion is a lifesaving practice, which explains why all these procedures results into safe and sufficient quality of blood supply.

\section{SARS Patients: Use of Convalescent Plasma Therapy in Rural Area}

\author{
Anjali Mathur*1, Mayank Mathur ${ }^{2,3}$ \\ ${ }^{1}$ Pathology, ${ }^{2}$ KD Medical College, Mathura, India, ${ }^{3}$ Surgery, KD \\ Medical College, Mathura, India
}

Aims \& Objectives: Aim and Objective- In order to evaluate the efficacy of convalescent plasma therapy in the treatment of patients with severe acute respiratory syndrome (SARS),

Patients/Materials \& Methods: Patients- 38 patients. Material and method- After the assessment of the donor, $200600 \mathrm{~mL}$ plasma can be collected with apheresis devices. Donor eligibility, Predonation evaluation of donors, Donor recruitment, Collection of convalescent plasma at apheresis centers.

Results: Results 37 SARS patients were given convalescent plasma at K.D MEDICAL COLLEGE MATHURA UP, between 09 SEPTEMBER and 13 OCTOBER 2020. Good outcome was defined as patient responded to plasma therapy in 24 to $48 \mathrm{~h}$ after infusion. Poor outcome was defined as no response or death after $48 \mathrm{~h}$ of infusion. Discussion \& Conclusion: Conclusion- CP seems to be a safe and probably effective treatment for critically ill patients with COVID19. It could also be used for prophylactic purposes but the safety and effectiveness of this approach should be tested in randomized prospective clinical trials.

\section{A Prospective, Observational Study to Compare Abo Isoagglutinin Titers by Three Different Methods in $O$ Blood Group Donors}

Prashant K. Pandey ${ }^{* 1}$, Divya Setya ${ }^{1}$, Shweta Ranjan ${ }^{1}$, Mukesh K. Singh ${ }^{1}$

${ }^{1}$ Transfusion Medicine, Jaypee Hospital, Noida, NOIDA, District - Gautam Budh Nagar, India

Aims \& Objectives: Manual method of titration has certain limitations. While automation has the advantage of objectivity, ease of use and reproducibility with well-defined end points of agglutination reaction, standardization of these methods have still not been possible. There is significant variation between methods and laboratories. [19-24] The aim of the study was to compare the results obtained by the age old manual CTT with semi-automated CAT and fully automated hemagglutination (HA)/SPRCA by calculating agreement, and correlation and comparing median titer anti-A and anti-B (IgG and IgM) titers obtained by CTT, CAT and HA/SPRCA.

Patients/Materials \& Methods: This was a prospective, observational study conducted from October 2018 to March 2020. All consecutive $\mathrm{O}$ group donors were included in the study. All samples were consecutively tested by CTT, CAT and HA/SPRCA and results were recorded. To reduce inter-observer bias for manual (CTT) and semi-automated (CAT) methods, each sample was given to two different personnel.
Results: A total of 2005 donors were included. 295 (14.71\%) samples had inter-observer variation by CTT and 51 (2.54\%) had interobserver variation by CAT. There was $43.4 \%$ and $40.3 \%$ agreement of anti-A IgM and IgG titers between CAT $(2+$ strength $)$ and CTT $(1+$ strength) respectively. There was $43.6 \%$ and $32 \%$ agreement of anti-B IgM and IgG titers between CAT $(2+$ strength $)$ and CTT $(1+$ strength) respectively. While IgM and IgG measurement of antiA and anti-B showed strong correlation between CTT $(1+$ strength $)$ and CAT $(1+, 2+$ strengths $)$, correlation between CTT $(1+$ strength $)$ and HA/SPRCA $(1+, 2+$ stengths $)$ were found to be weak. Median IgM titers for anti-A and anti-B were lower than median IgG titers.

Discussion \& Conclusion: In conclusion, we report that the semiautomated method (CAT) shows higher ability in detecting ABO isoagglutinins than the manual method (CTT) and automated method (HA/SPRCA) shows lower ability in detecting ABO isogglutinins than manual method (CTT). Transfusion services need to decide which method would benefit their patient population for which a measurement of clinical significance is necessary to discern the suitability as a replacement of the conventional, manual tube technique.

\section{Effect of Autologous Plasma Used as Eye Drops in Patients with Dry Eyes in Sjogrens Syndrome}

Diana S. Nathan ${ }^{* 1}$, Abhishekh Basavarajegowda ${ }^{1}$, Ramesh Babu ${ }^{2}$, Rashmi Kumari ${ }^{3}$

${ }^{1}$ Transfusion Medicine, ${ }^{2}$ Ophthalmology, ${ }^{3}$ Dermatology, JIPMER, Puducherry, India

Aims \& Objectives: Dye eye disorder in Sjogren s syndrome is very a debilitating condition to the patients as it affects their day-to- day activities, sleep quality and overall wellbeing. The conventional treatment options available so far such as artificial tears is found to be ineffectual in relieving symptoms and is economically not feasible for treatment of long duration. Our Aim was to study the effect of autologous plasma eye drops in reduction of dry eyes symptoms in patients with Sjogren s syndrome for over a period of three months with the SPEED questionnaire and DEWS SCORING criteria.

Patients/Materials \& Methods: Sixteen patients with Sjogrens syndrome dry eyes disease were enrolled for the study. They were given autologous plasma prepared as eye drops (in 20\% dilution as $5 \mathrm{ml}$ bottles) to be used for 6 times a day for over a period of 3 months. The outcomes were measured subjectively as SPEED score and objectively as DEWS score. These values were calculated for both the right eye (RE) and the left eye (LE) separately and on three occasions namely, pre intervention, after one month (post intervention 1) and three months (post intervention 2). The statistical analysis was carried out using SPSS.

Results: The Box and Whisker Chart was plotted with an IQR (interquartile range) of 1.5 . The outcome of the study was a statistically significant improvement in both symptoms (SPEED SCORE) and objective signs (DEWS SCORE) $(p<0.001)$ in both the eyes as shown in the table below. Our study showed that the plasma eye drops to be safe as there were no reported adverse effects including active ocular infections.

Discussion \& Conclusion: Autologous plasma eye drops can be used as a therapeutic option in patients with Sjogrens syndrome dry eye disorder.

Supporting Document: 


\begin{tabular}{|c|c|c|c|c|}
\hline Parameter & $\begin{array}{c}\text { Pre } \\
\text { intervention }\end{array}$ & $\begin{array}{c}\text { Post } \\
\text { intervention 1 }\end{array}$ & $\begin{array}{c}\text { Post } \\
\text { intervention 2 }\end{array}$ & p-value \\
\hline SPEED-RE & $18.68(4.22)$ & $12.68(5.92)$ & $7.443(3.22)$ & $<0.001$ \\
\hline SPEED-LE & $18.81(4.23)$ & $12.81(6.15)$ & $7.31(2.96)$ & $<0.001$ \\
\hline Parameter & Pre & Post & Post & p-value \\
\hline DEWWS-RE & $16.25(4.55)$ & $14.0(4.03)$ & $12.94(3.37)$ & $<0.001$ \\
\hline DEWS-LE & $16.43(5.24)$ & $14.31(4.75)$ & $13.0(3.38)$ & $<0.001$ \\
\hline
\end{tabular}

\section{Poster Abstract Papers}

\section{Acute Leukemia (Clinical)}

\section{Clinicopathological Features and Treatment Outcome of Acute Promyelocytic Leukemia Patients}

Mekhala Rao*1, Girish Kamat ${ }^{2}$, Deepak Goni ${ }^{2}$, Girish Balikai ${ }^{2}$, Vidisha Athanikar ${ }^{1}$

${ }^{1}$ Pathology, ${ }^{2}$ Hematology, SDM College of Medical Sciences and Hospital, Dharwad, India

Aims \& Objectives: To study the clinicopathological features and outcome of patients treated with Arsenic trioxide and all- trans retinoic acid based protocols in acute promyelocytic leukemia (APL) patients.

Patients/Materials \& Methods: Study design: Cross sectional study. 18 patients who were morphologically diagnosed to have APL and also had PML-RARA fusion gene by RT-PCR between January 2017 to August 2020 were included. High risk patients (total leucocyte count $>10,000 / \mathrm{cmm}$ ) were treated with APML 4 protocol. Low risk patients (total leucocyte count $<10,000$ ) were treated with protocol APL0406Intergroup Study AL WP GIMEMA-DSIL.

Results: The median age at presentation was 31.5 years. Male to female ratio was 1.25. Peripheral blood smear was suggestive of APL in $12(67 \%)$ cases. $9(50 \%)$ cases had a pancytopenia blood picture. During presentation, elevated prothrombin time, activated partial thromboplastin time and INR levels were seen in 15(83.3\%), $3(16.66 \%)$ and $18(100 \%)$ cases respectively. Bone marrow aspirate smears from 17 cases $(94.44 \%)$ were suggestive of APL and was inadequate in 1 case $(5.56 \%)$. Out of the 12 APL cases for which flow cytometry was done, $11(91.6 \%)$ cases were suggestive of APL and $1(8.4 \%)$ case was suggestive of acute myeloid leukemia(CD34 and HLA-DR positive) with aberrant CD7 expression. Chromosomal analysis was done in 11 APL patients and revealed 2(18.18\%) cases with additional cytogenetic abnormality including $46, X X$, add(7)(q36)t(15;17)(q22;q21)[16]/46,XX[(4] and 46,XY, $\operatorname{add}(4)(\mathrm{p} 16) \mathrm{t}(15 ; 17)(\mathrm{q} 22 ; \mathrm{q} 21)[20] .16$ APL patients could be followed up. Out of them, $15(93.75 \%)$ patients attained complete remission (CR) and was confirmed by post induction bone marrow study. $1(6.25 \%)$ patient died during induction therapy. Complications were seen in $10(55.55 \%)$ cases and included neutropenic sepsis ( 3 cases, $16.6 \%$ ), intracranial hemorrhage ( 2 cases, $11.11 \%$ ), differentiation syndrome ( 1 case, $5.5 \%$ ), deep vein thrombosis( 1 case, $5.5 \%$ ), pseudotumor cerebri(1 case, 5.5\%), QTc interval prolongation(1 case, $5.5 \%)$, \& pneumonia( 1 case, $5.5 \%$ ).

Discussion \& Conclusion: High rate of complete remission was seen in our study. There was a very low incidence of treatment related complications like differentiation syndrome, QTc prolongation and pseudotumor cerebri.

\section{CD3 Negative T-ALL/AUL: A Study of Three Cases and Review of Literature}

Simran Gilhotra ${ }^{* 1}$, Shashi Bansal ${ }^{1}$, Upendra Sharma ${ }^{2}$, Abhishek purohit $^{3}$, Syeda F. Jamil ${ }^{1}$

${ }^{1}$ Pathology, ${ }^{2}$ Medical Oncology, BMCHRC, Jaipur, Jaipur, ${ }^{3}$ Pathology, AIIMS Jodhpur, Jodhpur, India

Aims \& Objectives: Acute undifferentiated leukemia (AUL) is a rare entity and is defined by expression of no more than one membrane marker for any given lineage. AUL cases lack T-cell (cCD3), B- cell (CD19, cCD22, \& cCD79A), myeloid markers (MPO) \& monocytoid markers. They also lack the specific features of cells of other lineages, such as megakaryocytes or plasmacytoid dendritic cells. So the aim of the study is to emphasise for the establishment of lineage markers before labeling acute leukemia as acute undifferentiated leukemia as the treatment would be different for these different entities.

Patients/Materials \& Methods: We identified three cases of acute leukemia which on morphology were showing $90 \%$ blasts with condensed chromatin,inconspicuous nucleoli.Clinical features such as lymphadenopathy, hepatomegaly, splenomegaly were compared. Complete blood count revealed anemia, leucocytosis and thrombocytopenia.Lineage assessment of leukemic cells was established by use of comprehensive immunophenotypic study with associated cytogenetics and immunohistochemistry.

Results: In this case series, the blasts were positive for CD5, CD7, CD38, and TdT and negative for lineage specific markers of myeloid, monocytoid \& B-lymphoid \& cytCD3. The triumph of our case series is case three in which two populations were identified and one population( $12 \%$ of all blasts) revealed positivity of cCD3, CD5, CD7, CD33 \& CD34 leading towards T-ALL (ETP-ALL) while second population revealed expression of $\mathrm{CD} 5, \mathrm{CD} 7$ together with immature markers and negativity of $\mathrm{CCD} 3$ which might be indicative of very early T-ALL than that of Pro T-ALL/ETP-ALL. A diagnosis of AUL can be debatable for our cases, however positivity of CD5, CD7 (bright positive) rules out AUL (due to positivity of more than one surface markers of T-cell lineage).

Discussion \& Conclusion: Lineage assignment of leukemic cells should always be established by use of comprehensive immunophenotypic study with associated cytogenetic, immunohistochemistry, molecular studies along with clinical features, would be helpful in differential diagnosis of early T-ALL with AUL and this will lead to choice of the most appropriate treatment and management for such patients. 


\section{Hemophagocytic Lymphohistiocytosis with Acute Lymphoblastic Leukemia: A Wolf in Sheep's Clothing}

Darshan Rangaswamy ${ }^{1}$, Jaikumar G. Ramamoorthy ${ }^{* 1}$, Prabhu Manivannan $^{2}$, Dhandapany Gunasekaran ${ }^{1}$

${ }^{1}$ Pediatrics, ${ }^{2}$ Pathology, JIPMER, Puducherry, India

Aims \& Objectives: Hemophagocytic Lymphohistiocytosis (HLH) syndrome is characterised by unusual features: a protracted clinical course and liver or brain injury. We present a case of acute lymphoblastic leukemia (ALL) which presented with classical features of HLH.

Patients/Materials \& Methods: A 8 years old boy presented with hectic pattern fever and fatigue for 3 days.

Results: On examination hepatosplenomegaly $(6 \mathrm{~cm}$ below costal margin) without significant lymphadenopathy was observed. Hemoglobin was $7.9 \mathrm{gm} / \mathrm{dl}$, platelet counts of $59000 / \mu \mathrm{l}$ and total leucocyte count (TLC) was $3230 / \mu 1$ with $53 \%$ neutrophils in complete hemogram. Peripheral smear showed white blood cells were reduced without atypical/blast like cells. Liver function tests showed hypoalbuminemia $(2.8 \mathrm{gm} / \mathrm{dl})$, elevated levels of aspartate transaminase (245units/L) and alanine transaminase (67units/L), hyperbilirubinemia (total $-2.4 \mathrm{mg} / \mathrm{dl}$, direct $-1.1 \mathrm{mg} / \mathrm{dl}$ ) and prothrombin time of $21 \mathrm{~s}$. Tropical infections, viral infections and bacterial sepsis were ruled out with appropriate investigations over the next 4 days with persistent fever. Pancytopenia worsened further (Hemoglobin- $5.9 \mathrm{gm} / \mathrm{dl}$, TLC was $250 / \mu \mathrm{l}$ and platelet count was $13000 / \mu \mathrm{l})$. Investigation for HLH showed: serum fibrinogen- $88 \mathrm{mg} / \mathrm{dl}$, serum triglyceride- $320 \mathrm{mg} / \mathrm{dl}$ and serum ferritin- $3264 \mathrm{ng} / \mathrm{ml}$. Clinically he was diagnosed as HLH as per the HLH-2004 criteria. Bone marrow evaluation (BME) performed to evaluate pancytopenia showed: $82 \%$ lymphoid blast positive for CD34, CD38, CD19, CD10, CD20, CD22, CD79a and nTDT on flow cytometry suggesting precursor B-cell acute lymphoblastic leukaemia(ALL) surprisingly. Cytogenetics and Fluorescence in situ hybridisation evaluation were non-contributory. Child was initiated on Berlin- Franfurt-Munich protocol.

Discussion \& Conclusion: HLH-disease develops because of diverse etiologies including genetic (primary) or infectious, rheumatologic, immunodeficient and malignant disorders (secondary). The prevalence of malignancy triggered HLH (MTHLH) in children is estimated to be $8-11 \%$ and has increased mortality rates than nonmalignancy triggered HLH (N-MTHLH). MTHLH can be observed at onset or during therapy for malignancy. It is recommended to perform bone marrow examination and/or positron emission tomographycomputerised tomography (PET-CT) scan whenever the trigger for HLH is unclear and not to solely rely on HLH diagnostic criteria. This will prevent misdiagnosis of MTHLH as HLH disease and thereby outcome. Concurrent HLH at diagnosis of ALL has been reported in 2 cases of B cell ALL presenting with pancytopenia with blasts and septicaemia. Our index case had neither at presentation, a wolf in sheep's clothing. To conclude in all children with HLH syndrome performing BME and/or PET-CT scan to be performed whenever trigger for HLH is unknown.

\section{Nucleophosmin (NPM1) Gene Mutation in De-Novo Acute Myeloid Leukemia and Its Morphological, Immunophenotypic, Clinical Features and Prognostic Significance: A Retrospective Analysis of 9 Cases}

Sudarshan Pandit ${ }^{* 1}$, Nilesh wasekar ${ }^{1}$, Girish Badarkhe ${ }^{1}$, Raj Nagarkar ${ }^{2}$

${ }^{1}$ Department of Haematology, ${ }^{2}$ HCG MANAVATA CANCER CENTRE, NASHIK, India
Aims \& Objectives: The study presented here investigates the morphological, immunophenotyping,clinical characteristics, and prognosis of the NPM1 mutations in de novo AML patients.

Patients/Materials \& Methods: 40 patients (39 adults and 01 child) with confirmed diagnosis of AML admitted under the Department of Hematology,HCG- MANAVATA CANCER CENTRE, nashik, India, were retrospectively analysed in the study from the 1-6-2020 to 1-1-2019. Clinical data, including age, gender, whole blood cell count (WBC), hemoglobin ( $\mathrm{Hb})$, platelet count, and presence of organomegaly, were collected at the time of diagnosis. Immunophenotyping studies by flow cytometry done and PCR and DNA sequencing detected NPM1 exon 12 mutation. These patients got the treatment options depending on the guidelines, ECOG status and willingness of the family. The outcome of the treatments were analysed after completion of chemotherapy.

Results: Out Of 40 de novo AML patients included in the study, 9 were NPM1 mutation positive. The NPM1 mutation was found more frequently in adults as compared to children.Further stratification of age showed that frequency of NPM1 mutation increases with age, $77 \%(07 / 09)$ being the highest frequency observed in AML patients above 45 yrs of age. Typical morphological characteristics like Intranuclear cup like inclusions present and Auer Rod present in 7 out of $9(78 \%)$ patients. Immunophenotypically, NPM1 mutation was found to be associated with the lower positivity of CD34 HLA-DR expression. Out of the 9 de novo NPMc + AML patients 5 patients $(56 \%)$ patients associated with fms-like tyrosine kinase-3 (FLT3) internal tandem duplications (ITD) mutations. 2 patients were succumbed during induction chemotherapy. 3 patients had completed there consolidation chemotherapy Post consolidation chemotherapy AML-MRD was negative. FLT3 and NPM1 mutation status were negative. They are on regular follow-up. 2 patients are still in their consolidation phase and 2 patients are on azacytidine based chemotherapy.

Discussion \& Conclusion: The mechanistic role of NPM1 in the pathogenesis of AML has not been fully elucidated, and perhaps because of favorable prognosis of NPM1-mutated AML, targeted therapies have not been investigated. Identifying possible mechanisms that result in improved outcome could point toward potential targets to augment chemosensitivity in AML and perhaps other malignancies. More work is necessary to confirm NPMc + AML as a true disease entity with distinctive clinical and biological features and favourable outcomes.

\section{Experience of BFM 2002 Protocol in Acute} Lymphoblastic Leukaemia: Single Centre Experience

Prakash S. Shekhawat $^{* 1}$, Malini Garg ${ }^{1}$, Avriti Baveja ${ }^{1}$, Sumit Mitra $^{1}$, Subham Bhattacharya ${ }^{1}$, Shuvraneel Baul ${ }^{1}$, Prakas K. Mandal ${ }^{1}$, Rajib De ${ }^{1}$, Tuphan K. Dolai ${ }^{1}$

${ }^{1}$ Hematology, NRS Medical college and hospital, kolkata, India

Aims \& Objectives: To study the outcome of Acute Lymphoblastic Leukemia (ALL) patients treated with BFM 2002 protocol.

Patients/Materials \& Methods: Retrospective analysis of ALL patients $(\mathrm{n}=118)$ treated with BFM2002 protocol at NRSMCH was done between September 2016 to August 2020. Baseline characteristics were noted and analysed using Pearson correlation coefficient. Death, relapse, Pancreatitis(grade3,4), PRES, CVT, Neutropenic enterocolitis, treatment abandonment were considered significant event. Overall survival(OS) and Event free survival(EFS) was calculated using SPSS version 26.

Results: The median age was 10.5 (range, 2-30)year with male to female ratio of 1.5:1 and median TLC 12,800/cumm(range,700291800/cumm). B ALL was commonest $(89,75.42 \% ; \mathrm{n}=89)$, 
followed by $\mathrm{T} \operatorname{ALL} / \operatorname{LBL}(23.7 \% ; \mathrm{n}=28)$ and $\operatorname{MPAL}(0.8 \% ; \mathrm{n}=1)$. Diploidy was commonest $(90 \%)$ in evaluable karyotype $(\mathrm{n}=100)$. FISH(ph, MLL) study for ALL $(n=112)$ was negative in $95 \%$ cases followed by $3.9 \% \mathrm{ph}+$ ve and MLL $0.9 \%$. CNS was involved in $14(11.86 \%)$ cases. Intermediate risk category $(63.5 \% ; n=75)$ was commonest at baseline followed by standard $\operatorname{risk}(23.7 \% ; \mathrm{n}=28)$ and High risk $(12.7 \% ; n=15)$. Day 8 prednisolone good response was seen in $86.4 \%(102 / 118)$ cases. Poor Day 8 prednisolone response was significantly associated with day15 M2,M3 marrow $(p<0.05)$, however it had no significance with post induction remission status $(p>0.05)$. Induction mortality was $19.5 \%(23 / 118)$ while Postinduction mortality was 20\%(19/95). Initial risk status was significantly associated with increased Induction mortality, relapse rate and overall mortality $(p<0.05)$. Overall mortality was $35.6 \%(42 / 118)$ and had significant association with older age and male $\operatorname{sex}(p<0.05)$. Overall Relapse rate was $24.1 \%$ (23/95). Relapse was more in older age and male $\operatorname{sex}(p<0.05)$. Events were seen in $50 \%$ cases and were more in older age, patients with febrile neutropenia $(p<0.05)$. Median OS and EFS was 4.07 and 0.31 years, respectively. One-year OS and EFS was $66 \%$ and $22 \%$ respectively. Median OS of $\leq 18$ years was 4.06 years and $>18$ years was 0.9 years $(p<0.05)$. EFS at 1 year showed no difference $(p>0.05)$ for patients $\leq 18$ years $(23 \%)$ and $>18$ years $(12 \%)$.

Discussion \& Conclusion: Day 8 poor prednisolone response is not predictive of day 33 bone marrow remission status. Higher Initial risk status predicted increased induction mortality, relapse rate and overall mortality. Overall mortality was more in older age and male sex. Overall relapse rate was $24.1 \%$. Median OS was higher in paediatric population compared to adult without any significant EFS benefit. Supporting Document:

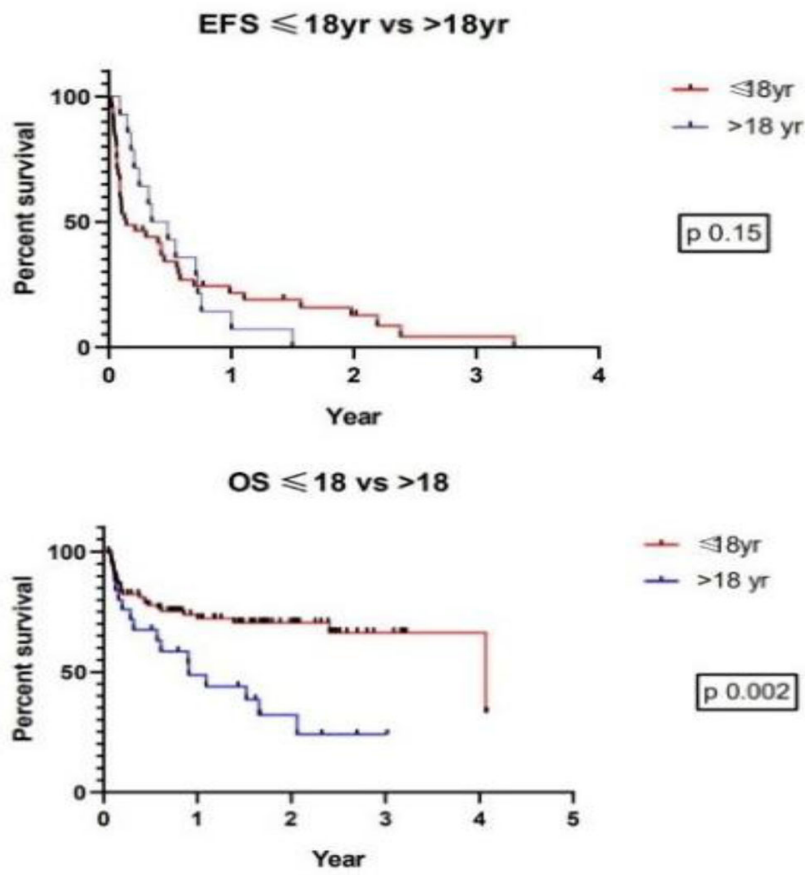

\section{Management of Acute Promyelocytic Leukaemia (APML) in a Resource Limited Setting}

Prakash S. Shekhawat ${ }^{* 1}$, Malini Garg ${ }^{1}$, Avriti Baveja ${ }^{1}$, Sumit Mitra $^{1}$, Subham Bhattacharya ${ }^{1}$, Shuvraneel Baul ${ }^{1}$, Prakas K. Mandal ${ }^{1}$, Rajib De ${ }^{1}$, Tuphan K. Dolai ${ }^{1}$

${ }^{1}$ Hematology, NRS Medical College and Hospital, Kolkata, India

Aims \& Objectives: To study the characteristics and outcome of APML patients.

Patients/Materials \& Methods: Retrospective analysis of APML patients $(\mathrm{n}=106)$ treated at NRSMCH from October 2009 to August 2020 was done. Patients were treated with ATRA \pm ATO \pm cytoreductive therapy in form of anthracycline depending on their risk stratification. Presenting symptoms, clinical spectrum and response to treatment was analyzed. Differentiation syndrome, pseudotumor cerebri, DIC, treatment abandonment, stroke, venous thrombus, relapse and death were taken as events. Overall survival(OS), event free survival (EFS) and relapse free survival (RFS) was calculated.

Results: The median age was 26.5 (range, 2-71)years with male to female ratio of 1.7:1. Majority presented with bleeding $(91.3 \%)$ followed by anaemia (84.4\%) and fever (78\%). Spleen was palpable in $5 \%$ cases. Median $\mathrm{Hb}$, TLC and platelet count were $9.5 \mathrm{~g} / \mathrm{dl}$, $5700 /$ cumm and 54500/cumm respectively. Standard risk(45.3\%) was commonest followed by Intermediate $\operatorname{risk}(29.2 \%)$ and high risk(25.5\%). Amongst PML RARA RT PCR, BCR1 was commonest (53.1\%) followed by BCR 3(43.8\%) and BCR 2 (3.1\%). ZBTB/ RARA $t(11 ; 17)$ was detected in 2 cases $(1.8 \%)$ and were treated with AML like therapy. Single agent ATRA induction was given to $21.7 \%$ patients, whereas, $37.7 \%$ received ATRA + ATO, and ATRA + chemotherapy was given to $40.6 \%$ patients. Induction mortality was $16.9 \%$ and it was significantly associated with low haemoglobin and low platelet $\operatorname{count}(p<0.05)$. Remission was attained in $83 \%$ patients, whereas $50 \%$ patients had an event(s). Events were significantly associated with older age $(p<0.05)$. Overall survival at 10 year was $78.9 \%$, whereas, median OS has not been reached. Median EFS was 4.01 year while 10 year EFS was $46.6 \%$. RFS was $66.2 \%$ at 10 year, however median RFS has not been reached. Extrapulmonary tuberculosis warranting treatment interruption was seen in $1.8 \%$ patients $(\mathrm{n}=2)$.

Discussion \& Conclusion: Most common presenting symptom was Bleeding (91.3\%). Majority of cases belonged to Standard risk $(45.3 \%)$. Induction mortality $(16.9 \%)$ was significantly associated with low haemoglobin and low platelet count. Overall survival and relapse free survival at 10 year was $78.9 \%$ and $66.2 \%$ respectively. Median EFS was 4.01 year while 10 year EFS was $46.6 \%$.

\section{Dexamethasone is Useful as Initial Cytoreduction Therapy in Acute Myeloid Leukemia}

\author{
Girish Kamat ${ }^{* 1}$, Mekhala Rao $^{2}$, Deepak Goni ${ }^{3}$, Girish Balikai ${ }^{3}$ \\ ${ }^{1}$ Hematology, SDM College of Medical Sciences and Hospital, \\ Dharwad, ${ }^{2}$ Pathology, ${ }^{3}$ Hematology, SDM College of Medical \\ Sciences and Hospital, Dharwad, India
}

Aims \& Objectives: Few in vivo and in vitro studies have shown the anti-leukemic effect of steroids in acute myeloid leukemia (AML). We present 2 cases of AML, which showed significant cytoreduction with use of dexamethasone. 
Patients/Materials \& Methods: At our institute, in view of nonavailability of in-house flow cytometer, as a routine, once morphologically bone marrow aspirate is reported as suggestive of acute lymphoblastic leukemia (ALL), patients are started on pre- induction steroids. Once flow cytometry report is available, which usually takes 3-4 days; patients are appropriately categorized into specific subtypes of leukemia and treated accordingly. In this retrospective analysis, we have included 2 cases of acute myeloid leukemia, bone marrow of which was reported as suggestive of ALL. These patients were started on dexamethasone $(10 \mathrm{mg} / \mathrm{m} 2)$. Daily complete blood counts were done in these cases. Responses were assessed in terms of reduction in total WBC count.

Results: Case 1: A 35 year old male patient had presented with fever, fatigue and cough. Following admission bone marrow was done and as it was reported as suggestive of ALL. Patient was started on dexamethasone. Total count, which was $25,300 / \mathrm{cmm}$ at presentation, dropped to $2640 / \mathrm{cmm}$ on day 5 of steroids ( 3 days of dexamethasone and 1 day of prednisolone). Case 2: A 14 year old boy had presented with fever, fatigue and body-ache. His initial WBC count was $1,51,350 / \mathrm{cmm}$. Bone marrow was suggestive of ALL. He was started on dexamethasone. After 3 days of steroid, his total WBC count dropped to $23,770 / \mathrm{cmm}$.

Discussion \& Conclusion: AML is a heterogeneous group of disorders and some of them probably respond to steroids. Several previous studies have shown that, dexamethasone or high dose methyl prednisolone are useful in induction therapy. Proper clinical trials are necessary to assess utility of steroids in treatment of AML.

\section{Dengue Infection Masking Acute Lymphoblastic Leukemia in an Adult: A Case Report}

Sneha Jawalkar*1, Ratnakar Potekar ${ }^{1}$

${ }^{1}$ Pathology, BLDE(DU) Shri B M Patil Medical College, Hospital \& Research Centre, Vijayapura, India

Aims \& Objectives: The aim of this case report was to diagnose acute lymphoblastic leukemia accurately especially when its presentation hints towards an infectious disease.

Patients/Materials \& Methods: A 58 years old male presented with 2 days history of fever and generalized weakness. Fever was moderate in grade, on and off, intermittent type, not associated with chills and rigors, relieved on taking medication. The patient had no other complaints and was not a known case of diabetes mellitus, hypertension, or tuberculosis. His physical examination was unremarkable. Results: Laboratory findings revealed Pancytopenia with a total leucocyte count (TLC) of 550cells/cumm, hemoglobin-6.0gm \% (normocytic normochromic anemia), platelet count $-0.58 \mathrm{lakh} /$ cumm. Differential leucocyte count showed $80 \%$ lymphocytes with few atypical/reactive lymphocytes on peripheral smear. Blood serology was positive for Dengue IgM antibodies but negative for IgG antibody, indicating primary dengue infection. His random blood glucose levels, liver and kidney function tests were normal. The test for HIV antibody was negative. His platelet count remained the same for the next 4 days. On day 5 of admission, the platelet count was reduced to $0.4 \mathrm{lakh} / \mathrm{cumm}$. As the patient showed no improvement in TLC and platelet count despite appropriate therapy for Dengue infection and showed persistent atypical lymphocytes on peripheral smear, a bone marrow study was advised. Bone marrow aspiration revealed, $45 \%$ blasts suggesting a diagnosis of Acute Leukemia. On bone marrow biopsy, marrow was largely replaced by blast cells.
With further IHC evaluation, a diagnosis of CALLA- positive BAcute lymphoblastic leukemia was given.

Discussion \& Conclusion: Acute lymphoid leukemia (ALL) is the least common type of leukemia in an adult. ALL can be complicated by Dengue fever (DF). Both illnesses can have a common presentation. As both can cause bone marrow suppression, it may result in a more severe cytopenia. Careful monitoring of hematological changes is needed to diagnose acute leukemia especially when it is complicated with other hematological infections like dengue. A peripheral blood smear can sometimes provide clues, but not until a bone marrow study is performed, the diagnosis of acute leukemia can be confirmed.

Keywords: Dengue, Pancytopenia, ALL.

\section{Buffy Coat Derived Granulocyte Transfusions During Intensive Chemotherapy of AML}

Jay Y. Sheth ${ }^{* 1}$, Arijit Nag ${ }^{1}$, Suvro S. Dutta ${ }^{2}$, Jeevan Kumar ${ }^{1}$, Mayur Parihar $^{3}$, Vivek Radhakrishnan ${ }^{1}$, Sabita Biswas ${ }^{2}$, Deepak K. Mishra ${ }^{3}$, Reena Nair ${ }^{1}$, Mammen Chandy ${ }^{1}$, Saurabh J. Bhave ${ }^{1}$

${ }^{1}$ Clinical Haematology and BMT, ${ }^{2}$ Transfusion Medicine and Blood Bank, ${ }^{3}$ Laboratory Medicine, Tata Medical Centre, Kolkata, India

Aims \& Objectives: Evaluate the safety and feasibility of Buffy coat (BC) granulocyte transfusion in patients with AML receiving intensive chemotherapy at a tertiary care cancer centre.

Patients/Materials \& Methods: This is a retrospective audit of the use of Buffy coat transfusions during intensive therapy of acute myeloid leukaemia. Transfusions were initiated prophylactically when the leucocyte count was $<1000 /$ cu.mm for patients not having evidence of an active infection. For patients presenting with sepsis (without significant pulmonary changes), granulocyte transfusions were initiated with a therapeutic intent. Granulocyte transfusion was stopped when ANC maintained > 500/cu.mm for 3 consecutive days, or with-held due to transfusion-related SAE. All patients received antibiotic therapy, transfusion support and growth factors as per standard institutional practice.

Results: Between Nov 2017 and Feb 2020, BC Granulocyte product was administered to 58 patients in a total of 3181 transfusions (median 45, range: 13-143). Baseline characteristics are mentioned in Table 1. BC transfusion was well tolerated by all the patients. No Grade 4-5 SAEs were observed. Only 5 patients $(8.62 \%)$ had adverse reactions (mostly Grade 1-2) attributable to $\mathrm{BC}$ granulocyte transfusions (Table 1). 18/58 (31.03\%) were shifted to ICU because of infection related complications. Out of the 12 patients shifted to ICU during neutropenic phase, resolution of index infection post BC granulocyte transfusion was seen in $8 / 12$ patients $(66.67 \%) .8 / 58$ (13.79\%) patients died due to infective complications, of which $5 / 58$ $(8.62 \%)$ died during induction chemotherapy. Background AML induction mortality reported from Indian centres vary from $18 \%$ $27 \%$. Outcomes in our study note a potentially significant reduction in this parameter.

Discussion \& Conclusion: BC Granulocyte transfusion is a feasible and reasonably safe approach in AML patients receiving intensive therapy and can potentially reduce infection related morbidity and mortality. Based on these results, a prospective study is being proposed. 
Supporting Document:

\begin{tabular}{|c|c|c|c|}
\hline & & $\mathbf{N}$ & $\%$ \\
\hline Age in years (Median range) & & $\begin{array}{c}42 \\
(18-69)\end{array}$ & \\
\hline \multirow[t]{2}{*}{ Gender } & Male & 31 & 53.4 \\
\hline & Female & 27 & 46.6 \\
\hline \multirow[t]{3}{*}{ Diagnosis } & AML, de novo & 52 & 89.65 \\
\hline & AML, transformed & 1 & 1.72 \\
\hline & Relapsed AML & 5 & 8.62 \\
\hline Induction chemotherapy & (Median cycles, range) & $1(1-2)$ & \\
\hline Consolidation chemotherapy & (Median cycles, range) & $3(0-3)$ & \\
\hline $\begin{array}{l}\text { No. of granulocyte } \\
\text { transfusions }\end{array}$ & (Median, Range) & $\begin{array}{c}45 \\
(13-143)\end{array}$ & \\
\hline ICU transfer & & 18 & 31.03 \\
\hline $\begin{array}{l}\text { Deaths due to infective } \\
\text { complication }\end{array}$ & & 8 & 13.78 \\
\hline $\begin{array}{l}\text { Deaths within } 30 \text { days of } \\
\text { induction }\end{array}$ & & 5 & \\
\hline \multirow[t]{2}{*}{$\begin{array}{l}\text { Adverse reactions due to } \\
\text { granulocyte transfusions }\end{array}$} & Grade IV / Grade V & 0 & 0 \\
\hline & $\begin{array}{l}\text { Grade I/ Grade II (Respiratory distress: } \\
2 ; \text { Fever: } 1 ; \text { Pruritic skin rash: } 1 ; \text { High } \\
\text { haemoglobin requiring phlebotomy: } 1 \text { ) }\end{array}$ & 5 & 8.62 \\
\hline
\end{tabular}

\section{"Swinging Heart": A Rare Case of T-Cell Lymphoblastic Lymphoma Presenting as Pericardial Tamponade}

Robin Singh ${ }^{* 1}$, Davinder Paul ${ }^{1}$, Gurbhej Singh ${ }^{2}$, Naved Aslam ${ }^{3}$, Suvir Singh ${ }^{4}$

${ }^{1}$ Medical Oncology, ${ }^{2}$ Cardiology, Dayanand Medical College and Hospital, ${ }^{3}$ Cardiology, Dayanand Medical College, Ludhiana, ${ }^{4}$ Clinical Hematology and Stem Cell Transplantation, Dayanand Medical College and Hospital, Ludhiana, India

Aims \& Objectives: Acute lymphoblastic lymphoma (LBL) is a variant of acute lymphoblastic leukemia (ALL) with no overt marrow involvement. T-LBL is usually presents with with fever, systemic symptoms or mediastinal mass.

Patients/Materials \& Methods: We report an unusual case of lymphoblastic lymphoma who presented with pericardial tamponade without any other systemic symptoms.

Results: A 17-year-old male presented with dyspnea and dry cough for 1 week. Initial examination was remarkable for pallor and tachycardia with otherwise stable vital signs. Physical examination revealed muffled heart sounds and decreased breath sounds in the left lung fields, which were also stony dull on percussion. Jugular venous pulsations were prominent. Chest X-ray revealed cardiomegaly with massive left pleural effusion along with mediastinal widening. ECG showed findings of electrical alternans especially in the lead aVF along with low QRS voltage and sinus tachycardia. A screening echocardiogram confirmed cardiac tamponade and an emergency pericardiocentesis was performed, which drained $1100 \mathrm{ml}$ of strawcolored fluid. It was exudative but negative for malignant cells or tuberculosis. Both bone marrow biopsy and lumbar puncture were negative for any leukemia or lymphomatous involvement. A positron emission tomographic (PET) scan revealed a confluent mass in anterior and middle mediastinum infiltrating mediastinal vascular structures (encasing aorta, SVC, pulmonary artery) with metabolic active disease involving supra and infra diaphragmatic lymph nodes. A diagnosis was made by a CT guided biopsy which showed features of lymphoma with positivity for LCA, CD3, TdT, CD10 with MIB1of $98 \%$. The patient was planned for BFM-90 Induction Chemotherapy Protocol. The pericardial tube was removed after 2 weeks following total drainage of about $2500 \mathrm{cc}$ fluid. In his follow-up cardiological examination at 2 weeks, echocardiography revealed pericardial thickening with minimal pericardial effusion. His pericardial effusion did not reappear and there were no signs of pericardial constriction.

Discussion \& Conclusion: Leukemia/Lymphoma, especially of T cell origin commonly presents with pleural effusion or mediastinal mass. Presentation primarily as pericardial tamponade is rare. Rapid stabilization by cardiac decompression and initiation of steroids after confirming diagnosis are the cornerstone of management and can ensure long term survival in these patients.

\section{Acute Lymphoblastic Leukaemia in an Adult Presenting as Massive Pleural Effusion: Diagnostic Dilemma}

\author{
Mamatha $\mathbf{K}^{* 1}$ \\ ${ }^{1}$ Pathology, BLDEDU,Shri.B.M.Patil Medical \\ College,Vijayapura, Vijayapura, India
}

Aims \& Objectives: This case highlights the rare initial presentation of ALL (Acute Lymphoblastic Leukaemia) as isolated pleural effusion in a 46 year old male patient.

Patients/Materials \& Methods: INTRODUCTION-Pleural effusion is the rare initial presentation in leukaemia. Solid tumours like lung carcinoma, lymphomas, breast carcinomas, Gastrointestinal malignancies are known to present with pleural effusion. Leukemic infiltration of the lungs may occur as a part of a systemic relapse or rarely as an isolated pulmonary leukemic infiltration. This case highlights the rare initial presentation of ALL (Acute Lymphoblastic Leukaemia) as isolated pleural effusion in a 46 year old male patient. Patient Case Report-A 46 year old male patient presented with complaints of dyspnoea since two months, dry cough and chest pain since one month. Past history-not a known case of diabetes and hypertension. Patient is a non smoker. Respiratory examination suggested a right pleural effusion which was confirmed on chest X-ray. Results: Thoracocentesis was done and approximately one litre fluid was aspirated. The fluid was subjected to routine biochemical and cytopathological examination. The fluid cytology showed lymphoblasts. The total leucocyte count was 8060, few atypical lymphocytes were present. Bone marrow aspiration was done and reported as Acute Leukaemia and confirmed with flow cytometry as ALL. This confirmed the cause of effusion as the ALL.

Discussion \& Conclusion: Pleural effusion as a initial clinical manifestation is rare and not been reported frequently in the literature. Pleural fluid cytology is usually an early step in the diagnosis of the malignant origin of the fluid, followed by biopsy.This case highlights the rare initial presentation of ALL as isolated pleural effusion.

\section{Granulocytic Sarcoma: A Diagnostic Conundrum- Two Case Reports}

Megha Verma ${ }^{* 1}$, Amrita Saraf ${ }^{2}$, Richa Chauhan ${ }^{2}$, Ajay Sharma ${ }^{1}$, Nitin Gupta ${ }^{1}$, Seema Rao ${ }^{3}$, Jasmita Dass ${ }^{4}$, Jyoti Kotwal ${ }^{2}$

${ }^{1}$ Clinical haematology, ${ }^{2}$ Haematology, ${ }^{3}$ Pathology, Sir Ganga Ram Hospital, ${ }^{4}$ Haematology, AIIMS, Delhi, India

Aims \& Objectives: Granulocytic sarcoma is a rare extramedullary presentation of Acute Myeloid Leukemia (AML). We here present 
two case reports of patients who presented to us with this diagnostic dilemma.

Patients/Materials \& Methods: Case 1- A 17-year old boy had presented with SVC syndrome to a tertiary care center in June 2018. CAT scan revealed mediastinal mass and cervical lymphadenopathy. A diagnosis of lymphoblastic lymphoma was made on cervical lymph node biopsy. He received 3 weekly therapy of 1 st cycle of induction chemotherapy as per BFM protocol. He then presented to our center in August 2018 with neutropenic fever. After stabilization, on hematological evaluation including bone marrow study and immunophenotyping, diagnosis of acute myeloid leukemia-M0 was made. Review of lymph node biopsy showed acute myeloid leukemia (granulocytic sarcoma) with aberrant expression of Tdt and CD7. The diagnosis was revised to acute myeloid leukemia with extramedullary disease. He then received AML induction therapy, followed by two high dose cytarabine consolidation. The disease progressed after the 2nd cycle followed by salvage therapy but developed severe sepsis and later succumbed. Case 2- A 43-year old lady with 3 months history of generalized weakness was found to have pallor, generalized lymphadenopathy with hepatosplenomegaly. A provisional diagnosis of lymphoproliferative disorder was made. CBC revealed $\mathrm{Hb}-4.8 \mathrm{~g} / \mathrm{dl}$, TLC- 1230/mm3 (DLC-N-6\%,L-60\%,M-26\%,Myelo-2\%,Meta-2\%, $2 \%$ abnormal cells), Platelet count-62,000 $\times 109 / \mathrm{L}$. FDG-PET CT revealed mildly FDG-avid bilateral supra/infradiaphragmatic lymphadenopathy and hepatosplenomegaly. Bone marrow study with immunophenotyping revealed the diagnosis of acute myeloid leukemia with monocytic differentiation. Lymph node biopsy was reported as leukemic infiltrate of monocytic/monoblastic lineage- suggestive of monocytic sarcoma. Cytogenetic study on bone marrow revealed karyotype-46XX; del7q; add 12p. The final diagnosis of acute myeloid leukemia with monocytic differentiation with extramedullary disease was made. She was treated with conventional AML induction and consolidation therapy and is in morphological remission 1 month after the last consolidation cycle.

Results: Both of our patients were provisionally suspected to have lymphoproliferative disorder. The correct diagnosis of granulocytic sarcoma could be made with integration of tissue morphology, immunohistochemistry and immunophenotyping.

Discussion \& Conclusion: Extramedullary presentation of Acute myeloid leukemia is rare and often misdiagnosed. An experienced morphologist, application of immunophenotyping and broad panel of immunohistochemistry markers can contribute in arriving at a correct diagnosis.

\section{Oxidative Stress in Children with Acute Lymphoblastic Leukemia}

Preety Chaudhary ${ }^{* 1}$, Pooja Dewan ${ }^{1}$, Sunil Gomber ${ }^{1}$, Mrinalini Kotru $^{2}$, Rafat Ahmed ${ }^{3}$

${ }^{1}$ Pediatrics, ${ }^{2}$ Pathology, ${ }^{3}$ Biochemistry, UCMS, Delhi, India

Aims \& Objectives: To compare the antioxidant levels [Total antioxidant capacity (TAC)] and oxidative stress (OS) markers [thiobarbituric acid reactive substances (TBARS) in plasma in children with acute lymphoblastic leukemia (ALL) compared to healthy controls and during different phases of treatment in children with ALL. To assess relationship between antioxidant status at diagnosis and chemotherapy tolerance by recording occurrence of febrile neutropenic episodes and hematological toxicity as per the CTC (common toxicity criteria) during initial treatment. To assess correlation between antioxidant status and vitamin B12/folate levels.

Patients/Materials \& Methods: Children $\leq 12$ years in age newly diagnosed with acute lymphoblastic leukemia.Children with ALL received chemotherapy treatment by modified MCP-841 protocol.
Baseline assessment of all children i.e. Anthropometry, laboratory invstigations were done, antioxidant capacity was measured by using TAC in plasma and oxidative stress by TBARS in plasma.

Results: We included 23 children with ALL aged $<12$ years and 19 age-sex matched children as controls. The oxidative stress (OS) in children with ALL at diagnosis was significantly higher than that in controls. TAC levels in children with ALL vs controls were $1.17(0.12) \mathrm{mM}$ vs $1.29(0.04) \mathrm{mM}[p<0.001]$, TBARS levels 311.33 (124.99) $\mathrm{nmol} / \mathrm{mL}$ vs $16.16(3.71) \mathrm{nmol} / \mathrm{mL}[p<0.001$. OS was highest at completion of induction-1 phase of chemotherapy in children with ALL. TAC levels 1.01 (0.18) mM, TBARS levels 472.52 (191.02) nmol/mL and 8OHdG levels $4.50(2.50) \mathrm{ng} / \mathrm{mL}$ at the end of induction-1. OS at completion of intensive chemotherapy (induction 2A-consolidation) was higher than at diagnosis despite clinical remission of disease. TAC levels at end of induction 2A-consolidation were $1.13(0.09) \mathrm{mM}$, TBARS levels 364.30 (112.26) nmol/mL which were significantly different from that at diagnosis and also from controls. A significant correlation was found between serum folate levels and total anti-oxidant capacity (TAC) at baseline $(p=0.02)$. No correlation was observed between OS and serum vitamin B12 levels, hematological toxicity, total febrile neutropenic episodes.

Discussion \& Conclusion: Oxidative stress (OS) in children with ALL is significantly higher compared to controls indicating that underlying disease affects the oxidative balance unfavourably. Persistent OS is due to chemotherapy.

\section{Clinical Profile and Outcomes in Patients with Acute Myeloid Leukemia at a Tertiary Care Center in North India}

Kapil L. Barbind ${ }^{* 1}$, Kundan Mishra ${ }^{2}$, Rajan Kapoor ${ }^{3}$, Suman K. Pramanik $^{3,4}$, Yanamandra Uday ${ }^{3,4}$, Deepti Mahajan ${ }^{5}$, Diksha Pandey $^{6}$, Ankur Ahuja ${ }^{7}$, Tathagata Chatterjee ${ }^{8}$

${ }^{1}$ General Medicine, Army Hospital, R\&R, ${ }^{2}$ Clinical Haematology \& Stem Cell Transplantation, Army Hospital R\&R, Delhi,

${ }^{3}$ Clinical Haematolgy, Command Hospital, Eastern Command, Kolkatta, ${ }^{4}$ Clinical Haematology \& Stem Cell Transplant, ${ }^{5}$ Army Hospital R\&R, Delhi, India, ${ }^{6}$ General Medicine, ${ }^{7}$ Dept of

Pathology, ${ }^{8}$ Dept of Lab Sciences and Molecular Medicine, Army Hospital R\&R, Delhi, India

Aims \& Objectives: The study was carried out with an aim to look into the clinical, cytogenetic profile \& outcomes of de novo, AML patients (non-AML-M3).

Patients/Materials \& Methods: In this prospective observational study, 100 patients with confirmed AML were enrolled from 01st June 2018 to 31st May 2019. Clinical parameters of the patients were recorded and they were evaluated for hematological, biochemical and cytogenetics profile. The outcomes were recorded in terms of survival and death.

Results: A total of $100(\mathrm{n}=100)$ patients were included in this study.Median age of study population was 46 years (range 16 75 years). There were $51(51 \%)$ males in this study. Morphological evaluation showed AML M1 to be the most common FAB type (57\%) followed by AML M4 (16\%). Based on cytogenetics, 32 patients had favourable risk while 41 had intermediate risk and remaining 17 had high risk disease. Most common presenting feature was fever (70\%), followed by pallor (52\%), gum hypertrophy $(22 \%)$ and petechia $(20 \%)$. Hepatosplenomegaly at presentation was seen in $83 \%$ of study population followed by gum hyperplasia (78\%). Complex karyotype was present in $16(16 \%)$ patients. Bone marrow study after induction chemotherapy showed compete remission in 38 (38\%) patients while 12 patients had either disease progression or partial remission only. Fifty $(50 \%)$ patients died during induction. Various infections (41\%) 
followed by progression of disease were the most common causes of mortality.

Discussion \& Conclusion: Despite improvement in supportive care and availability of targeted agents, the outcome of AML continues to be poor.

\section{Rare Presentation of Acute Lymphoblastic Leukemia as Maxillo Facial Tumor}

Akshay Lahoti ${ }^{*}{ }^{1}$, Prabodha K. Das ${ }^{1}$, Ashok Jena ${ }^{2}$, Saubhik Dasukil ${ }^{2}$, Amit K. Adhya ${ }^{3}$, Ashutosh Panigrahi ${ }^{1}$, Sonali Mohapatra ${ }^{1}$

${ }^{1}$ Hemato-Oncology, ${ }^{2}$ Dental, ${ }^{3}$ Pathology, AIIMS Bhubaneswar, Bhubaneswar, India

Aims \& Objectives: To be aware regarding atypical presentation of acute lymphoblastic leukemia.

Patients/Materials \& Methods: Acute lymphoblastic leukemia (ALL) is considered as the most common malignant neoplasm of childhood and the frequent cause of death from cancer before 20 -years. The facial swelling mimicking a maxillofacial tumor is rarely associated with leukemia. Clinicians should be aware of such rare manifestation of ALL. We present a case with an atypical mass in the facial region secondary to acute lymphoblastic lymphoma, which resulted in diagnostic dilemma. Reports of such atypical swelling in patients with ALL are occasional. The swelling was aggressive and the disease had a fulminant course.

Results: Patient succumbed within 3 weeks of starting chemotherapy with BFM-2002 protocol.

Discussion \& Conclusion: The extra-oral maxillo-facial representation in the present case was unusual, which preceded the diagnosis of acute lymphoblastic leukemia. Early diagnosis is a critical factor for the outcome of the disease. A clinician must be aware of the atypical presentation of this neoplasm for early diagnosis and treatment. Also such presentation should have an extensive genetic mapping including for Ph-like ALL at baseline.

\section{Acute Leukemia (Laboratory)}

\section{Hematocytological Evaluation of Acute Myeloid Leukemia Manifesting as Pleural Effusion}

Deepshikha Rana ${ }^{* 1}$, Swarnim Dalakoti ${ }^{2}$, Sucheta Malik ${ }^{2}$, Rajeev $\operatorname{Sen}^{2}$

${ }^{1}$ Pathology, PGIMS, Rohtak, Gurgaon, ${ }^{2}$ Pathology, PGIMS, Rohtak, Rohtak, India

Aims \& Objectives: Acute Myeloid Leukaemia (AML) with leukemic pleural effusion is an unusual entity. Its incidence is unclear and very few cases have been reported so far. We would like to present a case of AML manifesting as malignant pleural effusion. Acute myeloid leukaemias (AML) are defined as clonal expansions of myeloid blasts, most commonly in blood or bone marrow but occasionally presenting as tumour masses (myeloid sarcoma) in other tissues such as skin and lymph node. Pleural effusion caused by leukemic infiltration is an unusual extramedullary manifestation of the disease. We report a case of 54 years old female with AML with monocytic differentiation on chemotherapy who was admitted to the hospital with the complaint of shortness of breath and orthopnoea.

Patients/Materials \& Methods: In this case, 54-year-old man diagnosed as acute myeloid leukaemia with monocytic differentiation reported with pleural effusion. Peripheral blood smear examination at presentation showed a dimorphic predominantly macrocytic picture with Haemoglobin: $9.2 \mathrm{~g} \%$; Total Leukocyte Count: 9,900; Differential Leukocyte: Atypical cells: 58\%, Polymorphs: 22\%, Lymphocytes: 16\%, Monocytes: 04\%., Absolute platelet count: $80,000 / \mu \mathrm{l}$. Bone marrow aspirate favoured the diagnosis of acute myeloid leukaemia with monocytic differentiation. On flow cytometry, side scatter vs CD45 population $(68 \%)$ was positive for CD45, CD64, CD4, CD13, CD33, MPO, HLA-DR. We received $30 \mathrm{ml}$ of yellow turbid pleural fluid after 6 months of its diagnosis. Smears were prepared after centrifugation and stained with Leishman stain. Results: Smears prepared and examined showed the presence of atypical neoplastic cells admixed with lymphocytes, reactive mesothelial cells and macrophages in a haemorrhagic background. Morphologically cells favoured the diagnosis of AML and further staining using myeloperoxidase was positive.

Discussion \& Conclusion: Clinically significant pleural effusions, regardless of aetiology, are rarely encountered in patients with AML. However, the incidence of leukemic pleural effusions may be increasing as a result of longer survival with improved chemotherapies. Leukemic pleural effusions are rare but the incidence may be underestimated. The timing of development of pleural effusion varies in all patients so, cytogenetic studies must be done as a routine part of pleural fluid analysis in cases of AML. It is very important for us to evaluate pleural effusions in such patients to classify them as refractory disease or relapse in patients with marrow remission thus affecting its prognosis.

\section{Immunophenotyping of T Cell Acute Lymphoblastic Leukemia: Practical Hurdles}

Priya G. Pai ${ }^{* 1}$, Sushma Belurkar ${ }^{1}$, Sindhura Lakshmi Koulmane Laxminarayana $^{1}$

${ }^{1}$ Department of Pathology, Kasturba Medical College, Manipal, MAHE, Manipal, India

Aims \& Objectives: To study the immunophenotypic characteristics in cases of $\mathrm{T}$ cell Acute Lymphoblastic Leukemia (T-ALL) and to assess the diagnostic difficulties encountered in its subtyping.

Patients/Materials \& Methods: This is a 4 year retrospective study (October 2016 - September 2020) in which 37 cases of T-ALL were analyzed on flow cytometry using a $6 / 8$ color panel of monoclonal antibodies, which included B cell, T cell, myeloid and non- lineage markers like CD34 and HLA-DR. The cases were categorized as Early $\mathrm{T}$ cell phenotype ALL (ETP-ALL), Cortical T-ALL and Medullary T-ALL. The clinical details were retrieved from patient case files.

Results: A total of 37 T-ALL cases were analyzed during the study period. Age range was 1-64 years, with median age being 13 years. 23 patients were below the age of 18 years. Male to female ratio was 2.1:1. The most common clinical presentation was fever, seen in $70.3 \%$ cases $(n=26) .35 .1 \%$ cases $(n=13)$ were documented to have mediastinal mass and $67.6 \%$ cases $(n=25)$ had palpable splenomegaly. Blast percentage ranged from 39 to $98 \%$, with median of $90 \% .10 .8 \%$ cases $(n=4)$ were ETP-ALL, $27.0 \%$ cases $(n=10)$ were Cortical T- ALL and $27.0 \%$ cases $(\mathrm{n}=10)$ were Medullary T-ALL. $35.1 \%$ cases $(n=13)$ could not be definitely assigned to a specific category. The table attached denotes the frequency of expression of different immunophenotypic markers. $32.4 \%$ cases $(\mathrm{n}=12)$ were CALLA $+(\mathrm{CD} 10+)$. The aberrant expression of one or more myeloid markers were found in $27.0 \%$ cases $(n=10)$ and B-cell marker $(\mathrm{CD} 79 \mathrm{a})$ in $5.4 \%$ cases $(\mathrm{n}=2)$.

Discussion \& Conclusion: Although classically T-ALL is subtyped into 4 categories, definite criteria have not been established for each category. The WHO 2016 classification introduced ETP-ALL as a provisional entity and mentions that this includes most pre and pro 
T-ALL cases. However, information regarding its CD5 expression is vague. In our study, 13 cases could not be accurately classified due to antigenic overlap and due to lack of an extended panel of secondary markers. We thus conclude that a wider panel of markers is required to categorize the T-ALL cases, which has prognostic and therapeutic implications.

Supporting Document:

Number and percentage of cases showing positivity for various markers

\begin{tabular}{|c|c|c|c|}
\hline S.No & Marker & No. of cases positive & Percentage (\%) \\
\hline 1 & CD7 & 37 & 100 \\
\hline 2 & CCD3 & 37 & 100 \\
\hline 3 & SCD3 & 25 & 67.6 \\
\hline 4 & CD5 & 33 & 89.2 \\
\hline 5 & CD2 & 34 & 91.9 \\
\hline 6 & CD1a & 11 & 29.7 \\
\hline 7 & CD4 & 17 & 45.9 \\
\hline 8 & CD8 & 14 & 37.8 \\
\hline 9 & CD10 & 12 & 32.4 \\
\hline 10 & CD56 & 3 & 8.1 \\
\hline 11 & CD34 and/or HLA-DR & 21 & 56.8 \\
\hline 12 & Any myeloid marker & 10 & 27.0 \\
\hline 13 & Any B cell marker & 2 & 5.4 \\
\hline
\end{tabular}

\section{Estimation of Serum Vegf in Various Hematological and Solid Malignancies: A Pilot Study}

Sant Prakash Kataria ${ }^{* 1}$, Neha Yadav $^{2}$, Gajender Singh ${ }^{1}$, Sanjay Kumar $^{1}$, Sucheta Malik ${ }^{3}$, Rajeev Sen ${ }^{1}$

${ }^{1}$ Pathology, PGIMS, Rohtak, ${ }^{2}$ Pathology, Lal Path lab, Alwar, ${ }^{3}$ Pathology, BPS GMC Khanpur, Sonipat, India

Aims \& Objectives: Vascular endothelial growth factor (VEGF), basic fibroblast growth factor (b-FGF), IL-8 and various other cytokines are positive regulators of angiogenesis. The VEGF family plays an integral role in angiogenesis, lymphangiogenesis, and vasculogenesis. Increased levels in urine, serum, and plasma are associated with increased angiogenesis, growth and metastasis in solid tumors. The aim of the study was to investigate the role of Serum VEGF in various hematological malignancies and in solid tumors.

Patients/Materials \& Methods: A total of 62 patients with various newly diagnosed haematological malignancies and solid tumours with age ranging from 15 to 70 years were included in the present study, 38 of them were males and 24 were females. These 62 cases were divided into six groups. Group I in-cluded 20 patients of acute leukemia, group II -10 patients of Non- Hodgkin's lymphoma, group III10 patients of Chronic Myeloid Leukemia with blast crisis, group IV10 patient of multiple myeloma, group V -12 patients of solid tumours, (6 patients with Gastrointestinal malignancies and 6 patients of CNS tumours) and group VI - 10 cases of normal individual. Serum samples taken from these patients were stored at $-800 \mathrm{C}$ and serum VEGF levels were determined by Enzyme Linked Immunosorbent Assay (ELISA) method.

Results: Subgroup analysis of hematological malignancy group revealed that mean serum VEGF level in AML patients was $138.4 \pm 25.10 \mathrm{pg} / \mathrm{ml}, \quad 80.9 \pm 31.78 \mathrm{pg} / \mathrm{ml}$ in ALL patients, $65.75 \pm 40.51 \mathrm{pg} / \mathrm{ml}$ in CML with blast crisis, $156.20 \pm 110.8 \mathrm{pg} /$ $\mathrm{ml}$ in NHL patients, $156.50 \pm 16.26 \mathrm{pg} / \mathrm{ml}$ in multiple myeloma. Mean serum VEGF level in gastrointestinal malignancies was $135 \pm 39.05 \mathrm{pg} / \mathrm{ml}$ and $205 \pm 7.07 \mathrm{pg} / \mathrm{ml}$ in CNS tumors. Mean serum VEGF levels of the patients in study groups of hematological malignancies and solid tumors $(133.96 \pm 47.50 \mathrm{pg} / \mathrm{ml})$ were significantly higher than those of control group $(40.3 \pm 8.27 \mathrm{pg} / \mathrm{ml})$.

Discussion \& Conclusion: VEGF is regarded as the most important pro-angiogenic factor which is crucial for vascu-logenesis and angiogenesis and plays key role in the development and progression of vari-ous hematological malignancies and in solid tumors. Serum VEGF levels of the patients with hematological malignancies as well as solid tumors were higher compared to controls. Further studies are needed to determine the therapeutic implications of these findings.

\section{Role of Bone Marrow Microvessel Density in Hematological Malignancies}

\author{
Shipra Verma*1, Deepti Mutreja ${ }^{1}$ \\ ${ }^{1}$ Pathology, Armed Forces Medical College, Pune, India
}

Aims \& Objectives: The aim of this study was to assess the role of bone marrow microvessel density(MVD) in hematological malignancies. Our objectives were to estimate mean MVD in various hematological malignancies, to determine change in MVD following chemotherapy and study association between tumor burden and MVD if any.

Patients/Materials \& Methods: We analyzed 153 bone marrow(BM) biopsies of cases of acute leukemias(AL), myelodysplastic syndromes(MDS), myelodysplastic/myeloproliferative neoplasms(MDS/ MPN), chronic myeloproliferative neoplasms(CMPN), plasma cell neoplasms(PCN), lymphomas and aplastic anemia. CD34 stained BM biopsies were studied for morphometric assessment of MVD. Results were compared with 30 control BM samples with normal marrow morphology. MVD was quantified by three "hotspots" (areas with maximum number of microvessels) identified by scanning at $100 \times$ and microvessels were counted 400x magnification. MVD was reported as mean of three hotspots (microvessels per mm2). For statistical analysis, $p$ values $<0.05$ were considered statistically significant.

Results: Mean MVD was significantly higher in $\operatorname{AL}(p<0.001)$, MDS, $\operatorname{MDS} / \operatorname{MPN}(p=0.011), \quad \operatorname{CMPN}(p<0.001) \quad$ and $\operatorname{PCN}(p$ value $<0.001)$ cases compared to controls. Also, mean MVD significantly correlated with marrow fibrosis $(p=0.011)$ and peripheral blood blasts $(p=0.029)$ in AL cases. Mean MVD in new and relapse cases was found to be higher than remission cases in $\mathrm{AL}$, MDS, MDS/MPN and PCN cases. In PCN cases, mean MVD showed significant correlation with $\mathrm{BM}$ plasma cells $(p=0.003)$ and $\beta-2$ microglobulin $(p=0.005)$. Further, in cases of lymphoma, aplastic anemia, megaloblastic anemia and reactive marrows, we found that mean MVD was higher than controls.

Discussion \& Conclusion: Our study found significantly higher angiogenesis in not only hematological malignancies (AL, CMPN, MDS, MDS/MPN, PCN) but reactive marrows also. There was significant reduction in MVD after therapy in AL, MDS, MDS/MPN, PCN cases. Cellularity did not correlate with MVD, however marrow fibrosis and peripheral blood blasts/plasma cells correlated significantly in AL and PCN cases respectively. The study brings out a definite role of angiogenesis in pathophysiology of hematological malignancies. Further larger studies are required to establish exact mechanism and interaction of angiogenesis and BM microenvironment. Inhibitors of angiogenesis can serve as potential novel therapeutic strategy alone or in combination with the established chemotherapy regimens. Figure legend: Figure 1 Box and whisker plot showing comparison of MVD in various hematological malignancies, megaloblastic and reactive marrows with controls. 
Bone Marrow Necrosis in Acute Leukemia: A Study Done from 2014 to 2020 at a Tertiary Centre

Hitesha Bhandari* $^{* 1}$, Shashi bansal ${ }^{1}$

${ }^{1}$ Pathology, BMCHRC, Jaipur, India

Aims \& Objectives: Bone marrow necrosis (BMN) in Acute Leukemia is a rare histopathological entity at the time of initial diagnosis. However, it represents an important diagnostic and prognostic challenge. We studied all the cases of Acute Leukemia from 2014- 2020 to know the impact of recognition of necrosis in a small cohort of patients.

Patients/Materials \& Methods: We undertook a 7-year retrospective review of all bone marrow specimens with necrosis at our institution and found 9 cases with main clinical presentation of fever,weakness,fatigue with organomegaly. In all these cases patient was investigated and diagnosis were made based on the corroboration of complete blood count, Bone marrow examination and Flowcytometric immunophenotyping along with clinical findings.

Results: Among 9 cases of Acute Leukemias,6/9 (66.66\%) patients were diagnosed of ALL and 1(11.11\%) each for APML, AML and Undiagnosed Acute Leukemia respectively. Among 6 cases of ALL,3/ $6(50 \%)$ presented with grade I, $2 / 6(33.33 \%)$ with grade II and $1 / 6(16.66 \%)$ with grade III necrosis. Out of 9 cases, APML and Acute Leukemia Undiagnosed each contributed 1/9(11.11\%) case with grade III necrosis whereas $1 / 9(11.11 \%)$ case was interpreted as AML with grade I necrosis(Table). Diagnostic challenges were encountered in leukemias with Grade III necrosis specially with peripheral pancytopenia.In our study we found 2 such cases.The first case of Acute Leukemia didn't reveal excess blast on peripheral blood due to marked pancytopenia and marrow showed extensive necrosis so due to unavailability of required diagnostic material the case remained unclassified and failed to follow up. The other case presented with decreasing trend of leucocyte count on peripheral blood and marrow showed extensive necrosis that hindered all the advanced studies like flowcytometric immunophenotyping, IHC. So, flowcytometry was processed from peripheral blood that showed 18-20\% blast and diagnosis of B-ALL was established .

Discussion \& Conclusion: : Bone marrow necrosis is an important and infrequent clinicopathological entity however not a rare presentation in Leukemias, but its presence is suggestive of inferior response and poor prognosis whose timely recognition and prompt careful assessment may herald the way for more effective line of treatment approach.

\section{Monocytic Leukemia Masquerading as a Cutaneous Lesion}

Abhishek Singh ${ }^{* 1}$, Preeti Tripathi ${ }^{1}$, Arijit Sen ${ }^{1}$, Akriti Kashyap ${ }^{1}$

${ }^{1}$ Pathology, Command Hospital Air Force, Bangalore, India

Aims \& Objectives: Cutaneous lesions preceding a leukaemia are extremely rare and are referred to as 'Aleukaemic Leukaemia Cutis'. We present a case of Acute Monocytic Leukaemia where skin infiltration of leukemic cells preceded any blood or bone marrow evidence of leukaemia. The case is being reported for its rarity.

Patients/Materials \& Methods: A 67 year-old woman presented with multiple cutaneous nodules all over the body of 3 months duration. She reported to a nearby health facility where a general examination showed multiple erythematous papules and plaques present over the face, trunk, extremities and back. She was also seen to have gingival hyperplasia and was given a provisional diagnosis of ?Cutaneous NHL. Investigations revealed normal blood parameters (TLC- 7130/cmm, N-57\% L-34\% M-09\%). A skin biopsy done revealed infiltration of the dermis by atypical cells suggestive of a hematolymphoid malignancy. The patient was then shifted to our institution for tertiary care management. The blood counts over a period of one month showed gradually increasing TLC from an initial normal blood count to one showing absolute monocytosis (TLC$25500 / \mathrm{cmm}$, AMC-3825/cmm) and finally abnormally high TLC (TLC-154050/cmm) with $79 \%$ monoblasts and promonocytes suggestive of Acute Leukemia. A repeat skin biopsy again showed infiltration of atypical cells in the dermis. The patient had now started exhibiting systemic findings corroborating with the cutaneous lesions and skin biopsy.

Results: IHC done on the skin biopsy showed the atypical cells to be positive for monocytic markers(CD14, CD64). Bone marrow aspirate was hypercellular and showed replacement by monoblasts $(82 \%)$. Cellular morphology was suggestive of AML-M5. Bone marrow biopsy showed a diffuse replacement of marrow by immature cells/ blasts. Flow cytometry reports were also positive for monocytic markers. The patient was hence diagnosed as AML M5 with cutaneous metastasis/Leukemia cutis and was immediately started on chemotherapy $(3+7$ chemo). Post Induction phase, the patient was in remission and her skin lesions subsided. She was subsequently discharged and advised regular OPD follow up for maintenance chemotherapy.

Discussion \& Conclusion: Cutaneous infiltration before systemic signs of Leukaemia is a fairly rare occurrence. However, an early diagnosis specially preceding multisystem involvement will go a long way in improving the prognosis and survival rate of the patient.

\section{Variant APML T(1;17): Experience of a Rare Case}

Zai Wade ${ }^{* 1}$, Shashi Bansal ${ }^{2}$, Lalit Mohan Sharma ${ }^{3}$

${ }^{1}$ Pathology, Apex Hospital Jaipur, ${ }^{2}$ Pathology, ${ }^{3}$ Medicine, Bhagwan Mahaveer Cancer Hospital, Jaipur, India

Aims \& Objectives: APML is a curable hematological malignancy with the help of targeted therapies like All trans retinoic acid(ATRA) \& Arsenic trioxide(ATO). However variant APML carrying other than $\mathrm{t}(15 ; 17)$ has different therapeutic implications. They may or may not respond to ATRA ATO, depending on genes involved in mutation.

Patients/Materials \& Methods: 24 yrs old lady presented to us in 2013 with a report of AML (diagnosed outside). Bone marrow aspirate showed PM + blasts $98 \%$. Flowcytometry favoured possibility of APML, but RTPCR for PML-RARA was negative.Conventional chemotherapy (AraC) for AML was started and patient went on remission. In 2017, patient relapsed with right parotid swelling. CBC showed PM + blasts $70+5$. Repeat RTPCR done, was negative.Conventional cytogenetics revealed 46XX,t(1;17)(p22;q21), simple variant APML.FISH for RARA break apart probe was done, showed break apart signal in $45 \%$ cells. Patient was given ATRA ATO, but follow up marrow didnt show remission.So, again chemotherapy for AML was given \& remission achieved. In 2020 July, she presented with multiple breast nodules. CBC revealed $6 \%$ atypical myeloid cells. Trucut biopsy from breast showed infiltration by myeloid cells.Bone marrow aspiration done subsequently showed relapse. Again conventional chemotherapy was given \& remission achieved.

Results: patient of variant APML $\mathrm{t}(1 ; 17)$ responds to conventional chemotherapy given for AML, while didnt respond to ATRA ATO. Discussion \& Conclusion: As variant APML with $t(1 ; 17)$ is exceedingly rare entity and almost no literature is available regarding the entity, treatment approach was hit and trial based. As per our experience variant APML associated with $\mathrm{t}(1 ; 17)$ donot respond to ATRA and ATO. 
Lineage Switch of Acute Myeloid Leukemia to T-Cell Acute Lymphoblastic Leukemia: Unique Case Report

Syeda F. Jamil ${ }^{* 1}$, Shashi bansal ${ }^{2}$, Upendra Sharma ${ }^{3}$, Chaitali Singh ${ }^{1}$ ${ }^{1}$ Pathology, ${ }^{2}$ Pathogy, ${ }^{3}$ Medical Oncology, BMCHRC, Jaipur, India

Aims \& Objectives: "Lineage switch" is term described when leukemic cells on relapse exhibit a new phenotype, where loss of one lineage defining markers with simultaneous gain of another lineage defining markers occurs. This is known to be associated with poor prognosis, despite administration of chemotherapy. Sequential phenotyping and cytogenetic studies at initial and on relapse may capitulate for early detection of lineage switch and standard treatment initiation. Here, we present a rare case of lineage switch, from AML to T-ALL which consider of great value due to different treatment approach. So, with better understanding of the genetic basis of lineage conversion, new therapies can be designed to address this rare lineage switch phenomenon with improved insight.

Patients/Materials \& Methods: Here, an unusual case of 10 year old boy presented with fever \& constitutional symptoms in December 2014. The diagnosis of AML was made on bone marrow examination (BMEx) along with help of flowcytometric- immunophenotyping (FCM-IPT). Standard AML Chemotherapy was planned but because of financial constraints he was put on Metronomic chemotherapy (which is not the standard of care), was well tolerated from December 2014 to January 2017. However patient became symptomatic again in August 2018.

Results: On repeat investigation this case of AML switched to T-ALL upon relapse, which is very rare and not well documented till date in literature. The diagnosis was supported by morphologic, cytochemistry and flowcytometric immunophenotyping (FCM-IPT).

Discussion \& Conclusion: There are not much data on lineage conversion available (mostly single case reports), probably because of FCM-IPT is generally performed initially but not at the time of relapse and thus the actual incidence of these events might be underestimated. During the treatment, if there is change in blast morphology, the FCM-IPT should be perform to confirm the presence of any subclone or emergence of new clone, which are usually resistant to treatment. We recommended phenotyping and cytogenetic studies at the time of relapse to confirm these rare events before reinitiation of relapse treatment protocol or whenever there is suspicious change in morphology.

\section{Cell Population Data and Scattergram Driven Rapid Screening of Acute Promyelocytic Leukemia}

Shruthi Neelamegam Ramesh ${ }^{* 1}$, Shruti Mishra ${ }^{1}$, Somanath Padhi ${ }^{1}$, Gaurav Chhabra ${ }^{1}$

${ }^{1}$ Pathology and Lab Medicine, All India Institute of Medical Sciences, Bhubaneswar, India

Aims \& Objectives: Acute promyelocytic leukemia (APL) is the commonest hematological emergency associated with an increased risk of bleeding. The timely identification of APL based on morphology supplemented by immunophenotypic and molecular evaluation is crucial for the management of patients. Although definitive, this approach is challenged by the experience of the morphologist, lack of appropriate setup and technical expertise particularly in resource constrained settings. Recent advances in technology has allowed automated cell counter to provide other relevant information based on the morphological and functional characteristics of the cell in the scatter plots and leucocyte cell population data (CPD). Herein this study we aimed to evaluate the utility of leucocyte CPD and scattergrams in the rapid screening of APL.

Patients/Materials \& Methods: We evaluated the leucocyte cell population data for the positional-parameters on neutrophils (NE- X, NE-Y, NE-Z, NE-WX, NE-WY, NE-WZ), lymphocytes (LY-X, LYY, LY-Z, LY-WX, LY-WY, LY-WZ), and monocytes (MO-X, MO$\mathrm{Y}, \mathrm{MO}-\mathrm{Z}$ and, MO-WX, MO-WY, MO-WZ) and scattergrams generated by Sysmex XN-1000 analyzer on 22 consecutively diagnosed cases of APL and compared it with 100 normal and 52 reactive controls. The CPD parameters were also compared with data obtained from 79 cases of non-APL acute myeloid leukemia (AML).

Results: The scatter-plots of APL cases showed a characteristic pattern which helped to differentiate it from other AML cases. This pattern was consistent irrespective of the morphological variation. There were significant differences between the CPD parameters between the APL and control group- both normal and reactive. CPD parameters on the neutrophils were able to successfully differentiate APL from other non-APL acute myeloid leukemias. ROC curves were plotted and a cutoff is proposed for various parameters to differentiate between APL and controls and other AMLs.

Discussion \& Conclusion: We conclude that the new age automated cell counters provide numerous data that remains unexplored. The combined use of scatter plots as well as leucocytic cell population data generated by Sysmex XN 1000 hematology analyser could be a useful tool in screening patients with APL pre-microscopically, particularly, at primary level where high-end technical expertise is not available and would provide important diagnostic information even before specialized tests are available.

\section{Immunophenotypic Profile of Acute Leukemia and Its Comparison with Morphological Diagnosis}

Prerna Walia ${ }^{* 1}$, Shivani Puri ${ }^{1}$, Saurabh Donald ${ }^{1}$, Naveen Kakkar ${ }^{1}$, M Joseph John ${ }^{2}$

${ }^{1}$ Department of Pathology, ${ }^{2}$ Department of Clinical Hematology, Hemato-oncology and Bone Marrow (stem cell) Transplantation, Christian Medical College and Hospital, Ludhiana, India

Aims \& Objectives: (1). To analyze the immunophenotypic profile of acute leukemia including Acute myeloid leukemia (AML), Acute lymphoblastic leukemia (ALL), Biphenotypic Acute leukemia (BPAL) and mixed phenotype acute leukemia (MAPL). (2) To compare morphological diagnosis of acute leukemia with immunophenotypic diagnosis.

Patients/Materials \& Methods: This study was carried over a period of 2.5 years. Hundred and five patients with suspected/proven leukemia by peripheral blood and/or bone marrow were included. Immunophenotyping using peripheral blood or bone marrow was carried out in all cases. Cytochemistry was applied wherever possible. Antigen expression and the percentage of expression was noted in all cases. The immunophenotypic diagnosis was compared with morphology and test of agreement (kappa) was applied.

Results: Out of 105 patients, morphological diagnosis of AML was made in 44 patients followed by 26 cases of ALL and 35 cases could not be classified further. Final diagnosis was made after immunophenotyping in these 35 patients which included 17 cases of B-ALL, 9 cases of AML with minimal differentiation, 4 cases of T-ALL, 4 cases of AML, 3 cases of Acute undifferentiated leukemia, 1 case of AML with monocytic differentiation and 1 case of MPAL. These cases were not included in the comparative analysis between leukemia diagnosis by morphology and that by immunophenotyping. Cohen's Kappa statistic showed good agreement $($ kappa $=0.83)$ between morphology and immunophenotyping. 
Discussion \& Conclusion: Morphology remains a very important diagnostic tool in patients with acute leukemia and guides the antibody selection panel for immunophenotyping. However, there were cases where morphology could not provide specific diagnosis thereby affecting treatment decisions. Also, aberrant antigen detection could be possible only on immunophenotyping. Thus, immunophenotyping is not an optional investigation but an essential tool to morphological diagnosis of acute leukemia.

Supporting Document:

\begin{tabular}{|c|c|c|}
\hline $\begin{array}{l}\text { Immunophenotypic d } \\
(n=105)\end{array}$ & gnosis of & ded in the st \\
\hline $\begin{array}{l}\text { Final diagnosis on } \\
\text { immunophenotyping }\end{array}$ & Number & Percentage \\
\hline & AML & \\
\hline $\begin{array}{l}\text { AML with monocytic } \\
\text { differentiation }\end{array}$ & 22 & 20.9 \\
\hline AML-NOS & 21 & 20 \\
\hline $\begin{array}{l}\text { AML with minimal } \\
\text { differentiation }\end{array}$ & 7 & 6.7 \\
\hline APML & 5 & 4.8 \\
\hline $\begin{array}{l}\text { AML without } \\
\text { maturation }\end{array}$ & 1 & 0.9 \\
\hline & ALL & \\
\hline B-ALL & 39 & 37.2 \\
\hline T-ALL & 6 & 5.8 \\
\hline $\begin{array}{l}\text { Acute } \\
\text { undifferentiated } \\
\text { leukemia }\end{array}$ & 3 & 2.8 \\
\hline MPAL & 1 & 0.9 \\
\hline
\end{tabular}

\section{Aberrant Marker in Acute Leukemia's: Effect on Clinico Hematological Profile and Induction Outcome}

Dwarika Tiwari $^{* 1}$, Ankur Ahuja ${ }^{2}$, Kanwaljeet $\operatorname{singh}^{3}$, Tathagata Chatterjee $^{1}$, Venkatesan Somasundarum ${ }^{1}$

${ }^{1}$ Lab Sciences \& Molecular Medicine, Army Hospital Referal and Resarch, New Delhi, ${ }^{2}$ Army Hospital Referal and Resarch, New Delhi, ${ }^{3}$ Army Hospital Referal and Resarch, New Delhi, India

Aims \& Objectives: Aberrant phenotype is a phenomenon of abnormal expression or loss of expression of cell specific lineage marker not associated with specific cell type. As per literature, there is mixed opinion regarding effect of aberrant phenotype expression on induction outcome. The purpose of the study was to find out the occurrence of aberrant phenotypes in leukemia patients and its relation to clinico-hematological profile and induction outcome.

Patients/Materials \& Methods: Prospective and retrospective study at Army Hospital (Referral and Research) New Delhi. All age and sex groups were included in this study. We analyzed a total of 200 acute leukemia cases by multiparametric flow cytometry using an acute panel of monoclonal antibodies (MoAbs).

Results: Among the 200 cases, $100(50 \%)$ cases were AML, 18() were $\mathrm{T}$-ALL $=18 \& 82()$ were $\mathrm{B}-\mathrm{ALL}=82$. Aberrant phenotypes were found in $78(78 \%)$ cases of AML, $20(41.2 \%)$ cases of B-ALL and 09 (51.0\%) cases of T-ALL. CD7 was the most frequent lymphoid associated antigen found in $28 \%$ of AML cases while CD33 was the myeloid antigen most frequently detected in ALL (37.9\%) cases. Aberrant phenotype expression was found to be more in male patients in AML \& ALL in male: female ratio of 1.8:1 \& 2.1:1 respectively.
Aberrant expression of CD7 in cases of AML more common in extreme of ages $(<2 \mathrm{yrs} \&>65 \mathrm{yrs})$. But there was no significant correlation of aberrant markers with induction outcome in this study, however more studies in greater subset of patients are required to conclude the same.

Discussion \& Conclusion: Variability in aberrant phenotype expression was observed in different types of acute leukemia patients with more aberrant marker expression in male patients \& in extreme of ages, however no effect on with induction outcome was seen.

\section{HLA-DR Expression in T Lymphocytes: An Accessible and Promising Tool to Diagnose Primary HLH}

Sainadh Mukarjee ${ }^{1}$, Jaikumar G. Ramamoorthy ${ }^{* 1}$, Prabhu Manivannan $^{2}$

${ }^{1}$ Pediatrics, ${ }^{2}$ Pathology, JIPMER, Puducherry, India

Aims \& Objectives: Primary HLH, a multifaceted aggressive disorder with severe systemic hyperinflammation secondary to defect in granule dependent lymphocyte cytotoxicity or lymphocyte apoptosis. The HLH-2004 criteria is non-specific in differentiating primary and secondary types of HLH and the criteria is often satisfied by non-HLH disorders like malignancies, infections and autoimmune disorders. Utility of genetic testing to confirm primary HLH is limited by cost, availability and turn-around time (4-6 weeks). Hence a widely available biomarker to diagnose HLH will be critical in making diagnosis.

Patients/Materials \& Methods: An infant of 4 months age born to second degree consanguinity was admitted to our institute with complaints of fever, progressively increasing abdominal distension and pallor for 1 week.

Results: On examination infant had severe pallor \& moderate hepatosplenomegaly. Hemogram revealed: haemoglobin: $4.2 \mathrm{gm} / \mathrm{dl}$, total leucocyte count: $2.1 \times 103 / \mu 1$, platelet count: $21 \times 103 / \mu 1$ and atypical cells were absent in smear examination. Liver function test was normal except aspartate transaminases was 107 IU/L. Microbiological investigations (blood and urine culture, typhus and EpsteinBarr virus serology, NS1 antigen testing, polymerase chain reaction for cytomegalo virus) were non-contributory. Biochemical markers for Hemophagocytic Lympho-Histiocytosis (HLH) were: serum ferritin- $4600 \mathrm{ng} / \mathrm{ml}$, serum triglyceride- $650 \mathrm{mg} / \mathrm{dl}$ and serum fibrinogen- $82 \mathrm{mg} / \mathrm{dl}$. Peripheral blood flow cytometry for HLA-DR expression in CD4 T-lymphocytes was $14.3 \%$ and CD8 T-lymphocytes was $18.2 \%$. The infant was initiated on HLH-94 protocol. A homozygous missense variation was observed in exon 3 of perforin gene.

Discussion \& Conclusion: Rapid immunological screening test (granule release assay, perforin, SAP and XIAP protein expression) are more sensitive to diagnose primary HLH. But these tests are limited by their cost and availability in limited immunohematology laboratories across the country. Shipment of samples quite often adds to the cost and affects the viability. HLA-DR expression in CD4 + $>4.8 \%$ and CD8 + T-lymphocytes $>14.4 \%$ was able to diagnose 1 oHLH with $98 \%$ and $91 \%$ specificity respectively. Anti-HLA- DR immune-cytochrome is commonly utilised in leukaemia panel and is widely available in many haematology and oncology centres across country. Its higher specificity, shorter turn-around time of $<24 \mathrm{~h}$, ease to perform and wider availability makes it a potential immunological screening test to diagnose primary HLH. To conclude addition of flow cytometric protein expression correlates and functional assays of cytotoxicity to the existing HLH-2004 criteria will augment its specificity. HLA-DR expression in T-lymphocytes is a promising tool to diagnose HLH in resource limited setting because of its wider availability and ease to perform. 


\section{Diagnostic Role of Flow Cytometry in Immunophenotyping of Adult Acute Lymphoblastic Leukemia}

Basavaraju K. Vatsala ${ }^{* 1}$, Basavaiah H. Sridevi ${ }^{1}$, Murali Nirupama ${ }^{1}$, Ashok Vanishree $^{2}$, Khadilkar Urmila ${ }^{2}$

${ }^{1}$ Pathology, Kasturba Medical College Mangalore, ${ }^{2}$ Pathology, Manipal Hospital Mangalore, Mangalore, India.

Aims \& Objectives: To study the clinico-pathological findings and to evaluate the immunophenotypic features of adult ALL.

Patients/Materials \& Methods: A retrospective study of adult ALL cases, diagnosed immunophenotypically between November- 2015 to June-2020 was performed. Baseline clinical, hematological, and immunophenotypic findings were reviewed and analyzed. Importance was given to various spectrum of morphological and immunophenotype findings in different subtypes of adult ALL.

Results: A total of 54 adult ALL cases were diagnosed during the study period with a mean age of 42.7 years and a male:female ratio of 3.6:1. Clinically, patients presented most commonly with fever(28\%), followed by organomegaly $(21 \%)$ and mediastinal mass $(7.1 \%)$. Blasts with deeply clefted nuclei were not highly specific for T-ALL and were noted in B-ALL cases as well. Hand- mirror cells were seen in 3 cases $(5 \%)$. Immunophenotypically, with expression of specific antigens in variable proportions, there were 36 cases $(66 \%)$ of B-ALL and 18 cases $(33 \%)$ of T-cell lineage. Of these, $74 \%$ cases expressed CALLA positivity $(\mathrm{CD} 10+)$. The subtype distribution of cases were as follows: 1. B-ALL- pre B-ALL (33cases;61\%), pro B-ALL (3cases;5.5\%); 2. T-ALL- cortical T-ALL (7cases;12.9\%), pre T-ALL (5cases;9.2\%), medullary T-ALL (4cases;7.4\%) and a single case (1.8\%) each of near-early T precursor ALL, and T-ALL with prethymic immunophenotype. Aberrant expression was noted in $18.5 \%$ cases and was commonly seen in T-ALL. Aberrant markers noted in T-ALL were cCD79a and CD19. Expression of CD13 and CD33 were noted in both B- and T-ALL cases and was commonly seen in T-ALL. Aberrant markers noted in T-ALL were cCD79a and CD19. Expression of CD13 and CD33 were noted in both B- and T-ALL cases.

Discussion \& Conclusion: Assessment of lineage and the differentiation stages of lymphoblasts is crucial in the management of adult ALL as these patients fall into poor prognostic age group. Multiparameter flow cytometry based immunophenotyping enhances the traditional morphological diagnosis by delivering additional information related to lineage and the differentiation stage. This also aids in monitoring the disease during measurable residual disease assessment. To conclude, immunophenotyping will greatly contribute in treatment strategies of adult ALL.

\section{Detection of $T(12 ; 21), T(1 ; 19), T(4 ; 11)$ in Pediatric Acute Lymphoblastic Leukemia by FISH}

\section{Vaishali Yadav $^{* 1}$, Anita Nangia ${ }^{1}$, Jagdish Chandra ${ }^{1}$ \\ ${ }^{1}$ Pathology, Lady Hardinge Medical College, New Delhi, India}

Aims \& Objectives: To find the frequency of any of these $t(12 ; 21) /$ ETV6-RUNX1, $\mathrm{t}(1 ; 19) / T C F 3-P B X 1, \mathrm{t}(4 ; 11) / \mathrm{MLL}-\mathrm{AF} 4$ in paediatric acute lymphoblastic leukemia by fluorescence in situ hybridisation.

Patients/Materials \& Methods: A Hospital- based Descriptive Observational study was performed in forty-Five newly diagnosed paediatric ALL cases after bilingual written informed consent and detailed history. $1 \mathrm{ml}$ of sample (peripheral blood/bone marrow) of the cases was collected in a heparinised vial (before the commencement of induction therapy) and was subjected to FISH analysis. Vysis LSI dual color dual translocation probe for ETV6-RUNX1, TCF3PBX1 and Vysis LSI MLL dual color, break apart rearrangement probe was used and the signals were interpreted in interphase nuclei.
Results: The study found that four out of the forty-five ALL cases of interest $(8.8 \%)$ showed ETV6-RUNX1 fusion signals with typical $1 \mathrm{R} 1 \mathrm{G} 1 \mathrm{~F}$ pattern and one case out of forty-five ALL cases $(2.2 \%)$ showed MLL split signal with typical 1R1G1F pattern. All the four ETV6-RUNX1 positive cases had age $>5$ year with female predominance. $75 \%$ (3/4) cases had hepatosplenomegaly, 50\% (2/4) had lymphadenopathy, $75 \%$ had total leucocyte count $<50000$ cells/ul and all the cases had high blasts percentage. Immunophenotypically, All the four cases were CALLA positive B cell ALL. On follow up all the cases were alive and MRD negative. The 1 case of MLL split signal had hepatomegaly, lymphadenopathy, low hemoglobin, low platelet count. On follow up, child did not survive after three months of initial diagnosis.

Discussion \& Conclusion: This study shows a lower percentage (8.8\%) of ETV6-RUNX1 fusion protein in Indian pediatric population with ALL as compared to other studies could be because of female predominance. No statistical significance is established regarding MLL because of a single case was positive. This study suggest incorporating FISH as a mandatory diagnostic tool in pediatric ALL. This will help in risk stratification, prognostication and modifying treatment protocols in the patient.

\section{Clinico-Pathologic Review of 118 Cases of Acute Leukemia with Special Reference to Cytochemistry and Flowcytometry}

Kamal Malukani ${ }^{* 1}$, Devashish Chaudhary ${ }^{1}$, Avinash Raghuvanshi ${ }^{1}$, Prakash Chitalkar ${ }^{2}$, Amit Varma ${ }^{1}$

${ }^{1}$ Pathology, ${ }^{2}$ Medical Oncology, Sri Aurobindo Medical College \& PG Institute, Indore, India

Aims \& Objectives: (1) To study clinico-pathological features of acute leukemia the results with morphology and cytochemical findings. (2) To further subtype the cases with flowcytometry and to associate.

Patients/Materials \& Methods: All cases of acute leukemia sand chronic myeloid leukemia in blast crises, diagnosed on peripheral blood and/or bone marrow examination over a duration of three years, were further investigated. Patient's details and clinical findings were recorded. $\mathrm{CBC}$, peripheral blood films, bone marrow aspirates and bone marrow biopsy were studied in all cases and findings were correlated. Cytochemistry was done to detect Myeloperoxidase, Periodic acid Schiff and Non specific esterase reaction in leukocytes. Classification of acute leukemias was done based on cytochemistry in correlation with morphology. Flowcytometry was done for the confirmation of diagnosis and further subtyping.

Results: Out of 118 patients, $59.32 \%$ were male and $40.68 \%$ were female $(\mathrm{M}: \mathrm{F}=1.46: 1)$. Youngest patient was 3.5 months old male while eldest was 80 years old female with mean age being 30.19 years. Acute lymphoblastic leukemias $(50.85 \%)$ outnumbered acute myeloid leukemias (44.91\%) and $4.24 \%$ cases accounted for chronic myeloid leukemia with blast crises. Hepato-splenomegaly was reported in $57.62 \%$ cases while lymphadenopathy in $25.42 \%$ (50\% cases of ALL). In $93.88 \%$ cases, distinction between myeloid and lymphoid was possible based on morphology and cytochemistry, while flowcytometry was essential in $6.12 \%$ cases only. No statistically significant relation could be obtained between type of antigen expressed and age of patient/haemoglobin content/WBC count \& platelet count ( $p=0.08, p=0.15, p=0.06, p=0.18$ ).

Discussion \& Conclusion: In a setting of lack of facilities for immunophenotyping, morphology with cytochemical evaluation is sufficient for diagnosis of majority cases of acute leukemias. FCA helps in confirming diagnosis and further typing. It is especially useful in cases with negative cytochemistry. Aberrant Ags may be 
expressed in acute leukemias so single marker expression/or lack of $\mathrm{Ag}$ should not be considered as diagnostic or exclusion of specific lineage.

\section{Study of Aberrant Expression of Cd2, Cd7, Cd19, Cd25, Cd56 And Cd79a on Flowcytometry in Acute Myeloid Leukemia with Its Immunophenotypic Characterization: A Tertiary Care Centre Based Study}

\author{
Amandeep Talwar ${ }^{* 1}$, Shashi Bansal ${ }^{1}$ \\ ${ }^{1}$ Pathology, Bhagwan Mahaveer Cancer Hospital and Research \\ Centre, Jaipur, India
}

Aims \& Objectives: To evaluate the aberrant antigen expression of CD2, CD7, CD19, CD25, CD56 and CD79a in AML in the Indian context, including its immunophenotypic characterization and correlation of clinico-pathological features with aberrant antigen expression markers.

Patients/Materials \& Methods: Inclusion Criteria: (1) All immunophenotypically newly diagnosed cases of AML, during the study period (February 2019 to June 2020) at our institution. (2) All age group patients. Exclusion Criteria: (1) Patients with AML but without immunophenotyping. (2) Patients with ambiguous lineage AL. (3) Blast crisis in a known case of MPN, MDS, MDS-MPN. (4) All patients who refused to be a part of the study.

Results: AML was the most common AL, AML-M2 most common FAB subtype, more common in males than females. Bleeding manifestations with deranged coagulation profile and higher blast + blast equivalent percentage were more in APML. Distribution of markers CD11c, CD34, CD38, and HLA-DR was significantly higher in NonAPML and of markers CD13, CD64, and CD2 was higher in APML. Antigen aberrancy more in children than adults, higher in males than females. Aberrant antigen positivity was highest in AML-M5. Mean duration of illness was significantly higher in aberrant positive AML, particularly Non APML. Splenomegaly was significantly seen in the aberrant positive Non APML. Aberrant marker positivity was $58.5 \%$ with CD56 as the most common. CD2 + (aberrancy) was significantly higher in APML and associated with CD34 + in AML-M3v. $\mathrm{CD} 7+$ (aberrancy) was significantly higher in Non APML group. Significant CD34 + seen in $71 \%$ of aberrant antigen positive AML and in 50\% aberrant positive APML cases. CD34 + and HLA$\mathrm{DR}+$ was significantly seen with $\mathrm{t}(8 ; 21)$, HLA-DR + and CD4 + with inv16. CD34 + was significantly lower with NPM1 mutation. Based on cytogenetic profile, $40.7 \%$ cases belonged to favourable risk, $42.6 \%$ intermediate, while $16.67 \%$ adverse risk group. Significant association of FLT3 mutation seen in CD25 + cases. $\mathrm{CD} 25$ + was associated with poor response to treatment.

Discussion \& Conclusion: Flowcytometry helps in identification of blasts and its characterization, useful in diagnosis of AML-M0 and can also facilitate in rapid diagnosis of APML. This study also highlights the importance of identifying aberrant markers e.g. CD25 in FLT3 mutation.

\section{Aberrant Immunophenotypic Expressions in Acute Lymphoid Leukemia}

\author{
Monica Sivakumar ${ }^{* 1}$, Atoshi Basu ${ }^{1}$, Enam M. Khan ${ }^{1}$ \\ ${ }^{1}$ Pathology, Apollo Gleneagles Hospitals, Kolkata, India
}

Aims \& Objectives: (1) To identify the aberrant immunophenotypic expressions in acute lymphoid leukemia. (2) To find out the association between the expression of such aberrant markers with haematological parameters and molecular and cytogenetic studies where available.

Patients/Materials \& Methods: 75 patients of Acute Lymphoid Leukemia who presented to Apollo Gleneagles Hospital Kolkata from January 2014 and March 2019. Flow cytometry (comprehensive leukemia panel) was done on FC500 (Beckman coulter). In all ALL cases data of complete blood picture, bone marrow studies, flow cytometry, cytogenetics and molecular studies (if available) was collected and cases classified according to latest WHO classification. Results: Out of 75 cases of ALL, 23 cases (30.67\%) showed aberrant cross-lineage expression. The most common aberrant antigen expressed were of the myeloid lineage. Amongst the B-ALL cases, the most common aberrant antigen expressed were myeloid antigens 17 cases $(77.27 \%)$. Aberrant $\mathrm{T}$ - antigens were noted in 4 cases $(18.10 \%)$. Aberrant co-positivity of myeloid as well as T-antigens was seen in 1 case $(4.54 \%) .1$ out of 10 T-ALL cases (10\%) expressed aberrant antigens (cCD79a). The most common aberrant myeloid antigen expressed was CD33(77.7\%) followed by CD13 (22.2\%) and then CD15 (11.1\%). Co-positivity of CD13 and CD33 was noted in 2 cases. CD2 and CD33 co-positivity was noted in 1 case. The most frequently expressed aberrant $\mathrm{T}$-antigen was $\mathrm{CD} 2$ seen in 3 out of 5 cases (60\%). CD8 and CD7 expression were seen in 1 case each (20\%).

Discussion \& Conclusion: Flow cytometry detects aberrant antigen expressions in ALL. In B-ALL, the most common aberration was myeloid antigen positivity followed by cross-lineage $\mathrm{T}$-antigen expression. CD33 was the most common aberrant marker expressed. Aberrant CD33 expression was most frequently associated with $t(9 ; 22)$ followed by $t(12 ; 21)$. Aberrant CD33 was more common in adults. Aberrant CD15 was most frequently associated with $t(4 ; 11)$. Aberrant CD15 was most common in children. No significant increase in cytogenetic and molecular abnormality was noted in cases expressing aberrant antigen in comparison to cases not expressing aberrant antigens. Hemoglobin levels, Total leucocyte count, platelet count, $\mathrm{CBC}$ blast percentage and bone marrow blast percentage did not vary significantly between the two groups.

\section{A Case Series of Morphological and Immunophenotypical Spectrum of Burkitt Leukemia: In Tertiary Care Centre}

Prabhugouda $\mathbf{H}^{* 1}$, Devi Venkatesan ${ }^{1}$, Prabhu Manivannan ${ }^{1}$, Rakhee $\mathrm{Kar}^{1}$, Debdatta Basu ${ }^{1}$

\section{${ }^{1}$ Pathology, JIPMER Puducherry, Puducherry, India}

Aims \& Objectives: Burkitt Lymphoma presenting as leukemia is uncommon. Such cases were earlier classified under FAB classification as Acute Lymphoblastic Leukemia (ALL - L3). The present study was undertaken to characterize the presentation and diagnosis of Burkitt leukemia by a combination of cytomorphology, immunophenotyping and cytogenetics.

Patients/Materials \& Methods: Seven cases of Burkitt leukemia were identified from the archives of our department in the period from 2012 to 2020. Clinical details, biochemical parameters indicative of tumor lysis syndrome, complete blood counts, peripheral smear, bone marrow aspiration and biopsy findings were compiled. Immunophenotyping was done by immunocytochemistry/immunohistochemistry/ flow cytometry depending on the availability of test and markers.

Results: Of the seven cases, there were six males. Four presented in childhood, three were adults. The ages ranged from 08 to 45 year. Peripheral blood showed more than $25 \%$ atypical lymphoid cells in six cases. These were large cells with characteristic vacuolated basophilic cytoplasm with high $\mathrm{N}: \mathrm{C}$ ratio and increased mitotic index. Five cases presented with tumor lysis syndrome at initial presentation. 
Almost all of our cases had peripheral blood involvement except one case. The bone marrow aspiration and biopsy showed diffuse/near total replacement by these cells. Immunophenotyping showed positivity for CD10, CD20 and negativity for CD34 and Tdt in all cases. C-myc was positive in three cases. Surface IgM restriction was found in few cases. Ki-67 was more than $95 \%$ in all cases.

Discussion \& Conclusion: Burkitt leukemia, a counterpart of Burkitt lymphoma is rare, characterized by high cell proliferation and tends to involve the CNS early in the disease course. Burkitt leukemia is a medical emergency and a life-threatening disease, therefore a prompt diagnosis and treatment are decisive to avoid a fatal outcome.

Expression of Leukemia Associated Immunophenotype Markers at Diagnosis by 10 Color Flow Cytometry in B Cell Precursor Acute Lymphoblastic Leukemia for Optimization of Minimal Residual Disease Panel

Chandni Bhandary ${ }^{* 1}$, Prabhu $\mathrm{M}^{1}$, Rakhee $\mathrm{Kar}^{1}$, Debdatta Basu ${ }^{1}$

${ }^{1}$ Pathology, JIPMER, Pondicherry, India

Aims \& Objectives: To study the expression of leukemia associated immuophenotypic (LAIP) markers at diagnosis in B-cell precursor acute lymphoblastic leukemia (BCP-ALL) for further optimization of minimal residual disease (MRD) panel.

Patients/Materials \& Methods: This pilot study included newly diagnosed cases of BCP-ALL by 10 color flow cytometry from October 2019 to June $2020(\mathrm{n}=35)$. Bone marrow aspirate sample was processed by stain-lyse-wash method and the sample was acquired in a precaliberated flow cytometer. Four tubes consisting of 26 marker panel for immunophenotyping were analyzed-table 1 (Tube 1: CD45, CD34, HLA-DR, nTdT, cMPO, CD19, CD79a, cCD3, sCD3 \& CD7; Tube 2: CD45, CD19, CD10, CD20, CD38, CD34, CD81, CD123, CD 9; Tube 3: CD45, CD19, CD34, CD10, CD20, CD38, CD58, CD73, CD86 \& CD44; Tube 4: CD45, CD19, CD34, CD13, CD33, CD11c, CD11b, CD15 \& CRLF-2). The data was interpreted using Kaluza software. LAIP markers expressed in $>80 \%$ of the cases are considered for further optimization of MRD panel.

Results: Mean age of presentation was 23 years $(2-70 \mathrm{yrs})$ with male: female ratio of 1.25: 1 . Among the 35 cases markers such as CD9, CD81, CD73, CD86, CD58, CD123 and CD 44 was expressed in $96.6 \%, 94.2 \%, 88.2 \%, 85.7 \%, 74.2 \%, 69.5 \%$ and $46.4 \%$ respectively. Aberrant myeloid markers, CD33 and CD13 were expressed in $31.4 \%$ and $8 \%$ of the cases.

Discussion \& Conclusion: CD9, CD81, CD73 and CD86 were the most relevant markers which can be included in a single tube MRD panel (CD45, CD19, CD20, CD10, CD34, CD38, CD9, CD81, CD73 and CD86). This is cost efficient and reduces the number of LAIP markers currently used for MRD diagnosis. The study requires follow up validation with more number of cases for further optimization.
Supporting Document:

TABLE-1: Tube 1-4 is the routine panel. Tube 5 showing the optimized panel for

MRO.

\begin{tabular}{|l|l|l|l|l|l|l|l|l|l|l|}
\hline Tubes & FITC & PE & ECD & PC5.5 & PC7 & APC & APC700 & APC750 & PB & K0 \\
\hline 1 & nTd & MP0 & CD19 & CCD79 & CD34 & CCD3 & CD45 & CD7 & SCD3 & HLADR \\
\hline 2 & CD81 & & CD123 & CD19 & CD34 & CD10 & CD20 & CD38 & CD9 & CD45 \\
\hline 3 & CD58 & CD73 & CD19 & CD86 & CD34 & CD10 & CD20 & CD38 & CD44 & CD45 \\
\hline 4 & CD13 & CRLF- & CD19 & CD33 & CD34 & CD11C & CD45 & & CD11B CD15 & \\
\hline $\begin{array}{c}5- \\
\text { Optimi } \\
\text { zed } \\
\text { panel }\end{array}$ & CD81 & CD73 & CD19 & CD86 & CD34 & CD10 & CD20 & CD38 & CD9 & CD45 \\
\hline
\end{tabular}

\section{Mixed Phenotypic Acute Leukemia (MPAL): Characteristics and Outcomes of Patients on a Uniform Chemotherapy Protocol, at a Tertiary Care Centre in India}

Sarjana Tiwari ${ }^{* 1}$, Stitha P. Gudala ${ }^{1}$, Lakshita Singh ${ }^{1}$, Jasmita Dass ${ }^{1}$, Ganesh K. Viswanathan ${ }^{1}$, Mukul Aggarwal ${ }^{1}$, Rishi Dhawan ${ }^{1}$, Pradeep Kumar $^{1}$, Tulika Seth ${ }^{1}$, Seema Tyagi ${ }^{1}$, Manoranjan Mahapatra ${ }^{1}$

${ }^{1}$ Hematology, AIIMS, New Delhi, India

Aims \& Objectives: Mixed Phenotypic Acute Leukemia (MPAL) is a very rare neoplasm and accounts for $2-5 \%$ of all acute leukemias. Treatment protocol used worldwide is heterogenous and survival outcome is dismal. There is a paucity of data from Indian population. This is a retrospective analysis of all cases of MPAL, diagnosed at one of the largest tertiary care centres in India.

Patients/Materials \& Methods: We retrieved details of all consecutive patients of MPAL and analysed their demographic, treatment and outcome details. All the patients were diagnosed on the basis of WHO,2008 criteria. A uniform treatment protocol was administered to all these cases.

Results: Study period was between January 2016 and September 2020. Out of a total of 1626 newly diagnosed patients of acute leukemia, $27(1.7 \%)$ were characterised as MPAL. Median age was 21 years and male to female ratio was 4.4:1. B-Myeloid type were more common (59\%). Karyotyping details were available for 8 patients, among which 5 patients had normal cytogenetics and one patient had positive Philadelphia chromosome. Treatment details were available for 26 patients. Two patients did not take further treatment after diagnosis. Rest 23 patients were treated uniformly with acute lymphoblastic leukemia protocol. There were 14 induction deaths $(54 \%)$. One patient underwent bone marrow transplantation in remission. Seven patients were alive at the time of analysis. KaplanMeier estimate of all patients who survived induction phase revealed that at a median follow up of 15 months (3-38 months), 2 years overall survival was $65 \%( \pm 17 \%)$.

Discussion \& Conclusion: Incidence of MPAL is very low, similar to western population. We observed a high induction related mortality during the treatment. Prognosis is very poor with currently available chemotherapy protocols. A high degree of suspicion for diagnosis, 
optimal supportive care and intensification of therapy may help improve outcome.

\section{Study of Serial Scattergram Patterns of Sysmex Xn- 9000 in Acute Promyelocytic Leukemia}

\author{
Swati Singh $^{* 1}$, Tushar Sehgal ${ }^{1}$ \\ ${ }^{1}$ Department of Laboratory Medicine, AIIMS, New Delhi, Delhi, \\ India
}

Aims \& Objectives: To study and analyse the serial scatteroram patterns in acute promyelocytic leukemia (APL) patients. This study was conducted to assess the usefulness of scattergram of hematology analyser and the flaggings generated in acute promyelocytic leukemia (APL) patients at the time of admission and serial follow up during the course of therapy all-trans retinoic acid and arsenic trioxide. The main objective was to assess the diagnostic and prognostic utility of scattergrams in the setting of APL.

Patients/Materials \& Methods: This study presents a prospective evaluation and retrospective compilation of scattergram findings generated by XN-9000, used in our laboratory, which provided a clue to the early diagnosis of APL. The scattergram of five APL cases were studied. The APL cases were identified based on the sacttergram and flags generated with subsequent peripheral smear examination followed by confirmation on bone marrow examination combined with molecular techniques. follow-up of patient was done during the course of therapy and changes in Serial scattergram were studied.

Results: APL represents a hematologic emergency that enforces a rapid exclusion for a quick start of its treatment, even before molecular and cytogenetic diagnosis. The scattergram on hematology analyzers are very sensitive as well as specific for diagnosing acute leukemia. Abnormal promyelocytes in APL can be initially identified on scattergram pattern. Correlation to morphology is important as hyperganular APL blasts generally fall in the same region as normal granulocytes. In our study 2 cases showed characteristic pattern with increased lateral fluorescence in monocytic region, 3 cases showed less common pattern with high fluorescence in lymphoid region, which were further diagnosed on peripheral examination followed by molecular studies. Follow up showed serial changes in scattergram, mature cells started appearing within one week of initiation of therapy.

Discussion \& Conclusion: It is suggested that the laboratories with high throughput of samples, screening is important as it will let us know which samples are suspicious of leukemia. This is easy-to-use and quick method which can support the diagnosis of APL and the prompt early initiation of the appropriate treatment.

\section{Mixed Phenotypic Acute Leukemia: Immunophenotyping Pattern}

\author{
Monika Gupta ${ }^{* 1}$, Pooja Dhamija ${ }^{1}$, Nisha Marwah ${ }^{1}$, Rajeev Sen ${ }^{1}$ \\ ${ }^{1}$ Pathology, PGIMS, Rohtak, India
}

Aims \& Objectives: Multiparametric approaches are being used for the diagnosis and sub classification of Acute Leukemia (AL). However, even after extensive immunophenotyping, a rare and heterogeneous subgroup of AL which cannot be readily classified, is Mixed Phenotypic Acute leukemia (MPAL) in which the blasts exhibit the antigens of more than one lineage. MPAL can be $\mathrm{B} /$ Myeloid, T/Myeloid or B/T. The aim of this retrospective study was to analyze the incidence of MPAL and correlate the clinicopathological and immunophenotypic characteristics.
Patients/Materials \& Methods: This study was done in PT. B.D. Sharma institute of Health Sciences, Rohtak from 2016 to June 2020. Clinical details and morphological evaluation was done. Immunophenotyping was performed on FACS Canto II Flowcytometer (Becton-Dickinson, San Jose, CA) and analysed by FACS Diva software. The diagnosis of MPAL was based mainly on the WHO 2008 classification.

Results: We analysed 7 MPAL cases, which include 4 cases $(57.1 \%)$ of B/MPAL and 3 cases (42.8\%) of T/MPAL. Males to females ratio was $0.4: 1$. The mean age was 39 years (range $23-70$ years). Hematological parameters included mean $\mathrm{Hb} 6.3 \mathrm{~g} \%$ (4.3-8.0 g \%), mean Total leucocyte count (TLC) $137.8 \times 109 / \mathrm{L} \quad(27 \times 109 / \mathrm{L} \quad$ $330 \times 109 / \mathrm{L})$, mean Platelets $56.8 \times 109 / \mathrm{L}(20 \times 109 / \mathrm{L}-80 \times 109 /$ L), mean percentage of blasts $77 \%(66 \%-90 \%)$ in peripheral smear. On flow cytometry, all cases were positive for cMPO while B/Meyloid cases were positive for CD19 and at least on B cell marker. T/Myeloid cases were positive for $\mathrm{CCD} 3$ as well as $\mathrm{CD} 7$ in all cases besides other myeloid and T cell markers.

Discussion \& Conclusion: The frequency of MPAL in our study was found to be $2.7 \%(7 / 256)$ which is in concordance with published data $(2.2-2.6 \%)$. MPAL are rare leukemias with diagnostic and therapeutic challenge and worse prognosis due to origin from primitive multipotent progenitors which are resistant to treatment and lineage plasticity. Strict diagnostic criteria should be followed in the diagnosis. Immunophenotyping is absolutely essential and should include lineage specific markers along with the cytoplasmic markers for proper categorization of MPAL.

\section{Autopsy Findings in a Young Male with Alleged Unknown Bite Masking a Life Threatening Malignancy \\ Nisha Verma*1, Shipra Verma ${ }^{2}$, Puneet Baveja ${ }^{3}$, Deepti Mutreja ${ }^{4}$ \\ ${ }^{1}$ Depatment of Pathology, ${ }^{2}$ Dept of Pathology, Armed Forces Medical College, ${ }^{3}$ Department of Pathology, Armed Forces Medical, ${ }^{\mathbf{4}}$ Department of Pathology, Armed Forces Medical College, Pune, India}

Aims \& Objectives: This report brings out the autopsy findings of a young-male, with no known co-morbidities, who was transferred to our hospital as a case of disseminated intravascular coagulation (DIC) with multiple organ dysfunction syndrome (MODS) following a suspected bite of vasculotoxic snake.

Patients/Materials \& Methods: The patient was managed antemortem as a case of snake bite and had a short stormy clinical course. $\mathrm{He}$ had features of DIC, diffuse alveolar damage (DAD) and acute kidney injury (AKI). He succumbed despite being given antisnake venom, hemodialysis and transfusion support. An autopsy was performed and representative samples from the organs were fixed in $10 \%$ buffered formalin, paraffin embedded sectioned and stained with hematoxylin and eosin. Immunohistochemistry was performed to aid the diagnosis.

Results: Postmortem findings of bone marrow showed features of acute myeloid leukemia(AML) with leukemic infiltration of various organs. The peripheral blood smear was reviewed postmortem. Considering both the clinical and autopsy findings, the diagnosis of FAB AML-M3 was made, though the confirmatory test in the form of cytogenetics and molecular diagnostics could not be performed.

Discussion \& Conclusion: Acute Promyelocytic Leukemia (APML) is a subtype of AML characterized by PML - RARA fusion gene and is one hematological malignancy that is a medical emergency. APML requires a prompt diagnosis based on its very characteristic clinical, morphological, immunophenotypic and molecular features. The presentation of APML as a granulocytic sarcoma is rare. The symptomatology of this patient was confused with that of a snake bite 
in view of his presentation as a granulocytic sarcoma with early onset of DIC. Early and accurate diagnosis of APML is of utmost importance for early intervention to prevent the dreaded complications of coagulopathy. The diagnostic importance of an urgent PBS cannot be undermined. Clinicopathologic correlation of a case of APML that was clinically confused with a vasculotoxic snake bite is discussed.

\section{Early T-Cell Precursor Lymphoblastic Leukaemia: Old Entity with New Identity}

Pinki Devi ${ }^{* 1}$, Monika Gupta ${ }^{2}$, Anjali Bishlay ${ }^{1}$, Sant Prakash ${ }^{3}$, Rajeev $\operatorname{Sen}^{1}$

${ }^{1}$ Pathology, PGIMS, ROHTAK, ${ }^{2}$ Pathology, PGIMS,ROHTAK, ROHTAK, ${ }^{3}$ Pathology, PGIMS, ROHTAK, PGIMS, India

Aims \& Objectives: To report an immunophenotypically diagnosed case of ETP ALL.

Patients/Materials \& Methods: : A 15 years old male presented with history of low grade fever and weakness from last 15 days. There was no significant past or family history. On examination moderate pallor and lymphadenopathy was observed. Peripheral blood showed features of acute leukemia and was negative for Sudan staining. Immunophenotyping was performed on 8 Color Flowcytometer BD FACS Canto II (Becton-Dickinson, San Jose, CA).The CD panel used was $\mathrm{CD} 45, \mathrm{CD} 34, \mathrm{sCD} 3$, cyCD3, CD5, CD2, CD7, CD4, CD8, CD10, CD20, CD19, CD79a, Tdt, CD13, CD33, CD117, HLA-DR, MPO, CD64. The analysis was performed on FACS Diva software. Gating strategy was side scatter (SSC)/Forward scatter (FSC) and SSC/CD45 and results were expressed as the $\%$ of positive cells in the gate. Results: Immunophenotypic analysis showed positivity for cytCD3, CD7(T-cell Markers),HLA-DR,CD13(Myeloid marker-aberrant expression),sCD34,CD117(Stem cell markers),CD19(B-cell marker) and dim expression of CD45. Immunophenotyping showed negative expression of CD1a, CD3, CD5, CD2, CD4, CD8 and Tdt. These findings were consistent with immunophenotype of ETP- ALL.

Discussion \& Conclusion: This case report is a supportive data for identifying immunophenotypically cases of ETP ALL in centres where genetic study and other ancillary techniques are not available. It needs to be differentiated from Non ETP ALLs as this entity has been reported to show treatment failure with the treatment modalities for Non ETP ALLs.

\section{Hematocytological Evaluation of Acute Myeloid Leukemia Manifesting as Pleural Effusion}

Deepshikha Rana*1, Swarnim Dalakoti ${ }^{2}$, Sucheta Malik ${ }^{1}$, Rajeev $\mathrm{Sen}^{2}$

${ }^{1}$ Pathology, PGIMS, Rohtak, Gurgaon, ${ }^{2}$ Pathology, PGIMS, Rohtak, Rohtak, India

Aims \& Objectives: Acute Myeloid Leukaemia (AML) with leukemic pleural effusion is an unusual entity. Its incidence is unclear and very few cases have been reported so far. We would like to present a case of AML manifesting as malignant pleural effusion. Acute myeloid leukaemias (AML) are defined as clonal expansions of myeloid blasts, most commonly in blood or bone marrow but occasionally presenting as tumour masses (myeloid sarcoma) in other tissues such as skin and lymph node. Pleural effusion caused by leukemic infiltration is an unusual extramedullary manifestation of the disease. We report a case of 54 years old female with AML with monocytic differentiation on chemotherapy who was admitted to the hospital with the complaint of shortness of breath and orthopnoea.
Patients/Materials \& Methods: In this case, 54-year-old man diagnosed as acute myeloid leukaemia with monocytic differentiation reported with pleural effusion. Peripheral blood smear examination at presentation showed a dimorphic predominantly macrocytic picture with Haemoglobin: 9.2 g \%; Total Leukocyte Count: 9,900; Differential Leukocyte: Atypical cells: 58\%, Polymorphs: $22 \%$, Lymphocytes: 16\%, Monocytes: 04\%., Absolute platelet count: $80,000 / \mu \mathrm{l}$. Bone marrow aspirate favoured the diagnosis of acute myeloid leukaemia with monocytic differentiation. On flow cytometry, side scatter vs CD45 population (68\%) was positive for CD45, CD64, CD4, CD13, CD33, MPO, HLA-DR. We received $30 \mathrm{ml}$ of yellow turbid pleural fluid after 6 months of its diagnosis. Smears were prepared after centrifugation and stained with Leishman stain. Results: Smears prepared and examined showed the presence of atypical neoplastic cells admixed with lymphocytes, reactive mesothelial cells and macrophages in a haemorrhagic background. Morphologically cells favoured the diagnosis of AML and further staining using myeloperoxidase was positive.

Discussion \& Conclusion: Clinically significant pleural effusions, regardless of aetiology, are rarely encountered in patients with AML. However, the incidence of leukemic pleural effusions may be increasing as a result of longer survival with improved chemotherapies. Leukemic pleural effusions are rare but the incidence may be underestimated. The timing of development of pleural effusion varies in all patients so, cytogenetic studies must be done as a routine part of pleural fluid analysis in cases of AML. It is very important for us to evaluate pleural effusions in such patients to classify them as refractory disease or relapse in patients with marrow remission thus affecting its prognosis.

\section{CD34 Negative HLA-DR Negative Acute Myeloid Leukemia: A Higher Association with NPM1 Mutation}

Smeeta Gajendra ${ }^{* 1}$, Ritu Gupta ${ }^{1}$, Sanjeev K. Gupta ${ }^{1}$, Deepshi Thakral $^{1}$, Ranjit K. Sahoo ${ }^{2}$, Sameer Bakshi ${ }^{2}$, Atul Sharma ${ }^{2}$, Lalit Kumar $^{2}$

${ }^{1}$ Laboratory Oncology, ${ }^{2}$ Medical Oncology, Dr. BRA IRCH, All India Institute of Medical Sciences (AIIMS), New Delhi, India

Aims \& Objectives: The aims and objectives of this study were to analyse the incidence of CD34 \& HLA-DR negative cases and its association with morphologic, immunophenotypic, molecular and clinical characteristics.

Patients/Materials \& Methods: A total of 451 newly diagnosed cases of AML admitted in Dr. B R Ambedkar Institute Rotary Cancer Hospital, All India institute of Medical Sciences, New Delhi, from January 2018 to December 2019 were included in this study. A diagnosis of AML was made based on the results of morphology, cytochemistry, immunophenotype, cytogenetics, and/or molecular studies.

Results: Among the 451 AML patients (age range: 9 months to 75 years; M:F ratio: 1.5:1), 277 patients (61.4\%) had co-expression of CD34 and HLA-DR, 60 patients (13.3\%) were CD34negativeHLADRpositive, 31 (6.9\%) were CD34positiveHLADRnegative and $83(18.4 \%)$ were negative for both CD34 and HLA-DR. Out of these 83 CD34negativeHLADR negative cases, 41 were APL $(49.4 \%)$ and 42 were non-APL (50.6\%) patients. These 42 CD34negativeHLADR negative were further analysed which included FAB subtypes: M1 (10 patients), M2 (20 patients), M4 (4 patients), M5 (4 patient), M6 (1 patient) and M7 (3 patients). Aberrant immunophenotyic marker (CD7/CD4/CD56) expression were seen in 15 cases (35.7\%), CD56 being the most common aberrancy (23.8\%) followed by CD4 (16.7\%) and CD7 (14.2\%). None of these cases showed an expression of CD19, cCD79a or CD2. Molecular analysis was done in 
33 cases, out of which $19(57.6 \%)$ were NPM1 positive, 8 cases (24.2\%) were FLT3-ITD positive and 1 case (3\%) was FLT3-TKD positive. Post induction status was evaluated in 20 cases with a CR rate of $50 \%$. The median follow up duration was 6 months (range $<1$ to 20 months). Six CD34negativeHLADR negative patients have relapsed.

Discussion \& Conclusion: Diagnosis of Acute myeloid leukemia (AML) requires combination of cytomorphology, immunophenotyping, cytogenetics, and molecular analyses. CD34 and HLA-DR negativity are useful markers for distinguishing acute promyelocytic leukemia (APL) from other subtypes of AML, but non-APL cases without CD34 and HLA-DR antigen expression have also been reported. HLA-DR and CD34 negativity is a distinctive feature of "AML-cuplike" with FLT3-ITD [2] and in AML-M1 and AML-M2 subtypes with NPM1 mutation. To conclude, CD34 and HLA-DR negative AML accounts for $18.4 \%$ of newly diagnosed AML which includes approximately equal numbers of APL and non-APL cases. It is commonly seen in AML-M1 and AML- M2 subtypes. CD34negativeHLADR negative is highly associated with NPM1 mutation.

\section{Burkitt Lymphoma Like Morphology and "Tear Drop" Shaped Side Scatter Resembling Promyelocytes in Flow-Cytometry: Diagnostic Pitfalls in a Case of Acute Myeloid Leukemia}

Indrani Karmakar ${ }^{*}$, Safal Muhammed M K ${ }^{2}$, Reena Das ${ }^{1}$, Sreejesh Sreedharanunni ${ }^{1}$, Man U. Singh Sachdeva ${ }^{1}$, Shano Naseem ${ }^{1}$, Amita Trehan $^{2}$

${ }^{1}$ Department of Haematology, Post Graduate Institute of Medical Education and Research, ${ }^{2}$ Unit of Paediatric Haemato-oncology, Department of Paediatrics, Advanced Paediatric Centre, Postgraduate Institute of Medical Education and Research, Chandigarh, India

Aims \& Objectives: While, the morphology of Burkitt leukemia/lymphoma cells are characteristic with the presence of numerous coarse cytoplasmic vacuoles; such vacuoles are also described in acute myelomonocytic leukemia, acute monoblastic/monocytic leukemia, acute erythroid leukemia, acute megakaryoblastic leukemia, B-lineage acute lymphoblastic leukemia, and metastatic carcinoma. The side scatter of abnormal cells in acute promyelocytic leukemia is very characteristic and is rarely seen in other leukemias. We describe a case that highlights the limitations and pitfalls in both morphology and flow cytometry.

Patients/Materials \& Methods: We present the hematological workup (bone marrow morphology, cytochemistry, immunohistochemistry, flow cytometry, cytogenetics, and molecular genetics) of an 8 years old, female child,who presented with fever and bone pain on and off for 2 months, hepatosplenomegaly, and cervical lymphadenopathy and was clinically suspected to be a case of acute leukemia.

Results: Her complete hemogram revealed pancytopenia and blasts in the peripheral blood. Bone marrow aspirate showed $78 \%$ blasts of variable size, having many large distinct vacuoles in the cytoplasm raising the possibility of Burkitt lymphoma. PAS stain showed block positivity. Flow cytometry repeatedly showed only a minor population in the blast/lymphocyte region adding to the diagnostic dilemma. A careful review revealed the clustering of abnormal cells in CD45 dim, intermediate to high side scatter region corresponding to the region of promyelocytes and mature granulocytes with an expression of monocytic and myeloid markers. MPO cytochemistry was also reviewed which showed $4 \%$ positivity in the blasts with an occasional Auer rod. Immunohistochemistry on the bone marrow biopsy showed positivity for CD34, CD68, and MPO. FISH study showed amplification of cMYC gene (8 copies) and KMT2A translocation. The case was finally reported as acute myelomonocytic leukemia with KMT2A translocation and cMYC amplification. While KMT2A translocation is common, c-MYC amplification is an uncommon finding in acute myeloid leukemia often resulting from double minutes or ring chromosomes. The child received induction therapy for AML for one month; following which, her bone marrow was in morphological remission without any residual disease in high-sensitivity flow cytometry.

Discussion \& Conclusion: Appropriate management needs accurate diagnosis, which needs awareness about the pitfalls in each laboratory technique and careful correlation of morphology with various ancillary investigations.

\section{Leukemic Masquerade of Adult Alveolar Rhabdomyosarcoma Confined to Bone Marrow. A Rare Case}

Jasdeep Kaur ${ }^{* 1}$, Ashwin Philips², Joseph M. John3, Preethi A. M. Paul ${ }^{1}$

${ }^{1}$ Pathology, ${ }^{2}$ Medical Oncology, ${ }^{3}$ Clinical Hematology, Hematooncology and Bone Marrow (Stem Cell) Transplant Unit, Christian Medical College and Hospital, Ludhiana, Ludhiana, India

Aims \& Objectives: Alveolar rhabdomyosarcoma (ARMS) is a rare sarcoma occurring in adolescents and young adults, however, is uncommon in older adults. Only isolated cases of ARMS have been reported limited to the bone marrow (BM) with no identifiable primary. We describe a case of ARMS morphologically resembling acute leukemia, and confined to the BM with no primary tumour detectable despite extensive imaging.

Patients/Materials \& Methods: A 64-year-old lady presented with generalized weakness, ecchymotic patches, low backache and anemia for 20 days, and fever for three days. Systemic examination was unremarkable. CBC showed bicytopenia: $\mathrm{Hb} 9.8 \mathrm{gm} / \mathrm{dL}$, TLC 6,300/ $\mu \mathrm{L}$, platelets $26,000 / \mu \mathrm{L}$ and a rare atypical cell in the peripheral blood. BM aspiration and flow cytometry (FCM) was performed along with Immunohistochemistry (IHC) on BM trephine biopsy (BMTB).

Results: BM aspirate showed $73 \%$ atypical cells/blasts of size 14-21 $\mathrm{m}$, with high N/C ratio, coarse chromatin, inconspicuous nucleoli and vacuolated basophilic cytoplasm. SSC vs CD45 gating on FCM showed $63.7 \%$ cells in the erythroid region (CD45 negative), which were moderately positive for CD235a (98\%), and dim positive for MPO (61\%). Rest of the B- and T-lymphoid, myeloid and immaturity markers were negative. Due to presence of occasional clustering of cells on aspirate, extensive IHC was performed on BMTB, which showed positivity for desmin, nuclear MYF4 (myogenin) and CD56; MIB1 proliferative index was 60-70\%. Negative IHC markers included CD45, CK, EMA, CD138, CD99, FLI-1, TTF1, CD4, Synaptophysin, Chromogranin-A and S100. On wholebody PET CT, increased diffuse/heterogenous FDG uptake was seen involving almost all visualized bones, more prominently in the sacrum and dorsolumbar vertebrae, and the spleen. No definite mass lesion was identified. MRI brain and ultrasound pelvis were normal. A final diagnosis of primary BM ARMS was suggested. The patient is currently undergoing chemotherapy with Vincristine, Dactinomycin and Cyclophosphamide, and is transfusion independent for 15 days. Discussion \& Conclusion: We report this case for its rarity, occurrence in an adult with strong resemblance to acute leukemia and absence of primary tumour elsewhere. Presence of blast conglomerates can help suspect non-hematological malignancy, and IHC plays a vital role to avoid misdiagnosis. Integration with clinical and 
radiological findings is critical for a comprehensive diagnosis which helps guide treatment options.

\section{Aleukemic Myeloid Sarcoma in BCR ABL1-Positive Acute Undifferentiated Leukemia, Post ALLO-HSCT}

Akriti Sachdeva $^{* 1}$, Preethi A. M. Paul ${ }^{1}$, Ashish Varghese ${ }^{2}$, Joseph M. John ${ }^{3}$

${ }^{1}$ Pathology, ${ }^{2}$ ENT, ${ }^{3}$ Clinical Hematology, Hemato-oncology and Bone Marrow (Stem Cell) Transplant Unit, Christian Medical College and Hospital, Ludhiana, Ludhiana, India

Aims \& Objectives: Myeloid sarcoma (MS) is a rare manifestation of acute leukemia and can occur simultaneously with, or rarely can be the sole manifestation of relapse post hematopoietic stem cell transplantation (HSCT). We describe a case of BCR ABL1- positive acute undifferentiated leukemia (AUL) with isolated aleukemic MS relapse in the posterior auricular and mastoid region, post allogeneic HSCT (Allo-HSCT).

Patients/Materials \& Methods: A 28-year-old man came with thigh pain and weight loss since 2-3 months. CBC showed TLC 1,08,000/ mm3 with blasts up to $87 \%$, Hb $14.7 \mathrm{gm} \%$, platelets $3,39,000 / \mathrm{mm} 3$. Bone marrow (BM) aspirate showed $76 \%$ blasts, of size $12-16$ microns, with immature fine chromatin, inconspicuous nucleoli and scant agranular cytoplasm without Auer rods. MPO and PAS cytochemistry were negative. Flow cytometry with SSC vs CD45 gating showed $77 \%$ CD45-dim positive blasts, which were positive for CD34, HLA-DR, CD38 and CD13; the rest were negative. BCR ABL1 RQ-PCR was positive. With initial diagnosis of BCR ABL1positive AUL, patient was started on Daunorubicin and Cytosine, and subsequently taken up for Allo-HSCT after achieving BM remission. At 32 months post transplant, he presented with right-sided posterior auricular swelling for 2 months. Examination revealed an irregular, hard, fixed $10 \times 10 \mathrm{~cm}$ mass in the posterior auricular and mastoid region. Overlying skin and systemic examination was unremarkable. Patient was taken up for right cortical mastoidectomy and tissue was sent for histopathological examination (HPE).

Results: HPE revealed infiltrating small round cells with round to irregular nuclei, fine chromatin, small nucleoli and scant cytoplasm. IHC showed diffuse positivity for CD34, TdT and CD99, while CD20, CD3, PAX5 and anti-MPO were negative. MIB1 proliferative index was $90 \%$. Patient's BM revealed no evidence of residual leukemia; CSF examination was normal. A very low positive BCR/ABL was noted in RQ-PCR and he was continued on oral Imatinib. A diagnosis of isolated aleukemic MS was made. The patient was given radiotherapy, after which there was marked reduction in the size of swelling, and the patient is currently on follow up.

Discussion \& Conclusion: Isolated aleukemic MS may be the first sign of relapse of AUL post-HSCT. HPE and IHC play an important role in avoiding misdiagnosis, which is critical for a further decision of management

\section{CD19 Negative B Lineage Acute Lymphoblastic Leukemia: A Diagnostic Dilemma}

Nidhi Kaushik ${ }^{* 1}$, Monika Gupta ${ }^{1}$, Sunita Singh ${ }^{1}$, Rajeev Sen ${ }^{1}$

${ }^{1}$ Pathology, PGIMS, Rohtak, India

Aims \& Objectives: B lineage acute lymphoblastic leukemia (B ALL) is a common neoplasm in children. Flowcytometric immunophenotyping (FCI) plays a major role in the diagnosis and follow-up of these patients. Expression of CD19 on blasts is considered as the most reliable marker for assigning them to the $\mathrm{B}$ cell lineage because it is expressed in all stages of B- lymphocytes maturation including plasma cells. Loss of this antigen is very rare in precursor B-cell ALL. We present an unusual case of CD19 negative paediatric B ALL.

Patients/Materials \& Methods: This is a case report of a 3 year old female presented with complaints of generalised weakness, on and off fever, joint pain, and hepatosplenomegaly for 4 months. Complete hemogram, bone marrow aspiration/biopsy and immunophenotyping was performed on 8 Colour Flow cytometer BD FACS Canto II using CD45/SSC gating.

Results: Peripheral blood examination revealed hemoglobin of 6.4 gm $\%$, total leucocyte count of $62.3 \times 109 / \mu 1$ and a platelet count 30 $\mathrm{x} 109 / \mu \mathrm{l}$ with $90 \%$ circulating blasts. BMA showed near total replacement of marrow by blast cells. On FCI, blasts were positive for CD10, HLA-DR, Tdt, CD45 (dim), CD34, CD22 (dim) and CD79a while negative for $\mathrm{CD} 19$, cCD3, cMPO, CD5, CD7, CD13, and $\mathrm{CD} 33$. In the absence of other lineage-specific markers (cCD3 and MPO) but presence B-lineage-associated markers, diagnosis of CD19negative precursor B-ALL was suggested.

Discussion \& Conclusion: Lack of awareness and rarity of CD19 negative B cell type leukemia, can lead to incorrect immunophenotypic diagnosis, treatment, and monitoring. Therefore, proper diagnosis should involve a combination of clinical features, immunophenotypic profiles, immunohistochemistry findings, and molecular analysis.

Keywords: Flow cytometric immunophenotyping (FCI), B-cell acute lymphoblastic leukemia, CD19.

\section{Prevalence and Clinical Correlation of ETS Related Gene (ERG) Deletions in B-Cell Acute Lymphoblastic Leukemia: A Single Centre Experience}

Sanjeev K. Gupta ${ }^{* 1}$, Sameer Bakhshi ${ }^{2}$, Ritu Gupta ${ }^{1}$, Smeeta Gajendra $^{1}$, Preity Sharma ${ }^{1}$, Deepam Pushpam ${ }^{2}$

${ }^{1}$ Laboratory Oncology, Dr BRA IRCH, ${ }^{2}$ Medical Oncology, Dr BRA IRCH, All India Institute of Medical Sciences (AIIMS), New Delhi, India

Aims \& Objectives: Recently, ETS related gene (ERG) deletions in childhood B-ALL have been reported to have a good outcome using standard therapy, even in presence of IKZF1 deletions, a known poor prognostic marker in B-ALL. So, we studied the prevalence of ERG deletions, its co-existence with IKZF1 deletions and clinical correlation in our B-ALL cohort.

Patients/Materials \& Methods: 432 newly diagnosed (November 2013-March 2018), untreated B-ALL cases were ambispectively analyzed for ERG deletions. In addition to the standard morphological, cytochemical, immunophenotypic and cytogenetic/molecular work-up (BCR-ABL1, ETV6-RUNX1, KMT2A, TCF3-PBX1), multiplex ligation-dependent probe amplification (MLPA) was done using SALSA MLPA kits P327, P335, and P202 (MRC-Holland) for ERG deletions and IKZF1 deletions. The clinical follow up was included till September 2020.

Results: The study included 336 pediatric B-ALL patients with median age 6.5 years (2 months-18 years; M:F ratio:: 1.8:1) and 96 adults (median 28.5 years (19-67 years; M:F ratio: 2.2:1). Eight out of $432(1.85 \%)$ cases had ERG deletions including 7 (2.08\%) of 336 pediatric and $1(1.04 \%)$ of 96 adults. All the 8 cases with ERG deletions were negative for the tested recurrent translocations as well as aneuploidy. The IKZF1 deletions were detected in $64(19.1 \%)$ pediatric and $37(38.5 \%)$ adult B-ALL patients. However, none of the 7 pediatric cases with ERG deletions had co-existent IKZF1 deletions. The sole adult patient with ERG deletion had a co-existent IKZF1 deletion. The rate of post-induction remission $(83.3 \%$ vs $83.2 \%$; 
$p=0.735)$, median EFS (29.8 vs 44.2 months; $p=0.822)$ and OS (median not reached; $p=0.505$ ) in the pediatric patients with ERG deletions was similar to those without ERG deletions. The adult patient with co-existent ERG and IKZF1 deletion relapsed after 8 months of completing therapy.

Discussion \& Conclusion: ERG deletions were seen in $2.08 \%$ pediatric and $1.85 \%$ overall B-ALL cohort, compared to $3-7 \%$ childhood B-ALL in the reported literature. None of the 7 pediatric patients with ERG deletions had co-existent IKZF1 deletion. The pediatric patients with ERG deletions had outcomes similar to those without ERG deletions.

\section{Isolated CNS Relapse in a Case of CMML Post Allogeneic Stem Cell Transplant}

Shipra Verma ${ }^{* 1}$, Deepti Mutreja ${ }^{1}$, Sanjeevan Sharma ${ }^{2}$, Venkatesan Somasundaram $^{1}$, Satyaranjan Das ${ }^{2}$

${ }^{1}$ Pathology, Armed Forces Medical College, ${ }^{2}$ Hematology, Command Hospital, Pune, India

Aims \& Objectives: To present an extremely rare case of isolated central nervous system (CNS) relapse of Chronic Myelomonocytic Leukemia(CMML) post allogeneic stem cell transplant(SCT).

Patients/Materials \& Methods: A 47 years old female presented with weight loss, easy fatigability and loss of appetite for three months. Examination revealed pallor and generalized lymphadenopathy. Hemogram showed severe anemia (hemoglobin $5.4 \mathrm{~g} /$ dL)with total leucocyte count(TLC) of 23,000/uL. Bone marrow (BM) was hypercellular with myeloid preponderance, $7 \%$ blasts, dyserythropoiesis and dysmegakaryopoiesis. Lymph node biopsy showed extramedullary hematopoiesis. Patient was managed with hydroxyurea however she continued to have raised TLC with absolute monocytosis. At three months of follow up, she was opined as a case of CMML 2 in view of persistent monocytosis and BM blasts of $12 \%$ with Auer rods. Next generation sequencing showed FLT3 gene mutation.

Results: Patient was administered two cycles of Azacitidine, following which she achieved hematological remission (BM blasts 3\%). Matched allogeneic SCT with sibling as donor was done and posttransplant course was uneventful with $96 \%$ donor chimerism. Six months later, patient presented with acute quadriparesis and bulbar symptoms. Her blood counts were normal, and cerebrospinal fluid(CSF) showed positivity for blasts. MRI brain was normal. Flow cytometry of CSF sample confirmed CNS involvement by myelomonocytic leukemia. Patient was managed with intrathecal triple therapy with cytosar, dexamethasone, methotrexate. Subsequent CSF studies showed reduction in blasts. She is planned for cranial irradiation.

Discussion \& Conclusion: CMML is a clonal hematopoietic disorder classified under WHO 2016 as a part of Myelodysplastic/Myeloproliferative syndrome. CNS involvement post-transplant is less commonly described in cases of acute myeloid leukemia with few case reports in chronic myeloid leukemia. Extensive literature search did not reveal any cases of CNS relapse in CMML. Risk factors for CNS relapse and management are discussed. To our knowledge this is the first case of isolated CNS relapse in CMML post allogeneic SCT.
Hypoplastic Acute Myeloid Leukaemia: A Rare Presentation of Acute Leukaemia

Aradhana Harrison ${ }^{* 1}$, Sindhura Lakshmi Koulmane Laxminarayana $^{2}$, Seemitr Verma ${ }^{3}$

${ }^{1}$ Department of Pathology, Assistant Professor, Melaka Manipal Medical College, Manipal. Manipal Academy of Higher Education, ${ }^{2}$ Department of Pathology, Associate Professor, ${ }^{3}$ Department of Pathology, Assistant Professor, Kasturba Medical College, Manipal. Manipal Academy of Higher Education, Udupi, India

Aims \& Objectives: Reporting a rare case of Hypoplastic Acute Myeloid Leukaemia (H-AML).

Patients/Materials \& Methods: A 39 year old male presented with easy fatigability and appetite loss for 6 months. He was treated with multiple blood transfusions for anaemia over the last 2 months in an outside center. There was no other treatment history. On examination, he had pallor with axillary lymphadenopathy but there was no hepatosplenomegaly.

Results: Laboratory investigations showed Haemoglobin-6.2 g/dl, Total Leucocyte Count-2.9x103/cumm, Platelet Count-9x103/ cumm. Differential Leucocyte Count showed Neutrophils - 25\%, Lymphocytes - 65\%, Monocyte - 10\%. No blasts/abnormal cells were present in the peripheral blood. In view of pancytopenia, bone marrow examination was done which showed dilute aspirate with blasts$25 \%$. Dyserythropoiesis and dysmegakaryopoiesis were present. Ring sideroblasts were $<5 \%$. Bone marrow trephine biopsy showed hypocellular marrow spaces with $<20 \%$ cellularity. On multiparametric flow cytometry, blasts were positive for CD34, CD117, HLADR, CD13, CD33, CD64, CD15 and cMPO. Hence, a diagnosis of Hypoplastic Acute Myeloid Leukaemia (H- AML) was made. Further testing could not be performed as patient was lost to follow up.

Discussion \& Conclusion: Acute Leukaemia presenting with hypocellularity is atypical and uncommon, and poses a diagnostic challenge to differentiate it from its mimickers. H-AML accounts for only $5-7 \%$ of AML cases. The diagnosis of $\mathrm{H}-\mathrm{AML}$ entails the presence of $\geq 20 \%$ blasts in peripheral blood or bone marrow and $\leq 20 \%$ cellularity on bone marrow trephine biopsy. Previous cytotoxic/radiation therapy should be excluded before making a diagnosis of H-AML since hypocellular bone marrow can be present in therapy related AML. The outcome of H-AML does not differ from nonhypocellular AML, however, the importance of awareness of this rare entity lies in its considerable morphological overlap with other causes of hypocellular bone marrow such as myelodysplastic syndromes and aplastic anaemia, which can lead to misdiagnosis and mismanagement. Differentiation of H-AML from these causes of hypocellular marrow is essential as the treatment and prognosis differ remarkably. A comprehensive diagnostic work up including clinical features, thorough morphological examination, cytochemistry, immunophenotyping and genetic mutational analysis, together substantiate the diagnosis.

Oligoblastic Bone Marrow with Myelofibrosis in Down Syndrome: Terms Revisited According to WHO 2017

Riva Choudhary ${ }^{* 1}$, Sunita Sharma ${ }^{1}$, Vandana Puri ${ }^{1}$, Nupur Pathak ${ }^{2}$

${ }^{1}$ Department of Pathology, ${ }^{2}$ Department of Paediatrics, Lady Hardinge Medical College, New Delhi, India

Aims \& Objectives: WHO 2017 introduced "Myeloid proliferation associated with Down Syndrome" in its latest edition including TAM (Transient Abnormal Myelopoiesis) and Myeloid leukemia associated 
with Down syndrome. Here we present a case of Myeloid leukemia associated with Down syndrome.

Patients/Materials \& Methods: A 6-year-old male of Down syndrome presented with congestive heart failure with history of on and off fever for 2 months and global developmental delay. On examination, hepatosplenomegaly of $6 \mathrm{~cm}$ and $8 \mathrm{~cm}$ were present. Liver function test was normal. There was no lymphadenopathy.

Results: On CBC analysis, $\mathrm{Hb}$ was $6.8 \mathrm{~g} / \mathrm{dl}$, WBC was $2.61 \mathrm{x} 103 / \mu \mathrm{l}$, Platelets were $58 \times 103 / \mu 1$.Peripheral smear showed few atypical cells which were 2 to 4 times the size of small mature lymphocytes with high N/C ratio, scant agranular cytoplasm. Nucleus was round, with slightly irregular nuclear membrane with fine chromatin and 1 to 2 nucleoli. Bone marrow aspirate yielded dry tap twice, hence Bone marrow biopsy and imprint were sent to the lab. Imprint smears were scantly cellular containing many degenerated bare nuclei and few similar atypical cells. Bone marrow biopsy also showed the presence of atypical cells with similar morphology along with megakaryocytic hyperplasia. Few megakaryocytes also showed hypolobation with dispersed chromatin and atypia. Reticulin stain showed grade 3 fibrosis. On PAS staining megakaryocytes showed positivity suggesting dysplastic megakaryocytes/megakaryoblasts. On Flow cytometry, a population of 3-5\% blasts were gated showing positivity for CD41. These blasts were negative for CD10, CD19, CD34, CD3, CD5. Immunohistochemical examination also revealed CD61 positivity in dysplastic megakaryocytes/Megakaryoblasts.

Discussion \& Conclusion: Distinction between MDS, AML, AML with MDS related changes and Myelofibrosis requires careful microscopic examination and diagnostic differentiation. Since the number of blasts were $<20 \%$, with fibrosis on biopsy, perplexity regarding terminology for final diagnosis was there. Although individuals with Down syndrome show no biological differentiation between MDS and AML and also have no prognostic or therapeutic implications, the diagnosis was made as "Myeloid leukemia associated with down syndrome" which encompasses both MDS and AML.

\section{Hematogones: Mimicker and Morphological Confounder in a Case of Precursor T Lymphoblastic Leukemia}

Arunima Gupta ${ }^{* 1}$, Venkatesan Somasundaram ${ }^{1}$, Kanwaljeet Singh ${ }^{1}$, Vikram Singh $^{1}$, Tathagata Chatterjee ${ }^{1}$

${ }^{1}$ Department of Pathology, Army Hospital Research and Referral, DELHI, India

Aims \& Objectives: B-lymphocyte progenitors, namely the hematogones may pose problems in morphological assessment of bone marrow, especially so when evaluating a marrow for remission status following chemotherapy. Here we describe a case of Precursor $\mathrm{T}$ lymphoblastic leukemia (T-ALL) when evaluated for remission status revealed $16 \%$ blasts, which on immunophenotypic analysis turned out to be hematogones.

Patients/Materials \& Methods: A 03 yr old female child, who was on therapy for Precursor T lymphoblastic leukemia, was lost to follow up after induction chemotherapy due to the Covid-19 scenario. Subsequently when the patient turned up, bone marrow was done to look for remission status.

Results: Bone marrow on morphological assessment revealed $17 \%$ blasts and the disease was thought to have relapsed. However on clinical assessment, the patient was well preserved, her organomegaly had regressed with normal peripheral counts. Hence the marrow aspirate was subjected to flow cytometry, which revealed $12 \%$ cells in progenitor region, however these cells to our surprise turned out to be CD19 positive B-lymphoid progenitors and were negative for CD3 which is a specific marker for T cells. This was followed by T-ALL
MRD analysis which revealed MRD negative status. The patient was put on subsequent phases of chemotherapy.

Discussion \& Conclusion: Hematogones are non neoplastic precursors of B-lymphocytes and are increased at times, confusing the morphologists. Increased levels (more than 5\%) are seen to occur in cases following chemotherapy when marrow is recovering, posing a greater challenge in morphological assessment of remission status. Hemotogones are also known to increase in Non Hodgkin Lymphoma, following bone marrow transplantation, HIV cases and in thrombocytopenia cases. Highest number of hematogones (19\%) is seen in a T-ALL case following chemotherapy, which is similar to our case. A panel of antibodies on flow cytometry could distinguish these hematogones from the neoplastic B lymphoblasts, which is essential in management of acute leukemias.

\section{Trisomy 21, Transient Abnormal Myelopoiesis and Transposition of Great Arteries: A Rare Association}

Tushar Sehgal $^{* 1}$, Chandan Mishra ${ }^{1}$

${ }^{1}$ Laboratory Medicine, All India Institute of Medical Sciences New Delhi, Delhi, India

Aims \& Objectives: Introduction. Down syndrome (DS) is a well described chromosomal disorder. Children with DS have a unique predisposition to develop transient abnormal myelopoiesis (TAM) and AML. Congenital heart diseases (CHD) are also frequently described in these patients. We describe a case of a term male neonate with phenotype of DS who presented with failure to thrive. Blood picture was suggestive of TAM. Genetics confirmed trisomy 21 and GATA1mutation. Echocardiography revealed multiple cardiac anomalies of which transposition of the great arteries (TGA) was noted, which is extremely uncommon in DS. To date, this rare association of trisomy 21 , TAM and TGA has not been previously reported.

Patients/Materials \& Methods: CASE. 38-week of gestation male was born to 25-year-old mother. The neonate presented with failure to thrive.Pertinent physical exam revealed flat facies,telecanthus, clinodactyly and hypotonia.Echocardiography revealed multiple cardiac anomalies including d-TGA, PDA and VSD.CBC showed hyperleucocytosis of $121.96 \times 103 / \mu \mathrm{L}$ with normal haemoglobin and platelet count. Blood film (image panel 1-4; x1000, MGG stain) revealed increased of blasts (47\%) (image1) and presence of giant platelets (image2).Blasts with cytoplasmic "blebs" (image3) and a micromegakaryocyte (image4) were also noticed. Flow cytometry showed expression of cytoplasmic CD41 and CD61 antigens on blasts. Karyotype revealed 47,XY, + 21 (image5). NGS showed GATA1 mutation in intron 2 region. Combined with phenotype of DS, the blood findings were consistent with TAM.

Results: Discussion. TAM occurs in about $10 \%$ of DS newborns. Majority are asymptomatic with spontaneous resolution of disorder by three months. However, $10 \%$ of them die from multiorgan failure. About $20 \%$ of patients with TAM that survive the neonatal period subsequently develop AML. Morphologically the myeloblasts have megakaryocytic features. Molecular hallmark of DS-TAM is the presence of somatic mutations in the gene encoding the key megakaryocytic/erythroid transcription factor GATA1. CHD are quite commonly seen in patients with DS. Among them ASD is the most common, followed by VSD, ASD, PDA and TOF. Although TGA is one of the most common CHD, it is essentially absent in DS.

Discussion \& Conclusion: DS is the most common autosomal abnormality caused by trisomy of chromosome 21 . Its associations with myeloid proliferations and CHD are well described. Hitherto, an 
association between trisomy 21 , TAM and TGA has not been previously reported.

\section{Myeloid Sarcoma: Uncommon Presentation of a Common Blood Cancer}

Toyaja M. Jadhav ${ }^{* 1}$, Puneet Baveja ${ }^{1}$

${ }^{1}$ Dept of Pathology, Armed Forces Medical College, Pune, India

Aims \& Objectives: Myeloid sarcoma (MS) is a neoplasm of the myeloid cells and can arise before, concurrent with or following haematolymphoid malignancies. It is an uncommon extramedullary manifestation commonly associated with AML. We report 04 cases of MS, diagnosed in this institute over a period of 6 years, during various phases of their respective haematolymphoid malignancy.

Patients/Materials \& Methods: Cases retrieved from the data base of the past 06 years. Blocks and slides of the respective cases retrieved and restained and reviewed along with their respective IHC studies. Relevant clinical data along with the respective cytogenetic studies retrieved and correlated.

Results: Diagnosis of MS established based upon histomorphological, immunohistochemical, cytogenetic studies and the relevant clinical data available.

Discussion \& Conclusion: MS is a marker of unfavourable prognosis in leukaemia and indicates poor survival. Early intervention along with timely diagnosis, including complete staging of the disease coupled with accurate molecular study and appropriate management is crucial to improve survival. Additionally, newer therapeutic markers can also be used in the pathological assessment of the disease, which can further accentuate the survival.

\section{The Less Known Leukemia: Acute Undifferentiated Leukemia}

Seemitr Verma ${ }^{* 1}$, Sindhura L. Koulmane Laxminarayana ${ }^{1}$, Sushma Belurkar $^{1}$

\section{${ }^{1}$ Pathology, Kasturba Medical College, Manipal, India}

Aims \& Objectives: Introduction. Acute undifferentiated leukemia is a rare entity with age adjusted incidence rate of 1.34 per $1,000,000$ person per year. It is a diagnosis of exclusion and has potential to be misdiagnosed. According to WHO 2017 classification, they express no more than one membrane marker for any given lineage. They also lack the lineage specific markers for myeloid (cMPO), T-lymphoid (cCD3) and B- lymphoid (strong CD19 or cCD22, cCD79a). They also lack lineages markers for plasmacytoid dendritic cell precursors, NK-cell precursors, basophils or non-haematopoietic tumors. A close differential diagnosis is acute myeloid leukemia with minimal differentiation (cMPO negative, but at least two myeloid markers like CD13, CD33 or/and CD117 are positive). Case report. A one-year-old female child was brought with bluish rash over forehead and leg which appeared a month back, along with fever since 3 days. On examination, the child was pale, with ecchymotic patch over abdomen, forehead, back and right hand. Mild hepatosplenomegaly was present. Hemoglobin was $7.3 \mathrm{gm} / \mathrm{dl}$, total WBC count was $18.7 \times 103$ / $\mu \mathrm{l}$ and platelet count was $10 \times 103 / \mu \mathrm{l}$. Peripheral smear and bone marrow showed $26 \%$ and $44 \%$ blasts respectively. No significant dyspoiesis noted. The bone marrow cytochemistry/IHC report along with the flow-cytometry report are as mentioned in table 1 . Thus, a diagnosis of acute undifferentiated leukemia was made pending cytogenetic and molecular studies. However, these tests could not be performed due to financial issues. The child was treated with prednisolone, cytrabine and daunomycin. During this induction phase, the child developed fungal bronchopneumonia, low oxygen saturation and had to be supported by mechanical ventilator. She died on the 23rd day of admission beside the best efforts.

Patients/Materials \& Methods: NA.

Results: NA.

Discussion \& Conclusion: With less cases reported in literature and considering the poor prognosis involved, unclear treatment recommendations, lack of risk stratification, we intend to add to the literature. An extensive panel of monoclonal antibodies to assign lineage along with cytogenetic (for complex karyotype of myelodysplasia) and molecular studies (for NPM1 or double CEBPA mutation) is required for final diagnosis of acute undifferentiated leukemia.

\section{Fallacious Morphology in B-Cell Acute Lymphoblastic Leukemia (B-Cell ALL): A Case Report}

Khushbu Girdher ${ }^{* 1}$, Vandana Bhatti ${ }^{1}$

${ }^{1}$ Pathology, Christian Medical College, Ludhiana, India

Aims \& Objectives: Acute lymphoblastic leukemia (ALL) is the most common leukemia seen in children. B-cell origin of these blasts is more common than T-cell origin. Acute Promyelocytic Leukemia (APML) is a M3 subtype of Acute Myeloid Leukemia (AML) which needs immediate treatment. The hypogranular variant of APML has a peculiar morphology of promyelocytes with bilobed nuclei and hypogranular cytoplasm. We present this case of B-Cell ALL resembling hypogranular variant of APML on morphology.

Patients/Materials \& Methods: A 34 year old gentleman presented with backache intermittently for 2 years. Complete blood count and peripheral blood smear did not show any evidence of leukemia. Bone marrow examination was performed to rule out Multiple Myeloma; however, it was suggestive of acute leukemia with blast morphology mimicking that of hypogranular variant of APML. Later immunophenotyping was done suggesting a diagnosis of B-Cell ALL. Results: Complete blood count showed Hb9.9 gm/dl, TLC 2,500/ $\mu$, platelets $1,86,000 / \mu 1$. Bone marrow was hypercellular with depressed trilineage hematopoiesis and increase in blasts up to $85 \%$. The blasts were of size 18-21 microns with irregular nuclear margins, immature chromatin and 1-2 conspicuous nucleoli in some with most of the blasts showing indented or bilobed nucleus. No granules or auer rods were seen. Immunophenotyping was positive for CD19, CD22, cCD79a, CD10, HLADR and CD34. Immunohistochemistry performed on bone marrow trephine biopsy also showed blasts to be positive for CD20, CD10, CD34, Tdt. Anti- MPO was negative. MRI cervicodorsal spine showed wedge compression fracture of C4-C6, compression fracture of $\mathrm{T} 4$ vertebrae which explained the backache. Discussion \& Conclusion: The case is reported to stress that although morphology is a good guide to the nature of leukemia, at times it may lead to diagnostic difficulty. Immunophenotying should be performed in all cases of Acute leukemia.

\section{Acquired Robertsonian Translocation in AML-MRC with Basophilia: A Rare Case}

Shalini Goel ${ }^{* 1}$, Bhawna Jha ${ }^{1}$, Shivani Sangwan ${ }^{1}$, Akshay Gore ${ }^{1}$, Nitin Mathur ${ }^{1}$, Amrita Ramaswamy ${ }^{2}$, Renu Saxena ${ }^{1}$

${ }^{1}$ Department of Hematopathology, ${ }^{2}$ Department of Medical Oncology and Hematology, Medanta - The Medicity hospital, Gurugram, India

Aims \& Objectives: A rare case of acquired robertsonian translocation in AML with myelodysplasia related changes (AML-MRC) and basophilia. 
Patients/Materials \& Methods: A 58-year-old female presented with generalised weakness and hepatosplenomegaly, since 2 months with $\mathrm{h} / \mathrm{o}$ multiple transfusions. Peripheral blood showed pancytopenia with $13 \%$ blasts, $15 \%$ basophils, and significant dyspoiesis in neutrophils with few pseudo pelger-huet cells. The bone marrow examination (BME) revealed $22 \%$ blasts, $9 \%$ basophils, with significant trilineage dyspoiesis and increased marrow fibrosis on biopsy. Flow cytometry showed $11.5 \%$ blasts including $6.5 \%$ myeloblasts and 5\% monoblasts, 7.4\% basophils and abnormal position and antigenic maturation patterns on maturing granulocytes. Based on BME and Flow, a diagnosis of Acute myeloid leukemia (AML) was given. The differentials considered were AML with $t(6 ; 9)$, AML progressing from MPN or from MDS/MPN.

Results: MPN panel (MPL, CALR and JAK2V617F gene mutations) and BCR-ABL1 mutational studies were done and the results were negative. The AML molecular panel using RT-PCR for gene rearrangements such as PML-RARA, AML1- $\operatorname{ETO}(\mathrm{t}(8 ; 21)(\mathrm{q} 22 ; \mathrm{q} 22)$; RUNX1:RUNX1T1),

CBFB-MYH11(inv(16)(p13;q22)/ $\mathrm{t}(16 ; 16) \mathrm{p}(13 ; \mathrm{q} 22)$, BCR-ABL(p210), and gene mutations such as FLT3 including ITD and TKD (D835), NPM1 and KIT were reported as negative. The cytogenetic analysis of 20 metaphases shows del $(5 q)$, acquired robertsonian translocation of chromosome $21 \mathrm{q} 10$ and trisomy 21 in 18 metaphases. In view of presence of increased blasts, trilineage dyspoiesis, basophilia, increased marrow fibrosis with $\operatorname{del}(5 \mathrm{q})$, acquired robertsonian $\mathrm{t}(21 ; 21)(\mathrm{q} 10 ; \mathrm{q} 10)$ and trisomy 21 ; a final diagnosis of AML-MRC with basophilia was given. The patient was started on Decitabine and has till date received 2 cycles of the therapy. The latest $\mathrm{CBC}$ revealed pancytopenia with an increase in the percentage of basophils over the period of 3 months.

Discussion \& Conclusion: According to the Revised 4th WHO ed, $\operatorname{del}(5 q)$ is one of the MDS classifying mutation. Robertsonian translocations are the most common constitutional structural abnormalities but are rarely reported as acquired aberrations in hematologic malignancies, frequency of one in 300-400 patients has been noted. The $\mathrm{i}(21)(\mathrm{q} 10)$ is usually accompanied by loss of chromosomes 5 and/ or 7 , as in present case where it is accompanied by del(5q). Trisomy 21 , which can be seen in cases of MDS, is associated with increased progression to AML and poor prognosis.

\section{Extragonadal Germ Cell Tumor Associated with a Rare Hematological Malignancy}

Shaliya M. Jamal' ${ }^{* 1}$, Shubha Sudhakar ${ }^{1}$, Madan $\mathrm{K}^{1}$, Guruprasad Bhat $^{2}$

${ }^{1}$ Pathology, ${ }^{2}$ Oncology, Yenepoya medical college, Mangalore, MANGALORE, India

Aims \& Objectives: Extragonadal germ cell tumor associated with a rare hematological malignancy.

Patients/Materials \& Methods: A 20 year old adult male presented with fever and dyspnea admitted to the hospital with a known history of Germ cell neoplasm. Alfa-feto protein and beta Human chorionic gonadotropin levels were elevated. The patient underwent 5 cycles of chemotherapy followed by a mediastinal mass excision. Macroscopically, the specimen measured $17 \mathrm{cms}$ in largest dimension. Outer surface appeared congested, nodular with irregular areas, and cut surface showed solid to cystic with pale white and dark brown areas. Microscopically diagnosed as Mixed germ cell tumour with somatic type malignancy (Mature Teratoma- 65\%; yolk sac-10\%; necrosis$20 \%$ and sarcomatous component- 5\%).

Results: Post operatively, Presented with fever, tachycardia and multiple palpable blanched purpura. Post operative CT scan showed bilateral pleural effusion, hepatomegaly, splenomegaly with small hypodense non enhancing foci predominantly in subscapular region.
Patient was transfused with platelets for thrombocytopenia. Peripheral smear showed numerous atypical cells with blastoid morphology $(15 \%)$. SBB, PAS, MPO stains were negative. Bone marrow biopsy showed metastatic malignant germ cell tumour with bone marrow fibrosis and severe bone marrow hypoplasia. Flow cytometry was done.The CD45 dim positive cells showed bright expression of CD41 and CD61 along with dim expression of CD117. Hence a diagnosis of Acute megakaryoblastic Leukemia(AML-M7) was established.

Discussion \& Conclusion: Hematological malignancies associated with extragonadal germ cell tumors stand as one of the most distinctive and rare aspects of male germ cell carcinomas. The hematologic neoplasia with germ cell tumors run a very aggressive course and a substantial portion of the patients die before the treatment. Pluripotent primordial germ cells and hematologic stem cells share the site of embryologic origin, the yolk sac. During embryogenesis, stem cells and primordial germ cells migrate to the bone marrow and genital ridge respectively. Embryonic cells elaborate growth factors and stimulate proliferation and differentiation of Leukemic blast cells as well as normal cells. This explains the cause of acute megakaryocytic leukemia-M7 in germ cell tumours. The association of germ cell tumors and hematologic malignancies brings out interesting questions about the nature of malignant transformation of hematopoetic cells. However it is important for an early diagnosis, because the development of leukemia in patients with germ cell tumors favours a bad prognosis.

\section{Clinico Hematological Profile of Pediatric B: All-First Tertiary Care Experience from Punjab}

Arshnoor Kaur ${ }^{* 1}$, Vikram Narang ${ }^{1}$, Neena Sood ${ }^{1}$, Bhavna Malhotra $^{1}$, Shruti kakkar ${ }^{2}$

${ }^{1}$ Pathology, ${ }^{2}$ Paediatric, DMCH, Ludhiana, India

Aims \& Objectives: To analyse clinical and hematological parameters of pediatric B ALL.

Patients/Materials \& Methods: All the patients diagnosed over a period of 4 years as B ALL in pediatric age group were included for analysing clinical and hematological parameters.

Results: Of the total 125 patients of B-ALL, maximum number of patients (117 patients,93.6\%) were in 11-20 years age.Male: Female ratio was 2.1:1.Fever was the commonest presentation (124 patients, $99.2 \%$ ),followed by loss of weight and loss of appetite (95, $76 \%)$. History of bleeding was seen in $38(30.4 \%)$ patients and 23 patients $(18.4 \%)$ with generalized weakness. Thrombocytopenia was the most common haematological abnormality (122 patients,97.6\%) followed by anemia in 118 patients( $94.4 \%)$. 69 patients $(55.2 \%)$ had leukocytosis at presentation and 27 patients(21.6\%)had leukopenia while 29 patients $(23.2 \%)$ presented with normal TLC. Highest TLC noted was $236.5 \times 10^{3} /$ microliter and lowest was $0.8 \times 10^{3} /$ microliter.79 patients presented with severe thrombocytopenia $\left(<50 \times 10^{3} /\right.$ microliter $) .3$ patients had normal platelet count. 108 patients $(86.4 \%)$ had Normocytic Normochromic RBC picture while 12 had Microcytic hypochromic RBC picture. Peripheral smear show average blast percentage as $80 \%$. HLA-DR was positive in 122 patients(97.6\%). CD19 which was taken to be more specific for B-lineage was positive in 119 patients $(95.2 \%)$.Tdt was positive in 73 patients(58.4\%), CD79a in 87 patients(69.6\%) and CD22 in 80 patients(64\%). 95 patients(76\%) had CD34 positivity.

Discussion \& Conclusion: Most of the cases of B-ALL present in childhood. Fever is the most common symptom. In our study Thrombocytopenia is the most common haematological abnormality, followed by anemia.CD19 was found to be specific marker for B-lineage. 
RTPCR Negativity and PAS Block Positivity in APL with PML-Rara: Curious Findings in a Well

\section{Characterized Disease}

Neha Bhardwaj ${ }^{* 1}$, Ojas Gupta ${ }^{1}$, Nabhajit Mallik ${ }^{1}$, Sreejesh Sreedharanunni ${ }^{1}$, Shano Naseem ${ }^{1}$, Neelam Varma ${ }^{1}$, Pankaj Malhotra ${ }^{2}$

${ }^{1}$ Department of Hematology, ${ }^{2}$ Department of Internal Medicine, PGIMER, Chandigarh, India

Aims \& Objectives: Our case explores the rare event of absence of all three PML-RARA fusion transcripts (by RT-PCR) in acute promyelocytic leukemia (APL) with PML-RARA-t(15;17) being demonstrated by fluorescent in situ hybridization (FISH). We describe the possible mechanisms for this phenomenon in our case, which had multiple relapses. Additionally, we showcase a novel morphological finding-block positivity for periodic acid Schiff (PAS) staining in APL.

Patients/Materials \& Methods: A 36-year-old lady presented with menorrhagia and epistaxis for 1 week. She was diagnosed with APL at another centre four years ago and had already suffered two relapses. The current complaints prompted the clinical suspicion of another relapse and hence peripheral blood and bone marrow examination was advised.

Results: The peripheral blood counts revealed bicytopenia with haemoglobin $47 \mathrm{~g} / \mathrm{l}$, platelet count 21x 109/L and leucocytosis with TLC of 23.7x 109/L. The peripheral blood film showed 77\% abnormal immature cells. These cells constituted $81 \%$ of the bone marrow aspirate and showed heterogenous morphology, some with typical bilobed nuclei (as seen in abnormal promyelocytes) and some showing regular round to oval nuclei, occasionally with Auer rods. The cytochemical staining showed strong MPO positivity and astoundingly many of these abnormal cells displayed block positivity for PAS stain. Flowcytometry revealed typical findings for APL with high side scatter, CD34 and HLA-DR negativity. Molecular analysis using RTPCR was negative for all three PML-RARA fusion transcripts; however, FISH using dual color dual fusion probes showed the PML/ RARA fusion in $90 \%$ of the cells confirming it to be a third relapse in this APL case.

Discussion \& Conclusion: In cases of relapsed/refractory APL with RTPCR negative for PML-RARA fusion, one needs to be aware that in addition to the typical PML-RARA isoforms and variant RARA translocations, sporadic cases of $\mathrm{t}(15 ; 17)$ positive APL with atypical breakpoints are also known. The confirmation by two distinct techniques is of utmost value as observed in the index case. In addition, this case emphasizes that PAS block positivity is not limited to acute lymphoblastic leukemia alone.

\section{Autopsy Findings in Pre B Cell All-Relapse Presenting as Acute Bilateral Renal Failure-A Rare Entity}

Aman Kumar ${ }^{* 1}$, Isha Sharma ${ }^{1}$, Divya Shelly ${ }^{1}$, Venkatesan Somasundaram $^{1}$, Sanjeevan Sharma ${ }^{2}$

${ }^{1}$ Pathology, Armed Forces Medical College, Pune, ${ }^{2}$ Clinical Hematology, Command Hospital (Southern Command), Pune, India

Aims \& Objectives: Acute lymphoblastic leukemia (ALL) is a malignant disease originating from abnormal proliferation of $\mathrm{B}$ or $\mathrm{T}$ lymphocytes in bone marrow (BM). It is known to involve several extramedullary sites such as central nervous system (CNS), testis, mediastinum, kidney, liver, etc. Parenchymal infiltration of kidneys by leukemic cells is common, however a resultant injury to kidney occurs in only about one percent of patients and renal failure is even more rare. Bilateral involvement of kidneys is very rare and only few cases has been reported in world literature. We present a case of 34 year old male, a known case of Pre B-cell ALL, in remission who reported with azotemia and respiratory distress. The aim of this report is to highlight the rare presentation of a case of ALL with bilateral renal involvement.

Patients/Materials \& Methods: A complete pathological autopsy was performed after receiving the consent from patient's family. The sections were stained with $\mathrm{H} \& \mathrm{E}$ and IHCs done.

Results: Immunophenotyping by flow cytometry showed blasts which were $\mathrm{CD} 10+, \mathrm{CD} 19+, \mathrm{CD} 38+$, HLA-DR + and CD20-, CD34, CD22-, CD13-, CD33-, CD117-, cyto MPO-, CD2-, CD5-, CD7-, cyto CD3- with a final diagnosis of The patient was a diagnosed case of CD10 positive Pre B-cell-ALL with normal karyotype (46, XY). Mutational analysis was negative for BCR - ABL1 fusion, ETVRUNX1 fusion, MLL and E2A gene rearrangements. He was maintained on Hyper CVAD protocol $4 \mathrm{~A} / \mathrm{B}$, and relapsed with renal involvement and presented as bilateral acute renal failure. Extramedullary relapse of ALL in bilateral kidneys, subsequently leading to renal failure was noted which is very rarely seen with infiltration of kidney with leukemic cells. Left adrenal and spleen were infiltrated by blasts. Although, brain parenchyma was not involved but meningeal involvement was noted. Testis was not involved. Bone marrow was totally replaced by monomorphic population of blasts suggestive of relapse in a known case of Pre-B-cell ALL. Lungs revealed alveolar haemorrhage and Acute respiratory distress syndrome (ARDS). Discussion \& Conclusion: Renal failure following leukemic infiltration of kidneys leading to Acute kidney injury (AKI) is rare and is seen in less than one percent of cases. This case report brings out clinical and pathological correlation of such rare occurrence and role of autopsy in establishing the diagnosis of extramedullary relapse at rare sites.

\section{T Acute Lymphoblastic Leukemia (T-All) Lacking Immaturity Markers Viz Tdt, Cd34 and Hladr: A Diagnostic Challenge}

Neha Garg ${ }^{* 1}$, Richa gupta ${ }^{1}$, Dilip kumar ${ }^{2}$, Mrinalini Kotru ${ }^{1}$

${ }^{1}$ UCMS and GTBH, Delhi, ${ }^{2}$ Max Hospital, Saket, Delhi, India

Aims \& Objectives: T-ALL is a rare and aggressive neoplasm. Establishing precursor nature of blasts in T-ALL is required to distinguish these from reactive conditions and peripheral $\mathrm{T}$-cell leukemia's. The commonly used immaturity markers are Terminal deoxynucleotidyl transferase (TdT), CD34 and HLADR. TdT is expressed in $90 \%-95 \%$ cases of ALLs but recently rare cases of TdT negative T-ALL have been reported. In addition, absence of CD34 and HLADR further hinders the diagnosis. The frequency of T- ALL lacking all the 3 immaturity markers is not known in literature. So the present study aimed to study the immunophenotype of T- ALL in Indian population along with frequency and significance of T-ALLs lacking the three immaturity markers.

Patients/Materials \& Methods: Thirty-eight cases of T-ALL were evaluated over a period of two and a half years retrospectively. Immunophenotype of blasts was studied including aberrant markers and immaturity markers. Correlation of CD34 expression was also studied with respect to these markers. Qualitative data was expressed as frequency and percentage. Differences between groups were evaluated using Student's T-test for quantitative data and Chi square test for qualitative variables. $p$ value less than 0.05 was considered significant.

Results: The most common immaturity marker expressed was TdT in $39.5 \%$ cases followed by CD34 in $34.2 \%$ cases. Out of 38 cases, 13 (34.2\%) were negative for all three of the immaturity markers i.e. TdT-/CD34-/HLADR-. The most common aberrant B cell antigen 
expressed was CD79a (31.6\%) and the most common aberrant myeloid antigen expressed was CD33 (23.7\%). The CD34 negative group showed a significantly higher expression of one or more of the following aberrant B-cell antigens - CD10, CD19 and CD79a $(p=0.029)$, was significantly associated with absence of CD13 and CD33 ( $p=0.010$ and $p=0.040$ respectively) and HLADR negative T-ALL $(p=0.038)$

Discussion \& Conclusion: T-ALL lacking of all the three immaturity markers viz TdT, CD34 and HLADR is not uncommon and their absence does not exclude the diagnosis of T-ALL. Pathologists should be aware of this diagnostic pitfall. Larger studies with cytogenetics are needed to know the exact clinical significance of such cases and thus pave the way for future research.

\section{Atypical Morphology and Aberrant Immunophenotypic Expression: A Diagnostic Dilemma in Acute Promyelocytic Leukemia

\author{
Sharanjit Singh ${ }^{* 1}$, Paresh Singhal ${ }^{1}$ \\ ${ }^{1}$ Department of Pathology, AFMC, Pune, India
}

Aims \& Objectives: We present a rare and interesting case of APL with quite deceiving morphology and aberrant immunophenotypic expression, posing a great deal of difficulty in initial diagnosis. Owing to the aggressive nature of this malignancy, the diagnosis requires to be prompt and accurate. We need to be aware of the atypical presentations of this malignancy to get a clue and keep a high index of suspicion.

Patients/Materials \& Methods: We present a 26 years old female patient who presented with easy fatiguibility and low grade fever. Hemogram, peripheral blood smear examination followed by bone marrow studies, cytogenetic studies and flow cytometry was performed.

Results: Based upon peripheral blood smear and bone marrow examination diagnosis of Acute myeloid Leukemia(MPO Positive) was made.The flow cytometric diagnosis was consistent with AML with aberrant CD19 expression. However cytogenetics study showed translocation $\mathrm{t}(15 ; 17)$ and quantitative RT-PCRrevealed PMLRAR $\alpha$ (bcr-1 transcript) fusion.

Discussion \& Conclusion: Early and accurate diagnosis of APL is of utmost importance for an early intervention to prevent the dreaded complicationsof coagulopathy. The initial diagnosis is completely based on the morphology of cells and cytochemistry. Thus, along with the common morphological types, the rare variants should always be kept in mind. Hyperbasophilic variant is an extremely rare variant of this rare disease. The immunophenotyping by flow cytometry is instrumental in rapid diagnosis, but aberrant expression may be present which is misleading, as in the present case. This case report highlights the importance of a high index of suspicion of APL. Though definitive diagnosis can be made only by cytogenetics or molecular studies, the atypical morphological appearances and aberrant immunophenotypic expressions, as in our case, need to be kept in mind by a pathologist for correctly and timely diagnosing this highly curable malignancy which could otherwise be devastating, claiming a young life.
Clinical, Hematological and Immunophenotypic Profile of Mixed Phenotypic Acute Leukemia

Alpeshkumar B. Kapadia*1, Man Updesh Singh Sachdeva ${ }^{1}$, Parveen Bose $^{1}$, Jogeshwar Binota ${ }^{1}$, Nabhajit Mallik ${ }^{1}$, Sreejesh $\mathrm{S}^{1}$, Narender Kumar $^{1}$, Shano Naseem ${ }^{1}$, Prashant Sharma ${ }^{1}$, Jasmina Ahluwalia ${ }^{1}$, Reena Das ${ }^{1}$, Amita Trehan ${ }^{2}$, Pankaj Malhotra3, Neelam Varma ${ }^{1}$

${ }^{1}$ Hematology, ${ }^{2}$ Pediatric Hematology Oncology Unit, 3Adult Clinical Hematology Unit of Internal Medicine, Postgraduate Institute of Medical Education and Research, Chandigarh, India

Aims \& Objectives: To study the clinical, hematological and immunophenotypic profile of mixed phenotypic acute leukemia.

Patients/Materials \& Methods: Retrospective data mining of mixed phenotypic acute leukemia (MPAL) case was done from May- 2016 to March-2020. The clinical, bone marrow and flowcytometric profile including end of induction phase (EOI) measurable residual disease (MRD) status were noted.

Results: A total of 17 cases of MPAL were noted comprising of 10 adults and 7 pediatric cases. The median age was 17 years (2-62) and male to female ratio was 1.42: 1 . The median values (range) of hemoglobin, total leucocyte counts and platelet counts were $79 \mathrm{gm} / \mathrm{L}$ $(40-141), \quad 23.1 \times 109 / \mathrm{L} \quad(0.9-332 \times 109 / \mathrm{L})$ and $59 \times 109 / \mathrm{L}$ $(16-365 \times 109 / \mathrm{L})$. The peripheral blood showed blasts in 15 cases $(88.2 \%)$. Only two cases $(11.8 \%)$ showed two type of blasts on morphology. Cytochemical myeloperoxidase was positive in 12 cases (70.6\%). On immunophenotyping, 11 cases $(64.7 \%)$ were B-myeloid and 6 cases $(35.3 \%)$ were T-myeloid. Five cases $(29.4 \%)$ were positive for BCR-ABL1 rearrangement. End of induction(EOI) complete hematological response (CHR) was seen in 11 cases $(64.7 \%)$, CR with incomplete count recovery (CRi) in 4 cases $(23.5 \%)$ and no CHR in 2 cases $(11.8 \%)$. EOI MRD status $(\mathrm{n}=14)$ was positive in 8 cases $(57.1 \%)$ while negative in 6 cases $(42.9 \%)$. During median follow up of 7 months (0- 38 month), 6 cases developed relapse involving bone marrow in 5 and CNS in 1 case. Among these 6 cases, 3 were EOIMRD positive while 3 were EOI-MRD negative.

Discussion \& Conclusion: MPAL is rare hematological neoplasm with B-myeloid sub-type constituting majority of cases. BCR-ABL1 is most common cytogenic abnormality seen in MPAL.EOI-MRD positivity and early relapse rates are high.

Phenotypic Spectrum and the Role of Flow Cytometric Measurable Residual Disease in NPM1 Mutated Acute Myeloid Leukemia: An Experience of a Tertiary Care Center in Northern India

Soma Pradhan ${ }^{* 1}$, Richa Chauhan ${ }^{1}$, Surbhi Singh ${ }^{1}$, Ramesh Balasubramanian ${ }^{2}$, Vandana Arya ${ }^{1}$, Nitin Gupta ${ }^{2}$, Manas Kalra ${ }^{3}$, Ajay Sharma $^{2}$, Anupam Sachdeva ${ }^{3}$, Jyoti Kotwal ${ }^{1}$

${ }^{1}$ Department of Hematology, ${ }^{2}$ Department of Clinical Hematology, ${ }^{3}$ Department of Pediatric Hemato-Oncology, Sir Ganga Ram Hospital, New Delhi, India

Aims \& Objectives: Acute myeloid leukemia (AML) with mutated NPM1 is recently upgraded to a defined entity in the latest WHO classification. We retrospectively analyzed phenotypic characteristics of NPM1-mutated AML along with the utility of flowcytometric MRD testing post-induction (where available) in these patients diagnosed over the past 12 months.

Patients/Materials \& Methods: Peripheral smears and bone marrows were retrieved from October 2019-October 2020. Diagnostic phenotype was performed on BD FACSCanto II acute leukemia panel. An 8-color FlowMRD was performed on four cases postinduction using 'Leukemia-associated phenotype' and 'difference 
from normal' approach. Molecular workup for AML cases consisted of translocations AML-ETO, inversion 16, PML-RARA; detected by nested RT- PCR and NPM1, FLT-3 mutations by fragment analysis. Results: Over a 12 months period, 103 acute leukemias were diagnosed, 12/63 AMLs were found to have NPM1 mutation at baseline on molecular workup. Two cases revealed concomitant FLT3 clone at baseline. Male:female was 9:3 with median age 58 years, out of these 2 were pediatric cases. One of the pediatric cases had $<20 \%$ blasts. Categorizing the rest of our cases as per FAB classification we found AML M1(1), AML M2(4), AML M4(2) AML M5(5). Auer rods were present in $2 / 12$ cases. Characteristic cupping of nuclei was seen in 2/12 FLT3 comutated cases. Rest of the cases showed indentation of nucleus consistent with myelo- monocytic morphology. Dysplasia in erythroid and megakaryocytic series was seen in 4 and 3 cases respectively. Flowcytometry patterns- CD13brightCD33bright (9), monocytic differentiation (6), HLA-DRdim-negativeCD34dim-negative(M3) (2), HLA-DR + CD34dim-negative (4), Aberrant CD7 (4)2 with FLT3 clone. FlowMRD positivity based on HLA-DR$\mathrm{CD} 34+$ and CD13brightCD33bright patterns was detected in two FLT3 comutated cases. Aberrant CD7 was not useful as LAIP in both cases.

Discussion \& Conclusion: NPM-mutated AML was detected mostly in 5th-6th decades in our cohort with common phenotype being monocytic-AML-M5. CD13bright/CD33bright except the two with M3 phenotype. Discordant CD34/HLADR was also a common finding in our cases. The two FLT3 comutated cases revealed aberrant CD7 apart from the characteristic cupping of blast nucleus. Also, FlowMRD could pick up abnormal clones (bright CD13/33 and discordant HLADR/CD34) and thus can be useful tool in following NPM1 mutated AMLs.

\section{A Rare Case of Mixed Phenotype Acute Leukemia with Complex Karyotype}

Rincy Thomas ${ }^{* 1}$, Unni S. Pillai ${ }^{2}$, Bindu T. $G^{1}$

${ }^{1}$ Department of Laboratory Medicine, ${ }^{2}$ Department of Medical Oncology, Caritas Hospital, Kottayam, India

Aims \& Objectives: To report a rare case of Mixed phenotype acute leukaemia with complex karyotype.

Patients/Materials \& Methods: A 64 year old male presented with history of short febrile illness and elevated WBC counts. He was detected to have a few axillary and mediastinal lymph nodes (less than $1 \mathrm{~cm}$ short axis).

Results: Complete blood counts showed an elevated total WBC count of $29,000 / \mu \mathrm{L}$. Peripheral smear showed $39 \%$ blasts, $14 \%$ neutrophils, $25 \%$ lymphocytes, $10 \%$ monocytes, $1 \%$ eosinophils and $11 \%$ immature granulocytes.Many of the blasts showed myeloperoxidase positivity. Flow cytometry done on bone marrow aspirate at a referral Hospital laboratory showed blasts with expression of CD34, H L A DR, TdT, CD 99, MPO, CD 13, CD33 CD7, cCD 3, CD 58, CD38 and $\mathrm{CD}$ 64. Other markers were negative, The final report was suggestive of Mixed phenotype acute leukemia (T/Myeloid), Karyotype revealed 45, XY, del (2)(q31q36)[19], inv (8)(p11q13)[06],-16[20].

Discussion \& Conclusion: MPALs are categorised under ambiguous lineage leukaemias that account for less than $4 \%$ of all acute leukaemias. MPALs have blasts that express antigens of more than one lineage. This patient has a T Myeloid MPAL with complex karyotype,T myeloid MPAL with uncommon cytogenetics are considered to have poor prognosis though data are insufficient.
A Rare Case of Acute Promyelocytic Leukemia (APL) with Extensive Marrow Fibrosis

Sampath Kumar KJ ${ }^{* 1}$, Kavitha Bali $\mathrm{N}^{2}$, Geeta $\mathrm{J}^{1}$, Asima $\mathrm{Q}^{1}$, Atif $\mathrm{SM}^{1}$, Rashmi K ${ }^{1}$, Kalyan $\mathrm{Rao}^{2}$, Fathima $\mathrm{A}^{2}$, Neelamani $\mathrm{M}^{1}$

${ }^{1}$ Hematolgoy, ${ }^{2}$ Histopathology, Vijaya Diagnostic Centre, Hyderabad, India

Aims \& Objectives: Bone marrow fibrosis is associated with a number of neoplastic and non-neoplastic conditions. Acute myeloid leukemia may present with fibrosis; however, vanishingly rare cases have been reported of acute promyelocytic leukemia (APL) with associated marrow fibrosis.

Patients/Materials \& Methods: Here we present a case of APL with extensive fibrosis (APL-F). A 35-year-old woman presented pancytopenia. Bone marrow evaluation performed at outside institution showed a dry tap. Splenomegaly was absent. She was presumptively diagnosed with primary myelofibrosis.

Results: Peripheral smear showed occasional blasts/atypical promyelocytes. Similar cells were seen in imprint smears. Trephine biopsy showed extensive reticulin fibrosis and myeloid maturation arrest with scattered atypical large cell clusters. On Immunohistochemistry, these cells were positive for CD117 and Myeloperoxydase (MPO) and negative for CD34. Fluorescence in situ hybridization (FISH) showed a t(15;17); PML-RARA.

Discussion \& Conclusion: APL-F is a rare diagnosis and very few cases reported cases in the literature in PubMed databases. In nearly half of reported cases, patients had either a prior diagnosis of APL and the fibrosis was detected on post therapy evaluation, or history of prior therapy for unrelated neoplasms. APL-F may evade rapid diagnosis due to lack of adequate fresh aspirate material for expedited FISH analysis.

Keywords: APL, Pancytopenia, Fibrosis.

\section{IGH-DUX4 Fusion Transcript in B-Lineage Acute Lymphoblastic Leukemia}

Vishal O. Tayade ${ }^{* 1}$, Shilpi Saxena ${ }^{1}$, Sweta Rajpal ${ }^{1}$, Gaurav chatterji $^{1}$, Prashant Tembhare ${ }^{1}$, Papagudi Subramanian ${ }^{1}$, Chetan Dhamne $^{1}$, Sumeet Gujral ${ }^{1}$, Gaurav Narula ${ }^{1}$, Nikhil Patkar ${ }^{1}$

${ }^{1}$ Tata memorial centre, MUMBAI, India

Aims \& Objectives: The recently discovered DUX4-rearranged (DUX4r) B-lymphoblastic leukemia (B-ALL) account for 4-5\% of childhood B-ALL and has a favourable outcome, when treated with intensive chemotherapy. Very little literature is available on the clinico-pathological features of DUX4r-BALL. The aim of this study is to analyse the clinico-pathological features of this rare-entity.

Patients/Materials \& Methods: Cases of B-ALL for whom NGS requisition was raised in Tata memorial centre from Jan 2018 to November 2020 were subjected to customized RNA-sequencing panel using primers for ABL1, ABL2, CSF1R, CRLF2, DUX4, EPOR, ETV6, EBF1, FGFR1, IKZF1, JAK2, KMT2A, MLLT4, MEF2D, NTRK3, NUP214, NUP98, P2RY8, PAX5, PDGFRA, PDGFRB, RUNX1, TCF3, TYK2 and ZNF384 genes to detect their unknown fusion-partners. IlluminaMiseq (300-cycle chemistry) was used to perform the paired-end-sequencing. Data was analysed using Oncofuse and Fusion-Catcher. Custom-scripts and bedtools-2.25 were used to determine the coverage and construct the pipeline. Flowcytometry was performed using 10-color Navios- flow-cytometer (Beckman coulter) and data were analysed using Kaluza-software-1.3.

Results: Out of 201 cases, six cases of B-ALL with IGH-DUX4 fusion were identified (3\%) by RNA-sequencing. All of them occurred in paediatric age-group with a median age of 8-years (range 4-14 years). The median TLC was $17.7 \times 109 / \mathrm{L}$ at the time of 
diagnosis (range 2.14-75.1 × 109/1). On Flowcytometric-immunophenotyping, all six patients expressed common B-lineage markers such as CD10, CD19, CD22 and CD79a. CD 10 was variable in $4 / 6(66 \%)$ and moderate in 2/6 (33\%). CD45 was Dim-Neg in $4 / 6$ $(66 \%)$, Dim in 1/6 ((16\%) and negative in 1/6 (16\%) cases. CD20 was expressed in only one of the cases $(16 \%)$. All six patients expressed aberrant CD73 and CD123, however were negative for CD304 and CD25. CD2 was moderately expressed in 2/6 (33.3\%) and CD371 was positive in $1 / 2$ cases $(50 \%)$. At post-induction time-points, residual disease was measurable by flow-cytometry in $2 / 5$ cases $(40 \%)$. None of the patients has relapsed with a median follow-up of 8.5 months. The clinico-laboratory features are summarized in Table 1.

Discussion \& Conclusion: In the present study, we characterize the clinico-laboratory features of DUX4r-B-ALLs. Optimally designed targeted RNA-sequencing assays are sensitive and useful in picking up rarer-subtypes of B-ALL.

\section{Immunophenotyping, Cytogenetic and Molecular Characteristics in a Cohort of BCR-ABL Positive Acute Lymphoblastic Leukemia}

Udayakumar DS ${ }^{* 1}$, Ritu Chadha ${ }^{1}$, Bhawna $\mathrm{Jha}^{1}$, Shalini Goel ${ }^{1}$, Akshay Gore $^{1}$, Nitin Mathur ${ }^{1}$, Amrita Ramaswami ${ }^{2}$, Roshan Dikshit ${ }^{2}$, Nitin Sood ${ }^{2}$, Manorama Bhargava ${ }^{1}$, Renu Saxena ${ }^{1}$

${ }^{1}$ Hematopathology, ${ }^{2}$ Medical Oncology and Clinical Hematology, Medanta-The Medicity, Gurugram, India

Aims \& Objectives: To analyse the immunophenotyping, cytogenetic and molecular characteristics of BCR-ABL1 positive acute lymphoblastic leukemia ( $\mathrm{Ph}+\mathrm{ALL})$.

Patients/Materials \& Methods: A cohort of 15 patients diagnosed with Ph + ALL in Medanta- the Medicity from June 2018 to October 2020 were included in the study. Flowcytometric Immunophenotyping (FCI) was performed using 10 colour, three laser BD FACS Lyric/BD FACS Suite with a panel of antibodies including CD34, CD19, CD45, CD20, CD10, CD22, CD58, CD38, CD66c, CD13 + 33, CD15, CD73, CD81, CD200, CD123. Karyotyping on BMA/peripheral blood was done using standard protocols in 14 cases and reported as per ISCN 2016 nomenclature. Molecular confirmation of BCR-ABL transcripts was done by RT-PCR. Results: The cohort had a median age of 31 years (Age range: 16-73 years) with a male: female ratio of 1.14:1. All cases were NCI high risk at presentation. On FCI all cases had a common-B immunophenotype. The blasts expressed CD19, $\mathrm{CD} 10, \mathrm{CD} 34, \mathrm{CD} 22, \mathrm{CD} 24$ in all cases with variable expression of other antigens such as CD13 + 33(12/15, 80\%), CD66c(14/15, 93.3\%), CD15(2/15, 13.3\%), CD20(8/15, 53.3\%), CD73(12/15, 80\%), CD9(13/15, 86.6\%), CD123(10/15, 66.6\%) and CD7(3/15, 20\%). On karyotyping Philadelphia chromosome was identified in 13/14 cases $(92.8 \%)$ and additional cytogenetic abnormalities (ACA) were identified in $10 / 14$ cases $(71.42 \%)$ cases. The significant ACAs identified include $-7(4 / 14,28.57 \%),+\operatorname{der}(22)(2 / 14,14.28 \%)$, and one case each of del17p, high hyperdiploidy, del9p, $+8, \mathrm{i}(9 \mathrm{q})$ and complex karyotype. On RT-PCR 14/15(93.3\%) cases had minor BCR-ABL (p190) transcript and one case had major (p210) transcript.

Discussion \& Conclusion: In the cohort studied all cases conformed to the Common B immunophenotype. In comparison to $\mathrm{Ph}$ negative ALL the aberrant antigen CD66c (93.3\% vs 53.2\%), the combination of CD66c\&CD13 + 33 (73.3\% vs $19.04 \%)$ expression was significantly higher in $\mathrm{Ph}+\mathrm{ALL}$. CD66c had a significant negative predictive value of $94.4 \%$ suggesting the preemptive evidence for lack of BCR-ABL in cases without CD66c.71.42\% of cases had ACAs with Monosomy 7 being the most common. The minor (p190) transcript was seen in $93.3 \%$ of our cases. In a cohort of Ph + ALL patients from a tertiary care center the immunophenotyping and genetic findings are in concordance with the published literature.
Study of Clinico-Pathological and Cytogenetic Profile in Newly Diagnosed Cases of Acute Myeloid Leukemia Based on Post Induction Remission Status

Manjubala Muralidaran ${ }^{* 1}$, Rakhee $\mathrm{Kar}^{1}$, Smita Kayal ${ }^{2}$, Debdatta Basu $^{1}$

${ }^{1}$ Department of Pathology, ${ }^{2}$ Department of Medical Oncology, JIPMER, Puducherry, India

Aims \& Objectives: To study the clinico-pathological and cytogenetic profile of newly diagnosed cases of AML patients based on their post-induction remission status in a tertiary care centre.

Patients/Materials \& Methods: Newly diagnosed cases of AML from January 2017 to July 2020 estimated from the pathology records were 228. Among these, 152 individuals had a post-induction bone marrow (BM) and their remission status was recorded. The peripheral blood counts, diagnostic BM findings, immunophenotype, and cytogenetic profile of these patients wherever available were noted from the Haematology and Medical Oncology archives.

Results: Out of 152 individuals, 92 patients $(60.5 \%)$ attained remission and $60(39.4 \%)$ were not in remission. Clinical and cytogenetic data were available for $52 / 92$ patients in remission and for $31 / 60$ patients not in remission. The median age group in both groups was 32 years. There was a slight female preponderance (1.1:1) in remission cases and slight male preponderance $(1.3: 1)$ in not in remission cases. In remission cases, M2 and M3 were the most common subtypes, followed by M1 and M4. In not in remission cases M2 was the commonest subtype, followed by M1 and M0. Immunophenotypic aberrancy observed was expression of CD5, CD7 and TdT. No cases showed aberrant B-Lymphoid marker expression. Commonest cytogenetic pattern observed was translocations (40.4\%) in remission cases and normal cytogenetics $(32.2 \%)$ in not in remission cases. Table 1 enlists the different cytogenetics pattern observed. However these cytogenetic patterns did not show a statistically significant difference among the groups $(p>0.05)$.

Discussion \& Conclusion: AML is a heterogenous disease with regard to morphologic pattern and spectrum of cytogenetic changes and multiple factors can affect the post-induction remission status. Supporting Document:

Table 1: Cytogenetic patterns in newly diagnosed AML patients based on their remission status

\begin{tabular}{|l|l|l|}
\hline Cytogenetics & $\begin{array}{l}\text { Remission } \\
\text { cases (52) } \\
\mathrm{n}(\%)\end{array}$ & $\begin{array}{l}\text { Not in remission } \\
\text { cases (31) } \\
\mathrm{n}(\%)\end{array}$ \\
\hline Normal cytogenetics & $17(32.7)$ & $10(32.2)$ \\
\hline Translocations & $21(40.4)$ & $7(22.6)$ \\
\hline Complex karyotype & $4(7.7)$ & $4(12.9)$ \\
\hline Trisomy & $3(5.8)$ & $3(9.7)$ \\
\hline Inversion & $1(1.9)$ & 0 \\
\hline Deletion & $2(3.8)$ & $1(3.2)$ \\
\hline $\begin{array}{l}\text { Test not performed } \\
\text { due to lack of } \\
\text { metaphases }\end{array}$ & $4(7.7)$ & $6(19.4)$ \\
\hline
\end{tabular}




\section{Targeted RNA Gene Fusion Variant Sequencing Metrics and Clinical Perspective in Myeloid Neoplasms at a Tertiary Care Centre}

Kunjal Patel $^{* 1}$, Kallol Saha ${ }^{1}$, Vamshi Thamtam ${ }^{1}$, Saheli Banerjee ${ }^{1}$, Vivek Radhakrishnan ${ }^{2}$, Saurabh Bhave ${ }^{2}$, Jeevan Kumar $^{2}$, Reena Nair $^{2}$, Mammen Chandy ${ }^{2}$, Neeraj Arora ${ }^{3}$, Mayur Parihar ${ }^{1}$, Deepak K. Mishra $^{1}$

${ }^{1}$ Molecular Genetics, ${ }^{2}$ Clinical Hematology and Bone marrow transplant, Tata Medical Center, Kolkata, ${ }^{3}$ Molecular Genetics, Unipath speciality laboratory, Ahmedabad, India

Aims \& Objectives: The aim of the study was to assess implications and viability of RNA gene fusion panel in myeloid neoplasms. The objective was to elucidate the NGS metrics in RNA fusion variant sequencing.

Patients/Materials \& Methods: We performed RNA fusion variant sequencing from bone marrow or peripheral blood on 102 suspected myeloid neoplasms. Libraries were prepared according to manufacturer's instruction of Oncomine Myeloid Panel and sequencing was performed on ion torrent PGM, NGS platform with semiconductor technology. Variant call and annotations were executed on an ion reporter software. This panel measures more than 600 common fusions variant and, 5 expression markers were used as an internal control. The required quality parameters to call a reportable transcript as fusion variant was set to greater than 60 read length with a minimum of 6000 mapped fusion reads of a particular transcript. After thorough validations of the RNA sequencing panel, it was implemented into clinical settings adjunct to ancillary techniques and platforms like karyotyping, FISH and RT-PCR.

Results: We got fusion transcripts in six acute myeloid leukemia and two myeloproliferative neoplasms cases with a few uncommon fusion transcripts. All 8 fusion variant positive cases were confirmed prospectively or retrospectively by orthogonal methods. (Table) In RNA fusion sequencing, the average mapped fusion reads per sample was 106803. Among the positive samples, the minimum fusion transcript reads count was 350 with the maximum being 13211. The RPKM (Reads Per Kilobase of transcript per Million reads mapped) in the analyzed sample ranged from 23617 to 139503 . For corresponding DNA mutation analysis mean coverage, Q20 bases covered and mapped reads were $1527 x, 167602573$, 915677, respectively. Uniformity and on target of the analyzed amplicons was $>95 \%$ and $>80 \%$ respectively in all the sequenced cases.

Discussion \& Conclusion: RNA sequencing along with DNA sequencing can be performed on diagnostic samples in myeloid neoplasms as it provides better sensitivity and comprehensive genomic profiling. It also serves as a discovery platform, identifying rare cryptic fusions that can be missed on karyotyping and FISH. An added advantage of performing Oncomine Myeloid Panel is that apart from fusions, mutations can also be elucidated.
Supporting Document:

\begin{tabular}{|c|c|c|c|c|c|}
\hline & WHO classification & $\begin{array}{l}\text { Fusion detected on } \\
\text { NGS }\end{array}$ & Fusion reads & $\begin{array}{l}\text { Associated DNA } \\
\text { mutations }\end{array}$ & VAF \\
\hline 1 & $\begin{array}{l}\text { AML with t(8;21)(q22;q22.1); } \\
\text { RUNX1-RUNX1T1 }\end{array}$ & $\begin{array}{l}\mathrm{t}(8 ; 21) \text { RUNX1- } \\
\text { RUNX1T1 }\end{array}$ & 10227 & CKIT & $45 \%$ \\
\hline 2 & $\begin{array}{l}\text { AML with t(8;21)(q22;q22.1); } \\
\text { RUNX1-RUNX1T1 }\end{array}$ & $\begin{array}{l}\mathrm{t}(8 ; 21) \text { RUNX1- } \\
\text { RUNX1T1 }\end{array}$ & 1944 & - & \\
\hline 3 & $\begin{array}{c}\text { AML with } t(9 ; 11)(\text { p21.3; } ; 23.3) ; \\
\text { MLLT3-KMT2A }\end{array}$ & $\begin{array}{l}\mathrm{t}(9 ; 11) \text { KMT2A- } \\
\text { MLLT3 }\end{array}$ & 350 & BRAF & $07 \%$ \\
\hline 4 & $\begin{array}{c}\text { AML with t }(9 ; 11)(\text { p21.3; } ; \text { 223.3); } \\
\text { MLLT3-KMT2A }\end{array}$ & $\begin{array}{l}\mathrm{t}(9 ; 11) \text { KMT2A- } \\
\text { MLLT3 }\end{array}$ & 13211 & NRAS & $41 \%$ \\
\hline 5 & $\begin{array}{l}\text { AML with minimal } \\
\text { differentiation }\end{array}$ & $\begin{array}{l}\mathrm{t}(6 ; 11) \text { KMT2A- } \\
\text { MLLT4 }\end{array}$ & 8398 & NRAS & $47 \%$ \\
\hline 6 & $\begin{array}{l}\text { Therapy-related myeloid } \\
\text { neoplasms }\end{array}$ & $\begin{array}{c}\mathrm{t}(11 ; 19) \text { KMT2A- } \\
\text { ELL }\end{array}$ & 5552 & CBL & $13 \%$ \\
\hline 7 & $\begin{array}{l}\text { Chronic myeloid leukaemia, } \\
\qquad \text { BCR-ABL1+ }\end{array}$ & $t(9 ; 22) B C R-A B L 1$ & 12401 & $A B L 1, R U N X 1$ & $\begin{array}{l}40 \%, \\
43 \%\end{array}$ \\
\hline 8 & MPN, unclassifiable & $t(9 ; 12)$ ETV6-ABL1 & 9516 & - & \\
\hline
\end{tabular}

Disclosure of Interest: None Declared.

\section{Mixed Phenotypic Acute Leukemia: A Rare Diagnosis}

Ritwika Dabral $^{* 1}$, Jyoti Sawhney ${ }^{1}$, B. R Nagarjun ${ }^{1}$, Biren Parikh ${ }^{1}$, Birva Raiya ${ }^{1}$

\section{${ }^{1}$ Oncopathology, GCRI, Ahmedabad, India}

Aims \& Objectives: MPAL is a rare diagnosis representing about $2-5 \%$ of acute leukemia cases. It comes under Acute Leukemia of Ambiguous lineage. Morphologically it is of two types- biclonal \& bilineal.Previously diagnosed by EGIL criteria but now WHO 2016 criteria is used which is more stringent. Its diagnosis requires flow cytometry \& expertise in interpretation. Aim of the study is to present a few diagnostically challenging cases, summarize biological \& clinical features \& address diagnostic pitfalls.

Patients/Materials \& Methods: EDTA anticoagulated bone marrow aspirate samples of patients diagnosed as acute leukemia/CML- BC on the basis of morphology were utilized for flowcytometry. A comprehensive panel of monoclonal antibodies targeting myeloid, B cell \& $\mathrm{T}$ cell marker was utilized. Diagnosis was made based on WHO 2016 classification criteria.

Results: Two cases came out to be MPAL- B/Myeloid, MPAL with $\mathrm{t}(9 ; 22) \mathrm{BCR}$ ABL1. One as Chronic Myeloid Leukemia Blast Crisis with biclonal population.

Discussion \& Conclusion: The understanding of MPAL has vastly improved over the years, which is attributed to the advancement in diagnostic modalities. Diagnosis of MPAL also requires cytogenetic/molecular evaluation. Treatment of such cases is challenging \& need to be assessed during follow up to see the response \& alter therapy according to the diagnosis. Accurate \& early diagnosis is necessary because of poor prognosis of this unusual leukemias. 
ABL1 Amplification on BCR/ABL1 Fish in a Case Of T-Cell Acute Lymphoblastic Leukemia: A Case Report

Viyati Vithlani*1 $^{*}$, Harsha Jaykar ${ }^{1}$

${ }^{1}$ Pathology, Bharati Vidyapeeth (Deemed to be University) Medical College, Hospital and Research Centre, Pune, India

Aims \& Objectives: To demonstrate the importance of BCR/ABL1 FISH in T-cell ALL.

Patients/Materials \& Methods: A 9-year-old male presented with generalized lymphadenopathy and anasarca since 15 days before admission. Clinical and radiological examination displayed generalized lymphadenopathy, mediastinal enlargement, hepatosplenomegaly and right sided pleural effusion. The haemorrhagic pleural fluid showed nucleated cell count of $16.4 \times 10^{9} / \mathrm{L}$ with $80 \%$ atypical lymphocytes suggestive of lymphoproliferative disorder. Haematological data confirmed the diagnosis of acute leukemia : peripheral blood examination showing haemoglobin $108 \mathrm{~g} / \mathrm{L}$, TLC $82.6 \times 10^{9} / \mathrm{L}$, and platelet count $21 \times 10^{9} / \mathrm{L}$, with $70 \%$ blasts. Immunophenotyping on peripheral blood showed $85 \%$ blast cells expressing CD3, CD7 and weak positive for $\mathrm{CCD} 3$ and aberrant weak expression of CD117. The CD34 and other B cell, Myeloid and T cell markers were negative. Impression of T-cell ALL was given. Fluorescence in situ hybridization (FISH) for BCR/ABL translocation was done using VYSIS dual color dual fusion probe. Karyotype was performed on peripheral blood.

Results: FISH for BCR/ABL translocation demonstrated 5-30 extrachromosomal copies of ABL1 locus in 10\% interphase cells and was negative for $\mathrm{t}(9 ; 22)$ in $100 \%$ cells. The composite karyotype showed hypodiploidy $(42 \sim 45)$ with add(1p32) and i(17)(q10). No dmin or hsr was seen on the karyotype. The patient was started on Allopurinol, Vincristine, Dexamethasone and Ceftriaxone. The patient's condition worsened, he developed multi-organ failure along with sepsis and succumbed to death on the 13th day of hospital admission.

Discussion \& Conclusion: Routine karyotyping in T-cell ALL cases can detect abnormality in only $55-75 \%$ cases as most of the rearrangements are cryptic. Utilization of $\mathrm{FISH} /$ molecular techniques such as mutation analysis, epigenetic studies and single nucleotide polymorphism can increase the diagnostic rate of genetic abnormality. The NUP214-ABL fusion leads to formation of a protein, which is an activated tyrosine kinase implicated in the pathogenesis of T-cell ALL. It has similar mechanism of action as of BCR-ABL as it activates similar pathways and for its sensitivity to tyrosine kinase inhibitors. But the NUP214-ABL protein is less potent and thus requires amplification of the fusion gene for neoplastic transformation. ABL1 amplification detection is important as this T-cell ALL subset is sensitive to tyrosine kinase inhibitors. Targeted therapies may improve outcome of patients with T-cell ALL expressing NUP214-ABL1 fusion with ABL1 amplification but the clinical data is limited to conclude the efficacy. We conclude FISH is the investigation of choice that quickly detects ABL1 amplification. We recommend to perform BCR-ABL1. FISH in each case of T-cell ALL even though Philadelphia positivity is very rare in T-cell ALL.

\section{Leukemic Evolution of Double Expressor High Grade B Cell Lymphoma: Is It or Is It Not?}

Sushma Belurkar*1

${ }^{1}$ Pathology, Kasturba Medical College,MAHE,Manipal, Manipal, India

Aims \& Objectives: Introduction : Double expressor lymphomas (DHLs) are aggressive mature B-cell neoplasms which show over expression of both MYC and B-cell lymphoma-2 (BCL-2) proteins on immunohistochemistry. The importance of diagnosing this subtype is because of the fact that these lymphomas are known to have worse outcomes as compared to other high grade B cell lymphomas. Such double-hit events are extremely rare in B-cell acute lymphoblastic leukemia (B-ALL), especially in paediatric patients or young adults.And these cases are aslo known to have poor prognosis as compared to other B cell ALL. Here in we present a case which was initially diagnosed as double expressor B cell lymphoma but eventually progressed to B-ALL.

Patients/Materials \& Methods: Case report: A 47 year old male presented with high grade fever with chills and rigors and easy fatigability. There was no significant lymphadenopathy or organomegaly on physical examination. His complete blood count and peripheral smear showed pancytopenia without any abnormal cells. His bone marrow evaluation showed $30 \%$ abnormal lymphoid cells. Flowcytometric analysis showed expression of B cell markers with aberrant CD56 and a small population of cells LAMBDA restricted. Immunohistochemistry on bone marrow biopsy showed expression of BCL-2 and MYC in $>50 \%$ of cells but BCL-6 was negative. Mutation studies could not be done due to financial constraints. It was diagnosed as double expressor high grade B cell Lymphoma. But his follow up smear showed $97 \%$ abnormal cells and repeat flowcytometry showed B-ALL immunophenotype. Patient was started on adult GMALL protocol and patient is in remission for last one year.

Results: NA.

Discussion \& Conclusion: Discussion \& Conclusion: Double expressor or double hit B cell lymphoma/leukaemia are rare but it is important to diagnose these lymphomas particularly because it affects the treatment outcomes and the prognostication. B-ALL with double expression of MYC and BCL2 is even rare and much literature is not available for it. This case highlights the diagnostic challenges faced in such cases.

\section{Cytogenetic Risk Stratification of B-Acute Lymphoblastic Leukemia and Its Correlation with Other Prognostic Factors}

Ritu Chadha $^{* 1}$, Shivani Sangwan ${ }^{1}$, Udayakumar DS ${ }^{1}$, Akshay Gore ${ }^{1}$, Bhawna Jha ${ }^{1}$, Shalini Goel ${ }^{1}$, Nitin Mathur ${ }^{1}$, Neha Rastogi ${ }^{2}$, Amrita Ramaswami $^{3}$, Roshan Dikshit ${ }^{3}$, Nitin Sood ${ }^{3}$, Manorama Bhargava ${ }^{1}$, Satya Prakash Yadav ${ }^{2}$, Renu Saxena ${ }^{1}$

${ }^{1}$ Department of Hematopathology, ${ }^{2}$ Department of Paediatric Haematology \& BMT, ${ }^{3}$ Department of Medical Oncology \& Haematology, Medanta-The Medicity, Gurugram, India

Aims \& Objectives: Purpose of the current study was to categorize WHO defined B-ALL cases into 3 cytogenetic risk groups (good, intermediate $\&$ poor) and to see their correlation with age, NCI risk criteria \& treatment response.

Patients/Materials \& Methods: Clinical \& diagnostic details were collected for 57 newly diagnosed B-ALL patients registered at Medanta- the Medicity between May 2018 to September 2020. Flow cytometric immunophenotyping was performed on bone marrow samples by 3 laser BD FACS Lyric. Karyotyping was performed by G-banding \& reported in accordance with ISCN 2016. RT- PCR was performed by multi-gene PCR panel kit for BCR/ABL, ETV6RUNX1, TCF3-PBX1 \& KMT2A (MLL) rearrangements in all cases. FISH for cryptic chromosomal abnormalities using commercial fusion or break apart probes for ETV6-RUNX1, BCR-ABL1 \& MLL was done in cases detected positive by RT-PCR.

Results: Study cohort comprised 57 B-ALL patients, 30 (52.6\%) children including 2 infants and $27(47.3 \%)$ adults. Median age for paediatric group was 6 years ( 4 months -17 yrs) and for adults was 
39 years ( 18 to 75 yrs). Male to Female ratio was $1.4: 1$. Their median TLC was $6,000 / \mathrm{uL}(830-2,42,230 / \mathrm{uL})$. According to NCI risk criteria 41/55 (74.5\%) were high risk and $14 / 55(25.4 \%)$ were standard risk. Clonal chromosomal abnormality was detected in 43/57 cases (75.4\%), while 14/57 (24.5\%) cases showed normal karyotype. Secondary chromosomal abnormalities were detected in 34/57(59.6\%) cases. Good risk cytogenetics were more common in paediatric than adult group who had poor risk cytogenetics. Intermediate risk cytogenetics were equally distributed among children and adults. There was strong correlation between NCI- high risk with poor and intermediate cytogenetic risk groups. Post treatment follow-up was available in 35/57 cases of which 23 children had good prednisolone response assessed by day8 peripheral blood smear. On Day 15 bone marrow, 19/23 children had M1 status \& 4/23 children had M2/M3 status ( 3 belonged to good risk and 1 to poor risk cytogenetics). Post induction morphological remission was achieved in 30/35 cases (85.7\%), 1 case was not in remission \& 4 adults died during induction phase. End of induction flow MRD was negative in 22/35 (62.8\%) patients and positive in $9 / 35(25.7 \%)$ patients ( 2 belonged to good risk, 4 to intermediate \& 3 to poor cytogenetic risk group).

Discussion \& Conclusion: Cytogenetic risk groups showed strong correlation with age \& NCI risk criteria; However, treatment response varied across cytogenetic risk groups, thus suggesting role of tailoring treatment to suit different subsets of ALL patients followed by refining risk stratification during course of treatment.

\section{Cytogenetic Profile of Acute Leukemia in Coastal Karnataka}

Nagaraj V. Kulkarni ${ }^{* 1}$, D Prashanth Shetty ${ }^{1}$, Vijith Shetty ${ }^{2}$, Meenakshi A ${ }^{1}$, Reshma Shetty ${ }^{1}$, Akanksha A. Kalal ${ }^{1}$, Rajesh Krishna ${ }^{3}$

${ }^{1}$ KSHEMA Center for Genetic Services, ${ }^{2}$ Department of Oncology, K.S.Hegde Medical Academy, NITTE (Deemed to be) University., ${ }^{3}$ Department of Haematology, Yenepoya Medical College Hospital, Yenepoya (Deemed to be) University., Mangaluru, India

Aims \& Objectives: Acute leukemia (AL) has been traditionally prognosticated using Conventional karyotyping and Florescent In Situ Hybridization (FISH). We have profiled consecutive cases from 2 tertiary university hospitals in coastal Karnataka between 2018 to 2020 and assessed if the results obtained were comparable to national data.

Patients/Materials \& Methods: A total of 38 AL patients were included in the study. Cytogenetic analysis was carried out on bone marrow or peripheral blood samples using standard GTG banding technique. Analysis was done using GenASIs software version 8.1. Results were interpreted according to International System for Human Cytogenetic Nomenclature (ISCN) - 2013. FISH was done on the culture failure cases to confirm the abnormality, using specific probes for Acute Myeloid Leukemia (AML): MLL rearrangements (11q23), AML-ETO t(8;21), PML-RARA t $(15 ; 17)$, CBF $\beta$ inv(16)/t(16;16) and Acute Lymphoblastic Leukemia (ALL): MLL rearrangements (11q23), BCR-ABL t $(9 ; 22)$ were used.

Results: Cytogenetic evaluation of AL patients $(n=38)$ age ranging from 2 to 70 years, which constitutes AML-21 and ALL-17. Of these 38 cases, 27 cases with good index which were karyotyped, whereas 11 cases resulted into culture failure. Among 27 karyotyped cases, 17 cases showed normal karyotype and 10 cases were abnormal karyotype which includes hypodiploidy, hyperdiploidy, composite karyotype, trisomy 11 and 13. FISH was done on 11 culture failure cases, which showed negative expression for AML and ALL panel probes.
Discussion \& Conclusion: Conventional cytogenetics and FISH results from coastal Karnataka were overall consistent with rest of Indian data, however further molecular studies are indicated in this regard.

Keywords: Acute Myeloid Leukemia, Acute Lymphoblastic Leukemia, Karyotyping, FISH.

\section{An Acute Megakaryoblastic Leukemia; Rare Hematological Neoplasm, Case Report}

Vinita Paswan ${ }^{* 1}$, Dinesh Chandra ${ }^{1}$, Ruchi Gupta ${ }^{1}$, Khaliqur Rahman $^{1}$, Manish K. Singh ${ }^{1}$, Anshul Gupta ${ }^{1}$, Sanjeev Yadav ${ }^{1}$, Rajesh Kashyap $^{1}$, Soniya Nityanand ${ }^{1}$

${ }^{1}$ Hematology, Sanjay Gandhi Postgraduate Institute, Lucknow, India

Aims \& Objectives: Here we report the morphological and immunophenotypic characteristics of two cases of AMKL in the pediatric age group.

Patients/Materials \& Methods: A total of 952 AML cases were diagnosed from our institution over a period of seven years (2013 to 2019). Only two cases $(0.2 \%)$ of AML-M7 were reported during this period. The first patient was a nine-year-old male child who presented with complaints of abdominal pain with hepatospleenomegaly, while the other was a two-year-old male child presenting with multiple ecchymotic patches all over the body with hepatospleenomegaly. Peripheral blood and bone marrow aspiration and biopsy were performed. Immunophenotyping and karyotyping was performed on bone marrow aspirate sample.

Results: In both cases, complete hemogram revealed anemia and thrombocytopenia. Blasts in the peripheral blood blast were noted only in second case. Bone marrow aspiration was very difficult in both cases suggesting underlying myelofibrosis. The hemodiluted marrow smears and imprint smears showed the presence of $80 \%$ and $50 \%$ myeloblasts respectively. Bone marrow biopsy were fibocellular, showed increase presence of immature precursors. Immunophenotyping by flow cytometry on bone marrow samples in both the cases confirmed the presence of megakaryoblasts in which were negative for $\mathrm{CD} 45$ region and were expressing cytoCD41, cytoCD42 and CD36. GTG-banding analysis of 30 metaphases revealed normal karyotype. Both the patients received chemotherapy, which was commenced within $48 \mathrm{~h}$ of diagnosis, however both the patients were developed severe respiratory distress and sepsis during the course of chemotherapy and succumbed due to their illness within one month after a diagnosis.

Discussion \& Conclusion: AMKL is a rare leukemia with a very poor prognosis. A strong morphological suspicion is essential to facilitate the diagnosis of this entity by incorporating relevant markers in the diagnostic panel.

Keywords: Acute Megakaryoblastic Leukemia, Acute Myeloid Leukemia, Bone marrow morphology, Immunophenotyping.

\section{Morphological and Immunophenotypic Clues in the Diagnosis of Acute Myeloid Leukemia with $\mathbf{T}(8 ; 21)$}

Karuna Balakrishnan ${ }^{* 1}$, Devianu $\mathrm{K}^{1}$, Prabhu Manivannan ${ }^{1}$, Rakhee $\mathrm{Kar}^{1}$, Debdatta Basu ${ }^{1}$, Smita Kayal ${ }^{2}$

${ }^{1}$ Pathology, ${ }^{2}$ Medical Oncology, JIPMER, Puducherry, Puducherry, India

Aims \& Objectives: Acute myeloid leukemia with $\mathrm{t}(8 ; 21)$, found in $1-5 \%$ of cases of AML, carries good prognosis. The aim of this study 
was to assess the morphological and immunophenotypic features associated with the diagnosis of acute myeloid leukemia with $\mathrm{t}(8 ; 21)$. Patients/Materials \& Methods: All cases of acute myeloid leukemia with confirmed $t(8 ; 21)$ were collected from the archives of departments of Pathology and Medical Oncology over a period of 6 years (2015-2020). We analysed the morphology and immunophenotypic features of 29 cases.

Results: Out of the 29 cases, twenty cases showed FAB-M2 phenotype, six cases showed marrow eosinophilia, blast count was less than $20 \%$ in six cases and three cases presented with myeloid sarcoma. Other features seen in varying frequencies were perinuclear hoff with very large pseudo-Chediak-Higashi granules in blasts, long and sharp Auer rod, myeloid dyspoiesis (Pseudo- Pelger Huet cells, monolobated neutrophils, homogenous pink cytoplasm in neutrophils) and Auer rods in neutrophils. Immunophenotyping by flow cytometry showed aberrant expression of B lymphoid markers in eight cases.Additional cytogenetic abnormalities in the form of loss of sex chromosome was seen in eighteen cases and a complex three-way translocation involving chromosomes 8 and 21 was seen in 2 cases. One case had a variant $\mathrm{t}(8 ; 21)$. FLT3-ITD and NPM1 mutations were studied in 24 cases, all of which were negative for NPM1 and one was positive for FLT3-ITD. Fourteen cases achieved complete remission after first cycle of induction. There were three cases with diagnostic dilemma. One case was reported as myelodysplastic syndrome as the bone marrow blast percentage was only $4 \%$ and the peripheral blood blast percentage was $1 \%$; second as mixed phenotype acute leukemia based on immunophenotypic features and third as acute promyelocytic leukemia as there were many Faggot cells with bilobed nucleus seen morphologically.

Discussion \& Conclusion: Many well recognized features of AML with $\mathrm{t}(8 ; 21)$ were seen and based on this series of cases, morphological as well as immunophenotypic clues helped predict the diagnosis of AML with $t(8 ; 21)$, all of which were confirmed by karyotyping.

\section{Rare Translocation T(6;14)/IGH:ID4 Causing Extensive Bone Marrow Fibrosis in B Cell Precursor Acute Lymphoblastic Leukemia: A Report of Two Cases}

Ashish B. Gorantla*1, Tanvi Gupta ${ }^{1}$, Arun SR ${ }^{1}$, Sipra R. patel ${ }^{1}$, Deepak K. Mishra ${ }^{2}$, Niharendu Ghara ${ }^{3}$, Shekhar Krishnan ${ }^{3}$, Reghu $\mathrm{KS}^{3}$, Arpita Bhattacharya ${ }^{3}$, Mayur Parihar ${ }^{1}$

${ }^{1}$ Department of cytogenetics, ${ }^{2}$ Department of Haematopathology, ${ }^{3}$ Paediatric Haemato oncology, Tata Medical Center, Kolkata, India

Aims \& Objectives: We report two cases of BCP ALLs presenting as Pancytopenia with extensive bone marrow fibrosis and $t(6 ; 14)$. The $\mathrm{t}(6 ; 14)$ results in juxtaposition of ID4 gene to $\operatorname{IgH}$ gene resulting $\mathrm{n}$ over expression of ID4 gene.

Patients/Materials \& Methods: Karyotyping was done on bone marrow aspirate samples as per standard cytogenetic protocols and reported in accordance with the International System for Human Cytogenetic Nomenclature (ISCN) 2016. Interphase and Metaphase FISH was performed as per standard protocols.

Results: Of the total 722 BCP ALLs seen between 2011 to 2019, $\mathrm{t}(6 ; 14) / \operatorname{IgH}$ :ID4 was seen in 2 patients $(0.27 \%)$. Both the patients were pediatric BCP ALLs (UPN1: 9 years;UPN2:6 years) and presented with Pancytopenia. Bone marrow Aspirate (BMA) examination revealed $50 \%$ blasts in UPN1 and $90 \%$ in UPN2. The Karyotype examination showed multiple structural and numerical abnormalities with $\mathrm{t}(6 ; 14)$ in both the patients. UPN1 Karyotype $47, \mathrm{XY},+5$, $\mathrm{t}(6 ; 14)(\mathrm{p} 22 ; \mathrm{q} 32), ? \operatorname{der}(7), \mathrm{i}(9)(\mathrm{q} 10)[9] / 46$, idem,- $\quad 21,+\operatorname{mar}[5] /$
46,XY[6]; UPN2 Karyotype $46 \sim 51, X Y, \operatorname{dup}(1)(\mathrm{q} 21 \mathrm{q} 31), \operatorname{der}(1)$ $\mathrm{t}(1 ; ?)(\mathrm{q} 21 ; ?), \mathrm{t}(4 ; 9)(\mathrm{q} 23 ; \mathrm{p} 11), \mathrm{t}(6 ; 14)(\mathrm{p} 22 ; \mathrm{q} 32),+8, \mathrm{i}(9)(\mathrm{q} 10), ? \operatorname{der}(9)$ $\mathrm{t}(9 ; ?)(\mathrm{q} 33 ; ?), \mathrm{t}(12 ; 15)(\mathrm{p} 13 ; \mathrm{q} 21),+1 \sim 4 \operatorname{mar}[\mathrm{cp} 16] / 46, \mathrm{XY}[4] . \mathrm{t}(6 ; 14)$ with $\mathrm{IgH}$ gene rearrangement was confirmed using interphase and metaphase FISH analysis using IgH break apart probe. Both were treated on the ICiCLE 2014 protocol with good day 8 prednisolone response and negative minimal residual disease (MRD) at end of induction (EOI). The bone marrow biopsies of either patients showed extensive grade 2 fibrosis that regressed at end of induction. UPN1 relapsed at end of maintenance ( 24 months). The bone marrow fibrosis (grade2) reappeared at relapse with $50 \%$ bone marrow blasts. The patient also showed a concomitant testicular relapse.UPN 2 was in reemission at the last follow up time point and was on maintenance phase of therapy.

Discussion \& Conclusion: IGH rearranged BCP ALL accounts for $5 \%$ of total ALL cases including B \& T ALL, predominantly presenting in adolescents and young adults. We present this rare translocation and its specific association with Pancytopenia and extensive bone marrow fibrosis. The bone marrow fibrosis appeared and regressed with the presence and disappearance of blasts suggesting possible modulation of the bone marrow microenvironment by the leukemic blasts carrying the $\mathrm{t}(6 ; 14)$.

\section{Immunophenotyping Directed Fish Strategy to Identify Znf384 Fusions}

Arun SR ${ }^{* 1}$, Tanvi Gupta ${ }^{1}$, Sipra R. Patel ${ }^{1}$, Subhajit Brahma ${ }^{1}$, Niharendu Ghara ${ }^{2}$, Reghu KS ${ }^{2}$, Shekhar Krishnan ${ }^{2}$, Arpita Bhattacharya $^{2}$, Vivek S. Radhakrishnan ${ }^{3}$, Saurabh B. Jayant ${ }^{3}$, Deepak K. Mishra ${ }^{1}$, Mammen Chandy ${ }^{3}$, Mayur Parihar ${ }^{1}$

${ }^{1}$ Department of Cytogenetics and Lab Haematology, ${ }^{2}$ Department of Paediatric Haematology and Oncology, ${ }^{3}$ Department of Clinical Haematology, Tata Medical Center, Kolkata, India

Aims \& Objectives: Recent studies based on transcriptome profiling have identified numerous new fusions in B cell precursor acute lymphoblastic leukaemias (BCP ALLs). We describe a clinicopathological approach using immunophenotyping findings to identify ZNF384 fusions.

Patients/Materials \& Methods: Immunophenotypic analysis was done using bone marrow aspirate (BMA) samples on BD FACS CantoTM II flow cytometer as per our previous publication. Karyotyping and routine FISH analysis was performed on BMA samples as per standard cytogenetic protocols. A diagnosis of BCP ALL was made based on immunophenotypic features. Patient showing weak or negative CD10 and aberrant myeloid expression of CD13 and/or CD33 were subjected to FISH analysis using break apart probe for detection of ZNF384 gene rearrangement.

Results: Patient's details: Two of the patients ( 1 pediatric: 5 years $\&$ 1 adult: 29 years) with complaints of intermittent fever, joint swelling and mild cough were referred to our institute. Baseline laboratory investigations and bone marrow aspirate examination were suggestive of acute leukemia. Immunophenotype was consistent with Pro-B Cell Acute Lymphoblastic Leukemia in both the cases with aberrant CD33 expression in the former and CD13 and CD33 positivity in the latter patient. In view of flow cytometric findings, interphase FISH analysis to detect ZNF384 gene rearrangement was performed, which was positive in $75 \%$ and $80 \%$ cells respectively. FISH findings were further confirmed by conventional karyotyping which revealed a translocation between the short arm of chromosome 12 and the long arm of chromosome 17 resulting in a TAF15-ZNF384 fusion. Triple FISH analysis including KMT2A gene rearrangement was negative in both cases. 
Discussion \& Conclusion: ZNF384 rearranged BCP ALL is a rare and novel entity associated with weak or negative expression of CD10 and aberrant myeloid expression of CD13 \& CD33. We present two cases of ZNF384 gene rearrangements identified using immunophenotyping based FISH strategy. ZNF384 rearranged ALL has been reported to be associated with a relatively favorable prognosis in pediatric BCP-ALLs. Testing for ZNF384 fusions is recommended in Pro B cell ALLs with aberrant myeloid antigen expression after excluding KMT2A gene rearrangements.

\section{Blastic Plasmacytoid Dendritic Cell Neoplasm Presenting as Leukemia Without Skin Involvement: Role of Flow Cytometry in Diagnosis of a Rare Entity with Unusual Presentation}

Rakesh B. Demde ${ }^{* 1}$, Deepak K. Mishra ${ }^{1}$, Mayur Parihar ${ }^{1}$, Jeevan Kumar $^{2}$, Mammen Chandy ${ }^{2}$, Vivek Radhakrishnan ${ }^{2}$, Saurabh Bhave ${ }^{2}$, Neeraj Arora $^{3}$

${ }^{1}$ Laboratory Hematology, ${ }^{2}$ Clinical Hematology, TATA MEDICAL CENTER, kOLKATA, ${ }^{3}$ Molecular Genetics, Unipath Speciality Laboratory Ltd., Ahmedabad, India

Aims \& Objectives: To emphasize the role of immunophenotyping by flow cytometry in the diagnosis of Blastic Plasmacytoid Dendritic Cell Neoplasm (BPDCN) in a patient with no cutaneous lesion(s).

Patients/Materials \& Methods: A 22-year old female presented with complaints of fever and bilateral cervical swelling of 15 days duration. On extensive clinical evaluation there were no skin lesions documented.

Results: On peripheral blood smear evaluation there were $70 \%$ blasts, which were 1-2 times the size of small lymphocyte, the cytoplasm was abundant, pale and the nuclei showed open chromatin with 1-2 prominent nucleoli. Bone marrow aspirate showed $87 \%$ blasts. Trephine biopsy revealed an increase in CD4-positive, CD56-positive and MPO-negative, CD34-negative immature precursors. Flow cytometric analysis done on bone marrow aspirate revealed $74 \%$ blasts expressing CD45(dim), CD123(mod), HLA- DR(bright), CD4(mod), CD56(dim), CD7(mod) and CD117(dim). CD34, CD13, CD33, CD38, CD19, CD10, CD64, CD11b, CD11c, TDT, cCD3, cMPO, CD41, CD61 and CCD71 were negative in these blasts. The diagnosis of Blastic Plasmacytoid Dendritic Cell Neoplasm (BPDCN) was made on flow cytometry. Cytogenetics showed modal karyotype 46, $[X X]$. Molecular testing done for NPM1, FLT3-ITD/TKD and CEBPA gene mutations were negative. High-risk ALL treatment protocol was initiated, followed by Haplo-identical (sister) allogenic stem cell transplant. Post SCT patient was in morphological remission. There was a disease relapse 14 months after stem cell transplantation, in which the blasts showed the same immunophenotype as at diagnosis. The patient succumbed to the disease soon after the relapse.

Discussion \& Conclusion: Primary leukemic involvement of BPDCN without cutaneous involvement is a rare occurrence. Immunophenotyping by flow cytometry plays a vital role in the diagnosis of BPDCN, especially in a case like ours where there were no documented skin lesions.
Expression of B Lymphocyte Antigen in Pediatric B-Cell Precursor Acute Lymphoblastic Leukemia: A Marker to Sub-Stratify Standard Risk Patients?

Nivedita Pathak ${ }^{* 1}$, Rachna Seth ${ }^{1}$, Akhilesh Mishra ${ }^{2}$

${ }^{1}$ Pediatrics, ${ }^{\mathbf{2}}$ Radiation Oncology, All India Institute of Medical Sciences, New Delhi, India

Aims \& Objectives: To investigate and correlate the prognostic outcome with respect to surface protein expression of B cell antigenCD 20 on leukemic blasts of BCP-ALL patients.

Patients/Materials \& Methods: Immunophenotyping of blast cells at diagnosis was done by multiparametric flow cytometry. Expression of antigens on leukemic cells was determined by using a 8-dimensional space formed by 2 light scatter parameters (forward scatter [FSC] and side scatter [SSC]) and 6 fluorescence-associated characteristics. The existence of blast cell population was established on the basis of abnormal antigen expression profiles of the blasts as compared to the control.

Results: A total of 85 pediatric patients (median age 9 yrs, range 1-14 yrs; M: F 4:1; median TLC-11.4x109/1, range 0.9-810x109/1) were studied. CD20 positivity was defined as more than $20 \%$ of leukemia blasts expressing surface CD20. Expression of CD20 was present in $52 / 85(61 \%)$ patients with B ALL. A worse outcome has been observed in our patients expressing CD20 than those without the expression. Disease free survival at 36 months in CD20-positive and CD20-negative groups (37\% [95\% CI, 09-61] versus $82 \%$ [95\% CI, $45-86], p=0.004)$ was statistically significant. Overall survival at 26 months (45\% [95\% CI, 21-57] versus 61\% [95\% CI, 38-71], $p=0.03$ ) was also poorer in CD20-positive group than CD20-negative group.

Discussion \& Conclusion: Compared to the previous studies reported, in the pediatric B ALL patients here the expression of CD20 antigen of the leukemic blast cells is found to be higher. To correlate its prognostic value in the Indian scenario it warrants further studies to meaningfully sub-stratify the standard risk patients.

\section{Leukemia Cutis-A Case of Cutaneous Manifestation of Acute Monoblastic Leukemia}

Rhituparna Das ${ }^{* 1}$, Ankita P. Mandal ${ }^{1}$, Sriranjan Mukherjee ${ }^{1}$, Moumita Sengupta ${ }^{1}$, Keya Basu ${ }^{1}$, Uttara Chatterjee ${ }^{1}$

${ }^{1}$ Pathology, IPGME\&R, Kolkata, India

Aims \& Objectives: The case report highlights the skin lesion as an initial manifestation of Acute Leukemia.

Patients/Materials \& Methods: It includes evaluation of skin biopsy along with consecutive examination of peripheral blood smear and bone marrow aspiration and biopsy.

Results: A skin punch biopsy from one of the lesions on the forearm was performed showed angiocentric infiltrate of monotonous large cells in upper dermis. The overall features are suggestive of leukemia cutis. Immunohistochemistry for CD45 and CD68 reveal positive expression. Bone marrow analysis showed increase in myeloid and monocyte precursors with presence dysplastic features in $>10 \%$ myeloid precursors.Blasts $(21 \%)$ were identified. The overall features were suggestive of Acute Myeloid leukaemia morphologically AML -M5.Bone marrow biopsy revealed hypercellular marrow predominantly comprising of myeloid and monocyte cells. Possibility of blastic transformation of myeoloproliferative disorder/myelodysplastic syndrome (MPN/MDS).

Discussion \& Conclusion: Leukemia cutis (LC) is a comprehensive terminology for dermal manifestations of any type of leukemia either with accompanied or antecedent blood or bone marrow involvement. 
Although both myeloid and lymphoid neoplastic leukocytes can infiltrate skin, the frequency is higher among children with congenital myeloid leukemia. But the underlying pathogenesis of dermal tropism is not yet established. Clinical manifestation varies regarding appearance, site and numbers. Skin biopsy is essential for early establishment of the diagnosis and to guide for further testing and categorical management. Leukemia cutis should be considered as differential diagnosis in a case of widespread cutaneous lesion. Prompt histological diagnosis helps in early investigation for hematological malignancy and thus in categorical management.

\section{Blasts Showing Megakaryocytic Differentiation: Clinicopathological Profile from A Tertiary Care Centre}

Durga Devi ${ }^{* 1}$, Nabhajit Mallik ${ }^{1}$, Man Updesh S. Sachdeva ${ }^{1}$, Sreejesh Sreedharanunni ${ }^{1}$, Narender Kumar ${ }^{1}$, Shano Naseem ${ }^{1}$, Prashant Sharma ${ }^{1}$, Parveen Bose ${ }^{1}$, Jasmina Ahluwalia ${ }^{1}$, Reena Das ${ }^{1}$, Amita Trehan $^{2}$, Pankaj Malhotra ${ }^{3}$, Neelam Varma ${ }^{1}$

${ }^{1}$ Department of Hematology, ${ }^{2}$ Paediatric Hemato-Oncology unit of Department of Paediatrics, ${ }^{3}$ Adult Clinical Hematology unit of Department of Internal Medicine, Post Graduate Institute of Medical Education and Research, Chandigarh, India

Aims \& Objectives: Blasts showing evidence of differentiation towards the megakaryocytic lineage are noted in a variety of hematological conditions, albeit uncommonly. The current study aims to evaluate the clinico-pathological features of patients whose disease is characterized by an increase in blasts showing megakaryocytic differentiation.

Patients/Materials \& Methods: Retrospective analysis was performed by scrutinizing the archives of the Department of Hematology, PGIMER, Chandigarh over a period of two year and 10 months (January 2018 to October 2020) and noting the clinical and laboratory findings of cases having increased blasts with megakaryocytic differentiation.

Results: 20 cases were diagnosed during the period, with a median age of 24 years (range: 2 weeks-53 years) and male to female ratio of $1.1: 1$. The median $\mathrm{Hb}$, TLC and platelet counts were $7.9 \mathrm{~g} / \mathrm{dL}$ (range: 4.4-14.3-14.1 g/dL), 26.3×109/L (range: 1.1- 203.4x109/L) and 91x109/L (range: $15-974 \times 109 / \mathrm{L}$ ) respectively. The predominant clinical presentation was fever followed by easy fatiguability, bleeding manifestations and abdominal pain. Hepatosplenomegaly was seen in $25 \%$ cases and splenomegaly in $20 \%$. The median blast counts in peripheral blood and bone marrow were 25 (7-86) and 37 (12-82) respectively. Blast morphology revealed cytoplasmic blebs in $25 \%$ cases and cytoplasmic vacuolations in $20 \%$ cases. Marrow fibrosis (grade $2+$ to $3+$ ) was observed in $9 / 13(69 \%)$ cases. On flowcytometry, the blasts showed myeloid phenotype in $100 \%$ cases along with positivity for CD61 in 20 cases (100\%), CD41 in 17 cases (85\%) and CD42 in 6/6 (100\%) cases. The final diagnosis was AML with megakaryocytic differentiation in 9 cases $(45 \%)$ and CML blast crisis in 6 cases (30\%). Other diagnoses included essential thrombocythemia-blast crisis (1 case), transient abnormal myelopoiesis (1 case) and MPN accelerated phase (1 case). Two interesting cases of AML with multi-lineage expression (including megakaryocytic, monocytic and erythroid) were also noted.

Discussion \& Conclusion: The two hematological disorders most commonly associated with blasts showing megakaryocytic differentiation are AML and CML- blast crisis. Majority of these cases show bone marrow fibrosis. It must be borne in mind that rarely in some AML cases, megakaryocytic differentiation in blasts may be a part of multi-lineage marker expression.
Prevalence of Alloimmunization and Autoimmunization in Thalassemic Patients on Transfusion Therapy

Chiranjit Gope $^{* 1}$, Zeeba Z.- Ur-Rab ${ }^{1}$

${ }^{1}$ Paediatrics, JNMCH, AMU, Aligarh, Aligarh, India

Aims \& Objectives: Lifelong blood transfusion remains the mainstay of treatment in thalassemic patients. However, development of antired blood cell antibodies remains a major problem in these patients. Our primary objective was to study the prevalence and identify the alloantibodies and autoantibodies in thalassemic patients on transfusion therapy and secondary objective was to analyse the possible risk factors for alloimmunization and autoimmunization in these patients. Patients/Materials \& Methods: A hospital based observational, cross-sectional study was conducted among 80 registered thalassemia patients on blood transfusion in Paediatric Haematology clinic in collaboration with Blood and Components Bank. All clinical and transfusion records of patients were examined.Alloantibody screening and identification was done using indirect coombs test (ICT) 3 cell panel and 14 cell panel respectively (Immucor Inc,Germany). Autoantibody was detected by auto control testing (BioRad,Switzerland) and direct coombs test (DCT). Data were analysed by IBM SPSS Statistics 25 .

Results: $3.75 \%$ patients $(n=3)$ were alloantibody positive and $6.25 \%$ $(n=5)$ were autoantibody positive. Two patients were positive for both. Out of total 4 alloantibodies, $75 \%$ belong to Rh group and $25 \%$ belong to Kidd blood group. Alloimmunization was statistically more significant among female patients $(p=0.005)$. Mean total number of blood transfusions received was significantly lesser in autoantibody positive patients compared to autoantibody negative patients $(13.2 \pm 5.2$ vs. $58.3 \pm 52.4 ; p=0.000)$.

Discussion \& Conclusion: All patients on transfusion should be screened regularly for antibodies. Female patients are at increased risk for alloimmunization. Antibody positive patients should get blood matched for Rh, Kell and Kidd group.

\section{HB D-Punjab: A Single Centre Largest Experience on HPLC from Punjab}

Vikram Narang ${ }^{1}$, Anshul Jain ${ }^{* 2}$, Bhavna Garg ${ }^{2}$, Neena Sood ${ }^{2}$

${ }^{1}$ Department of Pathology, ${ }^{2}$ Pathology, DMCH, Ludhiana, India

Aims \& Objectives: To analyze the clinical, hematological and chromatographic parameters of patients with HbDPunjab syndromes. Patients/Materials \& Methods: A total of 3139 blood samples (both pediatric and adult) were screened for hemoglobinopathies and structural hemoglobin variants by using Bio-Rad D10 variant for evaluation of anemia and for family and antenatal screening studies. The clinical, hematological, and chromatographic parameters of individual HbDPunjab syndromes comprising of heterozygous $(\mathrm{HbA} /$ D) and compound heterozygous states $\beta$-thalassemia trait $(\mathrm{HbD} / \beta)$ were correlated and analyzed.

Results: A total of 405 cases of structural hemoglobin variants comprising of $\beta$ thalassemia, HbS, HbE, HbD-Punjab, HbD Iran, HbQ India and $\mathrm{HbJ}-$ Meerut were detected. HbDP syndromes constituted 42 cases $(10.3 \%)$ of all hemoglobin variants and $1.34 \%$ of all the samples screened for hemoglobinopathies. Heterozygous HbDP constituted 38 of $42(90.5 \%)$ cases and Compound heterozygous for HbD Punjab and beta thalassemia constituted 04 of 42 cases $(9.5 \%)$. HbD was detected among both young and old individuals with an age range of $1-54$ years. Clinically significant pallor was noted in $\mathrm{HbA} / \mathrm{D}$ and $\mathrm{HbD} / \beta$ groups. Icterus was present in one case both in $\mathrm{HbA} / \mathrm{D}$ and $\mathrm{HbD} / \beta$. Splenomegaly was seen in two of four $\mathrm{HbD} / \beta$ patients. Three cases of $\mathrm{HbA} / \mathrm{D}$ and one case of $\mathrm{HbD} / \beta$ required blood transfusion. 
Discussion \& Conclusion: $\mathrm{HbDP}$ is not an uncommon hemoglobinopathy in India. Heterozygous state is a clinically asymptomatic state. CE-HPLC has the advantage of rapid detection and accurate quantitation.

\section{Early Assessment of Liver Fibrosis in Patients Aged 6-18 Years with Beta Thalassemia Major}

Himani Aggarwal ${ }^{* 1}$, Kapil Bhalla ${ }^{1}$

${ }^{1}$ Pediatrics, Pandit Bhagwat Dayal Sharma, University of Health Sceinces, Rohtak, Rohtak, India

Aims \& Objectives: AIM:- To assess early markers of liver fibrosis in patients aged 6-18 years with beta- Thalessemia major OBJECTIVES :- 1 . To determine liver stiffness using transient elastography in patients with beta -Thalessemia major. 2. To measure APRI and FIB-4 indices.

Patients/Materials \& Methods: A prospective observational study was carried out where 35 patients with beta-Thalessemia major were screened for enrollment into the study over a span of six months.After taking a thorough history emphasising on demographic characterstics,disease duration,frequency of transfusion,iron chelation including type,dose,duration and compliance,each participant underwent routine biochemical investigations like complete hemogram,viral markers, liver function tests and ferritin levels.Fibrosis scores (APRI and FIB-4) were calculated.Transient elastography was performed to assess the severity of fibrosis,and patients were classified as stage F1 to F4, according to specific cut off values.

Results: Out of 35 enrolled, $n=9(25 \%)$ showed evidence of liver dysfunction with $\mathrm{n}=3(8.5 \%)$ showing moderate fibrosis (F2) on fibroscan.It also positively correlated with APRI and FIB-4 indices $(p<0.005)$.

Discussion \& Conclusion: This study thus combines biochemical and imaging tools so as to provide a robust background for prior and nearly accurate assessment of liver fibrosis in beta-Thalassemia patients.As the chance of maximum reversibility lies in timely recognition,this research could help in a sublime way in a resource limited nation such as ours.

\section{Hemolysis and Thrombosis in an Infertile Male}

Sherin S. John ${ }^{1}$, Aboobacker M. Rafi ${ }^{* 2}$, Deepak Charles ${ }^{3}$, Susheela J. Innah ${ }^{1}$

${ }^{1}$ Immunohaematology and Transfusion Medicine, Jubilee Mission Medical Hospital, ${ }^{2}$ Immunohaematology and Transfusion Medicine, Jubilee Medical Mission Hospital, ${ }^{3}$ Haematology, Aster Medicity, Thrissur, India

Aims \& Objectives: A case of an infertile male with haemolysis and thrombosis.

Patients/Materials \& Methods: 30-year-old male, who has been married for 3 years being evaluated for infertility presented to us with left lower limb pain and swelling. He gives a history of nausea and vomiting for 2 days. Evaluation revealed that his $\mathrm{Hb}$ was $5.1 \mathrm{gm} / \mathrm{dl}$ with a raised ESR and coombs test positivity. Further evaluation confirmed the presence of ANA, APLA and anticardiolipin antibody. Doppler evaluation confirmed the DVT of the leg.USG abdomen and pelvis along with Karyotyping confirmed the cause of infertility as Klinefelter's syndrome.

Results: He was started on Methyl Prednisolone and anticoagulants, along with Iron, vit B12 and folic acid supplementation and his symptoms started to improve. ANA profile and repeat testing of
APLA after 3 months was advised. He was also referred to an endocrinologist for further management of Klinefelter's syndrome.

Discussion \& Conclusion: Discussion: Patients with Klinefelter's syndrome (47, XXY) show a higher percent of autoimmune disorders like SLE and other skin related autoimmune diseases than the normal population. Sometimes, SLE may be the only presenting symptom of Klinefelter's syndrome and may lead to diagnosis of the syndrome. Systemic lupus erythematosus (SLE) is a potentially fatal multiorgan inflammatory and patients with SLE have an increased risk for premature thrombosis and/or atherosclerosis, with up to half experiencing a thrombotic event.

Conclusions: The frequency of Klinefelter's syndrome (47,XXY), often subclinical, is increased in men with SLE by approximately 14fold compared with its prevalence in men without SLE. Diagnostic vigilance for $47, \mathrm{XXY}$ in male patients with SLE is warranted. The risk of SLE in men with Klinefelter's syndrome is predicted to be consistent with the notion that SLE susceptibility is partly explained by an $\mathrm{X}$ chromosome gene-dose effect.

\section{Efficacy of Solubility Test In Screening of Sickle Cell Diseases: A Tertiary Care Centre Based Study}

Kinjal P. Chandpara*1 ${ }^{*}$, Mandakini M. Patel ${ }^{1}$, Ajay Tilala ${ }^{1}$, Ruchita Sanghani ${ }^{1}$

${ }^{1}$ Department of Pathology, Government Medical College, Surat, India

Aims \& Objectives: To find out specificity and sensitivity of solubility test in screening of sickle cell disease at tertiary care center, Surat.

Patients/Materials \& Methods: A cross-sectional observational study was carried out at tertiary care centre, Surat. A total of 983 samples were studied over a period of 1 year. All the samples were subjected to solubility test and were further analysed on HPLC BIORAD analyzer for confirmation along with the distinction of Sickle cell trait and Sickle cell disease.

Results: Out of 983 samples screened, 684 samples were found to be positive with the solubility test and 299 samples were solubility negative. Out of 684 solubility positive samples, 481 were diagnosed as sickle cell trait (heterozygous), 200 were diagnosed as Sickle Cell Disease (homozygous), 1 was diagnosed as Sickle cell- HbD disease and 2 were normal on HPLC. Out of 299 solubility negative samples, none showed Sickle cell disease or trait or any other sickle cell variants. on HPLC. We calculated sensitivity of $100 \%$. Specificity was $99.3 \%$, positive predictive value was $99.7 \%$ and negative predictive value was $100 \%$.

Discussion \& Conclusion: Solubility test is very effective as screening test due to high specificity and sensitivity. Though solubility test is very cheap, it cannot differentiate between Sickle Cell Trait and Sickle Cell Disease hence, confirmation is to be done by electrophoresis or HPLC.

\section{Utility of Peripheral Smear Examination in Anemia Evaluation: A One Year Study}

Sindhu $\mathrm{C}^{* 1}$

${ }^{1}$ Consultant Pathologist, Vivekananda Memorial hospital Laboratory, Vivekananda Memorial Hospital, Mysuru, India

Aims \& Objectives: (i) To evaluate anemia cases through peripheral smear examination (ii) To compare cell counter generated hematological parameters with peripheral smear findings. 
Patients/Materials \& Methods: This is a one year study carried from September 1st, 2019 to August 31st, 2020 in the VMH laboratory; Vivekananda Memorial Hospital, Saragur where anemia cases were evaluated with peripheral smear and compared with the cell counter generated hematological parameters.

Results: A total of 90 cases documented. Among 90 cases, 64 were females and 26 were males. The most common morphological type of anemia was Microcytic hypochromic anemia followed by Dimorphic anemia. 3 cases of hemolytic anemia also noted in peripheral smear but on electrophoresis around 6 cases were confirmed.1 case each of Acute Leukemia and Chronic Myeloid Leukemia were also documented.

Discussion \& Conclusion: Peripheral smear examination is an important diagnostic tool for evaluation of anemia. The coexistence of micronutrient deficiency and inherited anemias makes diagnosis difficult which requires higher investigations like HPLC/Hemoglobin electrophoresis.

Supporting Document:

\section{Table showing Morphological types of Anemia:}

\begin{tabular}{|l|l|}
\hline Morphological types & $\begin{array}{l}\text { No of cases(Total - } \\
90 \text { cases) }\end{array}$ \\
\hline Microcytic hypochromic anemia & 38 \\
\hline Dimorphic anemia & 30 \\
\hline Pancytopenia & 7 \\
\hline Normocytic hypochromic anemia & 5 \\
\hline Normocytic normochromic anemia & 5 \\
\hline Hemolytic anemia & 3 \\
\hline $\begin{array}{l}\text { Others: i) Acute Leukemia with } \\
\text { Normocytic hypochromic blood } \\
\text { picture } \\
\text { ii)CML with Normocytic } \\
\text { normochromic anemia }\end{array}$ & 1 \\
\hline
\end{tabular}

\section{An Overlap of Paroxysmal Nocturnal Hemoglobinuria(PNH) with Aplastic Anemia}

Ashis Gupta ${ }^{* 1}$, Kailash Kumar ${ }^{1}$, Nilesh Kumar ${ }^{1}$, Prachi Mohapatra ${ }^{1}$, Nidhi Singh ${ }^{1}$, Qausain Akhtar ${ }^{1}$

${ }^{1}$ Department of General Medicine, Institute of Medical Sciences, BHU, Varanasi, India

Aims \& Objectives: Paroxysmal Nocturnal Hemoglobinuria (PNH) is an acquired hemolytic anemia characterized by non-malignant clonal expansion of the stem cells of the bone marrow. PNH has heterogeneous presentation and is classified accordingly: 1) Classical PNH; 2) $\mathrm{PNH}$ in context of bone marrow failure; 3)Sub-clinical $\mathrm{PNH}$. Aiming to categorize patients into these 3 groups provides the basis for individualizing management.

Patients/Materials \& Methods: 23 years-old male presented with complaints of generalized weakness and easy fatigability from one month and blurring of vision in the right eye from 3 days. On examination, vitals were stable, pallor was present, and systemic examination revealed no abnormality. Fundus examination showed multiple flame shaped hemorrhages at the temporal side of the disc in the right eye. Laboratory investigations found pancytopenia with $\mathrm{Hb}$ $3.6 \mathrm{~g} / \mathrm{dL}(\mathrm{MCV}-120 \mathrm{fL}), \mathrm{TLC}-1,700 / \mathrm{mm} 3(\mathrm{~N} / \mathrm{L}=27 / 64)$ and a manual platelet count of $16,000 / \mathrm{mm} 3$. The corrected reticulocyte count was 0.75 and serum LDH-288 U/L. Other investigations were within normal limits. Bone marrow (BM) aspiration showed hyper-cellular marrow with megaloblastic hyperplasia, which was suggestive of Megaloblastic anemia. BM biopsy revealed hypo-cellular marrow spaces and occasional area of hot-spots with preserved hemopoiesis. On suspicion of PNH, samples for FLAER based flow cytometry was sent, which was negative for CD-55 and CD-59 on 5.9\% of neutrophils and $9.8 \%$ of monocytes. Therefore, on basis of bone marrow biopsy and immunophenotyping a final diagnosis of PNH with Aplastic anemia was made.

Results: With adequate transfusion support patient was given oral cyclosporine (100 mg/day), danazol (2 mg TID) and hematinics. Eculizumab was not advised due to the lesser number of PNH clones in our patient and no evidence of hemolysis. Patient's response to therapy was good with hemogram showing $\mathrm{Hb}-9.3 \mathrm{~g} / \mathrm{dl}$, TLC- 6,700/ $\mathrm{mm} 3$ and platelets count -1.0 lakh (10 months after therapy) and transfusion independent ( 5 months after initiation of therapy).

Discussion \& Conclusion: $\mathrm{PNH}$ is a rare disease with an estimated prevalence of approximately 5/million. BM failure complicates management of $\mathrm{PNH}$, because compromised erythropoiesis contributes a certain degree of anemia, in addition to complement mediated intravascular hemolysis. Immuno-suppressants salvage the patient but allogenic hematopoietic stem cell transplant remains the mainstay of treatment.

Supporting Document:

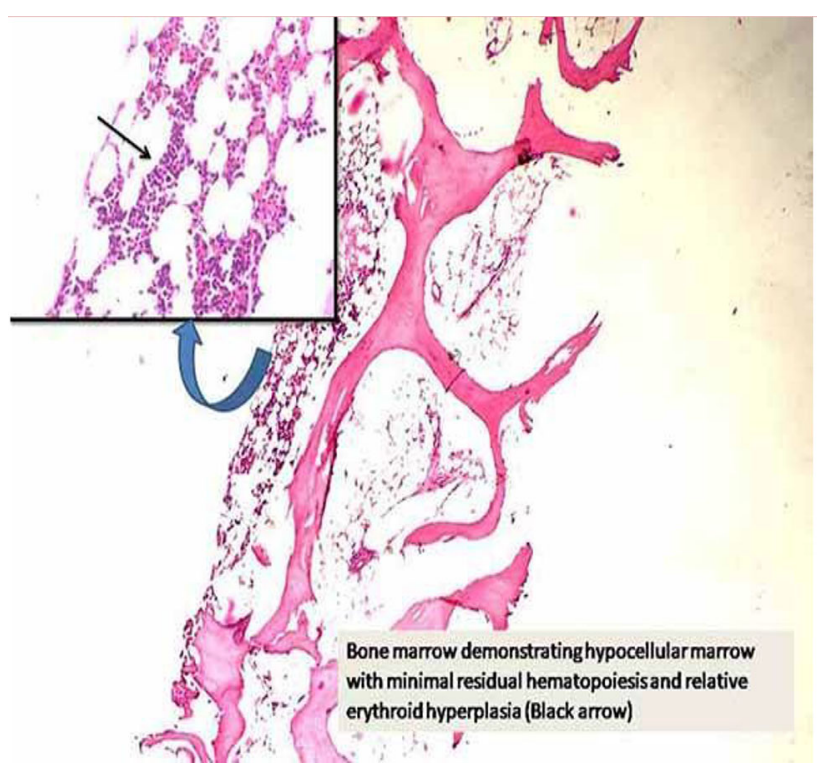

\section{Determination of Efficacy and Reliability of Various Discrimination Indices in Screening BTT}

Saima Khan ${ }^{* 1}$, Sayeedul H. Arif ${ }^{2}$

${ }^{1}$ Pathology, JNMCH, ${ }^{2}$ Pathology, JNMC, Aligarh, India

Aims \& Objectives: To find out the best discrimination index to screen out thalassemia trait cases so they can be subjected to Alkaline hemoglobin electrophoresis for confirmation to reduce the diagnostic cost and disease burden on the society. 
Patients/Materials \& Methods: 1600 cases of microcytic hypochromic anemia were selected. CBC and GBP were done and seven discrimination indices calculated then subjected to Iron studies/Alkaline hemoglobin electrophoresis accordingly.

Results: Mentzer index and Shine and Lal index had revealed better discriminative function compared to England and Fraser index, Srivastava index and Green and King Index.

Discussion \& Conclusion: In developing countries with limited facility for electrophoresis, poor health care resources and financial constraints, a practical approach should be established in screening Beta thalassemia trait cases based on discrimination indices. Hence, as observed Mentzer index and Shine and Lal index can be safely relied on for cost effective mass screening of microcytic hypochromic anemia.

Supporting Document:

TABLE V: Sensitivity, Specificity, Positive Predictive Value (PPV), Negative Predictive Value (NPV) And Youdens Index of Each Discrimination Index.

\begin{tabular}{|c|c|c|c|c|c|c}
\hline $\begin{array}{c}\text { S. } \\
n \\
0\end{array}$ & INDICES & SENSITIITY & SPECIFICITY & PPV & NPV & $\begin{array}{c}\text { YOUDENS } \\
\text { INDEX }\end{array}$ \\
\hline 1 & Mentzer & $90.3 \%$ & $93.4 \%$ & $77.7 \%$ & $97.4 \%$ & 83.7 \\
\hline 2 & England \& Fraser & $41.9 \%$ & $69.6 \%$ & $26.0 \%$ & $82.5 \%$ & 11.5 \\
\hline 3 & Srivastava & $77.4 \%$ & $73.7 \%$ & $42.8 \%$ & $92.7 \%$ & 51.5 \\
\hline 4 & Shine and tal & $74.1 \%$ & $96.7 \%$ & $85.1 \%$ & $93.6 \%$ & 70.8 \\
\hline 5 & RDWi & $83.8 \%$ & $73.7 \%$ & $44.8 \%$ & $94.7 \%$ & 57.5 \\
\hline 6 & Ricerca & $58.0 \%$ & $80.3 \%$ & $42.8 \%$ & $88.2 \%$ & 38.3 \\
\hline 7 & Green and King & $45.1 \%$ & $65.5 \%$ & $46.4 \%$ & $82.4 \%$ & 10.6 \\
\hline
\end{tabular}

\section{Methaemoglobinemia and G6PD in a Patient for Major} Surgery: Diagnostic Dilemma \& Therapeutic Enigmaa

Arun V. J ${ }^{* 1}$, Aboobacker M. Rafi ${ }^{1}$, Deepak Charles ${ }^{2}$, Susheela J. Innah $^{1}$

${ }^{1}$ Transfusion Medicine, JMMCRI, Thrissur, ${ }^{2}$ Haemato-Oncology, Aster Medicity, Kochi, India

Aims \& Objectives: Management of a patient with two rare mutations.

Patients/Materials \& Methods: 27-year old female presented with a history of swelling on the right side of the neck for 2 years with recurrent pain which made her approach our hospital for surgery. She was referred to haematology for fitness as the anaesthetist detected a saturation gap. A detailed evaluation revealed a history of dark brown coloured blood on cuts, giddiness and easy fatigability. On examination, she had cyanosis in the oral cavity and tongue. Considering all the positive findings including saturation gap, a decision was taken to evaluate for methaemoglobinemia. The radiological evaluation confirmed the diagnosis of a branchial cyst.Workup revealed Methaemoglobin levels of $68.47 \%(<2 \%)$ and NADH cytochrome B5 reductase $10.82(30-40 \mathrm{IUg} / \mathrm{Hb})$. Genetic analysis revealed a novel mutation (R192C) in CYBR3 gene (Arg192Cys) which is associated with an autosomal recessive congenital methaemoglobinemia type 1 . Vitamin C and Niacin were started \& samples were sent to analyse for
G6PD deficiency as the plan was to start methylene-blue during surgery. TheG6PD genetic study was suggestive of the G6PD KeralaKalyan pathogenic heterozygous variant ( 949 G- > A; 24.5\%) which deferred the use of methylene-blue. A multidisciplinary team decided to avoid methylene blue \& dehydration, along with monitoring for acidosis, hypoxia, sensorium \& renal function intra-operatively \& plan for hyperbaric oxygen therapy and exchange transfusion, if needed.

Results: She was reassessed after 1 month on being Vitamin-C and Niacin with a pulse-oximetry evaluation which showed a drastic improvement. She was prehydrated. Anaesthetic agents which could induce haemolysis were avoided. Propofol \& atracurium were used for induction \& muscle relaxation respectively. Maintenance of anaesthesia was achieved using oxygen, air, sevoflurane with a target saturation of $93 \%$. The surgery and post-operative period were uneventful. She was discharged on postoperative day 4 with the lab parameters within an acceptable range.

Discussion \& Conclusion: Even though the combined deficiency is rare, care should be taken to evaluate for G6PD deficiency in methaemoglobinemia, before initiating methylene blue as it may lead to life-threatening haemolysis. A multidisciplinary evaluation and management is necessary to avoid adverse outcome and thereby improve patient satisfaction.

\section{Role of Erythrocyte Eluate Technique in the Diagnosis of Direct Antiglobulin Test Negative Hemolytic Disease of Newborn}

Aneet Singh $^{* 1}$, Rajath Athreya ${ }^{2}$, Ankit Mathur ${ }^{3}$, Madhavi Babu ${ }^{4}$, Shantanu $S^{5}$

${ }^{1}$ Department of Pathology, ${ }^{2}$ Neonatology, Rainbow Children's Hospital, ${ }^{3}$ Blood Bank and Immunology, Rotary Club's TTK Blood bank, ${ }^{4}$ Departhemnt of Pathology, Rainbow Childrens Hospital, ${ }^{5}$ TTK Blood Bank, Bangalore, India

Aims \& Objectives: DAT (Direct Antiglobulin/coombs Test) has been the first line investigation for the diagnosis of hemolytic disease of newborn caused by erythrocyte immunization for decades. However, we observed DAT to be negative in some of the newborns with high clinical suspicion of hemolysis. We aimed to supplement the DAT with antibody elution to prove the hemolysis in newborns with ABO incompatibility.

Patients/Materials \& Methods: 20 patient samples with clinical suspicion of hemolysis were processed simultaneously for DAT and elution. Other parameters like Hemoglobin, bilirubin, reticulocyte count, peripheral smear and blood groups were compared.

Results: Out of 20 patients with clinical suspicion of hemolysis, 16 patients had a positive DAT and antibody identification on elution. 4 neonates had a Negative DAT with antibody identification on elution. The Positive predictive value of DAT was $100 \%$, however sensitivity was $80 \%$. The sensitivity of the DAT with Elution combined was $100 \%$ in the diagnosis of hemolytic disease of newborn. Anti B IgG was the most common antibody identified with elution which was missed by routine DAT.

Discussion \& Conclusion: DAT is requested in all ABO incompatible pregnancies which reduces the specificity of the test. For optimal use DAT should be requested only with a clinical suspicion of jaundice in newborns with Feto-maternal ABO incompatibility. A negative DAT does not rule out Hemolysis. Prozone phenomenon is one explanation for DAT to be negative when antibody titer is high. $\mathrm{IgG}$ antibody against rhesus antigen is detected by DAT, however IgG against $\mathrm{B}$ or $\mathrm{A}$ antigen is missed in $\mathrm{ABO}$ incompatibility scenarios. We recommend the use of Erythrocyte antibody elution, as a 
supplement test for the diagnosis of Hemolytic disease of newborn when there is a high clinical suspicion.

\section{Hereditary Hemochromatosis: A Case Report \\ Ani Baghla ${ }^{* 1}$, Shashi bansal ${ }^{1}$, Amandeep Talwar ${ }^{1}$ \\ ${ }^{1}$ Pathology, BMCHRC, Jaipur, India}

Aims \& Objectives: Hemochromatosis is an inborn error of metabolism resulting from increased absorption of iron from the diet leading to iron overload and eventual fibrosis and organ failure (cirrhosis, cardiomyopathy, diabetes, hypogonadism). Hemochromatosis can be primary (hereditary), secondary (acquired) or due to other causes (like long term hemodialysis, chronic liver disease etc.). Here, we report a case of hereditary hemochromatosis.

Patients/Materials \& Methods: A 34 year male presented to the OPD with complaints of slate grey discoloration of skin for 1.5 years. CBC revealed $\mathrm{Hb}-12.3 \mathrm{gm} / \mathrm{dl}$, TLC- $8400 /$ cumm, platelet220000/cumm, Renal and liver functions were deranged (urea$68.50 \mathrm{mg} / \mathrm{dl}$, creatinine- $2.74 \mathrm{mg} / \mathrm{dl}$, Bilirubin total: $4.30 \mathrm{mg} / \mathrm{dl}$, Bilirubin direct: $3.37 \mathrm{mg} / \mathrm{dl}$, AST: $231.42 \mathrm{IU} / \mathrm{L}$, ALT: $234.36 \mathrm{IU} / \mathrm{L}$, ALP: $141.29 \mathrm{IU} / \mathrm{L})$. Hence, iron studies were advised in view of clinical suspicion of iron overload.

Results: Iron studies revealed erratic result with Serum iron-504 $\mu \mathrm{g} /$ $\mathrm{dl}(\uparrow)$, ferritin-446 ng/ml ( $\uparrow)$, TIBC-992.1 $\mu \mathrm{g} / \mathrm{dl}(\uparrow)$. Then repeat iron study with modification was done which reveal corrected TIBC$462 \mu \mathrm{g} / \mathrm{dl}(\uparrow)$ and \% saturation-109\% (supersaturation).

Discussion \& Conclusion: Hereditary hemochromatosis is an Autosomal recessive disorder. It is the most common single gene disorder affecting Hemochromatosis gene (HFE). 60-93\% of the cases are homozygous for the mutation $\mathrm{C} 282 \mathrm{Y}$ (a cysteine-to- tyrosine substitution). The patients with abnormal iron studies should be evaluated for hemochromatosis, even in the absence of symptoms. In a patient with suggestive symptoms, physical findings, or family history, a combination of transferrin saturation and ferritin should be obtained rather than relying on a single test, If either is abnormal (TS- $45 \%$ or ferritin above the upper limit of normal), then HFE mutation analysis should be performed.

\section{Alternate Day Iron Supplements in Iron Deficiency Anemia: A Randomized Control Trial}

Elamparithi Pasupathy ${ }^{* 1}$, Aneesh Basheer ${ }^{1}$, Siddhant Thampi ${ }^{1}$, Kurien Thomas ${ }^{1}$

${ }^{1}$ General Medicine, Pondicherry Institute of Medical sciences, Puducherry, India

Aims \& Objectives: To determine the efficacy of alternate-day oral Iron therapy (given as $60 \mathrm{mg}$ elemental Iron for 2 months) compared to daily oral Iron therapy (at the same dose) in improving haemoglobin among adults with Iron Deficiency Anemia.

Patients/Materials \& Methods: In this 8 week, randomized, parallel-arm, double-blind trial, we assigned 200 patients with iron deficiency anemia and a baseline haemoglobin of less than $10 \mathrm{~g} / \mathrm{dL}$ in 1:1 ratio to receive oral iron (elemental iron $60 \mathrm{mg}$ ) once daily or on alternate days. The primary outcome was change in haemoglobin concentration from baseline at week 8 . Secondary outcomes included changes in reticulocyte response and ferritin levels at week 2 and 4 respectively from baseline.

Results: 98 patients in daily group and 96 patients in alternate day group completed the 8 week follow up. Baseline characteristics of patients in the two arms were similar. The mean baseline $\mathrm{Hb}$ of daily group was $6.682 \mathrm{~g} / \mathrm{dl}( \pm 1.8918)$ and that of alternate group was
$6.536 \mathrm{~g} / \mathrm{dl}( \pm 1.8924)$. The mean $\mathrm{Hb}$ at week 8 was $8.018 \mathrm{~g} / \mathrm{dl}$ $( \pm 1.8020)$ in daily group compared to $7.594 \mathrm{~g} / \mathrm{dl}( \pm 1.5837)$ in the alternate group $(p=0.083)$. The change in mean $\mathrm{Hb}$ from baseline at week 8 was $+1.336 \mathrm{~g} / \mathrm{dL}$ in daily iron group and $+1.058 \mathrm{~g} / \mathrm{dL}$ in the alternate group. $(p=0.083)$. The increase in mean reticulocyte count from baseline at week 2 was $0.3626 \%$ in daily group and $0.3459 \%$ in the alternate group. $(p=0.365)$ The rise in mean ferritin levels from baseline at week 4 was $18.1178 \mathrm{ng} / \mathrm{ml}$ in daily iron group and $32.4782 \mathrm{ng} / \mathrm{ml}$ in the alternate group. $(p=0.8)$.

Discussion \& Conclusion: Preliminary data from recent studies suggest that daily administration of oral iron increases hepcidin levels in the duodenal mucosa thereby reducing iron absorption. Alternate day supplementation was associated with lower hepcidin levels. There are no published randomized controlled trials addressing this to the best of our knowledge. We therefore hypothesised that alternate day oral iron supplementation would be effective in treatment of iron deficiency anemia compared to daily supplementation. However, this study was unable to demonstrate any statistically significant difference between the two strategies. We conclude that among adults with iron deficiency anemia, alternate day iron supplementation is as effective as daily supplementation in improving $\mathrm{Hb}$ at 8 weeks. This needs to be confirmed in larger trials with longer follow up as alternate day therapy has the potential to improve compliance with lower incidence of side effects.

\section{To Establish the Correlation Between Anemic Patients in Diabetes: A Retrospective Study}

Avnish Gaur ${ }^{* 1}$, Priyanka Saxena ${ }^{2}$

${ }^{1}$ Medicine, ${ }^{2}$ Pathology, Index Medical College, Indore, India

Aims \& Objectives: Diabetes mellitus is a global metabolic disorder characterized by persistent hyperglycemia. Often, DM is accompanied by mild-to-moderate anemia, called as anemia of inflammation or infection or anemia of chronic disease.

Patients/Materials \& Methods: This observational study was conducted in patients with type 2 diabetes mellitus in a tertiary health centres in the month of September over 30 diabetic patients. The patients were divided into 2 groups according to $\mathrm{HbAlc}$ levels: group 1, HbA1c levels $<10 \%$ (controlled) and group $2 \mathrm{HbA} 1 \mathrm{c}$ levels $>10 \%$ (uncontrolled). RBC count, Hemoglobin level, HCT and MCV were determined.

Results: Out of 30 patients included, the mean value of RBC count, $\mathrm{Hb}$ level HCT and MCV is lower in the second group $(\mathrm{HbA} 1 \mathrm{c}<10 \%)$ as compared to first group $(\mathrm{HbAlc}>10 \%)$ indicating a positive correlation between $\mathrm{HbAlc}$ and $\mathrm{Hb}$ level.

Discussion \& Conclusion: The findings suggest that diabetic patients with poor control are more prone to develop anemia, indicating the need for routine CBC examination for early detection and management of anemia in diabetic patients in tertiary health care centers, moreover these patients also presented with leucocytosis suggesting an independent role in inflammation.

\section{Burden of Hemoglobinopathies in Malwa Region: A Retrospective Study}

Utkarsha Singh $^{* 1}$, Priyanka Saxena ${ }^{2}$

${ }^{1}$ Pathology, Index Medical College \& RC, ${ }^{2}$ Pathology, Index Medical College, Indore, India

Aims \& Objectives: To find the type of hemoglobinopathies in cases who presented with microcytic hypochromic anemia. 
Patients/Materials \& Methods: The study was conducted on 517 individuals attending the OPD of a tertiary care centre between June 2018 to March 2020 with microcytic hypochromic anemia on routine CBC \& peripheral smear. Sickling test was conducted and subsequently all these cases were subjected to Hemoglobin electrophoresis. Results: 178 out of the total of $517(34.4 \%)$ cases were found to have one or the other form of hemoglobinopathy. Out of 178 cases $34.3 \%$ cases were of Sickle cell anemia, 29.8\% cases of Beta thalassemia, $20.8 \%$ cases of sickle cell Trait and $15.2 \%$ cases of sickle-beta thalassemia. Rest i.e. 339 (65.6\%) were subjected to further investigations like iron studies and revealed a mix of Iron Deficiency Anemia (80.5\%) and anemia of chronic disease (19.5\%).

Discussion \& Conclusion: Sickle Cell disorders has wide spread presence in different tribes of Central India. To reduce the burden of hemoglobinopathies screening of all anemic patients should be done in all areas and in all communities where the socioeconomic condition indicate frequent occurrence of genetic mutations.

\section{HB Electrophoresis as a Diagnostic Tool for Detection of Sickle Cell Disease in Tertiary Care Centre}

\author{
Sakar Saxena ${ }^{* 1}$, Utkarsha Singh ${ }^{1}$, Priyanka Saxena ${ }^{1}$ \\ ${ }^{1}$ Pathology, Index Medical College, Indore, India
}

Aims \& Objectives: To determine the prevalence of undiagnosed cases of sickle cell disease in the society.

Patients/Materials \& Methods: OPD \& IPD patients from departments of internal medicine, surgery, Ortho and Pediatrics with moderate to severe anemia suspected for sickle cell disease, at Index Medical College and Hospital, Indore, from the month of June 2019 to March 2020 were included in study. Sickling test was conducted on them and Hemoglobin electrophoresis was performed to confirm for presence of Sickle cell disease.

Results: Out of 109 samples included in study, 58 (53.2\%) cases were positive on sickling test $\& 51(46.7 \%)$ showed sickle test negative. On $\mathrm{Hb}$ electrophoresis, 69(63.3\%) cases were positive for sickle cell disease.

Discussion \& Conclusion: Sickle Cell disorders has wide spread presence in different tribes of Central India. Individuals with sickle cell trait have shown to develop sickling which leads to organ damage when they are exposed to hypoxia, excessive exercise, high altitude, dehydration etc. Genetic counselling should be offered to all adoloscents at risk of transferring the sickle cell disease to offsprings.

\section{Comparative Study Between HPLC and Capillary Zone Electrophoresis to Diagnose Hemoglobinopathies}

Pallavi Kommoju*1, Ratnakar M. Potekar ${ }^{1}$

${ }^{1}$ Pathology, Shri B M Patil Medical College(DEEMED to be University), Vijayapura, India

Aims \& Objectives: To compare 2 different modalities i.e. cation exchange HPLC and hemoglobin electrophoresis (Capillary Zone Electrophoresis) to assess the characterization of hemoglobin profile in hemoglobinopathies.

Patients/Materials \& Methods: A prospective hospital based study was conducted between 1st December 2018 to 31st May 2020 in the Department of pathology (Central laboratory), BLDE (Deemed to be University) Shri B M Patil Medical College, Hospital and Research Centre, Vijayapura. A total of 48 adults and children blood samples were examined for routine screening for hemoglobinopathies using both the modalities, excluding those patients who had undergone blood transfusion within a span of 12 weeks. All samples were analysed using automated cell counter (Sysmex XN-1000), HPLC (Biorad D-10) and capillary zone electrophoresis (CZE).

Results: Out of 41 cases, 24 cases were of thalassemia trait, 9 cases were normal, 3 cases of $\beta$ - thalassemia major, 2 cases of homozygous/double heterozygous for $\beta$-thalassemia and $\delta \beta$-thalassemia, 1 case of sickle cell anemia, 1 case of Compound heterozygous sickle cell anemia and $\beta$-thalassemia and 1 case of Compound heterozygous for $\mathrm{HbE} / \beta$-thalassemia. When $\mathrm{HbF}$ value is $>16.5 \%$ it merges with LHbA1c values in HPLC (Bio-Rad D-10) because of which it is very difficult to comment about exact values of $\mathrm{HbF}$ with the help of HPLC. So for such cases CZE helped us to evaluate exact value of $\mathrm{HbF}$. Remaining 7 cases CZE was not done.

Discussion \& Conclusion: Forty-eight cases were collected in which both HPLC and $\mathrm{Hb}$ electrophoresis was done on 41 samples. Both the modalities gave same results for 34 cases and remaining 7 cases we got different impressions. Advantage of $\mathrm{Hb}$ electrophoresis over HPLC is that it gives exact values of HbF which is very essential to differentiate thalassemia intermedia from thalassemia major and also it exactly evaluates percentage of $\mathrm{HbE}$ which elutes with $\mathrm{HbA}$ in case of HPLC. CZE is complimentary to HPLC, which is very helpful for evaluating exact values of $\mathrm{HbF}, \mathrm{HbS}$ and $\mathrm{HbE}$.

\section{Correlation of Transient Elastography with MRIT2* Values in Patients with Thalassemia Major}

Nupur Parakh ${ }^{* 1}$, Jagdish Chandra ${ }^{2}$

${ }^{1}$ Depatment of Pediatrics, ${ }^{2}$ Department of Pediatrics, Kalawati Saran Childrens Hospital, Delhi, India

Aims \& Objectives: Currently, Magnetic Resonance Imaging (MRI $\mathrm{T}^{*}$ ) is considered as the gold standard investigation to assess Iron Overload (IO) in children with Transfusion Dependent Thalassemia (T.D.T.). But its cost and availability, limit its universal use, specially in peripheral areas. Recently Transient Elastography (T.E.) is establishing its place in assessing I.O. related liver fibrosis in various transfusion-dependent hereditary anemias such as $\beta$-thalassemia, Sickel cell disease and also in heredietary hemochromatosis, as liver iron is the surrogate marker of the entire body I.O. status. To add to literature available from India, we investigated the correlation of T.E. with MRI T2* values and also with serum ferritin levels in patients with TDT.

Patients/Materials \& Methods: We conducted a retrospective observational study of 59 patients (34 male; mean age, $12.98 \pm 3.11$ years). The study was performed at a tertiary hospital in India from January 2013 to December 2018 after ethical approval. Clinical and biochemical parameters of enrolled patients were entered in pre-designed proforma. The TE was performed within 3 month of MRI T2* analysis of the liver. The Spearman correlation test and linear regression analysis were used to evaluate the correlation between TE liver stiffness measurements and $\mathrm{R} 2{ }^{*}$ MRI results or serum ferritin levels.

Results: The subjects mean total serum levels of hemoglobin, total bilirubin, indirect bilirubin, alanine aminotransferase, aspartate aminotransferase, and ATR were $9.33 \pm 0.63 \mathrm{mg} / \mathrm{dl}, 1.22 \pm 0.83$, $0.79 \pm 0.87, \quad 57.34 \pm 52.12 \mathrm{IU} / \mathrm{L}, \quad 53.14 \pm 39.32 \mathrm{IU} / \mathrm{L}, \quad$ and $136.03 \pm 17.98$ respectively. Mean liver stiffness measurement, MRI $\mathrm{T}^{*}(3 \mathrm{~T})$, corresponding MRI R2* $(3 \mathrm{~T})$, and ferritin values were $6.55 \pm 3.10 \mathrm{kPa}, \quad 3.4 \pm 4.58 \mathrm{~ms}, \quad 616.20 \pm 383.89 \mathrm{~Hz}, \quad$ and $2874.69 \pm 1570.86 \mathrm{ng} / \mathrm{ml}$ respectively. IO based on MRI R2 ${ }^{*}$ values, was present in $93.22 \%$ patients, out of which, $13.56 \%$ had mild, $22.03 \%$ had moderate, and $57.63 \%$ patients had severe IO. TE measurements correlated with MRI R2 ${ }^{*}$ values $(r=0.615 ; p=0.000)$. Significant correlation was also found between TE values and serum level of ferritin $(\mathrm{r}=0.587, p=0.000)$. 
Discussion \& Conclusion: Results of TE correlated with serum ferritin and MRI R2 ${ }^{*}$ values in TDT patients. TE is cheaper and more readily available than MRI and might be used to estimate hepatic iron overload, in patients with T.D.T. who require chronic transfusion specially in developing countries.

\section{Cold Agglutinin Disease Associated with Digital Thrombosis}

Anish K. Saha ${ }^{* 1}$, Mukti P. Meher ${ }^{1}$, Deepak K. Gautam ${ }^{1}$, Lalit P. Meena ${ }^{1}$, Saumya Gupta ${ }^{1}$, Abhishek Pandey ${ }^{1}$

${ }^{1}$ Department of General Medicine, Institute of Medical Sciences, Banaras Hindu University, Varanasi, India

Aims \& Objectives: Cold agglutinin disease (CAD) is a variant of haemolytic anaemia characterised by presence of autoantibody which agglutinate red blood cells at a temperature lower than body temperature. We present a case of a patient who presented with blackish discolouration of digits. On evaluation haemolytic anaemia was present along with digital thrombosis. Later the diagnosis of CAD was made.

Patients/Materials \& Methods: 57 year male presented with blackish discoloration of upper and lower limbs progressing from distal to proximal for 5 days, preceded by colour changes in fingers on cold exposure for 3 days. On examination, pallor was present. Blackish discoloration of all 4 limb along with sharp line of demarcation was present. Limbs were cool on palpation. Systemic examination was uneventful. There was a peculiar event of blood clotting in EDTA vial just after drawing of sample. Investigation revealed striking abnormalities of Hb-8, TRBC -2.8 x106, MCV98 fl,MCH-207 pg, MCHC-214 g/dl,HCT-2.7, LDH-1389 U/L, cRC$6.91 \%$. General blood picture revealed RBC agglutinates. Coomb's direct and indirect test were positive. Urine routine microscopy revealed hemoglobinuria suggestive of intravascular hemolysis. ANA, RA factor, serum cryoglobulin, IgM antibody for mycoplasma pneumonia, monospot test were negative. Color Doppler lower limb showed near total thrombosis of short saphenous vein. 2D echocardiography was negative for intra-cardiac thrombus. Cold agglutinin test was positive. The final diagnosis of CAD was made.

Results: The patient was started on IV Methyl Prednisolone. Rituximab was planned but the patient did not comply to treatment and left against medical advise.

Discussion \& Conclusion: Cold agglutinin disease is a rare form of autoimmune hemolytic anemia caused by cold reacting autoantibody. In $90 \%$ of cases the autoantibody is IgM; but may involve IgG or IgA. IgM autoantibodies activate complement system leading to predominantly extravascular hemolysis with minimal intravascular hemolysis. Primary cold agglutinin disease is Associated with monoclonal cold reacting autoantibodies. Secondary cold agglutinin disease is associated with either monoclonal or polyclonal cold reacting autoantibody. Causes include bacterial or viral infection, autoimmune disease and cancers. Management includes keeping all body parts warm. Immunosuppressive therapy like Rituximab, chlorambucil, cyclophosphamide, steroids are used. Plasmapheresis has also been used to successfully.
Early Markers for Atherosclerotic Risk in Children with Beta Thalassemia Major

Ekta Sharma $^{* 1}$, Kapil Bhalla ${ }^{1}$

${ }^{1}$ Pediatrics, Pt. B.D Sharma PGIMS, ROHTAK, India

Aims \& Objectives: A case control study was performed to identify early markers for premature atherosclerosis in children with $\beta$ Thalassemia major.

Patients/Materials \& Methods: 60 children were compared with 60 gender and age matched controls and underwent detailed history regarding the duration of illness, frequency of transfusion, duration of chelation therapy and periods of hospitalization. They were subjected to complete hemogram, serum ferritin levels, lipid profile, liver and renal function tests, and serum Apelin levels using a commercially available sandwich enzyme linked immunosorbent assay kit. Doppler USG of the carotid arteries was done on all subjects blindly by an experienced radiologist and CIMT (Carotid Intima Media Thickness) was evaluated.

Results: The mean age group was comparable between both groups. Thalassemic children had lower levels of cholesterol $(121.25 \pm 22.42 \mathrm{mg} / \mathrm{dl} \mathrm{Vs} 156.02 \pm 19.66 \mathrm{mg} / \mathrm{dl}$ in controls), HDL $(27.13 \pm 6.94 \mathrm{mg} / \mathrm{dl} \quad \mathrm{Vs} \quad 42.97 \pm 8.21 \mathrm{mg} / \mathrm{dl})$ and LDL $(67.22 \pm 14.95 \mathrm{mg} / \mathrm{dl}$ Vs $114.25 \pm 29.47 \mathrm{mg} / \mathrm{dl})$ with high levels of serum Triglycerides $(141.25 \pm 54.72 \mathrm{mg} / \mathrm{dl}$ Vs $99.10 \pm 17.44 \mathrm{mg} /$ dl). Mean CIMT of carotid artery was $0.481 \pm 0.051 \mathrm{~mm}$ in the study population and was significantly higher than the control population $(0.400 \pm 0.019 \mathrm{~mm} ; p$ value $<0.01)$ on the right side. CIMT strongly correlated to BMI, cardiac risk ratio (total cholesterol/HDL) and Diastolic BP. Mean serum Apelin was $1027.53 \pm 190.90 \mathrm{pg} / \mathrm{ml}$ compared to $793.47 \pm 106.92 \mathrm{pg} / \mathrm{ml}$ in control group and was significantly higher in thalassemic children $(p$ value $<0.001)$. Serum Apelin correlated strongly with age, duration of illness, duration of chelation, serum ferritin and Systolic BP. Both parameters were found significant in univariate analysis however, the independent predictor for risk of early atherosclerosis in thalassemic children was serum Apelin levels $(p$ value $=0.01)$ in the multiple logistic regression model.

Discussion \& Conclusion: Thalassemia is a chronic proinflammatory condition, increasing the risk of endothelial injury at a premature age. This study has been unprecedented to conclude positive corelation between serum Apelin levels and CIMT in thalassaemic children before any clinical evidence of vascular dysfunction and concludes this biomarker useful in assessment of vascular changes early during the course of their disease providing scope for primary prevention.

\section{Pediatric Cold Agglutination Disease: Series of 5 Cases}

Malvika Gaur ${ }^{* 1}$, Tushar Sehgal ${ }^{1}$

${ }^{1}$ Laboratory medicine, All india institute of medical sciences, new delhi, DELHI, India

Aims \& Objectives: Cold agglutinin disease is a rare hematological condition and uncommon form of autoimmune hemolytic anemia. Cold agglutinin are seen with the primary cold agglutinin disease (CAD) or secondary cold agglutinin syndrome (CAS) Secondary CAS is even rarer and can be seen in settings of infections, hematological malignancies, autoimmune disorders and other neoplasms. We describe a series of five cases of secondary CAS in pediatric age group.

Patients/Materials \& Methods: This study was done at the All India Institute of Medical Sciences, New Delhi.

Results: In this series of five pediatric CAS cases the median age was 9 years (range 3-15). Females were more than males. The clinical presentation was non-specific with generalized weakness, fever, 
cough and cold in all patients. The median hemoglobin was $6.8 \mathrm{gm} / \mathrm{dl}$ (range 5.9-10.3) and median RBC was 1.02 x106/ul (range 0.75-2.87). All patients had spurious macrocytosis. Mycoplasma pneumoniae was identified in three cases, B-Acute lymphoblastic leukemia in one case and one case was idiopathic. One case was complicated by coexisting chlamydial infection along with Mycoplasma pneumoniae. Blood film showed RBC agglutination with spherocytes. Direct coombs test was positive for all cases for complement C3d.

Discussion \& Conclusion: Cold agglutinins are $\operatorname{IgM}$ antibodies that recognize $\mathrm{RBC}$ antigens at temperature below normal core body temperature. They can cause agglutination of RBC and extravascular hemolysis resulting in anemia without hemoglobinuria. The target antigen is typically "I" or "i" on RBC surface. Secondary CAS is uncommon and seen in infections like Mycoplasma pneumonia and Epstein Barr virus, autoimmune disorders and hematological neoplasms. The two major presentations of cold agglutinin disease are cold-induced symptoms and AIHA and positive Coombs test for complement. RBC agglutination caused by cold agglutinin leads to spurious $\mathrm{CBC}$ results involving $\mathrm{RBC}$ count, $\mathrm{MCV}$ and $\mathrm{MCHC}$. The $\mathrm{CBC}$ results normalize by incubating the sample at $37{ }^{\circ} \mathrm{C}$ in a water bath for $30 \mathrm{~min}$. A thorough literature search showed only a few case studies associated with pediatric CAS. Delay in diagnosis is common in cold agglutinin disease. A clue to the latter may be a very high mean corpuscular volume due to RBC agglutination. Early recognition and evaluation of these cases can prevent complications due to peripheral vascular obstruction and surgery.

\section{Prevalance of Thyroid Dysfunction in Iron Deficiency Anemia}

Dinesh Sharma $^{* 1}$, Sunita Aggarwal ${ }^{1}$, Smita Kaushik ${ }^{2}$, Ravi Ahlawat ${ }^{1}$, Sandeep Garg ${ }^{1}$

${ }^{1}$ General Medicine, ${ }^{2}$ Biochemistry, Maulana Azad Medical College \& LNH, Delhi, India

Aims \& Objectives: (1) To study the prevalence of thyroid dysfunction in iron deficiency anaemia. (2) To study the association between Iron deficiency parameters with thyroid profile.

Patients/Materials \& Methods: An observational cross sectional study was conducted on 70 adult (age $>18$ years) patients diagnosed with Iron deficiency anaemia (Haemoglobin $<13 \mathrm{~g} \%$ in males, $<$ $12 \mathrm{~g} \%$ in females with microcytic hypochromic picture on peripheral smear). Detailed history and physical examination was done. Blood investigations including complete Hemogram (Hb, TLC, DLC, Platelet, HCT, MCV, MCH, MCHC), peripheral smear, LFT, KFT, Serum Ferritin, Serum Iron, TIBC, TSH, FT3 and FT4 done for all patients. Patients on treatment for thyroid dysfunction were excluded. Results: The Mean age of study subjects was $46.84 \pm 18.6$ years with $67.14 \%$ females and $32.86 \%$ males. Out of the 70 patients, $52.9 \%$ had moderate and $45.7 \%$ had severe anemia. The prevalence of thyroid dysfunction among IDA adults was $28.57 \%(n=20)$. Among them 7 patients had hyperthyroidism and 13 cases had hypothyroidism. Sixty percent patients of iron deficiency anemia had symptoms. The most common symptom was easy fatiguability $(47.1 \%)$ followed by generalised weakness $(41.4 \%)$. Seventy percent patients had signs of IDA. Most common sign was pallor $(65.7 \%)$ followed by koilonychia (22.9\%). On correlation analysis of thyroid enzymes with iron indices, significant positive correlation was seen between $\mathrm{TSH}(\mathrm{mIU} / \mathrm{L})$ and total iron binding capacity(TIBC) $(\mathrm{r}=0.273, p=0.023)$. Significant negative correlation was seen between TSH and serum ferritin( $\mathrm{r}=-0.261, p=0.029)$.

Discussion \& Conclusion: We report a high prevalence of $28.57 \%$ thyroid dysfunction among adult patients with iron deficiency anemia.
Thyroid peroxidase activity is dependent on iron for its action which is responsible for T3, T4 synthesis. Iron deficiency leads to decreased production of thyroid hormones. Hence, thyroid function test should be done in all patients of iron deficiency anemia for early diagnosis and treatment of associated hypothyroidism.

\section{Evaluation of Anemia in IBD: A Cross Sectional Study}

Neha Aggarwal $^{* 1}$, Sadhna Marwah ${ }^{2}$, Vaishali Bhardwaj ${ }^{3}$

${ }^{1}$ Pathology, ABVIMS and Dr RML Hospital, New Delhi, ${ }^{2}$ Pathology, ABVIMS \& Dr RML Hospital, New Delhi, ${ }^{3}$ Gastroenterology, ABVIMS \& Dr RML Hospital, New Delhi, New Delhi, India

Aims \& Objectives: To study incidence and type of anemia in newly diagnosed patients of inflammatory bowel disease(IBD) and to correlate it with disease activity.

Patients/Materials \& Methods: 95 adult patients were selected who attended out patient department at Department of Gastroenterology and hematological evaluation (Complete blood count, iron profile, serum vitamin B12,serum ferritin and serum folate levels) was carried out in Department of Pathology at ABVIMS and Dr RML Hospital, New Delhi.

Results: 74 (71\%) patients were found to be anemic. Most common type of anemia was iron deficiency anemia followed by anemia of chronic disease. Increase in disease severity was associated with increased incidence of anemia.

Discussion \& Conclusion: Anemia is commonly present in patients of IBD at the time of diagnosis and is positively associated with disease severity. Routine screening of patients for anemia should be undertaken to improve the quality of life of patients.

\section{RET-HE as a Novel Parameter for Assessment of Anemia in Hemodialysis Patients}

Tushar Sehgal ${ }^{1}$, Shilpa Anupurba ${ }^{* 1}$

${ }^{1}$ Laboratory Medicine, All India Institute Of Medical Sciences, Delhi, India

Aims \& Objectives: Introduction. Anemia is a common complication in CKD patients, especially those who are undergoing dialysis. Majority of anemia is due to insufficient erythropoietin levels but is also superimposed with iron deficiency. Early detection and treatment of such cases is of utmost importance. Biochemical markers like serum ferritin and transferrin saturation levels are used but their use is debatable. Other parameters that can directly estimate bone marrow iron availability have been investigated, such as percentage of hypochromic erythrocytes (\%hypo), reticulocyte hemoglobin content (CHr), and reticulocyte hemoglobin equivalent (RET-He). RET- He estimates the amount of hemoglobin in the reticulocyte, with good reflection of the iron available for erythropoiesis. It can be obtained with the help of a high-end hematology analyzer. Objectives. To assess the diagnostic value and investigate RET-He as an early indicator of iron deficiency.

Patients/Materials \& Methods: All patients within the age group of 18-65 years on hemodialysis were included between August 2020 and October 2020. Anemia was defined as per WHO criteria for anemia. Iron deficiency (CKD patients) was defined with respect to transferrin saturation $<20 \%$ and serum ferritin $<100 \mathrm{ng} / \mathrm{ml}$. CBC results including RET-He and parameters were obtained by Sysmex XN 9000 analyzer. Cut off for RET-He was taken as $31 \mathrm{pg} /$ cell. Differences between selected data sets were evaluated by performing a t-test for independent samples using Microsoft Excel Windows 
2010. Statistical differences between data sets were defined by a $p$ value of $<0.05$.

Results: 52 patients were enrolled till date. Ret-He is lower in patients with iron deficiency anemia in CKD patients vis-a-vis anemia due to other causes. The sensitivity of Ret-He as a parameter in detecting iron deficiency was $85 \%$ and specificity was $56.3 \%$ with data available. This is comparable to serum ferritin and transferrin saturation as parameters in evaluation of iron deficiency.

Discussion \& Conclusion: RET-He can be useful alternative parameter for iron deficiency anemia in regular hemodialysis patients due to good diagnostic performance, availability on hematology analyzer, lower cost, less labour intensive and lower turnaround time. Monitoring of response to iron therapy in these patients can also be assessed using RET-He.

\section{Iron Overload in Non Transfusion Dependent Thalassemia Patients}

Lekha Parikh*1, Sujata Sharma ${ }^{2}$, Nikita Shah ${ }^{3}$

${ }^{1}$ Lokmanya Tilak municipal medical college, sion, Mumbai, Maharashtra, India, ${ }^{2}$ Paediatrics and Head of hematoncology, ${ }^{3}$ Paediatrics, Lokmanya Tilak municipal medical college, sion, Mumbai, Maharashtra, India

Aims \& Objectives: To study the Iron overload in Non-Transfusion Dependent Thalassemia patients. To study the clinical profile of NTDT patients, To correlate Iron Overload with Number of PRC Transfusions received and with Serum Ferritin levels and Cardiac MRI. To study the mutation studies in NTDT patients.

Patients/Materials \& Methods: Retrospective and prospective study of 78 Non-Transfusion Dependent thalassemia patients registered in a tertiary care centre of Mumbai. Patients of Age group less than 12 years were included and Thalassemia patients who were on regular transfusions were excluded. The demographic data and parameters of these patients were studied. Number of PRC Transfusions received, Serum Ferritin levels and Special investigations (to measure the Liver Iron Concentration and to study the complications) like Cardiac MRI, 2DEcho \& DEXA scan were also studied.

Results: There is a positive significant correlation between the number of PRC Transfusions received and degree of Hepatic and Cardiac Iron Overload ( $p$ value being 0.03 and 0.04 respectively). As assessed by Cardiac MRI T2 ${ }^{*}$ values, Majority of the patients had Mild Hepatic Overload. Out of 56 patients; $41 \%$ had Mild Hepatic Overload, $11 \%$ had Moderate, $11 \%$ had Severe and $38 \%$ did Not have Liver Overload. Majority of the patients (84\%) had No cardiac overload and Only 9\% had Mild and 7\% had Moderate-Severe Cardiac Iron Overload. However, 17 patients $(30 \%)$ who had received even less than 10 PRC Transfusions had Hepatic Overload. About 13 such patients had Mild whereas 4 such patients had Moderate - Severe Iron Overload. There was a positive significant correlation between the number of PRC Transfusions received \& Serum Ferritin(SF) levels. There is No correlation between the SF \& Degree of Iron Overload as assessed by Cardiac MRI. $p$ value being 0.19 for Hepatic Iron Overload and SF levels whereas 0.51 for Cardiac Iron Overload and SF levels. Patients with even Moderate to Severe Iron Overload had SF levels $<500 \mathrm{ng} / \mathrm{ml}$, rather than having higher levels. The most common mutation among study patients was IVS 1-5 (G-C) \& CODON 26(G- A).

Discussion \& Conclusion: Iron overload is seen in NTDT patients even though they have received lesser PRC Tx. The reason for this Iron accumulation in NTDT patients is mainly secondary to Intestinal absorption. Ineffective erythropoiesis triggers suppression of Hepcidin synthesis leading to the up-regulation of Intestinal Iron absorption and Iron release from macrophages. SF levels is a non- reliable indicator for NTDT patients as it under-estimates the overload and delays the initiation of Iron Chelation Therapy. Cardiac MRI \& DEXA scan are better Indicators for assessment of Iron Overload in NTDT patients.

\section{Anthropometric Assessment in Transfusion Dependent Beta Thalassemia Patients}

Priti B. Kamble*1, Saiprasad Kavthekar ${ }^{1}$, Anil B. Kurane ${ }^{1}$

${ }^{1}$ Paediatrics, D Y Patil Hospital, Kolhapur, India

Aims \& Objectives: Aim: To assess anthropometric measurements in transfusion dependent beta thalassemia patients. Objectives: To measure anthropometric measurements like weight, height and body mass index. To correlate mean pre transfusion hemoglobin level with anthropometrical measurements.

Patients/Materials \& Methods: A cross sectional observational study was done at D Y Patil Medical College and Research Center, Kolhapur. This study included 50 patients of beta thalassemia which were transfusion dependent(thalassemia major) with age $>2$ years. Anthropometric measurements like Weight, Height were taken as per standard measurement protocol in $\mathrm{kg}$, $\mathrm{cm}$,Body Mass Index were calculated by weight $(\mathrm{kg}) /[$ height $(\mathrm{m})] 2$. Weight for age, Height for Age, and BMI were expressed in terms of Percentiles related to WHO 2007 and IAP child Growth Standards reference data. Mean pre transfusion hemoglobin of all patients were calculated.

Results: The present study showed that $44.9 \%$ children had weight below 3 rd percentile and $51 \%$ children had a height below 3 red percentile. Around $91.8 \%$ had BMI below normal. Those with low BMI were having low pretransfusion hemoglobin(less than $9 \mathrm{~g} / \mathrm{dl}$ ) which was statistically significant $(p$ value $<0.05)$.

Discussion \& Conclusion: Maintaining pre transfusion hemoglobin $>9 \mathrm{~g} / \mathrm{dl}$ and early identification of growth failure with close monitoring of growth pattern will help in achieving near normal weight and height.

\section{A Case Series of Cost Effective Management of Refractory Iron Deficiency Anaemia with Subclinical Megaloblastic Anaemia}

Neelanjali Jain *1, Maneesh Sulya ${ }^{2}$, Dr. R. K. Nigam ${ }^{3}$, Reeni Malik ${ }^{4}$

${ }^{1}$ P.G. Third Year, ${ }^{2}$ Dept. of Pathology, Associate Professor, ${ }^{3}$ Dept. of Pathology, Professor, ${ }^{4}$ Dept. of Pathology, Professor and Head, Gandhi medical college, Bhopal, India

Aims \& Objectives: Cost effective diagnosis and treatment of dimorphic anemia with high index of clinical suspicion of refractory iron deficiency anemia in resource limited healthcare services.

Patients/Materials \& Methods: We did a observational retrospective study for one year (2019-20). 10 cases of refractory iron deficiency anemia diagnosed on the basis of red cell indices and peripheral smear. Patients were given treatment for iron deficiency anemia but their symptoms did not respond to iron therapy given for three months. Later they were diagnosed as refractory anemia and sent for hematological evaluation and we did bone marrow examination.

Results: In our study out of 10 cases 6 cases were females and 4 cases were males. The mean age in our study was 35 years $\pm 16 \mathrm{SD}$. The mean hemoglobin level in our study was $5.4 \pm 1.7 \mathrm{gm} \%$. The mean corpuscular volume in our study was $65.75 \pm 7.14$ SD while Mean corpuscular hemoglobin concentration was $28.99 \pm 1.54 \mathrm{SD}$. The Peripheral smear of all the patient showed microcytic hypochromic picture, while on bone marrow aspiration done after iron therapy $50 \%$ of the cases showed dimorphic anemia while another $50 \%$ showed 
predominantly megaloblastic anemia. On giving combined therapy (Iron, vitamin B12 \& B9) all patients responded to the treatment. Discussion \& Conclusion: In our study we found that subclinical megaloblastic anemia is an important cause for refractory iron deficiency anemia. Thus a clinician should have a high index of suspicion for diagnosis of dimorphic anemia, when patient do not respond to treatment for iron deficiency anemia even if red cell indices and peripheral smear indicate Iron Deficiency Anemia. These patients often need to undergo bone marrow examination, delaying their treatment and making it expansive. We recommend combined iron and vitamin therapy for cases of subclinical megaloblastic anemia. This approach will decrease the burden of refractory cases of nutritional anemia and need of bone marrow examination, which will also be cost effective.

\section{Evaluation of T-Regulatory Cells in Beta Thalassemia Major}

Farah Choudhary $^{* 1}$, Mrinalini Kotru ${ }^{1}$, Sunil Gomber ${ }^{2}$, Pooja Dewan $^{2}$, Richa Gupta ${ }^{1}$, Meera Sikka ${ }^{1}$

${ }^{1}$ Pathology, ${ }^{2}$ Pediatrics, UCMS, Delhi, India

Aims \& Objectives: The study aimed to evaluate T-regulatory cells in $\beta$-Thalassemia major (BTM). The study also compared the Tregulatory levels with CD4 and CD8 T cell counts and correlated the T-regulatory levels with Serum Iron \& Serum ferritin.

Patients/Materials \& Methods: Study enrolled 20 cases and 20 controls. Cases were confirmed BTM patients who came in thalassemia OPD or in emergency for blood transfusions. Remaining 20 were healthy controls \pm 2 years of age from OPD. They were subjected to extensive haematological work-up, Iron studies and Flow Cytometry based work-up. Data was analyzed and statistically evaluated using SPSS-PC-19 version.

Results: T-reg were quantified by using two definitions as CD4/ CD25high and CD4/CD25/FOXP3 on CD4-T-lymphocyte gating. $\mathrm{CD} 4 / \mathrm{CD} 25$ high $\%$ ranged from 0.5 to $6.1 \%$ with mean $\pm \mathrm{SD}$ of $1.64 \pm 1.32 \%$ in cases and from 0.1 to $4.8 \%$ with mean \pm SD of $2.01 \pm 1.24 \%$ in controls. CD4/CD25/FOXP3\% ranged from 0.4 to $5 \%$ with mean \pm SD of $1.47 \pm 1.09 \%$ in cases and from 0.1 to $3.7 \%$ with mean $\pm \mathrm{SD}$ of $1.74 \pm 1.01 \%$ in controls. No significant difference was noted in CD4/CD25high and CD4/CD25/FOXP3 and T-lymphocytes of cases and controls $(p>0.05)$. CD4 T- lymphocytes are increased in BTM compared to controls. There was no correlation in between serum iron and T-reg cells. However, serum ferritin was negatively correlated with both T-reg markers used.

Discussion \& Conclusion: CD4 T- lymphocytes are increased in BTM possibly due to repeated antigenic stimulation because of blood transfusion. However, T-reg cells is not different between the two groups. As we know T-reg helps in suppressing the autoimmunity more studies on large number of patients need to be done to study the T-regs in allo-immunised versus non- alloimmunised patients of BTM to prove the role of T-regs in tolerance to allo-immunisation. In addition, more studies on varying $\mathrm{T}$ - reg levels during heterologous infections need to be done.
Prevalence of Metabolic Syndrome in Adolescents Attending Thalessemia Clinic in a Tertiary Care Institute of Haryana

Ankit Singla ${ }^{* 1}$, Anjali Sharma ${ }^{1}$, Kapil Bhalla ${ }^{1}$, Puneet Singla ${ }^{2}$, Nikhil Nagpal ${ }^{1}$, Gerish Atri ${ }^{3}$

${ }^{1}$ Department of Paediatrics, ${ }^{2}$ Department of Pulmonary and Critical Care Medicine, ${ }^{3}$ Department of Community Medicine, Pt. B.D. Sharma Post Graduate Institute of Medical Sciences, Rohtak, India

Aims \& Objectives: To investigate the association of metabolic syndrome with $\beta$-thalessemia major in Indian children.

Patients/Materials \& Methods: A cross-sectional study was conducted in Thalessemia clinic of Pt. B.D. Sharma PGIMS, Rohtak, a tertiary care institute in Haryana. A total of 80 subjects, i.e., 40 subjects with $\beta$-thalessemia major (cases) and 40 subjects without $\beta$ thalessemia as their age and gender match control, were enrolled in our study. Height, weight, systolic and diastolic BP, waist circumference of the subjects were recorded by a trained paediatrician. Fasting blood glucose and fasting serum lipid profiles were analyzed. WHO body mass index (BMI) curve and International Diabetic Federation (IDF) metabolic syndrome criteria were used as reference. SPSS software version 20.0 was used for analysis.

Results: 25 male and 15 female adolescents aged 10-16 years constituted the case group. $20 \%$ of the adolescents were overweight, $32.5 \%$ had central obesity. The prevalence of Metabolic syndrome was found to be $22.5 \%$. It was significantly higher in female as compared to male adolescents $(p=0.04)$ and was found to be significantly associated with hypertriglyceridemia $(p=0.006)$ and low HDL $(p=0.025)$.

Discussion \& Conclusion: As the management of $\beta$-thalessemia major patients is improving for last few decades, survival of those patients is also improving. But now there is an increasing concern about other long term therapeutic side-effects including cardiovascular ones. Metabolic syndrome is a condition that predisposes to early atherosclerosis and increases cardiovascular risk. This has not been studied much before in thalessemic children Thus this study is an attempt to understand the association of $\beta$ - thalessemia major with Metabolic syndrome so that an early intervention can be done at subclinical cases of the disease.

\section{New Erythrocyte and Reticulocyte Parameters: Indicators for Early Diagnosis of Iron Deficiency Anemia and Anemia of Chronic Disease}

Charu Agarwal $^{* 1}$, Shefali Gupta ${ }^{2}$, Mukta Pujani ${ }^{1}$, Varsha Chauhan ${ }^{3}$, Kanika Singh $^{1}$

${ }^{1}$ Associate Professor, Pathology, ESIC MEDICAL COLLEGE, Faridabad, ${ }^{2}$ MBBS IIIrd Prof Student, ESIC Medical College, Faridabad, ${ }^{3}$ Assistant Professor, Pathology, ESIC Medical College, Faridabad, India

Aims \& Objectives: The aim and objectives of the present study was to evaluate the utility of new erythrocyte and reticulocyte parameters for differentiation of iron deficiency anemia and anemia of chronic disease and to establish the role of new erythrocyte and reticulocyte parameters for early diagnosis of latent iron deficiency.

Patients/Materials \& Methods: Blood samples of adult females of reproductive age group (15-45 years) were collected and processed within $6 \mathrm{~h}$ of collection. Based on hematological and biochemical findings, patients were segregated into four subgroups: Iron deficiency anemia (IDA), Anemia of chronic disease (ACD), Latent iron deficiency and Controls. 
Results: The novel hematological parameters- Reticulocyte hemoglobin content (Ret-He), percentage of microcytic red blood cells (MicroR), hemoglobin content of erythrocytes (RBC-He) and percentage of hypochromic erythrocytes $(\mathrm{HypoHe})$ had a statistically significant difference between the four groups $(p<0.05)$. The correlation between reticulocyte indices and iron parameters depicted that levels of Ret-He among all study participants (160) increased as the serum iron and serum ferritin increased indicating a significant positive correlation. Ret-He and RBC-He demonstrated better predictive power as per area under curve of ROC as compared to MicroR and HypoHe indices for latent anemia identification.

Discussion \& Conclusion: The present study emphasized upon the importance of novel and cost effective routine new parameters in early and accurate diagnosis of IDA and ACD. Moreover Ret-He emerged as a sensitive and specific marker for diagnosis of latent iron deficiency.

Disclosure of Interest: None Declared.

\section{Malarial Anemia Revisited: Falciparum Induced Megaloblastic Anemia and Dimorphic Anemia; A Report Of 2 Cases \\ Ashima mishra ${ }^{* 1}$, Purna C. Karua ${ }^{1}$ \\ ${ }^{1}$ Internal Medicine, VIMSAR, Burla, India}

Aims \& Objectives: Direct destruction of infected and uninfected erythrocytes, dyserythropoesis and post artesunate delayed hemolysis contribute to malarial anemia.There were two cases of pancytopenia, diagnosed as plasmodium falciparum(pf) positive cases, who presented with severe anemia after a fortnight requiring additional transfusions. The aim of the case report is to suggest that falciparum malaria can cause megaloblastic anemia and dimorphic anemia besides hemolytic anemia and such cases should be followed up for complications so as to formulate the hypotheses, which can be studied further with larger sample size.

Patients/Materials \& Methods: CASE 1.A 16 year girl presented with fever and headache for 3 days. She had pallor, jaundice,pancytopenia.positive rapid antigen test for pf. She was treated with parenteral artesunate therapy and blood transfusions and discharged after 5 days with IFA,VITAMIN B complex supplements. She got readmitted after 15 days due to severe generalised weakness. Investigations revealed anemia,leucopenia,hyperbilirubinemia,,positive antigen test for pf,reticulocytopenia,hyperferritenemia. Bone marrow aspirate showed megaloblastic anemia. Serum vit B12 and folate levels were normal. Despite being administered methycobalamin injections, her haemoglobin levels kept dropping over next 1 week. She received urgent blood transfusions.Antibiotics were started as neutropenia persisted. She received a single dose of primaquine.Her blood parameters improved over next 2 months. She was diagnosed as falciparum induced megaloblastic anemia. CASE 2.Her mother developed fever on the second day of their first hospital stay, was found to be falciparum positive, had pancytopenia, hyperferritenemia and was prescribed antimalarial therapy. Later, she also complained of fatigue, retested positive for malaria parasite and was given antigametocidal. She was diagnosed as falciparum induced dimorphic anemia which resolved over 2 months.

Results: Malaria can alter blood parameters and mimic functional b12 and iron deficiency state. Untreated malarial anemia can be fatal. Malarial anemia is significant in endemic areas with seasonal transmission.

Discussion \& Conclusion: Dyserythropoesis hinders rapid recovery from anemia.Persistent antigenemia might indicate residual disease. Alterations of iron,b12 and folate metabolism occurs. Primaquine must be given in falciparum cases after ruling out G6PD deficency.
The falciparum cases must be followed up for early detection of anemia and appropriate management.

\section{Thalassemia Screening in Families of Known Cases of Thalassemia Major}

Prateek Mathur*1, Mala Kanthali ${ }^{2}$

${ }^{1}$ R.D. Gardi Medical College, ${ }^{2}$ Pathology, R.D. Gardi Medical College, Ujjain, India

Aims \& Objectives: Screening of family members of known cases of Thalassemia major. To know the pattern of Thalassemia in families. To correlate haematological findings with abnormal hemoglobin.

Patients/Materials \& Methods: It is a Prospective Observational study. Study done on 99 samples, which were collected after taking informed consent from parents of patients. $2 \mathrm{ml}$ blood was collected in vial containing ethylene diamine tetraacetic acid disodium salt (K3 EDTA salt). Complete blood count and $\mathrm{Hb}$ electrophoresis on cellulose acetate paper with alkaline buffer were carried out. Sample were collected under complete aseptic precautions.

Results: 99 samples of first degree relatives of Thalassemia major patients including mothers, fathers and siblings were subjected to complete blood count, peripheral smear examination and $\mathrm{Hb}$ electrophoresis. In these subjects $72(72.72 \%)$ were positive for variants of thalassemia; thalassemia trait 62 ,thalassemia intermedia 6 \& sickle thalassemia only 1. Families with both the parents having thalassemia trait or thalassemia intermedia had more prevalence of thalassemia major and other variants of thalassemia. 5 families having only one parent with thalassemia trait.Most of the siblings were having normal $\mathrm{Hb}$ electrophoresis pattern except the index cases. Slight female preponderance was observed in the study (female to male ratio 1.06). Mostly affected individuals were in the age group of 20-40 yrs which is a reproductive age group. On peripheral smear, target cells and microcytic hypochromic RBC's were seen.

Discussion \& Conclusion: Higher prevalence of thalassemia is seen in the families of known cases of Thalassemia major.So the families with history of Thalassemia major should be screened inspite of normal $\mathrm{CBC}$ to detect the trait. The counselling and proper screening of these members may reduce the incidence of Thalassemia. Advanced technology based investigations should be carried out in those normal subjects in these studies. In the families of known thalassemia major with microcytic hypochromic and target cells on peripheral smears should be screened for Thalassemia variants. Spleenomegaly though is considered as a sign of Thalassemia. It is not a common finding in trait.

\section{Missense C.569C > G, P. PRO190ARG Variation in SLC25A38 Gene Played an Important Role in Autosomal Recessive Inherited Sideroblastic Anemia in an Indian Patient}

Rashmi Dongerdiye ${ }^{* 1}$, Prabhakar Kedar ${ }^{1}$

${ }^{1}$ Haematogenetics, ICMR-National Institute of Immunohaematology, Mumbai, India

Aims \& Objectives: Sideroblastic anemias are a group of inherited and acquired hematological disorders, marked by the presence of ring sideroblasts in the bone marrow. Non- syndromic sideroblastic anemia is caused due to mutations in ALAS2 followed by SLC25A38, GLRX5, and HSPA9 genes. SLC25A38 (3p22.1) belongs to the family of mitochondrial membrane transporters protein, predicted to encode glycine transporters. We aim to describe a novel case of non- 
syndromic sideroblastic anemia associated with a biallelic mutation in the SLC25A38 gene.

Patients/Materials \& Methods: The proband is a two years old female child, born to consanguineous parents. She presented with severe microcytic transfusion-dependent anemia since early childhood. Initially, she was suspected to be a case of thalassemia major. Later, her bone marrow examination revealed ring sideroblasts $(++)$. Advanced molecular analysis (NGS) identified a disease-causing variant in the SLC25A38 gene, which was confirmed by DNA Sanger sequencing. The effect of the variant on the transmembrane structure of the SLC25A38 protein was studied using various bioinformatics tools and software.

Results: She clinically presented with hypochromic, microcytic (HB$6.5 \mathrm{~g} / \mathrm{dl}$, MCV-63.3 fl), transfusion-dependent anemia, with ring sideroblasts in the bone marrow. The genetic sequencing identified missense homozygous substitution c.569C > G, p. Pro190Arg in the SLC25A38 gene. Bioinformatics tools-SIFT, Polyphen2 supported the deleterious nature of the variant. The predicted structural model of SLC25A38 was constructed by SWISS-MODEL and visualized with PyMol. The SLC25A38 protein is one member of a large family of mitochondrial carrier proteins. Some of these carriers' transport in only one direction, but most exchange one substrate for another. In the case of SLC25A38, the arginine-aspartic acid (RD) dipeptide at 187-188 putatively identifies it as an amino acid transporter. From the structural study, we hypothesize that the missense mutation Pro190Arg affects the pivotal position for substrate binding and gate opening to the intermembrane space, thus affecting the transport function of the protein.

Discussion \& Conclusion: The missense substitution c.569C > G, p. Pro190Arg in the SLC25A38 gene causes severe autosomal- recessive sideroblastic anemia. Recent studies have identified the most common c.409dupG variant in the SLC25A38 gene in eleven unrelated Indian patients. A detailed investigation of this variant has shed light on structure-function relationships in the protein. The early identification of the causative gene has helped in shaping the diagnosis and future prognosis, where therapies are currently unavailable.

\section{Utility of Red Blood Cell Parameters in the Evaluation of Microcytosis Without Anemia or with Mild Anemia}

Paruvathavarthini Thambiraj ${ }^{* 1}$, Rakhee $\mathrm{Kar}^{1}$, Debdatta Basu ${ }^{1}$

${ }^{1}$ Pathology, JIPMER, Puducherry, India

Aims \& Objectives: To assess the utility of red blood cell parameters in the differential diagnosis of different causes of microcytosis without anemia or with mild anemia.

Patients/Materials \& Methods: Patients who had presented to JIPMER hospital during the study period from March 2019 to October 2020 for whom routine hemogram had been sent to main hematology laboratory which showed microcytosis $[\mathrm{MCV}<80 \mathrm{fL}]$ without anemia or with mild anemia $(10-10.9 \mathrm{~g} / \mathrm{dl})$ as per ICMR criteria were taken. Ethical clearance was obtained from the Institute Ethics Committee (Human Studies). Complete hemogram values (Sysmex $\mathrm{XT}$ 4000i) such as haemoglobin, RBC count, MCV, MCH, MCHC, RDW and peripheral smear were assessed. Based on these values derived RBC indices such as red cell distribution width index, Mentzer index, Ehsani index, Shine and Lal index, Sirdah index, Matos Carvalho index and Sehgal index were calculated and their diagnostic accuracy in thalassemia trait were evaluated. Using $\mathrm{Hb}$ HPLC (Biorad D10), haemoglobin variants such as $\mathrm{HbA}, \mathrm{HbA} 2, \mathrm{HbF}$ levels were assessed. All cases were divided into two groups based on microcytosis with normal and abnormal proportion of $\mathrm{Hb}$ variants.

Results: Total of 70 samples were evaluated. Twenty-two out of 70 samples had high HbA2 levels $(>4 \%), 03$ out of 70 showed borderline HbA2 levels $(3.5 \%-4 \%)$ and 45 out of 70 had normal HbA2 levels (2-3.5\%). Derived red cell indices were calculated of which Sehgal index showed higher sensitivity of $80 \%$ followed by Shine and Lal index and Mentzer index which showed sensitivity of $75 \%$ in identifying thalassemia trait. Sirdah index showed better specificity of $75 \%$.

Discussion \& Conclusion: Shine and Lal index, Sehgal index and Mentzer index helps in discriminating causes of microcytosis and also helps to detect subjects who have high probability of requiring appropriate follow up with sophisticated techniques like $\mathrm{Hb}$ HPLC/capillary electrophoresis, thereby reducing unnecessary investigative costs.

\section{Effect of Maternal Hemoglobin on Neonatal Birth Weight}

\author{
Varsha Mallavarapu ${ }^{* 1}$, Srinivas Pagadpally ${ }^{1}$ \\ ${ }^{1}$ Paediatrics, Vinayaka Mission's Medical College, Karaikal, \\ India
}

Aims \& Objectives: The main objective of this study is to determine the effect of maternal anemia on neonatal birth weight.

Patients/Materials \& Methods: A cross-sectional observational study was done on 116 pregnant mothers and their newborns at VMMCH, Karaikal. Maternal anemia was defined as $\mathrm{Hb}<11 \mathrm{gm} / \mathrm{dl}$. Neonatal birth weight $<2.5 \mathrm{~kg}$ was considered as LBW. Mean, standard deviation, and Student t-test were used for statistical analysis.

Results: Out of 116 cases, 33 cases had maternal $\mathrm{Hb}<10 \mathrm{gm} / \mathrm{dl}, 30$ cases had $\mathrm{Hb}$ between 10 and $11 \mathrm{gm} / \mathrm{dl}$ and 53 cases had $\mathrm{Hb} \geq 11 \mathrm{gm} /$ dl. The prevalence of LBW among anemic mothers with $\mathrm{Hb}<10 \mathrm{gm} /$ dl was $69.7 \%$ and with $\mathrm{Hb}$ between 10 and $11 \mathrm{gm} / \mathrm{dl}$ was $20 \%$. The prevalence of LBW babies among non-anemic mothers $(\mathrm{Hb} \geq 11 \mathrm{gm} /$ dl) was $16.98 \%$. Moderate to severe maternal anemia was found to have a statistically significant association with lower birth weight compared to mild anemia. ( $p$ value $<0.01$ ).

Discussion \& Conclusion: Maternal hemoglobin concentration of $<10 \mathrm{gm} / \mathrm{dl}$ (moderate to severe anemia) is associated with an increased risk of Low Birth Weight (LBW) in newborn compared to $\mathrm{Hb} 10-11 \mathrm{gm} / \mathrm{dl}$ (mild anemia). The incidence of LBW newborns in mother's with $\mathrm{Hb} 10-11 \mathrm{gm} / \mathrm{dl}$ (mild anemia) is comparatively less and is almost similar to that of non-anemic $(\mathrm{Hb}>11 \mathrm{gm} / \mathrm{dl})$ mother's.

\section{Impact of Oxidative Stress and Inflammation on Endothelial Cells Activation in Haemoglobinopathy Patients}

Manju S. Gorivale ${ }^{* 1}$, Anita H. Nadkarni ${ }^{1}$

${ }^{1}$ Haematogenetics, National Institute of Immunohaematology (NIIH-ICMR), Mumbai, India

Aims \& Objectives: Defective $\beta$-globin synthesis, leads to excess $\alpha$ globin chains that causes haemolysis and impair erythropoiesis. Hence the internal environment in these patients leads to oxidative damage causing inflammation and endothelial activation that can cause various complications such as thrombosis. Thus the aim of the study was to analyse the effect of various inflammatory markers in different haemoglobinopthy groups.

Patients/Materials \& Methods: Total 189 haemoglobinopathy samples (82 thalassemia major, 35 thalassemia intermedia, $32 \mathrm{HbS}$ homozygous, $20 \mathrm{HbS}-\beta$ thalassemia and $20 \mathrm{HbE}-\beta$ thalassemia) were recruited along with 80 age and sex matched normal controls. Haematological study was carried out on KX-21 analyzer. HbA2 and 
$\mathrm{HbF}$ were quantified on Bio-Rad variant II. Reactive Oxygen Species (ROS) was measured on Flow cytometer by treating RBCs with $0.4 \mathrm{mM}$ DCFH-DA. Ferritin was quantified by ELISA kit. Soluble endothelial markers in serum (sP-Selectin, sE-Selectin, sICAM-1, and sVCAM-1) were quantified on Flow Cytometer by using Aimplex Kit. Results: The present study showed that the ROS level was significantly high in thalassemia intermedia patients $(304 \pm 89.50)$ followed by other groups [HbE- $\beta$ thalassemia $(239.2 \pm 56.95)$ and HbS- $\beta$ thalassemia $(197.2 \pm 35.70)$ when compared with control groups $(172.9 \pm 32.47)] p$ value $<0.0010$. Serum Ferritin were positively correlated with ROS of less frequent transfusion dependent hemglobinopathies- thalasemia intermedia, HbS- $\beta$ thalassemia, HbE$\beta$ thalassemia and $\mathrm{HbS}$ homozygous ( $\mathrm{R}$ value: 0.3913 ) whereas it has been negatively correlated in thalassemia major patient ( $R$ value: 0.1359). The soluble endothelial molecules levels [sE-Selectin, sPSelectin, sICAM-1 and sVCAM-1] showed statically significant increased in patients group ( $p$ value: $<0.05)$ when compared with the control group.

Discussion \& Conclusion: Our current study of ROS highlights the severe degree of oxidative damage and inflammatory state in intermittently transfused thalassemia patients. This may have a role in development of cardiovascular diseases and thrombosis.

\section{The Potential Role of Hydroxyurea in the Reduction of Aberrant Erythrocyte Apoptosis in Thalassemia Patients}

\section{Neha Kargutkar ${ }^{* 1}$, Anita Nadkarni ${ }^{1}$ \\ ${ }^{1}$ Haematogenetics Department, ICMR-National Institute of Immunohaematology, Mumbai, India}

Aims \& Objectives: Thalassemia is a monogenic blood disorder results due to mutations in the $\beta$-globin gene. The major consequence of the disease is ineffective erythropoiesis, an effect contributing to anemia. Thalassemia patients are more sensitive to the erythrocytes depletion which may be due to aberrant exposure of apoptotic marker; phosphatidylserine (PS) on outerleaflet of RBC cell membrane. The exposed Phosphatidylserine receptor are recognized, bound and engulfed by macrophages/monocytes. Hydroxyurea $(\mathrm{HU})$ is an approved drug for thalassemia patients. It has been known to reduce erythrocyte apoptosis in patients, but the mechanism is not known hence we aim to study the effect of hydroxyurea in ameliorating RBC apoptosis in thalassemia major and intermedia patients. In this study we determine the percentage of phosphotidylserine exposure on RBC membrane in thalassemia patients mediated with hydroxyurea treatment.

Patients/Materials \& Methods: In the present study we recruited 17 $\beta$ - thalassemia major, $13 \beta$-thalassemia intermedia patients at baseline and after 3 and 6 months of HU treatment along with 25 healthy control group. The erythrocytes were stained with FITC labelled annexin $\mathrm{V}$ antibody and were analysed using flow cytometer.

Results: We found a significant higher exposure of Phosphotidylserine receptor on erythrocytes surface in both thalassemia major and intermedia patients as compared to control $(p<0.01)$. Among both the patient groups, thalassemia major patients showed significant higher $(p<0.01)$ exposure of PS as compared to thalassemia intermedia patients. We tried to study the effect of HU treatment in both thalassemia major and thalassemia intermedia group, and interestingly, we found a significant decrease in PS exposure among both the group $(p<0.01)$ after HU treatment.

Discussion \& Conclusion: In this study, we documented the role of $\mathrm{HU}$ could be the main factor involved in reduction of RBC apoptosis and thereby ameliorating clinical manifestation in thalassemia patients.
The Role of Haematological Indices in Predicting Early Iron Deficiency Among Pregnant Women in Index Medical College Indore

\author{
Deeksha Gupta*1 \\ ${ }^{1}$ Pathology, Index Medical College Indore, Indore, India
}

Aims \& Objectives: Early detection and treatment of iron deficiency during is crucial for optimum pregnancy outcomes. Anemia is a late indictor of iron deficiency measured as $\mathrm{Hb}<11 \mathrm{~g} / \mathrm{dl}$, and is widely used as a proxy for iron deficiency. We aimed to evaluate the role of red cell indices as a screening tool for early detection of iron deficiency among pregnant women in an index medical college indore. Patients/Materials \& Methods: study conducted in index medical college hospital and research centre indore from 30 sep 2019 to 30 sep 2020.A Cross-Sectional study was conducted among 100 apparently healthy pregnant women $<12$ weeks of gestation attending antenatal clinics in index medical college indore. Women already on nutritional supplements were excluded.Full blood count, serum ferritin(SF) and high sensitive C-reactive protein (hs-CRP) assessments were performed. The womenwith evidence of inflammation as indicator by hs$\mathrm{CRP}>10 \mathrm{mg} / \mathrm{L}$ were excluded $(\mathrm{N}=20)$ From data analysis.Anemia $(\mathrm{Hb}<11 \mathrm{~g} / \mathrm{dl})$ and iron deficiency $(\mathrm{SF}<30$ microgm/L)were defined according to WHO guide.operating characteristics curves were used to derive red blood cell indices that showed the optimal cut-offs in detecting early iron deficiency.

Results: Result of the 100 women, 70 (70\%) were iron deficient $(\mathrm{SF}<30 \mathrm{microgm} / \mathrm{L})$, out of whom $20(14 \%)$ were identified as having iron deficiency anemia $(\mathrm{Hb}<10 \mathrm{~g} / \mathrm{dl})$. A high sensitivity $(>70 \%)$ IN prediction of iron deficiency was obtained for the optimal cut-off values of $\mathrm{Hb}<12.2 \mathrm{~g} / \mathrm{dl}$, MCV $<83.2 \mathrm{FL}, \mathrm{MCH}<26.9 \mathrm{pg}$ and MCHC $33.2 \mathrm{~g} / \mathrm{dl}$ while maintaining a specificity $>40 \%$.

Discussion \& Conclusion: Iron deficiency can be predicted in early stages using $\mathrm{Hb}$ and red cell indices, which is much less expensive. This could be a useful method in areas with limited resoueses and a high prevalence of iron deficiency.

\section{Deferoxamine Intravenous Once a Month Injection Single Dose Versus Deferasirox Oral Daily: Iron Chelator in Thalassemia Major: An Open Label Randomized Parallel Group Active Control Interventional Trial}

\author{
Ratna D. Bhojak ${ }^{1}$, Jayendra R. Gohil ${ }^{* 1}$, Bibin Varghese ${ }^{1}$ \\ ${ }^{1}$ Pediatrics, GMC, Bhavnagar, Bhavnagar, India
}

Aims \& Objectives: Objective: Compare efficacy of Once a Month Single Dose Intravenous (Deferoxamine) with Daily Oral (Deferasirox) Iron Chelator in Thalassemia Major.

Patients/Materials \& Methods: Methodology: In this open label trials Patients were randomized by chit method after informed consent and allocated to two groups. Group A was administered Inj Deferoxamine by intravenous route once during monthly blood transfusions and Group B received Tab Deferasirox administered daily. The monitoring was done by serum Ferritin level every two months.

Results: Results: The primary outcome was decrease in ferritin level over 6 months from the baseline. Decrease in serum ferritin (\%) over six months for Group A was $46 \pm 18$ and Group B was $25 \pm 17$ with significant difference between the two groups $(p=0.001)$. The cost of treatment per kilo weight was not significantly different.

Discussion \& Conclusion: We observed that Intravenous Deferoxamine once a month is a better and cheaper iron chelator as compared to the daily oral regimen; and preferred by children. 


\section{Clinical, Hematological and Molecular Profiles of Patients with Sickle-Cell Disorders in A Non-endemic North Indian Region}

Diksha D. Yadav ${ }^{* 1}$, Jasbir K. Hira ${ }^{1}$, Sanjeev Chhabra ${ }^{1}$, Sreejesh Sreedharanunni ${ }^{1}$, Amita Trehan ${ }^{2}$, Alka R. Khadwal ${ }^{3}$, Pankaj Malhotra $^{3}$, Prashant Sharma ${ }^{1}$, Reena Das ${ }^{1}$

${ }^{1}$ Department of Hematology, ${ }^{2}$ Department of Pediatrics, ${ }^{3}$ Department of Internal Medicine, Post Graduate Institute of Medical Education and Research, Chandigarh, India

Aims \& Objectives: To study the frequencies, clinico-demographic profiles and laboratory features of sickle-cell disorders (SCD) at a north Indian referral hospital with an emphasis on the role of molecular genetic analysis.

Patients/Materials \& Methods: All SCD patients diagnosed between January 2009 to June 2020, based on cation-exchange high pressure liquid chromatography (CE-HPLC), and confirmed by sickling test and alkaline $\mathrm{pH}$ electrophoresis, were included. Clinical, demographic and laboratory data including molecular findings (HbS mutation analysis by PCR-RFLP/ARMS-PCR and $\beta$-mutation analysis by multiplex ARMS-PCR) were collated.

Results: Total 282 cases of SCD were identified: 166 sickle-cell trait, 48 sickle-cell anemia (SCA), 54 double-heterozygous sickle- $\beta$ - thalassemia $(\mathrm{S} \beta)$ and 14 double-heterozygous sickle-HbD-Punjab. Clinical, demographic and laboratory findings are summarised in Table 1. Median age at referral to our hospital was 19 and 14 years for S $\beta$ and SCA respectively. The states of Punjab and Jharkhand contributed highest numbers of S $\beta$ and SCA patients respectively. Clinically, SCA patients had more frequent pain and jaundice and slightly less frequent splenomegaly compared to S $\beta$ group. The SCA group was more likely to have required hospitalisation and received hydroxyurea than the $S \beta$ group. Among the laboratory parameters, $29 \%$ of the SCA's had microcytosis while $12.9 \%$ had $\mathrm{HbA} 2 \%$ $\geq 3.5 \%$. MCV $<80 \mathrm{fl}$ together with $\mathrm{HbA} 2 \% \geq 4.0 \%$ successfully distinguished S $\beta$ and SCA (81.2\% sensitivity, $96.7 \%$ specificity). However, this approach missed $18.7 \%(\mathrm{n}=9) \mathrm{S} \beta$ cases and mislabelled 3\% ( $\mathrm{n}=1)$ SCA cases as $\mathrm{S} \beta$. On molecular testing, the predominant thalassemic allele was HBB:c. $92+5 \mathrm{G}>\mathrm{C}$ mutation $(58 \%)$ followed by HBB:c.126_129delCTTT (15\%), NG_000007.3:g.71609_72227del619 (6\%), HBB:c.27_28insG (4\%) and HBB:c. $92+1 \mathrm{G}>\mathrm{T}(2 \%)$. Remaining cases $(\mathrm{n}=8,15 \%)$ showed uncommon mutations. In one case, the RFLP pattern suggested SS-state, however, ARMS-PCR revealed the Codon 5(-CT); HBB:c.17_18delCT thalassemic mutation altering the DdeI restriction site. Amongst the S $\beta$-group, HPLC-derived $\mathrm{HbA} 0$ was significantly lower $(p<0.001)$ and $\mathrm{HbA} 2(p=0.03)$ significantly higher in $\mathrm{S} \beta 0$ in comparison to $\mathrm{S} \beta+$ mutation groups.

Discussion \& Conclusion: SCD, especially $S \beta$, is not rare in northern India. Molecular testing aids accurate characterisation of cases where parental studies are not feasible, in transfused patients, and in cases with misleading RBC indices. ARMS-PCR is superior to PCR-RFLP for the detection of HBB:c.17_18delCT mutation.

\section{Pseudothrombocytosis in a Case Of Beta: Thalassaemia Intermedia Masquerading as Myeloproliferative Neoplasm}

\section{Asha Goyal ${ }^{* 1}$, Chandrika Gupta ${ }^{1}$, Dharamveer Yadav ${ }^{2}$, Abhishek Purohit $^{3}$ \\ ${ }^{1}$ Pathology, RUHS CMS, Jaipur, ${ }^{2}$ Biochemistry, ${ }^{3}$ Pathology, AIIMS, JODHPUR, India}

Aims \& Objectives: Introduction:-Finding of spurious thrombocytopenia is a common occurrence in clinical practice whereas pseudothrombocytosis is very uncommon event. Despite there being several technical advancements in automated haematology analyzers, a careful peripheral smear examination remains standard in cases of discrepancy.

Patients/Materials \& Methods: A 30 year-old lady presented to haematology clinic with a provisional diagnosis of myeloproliferative neoplasm for further evaluation. She had a six-month history of generalized weakness, easy fatigability and three-month history of lump and pain in left side of abdomen. Examination revealed pallor and moderate splenomegaly. There was no evidence of icterus, cyanosis, clubbing or lymphadenopathy. She had received multiple transfusions in last six months. Investigations showed that her haemoglobin, total leucocyte count and platelet count were $5.5 \mathrm{~g} / \mathrm{dL}$, $5.38 \times 103 /$ cumm and $1223 \times 103 /$ cumm. Red cell indices revealed mean corpuscular volume as $60.9 \mathrm{fL}$, mean corpuscular haemoglobin being $22 \mathrm{pg}$ and red cell distribution width (RDW-SD) was $23.2 \%$. Further, serum uric acid levels were $11.5 \mathrm{mg} / \mathrm{dL}$ and serum ferritin was $580.5 \mathrm{ng} / \mathrm{mL}$. Bone marrow examination revealed hypercellular marrow with marked erythroid prominence. Her haemoglobin high performance liquid chromatography (Hb- HPLC) report revealed heterozygosity for beta thalassaemia as the $\mathrm{HbA} 2$ was $4.5 \%$.

Results: Peripheral smear examination revealed marked anisopoikilocytosis and hypochromic microcytic red cells along with numerous fragmented red cells and low platelet count (70x103/cumm approximately). The fluorescent platelet count (Plt-F) was consistent with manual platelet count. Serum lactate dehydrogenase levels were 591 $\mathrm{U} / \mathrm{L}$ and there was indirect hyperbilirubinemia (Total bilirubin $2.75 \mathrm{mg} / \mathrm{dL}$ and indirect bilirubin being $2.33 \mathrm{mg} / \mathrm{dL}$ ). Further, detailed history was elicited and it was found that earlier Hb-HPLC was performed two days after packed red cell transfusion. Hence, we repeated the $\mathrm{Hb}$-HPLC and we observed that $\mathrm{HbF}$ and $\mathrm{HbA} 2$ were raised $(30.4 \%$ and $5.4 \%$ respectively) and an interpretation of homozygous beta thalassaemia was rendered on Hb- HPLC and a clinical diagnosis beta thalassaemia intermedia was offered.

Discussion \& Conclusion: Pseudothrombocytosis is not a common observation in the routine practice and falsely high platelet counts may be reported by automated analyzers in cases of fungal contamination or accidental heating of blood samples, malarial infestation, hyperlipidemia, tumour lysis in acute monocytic leukemia or fragmented red cells in haemolytic anaemias. This communication reinforces the importance of thorough clinical examination and peripheral smear examination in haematology.

\section{Coagulation Defects in Thalassemia Patients: A Study From Eastern Part Of India}

Malini Garg ${ }^{* 1}$, Prakash S. Shekhawat ${ }^{1}$, Avriti Baveja ${ }^{1}$, Subham Bhattacharyaa $^{1}$, Sumit Mitra ${ }^{1}$, Shuvra N. Baul ${ }^{1}$, Rajib De ${ }^{1}$, Prakas K. Mandal $^{1}$, Tuphan K. Dolai ${ }^{1}$

\section{${ }^{1}$ Hematology, Nil Ratan Sircar Medical College and Hospital, Kolkata, India}

Aims \& Objectives: To compare the coagulation profile in thalassemia patients versus control population and thalassemia major versus thalassemia intermedia. To study the association between iron overload with coagulation parameters in thalassemia patients.

Patients/Materials \& Methods: A cross sectional study was conducted between January 2020 to September 2020 in the Department of Hematology at NRS Medical college, Kolkata. Total 60 diagnosed cases of thalassemia and 20 cases of age and sex matched healthy controls were included. Prothrombin time(PT), Activated partial thromboplastin time(aPTT), Fibrinogen, Protein C and Antithrombin levels were done by using automated Sysmex CA600 analyser. These parameters were compared between thalassemia patients and healthy 
controls, and thalassemia major and intermedia patients using $\mathrm{T}$ test. Correlation of serum ferritin with coagulation parameters was studied using Pearson correlation coefficient.

Results: The mean age of the patients was 14.1(range 5-39) years, with male to female ratio of 1.4:1. Thalassemia major were $65 \%(\mathrm{n}=39)$ and thalassemia intermedia were $35 \%(\mathrm{n}=21)$. The baseline parameters included mean $\mathrm{Hb} 7.6 \pm 1.3 \mathrm{~g} / \mathrm{dl}$, mean MCV $71.3 \pm 8.06 \mathrm{fl}$, mean RDW $32.1 \%$ with mean age of presentation being 4.1(range, $0.8-15$ ) years. Eighty percent patients (48/60) were on iron chelation with mean age of starting chelation being 8.4 years(range,4-16). Mean values of PT (Test-14.1 $\pm 2 \mathrm{~s}$, control$12.9 \pm 1.8 \mathrm{~s}$ ) and aPTT (Test-31.8 $\pm 6 \mathrm{~s}$, control-28.9 $\pm 3.1 \mathrm{~s}$ ) were significantly higher $(p<0.05)$ in thalassemia patients compared to control population. Fibrinogen was low in $15 \%$ of thalassemia patients, whereas $81.6 \%$ of these patients had low Protein $\mathrm{C}$ and $45 \%$ patients had low antithrombin levels. Levels of Fibrinogen(Test$197.8 \pm 63 \mathrm{mg} / \mathrm{dl}$, control-383 $\pm 76.1 \mathrm{mg} / \mathrm{dl})$, Protein C(Test$56 \pm 30 \%$, control-95.1 $\pm 20.5 \%)$ and Antithrombin(Test$78 \pm 24 \%$, control-100.4 $\pm 15.6 \%)$ were significantly reduced $(p<$ 0.05 ) in thalassemia patients compared to control population. There was no difference in these coagulation parameters between thalassemia major and thalassemia intermedia $(p>0.05)$. These coagulation parameters did not show any correlation $(p>0.05)$ with serum ferritin $(1265 \pm 1193.1 \mathrm{ng} / \mathrm{ml})$.

Discussion \& Conclusion: Mean PT and aPTT were significantly increased in thalassemia patients compared to control population. Levels of fibrinogen, protein $\mathrm{C}$ and antithrombin were significantly reduced in thalassemia patients compared to control population. However, there was no difference in these coagulation parameters between thalassemia major and Thalassemia intermedia patients. Hence, thalassemia is a hypercoagulable state irrespective of transfusion dependence and iron overload status.

\section{Hereditary Pyropoikilocytosis Misdiagnosed As Congenital Dyserythropoietic Anemia: A Diagnostic Challenge}

Manu Jamwal $^{* 1}$, Anu Aggarwal ${ }^{1}$, Prashant Sharma ${ }^{1}$, Arindam Maitra $^{2}$, Deepak Bansal ${ }^{3}$, Amita Trehan ${ }^{3}$, Man Updesh Singh Sachdeva ${ }^{1}$, Reena Das ${ }^{1}$

${ }^{1}$ Hematology, PGIMER, Chandigarh, ${ }^{2}$ NIBMG, Kalyani, Kolkata, ${ }^{3}$ Pediatrics (Hematology-Oncology Unit), PGIMER, Chandigarh, India

Aims \& Objectives: Congenital dyserythropoietic anemias (CDA) are a rare group of inherited anemias characterized by reticulocytopenia, distinct erythroblast morphology in the marrow with/without splenomegaly. Clinical features often overlap with hemolytic anemias. Hereditary pyropoikilocytosis (HPP) is an extremely rare membranopathy caused by mutations in spectrin (SPTA1 and SPTB) or protein 4.1 genes. Targeted NGS-based resequencing has emerged as the technique-of-choice in hemolytic disorders with confusing/ obscure diagnoses.

Patients/Materials \& Methods: Two patients who were initially suspected to have CDA based on the clinical and bone marrow findings and were later found to have HPP on NGS are described.

Results: Two unrelated infants presented with anemia requiring transfusions, low reticulocyte counts and hepatosplenomegaly. Bone marrow features were suspicious of CDA (nuclear budding, karyorrhexis, and binucleation in $10 \%$ and $04 \%$ erythroblasts respectively). However, the common SEC23B exon 12 c.1385A $>$ G mutation was negative; hence both underwent NGS. DNA libraries prepared using TruSeq Custom Amplicon v1.5 panel (Illumina's) were sequenced on a HiSeq instrument. Diagnostic findings were as follows: Case 1 (6 m/Male): A novel homozygous SPTB variant p.Glu2075Gln was found. A pathogenic variant at the same amino acid position "Spectrin Chiang Mai" (p.Glu2075Gly) is previously reported in compound heterozygosity with other SPTB variants. The patient underwent splenectomy, but without reduction in transfusion requirement. His parents were both heterozygous of the variant and showed elliptocytes on peripheral smears corroborating the genetic findings. Case $2(6 \mathrm{~m} /$ Female): She had a complex SPTA1 genotype: She had homozygous $\alpha$ LELY variant c.[5572C $>$ G;6531-12C $>$ T] (a low- expression allele) along with a novel heterozygous splice donor variant c. $1112+2 \mathrm{~T}>\mathrm{A}$. Her mother also had homozygous $\alpha$ LELY and the same heterozygous splice donor variant. She inherited another novel heterozygous missense variant c.6145G $>$ C (p.Ala2052Pro) from her father who also had heterozygous $\alpha$ LELY. Her peripheral smear revealed microspherocytes with elliptocytes and erythroblasts. Blood film of her mother showed microspherocytes (consistent with hereditary spherocytosis) while her father showed elliptocytosis, strongly substantiating the genetic findings. Neither case showed any pathogenic variants in the genes implicated in CDAs.

Discussion \& Conclusion: These are the first molecularly confirmed cases of HPP reported from India. This severe, early-onset rare cause of hemolytic anemia can be confused easily with CDA. Early resort to NGS is possibly the best diagnostic option, as splenectomy has been described to be less useful in this disorder.

\section{Childhood Autoimmune Haemolytic Anemia: An Observational Study on Clinico-Haematological Profile and Consequences}

Shrutiprajna Kar ${ }^{* 1}$, Delhi K. C.G. ${ }^{1}$, Rakhee $\mathrm{Kar}^{2}$, Abhishekh B. Gowda $^{3}$

${ }^{1}$ Pediatrics, JIPMER, ${ }^{2}$ Pathology, India, ${ }^{3}$ Transfusion Medicine, JIPMER, Pondicherry, India

Aims \& Objectives: To analyze the clinico-haematological profile of childhood AIHA in our hospital setting. To explore the disease outcome in terms of treatment response, relapse rate and associated mortality.

Patients/Materials \& Methods: This was a retro-prospective observational study based in a tertiary healthcare setup. During 2013 to 2020, forty-six consecutive cases diagnosed with AIHA and positive Direct antiglobulin test (DAT), aged between 1 and 13 years, were included. A pre-structured proforma was used to document data on the clinical presentation, laboratory investigations, treatment and eventual outcome.

Results: Majority of the children manifested AIHA in the setting of an underlying systemic illness (29 cases; 67\%). The most common systemic etiology associated with AIHA was systemic lupus erythematosus (SLE) diagnosed in 13 children (28\%). The mean age of onset was 8.7 years. Fatigue $(80.4 \%)$, pallor $(100 \%)$, hepatomegaly (72\%) and splenomegaly (48\%) were the most common clinical findings. The symptom-complex of breathlessness, jaundice and CCF were significantly more common in the primary type. Correspondingly, laboratory parameters like LDH $(p=0.026)$ and unconjugated bilirubin $(p=0.014)$ were significantly higher in primary AIHA. Response to glucocorticoid therapy in the initial episode was noted in $85 \%$ of the cases. Intravenous immunoglobulin was administered concurrently in six children with secondary AIHA after establishing the primary diagnosis. In children with unsatisfactory response/relapse, additional immunosuppression was tried with rituximab, cyclophosphamide, mycophenolate mofetil, and azathioprine. At the end of our study period, 13 out of 17 children (71\%) with primary AIHA were in complete remission. Four of them required additional immunosuppression for control of disease activity. Of the children with secondary AIHA, 17 (59\%) were in partial remission with second-line immunosuppressive agents; four were in complete remission 
off drugs and seven had expired. On multinomial regression analysis, children more than five years and those with the mixed immunophenotype were at less risk of relapse on follow-up.

Discussion \& Conclusion: In conclusion, secondary AIHA in children was more common than the primary type. Whilst children with primary AIHA presented with severe hemolysis, the outcome of secondary type was complicated by multiple relapses, higher mortality rate and often required second-line immunosuppressive therapy for sustained remission.

\section{Anemia and Inflammation in Covid Patients: A Retrospective Study in a Tertiary Health Care Centre} Avnish Gaur ${ }^{* 1}$, Nikita Goyal ${ }^{1}$

\section{${ }^{1}$ Medicine, Index Medical College, Indore, Indore, India}

Aims \& Objectives: Anemia commonly aggravates the severity of all respiratory illness. There are few studies exploring the relationship between anemia and severity of Covid 19 .

Patients/Materials \& Methods: This single center retrospective observational study is based on recently published guidelines and imported according to PRISMA. The study was conducted in September 2020 covering 20 Covid positive anemic patients. We have used clinical characteristics and laboartory findings to analyse the prognosis.

Results: Covid positive anemic patients show more co- morbid conditions than non anemic patients. In Covid positive patients, there is an increase in ferritin levels, LDH and CRP levels. Ferritin is known to be elevated in inflammatory conditions, with hyperferritinemia being a key acute phase reactant used by the clinicians as a marker for key response. Moreover, many of the patients also developed cellulitis over time.

Discussion \& Conclusion: Anemia is an independent and very important risk factor for complicating the severity in Covid positive patients. Also, high ferritin levels are a major finding in elderly males who are hypertensive. Therefore, clinician should be more sensitive on level of hemoglobin on admission of the patients in order to avoid the complications due to anemia.

\section{Supporting Document:}

\begin{tabular}{|c|c|c|c|c|c|c|c|c|}
\hline S.No, & Age & Sex & $\mathrm{Hb}$ & $\mathrm{MCV}$ & $\mathrm{MCH}$ & RDW-CV & Ferritin & $\begin{array}{c}\text { CRP } \\
\text { Quantitative }\end{array}$ \\
\hline 1 & 68 & M & 11.5 & 75.1 & 24.5 & 18.9 & 1008 & 23.31 \\
\hline 2 & 62 & $\mathrm{~F}$ & 9.3 & 75.5 & 24.8 & 19.2 & 475.5 & 20.06 \\
\hline 3 & 38 & $\mathrm{~F}$ & 7,6 & 69.7 & 20.5 & 26.8 & 542 & 28.6 \\
\hline 4 & 60 & $F$ & 9.9 & 77.7 & 26.3 & 16.2 & 636 & 27.5 \\
\hline 5 & 63 & $\mathrm{~F}$ & 8.3 & 68.8 & 20.7 & 17.8 & 680 & 62.6 \\
\hline 6 & 25 & M & 7.6 & 76.3 & 25.4 & 16 & 311 & 71.4 \\
\hline 7 & 46 & $M$ & 9.7 & 69.9 & 22.1 & 16.1 & 137 & 31.5 \\
\hline 8 & 37 & $\mathrm{~F}$ & 10 & 55.8 & 18.8 & 17.5 & 709 & 2.06 \\
\hline 9 & 60 & $F$ & 9.6 & 62.4 & 19.5 & 16.7 & 1850 & 2.51 \\
\hline 10 & 60 & M & 11.8 & 70.5 & 20.2 & 18.5 & 1072 & 0.86 \\
\hline 11 & 20 & $\mathrm{~F}$ & 7.4 & 79.5 & 25.2 & 16.7 & 454 & 10.78 \\
\hline 12 & 8 & $F$ & 7.8 & 67.3 & 19.2 & 19.9 & 83.25 & 11.36 \\
\hline 13 & 70 & $F$ & 10.5 & 72.7 & 22.4 & 17.8 & 83.51 & 0.15 \\
\hline 14 & 30 & $\mathrm{M}$ & 7.3 & 58.2 & 20 & 18.7 & 1800 & 20.06 \\
\hline 15 & 60 & $M$ & 5.8 & 76 & 25 & 16.5 & 970 & 10.18 \\
\hline 16 & 66 & $M$ & 10 & 52.7 & 15.5 & 22.6 & 73.92 & 3.48 \\
\hline 17 & 63 & $\mathrm{~F}$ & 10.5 & 75.9 & 21.7 & 27.3 & 1750 & 2,02 \\
\hline 18 & 75 & M & 12.1 & 78.8 & 20.7 & 18.5 & 814.8 & 2.1 \\
\hline 19 & 54 & $\mathrm{~F}$ & 12.2 & 75.9 & 26.1 & 16.6 & 1061 & 15.33 \\
\hline 20 & 75 & $\mathrm{~F}$ & 10 & 72.3 & 26.6 & 16.8 & 869 & 18.7 \\
\hline
\end{tabular}

\section{The Accuracy of Physical Examination to Diagnose Anemia: A Systematic Review of the Literature}

Neha Vyas" ${ }^{* 1}$, Oluwatoyosi Onwuemene ${ }^{2}$, Stephanie Hendren ${ }^{2}$, Tushar Sehgal ${ }^{3}$, Charu Monga ${ }^{4}$, Rajeev Ranjan ${ }^{3}$, Harsh Chaturvedi ${ }^{4}$, Subramanian Arulselvi ${ }^{3}$, Vishal Vashistha ${ }^{5}$

${ }^{1}$ Duke University, ${ }^{2}$ Duke University Medical Center, Durham, United States, ${ }^{3}$ All India Institute of Medical Sciences, Delhi, ${ }^{4}$ Indian Institute of Technology, Guwahati, India, ${ }^{5}$ Hematology and Oncology, Raymon G. Murphy New Mexico Veterans Affairs Medical Center, Albuquerque, United States

Aims \& Objectives: Anemia remains a significant public health challenge, with a disproportionate impact on lower-income patients residing in areas of lesser healthcare resources. These patients frequently share limited access to routine laboratory testing, and as a result, physical examination still serves as a critical tool for anemia assessments. We sought to evaluate the accuracy of clinical exam techniques to diagnose anemia among patients 5 years or older.

Patients/Materials \& Methods: A systematic review of five databases (MEDLINE via OVID, EMBASE, Scopus, Global Health and Global Health Archives, and WHO Global Index Medicus) was conducted to evaluate studies published before February 2020. Studies that (1) compared non-invasive physical exam techniques with anemia diagnoses using standard laboratory measurements and (2) individually reported the diagnostic accuracy of physical exam techniques for patients 5 years or older were considered for inclusion. The diagnostic accuracies of individual and collective physical exam techniques to diagnose anemia were documented. This systematic review was registered with PROSPERO.

Results: The systemic literature search yielded 6,457 unique studies after removal of duplicates. Following screening of titles and abstracts, 97 studies were selected for full-text analysis. Of the 14 selected publications, 8 studies solely assessed pregnant females, 3 solely assessed hospitalized patients, and 3 evaluated the general population. Five studies were conducted in South Asia, and 4 were conducted in Africa. The most frequently evaluated physical exam technique was assessment of clinical pallor, and the anatomical sites most often examined were conjunctivae, nail bed, and palms. The diagnostic accuracy ranged widely for pallor assessments of conjunctivae (sensitivity: 19\% - 97\%, specificity: 65\% - 100\%), nailbed (sensitivity: $41 \%-65 \%$, specificity: $58 \%-93 \%$ ), and palms (sensitivity: $33 \%-91 \%$, specificity: 54\% - 93\%). Similarly, sensitivity and specificity of collectivizing at least two or more physical exam findings to diagnose anemia ranged from $26 \%$ to $96 \%$ and $13 \%$ to $99 \%$, respectively.

Discussion \& Conclusion: The wide range in previously reported associations between exam findings and anemia diagnoses is likely due to heterogenous populations being investigated. More robust studies are required to identify the most optimal exam technique or combination of techniques to screen and/or diagnose anemia among patients served in areas of lesser-resources.

\section{Degree and Severity of Anaemia in Children with Cerebral Palsy: A Case Control Study}

Namita Gwasikoti*1, Kapil Bhalla ${ }^{1,2}$

${ }^{1}$ Pediatrics, PT. B D Sharma, PGIMS, Rohtak, ${ }^{2}$ Pediatrics, PT. B D Sharma, PGIMS Rohtak, Rohtak, India

Aims \& Objectives: This study was planned to look for nutritional status and degree of anaemia by evaluating anthropometric indices, $\mathrm{RBC}$ indices and serum ferritin values in children with cerebral palsy and compare it with normal children of same age. 
Patients/Materials \& Methods: The present study was conducted in 60 children with cerebral palsy, presenting to the Pediatric OPD of a tertiary care hospital. 30 age matched controls were also taken with similar parametrs. Anthropometric analysis was done and blood samples were taken for RBC indices and serum ferritin levels.

Results: Serum ferritin and haemoglobin concentration was found to be significantly lower in children with $\mathrm{CP}$ as compared to age matched controls. These patients had a statistically significant difference in S. Ferritin levels and RBC indices when compared to the control group $(p=<.001)$. Children with $\mathrm{CP}$ also had high prevelance of wasting and stunting as per WHO norms.

Discussion \& Conclusion: Children with cerebral palsy had severe iron deficiency anemia which was accentuated with poor nutritional status in these children.

\section{Hereditary Spherocytosis}

Suresh Kaundiyal ${ }^{* 1}$, Abhinav Singh ${ }^{2}$, Kunal Das ${ }^{3}$, Mansi Kala ${ }^{4}$, Meghali Dhebane ${ }^{5}$, Anuradha Kusum ${ }^{6}$

${ }^{1}$ Resident pathology, ${ }^{2}$ Senior resident pathology, Himalyan institute of medical sciences, ${ }^{3}$ Associate professor pediatrics, Himalayan institute of medical sciences, ${ }^{4}$ Associate Professor Pathology, ${ }^{5}$ Assistant professor pathology, ${ }^{6}$ Professor Pathology, Himalyan Institute of Medical Sciences, Dehradun, India

Aims \& Objectives: To establish the role of clinicopathological correlation and molecular study in the diagnosis of hereditary spherocytosis.

Patients/Materials \& Methods: Forty year old male came with the complain of weakness and jaundice at our hospital. He was diagnosed as Gilberts disease outside at a tertiary care centre. Physical examination revealed splenomegaly. He was pale and had indirect hyperbilirubinemia. Liver enzymes were within normal limit. There was no family history of jaundice and anemia. Peripheral examination showed homogenous population of spherocytes. However serum iron studies, vitamin B12/folic acid levels and hemolytic work up, all were within normal limit. In view of above findings, a possibility of hemolytic anemia was suggested despite a normal hemolytic work up. Next generation sequencing was advised to rule out any concerned mutations.

Results: NGS detected a heterozygous splice region variant affecting the genomic sequence encompassing coordinates chr 14:65241110. This variant is an intronic variation as numbered according to metatranscript NM _ 001024858.2:c. $4973+5 \mathrm{G}>$ A of SPTB.

Discussion \& Conclusion: Hereditary spherocytosis (HS) is a hereditary hemolytic anemia, with wide heterogeneity in the severity of its clinical symptoms, membrane protein defects, and genetic patterns. HS is characterized by anemia, jaundice, splenomegaly, and cholelithiasis. There are 5 genes associated with HS, including $\alpha$ spectrin (SPTA1), $\beta$-spectrin (SPTB), ankyrin (ANK1), band 3 (SLC4A1), and protein 4.2 (EPB42), that are involved in the interaction between the erythrocyte membrane and the lipid bilayer. Test like osmotic fragility are neither sensitive nor specific for hereditary spherocytosis, hence a clinical suspicion should always be correlated with molecular studies whenever the laboratory findings are not contributory.
Accuracy of Clinical Pallor in The Diagnosis of Anemia in Children Aged 6 Months to 5 Years

Yamini. $\mathbf{S}^{* 1}$, Saiprasad O. Kavthekar ${ }^{2}$, Mohan Patil ${ }^{2}$

${ }^{1}$ Pediatrics, ${ }^{2}$ Pediatric, Dr.D.Y. Patil Educational Society and

Deemed University, Kolhapur, India

Aims \& Objectives: To study sensitivity, specificity and predictive value of pallor at palpebral conjunctiva, tongue, nail bed and palm in diagnosing anemia and correlate pallor with hemoglobin.

Patients/Materials \& Methods: 400 children aged between 6 months to 5 years out of which 200 having pallor(Case) and 200 no pallor(Control), admitted at Department of Pediatrics, D.Y.Patil Medical college and hospital, Kolhapur from October 2016 to March 2018 were included in this study. Palpebral conjunctiva, tongue, nailbed and palms were examined for pallor. Hemoglobin estimation were done by Drabkin's method in all children. Anemia was defined according to WHO criteria $(\mathrm{Hb}<11 \mathrm{~g} / \mathrm{dl}) 2$. Data was analyzed statistically.

Results: Among 200 children with pallor, tongue pallor was identified in maximum $(79 \%)$, palmar pallor in minimum $(61 \%)$. Mean hemoglobin in pallor group ranged from 8.7 to $9.04 \mathrm{~g} / \mathrm{dl}$ and 10.96 to $11.1 \mathrm{~g} / \mathrm{dl}$ in no pallor group. Hemoglobin was lowest with tongue pallor $(8.7 \mathrm{~g} / \mathrm{dl})$. Children with pallor group, $160(71 \%)$ had anemia and no pallor group $140(77 \%)$ had no anemia. Maximum sensitivity and specificity were for tongue pallor $(59.5 \%)$ and palmar pallor $(92.2 \%)$ respectively and maximum predictive value was for palmar pallor $(88.2 \%)$.

\begin{tabular}{lllll}
\hline Pallor site & Sensitivity Specificity & $\begin{array}{l}\text { Positive } \\
\text { Predictive } \\
\text { value }\end{array}$ & $\begin{array}{l}\text { Negative } \\
\text { Predictive } \\
\text { Value }\end{array}$ \\
\hline Conjunctiva & $55 \%$ & $87.22 \%$ & $85.80 \%$ & $61.30 \%$ \\
Tongue & $59.50 \%$ & $85.0 \%$ & $82.90 \%$ & $63.20 \%$ \\
Nail bed & $56.8 \%$ & $89.4 \%$ & $86.8 \%$ & $62.8 \%$ \\
Palm & $49.0 \%$ & $92.2 \%$ & $88.5 \%$ & $59.70 \%$ \\
\hline
\end{tabular}

Discussion \& Conclusion: Pallor was found to be very useful in detecting anemia also it had more specific than sensitive value. The best predictor site for pallor for diagnosing anemia was palm. This study would be of great help for Pediatricians and Medical Professionals who are working at remote areas where there is no laboratory facilities in diagnosing anemia by assessing pallor in children aged 6 months to 5 years.

\section{Unusual Presentation of a 'Not So Uncommon' Entity}

Khan Ummiya Afreen ${ }^{* 1}$, Ramya L. Veduruvada ${ }^{1}$, Shantveer Uppin 1, Swaroopa Reddy ${ }^{2}$, Tara R. Paul ${ }^{1}$

${ }^{1}$ Pathology, ${ }^{2}$ Internal Medicine, NIMS, Hyderabad, India

Aims \& Objectives: HbS/ß- Thalassemia is a common sickling disorder, clinically resembling sickle trait and presenting with splenomegaly. Patients presenting with bone and muscle pain and imageologic evidence of altered signal intensities in thoracolumbar spine, are rarely reported.

Patients/Materials \& Methods: An 18 year old male, with no significant family history, presented with fever, bone and muscle pains of 20 days duration. The pain was in the back, sacrum and bilateral 
thighs, non-radiating, not associated with any swelling. The fever was mild to moderate, not associated with chills or rigors. On examination, he had pallor, icterus and $2 \mathrm{~cm}$ palpable splenomegaly.

Results: Investigations revealed microcytic, hypochromic anemia with normal leucocytes and platelets. The smear also showed target cells, tear drop cells, polychromatophils and elongated RBCs but no sickle cells. Total bilirubin and LDH were mildly elevated. PET-CT and MRI revealed altered signal intensities in thoraco-lumbar vertebrae and were opined as marrow infiltrative disorder- possibly Infective (tuberculosis) or due to an MPN. Bone marrow aspirate was aparticulate with imprints showing varying cellularity. The biopsy revealed extensive areas of myelonecrosis, aggregates of histiocytes, but there was no evidence of granulomas or malignancy. Stains for AFB were negative. When the sections were reviewed, the vessels showed sickle cells. A sickling test and HPLC were requested. The sickling test was positive and HPLC revealed $\mathrm{Hb} \mathrm{S} / \mathrm{B}+$-thalassemia(HbA2- 5.9\%, HbS- 65.4\%, HbF- 3.8\% and HbA- 24.9\%). The patient had no significant past or family history. The reviewed smears did not show sickle cells. In $\mathrm{HbS} / \mathrm{ß}$-thalassemia, there may be pronounced microcytosis, variable hypochromia, and numerous target cells. Variable amounts of $\mathrm{Hb} \mathrm{A}$ dilute $\mathrm{Hb} \mathrm{S}$ then inhibit polymerizationinduced cellular damage; hence fewer sickle cells are seen. The patient was given symptomatic treatment and discharged.

Discussion \& Conclusion: Myelonecrosis occurs in a wide variety of neoplastic and non-neoplastic conditions. In sickle cell anemia, vascular occlussion can occur, leading to infaction. Bone infarcts can be associated with low grade fever and pain. This is a rare presentation of sickle cell- $\beta$ thalassemia, the formalin fixed trephine sections highlighted the sickle cells which were not picked up earlier.

\section{Echocardiography Changes in Patients of Age 6-18 Years in Beta Thalessemia Major}

Priyanka Chhari $^{* 1}$, Kapil Bhalla ${ }^{1}$

${ }^{1}$ Paediatrics, Pt. B D Sharma PGIMS, Rohtak, India

Aims \& Objectives: To assess cardiac abnormalities in patients age 6-18 years with transfusion dependent beta thalassemia major.

Patients/Materials \& Methods: A prospective observational study was conducted in thalassemia day care centre of a tertiary care hospital. 30 patients with transfusion dependent beta thalassemia major were enrolled and were evaluated in detail including history,clinical examination, serum ferritin levels and echocardiography.

Results: out of total 30 patients 18 were male and 12 female $(3: 2)$, diastolic dysfunction assessed by (E/A) ratio which was $>1.5$ in 19 patients $(63 \%)$ and serum ferritin level was raised in 20 patients(60\%).

Discussion \& Conclusion: Thalassemia major patients who are non compliant to iron chelation therapy and those having high serum ferritin levels have significant cardiac dysfunctiuon and diastolic dysfunction which is an early sign of myocardial injury.

\section{Systemic Lupus Erythematosus Mimicking Vaso- Occlusive Crisis In Sickle Cell Disease: A Case Report}

Rakesh Mohanty $^{* 1}$, Atanu Thakur ${ }^{1}$

${ }^{1}$ General Medicine, VSS Institute of Medical Science and Research, Burla, India

Aims \& Objectives: Sickle cell anaemia is one of the most prevalent genetic disorder. It is rarely associated with connective tissue disorders.Sickle cell disease patients often present with multiple joint pain and arthralgia attributed to vaso-occlusion. These symptoms are similar to those of patients with connective tissue disorder. This often results in delay in diagnosis of the connective tissue disorder associated with sickle cell disease. Herein, we report a case of 20 year-old female with known sickle cell disease who was later diagnosed to also have associated systemic lupus erythematosus.

Patients/Materials \& Methods: PATIENT- A 20 year-old female patient admitted to department of general medicine, VIMSAR, Burla. MATERIALS and METHODS. Place of study - VSS institute of medical science and research sickle cell disease was established by sickling slide test and haemoglobin electrophoresis. Systemic lupus erythematosus was established fulfilling the ACR/EULAR criteria.

Results: ANA and anti DsDNA titres were positive for systemic lupus erythematosus. Sickling slide test and Haemoglobin electrophoresis revealed sickle cell disease. She was then diagnosed as case osf systemic lupus erythematosus with sickle cell disease. After establishing systemic lupus erythematosus in known patient of sickle cell disease, she was started on steroids and hydroxychloroquine. Her symptoms improved and both her disease were under control.

Discussion \& Conclusion: DISCUSSION: In this case report we described a 20 year old female with coexistant systemic lupus erythematosus along with sickle cell disease. Systemic lupus erythematosus being a great imitator has a variety of clinical manifestations. Due to limited number of case reports of associated lupus erythematosus in patients with sickle cell disease the diagnosis of systemic lupus erythematosus is usually missed. Such patients usually lack skin manifestations as previously reported but usually had articular manifestations attributed wrongly to sickle cell crisis. Further the diagnosis of systemic lupus erythematosus had been established several years after the diagnosis of sickle cell disease was made owing to overlap of clinical features. CONCLUSION: This report illustrates the importance of searching for other associated diseases when a patient of sickle cell disease who is on appropriate therapy presents multiple times with the same complaints. Early initiation of appropriate treatment in these patients may decrease morbidity, mortality and improve the condition of living.

\section{A Case of Auto-Immune Hemolytic Anemia Associated with Covid-19}

Shreyas Hanmantgad*1, Rohith Chitrapur ${ }^{1}$, Mahalakshmi V. ${ }^{2}$, Girish T.S. ${ }^{2}$, Indu Nair ${ }^{2}$

${ }^{1}$ Haematology and Bone Marrow Transplant, ${ }^{2}$ Internal Medicine, Bangalore Baptist Hospital, Bengaluru, India

Aims \& Objectives: SARS-COV2 infection or COVID-19 usually presents as sore throats and dry cough leading on to pneumonia or respiratory distress in its worst forms. Auto-immune Hemolytic anemia with a positive direct coombs test associated with SARSCOV2 has been described in case reports. This is a case-report of a 28 years old man presenting with AIHA leading to a diagnosis of COVID-19. Very few of such cases have been reported so far from India.

Patients/Materials \& Methods: A 28 years old unmarried male with no known co-morbid illnesses presented to the OPD with history of jaundice and easy fatigue of 10 days. He denied history of sore throat, fever and other covid-19 related symptoms. He consumed a mixed diet with occasional consumption of alcohol. He denied history of taking alternative medicines for any symptoms in the present or past. On examination, he was afebrile but had tachycardia with a heart rate of $120 \mathrm{bpm}$ with normal blood pressure and oxygen saturation. He had icterus with mild pallor. Neck veins were engorged but the JVP was normal. He had no signs to suggest congestive cardiac failure. There were no enlarged lymph nodes. Liver and spleen were not palpable. 
Results: Investigations revealed a hemoglobin of $4 \mathrm{~g} / \mathrm{dL}$ with normal white blood cells and platelets. Peripheral smear showed predominant normocytic red blood cells with features of hemolysis. No abnormal cells or parasites were seen. Reticulocytosis was seen with a high proliferation index. He had indirect hyperbilirubinemia with an elevated lactate dehydrogenase. Direct Coombs test was $3+$ positive. No compatible red blood cells units could be found by coombs crossmatch. ANA screen was 2 + Fine speckled positive but no significant autoimmune antibodies could be identified in the profile. Bone marrow aspirate and biopsy showed mild megaloblastic changes but no marrow infiltrating disease. Mycoplasma pneumoniae IgM was negative. He developed high grade fever in the hospital. On probing, he admitted to having fever with cough prior to admission. RT PCR for SARS-COV2 was positive. He was transferred to an isolation ward and managed as per COVID -19 treatment guidelines. He was classified as mild COVID-19 and did not require steroids. He was transfused least incompatible packed red cell units. He was also treated with azithromycin to cover for atypical bacterial infections. He remained stable and staged a rapid recovery. On follow up after two weeks after discharge, he remained asymptomatic with a hemoglobin of $10.2 \mathrm{~g} / \mathrm{dL}$ and no evidence of hemolysis on the blood picture.

Discussion \& Conclusion: Autoimmune hemolysis can be one of the rare presentations of COVID-19. More studies are needed to understand the pathophysiology of the same.

\section{FNAC of Spleen in a Case of Hemophagocytic Lymphohistiocytosis Presenting as Puo with Pancytopenia}

\section{Subhash C. Jha ${ }^{* 1}$ \\ ${ }^{1}$ Hematology, New Gardiner Hospital, Patna, India}

Aims \& Objectives: To present rare hematological and immunological disorder Hemophagocytic lymphohistiocytosis with major hematological manifestation. Importance of FNAC of Spleen and bone marrow aspirate in diagnosis of this disease is especially shown in this paper.

Patients/Materials \& Methods: Hemophagocytic lymphohistiocytosis is due to abnormal and exaggerated immune response to infectious and non-infectious agents. The most common presentation is fever with palpable splenomegaly and pancytopenia. In this case, a young thin girl presented with moderate grade fever and huge splenomegaly (spleen palpable $24 \mathrm{cms}$ below left costal margin) and pancytopenia with severe normocytic anemia of six months duration.. Serological markers, parasitic a, fungal and bacterial cultures done, were normal.. However, her bone marrow and splenic aspirate smears were strongly positive for hemophagocytosis. These morphological findings were also confirmed by biochemical results of high triglycerides, serum ferritin and low plasma fibrinogen level.

Results: This rare case presented to hematology centre and finally diagnosed as Hemophagocytic syndrome and treated with HLH-94 protocol, she became afebrile just after one cycle of HLH 94 protocol and her splenic size was decreased markedly and pancytopenia was corrected gradually.

Discussion \& Conclusion: Now after completion of six cycles of therapy, she is asymptomatic for more than one year, however she still has mild splenomegaly and mild anemia, but her WBC and platelet counts were corrected completely. She is leading normal transfusion independent and afebrile life.

Keywords: Hemophagocytosis, bone marrow, splenomegaly, HLH 94 protocol.

Supporting Document:

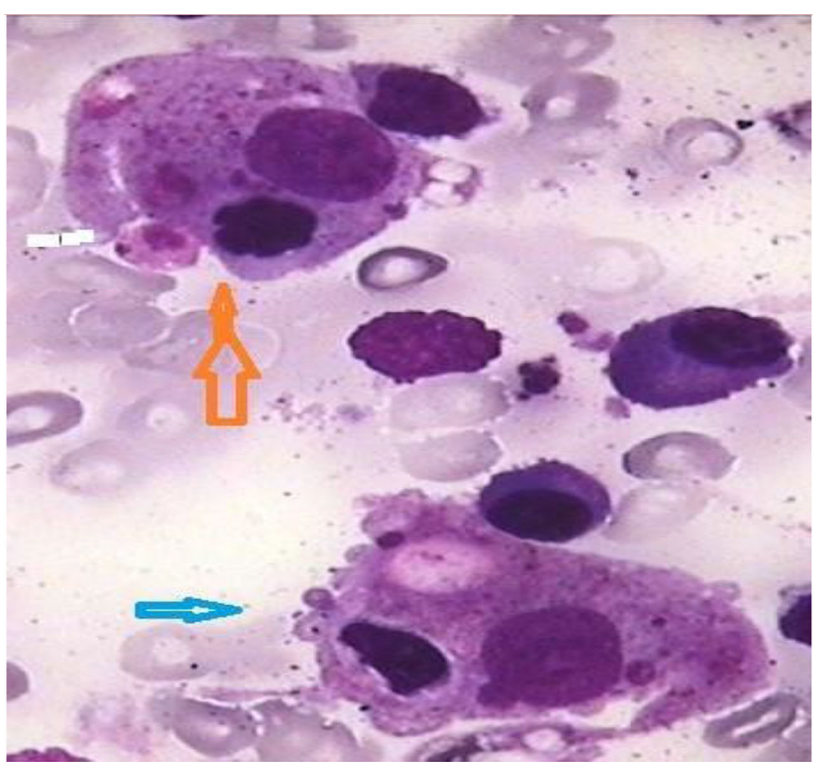

\section{An Atypical Presentation of Megaloblastic Anaemia Resembling MDS}

Sushil K. Gaur ${ }^{* 1}$, Dhiraj Kishore ${ }^{1}$, Satya P. Mahapatra ${ }^{1}$, Saket Ramraika $^{1}$, Anuj Kumar ${ }^{1}$

${ }^{1}$ Internal Medicine, IMS, BHU, Varanasi, India

Aims \& Objectives: Presenting an atypical case of megaloblastic anaemia resembling MDS.

Patients/Materials \& Methods: A 50 year male, resident of Balia, U.P., farmer by occupation presented with complaints of generalised weakness and easy fatiguability from 1 year, exertional dyspnea from 6 month, swelling of bilateral lower limb from 1 month. There was no other significant history. On examination: There was severe pallor, pitting edema, spleen and liver not palpable, no added heart sound, no crepts. Investigations: Baseline $\mathrm{Hb}$ was $1.4 \mathrm{~g} / \mathrm{dl}$, WBC-2600/mm3, N/L-58/40, Platelets- 10,000/mm3, MCV -138 fl, serum LDH- 1053, serum Fe/TIBC- 184/186, serum uric acid $-6.3 \mathrm{mg} / \mathrm{dL}$, serum ferritin2282 ,stool occult blood negative,thyroid profile-normal, corrected reticulocyte count $<1 \%$ GBP showed normochromic, polychromasia, anisopoikilocytosis, macro-ovalocytes, hypersegmented neutrophils few nucleated RBC s/o dimorphic anemia with pancytopenia. Bone marrow aspiration examination showed Hypercellular marrow, M:E- 2:1 with many giant metamyelocytes, giant band form, ringed neutrophils, few myeloid dysplastic cells, nuclear cytoplasmic asynchrony, with no of blast cells.serum vit b12 was 50.2, folic acid $7 \mathrm{ng} / \mathrm{ml}$. USG abdomen was within normal limit.

Results: Patient was managed with injection eldervit, 4 unit PRBC, 5 unit RDP and other conservative measures. Retic count on Day 3 of eldervit was $<1 \%$. LDH on day 5 of eldervit was 410 . after 4 units of rbc transfusion, 6 units of rdp transfusion, 5 days of eldervit -hb was 5.9 , tlc- 4819 , platelet- 20,000 , mcv -92 , rdw- $22 \%$. On day 8 of eldervit retic count was $18.2 \%$, hb-7.2, tlc-8330, platelet- 2.31 , mcv 96.

Discussion \& Conclusion: A 50 year male presenting with severe pancytopenia (hb-1.4gm/dl, plt-10 k, wbc-2600/micro lit), with no bleeding manifestations. after ruling out MDS, viral infections, drug induced, leukemias, eldervit trial was given. patient started to improve clinically biochemical parameters started to show improving 
trend. although uncommon, megaloblastic anemia can present with severe pancytopenia at elderly age with no neurological manifestations. Megaloblastic anemias is based on ineffective erythropoiesis usually caused by deficiency of either vit-b12 or folate deficiency and related to defect in DNA synthesis. The presence of unusual large, structurally abnormal immature red blood cells called megaloblast is the morphologic feature. Myeloidysplastic syndrome (MDS) disorder related to ineffective hematopoiesis, is characterised by bone marrow dysfunction. In elderly MDS and megaloblastic anemia are often difficult to distinguish from one another.

\section{Secondary Hemophagocytic Lymphohistiocytosis in an Infant with Wolman Disease}

Dhanashree Dave*1, Amit Nisal ${ }^{2}$, Sandip Bartakke ${ }^{3}$, Vibha Bafna ${ }^{3}$, Aashna Valecha ${ }^{3}$, Anjali Kelkar ${ }^{2}$, Ravindra C. Nimbargi ${ }^{2}$

${ }^{1}$ Department of Pathology, Bharati Vidyapeeth (Deemed to be University) Medical College, Hospital and Research Centre, ${ }^{2}$ Department of Pathology, ${ }^{3}$ Division of pediatric hematology and oncology, Bharati Vidyapeeth (Deemed to be University) Medical College, Hospital and Research Centre, Pune, India

Aims \& Objectives: Diagnosis of a case of anaemia in an infant with correlation of clinical presentation, bone marrow findings and other laboratory investigations.

Patients/Materials \& Methods: A 2-month-old male child, presented with 1 month history of pallor and poor weight gain. On physical examination, there was pallor, and moderate hepatosplenomegaly (liver $6 \mathrm{~cm}$ and spleen $4 \mathrm{~cm}$ palpable below the costal margin). Hemogram showed $\mathrm{Hb}$ of $5.0 \mathrm{gm} / \mathrm{dl}$, WBC count of $7800 /$ cumm, platelet count of 53000/cumm. Liver function tests were deranged with total bilirubin of $4.88 \mathrm{mg} / \mathrm{dl}$, AST $298 \mathrm{IU} / \mathrm{L}$ and ALT of $158 \mathrm{IU} /$ L. He had prolonged PT( $>180 \mathrm{~s})$ and APTT $(>180 \mathrm{~s})$ and low fibrinogen level of $108 \mathrm{mg} / \mathrm{dl}$. Serum triglycerides were $392 \mathrm{mg} / \mathrm{dl}$ and ferritin was markedly elevated at $8460 \mathrm{ng} / \mathrm{ml}$. In view of above findings, a diagnosis of Hemophagocytic Lymphohistiocytosis (HLH) was suspected. Bone marrow examination, enzyme studies and next generation DNA sequencing were done.

Results: Bone marrow examination revealed infiltration with macrophages which showed eccentric nuclei and vacuolated cytoplasm, highly suggestive of lipid storage disorder. Evidence of phagocytosis of erythroid precursor cells and lymphocytes by histiocytes was also seen (Fig.). Enzyme studies for Gaucher and Niemann-Pick disease were normal. The patient succumbed to progressive liver failure within 10 days of admission. Next generation sequencing from patient's stored DNA revealed a pathogenic homozygous mutation c.193C [T(p.Arg65Ter) in exon 3 of LIPA gene leading to the diagnosis of Wolman disease (WD).

Discussion \& Conclusion: Wolman disease is a rare autosomal recessive storage disorder resulting from complete deficiency of liposomal acid lipase enzyme. It presents in early infancy with clinical features similar to familial HLH. Similar clinical overlap has been reported previously and it is important that clinicians should be aware of this rare cause of secondary HLH. Moreover, histiocytes with marked clearing and vacuolation may not always signify the more common storage disorders like Gaucher and Niemann-Pick disease. Rarer enzyme deficiencies may also produce similar morphology picture which could raise diagnostic suspicion.
Anemia Secondary to Sodium Valproate Use in a 4 Year Old Child with Seizure Disorder

Khumanthem B. Devi*1 ${ }^{*}$, Irom A. Singh ${ }^{1}$

${ }^{1}$ Medicine, Jawaharlal Nehru institute of medical sciences, Imphal, India

Aims \& Objectives: Anemia secondary to sodium valproate use in a 4 year old child with seizure disorder.

Patients/Materials \& Methods: A case report of a 4 year old boy who was referred to hematology OPD for evaluation of anaemia. The boy had history of seizure disorder treated with sodium valproate diagnosed one year back. The parents noticed that child was feeling weak and gets tired easily, took him to a paediatrician, he noticed the child to have pallor. On investigation the child had anaemia.

Results: On investigation the child had anaemia. His cbc showed $\mathrm{Hb}$ of $4.2 \mathrm{gm} / \mathrm{dl}$, RBC of $1.64 \mathrm{million} / \mathrm{cumm}$, peripheral blood smear showed normocytic normochromic with macrocytic red cells, increased polychromasia and occasional nRBCs. The reticulocytes count was $1.2 \%$. serum ferritin was $200 \mathrm{ng} / \mathrm{mcl}$. He was given one unit of red blood cell, hb improved to $8.9 \mathrm{mg} / \mathrm{dl}$ but fall down again to $5.7 \mathrm{~g} / \mathrm{dl}$ after 1 month.

On referral to hematology OPD, possibility of drug induced anaemia and pure red cell aplasia was kept. Bone marrow aspirate and biopsy was done, showed adequate megakaryocyte with $12 \%$ hypolobated forms.erythropoeisis showed normoblastic to megaloblastic maturation. Normal myeloid erythroid ratio. Perl stain showed $2+$ and no sideroblast.The biopsy showed hypocellular marrow with preponderance of erythroid elements. The megakaryocytic and granulocytic lineage elements were proportionately represented.The bone marrow examination gave diagnosis of drug induced and viral etiology. Epstein-Barr virus was not detected. bone marrow examination showed drug induced.Valproate changed to leviteracetam. $\mathrm{Hb}$ raised to $10.8 \mathrm{gm} / \mathrm{dl}$. Follow up after one month showed hb of $8.2 \mathrm{~g} / \mathrm{dl}$ but after 2 months $\mathrm{Hb}$ improved to $11.8 \mathrm{~g} / \mathrm{dl}$.

Discussion \& Conclusion: Hematological side effects of sodium valproate has been reported occasionally from a pediatric patient. Our patient had only anaemia, the child started improving after stopping the standard dose of valproate for 12 months, his $\mathrm{Hb}$ normalised after 2 months of stopping. Similar studies has been reported in patients taking higher dose or for longer duration. Studies reported hypocellular marrow myelodysplastic nature, ours show hypocellular marrow with megakaryocytic dyspoeisis. Previous studies have confirmed the role of valproic acid to alter hematopoeisis by inhibition of erythroid differentiation in experimental K562 cell lineage.Thus we suspected sodium valproate caused mild dyserythropoiesis in our patient resulting in anemia.

\section{Haemoglobin-D Disease (Iran Subtype) in Adult Male: Rare Case Report}

Harsh S. Waingankar ${ }^{* 1}$, Badal Taori ${ }^{2}$, Manjari Shukla ${ }^{2}$, Amrit Kejriwal $^{3}$

${ }^{1}$ General Medicine, Junior Resident, ${ }^{2}$ General Medicine, ${ }^{3}$ General Medicine, Professor, MGM Hospital,Kamothe, Navi Mumbai, India

Aims \& Objectives: To report a rare case of hemoglobin-d disease(Iran subtype) in adult male.

Patients/Materials \& Methods: Hemoglobin D [Hb D]is a rare form of hemoglobinopathy in homozygous form. The heterozygous disease is silent clinically. A 50 year old male presented to the Emergency Room of MGM Medical College and Hospital, Kamothe, Navi Mumbai, with chief complaints of giddiness since four days and right 
shoulder pain with yellowish discoloration of sclera for a period of three months. On examination the patient had severe pallor and icterus. Peripheral smear showed dimorphic anemia with thrombocytopenia and High performance liquid chromatography [HPLC] was suggestive of $\mathrm{Hb} \mathrm{D}$ Iran subtype.

Results: Peripheral smear showed Dimorphic anemia with thrombocytopenia with microcytic hypochromic and normocytic normochromic RBCs with anisopoikilocytosis and occasional tear drop cells. A High performance liquid chromatography [HPLC] of haemoglobin done was suggestive of Hb D Iran.

Discussion \& Conclusion: Discussion: Itano in 1951 first found a group of hemoglobinopathies in a white family and named it as Hemoglobin D ( $\mathrm{Hb} \mathrm{D}$ ) [3]. Later, Rahbar found a substitution of Glutamic Acid $\rightarrow$ Glutamine (GAA $\rightarrow$ CAA) at $\beta 22$ and classified it as Haemoglobin D-Iran (Hb D-Iran) [4].It should be noted that three other substitutions have been described at $\beta 22$ position viz. E Saskatoon (Lys), G Coushatta (Ala) and G Taipei (Gly) [5].In India, both the homozygous and heterozygous forms of the disease are very rare. In homozygous cases, such as one reported by Thornburg CD et al., $\mathrm{Hb}$ D-Iran can present with anaemia, poikilocytosis and mild hemolysis [1]. In heterozygous forms the patient remains clinically silent and hence the case is missed and remains undetected. A case has been reported by Agrawal MG et al., where $\mathrm{Hb}$ D-Iran was coexisting with $\beta$-Thalassemia [6]. Another case was reported by Gupta et al., where Hb D-Iran was present along with Hb D-Punjab [2].The diagnosis still remains an incidental finding and may remain undetected unless it presents with symptoms of other co existing hemoglobinopathies like thalassemia, sickle cell disease etc. When identified,genetic sequencing still remains the gold standard. As most cases are silent clinically, no active intervention is required for the disease.Conclusion: Hemoglobin D Iran subtype is a very rare form of hemoglobinopathy. It is mostly an incidental diagnosis when a patient presents with complaints due to other common hemoglobinopathies for example thalassemia. With newer and relatively easier technologies like capillary electrophoresis the diagnosis of $\mathrm{HbD}$ disease will become less cumbersome. However genetic sequencing plays important role in diagnosis. In above case patient presented with lone diagnosis of Hemoglobin D Iran subtype it was not associated with any other hemoglobinopathy.

\section{Familial Haemolytic Anaemia: A Mysterious Case of Hereditary Pyropoikilocytosis}

Rose Mathew ${ }^{* 1}$, Shubha Sudhakar ${ }^{1}$, Renuka Patil ${ }^{1}$, Sahana $\mathrm{KS}^{2}$, Prakash Saldanha ${ }^{2}$, Rajesh krishna ${ }^{3}$

${ }^{1}$ Pathology, ${ }^{2}$ Paediatrics, ${ }^{3}$ Hematology, Yenepoya Medical college, Mangalore, India

Aims \& Objectives: (1) To detect a case of Hereditary Pyropoikilocytosis and to differentiate it from other other causes of hemolytic anemia due to RBC membrane defects. (2) Laboratory workup of Hereditary Pyropikilocytosis.

Patients/Materials \& Methods: A 4 month old infant presented with respiratory distress and icterus. On examination, a massive splenomegaly was palpated with hepatomegaly. The peripheral blood film findings showed a severe hemolytic picture when correlated with other hematological parameters, it confirmed the diagnosis of hemolytic anemia. On further work up of the case, osmotic fragility tests was performed on the child and her parents. The test showed a similar picture of early lysis in the child and the mother. The blood film of the mother presented with a major component of elliptocytes. The blood film and osmotic fragility of the father showed morphologically normal red cells in the film and had no lysis respectively. The diagnosis tainted towards Hereditary elliptocytosis, with the consideration of underlying features, the samples of the mother and child were further investigated by performing thermal sensitivity screening, that presented with a dramatic increase of bizarre poikilocytes in the blood film. Hence, the diagnosis of Hereditary Pyropoikilocytosis was confirmed and blood transfusion was initiated, with a follow up.

Results: Hereditary Pyropoikilocytosis is a rare, autosomal recessive condition, caused by a defect in the RBC membrane due to deficiency of alpha spectrin, characterized by bizarre poikilocytes that includes red cell fragments and microspherocytes. Although the disorder has been considered a separate entity, many researchers cite convincing evidence that HPP is a subtype or "aggravated" form of Hereditary Elliptocytosis. The defect leads to cell destabilization followed by erythrocyte fragmentation due to abnormal thermal sensitivity resulting in a severe hemolytic picture.

Discussion \& Conclusion: Although Hereditary Pyropoikilocytosis is a rare entity, possibility of such rare cases should be kept in mind for accurate diagnosis and appropriate treatment and management of the patient.

Supporting Document:

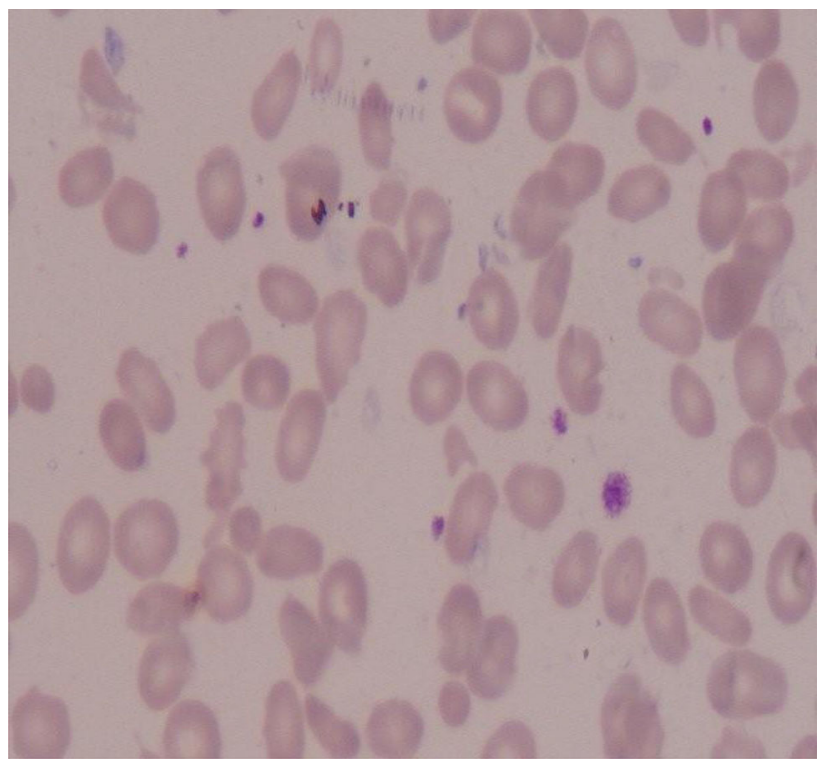

\section{Depression and Anxiety Among Children of Beta- Thalassemia: A Cross Sectional Study}

Vishal Rohilla*1, Kapil Bhalla ${ }^{1}$, Shradha Zutshi ${ }^{1}$

\section{${ }^{1}$ Pediatrics, PT.B.D. Sharma PGIMS, Rohtak, India}

Aims \& Objectives: To determine prevalence of depression and anxiety among children of beta-thalassemia receiving care at Thalassemia day care centre from caregiver's perspective.

Patients/Materials \& Methods: A cross-sectional study was done in thalassemia unit of a tertiary care hospital, which included 69 caregivers of beta thalessemia (BT) patients aged 5-14 years over a period of 3 months. RCADS-25P was used to assess all the children. $\mathrm{T}$ scores were used to assess depression and anxiety in children.

Results: Among 69 children evaluated, $43.4 \%$ were having T-scores above borderline threshold depression and $33.3 \%$ were having T-scores above clinical threshold depression. T-scores above Borderline threshold anxiety were seen in $57.9 \%$ children and above 
clinical threshold anxiety symptoms in $40.5 \%$ children.T-scores for both anxiety and depressive borderline threshold symptoms were above in $49.2 \%$ children and anxiety and borderline clinical threshold symptoms were above in $33.3 \%$ children.

Discussion \& Conclusion: Both anxiety and depressive borderline symptoms and clinical threshold symptoms are more common in patients of thalassemia major and these symptoms are associated with presence of adverse clinical factors and chronic duration of illness. Also, these symptoms were observed more common in girls as compared to boys.

\section{Beneficial Effect of Gluten Free Diet on Hemoglobin Level in Newly Diagnosed Celiac Disease Patients}

\author{
Kusum Lata ${ }^{* 1}$, Anjali Verma ${ }^{1}$ \\ ${ }^{1}$ Pediatrics, Post Graduate Institute of Medical Sciences, Rohtak, \\ Rohtak, India
}

Aims \& Objectives: To study effect of gluten free diet on hemoglobin level in newly diagnosed celiac disease patients.

Patients/Materials \& Methods: Sixty newly diagnosed celiac cases with IgA-tTG levels more than 10 folds of upper limit of normal were enrolled in the study and followed up at 6 months. Spectrum of various growth and clinical parameters were studied. Statistical analysis was performed by the SPSS version 21.0. $p$ value less than 0.05 was considered statistical significant.

Results: 60 cases were enrolled in study. After initiation of gluten free diet, hemoglobin level increased from baseline level of $7.30+1.91 \mathrm{~g} / \mathrm{dl}$ to $8.58+1.16 \mathrm{~g} / \mathrm{dl}$ after 6 months.

Discussion \& Conclusion: Gluten free diet has major role in improvement in growth parameters as well as anemia. So, early detection of celiac disease is an important step in prevention of morbidity associated with celiac disease.

\section{Beta Thalassemia Trait (BTT) With Coexistent Iron Deficiency Anaemia Versus BTT: Discriminant Parameters}

Gauri Gaur ${ }^{* 1}$, Namrata P. Awasthi ${ }^{1}$, Nuzhat Husain ${ }^{1}$, Pradyumn Singh $^{1}$

${ }^{1}$ Pathology, Dr Ram Manohar Lohia Institute of Medical Sciences, Lucknow, Lucknow, India

Aims \& Objectives: Beta Thalassemia trait (BTT) when encountered with coexistent iron deficiency anaemia (IDA) presents a diagnostic challenge. This study aims to evaluate the diagnostic utility of red cell parameters and discriminant functions (DF) to differentiate cases of BTT and BTT with coexistent IDA (BTT-IDA).

Patients/Materials \& Methods: It is a retrospective study of BTT cases where the patients were categorized into 2 groups, BTT $(\mathrm{n}=24)$ and BTT-IDA $(\mathrm{n}=23)$. Following data was retrospectively analysed and compared within the two groups: Haemoglobin $(\mathrm{Hb})$, haematocrit(HCT), red cell distribution width(RDW), red cell count, mean cell volume(MCV), mean cell corpuscular haemoglobin(MCHC), mean cell haemoglobin $(\mathrm{MCH})$. $\mathrm{CBC}$ was done on Sysmex XN 1000, Hb A2 estimation was performed on Biorad D-10 on $\mathrm{HbA} 2 / \mathrm{F}$ mode. To detect iron deficiency, serum iron, serum ferritin and total iron binding capacity were estimated on Beckman Coulter AU480 in cases with $\mathrm{Hb}<11 \mathrm{gm} / \mathrm{dl}$.Cases with $\mathrm{HbA} 2$ more than $3.5 \%$ were identified as BTT and cases with serum ferritin lower than $15 \mathrm{ng} / \mathrm{ml}$ with $\mathrm{HbA} 2>3.5$ as BTT-IDA. Following six DFs were evaluated: Shine and Lal (S \& L) index, Srivastava index (SI), England and Fraser (E \& F) index, Green and King index (G \& K),
Mentzer index (MI) and RDW index(RDWI). ROC curve analysis and diagnostics were performed on SPSS version 22.0 and Medcalc version 19.5.3

Results: Median age(range) for BTT and BTT-IDA was 24 years( 9 77 years $)$ and 25 years $(2-50$ years $)$ respectively. The male: female for BTT and BTT-IDA was 17:7 and 8:15 respectively. Results are given in table 1 . Among all the parameters studied, $\mathrm{Hb}, \mathrm{E} \& \mathrm{~F}$ index and red cell count(RBC count) resulted in highest sensitivity and specificity at the cut-off values of $<=10.9,>0.8$ and $<=5.3$ respectively for BTT- IDA.

Discussion \& Conclusion: A number of red cell parameters and DF have been reported to be helpful in discrimination of BTT and IDA, both of which present with microcytic hypochromic blood picture. However, co-existent IDA is frequently seen with BTT, especially in our country, making these parameters redundant. The current study highlights the usefulness of parameters $(\mathrm{Hb}, \mathrm{E} \& \mathrm{~F}$ index and RBC count) with newly derived cut off values for discrimination of these two conditions with very high sensitivity and specificity. These parameters can be used as a screening tool before performing specialized investigations.

Supporting Document:

\begin{tabular}{|c|c|c|c|c|c|}
\hline Inder & $\begin{array}{l}\text { Cut-off } \\
\text { value }\end{array}$ & $\begin{array}{l}\text { Area under } \\
\text { curve (ACC) }\end{array}$ & $\begin{array}{l}\text { Siginificant } \\
\text { Pvalue }\end{array}$ & Sensithitit(\%) & Specificity (\%) \\
\hline $\begin{array}{l}\text { E\&F } \\
\text { inder }\end{array}$ & 8.8 & 0.933 & 80.0001 & 95.65 & 83.33 \\
\hline $\begin{array}{l}\text { G\&K } \\
\text { inder }\end{array}$ & 375.71 & 0.855 & $\$ 0.0001$ & 73.91 & 87.5 \\
\hline $\mathrm{Bb}$ & $\Leftrightarrow 10.9$ & 0.958 & $\$ 0.0001$ & 100 & 91.67 \\
\hline $\mathrm{HCT}$ & $\Leftrightarrow 34.2$ & 0.957 & $\$ 0.0001$ & 86.96 & 95.83 \\
\hline III & $\$ 12.3$ & 0.861 & 0.0001 & 73.91 & 83.33 \\
\hline RDWII & 3236.5 & 0.841 & $\$ 0.0001$ & 69.57 & 91.67 \\
\hline $\begin{array}{l}\mathrm{RBC} \\
\text { count }\end{array}$ & $\Leftrightarrow=\int .3$ & 0.938 & 80.0001 & 91.3 & 87.5 \\
\hline SI & 34.11 & 0.766 & $\$ 0.0002$ & 56.52 & 87.5 \\
\hline
\end{tabular}

\section{High HB F Level in Sickle Beta Thalassemia Patient Leads to Milder Sickle Phenotype \\ Monika J. Gamit*1, Renuka Patil ${ }^{2}$, Sanila Rahim³ Rajesh Krishna ${ }^{4}$ \\ ${ }^{1}$ 1srt Year Post Graduate, Pathology, ${ }^{2}$ Assistant Professor, ${ }^{3}$ Assistant Professor, Pathology, Yenepoya Medical College, ${ }^{4}$ Consultant Hematologist, Hematology, Yenepoya Medical college Hospital, Mangalore, India}

Aims \& Objectives: High HB F level in sickle beta thalassemia patient leads to milder sickle phenotype.

Patients/Materials \& Methods: HbS- $\beta$ thalassemia is a sickling disorder of varying severity, which results from compound heterozygosity for sickle cell trait and $\beta$ thalassemia trait. clinical features and hematologic findings are highly variable depending on the severity of $\beta$ thalassemia.Herein, we report a case of $\mathrm{HbS}-\beta$ 
thalassemia with High Hb F level. A 12 year old boy has been followed up in our hospital for last 2 years. He is a known case of $\mathrm{HbS}-\beta$ thalassemia. Hematological parameters like CBC,Reticulocyte count and Peripheral blood smear was performed.Biochemical parameters like RFT,LFT and LDH was performed. He had recurrent admissions previously with complains of joint pains, fever accompanied by abdominal pain.6 months ago, he was started on hydroxyurea $500 \mathrm{mg}$ and this led to complete resolution of his symptoms. We also checked his $\mathrm{Hb}$ electrophoresis.

Results: His hemogram showed $\mathrm{Hb} 8.6 \mathrm{gm} / \mathrm{dl}, \mathrm{Mcv} 67.6 \mathrm{fl}, \mathrm{MCH}$ $22.5 \mathrm{pg}$, MCHC $33.2 \mathrm{gm} / \mathrm{dl}$, TLC $9.3 \times 10^{3}$, Platelet count $139 \times 10^{3}$. On peripheral smear, RBC picture showed Microcytes, target cells and few sickle cells.Reticulocyte was 5.24\%.Biochemical assessment showed Creatinine $0.4 \mathrm{mg} / \mathrm{dl}$, LDH $720 \mathrm{U} / \mathrm{L}$,Total bilirubin $4.3 \mathrm{mg} / \mathrm{dl}$,Direct bilirubin $0.5 \mathrm{mg} / \mathrm{dl}$ and indirect $3.8 \mathrm{mg} /$ d1.Hb electrophoresis showed $\mathrm{Hb}$ F- $33.3 \%, \mathrm{Hb}$ A2- 3.9\%, $\mathrm{Hb} \mathrm{S}-$ $50.4 \%$.

Discussion \& Conclusion: Discussion: Haemoglobinopathies occurs due to structural defect in the globin gene. Thalassemias are due to defect in the production of globin chain. The $\mathrm{Hb} \mathrm{S}$ is of clinical importance because of their worldwide prevalence. This is the most common form of sickle cell disease in people of Mediterranean descents.In India it is most prevelent in North-East. A case of Compound heterozygous for $\mathrm{Hb}$ S- $\beta$ thalassaemia was previously reported with severe anaemia, massive hepato-splenomegaly and acute Chest syndrome. But in our case patient showed large $\mathrm{Hb} \mathrm{F}$ response to hydroxyurea and there was significant improvement in his clinical symptoms. Conclusion: This patient shows large $\mathrm{Hb} F$ response to hydoxyurea. we postulate that this led to significant improvement in his clinical symptoms. We also wanted to discuss the morphology picture and $\mathrm{Hb}$ electrophoresis for academic purposes.

\section{A Rare Case of Haemoglobin J Diagnosed On High Performance Liquid Chromatography}

Manasi Dandekar ${ }^{* 1}$, Manish Sulya ${ }^{2}$, Rajendra Nigam ${ }^{3}$, Reeni Malik ${ }^{4}$

${ }^{1}$ II year JR Department of Pathology, ${ }^{2}$ Associate Professor Department of Pathology, ${ }^{3}$ Professor Departmrnt of Pathology, ${ }^{4}$ Professor and Head Department of Pathology, Gandhi Medical College, Bhopal, India

Aims \& Objectives: To determine the cause of anemia in a $4 \mathrm{yr}$ old boy.

Patients/Materials \& Methods: A 4 year old boy came to our laboratory for thalassemia screening. The parents and sibling of the patient were not known to have any hemoglobinopathy. His presenting complaints included short stature, loss of appetite, pallor and weakness $4 \mathrm{ml}$ Blood sample was collected in an EDTA vial and stored at 4 C. It was screened for hemoglobinopathies and hemoglobin variants by CE-HPLC (BIO-RAD D10). The hematological parameters were assessed by Mind-Ray Three Part Cell Counter.

Results: In hemoglobin electrophoresis of his blood sample by fully automated HPLC, Hb J Meerut was detected. Following findings were seen:

- There was a peak within P53 retention time window

- P3 value-23.3\%

- $\mathrm{Hb}$ A2- $0.8 \%$

- $\mathrm{Hb} \mathrm{A}-64.4 \%$

- $\mathrm{Hb}$ F-1.9\%

Discussion \& Conclusion: Fast moving hemoglobins are rare hemoglobin variants. They have a tendency to migrate anodally to hemoglobin A on alkaline gel electrophoresis. Because of the mutation in the globin genes, these hemoglobin variants are fast moving in nature. At present, hundreds of different variants of hemoglobin are known. Hemoglobin $\mathbf{J}$ was first described by Throup et al. in an African American patient in 1956. Since then more than 50 variants of $\mathrm{HbJ}$ have been described. Formation of $\mathrm{Hb} \mathrm{J}$ Meerut is due to $\mathrm{C}$ to A mutation (GCG > GAG) at codon 120 of alpha1 or alpha2 globin gene, changing the alanine to glutamic acid at residue 120 of the alpha chain. The first case of $\mathrm{Hb} \mathrm{J}$ Meerut was described in two sisters from Meerut, Uttar Pradesh and later in two Bangladeshi brothers living in Brimingham, England. Subsequently, the same abnormal hemoglobin was noted in a Japanese family and a Turkish family. U Srinivas et al. reported 7 cases of $\mathrm{Hb} \mathrm{J}$ Meerut from India. A majority of fast moving hemoglobins of $\mathbf{J}$ family do not show any clinical significance and are therefore accidently detected during family or antenatal screening by HPLC.

\section{Comparative Study Between CE-HPLC and Capillary} Zone Electrophoresis to Diagnose B-Thalassemia Major

Ratnakar M. Potekar ${ }^{1}$, Pallavi Kommoju ${ }^{* 1}$

${ }^{1}$ Patholgy, Shri B M Patil Medical College(DEEMED to be University), Vijayapura, India

Aims \& Objectives: To compare two modalities i.e., HPLC and Capillary zone electrophoresis in evaluating Beta thalassemia major. Patients/Materials \& Methods: A prospective hospital based study in the Department of Pathology (Central laboratory), BLDE (Deemed to be University) Shri B. M. Patil Medical College was conducted between 1st December 2018 to 31st May 2020.

Inclusion criteria:

- $\mathrm{MCV}<72 \mathrm{fL}$ and $\mathrm{MCH}<27 \mathrm{pg}$.

- Clinically suspected cases of hemoglobinopathy or $\beta$ thalassemia.

- First degree relatives of the cases.

Exclusion Criteria

- Patients who had undergone blood transfusion within a span of 12 weeks.

All samples were run on HPLC; Biorad D-10 and Capillary zone electrophoresis; Sebia Capillary 2 flex piercing to quantify $\mathrm{HbA} 2$, $\mathrm{HbF}, \mathrm{HbA}$, and screening of hemoglobin variants like $\mathrm{HbS}, \mathrm{HbD}$, $\mathrm{HbE}$, and $\mathrm{HbC}$.

Results: Total, we got 3 cases in our study with $\beta$-thalassemia major in which Capillary zone electrophoresis gave better results than HPLC in evaluating exact value of $\mathrm{HbF}$.

Discussion \& Conclusion: The HPLC (BIORAD D-10) variant, which we use in our laboratory, has its limitations in evaluating $\mathrm{HbF}$ values whose range is in between 0.8 and $16.5 \%$ above $16.5 \%$; it goes and elutes with HbLA1c, which is of no use for us in making a difference between thalassemia intermedia and major. As $\mathrm{HbF}$ value should be between 10 and $30 \%$ to call it as thalassemia intermedia and between 30 and $90 \%$ to call it as thalassemia major. CZE gives the exact value of $\mathrm{HbF}$, which helps to differentiate between $\beta$-thalassemia intermedia and $\beta$-thalassemia major.

\section{Mixed Type Evan's Syndrome: A Case Report}

Richard B. Michael ${ }^{* 1}$, Ashok K. Das ${ }^{1}$, Vasanthi $\mathrm{N}^{1}$, Indira Kumarappan $^{1}$

${ }^{1}$ General Medicine, Pondicherry Institute of Medical Sciences, Puducherry, India

Aims \& Objectives: Evan's Syndrome is a complex uncommon hematologic disease; it entails a sequential or simultaneous development of autoimmune hemolytic anemia (AIHA) and immune 
thrombocytopenia (ITP) and/or immune neutropenia. Exact frequency of Evan's Syndrome (autoimmune hemolytic anemia with simultaneous/sequential Immune thrombocytopenia) is unknown. Further uncommon is the Mixed Type Evan's Syndrome. This case report entails a patient with Mixed Type Evan Syndrome.

Patients/Materials \& Methods: A 47 year old lady, known Diabetic, came with easy fatigability for the past 2 weeks. Complete blood count showed a normal total leucocyte count with left shift, thrombocytopenia of 20,000 and severe anemia of $3.7 \mathrm{~g} / \mathrm{dl}$. Peripheral smear showed red cell agglutination at room temperature, predominantly normocytic with occasional microcytes with moderate anisopoikilocytosis with few tear drop cells and polychromatophils. Serum LDH was found to be 600. Ultrasound abdomen showed Hepatosplenomegaly (liver $16.2 \mathrm{~cm}$, Spleen $13.3 \mathrm{~cm}$ ) and cholelithiasis. Direct Antiglobulin Test was done which showed IgG $1+, \mathrm{C} 3 \mathrm{~d} 3+$ and Ig M was negative. Viral serology markers for $\mathrm{HIV}, \mathrm{HbsAg}$ and $\mathrm{HCV}$ were found to be negative. Malarial Antigen and Smear for Malarial parasite were negative. Scrub typhus, Dengue NS1 Antigen, and leptospira antigen were found to be negative. Paul Bunnel test for EBV virus was negative. Blood cultures and urine cultures were negative. ANA (by ELISA and Immunofluorescence) was found to be negative. Rheumatoid factor was negative. Cold Agglutination Test to detect heterophile antigens for mycoplasma pneumonia was found to be negative. Donath Landsteiner Antibody test for Paroxysmal Cold Hemoglobinuria was found to be negative. Bone marrow aspiration showed hypercellular marrow with prominent erythroid hyperplasia. Bone marrow biopsy showed hypercellular marrow with prominent erythroid hyperplasia and dimorphic erythropoiesis. Giant Platelets were noted. A Diagnosis of Mixed Type of Evans Syndrome was arrived at.

Results: The patient was initiated on prednisolone at $1 \mathrm{mg} / \mathrm{kg} / \mathrm{day}$ and responded to the provided treatment. Follow up hemoglobin was $10.3 \mathrm{mg} / \mathrm{dl}$ and platelet count was 1,63,000. Follow up LDH was found to be 252 and Liver function test was found to be normal. Hence it was concluded that the patient had Mixed type Evans's Syndrome.

Discussion \& Conclusion: She has been in remission on follow up for 12 months with maintenance dose of steroids. Mixed type of AIHA denotes the presence of both Warm and Cold AIHA. Concomitant or sequential presence of AIHA with Immune thrombocytopenia is termed Evan Syndrome.

\section{Evaluation of Diastolic Dysfunction \\ Echocardiographically in Multi-transfused Thalassemia Patients}

Mitsu P. Vithalani*
${ }^{\text {1} P e d i a t r i c s, ~ M ~ P ~ S h a h ~ G o v e r n m e n t ~ M e d i c a l ~ C o l l e g e, ~ J a m n a g a r, ~}$
India

Aims \& Objectives: To assess the systolic and diastolic right ventricular function of patients with TM using Tissue doppler imaging and to determine whether this echocardiographic technique is an adequate diagnostic tool for the screening and detection of subclinical cardiac dysfunction.

Patients/Materials \& Methods: 100 patients with Beta-thalassemia and transfusion burden $>10$ times/year in the age group of 2-18 years were included and the prospective study was carried out over a period of 1 year. Informed consent was taken and IEC approval was done. They were subjected to various tests, CXR and echocardiography. Parameters included in this study: M-mode: Left ventricular end diastolic and systolic dimensions, wall thickness, left ventricular mass and wall stress, fractional shortening and corrected mean velocity of circumferential shortening. Pulse Doppler: mitral inflow peak velocities and mitral deceleration time. Tissue Doppler: Measurement of E', A' and S wave from the AV ring at the ventricular free wall and interventricular septum. Color and continuous flow doppler: Severity and velocity of tricuspid and pulmonic regurgitant jets.

Results: Out of 100 patients, 14\%(14) had changes of diastolic dysfunction. Out of these, $22 \%$ (3) were below the age of 10 years and $78 \%(11)$ were above the age of 10 years. Out of these $14,72 \%(10)$ had ferritin levels $>2000 \mathrm{ng} / \mathrm{ml}$. Only 2 patients had signs of Cardiac dysfunction.

Discussion \& Conclusion: Diastolic dysfunction preceded the onset of systolic impairment. Early initiation of chelation therapy can prevent and reverse the cardiomyopathy.

\section{Utility of Low Hemoglobin Density (LHD \%) in Distinguishing Iron Deficiency Anaemia from Beta Thalassemia Trait}

Mary Raju ${ }^{* 1}$, Shiela Das ${ }^{1}$

${ }^{1}$ Hematopathology, Believers Church Medical College, Thiruvalla, India

Aims \& Objectives: To investigate the reliability of LHD in differentiating BTT from IDA.

Patients/Materials \& Methods: This project was conducted in Haematopathology section of Believers Church Medical College Hospital, Thiruvalla, Kerala. Hundred and sixty-six blood samples were received for detection of hemoglobinopathy. These blood samples belong to anaemic patients unresponsive to hematinics and others with low Mentzer index. All blood samples were run in Beckman Coulter Unicell DxH 800 from which RBC indices and reticulocyte parameters were noted.

Results: IDA comprised 97 cases, BTT-32 cases and other Hemoglobinopathy - 4 cases. Results: Of the 32 cases of BTT,34\% had LHD less than 10, 57\% had LHD 10-40,and 9\% LHD more than 40. In 15\% BTT associated with IDA, LHD ranged from $10.4 \%$ $28.4 \%$ Of the 97 cases of IDA, 89\%showed LHD more than 10.

Discussion \& Conclusion: DISUSSION: Iron deficiency anaemia (IDA) and Beta Thalassemia Trait (BTT) are two of the most common causes of microcytic hypochromic anaemia. It is essential to differentiate between the two so as to avoid unnecessary iron therapy.Jai Vigyan multicentric study (2013) in India showed that the overall prevalence of $\beta$-thalassemia trait is $2.78 \%$ which in 59 ethnic groups varied from $0 \%$ to $9.3 \%$ respectively. Mentzers Index (MI) with a sensitivity of $98.7 \%$ and specificity of $82.3 \%$ can help to distinguish between these two types of anaemia. However in the presence of BTT with IDA, it is not helpful. Recently Low hemoglobin density is a new parameter provided by Beckman-Coulter which is significantly increased in IDA.The reference range is $5.3-10.4 \%$. This study aims to investigate the reliability of LHD in differentiating BTT from IDA CONCLUSION:LHD appears to be an insensitive index in cases of BTT associated with IDA.

\section{Erythroid Maturation Patterns in Anemia: A Case Series}

Shrinza Gupta ${ }^{* 1}$, Mrinalini Kotru ${ }^{1}$, Ashish Goel ${ }^{2}$, Meera Sikka ${ }^{1}$, Richa Gupta ${ }^{1}$, Priyanka Gogoi ${ }^{1}$

${ }^{1}$ Pathology, ${ }^{2}$ Medicine, UCMS and GTB Hospital, Delhi, India

Aims \& Objectives: To study erythroid maturation patterns in anemia in different settings. 
Patients/Materials \& Methods: We conducted this study in Department of Pathology and Department of Medicine, UCMS and G.T.B Hospital, Delhi from November 2019 to March 2020 and included 4 cases with anemia. Patients with $\mathrm{Hb}<12 \mathrm{~g} / \mathrm{dl}$ in females and $<13 \mathrm{~g} / \mathrm{dl}$ in males were included and those who had hematinics and blood transfusion in last 14 days were excluded. A complete history and clinical data was collected and informed consent was taken before blood collection and bone marrow aspiration. Complete Hemogram, Peripheral smear (Wright's stain), Reticulocyte count, ESR, Biochemical markers of Iron, Vitamin B12 and Folate was done. Bone marrow aspirate (BMA) and Biopsy (BMB) were done for all the cases and flow cytometry for analysis of erythroid maturation was done using CD34, CD45, CD71, CD117 and CD235a antibodies. Results: Different staining patterns were identified in various causes of anemia: Megaloblastic anemia- CD71 showed heterogenous expression, $\mathrm{CD} 71+/ \mathrm{CD} 117$ + was nil and CD71 +/CD235a + were $85 \%$, suggesting predominantly intermediate erythroblast population. Regenerating marrow (post B-ALL)- CD71 +/ CD117 + cells were $25 \%$ and CD71 +/CD235a + were $7 \%$ and CD71 + were $67 \%$, suggesting a predominant proerythroblast population. Aplastic anemia- CD235a + cells were 98\%, CD71 +/ CD117 + were $0.6 \%$ and CD71 +/CD235a + were $1.4 \%$, suggesting a decreased production of erythroid precursors. Unexplained anemiaCD71 +/CD117 + cells were $0.5 \%$, CD71 +/CD235a + were nil, CD71 + cells were 95\%, suggesting a downregulation of CD235a on erythroid precursors.

Discussion \& Conclusion: We present flow cytometric findings of erythroid maturation patterns in 4 cases of anemia. Such findings can be useful in defining the shifts in normal bone marrow development and can be used in screening, although a larger population size with more erythroid markers would be better to define these changes.

\section{Sheehan's Syndrome}

Ramji Billa $^{* 1}$, Anil I. Singh ${ }^{1}$

${ }^{1}$ Dept of Medicine, JNIMS, Imphal, India

Aims \& Objectives: To uncover an unusual diagnosis with an usual presentation.

Patients/Materials \& Methods: A 28 year old female presented with symptoms related to chronic anemia with significant postnatal history which made us to evaluate briefly.

Results: Anemia though a rare presentation of sheehan 's syndrome it should be considered in workup of patient with anemia.

Discussion \& Conclusion: Anemia and hematological abnormalities might be the presentation of patient with sheehan's syndrome hence all chronic anemia should be evaluated promptly.

\section{Sickle Cell Disease: Presenting as Cirrhosis of Liver \\ Biraja P. Beura ${ }^{* 1}$, Purna C. Karua ${ }^{2}$ \\ ${ }^{1}$ Post Graduate in Department of General Medicine, ${ }^{2}$ Associate Professor Department of General Medicine, VIMSAR Burla, Odisha, Sambalpur, India}

Aims \& Objectives: Sickle Cell Disease- Presenting as Cirrhosis of Liver. Sickle cell disease is a common haemoglobinopathy which may present with multiple symptoms. The major features are related to haemolytic anemia and vasooclusion. We report a case of an undiagnosed sickle cell disease presenting with cirrhosis of liver.

Patients/Materials \& Methods: A 37 year non-alcoholic male presented with abdominal distension and yellowish discoloration of eyes and urine. On examination there was pallor,icterus and features of ascites. Laboratory investigations suggested severe anaemia, features of haemolytic jaundice, normal ferritin and elevated LDH. Peripheral blood smear showed sickle shaped RBCs and reticulocytosis. Haemoglobin electrophoresis showed 76.1\% HbS. HBsAg and HCV RNA were negative. Coomb's tests were negative. Ultrasound abdomen showed hepatomegaly with coarse hepatic echotexture with dilated portal vein and sludge filled gall bladder and gross ascites. Ascitic fluid analysis showed SAAG of 1.5. Upper GI Endoscopy showed grade II esophageal varices. There was history of 1 unit blood transfusion 2 years back.

Test with observed value and reference in the bracket Haemoglobin $5.6 \mathrm{gm} / \mathrm{dl}$ (male $14-17 \mathrm{gm} / \mathrm{dl}$ ) Reticulocyte count $10.5 \%(0.5-2.5 \%)$.

Total bilirubin $10.4 \mathrm{mg} / \mathrm{dl}(0.2-1.2 \mathrm{mg} / \mathrm{dl})$

Direct bilirubin $4.8 \mathrm{mg} / \mathrm{dl}(0-0.4 \mathrm{mg} / \mathrm{dl}$

SGOT 93 IU/L (0-40 IU/L) SGPT 59 IU/L (0-40 IU/L) ALP $115 \mathrm{IU} / \mathrm{L}(<250 \mathrm{IU} / \mathrm{L})$

Serum Urea $26 \mathrm{mg} / \mathrm{dl}(10-40 \mathrm{mg} / \mathrm{dl})$ Serum creatinine $1.2 \mathrm{mg} /$ $\mathrm{dl}(0.5-1.5 \mathrm{mg} / \mathrm{dl}) \mathrm{S}$. albumin $2.9 \mathrm{gm} / \mathrm{dl}(3.6-4.8 \mathrm{mg} / \mathrm{dl})$ Ascitic fluid albumin $1.4 \mathrm{gm} / \mathrm{dl}$

PT/INR $1.63(<1.5)$

S.LDH 760U/L (240-480 U/L)

S.Ferritin $54 \mathrm{ng} / \mathrm{ml}(20-250 \mathrm{ng} / \mathrm{ml})$ G6PD 24.78U/g Hb (6.4-8.7 $\mathrm{U} / \mathrm{g} \mathrm{Hb})$

Coombs test (direct \& indirect) Negative HIV Negative

HBsAg Negative HCV RNA Negative

Haemoglobin electrophoresis $\mathrm{HbS} 76.1 \%$

$\mathrm{HbF} 18.3 \%$

$\mathrm{HbA} 22.2 \%$

Ascitic fluid analysis Appearance clear

Total cell count- $420 / \mathrm{cmm}$ (mostly lymphocytes) Sugar $88 \mathrm{mg} / \mathrm{dl}$

Albumin $1.4 \mathrm{mg} / \mathrm{dl}$

ADA 18

Gram staying No organism seen ZN stain AFB not detected Chest xray - Normal

Results: The cirrhosis of Liver in our patient is due to sickle cell disease.

Discussion \& Conclusion: The heaptobiliary manifestations may vary from asymptomatic incidentally detected abnormal liver function tests to decompensated liver failure. It is important to recognise the rare presentations of the disease for better patient care.

\section{Serum Hepcidin: Good Predictor of Iron Defeciency}

Jyothi Allamsetti ${ }^{* 1}$, Suneetha Padala ${ }^{1}$, Ranjit K. CS ${ }^{2}$, Rajesh M. Gottipati $^{3}$, Anil Aribandi ${ }^{2}$

${ }^{1}$ Department of Biochemistry, Care Hospitals, ${ }^{2}$ Department of Haemato-oncology, American Oncology Institute, Hyderabad, ${ }^{3}$ Department of Haemato-oncology, American Oncology Institute, Vijayawada, India

Aims \& Objectives: Iron deficiency anaemia is the commonest nutritional problem responsible for about $50 \%$ of the cases of anaemia worldwide. The present study is aimed at determining and evaluating the serum hepcidin levels in relation to serum ferritin and other iron indices in individuals with iron deficiency.

Patients/Materials \& Methods: This study includes 30 individuals with iron deficiency based on their serum iron and ferritin levels. Individuals were enrolled from a tertiary hospital from December, 2019 to August, 2020. Serum ferritin, serum iron and other indices were assayed on the fully automated Beckman Coulter analyzer. Serum hepcidin biomarker was estimated by ELISA method using DRG-Hepcidin 25 bioactive ELISA kit. The statistical evaluation was done by using the SPSS Software 23.0 version. 
Results: Out of the 30 individuals with iron deficiency, 20 (66.6\%) were female while $10(33.3 \%)$ were male. Serum hepcidin was significantly low in individuals with iron deficiency, with a $p$ value $<0.0001$, which is highly significant. Table:

\begin{tabular}{llll}
\hline Variable & Mean \pm S.D & t-Value & $p$ value \\
\hline Hemoglobin $(\mathrm{gm} / \mathrm{dl})$ & $8.66 \pm 1.88$ & 25.08 & $<0.0001$ \\
MCV (fl) & $67.92 \pm 9.73$ & 38.20 & $<0.0001$ \\
MCH (pg) & $21.12 \pm 3.87$ & 29.82 & $<0.0001$ \\
RDW & $19.61 \pm 3.92$ & 27.33 & $<0.0001$ \\
Serum Hepcidin $(\mathrm{ng} / \mathrm{ml})$ & $16.47 \pm 4.45$ & 20.21 & $<0.0001$ \\
\hline
\end{tabular}

On ROC analysis hepcidin has sensitivity of $98 \%$ specificity of $72 \%$ at cut-off value of 13.4. AUC of hepcidin was 0.84 .

Discussion \& Conclusion: Serum hepcidin levels are significantly low in individuals with iron deficiency and may help diagnose iron deficiency even in patients without anaemia. Serum hepcidin levels may help differentiate iron deficiency anaemia and anaemia of inflammation, which sometimes can be challenging. Further studies are necessary to determine the reliable reference ranges and cut-off values in healthy subjects.

\section{Case of Deta-Beta Thalassemia}

Juhi Singh ${ }^{* 1}$, Ankitesh Kumar $^{1}$

${ }^{1}$ Medicine, MAMC, Delhi, India

Aims \& Objectives: Case study of homozygous delta-beta thalassemia.

Patients/Materials \& Methods: Fifteen years old boy born out of consanguineous marriage presented with yellowish discoloration of eyes and skin, fatigue and abdominal discomfort since two months. No history of fever, rash, bleeding from any site or drug intake. Similar complaints two years back for which blood transfusion done (two units).Family history with five siblings suggested younger sister expired at an age of five years with similar complaints.

Results: Physical examination revealed stable vitals, jaundice, palpable spleen $2 \mathrm{~cm}$ below costal margin. Complete blood count revealed $\mathrm{Hb} 9.6 \mathrm{gm} / \mathrm{dl}$, wbc $7.3 \times 109 / \mathrm{L}$, platelet count $156 \times 109 / \mathrm{L}$, peripheral blood smear revealed normocytic normochromic to microcytic hypochromic red cells with mild anisocytosis, corrected retic count $7 \%$.Liver function tests showed raised levels of serum total bilirubin $(4.8 \mathrm{mg} / \mathrm{dL})$ and indirect bilirubin $(4.0 \mathrm{mg} / \mathrm{dL})$. Bone marrow aspirate and biopsy revealed erythroid hyperplasia with Myeloid:Erythroid 1:6, bone maroow iron grade 3 to $4+$, without sideroblast. High-performance liquid chromatography (HPLC) showed $99.8 \% \mathrm{HbF}$ and an absence of $\mathrm{HbA}$ and $\mathrm{HbA} 2$.Patient was managed conservatively. CBC followed by HPLC performed for mother and father who were apparently healthy and had no history of blood transfusions normocytic normochromic to hypochromic anemia with $\mathrm{HbF}$ and $\mathrm{HbA} 218.9 \%, 2.7 \%$ and $10.9 \%, 3.2 \%$, respectively.

Discussion \& Conclusion: Both parents were heterozygotes for deltabeta thalassemia and patient found to be homozygous. Mutational analysis could not be performed because the patient was lost to follow-up. Joob et al. in a study from Brazil showed incidence of $\delta \beta$ thalassemia is less than 1:10,000, hence a rare cause of elevated HbF.
Eliciting Role of Mirnas in Hydroxyurea Induced Foetal Haemoglobin (HBF) in Sickle Cell Disease (SCD)

Smruti S. Biswal ${ }^{* 1}$, Anand Bodade ${ }^{2}$

${ }^{1}$ Life Science, NIT Rourkela, Rourkela, ${ }^{2}$ Haematogenetics, NIIH, ICMR, Mumbai, Mumbai, India

Aims \& Objectives: To elucidate the role of 50 differentially expressed miRNAs by in silico analysis of microarray data obtained through online databases, finding their targets and the biological functions enriched in it.

Patients/Materials \& Methods: Two datasets from Array Express database (GSE32035 and GSE11060) were collected. Identification of DE expressed miRNAs was performed on R-software. Targets of the identified DE miRNAs were established. Later, Gene set enrichment analysis of the gene targets was performed, followed by their pathway enrichment analysis using KEGG pathway.

Results: Out of the $50 \mathrm{DE}$ miRNAs chosen from the lot of 2000 produced by the software, $32 \mathrm{DE}$ miRNAs were identified as downregulated while the remaining were up-regulated. A total of 29000 genes were identified as targets for the DE miRNAs. Upon identification of targets, the gene set enrichment analysis was performed which identified around 125 enriched biological functions. KEGG pathway analysis revealed that up-regulated DEGs were mainly enriched in 48 pathways, including, B cell receptor signalling pathway, $\mathrm{T}$ cell receptor signalling pathway, B-cell receptor signalling pathway, Maturity onset diabetes of the young (MODY), endocytosis, apoptosis, MAPK Signalling pathway, Axon guidance, ErB Signalling pathway, renal cell carcinoma, pancreatic cancer and endocytosis pathways. Notably hsa-mir-126 was found to highly down-regulated in the control samples of healthy individuals, while being highly up-regulated in the samples where patients were treated with Hydroxyurea.

Discussion \& Conclusion: These miRNAs are plausibly involved in $\mathrm{HbF}$ induction process following $\mathrm{HU}$ treatment through the biological functions discovered by GSEA analysis. This information can be exploited to find out novel miRNA candidates upon which further investigation can be carried out for pinning the exact mechanism of miRNAs; posing a therapeutic target for the treatment of SCD using the recently developed genome editing and gene therapy tools.

\section{Clinico-Hematological Profile of Chronic Lymphoproliferative Disorders Presenting with Autoimmune Hemolytic Anemia}

Sushmitha Sathyanarayanan ${ }^{* 1}$, Abhiraami Muthuraman ${ }^{1}$, Abhishekh Gowda ${ }^{2}$, Rajendra Kulkarni ${ }^{2}$, Prabhu Manivannan ${ }^{1}$, Rakhee Kar ${ }^{1}$, Debdatta Basu ${ }^{1}$

${ }^{1}$ Pathology, ${ }^{2}$ Transfusion Medicine, JIPMER, Puducherry, India

Aims \& Objectives: The present study was undertaken to assess the clinicohematological profile of cases of chronic lymphoproliferative disorder (CLPD), associated with autoimmune haemolytic anemia (AIHA), seen in our centre over the last eight years.

Patients/Materials \& Methods: It was a record based study of cases of CLPD diagnosed in our department from 2012-2020. The complete blood counts, peripheral smear, bone marrow findings, immunophenotype, serum bilirubin, serum LDH and Direct Coomb's test results of these cases were analysed.

Results: Of the 142 cases of CLPD diagnosed during this period, chronic lymphocytic leukemia (CLL) was the most common (84\%). Five of the $142(3.5 \%)$ cases had autoimmune haemolytic anemia (AIHA) as evidenced by presence of spherocytes, agglutination and reticulocytosis in the peripheral blood, positive direct Coomb's test, indirect hyperbilirubinemia and elevated LDH. Of the five cases, four were CLL and was associated with warm type AIHA and there was 
one case of splenic marginal zone lymphoma which was of mixed warm and cold type with positivity for $\mathrm{IgG}, \operatorname{IgM}$ and $\mathrm{C} 3 \mathrm{~d}$. Hemoglobin ranged from 2.7 to $6.4 \mathrm{~g} / \mathrm{dL}$. Thrombocytopenia (30 to $110 \mathrm{X}$ $103 / \mathrm{mm} 3$ ) was noted in three and absolute lymphocytosis (7.9 to $403.2 \times 103 / \mathrm{mm} 3$ ) in all cases. All the cases were simultaneously diagnosed to have CLPD and AIHA at presentation. Bone marrow examination was performed in the case of splenic marginal zone lymphomas and showed nodular to interstitial infiltration. Two of the five cases are currently undergoing treatment and have improved with respect to their hemolysis.

Discussion \& Conclusion: The prevalence of AIHA in CLPD was found to be $3.5 \%$ which is comparable to other studies. Splenic marginal zone lymphoma was rare non-CLL CLPD associated with AIHA. A good look at the peripheral blood smear with presence of agglutinated RBCs and/or spherocytes and polychromasia along with absolute lymphocytosis were clues to clinching the diagnosis of AIHA associated with CLPD.

\section{Hemoglobin SE Disease: A Case Report from Kerala}

Mohan Varughese ${ }^{* 1}$, Sheila Das ${ }^{2}$

${ }^{1}$ General Medicine, ${ }^{2}$ Hematopathology Section, Department of Pathology, Believers Church Medical College, Thiruvalla, Kerala, Thiruvalla, India

Aims \& Objectives: To investigate hemoglobinopathy in a diabetic with normal $\mathrm{Hb}$ Alc.

Patients/Materials \& Methods: 49 year old male,from Pathanapuram, admitted for Diabetic control presented with hyperglycemia and anemia, his $\mathrm{Hb} \mathrm{A1c}$ was normal.

Results: $\mathrm{Hb} 11.5 \mathrm{~g} / \mathrm{dl}$, Retic $2.33 \%$ other blood counts normal, iron status normal Blood sugar $417 \mathrm{mg} / \mathrm{dl} \mathrm{HbA1c} 4.6 \%$ Indirect Bilirubin $0.96 \mathrm{mg} / \mathrm{dl}$ other biochemical parameters normal HPLC-variant testing : $\mathrm{Hb} \mathrm{S} 53 \%, \mathrm{Hb}$ A2 $24.4 \% \mathrm{R}$ time 3.06 .

Discussion \& Conclusion: Diagnosis : Double heterozygous for hemoglobin $\mathrm{S}$ and hemoglobin $\mathrm{E} A$ rare case of $\mathrm{Hb}$ SE disease is reported from Kerala.

Keyword: HbSE disease.

\section{Evaluation of Mutation Spectrum in Cases of Beta- Thalassaemia in Western Rajasthan}

Souvik Saha ${ }^{* 1}$, Abhishek Purohit ${ }^{1}$, Kuldeep Singh ${ }^{2}$, Anand R. Kalla ${ }^{3}$, Manju Bohra ${ }^{4}$, Mayank Kumar ${ }^{1}$, Shashikant Saini ${ }^{1}$

${ }^{1}$ Pathology, ${ }^{2}$ Pediatrics, AIIMS Jodhpur, ${ }^{3}$ Pathology, ${ }^{4}$ Immunohaematology and Blood Transfusion, Dr. SN Medical College, Jodhpur, India

Aims \& Objectives: In India, $\beta$-thalassaemia is the most common monogenic disorder. The disease is characterized by its genetic heterogeneity at the molecular level, and more than 300 mutations of the $\beta$ globin gene have been characterized all over the world, however, five mutations, codon $8 / 9(+\mathrm{G})$, codon $41 / 42$ (-TCTT), IVS I-1 $(\mathrm{G} \rightarrow \mathrm{T})$, IVS I-5(G $\rightarrow \mathrm{C})$, and 619 bp deletion at $3^{\prime}$ end of $\beta$ - globin gene, account for about $85-90 \%$ of $\beta$-thalassaemia mutations in Indian population. The aim of the present study was to screen known cases of $\beta$-thalassaemia in the Western part of Rajasthan state.

Patients/Materials \& Methods: The study included 144 known cases of $\beta$-thalassaemia of all clinical phenotypes including $\beta$ - thalassaemia major, $\beta$-thalassaemia minor and $\beta$-thalassaemia intermedia. Cases were diagnosed based on clinical features, haematology investigations including haemogram and Hb-HPLC (Haemoglobin-High performance liquid chromatography). Blood samples from all these cases were taken for mutation analysis. The mutations were characterized by the polymerase chain reaction method to study the five thalassaemia mutations including IVS-I-5 $(\mathrm{G} \rightarrow \mathrm{C})$, IVS-I-1 $(\mathrm{G} \rightarrow \mathrm{T}), \mathrm{CD}$ $41 / 42$ (-TCTT), CD 8/9 (+G) and 619 bp deletion from the $3^{\prime}$ end of the $\beta$-globin gene.

Results: Of all 144 cases, 74 (51.38\% of all) cases were of $\beta$-thalassaemia major, five $(3.4 \%$ of all) cases were of $\beta$-thalassaemia intermedia and $65(45.14 \%$ of all) cases were of $\beta$-thalassaemia minor. Mutation analysis revealed that five common mutations were present in 130 (90.27\% of all) cases. Among identified mutations, highest frequency of mutation was of IVS-I-5 $(\mathrm{G} \rightarrow \mathrm{C})$ identified in 73 cases $(50.7 \%$ of all cases). In 11 ( $7.63 \%$ of all) cases, more than one mutation was identified.

Discussion \& Conclusion: $\beta$-thalassaemias are common in Western part of Rajasthan state of India, however, there have been very few studies focusing this part of the country. In this study, we screened known cases of thalassaemia for five common mutations and found that these mutations are common in this part of the country also. These observations might help in forming the basis for comprehensive diagnostic database that would not only be useful for genetic counseling but also for prenatal diagnosis.

\section{Autoimmune Haemolytic Anaemia Associated with Covid-19 Infection: A Case Report from Kerala} Mary Raju*1, Shiela Das ${ }^{1}$

${ }^{1}$ Pathology, Believers church medical college, Thiruvalla, India

Aims \& Objectives: Autoimmune haemolytic anaemia associated with COVID-19 infection- A case report from Kerala.

Patients/Materials \& Methods: 59 year old female presented to emergency department with history of generalized weakness, shortness of breath and blood clots in stool. She was a known case of Parkinsonism. Routine blood counts showed anaemia and reticulocytosis.So peripheral smear was done which showed polychromasia, nucleated red blood cells, occasional shistocytes and mild clumping of RBCs.Due to high reticulocyte count, a coombs test was done and it came to $4+$.The antibodies to Ebstein Barr viral capsid, Parvovirus B and autoimmune antibodies such as ANA profile,p ANCA, c- PNH panel profile were screened and found to be negative. So it was considered that the patient developed secondary AIHA triggered by Covid-19. Since the patient had progressive Covid-19 infection he was started on favirapivir and steroids. Howevever the hemolysis was refractory to steroids and was started on blood transfusion and a single dose of Rituximab was administered to which patient become symptomatically better.

Results: it was considered that the patient developed secondary AIHA triggered by Covid- 19 .

Discussion \& Conclusion: While the main target of Covid-19 remains the lung, with respiratory failure and acute respiratory distress syndrome for the most severe cases, extra-pulmonary complications such as autoimmune hemolytic anemia (AIHA) is an unusual finding in COVID 19.This case is reported for its rarity.

\section{Sudden Onset Hearing Loss in Sickle Cell Disease: A Rare Case Report}

Dhirej K. Lenka ${ }^{* 1}$, Manoj K. Mohapatra ${ }^{2}$

${ }^{1}$ Junior Resident, Department of General medicine, Vimsar, Burla, ${ }^{2}$ Professor, Department of General medicine, VIMSAR BURLA, Sambalpur, India

Aims \& Objectives: Sickle cell disease is a chronic complex disease that can affect any organ of the body causing progressive organ 
damage.Sickle cell disease can affect internal ear due to intermittent vaso occlusive crisis leads to sensorineural hearing loss. We report a case of sudden onset sensorineural hearing loss in sickle cell disease due to vaso occlusive crisis.

Patients/Materials \& Methods: A 26 year male patient presented with the chief complaint of multiple joint pain and sudden onset hearing loss in both ear for 2 days.He is a known case of sickle cell disease on medication with hydroxyurea and folic acid.There is also no history of urti,head injury,drugs intake,loud noise exposure,tinnitus, vertigo,ear discharge,pre and post auricular pain.On examination there is presence of pallor and icterus.Investigations showed features of haemolytic anemia i.e. severe anemia,elevated bilirubin and LDH level.The patient then referred to ENT department for further examination.Extarnal ear and auditory canal is normal in both ear.Tympanic retraction was present on left ear.Weber test lateralized to left side.Pure tone audiometry showed moderate to severe high frequency sensorineural hearing loss in right ear and mild sensorineural hearing loss in left ear.Acoustic emission absent on right side.Tympanogram shows type A curve in both ear.MRI of the brain and internal ear is normal.Test showed Haemoglobin $6.6 \mathrm{gm} / \mathrm{dl}$ (male 14-17 gm/dl), Reticulocyte count 7.5\% (0.5-2.5\%), Total bilirubin $8 \mathrm{mg} / \mathrm{dl} \quad(0.2-1.2 \mathrm{mg} / \mathrm{dl})$, Direct bilirubin $4.8 \mathrm{mg} / \mathrm{dl} \quad(0-0.4 \mathrm{mg} /$ dl,SGOT $76 \mathrm{IU} / \mathrm{L} \quad$ (0-40 IU/L),SGPT $60 \mathrm{IU} / \mathrm{L} \quad$ (0-40 IU/L),ALP $105 \mathrm{IU} / \mathrm{L}(<250 \mathrm{IU} / \mathrm{L})$,Serum Urea $18 \mathrm{mg} / \mathrm{dl}(10-40 \mathrm{mg} / \mathrm{dl})$, Serum creatinine $1 \mathrm{mg} / \mathrm{dl}(0.5-1.5 \mathrm{mg} / \mathrm{dl}), \mathrm{S} . \mathrm{LDH} \quad 660 \quad \mathrm{U} / \mathrm{L} \quad(240-480$ U/L),HIV NegativeHBsAg NegativeHCV RNA Negative. patient received i.v fluid, analgesic, oral steroid for 7 days and recovered completely.

Results: Sudden onset sensorineural hearing loss is due to vaso occlusive crisis in sickle cell disease.

Discussion \& Conclusion: The cochlea is a high sensitive organ to ischemia as it is fed by an end artery called labyrinthian artery.Vascular occlusion of the end artery in SCD due to vaso occlusive crisis leads to cochlear ischemia and anoxia.Sensorineural hearing loss due to cochlear ischemia needs early diagnosis and treatment so that permanent hearing loss due to irreversible damage to cochlea can be prevented.

\section{Prevalence of Folate and Vitamin B12 Deficiency in Thalassemia Major}

Titiksha Agrawal ${ }^{* 1}$, Pooja Dewan ${ }^{1}$, Sunil Gomber ${ }^{1}$, Rachna Agarwal $^{2}$, Mrinalini Kotru ${ }^{3}$, Sangeeta Sharma ${ }^{4}$, Deepak Patel ${ }^{1}$

${ }^{1}$ Pediatrics, UCMS and GTB Hospital, ${ }^{2}$ Neurochemistry, IHBAS, ${ }^{3}$ Pathology, UCMS and GTB Hospital,

${ }^{4}$ Neuropsychopharmacology, IHBAS, New Delhi, India

Aims \& Objectives: To assess the prevalence of Folate and Vitamin B12 deficiency in transfusion dependent Thalassemia major (TDT) patients.

Patients/Materials \& Methods: Children with TDT aged 5-18 years were enrolled. Baseline assessment of all children was done, including anthropometry and dietary history. Participants were on Folic acid @ $5 \mathrm{mg}$ per day. $2 \mathrm{ml}$ sample was taken in plain vials before they received transfusion. Serum Vitamin B12 and Folate levels were measured using chemi-luminescence method.

Results: We included 102 TDT patients aged 5-18 years with $61.8 \%$ (63/102) males and 38.2\% (39/102) females. 5.9\% (6/102; 2 males, 4 females) were found to be folate deficient, $33.3 \%(2 / 6)$ of which were vegetarians and $66.7 \%(4 / 6)$ were non- vegetarians. $17.6 \%(18 / 102 ; 9$ males, 9 females) were found to be Vitamin B12 deficient, $44.8 \%$ (8/ $18)$ of which were vegetarians and $55.6 \%$ (10/18) were non- vegetarians.None of the ovo-vegetarians were found to be deficient in Vitamin B12 and folate $(2.9 \%, 3 / 102)$. A correlation was found between age and Vitamin B12 levels ( $\mathrm{r}=-0.332, p=0.001)$, younger children had higher serum Vitamin B12 levels. No correlation was found between age and folate levels $(r=-0.135, p=0.263)$ No correlation was found between serum levels of the vitamins and duration of transfusion.

Discussion \& Conclusion: Vitamin B12 and Folate deficiency are prevalent in Thalassemia patients, perhaps due to their dietary restrictions and increased RBC turnover. Egg consumption may have a protective effect against folate and vitamin B12 deficiency. Vitamin B12 deficiency is found to be more prevalent in older age. Currently, there are no guidelines for monitoring and supplementation of Vitamin B12 in TDT patients. Regular supplementation may be needed in such children.

Supporting Document:
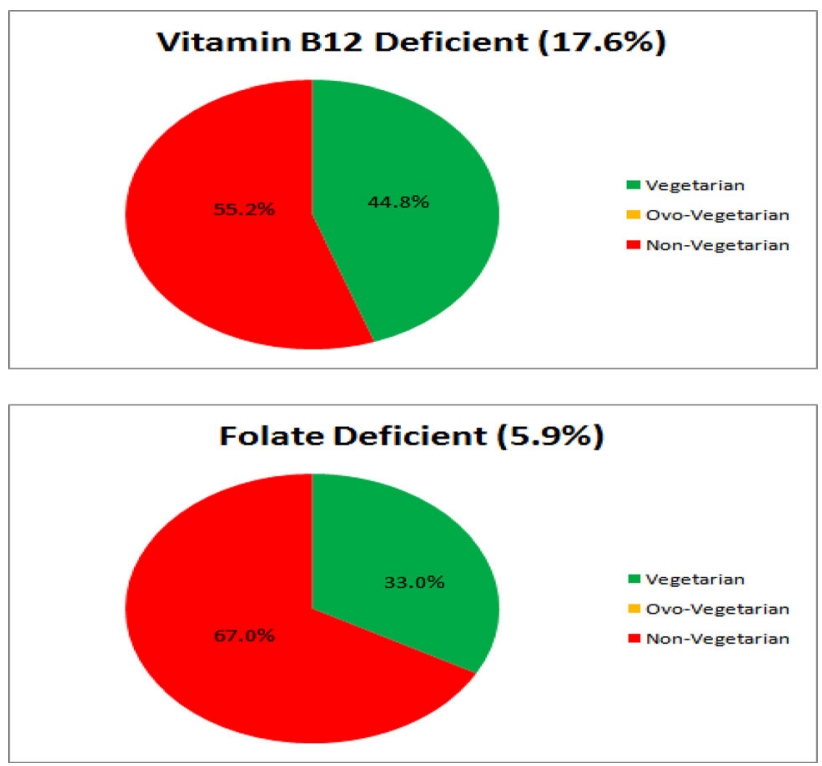

\section{Nutritional Status and Prevalence of Anemia in Tribal Children}

Somnath Mondal' ${ }^{* 1}$, Suman K. Maji ${ }^{2}$, Prakas K. Mandal ${ }^{1}$, Tuphan K. Dolai $^{1}$

${ }^{1}$ Hematology, Nil Ratan Sircar Medical College and Hospital, Kolkata, ${ }^{2}$ Microbiology, Purulia Medical College, Purulia, India

Aims \& Objectives: To assess the prevalence of nutritional anaemia in school going tribal children of various tribal areas of West Bengal. Patients/Materials \& Methods: Asymptomatic school going tribal children (6-12 years of age) of tribal area at west Bengalwere studied cross-sectionally.Anaemia was defined according to WHO criteria. The parameters studied are CBC (Complete blood count), serum ferritin, TIBC, Serum iron, Vitamin D3, VitaminB12 and folate. Sysmax XP100 Cell Counter, Chemiluminescence \& Accurex biochemistry analyzer were used to analyze the parameters.

Results: Out of total 1070children, male were $44.02 \%$ (471)while female were $55.98 \%$ (599). The district wise distribution of children are Midnapore 55.61\% (595), Purulia 25.05\% (268) \& Bankura $19.35 \%$ (207). Mean $( \pm$ SD)age, height \& weight were $9.51 \pm 2.25$ yrs, $119.69 \pm 27.24 \mathrm{~cm} \& 24.61 \pm 7.94 \mathrm{~kg}$ respectively. Mean $( \pm \mathrm{SD})$ haemoglobin $(\mathrm{Hb})$ was $11.55 \pm 0.90 \mathrm{~g} / \mathrm{dl}$. Anaemiawas found in $49.77 \%$ of children. From this study girls had a lower mean 
$\mathrm{Hb}$ value than that of the boys.According to the WHO/UNICEF/UNU protocolfrom this study prevalence of mild (11- $11.5 \mathrm{~g} / \mathrm{dl})$, moderate $(8-<11 \mathrm{~g} / \mathrm{dl})$ and severe $(<8 \mathrm{~g} / \mathrm{dl})$ anaemia were found to be $26.29 \%, 23.39 \%$, and $0.09 \%$ respectively.Mean $( \pm$ SD) HCT, MCV, $\mathrm{MCH}, \mathrm{MCHC}$ of the study population were $37.18 \pm 2.70 \%$, $73.53 \pm 6.77 \mathrm{fl}, \quad 22.99 \pm 3.50 \mathrm{pg}, \quad 31.04 \pm 1.36 \mathrm{~g} / \mathrm{dl}$.Also, $\mathrm{MCH}$, HCT, MCV \& MCHC of girls tribal children were low compared to boy's tribal children. Out of all anaemic study population, $12.54 \%$ (male $8.77 \%$ \&female $4.86 \%$ ) was found to have iron deficiency anaemia. A $35.73 \%$ (male $17.96 \%$ \&female $17.77 \%$ ) had folate deficiency anaemia, while $19.83 \%$ (male $9.82 \%$ \&female $10 \%$ ) had Vitamin B12 deficiency anaemia. 14.87\%(male $4.58 \%$ \&female $10.29 \%$ ) of children was found to have Vitamin D deficiency.

Discussion \& Conclusion: In this cohort, $49.77 \%$ of school tribal children are anemic.Nutritional anaemiahad $75.18 \%$ of the study population. Folate deficiency anemia $(37.84 \%)$ is the commonest cause anemia.

\section{Infection in An Infant Presenting as Steroid Refractory Aiha}

Mona Vijayaran*1, Mukul Aggarwal ${ }^{1}$, Stitha P. Gudala ${ }^{1}$, Sahitya S. Krishna ${ }^{1}$, Jasmita Dass ${ }^{2}$, Hem C. Pandey ${ }^{3}$, Tulika Seth ${ }^{1}$, Manoranjan Mahapatra ${ }^{1}$

${ }^{1}$ Clinical Haematology, ${ }^{2}$ Hematopathology, ${ }^{3}$ Transfusion Medicine, AIIMS, Delhi, India

Aims \& Objectives: Autoimmune haemolytic anemia (AIHA) is a rare hematologic disease in childhood with an incidence of $0.2 /$ 100,000 under 20 years of age. We report a case of an infant with steroid refractory AIHA secondary to CMV infection.

Patients/Materials \& Methods: A 6-month old male child presented with complaints of new onset progressive paleness of body and worsening respiratory difficulty from past 20 days. There was no history of fever. On examination, there was pallor, icterus with moderate hepatosplenomegaly. His investigations from an outside hospital were suggestive of hemolysis and he had no response to oral prednisolone.

Results: His CBC at admission showed severe anemia with thrombocytopenia with a hemoglobin of $3.1 \mathrm{~g} / \mathrm{dl}$ with platelet count of $43,000 / \mathrm{mm} 3$. The corrected total leucocyte count was $18,600 / \mathrm{mm} 3$ with differential showing neutrophil (30\%); lymphocyte $(68 \%)$; myelocyte and metamyelocyte (2\%). Peripheral smear showed numerous spherocytes; raised indirect hyperbilirubinemia; reticulocyte count of $23 \%$; LDH was $2150 \mathrm{U} / \mathrm{L}$ and direct coomb's test was positive $(4+)$ by gel card. DCT positivity was due to $\operatorname{IgG}$ (titer $>$ 1000 ) of subclasses IgG1 (titer $>100) \&$ IgG3 (titer $>100$ ) coating the red cells along with $\mathrm{C} 3 \mathrm{~d}$, very weak coating with $\mathrm{IgM}$ also seen. A diagnosis of autoimmune hemolytic anemia was made. He was further investigated for TORCH infections and autoimmune lymphoproliferative syndrome. He came positive for CMV IgM and CMV IgG. CMV PCR in blood and urine showed 200 copies and 2120 copies respectively. Flow cytometry for double negative $\mathrm{T}$ cells was negative. He was started on iv ganciclovir (@3 $3 \times$ BSA $\times$ CrCLS) daily for 10 days. He also received IVIg at $1 \mathrm{~g} / \mathrm{kg}$ daily for 2 days along with methylprednisolone at $2 \mathrm{mg} / \mathrm{kg}$ for 3 days followed by $2 \mathrm{mg} / \mathrm{kg}$ for 2 week followed by slow taper of $0.25 \mathrm{mg} / \mathrm{kg} / \mathrm{week}$. He continued to receive PRBC every $4-5$ days during his hospital admission. At discharge he was shifted to valgancyclovir. His hemolysis continued and was still requiring blood transfusion. He had poor response to steroid and rituximab was started weekly once for four weeks at a dose of $375 \mathrm{mg} / \mathrm{m} 2$. His CMV DNA PCR blood serology came negative after 3 weeks of valgancyclovir. His hemoglobin stabilized at $10.8 \mathrm{~g} / \mathrm{dl}$, platelet count normalized, LDH was $568 \mathrm{U} / 1$ and bilirubin was $0.8 \mathrm{mg} / \mathrm{dl}$ after 3 weeks of rituximab and valgancyclovir. He has remained transfusion independent till date.

Discussion \& Conclusion: AIHA may be primary or secondary to other diseases, including infections, autoimmune disorders, and malignancies. The mixed AIHA in our case was secondary to CMV infection. It was steroid refractory and required rituximab as second line treatment in addition to anti CMV medications. Some children less than two years exhibit resistance to steroids as per the literature. CMV infection is a treatable cause and should be considered when evaluating pediatric AIHA patients.

\section{Oxidant Damage Associated Hemolysis: A Case Report}

Bency $\mathbf{S}^{* 1}$, Nisha $\mathrm{TG}^{1}$, Rajesh Krishna ${ }^{2}$, Shubha Sudhakar ${ }^{3}$

${ }^{1}$ Pathology, ${ }^{2}$ Consultant Hematologist, Yenepoya Medical College, Karnataka, ${ }^{3}$ Pathology, Yenepoya Medical College, Mangalore, India

Aims \& Objectives: A case of oxidant damage associated hemolysis. Patients/Materials \& Methods: The oxidative status participates and modulates various normal physiological cell functions like, signal transduction. Oxidative stress, however, is cytotoxic-it oxidizes proteins, lipids and DNA - leading to cell death and organ damage. Although oxidative stress is not the primary etiology of different anemias, it mediates several of their pathologies, including hemolysis. A 2 year old male child came with complaints of loose stools and was irritable and subsequently developed vomiting and low grade fever. Further mother noticed yellowish discolouration of eyes and dark coloured urine. On admission $\mathrm{Hb}$ was $4 \mathrm{gm} / \mathrm{dl}$ for which two PRBCs were transfused.

Results: Results: Hematological parameters were estimated using Automated Sysmex XN-1000 and Biochemical parameters using Vitros 5600 fully automated chemistry analyzer. $\mathrm{Hb}$ was $9.6 \mathrm{gm} / \mathrm{dl}$, TLC was $7.47 \times 10^{3}$ and platelet count was $161 \times 10^{3}$. Peripheral smear examination showed anisopoikilocytosis with presence of schistocytes, blister cells, polychromatophils and nRBC, thus was reported as hemolytic anemia. LFT showed increase in SGOT and raised bilirubin. G6PD assay was done was found to be borderline normal. Patient has been started with steroids.Child improved symptomatically, hence being discharged with advice to avoid antimalarials (primaquine), sulphonamides and has been followed up as an outpatient.

Discussion \& Conclusion: Children presenting with severe anaemia must be evaluated for oxidant damage associated haemolytic anaemia. G6PD should be sent before blood transfusion and an urgent evaluation of blood film needs to be performed. With adequate precautions including avoidence of causative agent, further episodes of haemolysis can be prevented.

\section{Hematological and Molecular Spectrum of Deletional Hereditary Persistence of Fetal Hemoglobin and Delta- Beta Thalassemias: A Study From North India}

Ratneev Kaur ${ }^{* 1}$, Neetu Rani ${ }^{1}$, Jasbir K. Hira ${ }^{1}$, Sanjeev Chabbra ${ }^{1}$, Pankaj Malhotra ${ }^{2}$, Amita Trehan ${ }^{3}$, Rashmi Bagga ${ }^{4}$, Subhas C. Saha ${ }^{4}$, Prashant Sharma ${ }^{1}$, Reena Das ${ }^{1}$

${ }^{1}$ Department of Hematology, ${ }^{2}$ Department of Internal Medicine, ${ }^{3}$ Department of Pediatrics Hematology and Oncology, ${ }^{4}$ Department of Obstetrics and Gynaecology, PGIMER, Chandigarh, Chandigarh, India

Aims \& Objectives: Deletional hereditary persistence of fetal hemoglobin(HPFH) and $\delta \beta$-thalassemia are related disorders that 
result from large deletions involving the $\beta$-globin gene cluster and are characterized by high fetal hemoglobin (HbF) levels. They span a wide clincal spectrum ranging from transfusion-dependent $\beta$-thalassemia (TD $\beta$ T) and milder non-transfusion-dependent thalassemia (NTDT) to the asymptomatic $\beta$-thalassemia trait ( $\beta T T$ )-like phenotypes.Genotypically, these rare and extremely heterogeneous disorders exhibit myriad combinations of various deletions/mutations affecting the globin genes and/or their regulatory regions.

Patients/Materials \& Methods: We mined the departmental database for patients in whom a deletional HPFH or $\delta \beta$-thalassemia had been diagnosed on molecular genetic testing between 1996 to 2018 , with a view to defining their clinical spectrum and make genotypephenotype associations. Clinical phenotypes alongwith hemogram and high-performance liquid chromatographic data were collated. Gap-PCRs were done for seven specific HPFH deletions previously described among Asian populations, namely the HPFH-3 (Indian $\delta \beta 0$ ), HPFH-9, HPFH-10, $32.6 \mathrm{~kb}$ deletion, Asian-Indian inversion deletion, Corfu deletion and Chinese deletion. TD $\beta$ T and NTDT patients were screened for $\beta$-thalassemia mutations by ARMS-PCR. Results: We encountered total 59 cases (from 32 families) with high $\mathrm{HbF}$ after excluding homozygous/compound heterozygous $\beta$ - thalassemia and acquired causes of increased HbF. Among these 59, 19 index cases $(32.2 \%)$ were symptomatic while the remaining 40 were asymptomatic. Of the symptomatic patients, TD $\beta T$ phenotype was found in 8 index cases, all of whom had the Asian-Indian inversion deletion with a $\beta$-thalassemic mutation.The NTDT phenotype was present in 11 index cases. On genetic testing, they displayed compound heterozygosity for a $\beta$-thalassemic mutation with the AsianIndian inversion deletion $(\mathrm{n}=5)$, HPFH- $3(\mathrm{n}=3), 32.6 \mathrm{~kb}(\mathrm{n}=2)$ or HPFH-9 $(n=1)$ deletions. Family and antenatal screening revealed 40 asymptomatic persons with high $\mathrm{HbF}$. Among the 10 antenatal cases, 7 were heterozygous for the Asian-Indian inversion deletion while 3 were heterozygous for HPFH-3.

Discussion \& Conclusion: Co-inheritance of deletional HPFH with $\beta$-thalassemia leads to variable clinical phenotypes ranging from transfusion-dependent to completely normal states. In this north Indian cohort, the Asian-Indian inversion deletion was the commonest (66.1\%) followed by HPFH-3 (16.9\%), 32.6 kb deletion and HPFH-9 deletions $(8.5 \%$ each).

\section{Prevalence of Haemoglobinopathies in Western Rajasthan}

Madhubala R $\mathbf{R}^{\text {*1 }}$, Amit Kumar Gupta ${ }^{1}$, Mayank Kumar ${ }^{1}$, Sashikant Saini $^{1}$, Abhishek purohit ${ }^{1}$, Siyaram Didel ${ }^{2}$, Poonam Elhence ${ }^{1}$, Kuldeep Singh ${ }^{2}$

${ }^{1}$ Pathology, ${ }^{2}$ Paediatrics, All India Institute of Medical Sciences, Jodhpur, India

Aims \& Objectives: Haemoglobinopathies encompass the most common autosomal recessive genetically inherited monogenic red blood cell disorder, globally caused due to point mutation or elimination of the $\alpha / \beta$-globin genes resulting in abnormal globin chain synthesis. It has been estimated that in India 0.37 per 1,000 fetuses have $\mathrm{Hb}$ disorder. This is a retrospective observational study performed at AIIMS Jodhpur, to study the prevalence of haemoglobinopathies in western part of Rajasthan.

Patients/Materials \& Methods: All suspected case of haemoglobinopathies, from October 2017 to March 2020 were included in the study. Blood samples were collected in EDTA vials and analyzed with Sysmex XN1000 and peripheral blood smears were prepared. The haemoglobin HPLC for various haemoglobinopathies was performed on Biorad Variant D-10.
Results: In this retrospective study, we studied a total of 1668 cases. The mean age of the patients coming for HPLC testing was $24.78 \pm 12.62$ years with female preponderance of $4: 1$. On $\mathrm{Hb}$ HPLC, 225(13.48\%) cases revealed features of haemoglobinopathy while remaining $1443(86.52 \%)$ cases were normal. The most common $\mathrm{Hb}$ abnormality detected was $\beta$ thalassemia trait present in 181 $(10.85 \%)$ patients, followed by $\mathrm{Hb} \mathrm{D}$ Punjab trait in $17(1.01 \%)$ cases and $\beta$ thalassemia major $13(0.78 \%)$. One case $(0.06 \%)$ of $\mathrm{HbH}, \mathrm{E} \beta$ thalassemia and and $\mathrm{HbE}$ disease and two cases $(0.12 \%)$ of sickle- $\beta$ thalassemia, sickle-cell disease and $\mathrm{HbE}$ trait were observed.

Discussion \& Conclusion: In the present study, the prevalence of $\mathrm{Hb}$ disorders was found to be $13.48 \%$, and the most common $\mathrm{Hb}$ abnormality detected was that of $\beta$ thalassemia trait (10.85\%) which is in concordance with similar studies from other parts of the country. $\mathrm{Hb}-\mathrm{HPLC}$ is a reliable investigation for the early detection and management of hemoglobinopathies and variants, in view of the high incidence of beta thalassemia trait in the Indian subcontinent. Moreover, knowledge of common $\mathrm{Hb}$ patterns in this particular region helps to formulate appropriate preventive and therapeutic strategies.

\section{First Report of the Frequency of a Ly1-Antibody Reactive (Lyar)-Binding Motif Disruptive SNP in the Proximal Hbg1 Promoter (Rs368698783) Among Indian Transfusion-Dependent B-Thalassemia}

Namrata Singh ${ }^{* 1}$, Manu Jamwal ${ }^{1}$, Prashant Sharma ${ }^{1}$, Reena Das ${ }^{1}$, Alka R. Khadwal ${ }^{2}$, Amita Trehan ${ }^{3}$, Sanjeev Chhabra ${ }^{1}$

\section{${ }^{1}$ Hematology, ${ }^{2}$ Internal Medicine, ${ }^{3}$ Pediatrics (Hemato- Oncology), PGIMER, Chandigarh, India}

Aims \& Objectives: The Ly-1 antibody reactive clone (LYAR) protein is a repressor of $\gamma$-globin gene transcription that binds at the $5^{\prime}$ UTR of the A $\gamma$-globin gene. The rs368698783 SNP in A $\gamma$-globin gene $(\mathrm{G}>\mathrm{A})$ results in decreased binding efficiency of LYAR. Chen et al. in 2017 characterized the role of LYAR in ameliorating disease severity in $\beta$-thalassemia. They showed that attenuation of LYAR biding along with two repressive epigenetic regulators DNMT3A and PRMT5 from the HBG promoters mediates allele-biased $\gamma$-globin elevation by facilitating demethylation of $\mathrm{HBG}$ core promoter $\mathrm{CpG}$ sites in erythroid progenitors. We studied this for the first time in Indian $\beta$-thalassemia major and intermedia patients.

Patients/Materials \& Methods: One hundred symptomatic thalassemic patients were enrolled from the clinical hematology units. These comprised of $93 \beta$-thalassemia major patients and $7 \beta$-thalassemia intermedia patients. Thirty non-thalassemic controls were also included. All 130 subjects were screened for the rs368698783 polymorphism (NG_000007.3: g.47783G > A) by a customdesigned ARMS-PCR.

Results: Among controls, 11 were heterozygous (G/A), while 19 were homozygous wild-type $(\mathrm{G} / \mathrm{G})$, yielding an A-allele frequency of $18.3 \%$. Among the $\beta$-thalassemia major patients, 12 (12.9\%) were A/A, 27 (29.0\%) were G/G and 54 (58.1\%) were G/A genotypes. The A-allele frequency was $41.9 \%$. In case of $\beta$-thalassemia intermedia patients, all 7 were homozygous for wild-type allele (G/G).

Discussion \& Conclusion: The frequencies of the LYAR-binding motif (rs368698783) were defined for the first time among Indian patients, with a significantly higher allele frequency among thalassemia major and intermedia patients vis-à-vis controls. Further research will focus on assessing this genotypic marker in non-transfusion dependent $\beta$-thalassemia. 


\section{Profile of Autoimmune Haemolytic Anaemia: Analysis of 8 Years Data from a Single Center}

\author{
Avriti Baveja ${ }^{* 1}$, Prakas K. Mandal ${ }^{1}$, Shuvra N. Baul ${ }^{2}$, Sumit Mitra ${ }^{2}$, \\ Tuphan K. Dolai ${ }^{2}$ \\ ${ }^{1}$ Hematology, NRS, ${ }^{2}$ Hematology, Nrsmch, Kolkata, Kolkata, \\ India
}

Aims \& Objectives: Autoimmune haemolytic anaemia (AIHA) is defined as anaemia caused by the destruction of erythrocytes through autoantibodies directed against surface antigens of RBC. The present study conducted to know the clinical and laboratory profile and also the therapeutic outcome to different treatment modalities.

Patients/Materials \& Methods: This retrospective study analyzed the clinico-haematological profile and therapy outcome of 69 patients with autoimmune haemolytic anaemia (AIHA) from January 2012 to December 2019. The baseline characteristics and the therapy outcomes were assessed.

Results: This study identified 69 consecutive patients (56 primary; 13 secondary) of AIHA with a median age of 49 years. The common presentations included pallor $(98.5 \%)$ and jaundice $(84.5 \%)$ with presence of splenomegaly (56.5\%) and hepatomegaly (47.2\%). Direct Coombs' test was negative in two patients. Oral prednisolone produced remission in $91.04 \%$ patients with a median response duration of 28 days. Among responders, 28 patients relapsed after a median period of 31.3 months. The relapsed patients received steroid in most cases. Azathioprine, rituximab and splenectomy were given in eight, six and two patients respectively with an overall response rate of $62.65 \%, 66.6 \%$ and $100 \%$ respectively.

Discussion \& Conclusion: The common presentations in AIHA include pallor, jaundice and splenomegaly. Oral prednisolone produced remission in $91.04 \%$ patients. The relapsed patients received steroid in most cases; few others received azathioprine or rituximab and splenectomy done only in two patients.

\section{Red Cell Indices and Hemoglobin HPLC Parameters in a Spectrum of Thalassemias/Hemoglobinopathies in a Tertiary Care Hospital in Southern India}

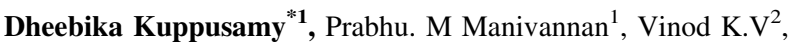
Dilli Kumar3, Rakhee Kar ${ }^{1}$

\section{${ }^{1}$ Department of Pathology, ${ }^{2}$ Department of Medicine, 3Department of Paediatrics, JIPMER, Puducherry, India}

Aims \& Objectives: Haemoglobinopathies are a heterogenous group of disorders ranging from various types of asymptomatic or mild carrier state with subtle hematological derangements to a full-blown disease state with hematological abnormalities. This study was undertaken to describe the hematological parameters in a varied spectrum of thalassemias/haemoglobinopathies diagnosed in a single referral centre.

Patients/Materials \& Methods: This was a cross sectional descriptive study over one year 10 months duration (Jan 2019 to October 2020) in a tertiary care centre in Southern India. The hemoglobin HPLC (Biorad D10, USA) and red cell parameters (XT 4000i, Sysmex corporation, Kobe, Japan) of all confirmed cases of thalassemias/ hemoglobinopathies were analyzed of which few were on follow-up. Results: A total of $107(47.7 \%$ Male and $52.3 \%$ female) positive cases were diagnosed on screening of 460 cases. A diverse group of hemoglobinopathies were seen. The most common diagnosis was heterozygous $\beta$-thalassemia seen in $43 \%$ cases. Among hemoglobinopathies, sickle hemoglobinopathies were commonest accounting to $26.2 \%$. A rare case of alpha hemoglobinopathy in four family members with an unknown peak of $16.8 \%$ at retention time (RT) of
4.34 min presenting with low $\mathrm{SpO} 2$ conforming with $\mathrm{Hb}$ Kirskey was identified. Another alpha hemoglobinopathy with unknown peak of $14.9 \%$ at RT of 3.9 min was observed. It matched with the RT of HbD Punjab, however the percentage of the unknown peak matched with an alpha hemoglobinopathy. We also encountered single cases of $\delta \beta$ thalassemia trait, $\mathrm{HbH}$ disease, $\mathrm{Hb} \mathrm{J}$-Meerut (coexisting nutritional deficiency) and $\mathrm{Hb}$-Lepore. The hematological findings of the various spectrum of thalassemialhemoglobinopathies diagnosed are summarized in table 1 . Majority of the cases were from Tamil Nadu $(61.7 \%)$ followed by West Bengal (20.6\%), Puducherry (10.3\%) and other states $(7.5 \%)$. Most of the thalassemias, sickle hemoglobinopathies and alpha hemoglobinopathies were from the local and regional population. In contrast most of the $\mathrm{Hb}-\mathrm{E}$ hemoglobinopathies were from West Bengal.

Discussion \& Conclusion: Although this region is not considered to be a part of typical thalassemia belt, we came across a variety of thalassemias/hemoglobinopathies. Most of these were from local regional population including several alpha thalassemia variants which would need molecular work-up for better characterization.

\section{Can Flaer be Used as a Standalone Reagent for the Testing of Paroxysmal Nocturnal Hemoglobinuria?}

Parveen Bose $^{* 1}$, Praveen Sharma ${ }^{1}$, Man Updesh S. Sachdeva ${ }^{1}$, Jasmina Ahluwalia ${ }^{1}$, Amita Trehan ${ }^{2}$, Pankaj Malhotra3, Neelam Varma $^{1}$

${ }^{1}$ Hematology, ${ }^{2}$ Pediatrics (Pediatric Hemato-oncology), 3Internal Medicine (Clinical Hematology), Postgraduate Institute of Medical Education and Research, Chandigarh, India

Aims \& Objectives: Flow cytometry is considered to be 'gold standard' for the diagnosis of paroxysmal nocturnal hemoglobinuria $(\mathrm{PNH})$ wherein there is loss of glycosylphosphatidylinositol (GPI)linked antigens from cell surface. Fluorescent aerolysin (FLAER) has specific binding to GPI moiety and it is known to increase the sensitivity of PNH detection assay. Consensus recommendations require identification of at least 2 GPI-deficient markers on at least neutrophils and monocytes. However, we hypothesize that FLAER as a standalone reagent may be equally effective for detection of $\mathrm{PNH}$ clone.

Patients/Materials \& Methods: A prospective study was carried out from September 2019-August 2020 including 163 patients referred to the flow cytometry facility of Hematology department. PNH assay was performed using pre-titrated cocktails of antibodies. "Routine panel" consisted of CD45, CD15, CD64, CD24, CD14 and FLAER. "Proposed panel" included CD45, CD15, CD64 and FLAER. Sample were processed using "lyse-wash-stain-wash" technique and acquired on Navios-Ex flow cytometer (Beckman Coulter Inc, USA). PNH clone was reported above $0.01 \%$.

Results: PNH clone was found in 30/163 patients $(18.4 \%)$ by both the panels tested. PNH clone range for granulocytes was $0.02 \%-87.71 \%$ and for monocyte was $0.04-86.46 \%$ in routine panel. The range in proposed panel for granulocyte was $0.02 \%-86.61 \%$ and monocyte was $0.05-87.58 \%$. There was a significant correlation between the size of PNH clone of granulocytes and monocytes among two panels tested (granulocytes $\mathrm{r}=1.000, p=0.00$; monocytes $\mathrm{r}=0.996$, $p=0.00)$. Also, on 'paired' t test analysis there was no statistical difference between two tubes for the identification of PNH (granulocytes $p=0.73$, monocytes $p=0.20$ ). The proposed panel did not identify any false positive PNH clone in any patient compared to the routine panel.

Discussion \& Conclusion: FLAER as a standalone marker, in our experience, is specific and sensitive for the identification of PNH 
clone in granulocytes and monocytes. The proposed 'test' panel is efficient and cost-effective for high sensitive PNH testing, especially in resource-limited settings.

\section{Emicizumab Improves the Quality of Life in Haemophilia a with Inhibitor: A Case Report}

\author{
Rohini T ${ }^{* 1,2}$, Sheena Mathew ${ }^{3}$, Vijayakumar N. Pillai ${ }^{4}$ \\ ${ }^{1}$ Professor, Samaritan College of Nursing, Ernakulam, \\ ${ }^{2}$ Hemophilia Treatment centre, ${ }^{3}$ Medical Officer, Hemophilia \\ Treatment Centre Aluva, ${ }^{4}$ Medical officer in-charge, Hemophilia \\ Treatment centre, Aluva, India
}

Aims \& Objectives: Haemophilia is an inherited bleeding disorder affecting one in 5000-10000 male births. Emicizumab has been approved in India for Haemophilia A with FVIII inhibitors. The aim of the study was to determine the effect of Emicizumab on the Quality of Life in a 12-year-old boy with haemophilia A with factor VIII inhibitor.

Patients/Materials \& Methods: Patient was born by full term normal delivery to non-consanguineous parents in 2007. He suffered recurrent bleeding in his joints. From 2007 till 2016 he was taking 'on demand factor replacement'. In 2016, child was tested positive for factor VIII inhibitor. When he suffers bleeds, he used to receive Inj. FEIBA (Factor eight inhibitor bypass activity) intravenously from HTC, Aluva. In 2019, he was admitted for six months in HTC, Aluva due to severe joint bleeds. The child was given 28 vials of Hemlibra (Emicizumab: $60 \mathrm{mg} / 0.04 \mathrm{ml}$-Roche products (India) Pvt. Ltd.) with the prescribed dosage. Weekly Emicizumab replacement was started on 19.10.2019 via subcutaneous in the forearm and continued till 23.04.2020.

Results: Parents exclaimed, "there are no words to tell about the wonderful efficacy of the drug". Child started to perform all his daily activities, just like any other normal child of his age. There was drastic improvement in his daily activities. He was able to walk effortlessly, free of any pain in his joint. He started playing with his younger sister. He went to school without any absenteeism. Compared to FEIBA child liked receiving Emicizumab infusion as it was given subcutaneously and there was no need for multiple pricks due to difficult vein access. As the trial has come to an end, the child and his parents are requesting, that they would be grateful if the drug would be continued.

Discussion \& Conclusion: Emicizumab is a chimeric bispecific humanised antibody directed against FIXa and FX, which mimics the co-factor function of FVIII. Its prophylactic use is widely accepted. Increased cost of this drug is an obstacle, which if tackled by the Government would promise a life of joy to these children who lead a miserable life with inhibitors.

\section{A Unique Case of CML Presenting with Acquired Von Willebrand Disease}

Satya P. Mahapatra ${ }^{* 1}$, Dhiraj Kishore ${ }^{1}$, Ranjan Bhattnagar ${ }^{1}$, Prachi Mohapatra $^{1}$, Sushil Gaur ${ }^{1}$, Satyam K. Singh ${ }^{1}$

${ }^{1}$ Internal Medicine, IMS, BHU, Varanasi, India

Aims \& Objectives: Presenting a case of CML in chronic phase with extreme thrombocytosis leading to acquired Von Willebrand Disease. Patients/Materials \& Methods: A 64 year old male, diagnosed case of CML in chronic phase in 2014 (baseline CBC- Hb-9.9 g/dl, TLC2.12L/ul, Plt-1.59L/ul, BCR-ABL-29.58\%, IS-18.5, Ph + cells$100 \%)$.The patient was on Imatinib since then with good compliance. Now the patient presented with complaints of black tarry stools for
7 days and epistaxis 1 episode 3 days back. On examination, vitals were stable, pallor was present and spleen was not palpable. Routine CBC showed anaemia (Hb-7.9 g/dl), mildly increased TLC (13200/ ul), N/L- 85/7, and increased platelets count (11.2 L/ul). LFT, RFT \& PT/INR were normal, aPTT was slightly prolonged (36.5 s). Peripheral blood smear showed mild leucocytosis with shift to left, no blast cells were seen and platelet counts were severely increased. Bone marrow aspiration was suggestive of CML in chronic phase. Upper GI endoscopy revealed duodenitis and antral gastritis. Repeat BCR-ABL was done which showed \% ratio- 9.66\%, IS-2.41(Major molecular response was not achieved). Imatinib resistance mutational analysis was done which was negative. Von Willebrand factor assay showed slightly decreased VWF Ag level(63\%), decreased VWF Ristocetin cofactor assay-VWF RCo(28.7\%) and decreased VWF RCo/Ag $(0.45)$ suggestive of acquired von Willebrand disease.

Results: Hence patient was diagnosed with CML in chronic phase with extreme thrombocytosis causing acquired Von Willebrand Disease. The patient was managed with 2 unit pRBC transfusion, PPIs, Nilotinib(300 mg BD), Hydoxyurea(1 g/day) \& Tranexamic $\operatorname{acid}(1.5 \mathrm{~g} /$ day). Patient improved clinically with reduction in platelet counts and control of bleeding.

Discussion \& Conclusion: CML is a clonal hematopoietic stem cell disorder. The disease is driven by the BCR-ABL1 chimeric gene product, that codes for a constitutively active tyrosine kinase, resulting from a reciprocal balanced translocation between the long arms of chromosomes 9 and $22, \mathrm{t}(9 ; 22)$, known as the Philadelphia chromosome $((\mathrm{Ph})$. Laboratory studies often reveal a significant leucocytosis with neutrophilia. A moderate thrombocytosis may be present, but is not usually problematic. Extreme thrombocytosis, defined as a platelet count $>10$ lakhs $/ \mathrm{mm} 3$, is rare in CML. Extreme thrombocytosis can cause acquired von Willebrand disease due to consumption of von Willebrand multimers by elevated platelet counts.

\section{Von Willebrand Disease Management During Pregnancy: A Case Report}

Divjot S. Lamba ${ }^{* 1}$, Manvi Talwar ${ }^{1}$, Rekha Hans ${ }^{1}$, Pooja Sikka ${ }^{2}$, Pankaj Malhotra ${ }^{3}$, Ratti R. Sharma ${ }^{1}$

${ }^{1}$ Transfusion Medicine, ${ }^{2}$ Obstetrics and Gynaecology, ${ }^{3}$ Internal Medicine, Post Graduate Institute of Medical Education and Research (PGIMER), Chandigarh, India

Aims \& Objectives: To present an interesting case of Von Willebrand disease management of a Pregnant female as during pregnancy and parturition various patterns of changes are seen in levels of both factor VIII (FVIII) and von Willebrand factor (VWF).

Patients/Materials \& Methods: PRESENTATION OF CASE We report a case of a 34 year old primigravida who was a known case of Von Willibrand Disease type 2 (non-type 2B). The patient enrolled at our institute at 22 weeks Period of gestation (P.O.G) for antenatal check up in view of her knowledge of her medical condition. Elective LSCS was planned for the patient keeping in view the breech presentation of the fetus with anterior grade III placenta and a L-sided uterine fibroid. Various investigations done during antenatal and postnatal period are mentioned in Table-I. PAST HISTORY It was significant with a single episode of bleeding per vaginum at the age of 20 years during which her $\mathrm{Hb}$ dropped to $2 \mathrm{gm} / \mathrm{dl}$ for which she was transfused with 2 units of packed red blood cells at a local hospital. She underwent tonsillectomy in 2003 in a private facility and had prolonged bleeding in the post-operative period which required suturing of the tonsillar bed.

Results: MANAGEMENT The patient was advised injection Immunate (plasma derived factor VIII with VWF) containing 250 IU FVIII/ 
$190 \mathrm{IU}$ VWF at a dose of $19 \mathrm{IU} / \mathrm{kg}$, with target RICOF activity of $50 \mathrm{IU}$ at the time of delivery Department of transfusion medicine was notified for prior arrangement of the required components (packed red blood cells and cryoprecipitates) as an additional supportive measure. LSCS and postoperative period went uneventful without any bleeding episode.

Discussion \& Conclusion: Transfusion therapy consisting of human derived and recombinant factors containing FVIII/VWF is currently considered the treatment of choice in patients of vwd with pregnancy. It is recommended to maintain the levels of VWF and Factor VIII above $50 \mathrm{IU} / \mathrm{dL}$ or higher during delivery and for 3-5 days after delivery to prevent primary PPH. A well characterisation of diseases type and subtype and a good and robust transfusion support mechanism ensures a safe and uneventful delivery in such cases.

Supporting Document:

Table-I: INVESTIGATIONS at our institute

\begin{tabular}{|c|c|c|c|}
\hline & 20 weeks & $31+5$ weeks & POST OP DAY 1 \\
\hline $\begin{array}{l}\text { Prothrombin time } \\
(11.0-12.5 \text { secs) }\end{array}$ & 11 seconds & 11.3 seconds & 14 seconds \\
\hline $\begin{array}{l}\text { PTI } \\
(85-100 \%)\end{array}$ & $100 \%$ & $100 \%$ & $99 \%$ \\
\hline $\begin{array}{l}\text { INR } \\
(0.8-1.1)\end{array}$ & 0.97 & Not done & 1.0 \\
\hline $\begin{array}{l}\text { PTTKIAPTT } \\
(30-40 \text { secs })\end{array}$ & 35 seconds & 33.5 seoonds & 40 seconds \\
\hline $\begin{array}{l}\text { Fibrinogen Assay } \\
(1.5-3 \mathrm{gms} / \mathrm{L})\end{array}$ & Not done & $4.72 \mathrm{gm} / \mathrm{L}$ & Not done \\
\hline $\begin{array}{l}\text { Factor VIII } \\
(50 \%-150 \%)\end{array}$ & Not done & $54 \%$ & Not done \\
\hline $\begin{array}{l}\text { Vwe Ag Assay } \\
\text { (3.5-26.6 ugiml) }\end{array}$ & Not done & 40.41 & Not done \\
\hline $\begin{array}{l}\text { Ristocetin Cofactor } \\
\text { Assay (RICOF) } \\
(50 \%-200 \%)\end{array}$ & Not done & $16.4 \%$ & Not done \\
\hline
\end{tabular}

\section{Evaluation of Thromboelastogram Parameters in Factor Deficient Plasma}

Geeta Vidyadharan*1, Aswathy Anil kumar ${ }^{1}$, Anu C. Jacob ${ }^{1}$, Karthika K. ${ }^{1}$, Bitty Kurian ${ }^{2}$, Vineeta Vijayakumar ${ }^{1}$, Geethu $\mathbf{M}^{1}$, Asha M.K ${ }^{1}$, Sunita $K^{1}$, Akhil K. K.G ${ }^{1}$, Rema $G^{3}$, Bhavani $M^{1}$

${ }^{1}$ Pathology, Amrita Institute of Medical Sciences, ${ }^{2}$ Pathology, Amrita Institute Of MedicalSciences, ${ }^{3}$ Clinical Hematology, Amrita Institute of Medical Sciences, Cochin, India

Aims \& Objectives: Aim: To study the various TEG parameters in factor deficient plasma. Objectives: (1) To observe change in each TEG parameter values in diluted plasmas with respect to control samples. (2) To assess the TEG parameter which best correlates with factor deficiencies in serially diluted plasma samples.

Patients/Materials \& Methods: The study was carried out as an experimental investigation in 2 phases: TEG study was compared with conventional coagulation studies. 10 normal healthy adult individuals ( 5 males and 5 females) with normal coagulation profiles were selected.Citrated whole blood and plasma samples were collected from each individual. The samples were diluted with Owren koller buffer into 5 discrete groups corresponding to normal, mild, moderate and severe factor deficiencies totaling up to 50 individual diluted samples. Classical clotting tests (aPTT, fibrinogen and Factor assays) and TEG testing was carried out on the plasma and citrated whole blood samples respectively. Each TEG parameter values viz R time, alpha angle, $\mathrm{K}$ time as well as MA and the lysis 30 were recorded in each dilution. The results obtained were analyzed statistically to determine the correlations of TEG values with the conventional clotting times as well as among themselves.

Results: Statistical comparison and correlation of the data. (1) Revealed direct correlations between aPTT and R time as well as between fibrinogen concentration and $\alpha$ angle. (2) Whereas an inverse linear correlation was established between the R time and FVIII: C, determining the sensitivity of the $\mathrm{R}$ time in detecting a factor deficiency at $\leq 25 \%$ of FVIII: C. (3) The study was also able to demonstrate an independent relationship of the $\mathrm{R}$ time with other TEG variables.

Discussion \& Conclusion: 1 . TEG variable $\mathrm{R}$ time could be used as an aid in expediting decision making regarding factor assay requirement in individuals with clinically unclassified coagulopathy especially in underpriviledged laboratories. 2. Also it could act a tool for prediction of the hemostatic adequacy in known hemophiliacs with ongoing factor product infusion, and can be utilised in place of repeated expensive factor measurements.

\section{Utility of Platelet Function Testing in Patients with Suspected Bleeding Disorders}

Karthika K V ${ }^{* 1}$, Geeta Vidyadharan ${ }^{1}$, Jyotsna Yesodharan ${ }^{1}$, Anu C. Jacob $^{1}$, Bitty Kurian ${ }^{1}$

${ }^{1}$ Department of Pathology, Amrita Institute of Medical Sciences, Kochi, India

Aims \& Objectives: This was a retrospective observational study to evaluate the utility of platelet function testing (PFT) by Light Transmission Aggregometry (LTA) as part of hemostatic workup and identify the spectrum of platelet function defects in a tertiary care centre in South India. The data was collected over a period of one year from August 2018 to July 2019.

Patients/Materials \& Methods: All patients who presented with suspected bleeding disorder and normal screening tests (Platelet count, PT, aPTT, TT, fibrinogen) were taken up for platelet function testing. Samples were collected in $3.2 \%$ sodium citrate vacutainers after overnight fasting. PFT was done on Platelet Rich Plasma (PRP) using PAP 8E LTA with healthy controls in every run. The agonists used were- Adenosine di-phosphate (ADP 10um/L), Epinephrine $(1 \mathrm{mg} / \mathrm{ml})$, Collagen $(2 \mathrm{ug} / \mathrm{ml})$, Arachidonic acid $(1 \mathrm{mM})$ and Ristocetin $(1.25 \mathrm{mg} / \mathrm{ml}$ and $0.5 \mathrm{mg} / \mathrm{L})$. von Willebrand antigen levels and Ristocetin cofactor activity were tested in required cases.

Results: Of the 200 patients who reported to coagulation laboratory, PFT was done in 55 cases. Of these 55 patients, 38 showed platelet function defects which included 21 adults and 17 children. Most of the patients had mucocutaneous bleeds such as epistaxis, oral bleed, ear bleed and hematuria. There were 13 cases of von Willebrand disease ( $\mathrm{vWD}$ ), 3 were acquired in association with a chronic myeloproliferative neoplasm. vWD was the most common abnormality on platelet function testing. Of these 13 cases, there were 7 cases of type 1 and 2 cases of type $2 b$ vWD. The rest of these cases did not undergo complete testing and were provisionally diagnosed as possible vWD- ?type1/2. Six patients (16\%) had Acquired Platelet Dysfunction with Eosinophilia (APDE), the most common abnormality was reduced response to ADP. There were 3 patients $(4 \%)$ with Glanzmann's Thrombasthenia with reduced response to all agonists except ristocetin. Rest of the cases showed unclassified platelet function defects with reduced response to one or more agonists - 2 of 
these were drug induced and one case was a known case of platelet signalling defect.

Discussion \& Conclusion: PFT by LTA has significantly helped us to diagnose and categorise platelet function defects such as vWD, APDE and Glanzmann's thrombasthenia and is essential for the workup of inherited and acquired platelet function defects.

\section{Spectrum of Platelet Function Disorders in a Tertiary Care Hospital}

\author{
Anupama Sinha*1 $^{*}$, Sushma Belurkar ${ }^{1}$ \\ ${ }^{1}$ Department of Pathology, Kasturba Medical College, MAHE, \\ Manipal, UDUPI, Karnataka, India
}

Aims \& Objectives: (1) To study the clinical and demographic aspects in platelet function disorders (PFD). (2) To study the spectrum of various platelet function disorders diagnosed based on platelet aggregation studies.

Patients/Materials \& Methods: Herein, we present a retrospective study of 45 cases of platelet function disorder diagnosed during a study period of 5 and 1/2 years from January 2015 to August 2020. Clinical data was collected from patient case files and laboratory parameters were obtained from laboratory information system (LIS) of the hospital.

Results: Based on the lab parameters studied, out of total of 45 cases, $24(53.3 \%)$ were classified as inherited PFDs, 17 (37.7\%) as acquired PFDs and 4 under unclassified category as they showed non-specific findings. Out of 24 cases of inherited PFDs, 17 were diagnosed as Glanzmann's thrombasthenia, 5 as von Willebrand's disease and 2 as platelet storage pool disorder. Among 17 acquired PFDs, 15 were drug induced, 1 was acquired secondary to eosinophilia and 1 secondary to myeloproliferative neoplasm. Patient age ranged from less than 1 year to 77 years of age with clinical presentation as minor as easy fatiguability to severe menorrhagia and hematemesis. 6 patients had associated severe anemia and 1 case was associated with Hermansky-Pudlak syndrome.

Discussion \& Conclusion: In our study, inherited PFD had higher incidence compared to acquired PFD, attributing cause could be under reporting of acquired PFD due to lack of methodical approach. Diagnosis of acquired PFD is important as they can be rectified with treatment of primary cause unlike inherited PFD which needs lifelong treatment. On other hand, inherited disorders can also lead to severe bleeding tendency if not diagnosed accurately for which newer entities like flow cytometric analysis and mutational studies will be helpful.

\section{Psychosocial Impact of Covid19 Pandemic on People with Haemophilia and their Concerns Beyond the Risk of Infection}

Ranvijay Singh ${ }^{* 1}$, Bhavana Mastebhakti ${ }^{2}$, Avishkar Agrawal ${ }^{1}$, Sunita Aggarwal ${ }^{1}$, Ankitesh Kumar ${ }^{1}$, Shikha Rawat ${ }^{3}$

${ }^{1}$ General Medicine, Maulana Azad Medical College and Lok Nayak Hospital, ${ }^{2}$ General Medicine, Maulanaa Azad Medical College and Lok Nayak Hospital, ${ }^{3}$ Haemophilia Day Care Centre, Lok Nayak Hospital, New Delhi, India

Aims \& Objectives: The worldwide SARSCoV2 pandemic can lead to either respiratory infection or isolation due to containment zones that produce a significant impact on chronic diseases such as inherited bleeding disorders- Haemophilia. The aim of the study was to evaluate the impact of COVID19 pandemic on patients with Haemophilia, regarding their psychosocial issues and financial concerns.
Patients/Materials \& Methods: A survey evaluating the impact of COVID19 pandemic on psychosocial health of people with Haemophilia was done. A questionnaire was developed for the survey that was taken up by 70 out of 100 new patients attending the Haemophilia day care center, Lok Nayak Hospital, Delhi during a two-month period from 1-6-2020 to 31-7-2020. The survey included information on demographic and clinical data, needs, psychosocial issues, financial concerns and experiences regarding medical care during COVID19 pandemic.

Results: In total, 73 patients with Haemophilia completed the survey (73\% response rate). Most patients suffered from Haemophilia A (73\%) while the others had Haemophilia B (37\%). $85 \%$ of the patients had psychosocial concerns which included insecurity regarding accessibility of factors in case of bleeding episodes, support and availability of the Haemophilia center personnel for assistance. Patients were also anxious and frustrated about regular drug production and supply. $68 \%$ of the patients had financial concerns which not only involved the fear of decreased earning hands in the family due to pandemic but mainly involved the fear of inaccessibility of appointments and cost-free factors in the government hospitals that have got converted into designated COVID centers. $74 \%$ of the patients had social concerns regarding the containment-associated isolation zones due to which the patients were not able to freely avail the medical services as and when required.

Discussion \& Conclusion: The corona virus pandemic has impact on patients with bleeding disorders such as Haemophilia in terms of thoughts and worries related to their underlying disease, accessibility and availability of affordable medical care and feelings caused by the coronavirus pandemic in general. Also, caregivers of these patients are equally concerned. Counselling and reassurance about COVID19 awareness and precautions among Haemophilia patients may reduce their worries and concerns. Because longterm effects of the pandemic on psychosocial health and financial burden on these patients are unknown, they should be examined in the followup.

\section{Evaluation of Plasma Von Willebrand Factor Level in Healthy Blood Donor Population and Its Association with Blood Group}

\author{
Nikita Agrawal ${ }^{* 1}$, Sangeeta Sindhwani ${ }^{1}$, Sunita Sharma ${ }^{1}$
}

${ }^{1}$ Pathology, LHMC, New delhi, India

Aims \& Objectives: To evaluate plasma von Willebrand factor level and fVIII level in healthy Indians along with its association with ABO blood group.

Patients/Materials \& Methods: The study was a hospital based Cross sectional study conducted from November 2018 to March 2020. Two thousand sixteen healthy adult donors who came for blood donation to Regional Blood Transfusion Centre, Lady Hardinge Medical College and Associated Hospitals were recruited in the study after excluding those on medications. von Willebrand factor antigen level (vWF:Ag) and fVIII coagulant assay along with $\mathrm{ABO}$ and $\mathrm{Rh}(\mathrm{D})$ blood group typing, complete blood count, prothrombin time, activated partial thromboplastin time were done in the donors.

Results: The age of the study population ranged from 18 years to 55 years. Maximum number of donors belonged to B Rh (D) positive blood group. Majority were males. None of them had personal or family history of bleeding manifestation. All the donors had hemoglobin levels and platelet counts within normal range. PT and aPTT were prolonged beyond the reference range in one and five out of two thousand sixteen donors respectively. vWF level of donors ranged from $24 \mathrm{IU} / \mathrm{dL}-186 \mathrm{IU} / \mathrm{dL}$ with mean of $96.31 \mathrm{IU} / \mathrm{dL}$. Low vWF antigen level (below $50 \mathrm{IU} / \mathrm{dl}$ ) was found in $2.5 \%$ of all donors while $0.1 \%$ donors $(2 / 2016)$ had level $<30 \mathrm{IU} / \mathrm{dL}$. Donors with $\mathrm{O} \mathrm{Rh}$ 
(D) positive blood group had lowest vWF level while A Rh(D) negative donors had highest vWF level. Factor VIII level of donor population ranged from $22 \%-174 \%$ with a mean of $98.82 \%$. Out of 2016 donors, $2.48 \%$ donors had f VIII levels below 50\%. vWF level showed a positive, statistically significant correlation with age and fVIII level $(p<0.05)$ but not with gender $(p=0.062)$.

Discussion \& Conclusion: Our study has shown that ABO blood group and age but not gender of an individual have significant influence on plasma levels of vWF: Ag while factor VIII level is influenced by blood group but not age of the individual. A positive correlation was observed between plasma vWF level and factor VIII level $(p<0.001)$.

\section{Role of Platelet Indices in the Diagnosis of Immune Thrombocytopenia (ITP) in a Tertiary Care Centre: A Case Series}

Karishma Makwane ${ }^{* 1}$, Maneesh Sulya ${ }^{2}$, R.K. Nigam ${ }^{3}$, Reeni Malik ${ }^{4}$

${ }^{1}$ P.G. Third Year, Dept. of Pathology, ${ }^{2}$ Assistant Professor, Dept. of Pathology, ${ }^{3}$ Professor, Dept. of Pathology, ${ }^{4}$ Professor and

Head, Dept. of Pathology, Gandhi Medical College, Bhopal, India

Aims \& Objectives: To assess the diagnostic value of platelet indices [Mean Platelet Volume (MPV), Platelet Distribution Width (PDW) and Platelet Large Cell-Ratio (P-LCR)] in cases of Immune thrombocytopenia so that it could assist in predicting and diagnosing patients of ITP and aid as an indicative tool in discriminating hyperdestructive and hypo-productive causes of thrombocytopenia, preventing the need of invasive procedures.

Patients/Materials \& Methods: The records of 15 patients with bone marrow aspiration(BMA) confirmed ITP, came with isolated thrombocytopenia from January 2020 to October 2020 were retrospectively reviewed, and were compared with 15 hypo productive causes of thrombocytopenia. Hematological analysis (complete blood count, platelet indices) done by Midray-3600 hematological analyzer were noted and evaluated along with the clinical details and bone marrow aspiration findings. The Data were collected from the hospital and pathology department, GMC, Bhopal, a tertiary care center.

Results: Out of 15 cases taken of ITP, 7-9 years was the most common age with female predominance. The most common presentation was skin rashes followed by gum bleed and epistaxis. Mean platelet count was 30,000/cumm with all the platelet indices significantly higher in patients of ITP(MPV,PDW and P-LCR) than in hypo productive thrombocytopenic causes (Myelofibrosis, AML, Megaloblastic anemia etc.) which shows normal/lower indices values. In particular MPV and P-LCR indicates a better predictive tool in differentiation the two. The bone marrow findings along with peripheral blood was consistent with ITP in all the patients (i.e. showing thrombocytopenia and giant platelets and increased/normal megakaryocytes in marrow) and almost all patients responded to corticosteroids.

Discussion \& Conclusion: This study suggest that if these platelet indices which are inexpensive to measure are used in a right direction along with the help of proper clinical details, it can discriminate ITP from hypo productive thrombocytopenia thereby, help in avoiding or delaying ITP patients from undergoing unnecessary, invasive bone marrow aspiration or prevent undesirable platelet transfusion.
Immune Tolerance Induction and Its Practical Limitations in Management of Hemophilia Patients with Inhibitor in Indian Scenario

Soumita Roy ${ }^{* 1}$, Arun K. De ${ }^{1}$

${ }^{1}$ Pediatrics, Midnapore Medical College, Midnapore, India

Aims \& Objectives: To find out the practical issues in administering ITI to the inhibitor patients in Indian scenario.

Patients/Materials \& Methods: A prospective, observational, unicentric study was conducted on haemophilia patients with inhibitors of 0-18yrs of age attending pediatric haemophilia treatment centre at Midnapore Medical college, Midnapore, during January 2020- June 2020, treated by low dose ITI thrice weekly, having no other platelet or vascular disorders or no h/o chronic illness. After recruitment, ITI was administered to the patients and outcome and practical issues were assessed.

Results: Out of thirty(30) severe haemophilia A patients, $6(20 \%)$ patients developed inhibitor. Among them, 4 (66.66\%) patients developed high titre inhibitors, $2(33.33 \%)$ patients developed low titre inhibitors as measured by Bethesda assay. ITI was started on the 4 patients with high titre inhibitors@50U/kg/d thrice a week and outcome was assessed by HJHS2.1 scoring showing no remarkable improvement in terms of joint score, bleeding frequency and quality of life in $3(75 \%)$ patients, with mild improvement in $1(25 \%)$ patient. Discussion \& Conclusion: Development of inhibitor was noted in $20 \%$ patients with severe haemophilia A, $66.66 \%$ having high titres. Low dose ITI was started on all four of them, showing no marked improvement in majority. Though patient's compliance is an important factor, role of treatment providers is also an important issue in terms of providing right amount of factor at right frequency, where fund crunch is an important issue. So even providing low dose ITI is a big challenge in our setup.

\section{Impact of Compliance to Prophylactic Therapy in Haemophilia Patients on Annual Joint Bleeding Rate}

\author{
Abhilash Chatterjee $^{* 1}$, Arun K. De ${ }^{1}$ \\ ${ }^{1}$ Pediatrics, Midnapore Medical College and Hospital, \\ Midnapore, India
}

Aims \& Objectives: The aim of this study is to assess the degree of annual joint bleeding rate with respect to the compliance to prophylactic therapy in Hemophilia patients attending the Hemophilia Treatment Centre in a tertiary care teaching hospital.

Patients/Materials \& Methods: A cross sectional observational study was conducted on 32 boys of age ranging from 4 to 18 years in the Pediatrics Department of Midnapore Medical College, Paschim Medinipur, West Bengal for prophylactic Factor VIII or Factor IX therapy from August 2019 to July 2020. Number of weeks patients were absent from taking prophylactic therapy was calculated through history taking and available records. Annual joint bleeding rate was estimated by calculating the total number of joint bleeds during the period of study through history taking and documents of hospital admission for joint bleeds wherever available. Data were analysed using Statistical Package for Social Sciences.

Results: The number of weeks for which patients remained absent from receiving prophylactic therapy ranged from 4 to 18 weeks. The mean of the annual joint bleeding rate in patients who were absent for more than 6 weeks was 7 episodes per year and that in patients who were absent for 6 weeks or less was 3 episodes per year.

Discussion \& Conclusion: Annual joint bleeding rate is markedly low in Hemophilia patients who are compliant to prophylactic therapy. 
Impact of Prophylactic Therapy on Rehabilitation Pattern of Haemophilia Patients

\author{
Asaduzzaman Laskar ${ }^{* 1}$, Arun K. De ${ }^{2}$ \\ ${ }^{1}$ Pediatrics, Midnapore Medical College and Hospital, \\ ${ }^{2}$ Paediatric, Midnapore Medical College \& Hospital, Midnapore, \\ India
}

Aims \& Objectives: Pattern and degree of rehabilitation of haemophilic patients taking prophylaxix.Incidence of arthropathy,joint destruction of patient taking prophylaxis.

Patients/Materials \& Methods: A cross sectional observational study done on 32 boys of age ranging from 1 to 18 years taking prophylaxis in the paediatric department of Midnapore medical college \&hospital, Medinipur from June 2019 to May 2020. The incidence of joint bleeding and its acute and chronic complications of these haemophiliacs was recorded during this study period. Their pattern and degree of rehabilitation in the form of schooling, participation in sports and other social activities were recorded. Results: Among 32 patients of taking prophylaxis only 6 patient (18.75\%)developed joint bleeding and only one(3.1\%) patient developed arthropathy. Rehabilitation pattern was improved in the form of more school attendance and more complex sports participation.

Discussion \& Conclusion: Prophylactic factor(OR) therapy decreases incidence of joint bleeding,arthropathy and decreses needs of rehabilitation or improves the quality of rehabilitation.

\section{An Objective Analysis of The Joint Health and Quality of Life of Paediatric Haemophiliac Patients at a Tertiary Care Centre in Gujarat}

Khushboo K. Taneja*1 ${ }^{* 1}$, Sheila Aiyer ${ }^{1}$

\section{${ }^{1}$ Pediatrics, Medical College, Vadodara, Vadodara, India}

Aims \& Objectives: To analyse the functional independence, joint health, and quality of life of paediatric haemophiliac patients using FISH (Functional Independence Score in Haemophilia), HJHS 2.0 (Haemophilia Joint Health Score), Haemo-QoL(Haemophilia Quality of Life) scores.

Patients/Materials \& Methods: A cross sectional study conducted at Medical College, Vadodara, included patients aged 4 to 8 years, attending the Haemophilia Clinic over a period of 1 year. Joint health was assessed using FISH, HJHS, and Annual Bleeding Rate (ABR), while quality of life was assessed using the Haemo-QoL questionnaire, after due permission. Scores were expressed in means, standard deviations, and appropriate statistical tests were used parametric and non-parametric data. $p$ value $<0.05$ was considered significant.

Results: Of 52 patients were enrolled, maximum belonged to 8 12 years group. Only $46 \%$ were diagnosed at less than 1-year age. Annual bleeding rate was highest in patients of severe Haemophilia A. Increasing age, severity of disease and presence of inhibitors negatively impacted FISH and HJHS $(p<0.05)$. The factors having highest negative impact on the quality of life (highest HaemoQoL score) of kids were the subscales- treatment (frequent injections), sports (less capacity than peers) and burden on the family. Similarly for parents, the negatively impacting factors were sports, global heath and future concerns. With increasing age and disease progression, subscales worst affected trend towards sports and physical health, bearing effect on schooling and peer adjustment.

Discussion \& Conclusion: Inhibitor positivity worsened outcome in patients in terms of quality of life and functional independence. Hence, this complication should be actively screened for. Different age groups valued different factors as worsening their quality of life, also differed between parent and child. FISH and HJHS can be instituted as regular, low cost monitoring tools for joint health, HaemoQoL for quality of life.

Supporting Document:

\section{Table 1 HaemoQoL Kid vs Parent comparison}

\begin{tabular}{|c|c|c|c|c|}
\hline \multirow{2}{*}{$\begin{array}{c}\text { Age } \\
\text { Group }\end{array}$} & \multicolumn{2}{|c|}{ Kid } & \multicolumn{2}{c|}{ Parent } \\
\cline { 2 - 5 } $\mathbf{4}$ to $\mathbf{7} \mathbf{y r s}$ & $45.9 \pm 8.6$ & $\begin{array}{c}\text { Worst } \\
\text { Subscale }\end{array}$ & Mean & $\begin{array}{c}\text { Worst } \\
\text { Subscale }\end{array}$ \\
\hline family, friends & SD & $43.7 \pm 6.6$ & $\begin{array}{c}\text { Dealing, } \\
\text { treatment, } \\
\text { family }\end{array}$ \\
\hline $\mathbf{8}$ to $\mathbf{1 2} \mathbf{y r s}$ & $44.9 \pm 6.4$ & $\begin{array}{c}\text { Sport, } \\
\text { perceived } \\
\text { support, } \\
\text { treatment }\end{array}$ & $45.4 \pm 7.6$ & $\begin{array}{c}\text { Global health, } \\
\text { sports, } \\
\text { friends }\end{array}$ \\
\hline $\mathbf{1 3}$ to $\mathbf{1 6} \mathbf{y r s}$ & $47.3 \pm 7.5$ & $\begin{array}{c}\text { sports, } \\
\text { treatment }\end{array}$ & $48.9 \pm 8.7$ & $\begin{array}{c}\text { Sports, global } \\
\text { health, } \\
\text { treatment }\end{array}$ \\
& & & & \\
\hline $\mathbf{1 7}$ to $\mathbf{1 8} \mathbf{y r s}$ & $50.17 \pm 6.92$ & $\begin{array}{c}\text { Sports, } \\
\text { family, } \\
\text { physical } \\
\text { health }\end{array}$ & $49.83 \pm 7.69$ & $\begin{array}{c}\text { Sports, } \\
\text { family, } \\
\text { physical } \\
\text { health }\end{array}$ \\
\hline
\end{tabular}

\section{Incidence of Neutralizing Antibody (Inhibitor) in Previously Untreated Patient (PUPS) With Severe Hemophilia A}

Shashikant Apte ${ }^{* 1}$, Kannan Subramanian ${ }^{1}$, Rajesh Phatale ${ }^{2}$, Aditi Joshi $^{1}$

${ }^{1}$ Hematology \& BMT, 2Laboratory Hematology, Sahyadri Speciality Hospital, Pune, India

Aims \& Objectives: (1) To Assess Incidence of Inhibitor in PUPs with severe Hemophilia A. (2) To assess F 8 mutation in PUPs who have Inhibitor. (3) To assess anamnestic response after 1 exposure and before second exposure who had Inhibitor to FVIII.

Patients/Materials \& Methods: Data was collected between April 2016 -September 2020.All were diagnostic samples. Very detailed history was elicited to confirm PUP status.

Number : 136.

Age : 4 months-60 months

Family H/O hemophilia A : 108/136 (79\%) History of Inhibitor in family : $27 / 136(19 \%)$

Results: 4/136 (2.9\%) patients had Inhibitor at PUP state. One patient had high titre (10.2 NBU) and three had Low titer (2.1,2.8, 1.9 NBU) Inhibitor. All 4 patients had Intron 22 mutation of $F 8$ gene. All four patients had family history of Inhibitor. All 4 patients had anamnestic High Responder state to one exposure of CFC with Inhibitor Titres of 32.6,76.5,116.2 \& 98.4 NBU before second ED.

Discussion \& Conclusion: (1) Exposure to maternal blood \& thus Factor VIII antigen,at the time of delivery may be the explanation for Inhibitor development in PUP. (2) Scientific community needs systematic data to address this issue. (3) This can have great influence on factor replacement policies for these patients. (4) These patients could be the candidates for Non-factor therapies for Hemophilia A or ITI at PUP status. All these patients were High Responders to one dose of CFC. (5) It may be worth to do genotype on PWH at diagnosis of Hemophilia and assess the High Risk mutation status to develop Inhibitor. (6)Larger data needed to address this issue. (7)PUP status was established only by history \& that can be the limitation. 
Study on Quality of Life of Haemophilic Children in a Tertiary Care Center in Central Madhya Pradesh

Madhuri Tejwani $^{* 1}$, Shweta sharma ${ }^{1}$, Pankaj Pal ${ }^{1}$

${ }^{1}$ Department of pediatrics, Gandhi medical college, Bhopal, India

Aims \& Objectives: A study on clinical profile of hemophilic patients and assessment of their quality of life.

Patients/Materials \& Methods: This was a hospital based observational study, in which all Children 4 to 12 year of age with factor VIII/IX deficiency attending pediatric ward for factor transfusion over one year in a tertiary care center in central Madhya Pradesh. A detailed clinical history was elicited from the accompanying parent/guardian and a HEMO-QoL questionnaire was used as the data collection tool.

Results: In our study haemophilia A is more common than haemophilia B. It was also seen that Proportions of patient who has an early diagnosis was significantly higher in 4-7 years when compared to $8-12$ years of age group of patients $(p<0.05)$. The result of this study revealed that mean overall scores on the Haemo-QoL for the age group 4-7 year and 8-12 year were $41.92 \pm 17.9$ and $51.84 \pm 16.3$, respectively. The highest impairment was in the physical health, school and sports and family dimensions of QOL. Mean transformed scale scores for Physical health, View of himself, View of Family, friends and others was significantly higher in the age group of 8-12 age group compared to 4-7 age group $(p<0.05)$.

Discussion \& Conclusion: Most of the studies including this showed that as the age advances quality of life of hemophilic patients becomes poorer so it is of utmost importance to increase the awareness of the disease among the children and their parents, so that appropriate measures could be taken to prevent morbidities and to bring improvement in quality of life of people living with haemophilia.

Supporting Document:

Transformed scale scores (TSS) for different Haemo-QoL dimensions

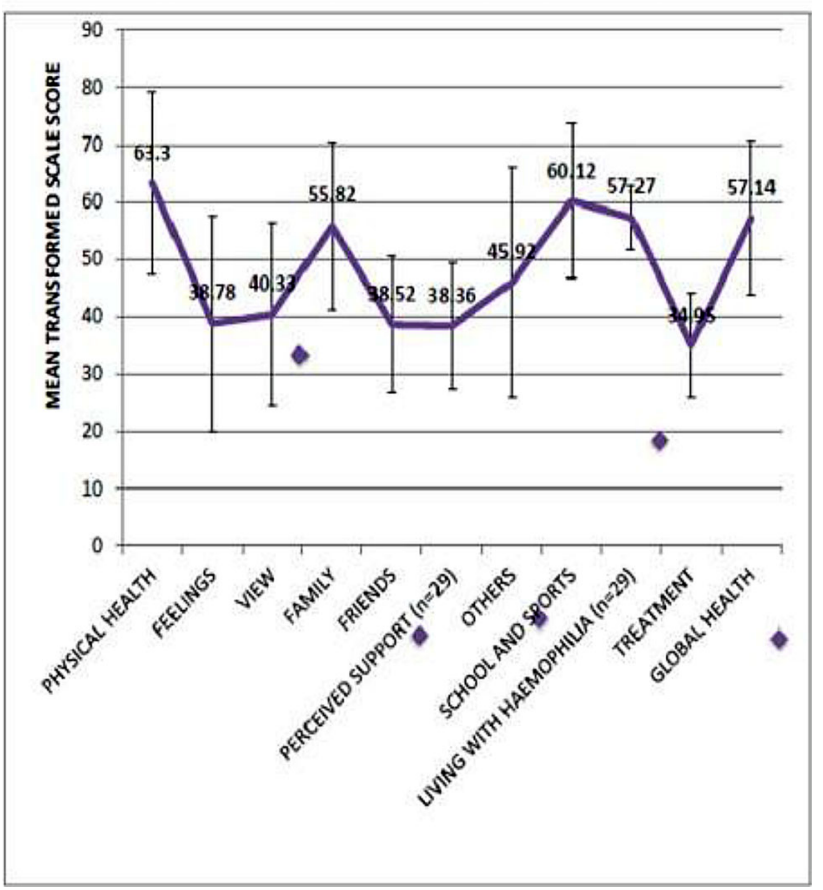

Clinical Use of Romiplostim for Second Line and Subsequent Therapy of Immune Thrombocytopenia

Komal Bhinder ${ }^{* 1}$, Suvir Singh ${ }^{1}$

${ }^{1}$ Clinical Haematology and Stem Cell Transplantation, Dayanand Medical College and Hospital, Ludhiana, India

Aims \& Objectives: Over 50\% of adults with ITP require second line therapy for persistent or chronic disease, but these options are plagued by low rates of response which are often delayed by $2-3$ months. Romiplostim is a TPO receptor agonist that has shown significant efficacy in this setting, and is now available in a generic formulation. No data on the efficacy and safety of the same is available in the India context.

Patients/Materials \& Methods: This study was conducted from August 2019 to July 2020. Patients who were either A. Steroid or IvIg refractory, or B. Not responding to other second line agents were included in the analysis. Platelet responses were defined by American Society of Hematology guidelines.

Results: A total of 11 patients were included in the analysis $(\mathrm{M}: \mathrm{F}=6: 5$ ) with a median age of 44 years (range, 18-67). Corticosteroids were the initial therapy of choice for all patients, of which nine $(81 \%)$ had an initial response. Second line therapy included Azathioprine for six patients (54\%) and IvIg for four (36\%) patients. All patients were refractory to second line therapy and were initiated on Romiplostim at a median dose of $3.68 \mathrm{ug} / \mathrm{kg} /$ week (range, 2.7 to 6.2). A sustained response was observed in 10 patients (90.9\%) after a median duration of 13 days (range, 2-32). One patient achieved an initial response which was lost after two weeks, and one patient was lost to follow up. At a median follow up of 5.5 months, nine patients $(81 \%)$ had stable platelet counts.

Discussion \& Conclusion: Initial data suggests that generic Romiplostim is an effective and safe second line medication for ITP and can potentially avoid splenectomy in steroid and IvIg refractory patients. Prospective follow up will provide a better perspective on efficacy and cost effectiveness of TPO-RAs in the Indian setting.

\section{Rare Case of Thrombocytopenia- Rifampicin Induced Thrombocytopenia}

Shubham Gupta ${ }^{* 1}$, Madhukar Rai ${ }^{2}$, Jaya Chakravarty ${ }^{3}$, Manaswi Chaubey $^{3}$, Naman M. Pathak ${ }^{3}$, Rupal Prasad ${ }^{4}$

${ }^{1}$ Department of General Medicine, Institute of Medical SciencesBHU, Varansi, ${ }^{2}$ Department of General Medicine, Institute of Medical Sciences, BHU, ${ }^{3}$ Department of General Medicine, Institute of Medical Sciences-BHU, ${ }^{4}$ Department of General Medicine, Institute of Medical Sciences, Varanasi, India

Aims \& Objectives: Rifampicin is an important constituent of antitubercular therapy (ATT) in both intensive and continuation phase. Adverse effects include hepatotoxicity, gastrointestinal upset, skin rash and flu like illness. Thrombocytopenia due to rifampicin is rare and first case was reported in 1970 .

Patients/Materials \& Methods: A 33-year old female presented to us with complaints of gum bleeding, black colored stools and rashes over body for 10 days. She was a known case of chronic hepatitis B and disseminated histoplasmosis and was on treatment with tenofovir and itraconazole. She was diagnosed as Intestinal tuberculosis in June 2019 and was started on intensive phase of ATT containing rifampicin. She missed her ATT therapy for 15 days and 2 days after restarting she had bleeding manifestations. On examination, bilateral pitting edema and petechial rashes over limbs and splenomegaly was present. 
Results: Itraconazole and tenofovir therapy was continued. She was transfused with 4 units of platelets and 1 unit of packed red blood cells. Since she had bleeding episodes after restarting ATT therapy and rest of her extensive workup didn't pointed towards a specific diagnosis so the cause of thrombocytopenia was considered as one of the ATT drugs. We withdrew her rifampicin and started her on modified ATT and on day 3 her bleeding improved and platelet counts started to rise with significant rise on day 10 . Hence thrombocytopenia was considered as rifampicin induced.

Discussion \& Conclusion: Rifampicin induced thrombocytopenia is very rare. Tuberculosis research center, Chennai reported only single case in more than 8000 tuberculosis patients treated over 30 years. Pathophysiology of drug induced thrombocytopenia is due to either decreased platelet production which is generally seen in chemotherapy drugs or due to immune mediated destruction which is seen with majority of drugs. Rifampicin induced thrombocytopenia is mostly seen when rifampicin therapy is intermittent or restarted after gap. Confirmation of drug induced thrombocytopenia is by demonstration of anti-platelet antibodies but it is most often not possible due to unavailability in most of the laboratories in India. Best evidence of drug induced thrombocytopenia is considered when patient has improvement in platelet counts after stopping the suspected drug. To conclude drug induced thrombocytopenia is a frequent complication and though rifampicin is very rarely associated with thrombocytopenia, one must be vigilant for bleeding and platelet count when patient is on multiple drugs like when on ATT, as prompt identification and withdrawal can be lifesaving and these individuals should not be restarted on rifampicin again.

Supporting Document:

\begin{tabular}{|c|c|c|}
\hline Investigation & Values & Normal range \\
\hline Hemoglobin & $7.2 \mathrm{gm} / \mathrm{dl}$ & $11-15$ \\
\hline Total leucocyte count & $4150 / 21$ & $4000-11000$ \\
\hline Neutrophils (\%)/L ymphocyte (\%) & $73 / 17$ & $50-70 / 20-40$ \\
\hline P1atelet count & $5000 / \mathrm{ul}$ & $150000-450000$ \\
\hline MCV & 94 & $80-100$ \\
\hline Lactate dehydrogenase & $233 \mathrm{U} / \mathrm{L}$ & $105-248$ \\
\hline Iron & $22 \mathrm{ug} / \mathrm{dl}$ & $50-170$ \\
\hline Ferritin & $296 \mathrm{ng} / \mathrm{ml}$ & $10-291$ \\
\hline Creatinine & $0.7 \mathrm{mg} / \mathrm{dl}$ & $0.5-1.4$ \\
\hline Tota1 Protein & $6.9 \mathrm{gm} / \mathrm{dl}$ & $6.4-8.5$ \\
\hline Albumin & $3.1 \mathrm{gm} / \mathrm{dl}$ & $3.2-5.5$ \\
\hline $\begin{array}{c}\text { Prothrombin time/ International } \\
\text { normalized ratio }\end{array}$ & 13 seconds $/ 1.1$ & $11-13 / 0.8-1.1$ \\
\hline Activated partial thromboplastin time & 38 seconds & $30-40$ \\
\hline Anti-Nuclear antibody & 0.7 & $<0.8$ \\
\hline Collagen vascular profile & Negative & \\
\hline HIV antibody and Anti HCV & Negative & \\
\hline General Blood Picture & \multicolumn{2}{|c|}{ Observation } \\
\hline White Blood cells & \multicolumn{2}{|c|}{ Occasional atypical lymphocytes } \\
\hline Red blood cells & \multicolumn{2}{|c|}{$\begin{array}{l}\text { Anisocytes and microcytes present with mild hypochromasia } \\
\text { with few macrocytes. }\end{array}$} \\
\hline Platelets & \multicolumn{2}{|c|}{$\begin{array}{l}\text { Markedly reduced } \\
\end{array}$} \\
\hline Reticulocyte count & \multicolumn{2}{|c|}{$4.4 \%$} \\
\hline Bone marrow examination & \multicolumn{2}{|c|}{ Observation } \\
\hline Cellularity & \multicolumn{2}{|c|}{ Normocellular } \\
\hline Myeloid: Erythroid & \multicolumn{2}{|c|}{$1.5: 1$} \\
\hline Megakaryocytes & \multicolumn{2}{|c|}{ Increased } \\
\hline Other & \multicolumn{2}{|c|}{$\begin{array}{l}\text { Histiocytes showing Histoplasma capsulatum and ill-defíned } \\
\text { granuloma }\end{array}$} \\
\hline Special stain & PAS stain & istoplamsa \\
\hline
\end{tabular}

Resolution of Target Joints (TJS) in Children with Severe Haemophilia A (HA) Treated WITH (N8GP)

Ranjan KM*1, Sonata S. Trakymiene ${ }^{2}$, Chunduo $\mathrm{Shen}^{3}$, Soraya Benchikh El Fegoun ${ }^{4}$, Andrea Landorph ${ }^{3}$

${ }^{1}$ Novo Nordisk India, Bangalore, India, ${ }^{2}$ Children's Hospital, Affiliate of Vilnius University Hospital Santaros Klinikos, Vilnius, Lithuania, ${ }^{3}$ Novo Nordisk A/S, Bagsværd, Denmark, ${ }^{4}$ Novo Nordisk Health Care AG, Zürich, Switzerland

Aims \& Objectives: TJs in haemophilia increase disease burden, impacting resource utilisation and costs. pathfinder 5 evaluated the safety/efficacy of prophylactic N8-GP, an extended half-life glycoPEGylated recombinant factor VIII, in previously treated children ( $<12$ years) with severe HA. Patients received N8-GP $\sim 60 \mathrm{IU} / \mathrm{kg}$ (range: $50-75 \mathrm{IU} / \mathrm{kg}$ ) twice weekly for prophylaxis. Written informed consent was obtained; the study was approved by relevant ethics committees. TJ ( $\geq 3$ bleeds/consecutive 6-month period) resolution was assessed according to strict criteria (Meunier et al., 2017). The aim of this study was to report the proportion of resolved baseline TJs, using protocol- and International Society of Thrombosis and Haemostasis (ISTH)-defined criteria, throughout the trial.

Patients/Materials \& Methods: TJs from patients participating in both main and extension phases of pathfinder $5(n=63)$ were assessed at baseline, during and at trial end; TJ resolution was defined in the protocol as no bleeds/consecutive 12-month period. The ISTH definition: $\leq 2$ bleeds/consecutive 12-month period (Blanchette et al., 2014). Annualised bleeding rate (ABR) in TJs for the whole population $(n=68)$ was also analysed.

Results: Twelve patients had 16 TJs at baseline (age: 3-11 years; four [33.3\%] were aged $\leq 5$ years), all of which resolved during the trial according to both protocol- and ISTH-defined criteria. One of these patients developed two new TJs (one of which developed after 6 months in trial, resolved at 20 months, was redefined as a TJ at 28 months and resolved again at 44 months), whilst his reporting of bleeds varied from the other patients. Five patients (41.7\%) with seven TJs at baseline had no TJ bleeds during the trial (mean 4.5 years) (Table 1 ). Median TJ ABR was 0.00 , and the poison estimate of the ABR 95\% CI was [0.09 (0.04-0.20)] in the total patient population.

Discussion \& Conclusion: N8-GP prophylaxis resolved all baseline TJs in previously treated children in pathfinder 5. These findings support early prophylaxis with N8-GP and its benefits in children with severe HA, even those with pre-existing TJs.

\section{Wiskott Aldrich Syndrome}

Ganesh CP*1, Anil I. Singh ${ }^{1}$

${ }^{1}$ Dept. of Medicine, JNIMS, IMPHAL, India

Aims \& Objectives: A case report of a WISKOTT aldrich syndrome. Patients/Materials \& Methods: A 10yrs old boy presented with patechia, rash over trunkand extremities(upper and lower limb) since 4 months of age repeatedly and was intensively evaluated for cause of thrombocytopenia.

Results: Our patient had WAS gene analysis positive with single based pair duplication in exon 4 of the WAS gene a variant (c-435 dupA)and immunoglobulins revealed normal value. Indiacting towards wiskott aldrich syndrome.

Discussion \& Conclusion: WAS exhibit wide spectrum of clinical severity.Autoimmune disorder and malignancy can occur duringchildhood but more in aldolescent and young adults with 
classicform of disorder. Thus,clinical diagnosis can be difficult and isusuallly supported by the detection of WAS gene mutation.

\section{A Canadian, Multi-center, Retrospective Study of Clinical Outcomes from Early Use Of N9-GP}

Rinz Paulose ${ }^{* 1}$, Davide Matino ${ }^{2}$, Alfonso Iorio ${ }^{2}$, Arun Keepanasseril $^{2}$, Federico Germini ${ }^{3}$, Alexandre Caillaud ${ }^{4}$, Manuel Carcao $^{5}$, Julia Hews-Girard ${ }^{6}$, Emma Iserman ${ }^{7}$, Paula James ${ }^{8}$, Adrienne Lee ${ }^{9}$, Chai W. Phua ${ }^{10}$, Haowei (Linda) Sun ${ }^{11}$, Jerry Teitel ${ }^{12}$, Man- Chiu Poon ${ }^{13}$

${ }^{\mathbf{1}}$ Novo Nordisk India, Bangalore, India, ${ }^{\mathbf{2} D e p a r t m e n t ~ o f ~ M e d i c i n e, ~}$ ${ }^{3}$ Department of Health Research Methods, Evidence, and Impact, McMaster University, Hamilton, ${ }^{4}$ BioPharm Medical Affairs, Mississauga, ${ }^{5}$ Department of Pediatrics, The Hospital for Sick Children, University of Toronto, Toronto, ${ }^{6}$ Foothills Medical Centre, Alberta Health Services, Calgary, ${ }^{7}$ McMaster University, Hamilton, ${ }^{8}$ Department of Medicine, Queen's University, Kingston, Kingston, ${ }^{9}$ Hematology, Department of Medicine, University of Calgary, Calgary, ${ }^{10}$ Department of Medicine, Western University, London, ${ }^{11}$ Department of Medicine, University of Alberta, Edmonton, ${ }^{12}$ St Michael's Hospital, Toronto, ${ }^{13}$ Departments of Medicine, Pediatrics and Oncology, Cumming School of Medicine, University of Calgary, Calgary, Canada

Aims \& Objectives: The Canadian Blood Disorders Registry (CBDR) captures data collected from hemophilia treatment centers and directly from patients. With the recent availability of nonacog beta pegol (N9-GP) in Canada, the CBDR provides a unique opportunity. The aim of the study was to assess real-world outcomes following N9-GP use in a clinical setting.

Patients/Materials \& Methods: This non-interventional, retrospective study used CBDR data to describe and analyze real-world outcomes in Canadian hemophilia B patients receiving N9-GP for $\geq 3$ months in any setting (prophylaxis, on-demand treatment, treatment of breakthrough bleeding [BTB]). For comparison with previously-used products, only patients for whom data existed in CBDR for the 6-month period pre-switch to N9-GP were included. Results: At the data cut-off (September 30, 2019), 40 patients were included in the analysis, with a median age of 44 years. Distribution of disease severity was $2.5 \%$ mild, $40 \%$ moderate, $55 \%$ severe, and $2.5 \%$ unknown. At study start, 10 target joints were present in 5 patients. Most patients had previously received $\mathrm{rFIXFc} \mathrm{(55 \%} \mathrm{versus}$ $40 \%$ rFIX), with most previously receiving prophylactic treatment ( $85 \%$ versus $15 \%$ on-demand). No patients had present or previous inhibitor development. During a median treatment period of 11.11 months on N9-GP, 106 BTBs were reported in 22 patients; $42 \%$ of patients reported zero bleeds. Median time from last recorded prophylactic injection to start of bleeding was 7.1 days and the mean number of injections required to treat a bleed was 1.23 . Figure 1 shows the comparative effectiveness of N9-GP prophylaxis versus previous product by type of bleed and previous product. The total median annualized factor consumption [kIU/year], for prophylaxis and BTBs was less for N9-GP versus the previous product.

Discussion \& Conclusion: This is the first study to investigate treatment patterns and clinical outcomes with N9-GP in hemophilia B patients in a real-world setting. Initial data suggest improved bleeding outcomes with lower factor consumption after switching to N9- GP, regardless of whether patients previously received standard or extended half-life products.
With New Drugs Comes New Complications: Nivolumab Induced Acquired Hemophilia $A$ in Relapsed Hodgkin Lymphoma

Sachin Bansal ${ }^{* 1}$, Rahul Bhargava ${ }^{1}$, Meet Kumar ${ }^{1}$, Santosh R. Kurmi $^{1}$, Udaya Kumar ${ }^{2}$, Renu Saxena ${ }^{2}$

${ }^{1}$ Hematology, FMRI, ${ }^{2}$ Hematology, Medanta, Gurugram, India

Aims \& Objectives: Relapsed/Refractory Hodgkin Lymphoma is not an untreatable disease now a days. Availabilty of new drugs Nivolumab, Brentuximab Vedotin and others in pipeline has opened new doors for treatment. Here we report a case of Nivolumab induced acquired haemophilia A in relapsed Hodgkin Lymphoma.

Patients/Materials \& Methods: Relapsed/Refractory Hodgkin Lymphoma is not an untreatable disease now a days. Availabilty of new drugs Nivolumab, Brentuximab Vedotin and others in pipeline has opened new doors for treatment. Here we report a case of Nivolumab induced acquired haemophilia $\mathrm{A}$ in relapsed Hodgkin Lymphoma.

Results: Relapsed/Refractory Hodgkin Lymphoma is not an untreatable disease now a days. Availabilty of new drugs Nivolumab, Brentuximab Vedotin and others in pipeline has opened new doors for treatment. Here we report a case of Nivolumab induced acquired haemophilia $\mathrm{A}$ in relapsed Hodgkin Lymphoma.

Discussion \& Conclusion: Nivolumab blocks PD-L1 from binding to PD1, allowing T cell to work. It has been used in treatment of melanoma, squamous non -small cell lung cancer and treatment of squamous cell lung cancer. Immune side effects of Nivolumab leading to acquired haemophilia $\mathrm{A}$ has been reported in literature as case reports. No case of acquired haemophilia A(AHA) has been reported as a side effect of Nivolumab induced AHA yet. We should be cautious while using newer modalities of treatment for our patients as these newer modalities are not time tested.

\section{A Cost Effective Strategy for Mutation Detection in Indian Hemophilia A Patients}

Sharda Shanbhag ${ }^{* 1}$, Nikesh Kawankar ${ }^{1}$, Rucha Patil ${ }^{1}$, Bipin Kulkarni ${ }^{1}$, Shrimati Shetty ${ }^{1}$

${ }^{1}$ Haemostasis and Thrombosis, National Institute of Immunohaematology, MUMBAI, India

Aims \& Objectives: Objective: The FVIII gene (F8) consists of 26 exons and spans $186 \mathrm{~kb}$; sequencing such a huge gene is a tedious task. Techniques for mutation detection such as Single Strand Conformational Polymorphism, Conformation Sensitive Gel Electrophoresis, Amplification and Mismatch Detection, Denaturing Gradient Gel Electrophoresis followed by DNA sequencing are available. However, none of them are suitable for routine diagnosis even in sophisticated laboratories because of technical complications. Aim: To deduce a simple time saving and cost effective direct sequencing strategy for genetic diagnosis of HA patients.

Patients/Materials \& Methods: The series consisted of HA families referred at our centre for carrier and antenatal diagnosis. Phenotypic assays and subsequent genetic analysis were done in all patients. Mutation analysis: Intron 1 and 22 inversions were detected using multiplex and inverse PCR respectively. Characterization of the mutation in families without inversions was performed by direct sequencing of genomic DNA using FVIII-specific oligonucleotide primers. Gross deletions were confirmed by MLPA technique.

Results: 551 HA families were analysed in the current series. 254 $(46.09 \%)$ patients were found to be inversion positive i.e. Intron 22 $(\mathrm{n}=243)$ and Intron $1(\mathrm{n}=11) .26(4.7 \%)$ patients showed deletions of different exons. Mutations could not be detected in 2 patients. 
Remaining 269 (48\%) patients exhibited a heterogeneous pattern of variations throughout F8. Based on the frequency of mutations in different exons we divided the entire F8 into 2 sets of exons; first set i.e. exons 4,7,8,9,10,11,13,14a, 14e, $14 \mathrm{~g}, 14 \mathrm{k}, 17,18,19,23$ and 24 where mutations could be detected in $202(75 \%)$ cases and second set i.e. $1,2,3,5,6,12,14 \mathrm{~b}, 14 \mathrm{c}, 14 \mathrm{~d}, 14 \mathrm{f}, 14 \mathrm{~h}, 14 \mathrm{j}, 15,16,20,21,22,25$ and 26 where only $67(25 \%)$ could be detected (Figure 1 ). Based on the findings, an effective strategy could be adopted in the laboratory as below.

- Screening for F8 Intron 22 \& 1 inversions

- Subsequent screening for gross deletions post PCR

- Direct sequencing of the most probable exons (Red bars in Figure 1)

Discussion \& Conclusion: The strategy adopted here is based on a large series of families and is currently being utilized in the routine diagnosis of HA families. By following this two-tier strategy in DNA sequencing step, a substantial reduction in the cost and turnaround time is feasible.

\section{'The Invincible ITP': An Interesting Case of Transient Antiglobulin Positive Steroid Resistant ITP}

Rajat P. Pande ${ }^{* 1}$, Durga Krishnan ${ }^{2}$, Mayilananthi $\mathrm{K}^{2}$, Vrinda $\mathrm{V}^{2}$, Senthilnathan $\mathrm{P}^{2}$, Rehab Y. Nainar ${ }^{2}$, Thejesh $\mathrm{C} \mathrm{V}^{3}$, V R Mohan Rao ${ }^{2}$

${ }^{1}$ General Medicine, Chettinad Hospital and Research Institute, ${ }^{2}$ General Medicine, Chettinad Hospital \& Research Institute, ${ }^{3}$ General Medicine, Chettinad Hospital \& Research Institute, Chennai, India

Aims \& Objectives: Immune thrombocytopenia (ITP) is an autoimmune disease with isolated thrombocytopenia, characterised by antibody-coated platelets being prematurely destroyed in the spleen or liver. High-dose corticosteroids are the initial treatment of choice; with their use, however, many patients with refractory disease cannot maintain "safe" platelet counts and require further therapy.

Patients/Materials \& Methods: A 24 year old unmarried female presented with history of gum bleeding and rashes all over the body for 5 days with no other positive present or past history. On investigating, platelets were 3000/cu.mm, Hb- $10.3 \mathrm{~g} / \mathrm{dl}$, with a normal LFT, coagulation profile, and undetectable ANA and APLA. Workup for common infectious diseases was found to be negative. USG Abdomen was normal.

Results: Diagnosing her as a classical case of ITP, she was treated with high dose steroids, which had no response in improving platelet counts. Instead, a fall in haemoglobin was noted, direct antiglobulin test was found out to be positive and a possibility of Evan's syndrome was thought off, but a repeatedly normal LFT and haptoglobin levels ruled it out. Bone marrow aspirate showed increased megakaryocytes. Patient was treated further with IvIG therapy followed by eltrombopag for 2 weeks, showing no response in sustaining 'safer' platelet levels. Likely to be a case of 'Steroid resistant - persistent ITP' a favourable response was achieved by a third line drug azathioprine, given for 3 months. The platelet count and hemoglobin normalized with a negative DAT on 3 months and 12 months follow up.

Discussion \& Conclusion: The antiglobulin test helps to distinguish immune from nonimmune hemolytic disorders. DAT positivity in acute ITP is rarely seen. Marie Hollenhorst et al. in their study on markers of autoimmunity in ITP found that DAT positivity in ITP has an effect on the remission and drug resistance. The possibility of high titres of antibodies in ITP patients causing a positive DAT test due to cross reactivity with reagent needs further evaluation. This case report questions the possible significance of a positive DAT test in understanding the remission and drug resistance in ITP. The report also intends to highlight the requirement of a trial of third line drugs in patients with persistent ITP.

Supporting Document:

PLATELET RESPONSE \& DRUGS ADMINISTERED

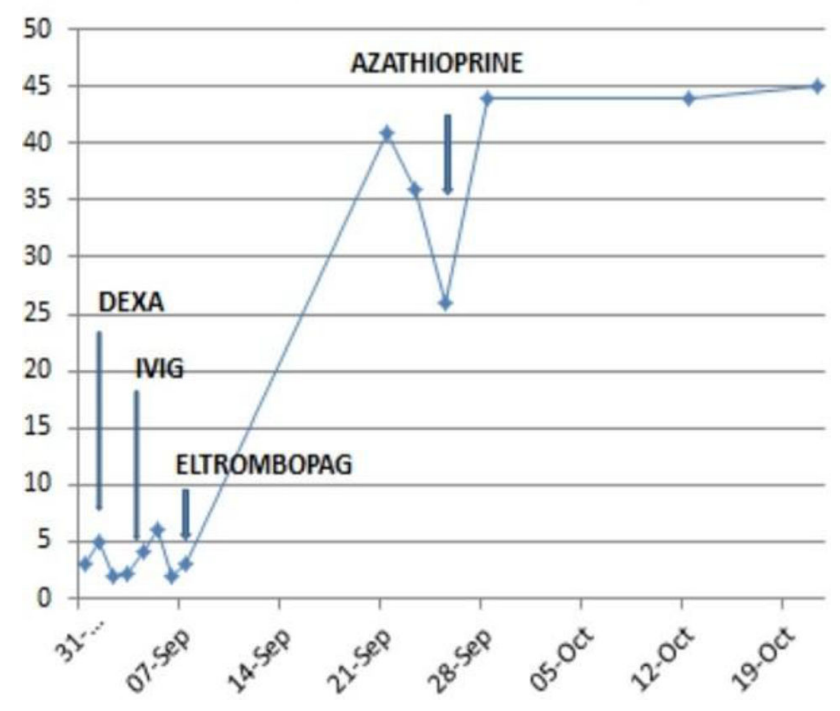

\section{Improvement in School Absence After Factor Replacement in Students with Haemophilia in Upper Assam}

\author{
Anupam Dutta ${ }^{* 1}$, Dipjyoti Boruah ${ }^{2}$ \\ ${ }^{1}$ Assam Medical College and Hospital, ${ }^{2}$ Department of Medicine, \\ Medicine, Dibrugarh, India
}

Aims \& Objectives: Patients with Haemophilia experience a lifetime burden of joint complications and loss of functional ability due to repeated bleeding episodes caused by the inadequate levels of clotting factor VIII or IX in blood. These complications have a large social consequences in day to day life, among which the active participation in school and academic study in children with haemophilia's $(\mathrm{CwH})$ is noticeable. Treatment with factor replacement therapy can help to prevent this, but access to this has been challenging in low-resource settings such as Northeast India. The study shows the impact of factor replacement therapy in bleeding episodes, joint complications and school absenteeism in these $\mathrm{CwH}$.

Patients/Materials \& Methods: A retrospective observational study was undertaken to examine the impact of receiving regular factor replacement therapy (prophylaxis or on demand) on school absences among $\mathrm{CwH}$ registered with the haemophilia treatment centre at Assam Medical College and Hospital. Annual bleed rate (ABR), Haemophilia Joint Health Score (HJHS) and Functional Independence Score in Haemophilia (FISH) were also assessed.

Results: Thirty-eight $\mathrm{CwH}$ were eligible for the study; 26 (68.4\%) were on prophylaxis therapy and $12(31.5 \%)$ on on-demand therapy. In the year before starting regular treatment, the mean annual bleed rate $(\mathrm{ABR})$ was $37.8(+20.0)$, HJHS was $31.1(+18.1)$ and mean FISH Score was $21.1(+4.2)$. At the end of the study period the mean $(+\mathrm{SD})$ annual bleed rate in prophylactic therapy was significantly lower $5.8(+4.6)(p<0.001)$ and the HJHS was significantly lower 4.7 (+4.6). FISH Score significantly improved to $27.9(+3.3)$ 
( $p<0.001$ ). Prophylaxis therapy showed better (but not significant) results in comparison to on-demand therapy.

Discussion \& Conclusion: Treatment with factor replacement significantly reduces school absence in $\mathrm{CwH}$ and correlates strongly with joint health and functional improvement, with the effect slightly better with prophylaxis therapy than on-demand. Low dose prophylaxis is a good treatment option in low-resource settings, but improvements are also needed in rates of diagnosis.

\section{Scorpion Sting Resulting into DIC Causing Multiple Ischemic Stroke and Thrombus in Peripheral Artery}

Raushan Kumar ${ }^{* 1,}$, , Raushan Kumar ${ }^{2}$, Jaishree Ghanekar ${ }^{2}$

${ }^{1}$ General Medicine, MGM Medical College Kamothe, ${ }^{2}$ General Medicine, MGM Medical College Kamothe Navi Mumbai, Navi Mumbai, India

Aims \& Objectives: To report case of Scorpion sting resulting into DIC,ischemic stroke and thrombus in peripheral artery.

Patients/Materials \& Methods: Retrospective study was done in Scorpion sting with atypical presentation of stroke and thrombus, all investigations done were analysed and rarity of presentation was reported.

Results: Patient succumbed to disease in ICU.

Discussion \& Conclusion: Ischaemic stroke is one of serious and rare complication of scorpion sting.Several mechanism of ischaemic stroke are autonomic dysfunction,myocarditis,hypoperfusion secondary to hypotension and shock and coagulopathy and DIC resulting in thrombosis. In our patient ischaemic stroke probable mechanism seems to be secondary to myocardial inflammation and severe inflammatory response syndrome resulting in arterial thrombosis of left upper limb and causing multiple ischemic lesion in brain.

\section{Effect of Fresh Frozen Plasma in Correcting Mildly Elevated PT-INR in Critically ILL Neonates}

\section{J Satya Mani Deepika Chilukuru*1, Nivedita Patil ${ }^{2}$ \\ ${ }^{1}$ Pediatrics, Dr. D Y Patil Educational Society and Deemed University, ${ }^{2}$ Pediatrics, Dr. D Y Patil Educational Society and Deemed University, Kolhapur, India}

Aims \& Objectives: To assess the effectiveness of fresh frozen plasma in correcting mildly elevated PT-INR in critically ill neonates. Patients/Materials \& Methods: This is a retrospective study done in neonates admitted to the NICU in DR D Y Patil hospital and research institute, Kolhapur during the period of January 2019 to January 2020. Sample size is 96.All patients who received frsh frozen plasma for mildly elevated Prothrombin time (PT)- INR levels $(\leq 1.5)$ and had post transfusion PT-INR values were studied retrospectively.

Results: Among 96 patients who had plasma transfusion with post transfusion PT-INR values, only $15 \%$ patients showed post transfusion normalization of PT-INR.the remaining $85 \%$ showed no difference there was no significant difference in their PT-INR values $(p=0.34)$ pre and post transfusion.

Discussion \& Conclusion: Fresh frozen plasma transfusion in critically ill neonates does not significantly correct mildly prolonged PTINR.So transfusion of plasma with an aim of preventing or controlling bleeding should be avoided.
Diffuse Alveolar Hemorrhage: A Rare Complication of Immune Thrombocytopenic Purpura

Anish K. Saha ${ }^{* 1}$, Deepak K. Gautam ${ }^{1}$

${ }^{1}$ Department of General Medicine, Institute of Medical Sciences, Banaras Hindu University, Varanasi, India

Aims \& Objectives: Diffuse Alveolar Hemorrhage is characterized by bleeding into the alveolar spaces of the lungs. Clinical presentation includes hemoptysis, anemia, usually accompanied by respiratory failure and diffuse alveolar infilterates. Association of DAH as a complication to Immune Thrombocytopenic Purpura (ITP) is very rare with very few cases reported in literature. We present a case of 43 year women with ITP, complicated with DAH.

Patients/Materials \& Methods: A 43 year old women, previously diagnosed as a case of ITP 1 year back, presented with complaints of per vaginal bleed, hemoptysis, petechial rash and shortness of breath for 4 days. She denied any history of fever, headache, chest pain and orthopnea. She had been on prednisone $10 \mathrm{mg}$ for ITP for 8 months, 1 year back. On admission, respiratory rate was $28 / \mathrm{min}$. Pallor was present along with scattered petechial rash over the body. There were fine crepitations over the chest bilaterally. $\mathrm{Hb}=8.4 \mathrm{gm} / \mathrm{dl}$, TLC$12300 / \mu \mathrm{L}$ and platelet count $<15,000 / \mu \mathrm{L}$, INR- 0.98, aPTT- $29.9 \mathrm{~s}$. Chest radiograph showed bilateral fluffy infilterates. CECT thorax was suggestive of Diffuse alveolar hemorrhage. ANA, Anti-dsDNA, Anti GBM, cANCA and pANCA were negative.

Results: Platelet transfusion, IV Methyl Prednisolone and Thrombopoietin agonist (Eltrombopag) were given. The patient showed clinical and radiological improvement.

Discussion \& Conclusion: The association of ITP with DAH is an extremely rare presentation. We have excluded other etiologies of alveolar hemorrhage to a reasonable degree, and our patient was treated successfully with steroids and eltrombopag for underlying ITP. DAH can present as a medical emergency, and early identification is of paramount importance.

\section{Quality of Life and Its Association with Arthropathy in Children Aged 5-18 Years with Hemophilia}

Daksh Bhutani $^{* 1}$, Anjali Verma ${ }^{1}$, Alka Yadav ${ }^{1}$

${ }^{1}$ Pediatrics, Pt. B. D. Sharma PGIMS, Rohtak, Rohtak, India

Aims \& Objectives: Health-related quality of life (HRQoL) is being used as an important health outcome measure in Hemophilia in multiple studies across globe. This study was conducted with an aim to evaluate Health Related Quality of life (HRQoL) in Indian children with Hemophilia, aged 5 to 18 years using Pediatric Quality of Life Inventory Version 4.0 (Peds QL 4.0) and determine its association with arthropathy.

Patients/Materials \& Methods: The study enrolled 100 children with moderate or severe hemophila A or B, aged 5 to 18 years. An equal number of age and gender matched children with no/minor illness were taken as control. Hemophilia children with other causes of arthropathy such as traumatic, rheumatic, psoriatic arthritis were excluded. Baseline characteristics of cases were recorded. Cases as well as controls were subjected to Peds QL 4.0 questionnaire. Joint health status of cases was analysed using Hemophilia Joint Health score (HJHS) version 2.1. Results were drawn and compared.

Results: Hemophilia children with arthropathy had scored lower in above mentioned domains when compared to those without arthropathy and the difference was statistically significant $(p<0.001)$. A significant correlation exists between HJHS 2.1 score and age of patient, frequency of bleeding and severity of the disease. 
Discussion \& Conclusion: Overall, Hemophilia has a significant impact on quality of life of children. Hemophilia in particular when associated with arthropathy has significant impact on HRQOL. Therefore, early diagnosis, timely management of bleeding episode and prophylaxis may help to reduce morbidity and improve quality of life in these patients.

\section{Ecchymosis and Eosinophilia: Are They Related?}

\section{Aboobacker Mohamed M. Rafi ${ }^{\mathbf{1}}$, Maglin Monica ${ }^{* 1}$, Arun V J ${ }^{1}$ \\ ${ }^{1}$ Transfusion Medicine, Jubilee Mission Medical College \& Research Institute, Thrissur, India}

Aims \& Objectives: Ecchymosis is one of the most common manifestation of any coagulation defect. The importance of a detailed history \& a complete hemogram along with a peripheral smear helps to clench the diagnosis before going into coagulation testing.

Patients/Materials \& Methods: A 9-year-old boy presents with complains of acute onset of bluish discoloration on right side of thigh and other areas of the body. He has no history of trauma, fever, or weight loss. He has no similar past or family history of bleeding disorders. He was not on any medication.

Results: The ISTH BAT score was 2. Hess Test turned out to be positive. The complete hemogram picked up Eosinophilia (23\%) with an AEC of 2400.The Bleeding time was prolonged $(9 \mathrm{~min}$ ) with normal Coagulation screen. Considering the history and the lab finding an empirical diagnosis of Acquired platelet dysfunction with eosinophilia (APDE) was made and the patient started on broad spectrum anti helminths. The patient improved and the ecchymosis disappeared.

Discussion \& Conclusion: Acquired platelet dysfunction with eosinophilia (APDE) is a syndrome which has transient state of platelet dysfunction in the presence of marked eosinophilia. This bleeding disorder, otherwise known as "non-thrombocytopenic purpura with eosinophilia", occurs commonly in children from South-East Asia. Platelet aggregation studies are usually advised. They would show a normal platelet aggregation with ristocetin, reduced aggregation with ADP and no aggregation with collagen. The importance of recognizing this benign condition, which usually does not require any specific therapy, is to avoid pitfalls of diagnosing more serious bleeding disorder in children with ecchymosis. The mechanism of APDE is not clearly understood. Previous studies have proposed that parasites induce eosinophils, leading to the production of antibodies and the formation of immune complexes, which can bind to platelets, resulting in abnormal secondary aggregation.This syndrome runs a benign course and resolves spontaneously within 6 months to a year. No specific treatment has been found to be useful except, in some instances, empirical use of anthelmintics is beneficial. Reassurance of the patient and their parents is instrumental in this syndrome. Further research is necessary to elucidate all the etiological factors that lead to APDE.

\section{Covid Fear Leads to Bleed: What is the Cause?}

Febe R. Suman ${ }^{* 1}$, Krishnaratnam Kannan ${ }^{2}$, Sri Gayathri ${ }^{1}$, Gayathri Kennedy ${ }^{1}$, Ganapathy Settu ${ }^{3}$, Uma Lakshmi ${ }^{3}$

${ }^{1}$ Pathology, ${ }^{2}$ Hematology, ${ }^{3}$ Sri Ramachandra Laboratory Services, SRMC, Chennai, India

Aims \& Objectives: Aim: To describe an unexpected clinical condition during the pandemic due to Covid 19 Objectives: (1) To understand the effects of allopathic and alternative medicines at inappropriate doses on coagulation. (2) To understand the need of full coagulation work up to provide proper treatment.

Patients/Materials \& Methods: Present : A 47 year old gentle man was referred to haematologist for prolonged coagulation screen and factor X deficiency. Past : He had echymoses in the lower limbs and hematuria 2 months back. Nephrology \& Urology opinion: Nil abnormality detected. Coagulation screen (prothrombin time \& partial thromoplastin time) prolonged. InvestigatIon for common pathway factors show low Factor X. 3 units of fresh frozen plasma transfused to arrest hematuria and echymoses. (These past events happened elsewhere and was verified from his case file).

Two years back he had plate implant without transfusion support for fracture femur which occurred due to road traffic accident. He has no comorbid conditions or bleeding history.

Family history: No significant illness or bleeding history.

Occupation: Pharmacist.

Drug history: (1) Diclofeanac paracetamol 2 tabs/day for two years for pain. (2) Antihiastamines and antifungals for echymoses. (3) Kabasurakudineer and many other decotions for fear of Covid 19.

Results: Diagnosis: Acquired Vitamin K dependent factor deficiencies. Treated with Vitamin K $10 \mathrm{mg}$ orally daily for 3 months. Follow up: coagulation screen within reference range.

Discussion \& Conclusion: 1.Diclofenac known to inhibit platelet aggregation will interfere with coagulation? As in rats 2.Alternate system medicines interfere with vitamin K? 3.Diclofenac and alternate medicines interaction cause vitamin $\mathrm{k}$ deficiency? 4.Lot of drugs causing malabsorption?

Medications of all streams need physician guidance and follow up. Full coagulation work up is diagnostic and aids in treatment.

\section{Romiplostim: In the Treatment of Primary Immune Thrombocytopaenia-An Observational Study}

Ranjit K. CS ${ }^{* 1}$, Ashok K. $\mathrm{K}^{1}$, Rajkishore $\mathrm{P}^{2}$, Rajesh M. Gottipati ${ }^{3}$, Anil Aribandi ${ }^{1}$

${ }^{1}$ Department of Haemato-oncology, American Oncology Institute, ${ }^{2}$ Department of Haemato-oncology, Amercan Institute Of Haematology, Hyderabad, ${ }^{3}$ Department of Haemato-oncology, American Oncology Institute, Vijayawada, India

Aims \& Objectives: Romiplostim, a thrombopoietin receptor mimetic, increases platelet count in patients with immune thrombocytopaenia, with few adverse effects.

Patients/Materials \& Methods: This is an observational study in primary immune thrombocytopaenic purpura regarding the role of Romiplostim beyond first line therapy after corticosteroids or intravenous immunoglobulins. 20 consecutive patient were enrolled in the study, to receive weekly subcutaneous injections of Romiplostim, 250 micrograms. Primary endpoints were rate of platelet response and toxicity. Secondary end point was treatment failure rate. Patients were followed for up to 15 weeks from enrollment Results: The study enrolled 20 consecutive patients. There was slight male preponderance $(55 \%$ Vs $45 \%)$ Median age of presentation was 39.6 years. About $50 \%$ of patients were initiated in the 2 nd line setting. The mean platelet count before initiating Romiplostim was $28500 / \mathrm{cu}$ mm. 19 out of $20(95 \%)$ patients showed response following two doses of Romiplostim (platelet count $>50,000 / \mathrm{cu} \mathrm{mm}$ ) Maximum response was observed around 5th week. $40 \%$ of the patients lost their response by 8 th -10 week. Commonly observed adverse effects were headache in $20 \%$, joint pains in about $15 \%$ and alopecia in 2 patients $(10 \%)$. The study enrolled 20 consecutive patients. There was slight male preponderance $(55 \%$ Vs $45 \%)$ Median age of presentation was 39.6 years. About $50 \%$ of patients were initiated in the 2nd line setting. The mean platelet count before initiating Romiplostim was $28500 / \mathrm{cu} \mathrm{mm} .19$ out of $20(95 \%)$ patients showed response following two doses of Romiplostim (platelet count $>50,000 / \mathrm{cu} \mathrm{mm}$ ) Maximum response was observed around 5th week. $40 \%$ of the patients lost their response by 8 th -10 week. Commonly observed adverse 
effects were headache in $20 \%$, joint pains in about $15 \%$ and alopecia in 2 patients $(10 \%)$

Discussion \& Conclusion: Patient treated with Romiplostim showed a fast response. However, we noticed that a considerable number of patients lost response with time. Due to the small numbers in our study, we recommend that this needs to be looked into in a larger study to ascertain the relevance. Romiplostim, however, is well tolerated.

\section{Spectrum of Bleeding Disorders in Pregnancy a Rare Occurrence with Varied Presentation and Outcome}

Prerna Arora*1,2, Akriti Khare ${ }^{2}$, Jasmita Dass ${ }^{2}$, Ganesh V. Kumar ${ }^{2}$, Mukul Aggarwal $^{2}$, Rishi Dhawan ${ }^{2}$, Tulika Seth ${ }^{2}$, Seema Tyagi ${ }^{2}$, Manoranjan Mahapatra ${ }^{2}$, Sunesh Kumar ${ }^{3}$

${ }^{1}$ AIIMS DELHI, Noida, ${ }^{2}$ Hematology, ${ }^{3}$ Obstetrics and Gyanecology, AIIMS DELHI, Delhi, India

Aims \& Objectives: To analyse the incidence and spectrum of inherited bleeding disorders in pregnancy and to correlate the findings with clinical features and outcome of pregnancy.

Patients/Materials \& Methods: We retrospectively studied the profile of 240 cases of pregnant females over a period of two year duration (September 2018 -September 2020) who presented in our institution with bleeding disorders. Patients already on anticoagulation therapy and isolated thrombocytopenia were excluded from the study.

Results: Out of 240 cases, 5 cases were detected to have bleeding disorders $(2.1 \%, 5 / 240)$. Of these 5 cases, most common cause was Glanzmann thrombasthenia (GT) found in 2 cases $(40 \%, 2 / 5)$ followed by 1 case each of factor $\mathrm{X}$ deficiency, type $3 \mathrm{Vwd}$ and carrier state of Haemophilia A (20\%). Successful pregnancy outcome and delivery of healthy neonate was found in 2 cases (GT and Factor X deficiency) while 1 intrauterine death was noted in case of GT.Case of haemophilic carrier was lost to follow up and case of vWD is yet to deliver. Discussion \& Conclusion: Pregnancy, childbirth and puerperium are hemostatically challenging to women with bleeding disorders. Bleeding disorders not only present problems for delivery and postpartum period but may have significant implications for foetus and neonate and result in miscarriage, placental abruption and foetal loss. Postpartum haemorrhage has been reported in $20-25 \%$ of women with Von Willebrand's disease (vWD), $2-11 \%$ in haemophilia carriers and $24 \%$ with factor XI deficiency. Rare bleeding disorders includes factor deficiencies other than factor VIII and IX and platelet disorders are rarer in pregnancy and there is paucity of data in Indian literature on bleeding risk and its management in women with bleeding disorders. $2(40 \%)$ cases of inherited bleeding disorder delivered successfully in the current study. Inherited bleeding disorders though rare must be kept as a differential in pregnant women presenting with bleeding or suggestive family history. The diagnosis of coagulation factor defects may be masked in pregnancy hence, the key to optimal management and safe delivery.

\section{Rare, Unclassified Platelet Function Disorders: How Rare are They? \\ Nikesh K. Kawankar ${ }^{* 1}$, Shrimati Shetty ${ }^{1}$, Bipin Kulkarni ${ }^{1}$ \\ ${ }^{1}$ Dept of Haemostasis and Thrombosis, National Institute of Immunohaematology (NIIH), ICMR, MUMBAI, India}

Aims \& Objectives: (1) To correctly diagnose and classify the patients having unexplained platelet function disorders and avoid misdiagnosis and mismanagement. (2) To identify the disease causing molecular pathologies and mechanisms underlying unexplained Platelet Function Disorders.
Patients/Materials \& Methods: The study was carried out on 28 referred patients with unclassified platelet function defects (UPFD). Light transmission aggregometry (LTA) was performed using undiluted PRP on platelet aggregometer with various platelet agonists (ADP:6 $\mu \mathrm{M}$, Ristocetin :1.25 mg/ml, Collagen: $1 \mu \mathrm{g} / \mathrm{ml}$, TRAP-6, TxA mimetic peptide). ATP secretion analysis was performed using ADP, Collagen, TRAP-6. Expression of glycoproteins (GPs), GPIX, GPIb, GPIIbIIIa and GPVI was assessed by flow cytometry (FACSAria, Becton-Dickinson, CA). Platelet activation status was studied using ADP, TRAP activation followed by assessment of CD62P, CD63 activation by flow-cytometry. Clinical exome sequencing was performed for UPFD with strong bleeding history (ISTH-BAT $\geq 3$ ). Insilico analysis of novel mutations was done using SIFT, Provean, Polyphen 2 and Mutation taster softwares, to predict its damaging effects.

Results: Coagulation screening (PT, APTT, TT) was normal in all patients. Out of 28 patients, 9 had mutations in RASGRP2 gene and other patients had mutations in NBEAL2, GFI1B, ACTN1, ACVRL1, MYH9, MYH13, WAS, TBXAS1, ANKRD26, ACD, ABCB4 and ITGB3 genes (figure 1). Sanger sequencing confirmation was done. In-silico analysis of variants using bioinformatics was done. Phenotypic analysis of platelet aggregation in response to ADP and collagen was severely reduced in RASGRP2 and ITGB3 cases. Moderately reduced platelet aggregation with ADP and collagen was found in GFI1B case. The expression GPIX, GPIb, GPIIbIIIa and GPVI, was normal in all cases. ATP secretion using ADP, collagen and TRAP-6 was variable. Platelet activation using CD62P was variable.

Discussion \& Conclusion: Rare platelet function disorders, especially with milder bleeding phenotypes, could be difficult to diagnose because of the lacuna in standardized diagnostic laboratory assays. General approach to identify such disorders is by whole exome sequencing. In this study, genotypic analysis have identified distinctly different sets of genes associated mainly with Gi signaling (50\%), secretion abnormalities (43\%) and few with platelet aggregation $(3.5 \%)$ and production $(3.5 \%)$ defects. RASGRP2 gene (Gi signaling defect) mutations were identified in $32.14 \%$ of the cases. As per published literature, only 20 mutations in 24 families are reported so far with RASGRP2 (Jae Won Yun et al. 2019). This study will not only aid in identifying the appropriate phenotypic assays that affect specific pathways, but also highlights the relatively high occurrence of defects that are otherwise considered rare in the published literature.

Supporting Document:

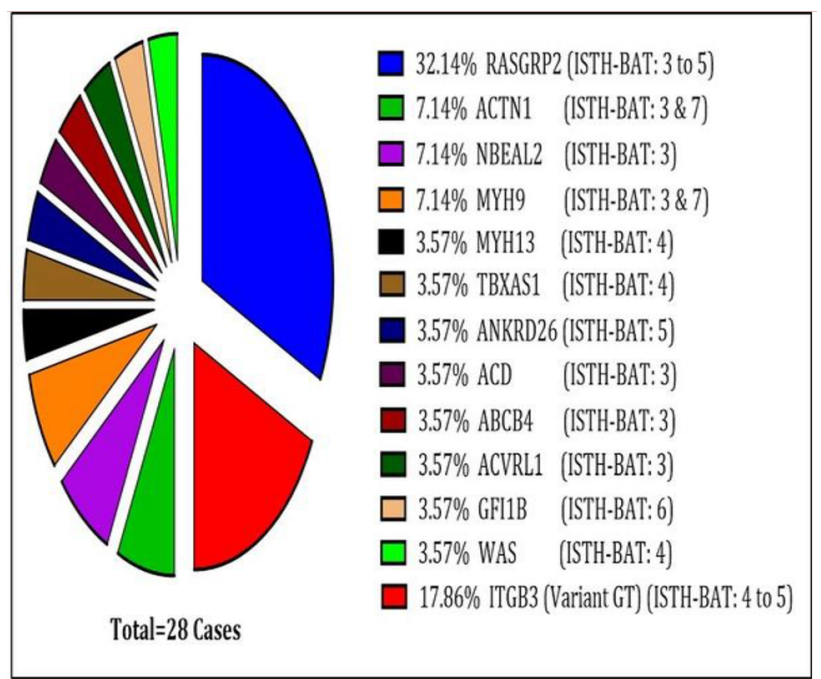

Figure 1: Clinical and Molecular profile of unclassified platelet function disorders. 
Clinico-Epidemiological Profile of Patients with Inherited Coagulopathies at a Paediatric Haemophilia Clinic of a Tertiary Care Centre in Gujarat

Khushboo K. Taneja ${ }^{* 1}$, Sheila Aiyer ${ }^{1}$

${ }^{1}$ Pediatrics, Medical College, Vadodara, Vadodara, India

Aims \& Objectives: To study the existent epidemiology, symptomatology, disease severity and bleeding profile of paediatric haemophiliac patients receiving on-demand rescue therapy.

Patients/Materials \& Methods: A prospective observational study conducted after 5 years of regular government supply of clotting factors at the Paediatric Haemophilia Clinic of Medical College, Vadodara. It included children diagnosed with Haemophilia A or B and Von Willebrand Disease (VWD) aged up to 18 years, receiving on-demand rescue therapy. Of the total 72 beneficiaries, 63 could be enrolled in the study after excluding those lost to follow up. After approval from the Ethics Committee, a written informed consent and a detailed history were taken from the parent-child dyad using the predesigned proforma. The patient record file was used for their bleeding profile and factor exposure. Data was analysed by applying appropriate standard statistical tests.

Results: Severe Haemophilia A formed $58.7 \%$ of the Haemophilia A patients. $9(14.3 \%)$ inhibitor positive patients were a part of the study, most were Haemophilia A. Majority wasn't diagnosed in the first year of life, nor received factors in infancy. Severe Haemophilia A had the highest days of school absenteeism and annual days wasted in hospitals, bearing consequences on their education. Common hindrances reported in factor procurement were long distance $(61.9 \%)$, time management $(41.3 \%)$ and lack of escort for the child (31.7\%). Most common bleeding sites reported in Haemophilia were knee (47.4\%), elbow $(19.3 \%)$ and oral mucosa $(10.5 \%)$. Annual bleeding rate and mean factor exposures were found to be the highest in Haemophilia A, varying significantly across the coagulopathy disorders $(p<0.05)$. $\mathrm{ABR}$ and total factor exposures have a directly proportional relationship with severity of Haemophilia A.

Discussion \& Conclusion: Analysis of problems of patients in procuring factors denotes that an effort to make factors available at more peripheral centres is a step in the right direction. Majority of bleeds in haemophiliacs were found to be post traumatic, and hence preventable. Common target joints like knees should be strengthened by physiotherapy to prevent disability. All paediatric haemophiliacs receiving factors should undergo biannual screening for inhibitors.

\section{Pseudotumor in Hypofibrinogenemia: A Rare Presentation}

Stitha P. Gudala*1 ${ }^{*}$, Mona Vijayaran ${ }^{1}$, Mukul Aggarwal ${ }^{1}$, Ganesh K. $\mathrm{V}^{2}$, Priyanka Naranje ${ }^{3}$, Tulika Seth ${ }^{4}$, Manoranjan Mahapatra ${ }^{4}$

${ }^{1}$ Clinical Hematology, ${ }^{2}$ Hematopathology, ${ }^{3}$ Radiodiagnosis, All India Institute of Medical Sciences, ${ }^{4}$ Clinical Hematology, AIIMS, New Delhi, India

Aims \& Objectives: Pseudotumor is known complication of hemophilia A and B. Pseudotumor is an expansive destructive encapsulated cyst/hematoma. They are an unusual complication of hemophilia, but pseudotumors can be seen in other rare bleeding disorders including deficiency of factors XI and VI and fibrinogen.

Patients/Materials \& Methods: A 32-year-old gentleman presented with gradually increasing painless swelling in the left thigh over past one month. There was no history of antecedent trauma. He gives history of prolonged bleeding after minimal trauma since childhood, but this was never evaluated. On examination, there was a $30 \mathrm{~cm} \times$
$14 \mathrm{~cm}$ non-tender firm swelling of the left thigh. There was no restriction of movement at the left hip/knee joint.

Results: His complete blood picture, liver, and kidney function tests were normal. His Prothrombin Time (PT) was $18 \mathrm{~s}$ (13 s), Activated Partial Thromboplastin Time (APTT) was $32 \mathrm{~s}$ (30 s), Thrombin time (TT) $31 \mathrm{~s}(16 \mathrm{~s})$ and fibrinogen was $50 \mathrm{mg} / \mathrm{dL}(150-400 \mathrm{mg} / \mathrm{dL})$. Radiograph of left femur revealed a homogenous soft tissue opacity in the medial thigh region and no bony injuries. Ultrasound showed a loculated collection of size $24 \mathrm{~cm} \times 14 \mathrm{~cm}$ in the intramuscular plane, anterior compartment of the left thigh along with complete thrombosis of the left popliteal vein. Magnetic resonance imaging (MRI) of the left thigh revealed a large well-defined hyperintense collection with internal septations, in the intramuscular region of vastus muscles. Imaging findings were consistent with pseudotumor(Figures A and B, Coronal T2-weighted fat-saturated (A) and T1weighted (B) images show large, sharply defined mass within vastus muscles with T1/T2 hyperintense signal. Multiple internal septations(black arrow) are seen. Surrounding muscles are compressed by mass effect and show edema (arrowhead). Mild reactive joint effusion is also noted in the knee joint (white arrow).C. Axial T2-weighted image show fluid-fluid levels (arrow) and septations within the collection suggestive of variable stages of hemorrhage. A dilated popliteal vein with loss of flow void is also noted suggesting thrombosis (arrowhead).). He was managed conservatively with regular FFP/cryoprecipitate transfusion.

Discussion \& Conclusion: The skeletal abnormalities observed in patients of fibrinogen abnormalities include bone cysts, intraosseous and extraosseous (soft tissue) pseudotumor and, rarely fractures. Radiologic assessment by CT, ultrasound, MRI, arteriogram if required. Biopsy is usually contraindicated. Management of pseudotumor includes conservative measures such as factor replacement, drainage or aspiration are indicated in small lesions. Surgery under blood/product cover is indicated for massive lesions, long bone fracture.

Family Screening for Thrombocytopenia in Voluntary Blood Donors with Thrombocytopenia in Kashmir Valley

Anil Kumar ${ }^{* 1}$, Parvaiz A. Koul ${ }^{2}$, Javid R. Bhat ${ }^{3}$

${ }^{1}$ Cardiology, SCB Medical College, Cuttack, ${ }^{2}$ Internal Medicine, ${ }^{3}$ Clinical Haematology, SKIMS Deemed University, Srinagar, India

Aims \& Objectives: To study familial thrombocytopenia in voluntary blood donors with thrombocytopenia in Kashmir valley.

Patients/Materials \& Methods: This was a single centre, prospective observational study conducted in SKIMS, Soura, Srinagar, India from August 2014 to May 2016. Blood samples from voluntary blood donors, families of blood donors with thrombocytopenia,and neonates reporting to SKIMS from different areas were taken by a 18 guage needle under all aseptic conditions and stored in an EDTA anticoagulant tube after taking proper consent from the subjects and analysed using 5 Part Differential (Sysmax XT 2000i) and peripheral blood film (PBF) examination to find out the prevalence of asymptomatic thrombocytopenia in the aforementioned population.Thrombocytopenia was defined was a platelet count of less than $150 \times 109$ per $\mathrm{L}$ on peripheral blood film examination. Blood donors were screened for viral serology (HBV, HCV, HIV) and antinuclear antibody (ANA).

Results: In our study, we found thrombocytopenia in about $44.6 \%$ of healthy blood donors of Kashmir Valley, with no identifiable acquired cause, and with absent bleeding symptoms.Majority of blood donors with thrombocytopenia have large platelets. After screening family 
members of voluntary blood donors having thrombocytopenia,a total of 100 families screened randomly,we found a high prevalence of familial thrombocytopenia( $64.1 \%)$ with autosomal dominant pattern of transmission and majority have large platelets. Thus kashmiris have a famililial thrombocytopenic disorder with large platelets. We designate this as "Kashmiri macrothrombocytopenic Syndrome." And we plan to exclude known causes of macro-thrombocytopenia so as to elucidate the etiology of the clinical disorder.

Discussion \& Conclusion: In our study, we found thrombocytopenia in about $44.6 \%$ of healthy blood donors of Kashmir Valley, with no identifiable acquired cause, and with absent bleeding symptoms.Majority of blood donors with thrombocytopenia have large platelets. After screening family members of voluntary blood donors having thrombocytopenia,a total of 100 families screened randomly,we found a high prevalence of familial thrombocytopenia(64.1\%) with autosomal dominant pattern of transmission and majority have large platelets. Thus kashmiris have a famililial thrombocytopenic disorder with large platelets. We designate this as "Kashmiri macrothrombocytopenic Syndrome." And we plan to exclude known causes of macro-thrombocytopenia so as to elucidate the etiology of the clinical disorder.

\section{Should We Still Do Bone Marrow Examination in Suspected Cases of ITP: A Study from Western Rajasthan}

\section{Arsha B.S ${ }^{* 1}$, Abhishek Purohit ${ }^{1}$, Gopal Krishana Bohra ${ }^{2}$, Siyaram Didel $^{3}$ \\ ${ }^{1}$ Pathology, ${ }^{2}$ Medicine, ${ }^{3}$ Paediatrics, All India Institute of Medical Sciences, Jodhpur, India}

Aims \& Objectives: Immune thrombocytopenia (ITP) is a diagnosis of exclusion and is characterized by immune-mediated accelerated platelet destruction and suppressed platelet production. ITP presents as primary (isolated) ITP or as secondary ITP in the context of other diseases such as autoimmune disorders and infections. Diagnosis is based on clinical suspicion and normal peripheral smear except for thrombocytopenia. Bone marrow examination is done to rule out leukaemia, myelodysplastic syndrome or aplastic anaemia. However, in most cases, clinical diagnosis is not altered after the bone marrow reports. This retrospective study was carried out to evaluate the justification for bone marrow examination in the suspected cases of immune thrombocytopenia.

Patients/Materials \& Methods: In this retrospective study, electronic records were reviewed for clinical details, laboratory investigations including bone marrow examination findings in suspected cases of ITP. All bone marrows examined between August 2016 and October 2020, were included in the study. Data were entered in excel sheet and analysed.

Results: In the study duration, a total of 55 suspected cases of ITP were subjected to bone marrow examination (with M:F being 0.44).Age wise distribution of cases ranges from less than 5 years $5 / 55(9 \%)$ to more than 60 years $9 / 55(15 \%)$. Most common presenting features were petechia and menorrhagia in our cohort. Of all cases, 10 patients presented with organomegaly (hepatomegaly and/or splenomegaly). None of these cases, on follow-up, have developed any other findings to suggest an alternate diagnosis. 25 (45\%) patients presented with a prior history of fever and $10(18 \%)$ with weakness in addition to bleeding symptoms. In all cases, bone marrow examination revealed normal haematopoietic elements and prominence of megakaryocytes including juvenile forms. None of the cases who presented with organomegaly and/or lymphadenopathy have developed features to suggest an alternate diagnosis on follow up.
Discussion \& Conclusion: This small retrospective study suggests that the routine use of bone marrow examination in the diagnostic workup of isolated thrombocytopenia may not be required, whereas thorough examination to rule out organomegaly and/or lymphadenopathy along with complete hemogram and peripheral smear examination is necessary.

\section{Genotype Spectrum of Factor VII Deficiency in North Indians: Next-Generation Sequencing-Based Approach}

Ritika Sharma $^{* 1}$, Manu Jamwal ${ }^{1}$, Hari Kishan Senee ${ }^{1}$, Varun Uppal $^{1}$, Chander Hans ${ }^{1}$, Deepak Bansal ${ }^{2}$, Amita Trehan ${ }^{2}$, Pankaj Malhotra $^{3}$, Narender Kumar ${ }^{1}$, Jasmina Ahluwalia ${ }^{1}$, Reena Das ${ }^{1}$

${ }^{1}$ Hematology, ${ }^{2}$ Pediatrics (Hematology-Oncology Unit), ${ }^{3}$ Internal Medicine, PGIMER, Chandigarh, India

Aims \& Objectives: The study aimed to characterize the clinical and genotypic spectrum of patients with inherited factor VII deficiency from north India using a targeted resequencing based approach followed by extensive validation using Sanger sequencing of the potential causal variants in index cases and family members.

Patients/Materials \& Methods: A total of 30 cases with prolonged prothrombin time (PT) were enrolled. Correction studies followed by factor VII (FVII) activity were performed. All of them had decreased FVII activity $(<50 \%)$ and were further subjected to targeted resequencing. Genomic DNA was extracted using a QIAamp blood mini kit followed by quantification and quality assessment using nanodrop and qubit fluorimeter. The library was prepared using Illumina AmpliSeq Custom panel as per manufacturer's instructions and sequenced in MiSeq. The output files from MiSeq (Fastq files) were aligned and the variant calling was done using local run manager software (Illumina). The probably caual variants found after data analysis were validated by Sanger sequencing and predicted for their pathogenicity using various in silico software.

Results: In the enrolled cases, twenty-one cases were diagnosed with FVII deficiency incidentally (70\%). Only two of our cases had intracranial bleeds and both of them died in early infancy. Positive family history was reported in two cases and consanguinity was present in three cases. FVII levels were $<1 \%$ in thirteen cases, $1-5 \%$ in five cases, and $>5 \%$ in eleven cases. Molecular characterization of the F7 gene revealed a total of 12 probably pathogenic variants in 23 $(77.6 \%)$ cases. These includes seven missense c. $215 \mathrm{C}>\mathrm{G}$, c. $1109 \mathrm{G}>\mathrm{T}$, c. $253 \mathrm{G}>\mathrm{C}$, c. $244 \mathrm{~T}>\mathrm{C}$, c. $961 \mathrm{C}>\mathrm{T}$, c. $904 \mathrm{G}>\mathrm{A}$, c.1211G > A, two deletions c.21delG, c.868_870delATC, one each of nonsense c. $634 \mathrm{C}>\mathrm{T}$, promoter region c.-30A $>\mathrm{C}$, and splice site c. $316+1 \mathrm{G}>$ A. Thirteen cases were homozygous, eight were compound heterozygous and in three cases only one probably causal variant was found. In our study the most common variant in eleven cases found was c.1109G $>\mathrm{T}$ (p.Cys370Phe) followed by c. $215 \mathrm{C}>\mathrm{G}$ (p.Ser72Cys) in six cases.

Discussion \& Conclusion: Exon 3 (c.215C $>$ G) and exon 9 (c.1109G $>$ T) were found to be the hotspot for F7 variants in our cohort. From the finding of targeted resequencing we conclude that due to recurrent variants in exon 3 and 9, these exons should be screened as the first line of molecular analysis for FVII deficiency using molecular tests like PCR-RFLP. 
Outcome of Inhibitor Screening in Haemophiliac Patients at a Tertiary Care Center in Rajasthan: A Move Towards Inhibition of The Inhibitors

B Tulasi Satyendra*1, Abhishek Purohit ${ }^{1}$, Gopal K. Bohra ${ }^{2}$, Siyaram Didel $^{3}$, Govind Patel ${ }^{4}$

${ }^{1}$ Pathology, ${ }^{2}$ Medicine, ${ }^{3}$ Paediatrics, All India Institute of Medical Sciences, Jodhpur, ${ }^{4}$ Clinical Haematology, Dr SN Medical College, JODHPUR, India

Aims \& Objectives: Determination of the incidence of inhibitors in known haemophiliac patients on factor replacement therapy.

Patients/Materials \& Methods: 128 Cases of haemophilia, on regular follow up at various centres in Jodhpur and receiving replacement therapy were included in this cross-sectional study. Blood samples were collected from all haemophiliac patients of the region on a single day and citrated plasmas were used for estimating factor VIII and factor IX on Sysmex CA1500 analyser using factor deficient plasmas. Subsequently inhibitor screening was done on all samples using normal control plasma.

Results: Total of 128 cases were studied. The mean age of the patients was 16.05 . Out of the total cases, $47(36.71 \%)$ cases were positive for presence of inhibitors while $73(57.03 \%)$ cases did not have immediate or delayed inhibitors. Eight cases were excluded due to technical reasons.

Discussion \& Conclusion: In the present study, the incidence of presence of inhibitors, immediate or delayed, was observed in $36.71 \%$ in concordance with similar studies from other parts of the country. However, to the best of our knowledge no similar studies were carried out in this part of Rajasthan.

Hemophilia A \& B causes severe morbidity. Despite availability of various factor replacement preparations, due to development of inhibitors, refractoriness to the therapy can occur. There can be variable incidence of inhibitor development. This variation may be due to the type of product used as treatment, intensity of treatment or the genetic characteristics of the patients. Screening for factor inhibitors is to be followed by Bethesda assay. Bethesda assay for all the cases is being planned at our Institute in a phased manner.

\section{Significance of Early Coagulation Parameters Screening in Predicting Severity of Acute Pancreatitis}

Nidhi Sharma ${ }^{* 1}$, Sandhya Gulati ${ }^{1}$, Gaurav K. Gupta ${ }^{2}$, Mohit Punjabi ${ }^{1}$ ${ }^{1}$ Pathology, ${ }^{2}$ Gastroenterology, SMS Medical College, Jaipur, India

Aims \& Objectives: Hemostasis pathways disturbances occur in acute pancreatitis (AP) due to crosslinkage between inflammation and coagulation. The study was done with the aim to find out the spectrum of early $(72 \mathrm{~h}$ ) alteration of coagulation profile \{prothrombin time (PT), activated partial thromboplastin time (APTT), Fibrinogen level $\}$ and Platelet count in AP, to see the correlation, if any, with severity of AP.

Patients/Materials \& Methods: 100 patients of AP fulfilling the inclusion and exclusion criteria were selected. Diagnosis of AP was based on history, clinical examination, serum amylase, lipase level, abdominal ultrasonography (USG) and Computed Tomography (CT). PT, APTT and serum fibrinogen level were processed in a fully automated coagulation analyser. Platelet count was analyzed in an automated hematology analyser.

Results: Age range was $06-81$ years. Patients were graded into mild, moderate and severe AP by Mortele modified CTSI scoring. There was significant difference among the mean of PT $\{22.2 \%$, $35.5 \%, 40 \%$ of mild, moderate severe AP respectively $\}$, while none in the mean of APTT, serum fibrinogen and platelet count in the three grades of AP. Severity index was positively correlated only with PT. A cut- off of $13.6 \mathrm{~s}$ for PT and $598 \mathrm{mg} / \mathrm{dl}$ for fibrinogen could discriminate mild AP from moderate plus severe cases with sensitivity of $85 \%$ and specificity of $67 \%$.

Discussion \& Conclusion: This study correlates the coagulation parameters with severity of AP. We evaluated that PT can be used as marker of severity in patients with AP. Deranged coagulation parameters at admission are an indication for early referral.

\section{Genotype and Phenotype Study of Inherited Factor VII Deficiency Patients from India}

Amit Sharma ${ }^{* 1}$, Kamal Kishor ${ }^{1}$, Ravi Ranjan ${ }^{1}$, Ravi Kumar ${ }^{1}$, Seema Tyagi $^{1}$, Renu Saxena ${ }^{2}$, Manoranjan Mahapatra ${ }^{1}$

${ }^{1}$ All India Institute of Medical Sciences, New Delhi, ${ }^{2}$ Lab Medicine, The Medicity Hospital, Gurgaon, Gurgaon, India

Aims \& Objectives: To identify the molecular defects underlying inherited deficiency of FVII in India.

Patients/Materials \& Methods: Nine patients (mean age 27 years, age of onset of bleeding 17.5 years) with FVII deficiency (activities: $<1-45 \%$ ), 5 females and 4 males were collected for molecular study. Mutations were identified by direct DNA sequencing of the F7 gene. Insilico studies were performed to understand the impact of detected mutations on protein structure and stability.

Results: The commonest bleeding symptom was menorrhagia observed in $44.4 \%$ cases followed by epistaxis (22\%) and easy bruisability $(22 \%)$. Three patients with FVII levels $<1 \%$ had mild bleeding while 2 had severe bleeding. Three patients with FVII levels above $20 \%$ reported bleeding symptoms while 1 was asymptomatic. We detected four missense mutations Arg302Asn, Arg413Gln, Gly425Ser and Cys370Phe and two polymorphic variants His175His and Glu95Glu in these patients. Free energy calculations performed on molecular modeling tools and servers like I-mutant 2.0 predicted decrease in protein stability for all mutations. The Cys370Phe mutation causes the ablation of a critical disulphide bond and also disruption of several side chain and backbone hydrogen bonds resulting in a major impact on protein stability.

Discussion \& Conclusion: Similar to previous results we did not observe a correlation between FVII levels and bleeding severity. The Cys370Phe substitution had highest deleterious effect on protein structure and stability.

\section{Clinico-Haematological Evaluation of Pancytopenia in a Tertiary Care Centre}

Deepshikha Verma ${ }^{* 1}$, Parul Murab ${ }^{1}$, Vedanti Newaskar ${ }^{1}$, Maneesh Sulya $^{2}$, R.K. Nigam ${ }^{3}$, Reeni Malik ${ }^{4}$

${ }^{1}$ PG 2nd year, Department of Pathology, ${ }^{2}$ Associate professor, Department of Pathology, ${ }^{3}$ Professor, Department of Pathology, ${ }^{4}$ Professor and Head, Department of Pathology, Gandhi medical College, Bhopal, India

Aims \& Objectives: Our study is directed to correlate the bone marrow findings and haematological picture with the spectrum of pancytopenia. It also describes the incidence of pancytopenia in a defined population.

Patients/Materials \& Methods: Over a period of 1 year from July 2019 to June 2020, a retrospective study was conducted in the department of pathology of GMC Bhopal, a tertiary care centre in which 63 cases were included. Patients of both sexes having pancytopenia were included. Series of tests were conducted to correlate the 
haematological picture and the bone marrow findings with the severity of pancytopenia.

Results: Out of the 63 cases taken, 15-45 years was the most common age group of presentation of pancytopenia. The most common symptom in these patients were fever and weakness. Males were affected more than females in a ratio of 2.2:1. The most common clinical signs were pallor and hepatosplenomegaly. On investigation hypercellular marrow and megaloblastic changes were the most common bone marrow aspirate findings. In more than $50 \%$ cases with megaloblastic anaemia like picture vitamin B 12 and folic acid levels were found to be low but these were normal in leukaemia/lymphoma cases.

Discussion \& Conclusion: For the diagnosis and proper management of pancytopenia patients, a detailed clinical history, complete physical examination followed by correlation with haematological picture and bone marrow aspiration findings is very important.

\section{Response to Equine Anti-thymocyte Globulin in Aplastic Anaemia: Study from a Tertiary Care Referral Centre}

Monisha Harimadhavan ${ }^{* 1}$, Nataraj $\mathrm{KS}^{1}$, Sunil Bhat ${ }^{2}$, Shilpa Prabhu $^{1}$, Aditi Shah ${ }^{1}$, Shiva K. Komaravelli ${ }^{1}$, Karthick RG ${ }^{1}$, Shobha Badiger $^{2}$, Vasundhara Raj ${ }^{2}$, Pooja P. Mallya ${ }^{2}$, Santosh Asangi ${ }^{2}$, Mohana Reddy ${ }^{2}$, Mohammad Salmaan ${ }^{2}$, Bharath Ram ${ }^{1}$, Sharat Damodar $^{1}$

${ }^{1}$ Hematology, ${ }^{2}$ Pediatric Hematology-oncology, Narayana Health City, Bangalore, India

Aims \& Objectives: To assess the response to Anti-Thymocyte Globulin (eATG) in patients with aplastic anaemia and to evaluate response to two different preparations of ATG(ATGAM and Thymogam)

Patients/Materials \& Methods: Data of all patients $(n=70)$ with aplastic anaemia admitted between Jan 2015 and July 2020 was extracted from hospital records. All patients received eATG followed by cyclosporine (CSA). Patients who did not achieve complete response, were started on stanazolol. Hematological response was assessed at 3,6 and 12 months after ATG.

Results: Overall response to treatment was seen in 52 patients (74.3\%), with 29 patients (55.8\%) showing a complete response and 23 patients (44.3\%) showing a partial response. Among patients who had not achieved complete response and received stanozolol $(\mathrm{n}=33)$ a complete response was attained in 14 patients $(42.4 \%)$. Age wise response rates were $50 \%$ in $<18$ years group $(\mathrm{n}=16), 80 \%$ in 19 to 39 years group $(\mathrm{n}=15), 76 \%$ in 40 to 60 years $(\mathrm{n}=25)$ and $93 \%>60$ years $(\mathrm{n}=14) .9$ patients $(12.9 \%)$ did not respond and 9 patients $(12.9 \%)$ expired. Death was due to infectious complications in all.. Median duration of follow up, in patients who survived was 18 months (range 2 to 60 months). Relapse rate was 10\% among patients who responded. Out of the 70 patients, 35 received ATGAM and 35 received Thymogam and the response was $74.3 \%$ to both preparations and there was no statistically significant difference in the response rates between the two preparations of ATG $(p=1.0)$.

Discussion \& Conclusion: Our study shows that Immunosuppressive therapy is a reasonable option for patients with aplastic anaemia showing an overall response rate of $74.3 \%$ comparable to that of western studies. Adult patients ( $>40$ years) receiving ATG had very good response rates in the range of 76 to $93 \%$ which is comparable to transplant outcomes. However our study is limited due to the short duration of follow up.

Supporting Document:
Table 1: Comparison of demographic characteristics and ATG use patterns between reponders and non-teponders.

\begin{tabular}{|l|l|l|}
\hline & Responders, $n(\%)$ & Non-Responders, $n(\%)$ \\
\hline Age & & \\
\hline $0-18$ years & $8(15.4 \%)$ & $8(44.4 \%)$ \\
\hline $19-39$ years & $12(23.1 \%)$ & $3(16.7 \%)$ \\
\hline $40-60$ years & $19(36.5 \%)$ & $6(33.3 \%)$ \\
\hline$>60$ years & $13(25 \%)$ & $1(5.6 \%)$ \\
\hline Males & $31(59.6 \%)$ & $12(66.7 \%)$ \\
\hline ATGAM use & $26(50 \%)$ & $9(50 \%)$ \\
\hline THYMOGAM use & $26(50 \%)$ & $9(50 \%)$ \\
\hline Mortality (n) & - & $9(50 \%)$ \\
\hline Total & $52(100 \%)$ & $18(100 \%)$ \\
\hline
\end{tabular}

\section{A Rare Case of Pancytopenia}

Kumari Priyanka $^{* 1}$, Jaya $\operatorname{tapadar}^{1}$, Manaswi chaubey ${ }^{1}$

${ }^{1}$ Medicine, IMS, BHU, Varanasi, India

Aims \& Objectives: Pancytopenia in elderly must be investigated properly as apart from nutritional causes and haematological malignancies, solid organ malignancy is an important aetiology which is usually missed. Solid malignancies have very little propensity for metastasis to bone marrow. Here, we present a case of an elderly male presenting with pancytopenia which on further evaluation came out to be a case of Gall bladder adenocarcinoma with bone marrow metastasis.

Patients/Materials \& Methods: A 60 year male, Ayurvedic practitioner by occupation presented with generalized weakness and easy fatigability and abdominal distension followed by abdominal pain for 3 months and backache for 1 month. On examination, pallor with pedal oedema and distended and tender abdomen with coarse crepitation over the bilateral basal area of lungs were present. Routine investigations showed pancytopenia which was confirmed on GBP. Persistent pancytopenia was noted beyond the usual clinical course hence bma was done which suggested metastatic deposits marrow (Mucoid adenocarcinoma) with associated hypoplastic changes. On further evaluation FDG PET/CT features suggestive of carcinoma of gall bladder (Ca GB) along with few calculi in the lumen of gall bladder with lytic lesion at the left iliac bone.

Results: Patient was managed conservatively and refer to surgical oncology for further management.

Discussion \& Conclusion: Ca GB presents with non-specifics symptoms that often lead to a delay in diagnosis..CaGB presenting with bony metastasis is rare hence bone scintigraphy does not form a part of routine work up for such patients hence pancytopenia secondary to metastasis from Ca GB is identified late and is associated with a poor prognosis. On the basis of above clinical features patient presented with pancytopenia there is a high index of suspicion of malignancy and final diagnosis was kept as $\mathrm{CaGB}$ with bone marrow metastasis. 


\section{Bone Marrow Failure as the First Presentation of Carcinoma Prostate}

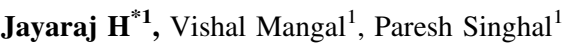 \\ ${ }^{1}$ Internal Medicine, Armed Forces Medical College, Pune, India
}

Aims \& Objectives: Prostate cancer is the most prevalent malignancy in men (after lung cancer). It commonly presents with lower urinary tract symptoms like urgency, hesitancy, poor stream besides some patients may present with bone pain. Anaemia is the most common presentation of solid tumours with bone marrow involvement, however pancytopenia as the presenting feature is rare.

Patients/Materials \& Methods: Here we describe a sixty-year-old man with significant comorbidities in the past who now presented with symptomatic anaemia without any urinary symptoms. On evaluation, he was found to have pancytopenia with elevated alkaline phosphatase levels. Work up for pancytopenia revealed metastatic deposits from the adenocarcinoma in the bone marrow. Further, he was diagnosed as a case of prostate cancer on transrectal prostate biopsy and managed with denosumab and maximal androgen blockade.

Results: Carcinoma Prostate diagnosed based on PBS findings, bone marrow findings, tumour markers and prostate biopsy findings.

Discussion \& Conclusion: The median age of diagnosis of prostate cancer is 67 ; only $35 \%$ of males are diagnosed between the ages of 55 and 63 . From an epidemiological standpoint, this patient was diagnosed relatively young at the age of 60 years. Patients at these ages thought to most benefit from prostate cancer screening and early definitive treatment. Patients with no urological complaints and patients who are not under urology follow up or who did not have PSA screening can present with advanced disease. The suppression of blood cell lines causing pancytopenia is usually a finding in the later course of the disease, compared to an osteoblastic/osteoclastic activity. This atypical presentation, like pancytopenia alone, would make diagnosing prostate adenocarcinoma difficult without the lower urinary tract symptoms. This patient presented with pancytopenia, so a bone marrow biopsy was obtained, which showed granular positivity for AMACR and negativity for CK7, CK20, and TTF-1 on IHC. Bone involvement by solid tumours can be diagnosed on CT, MRI, or bone scan. It is sporadic to detect non-hematologic malignancy while examining the bone marrow biopsy. This case highlights that there should be a low threshold for doing bone marrow examination in elderly patients presenting with pancytopenia.

\section{Dyserythropoiesis: A Morphology based Study on Bone Marrow Specimens}

Namrata Gautam ${ }^{* 1}$, Sunayana Misra ${ }^{1}$, Garima Baweja ${ }^{1}$, Sadhana Marwah $^{1}$, Vijay Kumar ${ }^{1}$

\section{${ }^{1}$ Pathology, ABVIMS and Dr RML Hospital, New Delhi, India}

Aims \& Objectives: Dyserythropoiesis is an altered or abnormal state of erythropoiesis in the bone marrow; classically seen in myelodysplastic syndrome (MDS) and congenital dyserythropoietic anemia (CDA). However, it can manifest in a variety of other disorders such as stress erythropoiesis resulting from intense erythropoietic proliferative drive. We aim to quantify and study light microscopic dyserythropoietic features in various reactive haematological as well as non-hematological disorders on bone marrow aspirates.

Patients/Materials \& Methods: Of the total of 150 bone marrow aspirate smears showing dyserythropoietic features, 132 smears met the adequacy criteria and were included in the study over one-year period. They were categorised into 7 broad groups based on bone marrow diagnosis. The percentage of erythroid cells showing dyserythropoiesis on bone marrow aspirate and the morphological features of dyserythropoiesis including nuclear budding, multinuclearity, internuclear bridging, karyorrhexis, megaloblastosis and cytoplasmic vacuoles were scored semiquantitatively in each of the cases. These features were then compared between the above mentioned 7 groups.

Results: The bone marrow diagnoses included erythroid hyperplasia, megaloblastic erythroid hyperplasia, cellular reactive marrow, megakaryocytic thrombocytopenia, acute lymphoblastic leukemia (ALL) in remission, hemophagocytic lymphohistiocytosis (HLH) and eosinophilia. The maximum dyserythropoietic changes were noted in erythroid and megaloblastic erythroid hyperplasia (75 to $90 \%$ ). Nuclear budding was the most frequent change seen through all groups while cytoplasmic vacuoles followed by internuclear bridging were less frequently observed.

Discussion \& Conclusion: Of all three hematopoietic lineages, erythroid series is most prone to dysplasia in the form of dyserythropoiesis. Erythroid hyperplasia, due to increased erythropoietic drive as seen in haemolytic and nutritional anemias can show prominent dyserythropoietic changes on bone marrow aspirate and is indicative of a reactive rather than neoplastic process. Therefore, the use of isolated dyserythropoiesis in diagnosing clonal disorders (MDS) warrants extreme caution.

\section{Outcome of Immunosuppressive Therapy in Patients with Idiopathic Aplastic Anaemia: Experience from a Tertiary Care Centre}

Rishu Vidhatri" ${ }^{* 1}$, Prakash S. Shekhawat ${ }^{1}$, Sumit Mitra ${ }^{1}$, Subham Bhattacharyaa $^{1}$, Shuvra N. Baul ${ }^{1}$, Prakas K. Mondal ${ }^{1}$, Rajib De ${ }^{1}$, Tuphan K. Dolai ${ }^{1}$

\section{${ }^{1}$ Department of Hematology, NRSMCH, Kolkata, India}

Aims \& Objectives: Aplastic anaemia (AA) is an immune-mediated bone marrow failure disorder, and most AA patients could not afford the HSCT due to non-availability of matched donor, co-morbidity and financial reasons. The aim of the study was to assess response of Horse Anti Thymocyte Globulin (ATG) and cyclosporine.

Patients/Materials \& Methods: This prospective study was conducted from year March'2011 to April'2020. AA was diagnosed as per established criteria. ATG was administered at the dose of $40 \mathrm{mg} /$ $\mathrm{kg} / \mathrm{day}$ for 4 days and cyclosporine at $5 \mathrm{mg} / \mathrm{kg}$ at two divided doses from day 14 onwards. Response assessment was done at 3 months and 6 months.

Results: Among 92 patients 32 patients received ATGAM and 60 patients received THYMOGAM, median age was 37 years (8-66 years), 28.2\% ( $\mathrm{n}=26)$ was female and 71.7\% $(\mathrm{n}=66)$ was male. Non severe AA was $11.9 \%(n=11)$, severe AA $81.5 \%(n=75)$ and very severe AA $6.52 \%(n=6)$. Median duration from diagnosis to ATG therapy was 378 days (10-1825 days). Complete response (CR) at 3 month was $3.2 \%$ and 6 months was $5.4 \%$. Overall response (OR) at 3 months $47.8 \%$ (44/92) and 6 months was $64.1 \%(59 / 92)$. OR at 6 months with ATGAM and THYMOGAM was $62.5 \%$ and $40.2 \%$ respectively. Overall survival (OS) at 6 months follow- up after IST was $91.3 \%$.

Discussion \& Conclusion: Our study shows most common type of AA is severe AA and majority of patients were in partial response. Three month OR was $47.8 \%$ and 6 month OR was $64.1 \%$. ATGAM had a better OR when compared with THYMOGAM. OS was $91.3 \%$ at a median follow-up of 6 months. 


\section{Dengue Fever with Hemophagocytic-Lympho- Histiocytosis (HLH)}

Nidhi Singh ${ }^{* 1}$, Kailash $\mathrm{Kumar}^{2}$, Nilesh Kumar ${ }^{1}$, Ashis Gupta ${ }^{2}$, PrachiMohapatra ${ }^{1}$

${ }^{1}$ Department of General Medicine, Institute of Medical Sciences, BHU, ${ }^{2}$ Department of General Medicine, Institute of Medical Sciences, BHU, Varanasi, India

Aims \& Objectives: HLH is an uncommon life-threatening hematological disease of severe hyperinflammation caused by uncontrolled benign proliferation of activated lymphocytes and macrophages that secrete high amount of inflammatory cytokines. If left untreated, patients with HLH survive for only a few months, due to progressive multi-organ failure. Hence high clinical suspicion and prompt diagnosis is must for patient survival.

Patients/Materials \& Methods: 18 years female presented with complaints of fever, vomiting from 5 days. On general examination patient was febrile, tacypnoeic and blood pressure was normal but postural drop in systolic BP was $>20 \mathrm{~mm} \mathrm{Hg}$, patient had pallor but no icterus and lymphadenopathy. On systemic examination mild tenderness was present in right hypochondrium. Rest of the examination was within normal limits. Routine investigations showed pancytopenia and deranged AST/ALT. Dengue IgM was positive and GBP showed anisocyotosis, hypochromasia, moderate leucopenia, few atypical lymphocytes and markedly reduced platelets. Due to persistence of pancytopenia beyond the usual clinical course bone marrow examination was done which showed normocellular reactive marrow with evidence of macrophage activation and hemophagocytosis suggesting the possibility of HLH. On further evaluation serum ferritin and triglycerides were raised. Since five out of the eight criteria of HLH (1994) were fulfilled hence diagnosis of Dengue fever with SECONDARY HLH was made.

Results: Patient was continued on IV fluids and in view of neutropenia prophylactic antibiotics was given to which she responded. She was monitored for signs of bone marrow failure and dengue haemorrhagic fever. Transfusion support was given to the patient. Since her clinical condition improved and disease markers of HLH activity like serum ferritin and D-dimer were on a declining trend hence disease specific therapy for HLH was not initiated.

Discussion \& Conclusion: The decision to start HLH specific treatment depends on clinical condition of the patient and values of rising serum ferritin, d-Dimer, liver enzyme, sCD25, or sCD163 levels. According to HLH-94 protocol dexamethasone and etoposide are recommended in tapering doses over 8 weeks with intrathecal methotrexate and hydrocortisone if CNS disease is present; Hematopaotic Stem cell Transplant being the only curative option. In this patient however, management of the underlying infection and transfusion support lead to improvement of symptoms and laboratory parameters and no cytotoxic therapy was required. Patient and her family members were advised for mutational analysis for HLH at the time of discharge.
Supporting Document:

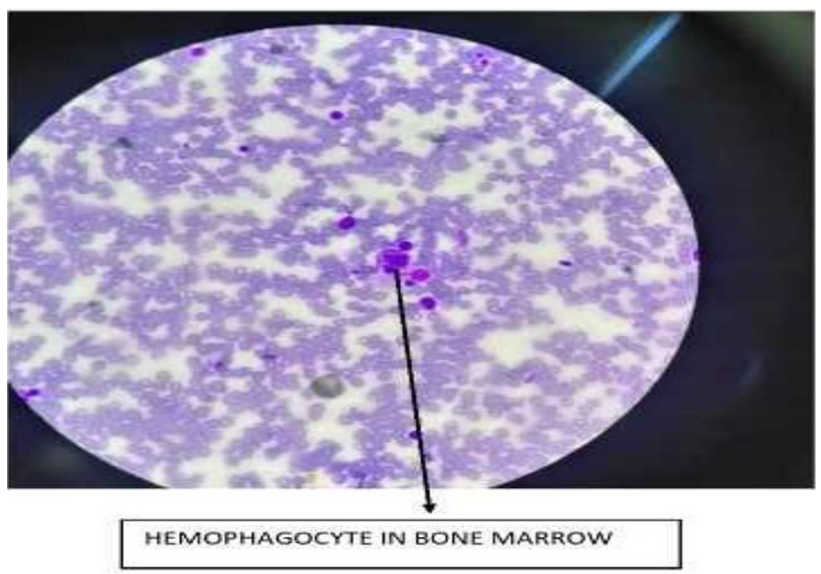

\section{Clinicopathological Study of New Onset Pancytopenia: Experience from a Hospital of Western India}

ShivaliAwasthi ${ }^{* 1}$, Paresh Singhal ${ }^{2}$, Ganesh Pendkur ${ }^{2}$

${ }^{1}$ Dept of Pathology, Armed Forces Medical College Pune, ${ }^{2}$ Dept of Pathology, Armed Forces Medical College, Pune, India

Aims \& Objectives: Pancytopenia, as per WHO, is defined as decreased haemoglobin (adult males $<13 \mathrm{gm} / \mathrm{dl}$, pregnant females $<10 \mathrm{gm} / \mathrm{dl}$, nonpregnant females and children $<11 \mathrm{gm} / \mathrm{dl}$ ), total leucocyte count $(<4 \times 109 / \mathrm{L})$ and platelet count $(<100 \times 109 / \mathrm{L})$. These patients may present with varied symptoms depending upon its severity. Vast majority of conditions causing pancytopenia are known. Therefore, a systematic approach to identify the underlying aetiology is a mandatory requirement. This paper aims to study clinicopathological background of new onset pancytopenia and to determine the frequency of different aetiologies causing pancytopenia.

Patients/Materials \& Methods: A prospective study of 451 cases who had pancytopenia and presented in emergency room of a tertiary care centre of Western India, from Jul 2017 to Sep 2019. Clinical data of patients were obtained and haematological investigations were analysed in Hematopathology section of Pathology Department. Bone marrow aspirates and biopsies were performed in 355 cases. Data were analysed using SPSS software.

Results: Patients age ranged from 6 months to 84 years with mean SD of $45.89 \pm 19.21$ years. Overall male to female ratio was 1.87:1. Hemato-lymphoid malignancy was the commonest cause ( $\mathrm{n}=137,30.37 \%)$ followed by nutritional anaemia $(\mathrm{n}=97,21.5 \%)$, infections $(n=68,15.07 \%)$, bone marrow failure $(n=66,14.63 \%)$, hypersplenism $\quad(\mathrm{n}=55,12.19 \%) \quad$ and autoimmune disorder $(\mathrm{n}=11,2.44 \%)$.

Discussion \& Conclusion: Pancytopenia is a common haematological entity with a plethora of underlying aetiological factors. It can present as a transient phenomenon or may even be life-threatening. Therefore, an accurate etiological break will further assist in appropriate management of these patients. Here in our study, Hematolymphoid malignancy was found to be the most common underlying cause of pancytopenia. 


\section{A Rare Presentation of Methotrexate Induced Pancytopenia}

Ashish A. Pushpakar ${ }^{* 1}$, Jaydeep R. DAMOR ${ }^{1}$, Satyam K. $\operatorname{singh}^{1}$, Ravi Tandon ${ }^{1}$, Ranjan Bhatnagar ${ }^{1}$

${ }^{1}$ General Medicine, Institute of Medical Sciences, Varanasi, India

Aims \& Objectives: presenting a case of methotrexate induced pancytopenia in a psoriatic patient.

Patients/Materials \& Methods: A 45 years old male with psoriasis for 15 years presented with complaints of generalized weakness for 2 months,painless bleeding oral ulcers for 2 months,multipleechymotic patches over body for 2 months and on \&off high grade fever.patient was taking methotrexate $20 \mathrm{mg}$ weekly since 15 years regularly but now had left from last 3 months. On examination severe pallor was present and multiple echymotic spots seen.no splenomegaly was present.routine :Hb 5.6,TLC 1700,platelet 12000,MCV 87 ,reticulocyte count $0.49 \%$, LDH 122 , LFT was normal. Dengue check and para check were negative.GBP suggestive ofpancytopenia.ANA test negative. Bone marrow aspirate showed hypocellular marrow with markedly reduced erythroid, megakaryocytes and myeloid cells.patient was admitted and managed with iv antibiotics, injcolistin $(300 \mathrm{mg})$, injleucovorin and tab eltrombopag (100 mg) along with blood and platelet transfusion and other supportive treatments were given. A bone marrow biopsy was done which suggest pancytopenia with hypocellular marrow, $10 \%$,with markedly reduced maturing myeloid and erythroid precursors. With no morphological e/o neoplastic hematolymphoid disorders.

Results: Hence from extensive diagnostic workup a diagnosis of psoriasis with methotrexate induced pancytopenia was made. Patient was treated with supportive treatment,methotrexate was stopped. TLC raised (4770),HB 6.4 gm,platelet were still 15000,a close monitoring was started but on request patient has been referred to Homi Baba cancer hospital for further management.

Discussion \& Conclusion: Myelosuppression is the major doselimiting side effect of high-dose MTX, but is infrequent in patients receiving low-dose therapy and should be kept in mind when patient presented with pancytopenia. The folic acid antagonist methotrexate has been used successfully in the treatment of psoriasis for over 50 years. the immunosuppressive effects of methotrexate on activated $\mathrm{T}$ cells controls psoriasis. With low-dose weekly therapy, hematologic toxicity with macrocytosis seen, but a more serious abnormality is the development of pancytopenia.hence a routine peripheral complete blood count should be performed at regular interval. Stop drug therapy as early recognition of bone marrow failure so that prevent further serious complications and mortality.

\section{A Rare Case Report of Myelodysplastic Syndrome with Myelofibrosis Showing Concomitant Deletion 5Q, Deletion 20Q and JAK2 Mutation: A Diagnostic Challenge}

ArpitaMathur $^{* 1}$, Shashi Bansal ${ }^{1}$, Ashish Gupta ${ }^{1}$, Upendra Sharma ${ }^{2}$ ${ }^{1}$ Pathology, ${ }^{2}$ Medical oncology, BMCHRC, Jaipur, India

Aims\&Objectives:Themyelodysplasticsyndromes(MDS)areaheterogeneousgroupofdisorderswithmultipleclonalcytogenetic abnormalities. $\operatorname{Del}(5 q),-7 / \operatorname{del}(7 q),+8$ and $-Y$ are among the most extensively studied cytogenetic abnormalities in MDS. JAK2 germline mutation is commonly associated with bcr-abl negative myeloproliferative neoplasms. MDS showing spectrum of cytogenetic abnormalities deletion $5 \mathrm{q}$, deletion $20 \mathrm{q}$ and JAK2 mutation is a rare phenomenon. MDS with isolated $5 \mathrm{q}$ deletion respondwelltotherapywhilelit-
tleisknownabouttheresponseoftherapyinMDSwithmultiplecytogeneticabnormalities

Patients/Materials \& Methods: A 62 year male was evaluated for generalized weakness, fatigue, weight loss and left hypochondrial fullness since 15 months. He was having transfusion dependent anemia since 10 months. On ultrasonography, spleen was $18 \mathrm{~cm}$. Peripheral smear revealed normocytic normochromic anemia, mild leucocytosis with $2 \%$ leucoblast and normal platelet count. Hb was $7.9 \mathrm{gm} / \mathrm{dl}$ and coombs test (both direct and indirect) was positive. Patient was subjected to bone marrow examination for further evaluation.

Results: Bone marrow aspiration revealed 7.5\% leucoblast. Myeloid series was suppressed. Erythroid series showed features of dysplasia. Megakaryocytes showed marked prominence with presence of non lobated nuclei (hypolobation and monolobation). On bone marrow biopsy, megakaryocytes were reaching uptoparatrabecular location. Fibrosis was seen with MF grade 2. On further analysis, it revealed JAK2V617F mutation and deletion of long arm of chromosome 5 and chromosome 20. Thus, the case was pinned as MDS with fibrosis having $5 \mathrm{q}$ deletion, $20 \mathrm{q}$ deletion and JAK2 mutation. The patient was given oral Lenalidomide therapy but after 4 months of treatment, he was lost to follow up.

Discussion \& Conclusion: Cytogenetic abnormalities form the basis for the IPSS-R (international prognostic scoring system) and are essential for therapeutic decision in MDS. MDS with myelofibrosis (MF grade2) showing concomitant deletion 5q, deletion 20q and JAK2 mutation is an extremely rare phenomenon. Superimposed autoimmune phenomenon can have a negative impact on overall survival of such type of cases. These cases could show potential benefit from Lenalidomide therapy. Our report suggests that such cases of MDS with rare complex cytogenetic abnormalities should be reported and studied to obtain a definitive therapeutic strategy.

\section{Diagnostic Approach for Suspected Myelodysplastic Syndrome in Refractory Cytopenias: Clinico Hematological Characteristics \& Risk Stratification}

JakkaSreedevi $^{{ }^{* 1} \text {, Minakshi Mishra }}{ }^{1}$, Radhika Narayan ${ }^{1}$, Farah $\mathrm{Rana}^{2}$, Anil Prasad ${ }^{2}$, Kaushik Saha ${ }^{2}$

\section{${ }^{1}$ Pathology, ${ }^{2}$ Dept of Pathology, Tata Main Hospital, EAST Singhbhum, India}

Aims \& Objectives: Myelodysplastic syndromes (MDSs) are clonal stem cell diseases characterized by ineffective hematopoiesis, multilineage dysplasia, and peripheral cytopenias with normocellular or hypercellular marrow. They represent a heterogeneous group of disorders with a varied spectrum of clinical, morphologic, biologic, and genetic characteristics. This study is aimed to determine the clinicohematological characteristics, cytogenetic abnormalities, and risk stratification of newly diagnosed de novo MDS patients.

Patients/Materials \& Methods: Retrospective observational study was done in the Department of pathology, Jamshedpur, Jharkhandfor3yearsfromJan2018totilldate. Thepatientswereselectedonthebasisofrefractorypancytopenia/bicytopeniawho did not respond to treatment and received multiple blood transfusions. All patients subjected to CBC, Peripheral smear, Serum B 12, Folate, ferrtin, Iron, $\mathrm{LDH}$, Reticulocyte count and followed by bone marrow examination. FISH MDS panel test was performed infewcases.RiskstratificationwasdoneinfewcasesusingtheRevisedInternationalPrognosticScoringSystem.

Results: A total of 59 cases of pancytopenia/bicytopenia were analyzed, including $38(64.4 \%)$ and 21 females $(35.5 \%)$. The most common presenting feature was anemia in $53(89.8 \%)$ patients, followed by anemia \& thrombocytopenia. 11 cases $(18 \%)$ showed 
trilineage dysplasia features favouring MDS, Out of which 6 cases were RCMD EBII. Blast transformation was seen in two cases.FISHMolecularstudiesdetected5q-,7q-trisomy 8clonalabnormalities.OnecaseshowedthatmutationspositiveinRUX1, ASL1 and EZH2genes.

Discussion \& Conclusion: : Pancytopenia is the common presentation in elders age more than 65 yrs. Risk stratification revealed a predominance of low-risk disease at the time of presentation. Keywords: Myelodysplastic syndrome, MDS - FISH cytogenetics, Revised International Prognostic Scoring System.

\section{Coexistence of Denovo Myelodysplastic Syndrome and Smouldering Myeloma in a Patient: A Case Report}

Aishwarya Iyer ${ }^{* 1}$, Divya Pavuluri ${ }^{1}$, Radhika Setty ${ }^{2}$, Shantveer Uppin $^{1}$, Tara R. Paul ${ }^{1}$

${ }^{1}$ Pathology, ${ }^{2}$ Hematology, NIMS, Hyderabad, India

Aims \& Objectives: Myelodysplastic syndrome with multilineage dysplasia (MDS-MLD) is a clonal hematopoietic stem cell disorder, characterized by cytopenias, multilineage dyspoiesis, ineffective hematopoiesis and genetic abnormalities. In smouldering plasma cell myeloma, patients have 10-60\% clonal plasma cells in the marrow with an M protein, without any myeloma defining events. Simultaneous occurrence of both these conditions in a patient is extremely rare.

Patients/Materials \& Methods: A 70 year old male, diabetic and hypertensive, presented with black coloured petechiae on skin, since 3 months. There was no h/o fever, loss of weight, loss of appetite. He was a pure vegetarian, non-smoker, non-alcoholic. There was no past history of blood transfusion or prior hospital admissions. O/E, patient was conscious, cooperative. He was afebrile, had pallor, no icterus, or palpable lymphadenopathy. He had petechiae over the skin. The abdomen was soft with no hepatomegaly or splenomegaly. He was found to have pancytopenia and bone marrow studies were performed.

Results: The peripheral blood examination revealed a reduced hemoglobin, normocytic, normochromic RBCs, ovalocytes and tear drop cells. There was leucopenia and thrombocytopenia. The marrow was hypercellular with trilineage dyspoiesis. Plasma cells accounted for 26\% and showed Kappa restriction. The karyotype was 46, XY del (20q) and the MDS-FISH panel was positive for del 20q. Protein electrophoresis revealed an M spike, IgG band and a Kappa band. B2 microglobulin- $4930 \mathrm{ng} / \mathrm{ml}$. The patient was put on hematinics and presented with similar complaints after 6 months. Repeat bone marrow study revealed trilineage dyspoiesis with increase in plasma cells to $45 \%$. On flow cytometry, these were found to be clonal plasma cells with Kappa restriction. The patient did not have any skeletal lesions or myeloma-defining events. Hence a diagnosis of MDS-MLD with smouldering myeloma was rendered.

Discussion \& Conclusion: Co-existence of MDS and plasma cell dyscrasia is extremely rare and this is limited to a few case reports. Occurrence of MDS/AML following treatment of MM is reported occasionally and this is due to the oncogenic effects of alkylating agents. However, this case had no prior h/o treatment with any drugs. Both conditions occur in the elderly. The case report is being presented due to its rarity.

\section{Cold Autoimmune Hemolytic Anemia with Myelodysplastic Syndrome: Not Just an Open and Shut Case}

\author{
Gourang Paliwal ${ }^{* 1}$, Deepti Mutreja ${ }^{1}$, Vishal Mangal ${ }^{2}$ \\ ${ }^{1}$ Dept of Pathology, ${ }^{2}$ Dept of Internal Medicine, Armed Forces \\ Medical College, Pune, India
}

Aims \& Objectives: Myelodysplastic syndromes (MDS) are clonal hematopoietic stem cell neoplasms characterized by marrow failure leading to ineffective hematopoiesis, dyspoiesis, and cytopenias with a risk of progression to acute leukaemia. There are reports of warm autoimmune haemolytic anemia with MDS, but cold AIHA is infrequent. We present an extremely rare case of cold AIHA with MDS. Patients/Materials \& Methods: An elderly male presented with fever and dyspnoea on exertion. He was being treated as a case of megaloblastic anemia in the past with no response to therapy. At present admission, lab investigations revealed cytopenias with elevated LDH, elevated blood sugar levels and features of acute kidney injury. The peripheral blood smear examination revealed marked red cell agglutination, thrombocytopenia and $4 \%$ blasts. Thermal agglutination was suggestive of cold AIHA. Bone marrow evaluation showed hypercellular marrow with erythroid hyperplasia, dyspoiesis and adequate megakaryocytes with $10 \%$ blasts. Cytogenetic studies revealed multiple abnormalities. Not only diagnosis but management of this patient was also challenging as steroid therapy had to be given with uncontrolled diabetes.

Results: The diagnosis of cold AIHA associated with MDS with excess blasts (MDS-EB2) was made based on the PBS findings, thermal agglutination test, bone marrow findings and cytogenetics by FISH.

Discussion \& Conclusion: Immunological syndromes have been reported to occur along with myelodysplastic syndrome(MDS) in literature, including vasculitides, connective tissue disorders, cytopenias and even asymptomatic serological laboratory abnormalities. Although AIHA in MDS is rare, and has been reported to occur with $3 \%$ patients only, autoantibodies against red cells are reported in up to $35 \%$ patients. An increased awareness of secondary autoimmune phenomena associated with MDS is required so that prompt action can be taken for earlier recognition of autoimmune phenomena, potentially leading to more positive outcomes for patients.

\section{Bone Marrow Oxalosis in a Patient with Recurrent Anemia}

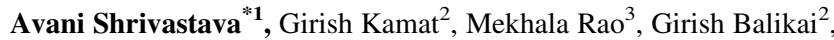
Deepak Goni $^{4}$, Anita Javalgi ${ }^{4}$, Vidisha Athanikar ${ }^{4}$

${ }^{1}$ Pathology, SDM College of Medical Sciences, ${ }^{2}$ Hematology, SDM College of Medical Science and Hospital, ${ }^{3}$ Pathology, SDM college of medical sciences and Hospital, ${ }^{4}$ Pathology, SDM College of Medical Science and Hospital, Dharwad, India

Aims \& Objectives: Bone marrow oxalosis is a type of systemic oxalosis. In this condition oxalate crystals are deposited in bone marrow. Systemic deposition of excess oxalate occurs in the bone and all organs and tissues except the liver.

Patients/Materials \& Methods: Here we report a case of bone marrow oxalosis in a patient with end stage renal disease and recurrent anemia.

Results: A 47 years old female patient who was a known case of hypertension, came with the complaints of fever, vomiting and weight loss. On evaluation her complete blood count revealed a $\mathrm{Hb}$ level of $6.3 \mathrm{~g} / \mathrm{dl}$, total WBC count of 4,100 cells/cumm and a platelet count of $90,000 /$ cumm. Her renal function tests revealed high serum creatinine 
(5.2 $\mathrm{mg} / \mathrm{dl})$. USG abdomen showed bilateral shrunken kidney with loss of corticomedullary differentiation without any calculi or nephrocalcinosis \& was suggestive of chronic kidney disease. CT scan revealed sclerosis of vertebral body. Hemodialysis was then started. Anemia persisted for 3 years and was not responding to treatment. Patient showed a pancytopenia blood picture after 3 years, for which a bone marrow aspiration \& biopsy was done. Bone marrow aspiration revealed normal erythropoiesis, myeloid hyperplasia with increased megakaryocyt. Bone marrow trephine biopsy showed multiple clusters of oxalate crystals arranged in radiating pattern. There was extensive fibrotic foreign body giant cell reaction. Polarising microscopy highlights birefringent polyhedral crystals in rosette formation. A diagnosis of bone marrow oxalosis was made.

Discussion \& Conclusion: Bone marrow oxalosis is a rare disease presenting with cytopenia, treatment resistent anemia and renal failure. The development of pancytopenia is a rare and late finding. The most common presentation is recurrent urolithiasis and nephrocalcinosis. However there was no nephrocalcinosis or recurrent urolithiasis in our case. Complications may occur due to cytopenias in bone marrow oxalosis. Early diagnosis of bone marrow oxalosis can avoid such complications. Bone marrow oxalosis should be considered as a differential in a patient with recurrent anemia and end stage renal disease even without nephrocalcinosis and recurrent urolithiasis.

\section{Malignant Melanoma Infiltrating the Bone Marrow: A Case Report}

\author{
Ruchee Khanna $^{* 1}$, Deepak Nayak ${ }^{1}$, Geetha $\mathrm{V}^{1}$ \\ ${ }^{1}$ Pathology, KMC, Manipal, Manipal, India
}

Aims \& Objectives: INTRODUCTION. The vascular nature of the bone marrow renders it one of the most common sites of hematogenous metastasis of a malignancy. Although the more common entities include prostate, breast and lung carcinomas, malignant melanoma infiltrating the marrow is exceedingly rare.1,2 Malignant melanoma accounts for less than $5 \%$ of all malignancies, but with an everincreasing incidence is being reported worldwide. Malignant melanoma with infiltration into the bone marrow is seldom reported in literature, for they are exceedingly rare. CASE REPORT. We present a case of a 50 year old male patient who presented with a one month history of fatigue and lower backache. He also gave a history of weight loss. On examination, the patient was pale. No lymphadenopathy or organomegaly was detected. On per rectal examination, a nodular mass was palpable in the anterior rectal wall. The mass was firm to hard in consistency. On colposcopic examination, a $3 \times 2 \mathrm{~cm}$ hyper-pigmented mass was noted with surface ulceration. The radiological examination by MRI showed several osteoblastic lesions in the pelvis including a pre-sacral mass. The biopsy of the same was reported as malignant melanoma, showing the malignant cells positivity for HMB-45 marker. Hematological findings showed anemia, thrombocytopenia and leukoerythroblastic blood picture. The marrow aspirates and trephine biopsy showed round to spindle-shaped malignant cells with intracytoplasmic brown-black coarse pigment, suggestive of melanin. The patient was diagnosed with stage IV melanoma, but was lost for follow-up.

Patients/Materials \& Methods: NA.

Results: NA.

Discussion \& Conclusion: CONCLUSION. The recognition of such an entity is important for both pathologists and clinicians alike. This case is being reported for the novelty of such an occurrence.

\section{Response to 1st Cycle Immunosuppressive Therapy (ATG \& Cyclosporine) in Children with Aplastic Anemia}

\author{
Nitish Kumar"1, Alok Hemal ${ }^{2}$ \\ ${ }^{1}$ Pediatrics, ABVIMS \& Dr. RML Hospital, ${ }^{2}$ Pediatrics, ABVIMS \\ \& Dr RML Hospital, New Delhi, India
}

Aims \& Objectives: This study was designed to assess the response to immunosuppressive therapy (ATG + Cyclosporine) in Indian children having Aplastic anemia who could not undergo HSCT.

Patients/Materials \& Methods: We did a prospective observational study on 35 children with Aplastic anemia from November 2018 to November 2019 at ABVIMS \& Dr.RML Hospital, New Delhi. Baseline parameters including age of presentation, sex, hematological data (Hemoglobin, Platelet count and absolute neutrophil count) at baseline and studied the response to immunosuppressive therapy (ATG + cyclosporine) after 4 months.

Results: In our study including 35 patients, 23 patients $(65.71 \%)$ had non-severe Aplastic anemia, 6 patients (17.4\%) each of severe and very severe Aplastic anemia. Our treatment showed a significant improvement in blood indices during follow-up. We observed that 19 patients $(54.28 \%)$ had partial response and 4 patients $(11.42 \%)$ had complete response. Our study also showed that 1 st cycle of immunosuppressive therapy (ATG + Cyclosporine) had a $65.7 \%$ of overall response rate (partial response + complete response) after 4 months of immunosuppressive therapy.

Discussion \& Conclusion: In resource-limited settings where HSCT is not feasible, role of immunosuppressive therapy is significant for the treatment of Aplastic anemia.

\section{A Curious Case of Pancytopenia: Drug Toxicity Mimicking Myelodysplasia}

Neha Suman ${ }^{* 1}$, Vandana Puri ${ }^{1}$, Sunita Sharma ${ }^{1}$, Brijesh Sharma ${ }^{2}$

${ }^{1}$ Pathology, Lady Hardinge Medical College, ${ }^{2}$ Medicine, RML Hospital, New Delhi, India

Aims \& Objectives: Pancytopenia with associated hypocellular marrow can occur due to various causes. Few of these include hypoplastic myelodysplastic syndrome and therapy-related myelodysplasia. It is very important to consider these possibilities as withdrawl of therapy results in improvement of pancytopenia and bone marrow morphology as well.

Patients/Materials \& Methods: A 30 year old male who presented to the medicine OPD with the complaints of fever and loose stool. The patient had mucosal ulcers and dermatitis. The patient was taking methotrexate for erythema nodosum for last 6 days.

Results: The blood examination of the patient revealed pancytopenia. Bone marrow aspirate were hypocellular and showed presence of few atypical cells alongwith suppression of myeloid series and features of dyserythopoiesis and few hypolobated forms of megakaryocytes. Though Perls Prussian blue stain for iron showed large granules in small clumps, no ring sideroblasts were seen. A possibility of hypoplastic myelodysplastic syndrome was suggested. But a drug history of methotrexate highlighted the possibility of bone marrow suppression by methotrexate. Further work-up and close follow-up was advised. Pancytopenia was improved and bone marrow aspirates were normal after 15 days after discontinuation of methotrexate. Flow cytometry showed presence of primitive stem cells/committed myeloid progenitors which were normal for age.

Discussion \& Conclusion: It is necessary to consider therapy related myelodysplasia a possibility in cases of sudden pancytopenia during immunosuppressive therapy. 
Recurrent Aplastic Anemia with Donor Type Aplasia: A Rare Occurrence in the Indian Subcontinent

\section{Ankur Majumder ${ }^{* 1}$, Sunayana Misra ${ }^{1}$, Umesh Tiwari ${ }^{1}$, Taruna Bansal $^{1}$, Vijay Kumar ${ }^{1}$}

\section{${ }^{1}$ Pathology, ABVIMS \& Dr RML Hospital, New Delhi, India}

Aims \& Objectives: To re-emphasize the importance of Donor Type Aplasia (DTA) as a rare yet importance cause of recurrent aplasia in bone marrow transplant subjects.

Patients/Materials \& Methods: A 17 year old male with recurrent bone marrow aplasia after a successful engraftment was brought to the pediatric department. Routine blood investigations, bone marrow and chimerism studies were done to rule out possible causes.

Results: Complete blood count and bone marrow studies showed "severe" aplastic anemia. Chimerism studies revealed $89 \%$ of the stem cells to have a "donor" origin. The patient succumbed to his illness before a second transplant could be attempted.

Discussion \& Conclusion: DTA is a condition where a an individual continues to be aplastic even after a successful engraftment of a Hematopoeitic Stem Cell Transplant with a majority of donor type cells in the bone marrow. This entity has been seen with varying frequency around the world, especially in South East Asia. However, its incidence in the Indian Subcontinent remains fairly low. Even though a variety of explanations have been offered, DTA remains a poorly understood/discussed topic. The awareness about the seriousness of this relatively rare condition, therefore, needs to be emphasized amongst clinicians and hemato-pathologists.

Supporting Document:

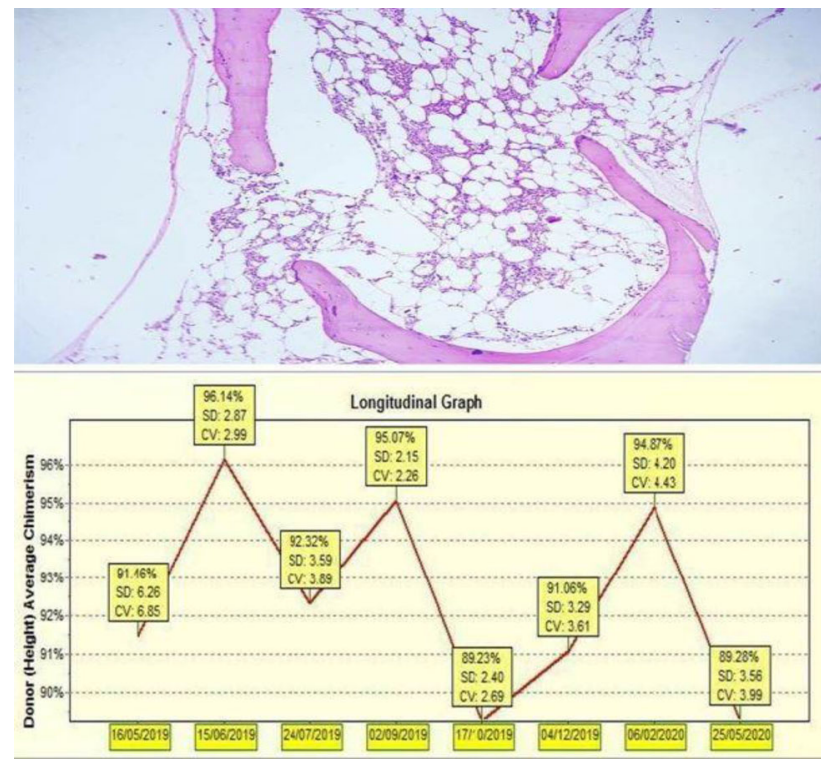

\section{When Clinico-Morphological and Molecular Studies Tell Different Stories: A Case Of MDS}

Niharika Jain ${ }^{* 1}$, Atul Tiwari ${ }^{1}$, Parul Gautam ${ }^{1}$, Anjana Mittal ${ }^{1}$, Naveen Gupta ${ }^{2}$, Mohan L. Yadav ${ }^{1}$

${ }^{1}$ Department of Pathology, ${ }^{2}$ Department of Clinical Haematology, Mahatma Gandhi Medical College and Hospital, Jaipur, India

Aims \& Objectives: Myelodysplastic Syndromes (MDS) are a very complex group of myeloid disorders characterized by peripheral blood cytopenias and morphologic evidence of dysplasia in bone marrow haematopoietic cellular elements. MDS is diagnosed based on the presence of dysplastic features in the bone marrow. Diagnosis is often supported by the presence of cytogenetic alterations. MDS with isolated $\operatorname{del}(5 \mathrm{q})$ is a distinct hematologic disorder with/without other cytopenias and/or thrombocytosis in which cytogenetic abnormality is $\operatorname{del}(5 \mathrm{q})$. These cases are not primarily defined by hematologic findings but by classic cytogenetic abnormality.

Patients/Materials \& Methods: We present a case of 66 years old male who presented with history of multiple packed red blood cells transfusions. Routine investigations, bone marrow aspiration and biopsy were done. The clinical and morphological findings raised the suspicion of MDS with isolated del(5q), so cytogenetics was done.

Results: When cytogenetics was done, there was a big mismatch in finding between clinical, morphological and molecular findings which brought a major change in prognosis as well as treatment.

Discussion \& Conclusion: It is, therefore, very essential to not rely only on the clinical and morphological findings to reach a diagnosis. Molecular findings play a pivotal role to come a final conclusion.

\section{Drug Reaction with Eosinophilia and Systemic Symptoms (Dress): A Rare Adverse Reaction to Imatinib}

\author{
Sukhjot Kaur ${ }^{* 1}$, Suvir Singh ${ }^{2}$, Robin Singh ${ }^{2}$, Palvi Singla ${ }^{1}$ \\ ${ }^{1}$ Dermatology, ${ }^{2}$ Clinical Haematology and Stem Cell \\ Transplantation, Dayanand Medical College and Hospital, \\ Ludhiana, India
}

Aims \& Objectives: Drug reaction with eosinophilia and systemic symptoms (DRESS) is a rare adverse drug reaction characterized by a triad of fever, skin rash, internal organ involvement and is reported in association with several drugs. Presentation of DRESS following exposure to a tyrosine kinase inhibitor (TKI) is rare and has significant treatment implications.

Patients/Materials \& Methods: We report a patient who presented with DRESS following imatinib and summarize evaluation and management of the same.

Results: A 55 year old gentleman diagnosed to have CML was initiated on Imatinib at $400 \mathrm{mg} / \mathrm{day}$. After 3 weeks, he developed high grade fever along with facial edema and a generalized blanchable maculopapular skin rash involving face, limbs, and trunk. Initial investigations revealed an $\mathrm{Hb}$ of $9.4 \mathrm{~g} / \mathrm{dl}$, WBC 12.7 X 109/L, (N $44 \%$, E 25\%, AEC 3170/ul). Skin biopsy revealed epidermal hyperkeratosis, follicular plugging, mild acanthosis, spongiosis, with a dense perivascular inflammatory infiltrate in dermis composed of lymphocytes, histiocytes and eosinophils. A diagnosis of Drug reaction with eosinophilia and systemic symptoms (DRESS) was considered and Imatinib was stopped, along with addition of prednisolone at $0.5 \mathrm{mg}$ per $\mathrm{kg} /$ day. RegiSCAR score for DRESS was 5, indicating probable DRESS and Naranjo score also indicated Imatinib as a probable culprit. A rechallenge with Imatinib after 4 weeks at $200 \mathrm{mg} /$ day resulted in recurrent symptoms and it was changed to Dasatinib at a dose of $100 \mathrm{mg} /$ day. Following the same, he developed mild papulopustular acneiform lesions over forehead and neck, controlled with low dose steroids. He continues to have a mild acneiform facial rash and has achieved good molecular control of CML with an RQPCR of $<1 \%$ at three months.

Discussion \& Conclusion: DRESS is a severe form of adverse drug reaction which must be promptly recognized by clinical and histological features and should immediately trigger removal of offending drug. DRESS with TKIs is rare, and as cost effective treatment options for CML are limited, a diagnosis of DRESS must be reliable before changing therapy. A rechallenge with a lower dose is 
reasonable, failing which an alternate TKI should be started to achieve expected responses.

Supporting Document:

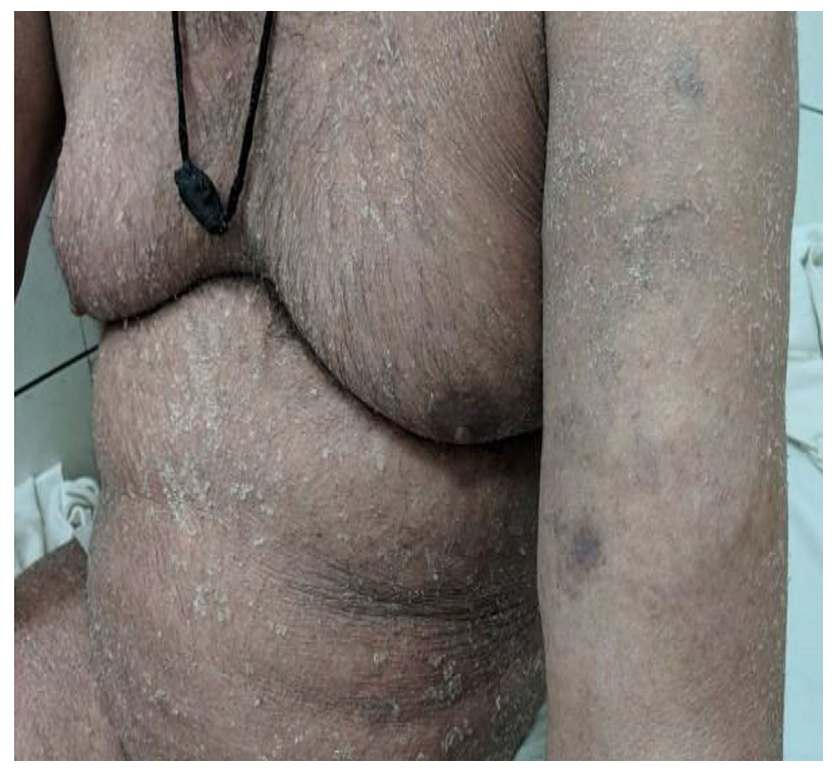

\section{An Unusual Extramedullary Site Involvement in Hairy Cell Leukemia}

\author{
Dibakar Podder $^{* 1}$, Nilanjan Sinha ${ }^{1}$, Siddhartha S. Roy ${ }^{1}$ \\ ${ }^{1}$ Institute Of Hematology And Transfusion Medicine, Medical \\ College and Hospital, Kolkata, India
}

Aims \& Objectives: Hairy cell leukemia is an extremely rare, low grade B cell leukemia characterized by proliferation of hairy cells in bone marrow, spleen, liver and rarely in lymph nodes. Most common presentation is splenomegaly, weight loss and fatigue. Skin involvement is extremely rare and the skin lesions are quite variable. Here we report 2 cases of hairy cell leukemia who presented with skin lesions. Patients/Materials \& Methods: It was a retrospective analysis from Nov, 2018 to March, 2020. Three cases of hairy cell Leukemia and 1 hairy cell Leukemia variant diagnosed over a period of 16 months. Results: Three cases of hairy cell leukemia were diagnosed in 2018-19 at our institute. Median age was 53 years. 2 males and 1 female. All the three cases presented with generalized weakness and fatigue. All the three patients had splenomegaly, one patient had mild splenomegaly, one patient had hepatomegaly. Two patients presented with maculopapular skin rash involving the lower limbs.

Discussion \& Conclusion: Here we describe two cases of hairy cell leukemia, one patient developed maculopapular skin rash and the other developed diffuse erythema involving both leg with limb swelling and edema. Both the patients were treated with injection Cladribine showed complete hematologic response, reduction in spleen size and disappearance of skin rash.

\section{Unusual Presentation of Hairy Cell Leukemia}

Sujata Kumari S P Prasad ${ }^{* 1}$, Monika Gupta ${ }^{1}$, Rajeev Sen ${ }^{1}$, Renuka Verma $^{1}$

\section{${ }^{1}$ Pathology, PGIMS, Rohtak, India}

Aims \& Objectives: Hairy cell leukemia (HCL) is an uncommon form of chronic B cell lymphoproliferative disorder comprising $2 \%$ of lymphoid leukemias. Patients are mostly middle aged and is characterised by typical findings of pancytopenia (or monocytopenia) and splenomegaly. The major sites of disease involvement are bone marrow, spleen and lymph node with rare or only a small number of leukemic cells in peripheral blood. We describe a case of a 70 years old male diagnosed with $\mathrm{HCl}$ involving mediastinal lymph node without classical feature of splenomegaly.

Patients/Materials \& Methods: 70 year old man presented in chest OPD with chief complaints of breathlessness and intermittent low grade fever. On examination, there was no organomegaly or lymphadenopathy. Radiological investigations revealed mediastinal lymphadenopathy along with no pulmonary lesion. Complete hemogram, bone marrow aspiration/biopsy and immunophenotyping was performed on 8 Colour Flow cytometer BD FACS Canto II using CD19/SSC gating.

Results: Hemogam showed anaemia, leukopenia (TLC-2500/ $\mathrm{mm}^{3}$ ) with normal platelet count and presence of $5-10 \%$ atypical lymphoid cells in peripheral smear. Bone marrow biopsy revealed marked suppression of trilineage hematopoeisis with near total replacement by diffuse sheets of monotonous population of atypical cells. On immunohistochemical staining, lymphoid cells revealed bright CD20 positivity and negative for CD3, suggesting of B cell - CLPD. Coombs test was within normal limits. Flowcytometric analysis revealed bright $\mathrm{CD} 200, \mathrm{CD} 25, \mathrm{CD} 11 \mathrm{c}, \mathrm{CD} 19, \mathrm{CD} 20$ expression, consistent with $\mathrm{HCl}$. The patient was started on Cladribine and achieved good response and is presently under follow up.

Discussion \& Conclusion: $\mathrm{HCl}$ is a rare entity. This case report is to emphasise that absence of splenomegaly does not exclude its diagnosis. Careful attention to morphological details is important while interpreting bone marrow biopsies, especially when low percentages of hairy cells are present in peripheral blood and bone marrow aspirate. Early diagnosis is essential to improve the prognosis, by obtaining maximum benefit from newer therapeutic options.

\section{Hairy Cell Leukemia or Hairy Cell Leukemia Variant: A Diagnostic Dilemma}

\author{
Anukriti Rastogi ${ }^{* 1}$, Deepti Mutreja ${ }^{1}$, Paresh Singhal ${ }^{1}$ \\ ${ }^{1}$ Pathology, Armed Forces medical College, Pune, India
}

Aims \& Objectives: Hairy cell leukemia (HCL) is a rare neoplasm of mature B lymphocytes which accounts for $2 \%$ of all lymphoid leukemias. The cells are characterized by hair like projections of cytoplasm. A neoplasm closely resembling HCL is hairy cell leukemia variant (HCL-v). Apart from the differences in cytology and immunophenotyping, it is important to differentiate between the two entities due to the resistance of the latter to conventional treatment. Through this case report and literature review, we aim to show the confounding ways in which a patient can present, the importance of differential diagnosis and detailed pathological examination essential for arriving at a correct diagnosis.

Patients/Materials \& Methods: A 75 year old male presented with urinary complaints since one month without constitutional symptoms. To rule out sepsis, a blood sample for complete blood count with peripheral smear (PBS) examination was sent. PBS revealed atypical lymphocytes with hair-like cytoplasmic projections and findings of urosepsis. Ultrasonography of the abdomen revealed 
hepatosplenomegaly and $\mathrm{BPH}$. After resolution of urosepsis, bone marrow studies (aspirate, biopsy and karyotyping) and flow cytometry analysis were performed and the diagnosis was confirmed by molecular studies.

Results: PBS findings and flow cytometry analysis suggested a Chronic lymphoproliferative neoplasm favouring HCL-v over HCL and splenic marginal zone lymphoma. However, immunohistochemistry on bone marrow was positive for Annexin A1 which pointed towards HCL. These findings have been tabulated in table 1. The diagnosis of HCL was further confirmed by molecular studies that showed presence of BRAF V600E mutation. The patient was subsequently started on chemotherapy and he responded well to the same. Discussion \& Conclusion: Owing to the unusual clinical presentation and contrasting features on histopathological, immunohistochemical examination and flow cytometry, this case was a diagnostic dilemma between HCL and HCL-v. It is essential to distinguish between them owing to the former requiring less aggressive therapy. A high index of suspicion must be kept while investigating such a case and each and every differential diagnosis should be ruled out using various modalities as discussed.

Supporting Document:

\section{Table 1 : Contrasting findings of HCL \& HCL-v in the} patient

\begin{tabular}{|c|c|c|}
\hline & $\begin{array}{l}\text { Features in favour of } \\
\mathrm{HCL}\end{array}$ & $\begin{array}{c}\text { Features in favour of } \\
\text { HCL-v }\end{array}$ \\
\hline Clinical presentation & & $\begin{array}{l}\text {-Older age : } 75 \text { years } \\
\text {-Absence of fatigue, } \\
\text { weight loss } \\
\text {-Moderate } \\
\text { splenomegaly }\end{array}$ \\
\hline $\mathrm{CBC}$ and PBS & $\begin{array}{l}\text {-Inconspicuous } \\
\text { nucleolus }\end{array}$ & $\begin{array}{l}\text { - Absence of } \\
\text { pancytopenia even after } \\
\text { urosepsis resolved } \\
\text { - Monocytopenia absent }\end{array}$ \\
\hline Bone marrow & & - Cellular and aspirable \\
\hline Immunohistochemistry & - Annexin A1 +ve & $\begin{array}{l}\text {-CD25-ve } \\
\cdot C D 123-v e\end{array}$ \\
\hline Flow cytometry & & $\begin{array}{l}\text {-CD25-ve } \\
\text { 'CD123-ve } \\
\text {-FMC7+ve }\end{array}$ \\
\hline Mutation studies & $\begin{array}{l}\text { •BRAFV600E mutation } \\
\text { present }\end{array}$ & \\
\hline
\end{tabular}

\section{A Study of Cytogenetic Anomalies Detected by Fish and Their Clinicopathological Correlations in CLL}

Bhawna Jha $^{* 1}$, Ritu Chadha ${ }^{1}$, Akshay Gore ${ }^{1}$, Shalini Goel ${ }^{1}$, Udaya Kumar DS ${ }^{1}$, Roshan Dikshit ${ }^{2}$, Amrita Ramaswami ${ }^{2}$, Nitin Sood ${ }^{2}$, Ashok Vaid ${ }^{2}$, Manorama Bhargava ${ }^{1}$, Renu Saxena ${ }^{1}$

${ }^{1}$ Department of Hematopathology, ${ }^{2}$ Department of Medical Oncology and Hematology, Medanta - The Medicity hospital, Gurugram, India

Aims \& Objectives: Chronic lymphocytic leukemia (CLL) is characterized by an extremely variable clinical course. Prognostic genetic testing can guide treatment decisions and predict outcomes for patients with CLL. The aim of the study was to detect (a) the frequencies of the recurrent aberrations in our series of patients with CLL using Fluorescence in situ hybridization(FISH) and to assess them regarding age, gender, disease status (stable versus progressive), clinical stage and CD38 expression and to evaluate the prognostic implications.

Patients/Materials \& Methods: Fifty-three patients (age range 39-90 years, Male:female 3.41:1) with CLL, diagnosed in the Department of haematopathology, Medanta, between May 2018 and September 2020 were included in this study. FISH was performed with panel of probes (METASYSTEMS, XL ATM/TP53, Dual colour probe and XL DLEU/LAMP/12cen tricolor probe), specific for the chromosome 12 centromere, 13q14.2 (RB1), 17p13.1 (p53) and 11q22.3 (ATM).

Results: FISH analysis revealed $79.2 \%$ patients had at least one chromosomal abnormality. No cytogenetic abnormality was seen in $20.8 \%$ cases. The most frequent one was del13q14 (52.8\%) of which biallelic (homozygous) deletion was observed in 4 cases. Trisomy 12 was found in $28.3 \%, 17 \mathrm{p} 13$ deletion in $9.4 \%$ cases and $11 \mathrm{q} 22$ deletion in $7.5 \%$ cases. Del17p was observed in three patients with previously treated CLL suggesting possible acquisition of CLL clones with $\operatorname{del}(17 p)$ during the course of treatment. Patients with unfavorable cytogenetic alterations, tended to be in the intermediate (Stage 1/2) and high (Stage 3/4) Rai stages $(81.2 \%)$. Additionally, patients with $17 \mathrm{p} 13$ more frequently express CD38 (80\% cases) and fell under high risk $(80 \%)$ and intermediate risk (20\%) Rai stages. In group with two simultaneous aberrations, $13 \mathrm{q}$ deletion was a major clone indicating $\operatorname{del}(13 q)$ as a primary event, possibly followed by $11 \mathrm{q}$ deletion, $17 \mathrm{p} 13$ deletion and trisomy 12 as secondary progressive events. Patients with del17p, Trisomy 12 and Coexistence of 2 aberrations more often had a progressive disease $(100 \%, 75 \%$ and $83.3 \%$ respectively) and del13q more often had a stable disease $(65 \%)$ (Table 1$)$.

Discussion \& Conclusion: It is concluded that prevalence of cytogenetic abnormalities in Indians is similar to that in the west and has similar prognostic significance and so should be carried out in all cases of CLL. Also, if possible, CD 38 testing should also be carried out for prognostication.

\section{An Interesting Paradox of Covid-19 Illness in Chronic Lymphocytic Leukemia}

Rehab Y. Nainar ${ }^{* 1}$, Durga Krishnan ${ }^{1}$, Mayilananthi Kaliannan ${ }^{1}$, Rajat P. Pande ${ }^{1}$, C V Thejesh ${ }^{1}$, V. R. Mohan Rao ${ }^{1}$

\section{${ }^{1}$ Department of General Medicine, Chettinad Hospital and} Research Institute, Chennai, India

Aims \& Objectives: A case report of 46 year old female with chronic lymphocytic leukemia manifested recently with Covid 19 disease.

Patients/Materials \& Methods: Chronic lymphocytic leukemia (CLL) is a monoclonal proliferation of mature B lymphocytes that is associated with an increased risk of COVID-19 infection and a worser outcome due to the immune dysregulation noted in them as well as use of immunosuppressive therapy. Hence, we report a case of chronic lymphocytic leukemia who has survived COVID-19 infection.

Results: A 46 year old female, known case of CLL treated four years back, was admitted in COVID care centre with complaints of high grade fever, cough and myalgia for 5 days. Spo 2 at the time of admission was $40 \%$ in room air. Laboratory investigations revealed lymphocytosis $(81 \%)$ and neutropenia. Inflammatory markers revealed increasing trend of D-dimer but a decreasing trend of serum ferritin, Interleukin-6 and CRP. HRCT Chest revealed CORADS V and CT severity score of $18 / 25$ with $70 \%$ lung involvement. RFT, LFT and coagulation profile was normal. She was started on Remdesivir, Clexane, Dexamethasone, and prolonged high flow nasal oxygen. She improved symptomatically and was discharged on Day 
10. At one month follow-up, patient was asymptomatic. Laboratory investigations given below in supporting document.

Discussion \& Conclusion: Typical COVID-19 presentations include neutrophilia, lymphopenia, and elevated inflammatory markers however, this patient had a different picture of increasing trend of total leucocyte count due to lymphocytosis and neutropenia. She had a severe COVID-19 disease that resolved with the above stated measures and did not receive any immunomodulators. She also did not have hypogammaglobinaemia which is a typical manifestation of severe CLL. In view of impending lymphocytic catastrophe, steroid was tapered shortly.

Keywords: CLL, Covid 19, Inflammatory markers.

Supporting Document:

\begin{tabular}{|c|c|c|c|c|}
\hline DAYOF AOMISSSION & DAY1 & DAYS & DAYS & After 1 month \\
\hline $\begin{array}{l}\text { Totat leucocyte count } \\
\text { (cells/cu,mm) }\end{array}$ & 46,700 & 71,600 & $1,39,800$ & 28,000 \\
\hline Hemoglobin $/ g / d|d|$ & 8.9 & 8.3 & 10.5 & 11 \\
\hline Platelet (lacc(cumm) & 1.17 & 1.26 & 1.75 & 1.78 \\
\hline Neutrophils $\mid(y)$ & 19 & 11.1 & 10.1 & 17 \\
\hline Lymphocytes $(y)$ & 81 & 88.7 & 89 & 79 \\
\hline D.OIMER $/ \mathrm{ng} / \mathrm{m} / \mathrm{m}$ & 855 & 4554 & & \\
\hline$\| .6(\mathrm{pg} / \mathrm{m} \|$ & 118.5 & 3.35 & & \\
\hline CRP $(m g / l)$ & $\$ 200$ & 33.15 & & \\
\hline FERRATIN (ng/mil) & 158.5 & 116.9 & & \\
\hline Globulin $|g / d| d \mid$ & 2.8 & 2.5 & 2.7 & \\
\hline
\end{tabular}

\section{Primary Granulocytic Sarcoma of Pancreas: A Rare Entity with Diagnostic Challenges}

Devika Bansal ${ }^{* 1}$, Saveena Jindal ${ }^{1}$, Sumit Grover ${ }^{1}$, Vikram Narang ${ }^{1}$, Harpreet Kaur ${ }^{1}$, Bhavna Garg ${ }^{1}$, Neena Sood ${ }^{1}$, Aminder Singh ${ }^{1}$

${ }^{1}$ Pathology, Dayanand Medical College \& Hospital, Ludhiana, India

Aims \& Objectives: To highlight the existence of granulocytic sarcoma as a rare etiology of pancreatic mass.

Patients/Materials \& Methods: A 60 year old female presented with pain in abdomen and jaundice. Computed tomography whole abdomen revealed poorly marginated heterogeneously enhancing soft tissue area measuring $2.5 \times 2.9 \mathrm{~cm}$ in the uncinate process and head of pancreas causing obstructive biliopathy. Whipple's surgery was performed and specimen was sent for histopathological examination.

Results: Grossly a thickened area was identified in duodenopancreatic region measuring $2 \times 1 \times 1 \mathrm{~cm}$. Microscopy revealed small to medium sized atypical cells arranged in sheets and perivascular arrangement having hyperchromatic nuclei and scant amount of cytoplasm infiltrating in a streak like pattern into the pancreatic tissue. I- mmunohistochemistry showed positivity for CD117, CD 99, CD 68 and LCA (weak positive). Markers for lymphoma, melanoma and neuroendocrine tumors were negative. Thus a diagnosis of granulocytic sarcoma of pancreas was rendered.

Discussion \& Conclusion: Granulocytic or myeloid sarcoma is an isolated extramedullary tumor mass consisting of immature myeloid cells. It is also called chloroma which refers to infrequent green colour observed as a result of myeloperoxidase action in neoplastic cells. It most commonly affects skin, lymph nodes and soft tissue. Tissue sampling followed by the use of ancillary studies and IHC is essential for diagnosis. These allow the distinction of myeloid sarcoma from B and T lymphoblastic leukemia/lymphoma, Burkitt lymphoma and Diffuse large B cell lymphoma. Moreover, bone marrow sampling is necessary to exclude marrow involvement. Abdominal region especially pancreatic involvement is a very rare occurrence. Hence the diagnosis of granulocytic sarcoma at such location is challenging and requires a high index of suspicion. We present this case because of the rare occurrence at this uncommon site.

\section{Extramedullary CNS Presentation in a Young CLL: A Case Report}

Chandan Mishra $^{* 1}$, Tushar Sehgal ${ }^{1}$

${ }^{1}$ Laboratory Medicine, AIIMS, New Delhi, New Delhi, India

Aims \& Objectives: Chronic lymphocytic leukemia (CLL) is a clonal disorder of $\mathrm{B}$ cell disorder and is the most common lymphoproliferative disorder in the West. CNS involvement in CLL is rare and even rarer is CLL presenting with upfront CNS symptoms. We report one such case that demonstrates an extremely rare form of CNS intraparenchymal involvement in a previously undiagnosed CLL patient. Patients/Materials \& Methods: Case Details.

Results: 42 years male presented to A \& E with altered sensorium and clonic movements of limbs. On examination he was disoriented (GCS score 6) with mild pallor. There was generalised lymphadenopathy in cervical, axillary and inguinal regions and splenomegaly. CBC showed hyperleukocytosis of $979 \times 103 / \mu \mathrm{L}$, with haemoglobin $5.4 \mathrm{~g} / \mathrm{dL}$ and platelets of $90 \times 103 / \mu \mathrm{L}$. His blood sugar and electrolytes were within normal limits. Blood film showed $68 \%$ mature lymphocytes with $35 \%$ prolymphocytes in background of smudge cells (image 1a). Immunophenotyping on peripheral blood showed 96\% kappa restricted B cell population expressing CD5, CD23, CD20, CD79a and CD200 suggestive of CLL. CT head revealed multiple hyper dense lesions in bilateral cortex, right thalamus, basal ganglia and bilateral cerebellar region suggestive of leukemic deposits (image 1b). Patient's condition deteriorated with respiratory distress and he succumbed to his illness on fourth day of his admission.

Discussion \& Conclusion: CLL is an indolent haematological neoplasm. The median age at diagnosis is seventh decade. Only very few $(\sim 10 \%)$ presents in the fifth decade. It is mostly an incidental finding on routine blood analysis in asymptomatic patients. Uncommonly patients may with present with lymphadenopathy, splenomegaly and anemia or thrombocytopenia. Extramedullary involvement of CNS is very uncommon. The sign and symptom of CNS involvement are heterogenous and include headache, cerebellar signs, cranial nerve palsies, visual problems, and motor or sensory deficits. The time from CLL diagnosis to CNS varies from 0 days to 15 years (median - 4.5 years). Our patient was a young CLL who 
presented with extramedullary involvement of brain parenchyma. Only a handful of cases have been reported in literature. CLL with CNS involvement at initial presentation is an uncommon manifestation. To our knowledge this is the fourth reported case of undiagnosed CLL presenting with CNS intra- parenchymal infiltration.

\section{Primary Follicles in Lymph Node Mimicking Follicular Lymphoma}

Sri Gayathri Shanmugam ${ }^{* 1}$, Rajendiran Swaminathan ${ }^{1}$, Priyathersini Nagarajan ${ }^{1}$

${ }^{1}$ Pathology, SRIHER, Chennai, India

Aims \& Objectives: To report an interesting finding in a follicular pattern of Lymphnode enlargement To discuss how it resembles follicular lymphoma and to differentiate the two lesions.

Patients/Materials \& Methods: We report a 25 yrs patient with retroperitoneal lymph node enlargement of $3 \mathrm{~cm} \mathrm{X4} \mathrm{cm} \mathrm{size.} \mathrm{The}$ histopathological examination of the core needle biopsy lymph node revealed closely placed follicles. Follicles were fairly uniform in size without any germinal centre formation and completely lacked tangible body macrophages. There was no zonation or mantle cell layer. Immunohistochemistry CD20, CD10, BCL2, BCL6, MUM1, Ki67, CD45, CD3, CD5 was done in that core needle biopsy Results: The histopathology of primary follicle and Follicular lymphoma -Low grade are similar without any difference. By immunohistochemistry both follicular lymphoma and primary follicle are positive for CD20, BCL2 and have low Ki 67. CD10 is negative in Primary follicle and positive in follicular lymphoma. But follicular lymphoma can also be negative for $\mathrm{CD} 10$.

Discussion \& Conclusion: Primary follicles are follicles in lymph node before the antigenic stimulus. As they lack the germinal centre of reactive follicle, they form a close mimicker of follicular lymphoma by morphology and IHC. A combination of clinical, morphology and IHC features are essential in the right diagnosis.

\section{Megakaryocyte Morphometry in Chronic Myeloid Leukemia and Its Association With Morphological Sub- type And Grades of Fibrosis}

Sreerag K ${ }^{* 1}$, Rakhee $\operatorname{Kar}^{1}$, Rajesh N. Ganesh ${ }^{1}$, Biswajit Dubashi ${ }^{2}$, Debdatta Basu ${ }^{1}$

${ }^{1}$ Pathology, ${ }^{2}$ Medical Oncology, Jawaharlal Institute of Postgraduate Medical Education and Research (JIPMER), Puducherry, India

Aims \& Objectives: Chronic myeloid leukemia (CML), is a myeloproliferative neoplasm characterised by reciprocal translocation of chromosome 9 and 22, $\mathrm{t}(9 ; 22)(\mathrm{q} 34 ; \mathrm{q} 11.2)$. Abnormal megakaryocyte morphology and fibrosis of bone marrow are histological features of CML. The study was undertaken to assess the megakaryocytic morphometry and to evaluate the association with grade of fibrosis as well as histomorphological subtype of CML.

Patients/Materials \& Methods: Sixty-seven newly diagnosed CML patients were included. $\mathrm{H}$ and $\mathrm{E}$ stained bone marrow biopsy sections were studied and subtyped as Granulocytic (CML-G) or granulocytic megakaryocytic (CML-GM). The number of megakaryocytes were counted in ten 400x field for each case. One 400X field represented
$0.23 \mathrm{~mm} 2$, hence megakaryocyte per millimetre square was obtained by dividing the number of cells per field by 0.23 . Ten megakaryocytes were analysed per case for morphometry making a total of 670 megakaryocytes. Morphometric parameters, including cytoplasmic major diameter (CMD), cytoplasmic area (CA), nuclear major diameter (NMD), nuclear area (NA) and nuclear cytoplasmic ratio (NCR) were studied using Olympus microscope BX 53 using ProgressR Capture Pro software. Grade of fibrosis was categorized as low (WHO grade 0 to 1 ) and high (WHO grade 2 to 3 ). Association of morphometric parameters with sub-type of CML and grade of fibrosis were done with independent sample $t$ test. Statistical analysis was carried out at 5\% level of significance and $p$ value $<0.05$ was considered as significant, using SPSS software v.19.

Results: A total of 670 megakaryocytes were studied. 42 cases were CML- $\mathrm{G}$ type and 25 were CML-GM. The megakaryocyte morphometry, especially CMD and CA, was significantly different between two groups. Mean \pm SD of CMD was $19 \pm 4.53$ and $20.8 \pm 5.46(p=0.014)$ and Mean \pm SD of CA is $204.7 \pm 76.75$ and $223.4 \pm 105.11(p=0.021)$ respectively in CML-G and CML GM. There were 33 cases with low grade and 34 with high grade fibrosis. However there were no statistical differences between low and high grades of fibrosis in any morphometric parameters.

Discussion \& Conclusion: Although megakaryocytes exhibit a varied morphology in CML with significant heterogeneity in morphometric parameters between both the groups, there is no significant difference in megakaryocyte morphometry between the grades of fibrosis.

\section{Comprehensive Characterization of BCR-ABL1 Tkd Mutations: A Single Center Experience}

Vamshi Krishna Thamtam ${ }^{* 1}$, Poonam Santra ${ }^{1}$, Biswajoy Pal ${ }^{1}$, Kunjal Patel ${ }^{1}$, Vivek Radhakrishnan ${ }^{2}$, Saurabh Bhave ${ }^{2}$, Reena Nair ${ }^{2}$, Mammen Chandy ${ }^{2}$, Neeraj Arora ${ }^{3}$, Mayur Parihar ${ }^{1}$, Deepak K. Mishra $^{1}$

${ }^{1}$ Molecular Genetics, ${ }^{2}$ Clinical Hematology \& Bone marrow transplant, Tata Medical Center, Kolkata, ${ }^{3}$ Molecular Genetics, Unipath Specialty Laboratory Ltd., Ahmedabad, India

Aims \& Objectives: The aim of this study was to enumerate and characterise the various mutations in the BCR-ABL1 kinase domain, seen in Philadelphia-positive Chronic myeloid leukemia (CML)/ Acute lymphoblastic leukemia (ALL) patients at our tertiary care cancer center.

Patients/Materials \& Methods: This is a retrospective observational study. A total of $153 \mathrm{CML} / \mathrm{ALL}$ patients were evaluated from September 2012 to September 2020 for BCR-ABL1 tyrosine kinase domain mutations. The clinical profile, disease characteristics and various KD mutations were documented. The patients' RNA was extracted from Peripheral Blood/Bone Marrow and reverse transcribed into complementary DNA. Nested PCR was performed and exons 4-10 of the ABL1 gene were amplified using 4 sets of primers, which were read after Sanger sequencing.

Results: In our study, patients' age ranged from $8-73$ years. The majority of our patients who underwent mutation analysis were in CML chronic phase $(\mathrm{n}=109 ; 71.24 \%)$, followed by accelerated phase $(\mathrm{n}=7 ; 4.5 \%)$ and blast phase $(\mathrm{n}=9 ; 5.8 \%)$. MPAL $(\mathrm{n}=1)$, AML with $\mathrm{t}(9 ; 22)(\mathrm{n}=1)$, and CML with extramedullary leukemia $(\mathrm{n}=1)$ were also seen among the patients evaluated. Patients with major transcript constituted $90.8 \%(\mathrm{n}=139)$, while $8.4 \%(\mathrm{n}=13)$ had minor BCR-ABL1 transcript. One patient had e14a3 BCR-ABL1 transcript. 
The sequence analysis showed $72.09 \%$ cases with substitution mutations $(n=31 / 43)$. Ten cases had deletion mutations and two cases showed insertion mutations. Double mutations were noted in 2 cases and one case had three mutations. The mutation rate was highest in CML cases which progressed from chronic to accelerated/blast phase $(6 / 10 ; 60 \%)$ and in ALL $(6 / 14 ; 42.8 \%)$. Most common mutation reported in our subset was the "gate keeper" mutation, T315I in 16/43 cases $(37.2 \%)$ followed by G250E in $3 / 43$ cases $(6.9 \%)$. Few novel mutations were also seen. Using semi-nested PCR technique we were able to detect a kinase domain mutation even at 840 BCR-ABL1 copies $(0.4 \%$ IS).

Discussion \& Conclusion: This study is a comprehensive characterisation of BCR-ABL1 kinase domain mutations at our tertiary cancer care center. Semi-nested PCR technique using 4 sets of forward and reverse primers is a sensitive technique to detect a BCRABL1 kinase domain mutation even at low numbers of BCR-ABL1 copies.

\section{Autoimmune Hemolytic Anemia Secondary to NK- Chronic Lymphoproliferative Disorder: A Case Report}

Rama Hariharan $^{* 1}$, Ganesh K V ${ }^{1}$, Jasmita Dass ${ }^{1}$, Mukul Aggarwal ${ }^{2}$, Jayastu Senapati $^{2}$, Rishi Dhawan ${ }^{2}$, Manoranjan Mahapatra ${ }^{3}$

${ }^{1}$ Hematopathology, ${ }^{2}$ Clinical Hematology, ${ }^{3}$ Hematology, AIIMS New Delhi, New Delhi, India

Aims \& Objectives: CLPD-NK constitutes approximately 5\% of LGL disorders and is an indolent hematologic disorder with a favorable prognosis. Although the most common cause of anemia in these patients is PRCA, some cases showed AIHA which is an unusual complication seen with a frequency ranging from 5 to $14 \%$.

Patients/Materials \& Methods: A 50-year-old lady presented with progressively increasing pallor and shortness of breath with abdominal fullness along with loss of weight and appetite. On examination patients had pallor with splenomegaly which was $12 \mathrm{~cm}$ below the left costal margin.

Results: CBC: Hb-5.3gm/d1, TLC-12,210/ul, Platelet count-77,000/ ul. Direct Coombs test with $\mathrm{IgG}$ and $\mathrm{C} 3 \mathrm{~d}$ was negative. Serum bilirubin- $2.35 \mathrm{mg} / \mathrm{dl}(\mathrm{T}) / 1.5 \mathrm{mg} / \mathrm{dl}$ (I), serum LDH-207U/L. Peripheral smear showed RBC anisocytosis with spherocytosis and polychromasia, reticulocyte count-42.3\%, nRBCs-6/100 WBCs, LGL-30\% and an absolute LGL count-3663/ul. Bone marrow aspirate showed clusters of atypical lymphoid cells.Bone marrow biopsy was hypercellular with intra-sinusoidal and trabecular pattern of infiltration by medium to large sized lymphoid cells. Flow cytometry immunophenotyping showed positivity for CD2, CD7, CD16 and negative for CD3, CD5, CD4, CD8, $\alpha \beta, \gamma \delta$, CD56 Patient was managed with immunosuppressants- Oral Prednisolone, Methotrexate and weekly Rituximab.

Discussion \& Conclusion: CLPD-NK is an indolent disease rarely seen in association with autoimmune diseases or other neoplasms, including haemato-lymphoid neoplasms. The most common NK-cell antigenic aberrancies in CLPD-NK include CD56 -/partial, CD7de- creased/dim, CD94bright, CD8uniform +, and evidence of KIR restriction. Immune-mediated phenomena, which include AIHA, have been described as a consequence of the increased levels of circulating immune complexes in LGLL including CLPD-NK. Treatments of anemic complications of LGLL should be considered based on the different mechanisms.

\section{Chronic Lymphocytic Leukaemia in a Case of Chronic Myeloid Leukaemia}

Ekta Rahul ${ }^{* 1}$, Smeeta Gajendra ${ }^{1}$, Ritu Gupta ${ }^{1}$, Ranjit K. Sahoo ${ }^{2}$, Lalit $\mathrm{Kumar}^{2}$

${ }^{1}$ Dept of Lab Oncology, BRA-IRCH, ${ }^{2}$ Dept of Medical Oncology, BRA-IRCH, AIIMS, New Delhi, India

Aims \& Objectives: The occurrence of Chronic Lymphocytic Leukemia (CLL) in a case of Chronic Myeloid Leukemia (CML) is rare as both are associated with separate genomic events. We report a patient who developed CLL 18 months after the diagnosis of a $\mathrm{Ph}$ positive chronic myelogenous leukemia-accelerated phase (CMLAP).

Patients/Materials \& Methods: We report a case of a 59-year-old gentleman who presented with fever, loss of appetite, weight loss, generalized weakness and dragging sensation over the left upper quadrant of abdomen for one year. On complete blood count, he had haemoglobin of $8.9 \mathrm{~g} / \mathrm{dL}$, total leucocyte counts of $427.49 \times 109 / \mathrm{L}$ and platelet count $484 \times 10$ 9/L. Peripheral blood smear $(14 \%$ blasts $\& 5 \%$ basophils) and bone marrow (12\% blasts and $12 \%$ basophils) findings were suggestive of CML-AP. Results: Cytogenetic analysis revealed 46, XY,t $(9 ; 22)(\mathrm{q} 34 ; \mathrm{q} 11)$. On quantitative real time polymerase chain reaction (qRT-PCR) protein p210 with IS of $35.3 \%$ was detected. The patient was started on imatinib followed by dasatinib followed by nilotinib. There was loss of complete haematological response after 18 months of diagnosis. Peripheral blood smear showed absolute lymphocytosis with $78 \%$ lymphoid cells along with mild shift to left, basophilia and thrombocytosis. On flow cytometric immunophenotyping, these lymphoid cells were positive for CD19, CD5, CD23, CD20 and negative for FMC7, CD10, CD3 which confirmed the diagnosis of CLL. Repeat qRT-PCR showed increasing $\mathrm{BCR}-\mathrm{ABL}$ fusion protein. Over all diagnosis of refractory CML (post 3 tyrosine kinase inhibitor) with CLL (Rai stage 0) was made.

Discussion \& Conclusion: Development of CLL in a patient of CML-AP is a rare event. In most cases reported in the literature, CLL preceded the development of CML or both the leukemias developed simultaneously. Very few cases of CLL in a patient of CML have been reported (Table-1). In majority of these cases, the patients were in the chronic phase of CML and CLL developed after 6- 152 months from the onset of CML. The age ranged from 45 to 88 years with mild male predominance. Only one case of CLL developed after CML-AP was reported.

Supporting Document: 
Table 1 (Cases of CLL developing in course of CML)

\begin{tabular}{|l|l|l|l|l|l|}
\hline Cases & $\begin{array}{l}\text { Age(years)/ } \\
\text { Sex }\end{array}$ & $\begin{array}{l}\text { Previous } \\
\text { diagnosis }\end{array}$ & $\begin{array}{l}\text { Treatment for } \\
\text { CML }\end{array}$ & $\begin{array}{l}\text { Interval } \\
\text { (months) }\end{array}$ & $\begin{array}{l}\text { Treatment for } \\
\text { CLL }\end{array}$ \\
\hline $\begin{array}{l}\text { Salim et al } \\
(2002)\end{array}$ & $54 /$ Female & CML-CP & $\begin{array}{l}\text { Hydroxyurea, } \\
\text { Interferon-alpha, } \\
\text { Imatinib }\end{array}$ & 36 & Observation \\
\hline $\begin{array}{l}\text { Chang et al } \\
(2004)\end{array}$ & 57/Male & CML-CP & $\begin{array}{l}\text { Hydroxyurea, } \\
\text { Imatinib }\end{array}$ & 6 & Observation \\
\hline $\begin{array}{l}\text { Gargola } \text { et al } \\
(2005)\end{array}$ & $88 /$ Female & CML-CP & $\begin{array}{l}\text { Chlorambucil, } \\
\text { Hydroxyurea }\end{array}$ & 20 & Not available \\
\hline $\begin{array}{l}\text { Bhagavati et al } \\
(2008)\end{array}$ & $71 /$ Male & CML-CP & Imatinib & 74 & Observation \\
\hline $\begin{array}{l}\text { Kumar et al } \\
(2013)\end{array}$ & $45 / F e m a l e$ & CML-AP & Imatinib & 8 & Chlorambucil \\
\hline $\begin{array}{l}\text { Manuela et al } \\
(2015)\end{array}$ & $54 /-$ & CML-CP & $\begin{array}{l}\text { Hydroxyurea, } \\
\text { Imatinib, }\end{array}$ & 60 & $\begin{array}{l}\text { Rituximab } \\
\text { based } \\
\text { chemotherapy }\end{array}$ \\
\hline $\begin{array}{l}\text { Lewandowski } \\
\text { et al (2016) }\end{array}$ & 68/Male & CML-CP & $\begin{array}{l}\text { Hydroxyurea, } \\
\text { Interferon-alpha, } \\
\text { cytosine } \\
\text { arabinoside, } \\
\text { Imatinib }\end{array}$ & 152 & Prednisone \\
\hline $\begin{array}{l}\text { Wei et al } \\
(2017)\end{array}$ & 53/Female & CML-AP & Imatinib & 25 & Ibrutinib \\
\hline $\begin{array}{l}\text { Salil et al } \\
(2018)\end{array}$ & 57/Male & CML-CP & Imatinib & 48 & Observation \\
\hline $\begin{array}{l}\text { Our case } \\
(2020)\end{array}$ & $59 /$ Male & CML-AP & $\begin{array}{l}\text { Imatinib/Dasatinib/ } \\
\text { Nilotinib }\end{array}$ & 18 & Observation \\
\hline
\end{tabular}

A Study of Incidence of Thrombocytopenia in Malarial Patients at a Tertiary Hospital

Bharatkumar M. Goyani*1

${ }^{1}$ Medicine, Geetanjali Medical College and Hospital, Udaipur, India

Aims \& Objectives: To find out incidence and degree of thrombocytopenia in malarial patients.

Patients/Materials \& Methods: This study was conducted at GEETANJALI HOSPITAL UDAIPUR, over a 6 months period. A total 500 malaria positive cases studied with thick \& thin smears stained with Field's stain and platelet count.
Results: In the study of 500 patients: 350 positive for P. Vivax, 150 positive for P. Falciparum Out of 350 cases of P. Vivax 300 cases had thrombocytopenia with most cases of Grade $1 \& 2$ and of 150 cases with P. Falciparum 125 cases had thrombocytopenia with most cases of Grade $3 \& 4$. So in our study, $85 \%$ malaria positive cases had thrombocytopenia.

Discussion \& Conclusion: Incidence of thrombocytopenia in a patient with acute febrile illness increases the probability of malaria. Findings can use to avoid unnecessary platelet transfusion in malarial patients. 
Lymphopeneia and Neutrophilia as a Marker of Severity in Patients with Covid 19

\author{
Ashish Bansal ${ }^{* 1}$ \\ ${ }^{1}$ Medicine, Geetanjali Hospital, Udaipur, India
}

Aims \& Objectives: Relationship between clinical outcomes and abnormal lymphocytes and neutrophil in covid 19 patients.

Patients/Materials \& Methods: This study was conducted in GMCH udaipur to identify the frequency of netrophilia and lymphopenia.s. Pooled odds ratios of clinical outcomes for each parameter were calculated using Comprehensive Meta-Analysis.

Results: Lymphopenia at admission was found to be significantly associated with increased odd of progression to severe disease (odds ratio $[\mathrm{OR}], 4.20 ; 95 \%$ confidence interval [95CI \%], 3.46- 5.09) and death (OR, 3.71; 95\%CI, 1.63-8.44). Neutrophilia at admission was also found to be significantly associated with increased odd of progression to severe disease (OR, 7.99; 95\%CI, 1.77-36.14) and death (OR, 7.87; 95\%CI, 1.75-35.35). Subgroup analysis revealed that COVID-19 patients with severe lymphopenia $(<0.5 \times 10 \times 9 / \mathrm{L})$ had 12 -fold increased odds of in-hospital mortality.

Discussion \& Conclusion: Admission lymphopenia and neutrophilia are associated with poor outcomes in patients with COVID-19. Regular monitoring and early and even more aggressive intervention shall hence be advisable in patients with low lymphocyte and high neutrophil counts.

\section{IGG Seropositivity for Sars-Cov-2 and Its Predictors Among Healthcare Workers of Eastern India}

Mala Mahto ${ }^{1}$, Bijit Biswas ${ }^{* 2}$, Ayan Banerjee ${ }^{1}$, Sushil Kumar ${ }^{1}$, Neeraj Agarwal ${ }^{2}$, Prabhat K. Singh ${ }^{3}$

${ }^{1}$ Biochemistry, ${ }^{2}$ Community and Family Medicine, ${ }^{3}$ Director, All India Institute of Medical Sciences-Patna, Patna, India

Aims \& Objectives: The study aimed to find out IgG seropositivity for severe acute respiratory syndrome coronavirus-2 (SARS-CoV-2) and its predictors among healthcare workers of a corona virus disease (COVID) dedicated tertiary care health facility of eastern India.

Patients/Materials \& Methods: It was a monocentric, observational study, cross-sectional in design conducted among healthcare workers of All India Institute of Medical Sciences, Patna, Bihar, India during the month of September, 2020. Serum IgG level was estimated using chemiluminescent immunoassay (CLIA) platform named 'ADVIA Centaur COV2G' in which IgG level of 1.00 or higher indicated seropositivity for SARS-CoV-2. Data were analysed using statistical package for social sciences (SPSS) (version 22.0).

Results: Among the 689 study subjects $13.5 \%$ [95\% confidence interval (CI): $11.1-16.2 \%$ )] were found to be IgG seropositive against SARS-CoV-2. The median IgG level in our study subjects was .03 with interquartile range (IQR) of .01-.08. Males were more likely to be IgG seropositive in comparison to females. Those who were nurses and working in intensive care units (ICUs) were least likely while those who were attendants and working in laboratories were most likely to be $\mathrm{IgG}$ seropositive. In univariate logistic regression analysis; gender, occupation, place of posting, use of full personal protective equipment (PPE), prior COVID-19 infection, influenza like illness (ILI), use of steam inhalation, consumption of azithromycin, zinc and vitamin $\mathrm{C}$ were the significant attributes affecting seropositivity for IgG against SARS-CoV-2. In multivariable logistic regression analysis; occupation, place of posting, prior COVID infection and ILI were significant determinants of seropositivity for IgG against SARS-CoV-2. Overall, the independent variables in the multivariable logistic regression model predicted $30.8 \%$ variability of the SARS-CoV-2 IgG seropositivity of the healthcare workers with high predictive accuracy rate $(88.1 \%)$.

Discussion \& Conclusion: SARS-CoV-2 IgG seropositivity among the healthcare workers in the present study was high in comparison to all the prior studies conducted in western countries till date. Occupation, place of posting, prior COVID-19 infection and ILI were found to be significant influencers of IgG seropositivity against SARS-CoV-2 in our study subjects.

\section{S. Ferritin, IL-6 and D-Dimer in Assessment and Prediction of Severity In Covid-19 Patients \\ Shivanee Sharma ${ }^{* 1}$, Vandana Agrawal ${ }^{1}$, K. B Mishra ${ }^{1}$ \\ ${ }^{1}$ Pathology, Laxmi Narayan Medical College and JK hospital, Bhopal, India}

Aims \& Objectives: Since December 2019, an outbreak caused by Severe Acute Respiratory Syndrome-Corona virus 2 (SARS- CO V 2) emerged in China and quickly turned into pandemic. India had 7.42 million and Madhya Pradesh had 157 thousand confirmed cases by 16 October 2020. This outbreak of novel corona virus has put health systems on high alert in India and across the world. Our study aimed to assess inflammatory markers (S. Ferritin and IL-6) and fibrin degradation product (D-Dimer) in the assessment of disease severity in COVID-19 patients and use of these investigations as prognostic markers.

Patients/Materials \& Methods: It is a retrospective single center study carried out at our institute. Data of 150 patients who were confirmed cases of COVID-19 were collected and analyzed. Cases were divided into mild, moderate $\&$ severe groups by using guidelines provided by MOHFW. Laboratory tests included are complete blood count, D-Dimer, S.Ferritin and IL-6 .

Results: 150 patient with laboratory RT-PCR confirmed COVID-19 infection of varying severity were assessed early after their admission. The average age of the patients was $50.02 \pm 16.1$ years. There were $76.7 \%$ aged 60 years or less and $23.3 \%$ aged above 60 years. Males were $105(70 \%)$ and females were $45(30 \%) .150$ subjects were classified as mild [46(30.7\%)], moderate [31(20.7\%)] and severe [73(48.7\%)]. Comparison across three groups mild, moderate and severe showed significant difference in D- Dimer. Inflammatory markers S.Ferritin and IL-6 were also found to be significantly raised across the severity spectrum as well.

Discussion \& Conclusion: We found that inflammatory markers and fibrin degradation product could assess the severity of patients with COVID-19 and further studies with robust methodologies must be performed to establish them as potential biomarkers in this illness.

Keywords: COVID-19, Inflammatory cytokines,D-Dimer,Disease severity. 
Supporting Document:

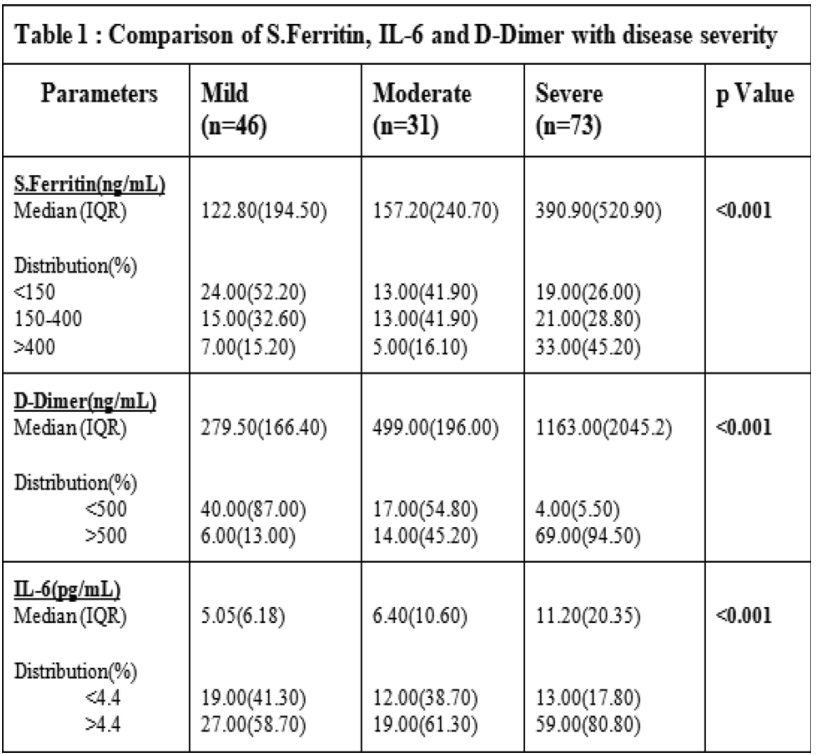

\section{MPV and RDW as Markers of Disease Severity in Psoriasis Vulgaris}

Anshu Palta ${ }^{* 1}$, Dr Navneet Deol ${ }^{1}$, Mala Bhalla ${ }^{2}$, Anita Tahlan ${ }^{1}$, Manveen kaur ${ }^{1}$

${ }^{1}$ Pathology, ${ }^{2}$ Dermatology and general diseases, GMCH32,Chandigarh, Chandigarh, India

Aims \& Objectives: To study the correlation of MPV and RDW with each other, with inflammatory markers and with the disease severity by using PASI scoring system in patients of psoriasis vulgaris.

Patients/Materials \& Methods: 50 cases clinically diagnosed with psoriasis vulgaris and 20 healthy controls were enrolled for the purpose of study. PASI score was calculated in each case and blood samples were taken for CBC with ESR and biochemical analysis.

Results: There was no statistically significant difference between MPV values in patients of psoriasis vulgaris and controls ( $p$ value $=0.319)$. There was no significant correlation of MPV with RDW (r value: - 0.187, $p$ value: 0.194 ) as well as with other inflammatory markers i.e. NLR (r value: $0.061, p$ value: 0.673$)$, ESR ( $\mathrm{r}$ value: $0.005, p$ value: 0.971 ) and CRP ( $\mathrm{r}$ value: $0.137, p$ value: 0.342 ). RDW was significantly higher in patients of psoriasis vulgaris as compared to controls ( $p$ value $=0.017$ ). However, increased RDW did not show any correlation with the severity of disease and with other inflammatory markers i.e. NLR $(p$ value $=0.753, \mathrm{r}$ value $=$ $0.046), \quad$ ESR $(p$ value $=0.215, \quad \mathrm{r} \quad$ value $=0.178)$ and $\mathrm{CRP}$ $(p$ value $=0.741, \mathrm{r}$ value $=-0.048)$.

Discussion \& Conclusion: In the present study, no significant difference was found between MPV value in patients of psoriasis vulgaris and the control group ( $p$ value $=0.319$ ). The study demonstrates that RDW showed significant increase in patients of psoriasis vulgaris as compared to controls ( $p$ value: 0.017 ). However, RDW failed to show any correlation with the severity of disease in patients of psoriasis vulgaris as well as with established inflammatory markers i.e. ESR and CRP. In future, a large database studies may be required to establish the role of MPV and RDW as markers of disease severity in patients of psoriasis vulgaris.

\section{NLR as a Prognostic Marker in COVID19}

Dharmendra S. Bhadouria*1, Vaibhav Koria ${ }^{2}$, Sanjeev Narang ${ }^{1}, \mathrm{~S}$ K. Nema ${ }^{1}$

${ }^{1}$ Pathology, Index Medical College, ${ }^{2}$ Pathology, Index Medical Collge, Indore, India

Aims \& Objectives: To analyze NLR as a surrogate prognostic marker for COVID 19.

Patients/Materials \& Methods: This is a prospective study done in Department of Pathology, Index Medical College, Indore from 07 may 2020 to 05 August 2020.In the present study, a total number of 517 patients are included. Out of which 299 are male and 218 are female. NLR is computed by Absolute Neutrophil count divided by absolute lymphocyte count.

Results: Majority of the patients are Asymptomatic (89.7\%), which is followed by Low risk (5.4\%),High risk (3.5) and Moderate risk (1.4\%). We have observed that 5 patients died due the covid-19 and these patients falls into severe or high risk group i.e. The Neutrophil lymphocyte ratio(NLR) of all these 5 patients was more than 7 .The minimum neutrophil lymphocyte ratio was 7.71 whereas the maximum neutrophil lymphocyte ration was 47.50 . In those patients who died because of COVID 19.,minimum absolute lymphocyte count is 278 whereas maximum absolute lymphocyte count is 2040 having standard deviation of 643.245 In our study we had observed NLR $>7$ $\&$ Absolute lymphocyte count (ALC) $<600$ indicates severe COVID19.

Discussion \& Conclusion: Complete Blood Count (CBC) is easily assessible hematological investigation at primary Health Care level in our country and NLR can be assesed for severity of COVID 19 and it will be surrogate prognostic marker and will be helpful in timely referral of critical patients to Dedicated Covid Hospitals.

\section{Role of Leukocyte Count, NLR And PLR as a Prognostic Marker in Pesticide Poisoning}

Shubham Chourishi ${ }^{* 1}$, Vijayalaxmi Patil ${ }^{1}$

${ }^{1}$ Pathology, Shri B.M. Patil Medical College, Hospital and Research Centre, Vijayapur, India

Aims \& Objectives: To assess the severity of Pesticide Poisoning by assessing the leukocyte count, neutrophil count, neutrophil- lymphocyte ratio and platelet-lymphocyte ratio and its comparison with levels of Plasma Cholinesterase as an aid to clinical diagnosis, for early treatment of the patients.

Patients/Materials \& Methods: A cross sectional study was done on 148 confirmed cases of pesticide poisoning admitted to the casualty in the period of 1.5 years (1st November 2018 - 30th May 2020). Blood samples were collected in EDTA and plain tubes within $24 \mathrm{~h}$ of admission and the values of total leukocyte Count (TLC), neutrophil count, neutrophil-lymphocyte ratio, platelet- lymphocyte ratio and plasma cholinesterase (PChE) were measured and compared. The severity of poisoning was assessed according to Peradeniya Organophosphorus (POP) scale.

Results: Majority of the patients were between 21 and 30 years. 67 $(45.3 \%)$ were males and $81(54.7 \%)$ were females. Approximately $89.86 \%$ (133 cases) of the poisoning cases were due to Organophosphorus compounds and remaining $10.14 \%$ (15 cases) were due to carbamate poisoning. The severely poisoned patients according to POP scale had increased levels of leukocyte count, neutrophil count, neutrophil-lymphocyte ratio and platelet lymphocyte ratio; and decreased plasma cholinesterase level as compared to the patients with mild and moderate poisoning.

Discussion \& Conclusion: After a positive history of pesticide consumption, the recognition of potent prognostic biomarkers is needed 
during the clinical management of acutely pesticide poisoned-patients to predict the outcome. Thus, total Leukocyte count, neutrophil count, neutrophil-lymphocyte ratio and platelet lymphocyte ratio are simple and easy to use parameters for estimating the severity of pesticide poisoning and assessing its prognosis.

\section{An In-House Lateral Flow Immunoassay: Point of Care Test for Covid-19 IGG/IGM Antibodies Detection}

\author{
Rucha Patil $^{* 1}$, Puja Soni ${ }^{1}$, Shrimati Shetty ${ }^{1}$ \\ ${ }^{1}$ Hemostasis and Thrombosis, ICMR-National Institute of \\ Immunohematology, Mumbai, India
}

Aims \& Objectives: The "gold-standard" test for the diagnosis of COVID-19 is real time PCR, not all cases with mild symptoms or asymptomatic cases are subjected to RT-PCR analysis. It is also not practically feasible in a country like India, considering the cost, number of subjects to be screened and the required technical expertise. The sero-conversion in case of COVID-19 generally occurs from 4 th or 5th day after the onset of symptoms. To develop a cost-effective, rapid kit for detection of IgG and IgM antibodies to COVID-19 using gold nanoparticle labeled antigen/antibody.

Patients/Materials \& Methods: Standardization of the LFIA antibody test involved many different experiments. Different size of gold nanoparticles were prepared and used. These GNPs were then adjusted to different $\mathrm{pH}$ for optimal binding: $\mathrm{pH}$ 6.0, 6.5, 7.0, 7.5, 8.0, 8.5, 9.0, 9.5, 10.0 using different buffers(PBS, Borate, carbonate, etc.). The pore size of membranes play an important role and thus different pore size membranes were use $(5,8,10,12,15 \mu \mathrm{m})$. Reaction temperature of $37 \mathrm{C}$ and RT were checked for maximum sensitivity. Different concentrations of the proteins ranging from $0.4 \mathrm{mg} / \mathrm{ml}$ to $100 \mathrm{mg} / \mathrm{ml}$ were tried. For the same, serum samples of 15 patients who were COVID-19 RT PCR positive having symptoms of fever, sore throat, etc. were collected between 7 to 14 days post symptoms and of 40 patients at least 28 days post symptoms. All the results were cross checked and compared with ICMR validated Rapid tests and ELISAs for IgG and IgM.

Results: Internal Validation: The results are in good accordance to ICMR validated Rapid tests and ELISAs where in ssensitivity of $89.6 \%$ and $91 \%$ were obtained for $\operatorname{IgG}$ and $\operatorname{IgM}$ respectively.

External Validation.

1: ICMR-National Institute of Virology

200 kits were sent for validation, Sensitivity, Specificity and cross reactivity with real-time RT-PCR positive for influenza A (H1N1) pdm09, influenza A (H3N2), human coronavirus OC43, rhinovirus, respiratory syncytial virus, influenza $B$, parainfluenza type 4 , hepatitis $B$ virus, hepatitis $C$ virus, etc. was done by ICMR -NIV. Sensitivity was $87.5 \%$ and $90 \%$ for $\operatorname{IgG}$ and $\operatorname{IgM}$, respectively and specificity was 97.12 for both.

2: Rajiv Gandhi Centre for Biotechnology (RGCB)-DBT 300 kits were sent for validation. Sensitivity was $96 \%$ and $90.67 \%$ and specificity was 97.12 for $\mathrm{IgG}$ and $\mathrm{IgM}$, respectively.

The working cost is around 120Rs. The kit has now gone to the Technology transfer and commercialization department at ICMR, New Delhi.

Discussion \& Conclusion: The antibody diagnosis approach is important in COVID-19 epidemic to diagnose the asymptomatic cases, to assess the magnitude of infection as well as to assess the number of people who are protected from infection, though it is still not clear about the protective effect of these antibodies for repeated infection. The in-house kit was found to be highly sensitive and specific with the working cost being 100-120 Rs.
Association of Neutrophil to Lymphocyte Ratio and DDimer with Severity of Covid- 19

\author{
Anjali Pandey ${ }^{* 1}$, Vandana Agarawal $^{2}$, Shailendra Meena ${ }^{1}$ \\ ${ }^{1}$ Pathology, L.N. Medical college, ${ }^{2}$ Pathology, L.N. Medical \\ College, Bhopal, Bhopal, India
}

Aims \& Objectives: 1. To assess the utility of Neutrophil Lymphocyte Ratio and D-dimer in identifying disease severity in Covid-19 patients. 2. To compare and correlate the NLR ratio and D-dimer value in patients with mild/moderate and severe Covid -19 infections. Patients/Materials \& Methods: A total of 100 patients admitted between 1st August 2020 and 30th September 2020 who were above 18 yrs and tested positive for Covid-19 with RT- PCR were enrolled in the study.The patients were classified into two groups : mild/moderate group and the severe group according to the guidelines of MOHFW. Medical records of the cases are analyzed retrospectively for demographic, clinical, laboratory data and radiological findings and were compared between the mild/moderate and severe COVID-19 groups. The receiver operating curve (ROC) and area under the curve (AUC) were calculated to compare the diagnostic performance of each parameter. A value of $p<0.05$ was considered statistically significant.

Results: 100 patients were enrolled, with a mean age of $51.77 \pm 16.4$ years, and Male :Female ratio was 31:19. The patients were classified into two groups : the mild/moderate group and the severe groups. Compared to the mild/moderate COVID-19 group, the absolute neutrophil count, NLR and D-dimer levels of severe group were significantly higher $(p<0.05)$. A ROC curve analysis was used to determine the efficacy of various parameters in predicting severe prognosis (Figure 1 and Table 1). D-dimer and NLR had the highest AUC in the ROC analysis. Of various indices, the highest AUC is D-dimer (0.798) followed by the NLR ratio (0.765).

Discussion \& Conclusion: The NLR and D-dimer values were found to be significantly higher in patients of COVID-19 with severe disease, reflecting an enhanced inflammatory process and poor prognosis. However a multicentric, prospective research is needed to further clarify the value of D-dimer and NLR in COVID-19.

\section{An Analysis of the Association of Iron Deficiency in Dengue Patients and Its Effect on The Severity}

Chitranga Kariyawasan ${ }^{* 1}$, Dinesh P. Ranasinghe ${ }^{1}$, Dulshani Gunaratne $^{1}$, Buddhika Mahesh ${ }^{2}$, Charith Ranatunga ${ }^{1}$

${ }^{1}$ Haematology, Sri Jayewardenepura General Hospital, ${ }^{2}$ Community Medicine, Ministry of Health, Colombo, Sri Lanka

Aims \& Objectives: To test the hypothesis of increased prevalence of dengue in patients with iron deficiencyl. If proven, iron treatment could reduce the incidence, morbidity and economic burden of dengue fever. General Objective - To analyze the association between iron deficiency and dengue infection among adult patients. Specific objectives -To determine any association between iron deficiency and severity of dengue fever.

Patients/Materials \& Methods: A retrospective analytical study conducted from January 2020 to June 2020 at the Sri Jayewardenepura General Hospital(SJGH). Sample size (273) was calculated using the formula by Charan \& Biswas (2013)8. Information from records of adult patients confirmed with dengue fever, was entered into a data sheet in SPSS version 23. Analysis done using the Pearson correlation and Chi square method.

Results: Of the total population (273), patients with and without iron deficiency were 81 and 192 respectively. Patients without iron deficiency were significantly higher.. Severe and non-severe forms of the 
infection (273) had normal iron status $(\mathrm{CHr}>26 \mathrm{pg})$ which was statistically insignificant ( $p$ value 0.651 )

Discussion \& Conclusion: The hypothesis "status of iron deficiency in the human population might contribute to the vectorial permissiveness to dengue virus, facilitating its spread by mosquitoes resulting in higher prevalence in patients with iron deficiency" 1 , was disapproved. Iron deficiency status had no association with the severity of the dengue infection.

Keywords: Dengue Infection, Iron deficiency, Reticulocyte Haemoglobin (RHe).

\section{Simple Smear-Tell Tales, Haematological Changes of Covid 19 in a Blood Smear}

\section{Nisha R. Maheshwari ${ }^{* 1}$ \\ ${ }^{1}$ Pathology, NMSS, Ranchi, India}

Aims \& Objectives: To establish correlation between subtle changes in peripheral blood smear (PBS) as an aid to complement diagnosis of SARS-COVID 19.

Patients/Materials \& Methods: Approximately over 300 PBS were studied from COVID suspected cases in a private hospital. Inclusion criteria was to take all samples from the fever clinic presenting with symptoms of COVID, also samples from known COVID contacts were considered. Detailed microscopic analysis of PBS of the selected slides during the hospital course was done.

Results: Significant numbers of slides showed characteristic changes in all cell lines, more so in WBCs. Before even when the well documented lymphopenia, high N/L ratio, the leucocytes exhibit peculiar morphological alterations which were never observed in any other disease. The neutrophils show coarse granulations, nuclear detachment, C-shaped or fetus like nuclei, abberant nuclear projection, elongated nucleoplasm, irregular cytoplasmic projections, cytoplasmic vacuolation, cytoplasmic blebs. The lymphocytes showed condensed chromatin, abundant light pale blue cytoplasm scalloping other adjacent cells, cytoplasmic pod formation. Monocytes showed prominent cytoplasmic vacuoles and nuclear dyspoiesis. Additionally these slides were kept separate to keep a tab on the RT-PCR report which followed after 48-72 h. Also other blood parameters like S.CRP, S.LDH, S.Ferritin, D-Dimer levels of these patients were noted. It was assuring to see how well the morphological changes in PBS correlated with the blood parameters, the HRCT and later confirmed by RT PCR.

Discussion \& Conclusion: A detailed and vigilant microscopic observation of a simple slide can provide a sea of information of the ongoing pathology. In a pandemic like this wherein in remote underresourced places where RT-PCR is out sourced or takes a longer turnaround-time, initiation of treatment merely based on symptoms can be challenging for the physicians. Thus the peculiar PBS findings can be exploited to help in diagnosis and to initiate earlier hospitalization and treatment for better outcome.

Disclaimer: No conflict of interest. This is a pilot study based on subjective observations of morphological features in PBF. More information and studies on a larger group are needed to establish the correlation.

\section{The Combined Role of NLR And PLR in Predicting the Severity of Covid 19 \\ Gopika $\mathbf{A}^{* 1}$, Veena $\mathrm{S}^{1}$, Chethan Sagar ${ }^{1}$, Ramesh Babu ${ }^{1}$ \\ ${ }^{1}$ Pathology, SIMS, Shivamogga, India}

Aims \& Objectives: To monitor the Neutrophil lymphocyte ratio (NLR) and Platelet lymphocyte ratio (PLR) in admitted COVID positive patients and their use as prognostic factors for predicting COVID 19 severity.

Patients/Materials \& Methods: A retrospective single center study on 500 COVID positive patients who had been hospitalized in between 12th August 2020 and 5th September 2020 was conducted. The demographic, clinical details and $\mathrm{CBC}$ which was determined using SYSMEX CELL COUNTER at admission were collected. NLR and PLR were calculated.

N/L R = ABSOLUTE NEUTROPHIL COUNT/ABSOULTE LYMPHOCYTE COUNT P/L R = PLATELET COUNT/ABSOLUTE LYMPHOCYTE COUNT

Data was entered in Microsoft Excel and analyzed.

Results: Of the 500 patients, 269 patients with no oxygen support, 164 patients under oxygen support and 67 patients were in ICU. NLR cut off value obtained $=3.84$.

PLR Cut off value obtained $=140$ Result table attached.

NLR and PLR were shown positively proportional to disease severity.

Discussion \& Conclusion: NLR and PLR ratios are easy to calculate which is novel, rapid \& used as a prognostic factor in the early screening of critical illness in confirmed COVID 19 patients and may help the physician to stratify patients into prognostic categories at its early stage. These ratios can be easily implemented even in primary health centers to monitor the patients and predict the severity. Supporting Document:

\begin{tabular}{|l|l|l|}
\hline $\begin{array}{l}\text { Number of } \\
\text { patients } \\
\text { (500) }\end{array}$ & High NLR (>3.84) & High PLR (>140) \\
\hline & Number, (\%) & Number, (\%) \\
\hline $\begin{array}{l}\text { Non o2 support- } \\
269\end{array}$ & $\begin{array}{l}63, \\
(23.4)\end{array}$ & $\begin{array}{l}92, \\
(34.2)\end{array}$ \\
\hline O2 support-164 & 131, \\
$(79.4)$ & $\begin{array}{l}97, \\
(59.1)\end{array}$ \\
\hline ICU-67 & & \\
\hline & $(67.1)$ & $\begin{array}{l}(61.1) \\
\end{array}$ \\
\hline
\end{tabular}

\section{Role of Platelet Indices in Severity of Dengue}

Anjali Sharma ${ }^{* 1}$, Ankit Singla ${ }^{1}$, Kapil Bhalla ${ }^{1}$, Puneet Singla ${ }^{2}$, Sailesh Khyalia ${ }^{1}$

${ }^{1}$ Department of Paediatrics, ${ }^{2}$ Department of Pulmonary and Critical Care Medicine, Pt. B.D. Sharma Post Graduate Institute of Medical Sciences, Rohtak, India

Aims \& Objectives: To study the role of platelet indices like platelet count, mean platelet volume, platelet distribution width, platecrit in patients with dengue fever and correlate with its severity.

Patients/Materials \& Methods: An observational study was conducted prospectively over a period of one month at Pt. B.D. Sharma PGIMS Rohtak. A total of 74 cases ranging from 2 to 14 years of age who were reported positive for NS1Ag were enrolled and classified on the basis of severity of dengue as per WHO Classification 2009. Platelet parameters were measured by CelltacES automated 
haematology analyser on venous sample collected in EDTA vacutainer from the cases and then analysed.

Results: Platelet indices were significantly altered in patients with severe dengue as compared to dengue fever without warning signs. Mean Platelet Volume (MPV) was found to be significantly higher in patients with severe dengue among the three groups. Similarly platelet index $(\mathrm{MPV} \times$ Platelet Distribution Width $(\mathrm{PDW}) / \mathrm{Platelet}$ Count $(\mathrm{PLC}) \times$ Platecrit), MPV/Platecrit, MPV/Platelet count, PDW/Platecrit ratio showed statistically significant difference among three groups.

Discussion \& Conclusion: Dengue fever is a benign syndrome caused by several arboviruses. Dengue is becoming endemic in India with reported case fatality rate of $3-5 \%$. Thrombocytopenia is the most common laboratory finding. Now recently platelet indices are gaining importance as one of the marker in evaluation of severity of illness in many critical illnesses in children like dengue.

Conclusion: Platelet indices are important to predict the severity of dengue fever.

\section{Utility of Clot Waveform Analysis (CWA) in Sepsis}

Sivaranjani Kalaichelvan ${ }^{* 1}$, Binoj $\mathrm{c}^{1}$, Febe Suman ${ }^{1}$, Suresh Varadarajan $^{2}$, Arthi Mohan ${ }^{1}$

${ }^{1}$ Pathology, ${ }^{2}$ Community Medicine, SRMC, chennai, India

Aims \& Objectives: AIM: To find out the utility of APTT clot wave as an early predictor of sepsis. OBJECTIVES: (i) To compare the various components of clot wave in sepsis and suspected sepsis (ii) To determine the parameters most useful to predict sepsis.

Patients/Materials \& Methods: (i) The patients whose blood samples were sent for culture as well as APTT were recruited for this study. (ii) The APTT was processed in fully automated coagulation analyser (CS2400, Sysmex Kobe, Japan). (iii) The components of clot wave namely $\min 1, \min 2, \max 2, \min 2 / \min 1$ and $\max 2 / \min 1$ are extracted from dat file. (iv) The culture reports were retrieved from laboratory information system. (v) Comparison of the parameters between culture positive (sepsis) and culture negative (SIRS - Systemic inflammatory response syndrome) groups were done. (vi) Statistical analysis was done using $\mathrm{R}$ base version 3.4.2 for Mac ( R Core Team2017). Wilcoxon rank sum test was applied. A $p$ value of $<0.5$ is considered to be significant. (vii) Institutional ethics committee approval was obtained prior.

Results: Refer Table.

Discussion \& Conclusion: Clot time is prolonged in sepsis. Min 1 and max 2 can differentiate sepsis from SIRS. The maximum velocity of clot formation is increased and maximum deceleration is decreased in sepsis. Min 1 can be a discriminating factor to predict sepsis. Turn around time of APTT is very short and reading the clot wave involves no extra economic burden.

\section{Platelet Leucocyte Aggregates and Protein Kinase C Delta Levels in Estimation of Sepsis Development in Obstetric and Gynaecologic Patients with Suspected Infection}

Saurabh J. Goswami ${ }^{* 1}$, Priyanka Gogoi ${ }^{1}$, Preeti Diwaker ${ }^{1}$, Satendra Sharma $^{1}$, Mrinalini Kotru ${ }^{1}$, Bindiya Gupta ${ }^{2}$

${ }^{1}$ Pathology, ${ }^{2}$ Obstetrics and Gynaecology, UCMS and GTB Hospital, Delhi, India

Aims \& Objectives: Aim: To evaluate the role of platelet leucocyte aggregates (PLA) and Protein kinase $\mathrm{C} \delta(\mathrm{PKC} \delta$ ) levels in estimation of sepsis development in obstetric and gynaecologic patients with suspected infection. Primary objective: To measure and compare the values of PLA and PKC $\delta$ in obstetric and gynaecologic patients with suspected infection and in those who develop sepsis.

Patients/Materials \& Methods: 100 obstetric and gynaecological patients who fulfilled criteria of suspected infection laid down by Third International Consensus Definitions Task Force were enrolled for the study. Each patient was assigned in one of the three groups: 1) Infection group 2) Sepsis group; based on Sequential Organ Failure Assessment (SOFA) Score, calculated at follow up after $48 \mathrm{~h}$. The sepsis patients were further followed up and those patients needing vasopressors to maintain a MAP of $65 \mathrm{~mm}$ of $\mathrm{Hg}$ and lactate more than $2 \mathrm{mmol} / \mathrm{L}$ despite adequate fluid resuscitation were classified into the septic shock group. Platelet Monocyte Aggregates (PMA), Platelet Neutrophil Aggregates (PNA) by flowcytometry, Protein Kinase $\mathrm{C} \delta$ (PKC $\delta$ ) by ELISA and $\mathrm{CBC}$ with platelet parameters were measured in all patients.

Results: PMA and PNA levels were significantly higher $(p<0.05)$ in infected patients than in sepsis patients. $\mathrm{PKC} \delta$ was not significantly different in sepsis patients compared to infected patients. None of the PMA, PNA and PKC $\delta$ were significantly different in sepsis patients compared to septic shock patients. There was no statistically significant correlation between PMA, PNA percentage and $\mathrm{PKC} \delta$ concentration.

Discussion \& Conclusion: Platelets are activated early in the course of an infection and subsequent sepsis development. PMA and PNA levels decrease in those infected patients who have high possibility of progression to sepsis. The cause of decreased levels of platelet leucocyte aggregates in those infected patients who are at high risk of progression to sepsis, may be because of sequestration of these aggregates in small vessels, which lead to microvascular occlusion and tissue ischaemia.

\section{Dynamic D Dimer as a Prognostic Indicator in Covid 19}

Immanuel Ratan ${ }^{* 1}$, Jayalakshmi Balasubramanian ${ }^{1}$, Febe R. Suman $^{1}$, Sowmya Gopalan ${ }^{2}$, Suresh Varadharajan ${ }^{3}$, Raj Kumar ${ }^{2}$, Sri Gayathri ${ }^{1}$, Siva Kumar ${ }^{1}$

${ }^{1}$ Pathology, ${ }^{2}$ General Medicine, ${ }^{3}$ Community medicine, SRMC, Chennai, India

Aims \& Objectives: AIM: To assess the utility of dynamic D-dimer as a prognostic marker in hospitalized patients with Covid-19. OBJECTIVE: (1) To correlate the initial D-dimer value with hospital stay and outcome of the patient. (2) To correlate the peak D-dimer value with hospital stay and outcome of the patient. (3) To correlate the initial D-dimer value with CT-chest score of the patient.

Patients/Materials \& Methods: MATERIAL \& METHODS: (1) The serial D-dimer values of patients admitted with RT-PCR positive for Covid-19 were retrieved. (2) The age, sex and number of days of hospital stay and the outcome of the patient were observed from hospital information system. (3) The age and sex of the patient were collected from hospital database. (4) Spearman's correlation and Mann- Whitney U test were done with R base version 3.4.2 for MAC ( $\mathrm{R}$ Core $\mathrm{T} 2017$ ) a $p$ value of $<0.5$ is considered significant .

Results: (1) Total no of patients :121. (2) The age of the patients ranged from 7 to 80 Years. (3) Mean \pm SD age is: $56.5 \pm 17.7$ years. (4) Sex: Male 89(73.6\%) Female 32 (26.4). (5) Male : female ratio 2.8:1. (6) The patients stayed in the hospital for a minimum of 1 day to 1 month. (7) The outcome of patients were good. (8) There was no correlation between the initial D-dimer value and duration of hospital stay inspite of elevated initial D dimer was noticed in patients who stayed long (Spearman's rho $=0.13$ ). (9) Also the peak D-dimer value was also not significant with duration of hospital stay. (10) (Spearman's rho $=0.28)$. (11) T D dimer value couldn't be correlated 
with adverse outcome since the numbers were less. (12) There was no correlation between initial D-dimer value and CT chest score (CORADS Score). (13) Spearman's rho $=0.21$.

Discussion \& Conclusion: Males are more commonly affected than females. Though statistically insignificant, the elevated initial D dimer value is associated with prolonged hospital stay in patients with Covid 19 infection.

\section{SARS-COV-2 Associated with CMV Reactivation- Proof of Immune Dysregulation;"Amalgamation of Past and Present"}

\section{Aditi Kudoo*1, Sabina Langer $^{1}$, Jyoti Kotwal ${ }^{1}$ \\ ${ }^{1}$ Department of Hematology, Sir Ganga Ram Hospital, New Delhi, India}

Aims \& Objectives: To study the immune profile of SARS-CoV-2 patients with recent \& active CMV infection.

Patients/Materials \& Methods: The immune profile of three SARSCoV-2 patients with persistent lymphopenia and increased CMV copy numbers were analysed using single platform flowcytometry, The patients were also evaluated for acute phase reactants such as IL-6, CRP, LDH and Ferritin levels. Other tests like PT, APTT, D-Dimer \& Fibrinogen for coagulopathies were also correlated. The patients were exhaustively evaluated for underling comorbities.

Results: The lymphocyte subset counts in SARS-CoV-2 patients showed markedly reduced CD4 and CD8 counts with mean CD4 counts 260 cells/microL and CD8 counts of 126 cells/microL. The patients were CMV positive with copy numbers ranging from 149 to $217 \mathrm{AU} / \mathrm{mL}$. The IL-6, CRP, Ferritin values were elevated in all with normal LDH and Procalcitonin values. D-dimer levels were elevated with normal PT, APTT\& Fibrinogen levels. The meanof IL-6,CRP and ferritin were : $286.86 \mathrm{pg} / \mathrm{mL}, 112 \mathrm{mg} / \mathrm{L}, 197.35 \mathrm{ng} / \mathrm{mL}$ respectively. All our patients had comorbidities like diabetes mellitus, hypertension and cardiovascular disease. These patients were all treated with injection Gancyclovir and patient showed steady improvement clinically and TLC counts reached the normal range within few days of initiating treatment with progressive reduction in CMV copies.

Discussion \& Conclusion: The common hematological feature of SARS-CoV-2 is leucocytosis with absolute lymphopenia \& high neutrophil to lymphocyte ratio. However, studies have shown that persistent leucopenia is associated with secondary and opportunistic infections. The novel corona virus affect the $\mathrm{T}$ cell subset especially $\mathrm{CD} 8+\mathrm{T}$ lymphocytes causing lymphopenia by either excessive recruitment to peripheral pathways, hyper activation by inflammatory cytokines or increased pro-apoptotic signaling by Caspase 3 and CD59. Immune dysregulation in SARS-CoV-2 patients may lead to reactivation of CMV. It has also been speculated that rampant use of anti IL-1 and anti-IL6 and immunomodifying drugs such as Favipravir might be playing an important role in reactivation of CMV virus

Conclusion: A CMV screening in all the high risk patients, persistent or increasing leucopenia and in ICU setting might help in reducing the percentage and degree of mortality and complications the patient might suffer from. A timely diagnosis and treatment with an eyesight for detection of early complication may help in this battle with SARSCoV-2.

Keywords: SARS-CoV-2, CMV reactivation, Lymphocytic subsets, immune dysregulation.
Cytomegalovirus Pneumonia in Immunocompromised Children (Non-bone Marrow Transplant): One Disease, Varied Presentations

Minakshi Bansal ${ }^{* 1}$, Sohini Chakraborty ${ }^{1}$, Mansi Sachdev ${ }^{1}$, Rahul Bhargava $^{1}$, Vikas Dua ${ }^{1}$

${ }^{1}$ Pediatric Hematology Oncology and Bone Marrow Transplant Unit, FMRI, Gurugram, India

Aims \& Objectives: Human cytomegalovirus (CMV) is a highly host-specific virus of the Herpesviridae family. It is probably one of the most common infections known to humans and is characterized by a self-limiting infection in healthy individuals. Symptomatic CMV disease can affect almost every organ of the body but CMV pneumonia is associated with a high mortality rate, especially when the timely diagnosis cannot be made and the initiation of treatment is delayed. CMV reactivation usually occurs in post stem cell transplant patients but rarely can be seen in pediatric hematological and oncological patients as well. We hereby, discuss four cases of CMV pneumonia with varied presentations who were successfully treated at our centre.

Patients/Materials \& Methods: We retrospectively analysed the records of all non-bone marrow transplant (BMT) patients admitted with pneumonia in the department of Pediatric Hematology/Oncology and BMT at our centre from April 2017 to March 2020. Flexible bronchoscopy was done and bronchoalveolar lavage (BAL) was sent for bacterial, viral and fungal cultures in majority of the cases.

Results: Of 52 non BMT patients admitted with pneumonia in our unit during this study period, 4 were found to have CMV as an underlying etiology (confirmed with qualitative CMV polymerase chain reaction test performed in BAL specimen). These patients were treated successfully with intravenous Ganciclovir and weekly doses of intravenous immunoglobulin (IVIG) in induction phase. Cidofovir was used in maintenance phase in 3 out of 4 patients while valganciclovir was used in 1 patient. Details are shown in table 1.

Discussion \& Conclusion: CMV pneumonia is difficult to diagnose as well as to treat specifically in immunocompromised patients. Delayed diagnosis and treatment can lead to poor outcomes. Therefore, a high index of suspicion along with specific diagnostic tests are required for timely diagnosis and management.

\section{Supporting Document:}

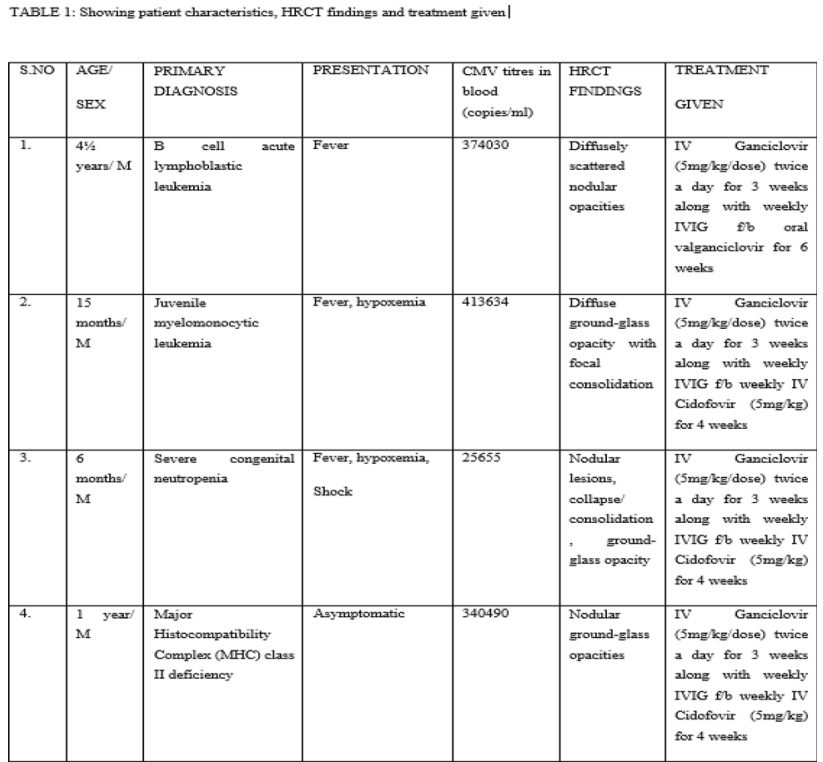


Diagnostic Utility of Bone Marrow Examination in the Evaluation of Pyrexia of Unknown Origin: A Retrospective Analysis from A Tertiary Care Centre

\author{
Akanksha Bhatia ${ }^{* 1}$, Nidhi Priya ${ }^{1}$, Sadhna Marwah ${ }^{1}$, Vijay Kumar ${ }^{1}$ \\ ${ }^{1}$ Pathology, ABVIMS, Dr. Ram Manohar Lohia Hospital, New \\ Delhi, India
}

Aims \& Objectives: Pyrexia of unknown origin (PUO) may pose a diagnostic dilemma in a clinical setup because of its various etiologies. Although invasive, bone marrow aspiration and biopsy can be a useful and affordable modality in such cases. Hence, the aim of this study was to evaluate the diagnostic utility of bone marrow examination in evaluation of PUO.

Patients/Materials \& Methods: This study was a retrospective analysis where bone marrow cases records presenting with PUO were reviewed and retrieved from 2014 to 2018. Detailed history and clinico-hematological parameters at presentation were recorded and data was analysed using descriptive statistics.

Results: A total of 194 cases were included where bone marrow aspiration and/or biopsy was done; of which a definitive diagnosis could be ascertained in 45 cases (23\%). This diagnostic yield increased to $31 \%$ in cases where both aspiration and biopsy was done. Among these, infective causes constituted 67\% (30 cases); malignancies accounted for 27\% (12 cases) and hypoplastic marrow accounted for the remaining 3 cases. Visceral leishmaniasis was the commonest infection seen in 13 cases.

Discussion \& Conclusion: Bone marrow examination forms an important part in the diagnostic workup of cases of PUO and can provide important clues to diagnosis especially if both aspiration and biopsy are performed.

\section{T-Regulatory Cells in Paediatric Tuberculosis}

Ranjith Kumar ${ }^{* 1}$, Richa Gupta ${ }^{2}$, Pooja Dewan ${ }^{3}$, Bineeta Kashyap ${ }^{4}$, Mrinalini Kotru ${ }^{2}$

${ }^{1}$ Junior Resident, ${ }^{2}$ Pathology, ${ }^{3}$ Paediatrics, ${ }^{4}$ Microbiology, University College of Medical Sciences, Delhi, India

Aims \& Objectives: AIM: To evaluate T-regulatory cells(Tregs) in paediatric tuberculosis(TB) patients using flow-cytometry. OBJECTIVES: Primary objective: To estimate the number of Tregs in paediatric TB. Secondary objectives: (1)To compare the number of Tregs in paediatric TB patients with age matched healthy controls. (2)To compare Tregs between severe TB and non severe TB.

Patients/Materials \& Methods: Study enrolled 40 paediatric patients(less than 12 years) newly diagnosed as TB based on clinical, radiological and/or microbiological criteria along with 20 age matched healthy controls(without any history of TB contact in family). TB patients were also classified as severe TB and non severe TB. They were subjected to extensive haematological work-up and flowcytometry based work up for Tregs. Data was analyzed and statistically evaluated using SPSS-PC-19 version.

Results: Tregs were quantified by using two definitions as CD4CD25 high and CD4FOXP3 cells on CD4-T lymphocyte gating. CD4CD25 high Tregs $(\%)$ ranged from 0.55 to 12.8 with a Mean \pm SD of $3.61 \pm 2.98$ in cases and 0.3-6.5 with a Mean \pm SD of $1.29 \pm 1.4$ in controls. CD4FoxP3 Tregs ranged from 0.02 to 13.44 with a Mean \pm SD of $3.56 \pm 2.76$ in cases and $0.33-2.59$ with a Mean \pm $\mathrm{SD}$ of $1.57 \pm 0.58$ in controls. There was a significant difference between cases and control group $(p<0.05)$. No statistically significant difference was observed in Tregs between severe TB and non severe TB.
Discussion \& Conclusion: During TB, T-cell responses helps in containing Mycobacterium tuberculosis infection invivo but it also causes collateral damage to host tissues. In order to control this damage, immune system sets various mechanisms to counteract the action of $\mathrm{T}$ cells, the most important of which are $\mathrm{T}$ regulatory cells. Therefore, our data of Tregs between TB patients and healthy controls in paediatric population suggests that during active $\mathrm{TB}$ and before anti-TB therapy, Tregs were increased in the peripheral blood. Supporting Document:

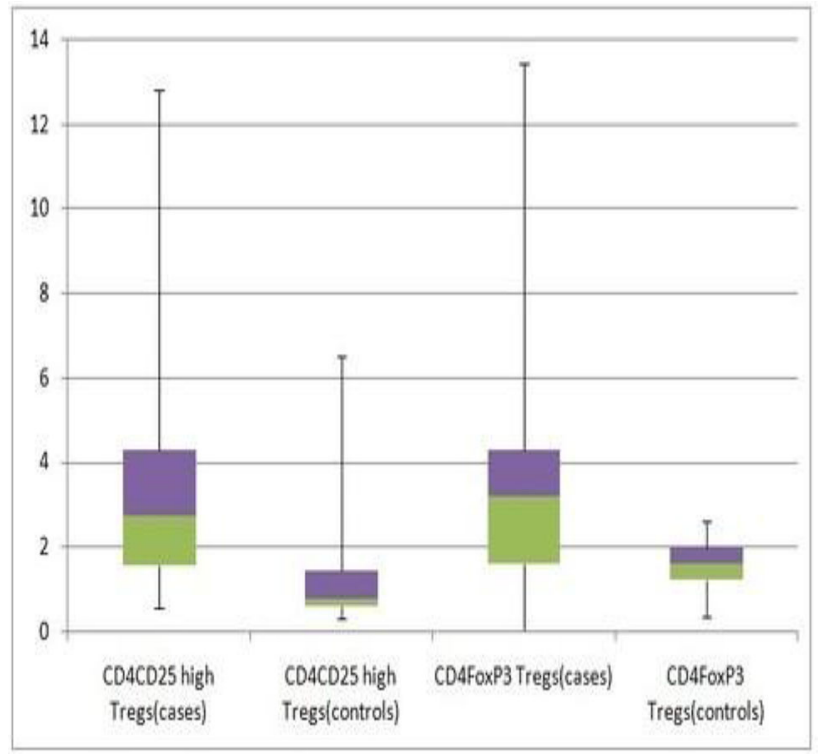

\section{Clinical Profile of Covid 19 Infection in Patients with Hematological Malignancies: A Single Centre Experience from North India}

Faheema Hasan $^{* 1}$, Anshul Gupta ${ }^{1}$, Soniya Nityanand ${ }^{1}$, Sanjeev yadav $^{1}$, Archit pandharipande ${ }^{1}$, Gopinathan mathiyazhagan ${ }^{1}$

${ }^{1}$ Dept of Hematology, SGPGI Rae Bareilly Rd, Lucknow, India

Aims \& Objectives: Patients with haematological malignancies are very different from other malignancies because of direct affection of immune system and therapy involving significant myelosuppression and lymphocyte depletion. Therefore, in this era of COVID 19 pandemic, where even healthy individuals can have severe COVID pneumonia, it is important to identify, how patients with haematological malignancies tolerated the disease. We analysed the clinical characteristics and risk factors of these patients of haematological malignancies and COVID 19.

Patients/Materials \& Methods: It is a retrospective study of patients of haematological malignancies who presented to our institute, between March 2020 till October 2020 with positive RT PCR reports for COVID 19 or who were referred to us for further management or who were incidentally found to be covid positive during admission in our hematology ward were included in the study. Asymptomatic patients and patients with mild COVID pneumonia were managed with home isolation and the rest were managed as per severity and protocol in Rajdhani Corona Hospital. The following clinical data was retrieved from medical records: clinical profile, baseline disease 
characteristics, hemogram, chemotherapy received and the severity of covid pneumonia were analysed.

Results: Out of 21 COVID positive patients encountered during the study period, male: female ratio was $1.3: 1$, with median age of 34 years. The most common underlying malignancy was Acute Lymphoblastic leukemia (ALL)(38\%), followed by Non Hodgkin Lymphoma(NHL) $(23.8 \%) 7(33.3 \%)$. Fever was the most common symptom present in almost all patients and diabetes (19\%) was the most common underlying co morbidity apart from the malignancy. Cytopenias, especially lymphopenia was associated with worse outcome. Seven patients $(33.3 \%)$ succumbed to COVID pneumonia despite protocolised measures in our covid speciality centre.

Discussion \& Conclusion: Our study highlights that hematological malignancies might be a poor prognostic factor for COVID19, especially in the presence of have a worse outcome cytopenias and that the risk of nosocomial infection of COVID19 is high. Our series data suggest that strong proactive measures are necessary to reduce likelihood of infection and improve early identification in this vulnerable patient population.

\section{A Plethora OF Diverse Lymphocytes in Peripheral Blood Smear of Dengue Patients: Rods, Motts, Downeys and Beyond}

Shreyam Acharya ${ }^{* 1}$, Aparna Ningombam ${ }^{1}$, Abhirup Sarkar ${ }^{1}$, Kundan Kumar

\section{${ }^{1}$ Laboratory Medicine, AIIMS, New Delhi, India}

Aims \& Objectives: Hal Downey and C.A. McKinlay first described the atypical lymphocytes seen in cases of infectious mononucleosis in 1923. They further categorized the atypical lymphocytes of various etiologies under three subtypes naming Type I, Type II and Type III. With the changing times, findings of atypical lymphocytes with the morphological description as was initially illustrated, have been observed in various infectious, auto-immune disorders and drug induced changes. Our objective is to document and re-emphasize the morphological heterogeneity of reactive lymphocytes in diagnosed seropositive dengue patients. We also aim to highlight on the numerous reactive lymphocytic changes which one seldom describes in non- malignant conditions but are frequently documented and given importance in malignant plasma cell disorders.

Patients/Materials \& Methods: A total of 30 patients with seropositive dengue infection were included in the study. Peripheral blood samples were collected at the time of hospital admission. Giemsa stained peripheral blood smears were made for each case and the smears were screened for reactive cells of lymphocytic lineage by two independent observers.

Results: Among the 30 patients, 25 cases had florid reactive changes in the peripheral blood smear while four (4) smears had very less populations of reactive cells and one (1) smear had no change at all. All three types of Downey cells were observed along with some other variants which included larger cells with deeply convoluted nucleus, cells with azurophilic rods and granules in cytoplasm, flame cells, mott cells and some intermediate forms whose morphology has not been defined by the already documented literature.

Discussion \& Conclusion: The benign lymphocyte morphology is varied and can alter more in reactive situations. Identification of atypical lymphocytes of reactive origin is important to reduce further unnecessary investigations and streamline the flow of diagnostics towards infectious etiology.
Impact of Covid 19 on Hematological Parameters: A Single Centre Study, India

Reshma Anegundi ${ }^{* 1}$, Rajeshwari $\mathrm{T}^{1}$, Arathi $\mathrm{C} \mathrm{A}^{1}$

${ }^{1}$ Pathology, PESIMSR, Kuppam, India

Aims \& Objectives: To analyze various hematological parameters in Covid19 infected patients.

Patients/Materials \& Methods: This is a retrospective, single centre cross sectional study conducted in a tertiary care hospital, located in a tri-state region. Data was collected from patients admitted in Covid 19 ward and ICU between 28 July to 7 September 2020, confirmed positive for Covid 19 infection. Basic demographic data was collected from Laboratory Information System(LIS) of our hospital. Whole blood EDTA sample sent from Covid 19 ward and ICU were processed in LH780/DXH 500(Beckman coulter) for complete blood counts. Further patients laboratory features were analyzed .

Results: Findings of 338 patients,both from ward and ICU, showed varied hematological parameters. Demographic data revealed age range from 1 day to 86 years. Males constituted 205(60.65\%) and females 133(39.34\%).Complete blood count showed Hemoglo$\operatorname{bin}(\mathrm{Hb})$ concentration ranged from $5.8-18.5 \mathrm{gm} / \mathrm{dL}$ and median value was $13.6 \mathrm{gm} / \mathrm{dL}$. Red cell distribution width(RDW) ranged from 11.4 to $39.9 \%$ with median value of $13.6 \%$,leucocytosis was observed in $13.9 \%$ of patients, high absolute neutrophil count in $15.6 \%$, high Neutrophil to Lymphocyte ratio(NLR) in $10.05 \%$ patients and eosinophil count ranged from 0 to $15.3 \%$.

Discussion \& Conclusion: Baseline alterations in CBC parameters of $10-15 \%$ of patients in the present study along with articles reviewed from various countries indicate SARS-CoV-2 infection causes systemic infection involving multiple organs and systems. Key words:COVID19, Leucocytosis, Absolute neutrophil count, Neutrophil - Lymphocyte ratio.

\section{White Blood Cell Scattergrams in Covid-19 Patients: A Rapid Screening Tool?}

\section{Zalak N. Parmar ${ }^{* 1}$, Mustafa F. Ranapurwala ${ }^{1}$}

${ }^{1}$ Pathology, Pramukhswami Medical College and Shree Krishna Hospital, Anand, India

Aims \& Objectives: The current study was undertaken to evaluate utility of the specific pattern of WBC scattergram as a screening tool for COVID-19 by establishing the sensitivity and specificity of the 'sandglass pattern'.

Patients/Materials \& Methods: The present study is a cross-sectional retrospective study undertaken at the Central Diagnostic Laboratory of Shree Krishna Hospital, Karamsad, a tertiary healthcare centre in Gujarat and a designated COVID centre during the recent pandemic. EDTA samples of patients admitted to the COVID centre, who had tested positive by RT-PCR were run on XN550 analyzer (SYSMEX $^{\circledR}$ (Sysmex Corporation) for complete blood counts, as a part of the initial assessment. WBC analyses were assessed blindly. Presence of the new pattern was considered WBCpositive $(\mathrm{WBC}+)$, all other patterns were considered negative (WBC -).

Results: WBC scattergrams from 750 samples from symptomatic patients admitted at our COVID centre were analysed for 'sandglass pattern'. The sensitivity and specificity of the sandglass pattern were $85 \%$ and $84 \%$ respectively.

Discussion \& Conclusion: WBC scattergram analysis can help quickly categorise symptomatic patients and provide a valuable screening tool for clinicians to expedite the management of suspected COVID-19 patients admitted in the hospital. 
Pelvic Osteomyelitis as a Complication of Bone Marrow Biopsy

\author{
Ashika Davis ${ }^{* 1}$, Aaron Lobo ${ }^{1}$, Seetharam Anandram ${ }^{2}$ \\ ${ }^{1}$ General Medicine, ${ }^{2}$ Haematology, St. Johns Medical College, \\ Bangalore, India
}

Aims \& Objectives: INTRODUCTION: Osteomyelitis is an infection of the bone, usually involving the tibia. Staphylococcus aureus is a commonly identified organism. Osteomyelitis of the pelvis is uncommon, and usually, the ilium is most commonly affected. Bone marrow biopsy is a widely performed investigation in haematology with an incidence of complications ranging between $0.05 \%$ and $0.07 \%$, and infections are sporadic. This rare case discusses a patient who developed osteomyelitis following a bone marrow biopsy.

Patients/Materials \& Methods: CASE REPORT: A 50-year-old male, with no prior co-morbidities presented with a one-month history of fever and generalized fatigue. On evaluation, he had pancytopenia. We performed a bone marrow biopsy in the right posterior super iliac spine, and the diagnosis of T cell Non- Hodgkin's Lymphoma based on morphology and Immuno-histochemistry(CD 5, CD 3, CD 8 and CD 4 positivity) made. He was started on chemotherapy (CHOP regimen). Four days later, the patient complained of pain and swelling at the biopsy site and ultrasound done showed features of a haematoma, managed conservatively, and the patient discharged. Following discharge, the patient continued to be symptomatic with pain. He was readmitted, and pus cultures revealed a growth of MRSA. The patient was treated with Vancomycin. An MRI Pelvis revealed features of osteomyelitis of the right iliac bone. The orthopaedic intervention was deferred initially because of pancytopenia and frailty.

Results: Over the next three years, the patient required multiple admissions, and subsequent MRIs showed sinus tract formation. However, the patient refused surgical intervention. On his last persistent the patient continued to have persistent discharge from the biopsy site.

Discussion \& Conclusion: DISCUSSION: In adults, pelvic osteomyelitis accounts for $2-11 \%$ of all cases of osteomyelitis. Literature review suggests this is only the third case of pelvic osteomyelitis following a bone marrow biopsy. In conclusion, while bone marrow biopsy is a safe procedure, it may cause unexpected complications like osteomyelitis. If a patient has prolonged complaints following a bone marrow biopsy, osteomyelitis must be considered and appropriately managed.

\section{Utility of Hematological Prognostic Marker in Predicting Disease Outcome in Indian Patients with Covid 19 Infection}

\author{
Bala Krishnan $^{* 1}$, Kumar Abhisheka ${ }^{1}$ \\ ${ }^{1}$ Medicine, Command Hospital Air Force, Bengaluru, India
}

\begin{abstract}
Aims \& Objectives: Aim: To establish role of hematological prognostic marker in predicting disease outcome in Indian patients with COVID 19 infection. Objective: To study the role of neutrophil lymphocyte ratio in predicting mortality in Indian patients with COVID 19 infection.

Patients/Materials \& Methods: Methods: 207 consecutive patients with COVID-19 infection admitted at our centre underwent evaluation. Descriptive analysis of demographic data was carried out and risk factor for various disease outcome(length of hospital stay, use of mechanical ventilation, mortality and severe covid) was analysed using multivariate linear or logistic regression as appropriate. Further neutrophil lymphocyte ratio was compared in patients with mortality with the rest using independent sample $t$ test.
\end{abstract}

Results: Results: Mean age of the studied patients was 58.48 (13.79) years with $67(32.2 \%)$ female patients. 74 patients $(35.6 \%)$ had diabetes. We found that in COVID-19 patients, diabetes (OR $2.37 \mathrm{CI}$ 1.07-5.20) and hypertension (OR 2.88 CI 1.28-6.44) are independent risk factors for severe pneumonia. The risk factors predicting mortality were age(OR 1.07 CI 1.03-1.12) malignancy(OR $11.52 \mathrm{CI}$ 2.84-46.85) and chronic kidney disease(OR 5.68 CI 1.25-25.86) and increased neutrophil lymphocyte ratio (OR 1.05 CI 1.01-1.09). Patients with mortality had significantly higher neutrophil lymphocyte ratio (16.17 vs $7.74 p$ 0.001).Patients with Co morbidities Cerebrovascular accident(OR 15.60 CI 2.62-92.79) malignancy (OR 22.05 CI 4.00-121.40), chronic kidney disease (OR 8.65 CI 1.47-50.75) and HIV infection (OR 41.30 CI 6.45-264.19) are more likely to require mechanical ventilation. The predictors of longer hospital stay were age ( $\mathrm{r}-0.15, p 0.017)$, chronic liver disease ( $\mathrm{r} 0.21$, $p 0.001)$ and fever at presentation ( $\mathrm{r} 0.19, p 0.003)$.

Discussion \& Conclusion: Our data revealed that neutrophil lymphocyte ratio is an independent risk factor for mortality in patients with Covid infection. Early identification of this risk factor can help in instituting corrective measures to improve mortality in patients with Covid infection.

Keywords: COVID-19, neutrophil lymphocyte ratio, mortality. Supporting Document:

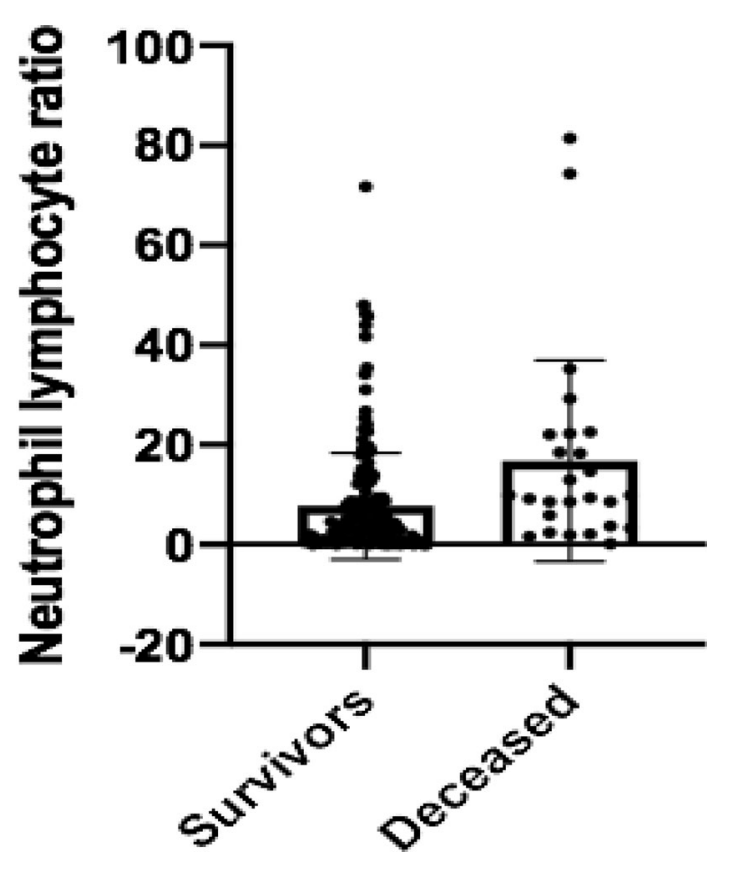

\section{A Case of Cyclical Neutropenia and Acquired Bartter Syndrome in a Patient with Brucellosis}

Prudhvi Dasari $^{* 1}$, Ratnamala Choudary ${ }^{1}$, Chaitanya H. Balakrishnan $^{1}$

\section{${ }^{1}$ General Medicine, St. Johns Medical College, Bangalore, India}

Aims \& Objectives: Cyclic neutropenia is a rare syndrome of recurrent neutropenia with a mutation in the ELANE gene, with an estimated frequency of one case per million in the general population. Acquired Bartter syndrome is a renal tubular salt-wasting disorder following drug therapy resulting from defective sodium chloride reabsorption in the medullary thick ascending loop of Henle. 
Brucellosis is a highly contagious zoonosis caused by Brucella species. Here we report all these three rare entities in the same individual. Patients/Materials \& Methods: This is a case description of a 21-year old female who presented with fever, chills and headache of 15 days duration. On clinical examination, she was febrile with pallor and cervical lymphadenopathy. Fundus showed early papilledema but systemic examination was otherwise unremarkable. Investigations showed anemia, severe leukopenia with an absolute neutrophil count of $<100 / \mathrm{mm} 3$. Peripheral smear showed no blast cells, all routine fever workup was negative, CSF panel showed Klebsiella and Acinetobacter species and bone marrow examination showed hypoplastic marrow. The patient was managed with broad-spectrum antibiotics and G-CSF with a working diagnosis of Neutropenic Sepsis with underlying Aplastic Anemia.

However, the patient had a torrid course with clinical worsening due to recurrent neutropenic sepsis(pan-resistant E. coli), requiring aminoglycosides, colistin, empirical amphotericin B. Three weeks into the hospital stay, her investigations showed dyselectrolytemia in the form of severe hypocalcemia, hypokalemia, hypomagnesemia, metabolic acidosis and after evaluation, it was attributed to antibiotic induced Bartter syndrome. The antibiotics were de-escalated and she improved with IV correction of the electrolyte imbalance.

Despite this, she continued to have persistent fevers. The pyrexia was re-evaluated and investigations revealed rising titres of Brucella Antibodies (1:360) for which she was treated with Rifampicin and Doxycycline for 6 weeks. She gradually improved and was discharged. On her follow-ups, it was noticed her neutropenia is recurrent, once in 3-4 weeks and hence she was continued on G-CSF twice weekly and she is currently doing well.

Results: After a detailed review of clinical presentation, examination and analysing investigations the diagnosis of Cyclical neutropenia with Drug induced Bartter syndrome in a patient suffering from Brucellosis was thus made.

Discussion \& Conclusion: In a patient with a hypoplastic marrow due to infections like Brucella, persistent neutropenia despite treatment may need evaluation for rare conditions like cyclic neutropenia. Keywords: Absolute neutrophil count, Cyclical neutropenia, Acquired Bartter syndrome, Brucellosis, ELANE gene.

\section{Changes in Blood Coagulation in Patients with Severe Coronavirus Disease 2019 (Covid19): A Study at Tertiary Care Centre}

Payal S. Bhatt ${ }^{* 1}$, Kirti J. Rathod ${ }^{1}$

${ }^{1}$ Pathology, Pramukh Swami Medical College, Anand, India

Aims \& Objectives: Coronavirus disease 2019 (COVID-19), first identified in Wuhan, China in December of 2019, has become a worldwide pandemic with widespread illness and mortality and a profound impact on society, culture, and the global economy. Although respiratory compromise is the cardinal feature of the disease, early studies have suggested that elevated circulating D- dimer levels are associated with mortality, suggesting a distinct coagulation disorder associated with COVID-19. Given the ongoing global pandemic, there is an urgent need to understand the rate of bleeding and thrombotic manifestations associated with COVID- 19 coagulopathy, as well as the clinical utility of abnormal coagulation testing to predict risk for bleeding, thrombosis, and severity of illness. (1)To establish relationship between outcome of the patient and coagulopathic complications by using these markers as predictors. (2) To explore the difference in blood coagulation parameters between severe and mild cases of COVID19.

Patients/Materials \& Methods: This study is being conducted in Shree Krishna Hospital, Karamsad. Patients with confirmed COVID19 from April 2020 till October 2020 were analysed. All the data of these patients were collected comparing information on coagulation parameters and severity of Covid-19. Patient data included age, sex, Prothrombin time (PT), Activated partial thromboplastin time (APTT), D-dimer, Platelet count and severity of covid-19. The pooled standardised mean difference (SMD) and 95\% confidence interval (CI) were computed.

Results: The degree of D-dimer elevation positively correlates with mortality in COVID-19 patients. Low platelet count is noted in severe cases as compared to the non-severe cases. PT and APTT are inconsistent with the severity of Covid-19.

Discussion \& Conclusion: Disseminated intravascular coagulopathy (DIC) and severe bleeding events are uncommon in COVID-19 patients. Here, we review the current state of knowledge of COVID19 and haemostasis.

\section{Thrombocytopenia in an Apparently Healthy Neonate:} An Unusual Report of Postnatally Acquired Dengue

ShradhaZutshi $^{* 1}$, Kapil bhalla ${ }^{1}$, Namitagwasikoti ${ }^{1}$

${ }^{1}$ Department of Pediatrics, PGIMS, Rohtak, India

Aims \& Objectives: To report an unusual case of postnatally acquired dengue infection in an apparently healthy newborn.

Patients/Materials \& Methods: : We report a case of 10 days old newborn, born to a primigravida mother (normal antenatal history), presented to a tertiary care hospital with complaints of fever for 2 days and macular rash all over the body. Examination findings revealed red colored, pin point macular rash while rest of general and systemic examination were unremarkable. Routine Sepsis work up was negative except presence of thrombocytopenia. Keeping in mind the endemicity and season of dengue, NS1 antigen of the baby was sent which was positive, which was further confirmed by IgM ELISA for dengue. However, the mother was asymptomatic and platelet count as well as NS1 antigen and IgM, IgG ELISA for dengue of the mother was negative.

Results: Dengue was diagnosed in a healthy new born with no history of any febrile illness in the mother and vertical transmission being ruled out.

Discussion \& Conclusion: Dengue is one of the commonest viral infections affecting general population in endemic zones every year. However, dengue is usually not reported in new born period as it is widely believed that infants are protected from serious viral infections in the first 6 months of life by presence of maternal antibodies. There are very few case reports of post natally acquired dengue in the newborn period, even less so in an apparently healthy newborn. Dengue should be kept in mind in the differential diagnosis of neonatal thrombocytopenia even in a well newborn, especially during dengue season, to ensure optimal management of the baby, even in absence of any dengue like fever in themother.

\section{Outcome of SARS-COV2 Infection in Hematological Disorders: A Tertiary-Care Cancer Center Experience}

Naveed Tamboli ${ }^{* 1}$, Arijit $\mathrm{Nag}^{1}$, VivekRadhakrishnan ${ }^{1}$, Jeevan Kumar $^{1}$, Saurabh Bhave ${ }^{1}$, RizwanJaved ${ }^{1}$, Sanjay Bhattacharya ${ }^{2}$, Gaurav $\mathrm{Goel}^{2}$, SoumyadipChatterji ${ }^{3}$, Sudipta Mukherjee ${ }^{4}$, Arna Das ${ }^{1}$, MayurParihar ${ }^{5}$, Deepak Mishra ${ }^{5}$, Reena Nair ${ }^{1}$, MammenChandy ${ }^{1}$

${ }^{1}$ Clinical Haematology and BMT, ${ }^{2}$ Microbiology, ${ }^{3}$ Infectious Disease, ${ }^{4}$ Critical Care, ${ }^{5}$ Laboratory Haematology, Tata Medical Center, Kolkata, India

Aims \& Objectives: We conducted a clinical audit of patients with hematological disorders and SARS-CoV2 infection. 
Patients/Materials \& Methods: A prospective registry was established in April 2020 for patients from the department of clinical hematology and HCT who were diagnosed to be positive for SARS$\mathrm{CoV} 2$ by PCR test. Out patients creening was symptom/contact-exposure driven, and in patients creening was symptomaticorpreemptive. This study is a part of ASHCOVID19 International collaborative and an interim analysis of the institute registry data from April 15, 2020 to October 7, 2020.

Results: 1201 new patients were registered and 9539 patient-visits were recorded in the out patient service of the department during this period. $91(0.08 \%)$ patients tested positive for SARS-CoV2. Baseline characteristics of the patients are listed in Table 1. $56(61.5 \%)$ patients needed hospitalization. The median absolute neutrophil count was 3700/cu.mm, while the median absolute lymphocyte count 900/cu.mm. Inflammatory markers $(\mathrm{n}=21)$ :median D-dimer was $2845 \mathrm{ng} / \mathrm{ml}$ (243-140643) and median CRP level was $14 \mathrm{mg} / \mathrm{dl}$ (3.334). Therapy directed against SARS-CoV2 included, Azithromycin $(\mathrm{n}=52,60.4 \%)$, Dexamethasone (27 patients,29.7\%), Remdesivir $(\mathrm{n}=10,11.1 \%)$ and doxycycline $(\mathrm{n}=9,9.9 \%)$. 2(2.2\%) patients received tocilizumab and 1 patient $(1.1 \%)$ received convalescent plasma. On univariate analysis, none of the therapies seemed to affect outcomes. The SARS-CoV2 infection mortality was 15\% (14/91). 2 patients died due to non-COVID related causes [tumor lysis syndrome $(\mathrm{n}=1)$, leukocytostasis with CVA $(\mathrm{n}=1)]$. 7/45(15.5\%) receiving anti-B-lymphocyte directed therapy died as compared to $7 / 46(15.21 \%)$ not receiving Anti-B-cell therapies. Patients with low grade B-NHL $(5 / 16,31.25 \%)$ had the highest mortality rate followed by high grade B-NHL $(7 / 23,30.4 \%)$.There was a trend to higher mortality in patients $>50$ years $(12 / 45,26.6 \%$ in those $>=50$ years age as compared to $4 / 30,13.33 \%$ in those $<50$ years age). With data available until the date of censoring, most patients were diagnosed in August $(\mathrm{n}=32,35.2 \%)$ with numbers tailing off in September ( $\mathrm{n}=20,22 \%)$.

Discussion \& Conclusion: In our subset of patients with hematological disorders, SARS-COV2 infectivity was found to be low, hospitalization rate was moderate, and mortality was high and commoner in patients $>50 \mathrm{yr}$ and a B-cell lymphoma diagnosis. A more aggressive screening approach will potentially improveoutcomes. Supporting Document:

\section{Bone Marrow Candidiasis in a Patient with Pulmonary Infiltrate and Secondary HLH}

\author{
Manisha Raj $^{* 1}$, Kanwaljeet Singh ${ }^{1}$, VenkatSomasundaram ${ }^{1}$, \\ Tathagata Chatterjee ${ }^{1}$
}

${ }^{1}$ Lab Sciences and Molecular Medicine, Army Hospital Research and Referral Institute, New Delhi, India

Aims \& Objectives: Aim is to highlight the role of Bone Marrow Biopsy and Aspirateindiagnosingr are fungal infections of bone marrow like Candidias is which can lead to secondary Hemophagocyticlymphohistiocytosis (HLH).

Patients/Materials \& Methods: A 34 year old male patient presented with unexplained intermediate to high grade fever and dry cough since one month. Examination didn't reveal organomegaly or lymphadenopathy. Patient was evaluated for fever and was found negative for malaria, dengueandtyphoid.Inviewofpersistentfeveranddrycough,patientwasinvestigatedforTuberculosis. Patient after under going battery of investigations, the mystery was unfolded by bone marrow aspirate and biopsy leading to final diagnosis of bone marrow candidiasis with secondary HLH.

Results: Bone marrow aspirate showed trilineage hematopoietic cells with increased histiocytes in bone marrow, showing significant hemophagocytosis (2-3/HPF) in the form of ingested hematopoietic cells. Few histiocytes showed presence of yeast forms which were PAS positive. Bone marrow biopsy showed normal distribution of erythroid and myeloid precursors. Increased histiocytes was noted, few of which showed presence of yeast forms with pseudohyphae which were better appreciated on PA Sand Grocott's (GMS) stain.

Discussion \& Conclusion: Bone marrow evaluation can be considered as an important diagnostic tool to delineate etiological diagnosis in certain infectious conditions such as candidiasis, particularly in cases presenting with PUO with cytopenias. This information can be well correlated with our case who presented with fever investigation and absence of any infective etiologyason investigations prompted the bone marrow aspirate andbiopsy. To conclude, this case report highlights the role of bone marrow examination as an important diagnostic modality for the etiological diagnosis of in a case of PUO and thereby leading to better management $\&$ prognostication in such cases.

\section{Correlation Between Fungal Blood Stream Infection (BSI) and Thrombocytopenia in Neonates}

J Satya Mani Deepika Chilukuru ${ }^{* 1}$, JinelaDesouza ${ }^{1}$, NiveditaPatil ${ }^{2}$

${ }^{1}$ Pediatrics, Dr. D Y Patil Educational Society and Deemed University, ${ }^{2}$ Pediatrics, Dr. D Y Patil Educational Society and Deemed University, Kolhapur, India

Aims \& Objectives: To assess the prevalence of neonatal fungal blood stream infection and risk factor associated mortality due to fungal septicemia and it's correlation withthrombocytopenia.

Patients/Materials \& Methods: This is are prospective studyd one in neonates admitted to the NICU at Dr DYPATIL hospital and research institute, Kolhapur during the period of Jan 2019 to Jan 2020. Total sample sizeis 131. Data collection was done based on the total number of blood culture sent and the medical records of culture proven blood stream infection and their corresponding CBCreports.wethenclassifiedthesepsisintofungalandbacterialandcorrelatedthereportswithcorrespondingthrombocytopenia.

Results: Out of 131 blood cultures sent, 49 blood culture reports were positive, out of which 15 were positive for fungal infection. Also, the neonates whose blood culture came positive, also showed corresponding decrease in platelet levels.about $90 \%$ neonates with fungal sepsis showed thrombocytopenia compared to bacterial sepsis in which only $25 \%$ showed thrombocytopenia.

Discussion \& Conclusion: Our study is an attempt to correlate sepsis with thrombocytopenia which serve as an early predictive marker in resource limited setting. Also, early prophylaxis of anti-fungal injectables can be started.

\section{Case of Dengue Fever with Secondary Hemophagocytic Lymphohistiocytosis}

Mayur S. Garg ${ }^{* 1}$, Sandeep Rai ${ }^{1}$

${ }^{1}$ General medicine, Mgm medical college and hospital, Navi Mumbai, India

Aims \& Objectives: To study the case of Dengue complicated by secondary HemophagocyticLymphohistiocytosis.

Patients/Materials\&Methods: Consent was taken from the relative of the patient before starting the case report.

Results: Were portacase of young man presenting with persistent fever, myalgia, bleeding gums and physical examination revealedhepatosplenomegaly.DiagnosedasDengueFeveronserological-

tests.Thepatientshowedprogressiveclinical deterioration even with intensive care. HLH was suspected and investigation showed the 
presence of pancytopenia with high ferritin and triglyceride levels. Hemophagocytosis on bone marrow biopsy confirmed the diagnosis of HLH. On initiation of intravenous corticosteroids, there was gradual recovery in clinical and biochemical parameters of the patient.

Discussion \& Conclusion: This case report contributes in understanding HLH syndrome even further and adds to the existing literature of the disease. It is imperative that physicians should promptly recognise and differentiate secondary HLH from severe dengue as HLH is usually missed since it is misdiagnosedas denguehe morrhagicsyndromeordengueshocksyndrome. Early recognition and treatment of this rare condition is important, as timely intervention can save significant morbidity and mortality.

\section{MDW as a Screening Tool for Early Detection of Sepsis in Emergency Departement Patients}

\author{
Jennifer Kabeer ${ }^{* 1}$, Sunithathomas ${ }^{2}$, Jaicobvarghese ${ }^{3}$ \\ ${ }^{1}$ Emergency Medicine, ${ }^{2}$ Pathology, ${ }^{3}$ Critical Care, Rajagiri \\ Hospital, Ernakulam, India
}

Aims \& Objectives: Primary Aim: To determine if a high MDW value can be used for early sepsis detection as defined by sepsis-2 criteria. Secondary Aim :- To determine a cutoff value of MDW for early detection of sepsis in Indian population.

Patients/Materials \& Methods: Adult patients in the age group18-89 years, presenting to the emergency departement, for whom a complete blood count with differential was done as part of the irroutinee valuation were included in the study $3 \mathrm{ml}$ of venous blood is collected in an EDTA vacutainer and analysed in a fully automated haematologyanalyser to obtain blood counts including white cell count and monocyte distribution width. Patients were divided into 4 categories- control, patients with SIRS criteria, patients with sepsis and patients with a source of infection without SIRS criteria. MDW value was evaluated in each of the above mentioned groups and compared.

Results: Based on ROC curve plotted, with MDW as the test indicator against sepsis or no sepsis, MDW value of 19.33 was found to have a sensitivity of $71.2 \%$ and specificity of $99.9 \%$. Mean MDW value in the study group having sepsis(SIRS with a source of infection) was 22.7 , as compared to the other study groups- control group with a mean value of 16.93 and patients with SIRS without a source of infection having a mean value of 18.57. Patients with a source of infection without SIRS criteria (due to confounding factors like prior hospital admission, use of antibiotics, use of antihypertensivesetc) also had an elevated mean MDW value of 20.09 .

Discussion \& Conclusion: This study concludes that MDW can be used as an indicator for early detection of patients with sepsis (as described by SEPSIS-2 criteria) in the ED The cut off value of MDW in our study population was found to be 19.33 with a sensitivity of $71.2 \%$ and specificity of $99.9 \%$.

Supporting Document:

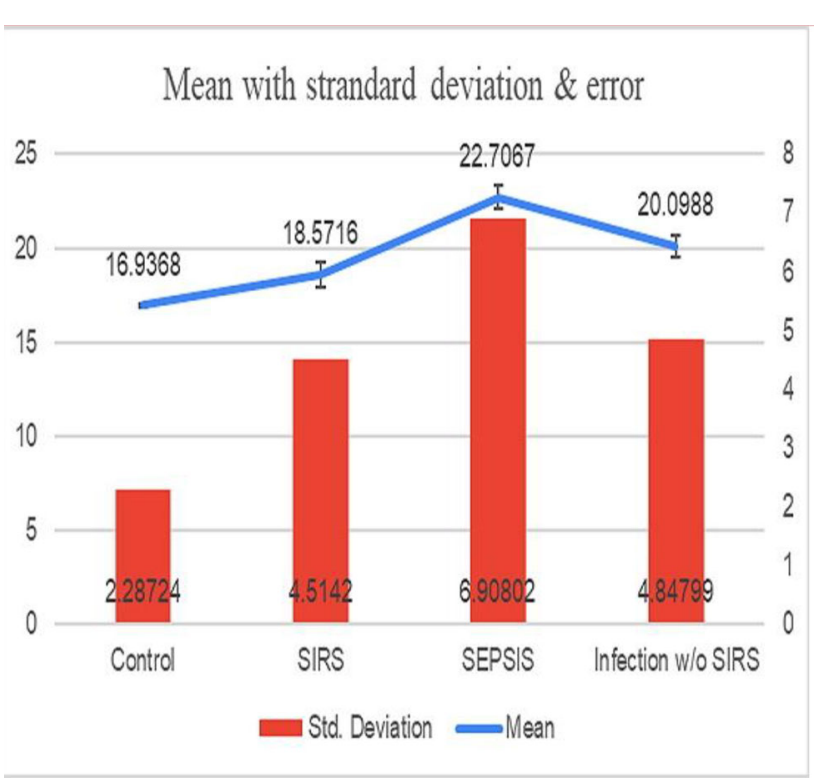

\section{Hemophagocytosis on Bone Marrow Examination: Clinicopathological Correlation}

Shruthi $\mathbf{V}^{* 1}$, MamtaSoni ${ }^{1}$

${ }^{1}$ Haematology, Apollo Hospitals, Chennai, India

Aims \& Objectives: This study was conducted to evaluate the correlation between presence of hemophagocytosis in bone marrow aspirates with laboratory findings and to investigate the possible associated etiology.

Patients/Materials \& Methods: The study was conducted in the departmen to hematology, which included review of 52 cases showinghemophagocytosisinbonemarrowaspirate.Clinicaldetailsandrelevantinvestigationswerenotedforeverycase.

Results: The data was collected on 52 cases showing hemophagocytosis. Meanage of the patients is 46 years. 2 cases were in pediatric group. 38 were males and 14 were females. The major presenting clinical symptom was fever which was present in about 50 of 52 cases. Splenomegaly was observed in 44 patients. Serumferrit in value $\geq 500 \mu \mathrm{g} / \mathrm{L}$ was observed in $89.13 \%$ (41/46) of the patients. Serum triglycerides value $\geq 265 \mathrm{mg} / \mathrm{dl}$ was observed in $30.95 \%$ (13/ 42 ) of the patients. Plasma fibrinogen value $<150 \mathrm{mg} / \mathrm{dl}$ was observed in $28.94 \%(11 / 38)$ of the patients. The associations found in our study with hemophagocytic syndrome were classified as autoimmune disease associated, leukemia associated, lymphoma associated, viral associated, post-transplant associated, HIV associatedetc.

Discussion \& Conclusion: HLH is a rare life-threatening disorder and promptalert is mandatory. Once the condition is suspected, all laboratory parameters must be evaluated and supporting clinical history must be collected for the confirmation of diagnosis. Immediate treatment should be instituted to suppress the severe hyperinflammation that is responsible for the life-threatening symptoms.

Supporting Document: 
TABLE 1. Disease association and distribution of cases.

\begin{tabular}{|c|c|c|c|}
\hline ASSOCIATIONS & NO. OF CASES & $\%$ OF CASES & DISTRIBUTION OF CASES \\
\hline SEPSIS & 8 & 15.38 & \\
\hline $\begin{array}{l}\text { ADULT ONSET STILLS } \\
\text { DISEASE }\end{array}$ & 2 & 3.84 & \\
\hline $\begin{array}{l}\text { AUTOIMMUNE } \\
\text { DISEASE }\end{array}$ & 3 & 9.61 & $\begin{array}{l}\text { SLE } \\
\text { MYASTHENIA GRAVIS - } 1\end{array}$ \\
\hline LEUKEMIA & 2 & 3.84 & $\begin{array}{l}\text { ALL RELAPSE ON } \\
\text { MAINTAINENCE - } 1 \\
\text { PHILADELPHIA POSITIVE ALL - } 1\end{array}$ \\
\hline VIRAL ASSOCIATED & 5 & 9.61 & $\begin{array}{l}\text { EBV - } 1 \\
\text { CMV - } 2 \\
\text { HIN1 PNEUMONITIS - } 1 \\
\text { COMMUNITY ACQUIRED VIR.AL } \\
\text { PNEUMONIA - } 1\end{array}$ \\
\hline LYMPHOMA & 2 & 3.84 & $\begin{array}{l}\text { HODGKIN LYMPHOMA - } 1 \\
\text { NHL ON CHEMOTHERAPY - } 1\end{array}$ \\
\hline TUBERCULOSIS & 3 & 5.76 & $\begin{array}{l}\text { DISSEMINATED TB }-2 \\
\text { TB PERITONITIS - } 1\end{array}$ \\
\hline SCRUB TYPHUS & 1 & 1.92 & \\
\hline POST TRANSPLANT & 4 & 7.69 & $\begin{array}{l}\text { LIVER TRANSPLANT - } 1 \\
\text { RENAL TRANSPLANT - } 3\end{array}$ \\
\hline HIV & 3 & 5.76 & \\
\hline $\begin{array}{l}\text { MULTI ORGAN } \\
\text { DYSFUNCTION } \\
\text { SYNDROME }\end{array}$ & 3 & 5.76 & \\
\hline DCLD & 3 & 5.76 & \\
\hline PUO & 9 & 17.30 & \\
\hline MDS ASSOCIATED & 1 & 1.92 & $\begin{array}{l}\text { REFRACTORY ANAEMIA WITH } \\
\text { MULTILINEAGE DYSPLASIA }\end{array}$ \\
\hline
\end{tabular}

\section{Hematologic Complications in Covid-19: Study from Kerala}

\author{
Sheila Das ${ }^{* 1}$, Samuel K. Mathew ${ }^{2}$, Renu Mathew ${ }^{3}$ \\ ${ }^{1}$ Hematopathology Section, Department Pathology, Believers \\ Church Medical College, ${ }^{2}$ Medicine, Believer's Church Medical \\ College, ${ }^{3}$ Microbiology, Believers Church Medical College, \\ Thiruvalla, Kerala, Thiruvalla, India
}

Aims \& Objectives: [a] To study hematologic parameters in COVID19 patients admitted in Believer's Church Medical College and Hospital Thiruvalla, Kerala [b] To find out whether these complications are more in the elderly.

Patients/Materials \& Methods: India has emerged as one of the worst hit countries by Coronavirus COVID-19. As per current news age group between 60 and 74 years have the highest mortality. Available literature has shown that this virus has a great impact on the hematopoietic system and haemostasis. We aimed to explore the hematologic complications of patients affected by this virus in our hospital over two and half month period 1st August-15th October 2020. Method- During this study period, 980 patients with fever were tested for COVID-19 Antigen and by RT PCR TRUENAT DUPLEX CHIP whenever indicated. Of these, $211(21.53 \%)$ were confirmed positive for the virus. Male :Female 1:1.1 The youngest was 11 days and the oldest 93 years. Both are females. Next complete blood counts and coagulation screen of these patients were recorded and analysed based on their admission to Intensive care units, High Dependency Units or Ward, by the Hematopathology Section of this hospital.

Results: Inthepresentseries $49.24 \%$ weremorethan60years, $29.6 \%$ in 20-49yearagegroup, $17.1 \%$ between 50 and 59 year and children $04 \%$. Derangedhematologic parameters are displayed in Figure 1. Of the total 12 patients died all of whom except two were more than 60 years. They presented with associated comorbidities like Chronic Renalfailure, Diabetes Mellitus, Hypertension, Coronary heart disease and Chronic Liver disease. In these cases deranged hematologic parameters in descending order were as follows, D-dimer $91.6 \%$, LDH in $66.6 \%$, Neutrophil lymphocyte ratio (NLR) $>4$ in $50 \%$, thrombocytopenia in $33.3 \%$ and Platelet lymphocyte ratio (PLR) in $25 \%$ cases. PT/APTT were mildly deranged or normal.

Discussion \& Conclusion: Senior citizens with comorbidities are at a higher risk for Hematologic complications which further precipitates a downhillcourse.

Keywords: COVID-19, NLR, PLR.

Supporting Document:

\section{Leukocyte Blue: Green Death Crystals: Early Mortality Marker In Blood Film}

Rajeev Ranjan*1, Tushar Sehgal ${ }^{1}$, Ranjan Yadav $^{1}$, VivekChaurasia $^{2}$, Upinder Singh $^{1}$

${ }^{1}$ Laboratory Medicine, ${ }^{2}$ Emergency Medicine, All India Institute of Medical Sciences, New Delhi, India

Aims \& Objectives: Called by many names (critical green inclusions, intra cytoplasmic green granules, and inform allyas crystals of death and green crystal of death) blue-green cytoplasmic inclusions in neutrophils are rarely seen on blood smears, but when present, mostly indicate a poor prognosis and impending death. They are refractive and irregular in shape, and are occasionally found in mono cytes also. This is a case report of a patient with a cute pancreatit is with pulmonary embolism whose blood smear demonstrated the deathcrystals. This case report aims to shed light on a rare finding of blue-green cytoplasmic inclusions associated with mortality in a case of acute pancreatitis.

Patients/Materials \& Methods: A 27-year-old male, symptomatic since last 20 days presented with epigastricpainabdo men, with spikes of fever. CT scan revealed diffusely oedematous pancreas with $>$ $50 \%$ necrosis and pulmonary thromboembolism. Patient was COVID negative and required in tubation for acutelung injury and shortness of breath. Patient's past medical history was significant for chronic alcoholintake.

Results: Sepsis markers and coagulation profile were marked lyelevated with deranged LFT and KFT values. CBC showed neutrophilia withd imorphicanaemia. Evaluation of peripheralsmear demonstrated blue-green inclusions "crystals" in the neutrophils and monocytes. These findings were acknowledged but not reported. Patient succumbed to death due to refractory septic shock caused by severe acute pancreatitis with pulmonary thromboembolism, shortly after finding the "death crystals" in the neutrophils and monocytes.

Discussion \& Conclusion: Condition of most patients with "Death Crystals" decline rapidly and death is imminent; this fact gives credibility to the importance of reporting these inclusions particularly in the case of sepsis, shock and SIRS. Further study of these blue-green crystals is necessary to evaluate the nature of the irappearance and the outcomef or the patient, which is yet to be understood completely. Though discussions are still ongoing regarding the importance of reporting these novelty findings, we propose that these inclusion bodies are associated with high mortality, and thus should be reported to the clinicians to facilitate a more diligent management of suchcases.

\section{The Relationship Between Drug Adherence to Antiretroviral Therapy and Viral Load in CLHIV}

Aditya K. Sangwan ${ }^{* 1}$, AlokHemal ${ }^{2}$

${ }^{1}$ Pediatrics, ABVIMS and Dr RML Hospital, New Deldi, ${ }^{2}$ Pediatrics, ABVIMS and DR RML Hospital, New Delhi, India

Aims \& Objectives: AIM: To study the relationship between viral load and drug adherence in children living with HIV. Primary objective: To determine the relationship between viral load and drug 
adherence in children living with HIV. Secondary objective: (1) To determine socio-demographic factors affecting adherence. (2) To determine common reasons for nonadherence.

Patients/Materials \& Methods: In this cross-sectional observational study, 70 children on ART were enrolled after applying the inclusion and exclusion criteria. All clinical, demographic details and investigations available were noted.Adherence was assessed by the pill counting method. Caregivers who reported missing doses were given the pediatric nonadherence questionnaire. Blood sample was analysed for viral load estimation. Appropriate statistical tests were used and analysis was done.

Results: The mean age of diagnosis in study subjects was $8.13 \pm 3.89$ years and mean duration of treatment was $4.24 \pm 3.05$ years. Majority, 61(87.1\%) patients had adherence more than $95 \%$. Among 9 non-adherent children, the main reason was that they forgot to take medicine in $8(88.9 \%)$ cases. The adherent group had significant virological suppression and higher CD4 count. Mean viral load in the adherent group was $2379 \pm 5996.74$ copies perml against $46529.22 \pm 86633.12$ copies perml in the non-adherent group.

Discussion \& Conclusion: We found that adherent grouphada higher CD4 countanda suppressed viral load and adherence to ART was unrelated to age, sex, duration of HIV infection and treatment, socioeconomic status, and route of transmission.

\section{A Case Report of Secondary Immune Thrombocytopenia and Neutropenia Post SARS CoV2 Infection}

VaishaliNagpal $^{* 1}$, Shubha Sudhakar ${ }^{1}$, Hassan Mubarak ${ }^{2}$, Rajesh Krishna $^{3}$

${ }^{1}$ Pathology, Yenepoya Medical College Hospital, ${ }^{2}$ Consultant Physician and Intensivist, Highland Hospital, ${ }^{3}$ Consultant Hematologist, Yeneoya Medical College Hospital, Mangalore, India

Aims \& Objectives: Title: A Case Report of Secondary Immune thrombocytopenia and neutropenia post SARS CoV2 infection.

Patients/Materials \& Methods: Introduction. Bicytopenia and pancytopenia has been described in patients infected with the new severe acute respiratory syndrome coronavirus 2, SARSCoV2 (COVID19 infection). In COVID thrombocytopenia may be due to disseminated intravascular coagulation (DIC), sepsis, immune mediated or cytokine mediated marrow suppression or druginduced. Neutropenia is described due to immune mediated or sepsis related or due to marrow suppression. We report a case where immune mediated thrombocytopenia and neutropenia was noted in a patient with COVID 19 infection. Case Report. A 66 year old male was admitted to the hospital with complaints of fever and shortness of breath for 7 days. Fever was high grade in nature, associated with cough and shortness of breath. No history of bleeding or bruising. On physical examination there was no evidence of organomegaly or lymphadenopathy.

Results: Hematological parameters were estimated using Automated Sysmex XN-1000 and Biochemical parameters using Vitros5600 fully automated chemistry analyzer. Hb was $9.4 \mathrm{gm} / \mathrm{dl}$, TLC was $1.7 \times 10^{3}$ and platelet count was $8 \times 10^{3}$. Peripheral smear examination showed neutropenia and true thrombocytopenia. LFT, clotting profile and creatinine levels were within normal range. Chest X-Ray was showed bilateral consolidation suggestive of Pneumonia. SARS CoV 2 RTPCR was interpreted as positive. CT chest/thorax revealed ground glass appearance in $60 \%$ of the pulmonary parenchyma. Bone marrow showed adequate megakaryocyte and myeloid precursors. Hence the cytopenias were presumed to be due to increased peripheral destruction. Patient was started on Oxygen, Remedesivir and dexamethasone for 1 week with excellent clinical improvement. At the end of 1 week, patient was taken off oxygen with no complaints of fever and an increased platelet count of $50 \times 10^{3}$ and neutrophils of $2 \times 10^{3} \mathrm{He}$ was discharged and has been followed up as an outpatient with further improvement in his counts.

Discussion \& Conclusion: It is important to note that Immuneme diated thrombocytopenia and neutropenia can occur in patients of SARSCoV2. This usually responds to steroids which are part of the treatmen to fth is condition.

\section{Post Malaria Secondary Hemophagocytic Lymphohistiocytosis}

Satyam K. Singh ${ }^{* 1}$, Satya P. Mahapatra ${ }^{1}$, Ravi Tandon ${ }^{1}$, Ashish A. Pushpakar $^{1}$, RanjanBhattnagar ${ }^{1}$

\section{${ }^{1}$ Internal Medicine, IMS, BHU, varanasi, India}

Aims \& Objectives: Presenting a case of post malaria secondary hemophagocyticlymphohistiocytosis.

Patients/Materials \& Methods: 19 years old male presented with complaints off ever high grade associated with chills and rigor, yellowish discoloration of eye and altered sensorium. On examination vitals are stable, mild pallor is present and spleen is palpable. Routine CBC showed $\mathrm{Hb}$ is $9.1 \mathrm{gm} / \mathrm{dl}$, TLC $10200 / \mu 1$, PLT $95000 / \mu 1$, creatinine $3.9 \mathrm{mg} / \mathrm{dl}$, urea $180 \mathrm{mg} / \mathrm{dl}$. LFT was deranged with SGOT/ SGPT $508 / 726 \mathrm{mg} / \mathrm{dl}$, total bilirubin $5.4 \mathrm{mg} / \mathrm{dl}$, direct bilirubin $4.1 \mathrm{mg} / \mathrm{dl}$ and LDH 2397U/L.Patient came positive for P.falciparum and P.vivax by rapid card test.Patient was treated with antimalarial,ceftriaxone,doxycycline and other supportive treatment but initially fever responded to treatment but again reappeared in range of 102-103f. Induecourse of time patient started developing thrombocytopenia $(60000 / \mu \mathrm{l})$, anemia $(\mathrm{HB}-7.0 \mathrm{gm} / \mathrm{dl})$ and leucopenia $(2200 /$ $\mu \mathrm{l})$ with absolute neutrophil count $730 / \mu \mathrm{l}$. His blood culture and urineculture report came out sterile with normal serumprocalcitoninlevel. In view of above finding we suspected HLH and send serumferritin and serumtriglyceride which came out $22544 \mathrm{ng} / \mathrm{ml}$ and TG306 mg/dl respectively. Bone marrow aspiration and biopsy showed hemophagocytes.So according to HLH-2004 protocol diagnosis of hemophagocyticlymphohistiocytosis secondary to malaria wasmade.

Results: Hence patient was diagnosed with HLH secondary to malaria. patient was treated with pulse steroid and cyclosporine A. patient fever responded and discharged on oral prednisone and cyclosporine A. After 2 weeks of treatment patient TLC increased and ferritin decreased.

Discussion \& Conclusion: Malaria is common in tropical countries, its association with HLH is rare but should be kept in mind. HLH should be suspected in patients presenting with high grade fever and multiorgan involvement. Secondary HLH is more common than primary HLH. Primary HLH is due to various genetic abnormalities but secondary HLH occure after infection, auto immuneor autoinflammatory disease, malignant disease, immuno suppression and AIDS. There is cytokine storm with over activation of antigen presenting cell and CD +8 cells. It is diagnosed by HLH 2004 protocol and treated by immunomodulatory therapy. Mortality is high in secondary HLH even in patient treated with HLH 2004 protocol, so early recognition and treatment of this disorder is essential. 
An Unusual Association of Haemophagocytic Lymphocytosis with Salmonella Typhi A Clinical Masquerader

\author{
Anil K. Behera ${ }^{* 1}$, H. Guruprasad ${ }^{1}$, G Navoday $^{1}, \mathrm{~K}_{\text {Divya }}{ }^{1}$ \\ ${ }^{1}$ Internal Medicine, Care Hospital, Banjara Hills, Hyderabad, \\ India
}

Aims \& Objectives: Haemophagocytic Lymphocytosis (HLH) secondary to an infection ia a great impersonator, caused by hyperimmune activation which leads to a wide array of haematological abnormalities. HLH is a rare hyperinflammatory condition characterized by haematological and immunological changes with haemophagocytosis and significant alterations in natural killer cells. While primary form of disease is familial, the secondary form can occur secondary to an infection, malignancy, autoimmune disorder or immune deficiency state. The life threatening condition is uniformly fatal if left untreated. Several infectious agents have been implicated in causing HLH but typhoid fever has been documented in very few handful of cases.

Patients/Materials \& Methods: A 45 year old patient came with history of fever with chills for 12 days with vomiting and looses tools. CBC showed pancytopenia, Renal parameters are normal, Liver parameters are deranged. Viral serology for Hepatitis B,C,HIV, Dengue viral serology were negative, nomal arial pararsite in blood smear. Empirical antibioticinj Ceftriax one was started and resuscitated with fluids. Patient remained febrile and blood lineages continued to decline. Blood cultures showed growth of Salmonellatyphi, sensitive tocephalosporins, how ever febrileepisodes persisted with looses tools, regimen was switched to ivpiperacillintazobactam with supplementation of zinc, vitamin b12 and zinc. Bone marrow aspiration was done for evaluation for pancytopenia suggested increased haemophagocytic activity and blast cells found to be $<5 \%$; picture suggestive of HLH. Serumtriglycerides and ferritin were quantified and found to behigh.

Results: Patient was diagnosed to have HLH as per HLH 2004 citeria (secondary HLH) primary beingun likely due to lack of family history and age of presentation, Patient was started with appropriate antibiotics, immunochemotherapy and transfusions as per protocol. Patient improved symptomatically and hence being discharged with regular followup.

Discussion \& Conclusion: HLH is characterized by low survival rate less than 10 percent in patients. HLH is associated with many infections; development of secondary HLH in the contex to typhoid fever is very rare. HLH also being clinical masqueraderpose difficulty in diagnosis and treatment for patients.

\section{Topic: Hemophagocytic Lymphohistiocytosis Secondary to SARS Covid 19: A Case Report}

Sumiah Syed $^{* 1,2}$, Sanilarahim ${ }^{1},{ }^{3}$, Hassan mubarak ${ }^{4}$, Rajesh Krishna ${ }^{5}$

${ }^{1}$ Pathology, Yenepoya, 22nd Year PG, Pathology, ${ }^{3}$ Assistant Professor, Pathology, Yenepoya Medical College, ${ }^{4}$ Physician and Intensivist, Highland Hospital, ${ }^{5}$ Haemotologist, Yenepoya Medical College, Mangalore, India

Aims \& Objectives: INTRODUCTION: Hemophagocyticlymphohistiocytosis (HLH) is a severe, life-threatening inflammatory syndrome associated with intensecytokinerelease (also known as a "cytokinestorm"). HLH can occur as familial (primary) or acquired (secondary) disorder that may be difficult to distinguish from one another. Secondary form is triggered by various causes including viral infections and is characterized by aggressive course leading to multiorganfailure.We plan to discuss the clinical and laboratory features of hemophagocyticlymphohistiocytosiss econdary to covid 19 .
Patients/Materials \& Methods: CASEREPORT: A 64 yr old female came with complain so fever, breath lessness and cough since 5 days. She was a known case hypertension and diabetesmellitus. She wastested SARSCoV-2-POSITIVE by conventional RT-PCR test. On further evaluation she showed feature sofbilateralpneumonia.

Results: Hematological parameters revealed Pancytopenia. Serum ferritin was raised $(603$ microgm/L) along with $\operatorname{ESR}(78 \mathrm{~mm} / \mathrm{hr})$. Bone marrow aspirate and biopsy showed features of hemophagocytosis. Patient was treated with dexamethasone, remdesivir, oxygen in intensive care with good clinical response.

Discussion \& Conclusion: CONCLUSION: Mortality of HLH is very high without early diagnosis and appropriate treatment.Hence, a screening of all COVID19 positive patients is highly recommended to analyze hyper inflammation using laboratory tests and Hs core which can improve the outcomes. A multidisciplinary team of experts is required to provide patients with appropriate treatment.

Keywords: HLH, Covid 19.

\section{Infection Profile During Cybord Induction Chemotherapy in Multiple Myeloma Patients}

GouthamGandham ${ }^{* 1}$, HridyaJayamohanan ${ }^{1}$, Arun Philip ${ }^{1}$, Wesley M Jose $^{1}$, KeechilatPavithran ${ }^{1}$

${ }^{1}$ Department of Medical Oncology, Amrita Institute of Medical Sciences, Kochi, India

Aims \& Objectives: The primary objective of this study was to describe the clinical and microbiological profile of infections during standard CyBor Dinduction chemotherapy in newly diagnosed multiplemyeloma patients.

Patients/Materials \& Methods: We retrospectively reviewed case records of 155 newly diagnosed multiple myeloma patients who have undergone CyBorD induction chemotherapy at a tertiary care hospital between January 2017 to December 2019.None of the patients were on antibiotic prophylaxis.All the patients were on acyclovir prophylaxis. Documented details of infection episodes during 16 weeks of CyBorD induction chemotherapy period were analysed.

Results: Among 155 newly diagnosed multiple myeloma cases reviewed in our study $22(14 \%)$ of them had clinically or microbiologically proven infection episodes during 16 weeks CyBorDinductionchemotherapyperiod. The most common presenting symptom was fever. The clinical sites of infections were lower respiratory $\operatorname{tract}(\mathrm{n}=7,32 \%)$, urinary $\operatorname{tract}(\mathrm{n}=7,32 \%)$ and upper respiratorytract $(n=5,22 \%) .1$ of the patients had aneutropenic fever. $3(14 \%)$ of the patients with fever had no identifiable focus of infection but microorganisms were isolated from blood. 3(2\%) of the total patients died of infection-related complications. Viral infections were noted in 2 patients, out of which 1 patient had herpes simplex virus infection and the other developed cytomegalo virus pneumonitis. Overall in 14 infection episodes microorganisms were isolated. Common site sofisolates were urine (7), bloodstream (5), and sputum (2). Gram negative infections accounted for $63 \%$ of total isolates with escherichia coli and klebsiellapneumoniae being the commonest. Gram positive micro organisms accounted for $32 \%$ of the total isolates. Candida tropicalis was isolated from the urine of 1 patient.

Discussion \& Conclusion: Infection is a major complication and a leading cause of death in patients with multiplemyeloma. The risk of infection in multiplemyeloma is due to a multifactorial immunodeficiency caused by the disease itself and the treatment regimens given during the different phases of therapy. The risk was greater during the first year following diagnosis and particularly during the first three months of therapy. In our study lower respiratory tract and urinary tract were the most common clinical sites of infection. Urinary tract infection was the most common site for positive bacterial isolates. Gram 
negative bacilli were the predominant cause of infections. Prophylactic antibiotic treatment in newly diagnosed multiplemyeloma patients during induction chemotherapy should be tailored to the locally prevalent pathogens and their susceptibility patterns. Further studies are warranted to assess the benefi to fantibioticprophylaxis to prevent infections in patients with newly diagnosed multiplemyeloma.

\section{Incidence of Covid-19 Infection in Haemato-Oncology Unit in a Non-COVID Hospital}

Ranjit K. CS ${ }^{* 1}$, Rajesh M. Gottipati ${ }^{2}$, Krishnamani KV ${ }^{1}$, Ashok K. $\mathrm{K}^{1}$, Arun Kumar Lingutla ${ }^{1}$, Anil Aribandi ${ }^{1}$

${ }^{1}$ Department of Haemato-oncology, American Oncology Institute, Hyderabad, ${ }^{2}$ Department of Haemato-oncology, American Oncology Institute, Vijayawada, India

Aims \& Objectives: The entire world is facing a severecrisis, regarding a new type of Coronavirus. Patients with oncological or hematological diseases have considered at high risk for severe COVID-19. Here in this observational study we present our data from a tertiary care cancerhospital.

Patients/Materials \& Methods: This study was conducted in atertiary Non-Covid cancer center betwee April 2020 to September 2020, was following the Lombardy experience we felt the incidence of COVID infection can be minimized among the vulnerable group. Data was collected prospectively. Every patient admission in haematology-oncology and bone marrow transplantation unit were strictly followed using the Lombardy guidelines. Every patient and one attendant under went COVIDRT PCR before admission and patient will had an additional plain CT chest. Before there sult they were segregated in a specialized isolation area with strict adherence. Results: During the study period a total of 374 [day care 214 and in patient 160] patients were admitted which included Acute leukemia, Lymphoma and Myeloma for day care chemotherapy, Aplastic anemia for Immunosuppressive therapy and Bone marrow transplantation (autologous and allogenic). Males were 191 and females were 183; median age was $43 y$ rs. Six [ 2 in day care and 4 in patient] patients out of 374 had COVID infection [0.9\% day care and $2.5 \%$ In patient] [ $1.6 \%$ over all], [4-AML;1 testicular lymphoma and 1 primary CNS lymphoma].Out of 6 patients 4 had moderate disease as per WHO definition requiring high flow oxygen support, remidisvir, steroids and anticoagulation. One patient with AML had life threatening infection and died. The remaining patients recovered with no morbidity and continued further treatment.

Discussion \& Conclusion: Our study indicates following strict adherence to social distancing and proper handling of cases, reduces the incidence of COVID infection and improves the final outcome of patients in continued Oncological care.

\section{Pantoea Agglomerans in Acute Myeloid Leukemia: A Rare Organism in Febrile Neutropenia}

JyothiGouthamKumar ${ }^{* 1}$, PriyankTripati ${ }^{2}$, SamuelL.koramati $^{2}$, Anjalimatani ${ }^{1}$, VishnupriyaMathivanan ${ }^{1}$, AmeyC $_{\text {panchal }}{ }^{3}$, Shobha K. Ganesan ${ }^{4}$, Sachin S.Jadhav

${ }^{1}$ DepartmentofHematology, ${ }^{2}$ Departmentofpharmacology, HCGcancerhospital,Bangalore, ${ }^{3}$ DepartmentofHematology, HCG Cancer hospital, Hospital, ${ }^{4}$ Department of Microbiology, ${ }^{5}$ HODDepartment of Hematology, HCG Cancer hospital, Bangalore, India

Aims \& Objectives: We submit a case report on febrileneutropenia caused by a rare bacterial species in patient diagnosed with AML.
Patients/Materials \& Methods: Retrospective chart review.

Results: A 40 year old female patient diagnosed with AML on treatment with induction $3+7$ regimen developed fever spikes in a "on and off" pattern on $6^{\text {th }}$ day of induction chemotherapy infusion. Complete blood count reported that patient's neutrophil count was $<100 /$ cells $/ \mu \mathrm{L}$ percentage when she developed fever. Blood cultures from blood an durine were sent for suspected organisms before she was started on empirical antibiotic treatment with colistin and meropenem. Blood cultures revealed that suspected organism causing bacteremia in this patient is pantoeaagglomerans. Based on the minimum inhibitory concentrations, we de-escalated the antibiotics to amikacin and meropenem. Patient was treated with antibiotics for the duration of 14 days and then discharged.

Discussion \& Conclusion: Pantoeaagglomerans is an agricultural organism that inhabits plants, soil, and water. According to previous reports, it is both commensal and pathogen of animals and humans. Although, secondary bacteremia and nosocomial infections with contaminated medical equipment, rare is the occurrence of spontaneous infections in immunocompromised cancer patients. We conclude that pantoeaagglomerans should be considered a bacterial threat in cancer patients who are undergoing a highly myelotoxic chemotherapy and should be treated before it causes severeinfection.

\section{Evaluation of Platelet Parameters in Serologically Positive Dengue Patients Based on Degree of Thrombocytopenia: An Institute Based Cross Sectional Study}

NiraimathiManickam ${ }^{* 1}$, IndiraGunasekaran ${ }^{2}$, RevathiM.Girish ${ }^{2}$,AnitaRamdas ${ }^{2}$

${ }^{1}$ Pathology, JIPMER, Karaikal, Karaikal, ${ }^{2}$ Pondicherry Institute of Medical Sciences, Puducherry, India

Aims\&Objectives:Theobjectiveofthisstudyistocorrelat evariousdegreesofplateletcountwithitsindicesindenguepatients. Patients/Materials\&Methods: This retrospective cross sectional study was conducted during dengue epidemic (October'20) in all serologically positive (NS1 or IgM positive) dengue patients in Pondicherry Institute of Medical Sciences. Patients were grouped according to the lowest platelet count during the hospital stay into very severe $(20 \times 103$ cu.mm \& less $)$, severe $(21 \times 103$ to $50 \times 103$ cu.mm), moderate $(51 \times 103$ to $100 \times 103$ cu.mm $)$ and mild $(100 \times 103$ to $150 \times 103$ cu.mm $)$ thrombocytopenia. Mean platelet parameters were analysed based on degree of thrombocytopenia along with correlation coefficient (r).

Results: A total of 105 patients were studied. Patients with very severe thrombocytopenia had the lowest mean MPV, PCT while PDW was highest (Table 1). PCT showed strong positive correlation with various degrees of thrombocytopenia while PDW showed negative correlation. On comparing MPV of pre \& post platelet transfusions, very severe thrombocytopenia with early recovery exhibited initial trend of decrease inMPV.

Discussion \& Conclusion: Discussion: There are various causes of thrombocytopenia hypothesized in dengue which are marrow suppression, destruction by antiplatelet antibody and peripheral consumption. Platelet parameters reflect the state of platelet activation and morphology during the course of illness that can be used to predict the recovery of platelet in patients with dengue fever. Serial measurement of Platelet counts with its change in indices give much information on disease courser at herthan much studied single value assessment. Correlation of these indices with various degree of platelet counts along with pre \& post transfusion changes helps in understanding the relationship among various platelet parameters with underlying the pathophysiology of dengue fever. Conclusion: 
Following the trends of platelet parameters during course of illness along with platelet count correlation helps in assessing the prognosis and response to treatment in dengue patients.

\section{Neutrophil to Lymphocyte Ratio (NLR): A Predictor of Outcome in Indian Covid-19 Patients}

\author{
ParulBarya $^{* 1}$, S. K. Nema ${ }^{1}$, Romi Shrivastava ${ }^{1}$ \\ ${ }^{1}$ Pathology, Index medical college, Indore, Indore, India
}

\begin{abstract}
Aims \& Objectives: Aims: To study the significance of NLR in outcome of COVID-19 patients. Objectives: To set the exact value of NLR of defining the severity of COVID-19 patients. To use NLR value for defining management protocol of COVID-19 patients. To
\end{abstract} use NLR value as prognostic marker in COVID-19 patients.

Patients/Materials \& Methods: 52COVID-19 patients admitted in our institute since May 2020, diagnosed by RT-PCR technique from naso-pharyngeal swab were included in study.

Clinical, demographic and laboratory data collected retrospectively. NLR was calculated by using absolute counts of each patient.

NLR $=$ Absolute Neutrophils count/Absolute Lymphocytes count. Results: Out of 52 patient analyzed $32(65 \%)$ were male, median age of infection was 51 year (12-78 years). 10 out of 52(19.2\%) patient had hypertension and 5 out of 52(9.6\%) patient had diabetes and one patient had bothasapre-existingco-morbidities. The median NLR was 4.15 .9 out of $52(17.3 \%)$ patient required ICU admission. Death of $4(7.6 \%)$ patient registered and 48 discharged after recovery. 8 out of $9(88.9 \%)$ who required ICU admission had NLR $>4$ and all 4 patient expired had NLR $>4$.

Discussion \& Conclusion: In our study NLR predicting the severity of infection as 8 out of 9 patient who admitted in ICU had NLR $>4$ and four patient who expired had NLR $>4$. So high NLR predicting the severity of infection and ICU admission retrospectively. NLR $<3$ predicting the less severity and improvement of COVID-19 infection. Although further studies with a larger sample size will be needed to properly assess this matter, in our study shows that NLR may be a rapid, less expensive, useful prognostic factor for categorizing severity and outcome of COVID-19 patients.

Supporting Document:

\section{Hematological Parameters in Covid-19 Illness: Emphasis on Platelet Indices as a Marker of Severity of Disease}

ChandniBhandary $\mathbf{P}^{* 1}$, Prattipati L. Agarkar ${ }^{1}$, PrabhuManivannan ${ }^{1}$, RakheeKar $^{1}$, DebdattaBasu ${ }^{1}$

${ }^{1}$ Pathology, JIPMER, Pondicherry, India

Aims\&Objectives: To study the hematological parameters and platele tindices and correlate with the severity of disease in COVID-19 positive patients.

Patients/Materials \& Methods: A record based study conducted in a tertiary care centre included 125 COVID-19 positive patients diagnosed by either RT-PCR orrapidantigentest. The clinical data was retrieved from the case records and the cases were categorized as mild, moderate, severe and critical based on the consensus guidelines. Hematological parameters studied were hemoglobin, RBC indices, total leucocyte count, neutrophil lymphocyte ratio (NLR), platelet count, platelet indices (plateletcrit, platelet distribution width, mean platelet volume and platelet large cell ratio) and D-dimer. Each of the parameters were correlated with these verity of the disease and statistical analysis was done using IBMSPSS software.
Results: The mean age was 53.3 years (range: 3-96 years) with male preponderance observed in $62.3 \%$ of cases. Of the 125 cases, 57 patients were categorized as mild disease, 42 moderate, 16 severe and 10 with critical illness. Cases with severe and critical COVID-19 illness were associated with eosinopenia and neutrophillymphocyte ratio more than 3.3 ( $p$ value of both $<0.05$ ). Platelet count was normal in $69 \%$ cases. Of the 39 patients with thrombocytopenia, as many as $59 \%$ had mild reduction (1.0-1.5 lakh/cumm). Among the various platelet parameters plateletcrit (PCT) was significantly lower in mild COVID illness in comparision with moderate to critical disease $(p<0.05)$ while mean platelet volume and platelet distribution width was higher in severe and critical COVID illness ( $p$ value $<0.05)$. PLCR did not show any statistical significance. Elevation of d-dimerlevels positively correlated with the severity of the disease. Patients with mild COVID-19 illness had mean D-dimer level of $0.86 \hat{A} \mu \mathrm{g} / \mathrm{dl}$ while patients with critical illness had $4.01 \hat{\mathrm{A}} \mu \mathrm{g} /$ dl.

Discussion \& Conclusion: In addition to the known hematologic paratmeters implicated in COVID-19 illness such as NLR, eosinopenia and D-dimer, platelet parameters (MPV, PDW and PCT) can also be used as biomarkers that indicate the severity of the disease.

\section{The Benign Masquerader: Two Case Reports of Chronic Epstein Barr Virus Infection-Related Lymphoproliferative Disease}

Surabhi Jain ${ }^{* 1}$, SaumyaranjanMallick ${ }^{1}$, Prashant Ramteke ${ }^{1}$, Manish Soneja ${ }^{2}$, A B Dey ${ }^{3}$, Ajay Gogia ${ }^{4}$, Sameer Bakhshi ${ }^{4}$, Mehar C. Sharma ${ }^{1}$

${ }^{1}$ Pathology, ${ }^{2}$ Internal Medicine, ${ }^{3}$ Geriatric Medicine, ${ }^{4}$ Medical Oncology, AIIMS, New Delhi, India

Aims \& Objectives: Here we describe two cases of Chronic Epstein Barrvirusrelatedlympho proliferative disease in two adult males.

Patients/Materials \& Methods: A 29-year old and a70-year old elderly patient both presented with complaints of fever and loss of weight for more than three months duration and with out any localising symptoms. They were diagnosed aspyrexiaofun known origin; however despite multiple therapies and investigations, the causative factor could not be ascertained. Both had generalised lymphadenopathy along with hepatosplenomegaly.

Results: Hemogram showed pancytopeniain the elderly with mildanemiain the young adult. Liver enzymes were on arisingtrend in the young male suggestive of hepatitis. Radiographic investigations confirmed the presence of heopatosplenomegaly and metabolically active nodes. Nodal biopsy from the young male showed maintained architecture with paracortical expansion and presence of non-necrotising granulomas. The lymphocytes were immunopositive for CD4(++),CD8(+),CD56(+),TIA(+),EBV-LMP1 and EBER. However, partial effacemen to farchitecture by multiple vaguenodules comprising of polymorphous population of histiocytes, plasma cells, mature lymphocytes and few large atypical cells was noted in the elderly. The large cells were immuno positive for CD30, CD20 and EBV-LMP1 with a background CD3 + T-cell population; on which a differential of Hodgkin lymphoma was given. However, the symptoms subsided with nonewlesions without any therapy after which quantitative-PCR for EBV was done and was indicative of high DNA titres $(>2 \times 100000$ copies). Bone marrow and liver biopsies were performed in the young male and showed increased lymphocytes and sinusoidal lymphoidaggregates (nonspecific hepatitis) respectively which were EBER positive. Flow cytometry in the young adults ho edapolyclonalB-cell population, normal CD4:CD8T-cell ratio and an increase in NK-cell population which showed a dim CD56 and strong 
CD94 expression. There was no evidence of any immunod eficiency or any other malignancy. Though the disease was cryptic in nature with a typically mphoproliferative diseaseas the main possibility; multimodality approach confirmed the diagnosis of systemic chronicactive EBV infection satisfying the WHO criteria.

Discussion \& Conclusion: CAEBV is a rare disease caused by the EBV infection. The diagnostic difficulty laysinthenon specific histopathologic and immunopheno typic features that overlap with infectious and inflammatory conditions as well as with T-and NK-cell LPDs. However, a multimodality approach is required for the correct diagnos is since the disease can rapidly turnfatal.

\section{To Study the Hematological Parameters in Covid-19 Positive Cases in Different Clinical Categories}

SeeratParmar ${ }^{* 1}$, Abhinav S. Kumar ${ }^{2}$, MeghaliDhebane ${ }^{3}$, Manish Raturi $^{4}$, Sohaib Ahmed ${ }^{5}$, KanikaMunish ${ }^{2}$, Mansi Kala ${ }^{4}$, AnuradhaKusum $^{6}$

${ }^{1}$ Resident Pathology, ${ }^{2}$ Senior Resident Pathology, ${ }^{3}$ Assistant Professor, ${ }^{4}$ Associate Professor, ${ }^{5}$ Professor General Medicine, ${ }^{6}$ Professor, Himalayan institute of medical sciences, Jolly Grant, Dehradun, India

Aims \& Objectives: Since it's origin in December 2019 Novel Corona virus infection has behaved in an unprecedented manner. A number of studies had been done so far to find out peculiar characteristics about this disease. However, few studies have shown varied hematological manifestations of this which have been found to have prognostic implications. In this study we aim to assess the hematological parameters of COVID-19 positive cases and in their different clinical categories (mild, moderate and severe).

Patients/Materials \& Methods: It is an observational study done in Himalayan Institute of medical sciences, Dehradun. (1) Number of cases included were 118 over 3 months duration (July, August, September-2020). (2) The relevant clinical details of all the COVID19 positive patients such as name, age, UHID were recorded. (3) EDTA sample was run on DXH800 (Beckmencoulter automated analyzer) and all parameters were recorded including the VCS. Inclusion criterion: Patients diagnosed with COVID-19 on Rapid antigentestor RTPCR of all age groups. Exclusioncriterion:Patientswithmissinghematologicalparameters.

Results: In our study, total number of cases included were 118, out of which $84(71.18 \%)$ were males and $34(28.81 \%)$ were females. Patients above 60 years were 35 (29.6\%). The patients were categorized into mild, moderate and severe on the basis of clinical findings and those findings were co-related with the VCS parameters. The significant findings include decreased absolutely mphocytecount in $43.2 \%$ cases and absolute eosinopenia in $83.05 \%$ of cases, absolute neutrophil count was increased in $44.9 \%$ cases and platelet count was reduced in $30.5 \%$ of the cases.

Discussion \& Conclusion: In major number of cases absolute lymphocyte count, absolute eosinophil count and neutrophil to lymphocyte ratio was found significant; so it is advised to categorize patients on the basis of clinical findings and related VCS parameters for early COVID-19 testing.
Reactive Hemophagocytosis Lymphohistiocytosis Associated with Thrombotic Thrombocytopenic Purpura Secondary to Dengue, CMV and EBV Infection

Ashimamishra $^{* 1}$, SagnikaTripathy ${ }^{1}$

${ }^{1}$ Internal Medicine, VIMSAR, BURLA, India

Aims \& Objectives: Secondary hemophagocytosislymphohistiocytosis(HLH) and Thrombotic Thrombocytopenic Purpura (TTP) are rare entities and can be fatal if not diagnosed and treated promptly. The clinical spectrum overlaps with sepsis and SIR Soften leading to delayed identification. We reporta case of virus induced HLH and TTP in a patient who had acute dengue and cmv infection and long standingebv infection who was extensively investigated and monitored. The aim of this case reportis to explore the possibility of such unusual hematological complications and its treatment protocol in the seviral infections.

Patients/Materials \& Methods: A young female, presenting with fever, vomiting, myalgia, shortness of breath, abdominal distension, skinrashes, jaundice, pallor, bleeding manifestations, lympadenopathy, hepatosplenomegaly had positive serologies for dengue IgM, CMVIgM, EBVIg Gand was given symptomatic care but she progressed to dengue hemorrhagic fever grade 3. Investigations revealed persistent pancytopenia, abnormal liver functiontests, normal ESR, hyperferritenemia, hypofibrinogenemia, hypoproteinemia, elevated lactatedehydrogenase, normalhaptoglobulin, negative coombs, elevated reticulocyte count, features of schistocytes on peripheralsmear. Bone marrow revealed erythroidhyperplasia with hemophagocytosis. A diagnosis of reactive HLH secondary to dengue, CMV andchronic EBV along with virus induced TTP was made. The patient was started on aggressive fluid resuscitation, multiple platelet sandcryoprecipitates, steroids, antiviral sand immunoglobulin and showed improvement in 1 week.

Results: Dengue, CMV, EBV can rarely precipitate HLH and TTP. CMV, EBV, Dengue coinfectionled to severe immunopathological dysfunction in this case.

Discussion \& Conclusion: Any prolonged febrile illness with severe or unusual progression of symptoms, should be evaluated for HLH.TTP can complicate viral infections. Treatment should be directed towards triggering disease and also include immunosuppression to prevent mortality.

\section{Evaluation of Hematological Parameters in Covid-19 Infected Patients}

TanimaDwivedi $^{*}$, Ritu Gupta ${ }^{2}$, BiswajeetSahoo ${ }^{1}$, Pawan Tiwari $^{3}$, Anant Mohan ${ }^{4}$, Saurabh Vig ${ }^{5}$, Sushma Bhatnagar ${ }^{6}$

${ }^{1}$ Laboratory Oncology, NCI-AIIMS, Delhi, ${ }^{2}$ Laboratory Oncology, AIIMS, New Delhi, ${ }^{3}$ Pulmonary, Critical Care and Sleep Medicine, AIIMS, Delhi, ${ }^{4}$ Pulmonary, Critical Care and Sleep Medicine, AIIMS, New Delhi, ${ }^{5}$ Onco-Anaesthesia and Palliative Medicine, NCI- AIIMS, Delhi, ${ }^{6}$ Onco-Anaesthesia and Palliative Medicine, AIIMS, New Delhi, India

Aims \& Objectives: The present study was undertaken to evaluate prognostic relevance of hematological parameters in COVID-19 infected patients for early identification of critical patients for early management.

Patients/Materials \& Methods: A retrospective study was conducted over a period of 2 months (01st July 2020 to 1st September 2020) on 912 admitted COVID-19 positive patients. $2 \mathrm{ml}$ venous blood was aseptically collected in EDTA vaccutainer and processed in automated hematology analyser (ADVIA 2120i). Patients with 
hematological malignancy were excluded from the study. Patients were assigned into three groups according to the severity of disease (mild/asymptomatic, moderate and severe) as well as on the basis of clinical outcome (discharged ordeath).

Results: To talleucocyte count, absolute neutrophil count, neutrophillymphocyte ratio, plateletlymphocyte ratio, reddistribution width were significantly higher $(p<0.05)$ is the decreased patients and in severe disease group where as hemoglobin and hematocr it was significant lower in these patients. Lymphopenia was recorded in decreased patient but was not statically significant.

Discussion \& Conclusion: COVID-19 patients with severe disease and poor outcome showed significant difference in ther outine hematological parameter such as total leucocyte count, absolute neutrophil count, absolute monocyte count, neutrophillymphocyte ratio, plateletlymphocyte ratio, reddistribution width and hematocrit which are readily available and can be helpful in the prediction of COVID-19 patien toutcome.

\section{A Case of Secondary Hemophagolymphohistiocytosis Post SARS Cov2 Infection}

VaishaliNagpal $^{* 1}$, Shubha Sudhakar ${ }^{1}$, Sahana K S ${ }^{2}$, Rajesh Krishna ${ }^{3}$

${ }^{1}$ Pathology, ${ }^{2}$ Paediatrics, Yenepoya Medical College Hospital, ${ }^{3}$ Consultant Hematologist, Yeneoya Medical College Hospital, Mangalore, India

Aims \& Objectives: Introduction. Coronavirus SARS-CoV-2, has been associated with a hyperinflammatory state. This has been described as a form of secondary haemophagocyticlymphohistiocytosis that may contribute to increased mortality and is characterised by a fulminant and fatal hypercytokinaemia with multiorgan failure. In adults, HLH is most commonly triggered by viral infections and sepsis. Cardinal features of HLH include unremitting fever, cytopenias, and hyperferritinaemia; pulmonary involvement (including ARDS).We report a case where secondary HLH was noted in a patient with COVID19 infection.

Patients/Materials \& Methods: Case Report. A 6 year old female child was admitted to the hospital with complaints of fever since 3 days. Fever was high grade in nature and of sudden onset with no previous respiratory symptoms. During her stay she developed nonproductive cough. The patient also had an episodeofhematemesis followed by 2 episodes offres hblood perrectum and 1 episode of seizure.Onphysicalexaminationpatient had tachypnea with pallor andhepatomegaly. Hematological parameters were estimated using Automated Sysmex XN-1000 and Biochemical parameters using Vitros 5600 fully automated chemistry analyzer.

Results: $\mathrm{Hb}$ was $5.1 \mathrm{gm} / \mathrm{dl}$, TLC was $21.2 \times 10^{3}$ and platelet count was $11 \times 10^{3}$. Peripheral smear examination showed normocyticnormo chromicanemia with neutrophilicleucocytosis and thrombocytopenia.Clotting profile and creatinine levels were with in normal range. LFT was deranged with an elevated ESR, LDH,DDimer and serumferritin levels.USG showed mildascites and bilateralminimalpleuraleffusion. MDCT revealed Bilateralconsolidation with airbronchogram. Dengue SPOT and IgG for SARSCoV2 was interpreted as positive. Bone marrow showed few large foamyhistiocytesexhibitingphagocytosis of blood cells. Hence the cytopenias were presumed to be due to increased peripheral destruction. Weilfelix reaction showed decreased titre and the patient was diagnosed to have Rickettsialfever. Patient was transfused with packed RBCS and platelets. She was started on antiepileptics, antibiotics and exoxaparinprophylactically. Dexamethasone was also given for 11 days for the management.

Discussion \& Conclusion: Conclusion: Mortality is increased in patients with Secondary HLHin COVID19. Screening for HLHis recommended in COVID19 patients presenting with bicytopeniaorpancytopenia.

\section{Neutrophil Oxidative Burst in HIV-Infected Children: A Case Control Study}

Nama Habib Ur Rehman ${ }^{* 1,2}$, Pooja Dewan ${ }^{1}$, Sunil Gomber ${ }^{1}$, Richa Gupta $^{2}$, AlpanaRaizada ${ }^{3}$

${ }^{1}$ Pediatrics, ${ }^{2}$ Pathology, ${ }^{3}$ Medicine, University College of Medical Sciences, New Delhi, India

Aims\&Objectives: Aim:To assess the effect of anti-retroviral therapy on neutro philphagocytic functions in HIV-infected children. Objectives: (1) To compare the neutrophil oxidative burst as determined by dihydrorhodamine (DHR) assay between HIV-infected children and healthy controls. (2) To correlate DHR assay with CD4 count, viral load, absolute neutrophil count (ANC), neutrophilto lymphocyteratio (NLR) and duration of anti-retroviral therapy (ART) in HIV-infected children.

Patients/Materials\&Methods: We evaluated 61 children aged between 18 months and 15 years consisting of 31 HIV-infected children receiving ART $>2$ years duration and 30 age-matched healthy controls, who were HIV-negative siblings of HIV positive children. We excluded children with critical illness, acute bacterial infection, chronic diseases, hepatitis $\mathrm{B} / \mathrm{C}$ co-infection, those receiving antibiotics, steroids $>1$ month duration, anti-tube rculousor Isoniazid preventive therapy. Baseline assessment included detailed history, clinical examination and laboratory evaluation for which $2 \mathrm{ml}$ of peripheral venous blood sample was drawn in EDTA vacuta inerfor estimation of hemogram and DHR assay (byflowcytometry).

Results: Out of 61 participants, 35 were boys and 26 were girls. Both groups had similar gender distribution. The mean (SD)ofHIV-infected children was 96.3 (37.7) months and that of healthy controls was 99.8(38) months, $p=0.72$. The median (IQR) CD4 count, viral load and duration of ART in the HIV-infected children was 1108(865-1510)cells $/ \mathrm{mm}^{3}, 0(0-5)$ copies $/ \mathrm{ml}$, and 40(29-62) months. The mean (SD) stimulated DHR was significantly higher in healthy controls [263.43(70.86)] as compared to HIV-infected children on ART [212.29 (126.99)]; $p=0.03$. The two groups had statistically comparable mean (SD) absolute neutrophil counts [3649 (1560) vs 4511 (1852); $p=0.05]$ and NLR [1.31(0.77) v/s 1.47(0.50); $p=0.33$. There was no correlation between stimulated DHR and ANC or CD4 count. There was a significant correlation between stimulated DHR assay and viral load $(\mathrm{r}=0.49 ; p=0.02)$.

Discussion \&Conclusion: Neutrophils from the HIV-infected childrenon ART exhibited significantly diminishedoxidative burst activity as compared to healthy control siblings although the ANC was statistically comparable. This suggests neutrophilsin HIV-infected children are dysfunctional which makes them more vulnerable to infections. 
Neutrophil/Lymphocyte Ratio an Important Prognostic Marker in SARS Cov 2 Pneumonia for Initiating LMWH and Steroid Therapy

\author{
Muzil Munshi ${ }^{* 1,2}$, Anu Singh ${ }^{3,4}$, Imran Patel $^{5}$, Rajesh Dere ${ }^{6,7}$ \\ ${ }^{1}$ Intensive Care, BKC Covid Centre, ${ }^{2}$ Cardiac Surgery, Aisn \\ Heart Institute, ${ }^{3}$ Hematology, BKC Covid Centre, \\ ${ }^{4}$ Hematopathology, Actrecmumbai, ${ }^{5}$ Clinicalresearch, ${ }^{6}$ Dean,BKC \\ Covid Centre, ${ }^{7}$ Forensic Medicine-HOD, Lokmanya Tilak \\ Hospital and Medical College, Mumbai, India
}

Aims \&Objectives: In this study we analysed the predictability of Neutrophil/Lymphocyteratio in patient with severe SARS-CoV-2 Acute Respiratory Distress Syndrome and role of steroid (Dexamethasone and Methylprednisolone) in controlling transition of inflammatory phase of pneumonia to hyper inflammatory phase ofARDS.

Patients/Materials \& Methods: This retrospective observational study includes $>250$ patients admitted in BKCCOVID Centre Mumbaifrom 1st September onwards (ongoing) who are RT-PCR positive for SARSCoV2 and presented with pneumonicpatchin Xray and breathlessness. All these patients were treated with standard of care treatment special with steroids (Dexamethasone $6 \mathrm{mg} /$ day or Methylprednisolone $80 \mathrm{mg} \mathrm{BD}$ ) and LMWH. Patients with high N/L Ratio $>5$ with GGOs in Xray were put on steroidsplus LMWH and amoxicillineplusclavulanic acid. While those who on admission with N/L $>7$ with distress were considered for MPSpulse therapy.

Results: Patient who were having N/L ratio $>7$ without any coverofsteroids and LMWH had poor disease outcome as compared to patients who are on steroids and LMWH. While those who were $\mathrm{N} / \mathrm{L}>5$ but were on Steroids and LMWH had better outcome even after increase in N/L ratio.

Discussion \&Conclusion: Patients with N/L $>5$ should be started with steroids with LMWH with broad spectrum antibiotics, while those with $\mathrm{N} / \mathrm{L}>7$ with respiratory distress should be considered for MPS pulsetherapy.

\section{Infection-Proof Fabrics to Prevent Secondary Infections in Immunocompromised Cancer Patients}

\author{
Yatee Gupta $^{* 1}$, Amar Ranjan ${ }^{2}$ \\ ${ }^{1}$ Fabiosys Innovations Pvt. Ltd., IIT Delhi, Delhi, ${ }^{2}$ IRCH, AIIMS \\ New Delhi, Delhi, India
}

Aims \& Objectives: Patients undergoing chemotherapy and radiotherapy are immunocompromised and become prone to severe secondary infections. One of the major modes of acquiring these infections is through direct contact with the contaminated surfaces. Textiles are one of the most abundant surfaces surrounding a patientinany health carefacility. It is well known that these textile surfaces such as bedsheets, uniforms and curtains are often a source of infections, especially in crowded healthcare facilities of developing countries like India \& often lead to Hospital Acquired Infections (HAIs) which prove fatal to immuno compromised patients. To tackle this problem, an Infection-proof fabric has been developed which has Durable Self-Sanitizing nature and capability to kill $99.9 \%$ of the pathogenic bacteria such as MRSA presenton its surface in Hospital wards. The fabric has been manufactured keeping inmind the everyday laundry requirements of the patient and the maintenance cycle of the laundry bythe hospitals.

Patients/Materials \& Methods: A statistical distribution of the type of pathogenic bacteria present on the bedsofin patients was studied using the secondary data from research publications. It was confirmed that there is a growing incidence of ESKAPE pathogens \& most commonly MRSA which are pathogenic and difficult to kill even by the common disinfectantsused. In order to tackle these pathogenic bacteria like MRSA, the Infection-proof fabricis designed. The basic cotton textile substrateis taken and chemically processed with proprietary nanomaterials and molecular antimicrobials to make the fabric wash-friendly, comfortable, non-toxic and biocompatible for the humanskin. This fabric is designed to be affordable for the middle-class Indian population using the affordable processing technology developed by IIT Delhi incubated company Fabiosys Innovations Pvt. Ltd.

Results: The fabric was tested as per the AATCC 100 Standards which gives the quantitative assessment of the degree of Antibacterial activity of the fabrics. The tests were done by Eurofins Product Testing India Pvt.Ltd. which showed that the fabric was abletokill $>99.9 \%$ of the resistant bacteria, ATCC6538 \& ATCC15380. The results showed that the number of colonies reduced from 470000 to 240 in case of ATCC6538 \& from 750000 to 420 in case of ATCC 15380 which resulted in the reduction of $99.95 \%$ and $99.94 \%$ respectively.

Discussion \& Conclusion: Usage of Infection-proof fabrics by the immuno compromised patients will prove useful for them in reducing both secondary infections acquired by them and possibly their hospital stay. Ournextgoal is to evaluate the relationship between decontamination of textile surfaces surrounding the patients subjected to chemotherapy \& radiotherapy and secondary infections acquired by them with a clinical study.

\section{Effect of Covid-19 on Hematological Services}

Bilal S. Kazi ${ }^{* 1}$, Prakash S. Shekhawat ${ }^{1}$, SumitMitra ${ }^{1}$, Subham Bhattacharya $^{1}$, Shuvra N. Baul ${ }^{1}$, Prakas K. Mandal ${ }^{1}$, Rajib De ${ }^{1}$, Tuphan K. Dolai ${ }^{1}$

\section{${ }^{1}$ Hematology, NRS Medical College, Kolkata, India}

Aims \& Objectives: To study the effect of COVID 19 on different hematological services.

Patients/Materials \& Methods: This observational study included 18 patients evaluated from June 2020 till September 2020 belonging to various age groups with a WHO defined hematological disease with laboratory-confirmed and symptomatic COVID-19. The primary outcome was mortality and evaluation of COVID-19 severity in different hematological diseases assessed on day 14 and 28. Patient characteristics, type of disease, symptomatology, HRCT findings and baseline qCRP levels were recorded and the need for oxygenation or in vasiveventilation with administration of various drugs and convalescent plasma and its consecutive effect in delay or nonadministration of chemotherapyevaluated.

Results: A total of 140 hematology patients were admitted of which 18 patients $(12.85 \%)$ were COVID-19 positive. 10 cases $(55.55 \%)$ were of B cell Acutelymphoblasticleukemia (B-ALL), 3(16.66\%) of acutemyeloidleukemia (AML) and $1(5.55 \%)$ each of acute promyelocytic leukemia (APL), T cell acute lymphoblastic leukemia (TALL), T- Lymphoblastic lymphoma (T-LBL), Hodgkins lymphomaandaplasticanaemia. Majority of patients had mild COVID-19 infection $(72.22 \%, \mathrm{n}=13), 4$ cases $(22.22 \%)$ were severely affected and 1 moderately affected. There was delay in administration in chemotherapy in 14 cases(77.77\%), while 3 patients continued to receive chemotherapy during the viralillness. Survival at day 14 and 28 in B-ALL was $100 \%$, survival in AML on day 14 and day 28 was $66.6 \%$, survivalin T-ALL on day 14 was $100 \%$ and $0 \%$ on day 28 , survivalin APL and T-LBL on day 14 was0\%, survivalin Hodgkinslymphomaandaplasticanemia on day 14 and day 28 was $100 \%$. 
Discussion \& Conclusion: The results indicate that $12.85 \%$ cases were COVID-19 positive. $72.22 \%$ had mild COVID-19 infection and $22.22 \%$ had severe COVID-19 infection. The most common affected was B-ALL (55.55\%). In $77.77 \%$ cases there was delay in administration of chemotherapy.

\section{Spectrum of Bacterial Infection from Hematological Setting in Tertiary Care Center in Eastern India}

Kusumita Mandal ${ }^{* 1}$, Malini Garg ${ }^{2}$, Prakash S. Shekhawat ${ }^{2}$, Arnab Chattopadhya $^{2}$, Prakash K. Mandal ${ }^{2}$, Rajib De ${ }^{2}$, Shuvra N. Baul ${ }^{2}$, Subham Bhattacharya ${ }^{2}$, SumitMitra ${ }^{2}$, Shyamali Dutta ${ }^{2}$, Tuphan K. Dolai $^{2}$

${ }^{1}$ Hematology, NRS Medical College and Hospital, ${ }^{2}$ Hematology, NRSMCH, Kolkata, India

Aims \&Objectives: To study the rate of blood culture positivity of the febrileneutropenic patients of different hemalological disorders and common causative organisms.

Patients/Materials \& Methods: Retrospective analysis of data of 52 patients of hematological disorders with febrileneutropenia during their hospital stay from Aprilto October 2020 was done. Culture from blood or central line tip were sentand tested in Bactalert 3D Biomeriuxanalyzer.

Results: Fifty-two cultures were sent and 22 samples came positive $(42.4 \%)$. Out of 22 positive culture, Acinetobactor sp. found in $6(27.2 \%)$, Klebsiellapneumoniae in 6(27.2\%), MRSA [Methicillinresistantstaphylococcusaureus] 2(9\%), Pseudomonusaeruginosa 2(9\%), Staphylococcusepidermidis 2(9\%), Candidasp. 2(9\%), Staphylococcushemolyticus $1(4.5 \%)$, and Stenotrophomonus maltophilia $1(4.5 \%)$

Discussion \& Conclusion: Rate of positivity in clinical sepsis is 42.4\%.Most common causative organisms were Acinetobactor sp. and Klebsiella pneumoniae.

\section{ICU Mortality in Neutropenic Sepsis}

Amey C. Panchal ${ }^{* 1}$, Sachin S. Jadhav ${ }^{1}$, Anjali Matani ${ }^{1}$, Vijay Krupesh $^{2}$, Vishnupriya $\mathrm{M}^{1}$, JyothiGoutham Kumar ${ }^{3}$, NishitOjha ${ }^{1}$

${ }^{1}$ Haematology and BMT, ${ }^{2}$ Intensive care unit, ${ }^{3}$ Clinical pharmacologist, Healthcare global enterprises limited, Bengaluru, India

Aims \& Objectives: To determine the determinants of mortality in patients with febrileneutropenia who required Intensive Care Unit (ICU) admission.

Patients/Materials \& Methods: Retrospective chart review of unselected patients, admitted for the treatment of hematologic malignancies and hematopoietic stem cell transplantation inatertiary care Center between September 2019 and August 2020. Analysis was done of those patients who had been transferred to the ICU for severesepsis.

Results: 143 patients with hematologicmalignancies were treated at our Centerduring this period. They had required 578 admissions. $347 / 578(60 \%)$ of the admissions were for neutropenic fever. $46 / 347(13.26 \%)$ admissions required ICU transfer. $4 / 46(8.7 \%)$ took inadequate treatment and were discharged against medical advice and were excluded from this analysis. The remaining 42 admissions received appropriate ICU treatment. Majority of the patients $(50 \%)$ who required ICU transfer had a primary diagnosis of leukemia. $33 / 42(78.57 \%)$ of ICU transfers were $>40$ years of age. This was significantly higher than those who were less than 40 years of age $(p=<0.00001) .14 / 42(33 \%)$ of the ICU patients died. Higher mortality was observed with high-dose chemotherapy regimens for leukemia and lymphoma salvage compared to low dose treatment regimens for myeloma and lymphoma $(p=0.0429)$. Mortality was also higher in patients who were older than 20 years of age, although this was not significant $(p=0.71)$.

Discussion \&Conclusion: Among the patients who are admitted for high-risk febrileneutropenia, those who are $>40$ years of age have a higher risk of requiring ICU care $(p=<0.00001)$. Among those who were transferred to the ICU, mortality was higher for those who have received high-dose chemotherapy compared to those who had received low-dose chemotherapy $(p=0.0429)$.

\section{Hematological Manifestations of Indian Patients Presenting with COVID 19}

Preethi Jeyaraman *1, Pronamee Borah ${ }^{2}$, Omendar Singh ${ }^{3}$, Arun Dewan $^{4}$, Rahul Naithani ${ }^{1}$

${ }^{1}$ Hematology and BMT, ${ }^{2}$ Max Superspecialty Hospital, New Delhi, India, ${ }^{3}$ Critical care, Max Superspecialty Hospital, ${ }^{4}$ Internal Medicine, Max SMART Superspecialty Hospital, New Delhi, India

Aims \& Objectives: Severe acute respiratory syndrome corona virus 2 (SARS-CoV-2) causing corona virus disease 2019 (COVID-19) can have significant impact on the haematological system in the form of cytopenia and disseminated intravascular coagulation. We intended to study the incidence and pattern of hematological involvement in patient with COVID 19. We also To study the association of hematological involvement with severity and outcomes like death and ICU stay.

Patients/Materials \& Methods: This retrospective study was conducted at Max Superspecialty Hospital, Saket, NewDelhi. All patients $>18$ years of age and who are admitted with COVID19 infection from 1st May 2020 to 1st June 2020 were enrolled in the study.

Results: A total of 440 patients were included in the study. The mean age of the study population was $47.57+15.83$ years. Two-third of patients were males. Fifity percent of patients had at least 1 underlying comorbidity. Mean duration of hospitals stay was $11.45+4.45$ days. Lymphopenia was observed in $24.3 \%$. Eosinopenia was seen in $44.3 \%$. Leukocytosis was seen in $8.2 \%$. Prolonged PT was observed in $60.5 \%$ of patients. Overall mortality was $3.40 \%$. Need for ICU stay was seen in $28.40 \%$. Severe disease was seen in $19.7 \%$. Age and presence of comorbidity was associated with severe disease, need for ICU stay and mortality. Male sex was associated with need for ICU stay and severe disease. Neutrophil Lymphocyte ratio was high in patients having severe disease, ICU admission and mortality. Multiple regression analysis showed that Ferritin and IL6 predicted mortality.

Discussion \& Conclusion: COVID19 infection is associated with significant changes in haematological system.

\section{Outcome of DLBCL After First Line Chemotherapy : Study in A Cancer Institute}

Zulfia Z. Chowdhury ${ }^{* 1}$, Tamanna Bahar ${ }^{1}$

\section{${ }^{1}$ NICRH, Dhaka, Bangladesh}

Aims \& Objectives: Diffuse large B-cell lymphoma (DLBCL) the single largest category of lymphoma, is a heterogeneous group of lymphomas with several morphologic features. Objective of this study is to know the outcome and survival of subtypes of DLBCL after firstline chemotherapy. 
Patients/Materials \& Methods: This is a retrospective study which includes all patients age $>18$ years, diagnosed with DLBCL and registered at the department of Hematology of National Institute of Cancer Research and Hospital, Bangladesh, between July 2016 to June 2019. We excluded secondary lymphoma, HIV associated lymphoma and patients with incomplete record.The Patient were classified as germinal center B cell like or activated B cell type using the Hans algorithm. Complete response, partial response, refractory, relapse were categorized according to international working group criteria for malignant lymphoma. Analysis carried out using SPSS version 23

Results: Total 126 cases were included in this retrospective data analysis. According to immune-histochemistry we divided the cases into three molecular sub categories to see the outcome and survival and their differences. We found non-GCB (Germinal Center B cell) types DLBCL were significant ( $p$ value: 0.004 ) and the prevalent one $(46 \%)$. Mean age was 47 years with a Standard deviation of 15 years. Males $(70.6 \%)$ were more in occurrence of DLBCL. Male-Female ratio is $2: 1$. No significant difference observed in chemotherapy response relating to age and sex among different types of DLBCL. We also subdivided the cases according to Ann-Arbor staging systems. We found, $68(54 \%)$ cases were of stage I and another 54 $(42.8 \%)$ cases were Stage II and stage III. Mean Serum LDH was 227 $\mathrm{U} / \mathrm{L}$ with a SD of $68 \mathrm{U} / \mathrm{L}$ and there was no significant difference observed among the molecular subtypes. Patients were categorized according to International Prognostic Index (IPI). We found more than half $(55 ; 43.7 \%)$ of subjects had low intermediate risk and there was no significant difference observed in risk categorization. Mean IPI score was $2(\mathrm{SD} \pm 1)$. Mean ECOG performance status of study subjects were $2(\mathrm{SD} \pm 1)$. Mean proliferating index (Ki67) was $71.3 \%$ (SD $\pm 16.1 \%$ ) and no significant difference observed in Ki67 among different types of DLBCL. Several types of first line chemotherapy used in management and 39\% complete remission with $10 \%$ death observed in overall outcome. No significant difference observed in outcome among different types of DLBCL after treatment. No significant difference observed in survival among different types of DLBCL and overall mean survival was 54 months

Discussion \& Conclusion: This limited database study of NICR\&H will help to ascertain the outcome and overall survival of DLBCL with first-line chemotherapy in Bangladesh.

\section{Study of Cases of Non-hodgkins Lymphoma with Emphasis on T-NHL's A Single Center Study \\ Dr Inara Abeer ${ }^{* 1}$, Shad S. Akthar ${ }^{2}$, Aida Kaisar ${ }^{3}$ \\ ${ }^{1}$ Pathology, ${ }^{2}$ Medical Oncology, Hakim Sanallulah Hospital and Cancer Center, Srinagar, India, ${ }^{3}$ Final year MBBS, Shaheed Ziaur-Rahman Medical College, Bogra, Bangladesh}

Aims \& Objectives: The aim of this study was to review the pattern of Non Hodgkin's Lymphoma (NHL), with emphasis on T-cell lymphomas (T-NHL), seen in our institution.

Patients/Materials \& Methods: The present study was conducted after approval from the Institutional Ethics Committee. All patients seen in our institution and satellite centre with a diagnosis of NHL were included. Data regarding the clinical features, stage of disease, subtype, availability of IHC (immunohistochemistry) and subtyping according to the revised 4th edition of WHO 2017 classification. The data was collected on a proforma, transferred to MsExcel, and analysed.

Results: A total of 125 cases of NHL's were seen during the period of study.114 were B-NHL's, $11(8.8 \%)$ were T-NHL's. Mean age of the patients was 51.3 years with a M:F ratio $2.4: 1$. A total of $62 \%$ of patients presented with constitutional symptoms, $38 \%$ showed generalized lymphadenopathy. Among the B-NHL's 23 cases were of DLBCL's, 4 were Follicular lymphomas, 3 each as Extranodal marginal zone B-cell Lymphoma(MALT type) and Plasmacytomas, 1 each as Mantle cell lymphoma and Burkitt lymphoma. Out of the T-NHL's 1 case each of primary cutaneous CD-30-positiveT-cell lymphoproliferative disorder, lymphoblastic lymphoma, angioimmunoblastic T-cell lymphomas, anaplastic large-cell lymphoma anaplastic lymphoma kinase (ALK) negative.

Discussion \& Conclusion: Non-Hodgkin lymphomas (NHLs) are a diverse group of malignancies arising from lymphocytes. B cell NHL can be broadly divided into low-grade (histologically indolent) and high-grade (histologically aggressive) subtypes. Low-grade B cell NHL usually grow relatively slowly and may not immediately need treatment. The peripheral Tcell lymphomas (PTCLs) are rare and heterogeneous. This intrinsic diversity and rarity, has inhibited progress in the disease as a subgroup of NHL they account for $6 \%$ to $10 \%$ of all cases of NHL, making them exceedingly rare. Our study highlights the necessity for subclassification of PTCLs for therapeutic and prognostic purposes, and can act as a stepping zone for further large-scale studies to favourably challenge the chemotherapy paradigms that have been used in the Tcell malignancies.

\section{Prevalence of Genetic Abnormalities in Patients with Multiple Myeloma and Its Clinical Relevance}

Aditi Shah ${ }^{* 1}$, Nataraj K. S. ${ }^{1}$, Sundareshan $\mathrm{TS}^{1,2}$, Shilpa Prabhu ${ }^{1}$, Bharath Ram ${ }^{1}$, Shiva Kumar $\mathrm{K}^{1}$, Monisha $\mathrm{H}^{1}$, Karthick R G ${ }^{1}$, Sharat Damodar $^{1}$

\section{${ }^{1}$ Hematology, Narayana Hrudayalaya, ${ }^{2}$ Clinical laboratory, Anand Laboratory, Bangalore, India}

Aims \& Objectives: To estimate the prevalence of genetic abnormalities by Fluorescent in situ hybridization (FISH) analysis in patients with Multiple Myeloma (MM). To compare the clinical outcome between high and standard risk groups.

Patients/Materials \& Methods: 61 patients were included from January 2016 to December 2019. Interphase FISH study was performed on bone marrow aspirate, with panel of probes consisting of CKS1B (1q21-22), CDKN2C (1p32.3), D13S319 (13q14.2/13q34), IGH (14q32.33), p53 (17p13.1) and trisomy (5p15/9q22/15q22). Patients were divided into 2 risk groups; 1) high risk group with presence of del(17p), del(13q), gain of (1q), del(1p) and two or more aberrations with either of these and 2) standard risk group with presence of hyperdiploidy or no genetic abnormality. There was no difference in chemotherapy regimen between the 2 groups.

Results: FISH analysis was done in 54 patients at diagnosis and 7 during relapse. $36.1 \%(22 / 61)$ patients had presence of genetic aberrations, with $16.39 \%(10 / 61)$ having 2 or more abnormalities. There was no significant increase in the number of genetic aberrations with increasing age ( $p$ value: 0.76 ). The frequency of different genetic aberrations was as follows; gain of 1q $(21.31 \%)$, del(13q) $(14.75 \%)$, hyperdiploidy $(11.47 \%)$, del(17p) $(6.55 \%), \quad \operatorname{IgH}$ rearrangement $(4.91 \%)$ and $\operatorname{del}(1 \mathrm{p})(1.6 \%)$. All 3 patients with IgH rearrangement also had presence of one or more other high-risk genetic aberration and hence were included in high risk group. $31.1 \%$ of the patients were high risk and $68.9 \%$ were standard risk. Patients with high risk genetic abnormality had significantly higher prevalence of R ISS stage 3 ( $p$ value 0.008 ). Patients with plasmablastic form on bone marrow morphology were associated with higher prevalence of highrisk genetic abnormalities ( $p$ value 0.003). The response to initial chemotherapy, incidence of relapse, time to 1 st relapse and total number of relapses are shown in (table;1) and there was no significant difference between high and standard risk group. Overall survival at 2 and 5 years was $89.6 \% \pm 5.8 \%$ and $78.6 \% \pm 13.9 \%$ in standard risk 
group and $60.7 \% \pm 13.2 \%$ and $29.5 \% \pm 23.1 \%$ in high risk group respectively ( $p$ value: 0.762 ).

Discussion \& Conclusion: $36 \%$ of the patients with MM had one or more genetic abnormality with gain of (1q21) being the most frequent. Patients with high risk genetic abnormality had significantly higher prevalence of RISS stage 3 disease and were significantly associated with plasmablastic form of MM, both of these indicating aggressive disease. Response to Induction chemotherapy and relapse rate was same in high and standard risk groups. Although overall survival was lower in high risk group it was not statistically significant. Our study highlights the importance of molecular genetic abnormality and its association with aggressive disease in patients with multiple myeloma.

Supporting Document:

\begin{tabular}{|c|c|c|c|}
\hline $\begin{array}{l}\text { Response to initial } \\
\text { chemotherapy }\end{array}$ & Standard Risk (N=38) & High Risk (N=18) & $P$ value. \\
\hline $\begin{array}{l}\text { Complete response (sCR } \\
\text { CR) }\end{array}$ & $18(47.37 \%))$ & $6(33.33 \%))$ & \multirow[t]{4}{*}{0.502} \\
\hline $\begin{array}{l}\text { Very good partial } \\
\text { response }\end{array}$ & $14(36.84 \%))$ & $7(38.89 \%))$ & \\
\hline Partial response & $2(5.26 \%))$ & $3(16.67 \%))$ & \\
\hline $\begin{array}{l}\text { Stable disease or } \\
\text { progression }\end{array}$ & $4(10.53 \%))$ & $2(11.11 \%))$ & \\
\hline Relapse & $\begin{array}{l}\text { Standard risk } \\
(\mathrm{N}=37)\end{array}$ & $\begin{array}{l}\text { High risk } \\
(\mathrm{N}=17)\end{array}$ & $P$ value \\
\hline Yes & $13(35.13 \%)$ & $7(41.18 \%)$ & \multirow[t]{2}{*}{0.669} \\
\hline No & $24(64.86 \%)$ & $10(58.82 \%)$ & \\
\hline Time to $1^{\text {st }}$ relapse & $\begin{array}{l}\text { Standard risk } \\
(\mathrm{N}=14)\end{array}$ & $\begin{array}{l}\text { High risk } \\
(\mathrm{N}=6)\end{array}$ & $P$ value \\
\hline $\begin{array}{l}\text { Mean duration in months } \\
\pm \mathrm{SD}\end{array}$ & $20.71 \pm 9.47$ & $20.5 \pm 11.29$ & 0.968 \\
\hline Number of relapses & Standard risk & High risk & P value \\
\hline $\begin{array}{l}\text { Mean number of relapses } \\
\pm \mathrm{SD}\end{array}$ & $1.69 \pm 0.7$ & $2.57 \pm 1.4$ & 0.081 \\
\hline Overall survival & Standard risk & High risk & P value \\
\hline 2 years & $89.6 \% \pm 5.8 \%$ & $60.7 \% \pm 13.2 \%$ & \multirow[t]{2}{*}{0.762} \\
\hline 5 years & $78.6 \% \pm 13.9 \%$ & $29.5 \% \pm 23.1 \%$ & \\
\hline
\end{tabular}

\section{Systemic AL Amyloidosis in 2020: The Heart is the Heart of the Matter}

Esha Kaul $^{* 1}$, Nivedita Dhingra ${ }^{2}$, Raman Arora $^{3}$, Sharique Ahmed ${ }^{3}$, Biswajit Paul ${ }^{4}$

${ }^{1}$ Hematology-Oncology/Bone Marrow Transplant, ${ }^{2}$ HematologyOncology/Bone Marrot Transplant, ${ }^{3}$ Histopathology,

${ }^{4}$ Cardiology, JayPee Hospital, Noida, India

Aims \& Objectives: Systemic AL amyloidosis is a rare and underdiagnosed hematological disorder characterised by end organ damage and traditionally believed to have a poor prognosis. Advances in plasma cell directed therapies, better risk stratification and increasing use of serum free light chain assay have changed the diagnostic and treatment paradigms.

Patients/Materials \& Methods: Data for 10 consecutive patients with biopsy proven systemic AL amyloidosis between June 2017 and October 2019 was retrospectively collected by chart review.

Results: 6 Male, 4 Female. Mean age was 60 y(39-83). 5/10 patients did not have an M-spike. 1 patient had concomitant myeloma. 5/10 patients had $<5 \%$ bone marrow plasma cells. 7/10 patients had a preserved EF on Echo though the mean IVS thickness was increased at $15 \mathrm{~mm}$ (9-21). Patients were staged per Mayo staging with Stage I-0 patients, II-3 patients, III-3 patients, IV-4 patients. Treatment: 9 patients received induction per CyBorD protocol (all with subcutaneous bortezomib), and 1 received Mel/Pred. One patient underwent autologous stem cell transplant. All patients who completed induction were maintained on bortezomib for at least one year. 8/10 patients who completed at least 1 cycle were evaluable for response assessment [Fig. 1]. 4/5 people who died did so within first 6 months of diagnosis and 1 patient died at close to 3 years from listeria meningitis. $5 / 10$ patients are alive to date including one patient who required significant inotropic support at presentation.

Discussion \& Conclusion: Serum Light Chain Assay is essential to employ when suspecting light chain amyloidosis since protein electrophoresis will miss majority of such patients. It is very common not to have significant plasmacytosis in the marrow. Bortezomib based regimens with subcutaneous use are well tolerated. Patients with early cardiac involvement can have good outcomes emphasizing the need for utilization of cardiac biomarkers rather than relying on EF which can remain preserved until advanced stage cardiac involvement. A high index of suspicion, bortezomib based therapies and good supportive care in the early months with sustained serological responses can result in improvement in organ function in several patients.

Supporting Document:

\section{Response to first line therapy}

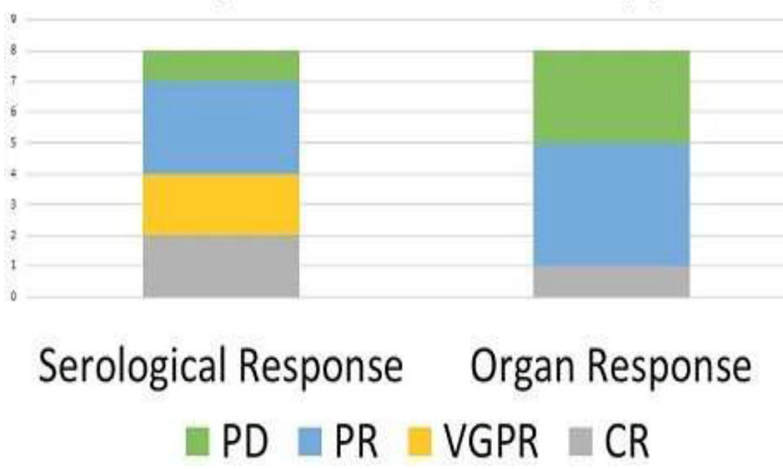

\section{Refractory Hypercalcemia-Coexistence of Multiple Myeloma and Hyperparathyroidism}

Avishkar Agrawal ${ }^{* 1}$, Ranvijay Singh ${ }^{1}$, Mastebhakti Bhavana ${ }^{1}$, Sunita Aggarwal ${ }^{1}$, Praveen K. Bharti ${ }^{1}$

${ }^{1}$ General Medicine, Maulana Azad Medical College \& LNH, New Delhi, India

Aims \& Objectives: Multiple Myeloma is a clonal plasma cell dyscrasia characterised by myeloma defining events: CRAB hyperCalcemia, Renal failure, Anemia, or lytic Bone lesions. Hypercalcemia is the most common metabolic complication of Multiple Myeloma. However, hypercalcemia is more commonly due to parathyroid adenoma and other malignancies. Lytic lesions are present in both hyperparathyroidism and MM. In MM, it is due to tumor cells infiltrating the bone and they show resolution on treatment. We present a case of hypercalcemia and multiple lytic lesion who had parathyroid adenoma and multiple myeloma. 
Patients/Materials \& Methods: A 48 years old female presented to general medicine OPD in Lok Nayak Hospital, New Delhi with complaints of low backache for 2 months with repeated episodes of headache and dizziness for past 1 month. Patient was diagnosed with Multiple Myeloma(MM) and was treated for the same. Patient achieved complete response to six cycles of chemotherapy(VRD regime). However 1 month later, patient presented again with similar complaints and symptomatic hypercalcemia. A complete workup of multiple myeloma was done in view of relapse, however, it was non diagnostic. This led us to workup for other causes of hypercalcemia which subsequently revealed parathyroid adenoma.

Results: At first presentation - Serum calcium was $12.6 \mathrm{~g} / \mathrm{dl}$, albumin-globulin ratio- 0.3, ESR-117, positive M-band in Serum protein electrophoresis, Bone marrow biopsy showed $60 \%$ plasma cells and, lambda/kappa ratio 58:1 in FLC assay. Serum PTH levels were on $52 \mathrm{pg} / \mathrm{ml}$. Skiagram showed multiple lytic lesions at skull and pelvis. Post chemotherapy- Serum calcium - $12.3 \mathrm{~g} / \mathrm{dl}$, s.phosphate$0.6 \mathrm{mmol} / \mathrm{L}$ and S.PTH - $112 \mathrm{pg} / \mathrm{mL}$. Skiagrams showed new lytic lesion in dorsal vertebrae and illium. Sestamibi scan showed a right inferior parathyroid adenoma and an ectopic parathyroid adenoma in right thorax. There was no evidence of MM.

Discussion \& Conclusion: Parathyroid adenoma is one of most common causes of hypercalcemia while multiple myeloma is not uncommon too. However, the coexistence of both of them has rarely been reported. It has been hypothesised that raised calcium might act as a mitogenic factor for cellular proliferation. Both entities present with similar presentation and a differential of parathyroid adenoma should be kept in mind in every case of hypercalcemia owing to the potentially treatable course and excellent prognosis.

\section{Treatment of Multiple Myeloma in the Covid19 Era: Experience from a Tertiary Care Cancer Centre in India}

Ishu Gupta ${ }^{* 1}$, Rasmi Palassery ${ }^{1}$, Vinayak Maka ${ }^{1}$, Nalini Kilara ${ }^{1}$, Santhosh K. Devadas ${ }^{1}$

${ }^{1}$ Medical Oncology, Ramaiah Medical College and Hospital, bangalore, India

Aims \& Objectives: There is limited data on the incidence and outcomes of COVID-19 infection in Multiple Myeloma (MM) patients. This study aimed to analyze the incidence, outcomes and risk factors for COVID-19 infection and mortality in patients with MM. Patients/Materials \& Methods: The study included all the MM patients receiving treatment at our center during a period of five months $(15 / 5 / 2020-15 / 10 / 2020 ; \mathrm{N}=52)$. All patients visiting our center undergo a COVID-19 RT-PCR at each visit, at least 14 days apart or earlier on clinical suspicion of infection. Patients who tested positive for COVID-19 infection were treated in an isolation facility. The primary end points were recovery from the infection and death due to the infection.

Results: We enrolled $52 \mathrm{MM}$ patients, of which 9 were newly diagnosed multiple myeloma (NDMM), 10 were relapsed refractory multiple myeloma (RRMM) and 33 patients were in remission. 13 patients were seen weekly (bortezomib + lenalidomide + dexamethasone; cyclophosphamide + bortezomib + dexamethasone; carfilzomib + pomalidomide + dexamethasone), 6 patients every two weekly (bortezomib) and 2 patients monthly (daratumumab or bendamustine). The remaining 29 patients were on oral therapy (lenalidomide, Lenalidomide + dexamethasone and Melphalan) with monthly OPD visits or tele-medicine consultation. 5 patients $(9.6 \%)$ contracted COVID-19 infection during the study period. 4 patients died (mortality rate $80 \%$ ) of which 3 patients had NDMM, and one patient had RRMM. The only patient who recovered from the infection was in remission. Among the patients who died, the mean time from the onset of symptoms to death was two days.

Discussion \& Conclusion: Patients with $\mathrm{MM}$ are at a high risk of death if infected with the SARS-CoV-2 virus. Patients with active disease are particularly prone to adverse outcomes with rapid progression of the infection and a very narrow window of opportunity for therapeutic interventions. Such patients may be switched to oral therapy whenever possible. Prevention, early detection and aggressive management of COVID-19 infection is very important in patients with MM.

\section{Death Audit in Hodgkins Lymphoma}

Sree Vamsee Chetana Panthula ${ }^{* 1}$, Wesley M. Jose ${ }^{1}$

${ }^{1}$ Medical Oncology, Amrita Institue of Medical Sciences, Kochi, India

Aims \& Objectives: To determine the causes of death in patients with Hodgkins Lymphoma.

Patients/Materials \& Methods: Patients between the age group of 18-85 years, diagnosed with Hodgkins lymphoma and who have expired during or after completion of the treatment between time period of 2011 to 2017 at our institute were included in the study. Various clinical parameters were collected. Deceased patient families were contacted and details collected retrospectively over telephone or from hospital records.

Results: A total of 98 patients were diagnosed with Hodgkins lymphoma between 2011 to 2017 based on the histopathological examination and IHC of the biopsy. Out of those 98 patients, 24 patients who had expired until writing of the abstract were included in the study group. The median age of death was 50 years. Male to Female ratio was 1.3 to 1 . In the study group, $50 \%$ of patients had comorbidities such as Diabetes,Hypertension, COPD,CAD and others. B symptoms were present in $61 \%$ of patients. $87 \%$ of patients were diagnosed with classical Hodgkins lymphoma. 55\% of expired patients were diagnosed with advanced disease. $46 \%$ of patients were noted to have a hasenclever score of 4 and above. Only $25 \%$ of patients underwent prechemotherapy PFT. All the patients received systemic chemotherapy with either ABVD or COPP. $42 \%$ of patients developed grade $3 / 4$ toxicities. 1 out 3 patients developed bleomycin toxicity requiring them to discontinue it. $29 \%$ of patients developed progressive disease and received second line of high dose chemotherapy. Among 9 patients who expired within 6 months, 5 patients had developed bleomycin toxicity. The common causes of death noted in decreasing order of frequency are ILD/Penumonia, Progressive disease, comorbidities related complications and unknown cause in some patients.

Discussion \& Conclusion: Hodgkins disease is one of the few malignancies with cure rate of $70-80 \%$. Mortality was $24.5 \%$ in our study. A middle aged male patient,presenting with B symptoms and in advanced stage was at a higher risk of death.The most common cause of death in our patients was ILD/Pneumonia. Bleomycin toxicty was present in every 1 out of 3 patients. As Bleomycin related toxicity leads to added disease burden,it is prudent that we evaluate all patients with pre chemotherapy PFT and obtain pulmonologist clearance prior to initiating chemotherapy. 
Supporting Document:

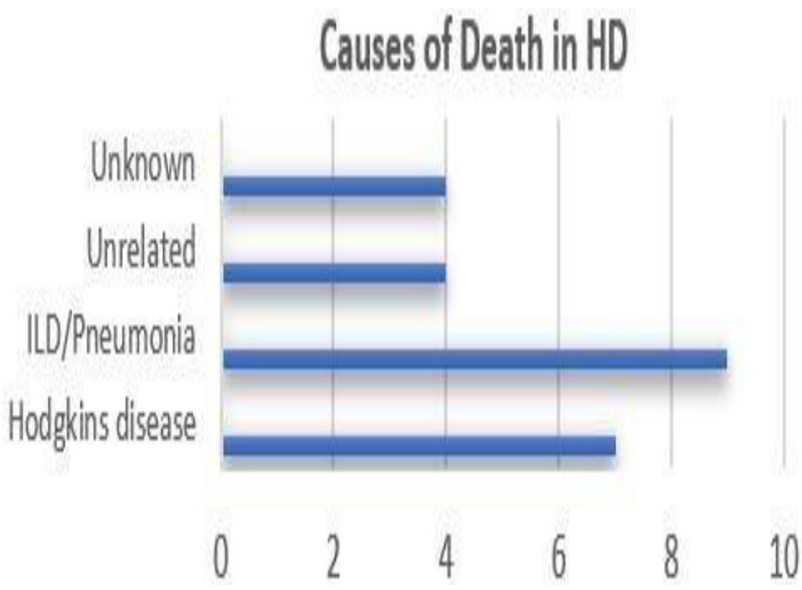

\section{Evaluation of Vitamin D Supplementation in Affecting The Outcome of Newly Diagnosed Patients with Lymphoma}

Arnab Bhattacharjee ${ }^{* 1}$, Smita Kayal ${ }^{1}$, Biswajit Dubashi ${ }^{1}$, Jaya P. $\mathrm{Sahoo}^{2}$, Sree R. J ${ }^{3}$, Harichandra K. KT ${ }^{4}$, Prasanth Ganesan ${ }^{1}$

${ }^{1}$ Medical Oncology, ${ }^{2}$ Endocrinology, ${ }^{3}$ Pathology, ${ }^{4}$ Department of Medical Biometrics and Infomatics, JIPMER, Pondicherry, India

Aims \& Objectives: Vitamin D deficiency in lymphomas are associated with inferior treatment outcome in recent studies. Vitamin D supplementation studies in lymphoma are sparse. We intended to evaluate the role of baseline Vitamin D levels and appropriate supplementation in deficient patients of newly diagnosed lymphoma in affecting treatment outcomes.

Patients/Materials \& Methods: Ours was a non randomised interventional study on newly diagnosed Lymphoma patients (both Hodgkin \& Non-Hodgkin) recruited from August 2018 to January 2020. Vitamin D measurements for all eligible patients were done at baseline, at 3 months and 6 months after diagnosis. Serum vitamin D level $<12 \mathrm{ng} / \mathrm{ml}$ was considered vitamin D deficient (VDD). All patients with VDD were supplemented with Vitamin D 6 lakh IU single intramuscular dose. Treatment for the primary diagnosis of lymphoma was done as per department policy. Primary objective was to check for difference in overall response rate (ORR) between baseline VDD vs nonVDD patients. Secondary end points were to compare chemotherapy toxicities \& EFS between the two groups.

Results: Total 151 patients were recruited; $56 \mathrm{HL}$ and 95 NHL. Median age was 48 years (7-80). VDD was seen in 37 patients $(24.5 \%)$ at baseline, and after supplementation $96 \%$ patients had correction in their Vitamin D level at 3 months and sustained it at 6 months. For patients where response was available $(n=134)$, there was no difference in ORR between VDD \& non-VDD in either Hodgkin $(83.3 \%$ vs. $88.6 \% ; p=0.678)$ or NHL $(78.9 \%$ vs. $68.4 \%$; $p=0.32$ ) sub groups. There was no difference in toxicities between VDD and non-DD patients. Median duration of follow up was 10.6 months. No significant difference in 18 months EFS was observed between VDD and non-VDD in the entire cohort $65.5 \%$ vs. $64.8 \%$ ), in Hodgkin $(59.8 \%$ vs. $79.8 \%$ ) or NHL (50\% vs. $68.3 \%$ ) as shown in attached figure.
Discussion \& Conclusion: No significant difference was observed in terms of ORR, toxicities \& EFS in VDD vs non-VDD lymphoma patients; possibly because of normalization of vitamin D in $96 \%$ patients after supplementation which would have overcome the adverse prognostic effect of baseline deficiency.

Supporting Document:

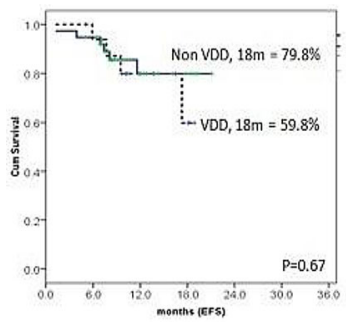

HL: Event Free Survival

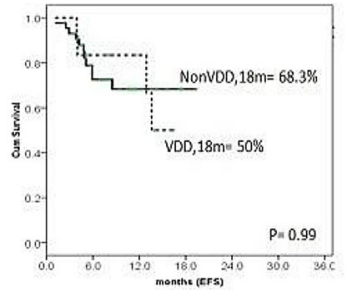

High grade B NHL: Event Free Survival

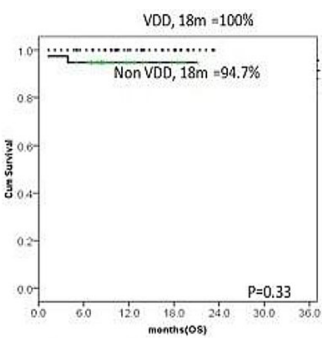

$\mathrm{HL}$ : Overall Survival

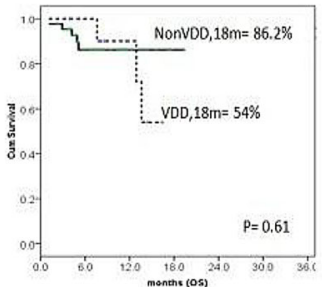

High grade B NHL : Overall Survival

\section{Evoloution of Autologous Stem Cell Transplantation for Multiple Myeloma: What has Changed Over the Last 23 Years at the Cancer Institute (WIA), Chennai}

Kiran Kumar Satti ${ }^{* 1}$, Nikita Mehra ${ }^{1}$, Jayachandran $\mathrm{PK}^{1}$, Parathan karunakaran $^{1}$, Sivasree kesana ${ }^{1}$, Venkatraman Radhakrishnan ${ }^{1}$, Manikandan Dhanushkodi ${ }^{1}$, Gangothri Selvarajan ${ }^{1}$, Shirleysunder singh $^{2}$, Arivazhagan $\mathrm{R}^{3}$, Rama Ranganathan ${ }^{4}$, Prasanth Ganesan ${ }^{5}, \mathrm{~T}$ S Ganesan ${ }^{1}$, Krishna Rathinam ${ }^{1}$, T G Sagar ${ }^{1}$

${ }^{1}$ Medical Oncology, ${ }^{2}$ Oncopathology, ${ }^{3}$ Clinical Biochemistry, ${ }^{4}$ Epidemiology and Biostatistics, Tumour Registry, Cancer Institute, Adyar,Chennai, Chennai, ${ }^{5}$ Medical Oncology, Jawaharlal Institute of Postgraduate Medicaleducation and Research, Puducherry, India

Aims \& Objectives: To study and analyse the evolution and trends in the outcomes of autologous stem cell transplantation (ASCT) for multiple myeloma (MM) at the Cancer Institute (W.I.A), Chennai. Objectives

- Progression-free survival (PFS)

- Evolution of transplant/peri-transplant treatment in myeloma Patients/Materials \& Methods: This is a retrospective analysis of patients diagnosed with MM who underwent ASCT from the first procedure done from 1996 to December 2019. Statistical analysis was done through SPSS version 20.

Results: One hundred and twenty-one patients underwent ASCT for multiple myeloma between 1996 and 2019. In 2012, the Tamil State health insurance scheme (CMCHIS) was introduced for ASCT. Of the 121 patients, 65 patients underwent ASCT under the CMCHIS fund. Notably, from 2012, among 85 patients, 65 (76\%) have been funded 
by the CMCHIS fund. Outcomes from 1996 to 2019. Among 121 patients, $117(96.6 \%)$ received ASCT as consolidation and 4(3.3\%) during relapse. Median age: 51(Range: 30-66) years; Males-78(64\%). ISS: ISS stage I-12(9\%), II- 23(19\%), III-65(53\%), stage not available- $21(19 \%)$ due to missing $\beta 2$ microglobulin. Induction regimen(s) prior to transplant: PI-IMiD-based triplet regimen: 36 (30\%), IMiD-based doublet alone-58(48\%), PI-based doublet$17(14 \%)$ and others-10(8\%). In 2015, induction chemotherapy for transplant-eligible patients was uniformly switched to PI- IMiD-based triplet regimen. Pre-transplant disease status: $\geq$ very good partial response (VGPR)-102 (84\%); partial response (PR)- 19(16\%). Melphalan was used as a high-dose conditioning regimen in all patients: Mel200-74(61\%), Mel <200-37(30\%) and Mel 140-10 (8.2\%). The median CD34 count was $4.6 \times 106$ cells $/ \mathrm{kg}(2.5-9 \times 106)$, and plerixafor was used in 23(18.9\%)patients. All the patients received non-cryopreserved stem cells. As a prophylactic and therapeutic measure for oral mucositis, laser therapy $(2 \mathrm{j} / \mathrm{cm} 2$ - prophylactic, $4 \mathrm{j} /$ cm2-therapeutic) was introduced in 2017 and was delivered in 36 patients $(30 \%)$. Laser was found to decrease the severity of mucositis by $11 \%$. Among the patients with PR prior to ASCT, 57\%achieved VGPR or better (11/19). Fourteen patients $(11.5 \%)$ received consolidation therapy after ASCT. Eighty-six $(70.5 \%)$ patients received maintenance therapy: thalidomide- 39 (45\%) lenalidomide- $31(36 \%)$, and bortezomib- $16(19 \%)$. The median follow-up of the study was 65 months (53.5-76.4). The median PFS was 52 months (45.6-58.3), and the median OS was 82 months (67.2-92.7). There were 2 treatment-related mortalities $(1.65 \%)$. Post-transplant day +100 flowbased MRD was started in 2018-14 (11.5\%) patients (12-negative, 2-positive).

Discussion \& Conclusion: We show the evolution of autologous stem cell transplant for multiple myeloma over 23 years at the Cancer Institute. With support from the State Health scheme, we have been able to provide the standard-of-care therapy to more number of patients with MM.

\section{Complications in Relapsed Mantle Cell Lymphoma}

Ekta Rahul ${ }^{1}$, Amar R. Singh ${ }^{* 1}$, Harshita Dubey ${ }^{1}$, Pranay Tanwar ${ }^{1}$

\section{${ }^{1}$ Lab Oncology, IRCH, AIIMS, New Delhi, NEW DELHI, India}

Aims \& Objectives: Mantle cell lymphoma (MCL) is an aggressive nonHodgkin lymphoma with median survival 3-4 years (1-2 year after relapse). It accounts for between 2- 10\% of all Non Hodgkin Lymphoma.

Patients/Materials \& Methods: It was retrieved from Hospital record.

Results: Clinical History: A 64 year old immunocompetent male presented with generalized lymphadenopathy for three months. Hemogram showed TLC 12200/cmm with $70 \%$ abnormal lymphoid cells in blood smear. Bone marrow (BM) aspirate smear showed 50\% abnormal lymphoid cells, which was supported by biopsy $(\mathrm{CD} 20+$, CD3-). Lymph node (LN) biopsy showed lymphoid cell positive for CD20, CD5 \& Cyclin D and negative for CD3 \& CD23; indicating MCL. Radiology showed bilateral lung nodule. Management with 6 \# Bendamustine \& Rutiximab resulted into clinical and hematological improvement. After 17 months of treatment free interval (TFI), he presented with Peripheral Neuropathy Grade -III with normal lab findings. Pregabalin was advised. Again after 2 months (19 months of TFI) he presented with left inguinal lymphadenopathy. LN \& BM Biopsy showed lymphoid cells positive for CD20, CD5 \& Cyclin D and negative for CD3, CD23, CD10 \& BCL-6; indicating MCL relapse. After 3 \# CHOP type II Diabetes was detected. After 6 \# CHOP chemotoxicity like fever, mucositis, oral ulcer etc. were noticed, which were managed conservatively along with
Linalidomide and Prednisolone. Follow up radiology showed nodular lesions in right upper lobe, which prompted to start therapy for Tuberculosis. Later a painless swelling in left thigh was noticed, for which radiotherapy showed partial relief. Doppler study suggested a partial thrombus in left Great saphanous vein. Possibly it was Lenalidomide induced and managed with anticoagulants. The case expired after some days.

Discussion \& Conclusion: No patient achieves complete response after relapse in MCL. Chemotherapy related complications are responsible for death.

\section{Image 001 Study Multicentric Study to Assess the Survival Statistics of Newly Diagnosed Multiple Myeloma Patients from Real World Settings}

Jeevan Kumar ${ }^{* 1}$, Dinesh Bhurani ${ }^{2}$, Rayaz Ahmed ${ }^{3}$, Saurabh J. Bhave $^{4}$, Reena Nair ${ }^{4}$, Gaurav Prakash ${ }^{5}$, Deepesh Lad ${ }^{5}$, Alka $\mathrm{Khadwal}^{5}$, Neelam Varma ${ }^{6}$, Aditya Jandial ${ }^{5}$, Subhash Varma ${ }^{5}$, Arihant Jain ${ }^{5}$, Suman Pramanik ${ }^{7}$, Rajan Kapoor ${ }^{8}$, Kundan Mishra ${ }^{8}$, Ajay Sharma $^{8}$, Sanjeevan Sharma ${ }^{7}$, Rajiv Kumar ${ }^{8}$, Uday Yanamandra ${ }^{9}$

${ }^{1}$ Department of Hemato-Oncology and BMT, Tata Medical Center, Kolkata, ${ }^{2}$ Rajiv Gandhi Cancer Institute and Research Centre, New Delhi, India, ${ }^{3}$ Department of Hemato-Oncology and BMT, Rajiv Gandhi Cancer Institute and Research Centre, New Delhi, ${ }^{4}$ Clinical Hematology Oncology \& HCT, Tata Medical Center, Kolkata, ${ }^{5}$ Department of Internal Medicine, ${ }^{6}$ Hematology, Postgraduate Institute of Medical Education and Research, Chandigarh, ${ }^{7}$ Army Hospital R\&R, New Delhi, India, ${ }^{8}$ Hematology, ${ }^{9}$ Department of Hemato-Oncology and BMT, Army Hospital R\&R, New Delhi, India

Aims \& Objectives: The outcomes of myeloma patients from realworld settings are often lacking in developing countries due to poor record maintenance. Publications from such settings are limited because of the retrospective nature of the data collection and heavy reliance on book audits, which can be bridged by real-time data maintenance. We present the real-time survival data of the myeloma patients from four different tertiary care centers who are part of Indian Myeloma Academic Groupe (IMAGe) using one such indigenously built hybrid application.

Patients/Materials \& Methods: All the patient characteristics were recorded contemporaneously in the hybrid application by the study centers under the IMAGe 001 study. We have analyzed only the survival of these and categorized them based on various disease characteristics at diagnosis. A total of 1361 patients were included in the study. All the data entered was counterchecked by the patient/their kin for appropriateness, and any discrepancies were clarified centrally by the secretarial research staff of the IMAGe society. All patients were managed based on the treating institution's protocols, and the consent of the patients was taken for entering the data on the Hybrid application (care4myeloma.in).

Results: The mean age of the study population was $60.98 \pm 10.9 y$ with male predominance $(64.22 \%)$. The median follow-up was 863 days. The mean BMI and BSA of the patients were $24.13 \pm 4.24 \mathrm{~kg} / \mathrm{m}$ and $1.69 \pm 0.18 \mathrm{~m}$ respectively. The baseline characteristics of the patients are enumerated in Table 1. One-third of the study population had co-morbidities, most prevalent being diabetes $(85 \%)$, and cardiovascular diseases $(40 \%)$. The calculated $10 \mathrm{y}$ overall survival (10y-OS) was $60.9 \%$. Cumulative OS stratified based on various characteristics is as shown in Fig. 1. Patients with lower stages based on the International staging system (ISS) and revised ISS (R-ISS) had a better survival (Fig. 1). 
Discussion \& Conclusion: The real-world data shows OS comparable to the published western literature. The ISS and R-ISS were prognostic even in real-world settings. Weight loss and presence of comorbidities at diagnosis were found to impact long term survival in our settings and need to be studied more deliberately. The use of such hybrid applications can aid in better data-keeping.

\section{Real World Experience of HIV Associated Lymphoma: A Single Centre Experience from Northern Part of India}

Urmimala Bhattacharjee ${ }^{* 1}$, Nishant Jindal ${ }^{1}$, Arihant Jain ${ }^{1}$, Ashwin Nair $^{1}$, Pulkit Rastogi ${ }^{2}$, Amanjit Bal ${ }^{2}$, Gaurav Prakash ${ }^{1}$, Alka $\mathrm{Khadwal}^{1}$, Deepesh Lad ${ }^{1}$, Neelam Verma ${ }^{3}$, Pankaj Malhotra ${ }^{1}$

${ }^{1}$ Internal Medicine, ${ }^{2}$ Histopathology, ${ }^{3}$ Hematology, Post Graduate Institute of Medical Education and Research, Chandigarh, Chandigarh, India

Aims \& Objectives: There is limited long-term data on epidemiology and outcome of AIDS related Lymphoma (ARL) from Indian setting. We retrospectively analyzed the outcomes of ARL from a tertiary care center of North India.

Patients/Materials \& Methods: All patients of age more than 12 years with confirmed diagnosis of HIV and lymphoma who presented at our center over the period of seven years were included and analysis of clinical and pathological characteristics and treatment outcomes was done.

Results: A total of 33 patients of lymphoma who had HIV coinfection were identified. The median age at lymphoma diagnosis was 44 years (16-70 years) with a M:F ratio of $2.3: 1$. DLBCL was the most common subtype seen in $45.5 \%$ followed by HL in $18.2 \%$, plasmablastic lymphoma in $12.1 \%$, Burkitt Lymphoma in 3\%, high grade NHL (NOS) in 3\%, EBV-LPD in $3 \%$ and PCNSL in $3 \%$. Other lymphoma subtypes included 2 patients with LPL, 1 with ALCL (ALK negative), 1 with T-LBL. Nineteen patients (59.4\%) had preexisting HIV diagnosis and 13 were diagnosed at the time of lymphoma diagnosis. Among patients with preexisting HIV diagnosis, the median interval between HIV diagnosis and lymphoma was 76 months (10-291 months). The median CD 4 count at diagnosis was 239 (20-704). 10 patients (38.5\%) had CD 4 less than 200 at time of diagnosis of lymphoma and 3 had CD 4 count less than $50 / \mathrm{mcl}$. Lymphadenopathy (45.5\%) and B symptoms (75\%) were the most common presenting complaints. Among patients with high grade NHL, GI was the most common extra-nodal site (30\%) followed by liver and bone (20\% each).Twenty-eight patients had received antilymphoma therapy with ART. Of the 17 patients high-grade B-NHL, 8 received CHOP, 6 RCHOP and 1 patient CVP while 2 patients lost follow up before completing therapy. Of the 13 patients evaluable patients, 8 patients achieved $\mathrm{CR} / \mathrm{CRu}(61.6 \%)$ and $5(38.5 \%)$ patients died during therapy. At a median follow up of 12 months (0-71 months) median PFS was 16 months and 2 year PFS was $44 \%$, and 2 year OS was $59.3 \%$. Of the 4 patients with PL, 3 received CHOP with Lenalidomide and 1 lost to follow up before therapy could be started. At a median follow up of 36 months (2-67 months), no patient had progressed with 2 year PFS and OS of $100 \%$. All 6 patients of HL received ABVD. 4 patients achieved CR, 1 CRu and 1 PR. At a median follow up of 18.5 months (9-72 months), no patient had progressed with 2 year PFS and OS of $100 \%$.

Discussion \& Conclusion: High grade DLBCL is the commonest subtype of HIV associated lymphoma in India. Combination of ART and anti-lymphoma chemo-immunotherapy can lead to remission in majority of patients.

\section{A Prospective Study of Response Evaluation with RCHOP Therapy in DLBCL Subtypes Using Hans Algorithm}

Sharat Chandra G ${ }^{* 1}$, Pratap kishore das ${ }^{2}$, Manish singhal ${ }^{2}$, Dipanjan panda $^{2}$, Atul sharma ${ }^{2}$, Shaunak valame ${ }^{2}$, Amarendra amar ${ }^{2}$, Jayant patowary $^{2}$

${ }^{1}$ Medical oncology, Apollo indraprastha hospitals, ${ }^{2}$ Medical oncology, Apollo indraprastha hospitals, Delhi, India

Aims \& Objectives: Aim: A prospective study of clinical and pathological correlation and response evaluation with $\mathrm{r}$-chop therapy in different subtypes of DLBCL (NHL) Patients.

Primary Objective: To determine the Response Rate (RR) based on Radiological Response after 1st line therapy with $\mathrm{R}-\mathrm{CHOP}$ regimen in DLBCL patients.

- To determine Progression Free Survival and Overall Survival in different subtypes

Secondary Objective:

- To determine different subtypes of DLBCL according to HANS algorithm.

- To study prognostic factors after 1st line therapy with R-CHOP Patients/Materials \& Methods: A prospective study of consecutive 74 newly diagnosed DLBCL patients, between Jan 2017 and Dec 2019 were included. Subtyping into GCB and ABC were done using IHC based on Hans' algorithm. All patients were treated with R-CHOP regimen for 6 cycles. Responses were evaluated using Lugano response criteria for NHL. Primary objective was to study clinical and prognostic factors along with response rates to chemotherapy. Secondary objective was to study Progression Free Survival (PFS) and Overall Survival (OS).

Results: Of 74 patients, median age was 56.98 years with $62 \%$ males. Fever (46\%), involuntary significant weight loss (38\%) and painless neck swelling $(30 \%)$ were most common presentations. Advanced stage comprised $67.5 \%$ of the cohort. NCCN IPI score was High in $16.2 \%$, High Intermediate in $33.7 \%$, Low Intermediate in $37.8 \%$, and Low in $12 \%$. Hans' algorithm classified $45.95 \%$ into GCB subtype while $54.05 \%$ were ABC subtype. Complete Metabolic Response (CMR) was seen in $83.7 \%$ overall, $94 \%$ in GCB, and $75 \%$ in $\mathrm{ABC}$. After a median follow up of 27 months, PFS was $81.3 \%$ and OS was $90.1 \%$ for overall cohort. PFS and OS were higher in GCB $(84.8 \%$ and $94 \%$ ) than in $\mathrm{ABC}$ subtype (77.9\% and $86.4 \%$ ). Using log-rank analysis,high IPI, ABC subtype, and not achieving CMR predicted poorer survival in this study.

Discussion \& Conclusion: In this single tertiary centre experience, Hans algorithm method based on IHC helped in delineating the patients into two prognostic groups of $\mathrm{GCB}$ and $\mathrm{ABC}$ subtypes. A longer follow up would further establish outcomes of poorer risk $A B C$ subtype using above strategy.

\section{Oncocollect Lymphoma Registry: Treatment Outcomes of Diffuse Large B-Cell Lymphoma}

\section{Pranita Mishra*1}

${ }^{1}$ Clinical Haematology, Tata Medical Center, Kolkata, India

Aims \& Objectives: To review disease characteristics, 5-year outcomes of patients with Diffuse large B cell lymphoma (DLBCL) treated in the rituximab era in a tertiary cancer center.

Patients/Materials \& Methods: 1396 lymphomas ( $\geq 18$ years) were entered in the OncoCollect data base of patients receiving 1st line treatment from May 2011 to December 2017. Demography, Clinical features, staging, prognostic stratification, associated co- morbidities, first line treatment response, outcomes of DLBCL patients treated at 
our center have been analyzed. The audit was internally validated by estimating disease free survival (DFS) for known prognostic markers such as age groups, stage and IPI. Response rates, DFS, overall survival (OS), follow up till September 2020 were evaluated.

Results: 608 patients (44\%) were treated for DLBCL. 12\% were < 40 years, $56 \%$ between 40 to 65 years, $32 \%$ were $\geq 65$ years. Gender ratio was 1.7:1. Median duration of symptoms was 3 months. Patients presented in early stage [I, II] 46\%, favorable IPI (0-2) in $63 \%$, extra nodal disease in $58 \%$. The commonest extra nodal sites were Gastro-intestinal (18\%), Head \& Neck (15\%), Central Nervous System (10\%), Genitourinary (8\%). Anthra-cycline based treatment was given to $470(77 \%)$ patients and Rituximab based treatment to $511(85 \%)$. Standard CHOP- R was used in $61 \%$ patients, with modifications in others. The overall response rate $(\mathrm{CR}+\mathrm{PR}+\mathrm{SD})$ $87.5 \%$, the progression on treatment $11 \%$. With a median follow-up of 39 months, the DFS is $70 \%$. DFS for patients above 65 years is $62 \%$ at 5 years, for those below 65 years is $76 \%(p=0.052)$. The 5 -year DFS for stage 1, 2, 3 and 4 are $81 \%, 78 \%, 66 \%$ and $65 \%$ respectively $(p=0.0064)$. The 5 -year DFS for IPI low, low-intermediate, high intermediate and high are $80 \%, 76 \%, 66 \%, 52 \%$ respectively $(p<0.0001)$. The OS at 5 year is $78 \%$.

Discussion \& Conclusion: Over the years Rituximab based therapy has impacted the survival outcomes in patients.

\section{Polatuzumab Vedotin in Relapsed/Refractory High- Grade B-Cell Non Hodgkin Lymphoma}

Rajat Pincha ${ }^{* 1}$, Vivek Radhakrishnan ${ }^{1}$, Arijit $\mathrm{Nag}^{1}$, Jeevan Kumar ${ }^{1}$, Saurabh J. Bhave ${ }^{1}$, Indu Arun ${ }^{2}$, M A Lateef Zameer ${ }^{2}$, Debdeep Dey ${ }^{2}$, Mammen Chandy ${ }^{1}$, Reena Nair ${ }^{1}$

${ }^{1}$ Clinical Haematology and BMT, ${ }^{2}$ Histopathology, TATA MEDICAL CENTER, Kolkata, India

Aims \& Objectives: To assess safety and feasibility of Polatuzumab based therapy in the setting of Relapsed/Refractory(R/R) high grade B-NHL.

Patients/Materials \& Methods: A chart review of R/R B-NHL patients receiving Polatuzumab based chemotherapy at our center between May 2019 and September 2020 was undertaken. Patients $>18$ years, failing 2 prior lines of therapy and receiving at least one dose of polatuzumab were included. Polatuzumab vedotin was provided on a compassionate access from Roche ${ }^{\circledR}$.

Results: Eight patients (7 male; DLBCL-6, PMBCL-1 and transformed B-NHL -1) received therapy. Median number of prior lines of therapy: 3 (range 2-4). Regimens used: Pola + BR-6, Single agent Polatuzumab-1, Pola-Pembrolizumab-Rituximab-1. Median number of polatuzumab doses received- 5 (range 1-8). Of the total 35 polatuzumab infusions, 28 were administered in-patient and 7 as daycare. Therapy was well-tolerated. Infusion related adverse event was noted in only 1 infusion (Grade 2). Hematological toxicities ( $>$ grade 3 or 4) were noted in 3 patients, all could continue treatment with supportive care. No Grade 3-4 non- hematological toxicities noted. 1 patient developed Grade 1 hepatic dysfunction. Response was evaluable in $7 / 8$ patients ( 1 patient died on day 8 of cycle 1 ). By the end of 3 cycles: $2 / 7(28.5 \%)$ and $4 / 7(57 \%)$ had Complete response (CR) and partial response (PR) respectively, 1 patient had progressive disease (PD) after 1st cycle. By the end-of- 6 cycles, best response: CR in $42 \%$ (3/7), PR: $28.5 \%$ (2/7), PD: $28.5 \%$ (2/7); treatment is withheld in one patient after cycle 3 (Tuberculosis) and he remains in PR. One patient in $\mathrm{CR}$ proceeded to an allogeneic hematopoietic cell transplant and remains in remission. At last follow up, 7 patients are alive.

Discussion \& Conclusion: In a subset of heavily pretreated R/R high grade B-NHL Polatuzumab based therapy was found to be safe, feasible and active. Larger prospective studies will provide more information.

\section{Angioimmunoblastic T Cell Lymphoma with Monoclonal Proliferation of Plasma Cells}

Sukanya Gogoi ${ }^{* 1}$, Christina $\operatorname{Cox}^{1}$, Mansour Ceesay ${ }^{1}$, Jon Salisbury ${ }^{2}$, Austin Kulasekararaj ${ }^{1}$

${ }^{1}$ Haematology, ${ }^{2}$ Histopathology, Kings College Hospital NHS Trust, London, United Kingdom

Aims \& Objectives: Angioimmunoblastic T cell Lymphoma, a subtype of peripheral $\mathrm{T}$ cell Lymphoma, is a very aggressive disease with distinctive clinical characteristics, pathology and immune phenomenon. This report tries to identify the pathogenesis in AITL with monoclonal proliferation of plasma cells.

Patients/Materials \& Methods: 74-year-old gentleman diagnosed with AITL stage IV B with initial presentation of generalised lymphadenopathy, pruritic rash, SIADH, dry cough. Patient received several cycle of chemotherapy CHOP, CHOEP (50\% Etoposide dose), Lenalidomide \& dexamethasone, Brentuximab. Later presented with a diffuse maculo-papular rash on trunk, neck and upper limbs. Skin biopsy reported as infiltration by cytologically atypical plasma cells, CD138 + , CD $38+$ and varied mitotic figures. Subsequently, patient died with rapid progressive disease and multi organ systemic involvement, even before having further management.

Results: Bone marrow trephine on diagnosis showed staining of CD2, CD5, CD7 with slight dominance of CD8 + over CD4(CD56, 57 negative). Mild increase in T-cells with CD8 + T-cell lymphocytosis. Lymph node biopsy reported strong positive staining for CD30, Ki67 90\%. PD1, CD10 and ICOS positive. EBER negative. Peripheral blood reported $30.7 \%$ of TNC's with B-cells $(\mathrm{CD} 19+) 0.2 \%$,T-cells $26.8 \%$ of TNC's.CD4/CD8 ratio of 2.6:1. NK Cells $2.7 \%$ of TNC's. No expanded B-cell or aberrant expression with abnormal $\mathrm{T}$ cell identified. Polyclonal Hyper-immunoglobulins reported $68 \mathrm{gr} / \mathrm{L}$ at the peak. BMAT on suspected relapse reported hypercellular and reactive (80-90\% cellularity) with all three haemopoietic cell lineages. No morphological/immunophenotypic evidence of lymphoma involvement. CD3 stained scattered clusters with single dispersed T cells. CSF reported majority of gated cells as $\mathrm{mCD} 3+\mathrm{CD} 5+\mathrm{CD} 4+$ mature $\mathrm{T}$ cells which were CD8-,CD10-, CD30-, TCR alpha/beta dim. No aberrant Lymphoma T cells. Skin biopsy done reported cytologically atypical plasma cells, CD138+, CD $38+$ and varied mitotic figures. Plasma cells reported as monocytic for Kappa immunoglobulin light chain with small infiltrate of reactive T Cells, CD2, CD3. CD4, CD5. Increased values of IL2, IL6, IL8, IL 10, Interferon Beta, Interferon Gamma reported.

Discussion \& Conclusion: There are only a few reported cases that has demonstrated AITL with marked polyclonal plasmacytosis, and monoclonal being more uncommon. With a comprehensive understanding and explanation, it is suggested that Interleukins and interferons have been involved in the pathogenesis of this patient's clinical condition along with monoclonal proliferation of plasma cells associated with Ig Kappa paraprotein.

Keywords: Bone marrow aspiration and biopsy(BMAT), Total nucleated Cells (TNC), Angioimmunoblastic $\mathrm{T}$ cell lymphoma(AITL), Syndrome of inappropriate antidiuretic hormone secretion (SIADH), Epstein Barr Virus (EBV), Cerebrospinal fluid (CSF). 


\section{Heterogeneity of Extranodal Involvement in Primary Nodal Non-hodgkin Lymphomas}

\author{
Victor Tomacinschii ${ }^{* 1}$, Maria Robu ${ }^{1}$, Sanda Buruiana ${ }^{1}$ \\ ${ }^{1}$ Nicolae Testemitanu State University of Medicine and Pharmacy \\ of the Republic of Moldova, Chisinau, Moldova, Republic of
}

Aims \& Objectives: Description of the frequency of extranodal involvement localisations, in the dissemination stages (III-IV) of nonHodgkin lymphomas(NHL) patients with primary lymph node involvement.

Patients/Materials \& Methods: A retrospective study was conducted on 78 patients with NHL with primary lymph nodes(LN) involvement aged between 60 and 84 years, examining the primary site of involvement and the sites of extranodal involvement. Results: It was determined that NHL with onset in the LN had developed more frequently in people aged from 60 to 70 years(73.1\%). NHL developed more often primarily in the peripheral $\mathrm{LN}(84.7 \%)$, less frequently in the mediastinal $\mathrm{LN}(6.4 \%)$, and abdominal $\mathrm{LN}(8.9 \%)$. Aggressive NHL predominated $(59.0 \%)$, indolent NHL was diagnosed in $41.0 \%$, more frequently in cases of affection to the cervical $\mathrm{LN}(47.4 \%)$, inguinal $\mathrm{LN}(41.7 \%)$, and abdominal $\mathrm{LN}(42.9 \%)$. Analysis of the frequency of involvement of various organs and tissues at the stage of generalization showed that extranodal metastases occurred more frequently in the liver (47.7\%), bone marrow (43.2\%), spleen $(29.5 \%)$. Less frequently in the tumor process were involved lung tissue $(11.3 \%)$, soft tissues $(11.3 \%)$, nasopharyngeal amygdala $(9.0 \%)$, pleura, and skin were affected in $2.3 \%$ cases each.

Discussion \& Conclusion: NHL with primary LN involvement developed more frequently among people aged 60 to 70 years. Among extranodal metastases the most frequent site were the liver $(47.7 \%)$, bone marrow $(43.2 \%)$ and spleen $(29.5 \%)$.

\section{Oral Domiciliary Therapy of Multiple Myeloma with Cyclophosphamide, Pomalidomide and Dexamethasone Regimen in the Covid Era: A Pilot Study}

Vishnupriya Mathivanan ${ }^{* 1}$, Anjali Matani ${ }^{1}$, Amey C. Panchal ${ }^{1}$, Jyothi Goutham Kumar ${ }^{2}$, Sachin S. Jadhav ${ }^{1}$

${ }^{1}$ Haematology and BMT, ${ }^{2}$ Clinical Pharmacology, HCG hospitals, BANGALORE, India

Aims \& Objectives: Aim: To observe the feasibility and efficacy of domiciliary, oral treatment with cyclophosphamide, pomalidomide and dexamethasone (Cy- Pom-Dex) for myeloma patients during the COVID-19 pandemic.

Objectives:

- To observe the feasibility of Cy-Pom-Dex for the treatment of newly diagnosed and relapsed myeloma patients.

- To assess the therapeutic efficacy of the same.

Patients/Materials \& Methods: Type of study: Prospective, observational, single-arm, pilot study Study period: from 1st March to 30th September 2020.

Study methodology: Patients who had newly-diagnosed or relapsed multiple myeloma during the above-mentioned time period at a tertiary care Centre in Bengaluru were started on oral treatment with CyPom-Dex during the lockdown mandated by the COVID-19 pandemic. This regimen was chosen as a replacement for cyclophosphamide-bortezomib-dexamethasone (CyBorD), which was the previous standard of care in our Centre. Dosage of the regimen: Cyclophosphamide $400 \mathrm{mg}$ PO on days 1,8 and 15; Pomalidomide $4 \mathrm{mg}$ PO from days 1 to 21 ; Dexamethasone $40 \mathrm{mg} \mathrm{PO} / 20 \mathrm{mg}$ PO on days 1,8,15 and 22; Cycle was repeated every 28 days.
Haematological and biochemical parameters of the patients were checked pretreatment. Weekly complete blood counts and biochemistry was checked with home collection of blood samples. This was combined with weekly video consultations. Face-to-face visits were conducted on a monthly basis and myeloma parameters were checked at the end of every 2 months.

Results: Out of 6 myeloma patients during the above-mentioned study period, 4 (66.67\%) had IgG kappa, 1(16.67\%) had IgA kappa and $1(16.67 \%)$ IgG lamda myeloma. $2(33.33 \%)$ had ISS stage II disease and $4(66.67 \%)$ ISS-III. $2(33.33 \%)$ patients were newly diagnosed and received Cy-Pom-Dex from the first cycle, while 2 $(33.33 \%)$ had hypercalcemia and renal failure at diagnosis, hence were given CyBorD as the first cycle which was then followed by Cy-PomDex from the second cycle. 2 (33.33\%) patients received Cy-PomDex for remission induction for relapse, post-autologous stem cell transplant. 1 patient who had received Cy-Pom-Dex from the first cycle was lost to follow-up (COVID-19 positive). Among the remaining 5 patients, $4(80 \%)$ achieved VGPR after 4 cycles and the last patient $(20 \%)$ achieved sCR. All the patients were subsequently started on lenalidomide maintenance.

Discussion \& Conclusion: Domiciliary treatment was feasible in all of our patients. This regimen achieved good disease control in all the evaluable patients, within 4 cycles. Larger number of patients is however required to draw definitive conclusions.

\section{Primary Plasma Cell Leukemia in Central India: A Case Report}

Deepshikha Verma* ${ }^{* 1}$, Parul Murab ${ }^{1}$, Vedanti Newaskar ${ }^{1}$, Maneesh Sulya $^{2}$, R.k. Nigam ${ }^{3}$, Reeni Malik ${ }^{4}$

${ }^{1}$ PG 2nd year, Department of Pathology, ${ }^{2}$ Associate professor, Department of Pathology, ${ }^{3}$ Professor, Department of Pathology, ${ }^{4}$ Professor and Head, Department of Pathology, Gandhi medical College, Bhopal, India

Aims \& Objectives: Our aim is to present a rare case of primary plasma cell leukaemia which was diagnosed and managed in our hospital producing good prognosis.

Patients/Materials \& Methods: Here we present a case, a 50-yearold male patient presented to the OPD of Gandhi memorial hospital Bhopal in 2019 with breathlessness and chest pain and decreased frequency of micturition. On examination of the patient mild pallor seen with toxic facies and No organomegaly. No previous history of drug abuse or chronic illness was found. MRI showed collapsed L1 vertebra with marrow oedema. On lab investigations the patient had the absolute plasma count of $2 \times 109 / \mathrm{L}$. on bone marrow extract $60 \%$ of the atypical plasma cells. All the investigations were suggestive of primary plasma cell leukaemia with involvement of both kidneys.

Results: MRI, Biochemical investigation:- Features of CRAB with Increased Rouleaux formation and Plasma cells in PS $>20 \%$ and Plasma cells in BM $>20 \%$ BUT less than $60 \%$ with BM histology shows osteoclasts and distortion of trabeculae.the patient was managed with Bortezomib-based chemotherapy, which improved the overall haematological and biochemical profile of the patient.

Discussion \& Conclusion: In this case report we describe the clinical and diagnostic features along with the management of primary plasma cell leukaemia. 


\section{Use of NLR And LMR as Prognostic Markers in Hodgkins Lymphoma}

\author{
Sree Vamsee Chetana Panthula ${ }^{* 1}$, Wesley M. Jose ${ }^{1}$ \\ ${ }^{1}$ Medical Oncology, Amrita Institue of Medical Sciences, Kochi, \\ India
}

Aims \& Objectives: To evaluate the prognostic value of NLR and LMR with respect to EBV, Stage, PFS and OS in patients of Hodgkins Lymphoma.

Patients/Materials \& Methods: Patients who were newly diagnosed as Hodgkins lymphoma between the age group of 18-85 years during the time period of 2011 to 2017 at our institute were included in the study. Diagnosis was based on histopathological examination and IHC. Clinico-etiological variables at the time of diagnosis were noted. NLR was calculated as absolute neutrophil count divided by absolute lymphocyte count. LMR was calculated at absolute lymphocyte count divided by absolute monocyte count. PFS and OS were determined using log rank tests and Kaplan-meier curves.

Results: A total of 98 patients with Hodgkins lymphoma were included in the study. Male to female ratio was 1.6 to 1 . The median age of diagnosis was 37 years. Majority $(57.2 \%)$ were diagnosed in advanced stage. Positive EBV-LMP on IHC was noted in $32 \%$ of patients. Out of 98 patients, progression was noted in 18 patients. Median NLR and LMR was calculated as 4.12 and 2.09 respectively in the study group. NLR $>4.1$ and LMR $<2.1$ was noted in $59 \%$ and $67 \%$ of patients with advanced disease respectively. 12 out of 21 patients with extranodal disease had NLR $>4.1$ and $\mathrm{LMR}<2.1$. $63 \%$ of patients with high NLR were noted to be negative for EBV. $70 \%$ of patients with negative EBV had LMR $<2.1$. PFS was better in patients with NLR $>4.1$ and LMR $<2.1$ at 84 and 85 months respectively $(p>0.05)$. OS was of longer duration in patients with NLR $<4.1$ and LMR $<2.1$ at 86 and 90 months respectively $(p>0.05)$.

Discussion \& Conclusion: In our study, we conclude that high NLR and low LMR are associated with advanced disease and extranodal disease. Negative EBV status also showed high NLR and low LMR in majority of patients. Prolonged PFS was noted with NLR $>4.1$ and LMR $<2.1$ OS was better with LMR $<2.1$. However, the results have not been in concordance with other international studies.

\section{Pulmonary Dysfunction in Pediatric Hodgkin Lymphoma Survivors: 5 yrs Experience}

\section{Mude Praneetha* ${ }^{*}$, Rachna Seth ${ }^{1}$, Sushil Kumar Kabra ${ }^{1}$, Aditya} Gupta $^{1}$, Jagdish Prasad Meena ${ }^{1}$

\section{${ }^{1}$ Pediatrics, AIIMS, New Delhi, India}

Aims \& Objectives: (1) To determine prevalence of pulmonary abnormalities as assessed by PFT (spirometry) in childhood Hodgkin lymphoma survivors of age 6-18 yrs, post completion of treatment. (2) To identify the risk factors for pulmonary abnormalities in childhood Hodgkin lymphoma survivors of age 6-18 yrs,post completion of treatment. (3) To determine the clinical spectrum of pulmonary abnormalities and pattern of PFT abnormalities in childhood HL survivors of age 6-18 yrs post completion of treatment.

Patients/Materials \& Methods: Hodgkin lymphoma survivors who were in follow up at the pediatric cancer survivors clinic and had completed treatment within last 5 years were evaluated prospectively. A detailed history of respiratory symptoms in the present and past, treatment history (chemotherapy and radiation dose exposure), disease related history was taken. They underwent a detailed physical and respiratory system examination followed by pulmonary function tests(spirometry) and 3 min step test were performed in all subjects under supervision of physiotherapist.

Results: 53 patients were evaluated. Majority were males with age range between 6 and 18yrs. The median period of follow up of 2 yrs(range was $1-5 \mathrm{yrs}$ ). 2 (3.7\%)survivors were symptomatic. All 53 patients received ABVD (4-6 cycles, cumulative dose of bleomycin ranging from 80 to 120 units), only 12 patients(22\%) received radiation (dose $25-30 \mathrm{~Gy} / 15 \#)$. Only 6 patients $(6.6 \%)$ patients showed restrictive pattern, $1(1.8 \%)$ showed obstructive pattern on pulmonary function testing at median time of 2.2yrs from diagnosis (4 patients received radiation,mean age at diagnosis 13 yrs).All the 53 subjects were able to complete $3 \mathrm{~min}$ step test.

Discussion \& Conclusion: Childhood cancer survivors are 8.8 times more likely to die of pulmonary cause when compared to general population: as aspect of concern with the growing population of childhood cancer survivors. Pulmonary dysfunction is the third leading cause of non recurrence related cause of death among Hodgkin lymphoma survivors. They may be asymptomatic and need to be evaluated by pulmonary function testing. Limited data has shown that radiation exposure is a risk factor for pulmonary dysfunction in Hodgkin lymphoma survivors.

\section{Case Series of HIV Associated Lymphomas: A Single Center Experience}

Deepika Gupta ${ }^{* 1}$, Nitin Gupta ${ }^{1}$, Kartik Purohit ${ }^{1}$, Ajay Sharma ${ }^{2}$

${ }^{1}$ Clinical Hematology, Sir Ganga Ram Hospital, ${ }^{2}$ Clinical Hematology, Sir Gangaram Hospital, New Delhi, India

Aims \& Objectives: We report a consecutive case series of seven patients with HIV associated lymphomas.

Patients/Materials \& Methods: A retrospective review of patients treated in clinical hematology department of SGRH with HIV associated Lymphomas was performed from 2015 to 2020. The diagnostic test utilized includes the lymph node excision biopsy, duodenal biopsy, oral incision biopsy, bone marrow aspiration and biopsy, flowcytometry in CSF. Imaging modalities includes the PET CT scan to stage the disease and for metabolic response after treatment.

Results: Seven patients were diagnosed and treated during the above period. Five had CD20 + diffuse large B cell lymphoma, one had $\mathrm{CD} 30$ + peripheral $\mathrm{T}$ cell Lymphoma and one had Plasmablastic Lymphoma. The median age of Lymphoma diagnosis was 36 years. The median CD4 count at diagnosis for DLBCL was $301 \mu 1,151 \mu \mathrm{l}$ for Plasmablastic lymphoma and $84 \mu \mathrm{l}$ for PTCL. Case 1-5: Three out of five with DLBCL had CNS disease and were treated with 6 cycles of RCHOP along with CNS directed therapy including intrathecal Methotrexate. One patient had CNS relapse and was further treated with 4 cycles of DeAngelis regimen but had progressive disease and is now on Ibrutinib with Rituximab therapy. Another patient with CNS disease succumbed to illness after 2 cycles of HDMTX with Procarbazine. One patient has complete metabolic response on PET CT and is in remission for 5 years. 2 patients are recently diagnosed and received one cycle of RCHOP. Case 6: He had CD30 + Peripheral T cell lymphoma and was treated with 6 cycles of RCHOP with partial response followed by 4 cycles of GDP and further consolidated with autologous stem cell transplant. $\mathrm{He}$ is in remission for 1 year. Case 7: One patient diagnosed with Plasmablastic Lymphoma was treated with 5 cycles of DA-EPOCHR following which he had CNS relapse. He was treated with 4 cycles of R-MVP. Post 4 cycles he succumbed to Neutropenic sepsis.

Discussion \& Conclusion: With the introduction of HAART and novel chemoimmunotherapy, the overall survival has increased in HIV associated Lymphoma. The CNS involvement is more common 
at diagnosis or during the course of treatment in HIV associated Lymphomas.

\section{Role of Fine Needle Aspiration Cytology in Diagnosis of Mediastinal Lymphomas}

\author{
Arvind Natarajan ${ }^{* 1}$, Chaithra GV ${ }^{1}$, Aditi Dixit ${ }^{1}$, Sharada Rai ${ }^{1}$ \\ ${ }^{1}$ Pathology, Kasturba Medical College, Mangalore, India
}

Aims \& Objectives: To study utility of Fine needle aspiration cytology in diagnosis of mediastinal lymphoma.

Patients/Materials \& Methods: Retrospective cross- sectional observational study wherein cases meeting the inclusion criteria in last eight -years duration were collected i:e period from January 2012 to July 2018. The study was conducted in department of Pathology, Kasturba Medical College, Mangalore in the cytology and histopathology section. All the patients with mediastinal lesions diagnosed as lymphoma on aspiration cytology or biopsy were studied. Patients with guided aspiration cytology samples from mediastinal lesions with excision biopsy report were included in this study. Aspirates from these twelve patients were studied in detail for various cytological features. Cytomorphological features were evaluated with biopsy report as standard.

Results: Total twelve cases were evaluated. Predominance of male patients was seen with ten out of twelve cases studied. Half of total cases studied were in the age group of 15-30 years. Total of five cases of Lymphoma were detected on cytology out of nine cases of Lymphoma diagnosed on histopathology. One out of two cases of Hodgkin lymphoma was detected on cytology. Four cases of Non Hodgkin's lymphoma was diagnosed on cytology out of seven cases of Non Hodgkin's lymphoma on histopathology. Five cases of lymphoma out of eight cases diagnosed on cytology were confirmed on histopathology.

Discussion \& Conclusion: Cytology is a reliable diagnostic method in evaluation of neoplastic mediastinal lesions. High accuracy seen in Non Hodgkin's lymphoma.

\section{Catch Me If You Can: Transformation of Myeloma to Plasmablastic Lymphoma}

Rohith Chitrapur $^{* 1}$, Shreyas hanmantgad ${ }^{1}$, Suneetha $\mathrm{K}^{1}$, Vishnu $\mathrm{R}^{1}$, $\mathrm{Saro} \mathrm{Jacob}^{2}$, Anupama R ${ }^{2}$, Goutham Sunny ${ }^{2}$

${ }^{1}$ Hematology and Bone Marrow Transplant, ${ }^{2}$ Radiotherapy, Bangalore Baptist Hospital, Bangalore, India

Aims \& Objectives: Catch me if you can-transformation of myeloma to plasmablastic lymphoma.

Patients/Materials \& Methods: Multiple myeloma (MM) a plasma cell neoplasm commonly seen in elderly patients characterized by Anaemia,Renal failure,Hypercalcaemiaby and bone lytic lesions. Younger patients the disease is aggressive and response to therapy is suboptimal and chances of transformation are likely. Transformation to Plasma blastic lymphoma is a rarity.

Results: 45 year old female evaluated for pain in the left upper limb from January 2020 following hit by a two wheeler. The $\times$ ray showed lytic lesion with pathological fracture to the left humerus. PET CT scan done showed multiple bone lytic lesion (left humerus, proximal left radius, head of left humerus, acromion of left scapula, right parietal bone, manubrium, sternum, few ribs bilaterally, several dorsal vertebra, sacrum, bilateral pelvic bones and bilateral femurs. She also had FDG avid lesion in the thyroid gland with left lobe of thyroid with SUVmax of 21.9.Biopsy from the left humerus suggestive of Plasmacytoma. Bone marrow aspiration and biopsy showed plasma cells
$25 \%$. Protein electrophoresis showed a peak in gamma region with paraprotein quantification of $4.26 \mathrm{~g} / \mathrm{dl}$ and gamma globulin $4.77 \mathrm{~g} / \mathrm{dl}$. $\mathrm{IgG}$ quantification was $6689 \mathrm{mg} / \mathrm{dl}$. She received 5 fractions of radiation to the left arm for pain. She received chemotherapy with Tab. Pomalidomide, Cyclophosphamide, Dexamethasone for a total of 6 cycles.Post 6 cycles patient presented with Para paresis in September 2020. She was started on Radiation for Cord compression. Discussion \& Conclusion: Results: Work up for Myeloma when she presented with paraparesis showed $\mathrm{M}$ band $-0.64 \mathrm{G} \%$, IFE-Monoclonal Gammapathy seen in IgG Lambda. PET CT scan showed hyper metabolic skeletal, bone marrow, spinal, pancreatic, thyroid lesions, muscular, subcutaneous deposits, skin thickening, axillary, inguinal and retroperitoneal lymph nodes.Viral markers(HIV/Hbsag,HCV)negative.LDH-698. Biopsy of the Lymph node and scalp lesion was done,IHC- CD138- Strong diffuse membranous expression seen in the atypical cells,CD3- Negative, CD20- Negative, Ki67- $>95 \%$ of the CD138 + cells- Plasmablastic Lymphoma.FNAC of the thyroid suggestive of Papillary carcinoma thyroid. Conclusions: Transformation of Myeloma to Plasmablastic Lymphoma is a rarity with a underlying Papillary carcinoma of thyroid.

\section{An Unusual Case of Discordant Lymphoma: Large B-Cell Lymphoma of the Skin and Peripheral T-Cell Lymphoma, NOS of the Lymph Node}

Rushreeta A. Deuri ${ }^{* 1}$, Dhivya Kuppuraman ${ }^{2}$, Soundarya Ravi ${ }^{2}$, Sree R. Jinkala ${ }^{2}$, Debdatta Basu², Rakhee $\mathrm{Kar}^{2}$, Neelaiah Siddaraju ${ }^{2}$, Debasis Gochhait $^{2}$, Prabhu Manivannan ${ }^{2}$

${ }^{1}$ JIPMER, Puducherry, India, ${ }^{2}$ Pathology, JIPMER, Puducherry, India

Aims \& Objectives: To report an unusual case of discordant lymphoma and discuss its histomorphological features.

Patients/Materials \& Methods: A 49-yr old male presented with intermittent fever associated with chills and rigors, cough, loss of appetite, easy fatigability and varied raised ecchymotic patches over the body for a duration of three months and pedal edema for one month. On examination, he was found to have massive hepatosplenomegaly and generalised lymphadenopathy. CECT thorax revealed, tree in bud nodules with few fibrotic bands in the upper lobe. Skin biopsy from ecchymotic patches, FNAC and lymph node (LN) biopsy of axillary LNs were done and studied. Bone-marrow biopsy was sent for staging.

Results: Skin biopsy from the anterior abdominal wall and left forearm showed dermis infiltrated by relatively monomorphous population of large atypical lymphoid cells with evidence of angiocentricity, extending into the subcutis. After immunohistochemical (IHC) work up, Large B-cell lymphoma was considered with differentials of lymphomatoid granulomatosis and EBV positive cutaneous diffuse large B-cell lymphoma (DLBCL). FNAC of bilateral axillary LNs showed necrosis with polymorphous population of lymphoid cells admixed with large atypical cells. ICC showed positivity for LCA and CD30 on these cells and a report of NHL of T-cell origin was suggested. Inguinal LN biopsy showed near total replacement of architecture with similar large atypical cells; angiocentricity and perinodal extension were identified. After IHC work up, a peripheral T-cell lymphoma (PTCL), NOS was suggested. IHC details are provided in the table. Bone marrow examination was positive for infiltration by T-cell lymphoma (CD3 positive).

Discussion \& Conclusion: Discordant lymphomas are extremely rare entities and involves occurrence of two distinct histological lymphomas at two different anatomical sites. There are only few cases reported worldwide and this was the first of its kind where the LN biopsy was reported as PTCL, NOS and skin biopsy as lymphomatoid 
granulomatosis/EBV positive cutaneous DLBCL. As they are extremely rare, there are no studies to determine the behaviour and prognosis of these cases.

\section{Ten Year Follow Up of Patients with Solitary Plasmacytoma; A Single Institution Study}

Gurram Sreeram $^{* 1,2}$, Pavithran $\mathrm{K}^{1}$, Wesley M. Jose ${ }^{2}$, Arun Philip ${ }^{2}$, Sumi Soman ${ }^{2}$

${ }^{1}$ Medical Oncology and Hematology, ${ }^{2}$ Medical Oncology, Amrita Institute of Medical Sciences, Ernakulam, India

Aims \& Objectives: To evaluate the clinical and radiological features and progression free survival involved in the progression of plasmacytoma to overt myeloma.

Patients/Materials \& Methods: It's a retrospective study at a single tertiary centre from 2005. After the detection of a plasmacytoma, patients underwent bone marrow studies, other biochemical parameters and PET CT for assessing bone involvement and to rule out multiple myeloma.

Results: A total of 45 patients were evaluated. The Male/Female ratio is $2.2: 1$ with males accounting for $68.9 \%(\mathrm{n}=31)$ and females accounting for $31.1 \%$ cases $(\mathrm{n}=14)$. The age range in males was 37 to $88 \mathrm{yrs}$ (median age $-64 \mathrm{yrs}$ ) and in females was 49 to 76 years (median age-63.5yrs). 37 patients have solitary plasmacytoma of the bone with dorsal vertebra being most common site followed by femur and 8 patients have solitary extramedullary plasmacytoma all accounting for different sites. Out of all patients, 16 patients received both radiation and surgery, 27 patients received radiation, 2 patients did not receive any form of treatment. Out of 45 patients, 20 patients progressed to myeloma, 5 patients had only local progression, 20 patients have no evidence of disease in follow up. At a median follow up period of 102 months we have a survival of $43 \%$. There was no gender difference in progression to myeloma. Median progression free survival (PFS) noted in our study was 84 months.

Discussion \& Conclusion: Being a rare entity, the diagnosis of Solitary plasmacytoma requires the exclusion of multiple myeloma and other plasma cell disorders. Patients requires periodic surveillance as the disease has increased risk of transformation of solitary plasmacytoma into multiple myeloma.

\section{Pancytopenia, A Rare Presentation of Waldenström's Macroglobulinemia: Approach to Diagnosis and a Brief Review of Literature}

Om Prakash', Om Prakash ${ }^{* 1}$

${ }^{1}$ Pathology, Dr RML Hospital, New Delhi, India

Aims \& Objectives: Waldenström's Macroglobulinemia (WM) is a low grade B cell neoplasm characterized by infiltration of the bone marrow by a lymphoplasmacytic infiltrate along with IgM monoclonal gammopathy.

Patients/Materials \& Methods: 70-year-old male who presented with pancytopenia and a relative prominence of lymphoplasmacytoid cells.

Results: Pancytopenia, a rare presentation of Waldenström's Macroglobulinemia.

Discussion \& Conclusion: Waldenström's Macroglobulinemia, Pancytopenia, IgM kappa monoclonality.
Monoclonal B Lymphocytosis in a Recovering Covid-19 Patient

Nandini Govindarajan ${ }^{* 1}$, Kala Kandhasamy ${ }^{1}$

${ }^{1}$ Dr. Kamakshi Memorial Hospital, Chennai, India

Aims \& Objectives: Monoclonal B lymphocytosis (MBL) is a recently recognised premalignant disease. In WHO 2016 revision of classification of hematolymphoid neoplasms it has been recognised as a distinct entity. MBL is defined as monoclonal B cell population $<5 \times 10^{9} / \mathrm{L}$ in otherwise asymptomatic patients.

Patients/Materials \& Methods: Case history: A 71 year old gentleman was admitted with difficulty in breathing and cough to our hospital. On further evaluation he was found to be COVID 19 positive. He did not show significant lymphopenia at admission. As he was recovering, he showed persistent relative lymphocytosis. Peripheral smear showed occasional smudge cells. On clinical examination and imaging he did not have any lymphadenopathy or splenomegaly.

Results: Immunophenotype: Bright positive for CD19, Moderate positive for CD200, CD22, CD5, CD23, Dim positive for CD 20 and negative for FMC 7, CD103, CD34, CD38, CD10, CD 11c, CD25, surface Ig and ZAP 70. Since the patient had monoclonal b lymphocytes less than $5 \times 10^{9} / \mathrm{L}$ and he did not have lymphadenopathy, hepatosplenomegaly or any extra medullary manifestations, he was diagnosed to have Monoclonal B lymphocytosis.

Discussion \& Conclusion: Monoclonal B-cell lymphocytosis (MBL) is defined as less than $5 \times 10^{9} / \mathrm{L}$ monoclonal B cells in the blood of otherwise healthy patients and is detected in $5 \%$ to $10 \%$ of individuals older than 60 years. The differential diagnosis between MBL, CLL (Chronic lymphocytic leukemia) and another related condition known as small lymphocytic lymphoma is based on peripheral blood B-cell counts and physical examinations on patient. The most common type is the CLL like MBL which has the same immunophenotype as typical CLL. MBL is further divided into low count and high count MBL with a cut off of $0.5 \times 10^{9} / \mathrm{L}$ of monoclonal $\mathrm{B}$ cells. MBL is a recognised premalignant disease and especially the high count MBL have the propensity to progress to CLL at a much higher rate. Hence it is important to evaluate persistent lymphocytosis in the elderly even when they are asymptomatic and total WBC count is within normal limits. The detection of smudge cells in the smear was an important clue for further evaluation in this case.

\section{A Cost-Comparison Model for the Different Treatment Options in Advanced Hodgkin's Lymphoma}

Luxitaa Goenka $^{* 1}$, Prasanth Ganesan ${ }^{1}$, Shankar Prinja ${ }^{2}$

${ }^{1}$ Medical Oncology, JIPMER, Pondicherry, ${ }^{2}$ Department of Community Medicine \& School of Public Health, PGIMER, Chandigarh, India

Aims \& Objectives: Advanced Hodgkin's lymphoma (aHL) is usually treated with 6 cycles of combination chemotherapy such as (doxorubicin, bleomycin, vincristine and dacarbazine) ABVD. Escalated BEACOPP (EB) yields higher disease control at the cost of increased toxicity and cost. Positron emission tomography with computerize tomography (PET-CT) (iPET2) guided response adapted therapy (RAT) strikes a balance between efficacy and toxicity. With RAT, one could start with ABVD (and escalate for positive iPET2) or EB (and de-escalate for -ve iPET2). Though the initial treatment costs are higher when using EB due to the increased requirement of supportive care, there are no models available to compare the costs of these approaches. We believe that such an analysis might help to 
understand the dynamics better and may help to guide decisions of physicians involved in treating aHL.

Patients/Materials \& Methods: We created a model for comparing the costs of various treatment approaches in aHL. In addition to the cost of initial treatment, we also factored in risks of future relapses and costs of salvage. We created a modelling table which assessed each phase of treatment.

Results: Using standard costs available from governmental insurance schemes in India, the model yielded overall costs of treatment for 100 patients for a center to be 1,22,17,450 (for 6 cycles ABVD, nonRAT), 1,04,92,495 (start with ABVD and then RAT), and $1,06,09,415$ (start with EB and then RAT). Despite higher initial costs, the RAT-models worked out to be less expensive in the long term and could possibly yield superior survival.

Discussion \& Conclusion: The tabular system of cost calculation can be used by any center to estimate their overall treatment costs for aHL using any of the 3 approaches. This model can help centers/institutions to factor in cost-considerations when making policy decisions regarding the treatment of aHL at their center.

\section{Uncovering a Mysterious Lesion}

Arthi Arun ${ }^{* 1}$, Meenakshi Sundaram Manickavasagam², Sri Gayathri Shanmugam $^{1}$, Rajendiran Swaminathan ${ }^{1}$

\section{${ }^{1}$ Pathology, ${ }^{2}$ Medical oncology, SRIHER, Chennai, India}

Aims \& Objectives: Non Hodgkin's lymphomas should always be included as a differential diagnosis in cases of poorly differentiated malignancies as the presentation may not be very typical in all cases. Here we discuss the need of consolidated clinical, histopathological, immunohistochemical and radiological evaluation for a definitive diagnosis as it is of great prognostic and theranostic value.

Patients/Materials \& Methods: A 24 year old male patient presented with fever, weight loss, breathlessness and right cervical lymph node enlargement. Patient initially presented to respiratory medicine department and core biopsy of the cervical node was done. Core biopsy of the mass revealed sheets of highly pleomorphic cells with marked atypia and abundant mitotic figures and focal areas of sclerosis. Histological differential diagnosis of poorly differentiated carcinoma, germ cell tumor, sarcoma or a anaplastic lymphoma(ALCL/ALBL/Anaplastic variant of DLBCL and Mediastinal B cell lymphoma) was considered.

Results: Primary IHC work up were positive for vimentin, CD30 and CD45 and negative for desmin, pax8,CK7, CK20, SMA,p63,sox10 and SALL4. Further markers CD3, CD20 and ALK were done to rule out ALCL. CD3 and ALK was negative and CD20 was positive in tumor cells, with this a diagnosis of primary mediastinal B cell lymphoma was made. On further radiological evaluation patient had a mediastinal mass and axillary lymphadenopathy. Echocardiography revealed a mild cardiac tamponade.

Discussion \& Conclusion: Clinical presentation can be subtle in certain cases, however a thorough histological and appropriate immunohistochemical markers can help one to arrive at a definitive diagnosis. This case is an example of how a poorly differentiated mysterious lesion lead to a final diagnosis of Primary mediastinal B-cell by ruling out the differentials with the help of immunohistochemistry.
Guillain-Barre Syndrome in a Patient with Mantle Cell Lymphoma: Paraneoplastic or Mere Co-Incidence?

Faheema Hasan ${ }^{* 1}$, Anshul Gupta ${ }^{1}$, Soniya Nityanand ${ }^{1}$, Sanjeev Yadav $^{1}$, Yatendra Parashar ${ }^{1}$, Sujay Rainchwar ${ }^{1}$

${ }^{1}$ Dept of Hematology, SGPGI, Lucknow, India

Aims \& Objectives: INTRODUCTION: Mantle cell Lymphoma(MCL) is a mature B cell lymphoma accounting for 3-10\% of all Non Hodgkin Lymphomas (NHL).Neurological signs or symptoms in MCL are very rare.Guillain-Barre Syndrome(GBS) is the most common form of acute flaccid paralysis all over the world. Its association with NHL's are very rare. We present the first case of GBS in a patient with MCL.

Patients/Materials \& Methods: CASE REPORT: A 43 year male presented with complaints of bilateral axillary and cervical swelling for 8 months associated with B symptoms and intermittent episodes of per rectal bleeding. On evaluation, he had generalised lymphadenopathy. Laboratory tests were normal except for raised LDH. Biopsy was performed from the axillary lymph node was consistent with the diagnosis of MCL. FDG PET CT showed a stage IV disease.He was planned for alternating cycles of R-CHOP/R-DHAP followed by ASCT. On day two of chemotherapy, he had complaints of tingling sensation of his feet and slight weakness in his lower limbs.The weakness progressed to and subsequently involved upperlimbs. A neurological examination revealed no cognitive deficits and normal CNS. Motor examination revealed a symmetrical flaccid quadriparesis (power $2 / 5$ to $3 / 5$ ) with areflexia. Sensory examination demonstrated a mild decrease in vibration sensation in both feet, as well as reduced sensation in all fingers and toes.

Results: The acute onset and rapid progression of symptoms was suggestive of GBS. Cerebro Spinal Fluid showed a TLC of 2 cells with normal glucose and an elevated protein of $1.7 \mathrm{~g} / \mathrm{L}$.. Nerve conduction studies revealed absent sensory and motor responses as well as decreased amplitude in the upper and lower limbs. A diagnosis of GBS, AMSAN variant was made. Treatment was initiated with intravenous human immunoglobulin $(400 \mathrm{mg} / \mathrm{m} 2 /$ day $)$ for five consecutive days. The patient developed respiratory failure requiring mechanical ventilation. The patient's respiratory function recovered and he was extubated 9 days later. The patient was then discharged and was followed up on an OPD basis. Now the patient has received 5 cycles of R Benda and is in complete remission and is planned for ASCT.

Discussion \& Conclusion: NHL's are extremely rare to be associated with GBS.There is still inadequate data as to the etiology of GBS in haematological malignancies, whether it is a paraneoplastic manifestation or is reflective of immune dysregulation considering the elevated levels of interleukin-6 in the CSF of patients with GBS. This case report highlights the rare association of GBS with NHL and that early diagnosis and prompt initiation of therapy can ensure recovery of patients and will not hinder timely administration of chemotherapy therby improving patient outcomes.

\section{Single Agent Cytarabine is a Safe and Effective Option for Adult Langerhans Cell Histiocytosis}

Suvir Singh ${ }^{* 1}$, Jagdeep Singh ${ }^{2}$, Davinder Paul ${ }^{2}$, Kunal Jain ${ }^{2}$

${ }^{1}$ Clinical Haematology and Stem Cell Transplantation, ${ }^{2}$ Medical Oncology, Dayanand Medical College and Hospital, Ludhiana, India

Aims \& Objectives: Langerhans Cell Histiocytosis (LCH) is characterized by tissue infiltration with CD1a positive dendritic cells and 
is rare in the adult population with very little prospective data on clinical presentation and therapeutic outcomes.

Patients/Materials \& Methods: We present a patient with multisystem LCH who responded well to single agent cytarabine with minimal toxicity.

Results: A 36 year old gentleman without any history of smoking presented with multiple cervical lymphadenopathy since 3-4 months without any systemic symptoms. There was no organomegaly or other palpable lymphadenopathy. A CT neck done elsewhere showed multiple posterior cervical and pretracheal nodes, from which an FNAC was suggestive of lymphoma. An excision node biopsy revealed sheets of large atypical cells, 10-15 um in size, with grooved and indented nuclei with fine chromatin and inconspicuous nucleoli. Multiple eosinophilic microabscesses were also noted. Immunohistochemistry was positive for CD3, CD20, CD68, CD1a, S100, CD4, Langerin (CD207) and CD45 with a Ki67 index of less than $10 \%$. Renal and liver function were normal and there were no features of diabetes insipidus. A PET-CT showed metabolically active lymph nodes in cervical, mediastinal and abdominal regions along with erosion of left mastoid air cells (Fig 1), which was confirmed by MRI to indicate mastoiditis with sclerosis, likely secondary to disease involvement. A bone marrow and CSF examination were normal, finalizing the diagnosis as multisystem $\mathrm{LCH}$ without any risk organ involvement. After reviewing treatment options, he was initiated on chemotherapy with single agent cytarabine at a dose of $100 \mathrm{mg} / \mathrm{m} 2$ five days a month with which he had significant symptomatic improvement. A PET scan done after three cycles showed near complete metabolic resolution of previously involved nodes and bony lesions. He has completed five cycles of the same and is doing well, with a plan to continue Cytarabine for a total of 12 to 14 months.

Discussion \& Conclusion: LCH in adults is a rare entity with less than 300 cases ever described, and no prospective treatment data is available. Single agent cytarabine has been found safe and effective and can be given on out patient basis. Our patient demonstrated significant disease resolution with the same, associated with minimal toxicity. Compilation of prospective data will further clarify the utility of this regimen in adult patients with $\mathrm{LCH}$.

\section{Treatment Outcomes of Patients with Hodgkin Lymphoma: The Oncocollect Lymphoma Registry \\ Susmita Dasgupta ${ }^{* 1}$ \\ ${ }^{1}$ Clinical Haematology, TATA MEDICAL CENTRE, KOLKATA, India}

Aims \& Objectives: To audit Hodgkin Lymphoma (HL) characteristics and 5-year outcomes in a tertiary cancer center.

Patients/Materials \& Methods: 1396 lymphomas ( $>18$ years) were entered in the Onco-Collect data base of patients receiving 1st line treatment from May 2011 to December 2017. Demography, Clinical features, staging, prognostic stratification, and first line treatment response and outcomes of $271 \mathrm{HL}$ patients treated at a tertiary center have been analyzed. The audit was internally validated by estimating disease free survival (DFS) for known prognostic markers such as age groups, stage, and presence of symptoms. A PET directed approach was followed for most patients receiving ABVD therapy. Response rates and DFS were evaluated for patients receiving 1st line treatment.These patients were followed till September 2020.

Results: HL formed $20 \%$ of the lymphoma cohort receiving 1st line treatment at our Institute. The age distribution was $51 \%, 40 \%$ and $9 \%$ respectively for $<40$ years, between 40 to 65 years and $\geq 65$ years of age. Gender ratio for male to female was 2.17:1. 38\% Patients presented in early stage [I \& II], and 54\% in late stage [III \&IV]. Most common protocol used was standard ABVD in $90 \%$ patients. The overall response rate $(\mathrm{CR}+\mathrm{PR}+\mathrm{SD})$ was $92.6 \%$, and the progression on treatment $5.86 \%$. With a median follow-up of 48 months, the DFS is $79.36 \%$. DFS for the patients above 65 years was $62 \%$ at 5 years and for those below 65 years was $76 \%(p=0.052)$. The 5-year DFS for stage 1, 2, 3 and 4 was $94.73 \%, 85.57 \%, 78.81 \%$ and $67.78 \%$ respectively $(p=0.017)$.

Discussion \& Conclusion: HL remains highly curable in early stage with standard chemotherapy, but advanced stage disease needs attention.

\section{Diagnostic Performance of Whole Body Low Dose Computed Tomography Compared to Conventional Skeletal Survey in Diagnosis and Management of Patients with Multiple Myeloma}

Mirza Saleem Amjad ${ }^{* 1}$, Gurpreet Kaur ${ }^{1}$, Manoranjan Mahapatra ${ }^{1}$, Rishi Dhawan ${ }^{1}$, Priyanka Naranje ${ }^{2}$, Ankur Goyal ${ }^{2}$, Tulika Seth ${ }^{1}$, Mukul Aggarwal ${ }^{1}$, Pradeep Kumar ${ }^{1}$, Seema Tyagi ${ }^{1}$, Jasmita Dass ${ }^{1}$, Ganesh $\mathrm{K} \mathrm{V}^{1}$

${ }^{1}$ Dept of Hematology, ${ }^{2}$ Dept of Radio Diagnosis, Aiims, New Delhi, India

Aims \& Objectives: - This study prospectively evaluated the diagnostic performance of Whole Body Low Dose CT (WBLDCT) and compared its performance with Conventional Skeletal Survey (CSS) in detecting osteolytic bone lesions in patients with SMM and MM. Patients/Materials \& Methods: All suspected Multiple myeloma patients $\geq 18$ years of age were prospectively evaluated and patients qualifying for SMM and MM as per updated IMWG criteria were included. Both WBLDCT and CSS were done for bone disease evaluation.

Results: A total of 36 patients were included in the study out of which 22 patients who underwent both WBLDCT and CSS were compared. - Osteolytic lesions were detected in additional 6 (27.3\%) patients with WBLDCT as compared to CSS (Cohen's $\mathrm{k}=0.241, p=0.221$ ). The higher detection rate was more pronounced in axial skeleton [Skull-5 (22.7\%), cervical spine and sacrum - 7(31.8\%), Thoracic and lumbar spine-9(40.9\%)]. A total of 03 out of 04 SMM patients $(75 \%)$ as per former criteria could be upstaged to MM based on detection of osteolytic lesion on WBLDCT as per updated IMWG diagnostic criteria. In comparision to WBLDCT, CSS had a Sensitivity of $62 \%$, Specificity of $67 \%$ and diagnostic accuracy of only $64 \%$ thus confirming increased sensitivity and superior diagnostic performance of WBLDCT over CSS in detecting osteolytic lesions in MM in our present study.

Discussion \& Conclusion: The updated IMWG criteria defining newly diagnosed Multiple myeloma needing treatment require a thorough imaging evaluation of the skeleton to detect bone (B of CRAB) and bone marrow (additional Myeloma defining event) lesions for which more sensitive cross sectional imaging modalities were included (WBLDCT, PET CT or MRI) in addition to conventional skeletal survey (CSS) which has been the only standard imaging modality for decades.

- Our study serves as validation study to the recent IMWG consensus recommendations on imaging in Plasma cell disorders for preferential use of WBLDCT instead of CSS in the diagnosis of Multiple Myeloma.

- As WBLDCT is easy, convenient, relatively cheap and widely available with comparable radiation dose we recommend for the upfront use of WBLDCT (alone or as part of PET CT) instead of CSS for evaluation of patients with suspected MM and follow up of SMM and MM. Efforts are to be taken to bring down the cost further and optimize low dose protocols locally to enable wide usage of this modality which is the new standard of care. 


\section{Supporting Document:}

\begin{tabular}{|c|c|c|c|c|c|}
\hline Region & सsreement & conter's ikappe & PValue & $\begin{array}{l}\text { WBLLCT present } \\
\text { but coss absert }\end{array}$ & $\begin{array}{l}\text { CS5 present but } \\
\text { wBingr absent }\end{array}$ \\
\hline Skull & Fair & 0.273 & 0.193 & $5(22.746)$ & $3(13.6 \%)$ \\
\hline Mandible & Moderate & 0.463 & 0.010 & $2(9.196)$ & 0 \\
\hline Cervical spine & Poor & 0.000 & - & $7(31.8 \%)$ & 0 \\
\hline Thoracic spine & Poor & 0.108 & 0.262 & $9(40.9 \%)$ & 0 \\
\hline Lumbar spine & Poor & 0.108 & 0.262 & $9(40.9 \%)$ & 0 \\
\hline Sacrum & Poor & 0.154 & 0.176 & $7(31.896)$ & 0 \\
\hline Sternum & Poor & 0.000 & 1.000 & $4(18.296)$ & 0 \\
\hline Rib cage & Near Perfect & 0.831 & $<0.001$ & $1(4.596)$ & 0 \\
\hline Ilium Right & Moderate & 0.433 & 0.014 & $5(22.7 \%)$ & 0 \\
\hline llium Left & Fair & 0.353 & 0.030 & $5(22.75)$ & 0 \\
\hline Pubic bone Rt & Poor & 0.185 & 0.134 & $6[27.3 \%)$ & 0 \\
\hline Pubic bone tt & Fair & 0.353 & 0.030 & $3(13.696)$ & 0 \\
\hline Ischium Right & Poor & 0.185 & 0.134 & $6[27.3 \% 6]$ & 0 \\
\hline Ischium Left & Fair & 0.353 & 0.030 & $3(13.6 \%)$ & 0 \\
\hline Clavicle Right & Fair & 0.225 & 0.095 & $5[22.736)$ & 0 \\
\hline Clavicle Left & Poor & 0.000 & 1.000 & $4(18.296)$ & 0 \\
\hline Scapula Right & Poor & 0.000 & - & $5(22.7 \%)$ & 0 \\
\hline Scapula Left & Poor & 0.000 & 1.000 & $4(18.296)$ & 0 \\
\hline Bumerus Right & Fair & 0.391 & 0.060 & $1(4.896)$ & $3(14.3 \%)$ \\
\hline Humerus left & Moderate & 0.463 & 0.028 & $2(10.0 \%)$ & $1[5.0 \% 6)$ \\
\hline Femur Right & Fair & 0.390 & 0.390 & $1[5.0 \% 6]$ & $4(20 \%)$ \\
\hline Femur left & Poor & 0.146 & 0.496 & $2(9.596)$ & $3(14.3 \%)$ \\
\hline Whole Body & Fair & 0.241 & 0.221 & $6[27.3 \% 6\}$ & $2[9.196]$ \\
\hline
\end{tabular}

\section{Outcome of Paediatric Hodgkin's Lymphoma: Single Centre Experience}

Rishu Vidhatri ${ }^{* 1}$, Prakash S. Shekhawat ${ }^{1}$, Sumit Mitra ${ }^{1}$, Subham Bhattacharyaa $^{1}$, Shuvra N. Baul ${ }^{1}$, Prakas K. Mondal ${ }^{1}$, Rajib De ${ }^{1}$, Tuphan K. Dolai ${ }^{1}$

\section{${ }^{1}$ Department of Hematology, NRSMCH, Kolkata, India}

Aims \& Objectives: Hodgkin's lymphoma (HL) in children is a highly curable disease. We aimed to assess the clinicopathological features and treatment outcome of paediatric HL at our centre.

Patients/Materials \& Methods: This prospective study was done from March 2017 to April 2020 on 30 previously untreated patients with classical HL of age $\leq 18$ years. Patients were treated with either adriamycin, bleomycin, vincristine, and dacarbazine(ABVD) or vincristine etoposide procarbazine prednisolone adriamycin/cyclophosphamide, vincristine, prednisone, procarbazine and dacarbazine (OEPPA/COPPDAC) chemotherapy initially, and radiation to bulky sites or a single site of residual disease when appropriate.

Results: The median age of presentation was 11 years (4-18) and $60 \%(18 / 30)$ were male. The extent of disease was stage I 13\% (4/30), II $20 \%$ (6/30), III 13\% (4/30) and IV 53\% (16/30). B symptoms were present in $43 \%(13 / 30)$ of the patients and $43 \%(12 / 30)$ had bulky disease at presentation. No of patients treated with ABVD was 13 and 17 received OEPPA/COPPDAC. Overall response (OR) at end of chemotherapy with ABVD and OEPPA/COPPDAC was $61.5 \%(8 / 13)$ and $70 \%(12 / 17)$ respectively. Six months overall survival (OS) was $96 \%$.

Discussion \& Conclusion: Paediatric HL in our population show male preponderance and majority of patients present with stage IV disease. Overall response is better with OEPPA/COPPDAC when compared with ABVD and overall survivor at 6 months follow up was $96 \%$.

\section{Multiple Myeloma Developing in a Patient with Chronic Lymphocytic Leukemia: Is It Serendipity or Biology?}

Ashwin Nair ${ }^{* 1}$, Arihant Jain ${ }^{1}$, Sreejesh $\mathrm{S}^{2}$, Ritambhra Nada ${ }^{3}$, Narender Kumar ${ }^{2}$, Pankaj malhotra ${ }^{1}$

\section{${ }^{1}$ Internal Medicine, ${ }^{2}$ Hematology, ${ }^{3}$ Histopathology, PGIMER,} Chandigarh, India

Aims \& Objectives: Both Chronic lymphocytic leukemia (CLL) and Multiple Myeloma (MM) are known to arise from a precursor neoplastic clone of mature B lymphoid cell. There is scarce literature on the natural history of patients diagnosed with concomitant MM and CLL. We describe our experience of managing a case of CLL who later developed MM.

Patients/Materials \& Methods: An 82 year old male, who was a diagnosed case of cytogenetically. normal Chronic Lymphocytic Leukemia RAI-stage 0 on observation presented 4 years later with c/o radicular pain and low back ache of 2 months duration. In the current presentation he had a Hb- $12.7 \mathrm{~g} / \mathrm{dl}, \mathrm{TLC}-27,100 \mathrm{cells} / \mathrm{mm} 3(70 \%$ lymphocytes), platelet $-1,83,000$, Calcium- $8.60 \mathrm{mg} / \mathrm{dl}$, S.Cr -0.95 and S.LDH- $150 \mathrm{mg} / \mathrm{dl}$ ) with peripheral blood film showing monotonous population of lymphoid cells with smudge cells. MRI spine revealed osteolytic lesions in L1 \& L3 vertebrae and a right renal lesion, while PET scan revealed FDG avid lytic lesions in vertebrae and right scapula with soft tissue component. However, there was no evidence of lymphadenothy or hepatosplenomegaly or B symptoms. His serum protein electrophoresis showed a positive IgG Kappa Mband $(1.64 \mathrm{~g} / \mathrm{L})$. Considering a possibility of a second malignancy such as Renal cell carcinoma, Multiple Myeloma vs Richter's transformation, a PET guided biopsy from vertebral lesion as well as a BM examination was done. Vertebral biopsy revealed sheets of plasma cells which were CD38 and CD 138 positive. BM examination revealed a dual population of $3 \%$ clonal kappa restricted plasma cells and $38 \%$ mature lymphoid cells, that were CD5/CD23 + on immunochemistry. The FISH from peripheral blood on $\mathrm{CD}$ $19+$ cells was negative for del13q,del11q,del17p and BM aspirate FISH on immuno-magnetically separated enriched plasma cells was negative for TP53 mutations, 1q abnormalities, $\mathrm{t}(14: 16), \mathrm{t}(4: 14)$. His SFLC was showing elevated kappa/lambda ratio. A final diagnosis of CLL RAI-0 BINET-A with IgG Kappa MM RISS-I was made. Considering his age he was managed with RVD-lite regimen (lenalidomide $15 \mathrm{mg}$, bortezomib $1 \mathrm{mg}$ and dexamethasone $20 \mathrm{mg}$ ) along with bisphosphonates. After 3 cycles he had clinical improvement in back pain and his M-band also reduced by $50 \%$. After 6 cycles of therapy he had achieved VGPR and also attained CR with respect to CLL according to IWG criteria.

Results: Standard treatment of MM may lead to response in CLL possibly due to overlapping activity of lenalidomide and corticosteroids on both the diseases. Further studies are required to determine the clonal relationship between the two mature B-cell malignancies. Discussion \& Conclusion: kindly note that this is a case report. there was no format available for case report submission. hence submitted like this.

\section{Aims \& Objectives: NA.}

\section{Patients/Materials \& Methods: NA.}

Results: A 49 year old male; a case of Hodgkin's Lymphoma Stage IVB presented with painful vesicular eruptions over his face after 2 days of first cycle ABVD chemotherapy. Examination revealed herpes zoster opthalmicus with disseminated zoster and he had Grade IV neutropenia (ANC 120/ $\mu$ l). During admission and subsequent stay in the hospital he developed high grade fever with septic cutaneous emboli both feet resembling Osler nodes. Punch biopsy of these lesions showed vasculitis with Staphylococcal colonies and Tzanck smear showed characteristic viral inclusions. Blood cultures were sterile and transthoracic echocardiography did not reveal any 
vegetations. He also developed hepatitis probably Zoster hepatitis with resolution of skin; eye lesions and hepatitis with acyclovir and vancomycin for staphylococcal septicemia. He made a complete recovery and is currently on secondary prophylaxis with acyclovir.

Discussion \& Conclusion: Herpes zoster prophlyaxis is not the standard of care during Hodkin's lymphoma chemotherapy. A rare presentation of disseminated zoster with staphylococcus septicemia is discussed.

\section{Auer Rods Like Inclusions in Multiple Myeloma: Report of Two Diagnostically Challenging Cases}

Shashi Bansal ${ }^{* 1}$, Arpita Mathur ${ }^{2}$, Upendra Sharma ${ }^{3}$, Amandeep Talwar $^{2}$

${ }^{1}$ Haematopathology, ${ }^{2}$ Pathology, ${ }^{3}$ Clinical Haematologisthaemato-oncologist, Bmchrc, jaipur, India

Aims \& Objectives: Non-immunoglobulin intracytoplasmic inclusions in plasma cells of multiple myeloma are very rare presentation. These are morphologically similar to Auer rods but chemically different from them.

Patients/Materials \& Methods: We studied two cases of multiple myeloma in a 60-year old woman and 45-year old man. Bone marrow examination, serum electrophoresis and immunofixation were done. Results: In both cases, plasma cells of bone marrow aspirate revealed multiple Auer Rods like Inclusions (ARLI). Sudan Black B (SBB) and Myeloperoxidase (MPO) cytochemistry were negative. Serum protein electrophoresis in both of them showed $\mathrm{M}$ spike, one with raised IgA-Kappa levels while other with raised IgG-Kappa levels.

Discussion \& Conclusion: Very few case reports have been published in literature and its prognostic implications are still unknown. Due to its rarity, it is important to distinguish such cases from acute myeloid leukemia and its exact incidence with its therapeutic and prognostic implications.

Supporting Document:

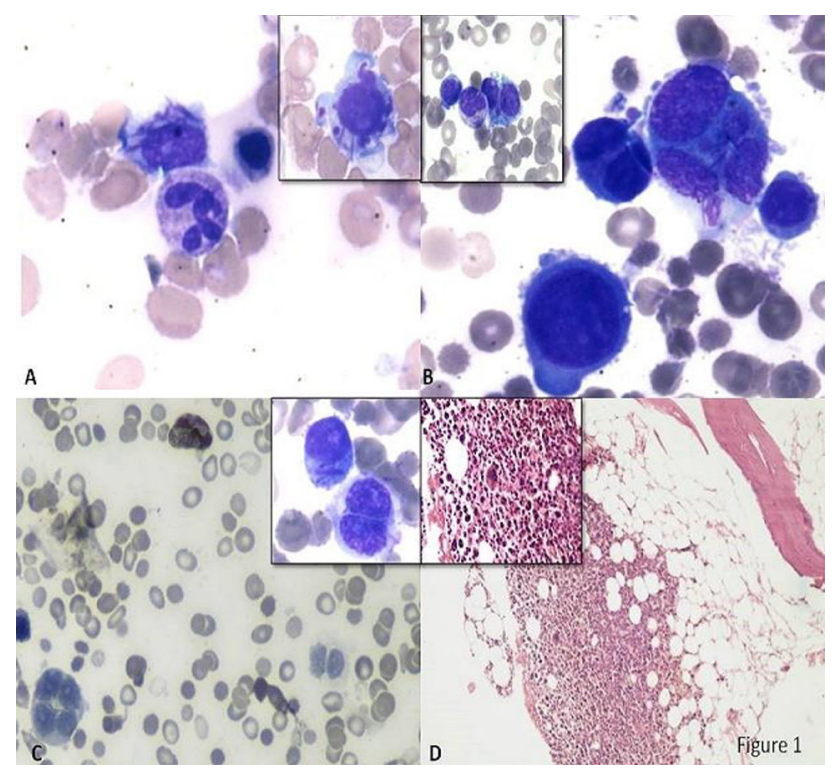

Immunophenotyping of Non Hodgkin Lymphomas: Flow Cytometric Analysis at Tertiary Health Care Centre

Manali Satiza ${ }^{* 1}$, Nisha Marwah ${ }^{1,2}$, Monika Gupta ${ }^{1}$, Sudhir Atri ${ }^{3}$, Rajeev $\operatorname{Sen}^{1}$

${ }^{1}$ Pathology, PGIMS Rohtak, Rohtak, ${ }^{2}$ Pathology, PGIMS Rohtak, India, ${ }^{3}$ Hematology, PGIMS Rohtak, Rohtak, India

Aims \& Objectives: Immunophenotyping of Non Hodgkin Lymphoma and staging by Flow Cytometry and further subtyping of cases using a minimum panel of monoclonal antibodies.

Patients/Materials \& Methods: This study was conducted over a period of two years at Dept of Pathology \& Clinical Hematology, PGIMS, Rohtak. All the clinically suspected, diagnosed cases of NonHodgkin Lymphoma (NHL) on Fine needle aspiration or Lymph node biopsy were evaluated for peripheral involvement by Flow Cytometry (FCM) on peripheral blood or bone marrow aspirate samples using a panel of monoclonal antibodies.

Results: All the cases in study comprised of B-cell NHL. These cases were further classified into Chronic Lymphocytic Leukemia (CLL) and Non-CLL group based on morphology and primary panel. An extended panel was used for further sub-typing of non-CLL cases which comprised of FMC7, CD200, CD25, CD103, CD123, CyclinD. FCM was able to diagnose most NHL including CLL. Atypical CLL, Mantle cell lymphoma, Hairy cell Leukemia and Marginal Zone Lymphoma while few of the cases remained unclassified. All the CLL cases were diagnosed using a primary panel of antibodies with a need for an extended panel for subtyping of non-CLL cases.

Discussion \& Conclusion: We propose a two-tier approach for immune-phenotypic analysis of newly diagnosed stage IV NHL cases by flow cytometry with a minimum primary panel including CD45,CD19, CD20, CD5, CD23, CD79, CD10, CD3, CD7,CD56 for differentiation into $\mathrm{B} / \mathrm{T} / \mathrm{NK}$ cell lymphomas, Classification of B-cell NHL further into CLL/non-CLL group and surface immunoglobulin (Kappa and lambda) for clonality assessment. An extended panel may be applied wherever required for further sub-typing and stratification of cases. This two tier approach using FCM in conjunction with morphology may be a cost-effective method and will avoid usage of unnecessary antibodies for sub-typing of cases.

Supporting Document:

\section{DISTRIBUTION OF CASES ON FLOW CYTOMETRY(FCM)}

\begin{tabular}{lll} 
FCM DIAGNOSIS & \multicolumn{2}{c}{ NUMBER OF CASES PERCENTAGE } \\
\hline \multicolumn{1}{|c}{ CLL } & $\mathbf{1 0}$ & $\mathbf{3 3 . 3} \%$ \\
\hline 1.CLL & 08 & $26.6 \%$ \\
2.Atypical CLL & 02 & $6.66 \%$ \\
NON-CLL & $\mathbf{2 0}$ & $\mathbf{6 6 . 6} \%$ \\
1.NHL(unclassified) & 15 & $50 \%$ \\
2.Mantle cell Lymphoma & 03 & $10 \%$ \\
3.Marginal zone Lymphoma & 01 & $3.33 \%$ \\
4.Hairy cell Leukemia & 01 & $3.33 \%$ \\
5.Follicular lymphoma & 00 & $0 \%$ \\
6.Diffuse large B cell lymphoma 00 & $0 \%$ \\
TOTAL CASES & $\mathbf{3 0}$ & $\mathbf{1 0 0} \%$
\end{tabular}




\section{Laboratory Parameters Associated with Bone Marrow Involvement in Non-Hodgkin Lymphoma}

Sinduja Raghuraman ${ }^{* 1}$, Sindhura Lakshmi Koulmane

${ }^{1}$ Pathology, Kasturba Medical College, Manipal, India

Aims \& Objectives: Bone marrow involvement (BMI) by primary nodal Non Hodgkin Lymphoma (NHL) indicates stage 4 disease as per the Lugano classification system.However, the staging heavily relies on FDG PET and CECT scans.Routine BM examination is no longer recommended, particularly in Diffuse large B- cell Lymphoma(DLBCL) subtype, indicated only if PET is negative and identifying a discordant histology is important for patient management.However,BM examination is still recommended for remaining subtypes along with immunophenotyping. A few studies have attempted to recognize predictive hematologic and biochemical parameters for BMI.These parameters provide important ancillary information in recognition and diagnosis of disease, histology, staging and prediction of morbidity and mortality.This study was aimed to assess the association of hematologic and biochemical parameters with BMI.

Patients/Materials \& Methods: This is a 3 and half year retrospective study that included consecutive cases of NHL for which pre- treatment BM examination was performed between January 2015 and June 2018. Complete Blood Count(CBC), biochemical parameters, peripheral smear, BMA and BMB were reviewed. The parameters were compared between the patients with and without BMI.Continuous variables were expressed in median (range).Student t- test was employed to study the association. $p$ value of $\leq 0.05$ was considered statistically significant.

Results: Total cases:199 (84 cases in BMI and 115 cases in Non-BMI groups).Table 1 results show that among the various laboratory parameters analyzed, $\mathrm{Hb}, \mathrm{Hct}$, \% Neutrophils, \% Basophils and Plt count in the BMI group were significantly lower than those in the non-BMI group ( $\mathrm{Hb}, p=0.03$; Hct, $p=0.02$; Neutrophils, $p<0.001$; Basophils, $p=0.04$; Plt count, $p<0.001$ ) whereas \% lymphocytes, PDW,S.LDH and the rate of PBI were significantly higher in the BMI group than in the non-BMI group (Lymphocytes, $p=0.01$, PDW, $p=0.005 ; \mathrm{S} . \mathrm{LDH}, p=0.04$;PBI, $p<0.001$ ).

Discussion \& Conclusion: In this study,decreased levels of $\mathrm{Hb}, \mathrm{Hct}, \mathrm{Plt}$ count, \% Neutrophis, \%Basophils along with increased levels of \% Lymphocytes, PDW,S.LDH levels and rate of PBI are suggested to be useful markers as predictors for BMI.These findings may be helpful for the diagnosis of BMI in clinical practice.

\section{Supporting Document:}

\begin{tabular}{|c|c|c|c|}
\hline Laboratory Parameters & $\begin{array}{l}\text { BMI Group } \\
\text { n=84 } \\
\text { Median(Min- } \\
\text { Max) }\end{array}$ & $\begin{array}{l}\text { Non BMI Group } \\
\mathrm{n}=115 \\
\text { Median(Min- } \\
\text { Max) }\end{array}$ & $\begin{array}{l}\text { P value } \\
\text { (Student } \\
\text { t test) }\end{array}$ \\
\hline Hemoglobin $(\mathrm{Hb}, \mathrm{g} / \mathrm{dL})$ & $11.1(5.6-18.3)$ & $11.8(4.4-16.7)$ & 0.03 \\
\hline Hematocrit(Het,\%) & $34.5(16.4-53.3)$ & $35.5(15.2-51.4)$ & 0.02 \\
\hline \multicolumn{4}{|l|}{ Differential Count(DC,\%) } \\
\hline Neutrophils & $59.7(12.2-89)$ & $65.2(35.5-93.6)$ & $<0.001$ \\
\hline Lymphocytes & $23.2(1-78)$ & $19.3(3-56)$ & 0.01 \\
\hline Basophils & $0.3(0-1.1)$ & $0.4(0-1.7)$ & 0.04 \\
\hline $\begin{array}{l}\text { Platelet count(PIt } \\
\left.\text { count, } x 10^{3} / \mu \mathrm{L}\right)\end{array}$ & $172.5(23-776)$ & $270(3-859)$ & $<0.001$ \\
\hline \begin{tabular}{l|} 
Platelet Distribution \\
Width(PDW $\%)$
\end{tabular} & $16.9(15.8-19.6)$ & $16.6(14.9-19.4)$ & 0.005 \\
\hline \multicolumn{4}{|l|}{$\begin{array}{l}\text { Peripheral Blood Involvement } \\
\text { (PBI,n(\%) }\end{array}$} \\
\hline Positive & $26(31)$ & $06(05)$ & $<0.001$ \\
\hline Negative & $58(69)$ & $109(95)$ & \\
\hline $\begin{array}{l}\text { Lactate } \\
\text { Dehydrogenase(LDH,IU/L) }\end{array}$ & $406(116-3303)$ & $260(135-5029)$ & 0.04 \\
\hline $\begin{array}{l}\text { Aspartate } \\
\text { Aminotransferase(AST,IU/L) }\end{array}$ & $24(10-397)$ & $24(11-701)$ & 0.4 \\
\hline $\begin{array}{l}\text { Alanine } \\
\text { Aminotransferase(ALT,IU/L) }\end{array}$ & $17.5(3-125)$ & $19(5-242)$ & 0.18 \\
\hline $\begin{array}{l}\text { Alkaline } \\
\text { phosphatase(ALP.U/L) }\end{array}$ & $101.5(51-1252)$ & $90(47-1363)$ & 0.5 \\
\hline Total Protein $(\mathrm{g} / \mathrm{dL})$ & $6.7(3.8-9.7)$ & $6.9(3.8-9.7)$ & 0.3 \\
\hline Serum Albumin $(\mathrm{g} / \mathrm{dL})$ & $3.9(1.6-4.9)$ & $3.9(1.9-5.4)$ & 0.4 \\
\hline Serum Globulin(g/dL) & $2.8(1.5-6.9)$ & $2.8(1.4-5.8)$ & 0.6 \\
\hline Serum Urea(mg/dL) & $22(8-84)$ & $20(8-128)$ & 0.3 \\
\hline & & $0.8(0,3-11$ & \\
\hline
\end{tabular}

\section{Post Transplant Lymphoproliferative Disease: Experience at a Tertiary Centre}

Nidhi Rathi ${ }^{* 1}$, Devika Gupta ${ }^{2}$, Kanwaljeet Singh ${ }^{3}$, Venkatesan Somasundaram $^{2}$, P S Mishra ${ }^{2}$, Tathagata Chatterjee ${ }^{2}$

${ }^{1}$ Dept of Lab Sciences \& Molecular Medicine, AHRR, Delhi Cantt, ${ }^{2}$ Dept of Lab sciences and molecular medicine, AHRR, New Delhi, ${ }^{3}$ Dept of Lab Sciences \& Molecular Medicine, AHRR Delhi Cantt, New Delhi, India

Aims \& Objectives: Post transplant Lymphoproliferative Disorders (PTLD) encompass a spectrum of lymphoid proliferations ranging from indolent, benign to malignant population of polyclonal to monoclonal cells. PTLD are defined as lymphomas arising in transplant recipients on immunosuppression. Recipients of both solid organ transplant (SOT) and Hematopoietic stem cell transplant (HSCT) have increased risk of developing lymphoproliferative disorders, mostly EBV related. We report here, the pattern of various categories of PTLDs in renal transplant cases, their diagnosis, treatment and outcome as observed at our centre.

Patients/Materials \& Methods: This was a retrospective analysis of 350 patients who underwent renal transplant at Army Hospital (Research \& Referral) over a period of last 06 years (2014-2019). We identified 06 cases who developed lymphoproliferative disease after kidney transplant during this period. All our 06 cases were confirmed as PTLD on organ specific biopsy, supported by immunohistochemistry.

Results: 06 cases were diagnosed with PTLDs out of a total of 350 renal transplant cases $(1.7 \%)$. All our 06 cases suffered from monomorphic B cell Non-Hodgkin's lymphoma. 02 cases presented within two years of renal transplant (early onset) whereas other 4 had late onset disease. Organ involvement was primarily extranodal (05 cases). Only one patient had involvement of the allograft by lymphoma whereas two suffered from Primary CNS lymphoma.

Discussion \& Conclusion: PTLDs are a major cause of increased mortality and morbidity in patients of solid organ transplant. PTLDs are rare diseases and hence a high index of suspicion on part of clinicians can help clinch the diagnosis in early stages and improve outcome.

\section{Role of Flow Cytometry in Diagnosis of Hematolymphoid Malignancies in Cytology Specimens}

Juhi Arora*1, Venkatesan Somasundram ${ }^{1}$, Ankur Ahuja ${ }^{1}$, Kanwaljeet Singh ${ }^{1}$, Rohit Tewari ${ }^{1}$, Vikram Singh ${ }^{1}$, Juhi Arora ${ }^{1}$, Tathagat Chatterjee ${ }^{1}$

${ }^{1}$ Dept of Lab Sciences \& Molecular Medicine, AHRR, Delhi Cantt, New Delhi, India

Aims \& Objectives: To observe the utility of flow cytometry (FCM) in diagnosis of hematolymphoid malignancies in cytology specimens. To observe the concordance between FCM with the conventional techniques such as cytology, cell block, tissue histopathology and immunohistochemistry (IHC).

Patients/Materials \& Methods: Cases of suspected hematolymphoid malignancy were subjected to cytological analysis after fine needle aspiration (FNA), Cerebrospinal fluid (CSF), pleural and peritoneal tap. The fluid specimens were also subjected to FCM analysis.

Results: We evaluated 16 cytology fluids by FCM. Total of 07 CSF were subjected to FCM, of which 05 cases were positive for blasts on morphology and the same was confirmed by FCM. One case had degenerated cells on morphology and no opinion could be offered on morphology, however FCM analysis revealed the presence of blasts. 
In this particular case, FCM had advantage over morphology. One case revealed reactive lymphocytes. Of the 5 lymph node aspirates, 01 had a relapse of B-ALL while other case was diagnosed as Follicular lymphoma and was confirmed on lymph node biopsy. Other 03 cases were reactive lymphadenoapthy. A mediastinal aspirate from $2 \mathrm{yr}$ old child was diagnosed as Precursor T lymphoblastic lymphoma. 2 pleural fluid aspirates subjected to FCM, 1 found involved by Chronic Lymphocytic Leukemia cells while other showed Burkitt lymphoma which was confirmed on cell block followed by IHC. An ascitic fluid was subjected to FCM and was negative for hematolymphoid malignancy.

Discussion \& Conclusion: The diagnosis of hematolymphoid malignancies in cytology specimens in the absence of ancillary techniques is challenging and error prone. Often one has to resort to cell block followed by immunohistochemistry or offer a provisional diagnosis and ask for a trucut/excision biopsy to arrive at a definitive diagnosis and these processes are time consuming. FCM, as brought out in this study can offer an accurate diagnosis in most of the haematological malignancies and this procedure can be carried out in cytology specimens such as body fluids, FNA specimens and at times the scrape cytology specimens of lymph nodes.

\section{Primary Extranodal NHL; Morphological Distribution Based on Recent WHO Classification \\ Nabanita De ${ }^{* 1}$, Asra N. Quadri ${ }^{1}$, Enam M. Khan ${ }^{1}$ \\ ${ }^{1}$ Pathology, Apollo Gleneagles Hospital (AGHL), Kolkata, India}

Aims \& Objectives: At least one-fourth of Non Hodgkin Lymphoma(NHL) arise from tissues other than lymph nodes and referred to as primary extranodal non Hodgkin lymphomas (pENL). In last two decades, incidence of pENL, has increased rapidly. The aim of this study is to evaluate the epidemiological and morphological trends and anatomical distribution of pENL in eastern India.

Patients/Materials \& Methods: This was a retrospective cross-sectional study conducted in pathology department of AGHL, Kolkata including pENL cases diagnosed in between 2016 and 2018. Relevant patient history, examination notes, detailed histopathological, immunohistochemical and radiological reports were obtained from hospital record. Old cases were reclassified according to latest 2016 WHO Classification.

Results: 26\% (50/186) of total NHL cases was pENL. M:F ratio was 1.8:1. Mean age was 57 years. Most common sites involeved were GIT $(30 \%)>$ Bone $(12 \%)>$ Brain and Spinal-Para spinal mass $(10 \%$ each). DLBCL was the commonest subtype. T cell lymphoma was rare (3\%). Among all DLBCL, NOS cases, 56\% was GCB type and $44 \%$ was non GCB type.

Discussion \& Conclusion: : Due to morphological mimics and varied clinical presentations correct diagnosis of pENL is frequently a challenge. In our study around 1/4th of total NHL was pENL, GIT was commonest site and DLBCL was commonest morphological subtype. These findings are comparable with some others studies.

\section{Footprints of CD200, CD79B \&CD43 in D/D Chronic Lymphocytic Leukemia \& It's Immunophenotypic Mimickers}

Sabina Langer ${ }^{* 1}$, Aditi Kundoo ${ }^{1}$, Kartik Purohit ${ }^{1}$, Nitin Gupta ${ }^{1}$, Jyoti Kotwal $^{1}$

${ }^{1}$ Hematology, Sir Ganga Ram Hospital, Delhi, India

Aims \& Objectives: To study expression patterns of CD200, CD79b and CD43 in differentiating classical CLL, atypical CLL and MCL.
Patients/Materials \& Methods: The cases reported as Classical CLL, Atypical CLL and MCL were retrieved from the records. The mean fluorescence intensities (MFIs) of antibodies were studied to identify pattern of expression. Level of significance of these markers in the differentiation of these sub groups was calculated by ANOVA test.

Results: From among the past 125 small lymphocytic (low grade NHLs) diagnosed on flowcytometry there were 76 classical CLL, 7 cases of atypical CLL \& 11 cases of MCL. The median MFIs of CD200, CD79b and CD43, in cases of classic CLL were 7646,366, 3813 respectively. In atypical CLL the median of markers in the above combination were 5114, 2184.5, 932.5 and in MCL MFIs were 152, 1695 and 1001. The MFI values of CD200, CD79b and CD43 were significantly different in the three subgroups $(p<0.05)$. When the MFIs of three antibodies were plotted on the histogram the pattern observed in classical CLL, atypical CLL and MCL were U shaped, step ladder and inverted $U$ shape respectively.

Discussion \& Conclusion: The flowcytometric immunophenotyping of chronic lymphocytic leukemia(CLL) has evolved over past decade. According to ERIC/ESCCA consensus for the diagnosis of CLL, CD19, CD5, CD23 and CD20 are the required markers and CD200, CD79b, CD43 are amongst the recommended markers. We selected cases of Classical CLL, Atypical CLL and Mantle Cell Lymphoma (MCL) and studied the expression of CD200,CD79b and CD43 in these 3 subgroups Conclusion: Flowcytometric immunophenotyping with this antibody cocktail show a unique pattern specific to each subgroup and can assist in the identification of these phenotypic mimickers. Key words: Atypical CLL, Mantle cell Lymphoma, Immunophenotypic pattern.

Supporting Document:
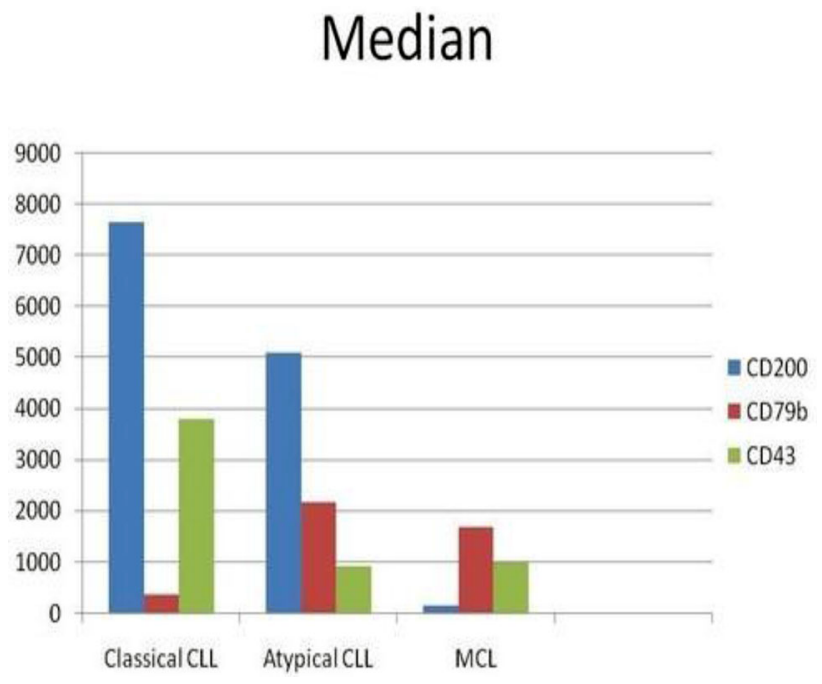

\section{A Rare Case of Round Blue Cell Tumour Primarly Presented as Metastasis in Bone Marrow \\ Prakrati R. Patel ${ }^{* 1}$, R. K. Nigam ${ }^{1}$, Reeni Malik ${ }^{1}$ \\ ${ }^{1}$ Pathology, Gandhi Medical College, Bhopal, India}

Aims \& Objectives: To present a case of a patient, presented with weakness, fatigue, bleeding manifestations, Roth's spots over sclera and to find out the cause by bone marrow aspiration.

Patients/Materials \& Methods: Peripheral smear and Bone marrow aspiration was done in Department of Pathology, GMC, Bhopal. Both 
peripheral blood and bone marrow were stained with Leishman stain and slides were examined microscopically by light microscope.

Results: Peripheral blood was suggestive of Dimorphic Anaemia with Thrombocytopenia. Bone marrow examination revealed metastatic deposits of round blue tumour cells forming Rosettes \& bare oval nuclei are also seen. Search for primary was advised.

Discussion \& Conclusion: In this case patient was advised bone marrow evaluation for bi-cytopenia i.e. anaemia, thrombocytopenia, which revealed metastatic deposits of round blue cells. On further evaluation, this case was diagnosed as small cell B cell lymphoma as primary, by histopathology and IHC studies.

\section{Multiple Myeloma: An Analysis of 70 Patients from a Tertiary Care Centre}

\section{Kamal Malukani ${ }^{* 1}$, Aditi Gupta ${ }^{1}$, Sushmita Tripathi ${ }^{1}$, Suvidhi Wankhede ${ }^{1}$ \\ ${ }^{1}$ Pathology, Sri Aurobindo Medical College \& PG Institute, Indore, India}

Aims \& Objectives: 1. To study the clinicodemographic factors like age, gender and clinical features of patients of Multiple Myeloma. (2) To depict hematological and biochemical parameters in cases of Multiple Myeloma. (3) To study radiological findings in patients of Multiple Myeloma. (4) To stage patients of Multiple Myeloma.

Patients/Materials \& Methods: This study included all cases of Multiple Myeloma diagnosed at SAIMS from July 2012 to June 2020. Clinical findings were recorded and relevant haematological and biochemical investigations done.

Results: Out of 70 patients, 48 were males and 22 females with a mean age of presentation 58.26 years. The most common clinical features observed were lower backache and bone pain $(95.71 \%)$ followed by generalized weakness $(49.6 \%) .17 .14 \%$ cases showed the presence of all the four features of CRAB criteria closely followed by cases $(15.71 \%)$ which presented with $\mathrm{R}+\mathrm{A}+\mathrm{B}+.83 .33 \%$ cases showed raised Beta-2 microglobulin levels levels ( $\geq 3 \mathrm{ug} / \mathrm{ml})$. However, on serum protein electrophoresis $\mathrm{M}$ band was present in $98.56 \%$ patients. Bence Jones proteinuria was detected in $10 \%$ patients only. The percentage of plasma cells was higher on the bone marrow trephine biopsy compared to the aspirate. Mature myeloma was the most common $(65.67 \%)$ morphologic subtype while plasmablastic myeloma was the least common (10.45\%). Plasmacytic morphology $(73.13 \%)$ was found to be more common than plasmablastic morphology (26.87\%). Most common pattern of infiltration on bone marrow trephine biopsy was interstitial $(42.86 \%)$ followed by diffuse pattern $(27.14 \%)$. Most of the patients presented in stage III disease $(75.71 \%)$. A higher incidence of hypercalcemia ( $\geq 11 \mathrm{mg} / \mathrm{dL})(50 \%)$, raised serum creatinine levels $(\geq 2 \mathrm{mg} / \mathrm{dL})(61.11 \%)$, hypoalbuminemia $(<3 \mathrm{~g} / \mathrm{dL})(83.33 \%)$ and anemia $(<10 \mathrm{gm} \%)(77.78 \%)$ were noted in plasmablastic group than in non- plasmablastic group. None of the prognostic variables studied i.e. haemoglobin levels, albumin levels, serum creatinine level, serum calcium level and age had any prognostic value independently.

Discussion \& Conclusion: The present study emphasizes not only the role of a bone marrow aspiration and biopsy in establishing a diagnosis of multiple myeloma, but also assessing the plasma cell burden in the marrow. Morphology of myeloma cells on bone marrow aspirate and biopsy was proved to be an important prognostic marker. Younger age of onset was noted in the index study. Some locoregional differences were remarkable.

\section{Extra Nodal Classical Hodgkin Lymphoma: A Series of 3 Cases at a Tertiary Care Centre}

Smriti Gupta ${ }^{1}$, Prabha S. Mishra ${ }^{* 1}$, Tathaghata Chatterjee ${ }^{1}$, Vikram Singh $^{1}$

\section{${ }^{1}$ Pathology, Army Hospital R\&R, New Delhi, India}

Aims \& Objectives: Reporting of increasingly more cases of extranodal classical Hodgkin lymphoma has generated much interest and debate. We report three such prospective cases of extra-nodal Hodgkin lymphoma diagnosed at our centre describing their important and rare clinicopathological attributes.

Patients/Materials \& Methods: We reported three cases of extranodal Hodgkin lymphoma in adult patients, one female \& two males, with very rare presentations in pleura, liver and nasopharynx respectively with no known involvement of nodes at the time of this writing.

Results: Case I. Thoracoscopic biopsy in a 38-year man showed multiple pleural lined soft tissue fragments with scattered Hodgkin Reed-Sternberg (HRS) like cells in a typical polymorphous inflammatory milieu. Case II. Liver nodule biopsy in a 32-year lady showed scattered large cells, few of them with classical RS-cell morphology, in a background of granulomatous inflammation and fibrosis. Case III. Nasopharyngeal biopsy in a 50-year man showed a soft tissue mass with dense polymorphous inflammatory infiltrates along with large lymphoid cells. In all three cases, neoplastic HRS cells were positive for CD15, CD30 \& MUM1 while negative for pan-CK, LCA, CD3, CD20 and HMB 45 among other markers.

Discussion \& Conclusion: Primary pleural Hodgkin lymphoma is a rare presentation of disease. More often, involvement of pleural nodes and soft tissue is seen in advanced stages of refractory disease. In our case, further work-up for involvement of other nodal or extra-nodal sites are underway at the time of this writing. Primary hepatic Hodgkin lymphoma is extremely rare. However, secondary liver along with splenic involvement can be seen in advanced disease. Ours is an exceptionally rare presentation of Hodgkin in a known case of sarcoidosis. Extra-nodal head and neck disease can be seen in less than $1 \%$ of cases of Hodgkin lymphoma and findings at nasopharyngeal biopsy are positive in about $20 \%$ of all such cases. However, nasopharyngeal Hodgkin in a diagnosed case of NHL is extremely rare.

\section{Utility of Multiparametric Flowcytometry in Bone Marrow Staging of Patients With B Cell: Non Hodgkins Lymphoma}

Thulasi Raman Ramalingam ${ }^{* 1}$, Lakshman Vaidhyanathan ${ }^{1}$, Anurekha Muthu ${ }^{1}$

\section{${ }^{1}$ Hematopathology, Apollo Hospital, Chennai, India}

Aims \& Objectives: BACKGROUND: Detection of lymphoma cells in staging marrow sample is crucial in cases of extramedullary B cell Non Hodgkins lymphoma (B-NHL). Though bone marrow aspiration (BMA) cytology, trephine biopsy (BMB) and positron emission tomography (PET) are done to assess marrow involvement by lymphoma, BMB stands to be the gold standard. The indolent nature of low grade lymphoma cells mimicking normal lymphocytes and no luxury of assessing architectural topography are the major drawbacks of BMA morphology. The drawbacks of BMB include inadequacy, obscurement by hemorrhage, crush artefacts and delayed results. AIMS AND OBJECTIVES: We studied the sensitivity and specificity of flowcytometry (FCM) in assessing marrow for lymphoma involvement. We also compared this with BMB and PET results. 
Patients/Materials \& Methods: PATIENTS AND METHODS: All B cell NHL staging marrows that were referred to our laboratory from January 2020 were enrolled $(\mathrm{N}=70)$. All were subjected to inclusion and exclusion criteria. Immunophenotyping was performed on Beckman coulter Navios cytometer with a simple seven color antibody panel. The samples were processed according to bulk lyse - stain - wash protocol and 1.5 million events were acquired.

Results: RESULTS: Bone marrow staging samples of 70 B-NHL cases were studied. The concordance rate between FCM and trephine biopsy was $89 \%$ (62 of 70: 48 both negative, 14 both positive). $10 \%$ of cases ( 8 of 70) showed discordance between FCM and biopsy. Seven of eight cases with marrow involvement were detected by FCM and was missed in biopsy analysis. One case which was negative by FCM was given equivocal in biopsy.

Discussion \& Conclusion: CONCLUSIONS: Multi parametric FCM is a simple, rapid and sensitive technique used in detecting minimal residual disease in acute leukemia, CLL and myeloma. Similarly minimal marrow involvement by lymphoma can be detected with clonality determined by restricted light chain either kappa or lambda using FCM. A skewed light chain ratio in mature B cells, strongly raises the suspicion of lymphoma involvement. Hence FCM is a more sensitive tool in detecting small number of lymphoma cells and also complements to trephine biopsy. (The FCM antibody panel and spectrum of B-NHL cases studied with PET findings will be presented and not discussed here due to space constrain).

\section{Monoclonal Plasma Cells Detection by Cytoplasmic Kappa Lambda Ratio by Flow Cytometry}

\author{
Indroneel Sen ${ }^{* 1}$, Sindhura K. L. Lakshmi ${ }^{1}$
}

\section{${ }^{1}$ Pathology, Kasturba Medical College, Manipal, Manipal, India}

Aims \& Objectives: Detection and quantification of monoclonal plasma cells is a prerequisite for the diagnosis of myeloma, Monoclonal gammopathy of undetermined significance (MGUS) and Monoclonal gammopathy of renal significance (MGRS). Monoclonality is determined by serum free light assay and multiparametric flow cytometry (MFC). A serum free kappa lambda ratio (KLR) of 1:4 or 3:1 is indicative of monoclonality. However, there is no established MFC KLR cut off for plasma cell neoplasms. This study aimed to: (1) Compare serum KLR with that of MFC (2) Determine the cut-off value for MFC KLR which determines diagnosis of monoclonality with highest sensitivity and specificity.

Patients/Materials \& Methods: This is was a retrospective observational study of consecutives cases of suspected myeloma. All cases for which concurrent flow cytometry ( 8 color single tube myeloma panel) and serum free light chain assay was performed between July 2017 and June 2020 were included. Demographic, clinical and biochemical data was recorded. Flow cytometry files were reviewed for cytoplasmic kappa and lambda ratio in plasma cells gated using CD45, CD38 and CD138. The values were compared between groups of myeloma/MGUS and those with reactive plasma cells. Continuous variables were expressed in median. The comparison of means was done by one sample T test. Correlation between serum KLR and MFC KLR was done by Pearson correlation. A $p$ value of $<0.05$ considered as statistically significant. ROC analysis was performed to obtain cut-off values.

Results: Total 179 cases were included in the study (129 myeloma, 16 MGUS and 34 reactive plasma cell proliferation). Male to female ratio was 2.08:1 with an overall median age of 63 years. The summary of analysis is given in table 1 . There was no correlation between serum KLR to MFC KLR (0.341). MFC KLR cut-off of $>3.8$ and $<0.4$ has an area under the curve of 0.610 with $90 \%$ sensitivity and $80 \%$ specificity.
Discussion \& Conclusion: Determination of MFC KLR is a valuable tool for detection of monoclonal plasma cells in bone marrow. To the best of knowledge, this is the largest study where cytoplasmic KLR by MFC is compared with serum KLR and ROC analysis is performed to obtain a cut-off value for MFC KLR.

Supporting Document:

\begin{tabular}{|c|c|c|c|}
\hline & PCM & MGUS & RPCP \\
\hline Free kappa (mg/dL) & 145 & 104.9 & 132.4 \\
\hline Free lambda (mg/dL) & 17 & 49 & 72.2 \\
\hline Serum kappa-lambda ratio & 8.7 & 1.7 & 1.4 \\
\hline MFC kappa (*BMPC events) & 1333 & 2093 & 513.5 \\
\hline MFC lambda (*BMPC events) & 66.5 & 1254 & 491 \\
\hline MFC kappa-lambda ratio & 59 & 1.5 & 1 \\
\hline Serum Creatinine (mg/dL) & 1.24 & 1 & 1.16 \\
\hline Blood Urea (mg/dL) & 33 & 35.5 & 28 \\
\hline Serum Calcium (mg/dL) & 8.9 & 8.6 & 8.3 \\
\hline Hemoglobin $(\mathrm{g} / \mathrm{dL})$ & 9.6 & 10 & 9.3 \\
\hline Red Blood Cells $(\times \mathbf{1 0} \mathbf{6} / \mathbf{\mu L})$ & 3.1 & 3.3 & 2.9 \\
\hline Serum Immunoglobulin G (mg/dL) & 2321 & 1293 & 1957 \\
\hline Serum Immunoglobulin M (mg/dL) & 25.5 & 41 & 82 \\
\hline Serum Immunoglobulin E (mg/dL) & 91.7 & 489.8 & 1292.8 \\
\hline
\end{tabular}

PCM: Plasma Cell Myeloma, MGUS: Monoclonal Gammopathy of undetermined Significance, RPCP: Reactive Plasma cell population, *BMPC events: Bone marrow plasma cell events, MFC: Multiparametric Flow Cytometry

\section{ORAL Submission}

Laboratory

\section{Lymphoma and Myeloma (Laboratory) (ORAL-667)}

\section{Spectrum of Lymphomas of The Spleen: What's in the Pandoras Box?}

Sujaya Mazumder ${ }^{* 1}$, Hemnath $E^{1}$, Rakhee Kar $^{1}$, Debdatta Basu ${ }^{1}$, Prabhu Manivannan ${ }^{1}$

\section{${ }^{1}$ Pathology, JIPMER, Puducherry, India}

Aims \& Objectives: To study the morphological and immunohistochemical patterns of splenic lymphomas in a tertiary care centre in South India.

Patients/Materials \& Methods: This was a retrospective study. The database of Department of Pathology, JIPMER, Puducherry was searched for splenectomy specimens and splenic biopsies from 2016 to 2020. All the lymphoma cases that were diagnosed in the spleen were included in the study. The morphology and immunohistochemistry were reviewed and analysed. Bone marrow aspiration, biopsy and flow cytometry immunophenotyping if available was correlated.

Results: Of 150 splenectomies and splenic biopsies received for various indications, $11(7 \%)$ were diagnosed as splenic lymphoma. The male to female ratio was 1.21 .0 , the mean age was 50 years (range 28-68 years), and all patients presented with massive splenomegaly. Most common peripheral blood abnormality was anaemia (five cases) followed by pancytopenia (three cases) and bicytopenia 
(three cases). There was peripheral blood lymphocytosis in six cases (54.5\%). Splenectomy was done in eight cases and three cases were diagnosed on splenic core biopsy. Four cases $(36.3 \%)$ were that of primary splenic lymphoma (PSL) i.e. confined to the spleen and splenic lymph nodes and the remaining seven cases $(63.7 \%)$ were predominantly splenic lymphoma (PdSL) showing prominent splenic involvement with additional involvement of other sites especially blood and bone marrow. By histomorphology and immunohistochemistry, the cases were classified as diffuse large B cell lymphoma (5 cases) being the most common diagnosis, hepatosplenic $\mathrm{T}$ cell lymphoma ( 3 cases), splenic marginal zone lymphoma ( 2 cases) and Hairy cell leukemia (1 case). All the cases were non-hodgkins lymphoma of which eight $(72.7 \%)$ were B cell type and three $(27.3 \%)$ were $\mathrm{T}$ cell type. Bone marrow aspiration and biopsy was done in nine cases and was positive for lymphoma infiltration in six of them $(66.6 \%)$.

Discussion \& Conclusion: Lymphomas in spleen are rare and can be primary or secondary infiltration. A thorough insight into the histomorphology and immunohistochemical spectrum of lymphomas involving the spleen is required for proper diagnosis and management of the cases.

\section{Standardization and Analysis Approach for Diagnosis and MRD Evaluation of Myelomas/Plasma Cell Dyscrasias Using a Single Tube 12-Color Antibody Panel}

Neha Seth $^{* 1}$, Kunal Sehgal ${ }^{1}$, Shweta Kedia ${ }^{1}$, Archana Sutar ${ }^{1}$, Florence Kunjachan ${ }^{1}$

\section{${ }^{1}$ Sehgal Path Lab, Mumbai, India}

Aims \& Objectives: Multicolor Flow cytometric(MFC) has high clinical relevance in diagnosis and risk stratification of multiple myeloma(MM). MFC detection of minimal residual disease(MRD) in $\mathrm{MM}$ is prognostic and predictive of response to therapy.As per consensus guidelines, it is recommended to maintain the exact same panel for evaluation of diagnostic and post-treatment MRD samples.

Patients/Materials \& Methods: We have standardized single tube 12-color Antibody panel for both diagnosis and MRD for myelomas.The panel includes cy-Kappa-FITC,cy-Lambda-PE, CD27PC5.5,CD19-PECY7,CD319-APC,CD45-APC700,CD81-

APCH7,CD138-BV421， CD56-BV510,CD38-BV605,CD28-BV711 and CD117-BV786 antibodies. A Stain Lyse wash protocol was used for all samples. "First-pull" bone marrow aspirates is recommended to avoid significant hemodilution.A target of 2-3 million cells to be acquired per cases was kept and if required,samples with low cellularity were concentrated prior to lysing.Samples were acquired on BD FACS LYRIC flow cytometer platform using BD FACS SUITE Software version 1.4 for analysis.

Results: Analysis template demonstrating the gating strategy using combination of different markers in few representative dot plots is shown in figure 1.Firstly all events were viewed on time gate $\mathrm{Vs}$ forward scatter height(FSC-H) to ensure stable acquisition followed by singlets gate and followed by "FSC SSC viable cell" gating excluding debris,red cells,platelet clumps and artefacts.Subsequently,Plasma cells(PC) were gated using boolean gating combining CD38Vs.CD45 and CD38Vs.CD138dot plots. We use hierarchial gating strategy for fine tuning PC and for detection of aberrant immunophenotypes with other markers and cytoplasmic-immunoglobulin light-chain restriction as recommended by International Consensus guidelines.CD319 is used as an additional PC gating marker for patients on Anti-CD38 therapy. Limit of Detection(LOD)\&Lower Limit of Quantification(LLOQ) of assay has detection sensitivity $10^{-5}$ as recommended by IMWG.We use tight cluster of 20-abnormal PCevents (for-LOD)and 50-events (for-LLOQ)and use the Viable-FSC-SSC cells as denominator.

Discussion \& Conclusion: The above panel has been found useful for evaluation at diagnosis of all types of PC Dyscrasia including MGUS, Plasmacytomas, Myelomas and MRD evaluation.Detailed case analyses with troubleshoots will be shared during the main presentation.

\section{CNS Involvement: A Rare Detour for Myeloma Cells}

Shifa Farheen ${ }^{* 1}$, Preeti Tripathi ${ }^{1}$, Arijit Sen ${ }^{1}$, Poonam Yadav ${ }^{1}$

${ }^{1}$ Pathology, Command Hospital Air Force Bangalore, Bangalore, India

Aims \& Objectives: Extramedullary involvement in Multiple Myeloma (MM) is seen in $7-18 \%$ of cases. Central nervous system (CNS) involvement is extremely rare and occurs in only about $1 \%$ of patients. We present an interesting case of non secretory multiple myeloma (NSMM) with atypical involvement of liver, spleen and central nervous system.

Patients/Materials \& Methods: A 59 year old female patient initially presented with low back ache. Skeletal survey showed multiple lytic bony lesions. An initial diagnosis of plasma cell neoplasm was made based on the biopsy and immunohistochemistry(IHC) of sacral lytic lesion. No monoclonal gammopathy was found in the serum or urine electrophoresis. A diagnosis of NSMM was made and the patient was started on bortezomib, dexamethasone, linaledomide (VRD regime). After about 1.5 months of starting chemotherapy, she started complaining of right frontal headache, decreased sensation on right side of face and angle of mouth and double vision and imaging showed lesions in liver, spleen, lungs as well as dural deposits. Such atypical organ involvement raised a suspicion of epithelial malignancy (? Concurrent).

Results: Cerebrospinal fluid (CSF) tap, liver biopsy and bone marrow $(\mathrm{BM})$ biopsy were done. Bone marrow was hypercellular and showed sheets of markedly pleomorphic cells (?plasmablasts). Liver biopsy showed multiple foci of similar sheets of plasmablastic cells along with normal preserved hepatocytes. IHC was performed individually on $\mathrm{BM}$ and liver biopsy which confirmed plasma cell origin and ruled out epithelial suspicion (CD 38/138 positive with lambda restriction and negative for PanCK, EMA, CD20, vimetin, CD99, Synaptophysin, melan A). CSF study showed cellular fluid with WBC count of 250 cells. Many atypical large plasmablasts with similar morphology as in bone marrow were noted. A diagnosis of highly aggressive NSMM with extramedullary plasmacytoma was made. Daratumumab was added to the VRD regime and she showed remarkable clinical improvement.

Discussion \& Conclusion: NSMM with uncommon organ involvement pose a diagnostic dilemma owing to its low incidence and scant supporting biochemical markers. It is essential for clinicians to keep an open mind for atypical presentations in such cases. Imaging and immunohistochemistry are cornerstones in diagnosing such challenging cases.

\section{Multiple Myeloma: Spectrum of Clinicohematological Presentation in Patients Younger than 40 Years}

Pallavi Sinha ${ }^{* 1}$, Chumbeni Ngullie ${ }^{1}$, Sarika Singh ${ }^{1}$, Lity Dhar ${ }^{1}$, Prerna Arora $^{1}$, Dharam P. Bhadoria ${ }^{2}$, Mradul K. Daga ${ }^{2}$, Sunita Aggarwal $^{2}$

${ }^{1}$ Pathology, ${ }^{2}$ Medicine, Maulana Azad Medical College, DELHI, India

Aims \& Objectives: To describe the clinical, biochemical and hematological profile of multiple myeloma in Indian patients and to 
find prevalence of MM in patients less than 40 years. This study also aims to compare variations in clinical, biochemical and hematological parameters in patients younger than 40 years and more than 40 years Multiple Myeloma (MM) patients.

Patients/Materials \& Methods: This study was conducted in a tertiary care hospital in Delhi. Eighty-seven patients diagnosed with MM over a period of 08 years (2013-2020) were included in the study. The study population was divided in 2 cohorts - patients with age $\leq 40$ years and patients with age $>40$ years. Clinical, pathological and biochemical characteristics of young MM patients were compared with elderly patients. Cases were studied regarding age, sex, presenting complaints, haemogram findings, KFT, serum calcium levels, LDH, CRAB features, radiological findings, percentage of plasma cells, plasma cell morphology, marrow fibrosis and pattern of infiltration of plasma cells in marrow biopsy.

Results: The age of patients ranged from 30 to 87 years with a Mean \pm SD of $54.8 \pm 12.6$ years. 15 patients (17.2\%) were less than 40 years of age, with the youngest being 30 years. Weakness, lower backache and nephropathy was seen more commonly in patients more than 40 years of age. Anaemia $(\leq 10 \mathrm{~g} / \mathrm{dl})$ was detected in $12(80 \%)$ of young patients and $64(88.8 \%)$ of older patients. Thrombocytopenia $(\leq 100 \times 109 / \mathrm{L})$ was seen in $6(40 \%)$ patients $<40$ years and was similar in patients $>40$ years $(38.8 \%)$. Plasma cell leukemia was present in $15(17.2 \%)$ patients and was less common in younger cohort $(6.7 \%)$. Plasmablasts were less in younger cohort (20\%) when compared to older cohort (38\%).

Discussion \& Conclusion: In India, age of presentation is much less compared to west and involves more males than females. In this study, anaemia and thrombocytopenia was seen in significant number of myeloma patients. Prognostically relevant variables like Hemoglobin, plasma cell number and morphology did not differ considerably among the two group.

\section{Reactive Bone Marrow Plasmacytosis: A Common Denominator with Diverse Etiology}

Sajida Batool $^{* 1}$, Umesh K. Tiwari ${ }^{1}$, Sadhna Marwah ${ }^{1}$, Vijay kumar ${ }^{1}$

${ }^{1}$ Hematology and Clinical Pathology Unit, Department of Pathology, ABVIMS, Dr Ram Manohar Lohia Hospital, New Delhi, India

Aims \& Objectives: We aim to quantify as well as observe the morphological features of reactive bone marrow plasmacytosis and to help distinguish them better from primary plasmacytic neoplasms.

Patients/Materials \& Methods: A consecutive series of 1409 bone marrow aspirates were analyzed for percentage of plasma cells along with associated hematological findings and clinical disease states. The study group included only those cases with bone marrow PRP; their polyclonality being supported either by serum protein electrophoresis (SPE) findings or by kappa/lambda immunohistochemistry on bone marrow biopsy. Clinical records, hemogram and bone marrow aspiration findings were reviewed in all cases. Morphological features of PRP cases were analysed in detail. Further, these cases were compared with 10 confirmed cases of PCD in an attempt to differentiate between the two on morphology.

Results: A total of 210bone marrow aspirates showed increased plasma cells $(>3.5 \%)$. Clonality could be proven in 135 case, of which 98 cases were polyclonal and 37 were PCD. Majority of patients with PRP were above 40 years with male predominance. The plasma cell concentration in PRP ranged from $4 \%$ to $25 \%$. The main associated diseases included infections followed by autoimmune diseases. There was considerable overlap in plasma cell percentages in PRP and PCD groups. However, on morphology, PRP revealed scattered, non-aggregated mature plasma cells while in PCDs, there was a mixture of both immature and mature forms including plasmablasts with frequent clustering.

Discussion \& Conclusion: Polyclonal reactive plasmacytosis (PRP) characterized by a diffuse distribution of mature non-clonal plasma cells in the bone marrow is known to occur in numerous disorders. These need to be differentiated from the clonal plasma cell dyscrasias (PCD).A number of diseases show increased percentage of plasma cells in bone marrow which may raise suspicion of PCD. In the proper clinical context, it may be challenging to differentiate reactive from neoplastic conditions as there is an overlap both in cell counts and morphology. Assessment of subtle morphological features along with associated clinical and laboratory findings are key for making a correct diagnosis

Keywords: Bone marrow examination, polyclonal reactive plasmacytosis, plasma cell dyscrasia.

\section{Angioimmunoblastic T-Cell Lymphoma: Histomorophological and Immunohistochemical Features}

Arthy Raman ${ }^{* 1}$, Sharmila Balajee ${ }^{1}$, Bidish Patel $^{1}$, Rakhee Kar ${ }^{1}$, Debdatta Basu ${ }^{1}$

${ }^{1}$ Department of Pathology, JIPMER, Pondicherry, India

Aims \& Objectives: To study the clinical, histomorphological and immunohistological features of Angioimmunoblastic T cell lymphoma (AITL) among nodal peripheral $\mathrm{T}$ cell lymphoma.

Patients/Materials \& Methods: A total 162 cases of nodal peripheral $\mathrm{T}$ cell lymphoma were diagnosed in the department in the last eight years. Of these 15 cases were reported as AITL. The details of the clinical, morphological and immunohistochemical features of these 14 cases were retrieved from the department archives.

Results: The mean age was 58 years, with a range of $40-70$ years and male to female ratio was $4: 1$. Common clinical features included generalized lymphadenopathy (100\%), hepatomegaly (33\%), splenomegaly $(13 \%)$ and polyclonal hypergammaglobulinemia $(40 \%)$. Microscopically, diffuse effacement of architectural patterns was seen in $100 \%$, with medium sized atypical clear cell infiltration in $93 \%$, increased vascularity in $100 \%$, increased mitosis seen in $50 \%$ of cases. Bone marrow infiltration was seen in $50 \%$ cases and $25 \%$ cases revealed plasmacytosis. Absence of follicles, polymorphous infiltrate, extra- follicular follicular dendritic cell (FDC) proliferation, high endothelial venules (HEV) prominence and neoplastic T-cells were the diagnostic features of AITL. CD10 positivity (46\%) and admixture with large size CD20 + B-immunoblasts $(60 \%)$, $\mathrm{T}$ cell downregulation was seen in CD5(100\%) followed by CD2 and CD4 were the immunohistological peculiarities.

Discussion \& Conclusion: Awareness of various morphological and immunophenotypic complexities of AITL involving downregulation of $\mathrm{T}$ cells markers offers diagnostic challenge and one must diligently study microscopic features and operate with a wisely selected panel of markers on nodal peripheral $\mathrm{T}$ cell lymphoma to arrive at a diagnosis of AITL. 


\section{Clinico-Pathological Profile of Patients with Plasma Cell Neoplasms with Special Reference to Bone Marrow Fibrosis and Amyloid Deposition}

Narasimhapriyan Kannan ${ }^{* 1}$, Sahitya Dangudubiyyam ${ }^{1}$, Jasmita Dass $^{1}$, Ganeshkumar Viswanathan ${ }^{1}$, Mukul Aggarwal ${ }^{1}$, Rishi

Dhawan ${ }^{1}$, Pradeep kumar ${ }^{1}$, Tulika Seth ${ }^{1}$, Manoranjan Mahapatra ${ }^{1}$, Seema Tyagi ${ }^{1}$

\section{${ }^{1}$ Haematology, AIIMS, Delhi, India}

Aims \& Objectives: The aim and objective is to study the clinicopathological profile of patients with plasma cell neoplasm, especially in patients with associated bone marrow fibrosis and amyloid deposition.

Patients/Materials \& Methods: This is a retrospective study done at the Department of Haematology, AIIMS, New Delhi. Patients who underwent bone marrow aspiration and biopsy with suspicion of plasma cell neoplasm like plasma cell myeloma, plasmacytoma, light chain amyloid deposition disease etc., were included in the study. Data collection was done from the year 2017 to 2019. The bone marrow findings were correlated with clinical profile of the patient along with biochemical parameters, cytogenetics, FISH wherever available. Treatment response was analysed in patients wherever available especially in the patients with bone marrow fibrosis and amyloid deposition.

Results: 266 bone marrow aspirates and biopsies were analysed for 247 patients. There were 165 male patients and 82 female patients (Male: Female $=2: 1$ ). Mean age at diagnosis was 56.4 years. 102 patients had bony pain (41.2\%), 22 had plasmacytomas (8.9\%), 26 had pathological fractures (10.5\%), 7 had bleeding manifestations $(2.8 \%), 3$ had associated systemic infections (1.2\%). Bone marrow fibrosis was noted in 12 patients at diagnosis (4.8\%). 21 patients had amyloid deposition in various organs including bone marrow, kidney, liver etc., (8.5\%). There were 210 cases of multiple myeloma (85\%), 12 cases of primary light chain amyloidosis and monoclonal gammopathy of undetermined significance (MGUS) each (4.8\%), 5 cases of plasmacytoma (2\%) and 4 cases of smoldering myeloma (1.6\%), 3 cases of POEMS syndrome (1.2\%), 1 case of extramedullary myeloma $(0.4 \%)$. Amyloidosis was associated with 9 cases of myeloma at diagnosis (4.2\%). Serum protein immunofixation showed immunoglobulin gamma as the most frequent heavy chain component, as seen in 101 patients $(40.8 \%), 42$ patients had immunoglobulin alpha (17.0\%), 27 patients had only either of the light chain component $(10.9 \%)$.

Discussion \& Conclusion: The incidence of fibrosis and amyloidosis associated with myeloma was much lower in our study as compared to published studies.

\section{LCA Negative P63 Positive Diffuse Large B Cell Lymphoma: A Diagnostic Conundrum}

Pradeep Arumugam ${ }^{* 1}$, Anandhi Anbalagan ${ }^{1}$, Rushreeta A. Deuri ${ }^{1}$, Soundarya Ravi ${ }^{1}$, Jinkala Sreerekha ${ }^{1}$, Debdatta Basu ${ }^{1}$

${ }^{1}$ Pathology, JIPMER, Puducherry, India

Aims \& Objectives: Introduction: Loss of LCA expression is usually observed in immature B-cell neoplasms, plasma cell dyscrasias and anaplastic T-cell lymphomas. However, it is uncommon in mature B-cell lymphoproliferative disorders. This case Diffuse Large B cell lymphoma (DLBCL) was CD45 negative and P63 positive and mistaken as metastatic poorly differentiated carcinoma posing diagnostic difficulty both morphologically and immunohistochemically.

Patients/Materials \& Methods: An elderly 59-year-oldmale presented with a left axillary lymphadenopathy measuring $3 \times 2 \mathrm{~cm}$. Pet
CT scan showed increased FDG uptake in multiple discrete heterogeneously enhancing lymph nodes in the left axillary region in all 3 levels with largest of size $3.2 \times 4.4 \mathrm{~cm}$. No other areas showed increased uptake. Lymphnode biopsy from axillary lymphnode done at an outside laboratory was reported with a possible differential diagnosis of metastatic poorly differentiated carcinoma or amelanotic melanoma. Metastatic workup including complete clinical examination with hematological profile, biochemical profile, and radiology was inconclusive.

Results: Pathological details: The slides were reviewed at our centre, showed a partially effaced lymph node with sheets of large cells arranged in syncytial pattern with round nucleus, markedly vesicular chromatin and prominent one to two tiny nucleoli. Numerous mitosis and apoptotic bodies were seen but there was no necrosis. Overall features were of a metastasis of poorly differentiated epithelial tumor. Immunohistochemistry (IHC) with a primary panel of antibodies included CK and, LCA. Both were negative. Further expanding our diagnostic profile EMA, Melan A, S100, Vimentin, PLAP P63 and CD 30 were done. All were negative except for a weak to focally strong staining with P63. However CK7 and CK5/6 were negative. Immature hematolymphoid markers like CD 34 and TdT were also negative. However the cells were strongly positive for CD 20.Subsequent profile showed positivity for CD79a, MUM1, BCL2, C- MYC and negative for CD3, BCL6, CD 10 \& CD138. A final diagnosis of diffuse large B cell lymphoma-activated B cell type with doubleexpression ( $\mathrm{C}$-myc \& $\mathrm{Bcl} 2)$ was made.

Discussion \& Conclusion: This case of DLBCL posed a real diagnostic challenge and is unique because of multiple reasons. The partial effaced architecture of the node with a syncytial pattern mimicking a metastasis; secondly the immunoprofile of LCA negativity couple with P63 (albeit of weak positivity) posed a practical diagnostic difficulty and needed the use of many immunohistochemical markers before arriving at a final diagnosis.

\section{EBV Positive Diffuse Large B Cell Lymphoma in a Young Adult : A Diagnostic Dilemma}

Vishesh Dhawan ${ }^{* 1}$, Mansi Kala ${ }^{2}$, Anuradha Kusum ${ }^{3}$, Meena Harsh ${ }^{3}$, Nadia Shirazi ${ }^{3}$, Kunal Das ${ }^{4}$

${ }^{1}$ Resident Pathology, ${ }^{2}$ Associate Professor Pathology, ${ }^{3}$ Professor Pathology, ${ }^{4}$ Associate Professor Paediatrics, Himalayan Institute Of Medical Sciences, Swami Rama Himalayan University, Swami Ram Nagar, Jolly Grant, Dehradun, Uttarakhand, India

Aims \& Objectives: • EBV-positive diffuse large B cell lymphoma, not otherwise specified was an entity confined to the elderly group in the recent past, however cases in young age group have also been identified.

- The neoplastic component in EBV-DLBCL imitates T cell rich B cell lymphoma both morphologically and immunohistochemically especially in young adults but EBV positivity differentiates these two conditions, also prognosis and age group are different in both these lesions. T cell rich B cell lymphoma often present in older age group and has worse prognosis.

- The EBV infected immunoblast also resemble RS cells and can confuse the pathologist, however a careful immunohistochemical examination can prove the cryptic nature of these RS cells unlike EBV infected immunoblast which are B lineage specific both molecularly and immunohistochemically.

- Importance lies in differentiating these conditions as treatment and prognosis differs.

Patients/Materials \& Methods: 17 year old boy with multiple cervical lymphadenopathy, occipital swelling and bone metastasis. 
FNAC revealed RS like cells. Lymph Node excision suggested Non Hodgkins Lymphoma versus Hodgkins lymphoma.

Results: - Immunohistochemistry revealed these RS like cells to be positive for CD-45, CD-20, CD-30, PAX5 and EBV and were negative for CD-3, CD-15, ALK and CD-21.

- A diagnosis of EBV-DLBCL was made.

Discussion \& Conclusion: Every RS cell should not conclude Hodgkin's lymphoma. Careful clinical history, immunohistochemistry and EBV using in situ hybridization is a must when differentiating these conditions.

\section{CD20 Positive Peripheral T Cell Lymphoma with Cutaneous Involvement and Concurrent B Cell Lymphoma in the Bone Marrow}

Sindhura Lakshmi Koulmane Laxminarayana ${ }^{* 1}$, Ranjini Kudva², Padmapriya Jayaprakash $^{2}$, Deepak Nayak $\mathrm{M}^{2}$, Anjali Vijay ${ }^{3}$

${ }^{1}$ Kasturba Medical College, Manipal, Manipal Academy of Higher Education, Udupi, India, ${ }^{2}$ Pathology, Kasturba Medical College, Manipal, Manipal Academy of Higher Education, ${ }^{3}$ Pathology, Melaka Manipal Medical College, Manipal, Manipal Academy of Higher Education, Udupi, India

Aims \& Objectives: Simultaneous occurrence of morphologically and genetically distinct lymphomas is rare and is known as composite lymphoma. We report a case of CD20 positive peripheral $\mathrm{T}$ cell lymphoma (PTCL) with simultaneous nodal and cutaneous involvement and concurrent B cell lymphoma in the bone marrow.

Patients/Materials \& Methods: A 57 year old gentleman presented with swelling in the left side of neck since a month, associated with painful blisters over right forearm, which exuded serous discharge upon rupture. There was no history of fever, exposure to plants or animals. On examination, he was pale, had multiple enlarged left cervical lymph nodes and palpable liver $2 \mathrm{~cm}$ below right subcostal margin.

Results: Histopathology of lymph node excision biopsy showed non Hodgkin lymphoma. The abnormal cells were positive for CD3, CD5 and CD20. Majority of abnormal cells were CD4 positive and small proportion was positive for CD8. Ki-67 index was $28 \%$. CD10 was negative. A diagnosis of peripheral $\mathrm{T}$ cell lymphoma, NOS with CD20 expression, was made. Cutaneous biopsy from right forearm plaque showed cutaneous infiltration by $\mathrm{T}$ cell lymphoma with $\mathrm{CD} 3$ and CD4 positive abnormal cells. Staging bone marrow aspirate was hemodilute, but showed $40 \%$ abnormal lymphoid cells. Flow cytometry of bone marrow aspirate revealed abnormal lymphoid cells (bright expression of CD45, CD19 and CD20, 64.7\% of all lymphoid cells) which were monoclonal surface lambda positive. They also expressed CD79b and CD38 moderately, but negative for CD5, CD10, CD200, CD23, CD103, CD123, CD25 and T cell markers. T cells were normal with a CD4/CD8 ratio of 0.8 . Hence, a diagnosis of bone marrow involvement by B cell lymphoma was given. Patient was treated with cyclophosphamide and was stable until last followup at 4 months from initial diagnosis.

Discussion \& Conclusion: PTCL (nodal and/or cutaneous) have been reported to express $\mathrm{B}$ cell markers suggesting a possible biological overlap with B cell precursors at origin. Concurrent bone marrow involvement with B cell lymphoma provides further insight into disease biology. To the best of our knowledge, this is first report of such a case.
Extranodal NK/T Cell Lymphoma: A Rare Entity

Ankita Goel $^{* 1}$, Ranjan Bhatnagar ${ }^{1}$, Ravi Tandon ${ }^{1}$, Mahima Yadav ${ }^{2}$, Amrita Ghosh $^{2}$

${ }^{1}$ Department of General Medicine, ${ }^{2}$ Department of Pathology, Institute of Medical Sciences, Varanasi, India

Aims \& Objectives: To highlight a rare case of extranodal NK/T cell lymphoma and discussing the clinicopathological, immunophenotypical findings and diagnostic challenges.

Patients/Materials \& Methods: A 52 year female presented with right side nasal mass for 6 months. Except pallor, there was no lymphadenopathy and hepatosplenomegaly. Local examination showed complete right nasal cavity filled with pinkish mass along with localised facial swelling. CBC showed normocytic normochromic anemia. CT-PNS was done for soft tissue swelling in the right nasal cavity. There were opacities of both maxillary sinuses with obstruction of both Osteomeatal Complexes. Patient underwent Right Medial Maxillectomy and the specimen was sent for histopathology. Dense heterogenous infiltrate of cells composed of atypical medium lymphocytes with prominent vascular invasion associated with large areas of necrosis were seen on biopsy. Immunophenotyping was positive for $\mathrm{CD} 2, \mathrm{CD} 3, \mathrm{CD} 43, \mathrm{CD} 56$ and negative for $\mathrm{CD} 20$. No extranasal lesion was identified on PET/CT. She got diagnosed as case of NATURAL KILLER/T-CELL LYMPHOMA NASAL TYPE (LIMITED- STAGE IE) and planned for GELOX regimen followed by radiotherapy and remaining cycles of chemotherapy.

Results: Natural Killer/T-Cell Lymphoma Nasal Type (LimitedStage IE).

Discussion \& Conclusion: Extranodal natural killer/T-cell lymphoma, nasal type (NKTL) is a rare disease with highly aggressive clinical course and poor prognosis. The most common site of presentation is upper aerodigestive tract. The disease may disseminate to various extranasal sites like intestine (37\%) and other organs but bone marrow infiltration is uncommon. Stage I disease is present in $81 \%$ of patients while stage II disease in $17 \%$ of patients. The treatment is largely determined by the extent of disease. Prompt recognition is essential as early treatment is necessary for survival. The efficacy of conventional anthracycline containing regimen is disappointing because of frequent expression of multidrug resistant $\mathrm{P}$ - glycoprotein. L-asparaginase containing regimens have significantly improved the efficacy and prognosis for patients with ENKTL.

\section{Detection of Focal Lesions in Clot Section with Negative Bone Marrow Aspirate and Trephine Biopsy}

Vandana Bhatti ${ }^{* 1}$, Naveen Kakkar ${ }^{1}$

${ }^{1}$ Pathology, Christian Medical College and Hospital, Ludhiana, India

Aims \& Objectives: To assess the importance of clot section in focal bone marrow lesions.

Patients/Materials \& Methods: We present five patients in whom granulomas/lymphomatous infiltration was detected in the clot section with negative bone marrow aspirate and trephine biopsy.

Results: In our case series comprising of 5 cases, granulomatous pathology was picked up only on clot section in 3 patients while lymphomatous infiltration was picked up in 2 patients on clot section alone.

Discussion \& Conclusion: Bone marrow aspiration and biopsy is an important procedure for diagnosing various hematological and non hematological disorders. Bone marrow examination includes studying aspirate smears, trephine biopsy, touch imprints and clot section. Out of these, trephine biopsy is considered most helpful in detecting focal lesions. In most of the centers the practice of making a clot section is 
not followed. Clot section is a useful adjunct to the study of bone marrow aspirate and trephine biopsy. It may be particularly useful in the detection of bone marrow lesions with a focal distribution. Clot sections must be studied alongside aspirate smears, touch smears and trephine biopsy to increase the diagnostic yield of this technique.

\section{Post-Transplant Lymphoproliferative Disorders. A Single Institute Experience}

Savita Singla ${ }^{* 1}$, Saurabh Donald ${ }^{1}$, Ashwin Philips ${ }^{2}$, Joseph M. John ${ }^{3}$, Roma Isaacs $^{1}$, Nalini Calton ${ }^{1}$, Preethi A. M. Paul ${ }^{1}$

${ }^{1}$ Pathology, ${ }^{2}$ Medical Oncology, ${ }^{3}$ Clinical Hematology, Hematooncology and Bone Marrow (Stem Cell) Transplant Unit, Christian Medical College and Hospital, Ludhiana, India

Aims \& Objectives: Post-transplant lymphoproliferative disorder (PTLD) is a rare complication following solid organ or hematopoietic stem cell transplant (HSCT) due to lymphoid cell proliferation in the immunosuppressed state. The incidence of PTLD after renal transplant ranges from 0.8 to $2.5 \%$, while in HSCT from matched unrelated donors (MUD) the incidence ranges from 4 to $10 \%$. Our objective was to study the clinicopathological and immunohistochemical (IHC) profile of PTLDs diagnosed in our hospital.

Patients/Materials \& Methods: All PTLDs diagnosed in our hospital over a four year period were retrospectively reviewed. Cases with relapse of similar disease were excluded. Clinical details including outcomes, histopathological examination (HPE) and IHC findings were analyzed.

Results: Three cases of PTLDs were identified. A 23-year-old man with chronic kidney disease (CKD) stage 5 underwent renal transplant. Donor was his brother. Immunosuppressive therapy given was Mycophenolate, Tacrolimus and Wysolone. Eight years later he presented with bronchopneumonia and pyrexia of unknown origin, however, succumbed to septic shock. IHC on post- mortem spleen and liver biopsies showed CD30-positive diffuse large B-cell lymphoma, non-germinal center B phenotype; EBV- LMP1 by IHC was negative. The second case was a 34-year-old man with CKD who received renal allograft from his mother. Cyclosporin with Omnacortil was given as immunosuppression. The patient presented with jejunal intussusception 13 years post- transplant and jejunal resection was done. HPE and IHC showed plasmablastic lymphoma (PBL); EBV-LMP1 and EBER-ISH were negative. The patient was given three cycles of CHOP, however, six months later developed recurrence of PBL on umbilical skin, and was subsequently lost to follow up. The third patient was a 6-year-old boy who underwent MUD HSCT for acute myeloid leukaemia (AML) M2 with monosomy 7. He was on prolonged immunosuppression with multiple agents for long standing graft versus host disease. Seven years later he presented with retroperitoneal lymph nodal mass. HPE and IHC showed EBVLMP1- positive low grade B-cell polymorphic PTLD. Four months following treatment with Rituximab, the patient had relapse of AML and succumbed during induction chemotherapy.

Discussion \& Conclusion: PTLD is a rare catastrophic complication of solid organ or HSCT and can have a wide clinicopathological spectrum with extranodal involvement. Integration of clinical details with histopathology and IHC is necessary for an accurate diagnosis of PTLD.

\section{Bone Marrow Involvement with Secondary HLH in Patients of Hodgkin's Lymphoma: A Rare Entity}

Niharika Mishra*1, Kanwaljeet Singh ${ }^{2}$, Venkat Somasundaram ${ }^{1}$, Tathagata chatterjee ${ }^{1}$

${ }^{1}$ Department of Lab Sciences and Molecular Medicine, Army Hospital (Research \& Referral) Delhi Cantt, New Delhi, ${ }^{2}$ Department of Lab Sciences and Molecular Biology, Army Hospital (Research \& Referral) Delhi Cantt, Delhi, India

Aims \& Objectives: Aim of this case report is to highlight the role of bone marrow biopsy and aspirate in diagnosing Hodgkin's lymphoma deposits with secondary hemophagocytic lymphohistiocytosis (HLH) in bone marrow, which is quite rare finding. We represent a case of 29 year old male patient who initially presented with mediastinal lymphadenopathy underwent battery of investigations and finally diagnosed as HL with lymphoma deposits with secondary HLH in bone marrow, which is quite rare finding.

Patients/Materials \& Methods: 29 year old patient with history of pyrexia of unknown origin (PUO) and mediastinal lymphadenopathy since one month. On examination, patient had pancytopenia with leukoerythroblastic picture. CT-guided FNAC of mediastinal lymph nodes was inconclusive. FDG-avid lesion was detected in bone marrow on PET scan.

Results: Bone marrow aspirate showed features of cellular reactive bone marrow with features of HLH. Bone marrow biopsy showed $>80 \%$ marrow cellularity with few marrow spaces showing areas of fibrosis and interspersed population of classical and mononuclear Reed Sternberg (RS) cells which were positive for CD30. Later CT guided mediastinal lymph node biopsy was done which showed Classical Hodgkin's Lymphoma (CHL) (Nodular sclerosis).

Discussion \& Conclusion: An accurate assessment of the stage of disease in patients with CHL is critical for appropriate therapy. Hodgkin's Lymphoma accounts for 10-15\% of lymphoma cases, almost entirely confined to the lymph nodes with extra nodal lymphomas accounting for $2-16 \%$ of HL. Moreover, metastasis to bone marrow is quite rare. To conclude, we want to stress upon the role of bone marrow biopsy in diagnosing Hodgkin's lymphoma deposits.

\section{Intraventricular Mass with Hydrocepahlus in an Immunocompetent Patient: A Rare Site for Lymphoma}

Rashim Sharma*1, Deepak Vedant ${ }^{1}$, Taruna Yadav², Jaskaran Singh $^{3}$, Poonam Elhence ${ }^{1}$

${ }^{1}$ Pathology, ${ }^{2}$ Diagnostic and Interventional Radiology, ${ }^{3}$ Neurosurgery, AIIMS Jodhpur, Jodhpur, India

Aims \& Objectives: Lymphomas in the central nervous system are seen in immunocompromised patients. Lymphoma in the intraventricular region in an immunocompetent patient is a very rare entity. Here, we report a 45-year-old immunocompetent male presenting with an episode of generalized tonic-clonic seizure (GTCS). The Computed Axial Tomography (CAT) scan showed an intraventricular mass which was further evaluated.

Patients/Materials \& Methods: A 45-year-old male presented with an episode of GTCS. He had a history of headache for the last three months. CAT scan showed multiple nodular, solid hyperdense masses in bilateral lateral ventricles with narrowed Foramen of Monroe. Patient was immunocompetent with non-reactive HIV, HBC and $\mathrm{HCV}$. There was no history of any previous malignancy. No organomegaly or lymphadenopathy noted in the systemic examination. He underwent septostomy and the biopsy was taken from the wall of left 
ventricle which was studded with multiple grey white nodular masses. The biopsy showed normal choroid plexus cells along with singly dispersed atypical cells having round vesicular nuclei, conspicuous nucleoli and moderate pale cytoplasm. Focal necrosis was also present. On immunohistochemistry tumour cells were positive for CD45, $\mathrm{CD} 20, \mathrm{CD} 10$ and $\mathrm{Bcl} 6$. Ki-67 index was nearly 95\%. The tumour cells were negative for TdT and CD3. CAM 5.2, Vimentin and S100p were positive in choroid plexus cells.

Results: In view of histomorphology and immunohistochemistry, diagnosis of Non-Hodgkin Lymphoma, possibly Diffuse Large B-cell type (DLBCL) was given. Patient was advised chemotherapy. However patient got lost to follow up.

Discussion \& Conclusion: A Non-Hodgkin lymphoma in the intraventricular region in an immunocompetent patent is a very rare entity. In this location, lesions that usually occur are choroid plexus papilloma, ependymomas, central neurocytoma, neurocysticercosis and meningiomas. Thus, while dealing with intraventricular mass lesions, possibility of DLBCL must be kept in differentials. Such cases should be dealt with rigorous chemotherapy with autologous stem cell transplant in cases resistance to chemotherapy.

\section{Typhoid Fever Masquerading as a Plasma Cell Neoplasm}

\section{Malvika Gaur*1, Tushar Sehgal ${ }^{1}$ \\ ${ }^{1}$ Laboratory Medicine, All India Institute of Medical Sciences, New Delhi, Delhi, India}

Aims \& Objectives: Introduction. Marked plasmacytosis in blood is an uncommon occurrence and is generally associated with a plasma cell dyscrasia/neoplasm. Rarely may it be seen with infections, inflammatory conditions, drug reactions, etc. We present a case of marked plasmacytosis in a non-neoplastic disorder.

Patients/Materials \& Methods: Case. 67-year-old male came to hematology clinic with low grade fever, backache and generalized fatigue since three weeks. On examination, he had pallor and was febrile. Blood findings showed hemoglobin of $90 \mathrm{~g} / \mathrm{l}$, total leucocyte count of $26.8 \times 109 / 1$ and platelet count of 214 x109/1. Blood film showed anemia, mild rouleaux with $29 \%$ plasma cells and $11 \%$ lymphoplasmacytoid cells(figure 1) Plasma cells were identified by an eccentric nucleus with a small perinuclear clear zone (hof) and a deeply basophilic cytoplasm.

Results: Serum creatinine was $1.2 \mathrm{mg} / \mathrm{dl}$, serum calcium was $9 \mathrm{mg} / \mathrm{dl}$ and total protein was $6.2 \mathrm{~g} / \mathrm{dl}$. Widal test was positive for Salmonella typhi with a titre of $>1: 320$.Blood culture confirmed Salmonella typhi infection. The bone marrow examination was unremarkable. Flow cytometry evaluation showed normal $\mathrm{T}$ cells and polyclonal $\mathrm{B}$ cells; abnormal cells were not identified. He was started on intravenous antibiotics and his condition improved gradually. The patient's fever resolved three days later, as did his plasmacytosis. This patient remains well six months later.

Discussion \& Conclusion: The incidence of plasma cell neoplasm generally increases after the fifth decade of life. It shows significant findings in a blood film such as rouleaux formation, lymphoplasmacytoid cells and/or plasma cells. Sometimes blood film plasmacytosis may also be seen in reactive conditions. However, extreme plasmacytosis is very rare. Irrespective of patient's age, blood plasmacytosis should be assessed to determine whether it is transient and related to an illness before proceeding for an extensive and invasive evaluation like bone marrow, etc. especially in resource finite settings. This is particularly important in tropical countries like India that are endemic for various infectious diseases. This may also help in reducing patient's hospital stay and mobilizing the resources where it is needed the most. To conclude laboratory physicians should be mindful and might consider infection-related plasmacytosis in the differential diagnosis of plasma cell neoplasm irrespective of patient's years.

\section{All Clival Masses are Not Chordoma- Multiple Myeloma Presenting as Clival Mass}

Pradeep $\mathbf{V}^{* 1}$, Vikram Singh ${ }^{1}$, Uday Yanamandra ${ }^{2}$, Ravi Roy ${ }^{3}$, Tathagata Chatterjee ${ }^{1}$, Venkatesan Somasundaram ${ }^{1}$, Kanwaljeet Singh ${ }^{1}$

${ }^{1}$ Dept of Pathology, ${ }^{2}$ Dept of Hematology, ${ }^{3}$ Dept of ENT, AHRR, Delhi, India

Aims \& Objectives: We report a rare case of multiple myeloma, presenting as a clival mass and created a diagnostic dilemma.

Patients/Materials \& Methods: A 52-year-old male, a known case of COPD, presented with diplopia left eye for six months, right hemi cranial headache for two months, mucopurulent discharge right ear and hearing loss for six months. He had pallor and splenomegaly. CNS examination revealed left fourth and bilateral sixth cranial nerve palsies.

Results: Haematological and biochemical parameters were normal. Magnetic resonance imaging (MRI) brain and orbit revealed clival mass involving left cavernous sinus measuring $5.8 \times 3.9 \times 3.1 \mathrm{~mm}$ and multiple lytic lesions of skull. CECT revealed lytic lesions on sternum, right ribs, lumbar vertebra (DV6) and iliac bone. Positron emission tomography scan revealed FDG avid lesions in the clival region, multiple lytic lesions in the sternum and skull. Multiple myeloma workup revealed light chain disease (SPEP): faint band in Kappa region, Kappa (K):2320, Lambda (L):21.3 with K/L ratio of 108.92, Beta-2-microglobulin:2412 ng/dL and SIFE revealed distortion in gamma region). Bone marrow was cellular marrow with no increase in plasma cells. Trans sphenoidal biopsy showed plasmacytoma with mature and immature plasma cells with mitoses of (6-8/ 10HPF). IHC showed CD38, CD138 positivity and Kappa (restriction). Ki 67 index was 15-20\%. Based on these findings, the diagnosis of MM with Clival plasmacytoma (C-R-A- B +) R-ISS III was made. The patient was treated with VCD chemotherapy (Inj Bortezomib and Inj Dexamethasone and Inj Thalidomide daily) along with local palliative radiotherapy, and he is responding well.

Discussion \& Conclusion: Clival tumors are extremely rare with overall incidence of 0.1 to $0.2 \%$ of all intracranial tumors, which predominantly includes clival chordoma, chondrosarcoma, plasmacytoma. At presentation, extramedullary $\mathrm{MM}$ is found in approximately $7 \%$ of patients, while another $6 \%$ may develop extramedullary lesions later in their disease course. Extramedullary Multiple myeloma, presenting as clival mass can create a great diagnostic dilemma, since these patients first come to ENT or neurosurgery OPDs as seen in this index case. High index of suspicion supported by an appropriate IHC panel is essential to arrive at the correct histological diagnosis for achieving best therapeutic outcome.

\section{Nodular Lymphocyte Predominant Hodgkin Lymphoma: Interesting Case Report of an Unusual Pattern}

\author{
Arupparna Sengupta*1, Seema Rao ${ }^{1}$ \\ ${ }^{1}$ Pathology, Sir Gangaram Hospital, New Delhi, India
}

Aims \& Objectives: We present a case where a 24 year old patient was treated as classical Hodgkin's Lymphoma for last 15 years. He was doing well till the present episode when he presented with supraclavicular and mediastinal lymphadenopathy. Extensive 
immunohistochemistry done on the present lymph node biopsy revealed his final diagnosis.

Patients/Materials \& Methods: Biopsy was done from one supraclavicular lymph node which showed a tumor with predominant central diffuse and peripherally nodular architecture. In the central areas, the tumor was composed of large, atypical lymphoid cells scattered singly in a reactive background. In the peripheral zones, similar tumor cells with T-cell collars are present within B-cell nodules, which show dendritic cell meshwork by CD21 expression. The tumor showed predominant Pattern E of NLPHL and lesser component of Pattern A [typical (B cell rich) nodular]. On IHC: Tumor cells were positive for CD20, PAX5, EMA, BOB1, Bcl-6 (weak), c-myc, MUM1 and BCl-2 and negative for CD30, CD3, CD5, CD10, CD15 and ALK-1.

Results: Based on the above histopathology and immunohistochemistry, a final diagnosis of Nodular Lymphocyte Predominant Hodgkin Lymphoma- Diffuse T cell Rich (TCRBCL) pattern was furnished. Discussion \& Conclusion: Nodular lymphocyte predominant Hodgkin lymphoma (NLPHL) is a B cell neoplasm characterised by a nodular or a nodular and diffuse proliferation of small reactive lymphocytes with singly scattered large neoplastic cells known as lymphocyte predominant (LP) or popcorn cells. Six distinct patterns are recognised - pattern A is typical (B cell rich) nodular; pattern B is serpinginous nodular: pattern $\mathrm{C}$ is nodular with prominent extranodular LP cells: pattern D is T-cell-rich nodular: pattern $\mathrm{E}$ is diffuse TCRBCL-like : pattern $F$ is diffuse B-cell-rich. TCRBCL-like NLPHL show morphological and molecular overlap with T-cell/histiocyte-rich large B cell lymphoma. CONCLUSION: Patients with NLPHL pattern E (TCRLBCL-like architecture) present with advanced clinical stages and develop relapses more frequently than patients with a typical nodular infiltrate.

\section{Plasma Cell Dyscrasia with Erythroblastic Morphology Masquerading as Acute Leukemia}

\section{Shilpi Dosi ${ }^{* 1}$, Kamal Malukani ${ }^{1}$, Prakash G. Chitalkar ${ }^{2}$, Amit V. Varma $^{1}$ \\ ${ }^{1}$ Pathology, ${ }^{2}$ Medical Oncolgy, Sri Aurobindo Medical College \& PGI, Indore, India}

Aims \& Objectives: We reported a rare case presentation of a case of Plasma Cell Dyscrasia with erythroblastic morphology masquerading as Acute Leukemia in a 55 year old female.

Patients/Materials \& Methods: A 55 year old female presented with lower back pain and unable to walk sine one month. Chest $\mathrm{X}$ - ray revealed mediastinal widening. History of multiple blood transfusion was present. Renal function tests were mildly deranged. Serum protein electrophoresis not revealed $M$ band spike. Serum calcium showed raised value.

Results: Haematological work up showed mild anaemia and leukocytosis (Haemoglobin-10.7 gm/dl, TLC_-21,900/cumm, Platelets$1.89 \mathrm{lacs} / \mathrm{cumm})$. Peripheral smear showed leukoerythroblastic blood picture with $35 \mathrm{nRBCs} / 100 \mathrm{WBC}$. Bone marrow aspiration smears showed $>80 \%$ Blasts like cells with erthroblastic morpholgy. Trephine biopsy showed diffuse sheets of blast like cells. There was no rouleaux formation and blue background staining in any of the smears. On the basis of these findings, there was diagnostic dilemma between Acute leukemia (M6) versus Plasma cell dyscrasia. Flow cytomery of Bone marrow revealed plasma cell myeloma .

Discussion \& Conclusion: Plasma cell myeloma is a bone marrow based multifocal neoplastic proliferation of plasma cells. Neoplastic plasma cells morphology can vary from mature forms to immature forms. Few cases have been reorted so far, of morphologic variants of plasma cell myeloma.Out of which lymphoplasmacytic, megakaryocytic, plasmacytic,lymphocytosis like and variant of hair cell leukemia like morphology. After an exhaustive review of literature,not found the reported case of plasma cell myeloma with erythroblastic morpholgy mimicking as Acute leukemia. Conclusion : To recognize the morphological variants of plasma cell myeloma and importance of differentiating it from Acute leukemia for proper treatment.

\section{Correlation of Plasma Cells with Normoblasts, Red Cell Count and Hemoglobin Levels in Myeloma Patients:}

\section{A Case Series}

Shamim A. Ansari ${ }^{* 1}$, R. K. Nigam ${ }^{1}$, Reeni Malik ${ }^{1}$, Maneesh Sulya ${ }^{1}$

${ }^{1}$ Department of Pathology, Gandhi Medical college and Hamidia Hospital, Bhopal, India

Aims \& Objectives: This study was carried out to know if there is any significant correlation between hemoglobin levels, bone marrow normoblast percentage, and marrow plasma cell percentage in patients with or without myeloma.

Patients/Materials \& Methods: We compared 07 samples (FBC and marrow smears) from patients with myeloma- Group 1 (diagnosed between March 2017-May 2020 by biochemical, clinical, pathological evidence or combination) with 07 samples (FBC and bone marrow smears) collected from patients- Group 2 with no evidence or clinical suspicion of myeloma. This group included patients with unexplained anemias, anemia of chronic disorders, hypoplastic/aplastic anemia, and pancytopenia. Group 1 patients included patients who were newly diagnosed cases of plasma cell neoplasm and were not on chemotherapy. The hemoglobin and RBC counts from both groups were estimated by Mindray-3600 and Mindray BC-5100 (Beckman Coulter) analyzer. Two Leishman stained bone marrow smears were evaluated and an average of three differentials from a 300 cell count was obtained.) Data were analyzed and interpreted.

Results: We have attempted to study closely between the relationship of hemoglobin, red cell count, and normoblasts with plasma cell percentage in both patients with and without myeloma. Results are compared with the help of Pearsons coefficient correlation formulae. There is a moderate negative correlation between plasma cells \% and normoblast $\%$ and a weak negative correlation between plasma cells with $\mathrm{RBC}$ counts and $\mathrm{Hb}$.

Discussion \& Conclusion: Our findings of a significant negative correlation between hemoglobin (and RBC count) and plasma cells in myeloma are not surprising and are due to the replacement of marrow by plasma cells with suppression of erythroid precursors. However, it is a fact that in the non-myeloma group there exists a significant negative correlation(although weak) between marrow normoblast and plasma cell percentage. This raises an important question as to whether plasma cells affect normoblast percentage with consequently affect on hemoglobin in both patients with and without myeloma.

\section{Aggressive Lymphoma with a Bad Prognosis}

Anjali V. S ${ }^{* 1}$, Ankitha Hebbar ${ }^{1}$, Ruchee Khanna ${ }^{2}$, Ranjini Kudva ${ }^{2}$, Sushma Belurkar ${ }^{2}$

${ }^{1}$ Patholgy, Melaka Manipal Medical College, MAHE, Manipal, ${ }^{2}$ Patholgy, Kasturba Medical College, Manipal, India

Aims \& Objectives: INTRODUCTION. Burkitt lymphoma is known to be the fastest growing tumour with a cell doubling time of 24-48 h. Its an aggressive non- Hodgkin B-cell lymphoma composed of diffuse sheet of monomorphic medium-sized B cells with high mitotic rate, high degree of apoptosis and starry-sky pattern. Its associated with 
Epstein Barr virus (EBV) and human immunodeficiency virus (HIV), and chromosomal translocations involving the oncogene C-MYC. According to World Health Organization (WHO) Classification, Burkitt lymphoma can have 3 clinical variants: endemic, sporadic and immunodeficiency-associated variant. CASE REPORT. A 13 year old boy presented with complaints of right sided neck swelling since 2 months duration with difficulty in chewing and swallowing. On physical examination, right side cervical lymphadenopathy was noted, which was firm on palpation. Clinician advised lymph node biopsy, which revealed diffuse infiltration by monotonous population of medium sized abnormal lymphoid cells with brisk mitotic activity and apoptotic debris with tingible body macrophages. On immunohistochemical evaluation, the tumour cells were found to be positive for CD20, CD10, Bcl2, Bc16, CMYC and Ki67 of 95\%. Subsequent bone marrow study revealed diffuse infiltration by medium sized abnormal lymphoid cells with starry sky pattern.

Patients/Materials \& Methods: Not applicable.

Results: Not applicable.

Discussion \& Conclusion: CONCLUSION. Pediatric Burkitt lymphoma is a curable disease, with intensive immunochemotherapy. Accurate diagnosis using bone marrow aspiration and biopsy, flow cytometry, immunohistochemistry, and cytogenetics is crucial and mandatory, as well as prompt treatment. The present case is a fitting example of the importance of timely diagnosis and prompt treatment which proved to be life saving for the child.

\section{Duodenal Plasmablastic Lymphoma in a Young Immunocompetent Female}

Nishav Garg ${ }^{* 1}$, Sumit Grover ${ }^{1}$, Vikram Narang ${ }^{1}$, Harpreet Kaur ${ }^{1}$, Bhavna Garg ${ }^{1}$, Neena Sood ${ }^{1}$, Ankita Soni ${ }^{1}$, Aminder Singh ${ }^{1}$

\section{${ }^{1}$ Pathology, Dayanand Medical College \& Hospital, Ludhiana,} India

Aims \& Objectives: To highlight the rare possible diagnosis of Plasmablastic Lymphoma in masses of GI Tract in Immunocompetent patients.

Patients/Materials \& Methods: A 38 year old immunocompetent female presented with pain abdomen for few weeks. Her MDCT scan showed circumferential wall thickening of 2 nd part of duodenum measuring $1.8 \mathrm{~cm}$. An associated mass lesion measuring $3.5 \times 4.2 \times 5.9 \mathrm{~cm}$ was noted lateral and posterior to the duodenum. Rest of the organs appeared to be unremarkable. Radiological possibility of adenocarcinoma was being kept. Duodenum brushings were non-diagnostic because of insufficient cellularity. A duodenal biopsy was performed.

Results: Duodenal biopsy on microscopy revealed sheets of large cells having vesicular nuclei, prominent nucleoli and moderate amount of cytoplasm with brisk mitosis mimicking undifferentiated malignancy. On immunohistochemisrty (IHC) the large cells were positive for $\mathrm{CD} 138$ with $\mathrm{Ki} 67$ proliferative index of $75 \%$. IHC for CK, LCA, CD3, CD20, CD79a, CD30, S-100, AlK1, CD7, DOG1 were negative. Thus diagnosis of Plasmablastic Non Hodgkin lymphoma was rendered.

Discussion \& Conclusion: Plasmablastic lymphoma is a very aggressive lymphoma which usually occurs in elderly immunocompromised patients. Most commonly it is seen in head and neck region. Morphologically it mimics poorly differentiated carcinoma and prognosis is generally poor. We present this case because of unusual occurrence of this rare high grade lymphoma in young immunocompetent patients.
Unicentric Castleman Disease: A Great Mimicker

Rashim Sharma ${ }^{* 1}$, Deepak Vedant ${ }^{1}$, Amit Gupta ${ }^{1}$, Himanshu Pandey $^{2}$, Pushpinder Khera ${ }^{3}$, Poonam Elhence ${ }^{1}$

${ }^{1}$ Pathology, ${ }^{2}$ Urology, ${ }^{3}$ Diagnostic and Interventional Radiology, AIIMS Jodhpur, Jodhpur, India

Aims \& Objectives: Castleman disease (CD), also known as angiofollicular lymph node hyperplasia encompasses a group of lymphoproliferative disorders which have common histomorphological features. Occurrence of CD in abdomen is itself an enigma. The diagnosis even becomes difficult when its morphology mimics other lymphoproliferative disorders. We present a 31 year old female with indolent para-adrenal mass.

Patients/Materials \& Methods: A 31 year old female presented with history of pain in right flank, dull in nature. A three year old CT scan showed well circumscribed heterogeneously hypo attenuating right supra-renal mass of size $5.9 \times 7.2 \times 6.2 \mathrm{~cm}$. Clinical examination showed no organomegaly or lymphadenopathy. A repeat CECT suggested a similar right suprarenal mass with size measuring $6.8 \times 5.8 \times 6.9 \mathrm{~cm}$. No evidence of invasion into vascular structures and adjacent organs noted. Possibilities of phaechromocytoma, ganglioneuroma and lymphoma were kept. Laparoscopic adrenalectomy with removal of mass was done.

Results: The received specimen was comprising of a large mass along with right adrenal gland, not adherent to it. Mass was well circumscribed, measuring $10 \times 6 \times 6 \mathrm{~cm}$ with grey white solid cut section. On microscopy, normal adrenal gland along with a large single lymph node showing effaced architecture with expansion of inter-follicular zone, expanded sinuses and regressing lymphoid follicles with atretic germinal centres traversed by prominent radially penetrating sclerotic vessels- lollipop vessels were seen. Pale and expanded mantle zone was seen with onion skin like layering of lymphocytes. Interfollicular areas showed extensive vascular proliferation with perivascular hyalinization and interspersed prominent plasma cells and a few eosinophils. No atypical mitosis, ReedSternberg cells, polykaryocytes, necrosis or epithelioid cell granulomas were noted. The case was thus reported as Castleman Disease: Hyaline Vascular Variant.

Discussion \& Conclusion: CD comprises a spectrum of lymphoproliferative disorders and its histomorphology can be misinterpreted as lymphoma. Histomorphologically, it is subdivided into Hyaline vascular, Plasma cell rich and mixed variants. The treatment modalities are limited. For localized form surgical excision is curative whereas for systemic form, no cure is available. Unicentric CD is rare and must be included in differentials of adrenal neoplasms.

\section{IGE Myeloma: The Secret Secretor}

Shiny Amber ${ }^{* 1}$, Sushma Belurkar ${ }^{1}$

${ }^{1}$ Pathology, MAHE, Manipal, India

Aims \& Objectives: Plasma cell myeloma (PCM) comprises 10-15\% of all haematopoietic neoplasms. The disease shows a male preponderance. Symptomatic patients present with signs of end organ damage. It is a consequences of abnormal plasma cell proliferation in the bone marrow. Serum protein electrophoresis remains a gold standard test and detects M protein spike in multiple myeloma (MM). Here, we report a rare case of IgE multiple myeloma with normal serum protein electrophoresis but elevated serum free light chains.

Patients/Materials \& Methods: CASE REPORT: 39-year-old male presented with abdominal discomfort, dyspnoea, and fatigue for 4 months along with palpitation for 2 months O/E: JVP was raised, right pleural effusion and ascites was noted along with hepatomegaly. ECHO revealed decreased effusion fraction. Diagnosis given was 
Heart failure due to restrictive cardiomyopathy and amyloidosis was considered as the probable cause. On evaluation for cause of Amyloidosis, 24-hour urine protein revealed nephritic range proteinuria. Serum protein electrophoresis showed absence of $M$ band, but free light chain assay showed significantly raised lambda light chain. Kappa lambda ratio was 0.04. As Multiple myeloma was suspected, bone marrow aspiration, biopsy, and Flow cytometry (FC) was advised. Bone marrow studies revealed $<10 \%$ plasma cells and on FC for myeloma panel confirmed monoclonality in plasma cells. As $\mathrm{M}$ band was absent probable diagnosis of light chain MGUS was considered. On further evaluation IgE levels were markedly increased i.e. $751.7 \mathrm{IU} / \mathrm{ml}$. IgG was normal; IgA and $\mathrm{M}$ were decreased. Elevated IgE along with end organ damage confirmed the diagnosis of IgE Multiple Myeloma.

\section{Results: N/A.}

Discussion \& Conclusion: IgE myeloma is a rare subtype of myeloma and it is known to have poor prognosis. It can pose a diagnostic challenge as it often presents with absent $\mathrm{M}$ band on protein electrophoresis. Further investigation such as immunoglobulin assay is warranted to evaluate and diagnose $\operatorname{IgE}$ myeloma in presence of an abnormal free light chain ratio.

\section{Cyclin D1 Expression in Plasma Cell Myeloma on Bone Marrow Trephine Biopsy}

Ankita Jaiswal $^{* 1}$, Priya Murthy ${ }^{2}$, Tejindar Singh ${ }^{1}$

${ }^{1}$ Haematopathology, Oncquest Laboratories Limited, Delhi, ${ }^{2}$ Haematopathology, Oncquest Laboratories Limited, Bangalore, India

Aims \& Objectives: Plasma cell Myeloma (PCM) is a bone marrow based multifocal neoplastic proliferation of plasma cells, usually associated with an $\mathrm{M}$ protein in serum and/or urine and evidence of end organ damage related to plasma cell neoplasm. CyclinD1 dysregulation is an early and unifying oncogenic event seen in 15-25\% cases of myeloma. These cases are associated with $\mathrm{t}(11 ; 14)$ translocations and carry a better overall survival and need to be differentiated from other lymphoproliferative disorders. We aimed to study the incidence of CyclinD1 expression in cases of PCM and its correlation with other histological features such as percentage of plasma cells (PC), pattern of involvement, PC morphology, type of light chain restriction and CD20 and CD56 immunohistochemistry (IHC) expression.

Patients/Materials \& Methods: Formalin fixed, decalcified bone marrow trephine biopsy specimen from 15 patients of PCM were subjected to CyclinD1 IHC by using a rabbit monoclonal antibody to CyclinD1 (Dako, clone EP12). PC distribution was subclassified as diffuse/partial replacement/nodular and interstitial. PC morphology was classified as Mature/Intermediate/Immature/Plasmablastic/Small lymphocyte-like/Spindled based on specific morphological features. IHC with CD138, kappa, lambda, CD56, CD20 and CyclinD1 was carried out.

Results: 8/15 cases of PCM (53\%) were positive for CyclinD1. Most cases had a diffuse invovement and all morphological types were seen. $3 / 8$ cases $(37.5 \%)$ had small lymphocyte like morphology and they also expressed CD20. 6/15 (40\%) expressed Kappa and 8/15 (53\%) expressed Lambda. 1 case was non-secretory. 8/15 (53\%) expressed CD56 and 4/15 (26\%) had CD20 expression. Discussion \& Conclusion: It is important to have a knowledge of CyclinD1 positive PCs, otherwise it may lead to a misdiagnosis of lymphoma, if additional testing is not done. It is of critical value in cases of myeloma with small lymphocyte-like morphology which also express CD20. As several studies have reported the correlation between CyclinD1 overexpression, IGH/CCND1 fusion with $\mathrm{t}(11 ; 14)$ and prognosis, use of CyclinD1 is a cost-effective method of indicating good prognosis in PCM cases.

\section{Plasma Cell Myeloma with Double M Bands on Serum Protein Electrophoresis: A Diagnostic Conundrum?}

Anu Krishnaa ${ }^{* 1}$, Sunayana Misra ${ }^{1}$, Garima Baweja ${ }^{1}$, Sadhna Marwah $^{1}$, Vijay Kumar ${ }^{1}$

${ }^{1}$ Pathology, ABVIMS and Dr RML Hospital, New Delhi, India

Aims \& Objectives: To distinguish between a true BGM(biclonal gammopathy) from an apparent monoclonal gammopathy presenting with two distinct band on serum protien electrophoresis.

Patients/Materials \& Methods: We have performed a serum protien electrophoresis on the provided sample.

Results: We report a case of a 61-year-old man, diagnosed with PCM and presenting with two discrete bands on SPE (simulating a BGM) which turned out to be monoclonal in nature.

Discussion \& Conclusion: Plasma cell myeloma (PCM) is a monoclonal gammopathy (MGM) characterized by proliferation of abnormal clone of plasma cells infiltrating the bone marrow with consequent end organ damage. The clonal plasma cells secrete a single clone of immunoglobulins (Ig) leading to presence of $\mathrm{M}$ protein in the serum and/or urine. The M- protein is appreciated as a discrete band on serum protein electrophoresis (SPE) in the gamma globulin region, also called the M-band. Biclonal gammopathy (BGM) occurs due to neoplastic transformation of a plasma cell clone undergoing Ig class switching or due to an independent neoplastic transformation event yielding proliferation of unrelated plasma cell clones, therefore resulting in two distinct M-bands on SPE. It is, however, vital to distinguish a true BGM from an apparent one (MGM presenting with two distinct bands on SPE) so as to make an accurate diagnosis.

Keywords: multiple myeloma, biclonal gammopathy, serum protein electrophoresis, IgG.

\section{Adult T Cell Leukemia/Lymphoma of Parotid Gland: A Diagnostic and Therapeutic Challenge}

Pooja K. Suresh ${ }^{* 1}$, Kshithi Kudlu ${ }^{2}$, Sridevi H. Basavaiah ${ }^{1}$, Suja Sreedharan $^{2}$, Sanyo Dsouza ${ }^{3}$

${ }^{1}$ Pathology, ${ }^{2}$ Otorhinolaryngology, Kasturba Medical College, Mangalore, Manipal Academy of Higher Education, ${ }^{3}$ Medical Oncology, Kasturba Medical College Hospital, Attavar, Mangalore, India

Aims \& Objectives: We report a rare case of ATLL involving parotid gland in a 70 year old woman who lack any history of travel to HTLV-1 endemic areas.

Patients/Materials \& Methods: A 70 year old woman presented with 2-month history of gradually progressive painful swelling in left infra-auricular region. Examination revealed a $9 \times 8 \mathrm{~cm}$ firm swelling in left parotid area fixed to underlying structures and skin. There was no organomegaly or mediastinal mass. Skin lesions in the form of erythematous rashes were noted.

Results: Routine blood investigations revealed hemoglobin $-11.9 \mathrm{~g} /$ $\mathrm{dL}$ and normal total leucocyte and platelet counts with an absolute lymphocyte count of $3.06 \times 109 / \mathrm{L}$. There was absence of any abnormal lymphoid cells in peripheral blood. Serum lactate dehydrogenase was increased(502 U/L) and calcium was normal. FDGPET scan revealed metabolically active mass epicentered in left parotid region with few discrete left level II,III and V cervical lymph nodes and multiple metabolically active marrow deposits. Excision 
biopsy showed salivary gland tissue diffusely infiltrated by monotonous population of abnormal lymphoid cells with absence of obvious cellular pleomorphism. On immunohistochemistry,lymphoid cells were $\mathrm{CD} 3+, \mathrm{,D} 5+, \mathrm{CD} 30+$ and were ALK- negative. Cells were negative for CD20,CD10 and TdT. During hospital course, patient progressed to develop CNS manifestations. At this time, peripheral smear showed presence of occasional atypical clefted lymphoid cells but marrow was uninvolved.CSF analysis revealed lymphocytic pleocytosis with presence of atypical lymphoid cells having deep nuclear clefts and abnormally clumped coarse chromatin.CSF immunophenotying revealed CD45 bright lymphoid cells that co-expressed CD4 and CD25 with loss of CD7,sCD3,CD8 and TCR $\alpha / \beta$. Cells lack CD19,CD20,CD10,CD22,kappa,lambda and CD56. Patient was tested positive for HTLV-1(reactive- 122 IU). Case was concluded as adult T-cell leukemia/lymphoma-lymphomatous subtype. Patient was started on CHOP regimen planned for a total of 6 cycles. Lesion improved dramatically, and at the last follow up after 2 nd cycle of treatment, the lesion had completely regressed clinically.

Discussion \& Conclusion: To conclude, the present case is unique by the presence of monotonous population of abnormal lymphoid cells (unlike the classic ATLL) and diffuse CD30 positivity. It highlights the importance of complete T-cell panel (IHC or flow cytometry) that needs to be considered in evaluating any T-cell lymphoma.

\section{Plasma Cell Myeloma in 56 Year Old Female with Atypical Cells: A Case Report \\ Ritika Bharti $^{* 1}$ \\ ${ }^{1}$ Pathology, Index Medical College Hospital and Research, Indore, India}

Aims \& Objectives: Plasma cell myeloma is multifocal neoplastic proliferation of plasma cells, usually associated with an increase in $\mathrm{M}$ protein and organ damage related to plasma cell neoplasm.We are presenting a case report of a 56/female patient with diagnosis of plasma cell myeloma where we find atypical cells which were suspicious of metastatic malignant cells.

Patients/Materials \& Methods: We performed hemogram, peripheral smear,B.M aspiration,serum electrophoresis,serum calcium, xray (skull and spine and $\mathrm{PBH}$ ).

Results: Hemogram showed pancytopenia, peripheral smear show rouleax formation, B.M show atypical cells and plasma cells > $10 \%$,spike in $\mathrm{M}$ band and high serum globulin, $3+$ proteinuria, $\mathrm{x}$ ray showed multiple lytic lesions in skull,spine and pelvis and multiple consolidation in bilateral lung.serum calcium was in normal limit.

Discussion \& Conclusion: Anemic patients especially elderly female are ignored in our country thinking simple nutritional anemia.malignancy should be kept in mind for older age patients.

\section{Extranodal Hodgkins Lymphoma as a Chest Wall Mass}

Priyathersini Nagarajan $^{* 1}$, Swaminathan Rajendiran ${ }^{1}$, Sri Gayathri Shanmugham ${ }^{1}$

\section{${ }^{1}$ Sri Ramachandra Medical College, Chennai, Chennai, India}

Aims \& Objectives: To present a a case of primary extranodal Hodgkins lymphoma as chest wall mass.

Patients/Materials \& Methods: A 32 year female patient presented with chest wall swelling of 6 months duration, diagnosed earlier as cold abscess in another hospital was on anti -tuberculous therapy. However the swelling persisted. On examination the swelling was in the right chest wall measuring $8 \times 5 \mathrm{~cm}$, firm to hard in consistency. Fine needle aspiration cytology revealed sheets of neutrophils. With a diagnosis of an abscess, she was started on antibiotics. However the swelling increased in size after 4 weeks. Excision biopsy was done. Results: Microscopic and immunohistochemical examination with CD 45, CD 15, CD 30,Ki 67, Alk, Vimentin showed features of Hodgkins lymphoma, nodular sclerosis type. Staging work up revealed involvement of mediastinal and left axillary lymph nodes, thus Stage II E. She was started on chemotherapy ABVD regimen. Follow up after one year showed complete remission.

Discussion \& Conclusion: Chest wall involvement can be either an initial manifestation of Hodgkin's lymphoma or a site of recurrence. Most commonly its due to infiltration of parasternal soft tissues by direct extension from anterior mediastinal nodes beneath or between the pectoral muscles without contiguous mediastinal or axillary adenopathy Thoracic spine involvement is frequently due to direct spread from posterior mediastinal nodes. Most have associated intrathoracic disease. However may occasionally be seen without evidence of intrathoracic disease. Such instances may present as a diagnostic challenge mimicking other soft tissue tumours.

\section{Waldenstrom's Macroglobulinemia with Isolated Calcification of Spleen: A Rare Case Report}

Fibah Bhat ${ }^{\text {*1, Rachana Meena }}{ }^{1}$, Sadhna Marwah ${ }^{1}$, Vijay Singh ${ }^{1}$, Mansi Singh ${ }^{2}$

${ }^{1}$ Pathology, ${ }^{2}$ Nephorolgy, ABVIMS \& Dr RML Hospital, New Delhi, India

Aims \& Objectives: Waldenstrom's Macroglobulinemia (WM) is a rare and slowly progressive malignancy of mature B cells with incidence of 0.38 per 100,000 persons per year. It accounts for about $1-2 \%$ of hematological malignancies.It is characterized by a monoclonal IgM in the serum and presence of Lymphoplasmacytic lymphoma (LPL) in the bone marrow.It presents usually with constitutional symptoms, organomegaly, cytopenias and hyperviscosity syndrome.

Patients/Materials \& Methods: Here we report a 53 year old male who presented in OPD with complaints of weakness, chronic headache,intermittent severe nausea and vomitting for 3 months. The patient had no lymphadenopathy,bony lesions or any organomegaly. However, ultrasonography of abdomen showed multiple hypoechoic and calcified lesions in the spleen parenchyma.

Results: On the basis of bone marrow morphology, immunohistochemistry,serum protein electrophoresis and markedly elevated levels of serum Ig M, the diagnosis of WM was made.

Discussion \& Conclusion: This case is unusual as isolated splenic parenchymal calcification has not been reported till date in the cases of WM. This case is presented due to its rarity.

Keywords: Waldenstron's Macroglobulinemia, Serum Ig M, Calcification,Spleen.

\section{Bone Marrow Immunophenotyping and Quantification of Clonal Circulating Plasma Cells in Plasma Cell Myeloma at Diagnosis by Multi-Parametric Flow Cytometry}

Sathya $\mathbf{P}^{* 1}$, Smita Kayal ${ }^{2}$, Srinivas $\mathrm{BH}^{1}$, Abdoul Hamide ${ }^{3}$, Rakhee $\mathrm{Kar}^{1}$

${ }^{1}$ Pathology, ${ }^{2}$ Medical Oncology, ${ }^{3}$ General Medicine, JIPMER, Pondicherry, India

Aims \& Objectives: Flow cytometric immunophenotyping (FC-IPT) is a valuable tool in the diagnosis of plasma cell myeloma (PCM). It is also a sensitive method to detect clonal circulating plasma cells (cPC) 
in PCM to define high-risk disease status. The aims of this study was to immunophenotype PCs and quantify clonal cPCs at diagnosis in PCM.

Patients/Materials \& Methods: Seventy-three patients diagnosed in the department of Pathology, JIPMER, Pondicherry from January 2018 to September 2020 were recruited. 73 Bone marrow (BM) aspirate and 65 Peripheral Blood (PB) samples collected in EDTA anti-coagulated tube were processed for IPT \& cPCs and acquired using Beckman-Coulter NAVIOS FC. These tests were performed on fresh BMA \& mononuclear cells were isolated from $\mathrm{PB}$ by density gradient centrifugation. An eight-color single/two tube technique was performed. This included antibodies against CD38, CD138, CD19, CD56, CD27, CD81, CD200, CD45, cytoplasmic kappa and lambda. A sequential gating strategy with CD45/CD38 and CD38/CD138 was used ( $>1 \times 105$ total events).

Results: The median age of patients was 55 (33-83) years with $64 \%$ male and $36 \%$ female. ISS stage-I was in 10\%, stage-II in $30 \%$ and stage-III in $60 \%$. The mean BM-PC \% by FC was $13.4 \%$ and that by morphology was $51.7 \%$. IPT showed strong expression of CD56 and CD200 in 89\% each and negative expression of CD19, CD27 \& CD81 in $90 \%, 80 \%$ and $87 \%$ respectively. Cytoplasmic Kappa \& lambda expression was seen in $92 \%$ \& $31 \%$ respectively. The mean $\mathrm{PBc}$ PC $\%$ by FC was 2.7 (7.5) \& that by morphology 3.86 (2.12). The mean $\mathrm{PB} \mathrm{cPC} \%$ by FC excluding four cases with plasma cell leukemia was $1.16(2.2)$. There was significant positive correlation between PB cPC \% by FC and morphology $(\mathrm{r}=0.6, p<0.0001) \&$ negative correlation with hemoglobin and platelet counts $(r=-0.4$, $p<0.01$ ). Weak negative correlation with serum albumin and positive correlation with $\beta 2$ microglobulin was also observed but was not statistically significant.

Discussion \& Conclusion: Inspite of lower number of PCs in the flow sample compared to BM which is due to inherent dilution. IPT and quantification of clonal $\mathrm{cPC}$ which can serve as potential prognostic markers in PCM.

\section{Hodgkin'S Lymphoma at an Unusal Site of Presentation : A Diagnostic Dilemma}

Durre Aden ${ }^{* 1}$, Swati Raj ${ }^{1}$, Rachana Meena ${ }^{1}$, Sadhna Marwah ${ }^{1}$, Minakshi Bhardwaj ${ }^{1}$, Vijay Kumar ${ }^{1}$

${ }^{1}$ Pathology, ABVIMS and DR RML Hospital, New Delhi, India

Aims \& Objectives: Hodgkin's lymphoma (HD) is a rare haematological malignancy which generally presents as an enlarged supraclavicular,cervical or axillary lymph node.It rarely presents as lymphadenopathy of mesenteric and inguinal lymph node with very few cases reported in the literature. We report a case of middle aged female who presented with ascites, mesenteric and inguinal lymphadenopathy suggestive of infectious etiology (tubercular). Her bone marrow aspiration and biopsy was performed and was reported as Hodgkin's lymphoma infiltration with coexisting granulomatous pathology.Later her inguinal lymph node excision was done which showed HD-mixed cellularity.The patient was referred to the haematologist for chemotherapy and she is getting treated.

Patients/Materials \& Methods: A 48 years old female patient presented to the emergency department with complaints of abdominal distension and dyspnoea. On examination there was marked ascites along with left inguinal lymphadenopathy of $2 \times 2 \mathrm{~cm}$. There was no other palpable lymph nodes. Her CBC showed severe anemia with leucocytosis with absolute neutrophilia and severe eosinophilia with normal platelet counts.USG abdomen and CT scan abdomen showed conglomerated mesenteric lymphnodes with presence of hepatosplenomegaly and a diagnosis of infectious ? tubercular etiology was made. The patient was started on anti tubercular drugs however,she did not improve and rather deteriorated further. Therefore,her bone marrow aspiration and biopsy was performed which showed extensive replacement of marrow by fibrosis with interspersed hodgkin's and Reed sternberg cells in mixed inflammatory background with focal areas showing granulomas. Thus,final diagnosis of Hodgkin's lymphoma infiltration with coexisting granulomatous pathology was given. This was confirmed on histopathology of inguinal lymph node.

Results: Result.

Discussion \& Conclusion: The evaluation of possible presence of lymphoma (Hodgkin's/Non Hodgkin's Lymphoma) should be considered in a patient presenting solely with mesenteric and inguinal lymphadenopathy especially in patients who do not show improvement after antibiotic/antitubercular treatment.This case is presented because of its unusual presentation.

Keywords: Inguinal lymph Node, Hodgkin's lymphoma,Granuloma.

\section{Peering into the Unknown: Artificial Intelligence for Lymphoma Diagnosis}

Aniruddha Mundhada ${ }^{* 1}$, Srigayathri S. ${ }^{1}$, Sandhya Sundaram ${ }^{1}$, Anurag M. ${ }^{2}$

${ }^{1}$ SRMC, Chennai, ${ }^{2}$ Insane AI, Bangalore, India

Aims \& Objectives: 1 . To visualise artificial intelligence model for diagnosis of lymphoma.

Patients/Materials \& Methods: Lymphoma H\&E slides were used to train AI algorithm classifier on Lymphoma cancer cell classification. We present quantitative accuracy metrics for classification. Further, we examine the visualisations of a convolutional neural network VGG16 at intermediate layers using the Grad CAM technique.

Results: Visualisations show how the model is able to visualise between tumor and non tumor regions during training. This will be useful for regulatory approvals for AI models in routine diagnosis in pathology laboratories.

Discussion \& Conclusion: Pathologist can diffrentiate and apply corrective actions while the AI model fails to do so. Still it can improve on a particular type of task when trained to do so. This is important in future for regulatory approvals and understanding the inner workings of an AI model.

\section{Multiparameter Flowcytometry; A Major Tool for Detection of Plasma Cell Neoplasm}

Subhajit Brahma* ${ }^{* 1}$, Deepak Mishra ${ }^{1}$, Sambhu N. Banerjee ${ }^{1}$, Rakesh Demde $^{1}$, Raka Hota ${ }^{1}$, Akshaya Mandloi ${ }^{1}$, Saurabh Bhave ${ }^{2}$, Vivek Radhakrishnan ${ }^{2}$, Mammen Chandy ${ }^{2}$, Reena Nair ${ }^{2}$, Jeevan Kumar ${ }^{2}$, Naveed Tamboli ${ }^{2}$

${ }^{1}$ Lab. Haematology, ${ }^{2}$ Clinical Haematology, Tata Medical Center, Kolkata, Kolkata, India

Aims \& Objectives: Plasma cell neoplasm (PCN) results from expansion of a clone of immunoglobulin secreting, heavy chainswitched, terminally differentiated B cells. Multiparameter flow cytometry plays a major role in diagnostic, prognostic and disease monitoring studies to determine clinical outcome. In the present study we tried to observe the details of marker expression in plasma cell disorders in diagnostic specimens.

Patients/Materials \& Methods: We retrospectively analyzed immunophenotypic data from consecutive cases $(n=48)$ of PCNs referred to our laboratory from January, 2018 to September, 2020. These cases were diagnosed based on morphology, 
immunophenotyping, biochemical (IFE, FLC and SPE) and Radiological parameters.

Results: Median age at diagnosis was 63 years; the male to female ratio was $3: 1$. PCN cases were diagnosed by morphology and other criteria based on WHO 2016 guidelines. Flowcytometry was done as an adjunct to document abnormal plasma cells. CD38/CD138 gating was used for selecting plasma cells. Abnormal plasma cells (aPC) expressed CD38/CD138 (dim to moderate) in all cases. CD56 (94\%), CD117(56\%), CD81 (35\%), CD28 (15\%), CD27 (10\%), CD19(10\%), CD20 (8\%) and CD45 (4.5\%) were expressed along with either cKappa $(70 \%)$ or cLambda (30\%) light chain. On the other hand, normal Plasma cell co-expressed (nPC) CD38/CD138 (moderate to bright), CD19, CD45, CD27, CD81and was polyclonal for cKappa and cLambda.

Discussion \& Conclusion: Multiparametric flowcytometry is required for immunophenotypic identification and characterization of aPCs and the same is also used for MRD monitoring.

\section{A Rare Case of Nasopharyngeal Extramedullary Plasmacytoma with Amyloidosis}

Elizabeth Joseph $^{\mathbf{1}}$, Jiby S. Ninan ${ }^{* 1}$, Nitin A. Thomas ${ }^{2}$, George Thomas $^{2}$

${ }^{1}$ Pathology, ${ }^{2}$ ENT, Believers Church Medical College Hospital, Thiruvalla, India

Aims \& Objectives: A rare case of extramedullary plasmacytoma with amyloidosis of the nasopharynx.

Patients/Materials \& Methods: A 54 year old male presented with a nasopharyngeal mass which was excised under general anaesthesia. Histopathological examination of the biopsied mass along with Immunohistochemical studies were done. Follow up of the patient with a peripheral smear, bone marrow examination and tests to rule out multiple myeloma and systemic amyloidosis was advised.

Results: Excised nasopharyngeal mass on histopathological examination showed eosinophilic acellular material with clusters of plasma cells and multinucleated giant cells scattered in between. Immunohistochemistry was strongly immunoreactive for CD138 and Kappa in the neoplastic plasma cells. The eosinophilic acellular material was salmon pink color on Congo red stain and showed apple green birefringence on polarized light microscopy. A diagnosis of extramedullary plasmacytoma with amyloidosis was made with the above findings.

Discussion \& Conclusion: High index of suspicion of an underlying plasma cell neoplasm need to be retained when encountering localised amyloid deposits in the head and neck region. Other differential diagnosis to be considered are B cell lymphoma and plasma cell neoplasms. Immunohistochemistry is mandatory to make a definitive diagnosis. Management of EMP should include surgical resection and radiotherapy with long term surveillance to rule out recurrence and progression to disseminated plasma cell myeloma .

\section{Role of Multiparametric Flow Cytometry in MGRS: Case Based Illustration}

Asish Rath ${ }^{* 1}$, Saleem Mirza ${ }^{1}$, Rishi Dhawan ${ }^{1}$, Mukul Agarwal ${ }^{1}$, Pradeep Kumar ${ }^{1}$, Ganesh K. V. ${ }^{1}$, Jasmita Dass ${ }^{1}$, Tulika Seth ${ }^{1}$, Manoranjan Mahapatra ${ }^{1}$, Seema Tyagi ${ }^{1}$

${ }^{1}$ Haematology, AIIMS,NEW DELHI, NEW DELHI, India

Aims \& Objectives: To identify small clonal plasma cell population by multiparametric flow cytometry(MFC) in suspected monoclonal gammopathy of renal significance (MGRS) cases.
Patients/Materials \& Methods: Bone marrow aspirate samples were processed by a bulk lysis protocol. Specimens were acquired using 6-color, 3-tubes multiparametric approach on 3-laser BD FACS Canto-II (BD Biosciences, San jose, CA) and analyzed on BD FACS Diva software ver. 8.0.

Results: Attached as supporting document.

Discussion \& Conclusion: Clonal identification is important as same renal lesions can be found in in different hematological disorders and treatment varies depending upon the type of clone. Characterization and clonality identification of plasma cells or B- cells in bone marrow by flow cytometry is a must in cases of MGRS as it is highly sensitive and guides in appropriate decision making in therapy.

Supporting Document:

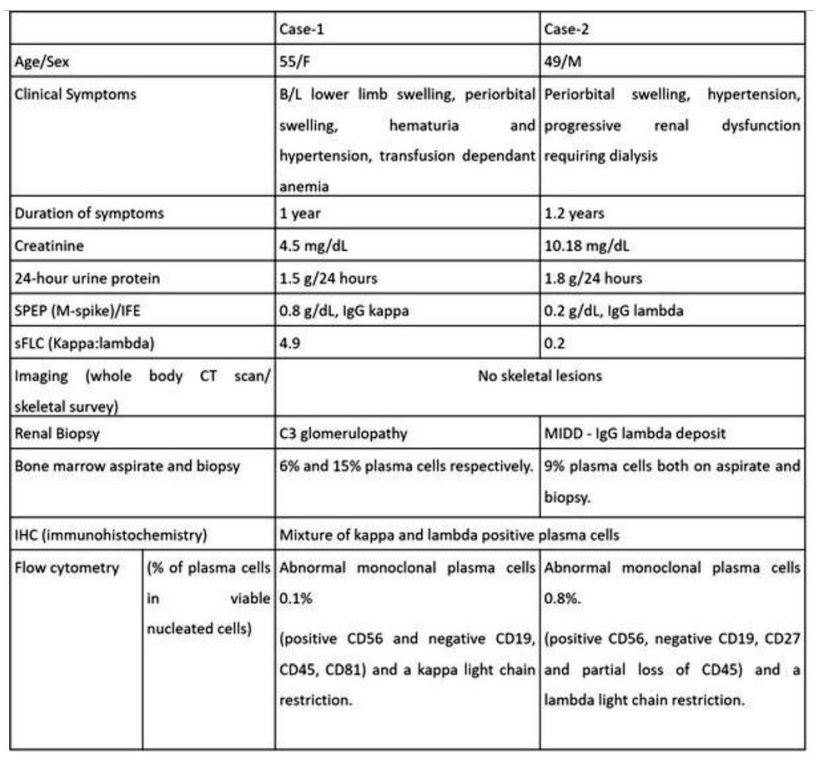

\section{Hairy Cell Leukaemia Sometimes a Hair Pulling Diagnosis: 5 Year Experience of a Tertiary Center with Diagnostic Challenges}

Abhishek Sharma ${ }^{* 1}$, Man Updesh S. Sachdeva ${ }^{1}$, Shano Naseem ${ }^{1}$, Nabhajit Mallik $^{1}$, Sreejesh $S^{1}$, Narender Kumar ${ }^{1}$, Prashant Sharma ${ }^{1}$, Parveen Bose ${ }^{1}$, Jogeshwar Binota ${ }^{1}$, Jasmina Ahluwalia ${ }^{1}$, Reena Das ${ }^{1}$, Pankaj Malhotra ${ }^{2}$, Neelam Varma ${ }^{1}$

${ }^{1}$ Hematology, ${ }^{2}$ Adult Clinical Hematology, PGIMER Chandigarh, Chandigarh, India

Aims \& Objectives: Despite having characteristic morphological, immunophenotypic and molecular features, hairy cell leukaemia (HCL), may present diagnostic challenges due to its varied clinical and phenotypic features. This study presents the clinical, haematological, immunophenotypic and molecular profiles of HCL patients seen over a period of five years in a tertiary care hospital with emphasis on diagnostic challenges and few atypical cases.

Patients/Materials \& Methods: HCL cases were analysed retrospectively based on clinical presentations, and laboratory findings from the archives of the Department of Hematology, PGIMER. Atypical findings were separately noted and described.

Results: There were 61 cases of HCL including 25 follow-ups out of the 505 B-lymphoproliferative neoplasms. Mean age of the patients was 53 years $(21-77 \mathrm{yr})$ with male predominance $(53 / 61)$. Weakness 
and fever $(70 \%)$ were the commonest presentations, followed by splenomegaly (64\%), hepatomegaly (20\%), and lymphadenopathy (18\%). $80 \%$ patients were pancytopenic (49/61). In $61 \%$ of the cases $(\mathrm{n}=37)$, peripheral blood showed hairy cells. Dry tap was observed in $46 \%(\mathrm{n}=28)$ cases. Bone marrow examination showed typical hairy cells in most of the cases (51/61). Trephine biopsy examination showed typical fried egg appearance in $81.9 \%$ cases $(n=50)$. Immunophenotyping showed expression of CD19, CD20, CD103, $\mathrm{CD} 25$ and $\mathrm{CD} 11 \mathrm{c}$ in majority of the cases, while positivity was seen for $\mathrm{CD} 79 \mathrm{~b}$ in $66 \%$, CD123 in 44\%, and CD79a in 31\% cases, whereas lambda and kappa light chain restrictions were seen in $73 \%$ and $27 \%$ cases, respectively. Notably, CD10 and CD23 expressions were seen in $18 \%(\mathrm{n}=11)$ and $11.5 \%(\mathrm{n}=7)$ cases. BRAF Mutation was detected in $86 \%(24 / 28)$ cases. $15 / 25$ follow-up cases were not in remission. In addition, one case showed intra-sinusoidal pattern of infiltration on trephine biopsy. Another case relapsed with generalised lymphadenopathy during the course of treatment. Cytology and flowcytometry showed presence of tumour cells in this case.

Discussion \& Conclusion: Unusual findings like absence of palpable spleen, presence of lymphadenopathy, normal/increased TLC, expression of CD10 and negative BRAF, is not rare in this otherwise classical disease entity. Thus it underlines the importance of awareness among hematopathologists regarding the diagnostic challenges and pitfalls for making correct diagnosis of HCL.

\section{Threads of Deception: Low Grade Lymphoma Presenting with Marrow Fibrosis and Aplasia}

Kanwarpal Singh ${ }^{* 1}$, Suvir Singh ${ }^{2}$, Jagdeep Singh ${ }^{3}$, Davinder Paul ${ }^{3}$, Kunal Jain ${ }^{3}$

${ }^{1}$ Dayanand Medical College and Hospital, Ludhiana, India, ${ }^{2}$ Clinical Haematology and Stem Cell Transplantation, ${ }^{3}$ Medical Oncology, Dayanand Medical College and Hospital, Ludhiana, India

Aims \& Objectives: Significant bone marrow fibrosis is commonly noted with myeloid malignancies and is rarely noted with lymphoma (exception: hairy cell leukemia). We report a patient who presented with atypical marrow findings associated with low grade lymphoma. Patients/Materials \& Methods: A 63 year old gentleman presented with generalized weakness and bicytopenia for one month, for which evaluation done elsewhere revealed a $\mathrm{Hb}$ of $7.9 \mathrm{~g} / \mathrm{dl}$, TC $1400 / \mathrm{ul}$ (N70, L19) and platelet count of 87000/ul with no atypical cells. He was referred to us for further evaluation.

Results: Initial examination was unremarkable except palpable hepatomegaly $2 \mathrm{~cm}$ below right costal margin. Liver and renal functions and autoimmune markers were negative. Bone marrow aspiration was a dry tap and biopsy revealed a hypocellular marrow with a cellularity of 10-20\% associated with absent megakaryocytes and grade 2-3 reticulin fibrosis. There was an interstitial infiltrate of lymphoid cells with round, clumped chromatin and inconspicuous nucleoli, which on IHC were positive for CD20 and BCL2, and negative for CD5, CD10, BCL6, Annexin A1, cyclin D1 and TdT. The final diagnosis was thus confirmed as CD5 negative low grade B cell lymphoma. Peripheral blood FISH panel for MDS and NGS Panel for MPN mutations sent initially were negative. PET Scan showed diffuse faint skeletal uptake but no other lesions. He was initiated on chemotherapy with Bendamustine- Rituximab, which was associated with significant symptomatic improvement. After 3 cycles, $\mathrm{Hb}$ improved to $10 \mathrm{~g} / \mathrm{dl}$, TC 2500 (N52, L18) and platelets 126,000/ul. Repeat bone marrow examination was also a dry tap and biopsy showed similar grade of fibrosis with improvement in cellularity to approximately $30 \%$ and appearance of myeloid precursors. He is clinically well and is planned for monthly BR for another 3 cycles followed by reassessment.

Discussion \& Conclusion: A hypocellular marrow with significant fibrosis should prompt initial investigation for myeloid malignancies such as MDS, MPN or MDS/MPN overlap. Approximately $6 \%$ patients with low grade lymphoma involving marrow have been noted to show a similar picture and evaluation must commence with exclusion of hairy cell leukemia. Identification of lymphoid aggregates and timely application of IHC can avoid diagnostic delays and expedite treatment.

\section{Atypical Double Hit Lymphoma Presenting as Massive Pericardial Fluid Effusion}

Shivani Sangwan ${ }^{* 1}$, Udayakumar $\mathrm{DS}^{1}$, Ritu Chadha ${ }^{1}$, Bhawna Jha ${ }^{1}$, Akshay Gore ${ }^{1}$, Amrita Ramaswami ${ }^{2}$, Roshan Dikshit ${ }^{2}$, Nitin Sood ${ }^{2}$, Shweta Gautam ${ }^{3}$

${ }^{1}$ Hematopathology, ${ }^{2}$ Medical Oncology and Clinical Hematology, ${ }^{3}$ Cardiology, Medanta-The Medicity, Gurugram, India

Aims \& Objectives: To report a case of Atypical Double Hit Lymphoma (DHL) presenting as Pericardial Fluid effusion.

Patients/Materials \& Methods: A 66 years old male, presented with complaints of memory loss and disorientation. Investigations including $\mathrm{CBC}$, Echocardiography, USG abdomen, and MRI Brain was done. ECHO revealed massive pericardial effusion. Pericardial fluid was evaluated for cytomorphology. Flowcytometric Immunophenotyping (FCI) was performed using 10 colour, three laser BD FACS Lyric/BD FACS Suite with a panel of antibodies including CD34, CD19, CD7, CD3, CD2, CD45,CD13, CD33, CD20, HLADR, CD138, CD10, CD38, CD5, CD14, CD64, CD117,Kappa, Lambda, SurfaceIgM. FISH studies were performed on pericardial fluid using BCL2, BCL6, IGH and MYC dual colour break apart probes. CT Chest and PET CT scan were also done.

Results: In a 66 years old male admitted in a debilitated state with complaints of memory loss and disorientation, pericardial fluid cytomorphology showed numerous atypical lymphoid cells. FCI was suggestive of B-lymphoproliferative disorder. Simultaneous FISH analysis showed BCL6-IGH rearrangement and extra copies of MYC. Following the diagnosis a PET CT revealed few deep seated intraabdominal PET avid lymph nodes.

Discussion \& Conclusion: We are aware of the Large B cell lymphomas with rearrangements in MYC, BCL2 and/or BCL6. Many large B cell lymphomas do not have rearrangements of two or three genes and instead have either extra copies of two/three of these genes or a combination of extra copies of one gene and rearrangement of the other genes. Such cases are designated as Atypical DHL/THL. Our case was an atypical DHL with an unusual clinical presentation of effusion and few small PET avid lymph nodes. Atypical DHL have a prognosis intermediate between double expressor (DE) and classical DHL/THL. Hence the need to diagnose these lymphomas which require treatment in parlance with classical ones. The case also describes the multifaceted approach in rapidly diagnosing a complex entity. 


\section{Evaluation of Cytogenetic Abnormalities in Multiple Myeloma Patients}

Akanksha A. Kalal ${ }^{* 1}$, D Prashanth Shetty ${ }^{1}$, Vijith Shetty ${ }^{1,}$, Padma Shetty $^{3}$, Reshma Shetty ${ }^{1}$, Meenakshi $A^{1}$, Nagaraj V. kulkarni ${ }^{1}$

${ }^{1}$ KSHEMA Centre for Genetic Services, ${ }^{2}$ Department of Oncology, ${ }^{3}$ Department of pathology, K S Hegde Medical Academy, NITTE (Deemed to be) University, Mangaluru, India

Aims \& Objectives: Multiple Myeloma (MM) is a haematological disorder characterized by clonal proliferation of plasma cell in the bone marrow producing excessive amounts of $\mathrm{M}$ protein. It accounts for $18 \%$ of haematological malignancies and $2 \%$ of cancer deaths. It is clinically heterogenous with spectrum of symptoms like bone failure, anaemia, renal dysfunction and hypercalcaemia. MM has two oncogenic pathways, one called hyperdiploid involving the trisomies of chromosomes and another non-hyperdiploid, involving in the translocation of immunoglobulin heavy chain (IGH) locus. The IGH translocations can be identified $10-15 \%$ by karyotyping and $50 \%$ by Interphase Fluorescence In Situ Hybridisation (iFISH). Cytogenetic study plays an important role in disease diagnosis and prognosis of the condition.

Patients/Materials \& Methods: A total of 20 Multiple Myeloma patients were included in the study. Cytogenetic analysis was done on bone marrow samples using standard GTG banding technique. 20 well spreaded metaphases were analysed using GENASIS software version 8.1. Results were interpreted according to International system of human cytogenetic nomenclature. FISH was performed using specific DNA probes for $\mathrm{t}(11 ; 14), \mathrm{t}(4 ; 14), \mathrm{t}(14 ; 20)$ and Del $13 \mathrm{q}$ with standard protocol. The FISH signals were visualized using different filters in the interphase and metaphases under Olympus BX53 fluorescence microscope.

Results: Cytogenetic evaluation of 20 patients, age ranging from 45 to 72 years was carried out. Staging was done according to International Staging System (ISS). Among 20 cases, 11 patients showed normal karyotype, 6 patients with abnormal karyotype, 3 patients having no metaphases due to culture failure. Abnormal karyotype constitutes hyperdiploid $(n=3)$ and hypodiploid $(n=3)$, whereas FISH findings showed positive for $\mathrm{t}(11 ; 14), \mathrm{t}(4 ; 14)$, trisomy 11 , trisomy 14 and monosomy 13 . One of the case with normal karyotype confirmed the $\mathrm{t}(4 ; 14)$ and monosomy 13 abnormality through FISH. Discussion \& Conclusion: Cytogenetic and FISH studies help in every aspect of the studies from evolution of malignancy to clinical presentation, prognosis and response to therapy, whereas FISH can be used as a valuable and reliable tool to tease out the specific abnormality in MM.

Keywords: Multiple Myeloma, Karyotyping, FISH, Hyperdiploidy, Hypodiploidy.

\section{A Leukemic Presentation of Double Hit Lymphoma in a Known Case of Plasmablastic Lymphoma}

Ajit Yadav ${ }^{* 1}$, Shanaz Khodaiji ${ }^{2}$, Vidisha Mahajan ${ }^{3}$, Sachin Almel $^{4}$, Satyakam Sawaimoon ${ }^{5}$, Pratibha Amare ${ }^{6}$

${ }^{1}$ Haematology, P.D Hinduja hospital and Research centre, 2Haematology, P.D.hinduja hospital, ${ }^{3}$ Haematology, ${ }^{4}$ Medical Oncology, ${ }^{5}$ Histopathology, P.D.Hinduja hospital and research centre, Mumbai, 'Clinical Genetics, Lilac insights, Navi Mumbai, India

Aims \& Objectives: B-cell Non Hodgkin Lymphomas (NHL) showing a plasmablastic transformation have been reported in less than 10 cases in the world. We report a rare case of a 44 year old female presenting with a plasmablastic lymphoma in the soft tissue around the ankle joint with simultaneous involvement of the bone marrow by B cell NHL.

Patients/Materials \& Methods: An immunocompetent 44 year old female presented to the hospital with left ankle swelling. Biopsy with IHC was done revealing plasmablastic lymphoma. Bone marrow findings and flow cytometry showed B cell NHL. Molecular studies demonstrated double hit lymphoma.

Results: Biopsy of the left ankle lesion revealed plasmacytoid cells,immunoreactive for CD138,CD38 and with kappa restriction.These cells were negative for CD45,CD20,CD19, CD30,CD99,HMB45 and positive Ki 67 of $90-95 \%$ favouring plasmablastic lymphoma. The bone marrow showed 30\% lymphocytes and 1\% plasma cells. The flow cytometry revealed 16\% kappa restricted clonal B lymphocytes which were CD19,CD20 and CD10 positive, while being negative for CD200,CD43,CD5 and lambda light chain suggestive of bone marrow involvement by B cell NHL. The FISH report on the bone marrow detected myc and bcl2 gene rearrangements suggestive of a double hit lymphoma. FDG PET showed an osseous lesion in the left ankle and uptake in inguinal, femoral lymph nodes. The patient was given 4 cycles VRD based chemotherapy. After a month the patient was readmitted in the hospital with a WBC count of $1,75,000 / \mathrm{cmm}$. The peripheral blood revealed $95 \%$ atypical lymphoid cells which on flow cytometry were medium to large sized and were CD 19,CD20,CD10 positive and kappa restricted, similar to bone marrow immunophenotype, suggestive of a high grade lymphoma.The patient is now receiving cyclophosphamide and Rituximab.

Discussion \& Conclusion: Our case documented the simultaneous identification of a B cell NHL involving the bone marrow with an extramedullary plasmablastic lymphoma in the ankle soft tissue. It presented a diagnostic challenge since both are reported as distinct entities with different treatment modalities and outcomes.

\section{A Rare Case of Primary Plasma Cell Leukemia with Multiple Organ Involved}

Ekta Rahul $^{* 1}$, Amar Ranjan ${ }^{1}$, Pranay Tanwar ${ }^{1}$

${ }^{1}$ Dept of Lab Oncology, BRA-IRCH, AIIMS, New Delhi, India

Aims \& Objectives: Plasma cell leukemia is the most aggressive form of plasma cell dysplasia that is characterised by clonal proliferation of malignant plasma cells in peripheral blood, accounting for $>20 \%$ of total leukocytes or absolute plasma cell count $>2000$ / cu.mm. Very rarely multiple organs are infiltrated by these clonal plasma cells. Here we present a case report of a patient who presented with increased peripheral blood and bone marrow clonal plasma cells along with bilateral plural effusion and hepatomegaly and splenomegaly.

Patients/Materials \& Methods: We are presenting a case of 64-year male who presented to us in January 2014 with complaints of pain in upper abdomen, weight loss, cough with expectoration. Complete blood count showed hyperleukocytosis with total count of 41360/cu.mm. and thrombocytopenia with platelets approximately $70000 / \mathrm{cu} . \mathrm{mm}$. On bone marrow aspirate, plasma cells were around $70 \%$ of total cellularity and $20 \%$ of total leukocyte on peripheral blood. On examination, liver was $13 \mathrm{~cm}$ below costal margin and tip of spleen was palpable. Bilateral wheeze was present. CECT of lung showed bilateral pleural effusion with ground glass opacity. Urine protein electrophoresis showed a narrow M-band however pleural fluid electrophoresis showed dense M-band. Urine protein was $15.4 \mathrm{mg} / \mathrm{dL}$. Serum free light chain assay showed kappa light chain of $664.4 \mathrm{mg} / \mathrm{L}$ and lambda light chain of $5.98 \mathrm{mg} / \mathrm{L}$. Kappa to lambda ratio of 111.11. Serum LDH was 1366 units/L. 
Results: A diagnosis of primary plasma cell leukemia was made and patient was started on 4 cycles of PAD regimen. On follow-up, patient relapsed with bone marrow aspirate findings of 50-60\% plasma cells of all hematopoietic cells and focally forming aggregates. Therapy was revised with 6 cycles of PAD, botizumib and dexamethasone. On repeat bone marrow aspirate, plasma cells were around $2 \%$ and PBS showed neutrophilia and adequate platelets and there was no M-band on urine protein electrophoresis and free light chain ratio came to 2.62. Patient is on follow up and doing well.

Discussion \& Conclusion: Primary plasma cell leukemia is an extremely rare type of plasma cell dysplasia with an aggressive course and poor prognosis. This case is being present because of its extreme rarity.

\section{Bone Marrow and Peripheral Blood Evaluation in Non- Hodgkin's Lymphoma}

\section{Arya $\mathbf{P} \mathbf{M}^{* 1}$}

${ }^{1}$ Department of Pathology, Government medical College, Thrissur, India

Aims \& Objectives: To evaluate the histological patterns of bone marrow involvement in different types of Non-Hodgkin lymphomas. And to evaluate the changes in peripheral blood counts including various types of cytopenias and presence of atypical lymphoid cells in different types of NHL.

Patients/Materials \& Methods: A Hospital based Cross sectional study was done in the Department of Pathology, Government Medical College, Thrissur, Kerala; from November, 2017 to August, 2019. A total of 78 patients with a prior histopathological diagnosis of NHL, belonging to all age groups and both sexes were included. Peripheral blood counts were performed on an automated haematology analyzer. Peripheral blood smears and bone marrow aspirate/imprint smears were stained using the Leishmans stain and a 500-cell differential leucocyte count were performed. Bone marrow trephine biopsies were fixed in Bouins fluid, decalcified using $4 \%$ nitric acid. 4 micron-thick sections were stained with hematoxylin and eosin.

Results: Bone marrow trephine biopsy showed involvement by lymphoma in $65.4 \%$ cases. The incidence of involvement was higher in B-cell lymphomas when compared with T-cell lymphomas, especially in low grade in B-cell lymphomas. The predominant pattern of involvement was interstitial pattern $(41.2 \%)$. Discordant histology between bone marrow and the primary anatomic site was found in $7.8 \%$ of the cases, which was seen more in diffuse large B-cell lymphomas. Adjuvant immunohistochemistry was performed in bone marrow biopsies with scant cellularity or crush artefact to discern the marrow involvement. Majority of the patients with bone marrow infiltration by NHL had anemia $(84.3 \%)$. Bicytopenia and pancytopenia were also observed. Careful and detailed examination of stained peripheral blood smear detected atypical lymphoid cells in $23 \%$ cases.

Discussion \& Conclusion: Bone marrow examination is an important aspect in the diagnosis of NHL, because of its both prognostic and therapeutic implications. Hence the presence of atypical lymphoid cells and other changes in the peripheral blood should be detected in these patients.
Evaluation of Hematological and Coagulation Parameters in Oral Squamous Cell Carcinomas: A Study at a Tertiary Care Centre

Varsha Chauhan $^{* 1}$, Kunal Jain ${ }^{2}$, Charu Agarwal ${ }^{1}$, Mukta Pujani ${ }^{3}$, Kanika Singh ${ }^{4}$, Mitasha Singh ${ }^{5}$, Rk Chandoke ${ }^{1}$

${ }^{1}$ Pathology, ESIC Medical College \& Hospital Faridabad Haryana, ${ }^{2}$ ESIC Medical College \& Faridabad Haryana, ${ }^{3}$ pathology, ESIC Medical College \& Hospital Faridabad, ${ }_{5}^{4}$ pathology, ESIC Medical College \& Hopital Faridabad Haryana, 5 community medicine, ESIC Medical College \& Hospital Faridabad Haryana, Faridabad, India

Aims \& Objectives: To evaluate PC, platelet indices and coagulation profile in patients of oral cancers and compare them with healthy controls. Also, to study the relationship of platelet indices and coagulation parameters with clinicopathological framework in oral cancers.

Patients/Materials \& Methods: It was a hospital based, cross sectional and analytical study conducted for a period of 3 months. 30 patients of oral cancers were included with similar number of age and gender matched controls. Venous blood samples were collected under aseptic conditions in the potassium EDTA and citrate vacutainers for estimation of hematological indices and coagulation parameters respectively. Samples were tested within $1 \mathrm{~h}$ of collection to minimize variations. Complete blood count with platelet indices estimation was done using 5 part hematology analyzer (Sysmex XN 1000).

Results: Statistically strongly significant differences on comparing health individuals with oral cancer patients were observed in $\mathrm{MCH}$, RBC, WBC, PDW, MPV and APTT. Furthermore RBC count emerged as a parameter of significance between different histological grades of oral cancer.

Discussion \& Conclusion: To summarise, simple and cost effective yet integral methods like platelet, haematological and coagulation parameters should be considered as an essential part of the work up of oral cancer patients in order to arrest delay in diagnosis henceforth propelling timely diagnosis, commencement of treatment and improving the overall prognosis of oral cancer patients.

\section{Use of Monoclonal Antibodies for Hematologic Conditions During Covid-19}

Anil Aribandi $^{* 1}$, Krishnamani KV ${ }^{1}$, Ranjit K. $\mathrm{CS}^{1}$, Ashok K. ${ }^{1}$, Arun K. Lingutla ${ }^{1}$, Rajkishore $\mathrm{P}^{1}$, Pravallika MRPL $^{1}$

${ }^{1}$ Department of Haemato-oncology, American Oncology Institute, Hyderabad, India

Aims \& Objectives: SARS- COV2, declared a pandemic by the World Health Organization (WHO), has affected 7.6 million people in India with close to 115000 deaths. COVID-19 has imposed restrictions on travel due to lockdown and affected treatments of patients, including those with hematologic malignancies. To study the pattern of use of monoclonal antibodies, delays in treatment, reasons for delay and treatment related complications during COVID-19.

Patients/Materials \& Methods: All patients with hematologic malignancies undergoing treatment with monoclonal antibodies in the Department of Haematology and Medical Oncology at the American Oncology Institute, Hyderabad from April 1 to October 152020 were included for analysis.

Results: A total of 36 patients, took cumulative 119 cycles of treatment during this period. Median age was 57.5 years and the number of males and females were 21 and 15 respectively. Diffuse large B cell Lymphoma (DLBCL), followed by Follicular Lymphoma (FL) 
and Chronic Lymphocytic Leukaemia (CLL), was seen in 10, 5 and 5 patients respectively. Rituximab and Daratumumab were used in 33 and 3 patients respectively. Three patients during this period turned out COVID RT-PCR positive and two were symptomatic with fever and one patient had COVID pneumonitis. All three patients recovered with no deaths during this period. Treatment delay was seen in 4 patients. One patient was delayed due to lockdown, while the three COVID positive patients had to postpone their treatment. There were no treatment related complications during this period. Post COVID recovery monoclonal antibodies were used in all the 3 patients for a total of 7 cycles. None of the patients had any complications.

Discussion \& Conclusion: Monoclonal antibodies in hematologic malignancies are safe during COVID-19 with no treatment related adverse events. Most patients adhered to treatment, and administering them in patients who recovered from COVID-19 did not pose any significant challenges.

\section{The Effect and Correlation of Smoking with Platelet Indices, Neutrophil Lymphocyte Ratio and Platelet Lymphocyte Ratio}

Kanika Singh $^{* 1}$, Mukta Pujani ${ }^{2}$, Varsha chauhan ${ }^{1}$, Samarth Rastogi ${ }^{1}$, Charu Agarwal ${ }^{1}$, Kamal Gera ${ }^{3}$

${ }^{1}$ ESIC Medical College and Hospital, Faridabad, Faridabad, India, ${ }^{2}$ Pathology, ESIC Medical College and Hospital, Faridabad, ${ }^{3}$ Respiratory Medicine, QRG Health City, Faridabad, India

Aims \& Objectives: To study the platelet indices, neutrophil lymphocyte ratio(NLR) and platelet lymphocyte ratio (PLR) in smokers and their correlation with smoking pack years.

Patients/Materials \& Methods: 110 smokers and 110 non-smokers were included. The smokers were grouped into three groups: mild $(<5$ pack years), moderate (5-10 pack years) and heavy ( $>10$ pack years). The platelet count, plateletcrit (PCT), mean platelet volume(MPV) and platelet distribution width(PDW) were noted. NLR and PLR were calculated and statistical analysis done using student's $\mathrm{t}$ test,ANOVA and Spearman's correlation coefficient.

Results: The platelet count, PCT and PDW were significantly higher with mean values $218.56 \pm 121.31$ vs $203.23 \pm 80.35$ ( $p$ value $0.038), 0.27 \pm 0.10$ vs $0.26 \pm 0.10(p$ value 0.041$)$ and $12.54 \pm 1.45$ vs $11.99 \pm 1.70(p$ value 0.001$)$ in smokers and non smokers respectively.. PLR differed significantly with mean values $119.40 \pm 84.81$ in smokers and $181.99 \pm 313.09$ in non-smokers and $p$ value 0.045 . A significant positive correlation was found between pack years of smoking and platelet count and PLR with pearson correlation coefficient as 0.250 and 0.198 and $p$ values 0.008 and 0.037 respectively. The Platelet Count, PCT, MPV and PDW varied significantly between mild, moderate and heavy smoker groups with $p$ values $0.045,0.010,0.015$ and 0.017 respectively.

Discussion \& Conclusion: The platelet indices and Inflammatory markers NLR and PLR are derived from routine blood investigations which are easily available and inexpensive. The monitoring of platelet indices along with PLR can be used as early predictors of morbidity in smokers.
Utility of IL6 for Prediction to Intervene with Tocilizumab in Severe SARS Cov 2 Pneumonia and Outcome of Tocilizumab Therapy

Anu Singh ${ }^{* 1}$, Dipsha Kriplani ${ }^{1}$, Janardan Nimbolkar ${ }^{2}$, Muzil Munshi $^{3}$, Vistasp Antia ${ }^{1}$

${ }^{1}$ Clinical Hematology, ${ }^{2}$ Medicine, Breachcandy Hospital, ${ }^{3}$ CVTS, Asian Heart Institute/BKC COVID Centre, Mumbai, India

Aims \& Objectives: In this study we analysed the utility of serial measurements of IL6 levels in patients with SARS-CoV-2 to intervene with Tocilizumab in SARS CoV2 severe ARDS, and to assess the role of tocilizumab and predictability of Interleukin 6 levels for use of Tocilizumab by which invasive Mechanical ventilation can be avoided in patients with severe ARDS.

Patients/Materials \& Methods: This retrospective observational study includes 39 adult patients with SARS CoV2 severe ARDS who were admitted to tertiary care centre, Breach Candy Hospital Mumbai between 17th of May and 8th of August 2020. All 39 patients were treated with the standard of care out of which 26 patients who were given Tocilizumab constituted study group and 13 patients who didn't receive Tocilizumab constituted control group. For study group serial levels of IL6 were done before giving tocilizumab and after it. Tocilizumab was given intravenously at $8 \mathrm{mg} / \mathrm{kg}$ bodyweight (up to a maximum of $800 \mathrm{mg}$ ) in two infusions, $12 \mathrm{~h}$ apart, The primary endpoint was a composite of invasive mechanical ventilation or death. Results: The mean age of the patients in tocilizumab group was $64.12 \pm 10.75$ whereas in control it was $68.15 \pm 15.67 .5$ deaths were observed in tocilizumab group versus 5 deaths in control group $(p=0.19)$. In study group, out of 12 patients who required ventilation support, 9 survived versus 1 in control group $(p=0.01)$ and was statistically significant. In tocilizumab group all the patient who were on BPAP or HFNC recovered well. No difference was found in IL-6 levels pre-treatment versus post-treatment $(p=2.17)$ in study group. Discussion \& Conclusion: Our study concluded that though IL6 is a good marker of cytokine storm but its predictivity to determine tocilizumab usage was not found significant. Tocilizumab when given early in severe acute respiratory distress patients improves the outcome when compared with control group irrespective of IL6 levels.

A Case of Adult Hemophagocytic Lymphohistiocytosis: A Diagnostic Conundrum

Ranjan Yadav ${ }^{* 1}$, Maneesh Sulya ${ }^{2}$, Rajendra K. Nigam ${ }^{3}$, Reeni Malik $^{4}$

${ }^{1}$ 2nd Year P.G. Resident Pathology, ${ }^{2}$ Associate Professor Department of Pathology, ${ }^{3}$ Professor Department of Pathology, ${ }^{4}$ Professor and Head Department of pathology, Gandhi Medical College, Bhopal, India

Aims \& Objectives: To find the cause of pancytopenia in a 65 year male.

Patients/Materials \& Methods: Patient came to haematology laboratory with complain of generalized weakness, fatigue, loss of appetite, breathlessness, abdominal pain since 4 months with history of preceding fever and cough few. A routine workup was advised whereby complete blood picture showed pancytopenia. Serum protein, serum albumin and renal function test were normal. Liver function test revealed high serum triglyceride and VLDL cholesterol. Serum ferritin was markedly raised. Ultrasonography of abdomen revealed hepatomegaly of size $16.5 \mathrm{~cm}$ and splenomegaly of size $17 \mathrm{~cm}$. On peripheral examination, an impression of pancytopenia was made. Thereby bone marrow aspiration done for further evaluation. 
Results: On bone marrow aspiration, a diluted particulated marrow was aspirated. On microscopy showed increased number of histiocytes with features of haemophagocytosis with increased number of megakaryocytes.

Discussion \& Conclusion: According to patients clinical details and laboratory findings along with bone marrow aspiration findings an impression of Hemophagocytic Lymphohistiocytosis was made. Hemophagocytic Lymphohistiocytosis (HLH) is a rare life threatening hyperinflammatory disease characterized by uncontrolled proliferation of histiocytes and $\mathrm{T}$ cells in the bone marrow, liver and spleen. These cells secrete inflammatory cytokines. Histiocytes demonstrate phagocytosis of hemopoietic cells, leading to multiorgan failure and, often, death. Although an early diagnosis is crucial to decrease mortality, the definitive diagnosis is often challenging because of the lack of specificity of currently accepted diagnostic criteria and the absence of confirmatory gold standards. Because of the wide range of laboratory assays involved in the diagnosis of HLH. Therefore, proper and timely evaluation is a must for appropriate diagnosis and management.

\section{Rare Case of Histiocytic Disorder Presenting as Neck Swelling in Young Girl: A Case Report}

Trisha Mandal' ${ }^{* 1}$, Saumyaranjan Mallick ${ }^{1}$, Surabhi Jain ${ }^{1}$, Prashant Ramteke $^{1}$, Sameer Bakhshi ${ }^{2}$, Deepam Pushpam ${ }^{2}$, Mehar Sharma ${ }^{1}$

${ }^{1}$ Pathology, ${ }^{2}$ Medical Oncology, All India Institute of Medical Sciences, New Delhi, India

Aims \& Objectives: Here we describe a rare case of histiocytic sarcoma arising in background of Rosai-Dorfman disease.

Patients/Materials \& Methods: A 10-year old girl presented with firm, $10 \times 10 \mathrm{~cm}$ cervical swelling for 9 months with history of intermittent low grade fever. Haematological, biochemical, radiological tests had been done. She had received anti-tubercular therapy for 6 months. Incisional biopsy from neck mass and trephine bone marrow biopsy were performed.

Results: Hemogram showed mild anaemia. Biochemical parameters were within normal limits. CT scan revealed multiple lymph nodes in neck and nodules in bilateral lungs. Sections from lymph node biopsy showed complete effacement of nodal architecture by atypical histiocytes with moderate cytoplasm and marked nuclear atypia. Few histiocytes showed emperipolesis with admixture of eosinophils and plasma cells in the background. Atypical mitosis was seen. The atypical histiocytes were immunopositive for CD68, CD163, S100 (focal) while negative for langerin, CD1a, CD15, CD30, EBV-LMP1, ALK, CD138, CD3 and CD20. The MIB1 proliferative index was $30 \%$. Features are those of a non Langerhans histiocytosis. In view of marked atypia and aggressive course of disease, a possibility of histiocytic sarcoma was considered in a background of Rosai-Dorfman disease.

Discussion \& Conclusion: Histiocytic disorders are mostly present as benign nodal and extranodal mass. Malignant histiocytosis is rare and mostly de-novo in origin. Secondary malignant transformation can also occur. Histiocytic sarcoma is a very rare histiocytic neoplasm and transformation of Rosai-Dorfman disease to a histiocytic sarcoma is an unusual finding. It is an aggressive neoplasm with poor response to therapy. Most patients present at advanced stage of disease. Whereas Rosai-Dorfman disease is considered a benign, non-clonal disease. Detailed clinical, radiological and immunohistochemical evaluation is required for proper diagnosis.
Dress Syndrome: A Rare Cause of Hypereosinophillia

Tanvi Arora ${ }^{* 1}$, Sruthi Pallekonda ${ }^{1}$, Shailaja Shukla ${ }^{1}$

${ }^{1}$ Pathology, Lady Hardinge Medical College, Delhi, India

Aims \& Objectives: Introduction. Hypereosinophillia (HE) requires a detailed clinical history, immunological, dermatological and infectious disease evaluation. Reactive causes such as drug history, helminthic infections, travel history, allergies and other health conditions such as Connective tissue disorder are often considered before a malignant disorder. Drug reaction with eosinophilia and systemic symptoms (DRESS) is a rare, potentially life-threatening, hypersensitivity reaction that includes skin eruption, eosinophilia \pm atypical lymphocytosis, lymphadenopathy, and internal organ involvement (liver, kidney, lung). DRESS is characterized by a latency of 2 to 8 weeks between drug exposure and disease onset and frequent relapses despite the discontinuation of the culprit drug.

Patients/Materials \& Methods: Case report. A 60-year-old lady presented medicine OPD with history of fever for one month. She had a single episode of seizure for which she was given phenytoin one and half a month back. She had been complaining of difficulty in breathing for 15 days. For past 6 days she noticed presence of rash over both her legs. General physical examination revealed pallor, cervical $(2 X 0.7 \mathrm{~cm})$ and axillary lymphadenopathy $(1 \mathrm{X} 1 \mathrm{~cm})$ and pedal edema. Respiratory examination, there were crepts on right side of chest. CECT chest was suggestive of pulmonary embolism and bilateral pleural effusion. Abdominal ultrasound revealed moderate ascites, hepatomegaly and mesenteric lymphadenopathy.

Results: $\mathrm{CBC}$ and peripheral smear examination revealed moderate anemia, severe eosinophilia $(\mathrm{AEC}=8110 / \mathrm{mm} 3)$ with normal platelet counts. No atypical cell or hemoparasite was seen. Bone marrow aspiration and biopsy smears were normocellular for age showing normoblastic erythroid series, normal maturation in myeloid series with increased eosinophillic precursors and adequate megakaryocytes. There were increased plasma cells and histiocytes and illformed granulomas.

Discussion \& Conclusion: Diagnosis. The present case was diagnosed as DRESS syndrome as per the RegiSCAR criteria (patient requiring hospitalization for a suspected drug related reaction presenting with rash and having 3/4 findings of fever, lymphadenopathy (2 sites), 1 internal organ involvement and eosinophilia/lymphocytosis or lymphopenia or thrombocytopenia). The patient succumbed to disease within 2 days of hospitalization. Conclusion. Detailed multisystemic work up is mandatory for all cases of HE. Possibility of DRESS syndrome should be considered in cases presenting with extensive rash to timely institute topical and systemic steroids that can be life saving.

\section{Comparative Analysis of Haematological}

and Endothelial Dysfunction In Hbs $\beta++\mathrm{T}$ and Sickle Cell Disease (SCD) Variants of Tertiary Care Hospital of Odisha

Sonali Dutta*1, Biswakanth $\mathrm{Kar}^{2}$, Prafulla K. Mohanty ${ }^{1}$, Rajeeb K. Nayak $^{3}$

${ }^{1}$ P.G Department of Zoology, Utkal University, ${ }^{2}$ Department of Pharmacoloy, SOA University, ${ }^{3}$ Department of Clinical hematology, SCB Medical College and Hospital, CUTTACK, India

Aims \& Objectives: In sickle cell disease and $\mathrm{HbS} \beta++\mathrm{T}$, hematological variation pose a great threat to the well-being of endothelial cells, thus causing endothelial dysfunction. This may lead to various clinical complications including vaso-occlusive crisis (VOC), splenic 
sequestration, osteomyelitis, acute chest syndrome (ACS);etc. This study was done to analyse the correlation between the hematological parameters and endothelial dysfunction in children with an age range of $10.07 \pm 0.17$ in boys and $11.7 \pm 0.24$ in girls.

Patients/Materials \& Methods: The patients attending the out-patient department (OPD) of Hematology and Paediatrics, Sri Ram Chandra Bhanja Medical College and Hospital (SCBMCH), Cuttack, India were screened. To select the number of standard population or sample size, the statistical formula proposed by Yamane was used. Cohort method of analysis was used where clinical reports and questionnaire were used to analyse the symptomatic parameter in the patients. : Children with $\mathrm{Hb}-\mathrm{S}-\beta$ - thalassemia who were within the inclusion criteria and attending the OPD were first educated about the procedure and then the same procedure was also explained to their parents. The consent of the parents was taken on the consent form as maximum patients selected were minor in age. For comparative analysis, one way ANOVA with $p>0.05$ level of significance was considered.

Results: When hematological parameters were analysed it was observed that there was no significant variation in $\mathrm{HbA} \%$ in $\mathrm{HbS} \beta++\mathrm{T}$ variants whereas in SCD patients a decline was observed in both the sexes. $\mathrm{HbA} 2 \%$ and $\mathrm{HbF} \%$ was slightly increased inn both the variants whereas in SCD patients, there was an increase in $\mathrm{HbS} \%$ with significant variation. HCT \% showed gradual decrease in both the variants with modif decrease in the $\mathrm{Hb}$ (gmdl-1). A significant decrease was also observed in $\mathrm{MCH}$ (pg) and MCV (fL) with signifies the presence of abnormal erythropoietic cells. A significant decrease in the flow mediated vasodilation (FMD) was observed in SCD whereas a moderate decrease was observed in $\mathrm{HbS} \beta++\mathrm{T}$ variants, thus, indicating endothelial dysfunction.

Discussion \& Conclusion: In the present study, correlation was observed in the decrease in the FMD values and clinical manifestations among the patients which indicates impairment in erythropoiesis.

Keywords: Endothelial dysfunction, Flow mediated vasodilation \& Vaso-occlusive crisis.

\section{Is Haemophagocytic Lymmphohistiocytosis a Rare Disease? A Study from Western Rajasthan}

Ismetara Begam ${ }^{* 1}$, Abhishek Purohit ${ }^{1}$, Poonam Elhence ${ }^{1}$, Gopal K. Bohra $^{2}$, Deepak Kumar ${ }^{3}$

${ }^{1}$ Pathology, AIl India Institute of Medical Sciences, Jodhpur, ${ }^{2}$ Medicine, AIIMS, Jodhpur, ${ }^{3}$ Medicine, AIl India Institute of Medical Sciences, Jodhpur, Jodhpur, India

Aims \& Objectives: Haemophagocytic lymphohistiocytosis designates a syndrome that may be familial or sporadic, with or without an associated viral infection, is presented as the prototype of a hemophagocytic syndrome, a condition in which there is uncontrolled activation of the cellular immune system. The purpose of this study was to correlate the findings of bone marrow haemophagocytosis and analyze the presenting features in patients with suspected hemophagocytic lymphohistiocytosis (HLH) and other causes of hemophagocytosis in bone marrow at a tertiary care hospital during the course of January 2017-September 2020.

Patients/Materials \& Methods: The haematology database of All India Institute of AIIMS Jodhpur, Rajasthan, India, was searched for the terms "haemophagocytosis" and "histiocytes" encompassing the period January 2017-September 2020. Bone marrow aspirate and biopsy specimens, along with ancillary laboratory studies, if available and presenting clinical features, were reviewed for each case.

Results: In this retrospective observational study, we included 33 cases. Of the $33 \mathrm{HLH}$ was suspected in 05 (15\%). Bone marrow haemophagocytosis was seen in a higher proportion of patients (75\%) who were not clinically suspected as having HLH also showed evidence of bone marrow HPC. The majority of the patients presented with pancytopenia and history of infectious etiology. In addition, bone marrow examination revealed dyserythropoiesis and increased plasma cells in majority of cases.

Discussion \& Conclusion: The study concludes that haemophagocytosis even if observed in single cell should always be documented in the bone marrow reports. It may be the only indicator of subtle infection in the marrow. Cytopenias and evidence of haemophagocytosis on bone marrow examination may be helpful in establishing an early differentiation of HLH and non HLH cases.

\section{Hypereosinophilia a Disease of Wavering Phenotype}

Akash Khandelwal $^{* 1}$, Rishi Dhawan ${ }^{2}$, Manoranjan Mahapatra ${ }^{1}$, Tulika Seth ${ }^{1}$, Pradeep Kumar ${ }^{1}$, Mukul Aggarwal ${ }^{1}$, Jasmita Das ${ }^{3}$, Ganesh Kumar ${ }^{1}$

${ }^{1}$ Haematology, AIIMS New Delhi, Delhi, ${ }^{2}$ Haematology, AIIMS New Delhi, Delhi, ${ }^{3}$ Haematology, AIIMS New Delhi, Delhi, India

Aims \& Objectives: Hypereosinophila is a common entity faced many clinician, chest physician and haematologist it has diverse causes, interest of haematologist are cases of lymphoid or myeloid neoplasms or $\mathrm{cml}$ or lymphoma presenting as hypereosinophilia. Some patients in which no specific cause is present are diagnosed as idiopathic hypereosinophilia or if present with end organ damage as hypereosinophilic syndrome.

Patients/Materials \& Methods: A 42 year female came to emergency with hepatosplenomegaly,Right sided pleural effusion, pedal oedema also complaining altered bowel habits and fever from 2 months, and rashes on bilateral lower limb on evaluation ECG was normal, trop $t$ was negative, raised tlc of $1,34,000$ with $90 \%$ dysplastic eosinophils present. No secondary cause of eosinophilia present microfilaria antigen negative, no other parasitic infestation, no history of asthama. Echo showed right ventricle cardiomyopathy in view of end organ damage and hypereosinophilia patient was started on methylprednisolone $1 \mathrm{mg} / \mathrm{kg}$ for 3 days followed by oral steroids even after 7 days of treatment patient persisted to have tlc of 90,000 with $90 \%$ eosinophils, patient was started on hydroxyurea $1 \mathrm{gm}$ TDS, her bone marrow was normal without any blasts though hypercellular her panel for PDGRF alpha and beta, bcrABL, jak2 was negative in view of her persisting eosinophilia she was started on imatinib $400 \mathrm{mg}$ in day 14 patient started having reduction in count her steroid and hydroxyurea was tapered off, her rash, abdominal symptoms and cardiac symptoms resolved, she became asymptomatic, hence patient was discharged on imatinib, her total lymphocyte count after $1 \frac{1}{2}$ month of therapy was 13,000 , haemoglobin and platelet count normal. Results: We would like to highlight that imatinib should be tried in such patients of hypereosinophilc syndrome which are refractory to steroid or cytoreductive agents even if they are pdgfr alpha negative. Discussion \& Conclusion: However, one should follow such patients as disease might evolve into any leukaemia or lymphoma over time. 
Rapidly Progressive Ascites, Generalized Lymphadenopathy and Osteosclerosis: A Rare Unifying Diagnosis

Va Arun ${ }^{* 1}$, Arihant Jain ${ }^{1}$, Pankaj Malhotra ${ }^{1}$

${ }^{1}$ Hematology, PGIMER, Chandigarh, India

Aims \& Objectives: NA.

Patients/Materials \& Methods: NA.

Results: Rapidly progressive ascites is a frequent clinical manifestation of advanced abdominal malignancies or portal hypertension due to liver diseases. We present a case of 61 year old male who presented with rapidly progressive ascites. The presence of ascites, generalized lymphadenopathy, osteosclerosis on imaging and hepatosplenomegaly initially pointed towards the diagnosis of advanced high grade lymphoma or accelerated myeloid neoplasm. Lymph node biopsy revealed infiltration by CD 45; c KIT; CD30; tryptase and toluidine blue positive mast cells. Bone marrow examination revealed infiltration by mast cells and next generation sequencing revealed the pathognomic exon $17 \mathrm{D} 816 \mathrm{~V}$ KIT mutation. The patient was started on weekly pegylated interferon and steroids with significant resolution of ascites, splenomegaly and lymphadenopathy over next 6 months.

Discussion \& Conclusion: Systemic mastocytosis(SM), should be considered as a differential diagnosis in a clinical case of ascites of unknown etiology even in the absence of typical skin manifestations especially if lymphadenopathy is prominent or otosclerosis is incidentally detected.

\section{Spectrum of Haematological Disorders in Geriatric Patients: Clinicohematological Audit at a Tertiary Care Centre}

Prerna Chadha*1, Sunayana Misra ${ }^{1}$, Taruna Bansal ${ }^{2}$, Sadhna Marwah $^{2}$, Vijay Kumar ${ }^{2}$

${ }^{1}$ Pathology, ${ }^{2}$ Hematology and Clinical Pathology Unit, Department of Pathology, ABVIMS and Dr RML Hospital, Delhi, India

Aims \& Objectives: Bone marrow examination occupies a vantage position in the diagnosis of several haematological and non haematological disorders. With an increasing number of geriatric patients, an increased incidence of both malignant and non malignant aetiologies is being observed. Scarce literature is available pertaining to the geriatric population exclusively. This study is aimed at evaluating the clinico-hematological parameters and bone marrow findings in the geriatric age group ( $\geq 60$ years) for early diagnosis and to facilitate better patient management.

Patients/Materials \& Methods: This study includes all consecutive cases of geriatric age group who presented between Nov 2017 and Nov 2019. Patients $<60$ years of age and bone marrows which were non diagnostic were excluded from the study. A detailed relevant clinical history and physical examination were noted. The peripheral blood smear and bone marrow aspiration smears along with biopsy sections (wherever available) were evaluated in these cases. Giemsa staining was performed for aspiration and biopsy imprint smears while H\&E stain was used for biopsy sections. The data was collected and evaluated by two pathologists and it was cross-checked by the principle investigator. Appropriate statistical analysis was performed. Results: A total of 898 bone marrow examinations were performed over the study period, of which geriatric patients comprised 127 cases. Most of the patients presented with multiple symptoms out of which generalised weakness was the most common complaint.The most common indication for performing an aspirate was a suspicion of plasma cell dyscrasia/multiple myeloma (38.6\%) and the pathological diagnosis rendered most often on marrow in this study was a cellular reactive marrow (25.2\%).Some cases showed a discordance between the marrow aspirate and biopsy diagnoses (8.7\%), of which granulomas and marrow fibrosis were the ones made exclusively on biopsies.

Discussion \& Conclusion: : A recent rise in the elderly population has made it imperative for more research in this area. Both bone marrow aspirate and biopsy are imperative for a high diagnostic yield.A detailed analysis of clinico-hematological profile along with bone marrow findings can provide valuable clues even in cases with a diagnostic dilemma and help in elucidating the etiological diagnosis which in turn will facilitate better patient management.

\section{Role of Platelet to Lymphocyte Ratio in The Prediction of Early Onset Neonatal Sepsis}

\section{Satish Arakeri ${ }^{* 1}$}

${ }^{1}$ Pathology, BLDE(Deemed to be university) Shri B M Patil Medical COllege Research Hospital, Vijayapur, India

Aims \& Objectives: To study the relevance of platelet lymphocyte ratio in the diagnosis of neonatal sepsis.

Patients/Materials \& Methods: Total 54 patients are involved in the study. All cases are diagnosed as early onset sepsis. Exclusion criteria: small for gestational age (SGA), intrauterine growth restriction (IUGR), perinatal asphyxia, congenital abnormality, congenital heart disease, chromosomal abnormality, preeclampsia. Investigations like WBC count, Absolute lymphocyte count, platelet count, Absolute Neutrophil count, $\mathrm{C}$ reactive protein, Platelet distribution width, blood culture has been done for all 54 patients.

Results: All cases except two, shows raised CRP values which are considered as gold standard for comparison. In such cases, platelet lymphocyte ratio $(>25)$ is seen in $>95 \%$ of cases where CRP is raised.

Discussion \& Conclusion: Platelet Lymphocyte ratio with cut off $>25$ can act as predictor marker for the neonatal sepsis.

\section{Leukoerythroblastosis in Covid 19 Infection: Incidence and Clinical Significance}

Shareh Naqvi*1 ${ }^{* 1}$ Namrata P. Awasthi ${ }^{2}$, Pravin K. Das ${ }^{3}$, Nuzhat Husain $^{2}$

${ }^{1}$ Emergency Laboratory, RPGN Covid Hospital, Dr. Ram Manohar Lohia Institue of Medical Sciences, ${ }^{2}$ Pathology,

${ }^{3}$ Anaesthesiology, Dr. Ram Manohar Lohia Institute of Medical Sciences, Lucknow, India

Aims \& Objectives: Peripheral blood abnormalities in COVID 19 infection have not been fully explained. We report 33 cases of COVID-19 infection with Leukoerythroblastic picture on peripheral blood smear examination. We intend to describe this unfamiliar finding in this novel viral infection and correlate it with the clinical outcome.

Patients/Materials \& Methods: Investigation records of COVID 19 cases admitted from 20th August 2020 to 30th September 2020 at COVID hospital, Dr. RMLIMS, LUCKNOW, were reviewed for hematologic tests performed. Peripheral blood smear examination was done on test results flagged for abnormalities on automated cell counter. A leukoerythroblastic blood picture was reported when the smears showed presence of granulocyte left shift as well as nucleated red blood cells. This finding was correlated with the clinical outcome of the patient. 
Results: Out of 280 slides reviewed, 33 (11.78\%) showed a leukoerythroblastic picture. Among the 33 cases, $72.72 \%$ of cases $(24 / 33)$ showed presence of metamyelocyte/myelocyte, $15.15 \%$ of cases $(5 /$ 33) showed presence of promyelocytes, and $12.12 \%$ (4/33) showed presence of blasts. NRBC varied from $0.5 \%$ to $10 \%$ in these cases. Most patients had comorbidities at presentation, which included hypertension (8/33), type 2 Diabetes Mellitus (7/33), hypothyroidism (3/33), pregnancy (3/33), chronic kidney disease (2/33). 13 patients $(39.4 \%)$ required mechanical ventilation. There were $17(51.5 \%)$ deaths. 11 patients $(33.33 \%)$ recovered and were discharged. 5 patients $(15.15 \%)$ were shifted to other hospitals whose follow up was not available.

Discussion \& Conclusion: Leukoerythroblastosis is an uncommon and unfamiliar finding in patients with COVID 19 infection. This Peripheral blood abnormality can occasionally provide insight into the underlying pathophysiologic processes. Furthermore, it seems to be an adverse prognostic factor and may help clinicians and intensivists to make prompt management decisions.

\section{Hematologic Biomarkers in Covid-19: Correlation with Disease Severity and Mortality}

Mukta Pujani $^{* 1}$, Sujata Raychaudhuri ${ }^{2}$, Manjula Jain ${ }^{2}$, R K Chandoke $^{3}$, Shivani Agarwal ${ }^{4}$, Kanika Singh ${ }^{3}$, Dipti Sidam ${ }^{2}$, Varsha Chauhan $^{2}$

${ }^{1}$ Pathology, ESIC Medical College Faridabad, ${ }^{2}$ Pathology, ESIC Medical College, Faridabad, ${ }^{3}$ Pathology, ESIC Medical College, Faridabad, ${ }^{4}$ Physiology, ESIC Medical College, Faridabad, Faridabad, India

Aims \& Objectives: The aims and objectives of present study were to evaluate the various Hematological parameters including Neutrophillymphocyte ratio (NLR), Platelet-lymphocyte ratio (PLR) in COVID19 patients and controls and to study correlation of these parameters with each other and with disease severity.

Patients/Materials \& Methods: A prospective cross sectional study was conducted on 506 Covid-19 positive patients and 50 controls over a period of two months (June to July 2020). The cases were subclassified based on disease severity into mild to moderate $(n=337)$, severe $(\mathrm{n}=118)$ and very severe $(\mathrm{n}=51)$ and into survivors $(n=473)$ and non survivors $(n=33)$ based on survivor status.

Results: There were statistically significant differences in WBC count, Absolute neutrophil count (ANC), Absolute lymphocyte count (ALC), absolute monocyte count (AMC), NLR, PLR, RDW SD and RDW CV between covid cases vs controls; among the clinical subgroups and among the survivors and non survivors. There was a significant strong positive correlation between various parameters, that is, NLR and MLR (r: 0.852, $p=0$ ), MPV and PDW (r: 0.912, $p=0$ ), MPV and PLCR (r:0.956, $p=0$ ), PDW and PLCR (r:0.893, $p=0$ ). NLR (AUC: $0.676, p=0$ ) was the best single parameter and NLR + RDW-CV was best combination parameter as per area under curve $(0.871)$ of ROC to distinguish severe from mild to moderate disease.

Discussion \& Conclusion: Leucocytosis, neutrophilia, lymphopenia and monocytosis were characteristic while NLR and NLR + RDW$\mathrm{CV}$ emerged as the most effective single and combination $\mathrm{CBC}$ parameters in distinguishing mild to moderate and severe cases respectively.
Utility of Routine Haematological Parameters and Infectious Biomarkers to Assess the Disease Severity in Covid-19 Positive Patients, Analysis and Early Trend from India

Shashank Singh ${ }^{* 1}$, Pomilla Singh ${ }^{1}$, Anjali Kelkar ${ }^{1}$

${ }^{1}$ Pathology, Bharati Vidyapeeth (Deemed to be University) Medical college Hospital and Research Centre, Pune, India

Aims \& Objectives: To assess utility of haematological parameters and infection biomarkers in identifying COVID19 disease severity. Patients/Materials \& Methods: The test results for complete blood counts, Coagulation screen and inflammatory markers of 100 covid 19 positive patients were recorded and analysed based on their admission to Intensive care units,High Dependency Units or Ward. Those parameters with significant differences were assessed by receiver operating curve and the best screening cut-off was selected.

Results: 100 adults were analysed, area under the curve for total leukocyte count (TLC), Absolute neutrophil count (ANC), Neutrophil-to-Lymphocyte ratio (NLR) was found $>0.800$, with $p<0.05$. The best cut-off value obtained for TLC was $8100 / \mathrm{cmm}$, with sensitivity and specificity of $91 \%$ and $62.5 \%$; ANC $-6739 / \mathrm{cmm}$, with $91.7 \%$ and $75 \%$ respectively; NLR -6.9 with sensitivity and specificity $91.7 \%$ and $87.5 \%$ respectively. On comparing data between ward and ICU admissions, those needing ICU admissions had significantly lower $\mathrm{Hb}$ and ALC, while TLC, ANC, NLR, PLR were significantly higher $(p<0.05)$. The hematological parameters have been compared in the table. Lymphocytopenia was a prominent and the most consistent feature in all affected patients, although ICU patients suffered from greater lymphocytopenia thus significantly associated with severity. We observed no thrombocytopenia in either ICU/HDU admissions or ward patients in our study.

Discussion \& Conclusion: Study suggests that in adults, using TLC, ANC and NLR, obtained from the basic preliminary workup may segregate patients requiring intensive care at the time of admission enabling risk stratification and guide intervention. Procalcitonin was also studied as a sepsis marker in these patients, though it showed a higher mean value in ICU patients but did not show statistical significance in these patients.

\section{A Rare Case of Gauchers Disease with Immune Thrombocytopenic Purpura}

Neha Kumari*1, Rajendra K. Nigam ${ }^{2}$, Reeni Malik ${ }^{3}$

12nd Year Post Graduate, Department of Pathology, Gandhi Medical Collge, ${ }^{2}$ Professor, Department of Pathology, Gandhi Medical College, ${ }^{3}$ Professor, Department of Pathology, Gandhi Medical College, Bhopal, India

Aims \& Objectives: To evaluate the cause of pancytopenia in a 4 month old male.

Patients/Materials \& Methods: A 4 month old male admitted to the pediatric ward with complaints of Petechaie all over abdomen and back since 15 days, Swelling in abdomen since 8 days, Loose motions and fever(mild) since 8 days. On routine blood investigations a picture of pancytopenia was seen with Hemoglobin-9.2 g/dl,Total leucocyte count-4300 cells/cmm, Differential Leucocyte count Polymorphs-30\%, Lymphocyte-62\%, Monocyte-04\%,Eosinophils04\%,Basophils-0\%,Platelets-30,000/cmm. On repeated platelet transfusions, it remained below $50,000 / \mathrm{cmm}$. Peripheral smear examination showed normocytic normochromic erythrocytes and 
thromobocytopenia with few giant platelets.On radiological investigations, Ectopic Left Kidney, Hepatomegaly, Spleenomegaly, Mild Ascites were noted.Bone marrow aspiration was done to ascertain the cause of pancytopenia.

Results: Bone marrow aspiration showed hypercellular marrow with Myeloid Erythroid ratio of 1:1, The megakaryocytes were increased in numbers with majority showing the presence of hypolobated nucleus, basophilic cytoplasm with smooth margins and loss of budding,few showing cytophagocytosis. Also reticulum cells were increased, few Gaucher's cells seen. A Beta glucosidase enzyme assay for gaucher's disease was done which showed severe enzyme deficiency $2.2 \mathrm{nmol} / \mathrm{hr} / \mathrm{mg}$. (Normal-5-22 nmol/hr/mg).

Discussion \& Conclusion: A bone marrow report of Gaucher's disease with Immune Thrombocytopenic Purpura was given.

Supporting Document:

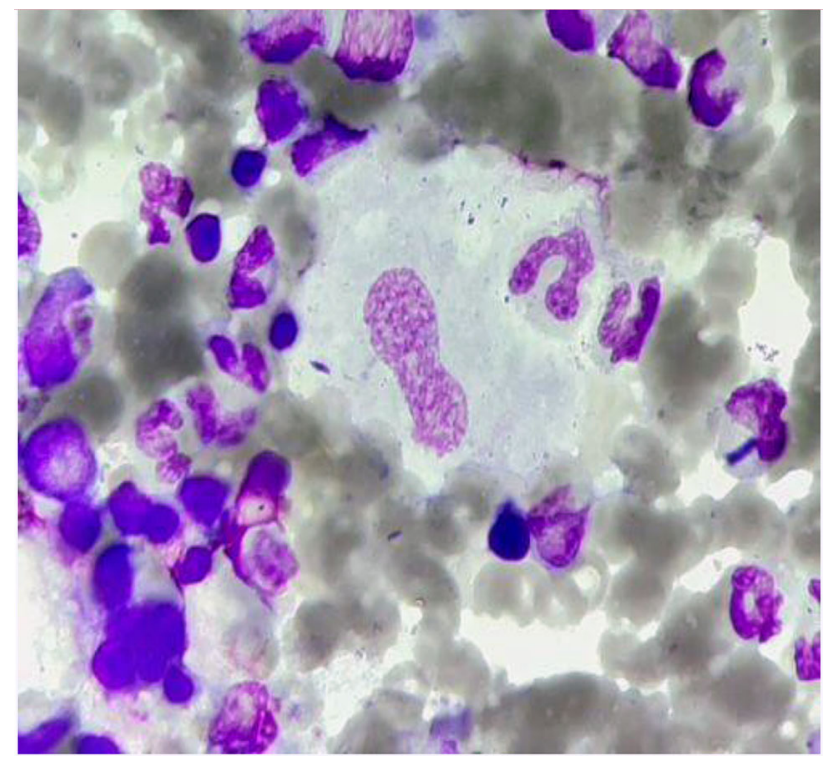

\section{Can Platelet Lymphocyte Ratio (PLR) Predict Severity of Aortic Stenosis?}

Sourabh Kumar ${ }^{* 1}$, Anita Tahlan $^{1}$, Sreenivas Reddy ${ }^{2}$, Anshu Palta ${ }^{1}$

${ }^{1}$ Department of Pathology, ${ }^{2}$ Department of Cardiology, Government Medical College \& Hospital, Sector 32, Chandigarh, India

Aims \& Objectives: 1.To assess the alteration of platelet indices (MPV, PDW \& PCT) in patients with aortic stenosis. 2.To correlate the platelet to lymphocyte ratio with severity of aortic stenosis.

Patients/Materials \& Methods: 40 Patients referred for echocardiography with severe AS (28 men, 12 women), mean age 58.2 years (12 to 93 years), peak transvalvular velocity greater than $4.0 \mathrm{~m} / \mathrm{s}$, or aortic valve replacement were studied. 40 subjects withno valvular heart disease served as controls mean age of 46.1 years (12 to79 years). A routine CBC (including platelet parametersMPV, PCT and PDW) was done along with echocardiography for all the patients. Platelet parameters \& Platelet:lymphocyteratio(PLR) were evaluated.
Results: he PLR ratio in the severe AS group ranged from 200 to 338 with a mean of $152.1 \pm 87.3$, and in the control group rangedfrom 320 to 980 with a mean of $173.7 \pm 164.9$. The difference was not statistically significant $(p$ value $>0.05$ ). The MPV in thesevere AS group ranged from 6.7 to $18.3 \mathrm{fl}$ with a mean of $10.9 \pm 2.4 \mathrm{fl}$, and in the control group ranged from 6.7 to $14.3 \mathrm{fl}$ with amean $9.6 \pm 1.2 \mathrm{fl}$. The difference was not statistically significant ( $p$ value $>0.05)$. The PCT in the severe AS group ranged from $0.1 \% 0.34$ with a mean $0.21 \pm 0.08 \%$, and in the control group ranged from $0.1-0.36 \%$ with a mean $0.26 \pm 0.06 \%$. The difference wasnot statistically significant ( $p$ value $>0.05$ ). PDW in the severe AS group ranged from 10 to $23.8 \%$ with a mean of $15 \pm 5.1 \%$, and inthe control group ranged from 10 to $15.9 \%$ with a mean of $15.9 \pm 2.7 \%$. We did not find any significant correlation with the severity of AS. The difference was not statistically significant ( $p$ value $>0.05)$.

Discussion \& Conclusion: PLR is now being recognized a new marker of prognostic importance that offers information regardingpathways that govern inflammation \& aggregation. An increased PLR has been reported associating it with severity and complexityof coronary artery disease. However in our study we could not find correlation of severity of stenosis to the platelet count and PLT parameters (statistically insignificant $(p$ value $>0.05$ ). Since this information can be gleaned from a routine CBC therefore a largerdatabase is required before negating its relevance.

\section{Identification and Quantification of Blood Cells in Digitised Peripheral Blood Smear Using Machine Learning}

Aniruddha Mundhada*1, Srigayathri S. ${ }^{1}$, Sandhya Sundaram ${ }^{1}$, Puneetha N. ${ }^{2}$, Ramakrishnan S. ${ }^{2}$

\section{${ }^{1}$ SRMC, ${ }^{2}$ IIT Madras, Chennai, India}

Aims \& Objectives: (1) To identify 5 different blood cell typesnamely neutrophil, eosinophil, lymphocyte, monocyte and basophil in a digitised peripheral blood smear from the Pathology department. (2) To train computer algorithm to identify the blood cells using classical computer vision techniques. (3) To train a deep learning model to classify blood cell type and measure its accuracy.

Patients/Materials \& Methods: Blood smear screening using 5 part differential counter is being supplanted with software to screen for blood cells based on morphology due to recent advances in machine learning and computer vision in the field of AI. Images of blood smear were used to segment blood cells using OpenCV software using classical Computer Vision techniques. An online database of over 1000 images of each class of blood cell was used to train a classifier to differentiate between each cell. Also this was used to differentiate amongst the cells from our Pathology department as a test set.

Results: Cells were segmented based on size. Classifier was used to identify the 5 classes of cells with reasonable accuracy.

Discussion \& Conclusion: The screening of blood cells can be supplemented using AI software as a screening tool to aid the pathologist and cut down on the time required for screening. This approach can be used to classify and segment various blood cells and also applied to leukemia cells too. 


\section{Supporting Document:}

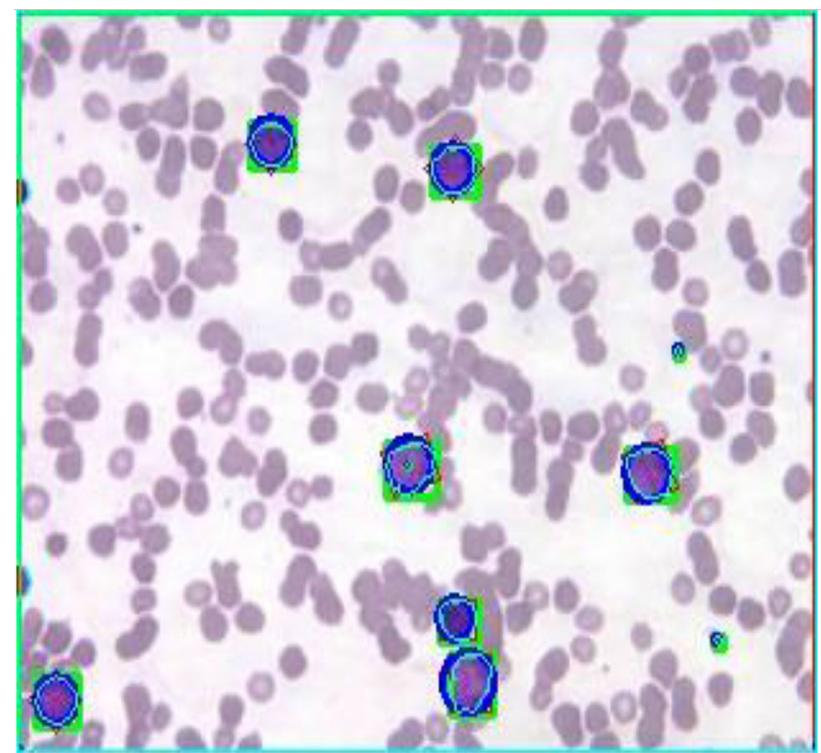

Utility of Flow Cytometry on Body Fluid Analysis

Riddhi Parmar ${ }^{* 1}$, Sindhura Lakshmi Koulmane Laxminarayana ${ }^{2}$

${ }^{1}$ Post Graduate Student in Department of Pathology, ${ }^{2}$ Associate Professor in Department of Pathology, Kasturba medical college, Manipal, Udupi, India

Aims \& Objectives: Multiparametric Flow Cytometry (MFC) is an indispensable tool for detection of abnormal cells, lymphocyte subset enumeration and monitoring therapy. In this study, we document the spectrum of immunophenotypic abnormalities in different types of body fluids where MFC was performed for suspected hematologic and non-hematologic diseases.

Patients/Materials \& Methods: All body fluid specimens submitted for MFC analysis between January 2016 and September 2020 were included in the study. Samples from hematopoietic system were excluded. The samples were processed for different MFC panels (acute leukemia, CLPD, myeloma, lymphocyte subset) depending on clinical suspicion. Demographic, clinical (organomegaly and lymphadenopathy), biochemical (fluid and serum), cytological (total nucleated cell count, differential count, cytomorphology) and MFC parameters were retrieved. The cases were grouped as per the MFC panels used and immunophenotypic abnormalities are documented.

Results: Total 34 samples were reanalysed. Male to female ratio:1.26:1; Median age was 44 years. Summary of results is depicted in table 1 .

Discussion \& Conclusion: MFC is an excellent tool for detection of abnormal cells in fluids even with low cell count. Analysis of CSF for abnormal cells is useful in detecting early relapse. It also aids in determination of $\mathrm{CD} 4 / \mathrm{CD} 8$ ratio in the diagnosis of interstitial lung disease.
Supporting Document:

\begin{tabular}{|l|l|l|l|l|}
\hline & CSF & $\begin{array}{l}\text { Ascitic } \\
\text { fluid }\end{array}$ & Pleural fluid & $\begin{array}{l}\text { Bronchoalveolar } \\
\text { lavage }\end{array}$ \\
\hline Number of cases & 10 & 5 & 9 & 10 \\
\hline Serum LDH in IU/L & $262(263.5)$ & $1000(1086)$ & $457.5(699)$ & NA \\
\hline $\begin{array}{l}\text { Cytomorphology positive } \\
\text { for abnormal cells }\end{array}$ & YES & YES & YES & NA \\
\hline $\begin{array}{l}\text { MFC positive for } \\
\text { abnormal cells }\end{array}$ & YES & YES & YES & NA \\
\hline $\begin{array}{l}\text { Detection rate by MFC in } \\
\%\end{array}$ & $\begin{array}{l}70 \% \\
(\text { Out of 10 } \\
\text { cases:7+, } \\
2 \text { for CD4) }\end{array}$ & 80 & 77.77 & \\
\hline Clinical correlation in \% & 87.5 & 80 & 77.77 & \\
\hline $\begin{array}{l}\text { Total nucleated cell count } \\
\text { / }\end{array}$ & $44(335.67)$ & $630(984)$ & $6080(8522.22)$ & $192(1289.11)$ \\
\hline $\begin{array}{l}\text { Differential count in } \\
\%[\text { median (range)] }\end{array}$ & \multicolumn{5}{|l|}{} \\
\hline Neutrophils & $72(69.25)$ & $85(81.74)$ & $73.4(62.54)$ & $68.5(69.96)$ \\
\hline Lymphocytes & $18.7(20.85)$ & $7(11.9)$ & $14.4(16)$ & $20.2(18.73)$ \\
\hline Monocytes & $9(8.7)$ & $3(5.12)$ & $8.5(10.28)$ & $8.75(8.68)$ \\
\hline Eosinophils & $0.7(1.06)$ & $1(1.12)$ & $0.4(0.733)$ & $0.95(2.21)$ \\
\hline Basophils & $0.3(0.27)$ & $0(0.12)$ & $0.4(0.444)$ & $0.35(0.42)$ \\
\hline $\begin{array}{l}\text { CD4/CD8 ratio (BAL } \\
\text { only) }\end{array}$ & NA & NA & NA & $0.74(2.238)$ \\
\hline MFC panels & \multicolumn{5}{|l|}{} \\
\hline Acute leukemia & 4 & - & 4 & - \\
\hline CLPD & 2 & 4 & 3 & - \\
\hline Myeloma & 2 & 1 & 2 & - \\
\hline Lymphocyte subset & 2 & - & - & 10 \\
\hline
\end{tabular}

\section{Evaluation of the Diagnostic Role of MPV in Thrombocytopenia: A Prospective Observational Study}

Akanksha Jaju ${ }^{* 1}$, Radhika Narayan ${ }^{1}$, Farah Rana $^{1}$, Minakshi Mishra ${ }^{1}$

\section{${ }^{1}$ Tata Main Hospital, Delhi, India}

Aims \& Objectives: Investigate the usefulness of MPV in discriminating between hyper-destructive thrombocytopenia and hypoproductive thrombocytopenia to differentiate different categories of thrombocytopenia. Assess the sensitivity and specificity, positive predictive value, negative predictive value and diagnostic accuracy of MPV to differentiate different categories of thrombocytopenia. Derive cutoff values in an attempt to consider the use of theses indices in the initial evaluation of thrombocytopenia.

Patients/Materials \& Methods: i. It was a prospective, observational Hospital based study, conducted in Department of Pathology, Tata Main Hospital, Jamshedpur from October 2017 to September 2020. It included newly diagnosed patients of thrombocytopenia under evaluation. We took 125 cases and 125 age and sex matched controls. Data analysis was done with excel Microsoft.

Results: - Mean platelet count and MPV for Hyper-destructive category were $23380.95 \pm 20332.54 \mathrm{mcL}, 11.93 \pm 2.77 \mathrm{fL}$ respectively. - Mean platelet count and MPV for Hypo-productive category were $36000 \pm 24326.25 \mathrm{mcL}, 9.29 \pm 2.67 \mathrm{fL}$ respectively.

- We used 5 cut off values to evaluate MPV as a diagnostic marker of hyper-destructive category, out of which $>9.7$ came out as the most accurate cut off with a sensitivity of $80.95 \%$, specificity of $61.45 \%$, Positive predictive value of $51.52 \%$, negative predictive value of $86.44 \%$ and AUC of 0.71 .

- As for Hypo-productive category for evaluating MPV as a diagnostic marker, $<10.95,<11.05$ showed similar results with a sensitivity of $75.64 \%$, specificity of $61.70 \%$, positive predictive value of $76.62 \%$, negative predictive value of $60.42 \%$, AUC of 0.69 , concluding $<10.95$ as the most appropriate cut off.

Discussion \& Conclusion: In the majority of patients, MPV could be helpful for distinguishing between thrombocytopenia due to an 
increase in the destruction of platelet (Hyper-destruction) and thrombocytopenia due to a reduction in platelet production (Hypoproduction). MPV should be combined with other platelet indices for more accurate results and further studies with combining these indices will definitely provide promising results.

\section{Thrombocytopenia in Coronavirus Disease 2019 (Covid-19) and Dengue Fever: Clinical Importance}

\author{
Naveen Bansal $^{* 1}$, Yashik Bansal ${ }^{2}$, Arti Ralta ${ }^{1}$ \\ ${ }^{1}$ Department of Laboratory, ESIC Model Hospital, Baddi, \\ Himachal Pradesh, ${ }^{2}$ Department of Microbiology, VCSG Govt. \\ Institute of Medical Science and Research, Srinagar Garhwal, \\ India
}

Aims \& Objectives: There are several common clinical and laboratory findings in dengue fever and coronavirus disease 2019 (COVID19) patients such as fever, bodyache along with thrombocytopenia on complete blood count.. We came across two case reports which reported false positive dengue serology in COVID-19 patients. In this study we investigated the complete blood count in confirmed cases of COVID-19 and also assessed whether false positive dengue serology is seen in patients of COVID-19.

Patients/Materials \& Methods: We performed complete blood count using automated cell counter (ABX Pentra DF 120; Horiba, Japan) in confirmed cases of COVID-19 (RT-PCR positive for SARS-CoV-2 of nasopharyngeal and throat swabs) admitted in the hospital which is a dedicated COVID Care Hospital in Himachal Pradesh, India. Dengue serology by rapid card test (Diagno Dengue Duo; Diagnocure, India) was performed in the patients in whom thrombocytopenia was seen on complete blood count examination. Results: Complete blood count was performed in 32 confirmed cases of COVID-19. Thrombocytopenia (platelet count $<1.5$ lacks/cumm) was seen in seven $(21.8 \%)$ patients. Dengue serology by rapid card test was performed in these seven patients. Dengue NS1 antigen and IgM antibody was positive in none of the patients. Dengue IgG antibody was positive in one patient which could signify past dengue infection. We found no false positive dengue serology in confirmed cases of COVID-19.

Discussion \& Conclusion: Early identification and isolation are important components in breaking the chain of transmission of COVID-19. Common clinical features and laboratory findings among COVID-19 and dengue infection patients can result in delay in making the correct diagnosis and result in intermixing of COVID-19 and non COVID-19 patients. There is a potential risk of transmission of COVID-19 to hospitalised patients and also to the health care workers. Thrombocytopenia is observed in both dengue and COVID19. However, false positive serology was not observed in COVID-19 patients. Occurrence of co-epidemic of dengue and COVID-19 can be a major public health problem in the coming months in India. Careful screening of patients is required for prompt isolation and appropriate management of the patients.

\section{Topic: Assessment of the Associationbetween Glucose Levels AND Serum Uric Acid in Healthy and Diabetic Individuals}

Ishita Sehgal $^{* 1}$

${ }^{1}$ Pathology, Sharda Hospital, Noida, India

Aims \& Objectives: To evaluate the serum uric acid and its correlation in type-2 Diabetes mellitus in North Indian population.

Patients/Materials \& Methods: A Prospective observational study was conducted in 100 individuals based on data collected during time from October 2019 to September 2020 at Sharda medical college. The enzymatic method was carried out for measurement of the Serum uric acid [(Uricase) and blood glucose (BG) (Hexokinase)] level, and $\mathrm{HbA1c}$ was determined by immune inhibition method.Diabetes and prediabetes were described as FBG level $\geq 126 \mathrm{mg} / \mathrm{dL}$ and $100-125 \mathrm{mg} / \mathrm{dL}$, respectively.

Results: Patients in the diabetic groups had lower mean Serum uric acid level (SUA) $(4.1 \pm 0.9 \mathrm{mg} / \mathrm{dL}$,$) compared to the healthy$ $(5.4 \pm 1.8 \mathrm{mg} / \mathrm{dL})$ group. The SUA was found significantly negative association with FBS $[\mathrm{r}=-0.295 ; p<0.001]$ and HbA1c $[\mathrm{r}=-$ $0.255 ; p<0.001]$. SUA was associated positively with TG,BMI, and TC.

Discussion \& Conclusion: It could be concluded that serum uric acid may be a determinant of changed glucose metabolism but not the potential predictor of prediabetes/diabetes in north Indian populations residing in Uttar Pradesh. Further studies are required to observe the dependability of using S. Uric Acid to predict diabetes.

\section{Understanding the Molecular Subtypes of Aplastic Anaemia Based on Characterization of Telomere Maintenance Pathway}

Sarmistha Adhikari*1, Paramita Mandal ${ }^{1}$, Kaustav Nayek ${ }^{2}$, Arghya Bandyopadhyay $^{3}$

${ }^{1}$ Zoology, The University of Burdwan, ${ }^{2}$ Pediatric Medicine, ${ }^{3}$ Pathology, Burdwan Medical College, Burdwan, India

Aims \& Objectives: Aplastic Anaemia (AA), a rare disorder with hypocellular marrow and peripheral pancytopenia. The study was undertaken to identify the molecular markers associated with telomere length maintenance pathway among aplastic anaemia patients compared to age matched healthy donors.

Patients/Materials \& Methods: For this study, twenty aplastic anaemia patients and twenty age matched control samples were recruited. The study was approved by Institutional Clinical ethical Committee of The University of Burdwan and informed written consent were taken from the participants. Peripheral blood samples were collected from the participants. DNA and RNA were isolated from peripheral blood samples and cDNA was prepared from RNA samples by reverse transcription method. Absolute telomere length (aTL) from DNA samples and gene expression of the telomere maintenance genes from cDNA samples were estimated by quantitative real time PCR.

Results: The telomere length was significantly $(p<0.001)$ shorter among aplastic anaemia patients compared to age matched healthy donors. The absolute telomere length was negatively correlated with age only among healthy donors but not among aplastic anaemia cases. Interestingly, one subgroup $(n=3)$ of aplastic anaemia have moderate telomere length which is comparable to age matched healthy donors. Based on the gene expression analysis of telomere maintenance pathway, NOP10 was significantly downregulated in both subgroups (patients with moderate telomere length and patients with shorter telomere length) of AA. In contrast, another differentially expressed gene, TERF2, was significantly downregulated only in the subgroup with shorter telomere length for the AA patients. An association between platelet count and NOP10 gene expression was also found to be significantly and positively correlated in one subgroup of cases with moderate telomere length.

Discussion \& Conclusion: Telomere length estimation gene expression pattern of the telomere length maintenance genes can be used as a biomarker for discriminating different subgroups of aplastic anaemia patients. 


\section{Utilization of Histogram and Cell Counter Patterns for Detection of Platelet Clumps or Large/Giant Platelets in Thrombocytopenia Patients}

Gayathiri K. Chellaiya ${ }^{* 1}$, Tulasi Geevar ${ }^{1}$, Rutvi G. Dave ${ }^{1}$, Mullai Saravanan $^{1}$, Sukesh C. Nair ${ }^{1}$

${ }^{1}$ Transfusion Medicine and Immunohaematology, Christian Medical College, Vellore, India

Aims \& Objectives: 1. To determine the diagnostic utility of cell counter parameters to reliably measure the platelet count in thrombocytopenia patients. 2. To utilize histogram and cell counter parameters for accurate estimation of platelet count in thrombocytopenia patients. 3 . To assess the reliability of cell counter patterns to detect platelet abnormalities (platelet clumps, large or giant platelets) and thus help reduce the smear review rates.

Patients/Materials \& Methods: This is a prospective study, conducted in Department of transfusion Medicine and Immunohaematology, Christian Medical College, Vellore. Patients with thrombocytopenia $(<150,000 / \mu \mathrm{L})$ in whom manual platelet count was requested were included. Samples were run in DXH -900. Specific patterns in platelet, RBC and WBC histograms, differential scattergram and $\mathrm{nRBC}$ channel scattergram were analysed to look for platelet distribution, presence of large/giant platelets, RBC interference and platelet clumps. Smears were made in all cases to assess platelet count, morphology, presence of platelet clumps and correlated with the cell counter interpretations.

Results: A total of 45 samples were included in this study. Median platelet count was $49,000 / \mu \mathrm{L}$. There is strong positive correlation between platelet count estimated by cell counter and smear manual count $(\mathrm{r}=0.918, p=0.000)$. According the cell counter patterns, these patients were categorised as normal platelet distribution $(n=8)$, presence of platelet clumps $(n=26)$ and presence of giant/large platelets $(n=11)$. Figure below described the various patterns seen in platelet clumps and large/giant platelets. The sensitivity and specificity of cell counter to detect platelet abnormalities was $100 \%$ and $53.3 \%$ respectively.

Discussion \& Conclusion: Constitutional macrothrombocytopaenia is common in North India. It is important to have accurate platelet count estimation to avoid unnecessary transfusions and slide reviews. In study there is good correlation between cell counter and manual platelet count even in the presence of large or giant platelets. From this study it is evident that based on cell counter patterns can be used to detect platelet clumps and large/giant platelets and thus reducing unnecessary slide review.

\section{Hemostatic Abnormalities in Liver Diseases}

Aravind $\mathbf{R}^{* 1}$, Preeti Tripathi ${ }^{1}$, Arijit Sen ${ }^{1}$

${ }^{1}$ Pathology, Command Hospital Air Force, Bangalore, India

Aims \& Objectives: The important role of liver in hemostasis is evidenced by various coagulation abnormalities seen in liver diseases. However, mostly, the coagulation abnormalities does not go hand in hand with clinical bleeding and/or thrombotic manifestations. We studied various coagulation parameters in liver disease cases, to ascertain correlation with clinical bleeding and/or thrombosis.

Patients/Materials \& Methods: 104 patients with various acute and chronic liver diseases, were enrolled in the study, after ascertaining brief clinical profile. Clinically, three cases had variceal bleeds, one had portal vein thrombosis, and another one had both. Segregation into mild(41/104), moderate(51/104) and severe(12/104) liver failure was based on their serum bilirubin $(<5 \mathrm{mg} / \mathrm{dl}, \quad 5-10 \mathrm{mg} / \mathrm{dl}$ $\&>10 \mathrm{mg} / \mathrm{dl}$ respectively), ALT/AST and ALP(3-5 times, 5-10 times \& $>10$ times respectively) levels. Various coagulation parameters were evaluated following standard testing protocols.

Results: The results, summarised below, showed significant variations proportional to severity of liver failure, with the mild group showing no significant deviations from normal range. PT, aPTT, and INR were prolonged with a mean of $19.1 \mathrm{~s}, 52.5 \mathrm{~s}, 1.68$ in severe $\operatorname{group}(66.7 \%$ cases $)$ and $15.3 \mathrm{~s}, 37.8 \mathrm{~s}, 1.48$ in moderate group $(37.3 \%$ cases) respectively. Fibrinogen levels showed mean of $173 \mathrm{mg} / \mathrm{dl}$ in severe group $(66.67 \%$ cases) as compared to mildly elevated levels of $432 \mathrm{mg} / \mathrm{dl}$ in moderate group $(25.5 \%$ cases $)$, as it's an acute phase reactant. Semiquantitative D-Dimer levels were increased with a mode of $800 \mathrm{ng} / \mathrm{ml}$ and $400 \mathrm{ng} / \mathrm{ml}$ in severe $(91.66 \%)$ and moderate groups $(41.2 \%)$ respectively. Factors II,V,VII,IX\&X showed depleted levels compared to increased Factor VIII levels(as it is not solely synthesized in liver); Factor VII levels were most affected with a mean of $49.5 \%$ and $75.4 \%$ in severe(all cases) and moderate groups $(64.7 \%)$ respectively. ProteinC,S,Antithrombin levels were also proportionately depleted with protein $\mathrm{C}$ being most affected with mean of $38.6 \%$ and $68.7 \%$ in severe(all cases) and moderate groups $(35.3 \%)$ respectively. On correlation with clinical profile of patients, all cases which had clinical bleeding and/or thrombotic manifestations belonged to severely affected group of patients.

Discussion \& Conclusion: In our study, vast majority of cases, except a few, had no significant bleeding or thrombotic manifestations, even with coexistent severely deranged coagulation abnormalities, further reinforcing that, abnormalities in coagulation parameters does not correlate with clinical bleeding and/or thrombotic manifestations, in liver disease patients.

\section{Dry Tap on Bone Marrow Aspiration: A Red Flag}

\author{
Saurabh Donald ${ }^{* 1}$, Naveen Kakkar ${ }^{2}$
}

${ }^{1}$ Pathology, Christian Medical College \& Hospital, Ludhiana, Ludhiana, ${ }^{2}$ Pathology, MM Medical College \& Hospital, Kumarhatti- 173229, Solan, Himachal Pradesh, India

Aims \& Objectives: To look for possible underlying bone marrow pathology in patients with a dry tap on bone marrow aspiration. Patients/Materials \& Methods: This study was carried out over a period of $5 \frac{1}{2}$ years in which 2768 bone marrow aspirates and trephine biopsies were reviewed. Of these, 223 patients with bone marrow aspiration which yielded no marrow particles (dry tap) were included. Patients in whom adequate trephine biopsy could not be obtained were excluded from the study. Relevant clinical details, complete blood count, touch imprints and trephine biopsy findings were recorded. The final diagnosis was made by studying the trephine biopsy and correlating it with ancillary investigations. Descriptive statistics were used to analyze the data.

Results: Of the 2768 bone marrow aspirations studied, dry tap was seen in $223(8.0 \%)$ patients. (Table 1) Of these, 209 (93.7\%) patients had an underlying bone marrow pathology while 14 (6.3\%) patients had dry tap due to faulty technique. Hematological malignancies were seen in $164(73.5 \%)$ patients, benign hematological disorders in 33 (14.9\%) patients while $4 \%$ of patients had nonhematological disorders. Among hematological malignancies, leukemia (acute and chronic) was the commonest cause of a dry tap seen in $40.3 \%$ of the patients followed by myeloproliferative and lymphoproliferative disorders. Non-hematological causes included metastasis and infections.

Discussion \& Conclusion: Dry tap on bone marrow aspirate usually causes alarm due to a failure to obtain diagnostic material from the aspirate smears. However, it should serve as a red flag for the hematologist rather than a failed procedure. In most cases, it indicates 
the possibility of underlying bone marrow pathology. Trephine biopsy should always be studied in such cases.

Supporting Document:

\begin{tabular}{|c|c|c|}
\hline Diagnosis & Number & Percentage \\
\hline \multicolumn{3}{|l|}{$\begin{array}{l}\text { HEMATOLOGICAL MALIGNANCIES } \\
\text { ACUte Leukemia }\end{array}$} \\
\hline All & 45 & 20.2 \\
\hline & 12 & 5.4 \\
\hline -Acute Leukemia (unciassified) & & \\
\hline \multirow{2}{*}{\multicolumn{3}{|c|}{$\begin{array}{l}\text { Chronic myeloprolifer ative neoplasms } \\
\text { Chronic Myeloid Leukemia (CML) }\end{array}$}} \\
\hline & & \\
\hline CML in chronic phase & 10 & 4.5 \\
\hline CML in accelerated phase & 02 & 0.9 \\
\hline CML in blast crisis & 11 & 4.9 \\
\hline Primary myelofibrasis & 20 & 9.0 \\
\hline Primary myelofibrosis-progression to AMI & 04 & 1.8 \\
\hline Polycythemia vera & 04 & 1.8 \\
\hline Essential thrombocythemia & 01 & 0.4 \\
\hline \multirow{2}{*}{\multicolumn{3}{|c|}{$\begin{array}{l}\text { Chronic Lymphoproliferative disorders } \\
\text { Lymphoma }\end{array}$}} \\
\hline & & \\
\hline NHL & 17 & 7.7 \\
\hline HD & 01 & 0.4 \\
\hline Chranic lymphocytic leukemia & 04 & 1.8 \\
\hline Hairy cell le ukemia & 04 & 1.8 \\
\hline \multicolumn{3}{|l|}{ Plasma cell dyscrasias } \\
\hline Multiple myeloma & 07 & 3.1 \\
\hline \multicolumn{3}{|l|}{ BENIGN HEMATOLOGICAL DISORDERS } \\
\hline Aplastic anemia/hypocellular marrow & 26 & 11.6 \\
\hline Nutritional anemia & 07 & 3.1 \\
\hline \multicolumn{3}{|l|}{ NON HEMATOLOGICAL DISORDERS } \\
\hline Metastatic disease & 06 & 2.7 \\
\hline Granulomatous involvement-marrow & 03 & 1.3 \\
\hline FAULTY TECHNIQUE & 14 & 6.3 \\
\hline MISCELLANEOUS" & 03 & 1.3 \\
\hline
\end{tabular}

\section{Study of Utility of Reticulocyte Indices by the Automated Method in the Diagnosis and Management of Pancytopenia}

Jonali Das ${ }^{* 1}$, Yookarin Khonglah ${ }^{1}$, Iadarilang Tiewsoh ${ }^{2}$, Zachariah Chowdhury ${ }^{1}$, Himesh Barman ${ }^{3}$

${ }^{1}$ Pathology, ${ }^{2}$ Medicine, ${ }^{3}$ Pediatrics, Neigrihms, Shillong, India

Aims \& Objectives: 1 . To study the utility of reticulocyte indices in pancytopenia. 2 . To form a diagnostic algorithm based on reticulocyte indices for the classification of cases of pancytopenia.

Patients/Materials \& Methods: Reticulocyte indices such as reticulocyte \%, Immature Reticulocyte Fraction (IRF) and Mean Reticulocyte Volume (MRV) were measured in the automated coulter on 154 pancytopenia cases and 154 control samples. Correlation with the relevant biochemical and bone marrow tests was done and these cases were stratified into different etiological groups. ROC curve analysis was done and a diagnostic algorithm for evaluating the cause of pancytopenia using reticulocyte indices was formulated.

Results: A total of 154 cases of pancytopenia were obtained fulfilling the inclusion and exclusion criteria. Patient's age ranged from 7 months to 87 years with a mean age of 42 years. M:F ratio was 1.08:1. The most common cause of pancytopenia was Megaloblastic anemia. Cut off values of $<0.91$ for reticulocyte $\%$ with sensitivity of $78.1 \%$ and specificity of $70 \%,>0.45$ for IRF with sensitivity of $76.7 \%$ and specificity of $64 \%$ and $>121.8 \mathrm{fl}$ for MRV with sensitivity of $83.6 \%$ and specificity of $80 \%$ was found in megaloblastic anemia. The IRF and MRV helps to differentiate between megaloblastic and dual deficiency anemia. MDS has the most immature and higher sized reticulocytes compared to other causes of macrocytic anemia.

Discussion \& Conclusion: Megaloblastic anemia is the commonest cause of pancytopenia. Reticulocyte indices are helpful for the diagnosis and classification of pancytopenia cases. Reticulocyte indices can segregate nutritional anemia from other causes of pancytopenia allowing presumptive treatment to be initiated and may obviate invasive procedures such as bone marrow examination.

\section{Haematological Findings in Severe and Non-severe Covid-19 Patients}

\author{
Rajvala Choudhary $^{* 1}$, Gajendra S. Rajpoot ${ }^{1}$ \\ ${ }^{1}$ Pathology, RUHS College of Medical Sciences, Jaipur, Jaipur, \\ India
}

Aims \& Objectives: In our study differences detected in hematological parameters between severe and non-severe patients and A blood workup as well as continuous tracking hematological changes can reveal the risks of disease progression so that patients at high risk can identify and guide more appropriate critical care.

Patients/Materials \& Methods: We analysed prospectively haematological parameters of 850 COVID19 cases with laboratory confirmation during may 2020 to September 2020 at RUHS College of Medical Sciences, Jaipur.

Results: When the disease progresses to severe stage, lymphopenia continues to aggravate. Increased neutrophil count and neutrophil-tolymphocyte ratio, and decreased haemoglobin concentration were identified as the risk factors of severe illness in patients with SARSCoV-2 infection. Elevated DDimer levels are consistently reported, whereas their gradual increase during disease course is particularly associated with disease worsening. Other coagulation abnormalities such as PT and aPTT prolongation, fibrin degradation products increase, with severe thrombocytopenia lead to lifethreatening disseminated intravascular coagulation (DIC), which necessitates continuous vigilance and prompt intervention.

Discussion \& Conclusion: Hematological abnormalities are not rare in COVID-19 patients including lymphopenia, neutrophilia, thrombocytopenia, and decline of haemoglobin. The activation of monocyte-macrophage system aggravates the immune damage of lung and other tissues, which leads to the increase of D-dimer, prothrombin time, and platelet consumption. Study indicate the onset of inflammatory cytokine storms in COVID-19 lead to progress to severe lung injury, respiratory distress, and multiple organ failure.

Keywords: SARS-CoV-2, COVID-19, Lymphopenia, Neutrophilia, Thrombocytopenia.

\section{In this Era Of Technology, Manual Platelet Estimation Still Holds The Key in Critical Cases} Anjali N. Patel
Mansuri $^{*}$

${ }^{1}$ Pathology, Pramukhswami Medical College, Anand, India

Aims \& Objectives: Platelet count estimation is an important element of the diagnostic and treatment process. Platelets are non-nucleated discoid 1-3 $\mu$ cells, produced by bone marrow megakaryocytes by fragmentation of cytoplasm1. In patients having thrombocytopenia where platelet transfusion is required, the reliability of the platelet count is highly desired and necessary to provide appropriate treatment. The aim of this study is to determine reliability and accuracy of the automated cell counter with manual microscopic count method. Patients/Materials \& Methods: This is an observational cross-sectional study done at Central Diagnostic Laboratory of Shree Krishna Hospital and Pramukh Swami Medical College, Karamsad during the period of 1st August to 20th October, 2020. Two hundred random blood samples collected in EDTA were analyzed for platelet count using five-part automated hematology analyzer Sysmex XN 350 \& 550 Depending on the platelet count of machines it has been divided 
in two groups. First group with platelet count more than $150000 / \mu 1$ and second group with platelet count less than 150000/ $\mu$ l. The agreement between the two methodologies were assessed using the paired t-test and correlation coefficient analyses.

Results: In this study statistically, there is significant difference between the number of samples estimated with low count $(<1,50,000 / \mu \mathrm{l})$ by the two methods $(p<0.001)$. However, in samples with high count $(>1,50,000 / \mu \mathrm{l})$, there is no significant statistical difference between the two methods $(p>0.001)$.

Discussion \& Conclusion: After this study we can say that platelet count should be verified manually on the peripheral smear in all the cases of thrombocytopenia even when machine is not showing any error.

Keywords: EDTA: Ethylenediaminetetraacetate.

\section{Hematological Profile of Chronic Kidney Diseases Patients in Pre-dialysis Stages and After Initiation of Hemodialysis}

\author{
Krishna M. Panchal ${ }^{* 1}$, Menka H. Shah ${ }^{1}$, Zalak N. Parmar ${ }^{1}$
}

${ }^{1}$ Pathology, Pramukhswami Medical College, Karamsad, Anand, India

Aims \& Objectives: Chronic kidney disease (CKD) is a global public health problem, where slowly progressive deterioration in kidney function leads to numerous hematological and biochemical dysfunctions which further make the patients vulnerable to cardiovascular morbidity and mortality, if appropriate measures are not taken for their control. The objective of present study was to find out the common hematological dysfunctions that may occur in the patients of chronic kidney disease (CKD) undergoing the process of dialysis and suggest appropriate measures for their management.

Patients/Materials \& Methods: This study is a retrospective study, done at Shree Krishna Hospital, Karamsad, Gujarat from June to October 2020. During this period, data of a total of 52 patients who were diagnosed to have chronic kidney disease and underwent for hemodialysis have been taken. Pre and post dialysis diagnostic test data of these patients was collected. Data were entered in Microsoft excel 2016 and further analyzed in SPSS 15.

Results: The total number of cases in this study were 52 . The mean age of patients is 50.03 , with a range of $2-88$ years. The mean total leucocyte count, hemoglobin, platelet count, MCV along with serum creatinine, potassium and sodium levels are significantly changed after the dialysis.

Discussion \& Conclusion: The study concludes significant changes in red cell indices, platelet count, serum creatinine levels and serum electrolyte levels comparing the pre- and post- dialysis diagnostic profiles of CKD patients. While there is significant anemia as observed, the patients are at a higher risk of developing related complications, requiring prompt therapeutic action and close monitoring. Other parameters are observed to be more or less insignificantly altered.
Supporting Document:

\begin{tabular}{|l|l|l|}
\hline Parameters & $\begin{array}{l}\text { Pre-dialysis } \\
{[\text { Mean(SD) }]}\end{array}$ & $\begin{array}{l}\text { Post-dialysis } \\
{[\text { Mean(SD) }]}\end{array}$ \\
\hline $\begin{array}{l}\text { Total leucocyte } \\
\text { count }\end{array}$ & $12.97(10.02)$ & $13.55(10.72)$ \\
\hline Hemoglobin & $8.58(2.15)$ & $8.25(2.00)$ \\
\hline MCV & $83.28(9.4)$ & $83.75(7.82)$ \\
\hline Platelet count & $246.34(141.9)$ & $216.96(122.0)$ \\
\hline Creatinine & $9.42(4.46)$ & $6.21(2.61)$ \\
\hline Potassium & $4.65(0.96)$ & $4.12(0.66)$ \\
\hline Sodium & $134.8(6.43)$ & $135.75(6.8)$ \\
\hline
\end{tabular}

\section{Study of Hematological Profile in Patients with Isolated Low Serum Vitamin B12}

Yogesh R. Vastani ${ }^{* 1}$, Kailash Inaniya ${ }^{1}$, Payal S. Bhatt ${ }^{1}$

${ }^{1}$ Pathology, Pramukh swami medical college, Anand, India

Patients/Materials \& Methods: This study is a retrospective study, done at Shree Krishna Hospital, Karamsad, Gujarat from April 2012 to March 2016. Parameters like serum Vitamin B12, serum iron, CBC, Peripheral smear for cell morphology and reticulocyte count were included. Data were entered in Microsoft excel 2016 and further analyzed in SPSS 15.

Results: In present study, among 119 patients analyzed, young adults (21 to 40 year of age) are most frequently affected by low serum Vitamin B12, that is $60 \%$ (72), childhood is the age which is least affected by Vit.B12 deficiency, that is $1.7 \%$ (2). Apart from these age groups, adolescents $11 \%$ (13), and elderly and middle aged (above 40 years) $27 \%$ (32) are affected by Vit.B12 deficiency.

Discussion \& Conclusion: Present study concludes that the young adults and middle aged groups are most commonly affected by Vit.B12 deficiency. Females are more commonly affected than males but males develop more severe Vit.B12. Among Vit.B12 deficient, macrocytic individuals shows more severe deficiency. The macrocytic anemia and overall anemia, both are more commonly seen in severe deficiency of Vit.B12. 
Supporting Document:

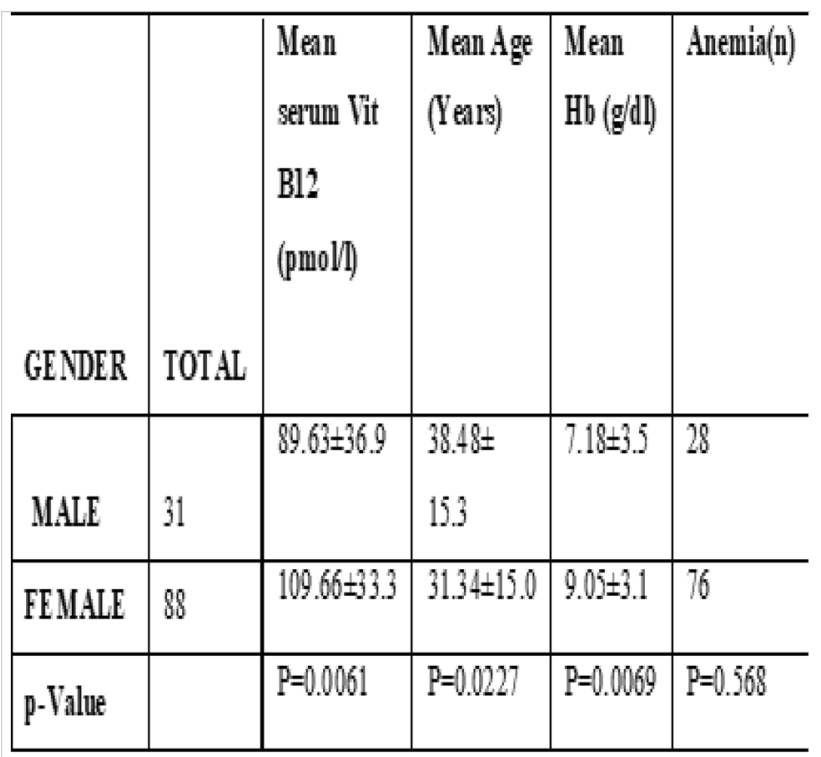

Aims \& Objectives: Vitamin B12 is a micronutrient essential for appropriate neuro-cognitive, hemopoetic and cardiovascular function. In vitamin B12 deficiency, multi-organ systems are affected concomitant with a wide spectrum of clinical manifestations. The clinical manifestations are usually non-specific and variable. 1. To find correlation between vitamin B12 deficiency and its hematological parameters. 2. To evaluate the vitamin B12 and folate status in the elderly residents living in long term care institutions. 3. To explored the association of vitamin B12 concentration with macrocytosis in the vitamin B12 deficient residents.

\section{Evaluation of Immature Granulocyte Count as the Earliest Biomarker For Sepsis: Experience at A Tertiary Care Hospital In North India}

\section{Nidhi Sharma*1, Sudhir Mehta ${ }^{2}$, Prabhav Bhansaly ${ }^{2}$ \\ ${ }^{1}$ Pathology, ${ }^{2}$ Medicine, SMS Medical College, Jaipur, India}

Aims \& Objectives: Sepsis is a serious condition and is a major cause of mortality. Early diagnosis has always been the key for successful management. To diagnose an infection rapidly and to accurately differentiate inflammatory response from sepsis, is challenging. This has necessitated studies to address the usefulness of various biomarkers to predict infection earlier. Various biomarkers are being used currently, but they are either expensive or not accessible. This study aims to evaluate usefulness of automated immature granulocyte count(IG\#) and immature granulocyte percentage (IG \%) as an early diagnostic marker of sepsis and compare it to other established predictive markers.

Patients/Materials \& Methods: In this prospective observational study, 137 consecutive eligible critically ill patients who did not have sepsis at time of intensive care unit(ICU) admission were analysed for automated complete blood count(CBC), IG\#, IG \%, serum procalcitonin(PCT), lactate(Lac) and sequential organ failure assessment(SOFA) score daily for the next 7 days. All the patients were followed for development of sepsis as defined by the new Sepsis-3 criteria. Those who developed sepsis were grouped under the sepsis group and rest were grouped under no-sepsis group. The study was divided into four time periods of $24 \mathrm{~h}$ each with respect to day of developing sepsis or day of developing systemic inflammatory response syndrome(SIRS) which was defined by change in SOFA $\geq 2$. Using receiver operator characteristic(ROC) and area under curve(AUC) methods, the best biomarker for prediction of sepsis in each time period was calculated.

Results: We found that IG\# and IG \% were earliest biomarkers to have a significant discriminating value (AUC of 0.81 and 0.82 respectively) as early as $24 \mathrm{~h}$ before clinical sepsis develops. Both IG\# and IG \% have a high diagnostic odds ratio (DOR) of 34.91 and 18.11 respectively when compared to PCT and Lac with DOR of 27.06 and 4.78 respectively on day of developing clinical sepsis. IG\# had the best diagnostic odds ratio in all the study time periods.

Discussion \& Conclusion: IG\# and IG \% are easily available, rapid and inexpensive tools to discriminate between septic and non- septic patients with high specificity and sensitivity and the earliest biomarker to show a significant rise in patients developing sepsis.

\section{Variation in The Haematological Indices in Various Types of Malaria}

Shivani Vora ${ }^{* 1}$, Jasmin Jasani ${ }^{1}$, Harsh Pandya ${ }^{1}$

${ }^{1}$ Pathology, SBKS MI \& RC, Vadodara, India

Aims \& Objectives: (1) To study the $\mathrm{Hb}, \mathrm{PCV}$ and morphology of RBCs in smears detected positive with malaria. (2) To make a Total count and Differential count of WBCs in the peripheral smears of malaria patients. (3) To establish a relationship of thrombocytopenia with malaria.

Patients/Materials \& Methods: All peripheral smear slides subjected for malarial parasite detection tested positive in Central Diagnostic Lab of DGH, BARODA. $4 \mathrm{ml}$ of blood is collected in an EDTA vacutainer. The blood drawn is fed into the automated cell counter where the Hb, PCV and Total counts of the WBCs are collected; with the remaining blood peripheral smears are made which are subjected to Leishmann's staining. The smears are evaluated and the RBC morphology, differential count of WBCs and platelet counts are done.

Results: Out of 100 cases, Plasmodium vivax infection is seen in $64 \%$ cases, plasmodium falciparum infection is seen in $33 \%$ of cases and $3 \%$ Patients shows mixed infection. $78 \%$ patients present with a short history ( $<7$ days) of fever. The most common complication seen was cerebral malaria mainly seen in falciparum infections. Severe anaemia was seen in 4 cases with 3 due to falciparum and 1 vivax infection. Majority of the patients had either mild (29\%) or moderate degree $(14 \%)$ of anaemia. Reduced MCV, MCH and MCHC values were more commonly seen in P.vivax infection in comparison to P.falciparum infection. Decreased platelet counts were a constant feature of both types of malaria with $84 \%$ of cases showing platelets less than 1.5 lacs. High parasitaemia was associated with marked thrombocytopenia in all except one case. Degree of parasitaemia was correlated with extent of thrombocytopenia.

Discussion \& Conclusion: Various haematological changes can occur in both falciparum and vivax infections, most commonly normocytic normochromic anaemia and thrombocytopenia. Degree of anaemia and thrombocytopenia usually correlates with parasite load. Changes in white blood cells are less dramatic and include leucopenia, Leucocytosis and occasionally presence of atypical lymphocytes. In conclusion the early diagnosis of malaria keeping in mind the various haematological changes and effective and aggressive therapy can limit the mortality and prevent further complications. 


\section{Clinico-Hematological Profile of CHS: Experience from Tertiary Care Center in North India}

Sharan K C ${ }^{* 1}$, Alpeshkumar B. Kapadia ${ }^{1}$, Jasmina Ahluwalia ${ }^{1}$, Reena Das ${ }^{1}$, Ankur K. Jindal ${ }^{2}$, Arun K. Baranwal ${ }^{2}$, Surjit Singh ${ }^{2}$, Neelam Varma

${ }^{1}$ Haematology, ${ }^{2}$ Paediatric Medicine, PGIMER, Chandigarh, India

Aims \& Objectives: To evaluate the clinical-haematological profile of Chediak-Higashi syndrome.

Patients/Materials \& Methods: The clinico-hematological profile of a series of 3 cases of CHS encountered at our centre over the last 4 years $(2016-2020)$ is presented. The diagnostic workup included complete hemogram with peripheral smear and bone marrow examination.

Results: The age of the patients ranged from 2 to 5 years. All the patients presented with fever and recurrent infections. Two of the patients had silvery hair and skin manifestations and all the three patients had hepatosplenomegaly. All three had anemia with normal total leucocyte count, two cases had thrombocytopenia. The cases showed large granules in the granulocytes on peripheral blood examination. Two patients showed significant hemophagocytic lymphohistiocytosis in bone marrow while one was stable. No history of any consanguinity seen in any of the cases.

Discussion \& Conclusion: The Morphological diagnostic hallmark of CHS is the presence of giant inclusion granules in the peripheral granulocytes and their marrow precursors. This case series is presented due to the rarity of the condition and its spectrum of clinical and hematological manifestations.

Disclosure of Interest: None Declared.

\section{Form Over Function: Pancytopenia Secondary to Renal Osteodystrophy}

Parul Verma ${ }^{* 1}$, Vikram Narang ${ }^{1}$, Suvir Singh ${ }^{2}$, Neena Sood ${ }^{1}$, Bhavna Garg ${ }^{1}$, Sumit Grover ${ }^{1}$

${ }^{1}$ Pathology, ${ }^{2}$ Departmenmt of Clinical Hematology, Dayanand Medical College \& Hospital, Ludhiana, India

Aims \& Objectives: To evaluate the cause of pancytopenia in patients with chronic kidney disease (CKD) secondary to long standing Diabetes.

Patients/Materials \& Methods: Patient 1-Mrs K, a 63 year old lady diagnosed to have diabetes mellitus related CKD Stage 4 was evaluated for incidentally detected pancytopenia (Hb-6.9 gm/dl, TLC$1900 / \mu \mathrm{l}$, Plt-70000/ $\mu \mathrm{l})$. No organomegaly or lymphadenopathy was found. PTH was $1136 \mathrm{pg} / \mathrm{ml}$. Possibilities considered were myelodysplastic syndrome (MDS), aplastic anemia or a low grade lymphoma. Patient $2-\mathrm{Mr} \mathrm{J}$, a 51 year old gentleman post renal transplant in January 2020 secondary to diabetes related end stage renal disease (ESRD) was treated with Prednisolone, Tacrolimus and Azathioprine. On investigations in February thrombocytopenia with counts up to $15000 / \mu \mathrm{l}$ was found. PTH was $1064 \mathrm{pg} / \mathrm{ml}$.

Results: Bone marrow biopsy was performed on both patients. In Patient 1 bone marrow biopsy revealed prominent bony trabeculae separated by fibrous tissue with cystic spaces and new bone formation. A significant increase in osteoblastic and osteoclastic activity was noted, consistent with a histological picture of renal osteodystrophy (Fig. 1). A sample for cytogenetics was not available due to a dry tap. Six months later, her counts continue to be stable with no serial worsening or onset of new symptoms.

In Patient 2 bone marrow examination biopsy foci of fibrosis with paratrabecular increase in reticulin and normal trilineage haematopoiesis. Karyotype was normal. The changes in the marrow were consistent with renal osteodystrophy and he was managed conservatively. His platelet counts stabilized between $45000 / \mu \mathrm{l}$ and $50000 / \mu 1$ and he had no further cytopenia.

Discussion \& Conclusion: Anemia of CKD is well characterized and known to occur due to a combination of impaired erythropoietin production, reduced red cell lifespan, and hepcidin mediated reduction in iron utilization. However, pancytopenia as a direct complication of CKD is uncommon and rarely reported. We present a short summary of two cases presenting with unexplained pancytopenia, which was eventually attributed to architectural marrow changes secondary to renal osteodystrophy.

\section{Recognising and Resolving ABO Discrepancies:Study of 191351 Cases}

Sunanda Bhardwaj ${ }^{* 1}$, Parul Chopra ${ }^{2}$, Anil Arora ${ }^{1}$

${ }^{1}$ Haematology, Dr Lal PathLabs, ${ }^{2}$ Hematology, Dr Lal PathLab, Delhi, India

Aims \& Objectives: ABO discrepancies occur when the results in forward and reverse grouping are in disagreement or when preceding and recent results do not match. If discrepancies are encountered, the interpretation has to be delayed until it has been resolved. This study aimed to recognise ABO discrepancy done through automated method and resolving the same.

Patients/Materials \& Methods: Blood Samples received in Department of Haematology and Immunology at Dr Lal PathLabs, National Reference laboratory, Rohini, Delhi for blood grouping was processed on QWALYS 3(DIAGAST, FRANCE). Samples showing any discrepancy in ABO between January 2018- February 2020 were included in study. To resolve discrepancies Conventional tube method was used.

Results: $217(0.11 \%)$ cases showed some sort of ABO discrepancy out of total 191351 samples. Of all the samples, no discrepancy was found in six $(n=6)$ samples on repeat testing with QWALYS, remaining 211 samples were retested with manual test tube methods. 211 samples were of $\mathrm{ABO}$ discrepancy. Most of the discrepancies found were due to Subgroup of the blood group (46\%), followed by alloantibodies $(21.3 \%)$ and cold autoantibody $(15.6 \%)$. Weak antibodies $(13.3 \%)$ and weak antigen $(2.4 \%)$ ) was seen in few cases. One case of Bombay blood group $(0.4 \%)$ was detected.

Discussion \& Conclusion: All cases of blood group discrepancies should be carefully recognised and such discrepancies can be resolved serologically by proper work up. ABO discrepancy can lead to haemolytic transfusion reactions and must be resolved before blood transfusion. Our approach should be to resort to the accurate methods so that these discrepancies are resolved serologically and high end investigations are avoided.

\section{Bone Marrow and Skeletal Metastasis in Carcinoma Gall Bladder}

Qausain Akhtar ${ }^{* 1}$, Nilesh kumar ${ }^{1}$, Kailash Kumar ${ }^{1}$, Ashis Gupta ${ }^{1}$, Vikash Maurya $^{1}$

${ }^{1}$ General Medicine, Institute of Medical Sciences, Varanasi, India

Aims \& Objectives: Carcinoma of the gall bladder presents with nonspecific symptoms that often lead to a delay in diagnosis. Usually detected when metastases to the liver and regional nodes have already occurred. Metastasis to bones and bone marrow are rare occurrence. We describe a rare case of bone marrow and bone metastasis from $\mathrm{Ca}-$ GB. 
Patients/Materials \& Methods: A 45 year married female presented with generalized weakness and easy fatigability, fever, lower backache for 3 months followed by generalized body swelling and dyspnoea on exertion for 15 days. On general examination, patient was severe anaemic, febrile, having bilateral pedal edema, without icterus or lymphadenopathy. On systemic examination she had bilateral fine inspiratory basal crept with mild tenderness in right upper quadrant of Abdomen. Routine investigation show anaemia (7.6 $\mathrm{gm} / \mathrm{dl})$ and thrombocytopenia $(40,000 / \mathrm{mm} 3)$ liver enzyme were deranged (ALT 292 unit/l, AST 375 unit/l), ALP (3993 unit per L), serum LDH (1428 unit/dl) and Ferritin (> 10000mcg per litre) were raised. Peripheral blood smear showed normocytic, normochromic anaemia, with marked thrombocytopenia. Persistent bicytopenia was noted beyond the usual clinical course hence bone marrow aspiration was done which showed cohesive clusters of large, atypical cells suggestive of metastatic tumour. Bone marrow biopsy showed metastatic carcinoma involving $90 \%$ of marrow spaces with proportionally reduced marrow with trilineage haematopoiesis. On further evaluation CECT abdomen features suggestive of carcinoma gall bladder (Probably Adenocarcinoma Stage 4,T3N0M1)with multiple lytic as well as sclerotic lesion noted involving thoraco lumbo- sacral vertebra and bilateral proximal femur.

Results: Patient was managed conservatively with packed RBC transfusion and diuretics for anaemia and de compensated heart failure. Pain was managed with Analgesics and Trans dermal anesthetic patch. For further management patient referred to surgical oncology.

Discussion \& Conclusion: Primary carcinoma of Gall bladder spread by extension and metastases, spread by extension occur earlier and more common. The most common site of extra-abdominal metastasis is the lung followed by the brain.skeletal and bone marrow metastasis in carcinoma Gall bladder are very rare. In presence of metastasis,the management is palliative and role of chemotherapy is limited for palliation symptoms.

\section{APTT Based Clot Waveform Analysis (CWA): A Useful Tool in Infections}

\author{
Keshav Sharma*1, Vikram Narang ${ }^{1}$, Neena Sood ${ }^{1}$, Bhavna Garg ${ }^{1}$ \\ ${ }^{1}$ Pathology, Dayanand Medical College and Hospital, Ludhiana, \\ LUDHIANA, India
}

Aims \& Objectives: INTRODUCTION. The Clot waveforms provided along with the first line of tests used for screening of the coagulopathies, namely Prothrombin Time (PT) and Activated partial thromboplastin time(aPTT) in the newer generation of coagulation analyzers have reported to provide useful information in early detection of disseminated intravascular coagulation and are also associated with detection of very low levels of coagulation factor VIII activity. AIMS AND OBJECTIVES: comparison of normal and Abnormal APTT-CWA and its usefulness as being an additional indicative marker for hemostatic dysfunction in dengue and Sepsis. Patients/Materials \& Methods: Materials and methods: APTTCWA of 25 samples with normal APTT and 25 samples with prolonged APTT processed on IL ACL Top 300 coagulation analyzer in the Pathology laboratory of Dayanand Medical College \& Hospital, Ludhiana were recorded. Clinical History was taken from the Laboratory Information system and the data was analyzed Results: RESULT: A total of 50 samples were analyzed out of which 25 were normal controls with standard baseline phase of APTT, Standard Acceleration and Standard Deceleration/plateau phase. Whereas out of the 25 samples with abnormal APTT value, there were 10(40\%) patients with Dengue, $8(32 \%)$ had history of sepsis and $3(12 \%)$ presented with hematoma/bleeds whereas clinical history was not available for 4(16\%). Mean APTT for the control group(n- = 25) was 28.04 whereas for sample with prolonged APTT $(\mathrm{n}=25)$ was 42.04 , on reviewing the APTT-CWA of both dengue and sepsis, the precoagulation phase of the curve was prolonged and presented with Extended stabile baseline for APTT although the CWA reaction curves for the first derivative and second derivatives exhibit normal first and second derivative curve patterns, the peaks of these CWA parameters were higher for sepsis and were lower for dengue.

Discussion \& Conclusion: CONCLUSION: The APTT-Clot waveform analysis can be useful in assessing the hemostatic activity of patients with Dengue and Sepsis. It can be used as a prognostic tool see the dynamic CWA changes over the course of infection within an individual patient to assess the hemostatic changes in hemorrhagic infections.

\section{In-Silico Analysis of Mutations in Gilbert's Syndrome Associated in Indian SCD Patients}

Sanya Sachdeva*1, Anand Bodade ${ }^{2}$

${ }^{1}$ Biotechnology, Amity University, Noida, New Delhi, 2NIIH ICMR Mumbai, Mumbai, India

Aims \& Objectives: To analyse and compare different genotypes of UGT1A1 responsible for Gilbert's syndrome in the Indian cohort of SCD patients by in silico approach.

Patients/Materials \& Methods: Genotypes of 266 patients from two case control studies via Human Gene Mutation Database were curetted and comparison was carried out for different genotypes of UGT1A1 responsible for GS in the Indian SCD patients. The association between genotypes and patient's status including GS with SCD, GS without SCD versus healthy controls were evaluated and indicated through Chi square and Pearson's test. Whole analysis was performed with SPSS 25 version. $p$ value $<0.05$ was considered as statistically significant.

Results: On comparison of three groups of our study i.e. Gilbert's Syndrome with Sickle Cell Disease, Gilbert's Syndrome without Sickle Cell Disease compared to Healthy Controls viz three genotypes of UGT1A1 mutations were extracted from Indian SCD patients. The genotypes commonly observed were (TA)6/(TA)6, (TA)6/(TA)7 and (TA)7/(TA)7. Higher total serum bilirubin levels were found in SCD patients with the (TA)7/(TA)7 and (TA)6/(TA)7 repeats as compared with SCD patients having the (TA)6/(TA)6 repeats.

Discussion \& Conclusion: In this in silico analysis, we observed that sample distribution of genotypes was observed significantly associated with the different groups in the study $(p<0.0001)$. These results will help us to know the genotype according to the patient's status. Supporting Document:

\section{Platelet Parameters, NLR, PLR, RDW: Novel Biomarkers in Evaluation of Severity Of Psoriasis}

Shivani Agarwal ${ }^{* 1}$, Mukta Pujani ${ }^{2}$, Charu Agarwal ${ }^{3}$, Varsha Chauhan $^{2}$, Kanika Singh ${ }^{4}$, Shanta Passi ${ }^{5}$

${ }^{1}$ Physiology, Associate Professor, Physiology, ESIC Medical College, Farid, ${ }^{2}$ Pathology, ESIC Medical College, Faridabad, ${ }^{3}$ Pathology, ESIC Medical College, Faridabad, ${ }^{4}$ Pathology, ESIC Medical College, Faridabad, ${ }^{5}$ Dermatology, ESIC Medical College, Faridabad, Faridabad, India

Aims \& Objectives: The aims and objectives of present study were to evaluate the various Hematological parameters including platelet indices (PC, PCT, MPV, PLCR, PDW), Neutrophil-lymphocyte ratio (NLR), Platelet-lymphocyte ratio (PLR) and RDW in Psoriasis 
patients and controls and to study whether any correlation exist between these indices and Psoriasis Area and Severity Index (PASI). Patients/Materials \& Methods: A cross sectional study was conducted on 50 Psoriasis patients and 50 healthy controls. Hematological parameters including platelet indices (PC, PCT, MPV, PLCR, PDW), Neutrophil-lymphocyte ratio (NLR), Platelet-lymphocyte ratio (PLR) and RDW were evaluated and correlation of these indices amongst themselves and with Psoriasis Area and Severity Index (PASI) analysed. Statistical analyses were performed using SPSS version 19.0 (SPSS Inc., Chicago, IL, USA).

Results: A statistically significant difference in RDW, platelet count, MPV, PDW, NLR and PLR was observed between psoriasis vs. controls and mild vs. moderate to severe psoriasis. There was a significant positive correlation between PASI and RDW, MPV, Platelets, PLR while ESR showed a significant correlation with MPV. MPV and RDW, RDW and NLR and RDW and PLR were also found to be correlated.

Discussion \& Conclusion: The present study assessed the role of simple and low cost parameters easily computed from routine tests like CBC as biomarkers for severity of Psoriasis. Mean values of MPV, RDW, NLR and PLR were found to be higher in psoriasis patients compared to controls. Moreover, significant correlation was observed between PASI and these novel markers. However, additional large scale, multicenter studies need to be conducted prior to application of these parameters in clinical practice.

\section{Osteosclerosis with Mast Cell Hyperplasia in Primary Hyperparathyroidism}

Surbhi Singh ${ }^{* 1}$, Richa Chauhan ${ }^{1}$, Ankur Ahuja ${ }^{2}$, Nitin Gupta ${ }^{3}$, Megha Verma ${ }^{3}$, Soma Pradhan ${ }^{1}$, Jyoti Kotwal ${ }^{1}$

\section{${ }^{1}$ Department of Hematology, Sir Ganga Ram Hospital, New Delhi, ${ }^{2}$ Department of Pathology, Command Hospital, Udhampur, ${ }^{3}$ Department of Clinical Hematology, Sir Ganga Ram Hospital, New Delhi, India}

Aims \& Objectives: Osteosclerosis is a rare presentation of hyperparathyroidism. We hereby present a case with altered vitamin D3 and calcium levels and mast cell hyperplasia concomitant with primary hyperparathyroidism.

Patients/Materials \& Methods: A 42-year old homemaker was referred for haematology consultation with complaints of low back ache, shoulder pain not responding to analgesics and history of itching over the body. There was no history of headache, blurring of vision, lumps anywhere. There was no organomegaly, lymphadenopathy, or lump over body. Her initial investigations revealed low Vitamin D3 $(2.2 \mathrm{ng} / \mathrm{ml})$ and normal serum calcium $(10.3 \mathrm{mg} / \mathrm{dl})$ levels. Complete blood count showed $\mathrm{Hb}: 9.4 \mathrm{~g} / \mathrm{dl}$; platelets: 3,51,000/ $\mu \mathrm{l}$; TLC:

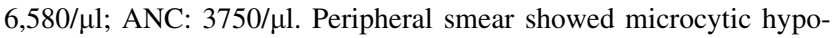
chromic red cells with mild anisocytosis. No rouleaux and no tear drop cells were seen. PET scan showed skeletal sclerosis with few illdefined lytic lucencies in bilateral iliac bones, suggesting a metabolic bone disease. Serum protein electrophoresis did not show any $M$ band. Bone marrow aspiration was hemodiluted showing prominence of normal-looking mast cells on imprints that were dispersed. The trephine biopsy showed diffuse fibrosis, osteosclerosis with narrowing of marrow spaces, prominent osteoblastic reaction, paratrabecular fibrosis, paratrabecular mast cell hyperplasia (confirmed by toluidine blue staining and immunohistochemistry with CD117). In view of the above findings, the possibilities of osteosclerosis secondary to hyperparathyroidism or systemic mastocytosis were considered. IHC with CD2 and CD25 were negative. On further evaluation Parathyroid hormone $(\mathrm{PTH})$ level was elevated $(590 \mathrm{pg} / \mathrm{ml})$. Serum tryptase levels were within normal range $(5.2 \mathrm{mcg} / \mathrm{L}) .99 \mathrm{~m}$ Tc-MIBI scan showed scintigraphic evidence of right lower parathyroid adenoma. Patient subsequently underwent parathyroidectomy. Thereafter, her sPTH fell to $189 \mathrm{pg} / \mathrm{mL}$.

Discussion \& Conclusion: Co-existing vitamin D deficiency in hyperparathyroidism can cause serum calcium level to fall into normal range as seen in our case, which can lead to diagnostic uncertainty. Osteosclerosis is a rare phenomenon in adults with primary hyperparathyroidism since the usual skeletal manifestation is generalized osteopenia. Mast cell accumulation (mimicking mastocytosis) around bony trabeculae in response to elevated PTH and their interaction with osteoblast lineage cells contribute to PTH-induced peritrabecular fibrosis.

\section{Age and Gender Based Study of Reference Range of Platelet Count, MPV and PDW in Healthy Eastern Indian Population}

\author{
Krupa V. Tarpara ${ }^{* 1}$, Enam M. Khan ${ }^{1}$, Atoshi Basu ${ }^{1}$ \\ ${ }^{1}$ Pathology, Apollo Gleneagles Hospitals, Kolkata, India
}

Aims \& Objectives: To study the platelet counts in healthy eastern Indian population and to define the biological reference interval for the same population.

Patients/Materials \& Methods: The prospective study was conducted on 4512 subjects attending health check-ups, or coming to donate blood or platelets at Apollo Gleneagles Hospital, Kolkata, during the period of January 2019 to December 2019. Complete blood count was collected in all cases using, Advia 2120i and Beckman Coulter LH 750, automated haematology analyser.

Results: Out of 4512 subjects, 3893 were males and 619 were females. Reference range of the platelet count for Eastern Indian population was $117.49 \times 1000 / \mu \mathrm{L}$ to $371.54 \times 1000 / \mu \mathrm{L}$, the lower limit of which is below the standard reference range values. Reference range of platelet count for males and females was $118.47 \times 1000 / \mu \mathrm{L}$ to $365.07 \times 1000 / \mu \mathrm{L}$ and $114.93 \times 1000 / \mu \mathrm{L}$ to $387.85 \times 1000 / \mu \mathrm{L}$ respectively. Reference range of platelet count for younger individuals in the study population was $122.51 \times 1000 / \mu \mathrm{Lto}$ $366.09 \times 1000 / \mu \mathrm{L}$ and for elderly individuals was $111.78 \times 1000 / \mu \mathrm{L}$ to $369.70 \times 1000 / \mu \mathrm{L}$. Reference range of MPV was $6.61 \mathrm{fl}$ to $15.85 \mathrm{fl}$. Reference range of MPV for males was $6.51 \mathrm{fl}$ to $15.65 \mathrm{fl}$ and for females" reference range was $7.05 \mathrm{fl}$ to $16.31 \mathrm{fl}$. Reference limit of MPV for younger subjects was 6.49 to $15.52 \mathrm{fl}$, for the elderly it was 6.7 to $16.11 \mathrm{fl}$. Reference range of PDW for the total study population was $15.14 \%$ to $18.20 \%$. Reference range of PDW for males was $15.17 \%$ to $18.14 \%$ and for females was $15.28 \%$ to $18 \%$. Reference limit of PDW for the younger subjects was $15.06 \%$ to $18.26 \%$. For the elderly reference range of PDW was $15.30 \%$ to $18.01 \%$

Discussion \& Conclusion: Reference range of haematological parameters may differ in different populations. Therefore it is important to establish a geographic region, gender and age specific reference ranges. We have established the reference ranges for platelet parameters for the Eastern Indian population stratified by gender and age: ${ }^{*}$ Reference range of the platelet count was found to be $117.49 \times 1000 / \mu \mathrm{L}$ to $371.54 \times 1000 / \mu \mathrm{L}$, lower limit of which is below the standard reference range values. *Reference range of platelet count for males and females was found to be $118.47 \times 1000 / \mu \mathrm{L}$ to $365.07 \times 1000 / \mu \mathrm{L}$ and $114.93 \times 1000 / \mu \mathrm{L}$ to $387.85 \times 1000 / \mu \mathrm{L}$ respectively. * Reference range of platelet count for younger individuals was $122.51 \times 1000 / \mu \mathrm{L}$ to $366.09 \times 1000 / \mu \mathrm{L}$ and for elderly individuals was $111.78 \times 10000 / \mu \mathrm{L}$ to $369.70 \times 1000 / \mu \mathrm{L}$. Lower reference limit of elderly subjects is quite beneath the standard reference limit. "Mean MPV for the total study population was $10.23 \mathrm{fl}$ and reference range was $6.61 \mathrm{fl}$ to $15.85 \mathrm{fl}$. Mean MPV is higher in 
Eastern Indian population than standard values. * Platelet count was negatively related with MPV and PDW. Suggesting that with decreasing platelet count both, MPV and PDW increase.

\section{Cellular Cannibalism in Bone Marrow-Two Cases of Malignancies with Different Origins}

Surbhi Singh ${ }^{* 1}$, Amrita Saraf ${ }^{1}$, Richa Chauhan ${ }^{1}$, Vandana Arya ${ }^{1}$, Ajay Sharma $^{2}$, Mitu Shrikhande ${ }^{3}$, Jyoti Kotwal ${ }^{1}$

${ }^{1}$ Department of Hematology, ${ }^{2}$ Department of Clinical Hematology, Sir Ganga Ram Hospital, ${ }^{3}$ Department of Clinical Hematology, Fortis Flt. Lt. Rajan Dhall Hospital, New Delhi, India

Aims \& Objectives: Cellular cannibalism is recognized as an adaptive phenomenon in which one cell engulfs another living cell of its own or another type. This phenomenon has been observed in several solid tumors, however, it is rarely seen in hematological malignancies and metastatic carcinoma in bone marrow. We are reporting two such cases exhibiting cellular cannibalism in bone marrow examination.

Patients/Materials \& Methods: Case report. Case 1- A 31-year-old male was diagnosed as NPM1 mutated Acute myeloid leukemia at our center in 2019. He had relapsed within 7 months with a new FLT3ITD clone (allele ratio 0.7). He was treated with FLAG-IDA, sorafenib. Post Day +5 sorafenib marrow had shown erythroid cannibalism with $\sim 6 \%$ blasts. Subsequently, work up for pre-allogenic hematopoietic stem cell transplant showed frank relapse with $\sim 39 \%$ circulating blasts and dysplastic NRBCs (12/100 WBCs) in peripheral blood. The marrow showed $\sim 38 \%$ blasts with several showing engulfment of RBCs, erythroblasts, other blasts and neutrophils, suggestive of cellular cannibalism. Case 2- A 64 year old male presented with weight loss and epistaxis with a history of chronic alcoholism. His CBC revealed anemia, thrombocytopenia and leukoerythroblastic picture. The bone marrow showed diffuse infiltration by tumor cells almost completely replacing the marrow, arranged in cohesive clusters, sheets and many exhibiting features of cellular cannibalism seen as engulfment of RBCs, neutrophils and tumor cells itself. A final diagnosis of metastatic tumor deposits was given and further investigations revealed the diagnosis of pancreatic carcinoma with metastasis.

Discussion \& Conclusion: The phenomenon of cellular cannibalism has been observed in some cases of leukemias such as AML with myelomonocytic or monocytic differentiation and erythroleukemia. Tumor cells eating their neighbouring cells is aimed at sustaining survival and their progression in an unfavorable microenvironment. Pancreatic cancer presenting as skeletal metastases is an uncommon occurrence and these metastasized tumor cells showing cellular cannibalism could be related to the aggressiveness, invasiveness, and metastatic disposition of the malignancy.

\section{Unusual Causes of Eosinophilia: A Report of Two Pediatric Cases}

Yogalakshmi Sivaprakasam ${ }^{* 1}$, Amrita Saraf ${ }^{1}$, Richa Chauhan ${ }^{1}$, Vandana Arya ${ }^{1}$, Manas Kalra ${ }^{2}$, Sabina Langer ${ }^{1}$, Anupam Sachdeva ${ }^{2}$, Jyoti Kotwal $^{1}$

\section{${ }^{1}$ Hematology, ${ }^{2}$ Pediatric Hemato-oncology, Sir Gangaram Hospital, New delhi, India}

Aims \& Objectives: Eosinophilia has been found in association with a wide spectrum of diseases. But determining the etiology of eosinophilia is cumbersome in certain cases even after extensive workup resulting in diagnosis of idiopathic eosinophilia. Here we describe two interesting cases of pediatric eosinophilia with varied etiology and presentation.

Patients/Materials \& Methods: Case 1: A 5-year male presented with complaints of fever, suprapubic pain, frequent urination, hematuria for 15 days and past history of passing stone in urine 6 months back. He was treated with antibiotics elsewhere with relief in urinary symptoms, however, fever persisted. Personal and family history of atopy was present. Examination was uneventful. CBC showed hyperleukocytosis with marked eosinophilia (AEC:82648/ $\mu \mathrm{L}$ ). Investigations to assess organ damage and infectious workup were normal. Bone marrow study and molecular analysis were done. Bone marrow was hypercellular with myeloid preponderance and increased eosinophils and its precursors $(65 \%)$ with some showing sparse granulation and hyposegmentation with increase in mast cells. Nested RTPCR revealed FIP1L1-PDGFRA fusion gene and a diagnosis of clonal FIP1L1-PDGFRA associated hypereosinophilia was made. During follow-up the patient responded to prednisolone. Case 2: A 6-year female child presented with chronic cough, intermittent fever and past history of delayed fall of umbilical cord, neonatal sepsis, recurrent infections and neutropenia since birth. Examination revealed pallor, clubbing, lymphadenopathy, hepato- splenomegaly with bronchial breath sounds on the left side of chest. Imaging showed consolidation and cavity in the left lower lung with airway malformation. $\mathrm{CBC}$ and peripheral smear showed anemia, $9 \%$ blasts (myeloblasts on flow cytometry), severe neutropenia, eosinophilia (AEC:2029/ $\mu \mathrm{L}$ ), basophilia and monocytosis. Bone marrow was hypercellular with $8 \%$ blasts, reduced granulocytic maturation with an increase in eosinophils, eosinophilic precursors, plasma cells and dysmegakaryopoiesis. Based on clinico-pathological findings and past history, severe congenital neutropenia with progression to MDS-EB was diagnosed. NGS revealed heterogenous missense variation in exon 3 of ELANE gene. Patient improved with antibiotics and G-CSF.

Discussion \& Conclusion: The diagnosis of PDGFRA associated eosinophilia and eosinophilia related to congenital neutropenia is possible due to improving diagnostic modalities. Molecular studies in all age groups is beneficial to reach a definitive diagnosis before a diagnosis of 'idiopathic eosinophilia' is made.

\section{Association \& Correlation of Von Willebrand Factor \& Severe Aortic Stenosis in North-West Indian Population}

Anita Tahlan ${ }^{* 1}$, Sourabh Kumar ${ }^{1}$, Sreenivas Reddy ${ }^{2}$, Anshu Palta ${ }^{1}$

${ }^{1}$ Department of Pathology, ${ }^{2}$ Department of Cardiology, Government Medical College \& Hospital, Sector 32, Chandigarh, India

Aims \& Objectives: To study the association of vWF Ag levels with aortic stenosis severity.

Patients/Materials \& Methods: The patients were enrolled into three groups. Group I consisted of Group I - 40 consecutive patients diagnosed with severe aortic stenosis (AS), Group II - 40 age and sex matched patients with any other valve disease other than AS, Group III - 40 age and sex matched normal healthy controls were enrolled for the purpose of study. The vWF antigen(VWF:Ag) assay and a measurement of vWF to collagen (vWF:CB) activity, was done in all the three groups. In addition grading of severe AS was done based on the arbitrary cut off value of aortic peak velocity as, "Severe" $(4.1-5 \mathrm{~m} / \mathrm{s})$ and "Critical" $(5.1-6 \mathrm{~m} / \mathrm{s})$.

Results: In our study comparing the $31 / 40$ severe \& $9 / 40$ critical AS, the vWF antigen (vWF:Ag) levels in group I ranged from 24.4 to $206.5 \mathrm{IU}$ with a mean of $126.9 \pm 52.3 \mathrm{IU}$, in group II it ranged from 61.1 to $200.8 \mathrm{IU}$ with a mean of $135.8 \pm 42.3 \mathrm{IU}$, in group III itranged from 21 to $200 \mathrm{IU}$ with a mean of $114.1 \pm 35.4 \mathrm{IU}$. The 
statistical variation between the groups was statistically insignifi$\operatorname{cant}(p$ value $>0.05)$. The $\mathrm{vWF}: \mathrm{CB}$ assay levels ranged from 5.0 to $206.6 \%$ with a mean of $186.4 \pm 39.1 \%$ in group I. In group II it ranged from 24.6 to $200 \%$ with a mean of $141.2 \pm 62.7$. In group III it ranged from 32.1 to $200 \%$ with a mean of $157.8 \pm 64.0 \%$. The statistical variation between group I and group II was statistically insignificant ( $p$ value $>0.05$ ). The correlation of vWF:CB with severity of AS found to be statistically significant, being increased in severe AS in comparison to critical AS ( $p$ value $<0.05$ ). The ratio of vWF:Ag: vWF:CB was statistically insignificant ( $p$ value $>0.05)$.

Discussion \& Conclusion: In the present study, vWF: Ag levels were not found to be statistically different among the three groups studied. Positive correlation was seen in the vWF:CB with severity of AS. However the numbers of patients analyzed are small and larger studies are needed to study the correlation and associations better. Supporting Document:

\section{Study of Mean Platelet Volume (MPV) and Neutrophil- Lymphocyte Ratio (NLR) in Patients of Knee Osteoarthritis and Their Correlation with Disease Severity}

\author{
Jyoti Mishra $^{* 1}$, Sadhana Marwah ${ }^{2}$, Vijay Kumar ${ }^{1}$, Ajay Shukla $^{3}$
}

${ }^{1}$ Pathology, ABVIMS and Dr. RML Hospital, ${ }^{2}$ Pathology, ABVIMS and Dr.RML Hospital, ${ }^{3}$ Orthopedics, ABVIMS and Dr. RML Hospital, New delhi, India

\begin{abstract}
Aims \& Objectives: We aim to study mean platelet volume (MPV) and neutrophil -lymphocyte ratio (NLR) in patients of knee osteoarthritis and their correlation with disease severity.

Patients/Materials \& Methods: The study type was cross-sectional observational study carried out in a period of one year (Nov 2018 to March 2020). Study population included 30 diagnosed cases of knee osteoarthritis as per ACR 2016 guidelines and 30 age and sex matched controls. Blood was collected in EDTA vial for CBC (complete blood count) including MPV and NLR; ESR and in plain vial for CRP examination

Results: The values of MPV and NLR were significantly higher in patients with OA compared to age and sex matched controls and is significantly correlated with the severity of OA.NLR $>2.7$ predicted KL Grade (Grade 3/4 from KL grade 1/2) with a sensitivity of $91 \%$, and a specificity of $86 \%$. ESR and CRP were higher in cases than controls but did not correlate with the severity of OA.

Discussion \& Conclusion: It is concluded that MPV and NLR are increased in the knee osteoarthritis and did correlate with the disease severity. Therefore, it is suggested that hematological parameters like MPV and NLR can be used as diagnostic inflammatory indicators like CRP and ESR in knee OA and can be used in diagnosing severity of OA. However, study including large number of patients with long term follow up is needed for conclusive results.
\end{abstract}

\section{Metastatic Neuroblastoma to Bone Marrow and Lymph Node at Presentation: A Series of Five Cases}

Roobashri $\mathbf{M}^{* 1}$, Monisha $\mathrm{C} \mathrm{R}^{1}$, Devi Anu $\mathrm{K}^{1}$, Prabhu $\mathrm{M}^{1}$, Rakhee $\mathrm{Kar}^{1}$, Debdatta Basu ${ }^{1}$

\section{${ }^{1}$ Pathology, JIPMER, Puducherry, India}

Aims \& Objectives: To study the clinico-pathological and histomorphological features of neuroblastoma cases metastasizing to bone marrow and lymph node at presentation.
Patients/Materials \& Methods: A record based search from departmental archives over the past five years revealed a total of five patients of neuroblastoma who presented with bone marrow and/or lymph node metastasis which preceded the diagnosis of neuroblastoma. The clinicopathological, histomorphological and immunophenotypic profile of these five cases were studied in detail. Results: Five cases were primarily diagnosed in bone marrow and/or lymph node biopsy after which the intra-abdominal mass was diagnosed. Four of them were boys with ages ranging from 2 to 10 years. The most common symptoms noted among the cases were fever and lymphadenopathy with additional proptosis in two cases. Clinicians suspected either acute leukaemia or non Hodgkin lymphoma in all the five cases. Complete hemogram showed microcytic anaemia in three cases, leukoerythroblastic blood picture in one and pancytopenia in the other. Histologically small round blue cell morphology was noted in all five cases with additional rosette formation in two. One of the cases with lymph node metastasis showed prominent Azzopardi effect. On Immunohistochemistry, tumour cells were positive for synaptophysin and chromogranin and negative for LCA and CD99. In all the five cases, following the diagnosis, a search revealed the primary site in the abdomen.

Discussion \& Conclusion: Metastasis to bone marrow and lymph node may be the initial presentation for neuroblastoma and acts as a mimic, both clinically and morphologically, for acute leukaemia or lymphoma. Careful assessment of the histomorphology and judicious use of immunohistochemistry helps clinch the diagnosis.

\section{Neutrophil-to-Lymphocyte Ratio and D-Dimer Assay in Prediction of Disease Severity in Covid Positive Indian Patients in A Tertiary Care Centre}

Shalini Goel*1, Bhawna Jha ${ }^{1}$, Udayakumar DS ${ }^{1}$, Ritu Chadha ${ }^{1}$, Manish K. Singh ${ }^{2}$, Yatin Mehta ${ }^{3}$, Renu Saxena ${ }^{1}$

${ }^{1}$ Department of Hematopathology, ${ }^{2}$ Medanta Institute of Education and Research, ${ }^{3}$ Institute of Anaesthesiology and Critical care, Medanta - The Medicity hospital, Gurugram, India

Aims \& Objectives: To evaluate role of hematological parameters in prediction of disease severity and also analyze the trends of NLR and D-Dimer during its course.

Patients/Materials \& Methods: A retrospective analysis of 83 patients diagnosed with COVID-19 by RT-PCR at Medanta-the Medicity hospital in June 2020 was done. The data included neutrophil-to-lymphocyte ratio (NLR), D-Dimer, PT/APTT and platelet count. The patients were divided into 20 critical patients and 63 Noncritical patients group, based on disease severity. The parameters were compared and trends analyzed.

Results: The COVID positive cases had a mean age of 56.7 years (Range: 7-84 years) with a male:female ratio of 2.6:1. The critical group had mean age of 64.7 years (Range: 42-76), versus 54.1 years (Range: 7-84) in non-critical group. At admission, the mean NLR in the critical and non-critical group was 12.26 and 5.7. Further, the critical and non-critical group had NLR $>3.13$ in 19 cases (95\%) and 49 cases $(77.8 \%)$, respectively. On receiver operating characteristic curve (ROC) analysis, predictive ability of NLR for detection of critical patients was $\operatorname{significant}(p$ value $=0.0001$; AUC:0.779) with optimal cut-off value of 6.01 , having $85 \%$ sensitivity, $68.9 \%$ specificity and $\sim 93.5 \%$ negative predictable value (NPV). The mean D-Dimer value in critical and non-critical group on Day-4 of admission was $18.89 \mathrm{mg} / \mathrm{L}$ and $2.48 \mathrm{mg} / \mathrm{L}$. Moreover, the D-Dimer $>0.55 \mathrm{mg} / \mathrm{L}$ were seen in 17 critical cases $(85 \%)$ in contrast with 35 non-critical cases $(55.5 \%)$. On ROC analysis, the ability of D-Dimer in predicting disease severity was signifi$\operatorname{cant}(p$ value $=0.0001 ;$ AUC:0.896) with optimal cut-off value of 
$2.27 \mathrm{mg} / \mathrm{L}$, having $85 \%$ sensitivity, $76.2 \%$ specificity and NPV $94.1 \%$. On trend analysis, it was observed that the D-Dimer and NLR showed a progressive upward trend in critical patients, whereas there were more of plateau/declining values in non-critical patients. PT was mildly prolonged in 14 critical patients $(70 \%)$ and 13 non- critical patients $(40.6 \%)$. The mean platelet counts were similar in both the groups.

Discussion \& Conclusion: The study shows that the severity of the disease is more in elderly (Mean age: 64.7 years). Also, at admission NLR $>6.05$ and Day-4 D-Dimer $>2.27 \mathrm{mg} / \mathrm{L}$ are significantly predictive of disease severity and such patients should receive prompt treatment to minimize further sequel and morbidities.

\section{Spectrum of Haematological Conditions WITH Secondary Myelofibrosis: A 3 Year Institutional Study}

Vidhya V. Balasubramaniam ${ }^{* 1}$, Udaya Sundarajan ${ }^{1}$, Prabhu Manivannan ${ }^{1}$, Rakhee Kar ${ }^{1}$, Debdatta Basu ${ }^{1}$

${ }^{1}$ Pathology, JIPMER, Pondicherry, India

Aims \& Objectives: Primary myelofibrosis is a clonal myeloproliferative neoplasm, whereas secondary myelofibrosis can be associated with many disease conditions in children and adults, both reactive and neoplastic. However, its prevalence and characteristics have not been well elucidated. The aim of this study was to elucidate the spectrum of haematological conditions associated with secondary myelofibrosis.

Patients/Materials \& Methods: This was a retrospective recordbased study over a period of three years (September 2017 to September 2020). All patients with various haematological conditions with associated myelofibrosis diagnosed in bone marrow trephine biopsy were included in this study. Both Gomori reticulin and Masson trichrome stains were used to study the grade of fibrosis. Myelofibrosis was graded from Grade 0 to 3 using the WHO grading system. Grade 1 to 3 is considered as increased fibrosis, Grade 3 specifically denotes collagenisation.

Results: A total of 126 cases had secondary myelofibrosis which included $69(55 \%)$ cases of chronic myeloid leukemia, two $(02 \%)$ cases of polycythemia vera and seven cases $(06 \%)$ of myelodysplastic syndrome. Amongst acute leukemias there were 13(10\%) cases of acute lymphoblastic and five $(04 \%)$ of acute myeloid leukemia. Two cases $(02 \%)$ of Hairy cell leukemia, seven $(06 \%)$ of Hodgkins lymphoma and four cases $(03 \%)$ of Non-Hodgkin lymphoma were also encountered. Twelve cases (10\%) of Multiple myeloma and two cases $(02 \%)$ of metastatic carcinoma were seen. Amongst benign conditions two $(02 \%)$ and one case $(01 \%)$ of pure red cell aplasia and renal osteodystrophy each were encountered.

Discussion \& Conclusion: The present study throws light upon the severity and grades of myelofibrosis in a wide spectrum of various haematological conditions.
Supporting Document:

\begin{tabular}{|l|l|l|}
\hline Secondary causes & $\begin{array}{l}\text { Grade } 1 \text { and 2 } \\
\text { fibrosis }\end{array}$ & Grade 3 fibrosis \\
\hline Chronic myeloid leukemia & $76 \%$ & $24 \%$ \\
\hline Polycythemia vera & $100 \%$ & - \\
\hline Myelodysplastic syndrome & $85 \%$ & $15 \%$ \\
\hline Acute myeloid leukemia & $80 \%$ & $20 \%$ \\
\hline Acute lymphoblastic leukemia & $100 \%$ & - \\
\hline Hairy cell leukemia & - & $100 \%$ \\
\hline Hodgkin's lymphoma & $71 \%$ & $29 \%$ \\
\hline Non-Hodgkin's lymphoma & $75 \%$ & $25 \%$ \\
\hline Multiple myeloma & $100 \%$ & - \\
\hline Metastasis & $50 \%$ & $50 \%$ \\
\hline Pure red cell aplasia & $50 \%$ & $50 \%$ \\
\hline Renal osteodystrophy & - & $100 \%$ \\
\hline
\end{tabular}

\section{Spectrum of Various Clinical Indications and Diseases Diagnosed on Bone Marrow Aspiration at a Tertiary Health Care Centre in Hilly State of Himachal Pradesh During The Period 1st June 2019-30th June 2020}

Akshita Mehta ${ }^{* 1}$, Pooja Murgai $^{1}$, Anchana Gulati ${ }^{1}$, Shobha Mahindroo ${ }^{1}$, Sarita Asotra ${ }^{1}$, Lalita Negi ${ }^{1}$, Kavita Mardi ${ }^{1}$, Sudarshan K. Sharma ${ }^{1}$

\section{${ }^{1}$ Pathology, IGMC, Shimla, India}

Aims \& Objectives: To study and compare the spectrum of clinical indication and disease diagnosed on bone marrow aspiration in the hilly state of Himachal pradesh during the period 1st June 2019 - 30th June 2020.

Patients/Materials \& Methods: This is a retrospective study of 1 year duration at a tertiary health care centre in Himachal Pradesh. Patients were aged from 1 to 89 years. A total of 226 cases were studied.

Results: Out of 226 patients 46 were in the pediatric age group and 180 were adults. In pediatric, 30 male $\& 16$ female. The most common indication of bone marrow in pediatric age group was for Minimal residual disease (MRD) in patient of acute leukemia constituting 32\% (15 case).Among female children most common indication was for MRD analysis and pancytopenia $31.25 \%$ each (5 cases each). In Male children the most common indication was for MRD analysis and PUO $33.3 \%$ each (10 case each). Diagnosis: In the pediatric age group $41.3 \%$ cases(19) with various clinical indications had normal morphology. The most common diagnosis was acute leukemia constituting $34.7 \%$ cases ( 16 case) with most common leukemia being ALL(12 case); male(7 case) and female(5 case). Other common diagnosis being erythroid hyperplasia(5) and megaloblastic erythropoiesis( 4 cases). Among adult patients 91 were male and 89 were female. The most common indication in adult was lower backache or AG reversal for clinical suspicion of Multiple myeloma (MM)$16.6 \%$ (30 case). In Female the most common indication was anemia $17.9 \%$ (16 case) while among men the most common indication was AG reversal and lower back for clinical suspicion of Multiple 
myeloma $17.5 \%$ (16 case).Diagnosis-In adults $50 \%$ patients (90 case) with various clinical indications showed normal bone marrow findings. The most common diagnosis in adults was erythroid hyperplasia $13.3 \%$ ( 24 case) followed by Plasma cell dyscrasia/Multiple Myeloma $8.8 \% /(16$ case).In female the most common diagnosis was erythroid hyperplasia $15.7 \%$ (14 case) while in male the most common diagnosis was plasma cell dysplasia/MM $13.2 \%$ (12 case).

Discussion \& Conclusion: Out of 226 bone marrow aspiration procedures performed during 1 year the most common overall indication of the procedure was pancytopenia $17.6 \%$ (40 case). Lower backache or AG reversal for clinical suspicion of $\mathrm{MM}$ being the most common indication among adults and MRD analysis for acute leukemia in children. Regarding spectrum of disease, $48.2 \%$ (109 case) with various clinical indications showed normal bone marrow findings. The most commonly diagnosed condition was erythroid hyperplasia $14.6 \%$ (33 case). In children the most common diagnosis being Acute lymphoblastic leukemia (ALL) with 12 new cases and in adults the most common diagnosed condition being erythroid hyperplasia and most commonly diagnosed malignancy being Plasma cell dyscrasias/ Multiple Myeloma with 12 new cases followed by Acute Myeloid leukemia with 9 new cases.

\section{Metastatic Non-hematological Tumors in Bone Marrow}

Gauri Nakra ${ }^{* 1}$, Pooja murgai ${ }^{1}$, Anchana Gulati ${ }^{1}$

${ }^{1}$ Department of Pathology, IGMC Shimla, Shimla, India

Aims \& Objectives: The aim of the current study was to analyse the clinicohematological presentation and morphological infiltration patterns of non-haematological tumor deposits in bone marrow.

Patients/Materials \& Methods: Two years retrospective analysis of MGG stained bone marrow aspiration and peripheral blood smears and $H \& E$ stained sections of bone marrow trephine biopsy was performed at the department of pathology in a tertiary care canter (June 2018 to June 2020). The morphological pattern of tumor infiltrations and clinicohematological presentation of patients was analysed. Exclusion criteria was presence of hemtolymphyoid malignancies.

Results: In total 6 cases studied, bone marrow aspiration was not useful in all cases for the diagnosis due to dry tap or inadequate material. Age of presentation was 26 years to 73 years with a slight male preponderance. Most common clinical presentation was pallor, bony pains, loss of weight, lower backache. Most common haematological presentation was anaemia and thrombocytopenia. Marked fibrosis in the bone marrow was seen in all the cases. 2 of the cases had an unknown primary tumor while two were a known case of prostatic carcinoma, one was a known case of nasopharyngeal carcinoma and one was a known case of adenocarcinoma lung.

Discussion \& Conclusion: DISCUSSION: Bone marrow examination is routinely done for evaluation of haemato-oncological disorders. Bone marrow metastasis by non-haematological tumors is a rare finding while examining bone marrow aspirate smears/bone biopsy sections. It is usually incidental which alarms the pathologists and the clinician to look for the primary tumor. Tumors which are capable of hematogenous spread could involve bone marrow. Most common tumors which metastasis to bone marrow are carcinoma of prostate, breast and lung in adults and neuroblastoma, Ewing's sarcoma and retinoblastoma in children. Detection of bone marrow metastatic deposits determine the stage of malignancy, prognosis and treatment response. Bone marrow metastasis is usually associated with anaemia, thrombocytopenia, leucocytosis, bony pains, pathological fractures etc. The aim of the current study was to analyse the clinicohematological presentation and morphological infiltration patterns of non-haematological tumor deposits in bone marrow.
Conclusion: Bone marrow examination is a cheap, easily accessible and a reliable diagnostic tool to detect metastasis of solid tumors to bone marrow and is useful in patients with haematological abnormalities to rule out metastasis in other unsuspected cases.

Supporting Document:

\begin{tabular}{|l|l|}
\hline PERIPHERAL BLOOD FINDINGS & $\begin{array}{l}\text { NO. OF } \\
\text { CASES }\end{array}$ \\
\hline Anaemia & 4 \\
\hline Thrombocytopenia & 4 \\
\hline Leucocytosis & 1 \\
\hline Leucoerythroblastic picture & 1 \\
\hline Pancytopenia & 1 \\
\hline
\end{tabular}

\begin{tabular}{|l|l|}
\hline BONE MARROW FINDINGS & $\begin{array}{l}\text { NO. OF } \\
\text { CASES }\end{array}$ \\
\hline $\begin{array}{l}\text { Metastatic tumor deposits in both a spiration smears } \\
\text { and trephinebiopsy sections }\end{array}$ & 3 \\
\hline $\begin{array}{l}\text { Metastatic tumor deposits only on trephine biopsy } \\
\text { sections }\end{array}$ & 3 \\
\hline Fibrosis in bone marrow & 6 \\
\hline Tumor cells in clusters & 5 \\
\hline Tumor cells in sheets & 1 \\
\hline Tumors cells in glandular pattem & 4 \\
\hline
\end{tabular}

\section{Evaluation for Paroxysmal Nocturnal Haemoglobinuria by Flowcytometry in Clinically Suspected Cases in Western Rajasthan}

\author{
Abhishek Purohit ${ }^{* 1}$, Gopal Krishana Bohra ${ }^{2}$, Siddhant Passey ${ }^{3}$ \\ ${ }^{1}$ Pathology, ${ }^{2}$ Medicine, ${ }^{3}$ Undergraduate student, All India \\ Institute of Medical Sciences, Jodhpur, India
}

Aims \& Objectives: Paroxysmal nocturnal haemoglobinuria (PNH) is a clonal haematopoietic stem cell disorder resulting from acquired mutation in the X-linked phosphatidylinositol glycan class-A (PIGA) gene. Clinically, patients present with intravascular haemolysis, thrombosis, bone marrow failure, smooth muscle dystonia and rarely myelodysplastic syndrome (MDS) or acute myeloid leukaemia (AML). There is no published literature from the state of Rajasthan, hence this study was conducted to evaluate presence of PNH clone in clinically suspected cases.

Patients/Materials \& Methods: In this prospective study, cases of unexplained anaemia, pancytopenia and thrombosis as well as cases of aplastic anaemia were included. Blood samples were processed with stain-lyse-wash technique using FLAER- Alexaflour488/CD14PE/CD24-PC5.5/CD33-APC/CD45-KromeOrange panel for granulocytes and monocytes and CD59- PE/CD235a-APCAlexaF750 for red blood cells and were acquired on Beckman Coulter Navious analyser. PNH clone was defined when double negative population on two lineages were more than one percent.

Results: 104 cases were enrolled during a duration of one year (April 2019 to March 2020). Mean age was 42 years with 37 females and 67 males. Of all cases, 71, 18 and 11 cases were of unexplained thrombosis, unexplained anaemia and pancytopenia respectively. Four cases were of aplastic anaemia. Out of total 71 cases of deep vein thrombosis, 3 cases (4.2\%) showed PNH clone of double negative population $>80 \%$ on both granulocytes and monocytes. Out of 
total 18 cases of unexplained anaemia, five cases $(27.77 \%)$ showed $\mathrm{PNH}$ clone of double negative population on both granulocytes and monocytes and the clone size varied from $2.38 \%$ to more than $90 \%$. In addition, PNH clone was also identified in one case of unexplained pancytopenia and three cases of aplastic anaemia.

Discussion \& Conclusion: Present study indicates that presence of $\mathrm{PNH}$ clones is not uncommon in clinically suspected cases in Western Rajasthan region, as 12 cases out of 104 enrolled cases $(11.54 \%$ of all) demonstrated large clones for $\mathrm{PNH}$, while 10 (9.62\%) cases demonstrated smaller clones. We believe that in Western Rajasthan region, screening for $\mathrm{PNH}$ should be done routinely in all suspected cases. Considerations should be made to add PNH screening in the panel of investigations done in cases of thrombosis.

\section{Leucocyte Cytoplasmic Inclusions and Their Utility in Diagnosis of Diseases}

Sarah John ${ }^{* 1}$, Vidhyalakshmi Rangarajan ${ }^{1}$, Prabhu ${ }^{1}{ }^{1}$, Rakhee $\operatorname{Kar}^{1}$, Debdatta Basu ${ }^{1}$

${ }^{1}$ Department of Pathology, Jawaharlal Institute of Postgraduate Medical Education and Research (JIPMER), Puducherry, India

Aims \& Objectives: To highlight the significance and utility of morphological changes observed in leucocytes in peripheral blood smear examination in diagnosis of diseases.

Patients/Materials \& Methods: Peripheral blood smears from EDTA anticoagulated blood samples were stained by Leishman and/ or Giemsa stain. The smears were studied in detail and examined for qualitative changes of the white blood cells such as cytoplasmic vacuolations, abnormal granulation or pigments. The cases were followed up and the diagnostic implications of each case were studied.

Results: The series is a report of 18 cases in which morphological changes observed in leucocytes in peripheral smear examination proved to be valuable diagnostic indicators to various underlying disorders. There were six cases in which the leucocytes showed abnormal giant cytoplasmic granules. Correlating with the clinical findings, a diagnosis of Chédiak Higashi syndrome was made. Examination of the peripheral smear in four cases of neonatal sepsis showed neutrophils with golden yellow crystals in the cytoplasm which were indicative of bilirubin crystals suggesting a coexisting hyperbilirubinemia. One patient had history of fever whose peripheral smear showed brownish pigmentation in neutrophils which prompted a detailed search of the smear and an occasional gametocyte of Plasmodium falciparum was picked up. In four cases, leucocyte vacuolations encountered in routine peripheral smear examination warranted further bone marrow evaluation confirming lipid storage disorders. In a neonate with suspected mucopolysaccharidosis, large and coarse granules were observed in the lymphocytes and monocytes suggestive of Alder-Reilly anomaly. In two cases of Acute Myeloid Leukemia (AML), Auer rods were noticed in hypogranular neutrophils. Cytogenetics revealed karyotypic abnormality of $\mathrm{t}(8 ; 21)$ with fusion of RUNX1-RUNX1T1. The presence of Auer rods in neutrophils can thus serve as a useful morphologic correlation in certain karyotypic abnormalities of Acute Myeloid Leukemia.

Discussion \& Conclusion: A careful and detailed study of leucocytes in an optimally stained peripheral smear is a simple yet valuable diagnostic exercise. In proper clinical setting, these morphologic clues aid in diagnosing a variety of diseases.
Evaluation of Bone Marrow Findings of Covid-19 by Minimally Invasive Autopsies: A Single Tertiary Care Centre Experience from India

Shruti Vaswani $^{* 1}$, Abhishek Purohit ${ }^{1}$, Vikarn Vishwajeet ${ }^{1}$, Deepak Kumar $^{2}$, Deepsikha Bhanja ${ }^{1}$, Parag Vijayvargiya ${ }^{2}$, Swapnil Tripathi ${ }^{2}$, Tanuj Kanchan ${ }^{3}$, Nikhil Kothari ${ }^{4}$, Naveen Dutt ${ }^{5}$, Poonam Elhence ${ }^{1}$, Pradeep K. Bhatia ${ }^{4}$, Vijaya L. $\mathrm{Nag}^{6}$, Mahendra K. Garg ${ }^{2}$, Sanjeev Misra $^{7}$

${ }^{1}$ Pathology, ${ }^{2}$ Medicine, ${ }^{3}$ Forensic Medicine and Toxicology, ${ }^{4}$ Anaesthesia, ${ }^{5}$ Pulmonary Medicine, ${ }^{6}$ Microbiology, ${ }^{7}$ Director, All India Institure of medical sciences, Jodhpur, India

Aims \& Objectives: The 2019 novel coronavirus (2019-nCoV) or the severe acute respiratory syndrome corona virus 2 (SARS-CoV- 2), originated in Wuhan City of Hubei Province of China. In India, first confirmed case of coronavirus disease (COVID-19) was reported on January 30, 2020 and since then the virus has spread across the country. More than 7.9 million cases of COVID-19 and more than 1.19 lakh deaths have been reported in India. The pathobiology of the disease is poorly known, and significant efforts have been made to understand the disease process worldwide. Clinical autopsies are known to have a vital role in developing an understanding of the disease process. The aim of the present study was to evaluate bone marrow findings of COVID-19 by minimally invasive autopsies.

Patients/Materials \& Methods: This prospective study was conducted at All India Institute of Medical Sciences, Jodhpur. After obtaining approval from Institute's ethics committee and consent from next of kins, minimally invasive autopsies were conducted within an hour after the death. Procedures were done with all biosafety measures. The tissue specimens were kept in neutral buffered formalin for $48 \mathrm{~h}$ and then processed with standard biosafety measures. Electronic medical records were reviewed retrospectively and patients' clinical details and results of laboratory investigations were noted.

Results: In this prospective study, bone marrow biopsy procedures were done in 37 COVID-19 minimally invasive autopsies. Mean age of these cases was 61.8 years (Range, 28-85 years) and male: female ratio was 2.36. Comorbidities were observed in $25(67.5 \%)$ of all cases. Histopathological analysis revealed hypercellular, normocellular and hypocellular marrow in 5, 25 and 5 cases respectively (two biopsies were inadequate). There was marked interstitial prominence of histiocytes in 24(68.5\%) cases. Out of these, evidence of haemophagocytosis (Figure 1) was observed in 14(40\%) cases, marked increase of haemosiderin laden macrophages in 20(57.1\%) cases. There was prominence of plasma cells in $28(80 \%)$ cases.

Discussion \& Conclusion: Incorporation of minimally invasive autopsies provides an effective method to study the pathological findings in COVID-19 deaths in resource constrained settings. Histopathological findings in bone marrow suggest indirect insult to bone marrow, presumably related to circulatory and/or hyperinflammatory response to viral infections.

\section{Evaluation of Frequency of Regulatory T Cells in Cases of Oral Squamous Cell Carcinoma}

Kanishka Chaurasia $^{* 1}$, Abhishek Purohit ${ }^{1}$, Darwin Kaushal ${ }^{2}$, Kapil Soni $^{2}$, Bikram Choudhary ${ }^{2}$, Amit Goyal ${ }^{2}$

${ }^{1}$ Pathology, ${ }^{2}$ Otorhinolaryngology, All India Institute of Medical Sciences, Jodhpur, India

Aims \& Objectives: Cancer of head and neck region is a significant global health burden particularly oral squa-mous cell carcinoma. The present prospective study was aimed to detect some immunoreg- 
ulatory changes with relation to regulatory $\mathrm{T}$ cells' frequency and phenotype in blood sam-ple of these patients by flowcytometric immunophenotyping.

Patients/Materials \& Methods: This prospective study enrolled cases of biopsy proven untreated cases of oral squamous cell carcinoma attending the outpatient department of the All India Institute of Medical Sciences, Jodhpur, India. Fifteen healthy volunteers served as normal controls. Staging of the tumour was done according to the American Joint Committee on Cancer (AJCC), tumour-node-metastasis (TNM) classification. Peripheral blood samples of cases and controls were processed and analysed on Beckman Coulter Navious analyser. After excluding doublets, lymphocytes were gated with the help of forward scatter- side scatter plots and CD45-side scatter plot. Further gating with the help of CD3 and CD4 yielded T-lymphocytes. Gated T- lymphocytes (CD3 + CD4 + cells) were analysed in subsequent CD4-CD25 plot scatter plot to obtain T-regulatory cells. These CD3 + CD4 + CD25 + cells were analysed in subsequent plots (CD25-Foxp3 plot and CD25-CD127 plots) to get different T-regulatory subsets $(\mathrm{CD} 4+\mathrm{CD} 25+\mathrm{CDFoxp} 3+$ and $\mathrm{CD} 4+$ CD25 + CD127Low respectively).

Results: The age of the patient ranged from 33 to 89 years. Among 48 patients, $42(87.5 \%)$ were males while $6(12.5 \%)$ were females. Late and early stages cancer were equally seen $(50 \%$ in each category). Most common affected site was buccal mucosa (27.08\%) followed by tongue $(20.83 \%)$. There was increased proportion of T-regulatory cells e.g. CD4 + CD25, CD4 + CD25 + Fox P3, CD4 + CD25 + CD127low in advanced stage of oral squamous cell carcinoma.

Discussion \& Conclusion: Present study emphasizes that the regulatory $\mathrm{T}$ cells are increased in OSCC patients. It clearly signifies the difference in their phenotypic and functional characteristics in the peripheral circulation of OSCC patients. The characterization of the Treg subpopulation could form a strong base in development of immunological targets for therapeutic management of cancer patients.

\section{Gelatinous Transformation of Bone Marrow in an HIV- Positive Paediatric Patient}

Rashmi Rajora*1, Shruti Vaswani ${ }^{1}$, Mohammed Ramzan ${ }^{2}$, Siyaram Didel $^{2}$, Abhishek Purohit ${ }^{1}$

${ }^{1}$ Pathology, ${ }^{2}$ Paediatrics, All India Institute of Medical Sciences, Jodhpur, India

Aims \& Objectives: The aim of this study was to look for causes of pancytopenia in a HIV positive paediatric patient which was found to be gelatinous marrow transformation.

Patients/Materials \& Methods: A 14 years old male child presented with two-month history of pain abdomen with bouts of vomiting and loose stools. Patient had also loss of appetite and weight since last two years. Medical history revealed him to be HIV-positive and on antiretroviral therapy since last one year. He had also received two courses of antitubercular therapy in the past. On examination, he had cachexic look with presence of pallor, pedal oedema, supraclavicular lymphadenopathy and moderate hepatosplenomegaly.

Results: On laboratory investigations, complete haemogram revealed bicytopenia (Haemoglobin: $8.8 \mathrm{~g} / \mathrm{dL}$, total leucocyte count: $8,500 / \mu \mathrm{L}$, platelet count: $93,000 / \mu \mathrm{L})$. Bone-marrow aspirate smears revealed hypocellular marrow fragments with deposition of extracellular gelatinous material (Figure). Bone biopsy revealed hypocellular marrow with suppression of haematopoiesis, adipose cell atrophy and deposition of gelatinous substance. There was no increase of plasma cells or evidence of karyorrhectic debris. Gelatinous substance revealed positivity for Alcian blue stain (Figure).

Discussion \& Conclusion: Gelatinous transformation of bone marrow (GMT) is a non-specific yet rare disorder of bone marrow of unknown pathogenesis. The deposited substance is mucopolysaccharides histochemically rich in hyaluronic acid. It is a morphologic sign of a severe generalized illness of a patient and common causes are anorexia nervosa, acute febrile states, AIDS, alcoholism and malignancies.

\section{Utility of Perforin Assay in the Era of Next Generation Sequencing}

Sabina Langer ${ }^{1}$, Divij Sachdeva ${ }^{* 2}$, Aditi Kundoo ${ }^{1}$, Jyoti Kotwal ${ }^{1}$, Anupam Sachdeva ${ }^{3}$

${ }^{1}$ Hematology, Sir Ganga Ram Hospital, Delhi, ${ }^{2}$ Paediatric hematooncology, Sir Ganga Ram Hospital, Delhi, ${ }^{3}$ Paediatric Hemato- oncology, Sir Ganga Ram Hospital, Delhi, India

Aims \& Objectives: Flowcytometric perforin assay in 2 cases with PRF1 mutations.

Patients/Materials \& Methods: Two cases of familial HLH, a 6 month old boy and 1 year old girl were admitted in the hospital. After the clinical profile and biochemical tests, a suspicion of primary HLH was made and next generation sequencing (NGS) for HLH panel was indicated. Also flowcytometric assessment for perforin expression on NK cells was done. The flowcytometric antibody panel used for perforin assay included CD45, CD4, CD3, CD8, CD56 \& perforin. The samples were processed using stain-lyse- wash technique and acquired on BD FACs Canto and analysed using DIVA software.

Results: In the 6 month old, The NGS panel was reported PRF1 (-) mutation in location Exon3, variant.1304C > T, homozygous familial HLH-2 with autosomal recessive inheritance pattern and was classified likely pathogenic. The flowcytometric assessment of perforin expression on NK cells was reduced to $-1.5 \%$ against the healthy control of $86 \%$.Thus reconfirming the NGS findings. The patient was treated as per HLH2 004 protocol and tested for HLA crossmatch to plan bone marrow transplant with sibling. However the child succumbed to disease. In the 1 year old, the mutation panel for primary HLH was reported to show PRF1 (-) mutation exon2,c.386G > C(p.Trp129ser), homozygous, familial-HLH autosomal recessive. Perforin expression on NK cells show reduced expression (4.1\%).The child was treated with HLH-2004 protocol and etoposide injection.

Discussion \& Conclusion: Back ground: There are currently 7 known genes responsible for primary HLH and the related pigmentary disorders (Griscelli syndrome type 2, Chediak Higashi syndrome, and Hermansky Pudak syndrome type 2). These genes include PRF1, which encodes the pore-forming protein perforin, and UNC13D, STX11, STXBP2, RAB27A, LYST, andAP3B1. It has been established that low perforin expression is extremely sensitive and specific for the detection of primary HLH when associated with biallelic PRF1 mutations. Discussion: With NGS panel it has become rapid and feasible to send for predesigned mutation panel for primary HLH. The flowcytometry on these cases further helped to corroborate by demonstrating reduced perforin expression in cases reported to show homozygous PRF1 mutation reported as likely cause of the HLH. The flowcytometry should therefore be the part of diagnostic algorithm of primary HLH.This will be more useful in cases of compound heterozygosity or uncommon variants. The addition of CD107a along with perforin in the flowcytometry panel can further help to NK cell activity and other familial HLH mutations. Conclusion: Clinico-biochemical presentation, NGS panel for primary HLH and Flowcytometry immunophenotyping can act as tripod to rest the diagnosis in the cases of familial HLH.

Keywords: Familial HLH,Perforin mutation,Perforin expression. 


\section{Hemophagocytic Lymphohistiocytosis: Entity not Explored Well}

Vindhyaprabha Hegde ${ }^{* 1}$, Sarika Singh ${ }^{1},{ }^{1}$, Parul Tanwar ${ }^{1}$, Pallavi Sinha $^{1}$, Suresh Kumar ${ }^{1}$, M K Daga ${ }^{1}$, Sunita Aggarwal ${ }^{1}$, Urmila Jhamb $^{1}$, Mukta Mantan ${ }^{1}$

\section{${ }^{1}$ Maulana Azad Medical College, New Delhi, India}

Aims \& Objectives: This study was conducted to assess the clinichematological presentation and etiology of HLH.

Patients/Materials \& Methods: A retrospective study was conducted over a period of 18 months (January 2019- July 2020) in the department of Pathology Maulana Azad Medical College New Delhi. All cases showing features of hemophagocytosis in the bone marrow were analyzed. Hemogram was conducted on Sysmex 2000i Automated Hematology analyzer. Giemsa stained peripheral blood smear; bone marrow examination smears \& sections were assessed. Biochemical evaluation was done for lipid profile, serum ferritin, fibrinogen and serum electrolytes along with radiological work up. Results: 22 patients with hemophagocytosis in the bone marrow were identified, out of 1100 bone marrows samples examined, comprising of 10 adults \& 11 pediatric patients. Age of presentation ranged from 3 to 75 years, with 11 males \& 11 females. Four cases showed normocellular marrow with histiocytosis, 4 cases showed presence of neoplastic process, 3 cases with suspicion of chronic inflammatory pathology with features of partially treated nutrition deficiency in one case, 3 cases showed megaloblastic anemia, 2 cases showed reactive marrow, 2 cases showed pancytopenia, 1 case each with ITP, Malarial parasite infestation, thrombocytopenia with normocellular marrow, pancytopenia and bilineage dysplasia were observed. Final diagnosis was made based on revised diagnostic criteria for HLH.

Discussion \& Conclusion: Pyrexia, cytopenia, hyperferritinemia with hemophagocytosis are very reliable markers of HLH. Since it is a fatal condition of obscure \& varied etiology involving the multiple organs, prompt diagnosis and high index of suspicion is a must to save the patients from near fatal condition.

\section{Varied Presentations of Essential Thrombocythemia: A Case Series}

Prachi Mohapatra ${ }^{* 1}$, Kailash Kumar ${ }^{1}$, Nilesh Kumar ${ }^{1}$, Satya P. Mahapatra $^{1}$, Ashis Gupta ${ }^{1}$, Nidhi Singh ${ }^{1}$

${ }^{1}$ Internal Medicine, Institute of Medical Sciences, BHU, Varanasi, India

Aims \& Objectives: To analyze different clinical presentations of Essential Thrombocythemia (ET).

Patients/Materials \& Methods: Case 1: 56 yr F with no co morbidities presented with throbbing left sided headache, nausea, photophobia $\times 4$ months; burning, redness \& swelling in tips of fingers \& toes $\times 4$ months. Examination- Vitals WNL, No pallor, mild splenomegaly. NCCT head WNL.Hb-12 g/dl, TLC-7800/ul, Plt.8.1L/ul. GBP showed thrombocytosis. Fe/TIBC/Ferritin/qCRP-WNL. JAK2V617F + ve. Bone marrow biopsy (BMB) s/o ET. Diagnosis-ET with migraine. Treatment-Aspirin $(75 \mathrm{mg} / \mathrm{d})$, Propranolol $(40 \mathrm{mg} / \mathrm{d})$. Case 2: $52 \mathrm{yr} \mathrm{M}$ with no co morbidities presented with heaviness in left upper abdomen $\times 1$ year, melaena $\times 10$ days. ExaminationVitals WNL, mild pallor, splenomegaly $(10 \mathrm{~cm}) . \mathrm{Hb}-10.2 \mathrm{~g} / \mathrm{dl}$, TLC10,030/ul, Plt.-7.1L/ul. GBP- normocytic normochromic anemia, thrombocytosis. Fe/TIBC/Ferritin/qCRP- WNL. PT/INR16/1.14,LFT/RFT- WNL, Stool occult blood +ve. UGI Endoscopy- 3 columns large, 2 column small esophageal varices. USG, color doppler abdomen-massive splenomegaly, EHPVO (chronic portal vein thrombosis with portal cavernoma formation). JAK2V617F +ve.
BMB s/o ET. Diagnosis-ET with EHPVO (d/t chronic portal vein thrombosis) with Portal Hypertension. Treatment-Endoscopic variceal ligation, Propranolol(40 mg/d), Hydroxyurea(1 g/d), Warfarin $(2 \mathrm{mg} /$ d). Case 3: $40 \mathrm{yr} \mathrm{M}$ with no comorbidities presented with severe left sided chest pain radiating to left shoulder with sweating $\times 2 \mathrm{~h}$. Examination- P 124/m, BP 134/90 mm Hg. ECG- ST elevation in V2V6 s/o anterior wall STEMI. Trop-I $>10$. CAG- $80 \%$ stenosis in proximal LAD. Hb- 14.6 g/dl, TLC- 14,350/ul, Plt.-10.2L/ul. GBPthrombocytosis, normal RBCs,WBCs. JAK2V617F + ve. BMB s/o ET. Diagnosis- ET with anterior wall STEMI. Treatment-PCI with stent to LAD, LMWH $\times 5 \mathrm{~d}, \operatorname{Aspirin}(150 \mathrm{mg})+$ clopidogrel(75 mg), Atorvastatin(40 mg), Hydroxyurea( $1 \mathrm{~g} / \mathrm{d})$. Case 4: $42 \mathrm{yr}$ $\mathrm{F}$ with no comorbidities presented with bleeding per rectum $\times 4$ days, gum bleeding $\times 2$ days. Examination- Vitals WNL.No pallor, LN, organomegaly. Proctoscopy- grade 2 internal hemorrhoids. Hb- 11 g/dl,TLC- 9800/ul, Plt.-18L/ul. GBP-marked thrombocytosis with multiple platelet clumps. PT/INR/aPTT- WNL. JAK2V617F -ve,CALR + ve. BMB s/o ET. vWF Ag- 75.2\%(normal), vWF Ristocetin cofactor assay(vWF Rco)-30\%(decreased), vWF Rco/vWF Ag- 0.39(decreased). Diagnosis- ET with acquired von Willebrand Disease. Treatment-Hydroxyurea $(1 \mathrm{~g} / \mathrm{d})$, tranexamic $\operatorname{acid}(1.5 \mathrm{~g} / \mathrm{d})$.

Results: Essential Thrombocythemia can have diverse clinical presentations ranging from asymptomatic, minor vaso-occlusive symptoms to life threatening thrombosis and even bleeding.

Discussion \& Conclusion: ET is a clonal myeloproliferative disorder characterized by excessive production of platelets. Mostly the patients are asymptomatic and the disease course is indolent, but it can present with major thrombotic complications and even bleeding. Early recognition and prompt management is life saving.

\section{Clinical Spectrum and Treament Response in Patient of CML in Correlation with ABO and RH Blood Group}

\author{
Mukti P. Meher ${ }^{* 1}$, Lalit P. Meena ${ }^{2}$, Saumya Gupta ${ }^{3}$
}

${ }^{1}$ General Medicine, Institute of Medical Sciences, Banaras HIndu University, ${ }^{2}$ GENERAL MEDICINE, IMS BHU, ${ }^{3}$ General Medicine, Institute of Medical sciences,BHU, Varanasi, India

Aims \& Objectives: (1) Distribution of CML in ABO blood group. (2) Clinical spectrum of CML in different blood group. (3) Treatment response of CML patient in correlation with $\mathrm{ABO}$ and anti $\mathrm{D}$ blood groups.

Patients/Materials \& Methods: All the patients included in the study were diagnosed on the basis of peripheral smears and bone marrow aspiration findings. RT-PCR and Cytogenetic analysis Blood grouping and $\mathrm{Rh}$ typing of each patient was done at the initiation of therapy. Patients were followed up using the. $\mathrm{CBC}$ and peripheral blood smear examination, RT-PCR for BCR-ABL. Symptoms of each patient were noted at initial presentation.

Results: Out of 100 included patients 58 were male and 42 were female patients.It was observed that $45(45 \%)$ patients were having $\mathrm{B}+$ blood group. $33 \%$ patients were having $\mathrm{O}+$ blood group, followed by $\mathrm{A}+(10 \%), \mathrm{AB}+(8 \%)$, $\mathrm{A}-(2 \%)$, B- $(1 \%)$ and $\mathrm{AB}-$ (1\%).Around $43.64 \%$ study subjects with $\mathrm{O}+$ blood groups showed complete cytogenetic response, followed by $\mathrm{B}+(41.82 \%)$, $\mathrm{A}+(10.91), \mathrm{A}-(1.82)$ and $\mathrm{AB}+(1.82)$. Equal number of patients (40\% each) with $\mathrm{O}+$ and $\mathrm{B}+$ blood groups, followed by $\mathrm{A}+(20 \%)$ showed undetectable BCR-ABL/ABLratio (\%). $75 \%$ patients showed CHR and 25\% showed PHR. Patients with B + and $\mathrm{O}+$ blood groups $(41.33 \%)$ showed complete haematological response, by.. It was observed that maximum number of patients suffered from symptom of abdominal mass (37\%),43.24\% patients 
with $\mathrm{B}+$ blood group showed abdominal mass, followed by $\mathrm{O}+(35.13 \%), \mathrm{A}+$ and $\mathrm{AB}+(8.11 \%$ each $)$.

Discussion \& Conclusion: The present study revealed higher association between CML and blood group $\mathrm{B}$ and $\mathrm{O}$, persons having $\mathrm{Rh}$ negative factor were less prone to CML. It was observed that study subjects with $\mathrm{B}+$ and $\mathrm{O}+$ blood groups showed better cytogenetic, molecular and haematological responses as compared to patients with other blood groups at 6 and 12 months of treatment with Imitanib.

\section{Masked Polycythemia Vera: A Diagnostic Dilemma \\ Nimisha Devpura ${ }^{* 1}$, Parul Gautam ${ }^{1}$, Naveen Gupta ${ }^{2}$, Anjana Mittal ${ }^{1}$, M. L. Yadav ${ }^{1}$ \\ ${ }^{1}$ Pathology, ${ }^{2}$ Clinical Haematology, Mahatma Gandhi Medical College and Hospital, Jaipur, India}

Aims \& Objectives: Polycythemia vera (PV) is one of the myeloproliferative neoplasms and its diagnosis requires elevated hemoglobin level ( $>16.5 \mathrm{mg} / \mathrm{dL}$ in men and $>16 \mathrm{mg} / \mathrm{dL}$ in women), bone marrow characteristics of PV (hypercellularity for age with trilineage development), and presence of JAK2 (Janus kinase 2) changes or diminutive erythropoietin level, yet there exists a subset of patients with standard hemoglobin and haematocrit either due to hemodilution, nutritional anemia or any other secondary cause but these patients still might have underlying PV. To timely diagnose masked polycythemia vera cases, especially in cases with iron deficiency anaemia which is very common in our country.JAK 2 differentiates polycythemia vera from reactive causes; however it cannot differentiate between BCR-ABL negative myeloproliferative neoplasms. Here comes the role of correct bone marrow assessment to differentiate between essential thrombocythemia and primary myelofibrosis from PV.

Patients/Materials \& Methods: We present to you three cases with different clinical findings which presented with normal haemoglobin level but were later diagnosed with masked PV. Eligibility criteria included the presence of clinical and laboratory parameters when considering further diagnostic workup emphasizing PV versus secondary erythrocytosis, JAK2V617F mutation, erythropoietin levels and bone marrow biopsies.

Results: In all the three patients diagnosed with masked polycythemia vera, Bone marrow biopsy features were consistent with the diagnosis of PV by showing age adjusted hypercellularity due to Panmyelosis including an increased number of megakaryocytes and minor increase in Bone marrow Reticulin fibrosis (Grade I).

Discussion \& Conclusion: Physicians ought to know about clinical indications of PV. There should be extensive workup in a patient with unusual site thrombosis including JAK2 mutation analysis even with normal blood counts. Consideration of bone marrow biopsy for the final diagnosis for masked PV. Masked PV patients receive less intense treatment due to delayed diagnosis leading to shortened survival as compared to overt PV.

\section{Evaluation of Visual Evoked Potential in Cases of Chronic Leukemias}

Avishkar Agrawal $^{* 1}$, Sunita Aggarwal ${ }^{1}$, Jawahar L. Goyal ${ }^{2}$, Sandeep Garg $^{1}$

${ }^{1}$ General Medicine, Maulana Azad Medical College \& LNH, ${ }^{2}$ Ophthalmology, Maulana Azad Medical College and Guru Nanak Eye Centre, New Delhi, India

Aims \& Objectives: (1) To evaluate the visual function by Visual Evoked Potential (VEP) in Chronic Myeloid Leukemia (CML) and determine prevalence of ophthalmic manifestations at the time of diagnosis. (2) To see the association between the ophthalmic findings and the hematological parameters.

Patients/Materials \& Methods: A case control study with 20 newly diagnosed cases of CML who presented in Lok Nayak Hospital, New delhi with age and sex-matched controls were taken. History and examination with ophthalmological evaluation was done. VEP of all the subject to objectively evaluate the visual pathway was performed. Abnormal VEP was defined as either prolonged latency ( $>110 \mathrm{~ms})$ or low amplitude $(<5 \mathrm{mV})$ of P100 wave. Complete external and internal opthalmic examination was done. Routine hematological test including hemogram, Tridot, KFT, LFT BCR/ABL analysis and bone marrow biopsy was done. Ultrasound of Spleen and Liver was also done.

Results: Sixty percent cases were in age-group of 31-50 years and $45 \%$ had symptoms of duration 1-3 months. The most common findings were fatigue (80\%), lump (75\%), Pallor (95\%), Splenomegaly $(100 \%)$. Average size of spleen and liver size was $17.83 \pm 1.6 \mathrm{~cm}$ and $14.9 \pm 1.4 \mathrm{~cm}$. While TLC \& blasts were significantly raised to values of $1,77,755 \pm 1,18,921 / \mathrm{mm} 3$ and $8.6 \pm 12.28 \%$ respectively, hemoglobin was low with mean of $11.23 \pm 1.96 \mathrm{~g} / \mathrm{dl}$. There were $70 \%$ cases in chronic while $15 \%$ each in accelerated and blast phase. All cases were positive for BCR/ABL mutation and negative for Hepatitis B/C and HIV. Only $30 \%$ of the cases had ophthalmic symptoms at diagnosis with most common being diminution of vision and only $5 \%$ had visual impairment of mild category $(>6 / 12)$. However, a significant $75 \%(15 / 20)$ cases had abnormal VEP. Most of abnormal VEP was associated with prolonged latency than a low amplitude at diagnosis. Also,60\% of cases had abnormal retinal examinations with most common finding being retinal hemorrhage, cottonwool spots, optic disc edema, roth spots. Rest of ophthalmic examination was normal. The mean hemoglobin was $11.02 \pm 1.78 \mathrm{~g} / \mathrm{dl}$ and mean TLC was $203646.67 \pm 125228.07 /$ $\mathrm{mm} 3$ in those with abnormal VEP compared to $11.86 \pm 2.56$ and $1,00,080 \pm 39,027.3$ respectively in those with normal VEP. The proportion of blasts, promyelocyte, metamyelocyte, myelocyte and platelets was-10.2 $\pm 13.79 \%, \quad 2.6 \pm 3.22 \%, \quad 2.33 \pm 2.44 \%$, $2.2 \pm 5.07 \%$ and $2.16 \pm 1.011 \mathrm{lac} / \mathrm{mL}$ respectively.

Discussion \& Conclusion: It was seen, a majority of patients had underlying abnormality in visual function however were asymptomatic with normal visual acuity at the time of diagnosis. Most of these patients also had retinal abnormalities at this asymptomatic stage. Hence, a baseline ophthalmological evaluation should be considered in evaluation of all patients at diagnosis. This abnormality was associated with high TLC, proportion of immature cells and low with hemoglobin. The derangement in latency more than amplitude points towards a demyelinating mechanism.

\section{Blastoid Variant of Mantle Cell Lymphoma: Imperative Role of IHC \& Flowcytometry in Rare Cases}

Anshum Bhalla*1, Arti Trehan ${ }^{2}$, Juhi Sondhi ${ }^{2}$, Kanwaljeet Singh ${ }^{2}$, Tathagata Chatterjee ${ }^{3}$

${ }^{1}$ Department of Pathology, Military Hospital, Mathura, ${ }^{2}$ Department of Pathology, ${ }^{3}$ Prof and Head, Dept of Lab Sciences and Molecular Medicine, Army Hospital R and R, Delhi, India

Aims \& Objectives: Mantle cell lymphoma, blastoid variant (BMCL), is a very rare type of non-Hodgkin's lymphoma exhibiting an aggressive clinical course.Distinction from acute lymphoblastic leukemia, B cell prolymphocytic leukemia (B-PLL) and leukemic phase of large cell lymphoma can be a diagnostic challenge. We aim to highlight the utility of cytomorphologic examination combined with 
flowcytometric (FCM) analysis to administer correct diagnosis and improve prognosis.

Patients/Materials \& Methods: Our patient was a 50 year old serving soldier known case of Diabetes Mellitus presenting with 2 months history of fever, generalized weakness and loss of weight. $\mathrm{He}$ was provisionally diagnosed as a case of Acute Leukemia on peripheral smear and was transferred to AHRR. After urgent and extensive work up in the form of cytomorphology and FCM, patient was diagnosed as a case of blastoid variant of mantle cell lymphoma (B-MCL). However within 2 days of admission, patient developed cardiac arrest and couldn't be revived. Postmortem was conducted.

Results: Antemortem clinical examination revealed cervical lymphadenopathy with massive splenomegaly. The lab findings showed a hemoglobin of $7.1 \mathrm{~g}, \mathrm{dl}$, TLC of 56,800 cells/cu mm, platelets value of $30,000 \mathrm{cu} \mathrm{mm}$. FCM on peripheral smear exhibited a positive CD 20 and CD5 and negative CD23 and Tdt suggestive of mature B cell neoplasm likely B-MCL. Post mortem salient gross findings showed hemorrhagic pleural and pericardial effusion with ascites, massively enlarged spleen with nodules and wedge shaped infarcts and multiple enlarged lymph nodes in mediastinum, perihilar, retroperitoneal and peripancreatic area associated with fish flesh appearance on cut surface.Microscopic examination of these enlarged lymph nodes exhibited small to intermediate size lymphoid cells which were CD20, CD5 and cyclin D1 positive and CD23 negative. Similar tumor deposits were noted in bone marrow, spleen, small and large intestine and pancreas.

Discussion \& Conclusion: B-MCL is a rare and clinically more aggressive variant than typical mantle cell lymphoma. Immunophenotypically, the conventional type and B-MCL is characterized by coexpression of CD5 and CD20 and lacking CD23.It is difficult to diagnose patients with B-MCL correctly without immunophenotyping. The present case demonstrates that cytomorphology combined with FCM analysis can be very helpful for timely diagnosis and further management protocol.

\section{JAK2V617F Exon 14 Mutation Driven Polycythemia Vera in an Asymptomatic 12-Year-Old Girl}

\author{
Arumugom Archana ${ }^{* 1}$, Jaikumar G. Ramamoorthy ${ }^{1}$, Venkatraman
} Ranjith Kumar ${ }^{1}$, Thirumurugesan Shapnalakshmi ${ }^{1}$

\section{${ }^{1}$ Pediatrics, JIPMER, Puducherry, India}

Aims \& Objectives: Polycythemia vera (PV) is a myeloproliferative disorder with very rare incidence among children. We report a 12 year old girl who had persistent erythrocytosis, thrombocytosis and hepatosplenomegaly who was otherwise asymptomatic.

Patients/Materials \& Methods: A 12 year old female child, first born of second degree consanguineous marriage, presented to us with persistently elevated haemoglobin and platelets on routine evaluation. History and examination was not suggestive of chronic lung disease, cyanotic heart disease, renal involvement, sleep apnoea or chronic medication use.

Results: Hemogram revealed: haemoglobin $=19.3 \mathrm{gm} / \mathrm{dl}$, haematocrit $=57.9 \%$, leucocytosis $(11060 / \mathrm{mm} 3)$, neutrophilia $(6600 / \mathrm{mm} 3)$ and thrombocytosis $(1116000 / \mathrm{mm} 3)$. Peripheral smear showed erythrocytosis with marked thrombocytosis. The following investigations: Ultrasonogram abdomen, chest roentgenogram, hemoglobin electrophoresis and echocardiogram were normal.Serum erythropoietin was low (1.02 IU/L).Reverse transcription PCR was not able to identify BCR-ABL transcripts. PCR could identify a somatic point mutation in exon 14 at residue 617 of JAK2 gene. A diagnosis of PV was made as all the 3 major criteria (elevated hematocrit, hypercellular marrow and JAK2 mutation) as per the World Health Organization (WHO) classification 2017. As the child had low risk disease (age $<65$ years and absence of thrombotic event) and asymptomatic without evidence of acquired von-Willebrand disease, anti-platelet dose aspirin was initiated. During the 2 years thrombotic or bleeding episodes, cutaneous symptoms and symptoms related to acute leukemia were not observed.

Discussion \& Conclusion: JAK2 mutation is observed in $95 \%$ of adults with PV. JAK2 mutation is observed in 30\% children with PV. The percentage of children positive for exon 14 mutation is very low as compared to adults, but that of exon 12 is identical. The understanding about this entity in children is very minimal as the reported cases are far and few. The median age of presentation in childhood is 11-12 years.Nearly $50 \%$ are incidentally found during evaluation for minor aliments as in our index case. The reason for lower incidence of symptomatic childhood PV in comparison to adults in still not well understood. Venesection was never performed as symptoms related to hyperviscosity were absent in our index patient. Only $50 \%$ of cases reported in literature have utilised venesection in management of childhood PV as majority are asymptomatic and doing these invasive procedures in children is deferred till adulthood unless symptomatic. To conclude the diagnosis of primary PV is established in children only after ruling out other secondary causes of polycythemia. JAK 2 mutation is observed only in one-third of children with PV. Nearly $50 \%$ of children with PV are asymptomatic. Venesection will be required in management in half of these children.

\section{Chronic Myeloid Leukemia Presenting as Myeloid Sarcoma: Rare Complication of Common Malignancy}

Bhavana Mastebhakti $^{* 1}$, Ranvijay Singh ${ }^{1}$, Avishkar Agrawal ${ }^{1}$, Sunita Aggarwal ${ }^{1}$, Praveen Bharti ${ }^{1}$, Sarika Singh ${ }^{2}$

\section{${ }^{1}$ General Medicine, ${ }^{2}$ Pathology, Maulana Azad Medical College} and Lok Nayak Hospital, New Delhi, India

Aims \& Objectives: Extramedullary blast crisis (Myeloid sarcoma or Chloroma) in chronic myeloid leukemia (CML) is a rare phenomenon and represents infiltration of leukemic blasts in areas other than bone marrow. The most common sites of extra medullary involvement by CML are lymph nodes, bones and CNS. Myeloid sarcoma as an initial presentation is commonly associated with blast and accelerated phase. We herein present two case reports of myeloid sarcoma in chronic phase of CML.

Patients/Materials \& Methods: CASE REPORT I-A 55-year-old male presented with generalized lymphadenopathy in the left cervical, left axillary and right inguinal region along with splenomegaly for 3 months. CASE REPORT II- A 35-year-old male a diagnosed case of $\mathrm{CML}$ in $\mathrm{CP}$ on Imatinib therapy for 4 years presented with features suggestive of compressive myelopathy.

Results: INVESTIGATIONS I-were suggestive of NCNC anemia, leukocytosis with myeloid bulge showing $4 \%$ blasts and adequate platelets in the peripheral smear with bone marrow examination suggestive of myeloproliferative neoplasm with $4 \%$ blasts and grade I myelofibrosis. BCR-ABL was positive. Lymph node biopsy showed leukemic infiltration with occasional CD34 + suggestive of myeloid sarcoma. The patient was treated with high dose imatinib and cytoreductive therapy after which patient got improved. INVESTIGATIONS II- Peripheral smear was suggestive of CML in CP with bicytopenia with leukocytosis with $5 \%$ blasts \& bone marrow examination was suggestive of grade 2 myelofibrosis with $<5 \%$ CD34 + cells. MRI Spine was suggestive of diffuse leukemic infiltration of the vertebral column with D10-L3 epidural myeloid sarcoma. The biopsy following decompression surgery with laminectomy was suggestive of myeloid sarcoma. The patient was treated with chemotherapy with high dose imatinib and steroids. Discussion \& Conclusion: Myeloid sarcoma is a rare clinical 
presentation of CML (percentage not documented in literature). It is more common in AML (3\% to 5\%). Treatment includes chemotherapy, radiotherapy and/or surgical decompression. Early diagnosis and intervention are the mainstay of therapy for long term survival.

\section{Application of Novel Research Parameters of Cell Population Densities on Sysmex: XN 3000 in Cases of Myelodysplastic Syndrome/Myeloproliferative Neoplasm (MDS/MPN)}

\section{Aditi Kundoo*1, Sabina Langer ${ }^{1}$, Jyoti Kotwal ${ }^{1}$ \\ ${ }^{1}$ Department of Hematology, Sir Ganga Ram Hospital, New Delhi, India}

Aims \& Objectives: To analyse and compare the new research parameters of granulocytes (NE-SSC, NE-SFL, NE-FSC, NE-WX, NE-WY, NE-WZ, MO-X, MO-Y\& MO-Z) in peripheral blood of the patients of MDS/MPN, megaloblastic anaemia and normal control sample.

Patients/Materials \& Methods: The novel research parameters from the peripheral blood of the three groups analysed in Sysmex $-\mathrm{XN}$ 3000 were retrieved and compared. The level of significance of these parameters in differentiating the three subgroups was calculated by the ANOVA test.

Results: Statistically significant results $(p<0.05)$ were found in the four new research parameter (NE-SSC, NE-FSC, NE-WX \& MO-X) between the samples of MDS/MPN, megaloblastic anaemia and normal control (refer to image). The ROC curve for each parameter was prepared by calculating the positive and negative results and comparing them with normal reference range published by Buoro et al.

Discussion \& Conclusion: With the advancement in haematology analyzers, novel research parameters characterizing different subgroups of leucocytes i.e. cell population data (CPD) are now available. The Sysmex XN 3000 can analyze 22 new research parameters such as NEW-X, NE-SSC, MO-X etc. These parameters depend on cell granularity, reactivity, fluorescence intensity and cell width dispersion among various other parameters. Myelodysplastic syndrome/Myeloproliferative neoplasm (MDS/MPN) constitutes a subcategory of proliferative disorders associated with dysplasia(s which can be mimicked by various reactive causes of dysplasia such as megaloblastic anaemia, viral infections, toxins/drugs etc.

Conclusion: These novel parameters can supplement our morphological findings and can be used as supportive feature. The new parameters help in the study of cell complexity, width of dispersion and fluorescence intensity among various leucocytes and can provide additional information to help in distinguishing MDS/MPNs like CMML, JMML and atypical CML from reactive samples or mimickers in the peripheral blood.

Keywords: Sysmex-XN-3000, Novel research parameters, MDS/ MPN,CPDs, Dysplasia.

Supporting Document:

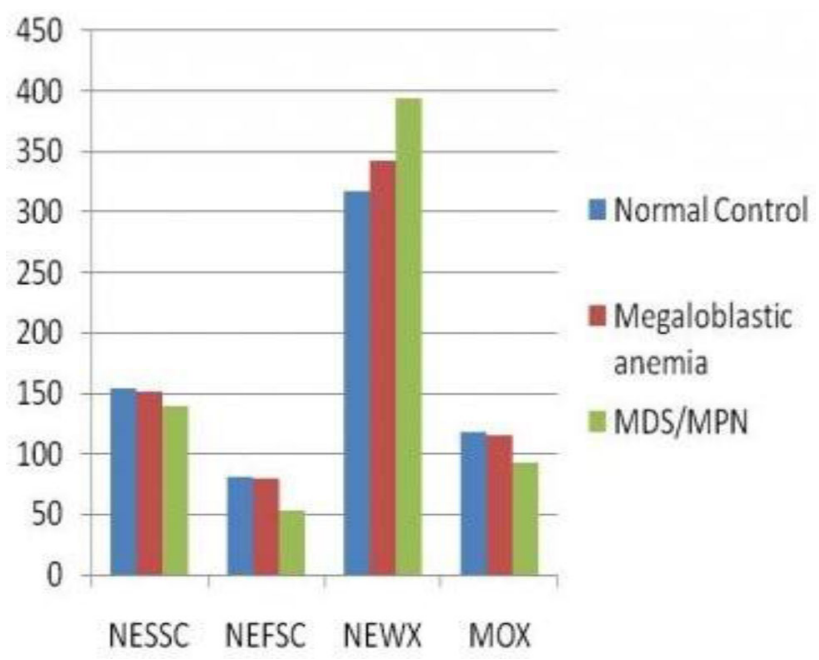

\section{Evaluation of Visual Evoked Potential in Treated Chronic Myeloid Leukemia}

Avishkar Agrawal ${ }^{* 1}$, Sunita Aggarwal ${ }^{1}$, Jawahar L. Goyal ${ }^{2}$, Sandeep Garg $^{1}$

${ }^{1}$ General Medicine, Maulana Azad Medical College \& LNH, ${ }^{2}$ Opthalmology, Maulana Azad Medical College \& Guru Nanak Eye Center, New Delhi, India

Aims \& Objectives: To see response to chemotherapy and correlate with hematological parameters.

Patients/Materials \& Methods: A case control study with 20 newly diagnosed cases of CML who presented in Lok Nayak Hospital with age and sex-matched controls were taken. History and examination with ophthalmological evaluation was done. Visual Evoked Potential(VEP) of all the subject to objectively evaluate the visual pathway was performed. Abnormal VEP was defined as either prolonged latency $(>110 \mathrm{~ms})$ or low amplitude $(<5 \mathrm{mV})$ of P100 wave. Routine hematological test including hemogram, Tridot, KFT, LFT BCR/ABL analysis and bone marrow biopsy was done. Ultrasound of Spleen and Liver was also done. Patients were treated with 6 weeks of imatinib and 10 patients with deranged VEP were followed up. Complete hematological and ophthalmic evaluation was repeated to asses response to chemotherapy.

Results: At diagnosis, 7 were in chronic, 3 in accelerated and 1 in blast phase. All patients achieved complete hematological remission. Most reported improvement in general and ophthalmic symptoms. The mean splenic size was $8.99 \pm 0.62 \mathrm{~cm}$ compared to $17.73 \pm 1.68 \mathrm{~cm}$ at diagnosis $(p<0.001)$ while the mean liver size$14.65 \pm 0.6 \mathrm{~cm}$ at the follow up. 1 out of 3 patients (33\%) with impaired visual acuity, showed improvement in visual acuity and had achieved a normal visual acuity at follow up. Thirty percent $(3 / 10)$ patients showed improvement in VEP findings at follow up $(p<0.02)$. The cases in whom VEP improved were also the cases who achieved hematological remission. The improvement in VEP was associated with significant increase in hemoglobin from $10.77 \pm 1.79 \mathrm{~g} / \mathrm{dL}$ at the time of initial diagnosis to $12.97 \pm 1.00 \mathrm{~g} /$ $\mathrm{dL}$ at the time of follow up and a significant reduction in TLC counts from an initial count of $2,05,824.29 \pm 1,13,015.25 / \mathrm{mm} 3$ to $9,122.86 \pm 2617.77 / \mathrm{mm} 3$ at follow up ( $p$ value $<0.004)$. However, no such significant difference was found in the reduction of immature cells $(p>0.05)$. The improvement in VEP was achieved mainly with 
normalisation of previously deranged latency. $75 \%$ of patients also showed improvement in retinal examination.

Discussion \& Conclusion: Patients responded well to Imatinib mesylate therapy with improvement in both general and ophthalmic symptoms and organomegaly. All patients achieved hematological remission within 6 weeks. This remission was associated with improvement in VEP functions and retinal findings. This improvement in VEP paralleled with a significant reduction in TLC and hemoglobin rise but not with immature cell reduction and was mostly due to normalisation of latency, indicating a hyperviscosity related ischemic demyelination being the primary process rather than immature cells related infiltrative mechanism. Hence, a baseline ophthalmic evaluation should be done for all patient of CML and early detection of abnormalities at diagnosis can lead to complete resolution with chemotherapy.

\section{Clinical and Molecular Correlation of Patients with Essential Thrombocythaemia: An Experience from Tertiary Care Centre}

Ramesh Balasubramanian ${ }^{* 1}$, Vandana Arya $^{2}$, Nitin Gupta ${ }^{1}$, Jyoti Kotwal $^{2}$

${ }^{1}$ Department of Clinical Hematology, ${ }^{2}$ Department of Hematology, Sir Ganga RAM Hospital, New Delhi, India

Aims \& Objectives: Essential thrombocythaemia(ET) is a Philadelphia-negative myeloproliferative neoplasm. Approximately 50-70\% of patients harbor JAK2 mutation while about $20-30 \%$ patients carries CALR mutations. We studied molecular and clinical features of ET patients presented to our hospital according to their mutation status.

Patients/Materials \& Methods: The patients presented to our hospital in last 3 years with incidentally detected thrombocytosis and were diagnosed as ET after bone marrow studies as per guidelines of WHO2016. Allele Specific PCR and fragment analysis was done to detect JAK2 (V617F), MPL(W515L/K) and CALRexon-9 mutations. Results: A total of 26 diagnosed ET cases with median age of 52 years $(30-83)$ were included in the study. Bone marrow biopsy megakaryocytic morphology satisfied the diagnostic criteria of ET as per guidelines of WHO2016. The study group comprises $42.3 \%$ males and $57.7 \%$ females. The median age was statistically significantly lower in CALR positive cases compared to the JAK2 positive cases(45.9 vs 60.9 years, $p=0.011)$. JAK2 mutation was found in $38.4 \%(10 / 26)$, CALR in $38.4 \%(10 / 26)$ and MPL in 3.8\%(1/26) cases. Triple negativity was found in $15.4 \%(5 / 26)$ cases. The median Platelet counts, TLC and $\mathrm{Hb}$ were not statistically different between JAK2 and CALR mutated groups. The median platetet and $\mathrm{Hb}$ counts were observed to be almost equal in both the group(11.0lakh and $11.75 \mathrm{~g} / \mathrm{dL}$ ). The TLC counts were slightly higher in CALR positive group compared to JAK2 positive group $\left(10450\right.$ cells $/ \mathrm{mm}^{3}$ and 9900 cells $\left./ \mathrm{mm}^{3}\right)$. However,triple negative cases showed the trend of higher median TLC(13000cells $\left./ \mathrm{mm}^{3}\right), \mathrm{Hb}(13 \mathrm{~g} / \mathrm{dL})$ and slightly lower platelets(10.8lakh) as compared to cases with driver mutations. Thrombosis occurred in two patients; one mutated for CALR and one for MPL(W515K).

Discussion \& Conclusion: We found frequency of JAK2 and CALR mutations equal in our study. Although CALR mutated patients were younger in age, the two groups were not different for hematological parameters. We observed that the triple negative population in our study had become intolerant to hydroxyurea after 1 year of therapy and shifted later to Anagrelide. We recommend larger multicentre study for further elucidation of these observations.
Study of Bone Marrow Findings, JAK2V617F Mutation and Serum Erythropoietin Levels in Individuals Presenting with Polycythemia

\author{
Aman Kumar ${ }^{* 1}$, Venkatesan Somasundaram ${ }^{1}$, Deepti Mutreja ${ }^{1}$ \\ ${ }^{1}$ Pathology, Armed Forces Medical College, Pune, Pune, India
}

Aims \& Objectives: We aimed to study bone marrow(BM) findings, JAK2V617F mutation status and serum erythropoietin(EPO) levels in individuals presenting with polycythemia. Our objectives were to study the clinical and hematologic profile of these patients and determine prevalence of JAK2V617F mutations.

Patients/Materials \& Methods: Our study was a unicentric, crosssectional, prospective study at a military tertiary care hospital. We enrolledallmale adult patients with two consecutive values of haemoglo$\operatorname{bin}(\mathrm{Hb})>16.5 \mathrm{gm} / \mathrm{dl}$.BM study, JAK2V617F mutation by RT- PCRand serum EPO was performed in all cases. Study population was evaluated with respect to history of smoking, stay in high altitude areas(HAA)and relevant clinical associated co-morbidities.Anthropometry, blood counts, BM cellularity, distribution of erythroid precursors \&megakaryocytes, megakaryocyte morphology and presence of marrow fibrosis were studied. Statistical analysis was done using SPSS software version 25.0, and $p$ values $<0.05$ were considered statistically significant.

Results: Of the total of 102 patients enrolled for study, 11(10.78\%) patients fulfilled $2016 \mathrm{WHO}$ diagnostic criteria of polycythemia vera (PV) and $91(89.21 \%)$ patientswere grouped into non PV group. JAK2V617Fmutation positivity was seen in only $9(8.8 \%)$ patients in our study and one of these did not fulfill the diagnostic criteria of PV. Within the PV group, three cases were JAK2V617F mutation negative. Within the non PVgroup, erythrocytosis could be attributed to secondary causes in $35(34.3 \%)$ cases and was idiopathic in 56 $(54.9 \%)$ cases. Mean values of total leucocyte counts $(p=0.004)$, platelets $(p=0.008)$, BM hypercellularity $(p<0.001)$, megakaryocyte morphology abnormalities $(p<0.001)$ and marrow fibrosis $(p<0.001)$ were significantly higher in the PV group.Mean $\mathrm{Hb}$, history of smoking and stay in HAA was found to be higher in Non-PV group.Panmyelosis and erythroid hyperplasia were consistently found in PV when compared to non-PV group.

Discussion \& Conclusion: In 102 patients with erythrocytosis with $\mathrm{Hb}>16.5 \mathrm{~g} / \mathrm{dL}, \mathrm{JAK} 2 \mathrm{~V} 617 \mathrm{~F}$ mutational positivity was found in only $9(8.8 \%)$ cases.11(10.78\%) patients were diagnosed as PV, erythrocytosis could be attributed to secondary causes in $35(34.3 \%)$ cases and was idiopathic in $56(54.9 \%)$ cases. We conclude that the 2016 WHO diagnostic criteria includes many causes of idiopathic and secondary erythrocytosis when cut offs of $\mathrm{Hb}>16.5 \mathrm{~g} / \mathrm{dL}$ are used. Supporting Document:

TABLE: Comparison of quantitative and qualitative variables among PV and Non PV groups

\begin{tabular}{|c|c|c|c|}
\hline & $P V(n=11)$ & Non $P V(n=102)$ & p value \\
\hline Age (Yr) mean \pm SD & $41.82 \pm 10.40$ & $38.51 \pm 9.31$ & 0.273 \\
\hline $\mathrm{BMI}$ mean $\pm \mathrm{SD}$ & $24.69 \pm 1.32$ & $24.61 \pm 1.94$ & 0.855 \\
\hline $\mathrm{Hb}(\mathrm{g} / \mathrm{dl})$ mean $\pm \mathrm{SD}$ & $17.44 \pm 2.1$ & $18.29 \pm 1.41$ & 0.213 \\
\hline Het mean \pm SD & $51.90 \pm 5.09$ & $51.21 \pm 4.28$ & 0.674 \\
\hline $\begin{array}{l}\text { RBC Count } \\
\left(x 10^{6} / \mathrm{LL}\right) \text { mean } \pm \mathrm{SD}\end{array}$ & $6.08 \pm 0.95$ & $5.73 \pm 0.62$ & 0.264 \\
\hline $\begin{array}{l}\text { WBC Count } \\
\left(\times 10^{3} / \mathrm{uL}\right) \text { mean } \pm \mathrm{SD}\end{array}$ & $10.69 \pm 4.7$ & $8.33 \pm 2.8$ & 0.004 \\
\hline Platelets $\left(\times 10^{3} / \mathrm{uL}\right)$ mean $\pm \mathrm{SD}$ & $387 \pm 217$ & $241 \pm 78$ & 0.008 \\
\hline Oxygen Saturation mean $\pm \mathrm{SD}$ & $96.09 \pm 1.38$ & $96.65 \pm 1.63$ & 0.235 \\
\hline Low serum EPO $\mathrm{n}(\%)$ & $10(90.9)$ & $14(15.38)$ & $<0.001$ \\
\hline JAK2V617F mutation, $\mathrm{n}(\%)$ & $8(72.72)$ & $1(1.09)$ & $<0.001$ \\
\hline Smokers, $n(\%)$ & $0(0)$ & $15(16.48)$ & $<0.146$ \\
\hline Stay in HAA, $n(\%)$ & $1(9.09)$ & $12(13.18)$ & $<0.701$ \\
\hline Marrow hypercellularity, $\mathrm{n}(\%)$ & $8(72.72)$ & $8(8.79)$ & $<0.001$ \\
\hline Megakaryocyte anisocytosis, $\mathrm{n}(\%)$ & $5(45.45)$ & $2(2.19)$ & $<0.001$ \\
\hline Marrow fibrosis, $\mathbf{n}(\%)$ & $6(54.54)$ & $6(6.59)$ & $<0.001$ \\
\hline
\end{tabular}


Eosinophilia, Basophilia and Mastocytosis in a case of Myeloproliferative Neoplasm with Very Low BCRABL1 Transcript: To Be or Not To Be CML

Seema Biswas ${ }^{* 1}$, Ruchi Gupta ${ }^{1}$, Dinesh Chandra ${ }^{1}$, Shalini Singh ${ }^{1}$, Khaliqur Rehman ${ }^{1}$, Manish K. Singh ${ }^{1}$, Sanjeev Yadav ${ }^{1}$, Anshul Gupta $^{1}$, Rajesh Kashyap ${ }^{1}$, Soniya Nityanand ${ }^{1}$

${ }^{1}$ Hematology, SGPGIMS, Lucknow, India

Aims \& Objectives: Basophilia and eosinophilia are common manifestations of chronic myeloid leukemia (CML). The diagnosis is usually established by demonstrating the presence of BCR-ABL1 fusion transcript. The presence of numerous mast cells in such scenario is very uncommon and should prompt further evaluation for systemic mastocytosis associated with myeloid or lymphoid hematologic diseases (SM-AHNMD). Here we report an unusual case of myeloproliferative neoplasm (MPN), which showed abundance of morphologically and immunophenotypically abnormal mast cells, thus confirming to the diagnosis of systemic mastocytosis with associated MPN, possibly chronic myeloid leukemia. Interestingly, the diagnostic sample showed a very low BCR-ABL1 transcript level. Patients/Materials \& Methods: A 66-year old male presented with a history of weight loss and mild splenomegaly of 1 month duration. Complete hemogram showed $\mathrm{Hb}$ of 5.9, TLC of 17,000/cumm and platelet count of 16,000 /cumm. Bone marrow (BM) examination was performed and subjected to flow cytometry and molecular analysis. Results: Peripheral smear examination showed marked eosinophilia (57\%) and basophilia (10\%) with absence of any shift cells, blasts or dyspoiesis. Bone marrow examination revealed marked proliferation of eosinophils (21\%) and basophils (4\%) with no excess of blasts. First round of Nested RT-PCR for BCR-ABL transcript (qualitative) analysis did not revealed any band; however second round revealed BCR-ABL1 p210 (e13a2) transcript band. The patient failed to respond to Imatinib. A repeat $\mathrm{BM}$ examination, performed after 2 months showed persistence of the findings with prominent basophilia $(14 \%)$ and no evidence of blast excess. However, the bone marrow biopsy showed aggregates of mast cells. An aberrant expression of CD25 on the mast cells was elicited on flow cytometry. Sample subjected for comprehensive NGS panel for myeloid neoplasms was negative for commonly known genetic aberrations including PDGFR-A, PDGFR-B, FGFR-1, KIT and JAK-2. Thus a diagnosis of systemic mastocytosis associated with myeloid diseases possibly CML was conferred. In view of inadequate response to imatinib, the patient was started on Dasatinib. Unfortunately the patient had a rapid downhill course, developed COVID and succumbed to death.

Discussion \& Conclusion: A diagnostic dilemma, presence of abnormal population of mast cells and a very low level of BCR-ABL1 transcript level will be discussed.

\section{HIV Negative Plasmablastic Lymphoma}

\author{
Ashish Bansal $^{* 1}$ \\ ${ }^{1}$ Medicine, Geetanjali Hospital, Udaipur, India
}

Aims \& Objectives: HIV Negative Plasmablastic Lymphoma. Patients/Materials \& Methods: Plasmablastic lymphoma (PBL) is a rare lymphoma associated with immunosuppression. It is strongly associated with immunosuppression (human immunodeficiency virus [HIV]) and often occurs within the oral cavity. PBL is also seen in patients receiving immunosuppressive therapy.

Results: 42 year old male patient presented with the chief complaints of multiple cervical lymphadenopathy and fever. HIV test was negative for him. CECT NECK done which shows multiple enlarged cervical lymph node on right side. Histopathology of cervical node shows Non Hodgkin Lymphoma, Diffuse large cell lymphoma. IHC was positive for LCA, CD10, CD79a, CD138, MUM1, C-Myc, Ki 67 index $70-80 \%$.

Discussion \& Conclusion: However, despite its predisposition for the immunocompromised patients, PBL has been diagnosed in immunocompetent patients.

\section{Classical BCR/ABL1 Negative Myeloproliferative Neoplasms (MPNS) in India}

Suvir Singh ${ }^{* 1}$, Komal Bhinder ${ }^{1}$, Davinder Paul ${ }^{2}$, Kunal Jain ${ }^{2}$, Jagdeep Singh ${ }^{2}$, Vikram Narang ${ }^{3}$, Bhavna Garg ${ }^{3}$, Neena Sood ${ }^{3}$, Barjinder Kaur Dhillon ${ }^{4}$

${ }^{1}$ Clinical Haematology and Stem Cell Transplantation, ${ }^{2}$ Medical Oncology, ${ }^{3}$ Pathology, ${ }^{4}$ Molecular Genetics, Dayanand Medical College and Hospital, Ludhiana, India

Aims \& Objectives: To evaluate clinical and molecular characteristics of patients with BCR/ABL1 negative myeloproliferative neoplasms (MPNs) in India.

Patients/Materials \& Methods: Patients referred for evaluation of suspected MPNs from May 2018 to August 2020 were screened and included after strictly following the WHO 2016 classification criteria. Somatic mutations were checked by targeted RT-PCR or next generation sequencing (NGS). Prefibrotic MF (PrePMF) was reported after retrospectively reviewing bone marrows.

Results: Out of a total of 233 patients, 63 met criteria for BCR/ABL1 negative MPNs and were included for analysis. The cohort included 39 males and 24 females, with a median age of 57 years (Range, $28-82)$ and $24(38 \%)$ patients younger than 50 . The presentation was incidental in 35 patients $(55.5 \%)$, followed by abdominal symptoms in $13(20 \%)$, fatiguability in $7(11 \%)$ and recent vascular events in 6 (9.5\%). The diagnosis was verified as PV in $27(43 \%)$, ET in 21 (33\%), PrePMF in $9(14 \%)$ and MF in $6(9.5 \%)$ patients. The spectrum of driver mutations was as follows: for PV, mutation in JAK2 in $20(75 \%)$ patients and CALR/MPL in nil; in ET, mutations in JAK2 in $7(33 \%)$ patients, MPL in one $(4.7 \%)$ and CALR in 7 (33\%) patients; for PrePMF, JAK2 in $6(66.6 \%)$ and CALR in $2(22 \%)$ patients. For $\mathrm{MF}$, mutation in MPL were seen in 2 patients and JAK2V617F and CALR in one each. Venesection was employed for 14 (51\%) patients with PV, while cytoreduction with hydroxyurea was used for 13 (48\%) patients with PV, 9 (42\%) with ET and 6 (66\%) with PrePMF. Ruxolitinib was used for three patients due to high symptom burden not controlled with hydroxyurea. Two patients progressed to AML after a duration of 38 months and 13 months, respectively. The remaining patients were stable and on regular follow up.

Discussion \& Conclusion: Our study highlights a relatively younger age of onset of BCR/ABL1 negative MPNs in Indian patients and a higher proportion of JAK2 unmutated disease. Strict adherence to diagnostic criteria and awareness about pre-fibrotic MF is essential for accurate diagnosis and risk stratification. Baseline bone marrow examination and somatic mutation testing is expected to improve diagnostic accuracy and treatment design. 
Supporting Document:

\section{Frequency of Muations in all MPNs combined (\%)}

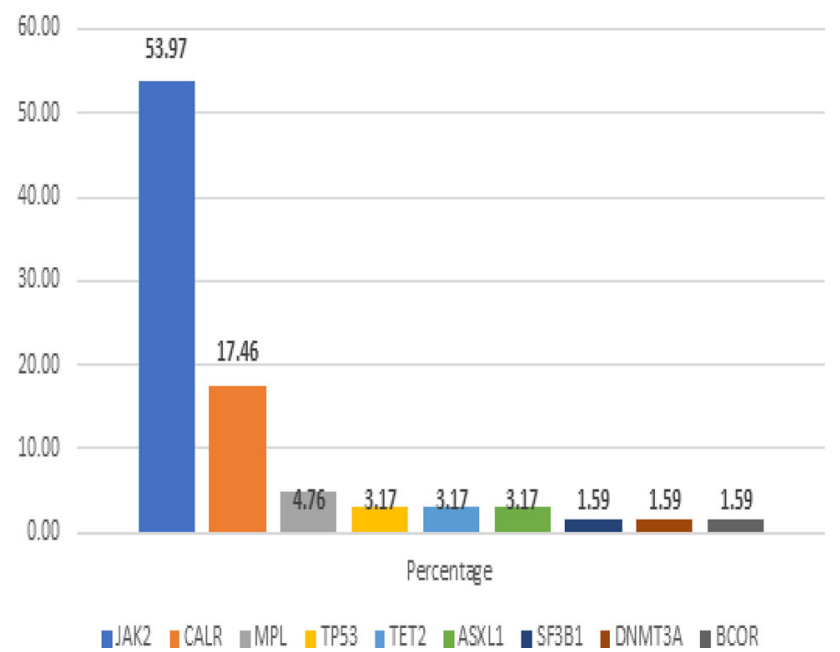

\section{Bone Marrow Metastasis in Lung Cancer: An Unusual Presentation}

\author{
Sanjay Kumar ${ }^{* 1}$, Pooja Dhamija ${ }^{1}$, Sant Prakash ${ }^{1}$, Rajeev Sen ${ }^{1}$ \\ ${ }^{1}$ Pathology, PGIMS, Rohtak, India
}

Aims \& Objectives: To study the unusual site of metastasis in patient of carcinoma lung.

Patients/Materials \& Methods: Lung cancer is the most frequently diagnosed malignancy and leading cause of mortality worldwide. Most $(>80 \%)$ of the lung cancer patients present at an advanced stage (stage III B) or metastatic stage. Most common site of metastasis is liver, bone, brain and adrenals. A 59 year old male presented with complaints of cough and easy fatigability. On examination, he was pale and had crepitations in the left infrascapular region. The chest X-ray revealed a right hilar mass, which was confirmed on CTScan. Patient showed microcytic hypochromic anaemia with low serum iron,low iron binding capacityand high serum ferritin levels.Bone marrow aspiration and biopsy revealed deposits from squamous cell carcinoma. Later on bronchoscopic guided biopsy was done.

Results: Bronchoscopy guided biopsy on histopatholgy confirmed squamous cell lung carcinoma. Serum iron profile reveals anaemia of chronic disease. On bone marrow examination, shows the presence of bone marrow metastasis from squamous cell carcinoma. A diagnosis of stage IV B squamous cell lung carcinoma with bone marrow metastasis was made and patient was started with chemotherapy. After the fourth cycle of chemotherapy, it was seen that overall tumour showed partial remission and haemoglobin began to rise.

Discussion \& Conclusion: Anemia in a patient of lung cancer can be multifactorial, and albeit rarely, metastasis of bone marrow can be one important contributor towards the same.
Aggressive Systemic Mastocytosis in Transformation to Mast Cell Leukemia. A Fatal Outcome

Sunakshi Sharma ${ }^{* 1}$, Ketan Modak ${ }^{2}$, Poojitha Byreddy ${ }^{2}$, Joseph M. John $^{2}$, Preethi A. M. Paul ${ }^{1}$

${ }^{1}$ Pathology, ${ }^{2}$ Clinical Hematology, Hemato-oncology and Bone Marrow (Stem Cell) Transplant Unit, Christian Medical College and Hospital, Ludhiana, India

Aims \& Objectives: Aggressive systemic mastocytosis (ASM) is a rare and fatal disorder. It can develop de novo or progress from indolent forms. ASM can progress to mast cell leukemia (MCL) or acute myeloid leukemia in $5-30 \%$ of cases. We present a patient with ASM in transformation to MCL having a rapidly deteriorating clinical course.

Patients/Materials \& Methods: A 65-year-old gentleman presented with generalized weakness, loss of weight and appetite, loose stools and high grade fever for 2 months. Systemic examination revealed mild pallor and mild hepatosplenomegaly. No skin or bony lytic lesions were identified. Patient was found to be HCV-positive, however liver function tests were normal. $\mathrm{CBC}$ showed pancytopenia; $\mathrm{Hb}$ $4.7 \mathrm{gm} \%$, TLC $3,400 / \mu \mathrm{L}$, platelets $15,000 / \mu \mathrm{L}$ and neutropenia ANC $1,734 / \mu \mathrm{L}$. In view of pancytopenia with constitutional symptoms, bone marrow (BM) was done.

Results: BM aspiration was a dry tap. Touch smears (TS) were paucicellular, however showed up to $34 \%$ mature mast cells along with lymphocytes, erythroid and myeloid series cells. BM trephine biopsy (BMTB) showed large nodular and paratrabecular aggregates of mature mast cells with a central core of small lymphoid cells. On immunohistochemistry (IHC) mast cells were positive for CD117, while the lymphoid collections showed an admixture of CD3, CD5, CD20, and BCL2-positive lymphocytes. CD2, CD30, CD23, CD10, BCL6 were negative and MIB1 labelling index was $<5 \%$ in the mast cells. KIT D816V mutation by PCR and gene sequence analysis was negative, and CD25 IHC done outside was positive. Serum tryptase levels were $181 \mu \mathrm{g} / \mathrm{L}$. Patient was started on Imatinib along with antiviral therapy for HCV and multiple transfusions. Due to worsening of cytopenias, a repeat BM was done to reassess disease status five months later. Rare mast cells were seen in the peripheral blood, with increase in mature and atypical mast cells on BM TS up to $29 \%$. IHC on BMTB showed mild increase in CD34-positive blasts along with sheets of CD117-positive mast cells. A diagnosis of mast cell leukemia was made. Patient succumbed to illness within eight months of initial diagnosis.

Discussion \& Conclusion: We report this case of KIT D816V mutation-negative ASM for its rarity, absence of skin lesions, rapid progression to MCL and fatal outcome. BM examination and IHC play an important role in accurately diagnosing mast cell disorders.

\section{JAK2 V617F Positive Essential Thrombocythemia Presenting as Portal Hypertension}

Mukti P. Meher ${ }^{* 1}$, Lalit P. Meena ${ }^{2}$, Saumya Gupta ${ }^{1}$

${ }^{\mathbf{1}}$ General Medicine, Institute of Medical Sciences, BHU, ${ }^{\mathbf{2}}$ General Medicine, Institute of Medical sciences,BHU, Varanasi, India

Aims \& Objectives: Essential thrombocytosis sometimes associated with abdominal complication like portal vein thrombosis, budd- chiari syndrome.In this case report present presented with splenomegaly with features of extrahepatic portal vein obstruction.her complete blood picture revealed increase platelet counts and color Doppler showing features of portal vein hypertension. patient was found positive for JAK2 mutation, following which diagnosis of essential thrombocytosis with portal vein thrombosis was made. 
Patients/Materials \& Methods: A 40 year female presented us with chief complaints of abdominal distension along with generalised body weakness for 2 month. She was also having loss of apetite for last 1 month. On clinical examination splenomegaly $(10 \mathrm{~cm})$ was present. Then she was advised for routine haematological profile and upper gastro intenstinal endoscopy.

Results: Her UGIE revealed 4 column grade 2 varices. Her color doppler of hepatic-portal system showed altered liver echotexture, portal vein showing thickening of its wall and attenuation of the color flow at porta hepatis. CBC revealed thrombocytosis (TPC- 6,87L). bone marrow aspiration showed hypercellular marrow with thrombocytosis and increased no of megakaryocytes. Patient was screened for JAK2 V617 mutation, which came out positive. Esophageal variceal ligation(EVL) was done. cytoreductive therapy (hydroxyurea) and antiplatelet therapy (aspirin) was started. After 1 month of cytoreductive therapy platelet count became normal (TPC-3.45 L) and spleen size reduced to $5 \mathrm{~cm}$ below subcostal margin.

Discussion \& Conclusion: Increase in the platelets count occur as a result of the continuous proliferation of megakaryocytes in essential thrombocythemia. This disease is characterized by splenic enlargement and presence of megakaryocyte in the bone marrow. Approximately $50 \%$ of the patients have JAK2 V617F mutation. Portal hypertension is one of the complication chronic liver disease and is responsible for many other complication like ascites, variceal haemorrhage and encephalopathy[1]. Sometime Portal hypertension may also present as a complication of the chronic myeloproliferative neoplasm. Patients with myeloproliferative neoplasm may present with severe portal hypertension, ascites, esophageal varices, as a result of the massively increased splenoportal blood flow.

\section{Clinical and Hematological Profile of Chronic Myeloid Leukemia in Sub-himalayan Region}

Pravesh Dhiman $^{1}$, Manish Gupta ${ }^{1}$, Vikas Fotedar ${ }^{1}$, Siddharth Vats ${ }^{1}$, Purnima Thakur ${ }^{1}$, Deepak Tuli ${ }^{1}$, Sunil Sharma ${ }^{1}$, Anup Negi ${ }^{1}$, Lalit C. Kant $^{1}$, Kapil M. Pal ${ }^{1}$, Shabnam Thakur ${ }^{1}$, Jyoti sharma ${ }^{1}$, Mrinalini Kumari $^{1}$, Kulbir Singh ${ }^{1}$, Rajeev Sandal ${ }^{* 1}$

${ }^{1}$ Department of Radiotherapy and Oncology, IGMC SHIMLA, SHIMLA, India

Aims \& Objectives: We studied the clinical and hematological parameters of patients with chronic myeloid leukemia who presented at regional cancer center, Indira Gandhi Medical College Shimla Himachal Pradesh (IGMC, Shimla).

Patients/Materials \& Methods: We retrospectively analyzed data of patients with newly diagnosed chronic myeloid leukemia who were registered in regional cancer centre IGMC Shimla from 2014 to 2019. Demographic data including age, place, gender, occupation, and socioeconomic status of patients were recorded. Detail history including duration of symptoms, any bleeding manifestations and baseline spleen size (below costal margin) were analyzed. The baseline investigations included complete blood counts, biochemistries, and bone marrow examination. CML was diagnosed by morphology and demonstration of the Philadelphia chromosome, or by the demonstration of the BCR-ABL transcript. EUTOS score was calculated for every patient. All patients in chronic phase were started on tablet imatinib $400 \mathrm{mg}$ once daily after confirmation of diagnosis. Adverse events during therapy were also analyzed.

Results: Out of fifty-one $(\mathrm{n}=51)$ patients thirty-seven were male and fourteen were female (M: F, 2.6:1). Median age of studied population were 44.5 years (range $15-68$ years) and $45.1 \%$ of patients $(n=37)$ were between the age 31 years to 60 years. $15.7 \%$ and $11.8 \%$ of patients were less than thirty and more than 60 years of age respectively. $49 \%$ of patients $(n=25)$ presented with abdomen discomfort or left upper quadrant pain. Two patients (3.9\%) were asymptomatic at time diagnosis. Other presenting symptoms were fatigue $(19.6 \%$, $\mathrm{n}=10)$, shortness of breath $(17.6 \%, \mathrm{n}=9)$ and lymphadenopathy $(3.9 \%, \mathrm{n}=2)$. Splenomegaly were seen in $76.5 \%$ of studies population $(n=39)$ at time of presentation. Median spleen size was $8 \mathrm{~cm}$ below left costal margin (range $0-15 \mathrm{~cm}$ ). Median $\mathrm{Hb}$ was $10.5 \mathrm{~g} / \mathrm{dl}$ (range, 6.3-15.2 g/dl) and median TLC was 81450/ul (range, $34000-332000 / \mathrm{ul})$ of studies cohort. $76.5 \%$ patients $(\mathrm{n}=39)$ were in chronic phase at the time of diagnosis. $15.7 \%(\mathrm{n}=8)$ and $7.8 \%$ $(n=4)$ of patients were accelerated and blast phase at time presentation.

Discussion \& Conclusion: We concluded that most of patients were of younger age group in our cohort. Abdomen discomfort was most common presenting symptom. Massive splenomegaly was most common physical finding on examination. Most of patient presented with anemia along with high TLC. Three-fourth of patients were in chronic phase at time of presentation.

\section{Bone Marrow Biopsy in Myeloproliferative Neoplasms Unclassifiable (MPN-U)}

Manjula Murari $^{* 1}$, Anamika Verma ${ }^{1}$, Seema Sharma ${ }^{1}$

${ }^{1}$ Pathology, SGPGIMS, Lucknow, India

Aims \& Objectives: WHO (2016) classification of myeloproliferative neoplasms (MPN) is applicable to most of the cases evaluated on blood and bone marrow examination. However some cases remain unclassifiable, this study presents the marrow histomorphology in MPN-U.

Patients/Materials \& Methods: This study included 464 cases of MPN from January 2012 to December 2014. Clinical features, full $\mathrm{CBC}$, bone marrow aspirate and biopsy findings were reviewed.

Results: There were 380 cases of CML and 84 cases of BCR-ABL negative MPN including ET 19 cases, PV12 cases, IMF 19 cases and 34 cases unclassifiable MPN. Unclassifiable MPN included 23 males and 11 females, mean age was 50.9 years, organomegaly in $64.7 \%$. Peripheral blood showed anemia $(55.8 \%)$, Leucocytosis $(76.4 \%)$, thrombocytosis (47\%), Basophilia (35.2\%), eosinophilia $(70.4 \%)$, blasts in 10 cases ranged from 2 to $8 \%$. Bone marrow was hypercellular in most ( $>80 \%$ cases, with packed pattern in 10 cases) with myeloid predominance, blasts were increased $(<10 \%)$ in 12 cases. Marked erythroid hyperplasia was noted in 5 cases. Megakaryocytes were increased in 29 cases $(85.2 \%)$ with moderate to marked increase in 23 cases that showed clustering and dysplastic morphology. Marrow fibrosis was evident in 13 cases using reticulin and collagen stains as grade I ( 5 cases) and grade II ( 8 cases).

Discussion \& Conclusion: This series of 464 cases showed $34(13.6 \%)$ unclassifiable MPN with hypercellular marrow in majority and morphology favouring PV, ET or cellular phase IMF, however, either peripheral blood diagnostic criteria were not met and/or marrow morphological overlap existed between various entities. In four cases biopsy morphology was suboptimal with variable degree of associated dysplastic changes. A diagnosis c/w MPN with further molecular tests and follow-up was advised in these cases.

\section{Transient Abnormal Myelopoiesis in Down Syndrome}

Sukanya Sarkar*1

${ }^{1}$ Pathology, Bharati Vidyapeeth (Deemed to be University) Medical College and Research Centre, Pune, India

Aims \& Objectives: Aim: To study Transient Abnormal Myelopoiesis in Down syndrome. Objective: Yo evaluate features of Transient 
Abnormal Myelopoiesis in Down syndrome and study myeloid proliferations related to Down syndrome.

Patients/Materials \& Methods: Single new born presented with Down syndrome with hypotonia and hepatosplenomegaly.

Results: Complete blood count showed marked leucocytosis (124700/ cu.mm) with $65 \%$ blasts (blasts were 20-24 micron in size with high $\mathrm{N}: \mathrm{C}$ ratio, moderate bluish cytoplasm. Nuclei were round to oval with fine nuclear chromatin. Few of these show 1-2 prominent y, intracytoplasmic vacuoles and cytoplasmic blebs. Blasts were MPO positive.) Flow results showed B tube: Negative for CD10, CD19, CD20. T tube was negative for CD3 and positive for CD7. Myeloid was positive for CD33 and CD117 and negative for CD13. Cytoplsamic weakly positive for MPO and CD61.

Discussion \& Conclusion: Children with Down syndrome have a markedly increased risk of developing acute myeloid leukemia (AML) known as ML-DS. ML-DS is preceded by a clonal neonatal preleukaemic disorder known as TAM which maybe clinically overt or silent. Trisomy 21 perturbs fetal haematopoiesis. Transformation of these fetal haematopoietic cells by acquired truncating mutations in GATA1 gene produces the clinical syndrome TAM.

\section{Outcome of a 10 Months Old Baby Treated for Seborrheic Dermatitis}

\author{
Virender K. Gehlawat ${ }^{* 1}$, Vandana Arya ${ }^{1}$ \\ ${ }^{1}$ Pediatrics, Pt B D Sharma PGIMS Rohtak, Rohtak, India
}

Aims \& Objectives: To describe the outcome of a 10 months old child treated for seborrheic dermatitis in the dermatology department. Patients/Materials \& Methods: We describe the clinical outcome and final diagnosis of an infant treated for seborrheic dermatitis.

Results: A 10 months old female child was treated for seborrheic dermatitis for around four months and was not improving clinically with the medications. In view of low grade fever on and off for one month, a pediatric opinion was sought.On examination, the child had pallor, rash, hepatosplenomegaly and cervical lymphadenopathy along with an exfoliating rash on scalp similar to seborrheic dermatitis. Investigations showed anemia (HB- $6 \mathrm{gm} / \mathrm{dl}$ ), normal leucocyte and platelet count with normal liver and kidney function tests. X ray skull showed lytic lesion. Skin biopsy revealed CD1a and S100 positivity. Bone marrow biopsy showed chancroid tissue, enclosing 4-5 intertrabecular spaces revealing hemorrhages and findings suggestive of LCH. CT chest showed extensive small air filled cysts scattered in both lungs. The child was transferred to haematoncology unit where she was started on multisystem high- risk disease regimen.

Discussion \& Conclusion: The present case highlights the need for a high index of suspicion for LCH among babies being treated for unresolving seborrheic dermatitis.

\section{A Case of Pediatric Myelofibrosis with Associated Lytic Lesions}

Sruthi Pallekonda ${ }^{* 1}$, Vandana Puri ${ }^{1}$, Sunita sharma ${ }^{1}$, Alok Hemal ${ }^{2}$

${ }^{1}$ Pathology, Lady Hardinge Medical Collge, ${ }^{2}$ Pediatrics, RML Hospital, Delhi, India

Aims \& Objectives: Primary myelofibrosis (MF) is a clonal myeloproliferative neoplasm occurring more commonly in adults in sixth to seventh decade and is very rare in pediatric age group.Blastic transformation occurs in 5-20\% and lytic lesions are very rare. Here we present a case of Pediatric MF with associated lytic lesions.
Patients/Materials \& Methods: A 2 year old male presented with fever for 2 months. Physical examination revealed pallor and bony tenderness in the limbs. There was no significant organomegaly or lymphadenopathy. CT scan revealed multiple ill defined permeative bony destructive lesions. A Differential diagnosis of Acute Leukemia or Langerhans cell histiocytosis was considered. Laboratory analysis showed $\mathrm{Hb}$ of $5.4 \mathrm{~g} / \mathrm{dl}$, WBC of $5.41 \times 103 / \mathrm{L}$, Platelets of $8 \times 103 /$ uL.Peripheral smear study showed shift to left with presence of $11 \%$ atypical cells which were 3-4 times the size of a small mature lymphocyte with scant agranular basophilic cytoplasm showing blebbing at places, round to oval nucleus, high N/C ratio, 1-2 nucleoli. Aspirate smears and Imprint smears were diluted, however showed the presence of few atypical cells. Immunophenotyping of BM aspirate revealed $30.3 \%$ of cells gated in blast window in a CD45/Ssc plot. The cells were positive for CD 41 and negative for MPO, HLA DR, CD34, CD13, CD33, CD79a, CD10 and were suggestive of Megakaryoblasts. Bone marrow biopsy showed grade 3 fibrosis (on Reticulin stain) and presence of few blasts. Immunohistochemistry revealed that these cells were positive for CD61, hence confirming the presence of megakaryoblasts.

Results: A diagnosis of Myelofibrosis with megakaryoblastic blast crises was given. Cytogenetic analysis for JAK-2 Mutation was suggested. The patient was referred to higher oncology center where he succumbed to his disease.

Discussion \& Conclusion: Extensive literature search revealed no similar cases in pediatric population. Thus the case is presented due to its rarity and the dilemma about the course of disease.

\section{An Unusual Cause of Respiratory Distress: CML Presenting with Leukostasis}

Aakash Chandran Chidambaram ${ }^{* 1}$, Jaikumar G. Ramamoorthy ${ }^{1}$, Narayanan Parameswaran ${ }^{1}$, Prabhu Manivannan ${ }^{2}$

\section{${ }^{1}$ Pediatrics, ${ }^{2}$ Pathology, Jipmer, Puducherry, India}

Aims \& Objectives: Hyperleukocytosis is conventionally defined as peripheral $\mathrm{WBC}>100000 / \mathrm{mm} 3$. The increased blood viscosity, secondary to leucocyte aggregates, results in stasis in smaller blood vessels predisposing to neurological, pulmonary or GI complications, resulting in morbidity and mortality.

Patients/Materials \& Methods: An 8 year old male child was brought with complaints of respiratory distress and fever for 2 day. On examination, child was found alert, afebrile with pallor and bilateral cervical and inguinal lymphadenopathy. His respiratory rate was $32 / \mathrm{min}$ and oxygen saturation (Spo2) in room air was $86 \%$ and improved to $93 \%$ with oxygen supplementation by face mask. Systemic examination revealed mild intercostal and subcostal retractions, with normal breath sounds and unremarkable cardiovascular exam. Abdominal examination revealed hepatomegaly of $6 \mathrm{~cm}$ (span $14 \mathrm{~cm}$ ) and massive splenomegaly of $12 \mathrm{~cm}$ below left costal margin.

Results: Emergency hemogram and electrolytes revealed a total White cell count of 7 lakh cells/ $\mu \mathrm{L}$, differential count N34L2M1B3 blast $1 \%$, myelocyte $37 \%$ promyelocyte $1 \%$ and metamyelocyte $12 \%$. His hemoglobin was $5.5 \mathrm{mg} / \mathrm{dl}$ and platelet count was 3.3 lakhs $/ \mu \mathrm{L}$. Peripheral smear revealed leukocytosis with myeloid bulge and entire spectrum of myeloid maturation seen with eosinophilia and basophilia. Allopurinol was administered along with liberal intravenous fluids for tumor lysis. Leukapheresis was then initiated and continued over $6 \mathrm{~h}$. Child improved symptomatically. Translocation $(9 ; 22)$ was identified in Fluorescence in- situ hybridization test. A diagnosis of Chronic myeloid leukemia in chronic phase with hyperleukocytosis was made and imatinib with hydroxyurea was initiated. 
Discussion \& Conclusion: A common diagnostic dilemma in these cases is between anemia with CCF and leukostasis. It is prudent to manage as leukostasis and start exchange transfusion or leukapheresis. Routine blood transfusion as part of treatment for anemia increases viscosity and clogs microcirculation. It is also important to avoid furosemide in these cases as it depletes intravascular volume. Serum potassium may be falsely high in these cases due to lysis of large amount of cells. The effect of leukapheresis is generally transient. Early initiation of chemotherapy is needed. Leucostasis can be an unusual cause of respiratory distress in children. Massive splenomegaly is one of the important clinical clue to suspect beyond respiratory infection in these children.

\section{A Rare Presentation of Chronic Myeloid Leukemia with Lymphoid Blast Crisis and Myelofibrosis}

Kusha Sharma ${ }^{* 1}$, Vandana Puri ${ }^{1}$, Sunita Sharma ${ }^{1}$, Shailaja Shukla ${ }^{1}$, Alok Hemal $^{2}$

${ }^{1}$ Pathology, Lady Hardinge Medical College, ${ }^{2}$ Pediatrics, Dr Ram Manohar Lohia Hospital, New Delhi, India

Aims \& Objectives: (1) To study the morphological and immunohistochemical features of chronic myeloid leukemia with lymphoid blast transformation and associated myelofibrosis in a young child on bone marrow imprint smears and bone marrow biopsy. (2) To study the flow cytometric immunophenotype and molecular profile in such cases and its role in establishing the diagnosis.

Patients/Materials \& Methods: A 7 yr old female child presented with pain abdomen, abdominal distension and fever since 3 months. Per abdominal examination revealed massive splenomegaly reaching up to the right iliac fossa. Hemogram revealed marked leucocytosis with presence of $10 \%$ blasts having lymphoblast morphology, myeloid bulge, basophilia and thrombocytopenia. Thus, provisional diagnosis of blastic transformation of CML was considered.

Results: RT-PCR revealed M-BCR(Major breakpoint cluster region) fusion protein BCR-ABL ex14-ABL ex2(b3a2):472 bp which confirmed the diagnosis. Bone marrow aspiration attempted resulted in dry tap. Bone marrow trephine biopsy was taken and imprint smears showed presence of blasts. Section from the bone marrow biopsy showed cellular marrow spaces with streaming of nuclei which on Reticulin stain demonstrated Grade 3 fibrosis. Clusters of blast cells were seen which on immunohistochemistry were positive for CD34, CD10, CD 19, CD20, Pax5 and CD79a. These were negative for T cell and myeloid markers. Flow cytometry performed on the peripheral blood showed blasts with expression of CD34, Tdt, HLADR, cCD79a and coexpression of CD10 and CD19. These were negative for CD3, CD5, CD7, cCD3, MPO, CD13, CD33, CD117, CD14. Hence, a diagnosis of lymphoid blast transformation in Chronic Myeloid Leukemia was made.

Discussion \& Conclusion: Chronic Myeloid Leukemia typically presents in older adults usually in chronic phase and may rarely progress to blast crisis if untreated. Blast phase commonly manifests as myeloid in $70 \%$ cases. Chronic myeloid leukemia with lymphoid blast crisis and myelofibrosis at presentation in children is rare and portends poor prognosis, thus reaching correct diagnosis is of paramount importance.

\section{Priapism as an Initial Manifestation in Chronic Myeloid Leukemia}

Thejesh $\mathbf{C ~ V}^{* 1}$, Mayilananthi Kaliannan ${ }^{1}$, Durga Krishnan ${ }^{1}$, Senthil Nathan $^{1}$, Vrinda $\mathrm{V}^{1}$

${ }^{1}$ General Medicine, Chettinad hospital and research institue, chennai, India

Aims \& Objectives: Priapism is a rare complication of leukemia. It is a medical and surgical emergency and an uncommon presenting feature of chronic myeloid leukemia (CML) and accounts for 50\% of all leukemic priapism. However, it is rarely reported as a presenting feature in CML patients.

Patients/Materials \& Methods: A 23-year-old man presented to the emergency department with history of an erect penis for over $12 \mathrm{~h}$ duration following sexual intercourse. He had a similar episode 6 months back, which subsided on its own. No h/o any drug intake. General examination was normal. Abdomen examination showed non tender hepatosplenomegaly. The penis was erect, firm and tender with superficial venous engorgement.

Results: Complete blood count analysis showed white blood count of $4,63,100 /$ cumm hemoglobin of $12 \mathrm{~g} / \mathrm{dl}$, hematocrit of $30.5 \%$, and platelet of 4.93 lac/c.mm. Peripheral smear showed increased total count with increase in basophils and eosinophils with band form-22\%, neutrophil-25\%, lymphocyte-02\%, monocyte-20\%, eosinophil- $06 \%$, basophil- $05 \%$, blasts-2\%, promyelocyte- $02 \%$, myelocyte- $20 \%$, metamyleocyte- $16 \%$, the peripheral picture was suggestive of chronic myleoproliferative disorder. Renal, hepatic and coagulation profile were normal. Bone marrow biopsy confirmed the diagnosis of CML with cytogenetic analysis revealed the presence of Philadelphia chromosome and molecular studies confirmed the presence of BCR$\mathrm{ABL}$ transcript. As the patient presented initially with priapism he was taken up for corporal aspiration and phenylephrine irrigation by the urology department. The erection gradually reduced following aspirations and washes. The total count reduced to 2.13 lakhs with imatinib given for 2 weeks.

Discussion \& Conclusion: Priapism is a urological emergency which unless timely intervened, results in erectile dysfunction. CML is one of the important differential diagnosis to be considered in evaluation of the patient with priapism. The pathophysiology of priapism in CML is related to hyperleucocytosis and hyperviscosity. Hence, early initiation of treatment with tyrosine kinase inhibitors or leukapheresis will reduce the total count and prevents the recurrence of priapism.

\section{Next Generation Sequencing Solves Diagnostic Dilemma in a Triple Negative Myeloproliferative Neoplasm: A Case Report}

Satyender S. Dharamdasani ${ }^{* 1}$, Richa Chauhan ${ }^{1}$, Amrita Saraf ${ }^{1}$, Vandana Arya ${ }^{1}$, Nitin Gupta ${ }^{2}$, Jyoti Kotwal ${ }^{1}$

${ }^{1}$ Hematology, ${ }^{2}$ Clinical Hematology, Sir Gangaram Hospital, New Delhi, India

Aims \& Objectives: Triple negative myeloproliferative neoplasms (TN-MPN), i.e. negative for three driver mutations- JAK2, CALR and MPL, comprise $\sim 10 \%$ of all MPNs. We present a case where a patient was treated like TN-MPN for 5 years before NGS revealed her final diagnosis.

Patients/Materials \& Methods: A 62-year old lady came for Hematology consultation in 2014 with complaints of fatigue, bilateral pedal edema, dragging sensation in the left upper abdomen and loss of appetite. On examination, there was massive hepatosplenomegaly. She had also required blood transfusions in the past. The hemogram showed an Hb of $9.5 \mathrm{~g} / \mathrm{dL}$, TLC- $18.4 \mathrm{X} 103 \mu \mathrm{L}$, platelets-615 X103 $\mu \mathrm{L}$, 
Hct-30.8\%， RBC-3.94 X106 $\mu \mathrm{L} ， \mathrm{MCV}-78.1 \mathrm{fL}, \quad \mathrm{MCH}-24.2 \mathrm{pg}$, MCHC-31 g/dL, RDW-CV 19.3\%. Peripheral smear showed leukoerythroblastic picture, $05 \%$ blasts and $06 \%$ basophils. Bone Marrow aspirate was hemodiluted with few hematopoietic elements. Bone marrow biopsy showed focal grade 2 myelofibrosis with focal osteosclerosis $(<30 \%$ area). Megakaryocytes showed atypia with abnormal paratrabecular localization forming tight clusters. As the RT-PCR was negative for BCR-ABL1, a provisional diagnosis of Philadelphia-negative MPN- Primary Myelofibrosis (PMF) was made and the patient was started on Hydroxyurea, allopurinol and low dose aspirin. MPN reflex panel for JAK2V617F, MPL W515K/L and CALR type $1 / 2$ was done in 2016 which was found to be negative. Hence, a diagnosis of TN-MPN (PMF) was made and the patient categorized as IPSS 2. She was put on Ruxolitinib $20 \mathrm{mg}$ BD. In 2019, the patient deteriorated and presented with fever, raised ESR, CRP and raised TLC of $90.25 \mathrm{X} 103 \mu \mathrm{L}$. Amoebic liver abscess was detected by radiological, microbiological and serological investigations. Bone marrow aspirate done to look for progression showed similar marrow findings as in 2014. This time an NGS panel was sent that revealed MPL W515K mutation in exon 10 and ASXL1 Q733X exon 13 mutation.

Discussion \& Conclusion: This case highlights the role of NGS in the diagnosis and appropriate classification of MPN.The MPL driver mutation was not detected in 2016 by RT-PCR, possibly because the mutation developed later in the course of the disease or the allele burden was low for the technique. MPL-PMF vs TN-MPN differ in their prognosis and disease course.

\section{Typically "Atypical”: A Case Series of Atypical Chronic Myeloid Leukemia}

Ramesh Balasubramanian ${ }^{* 1}$, Richa Chauhan ${ }^{2}$, Amrita Saraf ${ }^{2}$, Vandana Arya ${ }^{2}$, Nitin Gupta ${ }^{1}$, Mitu Shrikhande ${ }^{3}$, Ajay Sharma ${ }^{1}$, Jyoti Kotwal $^{2}$

${ }^{1}$ Department of Clinical Hematology, ${ }^{2}$ Department of Hematology, Sir Ganga RAM Hospital, ${ }^{3}$ Department of Clinical Hematology, Fortis Flt. Lt. Rajan Dhall Hospital, New Delhi, India

Aims \& Objectives: Atypical Chronic myeloid leukemia (aCML) is a rare subtype of MDS/MPN. The diagnosis of aCML has evolved over years with more evidence from cytogenetic and molecular studies. We report here a case series of three patients with aCML that was picked up based on morphology and molecular workup.

Patients/Materials \& Methods: Case 1: A 68-year male with incidental detection of leukocytosis on routine hemogram without

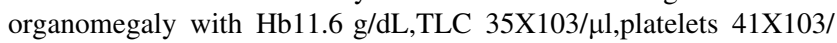
$\mu 1$,DLC My30MM5N53L8E3Baso1\%. Bone marrow was hypercellular with M:E ratio7.7:1, severe dysgranulopoiesis, 07\% blasts, and dysmegakaryopoiesis (>50\%). His extended MPN reflex panel (BCR-ABL1, JAK2, CALR, MPL) was negative. Provisional diagnosis of aCML was made. NGS panel revealed multiple mutations in ZRSR2, ASXL1, RUNX1, SF3B1, EZH2, TET2 genes. He has completed 6 cycles of Azacytidine and remains stable for 8 months since the diagnosis. Case 2: A 76-year old male with suspected CML presented with mild hepatosplenomegaly with $\mathrm{Hb} 8.9 \mathrm{~g} / \mathrm{dL}, \mathrm{TLC}$ 46X103/ $\mu 1$, platelets $75 X 103 / \mu 1$, DLC Blast3My12MM8 N66Ly8 Mono2Baso1\% and 2nRBC/100 WBCs. Bone marrow was hypercellular with M:E 6.3:1, significant dysgranulopoiesis $03 \%$ blasts, and dysmegakaryopoiesis. His extended MPN reflex panel was negative. NGS revealed mutations in ASXL1 and KRAS genes. After diagnosing aCML he was started on hydroxyurea and remains stable after 6 months of diagnosis. Case 3: A 71-year old female with suspected CML based on moderate splenomegaly and hemogram showed $\mathrm{Hb}$
$8.9 \mathrm{~g} / \mathrm{dL}, \mathrm{TLC} \quad 65.94 X 103 / \mu \mathrm{l}$, Platelets $45 \times 103 / \mu \mathrm{l}$ with DLC Blast1My29MM22N38Ly5Eo4Baso2\% Bone marrow was hypercellular with M:E 5.8:1, prominent left shift in granulocytic series, $01 \%$ blasts, no dysgranulopoiesis. There was significant dysmegakaryopoiesis. A provisional diagnosis of CML vs MDS/MPN was kept and was advised molecular workup. BCR-ABL1 and JAK2 mutation were negative. NGS panel revealed ASXL1 and SF3B1 gene mutations. Finally diagnosed as aCML and treated with Hydroxyurea. She developed undiagnosed fever during COVID19 lockdown and succumbed to her illness.

Discussion \& Conclusion: Atypical CML is a very rare entity with close differential diagnoses of accelerated phase of CML or MDS/ MPN-U. However as more awareness of this entity has emerged, more patients are subjected to NGS evaluation, thus contributing to the knowledge about this under-reported diagnosis from the third world.

\section{Tuberculosis Reactivation Following Ruxolitinib Therapy for Myelofibrosis}

Anil Aribandi $^{* 1}$, Ranjit K. CS ${ }^{1}$, Rajesh M. Gottipati ${ }^{2}$

${ }^{1}$ Department of Haemato-oncology, American Oncology Institute, Hyderabad, ${ }^{2}$ Department of Haemato-oncology, American Oncology Institute, Vijayawada, India

\begin{tabular}{llllllllll}
\hline Age in yrs & Sex & $\begin{array}{l}\text { Diag- } \\
\text { nosis }\end{array}$ & JAK2 & CALR & $\begin{array}{l}\text { Ruxolitinib } \\
\text { initiation }\end{array}$ & $\begin{array}{l}\text { TB } \\
\text { reacti- } \\
\text { vation }\end{array}$ & $\begin{array}{l}\text { Site of } \\
\text { reacti- } \\
\text { vation }\end{array}$ & $\begin{array}{l}\text { ATT } \\
\text { duration }\end{array}$ & Outcome \\
\hline 53 & F & $\begin{array}{c}\text { SMF/ } \\
\text { PV }\end{array}$ & + & - & Dec,2013 & 15 months & Abdomen & 6 months & Alive \\
33 & F & PMF & + & - & Feb,2016 & 14 months & Pulmonary & 6 months & Alive \\
40 & M & PMF & - & + & April,2017 & 4 months & Lymphnode & 6 months & Alive \\
54 & M & PMF & + & - & March,2017 & 11 months & Pulmonary & 6 months & Alive \\
\hline
\end{tabular}

\section{TISSUE IS AN ISSUE}

Janakiraman $\mathbf{K}^{* 1}$, Sharadamani $\mathrm{G}^{1}$

${ }^{1}$ Pathology, Vinayaka Mission's Medical College, Karaikal, India

Aims \& Objectives: Waldenström macroglobulinemia (WM) frequently presents with pancytopenia placing patients at risk of infection and bleeding. However, other less common complications can occur with similar presentations. This case highlights an apparent case of osteomyelitis and bleeding diathesis, that with a systematic diagnostic approach was identified as: (1) plasmacytoma-like infiltrative extramedullary WM and (2) acquired von Willebrand syndrome (aVWS) in a patient with progressive WM.

Patients/Materials \& Methods: A 73-year-old man with Waldenström macroglobulinemia, pancytopenia, and peripheral neuropathy presented with progressive dorsalgia, epistaxis, and 30- pound weight loss with initial imaging concerning for lumbar osteomyelitis. He reported no fever, headaches, vision changes, or weakness, and had no neurologic deficits on exam except for decreased peripheral pinprick sensation. Studies demonstrated hyperviscosity and an acquired von Willebrand syndrome (shown on right). Repeat MRI (shown on right) remained concerning for osteomyelitis. Infection continued to be the most likely etiology, but a biopsy was pursued to guide antimicrobial selection, and to provide diagnostic confirmation. Given the patient's 
hyperviscocity syndrome and aVWS, his surgical biopsy was delayed one week while he required plasmapheresis with cryoglobulin and VWF/FVIII concentrates (Humate- $\mathrm{P}^{\circledR}$ ). Vertebral biopsy revealed clusters of plasma cells indicative of infiltrative extramedullary WM. He was initiated on therapy for WM with bortezomib and dexamethasone. Rituximab was held due to elevated IgM.

Results: A Brief on Atypical Complications of Waldenström macroglobulinemia.

Discussion \& Conclusion: - Unlike multiple myeloma, there is no clear association between Waldenström macroglobulinemia and plasmacytoma. However infiltrative extramedullary WM may present on imaging as an infiltrative mass with pathology resembling plasmacytoma.

- It is estimated extramedullary disease is only present in $4.4 \%$ of WM cases with the common locations involving the lungs, CSF, soft tissue, and bone.

- There is a clear association between acquired von Willebrand syndrome (aVWS) and monoclonal gammopathies. A retrospective study shows an association between increasing IgM levels and risk of aVWS.

\section{Primary Myelofibrosis Presenting as a Case of Pyrexia of Unknown Origin: A Case Report}

\author{
Heshweaanth R D ${ }^{* 1}$, Mukthar Ahamed ${ }^{1}$, Vamshi Dharan $\mathrm{P}^{1}$ \\ ${ }^{1}$ General Medicine, Yenepoya medical college hospital, \\ Mangalore, India
}

\begin{abstract}
Aims \& Objectives: Primary myelofibrosis is one of the chronic myeloproliferative disorder, characterised by clonal proliferation of myeloid cells with variable morphologic maturity and hematopoietic efficiency. Primary myelofibrosis is the least frequent disease among myeloproliferative neoplasm with incidence of 1 in lakh population in India.
\end{abstract}

Patients/Materials \& Methods: A 62 years old male patient who is a known case of HTN, type 2 DM, presented to our hospital with history of high-grade fever of 15 days duration associated with chills and rigor, not subsiding to antipyretic therapy and complaints of generalised weakness and easy fatigability since the onset of fever. On general examination, vitals were stable, pallor was present, multiple axillary lymph nodes were palpable, bilateral pitting pedal oedema was present. Systemic examination revealed hepatosplenomegaly with large spleen crossing the umbilicus extending into Right Iliac Fossa, Other systems were within normal limits.

Results: Lab report showed haemoglobin- $8.7 \mathrm{~g} \%$, total counts-3140 cells/cumm, platelet-5000cells/cumm, with normal RBC indices, ESR-41 mm, with peripheral smear showing normocytic normochromic anaemia, leukopenia, thrombocytopenia. Serum iron profile, Vitamin B12, PT, aPTT, INR along with bleeding and clotting time were within normal limits, Urea- $98 \mathrm{mg} / \mathrm{dL}$, Creatinine- $1.3 \mathrm{mg} /$ $\mathrm{dL}$ with normal serum electrolytes, Corrected reticulocyte count -1.1 which showed hypo proliferative picture. MP smear and Dengue IgM ELISA were negative. Lepto IgM ELISA and scrub IgM ELISA were positive. Keeping the above investigation in mind, patient was initially suspected to have hemophagocytic lymphohistiocytosis secondary to infective etiology with $\mathrm{H}$ score of 168 points. Bone marrow examination was done in view of pancytopenia and to look for hemophagocytes. Patient was initiated on antibiotics and high dose corticosteroids pulse therapy and other supportive measures. Bone marrow biopsy report showed features of chronic myeloproliferative disease particularly cellular phase of myelofibrosis and cytogenetic analysis confirmed JAK2 V617F mutation. The patient improved with steroids therapy with no further complaints. His repeat investigations showed haemoglobin- $9 \mathrm{~g} \%$, total count-10,000cells/ cumm and platelet-79,000cells/cumm at time of discharge.

Discussion \& Conclusion: Primary myelofibrosis is an uncommon proliferative neoplasm which usually presents in older individuals. Our patient presented with history of high-grade fever with pancytopenia, hepatosplenomegaly and features of hemophagocytic lymphohistiocytosis. Bone marrow biopsy revealed hypercellular phase of myelofibrosis with positive JAK2 mutation and patient responded to high dose steroid therapy.

\section{Generalised Lymphadenopathy: An Unusual Presentation of Chronic Myeloid Leukemia}

Ankita Gupta ${ }^{* 1}$, Harsha Jaykar ${ }^{1}$, N.S. Mani ${ }^{1}$

${ }^{1}$ Pathology, Bharati Vidyapeeth Deemed to be University Medical College and Research Centre, Pune, India

Aims \& Objectives: Generalised lymphadenopathy—an unusual presentation of chronic myeloid leukemia.

Patients/Materials \& Methods: Case report: A 23 year old male presented with complaints of generalized lymphadenopathy involving bilateral cervical, axillary and inguinal lymphnodes which progressed gradually over the past 5-6 months. On clinical examination the patient had hepatosplenomegaly. Lymphnodes were firm in consistency with no secondary signs. FNAC of the cervical lymphnodes showed a mixed population of immature myeloid series of cells, megakaryocytes, erythroblasts and basophils in a background of reactive lymphoid hyperplasia. A possibility of Myeloproliferative neoplasm and Extramedullary Hematopoiesis was proposed. Hematological correlation confirmed Chronic Myeloid Leukemia in chronic phase due to persistent leukocytosis and demonstration of BCR- ABL abnormalities in FISH and RT-PCR.

Results: Conclusion: Lymphadenopathy is a rare and unusual presentation of Chronic Myeloid Leukemia. Extramedullary hematopoiesis in the background should prompt hematological investigations for a myeloproliferative neoplasm including bone marrow examination and molecular studies.

Discussion \& Conclusion: Discussion: Lymphadenopathy preceding in association with Chronic Myeloid Leukemia is rare. Most reports site the occurrence of Extramedullary Hematopoiesis in the background of Chronic Myeloid Leukemia. We report a case of Chronic Myeloid Leukemia presenting initially as generalized lymphadenopathy and cytological features of Extramedullary hematopoiesis in cervical lymphnode. Bone marrow examination revealed Grade II myelofibrosis. Extramedullary Hematopoiesis is likely to have resulted as a combination of myelofibrosis and replacement of marrow by malignant myeloid cells. Conclusion: Lymphadenopathy is a rare and unusual presentation of Chronic Myeloid Leukemia. Extramedullary hematopoiesis in the background should prompt hematological investigations for a myeloproliferative neoplasm including bone marrow examination and molecular studies.

\section{An Unusual Case of Chronic Myeloid Leukemia in an Adolescent Patient Presented with Priapism}

Anju G ${ }^{* 1}$, Abhishek Purohit ${ }^{1}$, Sujata Sarangi ${ }^{1}$, Deepak K. Sharma ${ }^{2}$, Gopal K. Bohra ${ }^{2}$, Souvik Saha ${ }^{1}$, Himanshu Pandey ${ }^{3}$

${ }^{1}$ Pathology, ${ }^{2}$ Medicine, ${ }^{3}$ Urology, All India Institute of Medical Sciences, Jodhpur, India

Aims \& Objectives: Chronic myeloid leukemia (CML), a common myeloproliferative neoplasm, usually occurs in 5th-7th decade of life and commonly presents with fatigue, weight loss and dragging 
sensation due to massive splenomegaly. The cases may be detected incidentally and uncommonly with priapism and neurologic deficit. We are presenting an adolescent CML patient who presented with priapism.

Patients/Materials \& Methods: A seventeen-year old male presented to the medical emergency with painful penile erection of twoday duration. There was no history of fever, chills, and trauma or of medications. On physical examination, the patient had stable vitals with pallor and moderate hepatosplenomegaly. Local examination revealed erect, firm and tender penis with prominent superficial veins. On investigation, urinalysis, liver and kidney function tests did not show any abnormality, however, serum lactate dehydrogenase levels were elevated (1009 IU/L). On haemogram, haemoglobin, total leucocyte count and platelet count were $9.2 \mathrm{gm} / \mathrm{dL}, 386,000 /$ cumm and $265,000 /$ cumm respectively. The patient was managed by corporal aspiration and irrigation with saline and there was reduction in the erection followed by complete detumescence.

Results: Later, case was seen in haematology clinic. His peripheral smear revealed marked neutrophilic leukocytosis with shift to left and basophilia, blasts comprised seven percent of all leucocytes. Bone marrow examination revealed hypercellular bone marrow with marked granulocytic prominence with presence of $11 \%$ blasts and a diagnosis of CML in accelerated phase was suggested. Molecular analysis revealed presence of chimeric p210 BCR-ABL1 fusion transcript. The patient was treated with imatinib mesylate and he achieved complete haematological response at the end of one month. Further, he remained in complete remission and achieved major molecular response. Currently, patient continues to report to us, is asymptomatic.

Discussion \& Conclusion: Priapism is full or partial erection which continues for more than four hours without any physical or psychological stimulation. Chronic myeloid leukemia (CML) presenting with this rare symptom is an uncommon phenomenonin adolescence. High total leucocyte counts and high blast count may be the underlying pathology for priapism. Prompt and coordinated management by urology and haematology team is of paramount importance.

\section{Metastatic Lung Tumour with Indiagnosed Case of Multiple Myeloma: A Rare Case Report}

Nency R. Narola*1, Sanjay N. Chaudhari ${ }^{1}$, Faruq I. Mulla ${ }^{1}$, Kailash Inaniya $^{1}$, Rohit K. Singh ${ }^{1}$, Dhara D. Ranoliya ${ }^{1}$

${ }^{1}$ Pathology, Pramukhswami Medical College, Anand, India

Aims \& Objectives: We aimed to describe rare presentation of multiple myeloma case with extramedullary metastasis where multiple myeloma actually diagnosed after lung biopsy revealed plasmacytosis.

Patients/Materials \& Methods: A 66 year old male without any co morbidities presented to our hospital with complaints of difficulty in walking and low oral intake since 3 months. There was no respiratory complain and other systemic examination was unremarkable. His hemogram showed hemoglobin: $9.0 \mathrm{gm} / \mathrm{dl}$, Total leukocyte count : 8400/ul, Platelet count : 316000/ul. Biochemical parameters were normal. CECT thorax revealed malignant mass lesion involving posterior segment of right upper lobe of lung with extension to spinal canal and mediastenum with multiple bony metastasis. MRI whole spine was suggestive of multiple abnormal foci in vertebral bodies. Patient underwent image guided lung biopsy which showed small cell tumour of lung which on immunohistochemistry was CD 138 and MUM-1 positive, consistent with diagnosis of multiple myeloma. Bone marrow aspiration and biopsy showed hypocellular marrow with plasmacytosis (25\%). Serum protein electrophoresis for $\mathrm{M}$ protein was negative and free light chain showed kappa: lambda ratio of 0.5 .

Results: The patient was diagnosed as Multiple Myeloma and is treated with palliative radiotherapy and chemotherapy.

Discussion \& Conclusion: : This patient laked other common clinical features of multiple myeloma like fatigue and renal failure(CRABHypercalcemia, renal failure, anemia and bone lesion). The laboratory work up initiated after diagnosis of lung plasmacytoma could establish underlying myeloma. We presenst this case for its unusual presentation.

\section{Evaluation of Distribution of Cases of Myeloproliferative Neoplasms at a Tertiary Care Centre of Western Rajasthan}

Deepsikha Dharamsaktu* ${ }^{* 1}$, Abhishek Purohit ${ }^{1}$, Gopal K. Bohra ${ }^{2}$, Deepak Kumar ${ }^{2}$, Mayank Soni ${ }^{3}$

${ }^{1}$ Pathology, ${ }^{2}$ Medicine, ${ }^{3}$ UG Student, All India Institute of Medical Sciences, Jodhpur, India

Aims \& Objectives: Myeloproliferative neoplasms (MPN) are clonal haematopoietic stem cell disorders characterised by the proliferation of cells of one or more of myeloid lineage and primarily affect adults mainly of fifth to seventh decade of life. The annual incidence rates are six cases per 100,000 populations. Despite an insidious onset, MPN has potential to undergo a stepwise progression that terminate in marrow failure due to myelofibrosis, ineffective haematopoiesis or transformation to blast phase. These changes can be seen in aspirate and biopsy. The aim of this study is to evaluate distribution of MPN cases at our centre and to look at the role of peripheral blood smear and bone marrow examination in final diagnosis.

Patients/Materials \& Methods: This is a retrospective observational study conducted in the Department of Pathology and Lab Medicine at AIIMS Jodhpur. All clinically suspected cases of myeloproliferative neoplasms (Between January 2018 and July 2020) were included in the study. Electronic records were reviewed to note clinical details, laboratory investigations and bone marrow findings in these cases.

Results: This is a retrospective observational study conducted in the Department of Pathology and Lab Medicine at AIIMS Jodhpur. All clinically suspected cases of myeloproliferative neoplasms (Between January 2018 and July 2020) were included in the study. Electronic records were reviewed to note clinical details, laboratory investigations and bone marrow findings in these cases.

Discussion \& Conclusion: WHO criteria for diagnosing myeloproliferative neoplasms mandate mutation analysis in addition to clinical features and bone marrow examination. In our study cohort, we also observed that final conclusive diagnosis often cannot be made in absence of molecular data in non-CML cases.

\section{Profile of Patients with Primary Myelofibrosis}

Bilal S. Kazi ${ }^{* 1}$, Prakash S. Shekhawat ${ }^{1}$, Sumit Mitra ${ }^{1}$, Subham Bhattacharya $^{1}$, Shuvra N. Baul ${ }^{1}$, Prakas K. Mandal ${ }^{1}$, Rajib De ${ }^{1}$, Tuphan K. Dolai ${ }^{1}$

\section{${ }^{1}$ Hematology, NRS Medical College, Kolkata, India}

Aims \& Objectives: Primary myelofibrosis (PMF) is a subcategoryof myeloproliferative neoplasm (MPN).We aim to share our experience with PMF.

Patients/Materials \& Methods: The study included a total of 24 patients seen from January 2019 to September 2020. The presence of any constitutional symptoms was noted and clinical, complete blood counts $(\mathrm{CBC})$, genetic evaluation and karyotyping of the cases was 
done. The DIPSS plus scoring system was applied for risk stratification.

Results: At initial diagnosis, the median age was 52.7 years (range 35 to $70 \mathrm{yrs})$, of which 14 were males $(58.3 \%)$. Fifteen patients $(62.5 \%)$ had a haemoglobin level $<100 \mathrm{~g} / \mathrm{L}, 5(20.83 \%)$ had a total leucocyte count of $>25 \times 109 / \mathrm{L}$, with $7(29.16 \%)$ had a platelet count of $<$ $100 \times 109 / \mathrm{L}$ and $7(29.16 \%)$ showing blasts $>1 \%$ on peripheral smear,13 $(54.16 \%)$ had splenic enlargement of $>10 \mathrm{~cm}$. Seven patients $(29.16 \%)$ required blood transfusion. Genetic mutations of the cases were assessed for JAK2 V617F, CALR and MPL, wherein 20 cases $(80.33 \%)$ were positive for JAK2V617F and $4(16.66 \%)$ for CALR, with no case identified for MPL mutation. None of the cases had unfavourable karyotype. Risk stratification was done using the DIPSS plus score in which 4 cases $(16.66 \%)$ were of low risk, 6 $(25 \%)$ of intermediate 1 risk, 11 cases $(45.83 \%)$ were of intermediate 2 risk and $3(12.5 \%)$ patients belonging to high risk group. 5 cases $(20.83 \%)$ received only ruxolitinib and the remaining 19 patients $(79.16 \%)$ received various combinations of hydroxyurea, prednisolone, danazol and thalidomide.

Discussion \& Conclusion: The results indicate that the disease affects primarily older patients (median age at diagnosis, 52.7 years) and has a slight male preponderance (58.3\% males). $54.16 \%$ patients presented with massive splenomegaly. $80.33 \%$ of PMF were positive for JAK2V617F. $45.83 \%$ were of intermediate 2 risk as per DIPPS plus score.

\section{Chronic Myeloid Leukemia: Blast Phase: Hematological, Immunophenotypic and Molecular Profile from Single Tertiary Care Center}

Jesty P. Tom ${ }^{* 1}$, Alpeshkumar B. Kapadia ${ }^{1}$, Man Updesh S. Sachdeva ${ }^{1}$, Shano Naseem ${ }^{1}$, Nabhajit Mallik ${ }^{1}$, Sreejesh Sreedharanunni ${ }^{1}$, Narender Kumar ${ }^{1}$, Prashant Sharma ${ }^{1}$, Parveen Bose $^{1}$, Jogeshwar Binota ${ }^{1}$, Jasmina Ahluwalia ${ }^{1}$, Reena Das ${ }^{1}$, Pankaj Malhotra $^{2}$, Neelam Varma ${ }^{1}$

${ }^{1}$ Department of Hematology, ${ }^{2}$ Adult Clinical Hematology Unit of Internal Medicine, Post Graduate Institute of Medical Education and Research, Chandigarh, India

Aims \& Objectives: To study the hematological, molecular and immunophenotypic profile of patients of chronic myeloid leukemia (CML) in blast phase.

Patients/Materials \& Methods: Patient-records over a period of one year and 10 months (January 2019 to October 2020) were retrieved and their clinical, hematological, molecular and immunophenotypic data were analyzed.

Results: The CML cases diagnosed during the study period were 776 in numbers of which 30 cases $(3.9 \%)$ were in blast phase. Among these 30 cases, $15(50 \%)$ were in blast crisis at the time of presentation and $15(50 \%)$ were of CML- chronic phase that progressed to blast phase over a median period of 34 months (range: 3-144 months). The median age was 36 years (range: 16-64 years) and male to female ratio was 3.3:1. The median hemoglobin, total leucocyte count and platelet counts were $7.8 \mathrm{~g} / \mathrm{L}$ (range: $5.3-14.1 \mathrm{~g} /$ L), $88.4 \times 109 / \mathrm{L}$ (range: $1.8-527.2 \times 109 / \mathrm{L}$ ) and $47 \times 109 / \mathrm{L}$ (range: $5-251 \times 109 / \mathrm{L}$ ), respectively. The median blast counts in peripheral blood and bone marrow were $31 \%(0-97 \%)$ and $46 \%$ $(0-94 \%)$ respectively. Reverse transcriptase polymerase chain reaction (RT-PCR) for BCR-ABL1 showed presence of major fusion transcript of $\mathrm{b} 3 \mathrm{a} 2$ or $\mathrm{b} 2 \mathrm{a} 2$. The flow cytometric analysis revealed myeloid immunophenotype in 18 cases $(60 \%)$, lymphoid in 9 cases (30\%) and biphenotypic in 2 cases $(6.6 \%)$, while for one case no lineage specific markers were identified. The myeloid blasts showed CD7 (72.2\%), CD56 (38.9\%), CD36 (38.9\%), CD19 (11.1\%),
cCD79a (11.1\%) and CD20 (5.6\%) as aberrantly expressed markers. The lymphoid blasts showed aberrant expression of CD33(55.5\%), CD13(33.3\%), CD36 (33.3\%) and CD56(11.1\%).

Discussion \& Conclusion: The incidence of blast phase in chronic myeloid leukemia is $3.9 \%$ in our study. The Immunophenotyping helps in precise characterization of blasts as well as cross-lineage antigen expression. The mixed phenotypic blasts crisis also can rarely occur.

\section{Outcome of Myeloma Patients on Non Bortezomib Based Protocol from a Tertiary Care Hematology Center of Eastern India}

Kusumita Mandal ${ }^{* 1}$, Malini Gorge ${ }^{2}$, Prakash S. Shekhawat ${ }^{2}$, Arnab Chattopadhyay $^{2}$, Prakash K. Mandal ${ }^{2}$, Rajib De ${ }^{2}$, Shuvra N. Baul ${ }^{2}$, Shubham Bhattacharya ${ }^{2}$, Sumit Mitra ${ }^{2}$, Shyamali Dutta ${ }^{2}$, Tuphan K. Dolai $^{2}$, Shyamali Dutta ${ }^{2}$

${ }^{1}$ Hematology Department, NRS Medical College and Hospital, ${ }^{2}$ Hematology, NRSMCH, Kolkata, India

Aims \& Objectives: To study the treatment outcome of non bortezomib regimen including Thalidomide-Dexamethasone(TD), Lenalidomide-Dexamethasone (LD), Melphalan-Prednisolone(MP), Cyclophosphamide Thalidomide Dexamethasone (CTD) for a period of 10 yrs.

Patients/Materials \& Methods: Retrospective analysis of data of 62 Multiple Myeloma pt. treated with non bortezomib regimen, collected between 2007 and 2018 was done regarding overall response rate (ORR) after 12 months and relapse rate (RR) with in 5 yrs of TD,LD,MP and CTD therapy.

Results: Data from 62 patients aged 23 to 76 years ( 29 women) was collected out of them $26,8,18$ and 10 patients were treated with TD,LD,CTD and MP respectively. Overall response rate of TD was $38.4 \%(10 / 26)$, LD was $62.5 \%(5 / 8)$, CTD was $66.6 \%(12 / 18)$ and MP was $80 \%(8 / 10)$. Out of 62 pt. 7(11.2\%)got relapsed with in 5 yrs. Out of 7 relapsed pt. 6 got TD $(85.7 \%)$ and 1 got $\operatorname{LD}(14.2 \%)$. Out of 26 TD treated pt. $6(23 \%)$ and 1 out of $8(12.5 \%)$ LD treated pt. got relapsed.

Discussion \& Conclusion: Overall response rate among non bortezomib based regimen is better in MP therapy followed by CTD. Relapse rate is more with thalidomide dexamethasone based chemotherapy.

\section{Rare Causes of Eosinophilia with Interesting Presentations: Two Case Reports}

Loveena Rastogi $^{* 1}$, Sachin Bansal ${ }^{2}$, Sachin Jain ${ }^{1}$, Rahul Bhargava ${ }^{2}$

${ }^{1}$ Hematopathology, ${ }^{2}$ Clinical Hematology, FMRI, Gurgaon, Gurgaon, India

Aims \& Objectives: Eosinophilia is a common peripheral blood finding and is seen in a wide variety of diseases. Common causes include infections followed by allergic, neoplastic and autoimmune disorders. Although worldwide most common cause of marked eosinophilia is helminth (worm) infections, the diagnostic approach must include non-infectious (non-parasitic) causes of marked eosinophilia as well. We herein report two cases of marked leucocytosis and eosinophilia reaching two rare diagnoses.

Patients/Materials \& Methods: Case 1. A 25-year-old female presented with high grade fever, breathlessness, difficulty in swallowing, altered taste sensation, abdominal distension with left sided abdominal pain, progressively increasing weakness and difficulty in walking for 5 months. She had similar history of fever, weakness and 
abdominal distension 5 years ago. On examination, patient was found to have fever, pallor, gum hypertrophy, bilateral crepitations, hepatosplenomegaly, abdominal and pleural effusions. The peripheral blood showed marked leucocytosis and eosinophilia. Meanwhile a thorough investigation including HRCT chest, MRI brain and abdomen and nerve conduction test were performed all of which showed significant changes and abnormalities. Bone marrow was done to rule out myeloproliferative neoplasm. BCR-ABL fusion gene, CALR, MPL, PDGFRA and FGFR1 rearrangement by FISH were negative. However, cytogenetic analysis showed TEL-PDGFRB gene fusion with $\mathrm{t}(5 ; 12)$ translocation. Patient was started on hydroxyurea followed by low dose Imatinib to which she responded very well. After three months of her therapy, all her symptoms improved with no palpable hepatosplenomegaly, normal $\mathrm{CBC}$ and complete overall improvement. Case 2. A 42-year-old male presented with oral ulcers and was evaluated for the same. The peripheral blood revealed leucocytosis with high WBC count and eosinophilia. He was treated with antihelminthics but leucocytosis and eosinophilia persisted. Patient was later diagnosed with Behcet's disease and was started on oral corticosteroids. Patient started improving and his total leucocyte count also started declining. However, on tapering the dose his total count started to increase again. Though bone marrow examination was suggestive of CML-CP, the BCR-ABL fusion gene was negative. The complete MPN reflex panel was negative. He was further evaluated for clonal causes of eosinophilia and FIP1L1-PDGFRA fusion gene was confirmed by FISH. He was started on low dose Imatinib and responded well.

Results: Marked leucocytosis with eosinophilia diagnosed as PDGFRB rearrangement in a young female and PDGFRA gene fusion in an adult male.

Discussion \& Conclusion: Conclusion: Marked persistent eosinophilia requires a thorough investigation and non-infectious conditions should also be considered. Low dose imatinib results in an excellent and sustained response.

\section{Outcomes of HSCT in Pediatric Primary Hemophagocytic Lymphohistiocytosis}

Pooja Prakash Mallya*1, Shobha Badiger ${ }^{1}$, Vasundhara Kailasnath ${ }^{1}$, Sharat Damodar ${ }^{2}$, Nataraj KS ${ }^{2}$, Mohana Reddy ${ }^{1}$, Mohammed Salmaan ${ }^{1}$, Santhosh Asangi ${ }^{1}$, Sunil Bhat ${ }^{1}$

${ }^{1}$ Pediatric Hematology, Oncology and BMT, ${ }^{2}$ Hematology and BMT, Mazumdar Shaw Medical Center, NH Health City, Bangalore, India

Aims \& Objectives: Primary hemophagocytic lymphohistiocytosis (HLH) includes familial and several primary immunodeficiencies which exhibit an autosomal recessive inheritance and usually occur in infancy. Hematopoietic stem cell transplantation stands to be the only curative option1.

Patients/Materials \& Methods: Eleven pediatric patients were diagnosed with primary HLH. All patients were initiated with HLH 2004 protocol. Retrospective analysis of the patient records was done. Results: Eleven patients were diagnosed with Primary HLH. Four patients had Griscelli syndrome and one had Chediak Higashi syndrome. The mean age of HSCT was 11 months, (range, 4-84 months). Three patients had CNS manifestations prior to HSCT. One patient had CMV and another EBV viremia prior to HSCT. Four patients underwent matched sibling donor HSCT with bone marrow as the stem cell source. These patients received cyclosporine and methotrexate for Gvhd prophylaxis. Seven patients underwent haploidentical HSCT with TCR depletion for Gvhd prophylaxis and one receiving post-transplant cyclophosphamide. Thiotepa, fludarabine, treosulphan and ATG was the most common conditioning used. The mean MNC and CD34 count was 9.05 (range of 2.7-41) $\times 108$ and $16(7.20-22.8) \times 106 / \mathrm{kg}$ respectively. The mean time to neutrophil and platelet engraftment was 12 and 11 days, respectively. Four patients had CMV reactivation. Sepsis was the most manifestation with 6 patients requiring PICU support. All these patients succumbed to their illness. One patient had grade 1 skin Gvhd. Child with Chediak Higashi syndrome rejected the transplant. Hence a second transplant was attempted but the child failed to engraft and died. Six patients died with 4 of them having associated primary immunodeficiency disorder. Five $(45 \%)$ of the eleven patients are alive till date (see table 1).

Discussion \& Conclusion: Conclusions: HSCT is the only curative option for primary HLH. Early identification of primary HLH is needed to refer for HSCT. Haploidentical HSCT is a feasible option for those with no fully matched donors.

\section{References}

1. Messina C, Zecca M, Fagioli F, et al. Outcomes of children with hemophagocytic lymphohistiocytosis given allogeneic hematopoietic stem cell transplantation in Italy. Biol Blood Marrow Transplant 2018; 24:1223-31.

Supporting Document:

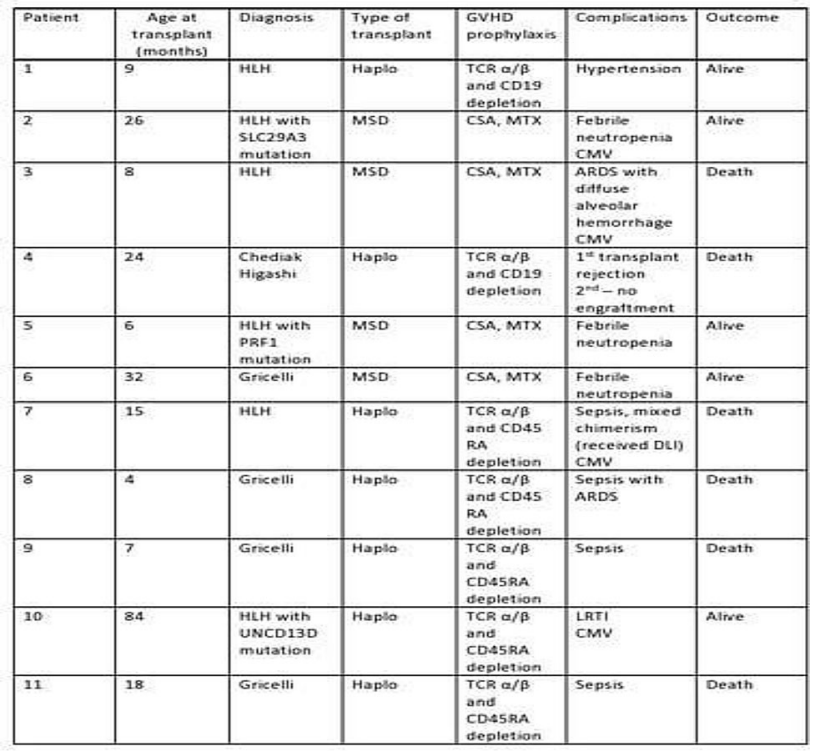

TABLE 1: PATIENT TRANSPLANT DETAILS AND OUTCOME

\section{HEMATOPOIETIC STEM CELL TRANSPLANTATION (HSCT) FOR ALPHA-MANNOSIDOSIS: A CASE REPORT}

Pooja Prakash Mallya ${ }^{* 1}$, Shobha Badiger ${ }^{1}$, Vasundhara Kailasnath ${ }^{1}$, Mohana Reddy ${ }^{1}$, Mohammed Salmaan ${ }^{1}$, Santhosh Asangi ${ }^{1}$, Sunil Bhat $^{1}$

${ }^{1}$ Pediatric Hematology, Oncology and BMT, Mazumdar Shaw Medical Center, NH Health city, BANGALORE, India

Aims \& Objectives: Alpha mannosidosis is a lysosomal storage disorder caused by mutations in MAN2B1 gene which codes for the enzyme alpha-mannosidase. Deficiency of this enzyme leads to lysosomal accumulation of mannose rich oligosaccharides leading to impaired cell function and apoptosis1. Patients present with intellectual disability, skeletal abnormalities, coarse facial features, 
hearing loss and immune deficiency. The symptoms worsen with time. There are three types - type 1 (mild), type 2 (moderate) and type 3 (severe). Enzyme replacement therapy and HSCT are the only curative options for this disease. Studies on HSCT are anecdotal to date.

Patients/Materials \& Methods: We report a 4-year-old boy diagnosed at the age of 3 years with alpha mannosidosis. He presented with development delay and skeletal changes. He was found to have MAN2B1 mutation and was classified as the mild form based on his disease severity. He underwent haploidentical HSCT.

Results: He underwent haploidentical HSCT with father was the donor. Peripheral blood was the stem cell source. TCR alpha/beta and CD45RA depletion was used for GvHD prophylaxis. He also received three courses of pre transplant immunosuppressive therapy with cyclophosphamide (only in the first course) fludarabine and dexamethasone. He received busulphan, fludarabine, ATG and cyclophoshamide based conditioning. The absolute CD34 count is $12.3 \mathrm{X} 106 / \mathrm{kg}$. He achieved neutrophil and platelet engraftment on 13 and 10 days respectively. Post-transplant he developed adenoviremia which resolved with appropriate treatment. He had complete chimerism at day 28 and it remains to be same till date. He is 22 months post HSCT with normalised enzyme levels of 151.2 and $456 \mathrm{nmol} / \mathrm{hr} /$ $\mathrm{mg}$ at 6 months and 1 year respectively. There is no progression of the skeletal manifestations and he has shown developmental progress. Discussion \& Conclusion: HSCT is a feasible therapeutic option for alpha mannosidosis.

\section{References}

1. Mynarek M, Tolar J, Albert MH, Escolar ML, Boelens JJ, Cowan MJ, Finnegan N, Glomstein A, Jacobsohn DA, Kühl JS, Yabe H, Kurtzberg J, Malm D, Orchard PJ, Klein C, Lücke T, Sykora KW. Allogeneic hematopoietic SCT for alpha-mannosidosis: an analysis of 17 patients. Bone Marrow Transplant. 2012 Mar;47(3):352-9.

\section{Should We Do Hematopoietic Stem Cell Transplant in High Risk Pediatric Leukemia and Myelodysplastic Syndrome Patients in the Developing World?}

Sohini Chakraborty ${ }^{* 1}$, Mansi Sachdev ${ }^{1}$, Minakshi Bansal ${ }^{1}$, Rahul Bhargava $^{1}$, Vikas Dua ${ }^{1}$

\section{${ }^{1}$ Hematology, Pediatric Hemato-Oncology and Bone marrow transplant, Fortis Memorial Research Institute, Gurugram, India}

Aims \& Objectives: Hematopoietic stem cell transplant (HSCT) is the only curative modality in high risk and relapsed pediatric leukemia and Myelodysplastic syndrome (MDS) patients. This study was done to describe the outcome of HSCT for these indications at our center.

Patients/Materials \& Methods: Retrospective data analysis was done of pediatric patients with leukemia and MDS who underwent HSCT at our Center from July 2014 to October 2019.

Results: Fifty-three patients who underwent a total of 57 transplants were included in the study. The median age of the study group was 9.5 years (range 1.5 to 18 years). There were 28 patients with acute lymphoblastic leukemia (ALL), 18 patients with acute myeloid leukemia (AML), 3 patients with MDS and 2 each with Juvenile myelomonocytic leukemia (JMML) and chronic myeloid leukemia (CML). Thirty-three underwent matched related donor (MRD) HSCT, 2 matched unrelated donor (MUD) HSCT, 21 haploidentical HSCT while 1 patient underwent autologous HSCT. Myeloablative conditioning was used in 49/57 (85.9\%) transplants. Graft source was peripheral blood in all except one in whom bone marrow was used as the source. Hematological recovery was achieved in $89.4 \%$ transplants. Acute GVHD occurred in 17 (33.3\%) while $3(5.9 \%)$ patients were found to have chronic GVHD. Relapse was seen in 6 patients. A total of 14 deaths occurred in the group (haploidentical- 12, MRD- 2). Cause of death was relapse in 3, infection in 7, GVHD in 2, pulmonary haemorrhage in 1 and primary graft failure in 1 patient. The probability of overall survival (OS) and disease free survival (DFS) at the end of 1 year was $67.68 \% \pm 7.57 \%$ and $61.37 \% \pm 7.77 \%$ respectively. When comparing outcomes based on the type of HSCT the OS and DFS at the end of 1 year for MRD group was $91.04 \% \pm 6.07 \%$ and $81.00 \% \pm 8.74 \%$ while for haploidentical group it was $30.19 \% \pm 11.97 \%$ and $27.09 \% \pm 11.18 \%$ respectively. Discussion \& Conclusion: Many patients of pediatric high risk/relapsed leukemia are not given the option of HSCT in our country. Our study suggests that majority of them can be salvaged with this modality of treatment.

\section{Successful Preimplantation Genetic Testing For: Thalassemia and HLA Haplotyping with Live Birth of a Savior Sibling and Cryopreservation of Umbilical Cord Blood Stem Cells For Future Use: A Case Report}

Firuza Parikh ${ }^{* 1}$, Arundhati Athalye ${ }^{1}$, Dattatray Naik ${ }^{1}$, Nandkishor Naik $^{1}$, Mangesh Sanap ${ }^{1}$, Rupesh Sanap ${ }^{1}$, Dhanashree Warang ${ }^{1}$, Suresh Dhumal ${ }^{1}$, Prashant Padyal ${ }^{1}$, Anahita Pandole ${ }^{1}$, Sangeeta Deshmukh $^{1}$, Prochi Madon ${ }^{1}$

\section{${ }^{1}$ Jaslok Hospital and Research Centre, Mumbai, India}

Aims \& Objectives: To carry out PGT of embryos for -thalassemia c.126_129delCTTT mutation with HLA matching the affected child. Patients/Materials \& Methods: After genetic counseling and prePGT workup, the couple underwent three IVF cycles between June 2019 and November 2019. Trophectoderm biopsy at blastocyst stage was performed and each embryo was vitrified individually. The biopsied cells were further analyzed for -thalassemia c.126_129delCTTT mutation and HLA matching followed by aneuploidy screening. A single HLA-matched, unaffected, chromosomally normal embryo was used for implantation in January 2020.

Results: Table 1 summarizes the analysis of 14 embryos biopsied from 3 IVF-ICSI cycles. Out of 14 embryos biopsied, one was uninformative, 10 were unaffected with -thalassemia, but only 3 were HLA matched and finally one of these was euploid which was used for transfer. 5/7 unaffected embryos were euploid but not HLA matched. The patient conceived and delivered a healthy boy in September 2020.

Discussion \& Conclusion: Preimplantation Genetic Testing (PGT) through in vitro fertilization (IVF) technology is useful to select embryos unaffected with a familial genetic abnormality for implantation. PGT with HLA haplotyping is a recent advance in reproductive medicine, hematology, and hematopoietic stem cell transplant (HSCT) medicine. It allows the preselection of HLA- identical embryos for curing a child affected with -thalassemia or other hematological conditions. We report a successful live birth of a boy free of the -thalassemia mutation c.126_129delCTTT with a $100 \%$ HLA match to his elder affected sib. The umbilical cord blood stem cells have been cryopreserved for HSCT at a later date. This is the first birth in Mumbai, Maharashtra and the second in India after PGT for HLA matching. This form of medical treatment is life saving for the affected elder sibling and cost effective. The role of PGT is shifting from diagnosis to treatment. It is now used to select unaffected HLA matched embryos as potential healthy donors for hematopoietic stem cell transplantation. The medical community and society at large should be aware of the availability of these advanced techniques in India for children affected with genetic and acquired conditions which currently have no cure. 


\section{Supporting Document:}

\begin{tabular}{|c|c|c|c|c|c|}
\hline $\begin{array}{l}\text { Embryo } \\
\text { No. }\end{array}$ & $\begin{array}{l}\text { PGT-M } \\
\text { (Mutation } \\
\text { analysis) } \\
\text { results }\end{array}$ & $\begin{array}{l}\text { HLA } \\
\text { matched } \\
\text { results }\end{array}$ & $\begin{array}{c}\text { PGT-A } \\
\text { (aneuploidy } \\
\text { screening) } \\
\text { results }\end{array}$ & $\begin{array}{l}\text { Sultable for } \\
\text { Embryo } \\
\text { Transter? }\end{array}$ & $\begin{array}{l}\text { Used } \\
\text { for ET }\end{array}$ \\
\hline 1 & Normal & Non-Match & $\begin{array}{c}\text { Low mosaic } \\
\text { aneuploidy: }+14\end{array}$ & NO & No \\
\hline 2 & Abnormal & Non-Match & NA & NO & No \\
\hline 3 & Carrier & Non-Match & $\begin{array}{c}\text { Aneuploid: }+1 q \text {. } \\
-19\end{array}$ & NO & No \\
\hline 4 & Carner & Non-Match & Normal & $\begin{array}{c}\text { YES (No HLA } \\
\text { Match) }\end{array}$ & No \\
\hline 5 & Carrier & Non-Match & Normal & $\begin{array}{l}\text { YES (No HLA } \\
\text { Match) }\end{array}$ & No \\
\hline 6 & Carrier & Match & Aneuploid: -10 & NO & No \\
\hline 7 & Carrier & Non-Match & Normal & $\begin{array}{c}\text { YES (No HLA } \\
\text { Match) }\end{array}$ & No \\
\hline 8 & Normal & Non-Match & Normal & $\begin{array}{l}\text { YES (No HLA } \\
\text { Match) }\end{array}$ & No \\
\hline 9 & Abnormal & Non-Match & NA & NO & No \\
\hline 10 & Carrier & Non-Match & Normal & $\begin{array}{c}\text { YES (No HLA } \\
\text { Match) }\end{array}$ & No \\
\hline 11 & Abnormal & Non-Match & NA & NO & No \\
\hline 12 & Carrier & Match & Normal & $\begin{array}{c}\text { YES (HLA } \\
\text { match) }\end{array}$ & YES \\
\hline 13 & Normal & Match & Aneuploid: $-14 q$ & NO & No \\
\hline 14 & No DNA & NO DNA & No DNA & Uninformative & No \\
\hline
\end{tabular}

\section{Allogeneic Hematopoietic Stem Cell Transplant Offer Good Outcomes in Pediatric Aplastic Anemia: A Single Center Experience}

Mansi Sachdev $^{* 1}$, Sohini Chakraborty ${ }^{1}$, Minakshi Bansal ${ }^{1}$, Rahul Bhargava $^{2}$, Vikas Dua ${ }^{1}$

${ }^{1}$ Pediatric Hematology/Oncology/BMT, ${ }^{2}$ Hematology and BMT, FMRI, Gurgaon, India

Aims \& Objectives: Hematopoietic stem cell transplantation (HSCT) is the preferred first line treatment strategy for aplastic anemia (AA) in children when a matched related or unrelated donor is available. We conducted this study to analyze the outcomes of matched sibling donor (MSD) and matched unrelated donor (MUD) HSCT in pediatric AA patients at our center.

Patients/Materials \& Methods: Retrospective analysis of pediatric patients with aplastic anemia (acquired and inherited) undergoing full-match HSCT at our center between August 2014 to March 2020. Results: Out of 31 patients with severe aplastic anemia (SAA), 19 had acquired aplastic anemia, 2 had Diamond Blackfan anemia (DBA) and 10 had Fanconi anemia (FA). Donors were either matched sibling $(n=29)$, related donors $(n=2)$ or unrelated donors $(n=3)$. Peripheral blood stem cells (PBSC) was the graft source in all the cases except one. The median CD34 + stem cell dose was $5.4 \times 106 / \mathrm{kg}$ (range: 3.5 - 6.5). Fludarabine- based reduced intensity conditioning (RIC) was used in all except for patients with a diagnosis of DBA. Patients with DBA received myeloablative conditioning with Thiotepa, Treosulfan and Fludarabine. All patients except one achieved hematological recovery in the form of neutrophil engraftment at 13 days (range-9-17) while platelet engraftment occurred at 14 days (range-10-18). Graft vs host disease (GvHD) prophylaxis consisted of cyclosporine (CSA) and methotrexate (MTX) \pm antithymocyte globulin (horse/rabbit). Acute GvHD developed in $12.9 \%$ patients while no patients developed chronic GvHD till the time of last follow-up. The 2-yr overall survival for the entire cohort was $93.21 \pm 4.6 \%$.
Discussion \& Conclusion: In patients with SAA, allogeneic stem cell transplant using fludarabine based conditioning regimen is very well tolerated and have excellent outcomes in a full match setting.

\section{Infections in Autologous Stem Cell Transplant (ASCT) in non Hepa Filter Setting: Single Centre Experience}

Malini Garg ${ }^{* 1}$, Prakash S. Shekhawat ${ }^{1}$, Avriti Baveja ${ }^{1}$, Subham Bhattacharyaa $^{1}$, Sumit Mitra ${ }^{1}$, Shuvra N. Baul ${ }^{1}$, Prakas K. Mandal ${ }^{1}$, Rajib De ${ }^{1}$, Tuphan K. Dolai ${ }^{1}$

\section{${ }^{1}$ Hematology, Nil Ratan Sircar Medical College and Hospital, Kolkata, India}

Aims \& Objectives: To study the infection characteristics of patients undergoing ASCT in a non HEPA setting.

Patients/Materials \& Methods: Retrospective analysis of data of 48 patients, who underwent ASCT from September 2016 to August 2020 at hematology department, NRSMCH, Kolkata was done. All patients received recommended prophylaxis. Cultures were sent from various sites during febrile neutropenic episodes and tested in Bactalert 3D Biomeriux analyzer. Positive cultures were compared with period of neutropenia, antibiotics duration and hospital stay following transplant using Pearsons' correlation coefficient.

Results: The mean age was 45.1 (range, 12-66) years with male to female ratio of $2: 1$. Most common diagnosis was plasma cell dyscrasia $(72.9 \% ; \mathrm{n}=35)$ followed by $\operatorname{lymphoma}(22.9 \%, \mathrm{n}=11)$ and relapsed AML $(4.1 \%, \mathrm{n}=2)$. A total of 363 cultures, including throat $\operatorname{swab}(11 \%)$, blood culture from venipuncture $(16.5 \%)$ and from venous catheters $(72.4 \%)$ were sent. Positive cultures were $33(9.1 \%)$, wherein majority were from venous catheters(14,42.4\%). Amongst catheters, Cultures from right internal jugular vein catheter were commonest $(6 / 14,42.8 \%)$. MDR Klebsiella(24.4\%) was commonest isolate, followed by Pseudomonas aeruginosa(15.1\%), $\operatorname{MRSA}(12.1 \%)$, and CONS(12.1\%). Period of neutropenia $($ Mean $=$ 11 days) was significantly associated with positive cultures $(9.1 \%)(p<0.05)$. However it was also significantly associated with low preinfusion CD 34 doses $\left(\right.$ Mean $=5.03 \times 10^{*} 6 / \mathrm{kg}, p<0.05$ ), prolonged antibiotic usage (Mean $=17.4$ days, $p<0.05)$ and increased hospital stay (Mean $=21.4$ days, $p<0.05)$. Patients with positive cultures $(9.1 \%)$ had prolonged antibiotic usage and increased hospital stay $(p<0.05)$. Four patients had respiratory symptoms, for which HRCT thorax was done which was not suggestive of any infections. One patient with delayed engraftment died of septic shock. Discussion \& Conclusion: Overall, positive cultures were $9.1 \%$, wherein majority were venous catheter related. Most common organism was MDR Klebsiella followed by Pseudomonas with no documented fungal infection. Hence, ASCT can safely be done in a non HEPA setting with minimal risk of infection.

\section{Preimplantation Genetic Testing (PGT) For Beta Thalassemia and Other Hematological Disorders}

Arundhati Athalye ${ }^{* 1}$, Dattatray Naik ${ }^{1}$, Rupesh Sanap ${ }^{1}$, Nandkishor Naik $^{1}$, Mangesh Sanap ${ }^{1}$, Dhanashree Warang ${ }^{1}$, Suresh Dhumal ${ }^{1}$, Prashant Padyal ${ }^{1}$, Sona Nair ${ }^{1}$, Prochi Madon ${ }^{1}$, Firuza Parikh ${ }^{1}$

${ }^{1}$ Jaslok Hospital and Research Centre, Mumbai, Mumbai, India

Aims \& Objectives: The main objectives were to offer Preimplantation Genetic Testing (PGT) to Beta-thalassemia, sickle-cell anemia and G6PD mutations carrier couples at high risk of an affected child and use this technology for selection of HLA matched unaffected euploid embryos. 
Patients/Materials \& Methods: For 16 couples diagnosed elsewhere with heterozygous status of hematological disorders, repeat $\mathrm{Hb}$ electrophoresis and mutation analysis showed that 14 were at high risk. At our IVF centre, PGT was carried out for nine couples who were carriers of beta-thalassemia, three for sickle cell anemia, and one each for hemochromatosis and G6PD deficiency, besides other monogenic disorders. During the IVF cycle, trophectoderm cells biopsied from blastocyst stage embryos were subjected to mutation analysis followed by aneuploidy screening. Unaffected euploid embryos were selected for transfer. Prenatal diagnosis reconfirmed the PGT results. For one case with beta-thalassemia, HLA matching of embryos with the affected child was carried out followed by transfer of the single HLA matched unaffected euploid embryo.

Results: Of 16 couples retested, two were normal and did not need PGT. The results of PGT for 14 couples are summarized in Table 1. For sickle cell anemia, both women delivered healthy babies. For G6PD mutation, a twin pregnancy occurred but miscarried in the first trimester. For beta-thalassemia, 6/7 (85.7\%) couples delivered healthy babies. One of these was a HLA identical baby ensuring a future savior sib for the elder affected child. This is the first reported HLA matched birth from Maharashtra and the second in India.

Discussion \& Conclusion: Beta-thalassemia, sickle-cell anemia and G6PD deficiency are common disorders in India. Termination of multiple affected pregnancies is traumatic, hence PGT during IVF is useful to select unaffected euploid embryos for implantation. To cure an affected child by hematopoietic stem cell transplantation (HSCT), PGT can be used to select HLA matched unaffected euploid embryos to get a 'savior sibling' if a suitable donor is not available. When a couple opts for PGT, molecular re-confirmation of variants is important during pre-PGT work-up. To get unaffected, even HLA matched, chromosomally normal children, PGT through IVF is now available in India for hematological and other disorders. Awareness of this facility needs to be spread.

Supporting Document:

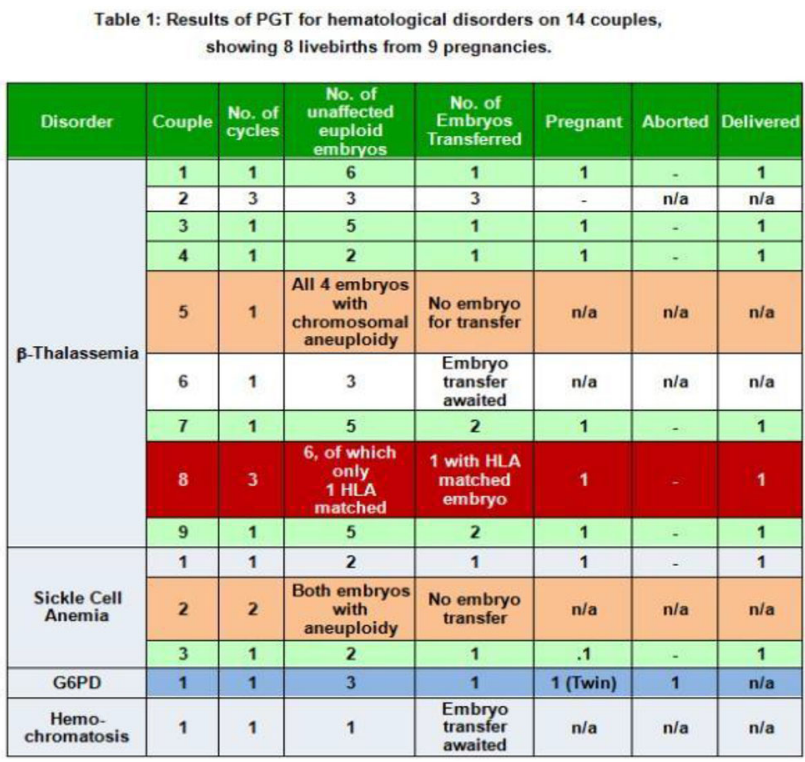

Factors Associated with the Adequacy of Stem Cell Harvest in Patients Undergoing Hematopoietic Stem Cell Transplantation: A Pilot Study from a Tertiary Care Centre in Southern India

Naadia F. Nadeem ${ }^{* 1}$, Prabhu $M^{1}$, Rakhee Kar $^{1}$, Abhishekh B ${ }^{2}$, Smita Kayal $^{3}$

${ }^{1}$ Department of Pathology, ${ }^{2}$ Department of Transfusion Medicine, ${ }^{3}$ Department of Medical Oncology, JIPMER, Puducherry, Puducherry, India

Aims \& Objectives: To determine the correlation between pre-harvest peripheral blood (PB) $\mathrm{CD} 34$ + cells and the final leukapheresis stem cell harvest and to study the factors associated with the adequacy of final stem cell harvest.

Patients/Materials \& Methods: This was a retrospective study with clinical and laboratory data collected from January 2019 till September 2020, from the Department of Pathology, Transfusion Medicine and Medical Oncology,JIPMER. Patients undergoing hematopoietic stem cell transplantation (HSCT), both autologous and allogenic were included. Demographic details and peripheral blood parameters like total leukocyte count (TLC), mononuclear cell count (MNC), platelet count and PB CD34 + count was collected prior to apheresis. Baseline characteristics such as weight, height, BMI, serum albumin and indication for transplant was noted. Pre- harvest PB CD34 + cells and the final harvest CD34 + cells enumeration was done by flow cytometry according to ISHAGE guidelines. Correlation was obtained using Pearson's correlation and a $p$ value of $<0.05$ was taken as significant. Results: Totally 40 patients underwent HSCT, of which 26 were autologous and 14 were allogenic. The male to female ratio was 3:1 and age group ranged from 6 to 61 years. For autologous transplant, the most common indication was multiple myeloma $(57.7 \%)(15 / 26)$, followed by lymphoma $(42.3 \%)(11 / 26)$ in which relapsed Hodgkin's lymphoma was most common $(45.4 \%)(5 / 11)$. Allogenic HSCT were performed for ALL(4), AML(8), MPAL(1) and aplastic anemia(1). The mean pre-harvest PB CD34 + count was 35.81 cells $/ \mu 1$ with an average co-efficient of variation $(\mathrm{CV})$ of $8.48 \%$. The mean CD34+ count in the final stem cell harvest was 1461.7 cells $/ \mu$ with an average $\mathrm{CV}$ of $5.83 \%$. The correlation coefficient ( $\mathrm{r}$ ) and $p$ value were 0.694 and $<0.001$ respectively for pre- harvest PB CD34 + count (with a standard cut-off of 10 cells $/ \mu \mathrm{l}$ ) and final harvest CD34 count, implying statistically significant correlation. Nine out of the 40 cases $(22.5 \%)$ had PB CD34 + count less than 10 cells/ $\mu$ l. Majority of these patients were undergoing autologous transplant for multiple myeloma and required plerixafor before harvest for adequate mobilisation. Among the baseline characteristics, pre-harvest weight, BMI and platelet count also showed statistical correlation with final CD34 count with $p$ values of $0.008,0.003$ and 0.023 respectively.

Discussion \& Conclusion: There is statistically significant correlation between pre-harvest weight, BMI and PB CD34 + cells and the final leukapheresis stem cell harvest and pre-harvest CD34 + count predicted the adequacy of final stem cell harvest.

\section{Response to TA-TMA with Eculizumab in a Post Transplant Patient with Chronic Idiopathic Neutropenia with Variation in CECR1 Gene}

Karthick R G ${ }^{* 1}$, Nataraj K S ${ }^{1}$, Bharath Ram ${ }^{1}$, Shilpa Prabhu ${ }^{1}$, Shiva K. Komaravelli ${ }^{1}$, Aditi Shah ${ }^{1}$, Monisha $\mathrm{H}^{1}$, Sharat Damodar ${ }^{1}$

${ }^{1}$ Haematology \& BMT, Narayana Hrudayalaya, Bangalore, India

Aims \& Objectives: TA-TMA post allogeneic transplant is one of the most serious complications associated with $80-90 \%$ mortality rate. Eculizumab is an effective therapeutic option for high risk TA-TMA 
with improved survival. Herewith presenting a patient with chronic idiopathic neutropenia with a rare variation in CECR1 gene, who underwent allogeneic transplant with matched sibling donor and developed TA-TMA in the post transplant period.

Patients/Materials \& Methods: A 34 years old male presented with the following history: Initial presentation: His parents had consanguineous marriage and mother had history of rheumatoid arthritis with Sjogren syndrome. The patient was said to have PUO at the age of 11 years for which he was extensively evaluated then. Bone marrow at that time was normocellular to hypercellular with relative lymphocytosis. He had recurrent fever episodes since then. In 2005, he was diagnosed to have left upper lobe pneumonia and Mantouxpositive and was started on ATT, which was dose adjusted later in view of deranged liver functions. In 2016, he had right hand weakness and on evaluation, was found to have acute infarct- left brain and was treated for stroke. Prothrombotic workup done at that time was negative. In March 2019, he had complaints of recurrent aphthous ulcers and was found to have TLC-1000. Transplant and post transplant : In July 2019 , he presented to us with fever and leucopenia. Bone marrow examination showed normocellular marrow with myeloid maturation arrest at metamyelocyte stage. In view of chronic idiopathic neutropenia, he underwent matched sibling donor PBSCT with his brother as donor who was a complete match. During pre transplant workup, he was found to have variation in CECR1 gene by NGS. Post transplant, he developed acute gut and skin GVHD. Skin GVHD responded with steroids; as the gut GVHD progressed, he was started on second line treatment with etanercept and ruxolitinib.

Results: He developed hypertension and increased creatinine; on evaluation, peripheral smear showed thrombocytopenia and schistocytes; hence, a diagnosis of TA-TMA was suspected; ADAMTS 13 levels-normal; CMV-negative; Given plasma exchange-7 times;since there was no response along with increasing creatinine, dialysis was initiated and rituximab 2 doses were given. Eculizumab was started following which his creatinine levels stabilised and platelet counts improved. He did not require further dialysis. Has completed 9 doses of eculizumab.

Discussion \& Conclusion: Eculizumab is observed to be a better option in post transplant TA-TMA patients. CECR1 mutations encode ADA2, reportedly cause vasculitis and patients can present with stroke and rashes. This is a rare gene variation seen in this patient associated with chronic idiopathic neutropenia.

\section{Mean Platelet Volume as A Predictive Marker for Cancer Associated Deep Vein Thrombosis}

\section{Chaitanya Patil ${ }^{* 1}$, Shrikant Atreya ${ }^{2}$ \\ ${ }^{1}$ Department of Pain and Palliative Medicine, Kolhapur Cancer Centre, Kolhapur, ${ }^{2}$ Palliative care and Pschooncolgoy, Tata Medical Center, Kolkata, India}

Aims \& Objectives: To find the association between mean platelet volume (MPV) with cancer associated DVT in a palliative care setting and find the best cut off to predict DVT using MPV.

Patients/Materials \& Methods: A cross sectional study was conducted on the patients attending the palliative care clinic at Tata Medical Center, Kolkata. The study was conducted for a period of 3 months (January 2019 to March 2019). The patients with unilateral or bilateral limb edema were screened for DVT as per protocol using ultrasound Doppler. The mean platelet volume of the same patient at the time of screening was noted. The MPV of the patients with DVT and without DVT was compared and Receiver Operating Characteristic (ROC) curves were used to find the best cut off of MPV to predict DVT.
Results: About $26.72 \%$ of the study sample had deep vein thrombosis. The mean platelet volume of study subjects with DVT was $10.32 \pm 1.64$ and without DVT was $9.13 \pm 1.33$ and this difference was statistically significant $(p<0.05)$. After ROC analysis, we found $9.25 \mathrm{fl}$ as the best cut off to predict DVT in our patients. The sensitivity, specificity, positive predictive value, negative predictive value and diagnostic accuracy of this cut off was $70.97 \%, 57.65 \%, 37.63 \%$, $84.48 \%$ and $61.21 \%$ respectively.

Discussion \& Conclusion: More than $1 / 4$ of the sample had DVT in the present study. The MPV of the patients with DVT was significantly higher than patients without DVT. The sensitivity and specificity of MPV in diagnosing DVT was fair but had high negative predictive value. MPV can be used as a additive marker of underlying DVT in the patients attending a palliative care setting.

\section{Evaluation of WBC and Platelet Indices as Prognostic and Risk Factor in Coronary Artery Disease}

Ankur Ahuja ${ }^{* 1}$, Pradeep $V^{2}$, Kanwaljeet Singh², Venkatesan Somasundaram $^{2}$, Kavita Sahai3, Tathagata Chatterjee ${ }^{2}$

${ }^{1}$ Dept of Lab, Government Hospital, Srinagar, 2Dept of Lab \& Mol Medicine, Army Hospital RR, Delhi, 3Dept of Lab, Government Hospital, Udhampur, India

Aims \& Objectives: Aim: To evaluate CBC parameters including Platelet indices, Platelet to lymphocyte ratio, neutrophil to lymphocyte ratio and GRACE risk score in patients with coronary artery diseases. Objectives: (1) To find the values of mean platelet volume (MPV), platelet -Large cell ratio (P-LCR), Platelet distribution width (PDW), platelet to lymphocyte ratio (PLR), neutrophil to lymphocyte ratio (NLR) in patients with coronary artery diseases. (2) To compare the above values with clinical parameters, survival of patients and GRACE risk score in coronary artery diseases and assess the outcome measures in form of mortality were evaluated at 6 months.

Patients/Materials \& Methods: Materials and methods: A total 330 cases of proven Coronary artery disease and 200 matched cases of healthy controls were studied and followed up for 6 months. MPV, P-LCR, PDW, PLR \& NLR were analyzed in an automated hematology analyser.

Results: Among 330 patients, 245 males and 85 females with 324 CAD cases and 4 NSTEMI and 2 Unstable angina cases. There were $34(10.3 \%)$ deaths with $138(40 \%)$ relapses at 6 months as seen in Table 1.

Table 1 Comparison of Parameters between patients and controls

\begin{tabular}{llllll}
\hline Parameter & Cases $(\mathrm{n}=330)$ & Control $(\mathrm{n}=200)$ & \multicolumn{2}{l}{$95 \%$ C.I. of the Difference } & $p$ value \\
\cline { 4 - 5 } & & & Lower & Upper & \\
\hline Plt & $273848 \pm 181623$ & $266880 \pm 183552$ & 25133 & 39070 & 0.670 \\
PDW & $14.3 \pm 3.1$ & $10.4 \pm 2.5$ & 3.39 & 4.41 & 0.0001 \\
MPV & $11.6 \pm 3.6$ & $8.4 \pm 0.8$ & 0.12 & 2.67 & 0.0001 \\
P-LCR & $34.3 \pm 9.9$ & $25.9 \pm 8.9$ & 6.67 & 10.04 & 0.0001 \\
Mean NLR & $2.6 \pm 1.4$ & $1.6 \pm 0.9$ & 0.76 & 1.21 & 0.0001 \\
Mean PLR & $108.8 \pm 70.4$ & $102.9 \pm 42.6$ & 4.93 & 16.70 & 0.286 \\
\hline
\end{tabular}

Similarly, the parameters were corelated with Grace risk score used commonly in cardiovascular disorders and were found to be statistically significant.

Discussion \& Conclusion: In our study we have observed significant correlation of high MPV, PDW, PLCR, NLR in predicting the risk for CAD cases It was also observed that there was positive correlation of 
GRACE risk score with MPV,PDW, PLCR,NLR and PLR in clinical outcome in the form relapse and mortality at 6 month follow up. This study may open new horizons for clinicians to prognosticate and further setting of treatment strategy in CAD cases especially when all these parameters are readily available in all hospitals in few minutes.

\section{A Study to Identify, Classify and Characterize TTP From Other TMAS}

\author{
Sneha S. Yadav ${ }^{* 1}$, Shrimati Shetty ${ }^{1}$, Bipin Kulkarni ${ }^{1}$ \\ ${ }^{1}$ Thrombosis haemostasis, NIIH-ICMR, Mumbai, India
}

Aims \& Objectives: To conduct detailed and comprehensive study of TTP.

-Distinguish between TTP from other TMAs like HUS

- Classify them as congenital or acquired

- Compare ADAMTS13 antigen levels with activity

-Validate PLASMIC score to estimate the probability of TTP in patients with TMA.

Patients/Materials \& Methods: TTP Suspected patients samples were tested for plasma ADAMTS13 antigen by ELISA, ADAMTS13 activity by immunofluorescence, plasma concentration of ADAMTS13 auto antibody was estimated by ELISA and genetic analysis of ADAMTS13 gene by direct DNA sequencing of the 29 exons and their flanking intronic regions by Sanger sequencing. We retrospectively evaluated the data of 130 patients presented with TMA (2016-2020). PLASMIC score of these patients was calculated. Patients with score of $0-4$ were considered as low risk, score 5 as intermediate risk and score 6-7 as high risk group.

Results: 130 TTP-HUS suspected patients were screened, of which 56 patients had low ADAMTS13 Activity, of which 47 patients had autoantibody against ADAMTS13 (Table 1). 6 patients having ADAMTS13 activity low and no auto antibody had ADAMTS13 mutation of which 2 were novel mutations. 3 patients showed low ADAMTS13 activity and no auto antibody nor mutation in them. When ADAMTS13 antigen levels were compared with activity, all congenital cases had activity $<10 \%$, had no detectable antigen levels or the level was $<100 \mathrm{ng} / \mathrm{ml}$. Acquired TTP cases had variable TTP activity and antigen levels while some acquired cases showed normal antigen levels. Plasmic score of only 89 patients could be calculated others were excluded due to lack of data. 30 of these 89 patients were in high risk group, 32 in intermediate risk and 27 in low risk. All 6 congenital TTP were in high risk group, along with 20 acquired TTP. 24 acquired TTP in intermediate and 2 in low risk were observed.

Discussion \& Conclusion: • Of 130 cases, 56 TTP cases were diagnosed of which 6 were congenital and 47 were acquired.

-ADAMTS13 antigen and activity showed correlation. However, patients with low titre antibody with normal level of ADAMTS13, needs to be monitored further as they may develop an acquired deficiency in due course.

-PLASMIC score may improve diagnostic approaches in TMA patients, as empirical treatment with Plasma Exchange can be started with the patients who are at high risk PLASMIC Score.
Supporting Document:

\begin{tabular}{|l|l|l|l|l|}
\hline $\begin{array}{l}\text { Sr. } \\
\text { No. }\end{array}$ & Clinical Manifestation & No. of cases & Relapsecases & $\begin{array}{l}\text { No. of patients } \\
\text { deaths }\end{array}$ \\
\hline 1 & Pregnancyassociated TP & 9 & 4 & 1 \\
\hline 2 & Cancer associated TP & 10 & 2 & 1 \\
\hline 3 & Dengue associated TP & 4 & 1 & 1 \\
\hline 4 & Transplantassociated TP & 8 & 0 & 1 \\
\hline 5 & Other Secondary TP & 16 & 5 & 2 \\
\hline
\end{tabular}

Table l.Acquired TTP cases and classifing them based on clinical details

\section{Thromboembolic Complications Following Recovery from Covid-19 Disease: A Case Series}

\author{
Ankit Kumar $^{* 1}$, Saurabh Dawra ${ }^{2}$ \\ ${ }^{1}$ Internal Medicine, AFMC, ${ }^{2}$ Internal Medicine, Command \\ Hospital, Pune, India
}

Aims \& Objectives: To look for thromboembolic complications in patients recovered from COVID-19 disease. To study clinical and biochemical profile of these patients. And to study the role of D-dimer in prediction and follow up of patients recovered from COVID- 19 disease.

Patients/Materials \& Methods: All COVID- 19 cases readmitted after discharge from the hospital after initial recovery from COVID19 at a tertiary care center in western Maharashtra over a period of two months(August and September 2020).

Results: Out of 384 total admissions of severe COVID-19 pneumonia at a tertiary care centre $4.15 \%(n=16)$ patients were readmitted with sequelae after COVID-19 recovery over a period of 02 months. Out of these $31.25 \%(n=5)$ had thromboembolic complication post COVID-19 infection recovery. All the patients were elderly (mean age:66 years) with multiple co-morbidities (Mean 'Charlson Co Morbidity Index': 3.5 ). 02 were initially managed at another COVID care facility and discharged. They were admitted within 01 week of discharge. 01 patient was discharged and re admitted at our centre. The other 02 had recovered from their illness and were planned for discharge. (Mean duration of hospital stay in initial admission: 14.4 days). All presented within 01 week of clinical and microbiological recovery (Mean: 4.2 days). All were managed as per established protocols and all were on adequate anticoagulation during initial presentation. All these patients had raised D- Dimer levels, (03 suffered sudden cardiac arrest, 01 had a confirmed Pulmonary Thromboembolism (PTE) 01 had a coronary event (Acute ST Elevation Myocardial Infarction).

Discussion \& Conclusion: Thromboembolic complications should be considered an important differential diagnosis in all patients who present with any complication in the immediate follow up period from COVID -19 disease. Repeat D- Dimer levels at follow up may be considered in those with recovery following severe disease. Extended period of anticoagulation and close follow up should be considered in all patients with COVID -19 who are at high risk of developing thromboembolic complications. 


\section{Correlation of Platelet Indices \& Calcium Levels with Serum Cholesterol Levels in Cardiac Patients}

Priyanka Nema ${ }^{* 1}$, Radhika Sharma ${ }^{2}$, Anoop Nigam ${ }^{3}$, Mrs. Manju R. Purohit $^{3}$

${ }^{1}$ Pathology, R.D. Gardi Medical College, ${ }^{2}$ Pathology, R.D. Gardi Mrdical College, ${ }^{3}$ Pathology, R.D.Gardi Medical College, Ujjain, India

\section{Aims \& Objectives:}

-To study correlation of serum calcium levels with serum cholesterol levels in cardiac patients.

-To study correlation of platelet indices with various heart diseases \& platelet indices with serum cholesterol in cardiac patients.

-To study correlation of serum cholesterol and serum calcium with various heart diseases.

Patients/Materials \& Methods: A prospective and observational study on cardiac patients admitted in I.C.U of a tertiary care centre from 2017 to 2019 .

Inclusion criteria:

All cardiac patients admitted in I.C.U. Exclusion criteria:

Patients not willing to provide history or to give blood samples. Procedure:

Clinical history was recorded from case sheet of patients. Data regarding BP, HR, JVP, cardiac markers, E.C.G, Echocardiography were recorded. Normal findings were coded as 0 and abnormal as 1 .

Blood samples for CBC and platelet parameters with automated hematology analyzer and for serum calcium and serum cholesterol were taken.

Results: Out of 153 majority of patients were in the age group of 51-60 years with male preponderance. Majority were diagnosed with CAD (26.1\%) followed by MI. Cholesterol was significantly higher in MI patients as compared to other cardiac conditions. Significant correlation between calcium levels in different CVD was seen. Similar finding was seen for Platelet indices as revealed by the insignificant $p$ value of $>0.05$. On Pearson Correlation, calcium level was positively correlated with cholesterol level which was significant. Calcium values have no correlation with PDW, MPV and P-LCR. Cholesterol was positively correlated with PDW, MPV and P-LCR. That means with increasing cholesterol levels PDW, MPV and P-LCR also increases. Cholesterol was significantly higher in MI patients as compared to other cardiac conditions.

Discussion \& Conclusion: Cardiac problems are more prevalent in male who were in fifth to seventh decade of their life. CAD and MI were more prevalent suggesting importance of early markers for secondary prevention. Strong association between cholesterol and calcium levels \& cholesterol and platelet indices was seen. Calcium supplements must be carefully prescribed. Cholesterol was significantly associated with PDW, MPV and P-LCR indicating them to be an important tool for assessing the risk for developing CVD.

\section{Antiphospholipid Antibodies in Young Adults with Myocardial Infarction}

Karishma Tahlan ${ }^{* 1}$, Meera Sikka ${ }^{1}$, Rajnish Avasthi ${ }^{2}$, Mrinalini Kotru $^{1}$, Priyanka Gogoi ${ }^{1}$, Richa Gupta ${ }^{1}$

${ }^{1}$ Pathology, ${ }^{2}$ Medicine, University College of Medical Sciences, Delhi, India

Aims \& Objectives: This study aimed to assess the prevalence of antiphospholipid antibodies in young $(<45$ years $)$ patients with myocardial infarction (MI) and to find an association between antiphospholipid antibodies and conventional risk factors.
Patients/Materials \& Methods: This case control study was done on 40 young adults diagnosed with acute $\mathrm{MI}$ and 30 age ( \pm 2 years) and sex matched healthy controls. Inclusion criteria required patients to be less than 45 years of age and clinically diagnosed with acute MI. Patients with a family history of heart disease/any other thrombotic event were excluded from the study.Venous blood was collected from patients and controls for complete blood counts (automated hematology analyzer), prothrombin time, activated partial thromboplastin time (automated coagulometer), test for antiphospholipid antibodies (APLAs): Lupus anticoagulant (LA), Anticardiolipin antibodies IgG and IgM and Anti- $\beta 2$-glycoprotein I IgG. SPSS (20) software was used for calculation of the quantitative and qualitative parameters. Positivity for APLAs was expressed in percentages. Association between APLAs and other risk factors was done using Chi square test and Fisher's exact test and Odds Ratio was calculated for various risk factors. $p$ less than 0.05 was considered statistically significant.

Results: Conventional risk factors like smoking, obesity, diabetes mellitus, hypertension and dyslipidemia were present in all patients. APLAs were detected in $6(15 \%)$ patients and $1(3.3 \%)$ control and remained elevated on repeat testing at 12 weeks. Two patients each were LA positive, had elevated levels of IgM Anticardiolipin antibodies and IgG Anti- $\beta 2$-glycoprotein I. IgG Anti- $\beta 2$ - glycoprotein I was elevated in 1control. Multiple risk factors were present in APLA positive patients. Five $(83.3 \%)$ of 6 APLA positive patients were smokers, $1(16.6 \%)$ patient was hypertensive, $2(33.3 \%)$ patients were obese and all $6(100 \%)$ had dyslipidemia. No significant association however was noted between APLAs and conventional risk factors.

Discussion \& Conclusion: The study identified APLAs in 15\% young MI patients all of whom had conventional risk factors. Co- existence of APLAs and conventional risk factors further increases the risk of thrombosis which presents as MI and maybe recurrent. Hence a workup for APLAs may be included in these patients.

\section{Deciphering Antithrombotic Potential of Plant Secondary Metabolites}

Swati Sharma ${ }^{* 1}$, Aastha Mishra ${ }^{1}$, Sayeed Ahmad ${ }^{2}$, Mohammad Z. Ashraf $^{1}$

${ }^{1}$ Biotechnology, Jamia Millia Islamia, ${ }^{2}$ Pharmacognosy and Phytochemistry, Jamia Hamdard, Delhi, India

Aims \& Objectives: Natural compounds chiefly plant secondary metabolites can reduce thrombotic risk and can be used to treat various cardiovascular complications. The natural products can broadly be divided into three categories (i) anticoagulants, (ii) antiplatelet agents, and (iii) fibrinolytic drugs, as conventional drugs to treat thrombosis. In the present study we intend to investigate the antithrombotic effects of secondary metabolites of ginger, garlic, guggul and papya based on their influence on human genomic pathways

Patients/Materials \& Methods: We established SMILES Format File for active ingredients in all four plants viz. ginger, garlic, guggul and papaya. The potential targets for all the active ingredients was predicted via Swiss Target Prediction (http://www. swisstargetprediction.ch/). Then we did mapping of thrombus-related targets and functional enrichment analysis of the potential action targets by Metascape (http://metascape.org). The next step was construction of Component-Target Network and Component-TargetPathway Network which was done by Cytoscape.

Results: The different active compounds in of plants under consideration could act on the same target, and the same active compound could also act on different targets, reflecting multi-component and multitarget action mode of plant metabolites. Total of 83 unique gene targets were predicted from the 33 identified active components using 
the Swiss Target Prediction analysis platform. The gene targets were also mapped to 743 genes possibly related to thrombosis in the CooLGeN database, and to 725 genes associated with the occurrence and development of thrombus in the GeneCards database. Among them, 25 gene targets were also found in the GeneCards database, and 35 gene targets were found in the CooLGeN database. The combination of these results in a total of 27 potential antithrombotic gene targets the active components including PTGS2, JUN, EDNRA KDR, MMP2, MMP9, MMP13, MMP1, MMP10, MAPT, EGFR, FYN, SRC, PTPN1, PRKCA, PTGS1, and ALOX12.

Discussion \& Conclusion: According to the initial screening results, the candidate targets for the active components of these plants were involved in the biological process of response to oxidative stress, which plays a key role in development of thrombus by triggering inflammation. Also, the regulate response to toxic substance, and response to inorganic substance. In terms of the molecular function, the targets were mainly related to metallo endopeptidase activity, endopeptidase activity, and protein domain specific binding. As for the cellular components, the extracellular matrix and membrane micro domain were the main components related to the targets. These initial findings may be helpful for better translational application of these compounds

Funding: Ministry of AYUSH, Grant: S-14013/2/2018-SCHEME.

\section{Factors Influencing Physician Preference in Using Oral Anticoagulant Agents}

Kanwarpal Singh $^{* 1}$, Suvir Singh ${ }^{1}$

${ }^{1}$ Clinical Haematology and Stem Cell Transplantation, Dayanand Medical College and Hospital, Ludhiana, India

Aims \& Objectives: Newer oral anticoagulants are characterized by a predictable pharmacokinetic profile and lower rates of bleeding compared to vitamin $\mathrm{K}$ antagonists (VKAs). However, there is very little data on physician preference for choice of initial anticoagulation and factors influencing the same.

Patients/Materials \& Methods: This study was performed as a cross sectional survey with an aim to include physicians from internal medicine and subspecialties. A 25 part questionnaire was sent to all physicians through Google forms and data was subsequently analysed.

Results: Out of a total of 150 physicians contacted, completed responses were obtained from 50 participants. The primary qualifications included DM/MCh in 57\%, MD in $31 \%$ and other fellowships in $10.6 \%$ respondents. The site of practice included non- teaching private hospital in $38 \%$, private medical college in $34 \%$, government medical college in $21.2 \%$ and private practice in $6.3 \%$. A majority of physicians $(42 \%)$ saw between 2 to 5 patients on anticoagulation drugs each week, with $25 \%$ having more than 10 . Choice of initial anticoagulant for unprovoked VTE was VKA in $46.8 \%$, Dabigatran in $23 \%$, Apixaban in $4.3 \%$ and Rivaroxaban in $17 \%$. Approximately $87 \%$ respondents considered NOACs to have better safety and lower bleeding rates, and $42 \%$ perceived VKAs to have superior efficacy. Over $64 \%$ declared inconsistent PT/INR monitoring by patients at each visit. The most common perceived problem with VKAs was need of regular monitoring, noted by $80.1 \%$, followed by drug interactions in $14.8 \%$. Approximately $80 \%$ participants cited cost as the most important deterrent of NOAC usage, with only $17 \%$ citing lack of complete knowledge. On problem based questions on preoperative use of NOACs, $65 \%$ answered correctly, and $76 \%$ affirmed careful usage in renal disease.

Discussion \& Conclusion: Our study indicates a wide variation in the choice of initial anticoagulant agent among physicians. The most important drawbacks with VKAs and NOACs were need of regular laboratory monitoring, and cost, respectively. Reduced risk of bleeding and lack of regular laboratory monitoring were perceived as the most positive features of NOACs among physicians. Ostensibly, costs, and not lack of knowledge are more likely deterrents of more common usage of NOACs.

\section{Microrna Dependent Regulation of Hypoxia Inducible Factor and Coagulation Pathway}

Anju A. Hembrom ${ }^{* 1}$, Swati Srivastava ${ }^{1}$, Iti Garg $^{1}$, Babita Kumari ${ }^{1}$, Vinay Kumar ${ }^{1}$, Nilanjana Ghosh ${ }^{1}$, Lilly Ganju ${ }^{1}$, Rajeev Varshney ${ }^{1}$

\section{${ }^{1}$ Defence Institute of Physiology and Allied Sciences (DIPAS),} DRDO, New Delhi, India

Aims \& Objectives: Human body maintains homeostasis by preserving an equlibrium between the natural procoagulants and anticoagulants in the blood. Any shift in this equilibrium, can predispose a person towards a thrombotic milieu. Hypoxia, or oxygen deprivation, is an established independent risk factor causing thrombosis. Hypoxic conditions increases the viscosity of blood by increasing pro-coagulatory factors and platelet aggregation, which may lead to formation of sudden blood clots/thrombus in veins, a condition referred as venous thrombosis (VT). The major transcription factor responsible for adaptation to hypoxia is Hypoxia Inducible Factor (HIF). Very scanty data is available on epigenetic regulation of hypoxia-induced coagulation mechanism. MicroRNAs (miRNAs) are potent epigenetic regulators of gene expression, and their role has been vividly studied in cardiovascular diseases. Present In-silico study is an attempt to identify the potential miRNAs that might play a significant role in hypoxia-induced coagulation.

Patients/Materials \& Methods: A list of the 5 major HIF family genes (HIF-1 $\alpha$, HIF-2 $\alpha$, HIF-3 $\alpha$, ARNT, and ARNT2), and Coagulation genes $(n=42)$ was prepared. Their target microRNAs were listed with the help of three online databases (MirNet, MirWalk, MirTargetLink Human). The lists were sorted after removing duplicate entries manually and prioritized on the basis of miRNAs targeting maximum genes of HIF family and coagulation pathway. To further validate the role of these miRNAs in hypoxia and coagulation they were subjected to gene ontology (GO) (using online software ShinyGO) and pathway analysis (using Panther pathways).

Results: In-silico analysis identified three miRNAs (hsa-mir-4433a$3 p$, hsa-mir-4667-5p, hsa-mir-6735-5p) that may potentially regulate the hypoxia-induced coagulation. These candidate regulatory miRNAs overlap in function between adaptation to hypoxia and maintaining homeostasis. GO and pathway analysis revealed that they specifically target genes of major biological processes like blood coagulation, hypoxia response via HIF activation, biological adhesion, etc., which confirms their regulatory input in hypoxia and coagulation mechanism.

Discussion \& Conclusion: The findings of this study present a panel of miRNAs that are common between physiological response to hypoxia and maintaining homeostatic balance. In-vitro validation of these predicted miRNAs may prove their potential as biomarkers in hypoxia-induced coagulation.

Keywords: MiRNA, HIF, Venous thrombosis. 


\section{Genetic Variations in Thrombin}

Activatable Fibrinolysis Inhibitor and Risk of Venous Thrombosis

Babita Kumari ${ }^{\text {*1 }}$, Iti Garg ${ }^{1}$, Swati Srivastava ${ }^{1}$, Vinay Kumar ${ }^{1}$, Uday Yanamandra $^{2}$, Lilly Ganju ${ }^{1}$, Rajeev Varshney ${ }^{1}$

${ }^{1}$ Ministry of Defence, DRDO, DIPAS, ${ }^{2}$ Army Hospital Research \& Referral \& Referral, Hematology, Delhi, India

Aims \& Objectives: Venous thrombosis (VT) is a preventable medical condition, wherein blood clot or 'Thrombus' often initiates in deep veins of leg or arm (deep vein thrombosis, DVT), which may later dislodge from its site of formation and travel to lungs causing pulmonary embolism (PE), a potentially fatal condition. Pathophysiology of VT is complex, as it involves various inherited and acquired risk factors. This multifactorial disease is the consequence of innate thrombotic tendency and non-genetic triggers. Several studies have established the association of genetic variants in coagulation factors, anticoagulants and fibrinolytic factors with risk of VT. Thrombinactivatable fibrinolysis inhibitor (TAFI), a metallocarboxypeptidase when activated, cleaves $\mathrm{C}$-terminal lysine or arginine residues from peptide substrates. This cleavage induces partial degradation of fibrin and a reduction in plasmin formation, with a subsequent attenuation of fibrinolysis. Since TAFI plays an important role in fibrinolysis inhibition thereby promoting clot formation, in the present study, common single nucleotide polymorphisms (SNPs) in TAFI gene have been analyzed in VT patients in comparison to healthy controls.

Patients/Materials \& Methods: This study was performed on human subjects as per institutional ethical guidelines and written consent was obtained from each volunteer before recruiting them for study. Peripheral blood was collected from VT patients along with sex and aged matched healthy control subjects. Basic demographic and physiological information was also collected in both the groups. Two SNPs in the coding region of TAFI gene, G505A (Ala147Thr) rs 3742264 and $1040 \mathrm{C} / \mathrm{T}$ (Thr325Ile) rs 2296642 were analyzed by PCR-RFLP method in VTE patients $(\mathrm{n}=50)$ and subsequently compared to healthy controls $(n=100)$.

Results: Statistically significant variations were observed in allelic frequency of both SNPs, G505A (Ala147Thr) and 1040C/T (Thr325Ile) ( $p$ value $<0.0001$ ). The frequency of occurrence of mutant allele was higher in control subjects than in VT patients.

Discussion \& Conclusion: Findings of present investigation revealed that mutations in TAFI gene could be protective against VT in nature. However, this observation needs to be validated in larger cohort. In addition, this study also suggests that TAFI gene variations may play a noteworthy role in genetic predisposition of an individual towards venous thrombosis.

\section{Apolipoprotein A1 Polymorphisms and Risk of Cardiovascular Diseases: A Meta-Analysis}

Vinay Kumar ${ }^{* 1}$, Iti Garg ${ }^{1}$, Chhavi Rai ${ }^{1}$, Swati Srivastava ${ }^{1}$, Lilly Ganju ${ }^{1}$, Rajeev Varshney ${ }^{1}$

\section{${ }^{1}$ Ministry of Defence, DRDO, DIPAS, Delhi, India}

Aims \& Objectives: Cardiovascular disease (CVD) is the main cause of mortality and it is increasing rapidly across the world with various risk factors like dyslipidemia, hypertension etc. Deep vein thrombosis (DVT), is a third major CVD, a clinical manifestation of venous thromboembolism (VTE) which has high annual incidence rate in adult population worldwide. High density lipoprotein (HDL) modulates the expression of various coagulation factors in various CVDs but these results are inconsistent in DVT. HDL is the lipid carrying molecule that plays a key role in prevention of many diseases through its main protein component ApoA1 which has anti- inflammatory, anti-oxidant and anti-thrombotic properties. Genetic variants plays crucial role in proper functioning of various genes associated with several diseases. In the present study, meta-analysis was done in comprehensive manner to find the association between APOA1 gene polymorphisms (G-75A and $\mathrm{C}+83 \mathrm{~T}$ ) and $\mathrm{CVD}$ using different genetic models.

Patients/Materials \& Methods: Meta-analysis was performed according to the guidelines of the PRISMA and literature was collected from various databases for APOA1 polymorphisms and CVD. Genotypic and allelic frequencies from all the selected human casecontrol studies were extracted. Association was assessed by odds ratio (OR) corresponding to $95 \%$-CI in allelic, homozygous, heterozygous, dominant and recessive genetic models using GraphPad Prism.

Results: From various databases, 2,362 publications were retrieved. These were screened according to exclusion and inclusion criteria and finally twelve studies were considered for meta-analysis. No publication bias between studies was found. Significant heterogeneity was found in all genetic models. However, pooled OR with $95 \% \mathrm{CI}$ indicated that APO1 gene polymorphism and CVD are not linked significantly.

Discussion \& Conclusion: Clinical reports showed that APOA1 gene polymorphism might influence the individual susceptibility to CVD being a crucial regulator of HDL functions. Association of APOA1 gene polymorphisms with CVD was assessed through meta- analysis in five different genetic models which demonstrate that APOA1 Polymorphism may not be an independent predisposing factor for the risk of CVD. These observations necessitates a human case-control study with large sample size to assess the association of APOA1 gene polymorphisms in pathophysiology of venous thrombosis.

\section{The Uselfulness of the Khorana Score in Non-Hodgkin's Lymphomas}

Buruiana Sanda $^{* 1}$, Robu Maria ${ }^{1}$, Tomacinschii Victor ${ }^{1}$, Mazur Minodora $^{1}$

${ }^{1}$ Department of Internal Medicine, SUMPh, Nicolae Testemiţanu”, Chişinau, Moldova, Republic of

Aims \& Objectives: The objective of the study was to assess the risk of thromboembolism in patients with NHL according to the Khorana score.

Patients/Materials \& Methods: The study included 67 patients with NHL (40-aggressive, 27-indolent), 58.2 was the average, 36- women, 31-men. The risk of thromboembolism was assessed according to the Khorana Score according to the NHL type.

Results: According to the Khorana score in aggressive NHL intermediate risk was in $74.5 \%$, high risk- $25.5 \%$; in indolent NHL the intermediate risk was in $85 \%$, the high risk-15\%. The low risk of thrombosis was not determined in any patient. Independent of the NHL type (90\% in aggressive NHL, $77.8 \%$ in indolent NHL), the risk was conditioned by the parameters's association. In aggressive NHL thrombocytosis-31.3\% and $11.1 \%$-indolent NHL. Leukocytosis predominated in indolent NHL compared to aggressive NHL $(67.8 \%$ and $19.5 \%$ ). Independent of the NHL type, in $20 \%$ was mixed anemia: metaplastic or autoimmune. Body mass index (BMI) $\geq 35 \mathrm{~kg} / \mathrm{m} 2$ was recorded in 9 patients (13.4\%) and was not NHL-dependent.

Discussion \& Conclusion: According to the Khorana score, intermediate risk predominated in indolent NHLs $(85 \%)$ and hight riskaggressive NHLs (90\%). Regardless of type of NHL, the presence of several risk factors with a predominance of leukocytosis $(67.8 \%)$ in indolent NHL was determined and of thrombocytosis (31.3\%) in aggressive NHL. The prevalence of anemia and BMI did not depend on the type of NHL. Only these risk factors do not allow adequate 
assessment of individual thromboembolic risk. It is necessary to develop individual instruments, adapted to each patient, to assess the risk of thromboembolic complications.

\section{Peripartum Cardiomyopathy with Left Ventricle Clot: A Rare and Challenging Case}

\author{
Arcot K. Kishore ${ }^{* 1}$, Sandeep Rai ${ }^{2}$ \\ ${ }^{1}$ General Medicine, MGM Hospital, Kamothe, ${ }^{2}$ General \\ Medicine, MGM Medical College, Mumbai, India
}

Aims \& Objectives: To describe a case of peripartum cardiomyopathy with left ventricle thrombus.

Patients/Materials \& Methods: Patient consent taken on admission $2 \mathrm{~d}$ echo to look for thrombus. Necessary lab and radiological investigations done.

Results: Patient discharged on day 40 with improvement in symtoms and on newer oral anicoagulants.

Discussion \& Conclusion: This case report highlights the diagnostic and therapeutic dilemma that physicians face when encountering patients with the rare, but interesting condition of peripartum cardiomyopathy which warrants prompt identification and treatment. PCM usually presents with classical symptoms and sign of systolic heart failure with ventricular enlargement and dysfunction seen on echocardiography. Often there is significant mitral and tricuspid regurgitation [11]. Unusual presentations include thromboembolism or hepatic failure secondary to heart failure. The development of heart failure and the usual time of diagnosis are during the post-partum period in more than $90 \%$ of the cases. PCM can occur at any age with a higher incidence in women older than 30 years. The diagnosis of PCM is challenging because most women in the last months of a normal pregnancy or soon after the delivery experience dyspnoea, fatigue and pedal oedema. Symptoms and signs which should raise the suspicion of heart failure and could help the clinicians in the diagnosis, are the presence of paroxysmal nocturnal dyspnoea, nocturnal cough, new regurgitant murmurs, pulmonary crackles, jugular venous distention and hepatomegaly. PCM is a relatively rare disease, which can have devasting consequences and should be promptly identified and correctly treated. Early diagnosis is important and therefore women who develop symptoms of heart failure during pregnancy or shortly after should be investigated for this condition. Effective treatment reduces mortality rates and increases the chance of complete recovery of ventricular systolic function.

\section{Clinical Diagnosis and Management of Disseminated Intravascular Coagulation in Pregnancy Infected with Sars-Cov2: A Rare Case Report}

Avinash S. Itagi ${ }^{* 1}$, Ishwar Hasabi ${ }^{1}$, Jitendra Kumar Hiremath ${ }^{1}$, Anudeep $\mathrm{M}^{1}$, Pooja V. Biradarpatil ${ }^{1}$

${ }^{1}$ Department of General Medicine, Karnataka Institute of Medical Sciences, Hubballi, India

\footnotetext{
Aims \& Objectives: Professional organizations such as WHO have advised caution in diagnosis and management of pregnant COVID-19 patients. However, the optimal management of pregnant women with COVID-19 has multiple challenges, ranging from screening for the virus on admission to labor to management of the acutely ill parturient, anesthesia, and protection of healthcare personnel. There is scarcity of literature on the management of pregnant COVID-19 patients. Here is a case report of severe presentation of COVID19 in pregnancy with DIC and its successful outcome by a multidisciplinary approach.
}

Patients/Materials \& Methods: A 21-year old female primigravida with 5 months of amenorrhea presented to the Emergency room with complaints of fever and dry cough since two days. Nasopharyngeal swab for SARS CoV2 (COVID 19) was taken for RT PCR testing which was reported positive for COVID 19.

Results: On the day of admission vitals and oxygen saturation was normal. Laboratory parameters showed features of acute kidney injury which were worsening. She further developed thrombocytopenia and hypokalemia. On Day-4 repeat investigations showed worsening AKI with dyselectrolytemia and thrombocytopenia. Chest X-ray with shield was also taken which showed non homogenous opacity in bilateral mid and lower zones. USG showed bilateral grade1 acute renal parenchymal changes and a single live intrauterine pregnancy of approximately 21 weeks of gestational age. On day 5, D Dimer was $>10,000 \mathrm{ng} / \mathrm{mL}$. Termination of pregnancy in the view of DIC and underlying CKD was considered. LMW Heparin $40 \mathrm{mg} \mathrm{s} / \mathrm{c}$ was initiated. On Day-8, 1 unit PRBC and 4 units FFP were transfused and termination of pregnancy was done using Tablet Misoprostol p.v. On day 10, D Dimer was $8654.62 \mathrm{ng} / \mathrm{mL}$. Patient's condition stabilized over the next five days with an increasing platelet count. Chest $\mathrm{X}$-ray was repeated before discharge which was found to be normal with normal D Dimer levels. Patient was diagnosed to have recovered from COVID-19 and discharged in stable condition.

Discussion \& Conclusion: We report a rare case of pregnancy with COVID-19 where the patient had mild symptoms initially but later develops complications of DIC, AKI and septic shock and then completely recovers from COVID 19. Limited studies are reported on COVID-19 affected pregnancy at second trimester stage. This case report may add to understanding, monitoring and treating COVID-19 positive pregnancy including complications.

\section{Quantification of Adamts13 Antigen Levels in Healthy Controls \& Patients with TTP by In-House Elisa}

Sneha S. Yadav ${ }^{* 1}$, Omkar Sawant ${ }^{1}$, Shrimati SHETTY ${ }^{1}$, Bipin Kulkarni ${ }^{1}$

${ }^{1}$ Thrombosis Haemostasis, NIIH-ICMR, Mumbai, India

Aims \& Objectives: To develop an in-house ADAMTS13 antigen ELISA for diagnosis of TTP.

Patients/Materials \& Methods: ELISA was developed using mouse monoclonal anti-human ADAMTS13 IgG to capture the human plasmatic ADAMTS13. The same antibody, labeled with horseradishperoxidase was used for detection. Quantification of bound ADAMTS13 was performed using recombinant human ADAMTS13 as standard.

Results: In total we analyzed 48 individuals, 20 healthy donors and 28 patients with the clinical diagnosis of TMA. In the TMA group 5 patients were suffering from hereditary TTP, 15 patients had acquired TTP and 8 patients had HUS. In healthy donors the ADAMTS13 antigen levels was between the range 460-1500 ng/ml (mean $984 \mathrm{ng} /$ $\mathrm{ml} \mathrm{S.D.} \mathrm{260.93).} \mathrm{The} \mathrm{assay} \mathrm{was} \mathrm{found} \mathrm{to} \mathrm{have} 95 \%$ Specificity and 96\% Sensitivity. Patients with diagnosis of hereditary TTP who had low or undetectable ADAMTS13 activities showed antigen levels $<100 \mathrm{ng} / \mathrm{ml}$, Patients with acquired TTP $(\mathrm{n}=10)$ due to autoantibodies had variable ADAMTS13 antigen levels. Some $(n=4)$ acquired TTP patients had very low or undetectable antigen levels $(<100 \mathrm{ng} / \mathrm{ml})$ in correlation with undetectable ADAMTS13 activities, indicating an enhancing effect of ADAMTS13 clearance caused by the antibodies. This in- house ELISA results correlated well with commercial kits and also costs less than half of that of the commercial kit. 
Discussion \& Conclusion: We could successfully develop an inhouse ADAMTS13 ELISA, that is rapid, sensitive and cost effective test for TTP diagnosis.

\section{Recurrent Hemorrhagic Bullae on Tongue: A Rare Hemorrhagic Manifestation of Primary Antiphospholipid Syndrome}

\author{
Aditi Kudoo $^{1}$, Sabina Langer ${ }^{1}$, Rupali Sharma ${ }^{* 2}$, Jyoti Kotwal ${ }^{1}$ \\ ${ }^{1}$ Department of Hematology, ${ }^{2}$ Department of Pathology, Sir \\ Ganga Ram Hospital, New Delhi, India
}

Aims \& Objectives: We present a case of recurrent hemorrhagic bullae on tongue with unexpected laboratory findings.

Patients/Materials \& Methods: A 29 year old non smoker healthy man, presented to the Hematology OPD with recurrent hemorrhagic bullae on tongue associated with acute pain.

Results: No significant medical or family history was present. No other mouth lesion/ulcer was seen. The CBC showed Hb- $140 \mathrm{~g} / \mathrm{L}$, WBC-6.5x109/L, Platelet- $350 \times 109 / \mathrm{L}$. The coagulations studies show normal prothrombin time (PT), thrombin time (TT), fibrinogen levels with elevated activated partial thromboplastin time (APTT). The factor assay for factor VIII, factor IX, von Willebrand antigen, ristocetin cofactor assay and ratio were normal. The APTT mixing studies revealed partial correction of $45 \%$ (cut off of our lab- 58\%) indicating a circulating inhibitor. The lupus anticoagulant tests DRVVT and SCT were positive suggesting presence of lupus anticoagulant. The serology for anticardiolipin antibodies IgM was positive with negative IgG. The serology for Beta-2 GPI antibodies was negative. The DRVVT \& SCT repeated after 12 weeks came moderately positive for lupus anticoagulant and anticardiolipin antibodies. The secondary causes of APS were found to be negative. Thus final diagnosis of primary APS with moderately positive LAC and presence of $\operatorname{IgM}$ anticardiolipin was considered. No thrombosis seen in radiology \& his D-Dimer levels were normal. He was given only local steroid application along with micronutrients and supplements and is in uneventful regular follow-up.

Discussion \& Conclusion: An Antiphopholipid syndrome (APS) clinically presents either with thrombosis or obstetric complications. The hemorrhagic clinical manifestations are only rarely reported. The hemorrhagic manifestations are explained in the literature by presence of either acquired factor VIII inhibitors or presence of non-neutralizing antiprothrombin/antiphosphotidyl serine antibodies (aPT/aPS) causing rapid clearance of antigen-antibody complexes. Conclusion: Rarely antiphospholipid syndrome can present with hemorrhagic manifestation. The APTT mixing studies can help in suspecting inhibitor in such cases and further tests should be ordered accordingly.

Keywords: Antiphospholipid syndrome, hemorrhagic manifestations, DRVVT,SCT, APTT mixing.

\section{HDL Associated Micrornas Co-Regulate Coagulation Pathway: An In-Sillico Study}

Chhavi Rai ${ }^{* 1}$, Vinay Kumar ${ }^{1}$, Anju A Hembrom ${ }^{1}$, Lilly Ganju ${ }^{1}$, Swati Srivastava ${ }^{1}$, Iti Garg ${ }^{1}$

\section{${ }^{1}$ Ministry of Defence, DRDO, DIPAS, Delhi, India}

Aims \& Objectives: HDL associated MicroRNAs Co-regulate Coagulation Pathway: An in-sillico study.

Patients/Materials \& Methods: Venous thrombo-embolism (VTE), a cardiovascular disease, is the third major cause of mortality and morbidity worldwide. VTE starts as a blood clot formed mostly in deep situated veins (deep vein thrombosis, DVT). This blood clot dislodges itself from the site of formation and travel to lungs through blood stream which results in sudden death (a condition referred as pulmonary embolism, PE). Several lipoproteins have been demonstrated to modulate coagulation and fibrinolytic pathway genes in VTE patients. MicroRNAs are known to regulate genes by posttranslational modification in several complex diseases. In this study, attempt was made to identify the common miRNAs that co-regulate high density lipoprotein (HDL) associated genes and coagulation and complement cascade.

Results: Literature review and database search was done to extract coagulation and complement cascade genes and HDL-associated lipoproteins, viz. apolipoprotein A1 (APOA1), apolipoprotein E (APOE) and paraoxonase (PON1). Thorough search of miRNA databases, sorting, screening, and prioritization was done to obtain common regulatory miRNA candidates. The target gene list of the candidate miRNAs was prepared using online databases, followed by gene enrichment analysis and pathway analysis.

Discussion \& Conclusion: Present In-sillico analysis identified HDL associated miRNAs that are potentially linked with various gene targets involved in blood coagulation, endothelial dysfunction, platelet activation/degranulation and hemostasis. This study comprehensively provide a systematic viewpoint for further research exploring the modulation of HDL associated miRNAs in thrombosis patient to get an insight into pathophysiology of VTE.

\section{Bone Marrow Necrosis in Antiphospholipid Syndrome with Concomitant Homozygous MTHFR C667t Polymorphism}

Soma Pradhan ${ }^{* 1}$, Richa Chauhan ${ }^{1}$, Surbhi Singh ${ }^{1}$, Vandana Arya ${ }^{2}$, Nitin Gupta ${ }^{3}$, Jyoti Kotwal ${ }^{1}$

${ }^{1}$ Department of Hematology, ${ }^{2}$ Department of Hematolgy, ${ }^{3}$ Department of Clinical Hematology, Sir Ganga Ram Hospital, New Delhi, India

Aims \& Objectives: Antiphospholipid Syndrome (APS) is characterized by arterial or venous thrombosis and or pregnancy morbidity accompanied by persistently positive tests for antiphospholipid antibodies. Bone marrow necrosis is a relatively rare finding associated with poor prognosis in cases of APS.

Patients/Materials \& Methods: A 30-year old female presented to emergency with fever, severe anemia and thrombocytopenia. No organomegaly was noted. The patient had past history of recurrent abortions and bilateral deep vein thrombosis and paraparesis. The patient was initially on Dalteparin, thereafter started on Fondaparinux 3 days prior to admission. The bone marrow studies were sent with clinical suspicion of tuberculosis. The complete blood count $\mathrm{Hb}$ :

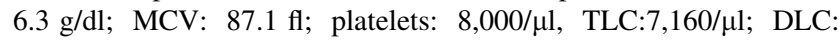
Myelocytes3\%Metamyelocytes1\% N72\%L17.5\%M4\%E0.5\% The peripheral blood smear examination revealed leucoerythroblastic picture with thrombocytopenia and schistocytes $(1.5 \%)$. The bone marrow aspirate and biopsy showed extensive necrosis. Morphology of hematopoietic cells was not discernible. Immunological workup for APS was advised on the basis of clinical presentation and microangiopathic hemolytic blood picture with bone marrow necrosis. The investigations revealed: PT 16.2secs (INR-1.41), APTT 60.5 s.The D-dimer $2.36 \mu \mathrm{g} / \mathrm{L}$, plasma fibrinogen $3.12 \mathrm{~g} / \mathrm{L}$, negative Anti p-ANCA and anti c-ANA, normal Antithrombin, Protein $C$, free Protein S activity, Activated ProteinC Resistance ratio. Both dilute Russell viper venom time and silica clotting time done just before the next dose of fondaparinux was strongly positive for lupus anticoagulant. Anticardiolipin antibodies-IgM were elevated (28.1 MPU/ml,lab cutoff $<18 \mathrm{MPU})$. The molecular workup for thrombophilia showed 
polymorphism for Methylenetetrahydrofolate reductase gene(MTHFR). Patient was lost to follow up for some time and then later on presented to nephrology with renal failure and succumbed to the disease.

Discussion \& Conclusion: The characteristic obstetrics history of recurrent pregnancy loss along with the positivity of lupus anticoagulants led to the diagnosis of APS in this case. The microangiopathic hemolytic blood picture along with bone marrow necrosis is an unusual non-criteria manifestation of APS. MTHFR polymorphism can also contribute to thrombosis and increase the patient's susceptibility to thrombosis.

\section{Evaluation of Procoagulant \& Anticoagulant Activity in Covid-19}

Kartik Jain ${ }^{* 1}$, Abhishek Purohit ${ }^{1}$, Gopal K. Bohra ${ }^{2}$, Mahendra K. Garg $^{2}$, Naresh Midha ${ }^{2}$, Deepak Kumar ${ }^{2}$, Ravi Manglia ${ }^{2}$, Praveen Sharma $^{3}$, Vijayalakshami $\mathrm{Nag}^{4}$, Sanjeev Misra ${ }^{5}$

${ }^{1}$ Pathology, ${ }^{2}$ Medicine, ${ }^{3}$ Biochemistry, ${ }^{4}$ Microbiology, ${ }^{5}$ Surgical Oncology, All India Institute of Medical Sciences, Jodhpur, India

Aims \& Objectives: To study coagulation parameters and its effect on outcome in patients with COVID-19.

Patients/Materials \& Methods: : It was an observational crosssectional study conducted in single tertiary hospital. The study was conducted on samples from 230 patients. All the patients were positive for SARS-CoV-2 by RT-PCR. Patients were categorized as asymptomatic, mild, moderate, or severe according to the guidelines published by the Ministry of Health and Family Welfare (MoHFW), Government of India and World Health Organisation. Following parameters were analysed for these patients- Prothrombin time (PT), activated partial thromboplastin time (APTT), D-dimer, fibrinogen, factor-VIII, protein-C (PC), protein-S (PS) and antithrombin.

Results: Our study conducted on 230 adults showed that there is increase in coagulation abnormalities with increase in severity of COVID-19. There is increase in pro-coagulant and anticoagulant activity with increase in severity of disease. 230 patients included 132 $(57.4 \%)$ males and had mean age $51.82 \pm 16.6$ years. In this cohort, $61.3 \%$ of patients were either asymptomatic $(34.3 \%)$ or had mild disease $(27 \%)$, whereas $20 \%$ and $18.7 \%$ patients had moderate or severe disease, respectively. $12(5.2 \%)$ and $26(11.3 \%)$ patients had prolongation of PT and APTT, respectively. D-dimer levels increased with increasing disease severity and were significantly higher in nonsurvivors when compared to survivors $(6.32 \pm 7.62$ vs $1.69 \pm 3.8 \mu \mathrm{g} / \mathrm{mL} ; p<0.0001)$. Mean levels of fibrinogen, PC, PS and antithrombin levels though remained in normal range but increased from asymptomatic to moderate disease. However, factor VIII showed significant increase in all stages.

Discussion \& Conclusion: Number of patients with abnormal procoagulant and anticoagulant parameters increased with increasing severity but didn't differ among survivor and non-survivors except PS. This study reveals age, D-dimer, APTT, fibrinogen and Factor VIII levels were significantly higher in patients with moderate and severe disease as compared to asymptomatic and mild disease.

\section{Evaluation of Anti Thrombin \& Fibrinogen Levels in Type 2 Diabetes: A Hypercoagulable State}

Prabin K. Phukan ${ }^{* 1}$, Abhishek Purohit ${ }^{1,2}$, Poonam A. Elhence ${ }^{1}$

${ }^{1}$ Pathology, ${ }^{2}$ AIIMS, Jodhpur, India

Aims \& Objectives: Patients with diabetes mellitus have a high risk of atherothrombotic events. Many studies have shown a variety of diabetes mellitus related abnormalities in haemostasis and thrombosis 1,2. Venous thrombosis has also been found to occur more frequently in diabetics. Evidence suggests that fibrinogen and antithrombin has a role both in early stages of atheromatous plaque formation and late complications of cardiovascular diseases. Aim of the study is to access the status of fibrinogen and antithrombin in type II diabetes mellitus and to know about their role in increased thrombogenesis.

Patients/Materials \& Methods: This prospective study is being carried out in the department of Pathology and Laboratory Medicine at All India Institute of Medical Sciences, Jodhpur. Patients of type II diabetes mellitus were included in the study. Plasma fibrinogen (by Clauss method) and antithrombin levels were performed using citrated blood samples on Sysmex CA1500 analyser. Patients' clinical details and fasting blood glucose and glycosylated haemoglobin (HbA1c) levels were noted. Data were entered in excel sheet and analysed.

Results: In this prospective study, 80 patients have been enrolled so far. Pertinent parameters of the cases we're as follows: mean HbA1c was $9.04 \%$ (range 6.6 to $14.5 \%$ ), mean blood sugar was $184.7 \mathrm{mg} \%$ (range 103 to $380 \mathrm{mg} \%$ ), mean plasma fibrinogen level was $328 \mathrm{mg} \%$ (range 50.3 to $601.5 \mathrm{mg} \%$ ), mean antithrombin was 98.3 (range 19.1 to 127$)$. Out of 80 cases $25(30 \%)$ showed higher fibrinogen level above cut off value of $400 \mathrm{mg} / \mathrm{dl}$ and 12 cases (15\%) showed lower antithrombin below 70. Patients are being followed up for any clinical events. However, till date no thromboembolic complications are reported.

Discussion \& Conclusion: Atheroembolic complications are commonly reported in type 2 diabetes patients. This study was conducted to evaluate hypercoagulable state by measuring these two parameters. We observed that $30 \%$ cases showed higher values of fibrinogen and $15 \%$ cases showed lower values of antithrombin. In comparison to normal controls, good number of patients with type 2 diabetes showed higher values of fibrinogen and slightly lesser patients showed lower values of antithrombin. Nevertheless, both being simple tests that is performed on OPD basis, these may help to find high risk cases of thromboembolism.

\section{Thalassemia Day Care}

Chalana *1

${ }^{1}$ Transfusion Medicne/Blood Bank, Rotary International Global Hospital Blood Bank,Radha Mohan Mehrothra Global Hospital Trauma Centre, Abu Road, India

Aims \& Objectives: Introduction: Thalassemias are a diverse group of genetically transmitted autosomal recessive haemolytic disorders characterized by quantitative abnormality of globin chain synthesis of haemoglobin. The clinical spectrum of this disease can vary in severity from asymptomatic laboratory abnormalities to death in utero. Aims and Objectives: Audit of Thalassemia management and care in our centre.

Patients/Materials \& Methods: A restrospective study conducted in our Thalassemia Day Care Centre by retrieval of records from December 2019 to August 2020.

Results: Total patients registered 34,majority being males, in the age group of 8 months to 23.6 years from the states of Rajasthan and Gujarat diagnosed with Thalassemia Major and other haemoglobinopathies. Management is mainly by packed red cell transfusion and or hydroxyurea depending on the type of haemoglobinopathy and severity of anaemia.

Discussion \& Conclusion: Thalassemia Major is a major public health problem. Management of thalassemic patients is extortionatefrequent blood transfusions,myelosuppressives, treatment of iron 
overload,splenectomy and bone marrow transplantation with associated complications. Hence it is vitally important to offer prospective genetic counselling and screening.

\section{Seroprevalence of HIV, HBV, HCV \& Syphilis Among Blood Donors in KIMS Blood Bank, Hubballi}

Sindhu S. $\mathbf{N}^{* 1}$, Sindhu S. $\mathrm{N}^{1}$

${ }^{1}$ Pathology, Karnataka Institute Of Medical Sciences, Hubballi, India

Aims \& Objectives: The aim of our study was to find out the seroprevalence of HIV, HBV, HCV \& Syphilis among the blood donors. Patients/Materials \& Methods: A 9 years retrospective study was carried out in the blood bank of KIMS, Hubballi, Karnataka, from January 2007 to December 2015. From the donor's blood units, $5 \mathrm{ml}$ blood samples were obtained for serological testing. All the samples were screened for HIV, HCV, HBV by ELISA and RPR for syphilis. Results: A total of 80312 apparently healthy donors were analyzed for prevalence of TTIs of which $50735(63.17 \%)$ were replacement donors and $29577(36.83 \%)$ were voluntary donors. The overall prevalence of TTIs was 2047/80312 (2.5\%). The overall prevalence of HIV, HBV, HCV and syphilis among donors were 283(0.35\%), 1556 $(1.9 \%), 195(0.2 \%) \& 13(0.01 \%)$ respectively.

Discussion \& Conclusion: In the western world the transmission rates of $\mathrm{HIV}, \mathrm{HBV}, \mathrm{HCV}$ and syphilis through blood transfusion have been reported to be around 1 in $2-5$ million, 1 in $0.5-1$ million, 1 in 2-4 million, 6 in a million respectively. In the present study, prevalence of HIV was found to be $0.35 \%$. Seroprevalence of HIV in various recent Indian studies reported to range between $0.06-0.6 \%$. Seroprevalence of HIV in Karnataka according to various studies range between 0.06 and $0.44 \%$. Seroprevalence of $\mathrm{HBV}$ in our blood donors was $1.9 \%$. Prevalence of HBV in various recent Indian studies range between 0.45 to $1.9 \%$. Seroprevalence of HBV in Karnataka according to various studies range between 0.34 and $1.9 \%$. Prevalence of $\mathrm{HCV}$ in various recent Indian studies range between 0.098 and $0.85 \%$. Seroprevalence of $\mathrm{HCV}$ in Karnataka according to various studies range between 0.03 and $0.23 \%$. In the present study, prevalence of $\mathrm{HCV}$ was found to be $0.2 \%$. Overall VDRL reactivity in present study is $0.01 \%$, which is comparable to various studies in Karnataka which varies between 0.01 and $0.28 \%$ and other recent Indian studies which varies between $0.01-0.89 \%$. The current infectious disease pattern and trends in donor population can help in planning of future blood transfusion related health challenges. Encouraging female population as well as voluntary blood donors for blood donation will increase the number of donors and safe donor pool.

\section{Alloimmunization Rate in Rhesus Positive Antenatal Women in Uttarakhand}

Anchal Tandon*1 ${ }^{* 1}$ Aparna Bhardwaj ${ }^{1}$, Vineeta Gupta ${ }^{2}$, Gaurav Raturi $^{1}$

${ }^{1}$ Pathology, ${ }^{2}$ Obstetric and Gynaecology, SGRRIMHS, Dehradun, India

Aims \& Objectives: To study alloimmunization rate in Rhesus positive antenatal women in tertiary care center in Dehradun, Uttarakhand.

Patients/Materials \& Methods: A prospective hospital based study was conducted on antenatal Rhesus positive patients attending outpatient department and patient admitted in-patient department of Obstetrics and Gynaecology and being evaluated for hematological

investigations in Pathology laboratory and Blood Bank, Shri Mahant Indiresh Hospital, Dehradun over a period 18 months after approval from Institutional Ethics Committee. Blood samples were taken in EDTA and plain serum separator for blood grouping and IAT(indirect agglutination test) respectively. IAT was done by gel card method.

Results: Alloimmunization rate in screened antenatal women in the tertiary care centre in Dehradun, Uttarakhand was 0.9\%.It was statistically significant with a $p$ value of $<0.04$.

Discussion \& Conclusion: All antenatal women irrespective of Rhesus status should be screened for presence of alloantibodies for RBC antigens.

Supporting Document:

\section{Distribution of IAT positive cases in Rhesus positive Antenatal women in Uttarakhand.}

- IAT positive =IAT negative

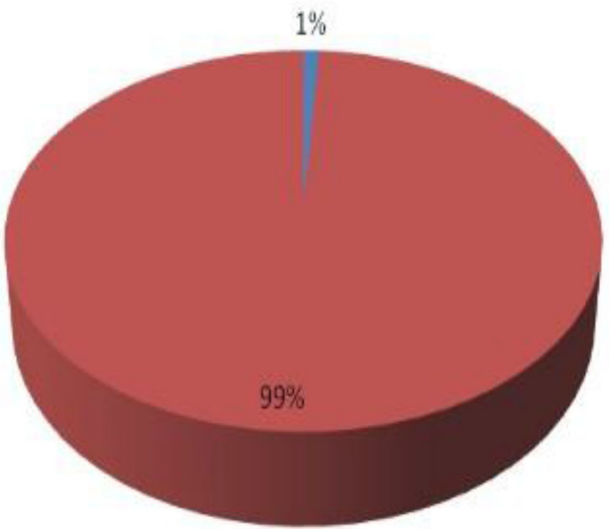

\section{Assessment of Donor Safety in Long Term Plateletpheresis Donation}

Rekha Hans ${ }^{* 1}$, Jyotdeep Kaur ${ }^{2}$, Biman Saikia ${ }^{3}$, Ratti Ram Sharma ${ }^{1}$, Neelam Marwaha ${ }^{1}$

${ }^{1}$ Transfusion Medicine, ${ }^{2}$ Biochemistry, ${ }^{3}$ Immunopathology, Post Graduate Institute Of Medical Education And Research, Chandigarh, Chandigarh, India

Aims \& Objectives: To study the long-term effects of regular apheresis platelet donation on donor; hematological, biochemical and immunological profiles.

Patients/Materials \& Methods: Thirty-three healthy voluntary regular repeat apheresis donors presenting for platelet donation, fulfilling the requisite donor selection criteria as per the drugs and cosmetics rules, in the apheresis section of the Department of Transfusion Medicine underwent sequential (3 months, 6 months and at 12 months) hematological, biochemical and immunological profile assessment over a period of three years.

Results: A total of 33 regular repeat donors were enrolled in the study, of which 22 could be followed up to 3 months and 12 up to 6 months and 10 donors up to 12 months for their hematological, biochemical and immunological parameters. There was no significant change observed in hematological parameters like $(\mathrm{Hb}$, Hematocrit, Total Leucocyte Count) however there was significant increase in 
platelet count at 3 month $(p=0.023)$ with no significant difference from baseline at 6 and 12 months. Mean platelet volume increased significantly from baseline at 12 months $(p=0.00)$. There was no significant change observed in serum protein and serum ferritin over 12 months of follow up, but there was significant decrease $(p=0.00)$ in serum calcium and increase in serum magnesium from baseline $(p=0.03)$ at 12 months. We observed a significant increase $(p=0.010)$ in serum Thrombopoietin at 3 months and serum erythropoietin at 6 months $(p=0.01)$. The CD3, CD4, CD8, CD19 and CD56 levels were not changed significantly.

Discussion \& Conclusion: Apheresis platelet donations do not predispose regular apheresis donors to significant alterations in their hematological \& immunological laboratory profiles. However, they should be encouraged to take calcium supplementation in between and advised to undergo bone scanning.

\section{Performance Indicators Review in Blood Donation Section at Department of Transfusion Medicine}

Saurabh Lahare ${ }^{* 1}$, Minal Wasnik ${ }^{1}$, Ramesh Chandrakar ${ }^{1}$, Sankalp Sharma ${ }^{1}$, Jaisingh Thakur ${ }^{1}$, Manish Sahu ${ }^{1}$

${ }^{1}$ Dept. of Transfusion Medicine \& Blood Bank, AIIMS Raipur, C.G., Raipur, India

Aims \& Objectives: This study was aimed to evaluate various performance indicators and analyze the blood donation section of our department.

Patients/Materials \& Methods: A retrospective study was conducted for 1 year and 06 months from January 2019 to June 2020 in the Department of Transfusion Medicine and Blood Bank, AIIMS Raipur, C.G. The parameters used were performance indicators defined by NABH and from several other studies.

Results: Total registrations for blood donation during the period between January 2019 to June 2020 were 6600 with 4385 blood donors accepted for donation. Total voluntary donations were 1021 $(23.3 \%) \&$ replacement donations were 3364 (76.7\%). In 2019 \& 2020 voluntary donations were $579(21.9 \%)$ \& $442(25.4 \%)$ \& replacement donations $2062(78.1 \%) \& 1301(74.6 \%)$. Total deferrals were $2202(33.4 \%)$. Total male donors were 4187 (95.5\%) \& female donors 197 (4.5\%). In 2019 \& 2020, 2564 (97.04\%) \& $1623(93.1 \%)$ male donors and $77(2.91 \%)$ \& $120(6.9 \%)$ female donors. Total voluntary blood donation camps were 22 with 18 in 2019 \& 04 in 2020. Total 1st time donors were 1703 (38.84\%) and repeat donors were $2682(61.2 \%)$. In $2019 \& 2020$ 1st time donors were 977 $(37.0 \%) \& 726(41.7 \%)$ and repeat donors were $1665(63.0 \%) \& 1017$ $(58.3 \%)$. Adverse donor reactions (ADR) were recorded only in the month of April in 2019, with total 05 cases $(2.4 \%),(\%$ ADR $=$ Total ADR in a month/Total donations in the month $X$ 100). In 2020, 14 adverse donor reactions were recorded, in January (03 cases, $0.67 \%$ ), February (04 cases, $0.92 \%$ ), May (02 cases, $0.90 \%)$, June (05 cases, $2.36 \%$ ). Total Low volume donations were 365 (8.3\%) with 225 $(8.5 \%)$ in $2019 \& 140(8.3 \%)$ in 2020 . There were no complaints from blood donors and maximum donation time recorded for a donor (from entry to exit) was $60 \mathrm{~min}$.

Discussion \& Conclusion: Performance indicators should be implemented to improve quality performance. Due to COVID in 2020, blood donation camps were reduced, but female donors raised in 2020 due to strict replacement policy. This study showed effectiveness of blood donation services at our department .

\section{Comparison of Platelet Indices in Dengue Fever Patients Based on Platelet Transfusion}

Asha J*1 ${ }^{*}$, Nithya M. Baiju ${ }^{1}$, Muhammad A. Rafi ${ }^{1}$, Susheela J. Innah ${ }^{1}$, Ramesh Bhaskaran ${ }^{1}$

\section{${ }^{1}$ IHBT, JMMC \& RI, Thrissur, India}

Aims \& Objectives: Aims: To compare platelet indices in patients with dengue fever to assess their role in the outcome such as hospital stay and platelet transfusion requirements.

Patients/Materials \& Methods: A group of 250 dengue patients were studies over a period of 18 months. The platelet parameters [Platelet count, mean platelet volume (MPV), platelet distribution width(PDW), platelet large cell ratio(PLCR), plateletcrit(PCT) and immature platelet fraction(IPF)]were measured with Sysmex XN1000 and followed up every $24 \mathrm{~h}$. The clinical features, duration of hospital stay, platelet transfusion requirements details were collected.Statistical analysis used: Independent $\mathrm{t}$ test, Chi square test,Karl Pearson Correlation Coefficient.Settings and Design: Prospective observational study in a tertiary care centre, Thrissur, Kerala.

Results: The study showed normal PDW and MPV, low platelet count and PCT, high PLCR and IPF in dengue patients. There were significant differences in platelet indices (lower platelet count and PCT, higher MPV, PDW, PLCR and IPF) on comparison between dengue patients based on platelet transfusion.

Discussion \& Conclusion: Platelet indices (PI) may act as a predictive tool in the diagnosis and predicting outcomes in dengue fever. Low platelet count and PCT, high PDW, MPV, PLCR, and IPF in transfused dengue patients were found to be statistically significant. Clinicians need to be sensitized about the utility and limitations of these indices and rationalizing the need for red cell and platelet transfusions in dengue.

\section{Blood Supply Management AMIDST Covid-19 Pandemic}

Kirti Devgan $^{* 1}$, Vijay K. Sharma ${ }^{2}$, Subrat C. Gupta ${ }^{3}$

${ }^{1}$ Transfusion Medicine, Dr.Ram Manohar Lohia Institute of Medical Sceinces, Lucknow, ${ }^{2}$ Blood Bank, Ram Manohar Lohia Combined Hospital, ${ }^{3}$ Transfusion Medicine, Dr. Ram Manohar Lohia Institute of Medical Sciences, Lucknow, India

Aims \& Objectives: we intend to assess the effect of this mass lockdown on our blood supply management in two phases [phase-I prior to the outbreak] and phase-II[during the outbreak].

Patients/Materials \& Methods: This is a retrospective study of twelve months of a blood bank supplying to a multi-specialty tertiary care academic hospital in Lucknow. The study was divided into two phases namely:

- Phase-I: pre-pandemic phase [Oct'19 to Feb'20]

- Phase-II: the full-blown pandemic phase [Mar 20-sept 20]

Results: The whole blood (WB) collected in phase I and phase II were 5517 and 5379 respectively. In Phase I 27 VBDC collected 1153 units i.e. $69 \%$ whereas in Phase II only 2 VBDC collected 93 units making it only $6.5 \%$ to total collection. The OBDE units in phase I were 218 i.e. $10.9 \%$ while in phase II 321 i.e. $23 \%$ were discarded. The PRBC demand in phase I was around 296/month for 5 months and was reduced to 284 /month in 7 months.Exclusive supply to covid patients in last 5 months were 69 units.

Discussion \& Conclusion: In phase I the WB collected was more and the discarded units were less as compared to phase II. The overall collection was reduced in phase II including the monthly demand of PRBC.With little insight of the disease and everyday learning, by 
motivating more voluntary donors and health care workers the efficient chain of blood supply and demand can be maintained as the virus is to stay with us for a long time.

\section{Significant Improvement in Patient Blood Management (PBM) Through Incorporation of Transfusion Practitioner}

\author{
Neelesh Jain ${ }^{* 1}$, Dibyendu De ${ }^{1}$ \\ ${ }^{1}$ Balco Medical Centre, Naya Raipur, Raipur, Chhattisgarh, \\ Sector-36, Naya Raipur, India
}

Aims \& Objectives: Aim: To ensure rational and safe blood transfusion practices in the hospital. Objectives: To improve transfusion reaction reporting mechanism. To minimize communication gap between clinical staff \& blood centre. To reduce clerical errors. To improve blood transfusion related general awareness amongst nursing staff and RMO's.

Patients/Materials \& Methods: Daily at 9 am we review the blood requisitions from various users along with $\mathrm{TP}$, we note down the observations. Then TP goes to the ward round with the clinical team, where he note down the relevant issues. After that, TP quickly interacted with the RMO \& duty nurses at patient bed side without wasting their time and submit his observations to the HOD Transfusion Medicine. We compile the information and organize interim blood transfusion committee meeting with all the stake holders to discuss the issues related to PBM improvement and rectify the process accordingly giving prime importance to the patient safety.

Results: In just 12 months' time we observed significant changes in our transfusion practice in terms of reduction in the number of unnecessary PRBC \& FFP (43 PRBC \& 74 FFP) transfusions, turnaround time for blood delivery, significant reduction in the clerical errors, improvement in transfusion reaction reporting with overall reduction in transfusion reactions. It has significantly improved the communications gap between clinical staff \& blood centre. Comparison was made through historical arm.

Discussion \& Conclusion: Although transfusion and patient blood management (PBM) processes are complex, The TP is often seen as driving force for change, working in a multidisciplinary capacity across the clinical spectrum to promote safe and rational transfusion practice. In our centre, we nominated a paramedical person with experience in transfusion services to play this role to bridge the communication gap amongst various stake holders. Conclusion: Transfusion practioner is a key link in the multidisciplinary chain and require strong support and leadership to effectively incorporate the positive changes in the existing process of patient blood management. This is relatively a newer concept in our country, more and more centers shall incorporate such practice to improve $\&$ enhance patient safety.

\section{Reasons for Discarding Whole Blood and Blood Components: A Crossectional Study}

Rakshitha HB ${ }^{* 1}$, Aneesha A. Kumar ${ }^{2}$

${ }^{1}$ Pathology, Adichunchanagiri Institute of Medical Sciences, Adichunchanagiri University, BG Nagara, Mandya, Mandya, ${ }^{2}$ Pathology, MES Medical College, Kerala, Malappuram, India

Aims \& Objectives: To find out the reasons for discarding blood and its components.

Patients/Materials \& Methods: This is a retrospective cross sectional study conducted over a duration of 02 years between January 2018 up to December 2019 at the MES Medical college blood bank,
Kerala. The details of whole blood and blood components collected and discarded during the study period were retrieved from the blood bank records and the reasons for discarding blood and its components were analysed.

Results: A total of 7,716 blood units were collected during the study period. Out of 7,716 blood units collected, 770 were whole blood and the remaining 6,946 were made into components such as Packed red cells 6946, Fresh frozen plasma 6946, platelet concentrate 2,794 and cryoprecipitate 106. Out of 7716 units of blood, 1110 units of blood and blood components were discarded. Discard rate was highest for platelet concentrate $(53.1 \%)$. Expired units formed the main reason for discarding units $(82 \%)$.

Discussion \& Conclusion: Blood and blood components should be utilized judiciously. Proper implementation of blood transfusion policies is required to reduce the discard rates of blood and its components.

\section{Role of Transfusion Support in Sepsis}

Ashwin Vaidya*1, Shamee Shastry ${ }^{1}$, Ganesh Mohan ${ }^{1}$

${ }^{1}$ Immunohaematology and Blood Transfusion, Kasturba Medical College, Manipal, Udupi, India

Aims \& Objectives: To identify the role of transfusion support in sepsis.

Patients/Materials \& Methods: We conducted a retrospective observational study in a tertiary care hospital in Karnataka over a period of 1 year (2018). Patients above 18 years of age admitted in Intensive care units diagnosed with sepsis were included. Sepsis was diagnosed based on SOFA score. Haematological parameters like haemoglobin, haematocrit and platelet count, coagulation parameters like PT, aPTT were collected at the time of diagnosis of sepsis, before transfusion and post transfusion support. The number of blood products transfused in sepsis patients was evaluated. Data was collected from laboratory and blood centre software. The data was analysed using SPSS 22.0.

Results: A total of 118 patients were diagnosed with sepsis. The male to female ratio was $3: 2$, mean age was $53.92 \pm 15.9$ years. Transfusion support was required in $47.45 \%(n=56$, group 1$)$ of the patients. Median number of blood components transfused were, PRBC: 2 (106 units), FFP: 4 (178 units), platelet: 5 (169 units) and cryoprecipitate: 10 (65 units). There was significant increment in haemoglobin $(p=0.001)$, haematocrit $(p=0.0002)$, and platelet count $(p=0.034)$ after transfusion. Patient who were transfused had a prolonged ICU (5 vs $4, p=0.008$ ) and hospital stay (7 vs 4 , $p=0.001)$ compared to non-transfused patients. The risk of receiving transfusion was more in the presence of coagulopathy (OR-8.96, $\mathrm{Z}=4.13, p<0.0001)$. Mechanical ventilation support, septic shock, MODS, and mortality rate was comparable between both the groups. Discussion \& Conclusion: Transfusion support is very critical in sepsis especially in the presence of sepsis induced coagulopathy and judicious usage of blood components is advised.

\section{Clinical Profile of Bleeding Patients Managed with Goal-Based Approach}

Prethika P A ${ }^{* 1}$, Shamee Shastry ${ }^{1}$, Ganesh Mohan ${ }^{1}$, Ashwinkumar Vaidya $^{1}$

${ }^{1}$ Immunohematology and Bood Transfusion, Kasturba Medical College, Udupi, India

Aims \& Objectives: To assess the clinical profile of hemorrhagic patients managed with Goal based transfusion approach. 
Patients/Materials \& Methods: We conducted a prospective study over a period of one year (2019) involving hemorrhagic patients receiving goal based blood transfusion. Data collected included indications for transfusion, the laboratory parameters (haematological, biochemistry and coagulation) collected on admission and $24 \mathrm{~h}$ post transfusion, number of blood components utilised, their clinical outcome based on the length of hospital stay, complications developed and mortality. The data were collected from hospital and blood bank software. The patients were followed up during the hospital stay; 24-hour mortality; complications developed post massive transfusion were documented. The data was analysed in SPSS software version 22.0

Results: A total of 109 patients were included in the study. The common causes for massive bleeding were trauma (51), Upper Gastro-intestinal bleed (24), Post-partum haemorrhage (14), Surgical causes (14) and Other causes (6). The male-female ratio was 2:1. On comparing the pre and post massive transfusion - haemoglobin, platelet count and coagulation screen improved significantly $(p<0.05)$ for treating massively bleeding patients. The number of blood components used were PRBC 4 (IQR 4, N = 488 units), FFP 4 (IQR 4; $\mathrm{N}=444$ units), Platelet 5 (IQR $1, \mathrm{~N}=288$ units), Cryoprecipitate 10 (IQR 5, N = 340 units). The Overall Length of hospital stay was 14.39 days; $82(75.2 \%)$ patients were free of transfusion at the end of $24 \mathrm{~h}$; the survival rate was $66.9 \%$. The overall mortality rate was $27.5 \%$ (30) and Discharged Against Medical Advice (DAMA) were $5.5 \%$ (6). We observed Metabolic acidosis in 28 patients $(51.9 \%)$, hypocalcemia in 29 patients $(72.5 \%)$ and Coagulopathy in 6 patients $(6.9 \%)$ post transfusion.

Discussion \& Conclusion: Timely administration of blood components using goal based massive transfusion helps in better patient blood management and improved clinical outcome in hemorrhagic patients.

\section{Trends and Prevalence of Transfusion Transmissible Infections Among Blood Donors in a District Hospital Blood Bank: A Five Year Study}

Chandrashree $\mathbf{J}^{* 1}$, Geethalakshmi $\mathrm{U}^{1}$, Ramesh B. $\mathrm{K}^{1}$

${ }^{1}$ Pathology, Shimoga Institute of Medical Sciences, Shimoga, India

Aims \& Objectives: To estimate the prevalence and to assess trends of TTI among blood donors.

Patients/Materials \& Methods: This is a retrospective study based on the records of all the blood donors (voluntary and replacement) of district hospital blood bank from January 2012 to December 2016. The donated samples were tested for HBV, HIV, HCV by both Enzyme Linked Immunosorbent Assay (ELISA) and Individual Donor Nucleic Acid Test (ID-NAT), Syphilis by VDRL test and Malaria by Slide \& card method. Appropriate statistical test was applied using SPSS software.

Results: Of the total 33,824 blood donations 30,451(90.03\%) were voluntary and 3,373(9.97\%) were replacement donors. Overall seroprevalence of $\mathrm{HBV}, \mathrm{HIV}, \mathrm{HCV}$ and syphilis in our study was $1.02 \%$, $0.16 \%, 0.06 \%$ and 0.03 respectively. Trend for each TTI was fluctuating except for Syphilis.

Discussion \& Conclusion: Transfusion transmitted infections are still a major threat to blood transfusion practices. It requires awareness, strict voluntary blood donation practices and vigilant screening of blood. Present study highlights the importance of vigilant donor selection, standard screening protocols and emphasizing voluntary blood donation along with post donation counselling to to achieve maximum blood safety.
Supporting Document:

\begin{tabular}{|c|c|c|c|c|c|c|}
\hline YEAR & 2016 & 2015 & 2014 & 2013 & 2012 & TOTAL \\
\hline $\begin{array}{l}\text { TOTAL } \\
\text { DONATIONS }\end{array}$ & $\begin{array}{l}7433 \\
\mathbf{N}(\%)\end{array}$ & $\begin{array}{l}7183 \\
\text { N }(\%)\end{array}$ & $\begin{array}{l}6757 \\
\text { N }(\%)\end{array}$ & $\begin{array}{l}5882 \\
\mathbf{N}(\%)\end{array}$ & $\begin{array}{l}6569 \\
\text { N }(\%)\end{array}$ & $\begin{array}{l}33824 \\
\mathbf{N}(\%)\end{array}$ \\
\hline \multicolumn{7}{|l|}{ GENDER } \\
\hline MALE & $\begin{array}{l}7148 \\
(96.16)\end{array}$ & $\begin{array}{l}7058 \\
(98.25)\end{array}$ & $\begin{array}{l}6598 \\
(97.65)\end{array}$ & $\begin{array}{l}5724 \\
(97.32)\end{array}$ & $\begin{array}{l}6401 \\
(97.45)\end{array}$ & $\begin{array}{l}32929 \\
(97.35)\end{array}$ \\
\hline FEMALE & $\begin{array}{l}285 \\
(3.84)\end{array}$ & $\begin{array}{l}125 \\
(1.75)\end{array}$ & $\begin{array}{l}159 \\
(2.35)\end{array}$ & $\begin{array}{l}158 \\
(2.68)\end{array}$ & $\begin{array}{l}168 \\
(2.55)\end{array}$ & $\begin{array}{l}895 \\
(2.65)\end{array}$ \\
\hline
\end{tabular}

DEMOGRAPHIC CHARACTERISTICS OF BLOOD DONORS IN DISTRICT HOSPITAL BLOOD BANK IN THE PERIOD 2012-2016

\section{Seroprevelance and Trends in Tranfusion Transmiccible Infections Among Blood Donors in Blood Bank of Sharda Hospital}

Tripti Kashyap ${ }^{* 1}$

${ }^{1}$ Pathology, Sharda Hospital, Greater Noida, India

Aims \& Objectives: To review the seroprevelance and trends of TTI'S among blood donors of sharda hospital.

Patients/Materials \& Methods: Samples collected in blood bank for screening of HIV, HBV,HCV,Syphillis \& malarial antigen using Elisa method.

Results: Out of 11458 screened donors 417 were infected with at least one infective agent. HBV-177, VDRL-126, HCV-105,HIV-13, MP-2. Discussion \& Conclusion: The pattern has shown rising trends in HBV,HCV \& VDRL infections.

\section{Changes in Haematological Indices of Red Cell Concentrates During Leukoreduction}

Deep Madkaiker*1, Shashank Ojha ${ }^{2}$, Sunil Rajadhyaksha ${ }^{1}$, Kalpesh Chawan $^{2}$

${ }^{1}$ Transfusion Medicine, Tata Memorial Center, ${ }^{2}$ Transfusion Medicine, ACTREC, Mumbai, India

Aims \& Objectives: To evaluate changes in haematology indices of red cell concentrates(RCC) after leukoreduction.

Patients/Materials \& Methods: Leukoreduction(LR) is performed in our department using Macopharma Leucolab LCG2b leukofilter as per departmental Standard Operating Protocols to obtain LR RCC especially for haemato-oncology patients. This was conducted as a retrospective study wherein complete blood count $(\mathrm{CBC})$ coulter reports obtained from ADVIA 2120i 5-part analyser on pre-LR and post-LR samples of RCCs were analysed for changes in the various haematology indices.

Results: A total of 93 pairs of CBC reports (Pre-LR and Post-LR) were analyzed in this study. Mean(SD) reduction in WBC count was $99.77(0.33) \%$ equivalent to $2-3 \log$ reduction although estimation 
by Naegotte's method gave a $99.99 \%$ reduction (4 Log). Additionally mean(SD) platelet reduction was $90.85(18.4) \%$ equivalent to a $1 \log$ reduction. Mean(SD) red cell volume loss during the process was estimated to be $9.72(3.29) \%$. In addition to the desired effect of leukoreduction, significant changes were also observed on several red cell indices such as RBC count, $\mathrm{MCV}, \mathrm{MCH}, \mathrm{CHCM}, \mathrm{CH}$ and Cell HB. The relative change in the different indices was analyzed for correlation with Pre-LR parameters. The Pre-LR haematocrit was found to be significantly correlated to the changes in 7 out of 13 indices measured.

Discussion \& Conclusion: Pre-storage leukoreduction of blood components is a valuable technique to mitigate the adverse effects of leukocytes in transfusion recipients. This study validates the efficacy of leukofilters to reduce the leukocyte load of a red cell concentrate to sufficiently low levels (4-5 log reduction) while minimising red cell loss to $<15 \%$ of the total volume filtered as mandated by regulatory guidelines. A $1 \log$ reduction of platelet content was also achieved and may provide additional benefits. Changes in Post LR red cell indices were noted which could result from the passage of RBCs through the narrow leukofilter pores. Furthermore the relative change in the haematological indices were found to be dependent on the PreLR parameters which may affect the efficacy of the filter used for leukoreduction.

Supporting Document:

Summary of changes in haematological indices of RCC

\begin{tabular}{|c|c|c|c|}
\hline Parameters & Pre-LR & Post-LR & $\begin{array}{c}\text { Results } \\
\text { expressed as }\end{array}$ \\
\hline WBC $\left(10^{3} / \mathrm{ul}\right)$ & $6.34(4.935,7.6)$ & $0.01(0.005,0.02)$ & \multirow{8}{*}{$\begin{array}{c}\text { Median } \\
\text { (Interquartile } \\
\text { range) }\end{array}$} \\
\hline $\operatorname{RBC}\left(10^{6} / \mathrm{ul}\right)$ & $6.12(5.765,6.535)$ & $6.04(5.7,6.235)$ & \\
\hline $\operatorname{MCV}(f)$ & $96.3(92.8,100.1)$ & $96.5(93.1,101.5)$ & \\
\hline MCH (pg) & $29.7(28.3,30.7)$ & $29.8(28.7,30.9)$ & \\
\hline $\mathrm{CHCM}(\mathrm{g} / \mathrm{dl})$ & $30.1(29.2,31.1)$ & $29.7(28.6,30.65)$ & \\
\hline $\mathrm{CH}(\mathrm{pg})$ & $28.8(27.35,30)$ & $28.8(27.4,29.8)$ & \\
\hline PLT (103/ul) & $83(61,162)$ & $2(1,8.5)$ & \\
\hline $\operatorname{MPV}(f)$ & $9.7(8.65,10.9)$ & $8.1(6.9,9.95)$ & \\
\hline Cell HB (g/dl) & $18.12+/-1.32$ & $17.93+/-1.23$ & \multirow[b]{2}{*}{ Mean $+/$-SD } \\
\hline Volume Loss (\%) & - & $9.72+/-3.29$ & \\
\hline
\end{tabular}

\section{Immune-Hematological Consequence of Intravenous Drug Abuse? A Case Report}

\section{Aikaj Jindal $^{* 1}$ \\ ${ }^{1}$ Transfusion Medicine, SPS Hospitals, Ludhiana, India}

Aims \& Objectives: We present here a case of 28-year-old man who presented with a rather unique predicament of having developed four different alloantibodies after exposure to allogenic blood through IV drug abuse. To the best of our knowledge and literature search, this is the first of its kind case ever reported from India and has no precedence.

Patients/Materials \& Methods: Blood Grouping and Irregular Antibody Screening Antibody Identification. Cross Match and usage of Select Cells Enzyme treatment.
Results: Four select cells (Table-3) from Resolve Panel B (Ortho Clinical Diagnostics, Johnson and Johnson, USA, Lot No. RB481 \& RB513) were used to confirm the presence of Anti-C, Anti S and antiLeb alloantibody and to rule out Anti- E. Further, enzyme- treated cells 6, 9 and 10 (all D0 C0) of Panel-A: decreased agglutination reaction strength confirmed anti-S as shown in Table 2.

Discussion \& Conclusion: The intravenous drug abuse is prevalent in the today's youth in North Western India, especially in states of Punjab and Haryana. Apart from being a medium for transmission of various blood borne infections it has also led to emergence of previously rarely seen phenomenon-alloantibodies in previously nontransfused patients. Alloantibodies have been found often in multi transfused patients like thalassemia and patients suffering from leukaemia, lymphoma etc. This patient had developed multiple alloantibodies despite having no history of transfusion due to admitted use of intravenous drugs using shared needle. The habit of flushing out the adherent IV drug from the syringe by flushing the syringe by withdrawing his own blood into the syringe and then pushing it back in the body, a technique used by all his friends, had exposed him to the antigens of other drug abusers. This case was detected promptly due to the routine usage of Type and Screen policy for all the patients receiving transfusion. Such screening for atypical antibodies must be instituted to pre-emptively identify these antibodies as in case of dosage they might be missed during routine crossmatch. The role of the reference Lab has been an illuminating one. With access to extensive repertoire of Select Cells, Rare antisera and Enzyme treatment along with advanced Immuno-haematological tools, the reference labs can provide support in difficult and tricky cases.

\section{Effectiveness of Goal Directed Therapy VS Massive Transfusion Protocol in a Tertiary Care Centre in South India}

Arun V J ${ }^{* 1}$, Aboobacker M. Rafi ${ }^{1}$, Susheela J. Innah ${ }^{1}$, Ramesh Bhaskaran $^{1}$, Divya Venugopal ${ }^{2}$

${ }^{1}$ Transfusion Medicine, JMMCRI, Thrissur, ${ }^{2}$ Transfusion Medicine, District Hospital, Idukki, India

Aims \& Objectives: To compare the blood component utilization, morbidity \& mortality in patients managed by an institutional massive transfusion protocol (MTP) versus goal-directed therapy (GDT) using Rotational thromboelastometry (ROTEM).

Patients/Materials \& Methods: A comparative study was done, wherein the retrospective data was collected from the institutional MTP registry and compared with prospective data of patients managed by GDT using ROTEM. Massive transfusion was defined as per standard guidelines. Goal-Directed therapy was as per the temogram from ROTEM, Which shows clear indication for transfusion of FFP, Platelet, or cryoprecipitate. Red cell transfusion is based on clinical and other lab parameters. Data pertaining to patient demographics, mode of admission, indication for massive transfusion, blood component usage, Morbidity, and mortality were collected and analyzed. Results: The study population comprised a total of 122 patients (61 in each protocol). The mean age of patients managed by MTP \& GDT was $33.36 \& 46.52$ years respectively. There was no statistical difference in gender, criteria of admission among the comparison groups. MTP was activated for PPH, Trauma \& surgery at a rate of $45 \%, 36.1 \%$ \& $11.5 \%$. Goal-directed Therapy was initiated mainly for Trauma, surgery, PPH \& snake bite at a rate of $42.6 \%, 23 \%, 19.7 \%$, $4.9 \%$ respectively. There was a significant reduction in blood component utilization for PRBC, FFP \& platelet $(p<0.05)$ in GDT, but cryoprecipitate utilization remained the same. There was a significant reduction in length of hospital stay $(\mathrm{Sig}=0.038)$ for patient managed 
by GDT (9.39 days) in comparison to those managed by MTP (17.05 days). There was no statistical difference in mortality among the comparison groups.

Discussion \& Conclusion: Goal-directed therapy shows a significant reduction in blood component utilization \& morbidity without a significant change in mortality among patients in this study. GDT has the potential to impact the care of critically ill patients in many ways, but current evidence for their use is limited. Further high-quality research, including randomized controlled trials are needed to elucidate the role of GDT.

\section{A Study of Knowledge, Attitude and Practice (KAP) Regarding Voluntary Blood Donation Among Health Care Professionals in Dhiraj Hospital, Vadodara}

Raxit Suthar $^{* 1}$, Kuntal Patel1, Gunjan Rathod ${ }^{2}$

${ }^{1}$ Pathology, SBKS MI\&RC, Sumandeep Vidyapeeth, ${ }^{2}$ Pathology, SBKS \& MIRC, Sumandeep Vidyapeeth, Vadodara, India

Aims \& Objectives: To study the Knowledge, Attitude and Practice (KAP) regarding voluntary blood donation among health care professionals in Dhiraj Hospital- Vadodara.

Patients/Materials \& Methods: Our study which is a cross sectional study includes total of around 80 health care workers of Dhiraj Hospital - Vadodara. On the basis of socio-demographic characteristics and according to the levels of Knowledge, Attitude and Practice among participants data were collected using a self-administered questionnaire. A structured questionnaire was given to all the health care workers working in the Dhiraj hospital were taken into consideration.

Results: Eighty health care workers were involved in our study which was conducted at Dhiraj hospital Vadodara among which it was found that about $55 \%$ donated to serve for humanity. A large group of around $15 \%$ were donating as a replacement for their kith and kin. Around $10 \%$ were because of self motivated decisions. And on the other side the reasons for non-donation by non- donors was that nobody approached to them and it constituted to about 6\%. Around $57 \%$ non donated due to some health related reasons while rest of them non donated due to fear of disease transmission and fear of needle which constituted to about $17 \%$.

Discussion \& Conclusion: The health care workers of Dhiraj hospital were having overall level of knowledge, attitude and practice was satisfactory. Health Professionals, various hospitals and blood banks services are demanded to develop new ways so to make blood donation services more reliable and accessible.

\section{A Retrospective Study on Seroprevalence of Transfusion Transmitted Infections in Blood Donors at Dhiraj Hospital, Vadodara \\ Gunjan Rathod ${ }^{* 1}$, Rakesh Tandon ${ }^{1}$, Raxit Suthar ${ }^{1}$ \\ ${ }^{1}$ Pathology, SBKS MI\&RC, Sumandeep Vidyapeeth, Vadodara, India}

Aims \& Objectives: To study the seroprevalence of Transfusion Transmitted Infections in blood donors at Dhiraj Hospital, Vadodara. Patients/Materials \& Methods: A retrospective study was undertaken to determine the seroprevalence of TTI among the blood donors who donated blood to Dhiraj Hospital Blood Bank between January 2015 and December 2019.The TTI reports of the donors was obtained from the blood donor data records of Dhiraj hospital. Each blood unit was tested by Enzyme-Linked Immune Sorbent Assay (ELISA) method for Human Immune Deficiency Virus, and Hepatitis B virus and Hepatitis $C$ virus. Syphilis was tested by Rapid Plasma Reagin (RPR) card test. Malaria was tested by antigen rapid diagnostic test. As is the practice in the Blood Bank, all positive samples were subject to repeat test for confirmation, before the seropositive blood unit is discarded. The information extracted from the Dhiraj Hospital blood bank database also included donor Id, Age, Sex, Residential address, donation type, donation frequency.

Results: The data analysed in the study consisted of a total 20,711 blood donations, of which $2728(13.1 \%)$ were voluntary and 18,288 $(86.9 \%)$ were replacement donation. The seroprevalence rate of HIV was 0.1 , that of HBV was 1.4 , HCV 0.1 , syphilis was 0.5 and Malaria was 0.3 among all the blood donors. The TTIs were more frequently found in replacement donors in comparison to volunteer donors.

Discussion \& Conclusion: The prevalence of HIV, HBV, HCV, Malaria and Syphilis infections is higher in replacement blood donors than voluntary blood donors, hence collection of blood from replacement donors should be at least reduced, if not totally eliminated. Stringent criteria need to be used for selection of blood donors. There is a need for creating awareness in the public about blood donation. Voluntary blood donation activity needs to be promoted within the local community. Potential Voluntary blood donors need to be identified and motivated to donate blood regularly.

\section{Does RH and Kell Phenotyping of Donor Units Complement Type and Screen Method of Compatibility Testing? \\ Divya Setya ${ }^{* 1}$, Prashant K. Pandey ${ }^{1}$, Shweta Ranjan ${ }^{1}$, Mukesh K. Singh $^{1}$ \\ ${ }^{1}$ Transfusion Medicine, Jaypee Hospital, Noida, NOIDA, District - Gautam Budh Nagar, India}

Aims \& Objectives: Despite knowing benefits of extended phenotyping, a vast majority feel that phenotype matched units add to cost of blood banking. The purpose of this study was to discuss the advantages and disadvantages of performing Rh Kell phenotyping in Indian scenario by assessing: (a) Prevalence of C, c, E, e, K antigen frequency in donors and degree of patient and donor Rh-Kell phenotype matching. (b) Prevalence of clinically significant Rh-Kell antibodies in patients. (c) Follow-up of patients transfused prophylactically antigen matched red cell units for alloimmunization. (d) Laboratory and cost-benefit of performing Rh-Kell phenotyping in resource constraint settings.

Patients/Materials \& Methods: This was a prospective, observational study conducted between July 2014 and February 2020. All consecutive donors and all consecutive patients whose samples were sent for Rh-Kell phenotyping were included for calculating antigen, phenotype and gene frequencies. For rate of alloimmunization in patients transfused with phenotype matched units, all patients who were given Rh-Kell phenotype matched transfusions were included in the prophylactic antigen matched (PAM) category and those who were given random units were included in the non-PAM category.

Results: A total of 37,588 donors and 258 patients were included for calculation of antigen, phenotype and gene frequencies. Percentage similarity of phenotypes between patient and donor populations was $67.69 \%$. For rate of alloimmunization, results of a total of 31,991 patient samples revealed $0.94 \%$ prevalence of unexpected antibodies; highest against the $\mathrm{Rh}$ system $(70.73 \%, 145$ of 205$)$. Of the 258 patients who were Rh-Kell phenotyped and followed up for alloimmunization, there were 151 patients in PAM category and 107 in the non-PAM category. A total of 439 and 372 units were transfused to patients belonging to PAM and non- PAM categories respectively. Three patients in the non-PAM category and one in the PAM category 
were alloimmunized during follow-up. Significant clinical and laboratory impact of phenotyping was observed in terms of reduced turnaround time and consumption of resources.

Discussion \& Conclusion: Rh-Kell phenotyping of donors can prevent alloimmunization, reduce cost burden on the patient and the laboratory, help the laboratory personnel in smooth routine testing and at the same time be cost effective even in a resource constraint setting.

Supporting Document:

\section{Massive Transfusion in the Times of the Covid-19 Pandemic}

Dharti P. Padharia ${ }^{* 1}$, Keyuri Patel ${ }^{1}$, Rohit K. Singh ${ }^{1}$, Neelofarbanu Mansuri $^{1}$

${ }^{1}$ Pathology, Pramukhswami Medical College, Anand, India

Aims \& Objectives: In the time of COVID-19 pandemic when most of blood transfusion was facing a crisis of blood shortage.

- To evaluate the number of massive transfusion protocol activated at the A D Gorwala Blood Bank, Shree Krishna Hospital, Karamsad from the month of January to September, 2020 (COVID pandemic).

- To infer the number of products dispatched, outcome of the patients and the management of the donor pool to meet the demand. Patients/Materials \& Methods: Massive transfusion is defined as transfusion of more than one blood volume, i.e., $\geq 10$ [Red Blood Cell (RBC)] units in $24 \mathrm{~h}(\mathrm{~h})$ or replacement of $>50 \%$ of total blood volume by blood products within $3 \mathrm{~h}$ or $>4 \mathrm{RBC}$ units in $1 \mathrm{~h}$, with anticipation of continued need for product support in adults. The patients with utmost need of this are those suffering severe blood loss like major road traffic accidents, severe trauma, post partum hemorrhage and other gynecological and obstetrical emergencies. Transfusion on such a large scale is definitely a risky process but the benefits outweigh the risks. In the COVID-19 pandemic, with the norms of social distancing, avoidance of social gatherings and the situations of lock down, remarkable effort goes into maintaining the donor pool.

Results: From the month of January to September, 2020; a total of 25 Massive transfusion protocols have been activated with the maximum of 29 products transfused to the patient having abruptio placenta with IUFD. On an average, each patient required at least 12 products. Out of these 25 patients, 18 patients could be saved.

Discussion \& Conclusion: In the times of COVID-19, each instituition should have a massive transfusion protocol incorporating practice points which should include donor motivation.

\section{Effect of Autologous Plasma Used as Eye Drops in Patients with Dry Eyes in Sjogrens Syndrome}

Diana S. Nathan ${ }^{* 1}$, Abhishekh Basavarajegowda ${ }^{1}$, Ramesh Babu ${ }^{2}$, Rashmi Kumari ${ }^{3}$

${ }^{1}$ Transfusion Medicine, ${ }^{2}$ Ophthalmology, ${ }^{3}$ Dermatology, JIPMER, Puducherry, India

Aims \& Objectives: Dye eye disorder in Sjogren s syndrome is very a debilitating condition to the patients as it affects their day-to- day activities, sleep quality and overall wellbeing. The conventional treatment options available so far such as artificial tears is found to be ineffectual in relieving symptoms and is economically not feasible for treatment of long duration. Our Aim was to study the effect of autologous plasma eye drops in reduction of dry eyes symptoms in patients with Sjogren s syndrome for over a period of three months with the SPEED questionnaire and DEWS SCORING criteria.
Patients/Materials \& Methods: Sixteen patients with Sjogrens syndrome dry eyes disease were enrolled for the study. They were given autologous plasma prepared as eye drops (in $20 \%$ dilution as $5 \mathrm{ml}$ bottles) to be used for 6 times a day for over a period of 3 months. The outcomes were measured subjectively as SPEED score and objectively as DEWS score. These values were calculated for both the right eye (RE) and the left eye (LE) separately and on three occasions namely, pre intervention, after one month (post intervention 1) and three months (post intervention 2). The statistical analysis was carried out using SPSS.

Results: The Box and Whisker Chart was plotted with an IQR (interquartile range) of 1.5 . The outcome of the study was a statistically significant improvement in both symptoms (SPEED SCORE) and objective signs (DEWS SCORE) $(p<0.001)$ in both the eyes as shown in the table below. Our study showed that the plasma eye drops to be safe as there were no reported adverse effects including active ocular infections.

Discussion \& Conclusion: Autologous plasma eye drops can be used as a therapeutic option in patients with Sjogrens syndrome dry eye disorder.

Supporting Document:

\begin{tabular}{|c|c|c|c|c|}
\hline Parameter & $\begin{array}{c}\text { Pre } \\
\text { intervention }\end{array}$ & $\begin{array}{c}\text { Post } \\
\text { intervention 1 }\end{array}$ & $\begin{array}{c}\text { Post } \\
\text { intervention 2 }\end{array}$ & p-value \\
\hline SPEED-RE & $18.68(4.22)$ & $12.68(5.92)$ & $7.443(3.22)$ & $<0.001$ \\
\hline SPEED-LE & $18.81(4.23)$ & $12.81(6.15)$ & $7.31(2.96)$ & $<0.001$ \\
\hline Parameter & Pre & Post & Post & p-value \\
\hline DEIVS-RE & $16.25(4.95)$ & $14.0(4.03)$ & $12.94(3.37)$ & $<0.001$ \\
\hline DEWWS-LE & $16.43(5.24)$ & $14.31(4.75)$ & $13.0(3.38)$ & $<0.001$ \\
\hline
\end{tabular}

\section{Analysis of Pre Donation Deferral Causes in Blood Donors}

Nidhi A. Patel ${ }^{* 1}$, Rakesh Tandon ${ }^{2}$

${ }^{1}$ Pathology, ${ }^{2}$ SMT. BK Shah medical institute \& research centre, Vadodara, India

Aims \& Objectives: $\bullet$ To find out the deferral rate of blood donors at our centre.

- To evaluate the different causes of donor deferral.

- To compare the blood donor deferral reasons and rates with different part of nation and world.

- Apply relevant findings to modify recruitment strategy for blood donors.

- To take proper follow up measurements for temporarily deferred donors.

- Try to achieve $100 \%$ acceptance of donation. 
Patients/Materials \& Methods: This was a prospective study carried out at the department of pathology, smt. BK Shah Medical Institute \& Research Centre, Sumandeep Vidyapeeth Deemed to be University, Piparia, 391760, Vadodara for a period of 1 year. Important donor details were obtained in a proforma and data was prepared. Data obtained was tabulated and analysed. Deferred donors from blood bank were included in study. Study was carried out from September 2019 to September 2020. Standard operating procedure was followed for donor selection. Donor screening and examination was done by a set of questionnaire, physical examination and haemoglobin level testing by copper sulphate specific gravity method. Donor deferral data were analysed from donor forms and registers maintained in blood bank.

Results: Total 3000 donors were registered. Among them, $98.11 \%$ were males, while $1.89 \%$ were females. Out of total registered donors, $94.20 \%$ were accepted for blood donation and $5.80 \%$ deferred. Overall deferral rate for male and female donors were $5.12 \%$ and $0.69 \%$ respectively. Males were deferred more than females. However, with respect to gender specific data, females had higher deferral rate as compared to males. Total registered donors with accepted and deferred donors: Accepted, 94\% Deferred, 6\%.

Discussion \& Conclusion: Males were deferred more than females. Temporary deferral rate was greater than the permanent deferral rate. Most of the deferred donors were in the age group of 18-30 years in both males and females. The present study showed that the donor deferral rate varies in different populations; the reasons for variation may be due to difference in socioeconomic status and environmental factors.

\section{Platelet Function Analysis of Stored Platelet Concentrate and Freshly Prepared Platelet Rich Plasma}

Tapasyapreeti Mukhopadhyay ${ }^{* 1}$, Arulselvi Subramanian ${ }^{1}$, Hara Prasad Pati ${ }^{2}$, Anand Kumar ${ }^{1}$, Vedanand Arya ${ }^{3}$, Sulekha Karjee ${ }^{3}$, Venencia Albert ${ }^{1}$

${ }^{1}$ Laboratory Medicine, ${ }^{2}$ Hematology, ${ }^{3}$ Transfusion Medicine, AIIMS, New Delhi, India

Aims \& Objectives: To compare the platelet function of platelets in stored blood component platelet rich plasma - platelet concentrate (PRP-PC) with platelets in freshly prepared platelet rich plasma (PRP) by flowcytometry.

Patients/Materials \& Methods: The study was conducted in the Department of Laboratory Medicine, Jai Prakash Narayan Apex Trauma Centre, AIIMS, New Delhi. Whole blood was collected from random donors in 450-ml bags containing CPDA1. PRP-PC was prepared within $4 \mathrm{~h}$ and stored at $20-24^{\circ} \mathrm{C}$ with continuous agitation until the time of issue. About $2-3 \mathrm{ml}$ of sample from one day old PRP-PC was collected aseptically in a plain vial for platelet function analysis by flowcytometry. For obtaining PRP, whole blood from healthy volunteer was collected in citrate vial and centrifuged for $15 \mathrm{~min}$ at $210 \mathrm{~g}$. The following parameters were assessed: platelet count, platelet activation and platelet aggregation. Platelet count was assessed by SysmexXE 2100 haematology autoanalyzer. Platelet function was studied by BD Accuri C6 flowcytometer and analyzed with BD software.

Results: Platelet function analysis was done in 15 stored PRP-PC and 10 freshly prepared PRP. The mean platelet count of PRP-PC was $738.8 \pm 312.9 \times 103 / \mu \mathrm{L}$. The median platelet activation of PRP-PC was significantly lower than the PRP $[0.4(0.1-42) \%$ v/s 10.5 $(0.8-32.4) \% ; p$ 0.002]. The median platelet aggregation of PRP-PC was similar to the PRP before adding agonist [56.56(14.47-92.00) v/s 41.82(23.44-86.67) \%; $p$ 0.374] However, the median platelet aggregation of PRP-PC was significantly higher than PRP [33.41(4.99-55.81) \% v/s 62.09(26.79-88.79); $p$ 0.003] at $3 \mathrm{~min}$ after adding agonist.

Discussion \& Conclusion: The platelets in stored PRP-PC were less active than in the freshly PRP. However, significantly higher platelet aggregation was observed in PRC-PC on adding agonist. Effect of storage changes on platelet activation of the platelets in PRC-PC need to be explored further. Larger studies are required to confirm the findings.

\section{Impact of COVID-19 Pandemic on Blood Transfusion Services: Strategies to Overcome}

Minal Wasnik ${ }^{* 1}$, Mili Patel ${ }^{1}$, Ramesh Chandrakar ${ }^{1}$, Saurabh Lahare ${ }^{1}$, Anil Verma ${ }^{1}$, Ruchi Sharma ${ }^{1}$, Sankalp Sharma ${ }^{1}$, Girish Singh Kshatriya $^{1}$, Ankit Jain ${ }^{1}$, Dhananjay Sahu ${ }^{1}$, Himabindu $\mathrm{P}^{1}$, Nitin Kashyap $^{2}$

${ }^{1}$ Transfusion Medicine \& Blood Bank, ${ }^{2}$ Cardio vascular \& Thoracic Surgery, All India Institute of Medical Sciences, Raipur, India

Aims \& Objectives: In December 2019, pneumonia of unknown origin was detected in Wuhan, China; later named by the International Committee on Taxonomy of Viruses as severe acute respiratory syndrome coronavirus 2 (SARS-CoV-2), and its associated condition named as coronavirus disease-2019 (COVID-19) by WHO. It was declared as a pandemic on 11th March 2020. First confirmed case was detected on 30th January'20 in India, whereas in our state (Chhattisgarh) on 19th March'20. Though there is no evidence of its spread by blood transfusion, yet the pandemic has severely affected blood transfusion services (BTS). This study was done with objective to see the impact of pandemic on blood donation and utilization along with strategies to effectively manage BTS during this pandemic, and other future health emergencies.

Patients/Materials \& Methods: We performed this study in Blood Bank of a teaching hospital of central India involved in pandemic management in the region. We analyzed retrospective 18-months data of blood donation and utilization before (April-December'19) and during (January-September'20) COVID-19 pandemic.

Results: During 9-months before pandemic (April-December'19), total number of donations were 2089 with 460 voluntary donations (in-house \& outdoor). To compensate for increased demands, we increased our voluntary donation leading to collection of 1123 units within first 3-months of 2020. With pandemic spread within the country and state, collection in next 6-months was 1183 . However, voluntary donation reduced from 291 in first 3-months to 119 in last 6-months with as less as three in September due to lockdown. As we maintain registry of voluntary donors who came and donated for patients, they were considered replacement donations, thus increasing its number. Blood utilization before was 3693, and 3939 during pandemic $(p<0.05)$; showing increased demand.

Discussion \& Conclusion: Our strategies such as maintaining voluntary donor registry, calling them on-demand, issuing travel passes for donation during lockdown, conduction of small in-house camps (10-12 donors), motivating hospital-staff, hostel students for donation, counseling patient's healthy attendants, strictly monitoring blood demand and utilization, and avoiding wastage helped us to maintain our inventory and overcome sudden burden on BTS during pandemic. 
Isolated Palmoplantar Hyperhidrosis During Blood Donation: A Case for Illustration

\author{
Amita R. Nair ${ }^{* 1}$, Debasish Gupta ${ }^{1}$ \\ ${ }^{1}$ Transfusion Medicine, SCTIMST, Trivandum, India
}

Aims \& Objectives: Vasovagal reactions (VVR) are the commonest adverse reaction to blood donation. Poor hydration is known to cause VVRs. We illustrate the case of 33 year male, with isolated palmoplantar hyperhidrosis, who had an uneventful donation. A prior knowledge helped us in anticipating VVR and preventing its occurrence, leading to a successful blood donation.

Patients/Materials \& Methods: A 33 year old man, came for blood donation. His medical examination revealed no abnormality, except mild sweating of the palms. Upon enquiry, he gave history of excessive sweating of palms during activities. He had sought consultation from a dermatologist and was investigated and diagnosed as having isolated palmoplantar hyperhidrosis. He was currently not on any medication. Vitals were stable. He was well hydrated and explained about the blood donation process and accepted for blood donation.

Results: During donation, he was profusely sweating from the palms and feet (Figure 1). His vitals were stable and the donation went uneventful, with $450 \mathrm{ml}$ blood collected within $6 \mathrm{~min} 36 \mathrm{~s}$. Post donation, he was rested for $15 \mathrm{~min}$ with oral hydration and refreshments and post donation instructions given. Donor promptly returned for his next blood donation after 4 months, which was also uneventful.

Discussion \& Conclusion: Discussion: Hyperhidrosis or abnormal increased sweating has been called a silent handicap due to its psychological impact. Hyperhidrosis can be generalised or focal. Patients with generalised hyperhidrosis should be evaluated for any underlying causes1. Focal hyperhidrosis, which is more common, develops idiopathically in otherwise healthy individuals 2 . It is due to overactivity of the sympathetic nervous system, stimulating the eccrine sweat glands with increase in vasomotor tone 3. Sweating during VVR is due to vasodilatation and activation of the parasympathetic system leading to hypotension4,5. Conclusion: Individual with idiopathic focal hyperhidrosis can be safely taken up for blood donation as illustrated with this case. A pre-donation counselling with emphasis on alleviating the anxieties along with good hydration pre and post donation and conduction of blood donation in an air-conditioned room will help in preventing dehydration and subsequent donor reactions. A pleasant blood donation experience also helps in motivating the donor for future blood donation.

\section{Trends of Blood Transfusion in Covid and Non-covid Patients: A Comparison}

Mili Patel $^{* 1}$, Minal Wasnik ${ }^{1}$, Ramesh Chandrakar ${ }^{1}$, Anil K. Verma ${ }^{1}$, Ruchi Sharma $^{1}$, Saurabh Lahre ${ }^{1}$, Girish Singh ${ }^{1}$, Ankit Jain ${ }^{1}$, Dhananjay Sahu ${ }^{1}$

\section{${ }^{1}$ Department of Transfusion Medicine \& Blood Bank, AIIMS, Raipur, Raipur, India}

Aims \& Objectives: COVID-19, the disease caused by SARS-COV2 virus, has led to a global pandemic. Elderly and those with comorbidities have proven to be more vulnerable to severe disease. We performed a study to analyse the blood transfusion requirement in Covid-19 versus Non-Covid patients hospitalised in our institution.

Patients/Materials \& Methods: A retrospective observational study of blood and blood products utilisation in Covid-19 patients hospitalised at our centre from January-September'20 was done by analysing the monthly transfusion records maintained in the
Department of Transfusion Medicine and Blood Bank, AIIMS Raipur. Transfusion of each blood component were compared in Covid- 19 wards versus non-Covid wards during the specified period. Covid patients were segregated according to blood group, age and gender. Results: 3754 Covid patients have been hospitalised in AIIMS Raipur since the first case diagnosed in Chhattisgarh during the mid of March 2020. $36(0.95 \%)$ patients out of 3754 hospitalized COVID-19 patients received transfusions with $0.77 \%$ receiving Packed Red Blood Cells (PRBCs), 0.08\% platelets (PLTs), 0.23\% Fresh Frozen Plasma (FFP). Covid-19 wards had significantly lower transfusion rates compared to non-Covid wards since total PRBC units issued to Covid wards was 44 as compared to 1899 units dispensed to the nonCovid wards. As was FFP, 33 to Covid wards and 1134 to non-Covid wards, same was the case with Platelets, where only 10 units were issued to Covid wards in comparison to 513 units issued to the NonCovid wards. $\mathrm{O}+$ ve patients received the highest number of transfusions amongst the Covid patients, followed by $\mathrm{A}+\mathrm{ve}, \mathrm{B}+\mathrm{ve}$ and $\mathrm{AB}+\mathrm{ve}$. Patients in the age group of $16-30 \mathrm{yrs}$ and $>45 \mathrm{yrs}$ were the ones who showed maximum transfusion requirement.

Discussion \& Conclusion: Transfusion requirement by Covid-19 inpatients was fewer as compared to the other hospitalized patients. Only the critically ill patients required transfusion, while the ones with mild to moderate disease were cured by conservative management, at our institute. This data will help our department to plan and prepare for blood resource utilisation during the ongoing pandemic.

\section{Investigating an Episode of 'Cranberry Coloured' Urine in an Indian Male Post Blood Transfusion}

\author{
Manish Raturi $^{* 1}$, Mansi Kala ${ }^{2}$, Anuradha Kusum ${ }^{2}$
}

${ }^{1}$ Immunohematology and Blood Transfusion, ${ }^{2}$ Pathology, Swami Rama Himalayan University, Dehradun, India

Aims \& Objectives: Acute hemolytic transfusion reaction [AHTR] is the rapid clearance or lysis of the transfused red blood cells [RBCs] due to an immunologic mismatch between the donor and their recipient/s that happens within $24 \mathrm{~h}$. Besides on the basis of causative mechanisms involved, it may further be classified as immunological [AIHTR] or non-immunologic [ANIHTR] in nature. Herein, we describe a clinical case of ANIHTR in a young male recipient of a donor unit. Our primary aim was to do a root cause analysis [RCA], on reasons for ANIHTR in the recipient.

Patients/Materials \& Methods: Red cell typing for ABO and Rh was performed using reagents [Resolve antisera, Tulip diagnostics $(\mathrm{P}) \mathrm{Ltd}$, Goa] and supplies in accordance with the manufacturers' instructions. Pre-transfusion work up was performed according to our department standard operating procedures [SOP]. We also performed the direct anti-globulin test, serologic cross-matches and antibody screening using poly-specific (anti-IgG,-C3d) column agglutination test [CAT]; Ortho Clinical Diagnostics, New Jersey, USA.

Results: A 16 year old Indian male received one unit of packed red blood cell [PRBC] after undergoing an urgent orchiectomy of his left testis at our hospital. Barring one episode of cranberry coloured urine [fig 1], all the other clinical and laboratory findings, namely, an uneventful blood transfusion, no $\mathrm{ABO}$ and $\mathrm{Rh}$ discrepancy, no unexpected allo-antibodies and negative DAT ruled out AIHTR. His serum bilirubin was $4.75 \mathrm{mg} / \mathrm{dL}$, lactate dehydrogenase level was 813 $\mathrm{U} / \mathrm{L}$ and urine was positive for hemoglobin [confirmed on saturating $5 \mathrm{~mL}$ urine with $2.8 \mathrm{~g}$ ammonium sulphate with the appearance of clear supernatant post centrifugation].

Discussion \& Conclusion: On RCA, it was found that the nursing staff had inadvertently kept the donor unit near the room heater on a window sill to avoid 'giving cold blood transfusion'. This, perhaps, presented us the clue as to why his urine was "cranberry coloured". 
With a presumptive diagnosis of DAT negative ANIHTR, the patient was given Prednisolone $60 \mathrm{mg} / \mathrm{m}^{2} / \mathrm{d}$. There was a dramatic improvement with a raise in his hematocrit to $29.5 \%$. During discharge, he was clinically stable. Currently, the patient has been advised for a regular follow-up at our hospital.

Keywords: Acute scrotum; Testicular torsion; Red Blood Cells; Hemolysis; Direct Antiglobulin Test; Hemoglobinuria

\section{Demographic \& Blood Group Characteristics of Hospitalized Covid-19: Experience of Private Hospital \\ Minal Wasnik ${ }^{* 1}$, Dhananjay Prasad ${ }^{2}$ \\ ${ }^{1}$ Transfusion Medicine \& Blood Bank, All India Institute of Medical Sciences, ${ }^{2}$ Laboratory Medicine, Shri Narayana Hospital, Raipur, India}

Aims \& Objectives: SARS-CoV-2 infection has been rapidly increasing around the world and has already been declared pandemic by the WHO. First case in Chhattisgarh was reported in MidMarch'20. Since then the number is increasing at an alarming rate. Though majority of the cases are mild-moderate who are managed by home isolation and conservative management; patients having severe disease require hospital admission, with several requiring intensive care. Initially, majority admissions were handled by governmenthospitals, but with increasing burden the private-hospitals were also roped in. We studied the demographic and blood grouping profile of hospitalized Covid-19 patients and relation with mortality.

Patients/Materials \& Methods: We studied 6-months data (AprilSeptember'20) of a private-hospital in Chhattisgarh involved in management of Covid-19 patients. Demographic and blood group data maintained in the Laboratory Medicine department of hospital was analyzed. This was correlated with mortality in these patients.

Results: Within 6-months, total 403 patients were hospitalized with $72 \%$ males and rest females. Most were in age-group of 41-60 years $(49 \%)$, followed by $\geq 61$ years $(33 \%)$. B + ve $(38 \%)$ was commonest blood group followed by $\mathrm{O}+\mathrm{ve}, \mathrm{A}+\mathrm{ve}, \mathrm{AB}+\mathrm{ve}$. Out of 403,34 patients $(8.4 \%)$ died, where $70 \%$ were males, mostly $\geq 61$ years. Blood group of those who died was $B+$ ve $(41 \%)$, followed by $\mathrm{O}+\mathrm{ve}(29 \%), \mathrm{A}+\mathrm{ve}(20 \%), \mathrm{AB}+\mathrm{ve}(5 \%)$ and 1 death in B-ve. However, the calculated relative risk showed $\mathrm{B}+\mathrm{ve}(\mathrm{RR}=1$, $95 \% \mathrm{CI}), \mathrm{O}+\mathrm{ve}(\mathrm{RR}=0.9,95 \% \mathrm{CI}), \mathrm{A}+\mathrm{ve}(\mathrm{RR}=0.9,95 \% \mathrm{CI})$, showing no increased risk of mortality in association with particular blood group.

Discussion \& Conclusion: Several western studies suggest that Covid-19 infection is commonly associated with blood group A. However, no studies were found in India. Our findings show B + ve to be most common, with no significant risk of increased mortality among particular blood group. This difference can be due to genetic, immunological variations and individual susceptibility for infection from western population. However, as we studied limited data, the generalization of these findings should be avoided. The preventive measures like hand hygiene, face masks, social distancing norms should be followed irrespective of your blood type
Malignancy Presenting as Blood Group Discrepancy

Aboobacker M. Rafi ${ }^{1}$, Athira Sasidharan ${ }^{* 2}$, Arun V J ${ }^{1}$, Maglin Monica $^{1}$

${ }^{1}$ Transfusion Medicine, Jubilee Mission Medical College \& Research Institute, ${ }^{2}$ Transfusion Medicine, Jubilee Mission Hospital, Thrissur, India

Aims \& Objectives: Blood grouping discrepancy is defined as a situation when the forward grouping doesn't match the reverse grouping. This abstract reports a case which was detected as malignancy when she was evaluated for Blood group discrepancy.

Patients/Materials \& Methods: A 46-year-old female patient came with complaints of vomiting, anorexia abdominal pain associated with intermittent fever and weight loss for the past 2 months. No history of blood transfusion or bleeding. She was not on any regular medications. USG neck and abdomen showing multiple enlarged lymph nodes.

Results: She was evaluated and her basic investigations showed Anemia with leukocytosis, deranged LFT \& RFT with A/G reversal. A Blood Grouping discrepancy was noted with auto agglutination in reverse grouping. Coombs test was strong positive. Bone marrow biopsy followed by IHC confirmed the diagnosis of High Grade T Cell Lymphoma.

Discussion \& Conclusion: Lymphoproliferative diseases are the most common disorders associated with blood grouping and autoimmune disturbances. Previous studies have demonstrated that lymphoproliferative disorders interfere with antiglobulin testing and other tests for platelet autoantibodies, ANA \& APLA and lupus anticoagulant. Prognosis and remission rates based on these markers requires a larger series of patients to be be investigated in future studies.

\section{A Rare Presentation of Blood Group Change in A 65 Year Old Patient with Myelodysplastic Syndrome}

Kanwaljeet Singh $^{* 1}$, Ankur Ahuja ${ }^{2}$, Venkatesan Somasundarum ${ }^{3}$, Dwarika Tiwari $^{3}$, Tathagat Chatterjee ${ }^{3}$

${ }^{1}$ Dept of Lab Sciences \& Molecular Medicine, Army Hospital (R\&R), Delhi Cantt, New Delhi, ${ }^{2}$ Dept of Pathology 92 BH, 92 BH, Srinagar, ${ }^{3}$ Dept of Lab Sciences \& Molecular Medicine, AHRR Delhi Cantt, New Delhi, India

Aims \& Objectives: The change of blood group, though rare, can be seen in certain disorders e.g. leukemia, lymphoma's, epithelial malignancies, immunosuppression, age related and patients on chemotherapy due to modification of these antigens. This case report is to highlight the impact of change in blood group in such cases and setting up proper transfusion protocol.

Patients/Materials \& Methods: : Here we report 65 years old case of $\mathrm{ABH}$ red cell antigenic modification in a patient with myelodysplastic syndrome with excess blasts (MDS- EB 2).

Results: Blood group of this patient at time of diagnosis was B positive and he was given two cross matched B positive packed irradiated RBCs and discharged with course of Decitabine. After 16 cycles of Decitabine, his follow up hemoglobin was $6.5 \mathrm{mg} / \mathrm{dl}$. When his blood sample was sent for cross matching and planned for further blood transfusion, it was found that patient's blood group was changed to $\mathrm{O}$ positive on both forward and reverse cross matching and this procedure was repeated twice to confirm the same. Finally he was transfused with $\mathrm{O}$ positive blood group \& he didn't have to be reported any transfusion reactions.

Discussion \& Conclusion: Alteration of $\mathrm{ABH}$ antigens on the RBC surface membrane have been reported to occur in hematological and few solid malignancies. The mechanisms involved in hematopoietic malignancies include stem cell mutation affecting antigenic 
production, inactivation of $\mathrm{A}$ and $\mathrm{B}$ transferases/H transferase and the role of epigenetics and genomic imprinting.

\section{A Pilot Study on Screening of Blood Donors for Emerging Transfusion Transmissible Leptospirosis Infection in an Endemic Area}

\author{
Deepika Chenna ${ }^{* 1}$, Shamee Shastry ${ }^{1}$, Sangthang Singson ${ }^{1}$ \\ ${ }^{1}$ Immunohematology and Blood Transfusion, Kasturba Medical \\ College, Manipal, Manipal Academy of Higher Education, \\ Manipal, Manipal, India
}

Aims \& Objectives: To study the prevalence of emerging transfusion-transmitted Leptospirosis infection among healthy voluntary blood donors.

Patients/Materials \& Methods: A prospective cross-sectional observational study on screening of blood donors for leptospira infection was conducted over 2 months. Donors who are eligible for blood donation as per department standard operating procedures were included in the study after obtaining written informed consent. The blood sample collected for the screening of transfusion transmissible infections (HIV, HBV, HCV, Malaria, and Syphilis) was used for detection of leptospira specific Ig M antibodies by the ELISA method. A sample tested positive for leptospira specific Ig M antibodies was sent to the Department of Microbiology to confirm the test results. If the confirmatory test is positive, the unit was discarded else was taken to inventory. Donors with confirmed positive results were contacted telephonically with respect to any symptoms related to leptospira infection for further advice.

Results: A total of 51 donors were screened for leptospira specific Ig $\mathrm{M}$ antibodies. The age range of the donors varied from 21 to 57 years (Mean: 34.5 and SD: 8.07) and the male: female ratio was 25:1. The blood group frequency of donors was $23.52 \%(12 / 51)$ each of $\mathrm{A}$ and $\mathrm{B}$, $5.88 \%(3 / 51)$ for $\mathrm{AB}$, and $45.1 \%(23 / 51)$ of $\mathrm{O}$. A total of $9.8 \%(5 / 51)$ were identified to be positive during the initial screening, and $5.88 \%(3 / 51)$ were found to be positive, $3.9 \%(2 / 51)$ were found to be negative upon repeat testing. All the donors were contacted telephonically for the development of any symptoms of infection like fever, headache, chills, muscle ache, etc. None of the donors developed any symptoms of leptospira infection which could be due to an asymptomatic carrier state.

Discussion \& Conclusion: Transfusion transmission of leptospira is possible because of the leptospiremia in the donated blood of asymptomatic donors. The present study shows a high prevalence of donors being asymptomatic and positive for IgM antibodies against leptospira. Hence, we recommend further studies on a larger population in endemic areas to elicit the true prevalence and to develop guidelines for screening blood donors.

\section{A Pilot Study on Evaluation of Hematological Profile of Healthy Blood Donors with High Hemoglobin in Southern Karnataka, India}

\author{
Dhivya Kandasamy ${ }^{* 1}$, Shamee Shastry ${ }^{1}$ \\ ${ }^{1}$ Immunohematology and Blood Transfusion, Kasturba Medical \\ College,Manipal, Udupi, India
}

Aims \& Objectives: This study aims at the analysis of the hematological profile of blood donors with high hemoglobin $(\mathrm{Hb}>18 \mathrm{~g} / \mathrm{dl})$ in our blood center to ensure donor safety.

Patients/Materials \& Methods: A cross-sectional observational analysis of the hematological profile of apparently healthy blood donors was performed in our center. After obtaining informed consent, a total of 60 blood donors were randomly recruited for the study which comprises 30 blood donors with high hemoglobin ( $>18 \mathrm{~g} / \mathrm{dl})$ group 1 and 30 donors with normal hemoglobin range (12.5 to $18 \mathrm{~g}$ / dl) for blood donation - group 2. About $2 \mathrm{ml}$ venous blood sample was collected in EDTA vial from each donor and subjected for complete blood count estimation using an automated hematology analyzer SYSMEX-KX21. The hematological parameters obtained were entered in MS Excel. Comparison of mean of hematological parameters between two groups was performed using Student ' $t$ ' test and $p$ value $<0.05$ was considered statistically significant.

Results: The median Hemoglobin was $14.8 \mathrm{~g} / \mathrm{dl}$ ranging from 13.4 to $17.3 \mathrm{~g} / \mathrm{dl}$ in group 1 and $19 \mathrm{~g} / \mathrm{dl}$ ranging from 18.2 to $22.4 \mathrm{~g} / \mathrm{dl}$ in group 2 . The blood picture demonstrated a statistically significant difference in mean value between the two groups for the following parameters: Hematocrit ( $p$ value 0.00001), red cell count ( $p$ value $0.00001)$, mean corpuscular volume ( $p$ value 0.00191$)$, red cell distribution width ( $p$ value 0.02575$)$, mean platelet volume ( $p$ value $0.00426)$, P-LCR ( $p$ value 0.0037 ), and platelet distribution width ( $p$ value 0.03862 ). The mean $\mathrm{MCH}$ and $\mathrm{MCHC}$ were not found to be statistically significant between the two groups.

Discussion \& Conclusion: The recruitment of healthy donors is the primary concern of the blood transfusion center. The significant hematological derangement noted among blood donors with high hemoglobin in our study warrants further extensive investigations including JAK-2 mutation and serum erythropoietin to identify the likely causes of high hemoglobin among apparently healthy blood donors and thereby plan for appropriate management of these donors.

\section{Emphasizing the Inclusion of Red Cell Antibody Screening In RH (D) Positive Pregnancies in India}

Ayushi Kediya ${ }^{* 1}$, Manish Raturi ${ }^{2}$, Mansi Kala ${ }^{1}$, Anuradha Kusum ${ }^{1}$

${ }^{1}$ Pathology, ${ }^{2}$ Immunohematology and Blood Transfusion, Swami Rama Himalayan University, Dehradun, India

Aims \& Objectives: Rhesus (Rh) D antigen associated hemolytic disease of the newborn (HDN) is a known entity. Nevertheless, a variety of Rh non-(D) red blood cell (RBCs) antibodies like anti-C, c, $\mathrm{E}$ and $\mathrm{e}$ are implicated too. We aimed to understand the reasons for unexplained spontaneous recurrent abortion (RSA) in a mother, likely due to an immunohematological (IH) mismatch between the $\mathrm{Rh}$ typing of her and her fetuses.

Patients/Materials \& Methods: RBC typing (ABO \& Rh) was performed using reagents (Tulip diagnostics (P) Ltd, Goa). Her IH work up included direct \& indirect anti-globulin test (DAT \& IAT), autologous control (Ac) and antibody detection using poly-specific (anti-IgG,-C3d) column agglutination test ((CAT); Ortho Clinical Diagnostics, USA).

Results: A 33y old G4P3A3L1 Indian female, presented to our hospital with her third spontaneous abortion. Barring the first live healthy baby, she experienced a poor obstetric history in the form of RSA (30 to 34 weeks) of all the three viable fetuses. There was no history of hypertension, contraceptive use, trauma and/or RSA in her family. She had undergone all the relevant investigations which were absent including antiphospholipid antibodies. Additionally, she was managed using enoxaparin, considering some underlying coagulation disorders as a probable differential for the unexplained RSA. Her unusual history merited a detailed $\mathrm{IH}$ work- up. Her blood type was found to be $\mathrm{B} R \mathrm{Rh}(\mathrm{D})$ positive with minor phenotyping (D $+\mathrm{C}+\mathrm{c}$ E- e +). Although the DAT and Ac were negative, her IAT was positive (fig. 1). On further workup, a high titer anti-c (1:128) was the culprit antibody identified. 
Discussion \& Conclusion: HDN occurs when IgG maternal antibodies destroy the fetal RBCs. This case is peculiar in the fact that the presence of high IgG titer of anti-c was distinctively missed in all the four pregnancies that led to three RSA. The antibody screening performed in India is mostly restricted to $\mathrm{Rh}(\mathrm{D})$ negative pregnancies. To sum up, we strongly recommend including routine red cell IAT for even Rh (D) positive mothers' right at their first antenatal check-up. Presently, she is doing well and dearly wishes to have another child. Keywords: Hemolytic Disease of the Newborn; Antibody Detection; Coombs; IgG Titer; Fetal Loss; Unexplained Recurrent Spontaneous Abortion.

\section{Evaluation of Erythrocyte Magnetization Technique for Measurement of Abo Isoagglutinin Titres}

Parul Chopra $^{* 1}$, Sunanda Bhardwaj ${ }^{1}$, Ajay Samkaria ${ }^{2}$, Asha Amoli ${ }^{1}$, Anil Arora ${ }^{1}$

${ }^{1}$ Hematology and Immunology, Dr. Lal PathLabs Ltd., ${ }^{2}$ DIAGAST, Delhi, India

Aims \& Objectives: A variation in the measurement of the $\mathrm{ABO}$ antibody titre has been seen among different laboratories due to lack of standardisation. In our study we aim to evaluate automated $\mathrm{ABO}$ isoagglutinin titre measurements by Erythro-Magnetic Technology (EMT) and compare with conventional tube technique (TT)

Patients/Materials \& Methods: We performed ABO isoagglutinin titration on samples received in a reference laboratory during a period of two months. A total of 134 tests for IgG titre and 116 for IgM for Anti-A or Anti-B were included in the study. Samples were processed for ABO isoagglutination titres by both TT and EMT by QWALYS-3 (DIAGAST, France). Microsoft Excel was used to compile data, for all calculations and to draw graphs and plots.

Results: Median titres and their ranges obtained by EMT were higher or equal to that by TT for all IgM and IgG ABO-antibodies in all BGs except anti-A IgM in BG O that was lower by EMT [32 (4:128)] than TT [48 (8:256)]. The number and percentage of cases within 1,2 or 3 titre difference (TT-EMT) were calculated. One twenty-one (121/134, $90.3 \%$ ) cases of $\operatorname{IgG}$ titre showed an agreement by both methods (within one titre difference). One hundred seven cases (107/116, $92.2 \%$ ) for IgM titre were within one titre difference by both the methods. Box plot showing frequency distribution, minimum, first quartile, median, third quartile and maximum $\operatorname{IgG}$ and $\operatorname{IgM}$ isoagglutinin titres of anti-B of BG A, anti-A of BG B, anti-A of BG O and anti-B of BG O obtained by the tube technique (TT) and ErythroMagnetic Technology (EMT) are as shown in figure.

Discussion \& Conclusion: EMT-based automated instrument QWALYS-3 is more sensitive than the conventional TT and their results may vary by one titre dilution in majority of the cases. Use of consistent method for patient management is therefore advised.

\section{Does High Serum Ferritin Predict Abnormal Thyroid Function in Transfusion Dependent Thalassemia}

Pankhudi Priya $^{* 1}$, Priyanka Aggarwal ${ }^{1}$, Vineeta Gupta ${ }^{1}$

${ }^{1}$ Department of Pediatrics, Institute of Medical Sciences, Varanasi, India

Aims \& Objectives: Thalassemia is the most common genetic disorder worldwide. The estimation of serum ferritin levels is the most commonly employed test to evaluate iron overload in $\beta$-thalassemia major. There is a possibility that there may be high prevalence of hypothyroidism in thalassemic children. We evaluated the thyroid function tests in thalassemia major patients and correlated the results with serum ferritin level.

Patients/Materials \& Methods: 43 patients with Thalassemia major on regular blood transfusion every 2-4 weeks were selected for this cross sectional analytical study.Blood samples were collected and analyzed for T3,T4 and TSH. According to values of the T3, T4 and $\mathrm{TSH}$, patients were categorized in two groups(Euthyroid and hypothyroid).Data was analyzed statistically using statistical software SPSS version 23. Mean values with standard deviation were presented for continuous variables and numbers with percentages for categorical variables. $p$ value was considered as a parameter to assess significance of various observations and results. $p$ value $<0.05$ was taken as significant.

Results: In this study mean serum ferritin in euthyroid patients was $2442.2 \pm 1434.77 \mathrm{ng} / \mathrm{ml}$ and in hypothyroid patients was $2124.24 \pm 745.37 \mathrm{ng} / \mathrm{ml}$ which was statistically insignificant $(p>0.05)$.

Discussion \& Conclusion: From present study it can be concluded that hypothyroidism is an important complication of Thalassemia major patient, occurring in about $19 \%$ children. Further, there was no direct correlation between serum ferritin and thyroid dysfunction in these patients. The findings suggest the possibility that single serum ferritin level alone may not be sufficient to determine the implication of chronic iron exposure in developing thyroid dysfunctions.Most of hypothyroid patients were subclinical cases, so their regular physical examination for any clinical signs of hypothyroidism along with their periodic thyroid function assessment must be done for early recognition of cases with overt hypothyroidism who need replacement therapy.

\section{Assessment of Hemosiderosis in Thalassemia Major Patients Attending a Tertiary Care Hospital}

Mallikarjun A. Pattanashetti ${ }^{* 1}$, Ganga S. Pilli ${ }^{2}$

${ }^{1}$ Pathology, Gadag Institute of Medical Sciences, Gadag, ${ }^{2}$ Pathology, Jawaharlal Nehru Medical College, Belagavi, India

Aims \& Objectives: Beta Thalassemia major is an autosomal recessive inherited hemolytic anemia which is treated with multiple blood transfusions. In this disease frequent blood transfusions, ineffective erythropoiesis and increased iron absorption leads to hemosideosis. Iron overload can be determined by serum ferritin measurement. Hence, the present study is done to assess the hemosiderosis by measuring serum ferritin levels in multi-transfused thalassemia major patients.

Patients/Materials \& Methods: The study was done at a superspeciality hospital for a duration of one year. A total of 31 thalassemia major patients who received blood transfusions in the superspeciality hospital were included. Clinical examination findings were noted and serum ferritin estimation was done to assess iron overload. The data collected was analysed and expressed in terms of rates, ratios, percentage. Continuous data was expressed as Mean \pm standard deviation, median and range.

Results: In present study, about $58.06 \%$ of the patients were aged between 10 to 12 years. The mean age was $12.45 \pm 2.38$ years and median age was 12 years with younger patients being 10 years and oldest being 18 years. The male to female ratio of $5: 1$. Splenomegaly was the most common clinical finding among the study population, followed by hepatomegaly. Mean serum ferritin of all patients was markedly raised $3786 \pm 2382 \mathrm{ng} / \mathrm{mL}$ with mean ferritin higher among females $(4411 \pm 1299 \mathrm{ng} / \mathrm{mL})$ as compared to males $(3636 \pm 2572 \mathrm{ng} / \mathrm{mL})$. The mean serum ferritin in Non-Chelated patients group was $4505 \pm 2633 \mathrm{ng} / \mathrm{mL}$ which is much higher as compared to mean serum ferritin in Chelated patients group 
$2479 \pm 963 \mathrm{ng} / \mathrm{mL}$ Hence,iron chelation is definitely effective in preventing complications of iron overload.

Discussion \& Conclusion: The patients with transfusion dependent thalassemia major are at risk of developing severe anemia and other complications due to iron overload. Hence, iron overload status for each thalassemia patient has to be regularly maintained by estimating serum ferritin as per the international guidelines.

\section{Determining the Usage and Efficacy of Covid-19 Convalescent Plasma Harvested at Our Blood Centre}

\author{
Heeya Gupta ${ }^{* 1}$, Manish Raturi ${ }^{2}$, Anuradha Kusum ${ }^{1}$
}

${ }^{1}$ Pathology, ${ }^{2}$ Immunohematology and Blood Transfusion, Swami Rama Himalayan University, Dehradun, India

Aims \& Objectives: COVID-19 convalescent plasma (CCP) has gathered momentum as a potential therapeutic option for patients hospitalized with the novel coronavirus [nCoV] especially as an "offlabel" therapy approved by the Indian Council of Medical Research (ICMR). Our principal aim was to evaluate the usage and efficacy of CCP harvested at our blood centre.

Patients/Materials \& Methods: Donors: All the donors were selected based on the guidelines given by ICMR. The CCP was harvested by apheresis equipment [MCS Haemonetics, U.K. Ltd]. The product was aliquoted in two parts of $200 \mathrm{~mL}$ each and frozen below $-30{ }^{\circ} \mathrm{C}$. Prior to issue it was thawed at $37{ }^{\circ} \mathrm{C}$ in accordance to our department SOP. Patients: All the RT-PCR COVID-19 positive patients during one and a half months [1st Sept'220 to 17th Oct'20] were included. This pilot study was approved by the institutional ethics committee and an informed consent was received from the patients prior to $\mathrm{CCP}$ administration.

Results: In total ten voluntary donors $[\mathrm{M}: \mathrm{F}=9: 1]$ consented for the CCP donation. Their average age in years was $35 \pm 7.6$ (25 to 48 ). The average length of first CCP harvest from the date of recovery [in days] was $41 \pm 16.3$ (26 to 75 ). The average signal/cutoff value by Chemiluminescence for the anti-IgG neutralizing antibody [Nabs] against spike protein was $34 \pm 10.5$ (16.4 to 47.3$)$ and the mean total serum protein level [in $\mathrm{g} / \mathrm{dL}$ ] was $7.2 \pm 0.53$ (6.5 to 8.4 ). The infection results and antibody screening were negative. Interestingly, among the eligible donors, around five were unwilling to donate CCP. All the donor-patient pairs were $\mathrm{ABO}$ compatible. The recipient details are reported (Table 1).

Discussion \& Conclusion: CCP therapy can grant the recipient, immunity in the form of Nabs against the $\mathrm{nCoV}$. Yet, one of the major limitations in this study was the small data sub-set to comment on the actual efficacy of the CCP in the recipients. To sum up, the recovery rates were much better when the $\mathrm{CCP}$ was given early [i.e. within five days of diagnosis]. Motivation for donating CCP needs to be stressed right at the time of recovery and discharge of such patients.

Keywords: Coronavirus Infection; Pandemic; Convalescent Plasma therapy; COVID-19; Transfusion Medicine; Blood Centre.

\section{A Retrospective Analysis of Patterns of Blood Donor Deferral in a Tertiary Care Institute}

Gajender Singh $^{* 1}$, Dimple Mehrotra ${ }^{2}$, Pooja Pawaria ${ }^{2}$

${ }^{1}$ Blood Transfusion, ${ }^{2}$ Blood Transfusion, Pt.B.D.Sharma,PGIMS, Rohtak, India

Aims \& Objectives: To study the blood donor deferral patterns at Deptt. of Blood Transfusion, Pt.B.D.Sharma PGIMS Rohtak and to look for the most common cause of Donor Deferral and the possible treatment modalities which can be offered to reduce the number of donor deferrals.

Patients/Materials \& Methods: The present study was a retrospective study conducted at Deptt. of Immunohaematology and Blood Transfusion Pt. B.D.Sharma, PGIMS, Rohtak in which donor deferral data was procured from the medical records and analyzed. The duration of the study was 45 months from January 2017 to September 2020. Blood donor selection criteria was as per the current WHO guidelines and NACO deferral guidelines were followed while temporarily or permanently deferring a donor.

Results: During the period of study a total of $1,50,732$ blood donations were received out of which 1,50,333 were males and 399 were females. The total number of donors who were deferred during this period were 10,953 out of which 7888 were males and 3065 were females. Year wise distribution of the number of deferred cases were 2090, 3183, 3581 and 2099 in the year 2017, 2018, 2019, 2020 (up to Sep.) respectively. The most common cause of deferral in the year 2017 and 2020 (up to Sep.) was medical/surgical cause and in 2018 and 2019 was Anaemia. Table 1 depicts the year wise distribution of Donor deferral based on the reason of deferral and the Gender wise distribution.

Table 1 Gender wise distribution of Deferral Cases Anaemia Under wt/Underage Med./Sx. Others

\begin{tabular}{lllllllll}
\hline & M & F & M & F & M & F & M & F \\
\hline 2017 & 286 & 319 & 301 & 40 & 606 & 27 & 265 & 36 \\
2018 & 415 & 472 & 466 & 150 & 540 & 207 & 353 & 134 \\
2019 & 458 & 494 & 306 & 124 & 616 & 247 & 532 & 136 \\
2020 & 327 & 214 & 208 & 17 & 686 & 65 & 278 & 13 \\
\hline
\end{tabular}

Discussion \& Conclusion: As per WHO norms, it is estimated that blood donation by $1 \%$ of the population is the minimum need to meet a nation's most basic requirement for blood. In 2016, the MOHFW reported a donation of 10.9 million units against a requirement of 12 million units. Therefore, such as study to identify the most common reasons for donor deferral is important as the aim towards our goal of $100 \%$ voluntary blood donations by $1 \%$ of India's population.

\section{Role of Therapeutic Plasma Exchange in Managing Post-Partum Thrombotic Microangiopathy: Case Series}

Ganesh Mohan ${ }^{* 1}$, Ashwinkumar Vaidya ${ }^{1}$, Prethika P. A. ${ }^{1}$, Shamee Shastry ${ }^{1}$

${ }^{1}$ Immunohematology and Blood Transfusion, Kasturba Medical College Manipal, Manipal, India

Aims \& Objectives: Utility of Therapeutic plasma exchange in the clinical management of Post-Partum Thrombotic microangiopathy. Patients/Materials \& Methods: We did a retrospective analysis of data on therapeutic plasma exchange procedures performed on patients with confirmed PP-TMA over a period of 2 years (2019 to 2020). The patient's pre and post procedure hematological and biochemical parameters were recorded and analyzed. All the TPE was carried out as per the ASFA guidelines, replacement fluids used were $0.9 \%$ normal saline, Fresh frozen plasma and 5\% human albumin solution. hemodialysis was carried out concurrently for the AKI. End point for TPE was Platelet count more than $100,00 / \mathrm{uL}$ and a LDH of less than $250 \mathrm{Iu} / \mathrm{L}$. The patients were followed up after discharge from hospital for 6 months. 
Results: TMA was diagnosed in post partum period ranging from 2 days to 5 days. Clinical status showed improvement in 4 out of 5 patients. Average volume processed was $3200 \mathrm{~mL}$ per cycle for a total of 16 cycles of TPE was done in 5 patients. Median length of hospital stay was 25 days and median hemodialysis required was 6 . There was significant improvement in Hb, HCT, Platelet count and LDH $(p<0.01)$. None of the patient went into TMA relapse. Full recovery of renal function was observed in two patients in 6 weeks time and 2 other patients recovered fully in 6 months time, one patient succumbed to death due to septic shock.

Discussion \& Conclusion: Current case series suggests that early initiation of TPE will play a major role in reducing mortality and morbidity of such cases.

\section{To Study Acute Blood Transfusion Reactions in Patients of Beta Thalassemia}

\author{
Narahari V. Susmitha ${ }^{* 1}$, Priti Kamble ${ }^{1}$, Suhas Kulkarni ${ }^{1}$ \\ ${ }^{1}$ Paediatrics, Dr D Y Patil Educational Society and Deemed \\ University, Kolhapur, India
}

Aims \& Objectives: To study acute blood transfusion reactions in patients of beta Thalassemia. To correlate relation of pre transfusion hemoglobin and acute transfusion reactions in beta Thalassemia patients.

Patients/Materials \& Methods: This cross sectional study included 54 beta Thalassemia patients whose age ranged between 1 year to 20 years. Acute blood transfusion reactions were noted in detail. Pre transfusion hemoglobin assessment was done for all patients.

Results: Most common transfusion reaction was fever. By fisher exact test, significant association is observed between pre transfusion hemoglobin level and reaction. It is observed that \% of reaction is more $(22 \%)$ in patients with hemoglobin level $<7.5$, than in patients with hemoglobin level $>7.5(6 \%)$, which is statistically significant $(p$ value $<0.0001)$

Discussion \& Conclusion: Acute febrile non haemolytic reactions are common among patients with low pre transfusion hemoglobin.

\section{Sero-Prevalence of Transfusion Transmitted Infections Among Blood Donors at AIIMS Raipur}

Saurabh Lahare ${ }^{* 1}$, Minal Wasnik ${ }^{1}$, Ramesh Chandrakar ${ }^{1}$, Sankalp Sharma $^{1}$, Mili Patel ${ }^{1}$, Jaisingh Thakur ${ }^{1}$, Manish Sahu ${ }^{1}$

${ }^{1}$ Transfusion Medicine \& Blood Bank, AIIMS Raipur, C.G., Raipur, India

Aims \& Objectives: (1) To assess the trend of transfusion transmitted infections (TTIs) among blood donors from the records (January 2017-September 2020) at blood bank, AIIMS, Raipur, C.G. (2) To study the sero-prevalence of TTIs among blood donors from the records. (3) To study the prevalence of seroreactivity amongst voluntary and replacement blood donors.

Patients/Materials \& Methods: In India blood is screened for five diseases which could be transmitted through blood and produce serious illness- HIV, Hepatitis B, Hepatitis C, Syphilis, and Malaria. A record based retrospective study was conducted from January 2017September 2020. Data were collected from the records of blood bank which also included data of blood donation camps. Data regarding TTIs prevalence in total blood donors and in replacement and voluntary donors was conducted.

Results: Out of total 6624 blood donors, voluntary donors were 1351 $(20.39 \%)$ and replacement donors were $5325(80.38 \%)$. Out of all TTIs, prevalence of HBV (38 cases $(0.57 \%)$ ) was highest followed by
HCV \& Syphilis having 09 cases $(0.14 \%)$, HIV had 7 cases $(0.11 \%)$ and no cases of Malaria were reported. Prevalence of TTIs in voluntary donors was $0.59 \%$ (08 cases) and in replacement donors was $1.05 \%$ (56 cases). Total $06(0.89 \%)$ TTI cases in 2017, 14 cases $(1.38 \%)$ in 2018,19 cases $(0.71 \%)$ in 2019 \& 24 cases $(1.04 \%)$ in 2020. Total voluntary male donors were 1167 and total voluntary female donors were 184 .

Discussion \& Conclusion: From the above conducted retrospective study it was found that TTIs were more prevalent in replacement donors than voluntary donors. There was year wise raise in total TTI cases (blood donation also raised yearly). Prevalence of HBV was highest and no cases of Malaria were detected (Nil malaria cases were probably due to Antigen testing kit for malaria antigens \& strict donor counseling). The number of voluntary donors has risen from 2017 2020 , but there is male preponderance in both voluntary and replacement donors than females. Numbers of blood donations were comparatively reduced in 2020 due to COVID scenario leading to cancellation of most of the outdoor blood donation camps.

\section{Screening of Thalassemia Patients for Hyper- Coagulation Status Using the Viscoelastic Method}

Shamee Shastry $^{* 1}$, Prethika Sarathy ${ }^{2}$, Ganesh Mohan ${ }^{1}$

${ }^{1}$ IImmunohematology and Blood Transfusion, Kasturba Medical College, Manipal. manipal Academy of Higher Education, Manipal., Udupi, ${ }^{2}$ IImmunohematology and Blood Transfusion, KMC, Manipal, Manipal, India

Aims \& Objectives: To identify the risk of hypercoagulability in patients with $\beta$ - thalassemia using Thromboelastography.

Patients/Materials \& Methods: A prospective study was conducted on patients with transfusion-dependent $\beta$ - thalassemia major over 6 months in a tertiary care center. The detailed history and clinical status of the patient were collected from the case file. The laboratory data on the pre-transfusion hemoglobin level, the iron status, and data on other laboratory parameters were obtained from the laboratory information system. Following the informed consent, the TEG was performed and categorised them into patients showing features of hypercoagulable state and patients with normal coagulation status. The TEG values of the study group were compared with that of the age-matched normal control group. The risk factors were identified by comparing the groups.

Results: We studied 34 patients with thalassemia major with a mean age of 10.6 years (Range: 2-20 years) with a male to female ratio of 1.27. The mean age at diagnosis was 8 months and the mean years on transfusion for the study group are 10 years (SD; 5.83). The study group received an average of $152 \mathrm{ml} / \mathrm{kg} /$ year (59-278) of red blood cell transfusion. A total of 44 TEG tracings were analyzed. A hypercoagulable state was noted in 22 patients $(64 \%)$. The mean values of the TEG parameters of the study group such as ' $R$ ' value was shorter and alpha angle, MA, CI, Lysis 30 were significantly higher in comparison to that of the control group. No clinical thromboembolic events were observed during the study period.

Discussion \& Conclusion: Thalassemia is a hypercoagulable condition. The present pilot study shows that implementing TEG as one of the screening tools might help in identifying high-risk patients and in the prevention of thromboembolic events. 
Evaluating Three Methods for Enumeration of Residual WBCS in Single Donor Apheresis Platelets: A Pilot Study From Eastern India as a Part of Blood Components Quality Monitoring Process

\author{
Nasir N. Naikoo ${ }^{* 1}$, Deepak K. Mishra ${ }^{2}$, Sabita Basu ${ }^{1}$, Suvro S. Datta ${ }^{1}$ \\ ${ }^{1}$ Transfusion Medicine, ${ }^{2}$ Lab Haematology and Molecular \\ Genetics, Tata Medical Center, Kolkata, India
}

Aims \& Objectives: The need for quality control of leucoreduction of blood products has led to the development of various methods to count low levels of residual leucocytes (rWBCs) in blood components. Unfortunately, the concentration of rWBCs in leucoreduced blood components is below the level of accurate detection by standard hematology analyzers and presents a technical challenge. The objective of this study was to compare use of Nageotte hemocytometry, Flow cytometry and standard hematology analyzer for enumeration of rWBCs in single donor apheresis platelets (SDAP) as a part of quality control process.

Patients/Materials \& Methods: A prospective, observational study was conducted in a tertiary care oncology center during January' 20 to March'20. A total of 36 single donor platelets collected by apheresis from healthy blood donors were subjected to testing by Nageotte hemocytometer, flow cytometry and hematology analyzer for enumeration of rWBC. All tests were performed within $24 \mathrm{~h}$ of collection and according to manufacturers' recommended methods. Results of Nageotte hemocytometer was obtained by: $\mathrm{rWBC} / \mu \mathrm{l}=[$ Cells counted $\mathrm{X}$ Dilution/Gridded area volume $(50 \mu \mathrm{l})]$ and flow cytometry calculation was performed by: $\mathrm{rWBC} / \mu \mathrm{l}=[$ Total beads $\mathrm{X}$ Number of Leukocyte Events/Total Beads Acquired X Total Sample Volume].

Results: Mean age of donor was 32 years (Male: 33, Female:3) and mean volume of SDAP was $262 \mathrm{~mL}$. Number of rWBC detected by flow cytometer were between $0.1 \mathrm{WBC} / \mu 1$ and $8 \mathrm{WBCs} / \mu 1$ whereas that detected by Nageotte's chamber were between $0.3 \mathrm{WBC} / \mu \mathrm{l}$ and $6.2 \mathrm{WBCs} / \mu \mathrm{l}$. The range of $\mathrm{rWBC}$ detected by hematology analyzer was $10 \mathrm{WBC} / \mu \mathrm{l}$ to $270 \mathrm{WBCs} / \mu 1$. There was no correlation observed between the results obtained by hematology analyzer with any of the other two methods. The concordance correlation coefficient between three methods was measured by kappa analysis and found 0.7 between Nageotte hemocytometry and flow cytometry. The kappa value was zero between hematology analyzer and flow cytometry.

Discussion \& Conclusion: Flow cytometry followed by Nageotte hemocytometry are better methods compared to standard hematology analyzer for enumeration of rWBCs in SDAP. To manage the cost of flow cytometry a centralized quality monitoring system for leukoreduced blood components could be considered in India.

\section{Impact of Donor Variables on The Yield OF Granulocyte Aphersis By GCSF and Dexamethasone Mobilisation Regimen}

Ranjit Kumar C.S ${ }^{\mathbf{1}}$, Sangeetha Gayam ${ }^{* 2}$, Anil aribandi ${ }^{1}$, Ashok Kumar K ${ }^{1}$, Rajesh Mallik Gottipati ${ }^{3}$

${ }^{1}$ Department of Haemato-Oncology, ${ }^{2}$ Department of Transfusion Medicine, American Oncology Institute, Hyderabad, ${ }^{3}$ Department of Haemato-Oncology, American Oncology Institute, Vijayawada, India

Aims \& Objectives: Administration of GCSF and Dexamethasone to normal donors leads to higher preharvest neutrophil counts and consequently larger granulocyte yield of the product.

Patients/Materials \& Methods: This is a retrospective study with a study population of 160 healthy voluntary donors who donated granulocytes at our teritiary care oncology centre over a period of
2 years. It is an interventional study analysing the variables of the donor affecting the yield of granulocyte collection. All the donors were selected following the DGHS donor selection criteria. All the donors were given uniform dose of Inj GCSF ( $5 \mu \mathrm{gm} / \mathrm{kg}$ body wt)subcutaneously and Dexamethasone $8 \mathrm{mg}$ orally $12 \mathrm{~h}$ prior to collection. All the collections were done on COBE SPECTRA continuous flow apheresis platform. More than One total blood volume of the donor is processed. Data was collected using excel and analysed the final product yield with respect to donor variable and correlation was done by using SPSS software.

Results: The data was analysed and found the following results. In the study all donors were male $(100 \%)$ with mean age of donor was 31 years, mean volume of product collected was 393 ml,mean ANC of donor post mobilization and preapheresis was $31,000 / \mathrm{cmm}$, mean haemoglobin concentration was $15.2 \mathrm{gm} / \mathrm{dl}$. Apheresis product mean volume was $393 \mathrm{ml}$,product mean haemoglobin concentration was $3.2 \mathrm{gm} / \mathrm{dl}$, mean Total leukocyte count was $109.5 \times 103 . \mathrm{cmm}$ and mean ANC of the final product was $94 \times 103 / \mathrm{cmm}$ [.Pre harvest value (Mean) Product value(mean) Total WBC $32,800 / \mathrm{cmm} 109.5 \times 103 / \mathrm{cmm}$ ANC $31,000 / \mathrm{cmm} 94 \times 103 / \mathrm{cmm}$ [3.69x1010/unit] Hemoglobin 15.2gm/dl $3.2 \mathrm{gm} / \mathrm{dl}$ Correlation was done between preapheresis ANC and product yeild; preapheresis haemoglobin concentration of donor and product yield. It was found to have correlation between pre-apheresis $\mathrm{hb}$ of donor but is not significant. Preapheresis ANC and yield of product has strong and significant correlation.It is also observed that product hb is positively correlated with the yield of the product. Age of the donor is also significantly correlated to the yield of the product. Discussion \& Conclusion: Our retrospective study found donors who have better mobilization have a better yield of product ANC. It was also observed that the RBC contamination of the product is not affecting the yield of the product instead it shows a positive effect but the donor hemoglobin level does not impact the product yield. It can also be concluded that the age of the donor may also effect the yield of the product, Hence donor variables such as age, $\mathrm{Hb}$, ANC are the predicting factors to expect the better yield which in turn may effect the clinical outcome of the patient.

\section{Efficacy and Safety of Intermittent Flow Therapeutic Plasma Exchange from a Tertiary Care Blood Centre}

Anjali Ahlawat ${ }^{* 1}$, Manish Raturi ${ }^{2}$, Mansi Kala ${ }^{1}$, Anuradha Kusum ${ }^{1}$

${ }^{1}$ Pathology, ${ }^{2}$ Immunohematology and Blood Transfusion, Swami Rama Himalayan University, Dehradun, India

Aims \& Objectives: Therapeutic plasma exchange [TPE] aims at the removal of harmful substances from the plasma and has been used as a therapy to treat a variety of diseases. The present study was undertaken to study various aspects of the TPE facility at our tertiary care blood centre.

Patients/Materials \& Methods: A retrospective analysis of the patients who underwent TPE for various indications was undertaken at our department from Oct'17 through Sept'20. The relevant patients' demographics (age, body weight, gender), clinical history, laboratory investigations, TPE procedural notes [total time taken, blood volume processed, any adverse events (AE) noted, and the type and volume of the substitution fluids] was performed. All the indications were adjudged for compliance based on the American Society for Apheresis [ASFA] guidelines.

Results: Total 57 TPE procedures were performed in 16 patients [M: $\mathrm{F}=2.2: 1]$. The patients' mean age [years] and body weight $[\mathrm{kg}]$ were $38.6 \pm 14.8$ [10 to 74$]$ and $64.94 \pm 10.2$ [45 to 84$]$ respectively. The average pre-procedure hemoglobin $[\mathrm{g} / \mathrm{dL}]$ and serum calcium $[\mathrm{mg} /$ $\mathrm{dL}$ ] were $13.1 \pm 2.3[7.5$ to 15.4$]$ and $8.32 \pm 0.6[6.8$ to 9.8$]$ respectively. The average total blood volume processed $[\mathrm{mL}]$ was 
$6464.72 \pm 1462.9$ [2800 to 8870 ]. In substitution fluids use of crystalloids [0.9\% normal saline] to colloids [FFP and 5\% albumin] was 2:1. The average time taken for TPE [minutes] was $235 \pm 53.8$ [53.8 to 382]. Among the indications, maximum TPE procedures were done for Guillain-Barre syndrome [ $=41]$, peripheral neuropathy $[n=7]$, thrombotic thrombocytopenic purpura $[n=6]$, Reduction of donorspecific anti-HLA antibodies prior to hematopoietic stem cell transplant $[\mathrm{n}=2]$ and one case of quadriparesis with respiratory involvement. All the indications were $100 \%$ compliant with ASFA guidelines. The most common AE noticed was urticaria $(n=7)$, followed by severe anaphylaxis, including bronchospasm $[\mathrm{n}=1]$. Most AE were mild in nature and were managed appropriately. Total fifteen patients improved whereas, one died.

Discussion \& Conclusion: TPE has proved to be an efficacious procedure in $94 \%$ [ $n=15 / 16$ ] cases resulting in quicker recovery and better clinical outcomes. One patient of TTP did not show immediate improvement, but recovered after some delay.

Keywords: Therapeutic plasma exchange; Therapeutic Indications; ASFA guidelines; Adverse Events.

\section{Convalescent Plasma Donation: Overcoming the Challenges: TQM Approach}

Siddharth Sagar ${ }^{* 1}$, Minakshi Mishra ${ }^{1}$, Farah Rana ${ }^{1}$, Nilam Mehta ${ }^{1}$

${ }^{1}$ Pathology, Tata Mains Hospital, Jamshedpur, India

Aims \& Objectives: - Select appropriate donors

- Ensure availability of Convalescent Plasma for Covid19 positive patients admitted in our hospital with moderate symptoms

Patients/Materials \& Methods: -Total Quality Management Approach

All the data of positive recovered patient from COVID 19 were retrieved and analysed for potent donors. Donor selection criteria were as per guidelines laid by ICMR. All potent donors were contacted through telephonic conversation and were counselled for plasma donation.
Results: We have successfully conducted 143 plasma apheresis donation in our blood bank and have issued 104 units of convalescent plasma to patients admitted in CCU in our hospital till date as per categorisation on severity of symptoms.

Discussion \& Conclusion: It was a challenging job to council healthy donors for donation during peak time of this pandemic of COVID19. Initially there was $5 \%$ of donation in month of August which rose to $59.28 \%$ in month of September with changes in criteria and increased participation through creating awareness among the COVID 19 positive patients. $40 \%$ of patients on Plasma therapy in critical care turned RTPCR negative.

Publisher's Note Springer Nature remains neutral with regard to jurisdictional claims in published maps and institutional affiliations. 